
2.18 32.

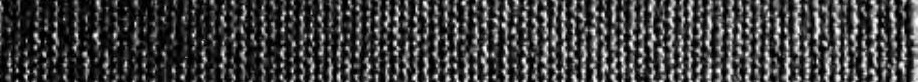

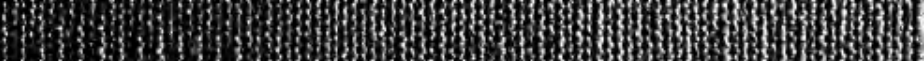

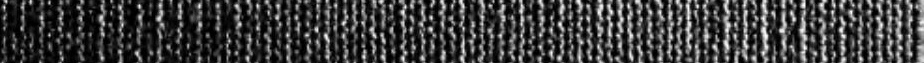

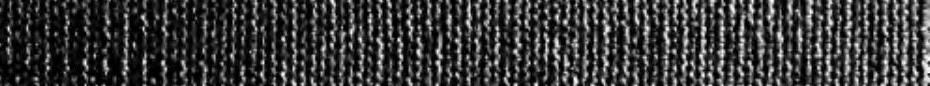

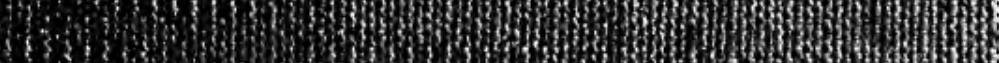

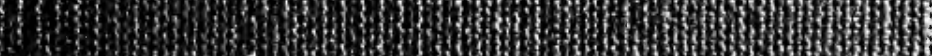

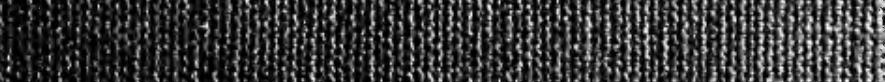

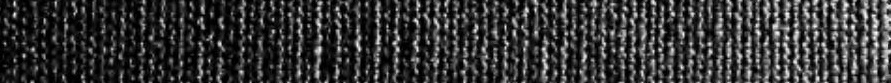

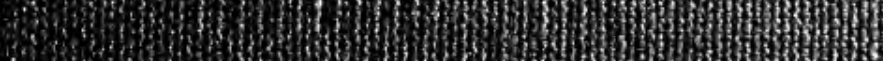
103 (10) B.6. 6ing

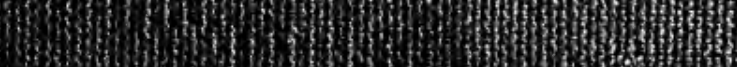
3.

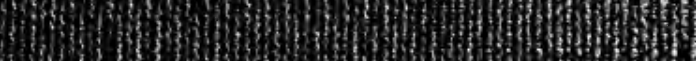
(1)

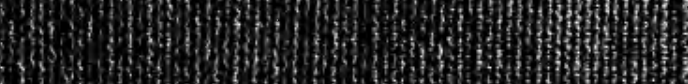
3.

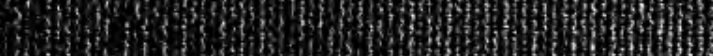

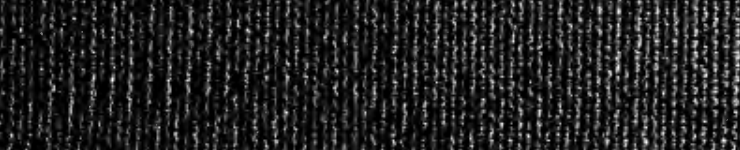
How

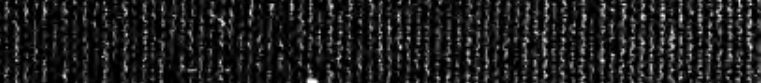

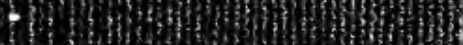
(t) intoly

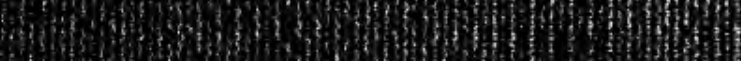
696 (6) Wo Whas

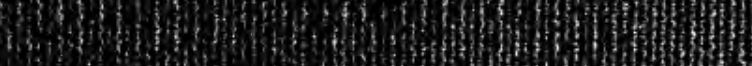
1. (1)

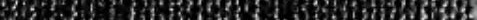




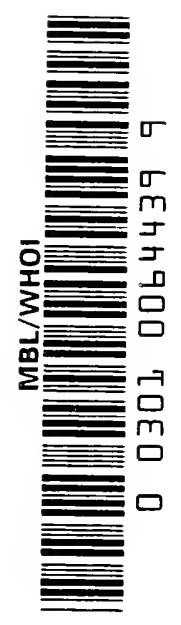


$\checkmark$ 




\section{Comparative ANIMAL PHYSIOLOGY}

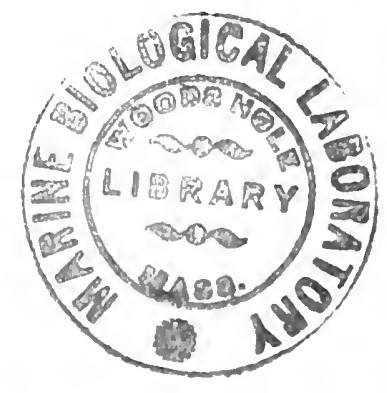





\section{Comparative}

\section{ANIMAL PHYSIOLOGY}

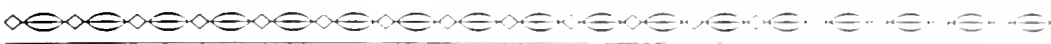

C. LADD PROSSER, Editor

$$
B_{y}
$$

DAVID W. BISHOP

Professor of Physiology, Lniversity of Mas-achurelt

FRANK A. BROWN, Jr.

Professor of Biology, Northwestern Lniversity

\section{THEODORE L. JAHN}

Professor of Zoology, University of California at Los Angelte

\section{LADD PROSSER}

Professor of Physiology, Lniversity of lllineis

\section{VERNER J. WULFF}

Assistant Professor of Physiology, Lniversity of Illinois

Illustrated

\section{W. B. SAUNDERS COMPANY}

Philadelphia $\cdot 1950 \cdot$ London 
COPYRIGHT, 1950, BY W. B. SAUNDERS COMPANY

COPYRIGHT UNDER THE INTERNATIONAL COPYRIGHT UNION

All rights reserved. This book is protected by copyright. No part of it may be duplicated or reproduced in any manner without written permission from the publisher. Made in the United States of America at the Press of W. B. Saunders Company, Philadelphia. 


\section{Preface}

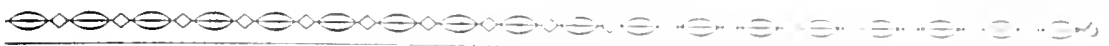

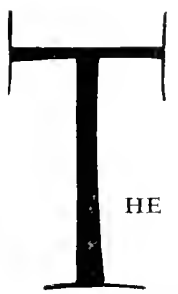

HE OBJECTIVES of comparative physiology are: $t$ w dexribe dhe diverse ways in which different hinels of animals macer sheir functional requirements; 2, to elucidate exolutionar felation ships of animals by comparing plasiological and bioxhemical characterustics. (3) to provide the physiological basis of ecology, describing the mechanisms of tolerance of the stresses of particular habitats and the functional adaptations underlying extension of the range of a population: $t$ to call altention to animal preparations particularly suitable for demonstrating specific functions. and (5) to lead to broad biological generalizations arising from the use of hind of animal as one experimental variable.

The present book attempts to serve wo purposes: 1. as a wathouk for use in courses in comparative physiology at the advanced undergraduate carly graduate level by students with some backgroumel in zoolog! and mammalian physiology; (2) as a source book of sufficient detail and bibliography adeguate to introduce investigators to particular branches of the subject. No antempe is made to cover in detail the ficlds of cellular and mammalian physiolog!, although a certain amount from each is utilized: experience tar ar the separation of courses in comparative, cellular and mammalian phrsiolesen. The index is arranged to provide zoologists with material on the phyioleges of particular animal groups, and this book might well give the phisiolegical material for courses in invertebrate zoology, protozoolog!s helmintholims, ento mology, ichthyology, herpetology, ormithology, and mammalog!.

The enormous literature of comparative physolog! cxists mainl! in jumrnals and monographs, and the only truly comprelensire account in recent lears is Buddenbrock's Grundriss der vergleichenden Phy siologie. The bibliography. of the present book, although extensive, is highly selective and therefore not comprehensive; it does not include many papers published since August 1949. No effort is made to cite the earliest reference for a giren fact. luut rather the recent important references are cited, and interested reatere can trace earlier literature from these.

Some animals are designated by two names and the same name in not always used throughout the book. This results from changes in momencta ture introduced by systematists who, for reasons of prionity or reclassification at the generic or specific levels, find it necessary to discard names which hase been used extensively in experimental papers. In this book, with a kw exceptions, animal names are given as used in the original experimental 
papers. I few examples of important synonymous genera are given with the older name in parentheses: Amoeba mira (Flabellula mira), Aurellia Aurelia, Neanthe's virens Nereis virens), Xiphosura (Limulus), Crago Crangon, (rangon (Aphacus), Lla (Celasimus), Ambystoma (Amblystomial.

This book was written to satisfy the need for a systematic organization of the diverse facts in the literature of comparative physiology and to emphasize and extend the comparative riewpoint in physiology as a, whole. Generalizations are drawn only when the existing data seem to warrant, but new gencralizations have emerged from the organized lacts. Every chapter includes numerous suggestions of unknowns, problems which need to be solved in the near luture.

The authors are grateful to their many colleagues who have contributed in discussions of the various topics. In particular they express their gratitude to the following, each of whom has critically read one or more chapters: E. I. Boell, I. B. Buch, I. H. Bullock, H. E. Carter, Peter Du Bruyn, Gottfried Fraenkel, E. \. I larvey, S. C. Kendeigh, Rudolph Kempton. George Kidder, Lewis Kleinholz, Peter Mlorrison, O. P. Pearson, Robert Ramsey, A. C. Redfreld, II. B. Steinbach, C. D. Turner, and Albert Tyler.

Undoubtedly many crrors of fact and interpretation still remain, and the authors hope that attention may be called to these by teachers, students and investigators who use this book.

The Authors

September, 1950

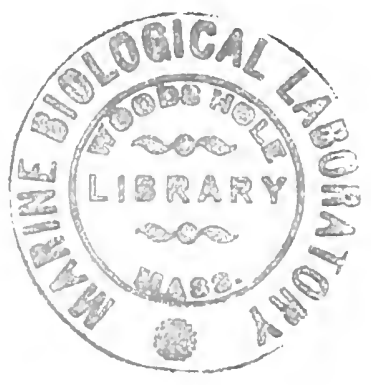




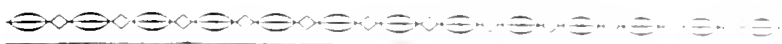

\section{Chapter I}

INTRODUCTION

C. LADD PROSSER

Chapter 2

IVATER

C. LADD PROSSER

\section{Chapter i}

INORGANIC IONS

C. LADD PROSSER

Chapter $t$

PROTE:X SPECIFICITY

C. LADD PROSSER

(hapter ;

NUTRITION

C. LADD PROSSER

Chapter 6

FEEDING AND DIGESTION

C. LADD PROSSER

\section{(Tapter T}

NITROGEN EXCRETION

C. LADD PROSSER

Chapter is

RESPIRATION AND METABOLISN DAVID W. BISHOP 
Chapter 9

RESPIRATORY FUNCTIONS OF BODY FLUIDS

C. LADD PROSSER

Chapter 10

TEMPERATURE: METABOLIC ASPECTS AND PERCEPTION 341 C. LADD PROSSER

Chapter 11

PHOTORECEPTION

THEODORE L. J AHN AND VERNER J. WULFF

Chapter 12

CHEMIORECEPTION

TIIEODORE L. JAHN AND VERNER J. WULFF

Chapter 13

PHONORECEP'TION

THEODORE L. JAHN AND VERNER J. WULFF

Clapter 14

MECH IANO- AND EQUILIBRIUIM-RECEPTION . . . . . . 502 I'ERNER J. WULFF AND C. LADD PROSSER

Chapter 15

CIRCLILATION OF BODY FLUIDS

C. I.ADD PROSSER

Chapter 16

MUSCLE AND ELECTRIC ORGANS . . . . . . . . . . . 576

C. I $\triangle$ DD PROSSER

Chapter 17

AMOEBOID MOVEMENT .

C. LADD PROSSER

Chapter 18

CILIA

IRANK A, BROWN, JR.

Chapter 19

TRICHOCYSTS ANI) NEMATOCYSTS 654

IJANK A. BROWN, JR. 


\section{Chapter 20)}

BIOLUMINESCENCE

FRANK A. BROWN, jR.

$$
\text { (hapter } 21
$$

CHROMATOPHORES AND COI_OR C I I INC IE 0,77 FRANK A. BROWN, JR.

\section{Chapter 22}

ENDOCRINE MECHIANISMIS

FRANK A. BROWN, JR.

\section{Chapter 23}

NERVOUS SYSTEMS

C. LADD PROSSER 



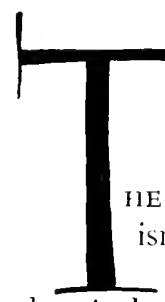

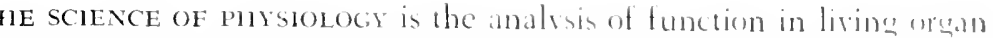
isms. One of the prerequisites for its study is a hnomkedere of morph ology. Physiology is a syntheric science which applice phrsical and chemical methods to biology:

The Fields of Physiology. For practical purposes, phrsiokegy can be divided into three categories, as follows.

Comparative Plysiology. Comparative phrviology treats of organ function in a wide range of groups of organisms. Comprative animal phrsiolese in tegrates and coordinates functional relutionships which tranecend special groups of animals. It is concerned with the ways in which divere oremism perform similar functions. Genetically dissimilar organisms mus show striking similarities in characteristics and response to the same en iromental stimulur. By the same tolien closely related animals frequently react very differenty we their surroundings. While other branches of physiolog! use such valtiallen dis light, temperature, oxyen tension, and hormone balance, compurative phy sint ogy uses, in addition, species or animal type ars a rariable for each function. The generalizations which comerge from this experimental approsts are $\mathrm{jm}$ portant as biological principles which of ten aid in solving problems of cellulur and special group physiology:

Physiology of Special Groups. The phrsiology of special groups of orean isms treats of functional characteristics in particular linds of plants and ani mals. Traditionally, the basic animal physiology is human and mammalion physiology, and this science provides the rational basis for much of medicine and animal husbandry. The physiolog! of higher plants is equally specialied and important as a basis for plant agriculure. Insect phrsiology is respidly becoming a special group physiology.

Cellutar Physiology. Cellular or general physiolog treats of those belsie characteristics common to most living organisms. I vast amount of biochemi cal evolution occurred in protoplasm before multicellular organisms appeared. and cells are exceedingly complicated in their functional organization. In an! cell-yeast, muscle fiber, or leaf parenchyma cell-the fundumental propertices

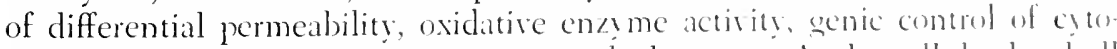
plasm, and many other properties are nuch the sume. At the cellular level all organisms have more in common than in difference, and this basic similarit? should form the starting point for erolutionary theory. Celluks spectalization has led to some diversity of cell types and has often brought with it the loss of one function with emphasis on another. In this sense one mas speath of a comparative physology of the cells in one organism. The characters trated in cellular phrisiology are nearly universal and are extremely stable with 
respect to the environment; these characters will not be discussed in this book.

The Organism and the Environment. Foremost among general principles which emerge from a study of comparative physiology is the functional adaptation of organisms to their environment. The distribution of a species is determined through natural selection by its limits of tolerance. Every species can live within certain limits of variation of each environmental factor. One environmental factor may limit the distribution of one group, and another may limit another group. Salinity of an aquatic habitat limits some animals, oxygen tension limits some, and temperature extremes limit others. Explanations of both restricted distribution and widely diversified distribution can be obtained by examining physiological reactions to environmental stress. The extent to which adaptation to a changed environment can occur and the mechanism by which such adaptation occurs provide a basis on which to account for existing distribution and to predict the possibilities of future migrations. Animals can respond to environmental stresses either by altering themselves (adjusting) to correspond with the environment, or by regulating themselves by means of protective mechanisms and thus maintaining a certain internal constancy. Each type of response permits survival and is, therefore, adaptive. For example, some aquatic animals follow osmotic changes in the medium by proportionate internal changes, whereas others maintain a constant internal osmolar concentration. Most animals are at the temperature of the external environment (adjustment), whereas a few show temperature regulation. When an environmental stress is removed an animal tends to return to its previous mean state; that is, recovery stops at a point of balance of body functions.

Different groups of animals solve a given problem of environmental stress by different means. For example, methods of obtaining oxygen (external respiration) and methods of oxygen transport show wide variation within single groups, as in insects. Conversely there are examples of physiological convergence. One respiratory pigment, hemoglobin, has arisen a number of times in totally unrelated groups. Animals never react to a complex environmental situation with a single organ system. It has often been stated that the organism as a whole is more than the sum of its parts, and out of the whole organism emerge characters not present in any of the parts. Animals react as integrated wholes, many organ systems combining in single responses.

Adaptive reactions to environmental stress may be of two types, physiological (within the individual) or genetic. Plysiological adaptations are those responses of an individual which occur within the genic limits of lability. The genetic limits for physiological response are much wider than is sometimes recognized. Modifying effects of the environment on morphological development are well known, as in the effects of oxygen tension on gill size in tadpoles, or of salinity on anal gills of mosquito larvac, but environmental modification is much better seen in physiological characters. When there are environmental changes in oxygen tension, salinity, or temperature, a variety of compensating or restorative reactions may occur, some even involving changes in enzymatic pattern. For example, lethal temperatures depend on acclimatization temperature; the nature of nitrogen excretion depends in some species on the amount of available water. It is one task of comparative physiology to learn the extent and kinds of lability permitted by a given genotype. 
The second or genetic type of adaptive reactions invelves selection of gente mutations. There is no evidence that crsvironmental stress pere se con induce genic change, but physiological selection of mututions dress aceur. 'I lume organisms which, by mutation, extend the limits of their physinlegical latilits widen the range of a species. Within a species there are mom genic differ ences, and it is the combined task of the comparatioc physinlogist, ecollogict, and geneticist to learn whether an extreme adaptive cappocity of certain measured individual animals represents a nomal latbilits for a given gene complex or represents a mutation. This can be learned only by suljectinn several generations to the environmental stress. Only those addiptatiom which have a genetic basis are used in evolution. Extensive crmbined genetic and physiological studies on animals found at the limits of their nomal range are needed. Many problems are implicit in such an approach, for example, the interplay of genetic potentialities with acclimatization capacity of organisms, the factors underlying change in habitat within groups as in mosements from water to land, and the basis for amual migration and hibernation of certain kinds of animals.

Ecological Aspects of Comparative Physiology. Ecology is closely reluted to plyylogeny; where an animal is able to live depends to a considerable cxtent on where its ancestors lived. Comparative physiology can help to answer such ecological questions as: Why do all echinoderms inhabit the eccems? Why are there practically no marine insects or amphibians? Why are some fish restricted to fast-flowing water? How can eels and salmon live in both ocean and fresh water?

There is no simple way to summarize all of the environmental factors influencing an organism, but we can distinguish four main halbitats: marine, fresh-water, terrestrial, and endoparasitic. Each of these can be extensively subdivided, and terrestrial habitats have been most completely classified hy zoogeographers and ecologists.

The comparative physiologist considers the organism in its cnrironment, played upon by a variety of environmental components-Water, inorganic ions, organic food, oxygen and carbon dioxide, light, high and low frequency mechanical stimuli, gravity, pressure, and temperature. W'c are concerned with the adaptive responses to these environmental factors. In adition, an animal is influenced in its environment by otler orginisms-plants and animals. A consideration of the biotic enviromment leads us beyond the realm of present-day physiology. Animal beharior will be eliscussed in this book only as it throws light upon sensory, central nervous, and loconotor mechanims.

The characters which compose the bullk of physiological adaptations in different environments are often unstable within an animal groups that is, they show great lability. These are the physiological characters which provide the basis for the ecological distribution of animals. Best eximples are found in respiration, nitrogen excretion, osmotic regulation, and circulatory and metabolic responses to temperature extremes.

Phylogenetic Aspects of Comparative Physiology. The physiology of any group of animals represents a point in the history of that group. The com parative animal physiologist needs, therefore, to know something of plyylogen! and taxonomy. The phylogenist uses the data of paleontology when they are available but also relies on taxonomy and comparative embryolog:. During 
recent years many physiological characters have been examined with respect to their crolutionary significance. Physiological and biochemical homologies and analogies can be established and are as striking as morphological ones. Evidences of embryonic retention, of convergence and divergence, of loss of function without return have also been presented for biochemical characters,

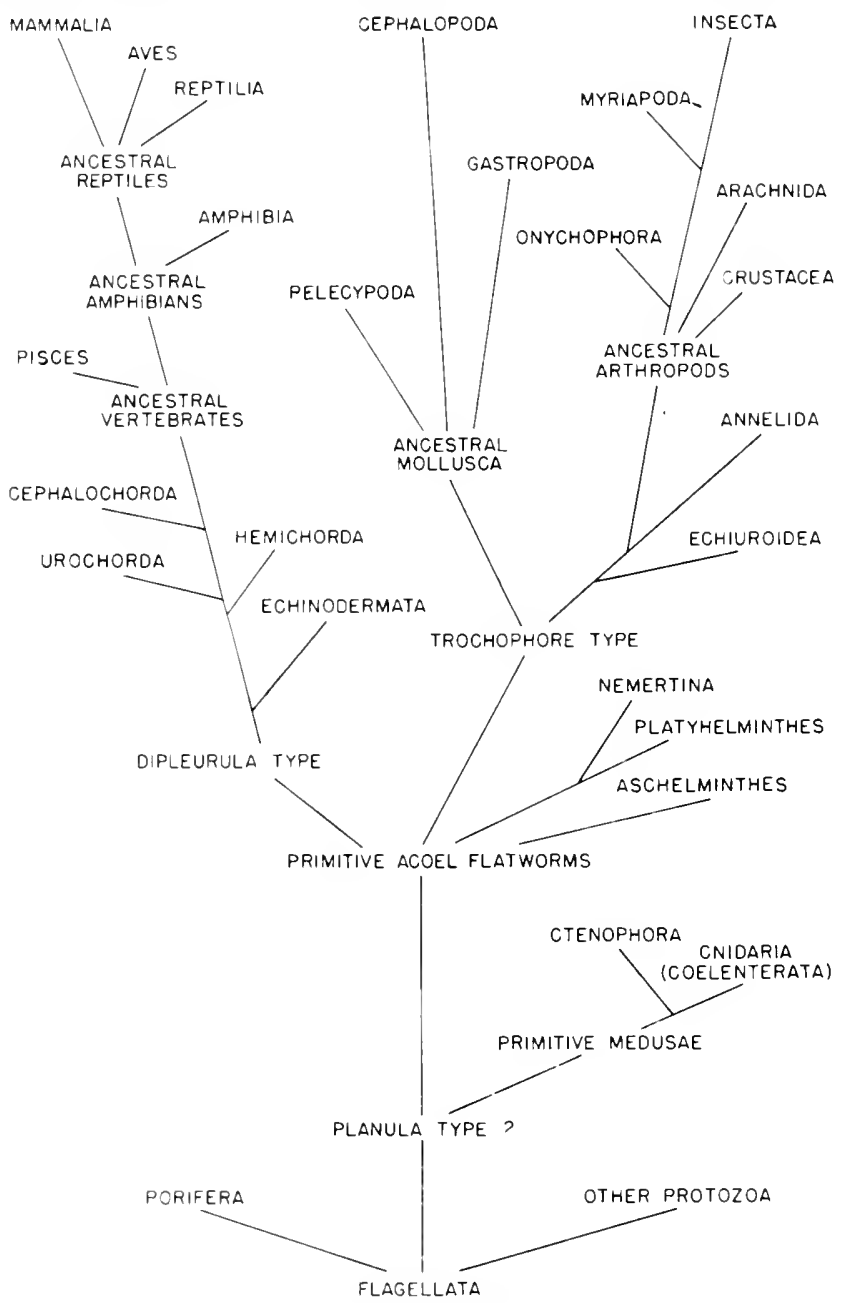

Fig. I. Phylogenetic tree modified from Hyman.'

particularly in embryos. It is important to learn how far functional evidence agrees with the traditional morphological evidence for animal kinships.

To provide a reference point for phylogenetic discussions a brief summary of present views regarding the history of the principal animal phyla is necessary. A "tree" of the animal kingdom is given in Figure 1. There is good agreement that at the level of the lower worms there occurred a divergence which resulted in two important parallel lines. On the one side there are the annelids, arthropods, and molluses; on the other side are the echinoderms 


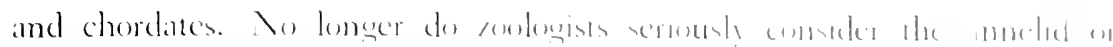

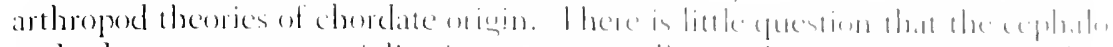

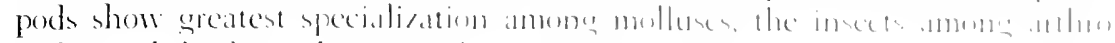

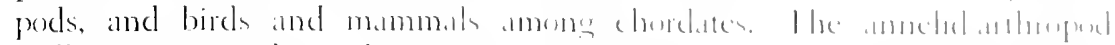

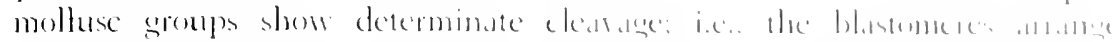

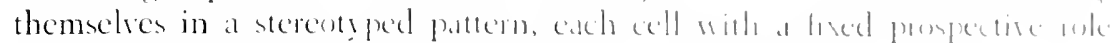

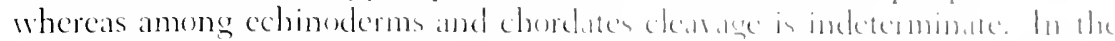

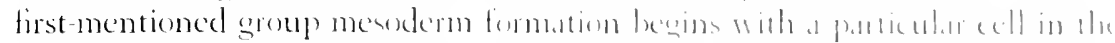

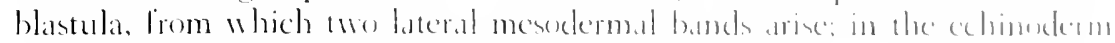

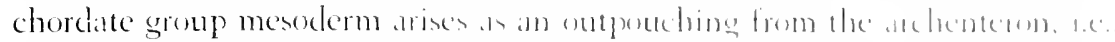

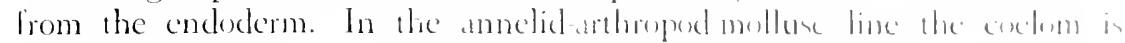

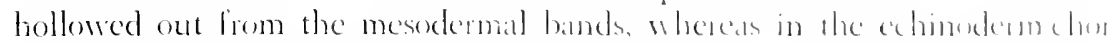
dates the coelom pushes out from the archenteron. In the annelid anthoper

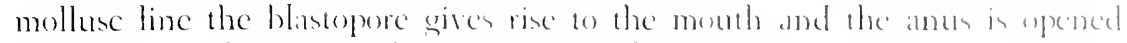

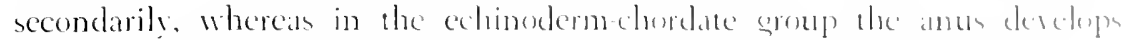
from or in the resion of the blastupore and the month opem secondarils.

Species may be considered as reprodactivel! isolated population which

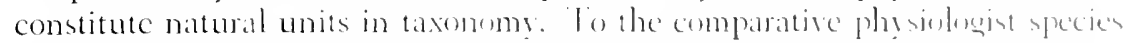
are not rigidly fixed populations; rather the individuals within a species stum

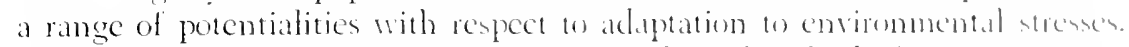
By ascertaining which adaptations are physological and which senetic it mats be possible to picture the evolution of small groups-the oriegin at bureties and subspecies. In its larger aspects comparative physubes supperts the well established lines of organic coolution. Centining the biluration of the phe lis genetic tree into two main portions and the specialisation of cephatepreds. insects, birds, and mammals as terminal groups are two evolutiomar! conelu sions which are amply supported by physological evidence.

The characters which are most useful in establishing phylogenetic relation are not as universal as cellular characters, but they are nidespread ammen many animal groups. Gord examples will be found in the fields of diencrion. visual pigments, nitrogen excretion, and in heart, muscle, and norve fihysiology.

Summary. Comparative physolog! is an integrating subject which provides the cridence for many biological generalizations. It is concemed par ticularly with functional adaptations to envirommental stresses and cmplosies kind of animal as one experimental variable. In combination with lickel and genetic studies, comparative phrsiology hels predictive value with respect to distribution and the crolution of species. There are many unknowns in this field, and each chapter in this book poses numerous unansivered questions.

\section{REFERENCE}

1. Hranan, L. H., The Invertebrates: Protozoa through Cienophura (1940, New York, McGraw-Hill, $726 \mathrm{pl}$. 


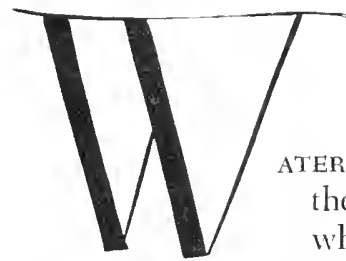

ATER IS AN ESSENTIAL constituent of all living things; it is the universal biological solvent, the diffuse phase in which most of the cellular reactions of metabolism occur, and the most necessary to life of all environmental constituents. Life undoubtedly began in a watery medium. Numerous exits from water to land have been made in the course of evolution, but only a few groups of animals have been successful in maintaining themselves out of water. Each group which has made the exit from water has used its own set of adaptations to life in air, some being more successful than others. Only the insects have made the exit complete, and some of them return to water for at least part of their life cycle. All other animals, including birds and mammals, return to a watery medium at least for embryonic life.

One problem of animal life is to maintain inside the organism just the proper amount of water-not too much, not too little. Terrestrial animals must retain and use what water is available; fresh-water animals must exclude water to prevent self-dilution; some marine and parasitic forms are in osmotic equilibrium with their medium, whereas others are more dilute and have the problem of taking in enough water while living in a plenitude of it.

Nearly all fresh-water and terrestrial plants, by virtue of their cellulose walls and active plasma membranes, maintain their cellular constituents, particularly their vacuolar sap, at concentrations higher than those of the fluids which bathe their tissues. The cells are continually more concentrated than the tissue fluids and hence turgid. In animals, however, cellulose walls are absent, and the effective intracellular concentration equals, or slightly exceeds, the concentration of body fluids. Regulation of osmotic concentration in animals takes place, then, not in single cells, as in plants, but in the organism as a whole.

Physical Factors. If the body fluids of an animal are more concentrated than the outside medium, the organism is said to be hypertonic; if they are more dilute the organism is hypotonic to the medium; if the concentrations inside and out are equal, the organism and the medium are isotonic. If a cell is hypertonic to the medium and is surrounded by a semipermeable membrane, that is, one which will pass water but not solute, water will tend to enter from the dilute medium. The pressure necessary to prevent such entrance of water is equal to the force by which water tends to enter and is termed osmotic pressure. Strictly semipermeable membranes rarely if ever exist in living organisms, otherwise there would be no exchange of solutes, but for the present discussion we can consider all animal cell membranes as essentially semipermeable. The osmotic pressure of a solution is proportional 
to the total number of particles of solute, irrespective of their hinel and sice.

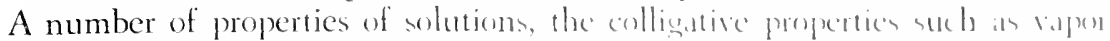

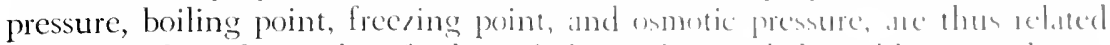
to the number of particles of solute. Dilute solutions behase like gersen; lacme the osmotic pressure $(\pi)$ equals the molal concentiation (' mulnplied hy the gas constant $(R=0.082$ liter atmosplicese, and the alsolute temperature $(\mathrm{T}),(\pi=\mathrm{CRT})$. The higher the concentration of solute, the ureater atre the

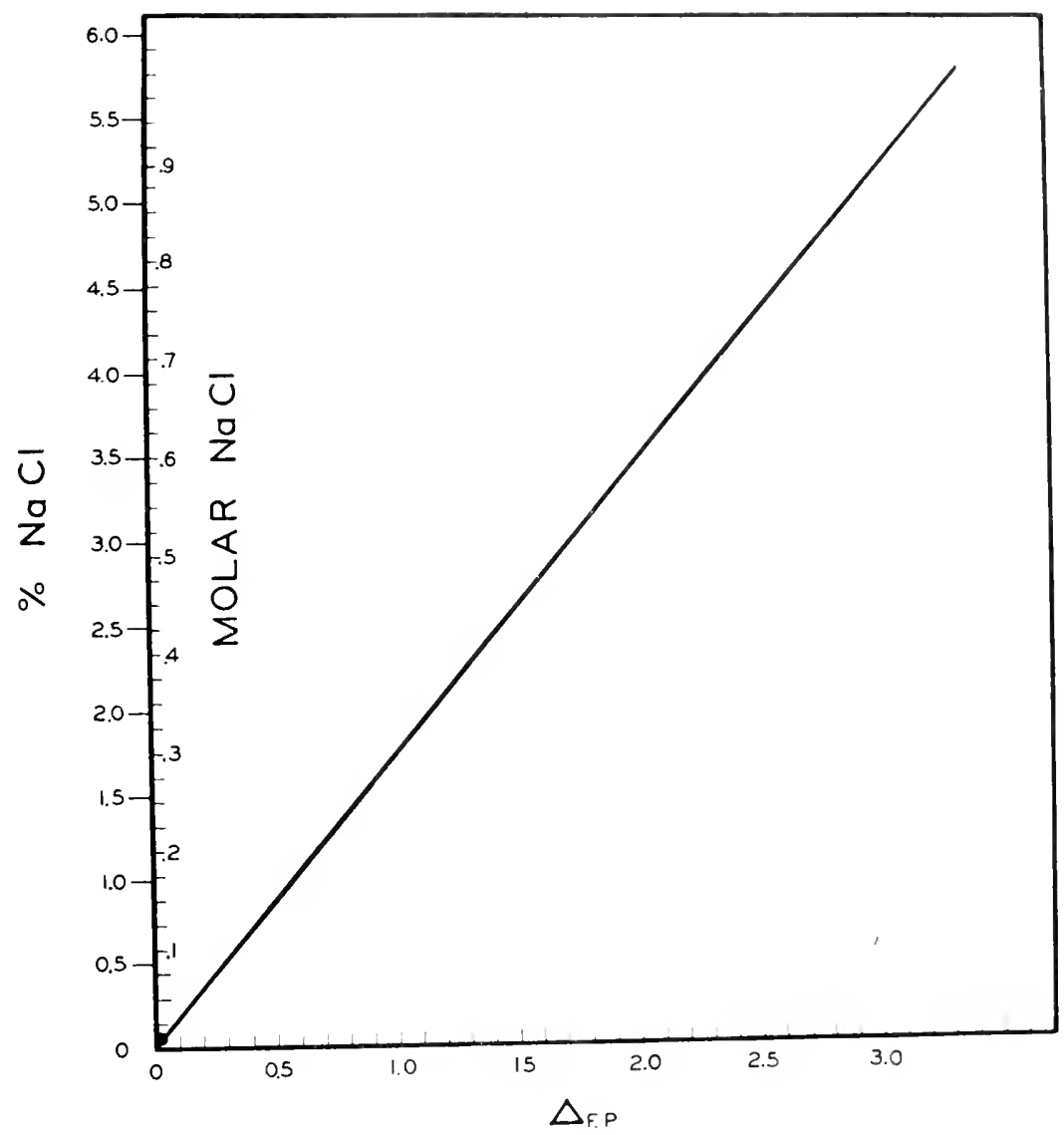

Fig. 2. The relation between lowering of the freesing point and concentration of $\mathrm{NaCl}$ in molarity and gm./100 ec.

osmotic pressure, the decrease in vapor pressure, the elevation of the boiling point, and the depression of the freesing point of the solution.

Any one of the colligative properties can be calculated from any of the others. In practice osmotic pressure of body fluids is not ordinarily measured directly, but usually either the depression of the freezing point Afr. or the vapor pressure is measured. $\$ molal anueous solution of nonclectrolyte freezes at $-1.86^{\circ} \mathrm{C}$. The symbol $\triangle$ indicates lowering of a freezing point; $\Sigma_{n}$ refers to the medium ( $=$ =outside), $\Delta_{i}$ refers to the blood $\left(i=\right.$ inside), $\Delta_{n}$ refers to urine. 
One common method of osmotic measurement based on vapor pressure is measurement of the size of a drop of the unknown solution in a capillary tube when on either side, separated by air, are drops of a known concentration (Barger's method, or modification by Krogh ${ }^{4: 3}$ ). If the unknown is a more concentrated fluid its drop will decrease in size; if it is less concentrated the drop will become larger; if the fluid is of the same concentration, or isotonic, the size of the drop will not change. Another method based on vapor pressure is to place a clrop of the unknown solution on one junction of a thermocouple and a drop of known concentration (usually sodium chloride solution) on the other (Baldes-Hill method ${ }^{17}$ ). Changes in temperature due to evaporation and condensation from one junction to the other may then be recorded.

For practical considerations, since salts dissociate into ions, each of which is as effective osmotically as an undissociated molecule, organic substances contribute only a small fraction to the osmotic concentration of most body fluids and practically none to most aqueous modia. The most easily determined and the most abundant biological anion is chloride. Figure 2 shows the relation between lowering of the freczing point and concentration of sodium chloride.

Patterns of Biological Response to Osmotic Conditions. The water content of the tissues of animals gives very little indication of their osmotic properties. In man, for example, the total water content is $63 \pm+$ per cent; the water content of the nonbony tissues is about 75-\$0 per cent, and of bone and fat 25-29 per cent, yet the osmoic pressures are fairly uniform throughout the body. A jellyfish may have a water content of 95 per cent or more and yet its osmotic concentration may be higher than that of a fist which is only 70 per cent water but which contains a greater proportion of organic material. Some adult insects contain as little as t6 per cent water and some larval insects as much as 92 per cent. Similarly the density or specific gravity of a solution is not a direct function of osmotic concentration, but depends on the nature of the solute, its concentration, the temperature, and the barometric pressure.

Some aquatic animals show osmotic lability, that is, the concentration of their body fluid changes when the medium changes; they adjust osmotically. Other animals are osmotically stable, that is, their hypertonicity or hypotonicity is maintained, irrespective of the environment; they regulate osmotically. The terms poikilosmotic and homoiosmotic are often applied to osmolabile and osmostable animals. There are, however, all gradations between the extreme conditions of flexibility and constancr. An animal may be homoiosmotic in one concentration range and poikilosmotic in another. Osmotic change may call into play gain or loss of water and thus be accompanied by rolume changes; if salt transfer also occurs, the body volume is liept constant when ever the concentration is changed with the modium.

Osmoregulation, or maintenance of relatively constant internal concentrat tion, implies volume regulation as well. Osmotic concentration may be liept constant by any of the following mechanisms:

1) Limited permeability to water

2) Limited permeability to salts (or other solutes)

3) Secretion (in or out) of salt against a gradient

t) Secretion (in or out) of water against a gradient

5) Storage of water or solute. 
Several of these mechanisms require energy, hence work is dome in mmonic regulation. The relative importance of each mechanion difter with the animal.

Animals tend to maintain an "optimum" ssmolic concenstration tor a given environment. Adolph ' has shown that in mans species, upm return to normal enviromment after a period of dehe dration, water is talen up and, alter

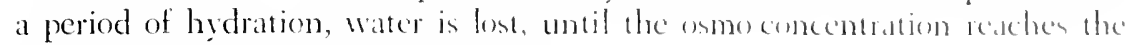
"optimum" lor the particular animal.

There are numerous variations in osmotic propertices of animak with seann,

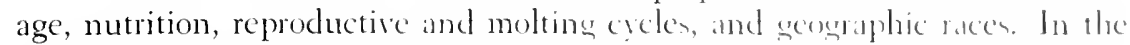
following account an attempt is made to select compurable material but it must be emphasized that measured values for a given species may difter with condi tions. There may be acclimatization of individuats to mometic condition amel there mav be genetic acclimatization which operates by selection.

$A$ careful summary of the mechanisms of unnotic respone in each phulum of animals which has been studied is given by lirogh. $14:$ Nie shall, aherefore. confine our discussion to a scarch for phylogenetic and ecological implications and applications of the subject. Ecologically, few factors in the envitumment so limit the distribution of animals as does the avalability of water. Plinge. genetically, so many separate exits have been made from the ocean we trent water, and from water to land, and so many separate geinvasions of waller hase been made that evolution with respect to water is a tree of mant trunks. Nature is carying out experiments in osmotic adaptation which we can observe at the present time, and species may be determined by the range ot osmotic lability of a population.

The ocean is the ancestral animal home. The composition of sals in seit water differs in different parts of the world and has changed considerabls during biological history. The total osmotically offective concentration of the ocean has increased since the earliest appearance of lite. The water of mid. ocean is concentrated, smaller seas and bass are diluted by intlow of fresh water, and in estuaries and tributary mouth brackish water mereses with fresh water. In seas such as the Nediterranean, where the evaporaten is high, the salinity exceeds that of the open ocean. Fresh witter dilutions of acean water are often expressed in per cont sea water. Is an arerage figure lor refierence we can consider sea water as equiralent wa 3.4 per cent sodium chloride solution, i.e. it has a salinity of 3.4 per cent of a chlorinits of 1.9 per cent. Sea water freezes at approximately -1.8 C and is, theretore, nearls molal in its osmotic concentration. Compare his with a fresh water pond which ma! show a freezing point dejecsion of 0.02 C. or less, or with salt hake where phytollagellates swim in water which freeres at -7.5 C.

All phyla and a majorits of the classes of animals have repreantation which have made the adjustments necendry for marime life: wme liase remained in the ancestril home, others hase returned to it. Animale lom fewer phyla have ventured into brachish water and only a lew hase suflicient osmoregulation for life in fresh water. Some animals hare invaded land directly from the ocem, whers through the anenues of esturine and fresh water. The parasitic habil has been assumed hy marine, fresh watere and soil dwelling groups. Evidence regarding the osmotic limitation to distribution of a group of animals can be obtained by observing the responses to osmotic stress. 


\section{OSMOTIC ADJUSTMENT TO THE MEDIUM; POIKILOSMOTIC ANIMALS}

The statement is sometimes made in textbooks that all marine invertebrates are isotonic with the ocean; this was found true for a great variety of species. ${ }^{46},{ }^{47}, 73$ Some crustaceans and a few annelids show deviation in osmoconcentrations, but most marine invertebrates are isotonic and adapt their

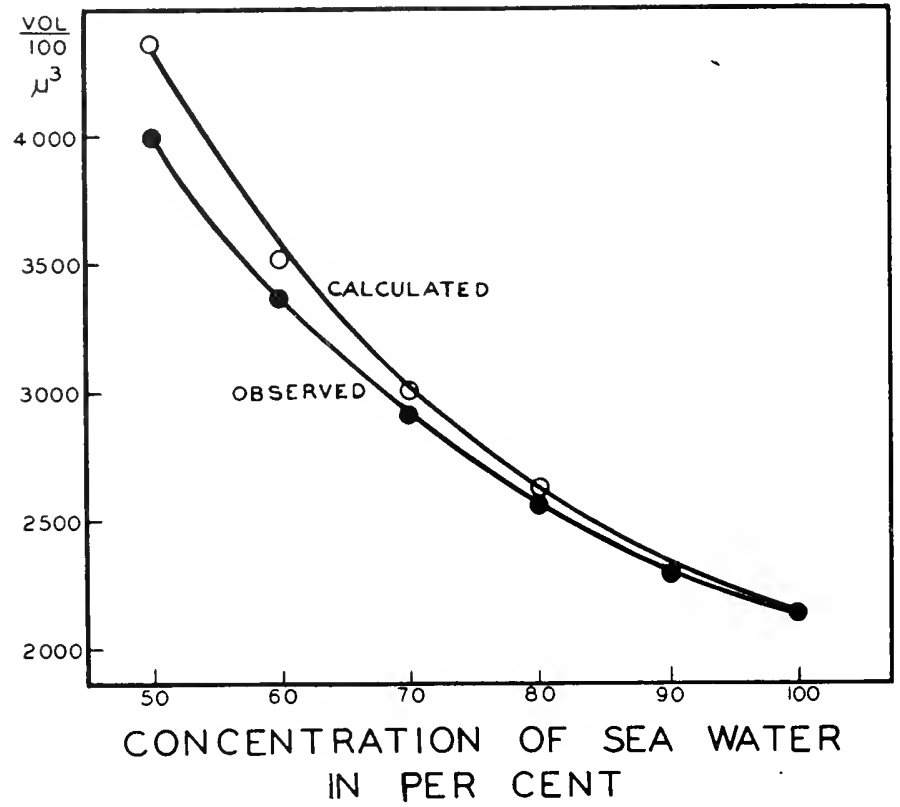

Fig. 3. Volume changes in cubic micra $\left(\times 10^{2}\right)$ in fertilized Arbacia eggs as a function of dilution of sea water as per cent normal sea water. - Lower curve, observed volumes; $\bigcirc$, upper curve, volumes calculated on basis of gas laws. From Lucké and McCutcheon. ${ }^{154}$

internal concentration to external changes. The limitation on the distribution of all osmolabile animals is probably the dilution at which their enzyme systems can still carry out sufficient metabolism for active life. The simplest and most economical osmotic adjustment is found in marine and parasitic organisms, which are highly permeable to water and gain or lose water according to the concentration of the medium. No osmotic work is done, and body volume is not regulated.

Osmotic Change without Volume Regulation: Unicellular Animals. Much evidence indicates that marine Protozoa are isotonic with sea water. The flagellate, Noctiluca, is in osmotic equilibrium with sea water but may have a lower specific gravity owing to the presence of considerable amounts of a salt of lower specifie gravity than sodium chloride (Goethard and Hensius, quoted by Krogh 14:3). This salt is probably ammonium chloride, which is ionic at the remarkably low pH of 3.0 in the cytoplasm. ${ }^{92}$ When Noctiluca is put into diluted sea water it swells and finally bursts at concentrations corresponding to a specific gravity between 1.007 and $1.012 .^{1+3}$ Thus the only adapta- 
tion tending to keep the cell isotonic is water exchange. II e shall consider the role of the contractile vacuole in marine Prowzoal later.

Several gregarines from the gut of mealworms swell and shrink wcording to the tonicity of the medium." "The gregarines have a lask content of glycogen, which may cause as much as 70 to 80 per cent of their volume to be osmotically inactive.

The organisms which have been most studied in respect to their ability to swell or shrink in changing tonicity are the eggs of marine invertebrates, particularly echinoderms and annelids. In general, when the volume of at marine invertebrate egg is measured in different dilutions and concentrations of sea water the cell is found to be a good osmometer, that is, it follow's Boyle's law over a narrow range: pressure $(P) \times$ volume $(V)=a$ constant $(K)$, $(\mathrm{PV}=\mathrm{K})$. In very dilute sea water it does not swell as much as would be expected if it followed the gas laws in proportionality of volume and pressure (Fig. 3). The explanation 154 of this deviation seems to be that a certain portion of the cell volume is osmotically inactive and thus does not take up water. Large protein and fat molecules, together with bound water, are osmotically insignificant, yet occupy a much greater relative volume than inorganic salts. When a correction is made for this osmotically inactive volume (b) and the corrected osmotically active volume (V - b) is plotted against external pressure, the gas laws as applied to dilute solutions are obeyed (Fig. 3 ). The osmotically inactive volume ${ }^{223}$ is 7.3 per cent of the initial cell volume in unfertilized Arbacia eggs, 27.4 per cent in fertilized eggs. ${ }^{223}$ Isolated nuclei of Arbacia eggs conform better to the gas laws, ${ }^{52}$ the osmo tically inactive volume is negligibly small.

A second explanation of the failure of cells to swell as much as predicted in dilute media might be leakage of salt., i.e., failure of the semipermeable nature of the membrane. Salt leakage apparently does not occur or is negligible over the range of rapid reversible changes, but it may be important in extreme dilutions, where injury occurs. Permeability to salt is very low in most developing aquatic embryos. ${ }^{185}$

In Protozoa which lack contractile vacuoles and in marine eggs, then, the rate of gain or loss of water is sufficiently great to provide a simple method of adjustment to changing external concentration.

Multicellular Animals. Osmotic adjustment by volume change is also shown by the marine sipunculid worm, Phascolosoma, whose body surface behaves like a semipermeable membrane. This worm is able to survive indefinitely in concentrated ( 160 per cent) sea water. ${ }^{6}$ Approximately 23 per cent of the body volume of Phascolosoma is osmotically inactive. The body weight decreases or increases rapidly on transfer to a medium of different tonicity and reaches equilibrium in 2 to 8 hours. On return to normal sea water after hydration or dehydration, the original volume is reached in a few hours. ' The rate at which water enters exceeds the rate at which it leaves. Apparently' there is little or no salt transfer because the volume of the animal remains high or low, according to the concentration of the medium, for several days. When salt solutions are injected, similar volume changes occur corresponding to the concentrations injected; some days later there is a tendency for body volume to return toward normal, indicating that there may be a very slow and delayed 
salt movement. LInder ordinary conditions, then, Phascolosoma behaves like an osmometer and show's no volume regulation. It is probable that Sipunculus behaves similarly. :":

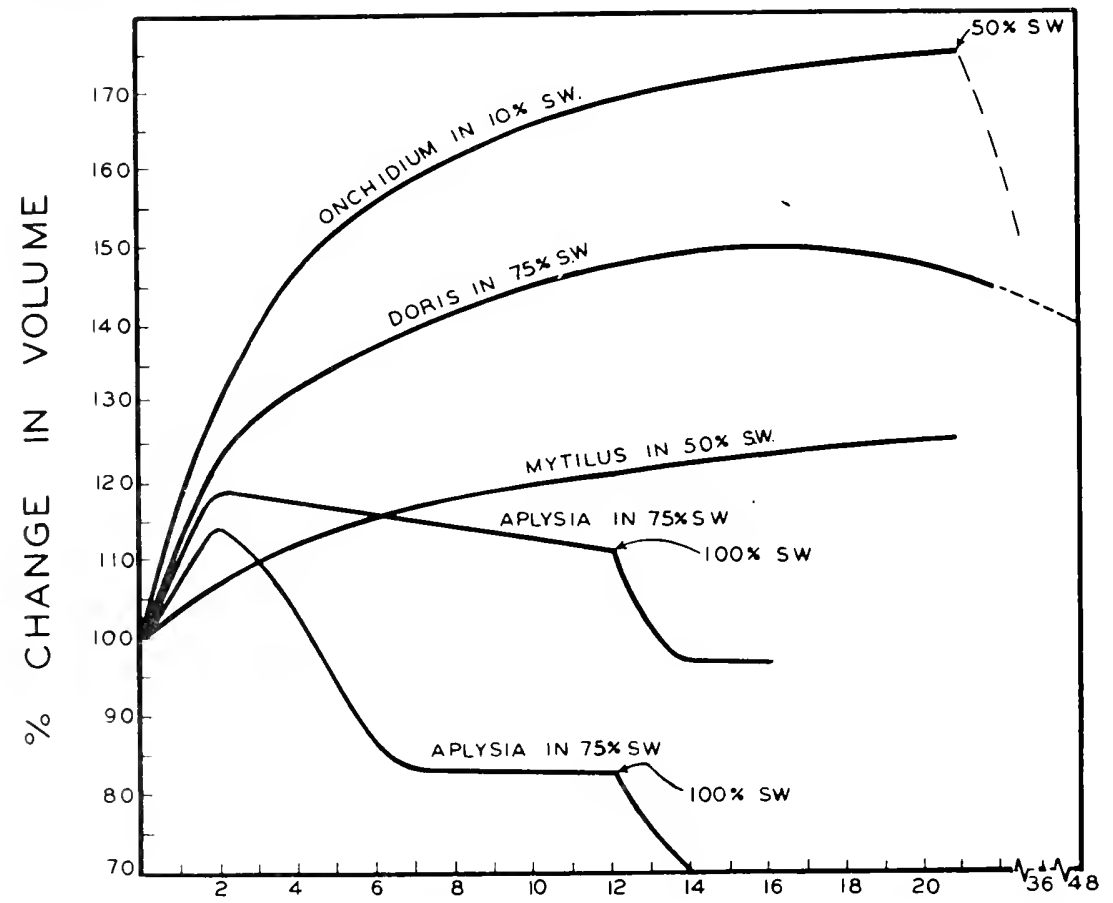

HOURS

Fig. 4. Percentage change in volume of four molluses when transferred at zero hours to the dilutions of sea water indicated. Onchidium changed from 10 per cent to 50 per cent sea water and Aplysia from 75 per cent to 100 per cent sea water at time indicated by arrows. Two paterns of response of Aplysia are shown. Onchidinm data from Dakin and Edmonds, Doris and Aplysia data from Bethe," Mytilus from Maloeuf.

The polychactes, Arenicola marina, Nereis pelagica, and Nereis cultrifera, show osmotic lability and some swelling in clilute media. The following data $21: 3$ (atso Fig. 6) show that Arenicola adjusts in osmoconcentration to its medium both in its natural habitat and in the laboratory:

$\begin{array}{ll}\triangle_{0} & \triangle_{1} \\ 0.29 & 0.28-0.30 \\ 0.75 & 0.76-0.77 \\ 1.72 & 1.70\end{array}$

\section{Habitat}

experimental

Kiel Canal

Helgoland

Thus Arenicola must be able to survive considerable dilution of its tissues. The sodium chloride content of the blood of both Sipunculus and Arenicola is nearly the same as that of sea water, and since both organisms are isotonic with the ocean the orginic solutes must be very dilute. "i:

Sereral molluscs also show little or no volume regulation (Fig. 4). The nudibranch Doris swells rapidly in a dilute medium, and remains swollen for 
at least 27 hours, although there may be a slight volume decrease it 48 hours (Fig. 4). Chloride concentration in the animal fell be 16 pere ent in the sume time. $"$ it Another gastropod, Onchidium, shows similar suclling. is In a mixture of one-third sea water and twrethirds isotennic sucrese the volume fell by 33 per cent in 14 hours; this shows that salt can be lust. I lonerer, (Mnchidi um regained its original weight when retumed to sea witer from dilute sea water, hence salt loss must have been negligihle. Doris and ()nchidinm show practically no volume regulation within a period of a day.

Mytilus edulis is a bivalse which cun live in a wide sulinits range. 'The hemolymph of Mytilus edulis adjusts ${ }^{21: 3}$ to the medium as follows:

$\begin{array}{ll}\Delta & \Delta 1 \\ 0.94 & 0.95 \\ 0.77 & 0.77 \\ 0.37 & 0.35\end{array}$

Osmotic adjustment occurred in Mytilus in sea water cliluted by 25 to 41 per cent, although the hemolymph did not become diluted proportionately when the sea water concentration was reduced by 70 per cent. "It If the values are open, Mytilus gains weight in a hypotonic medium and show's no tendency to return to its original solume during 50 hours Fig. t. . Hor llowerer, if the valves are closed the weight is unchanged: 140 in l'enus with values closed no dilution of the blood occurs during many dus in hypotonic sea water. "t The chloride concentration decreases in $1 /$, tilus in dilute sea water and increases in concentrated sea water ${ }^{i 1}$ along a curve smilar to that of the volume change. ${ }^{160}$ In 52 per cent sea water weight increased by 25 per cent; at the same time the total tissue chloride content decreased by $+t$ per cent and the total water content increased by 18 per cent. ${ }^{i 1}$ Krogh ${ }^{143}$ reported similar changes in water and chloride content in 76 per cent seal water, but in 30 per cent sea water he reported an increase of about 32 per cent in water content and a decrease of 71 per cent in chloride content of hemolymph. Unfortunately, data on weight, osmotic concentration, and chloride are not available for the same individuals, but evidently Mytilus adjusts to its medium osmotically, part of the change being by water uptake or loss and a larger part by chloride loss or uptake, the chloride loss or gain occurring at approximately the same rate as the water exchange, body volume being incompletely regulated.

The holothurian, Candina, swells in dilute sea water and shrinks when transferred from dilute to normal sea water. ${ }^{13:}$ The volume change in each transfer is less than half the value predicted if the body wall were semipermeable. Actually chloride is transferred in and out, but the rate of water exchange is greater than that of the gain or loss of chloride, and volume changes persist.

Osmotic Adjustment with Volume Regulation. Many marine intertebrates adjust osmotically to the concentration of the medium, but, in addition to gain or loss of water, they also gain or lose salts so that the body volume is kept relatively constant.

The blood concentration of those marine molluses which have been examined is equal osmotically to the concentration of their medium. Bethe $3.35,36.37$ studied weight and ionic changes of the gastropod, $A p l$ sia. On transfer to dilute sea water the weight rises at first (Fig. H), as water is taken up, and 
then falls as salt is lost and, with it, water. As much as 37 per cent of the body salt may be lost. After equilibrium is approached at a new low level, of ten below the original weight, return to 100 per cent sea water causes loss in weight because the normal sea water is now hypertonic. By ligating the mouth it is shown that the body surface of Aplysia is permeable to both water and salts.

The echinoderms are an entirely marine group and are adapted osmotically to the ocean. Indeed, in all except holothurians the ambulacral fluid is very similar to sea water. The body fluid of Asterias freezes at the same temperature as the sea water and becomes dilute when the starfish is put into dilute sea water. ${ }^{-1}$ In 50 per cent sea water ${ }^{160}$ starfish increased in weight by about 25 per cent in 8 hours, but returned nearly to the original weight in 24 hours. Starfish from the North Sea were unable to survive in 50 per cent sea water, but animals from the brackish Kiel Canal survived in greater dilutions. ${ }^{215}$ Echinoids have left the Baltic because of its low salinity. ${ }^{62}$ Echinoids and asteroids, because of their exoskeleton, are unable to swell as much as the soft-walled animals such as Aplysia, hence volume changes are limited and the animals must lose some salt to a hypotonic medium. ${ }^{3 \pi}$

Osmotic Adjustments by Cnidaria and by Ascidians. The osmotic concentration of jellyfish has been measured by both freezing point and by vapor pressure methods; jellyfish are composed of only 3 to 5 per cent solids ${ }^{117}$ and have the same or very nearly the same osmotic concentration as the ocean. ${ }^{19}$, 46, 47, 73, 74 After 36 hours in 75 per cent sea water the jelly disc of Cyanea differed in concentration from the medium by a negligible amount. ${ }^{19}$ Some corals and ctenophores tolerate a 20 per cent reduction in salinity. ${ }^{116,} 236$

The blood of an ascidian (Ascidia mentula) is approximately isotonic with the ocean, ${ }^{63}$ although the body fluid of Molgula is slightly hypotonic and its renal fluid hypertonic to sea water. ${ }^{5 s}$

In summary, some marine invertebrate eggs and some marine and parasitic Protozoa and worms such as Phascolosonia are true osmometers when allowance is made for osmotically inactive volume. They swell and shrink and show no salt exchange over periods of many hours; they show neither volume nor osmotic constancy. Numerous others, e.g., Nereis pelagica and N. cultrifera, Doris, Onchidinm, the holothurian Candina, and Mytilus, likewise remain swollen in dilute sea water. The amount of swelling is greater in some (Doris) than in others (Mytilus); when the swelling is considerably less than the amount predicted osmotically, salt may have been lost. Finally, many animals such as Aplysia, and to a less extent Asterias, swell initially in a dilute medium and then shrink as salt is lost; they regulate their volume while changing their concentration. The regulation of volume and that of osmotic concentration may, therclore, be separate functions.

\section{OSMO'IC REGULATION TO THE MEDIUM; HOMOIOSMOTIC ANIMALS}

Simple 'Types of Osmotic Regulation: Nereis diversicolor. Osmoregulation at a low level of effectiveness is shown by Nereis diversicolor, as contrasted to simple adjustment in dilute media shown by $N$. pelagica and $N$. cultrifera. Due to its regulatory ability $N$. diversicolor is able to live in brackish water (0.4 per cent silinity), whereas $N$. pelagica is restricted to the open sea. ${ }^{213}$ 
When transferred from 100 per eent to dilute scid witcer, the werglut of $\mathrm{N}$.

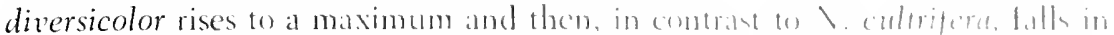

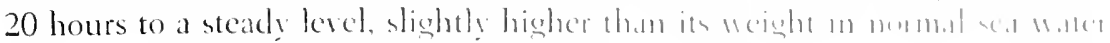

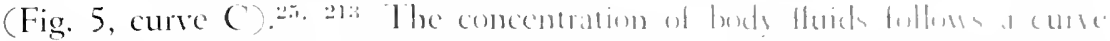

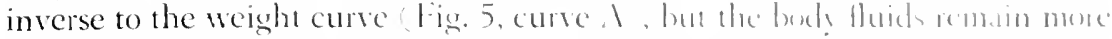

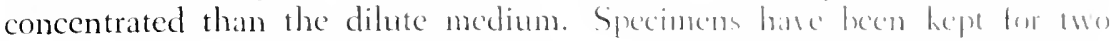

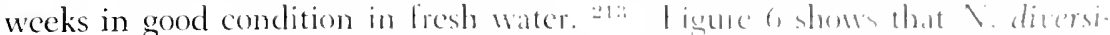
color, in contrast to $N$. pelagica, remains he pertonic in dilute se, water hut is unable to maintain the same concentration in a dilute medium that it hat in normal sea water. Its concentration curse steadity declines on the dilute sieke,

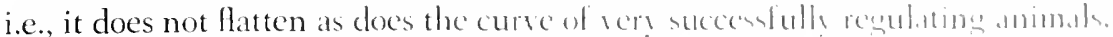

In order to carry out active esmoregulation, wonk must be done. Schleper

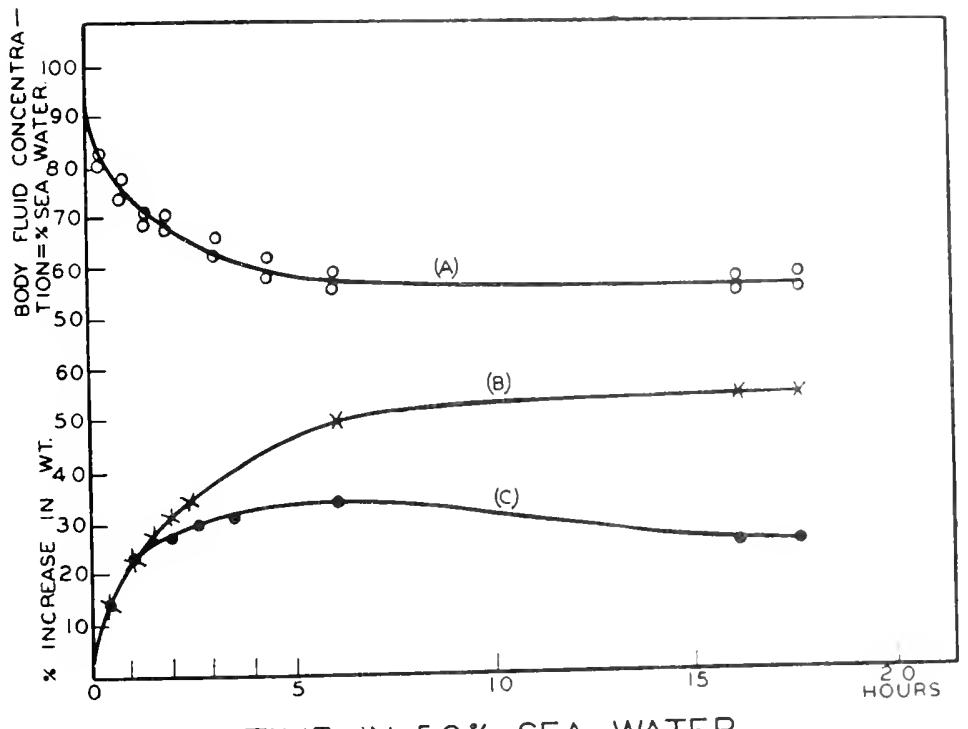

TIME IN 50\% SEA WATER

Fig. 5. Weight changes observed curve ( and calculuted at it there mere no salt loss or excretion (curve B); body flud concentration (cume $A$; in Nereis diversicolor in 50 per cent sea water from exro hours. From Beadle.

213 observed an increase of 81017 per cont in oxigen comsumption of $\backslash$. diversicolor in dilute sea water, but after an hour he oxy gen comsumption was reduced. Beadle 23.25 attributed the initial increase in respitation rate to

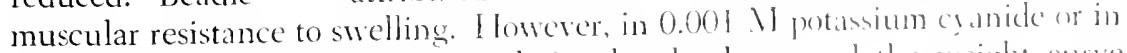
anaerobic conditions, osmotic regulation breats down and the weight curve approaches that of $N$. cultrifera.

Calcium is important in regulating salt intalic and loss. 20 per cent sea water the weight initially increases, llen recosers lig. T, curve B), whereas in 20 per cent sea water free of calcium the wom remains swollen (Fig. 7, curve A). Also a worm which has hecome aljusted wo nomal 20 per cent sea water gains weight rapidly when tramferred on sea 11 atcr of the same dilution but lacking calcium. No such water exalsomption cxcurs when the water lacks sodium, potassium, or magnesium. Mhen a menm 
adjusted to 20 per cent sea water was put back into 100 per cent sea water there was water loss and salt intake so that the worm soon had only 80 per cent of the weight it initially had in 100 per cent sea water. By repeated transfer from 100 per cent to 20 per cent sea water and back, the weight in 100 per cent sea water could be reduced to half. This indicates that water is transferred more rapidly than salt. The ratio of chloride output to water intake in 20 per cent sea water is nearly double the ratio of the exchanges in 100 per cent sea water, hence swelling is much less than in $N$. cultrifera, where the

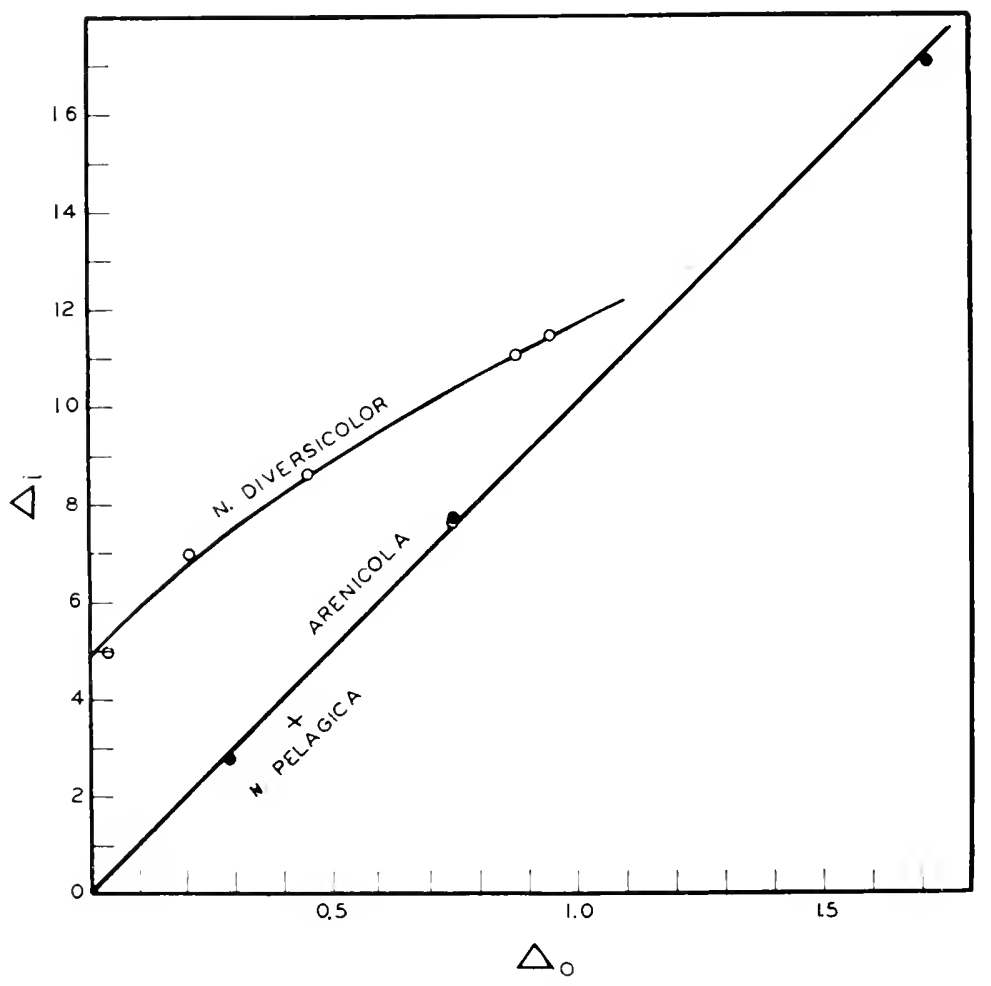

Fig. 6. Freezing point of body fluids, $\Delta_{1}: \bigcirc$, Nereis diversicolor, $\bullet$, Arenicola marina, and $t, N$. pelagicc; animals adapted to dilutions of sea water indicated by freezing points, $\triangle \ldots$ Data from Schlieper. ${ }^{213}$

chloride loss is very slow. Whether the avenue of chloride loss is through the nephridia or not is unknown.

Several mechanisms favor osmoregulation in $N$. diversicolor. The permeability of the skin is important in rolume regulation. Calcium decreases the skin permeability to water and thus prevents a continued rise in volume. Body wall contraction opposes swelling. After an initial increase the volume decreases as chlorick is lost and hypertonicity is maintained; this must, as Krogh points out, necessitate the elimination of hypotonic fluid, possibly by way of nephridia, and is an active process requiring energy. There may, in addition, be active salt absorption from the dilute medium. The mechanism of osmo- 


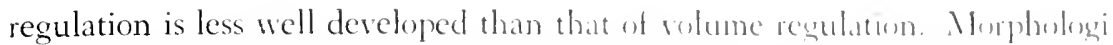
cal evidence for a mechanism of salt reabouption in the nephoidia in sum marized by Jürgens, ${ }^{12: 3}$ who shows that the nephrielial cand of $\backslash$. dinersi color is long and coiled, prosiding ample epithelium for reabur pum, whereas the nephridium of N. cultrifera is a simple sale Tin. $s$, .

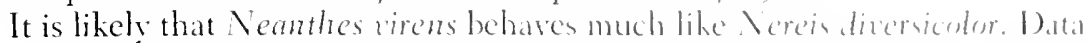
on blood concentration are not arailable, but studies hase shom that in transfer to 20 per cent sea water weight intially increased and then showly declined; when the worms were replaced in 100 per cent sea water the weight went below the original level. "\#11 When transferred through gradual stiges of salinity, Neanthes virens could erentually live for $1+$ days in 12.5 per cent sea water. A significant increase in oxygen consumption occurred in $N$ virens transferred to dilute solutions; ${ }^{23: 3}$ the weight remained constant with

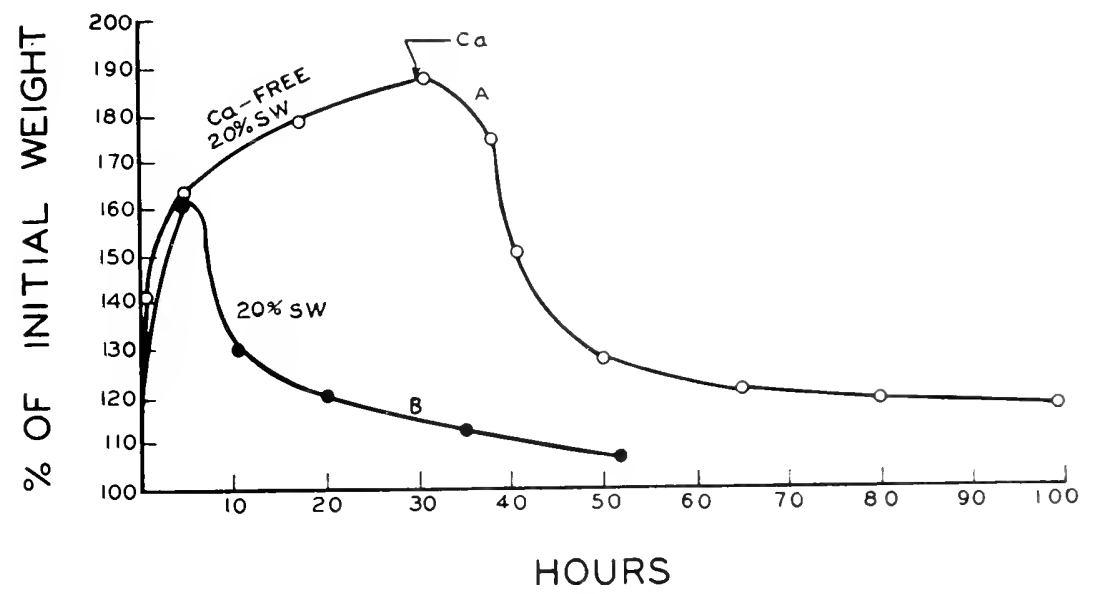

Fig. 7. Weight changes as per cent of initial weight in Nereis liversicolor when transferred from 100 per cent sea water to normal 20 per cent sea water (curve B; ; or to 20 per cent sea water lacking calcium (curve A), calcium added to 20 per cent sea water at arrow. From Ellis. ${ }^{8 \theta}$

dilution until the animals were in about 16 per cent sea water, when weight increased and oxygen consumption declined.

Among the marine worms there are sereral lesels of osmotic function. Phascolosoma, Sipunculus, and probably Nereis cultrifera adapt in body concentration by swelling and do not shrink during at least a day in dilute seal water. Nereis pelagica also changes in concentration but loses a little salt so that there is slight volume regulation. Vereis diversicolor and robably Neanthes virens show a limited regulation of hoth bod! concentration and volume.

Parasitic Worms. Parasitic worms are usually assumed to be at osmotic equilibrium with host tissues. Intestinal parasites may be subjected to considerable variations in medium from time to time. The coelomic Aluid of Ascaris megalocephala and the tissues of A. humbricoides are isotonic or slight ly hypotonic to the intestinal fluid in which the worms live. $=1$ Cestodes 
from mammals and from fresh-water and marine fishes are also isotonic with or slightly hypotonic to host tissues. However, the concentration of the trematode Fasciola hepatica was 40 per cent greater, as judged by freezing point, than that of the bile of the host. ${ }^{21}$ Ascaris in hypotonic solutions decreased rapidly in internal concentration; the worms swelled in dilute and shrank in hypertonic solutions. Volume changes were initially rapid, but the curves leveled at 6 to 10 hours and there was no tendency to return to original volumes in 18 hours. The body wall is, therefore, permeable to water. Maximum rolume changes were about 50 per cent; the osmotically inactive volume of the worm is probably large, but certainly the volume changes are less than if there were no regulation. Failure of recovery in hypotonic media indicates that there is little or no salt loss. It is likely that in a dilute medium the excretory organs are getting rid of water. The acanthocephalan, Neoechinorhynchus imydis, from the intestine of turtles is normally flat and wrinkled but it be-

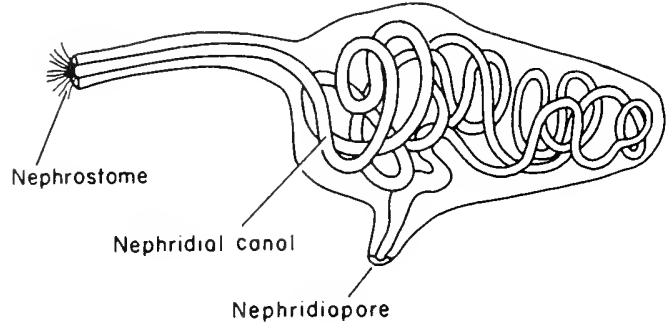

A

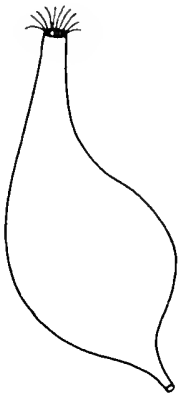

B

Fig. 8. Drawings of nephridia of Nereis diversicolor (A), and of N. cultrifera (B). Modified from Jürgens. ${ }^{123}$

comes swollen and turgid in salinities of less than 0.85 per cent sodium chloride. 235

In Cysticercus temicollis, a cestode larva from the abdominal cavity of sheep, volume changes are small (20 per cent swelling in a medium of onefourth the normal concentration), but as in Ascaris there is no tendency to return to the original weight during approximately a day in dilute or hypertonic media. "..2 Freczing point measurements show, however, that Cyst $i$ cercus has considerable osmoregulating ability, maintaining itself hypertonic in dilute media and hypotonic in concentrated media. Schistocephalus, a fish tapeworm, has an osmotic concentration similar to that of the host $\left(\triangle_{\mathrm{r} . \mathrm{p}}=\right.$ 0.44 ; the worms maintain constant weight in 0.75 per cent sodium chloride solution but gain in more dilute and lose in more concentrated solutions. 22x More accurate data are needed regarding the salt and water exchange and the osmotic concentration of various parasitic worms with and without excretory organs. It is probable that many degrees of regulation will be discovered among them.

Osmotic Regulation by Storage of Water: Gunda ulvae. The flatworm Cunda ulvere lives in estuaries at the intertidal zone in England and may be 


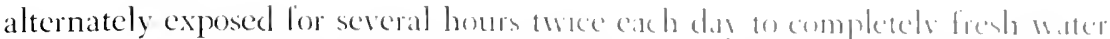

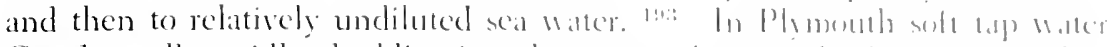
Gunda swells rapidly, doubling its volume in an hour, and it lete it dies within 48 hours. In dilute sea water or in strean water suctling excum but is much less than in tap water. Ihe calcium of the stresm water kners the permeshils ty of the body surface to water, and this actards swelling. Adelition of such substances as sodium chloride. sodium bicarbondte, or glycerol, to distilled or tap water does not retard the swelling. "at In dilute solutions non only is water taken up but salts alle lost. The worms lose 25 per cont w their londs salts while imbibing water equiralent to their initial whlume. 1:53 ln fresh water lacking calcium, as in clistilled water, salt lous is rapicl and general cytolysis occurs.

When Gunda is in dilute sea water. Water pusses onmotically through the cetoderm to the parenehyma, which swells during the tirst hour." then the water colleets in vacuoles in endodermal cells lining the sut, and the body volume remains relatively eonstant. Thus other body cells are kept from becoming diluted and normal activities of the worm can continue. I Jere, then, is osmotic regulation by storage of water in endodemal vacuoles, but bedy volume remains high. The vacuoles remain as long as the worm is in a dilute medium. Why they do not continue to grow berond a certain size is not entircly clear. Beadle cut worms behind the pharynx and found that the ectoderm closed over the wound, thus preventing any opening to the exterior from the gut. Such posterior ends swelled and reached equilibrium size in almost the same manner as normal worms in water containing calcium. Thus the water must normally enter through the ectoderm and cannot leave by way of the gut. Oxigen consumption is greater when the woms have reached equilibrium in dilute sea water. $"$ Neso, in anacrobic conditions or in the presence of cyanide, swelling is greater than in normal acrobic conditions. Beadle thinks that the active process which oecurs on adjustment at a given volume is due to energy expended in decreasing permeability of the ectoderm, although, as Krogh points out, Beadle has not prowed that the excretory organs are not used. Also energy must be expended by the gut cells to keep the vacuoles dilute. In any case. Gunda swells in fresh water; the excess water is taken up by vacuoles in the gut cells, and then an active process, either decreased permeability or increased excretion, takes orer to prevent further volume increase, and even allow's some decrease in size.

Osmotic Regulation by Exclusion of Water. The eggs of marine inverte brates swell and shrink to correspond with changes in the medium. The egges of marine teleosts are hypotonic to sea water, whereas all fresh-water eggs are hypertonic to their medium. Osmotic relations of embryos have been discussed by Needham, ${ }^{1 \times 6,}$ ist and by Krogh. ${ }^{1+3}$

The egg of the killifish, Fundulus, is nomally more dilute than its marine medium. ${ }^{152}$ These eggs are hypertonic in dilute and hypotonic in concen. trated media, isotonicity occurring at about $\Delta_{r, p}=0.76$. Such small changes as occur in different tonicities are attributed to adsorption by the outer membranes, ${ }^{152}$ and permeability to water is ver! low. Eggs of the plaice show limited permeability to both water and chloride. ${ }^{1+1}$ Krogh found that the extra-embryonic fluid of Nerophis was in equilibrium with sea water ( 412 $\mathrm{mM})$, whereas the embryo was much more dilute $(180 \mathrm{mM})$. 
The eggs of fresh-water fish take up a little water during the first 1 to 6 hours after oviposition, but thereafter they are impermeable to water. ${ }^{1+6.16 ; 6 ;}$ Oxygen can penetrate eggs which are impermeable to water and salts. The barrier which keeps water from entering the embryo is the vitelline membrane, the shell or chorionic membrane being freely permeable. ${ }^{\$ 9 .}{ }^{230}$ Impermeability of the vitelline membrane is maintained unless the membrane is injured or calcium removed from the medium. "s!

Eggs of Daphnia (data of Przylecki, quoted by Krogh ${ }^{1+3}$ ) and of Limnea stagnalis $^{207}$ are permeable to water and swell considerably ( 45 per cent, Limnea) during the first few hours after oviposition. Similarly frog eggs are initially permeable to water and then much less permeable. The osmotic concentration falls from about $120 \mathrm{mM}$ at the time of laying to $80 \mathrm{mM}$ by the time the blastopore closes, because of water uptake. ${ }^{1+\bar{\pi}}$ In the tail-bud stage osmotic concentration again increases, owing primarily to accumulation of organic products. After hatching, the embryos can grow for a time by water uptake; chloride absorption begins as soon as external gills are present.

The exceedingly low or negligible permeability of the vitelline membrane of many eggs protects the embryos against osmotic stresses until normal osmoregulatory mechanisms can function.

\section{AN INVASION OF FRESH WATER, LAND, AND SALT LAKES: CRUSTACEA}

Crustacea occur in water of a wide range of salinities, from the most dilute fresh-water ponds, through all degrees of brackishness, to the ocean, which is a 3.5 per cent salt solution, and even in salt lakes of some 22 per cent salt concentration. Crustacea are of marine origin and have invaded fresh water at numerous times and places. In fact, some forms seem to be on their way from ocean to fresh water at present. By comparing Crustacea of different osmotic capacities it is possible to picture the changes which have made possible their distribution and to predict which groups are likely to venture into new conditions of salinity.

The Crustacea can be arranged in a series with respect to osmotic capacities. The following seven groups are distinguished by their habitats and the responses of their blood concentration to changes in the concentration of the medium (Figs. 10, 12, 13, 14):

1. Animals limited to sea water, sometimes called stenohaline; ${ }^{*}$ they show no osmoregulation when put into dilute sca water, but they do show volume regulation. Example: Maja.

2. Crabs which regulate their osmotic concentration to a limited degree and are therefore able to venture into slightly brackish water. Examples: Cancer and Eriphia.

3. Animals which regulate better in dilute sea water and are common in regions of the shore near where fresh-water streams empty (euryhaline*). Their body fluids are adjusted to the medium in high concentrations and become regulated by remaining hypertonic in low concentrations. Examples: Carcinus and Ilemigrapsus.

* The terms stenohaline and curyhaline refer, respectively, to animals tolerating only slight changes in salinity and to animals tolerating considerable dilution. Since all degrees of intergradition eccur, the terms are of little practical value. 
4. Highly regulating crabs which not only maintain al stute of hepertonicity in dilute sea water but also regulate in a medium mere concentrated than normal sea water by keeping their blood more dilute than the medium. Il hey may live in very dilute brackish water and mony ene ento land. Such regula

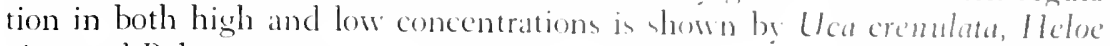
cins, and Palaemonetes.

5. Animals with ability to maintain hypotonicity in concentrated and hyper tonicity in dilute media to such an extent that they can live indefinitely in either the ocean or pure fresh water. For example. Lrincheir breeds in the ocean but migrates up European rivers where it grosts to maturits

6. Strictly fresh-water crustaccans which do not return wh the oxean. Examples: crayfishes and fresh-water microcrustacea.

7. Animals adapted to salinities greater than that of the ocean. Example: the brine shrimp, Artemia.

No Osmoregulation: Marine Crabs. Maje is a marine spider crab which survives only a few hours in sea water diluted br more than about 20 per

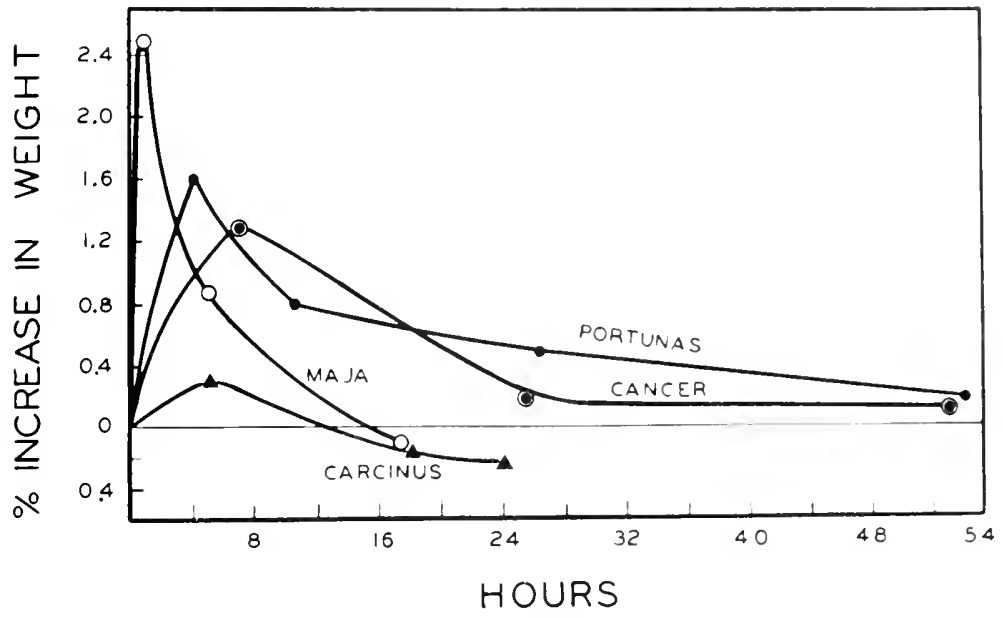

Fig. 9. Percentage increase in weight in crabs transferred to dilute sea water at zero hour. O, Maja in 75 per cent sea water, $\boldsymbol{\Delta}$. Carcinus in 75 per cent sea water (Schwabe - Portunas in 66 per cent sea waler: C. Cancer in 66 per cent sea water (Hukada $\left.{ }^{11 \overline{3}}\right)$.

cent. "2:2 Maja araneus, Maja bufo, and H, tha arameus are usuatly taken at an ocean depth of 15 to 100 fathoms. 2065

Maja placed in diluted ( 80 per cent, sea water first swelled but within 3 hours the weight decreased, owing to loss of salt and accompanying water (Fig. 9). 22 By the time the weight was back to momal the concentration of the blood was the same as that of the medium (Fig. 12. The rate of water intake was initially greater than the rate of salt loss. Conversely, When Maja was put into a more concentrated medium, salt was taken up and the body fluids again became adjusted to the external concentration (Fig. 11). Maja rapidly comes into isotonic equilibrium with a dilute medium (Fig. 10, curves $\mathrm{C}$ and $\mathrm{D}$ ). 
Even at equilibrium Maja forms 2 to $2.5 \mathrm{ml}$. of urine per hour, or the equivalent of 3 per cent of its body weight in 24 hours. ${ }^{39}$ In dilute sea water Maja becomes sluggish and its metabolism actually falls as its blood becomes dilute. When the blood is isotonic with the medium the water excreted cannot have entered osmotically and it is likely that water is actively absorbed and cxcreted in the process of ionic regulation. 215

Numerous other marine crabs behave similarly-Palinurus vulgaris (Fig. 12), ${ }^{6: 2}$ Portunas puber, ${ }^{11 . \overline{1}}$ 16: Portmas depurator (Fig. 10, curve E; Fig. 12), Ifix Cancer antennarius, Lophopanopeus heathii, Speocarcinus californiensis, ${ }^{122}$ Hyas aranea (Fig. 12), ${ }^{1 * t}$ and Pagurus longicarpus. ${ }^{160}$ When Hyas aranea is put into a mixture of sea water and isotonic sugar, ${ }^{184}$ there is a brief rapid weight loss and then a slow rise in weight; thus salt is lost more rapidly than sugar can enter.

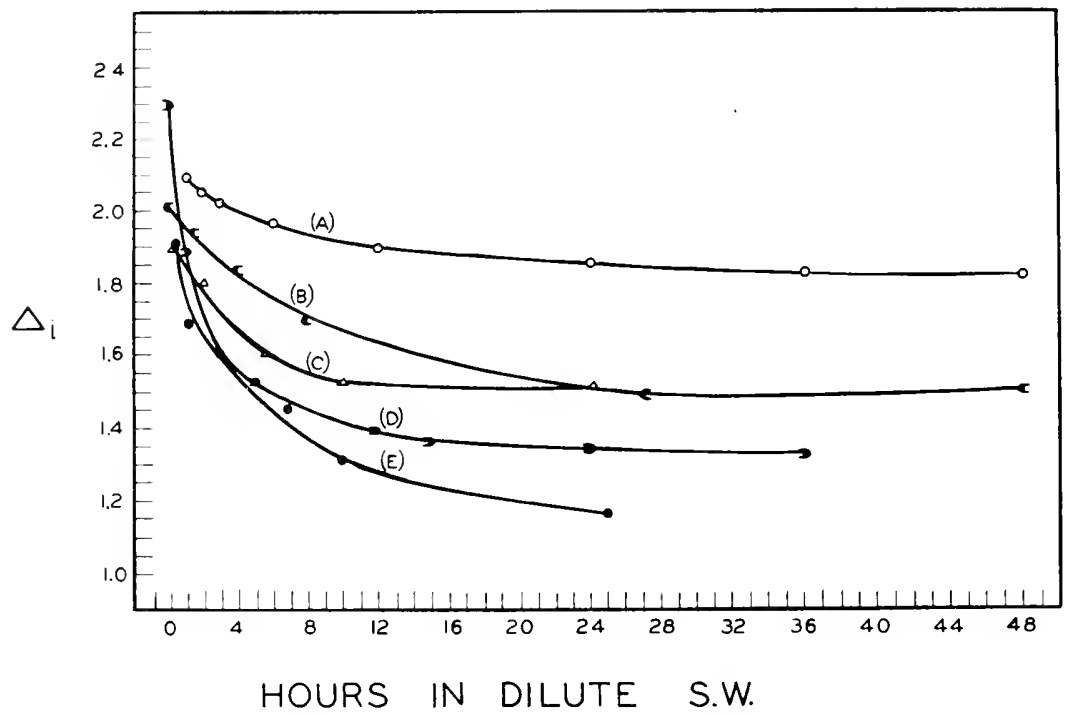

Fig. 10. Blood concentrations as lreezing points $\left(\triangle_{1}\right)$ of several marine crustacea after transferring to dilute sea water at time 0 . (A) Carcinus from $\triangle_{0}=2.33$ to $\triangle_{0}=1.33$. (B) Carcinus from $\triangle_{n}=2.2$ to $\triangle_{n}=1.07$. (C) Maja from $\Delta_{0}=2.2$ to $\triangle_{0}=1.54$. (D) Maja from $\triangle_{n}=2.33$ to $\triangle_{n}=1.33$. (E) Portunas from $\triangle_{0}=2.1$ to $\triangle_{0}=1.07$. Data for curves (A) and (D) from Schwabe; ;2: $^{2:}$ data for curves (B), (C), and (E) from Margaria. ${ }^{16 n}$

An interesting variant of an osmolabile crustacean is Lemaeocera branchialis, a copepod parasitic on codfish. The fish blood is hypotonic (equivalent to $1.4+3$ per cent sodium chloride), and while the copepod is attached to its host it also is hypotonic to the sea water (equivalent to 2.0 to 2.8 per cent sodium (hloride), but when separated from the host it becomes isotonic with the sea water ( 3.5 per cent saline). ${ }^{192}$ 


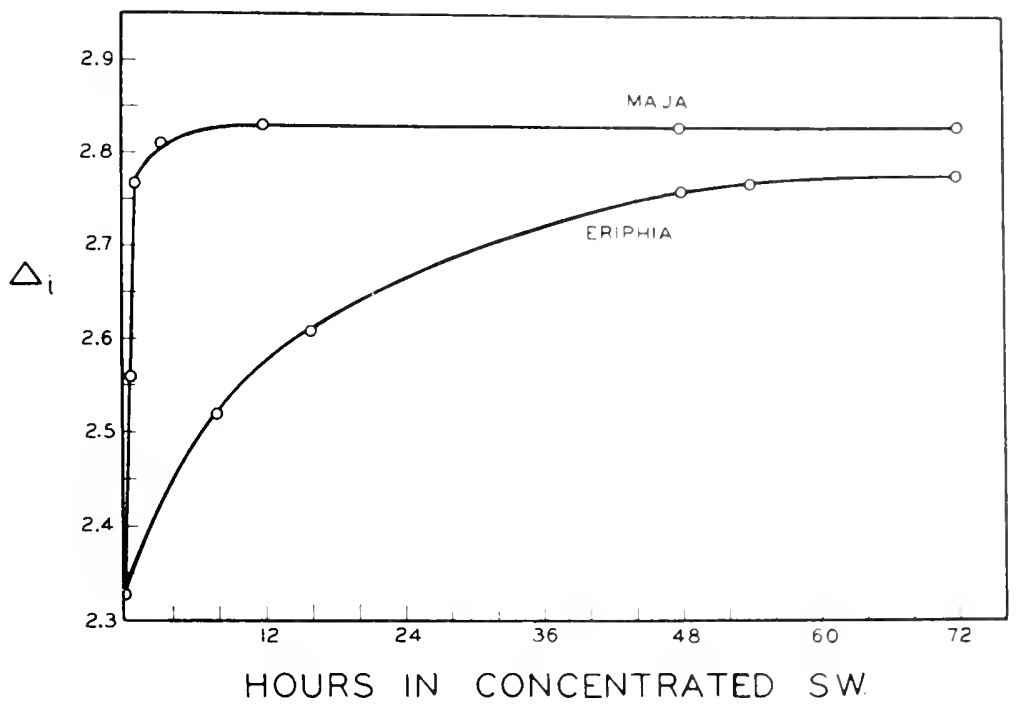

Fig. 11. Blood freezing point $\left(\triangle_{i}\right)$ of Maja and Eriphia after being transferred at zero hours from sea water of $\triangle_{0}=2.33$ to sea water of $\triangle_{0}=2.83$ (Schwabe ${ }^{292}$ ).

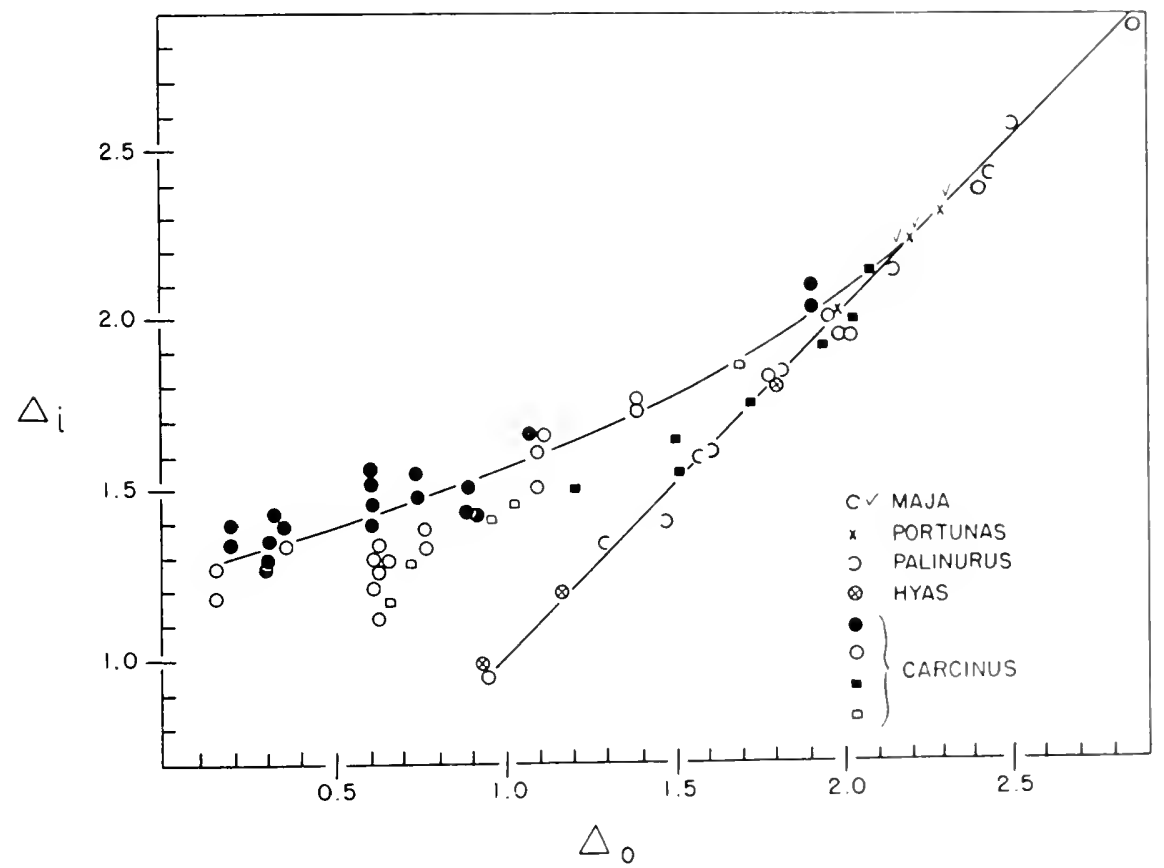

Fig. 12. Blood concentrations $\left(\triangle_{i}\right)$ of several marine Crustacea as a function of external concentrations $\left(\Delta_{0}\right)$. Data for Carcinus: - (Schlieper ${ }^{213}, \square\left(\right.$ (uget $\left.^{1-1}\right), 0$

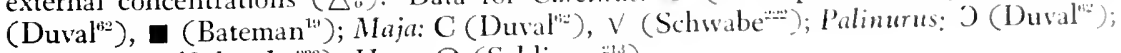
Portunas: $\times\left(\right.$ Schwabe $\left.^{22.2}\right)$; Hyas: $\otimes\left(\right.$ Schlieper $\left.^{-1.3}\right)$. 
Limited Osmoregulation: Invaders of Brackish Water. Eriphia spiniformis and Pachygrapsus marmoratus, -2-2- Cancer pagurus (Platycarcimus, Fig. 13 curve E), 21.5 and Cancer magister, Hemigrapsus nudus (Fig. 13, curve D), and Henigrapsus oregonensis (Fig. 13, curre B) 122 show a slight ability to remain hypertonic in dilute sea water (Fig. 13, curves B and D; Fig. 14, curve E). These crabs are isotonic or nearly so in sea water and in higher concentrations, and hypertonic but not constant in blood concentration in increasingly dilute media. They are rock crabs occurring along the shore, sometimes in tidal pools. One important factor in their regulation is low permeability to water and salts. Figure 9 show's that in 66 per cent sea water Cancer swells

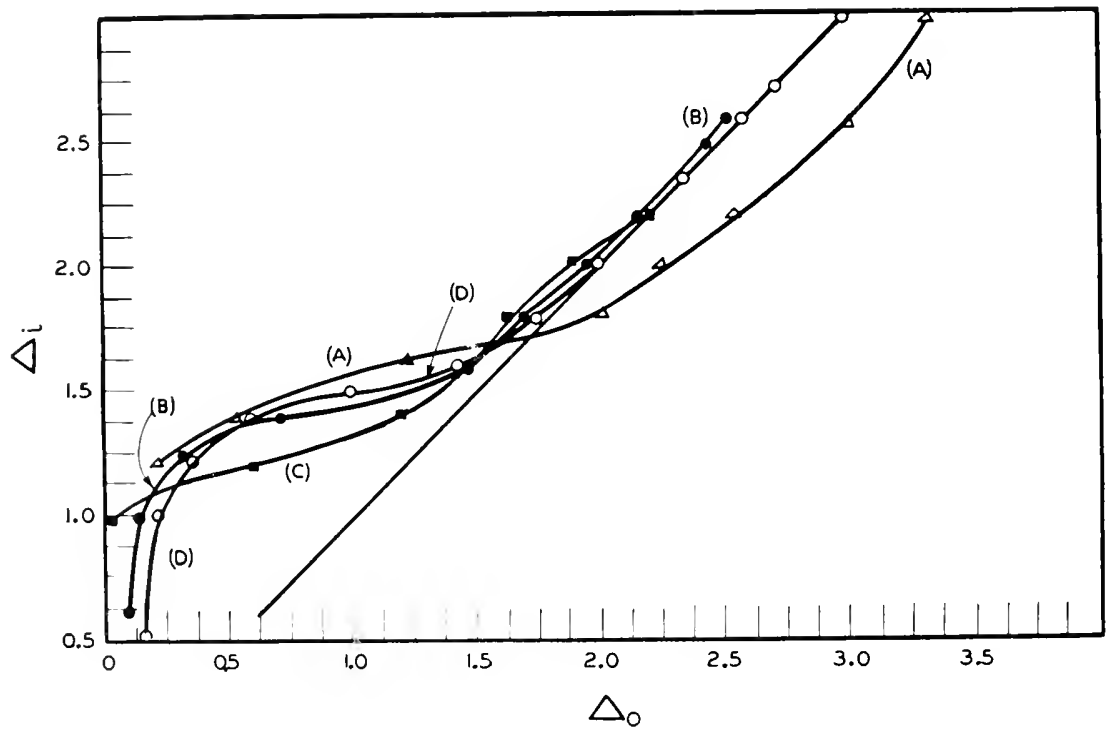

Fig. 13. Blood concentrations $\left(\triangle_{i}\right)$ as a function of external concentrations $\left(\triangle_{0}\right)$ in several Crustacea which are labile in hypertonic media and show some regulation in hypotonic media. $\triangle$ Pachygrapsus crassipes; - Hemigrapsus oregonensis; Rhithropanopeus harrisii; $\bigcirc$ Hemigrapsus mudus. Data from Jones. ${ }^{1{ }^{12}}$

less than does Maja in 75 per cent sea water, but that Cancer recovers more slowly in volume. Hence Cancer is less permeable to both water and salt. In a hypertonic medium ( 5.1 per cent salinity) the blood of Eriplia reached a concentration equal to that of the medium only after 72 hours, whereas Maja reached the same concentration as the medium in 12 hours (Fig. 11).222

A useful method for investigating salt permeability is to place crabs in sea water containing small amounts of iodine and to measure the amount taken up. When Cancer was bathed in seal water containing 0.5 per cent iodine, the crab took up 0.35 to $0.48 \mathrm{mg}$. iodine ce. of blood after 7 to 8 hours. ${ }^{34}$ When the intestine was plugged the uptalie was the same. When a tube containing 0.75 per cent soclimm iodide was placed on the surface of the carapace, Cancer took up in 50 hours an anount corresponding to $0.048 \mathrm{mg}$. cc. of blood. I lence there may be some ionic uptake directly through the shell but most of the exchange is across the gills. The poikilosmotic crabs Portunas and Hyas 
are more permeable to iodide than the weahly homomomotic Cuncer Fig.

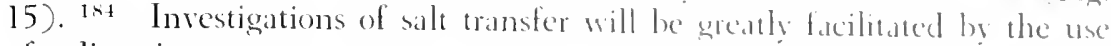
of radioactive tracers.

The fact that Cancer swells less than Maju also indicintes a lower permeat bility to water. Huf 114 fastened a tube to a hrole in the carrapace of (incer

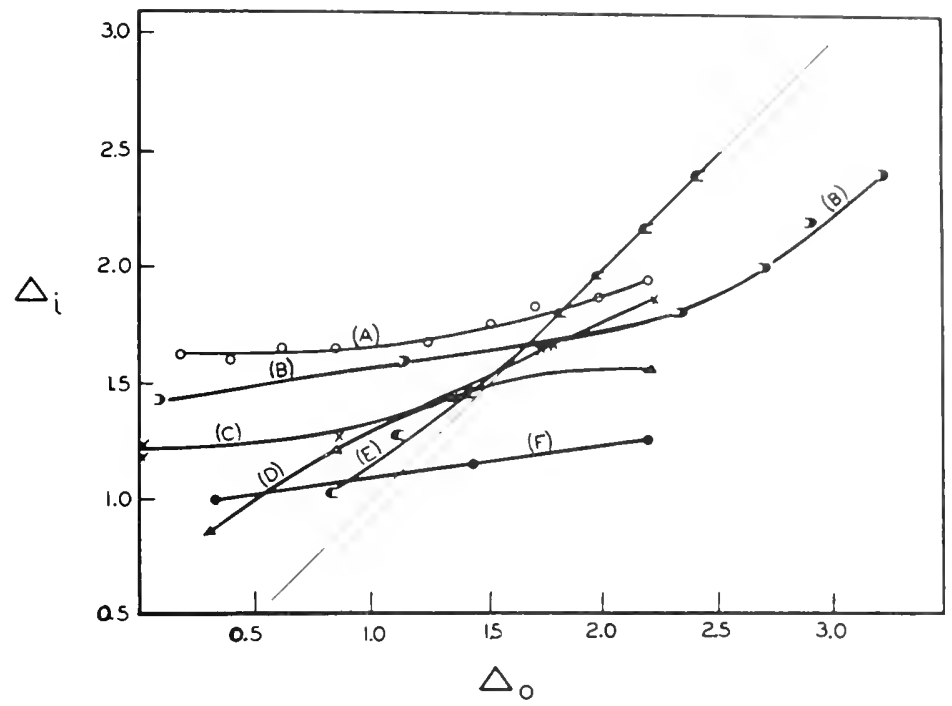

Fig. 14. Blood concentrations $\left(\Delta_{i}\right)$ as a function of external concentrations $\left(\triangle_{0}\right)$ in a series of crabs which regulate in both hypertonic and hypotonic media: Heloecius (Edmonds ${ }^{6 *}$ ); Uca crenulata (Jones ${ }^{102}$ ); $\times$ Eriocheir (Scholles ${ }^{2 * 21}$ ); $\triangle$ Leander (Panik-

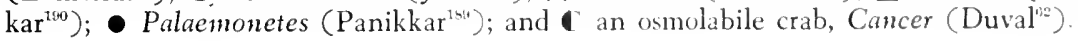

$$
\frac{I \text { BLOOD }}{I_{\text {MEOIUM }}} \cdot 100
$$

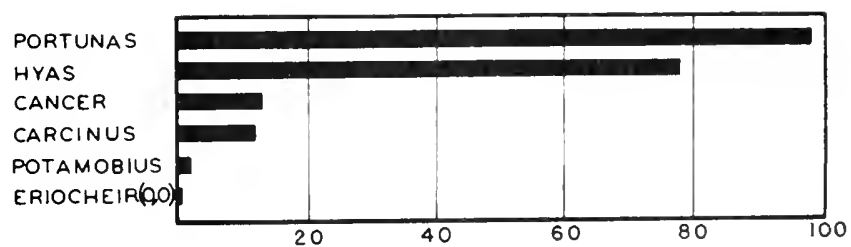

Fig. 15. Ratio of jodine in the blood to that in the medium after two and a half hours in sea water containing $\mathrm{NaI}$. From Nagel."nt

and measured the rise in water level in this tube when the crabs were put into dilute sea water. In 50 per cent sea water he recorded a pressure rise of 1.16 $\mathrm{cm}$; in crabs with kidneys closed the pressure rise 11 as $2.3 \mathrm{~cm}$. The relation between excretion and osmotic stress has not been eximined in this group of crabs; it would be of interest to know what regulating mechanism fails as the medium becomes more dilute. 
Limulus, an arachnoid, shows some regulation of ormotic concentration. It survives for a week or two in dilutions as low as one-fourth sea water but dies in one-eighth sea water. One Indian species occurs in brackish water. ${ }^{194}$ When Limulus was transferred from a medium of $\triangle_{0}=1.82$ to one of $\Delta_{0}=1.02$, the blood concentration reached $\triangle_{i}=1.12$ after 52 hours. ${ }^{81}$ The marine isopod Mesidotea also appears to maintain a hypertonic blood in dilute sea water but to adjust to the external medium in concentrations greater than its normal habitat. ${ }^{+1}$

Osmoregulation in Dilute Media: Shore Crabs. The shore crab, Carcinus, is capable of considerable osmotic regulation and hence is found in more dilute waters than any of the previously considered crabs. It can survive indefinitely in dilute sea water of $\Delta_{0}=0.60 .{ }^{62}$ C. maenas is found from the high-water mark and tidal pools out to depths of a few fathoms, although it occurs in the Elbc river at a freezing point of $-0.682^{\circ} .212$ Figure 12 shows that in ordinary and in concentrated sea water Carcinus changes in internal concentration and may be slightly hypertonic, but that in dilute sea water it maintains itself with some constancy in a more concentrated condition. ${ }^{62}$ In brackish water Carcinus remains hypertonic by as much as two times the external concentration.

When Carcinns is placed in a dilute medium it swells hardly at all (Fig. 9). ${ }^{2} 22$ Yet approximately 12 hours is required for the blood to come to a constant hypertonic concentration (Fig. 10, curve A). ${ }^{168}$ This must mean that the permeability to water is very low. Chloride is gradually lost from Carcinus in a dilute medium. ${ }^{184}$ In 50 per cent sea water the crab's weight increased less than 0.5 per cent in 21/2 hours. ${ }^{114}$ When, however, the openings of the antennal glands (kidneys) were closed, the crabs in 50 per cent sea water gained 2.2 per cent in weight in 1 hour and 5.5 per cent in weight in 3 hours. Apparently the gills are not impermeable to water, but the kidneys excrete water as fast as it comes in. Nagel ${ }^{184}$ measured the urine output by plugging the lidneys for 10-hour periods and then measuring the loss after removing the plugs. He found urine output as follows:

$\begin{array}{cc}\text { Urine output } & \\ \text { (ml./24 hrs./ } & \text { Salinity of medium } \\ 50 \mathrm{gm} . \text { body wt.) } & \text { (per cent) } \\ 5.1 & 3.3 \\ 6.3 & 1.62 \\ 8.5 & 1.57\end{array}$

Urine excretion increases with dilution of the medium, but not enough to account for the increased water intake. There was greater weight increase in 66 per cent sea water with both antennal glands and mouth plugged (3.4 per cent in 4 hours) than with only the antennal glands closed (2.7 per cent in $t$ hours), lience water is eliminated by the mouth. ${ }^{3 *}$ Carcinus gives off water by kicheys and gastrointestinal tract as fast as it takes it up in a hypotonic medium.

Carcinus has low permeability to water and also to salts, both incoming and outgoing; it takes up less iodine from the medium than does Portunas, Hyas, or Cancer (Fig. 15). 1n Also, when iodine was injected into Hyas, after 11/4 hours 50 to 94 per cent of the amount injected had been lost to the outside medium, while Carcinus lost only 5 to 16 per cent of the injected iodine in 
11/4 hours. Carcinus took up less than one serenth an much andine as ('ancer through a similar area of the dorsal carapace. 31

The urine of Carcinus may be hypertonic, bypotonic, or isotonic an ila blood when in sea water, and when in dilute seat water it mals be slighly hypotonic to the blood but mone concentrated than the mediums an las I lue high urine output in normal sea water cannos be esmotic water but rather appears to be a mechanisn of ionic regulation, and water must be talken in non-osmotically when the crabs are isotonic with the medium. Witce is necessary for the excretion of undesirable ions like $\mathrm{Ig}_{\mathrm{g}}$ and $\mathrm{S}()_{1}$. 2 th It is probable, therefore, that the kicheys of marine Crustacea developed als salt-regulating mechanisms.

In a dilute medium Carcinus consumes considerably more oxyeren than in

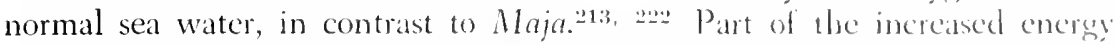
must go toward mantenance of the high concentration gradient. Ihe con centration gradient is maintained partly by active salt absorption. 1nt Starred specimens maintain their conecntration of body salts at a constant level despite continued urine excretion. Niso, when transferred from a medium of low to one of higher salinity, the crabs take up chloride even against a gradient to their more concentrated blood. For example, when they were transterred from a medium with $8.57 \mathrm{mg}$. Cl $\mathrm{ml}$. to one with $1 \mathrm{l} .45 \mathrm{mg}$. Cl $\mathrm{ml}$, their blom concentration increased from 12.0 to $1+\mathrm{mg}$. inl. The transfer and uptake took place with the digestive tract closed, hence the gills were probably actively absorbing chloricle. ${ }^{1 s t}$

Three mechanisms play a part in osmoregulation of Carcinus in dilute medium: low permeability to both water and salts, increased fluid output with increased osmotic gradient, and active salt absorption. It is impossible to separate osmotic regulation from ionic regulation except by degree, in different media.

It is likely that Rhithropanopeus harrisii is similar to Carcinus in osmotic regulation (Fig. 13, curve C). 1:2

Regulation in Both Hypertonic and Hypotonic Mccia. In Pachygrapsus crassipes (Fig. 13, curve A), Leptograpsus variegatus, ${ }^{6.1}$ Sesuma erythodactyla, 12: Heloecius cordiformis (Fig. 1t, curve 1 ), "it (la cremulata (Fig. 14, curve B), 1': Palaemonetes varians val. microgenita (Fig. 1t. curre F), 189 and Leander serratus (Fig. 14, curve I)), Ist the blood concentration is even more constant with changes in the modium than it is in Carcinms. These crabs remain hypotonic in a concentrated medium. Pachystapsus, which lives in sea water near the shore, is nomally more dilute than seat water."., 1:2 Some crabs, such as Pachlygapsus mamoratus, leave the water many times a day. Palaemonetes rarians var. microgenita can live in brack ish, salt, or nearly fresh water, and Crago unlgaris lives in the Elbe at a salinity of 0.43 per cent. $\because 12$

Curves relating internal to external concentration in these animals are nearly flat, with hypertonicity of the animal in dilute medium and hypotonicity in concentrated medium (Fig. 14). 12.2 At extreme dilutions a rapid fall in blomel concentration occurs and the condition of the animal is poor. The maximum concentration at which the downward Hexion of the $\Delta_{i} \Delta_{\text {n }}$ curve oceurs must limit the freshness of water which can be entered by the crabs. lnetestigat tion of the means by which osmoregulation thus breaks down at low concen 
trations would be of interest, as well as investigation of the mechanism of hypotonicity at high concentrations.

During the molting cycle changes occur in osmotic concentration of the blood of Pachygrapsus crassipes. In sea water of $\Delta_{1}=1.975$ the hard intermolt crabs showed a $\triangle_{i}$ of 1.327 ; in those about to molt $\triangle_{i}=2.601$, in those newly molted $\triangle_{i}=2.193$, and in crabs at the paper-shell stage $\triangle_{i}=1.76 .22$ Water and sugar content also changed during the molt. ${ }^{21}$

In some parts of the world certain crabs live on land most of the time, usually returning to salt water to breed. Their most interesting adaptations are respiratory. Pearse 195,196 found the blood of several land crabs to be more dilute than that of marine crabs while in one the concentration was about the same. Their chitinous shells are good protection against evaporation. Hypoosmotic forms such as $U_{c a}$ can live longer in air than can poikilosmotic crabs like Cancer, whose blood concentration increases markedly when they are exposed to air. ${ }^{122}$ All crabs which show hypo-osmotic regulation are grapsoid crabs, and all land crabs are also grapsoids. It is probable that the hypo-osmotic condition permits regulation against increased osmotic concentration of water in the branchial chamber when the crab is in air. ${ }^{122}$ However, many grapsoid crabs remain in sea water, and it is unlikely that for these the ability to maintain hypotonicity can have adaptive value.

Good Regulation Permitting Life in Either Fresh or Sea Water. A few crabs live successfully in either fresh or sea water. Young Eriocheir sinensis, according to Krogh, 14:3 ascend some of the rivers of Europe in the spring; they mature in fresh water, but in the autumn the adults return to the sea to breed.

When Eriocheir is transferred to media of different tonicities there are no volume changes but the blood concentration does rise and fall slightly. Animals living in North Sea water with $\triangle_{\text {. }}$ of 1.72 showed a $\triangle_{i}$ of 1.66 , while those in fresh water showed a $\triangle_{i}$ of 1.22. ${ }^{215} \quad$ Eriocheir is hypertonic in dilute and hypotonic in concentrated media (Fig. 14). However, Eriocheir differs from Hemigrapsus, Pachygrapsus, and $U_{c a}$ in not having a steep and lethal fall in its osmotic concentration in very dilute brackish or fresh water.

The permeability of the gills of Eriocheir is extremely low. This crab takes up iodine even more slowly than does the fresh-water crayfish (Fig 15). ${ }^{184}$

The urine output is at least $3.5 \mathrm{ml}$./day from a $60 \mathrm{gm}$. Eriocheir in fresh water. ${ }^{2211}$ The urine is hypertonic or isotonic to the blood, $\cdots$ of the medium:

\begin{tabular}{ccl} 
Blood & Urine & \multicolumn{1}{c}{ Medium } \\
$\Delta_{1}$ & $\Delta_{u}$ & \\
1.18 & 1.23 & fresh water \\
1.85 & 1.91 & Sea water of $\Delta_{0} 2.23$
\end{tabular}

The salt composition of the urine differs from that of the blood. The kidneys, therefore, put out much salt in the urine.

In fresh water, Eriocheir replaces the salt it loses by actively absorbing some salt through the gills. Krogh ${ }^{1+2}$ observed that a crab loses salt rapidly when transferrect from fresh to distilled water. After 24 hours the salt loss in distilled water was at the rate of $108 \mathrm{mM} / \mathrm{hr}$. with the kidneys open and 82 $\mathrm{mMl}$ hr. with the kidneys closed. The rate of ammonia loss was the same whether the kidneys were open or closed; hence the loss of most of the chloride 
and of all of the ammonia appears to occur by way of the gills. When at specimen has had its chloride content reduced hy protonged immersion in frequently changed distilled water and is then put back into pomel water on dilute salt solutions it absorbs salt very rapidly against a concentration gradient. The crab fails to reduce the concentration of the medium below 0.20 .t millimolar. No difference in respiration in salt and in fresh water was detected. $22: 2$

In Eriocheir, then, the kidneys have no osmoregulatory function hut do function in ion regulation. The crab is able to live in fresh water because of its low salt and water permeability and the ability of its gills to allsurb chloride. Possible extrarenal routes of vater excretion have not been investigated.

Fresh-Water Crustaceans. The Italian crab, Telphusa fluricutilis, adjusts to high concentrations of its medium but regulates well at lower concentrations, maintaining a $\triangle_{i}$ of 1.17 in fresh water. ${ }^{62}$ ' The urine of Telphusa is isotonic with blood. $" 15$ Changes in blood concentration occur morc slowly than in marine animals.

In fresh-water crayfish the blood freezes at about $-0.8 \mathrm{C}$, which corre sponds to a salinity of 1.4 per cent. There may be a normal fluctuation of 10 per cent in blood concentration during a period of a week or two. 114 When crayfish are put into dilute sea water ( 50 per cent or less) their internal concentration rises and they continue to be hypertonic, but in higher concentrations of sea water the blood concentration is isotonic with or slightly lower than that of the medium. Crayfish lived for a month in 66 per cent sea water and for three months in 50 per cent sea water.'2. 102

In isotonic ( 1.5 per cent) saline (diluted sea water) there was no weight change in crayfish, either normal or with antennal glands plugged, but in more dilute media there was a considerable increase in weight in those with the kidneys plugged; in more concentrated (hypertonic) media there was a loss of weight by both groups. ${ }^{102}$ Apparently, then, nomal crayfish can regulate their volume in dilute or isotonic media but not in hypertonic concentrations.

The permeability of crayfish to both water and salts is luw but not negligible. The volume increase in fresh water of crayfish with antennal glands plugged may be 5.5 per cent of body weight in 48 hours. ${ }^{102}$ Permeability to iodine is low (Fig. 15). Most of the salt and water exchange occurs through the gilts rather than through the body wall. Since weight remains constant the small increase in concentration in dilute sea water must be due to uptake of salts.

Crayfish urine is much more dilute than urine of marinc Crustacea or of Eriocheir. The total urine output of crayfish in per cent of the body weight per 24 hours is 3.8 per cent, 1025.2 per cent, ${ }^{1.1}$ or 4.0 per cent, ${ }^{2.20}$ an excretion volume not much higher than that in many marine crabs. The crayfish urine, however, is very dilute. Nean freezing points of blood and urine in crayfish from fresh water $\left(\triangle_{0}=0.018^{\circ}\right)$ are as follows:

\begin{tabular}{|c|c|c|}
\hline & Blood & Urine \\
\hline Cambarus clarkii ${ }^{151}$ & $\begin{array}{l}\triangle \text { i.p. } \\
0.6+t^{\circ} \mathrm{C} .\end{array}$ & $\begin{array}{l}\triangle \text { i.p. } \\
0.065^{\circ} \mathrm{C} .\end{array}$ \\
\hline Astacus fluviatilis: & $0.81{ }^{\circ} \mathrm{C}$. & $0.09{ }^{\circ} \mathrm{C}$. \\
\hline Astacus fluviatilis & $0.80^{\circ} \mathrm{C}$. & $0.16{ }^{\circ} \mathrm{C}$. \\
\hline
\end{tabular}

When salt is added to the medium the urine concentration increases; in isotonic and slightly hypertonic solutions the urine output declines to zero. 
Chloride concentrations in blood and urine, recalculated into millimols per liter by Krogh, are as follows:

Astacus fluviatilis ${ }^{250}$

Astacus fluviatilis ${ }^{\text {ins }}$

Blood $\mathrm{Cl}-$
$\mathrm{mM} / \mathrm{l}$.
195
196
117
Urine $\mathrm{Cl}-$ $\mathrm{mM} / \mathrm{l}$.
10
10.6
9.6

Cambarus clarkititist

117

This difference suggests cither that salt is reabsorbed into the blood or that water is secreted by the kidney. In Eriocheir in fresh water certain substances

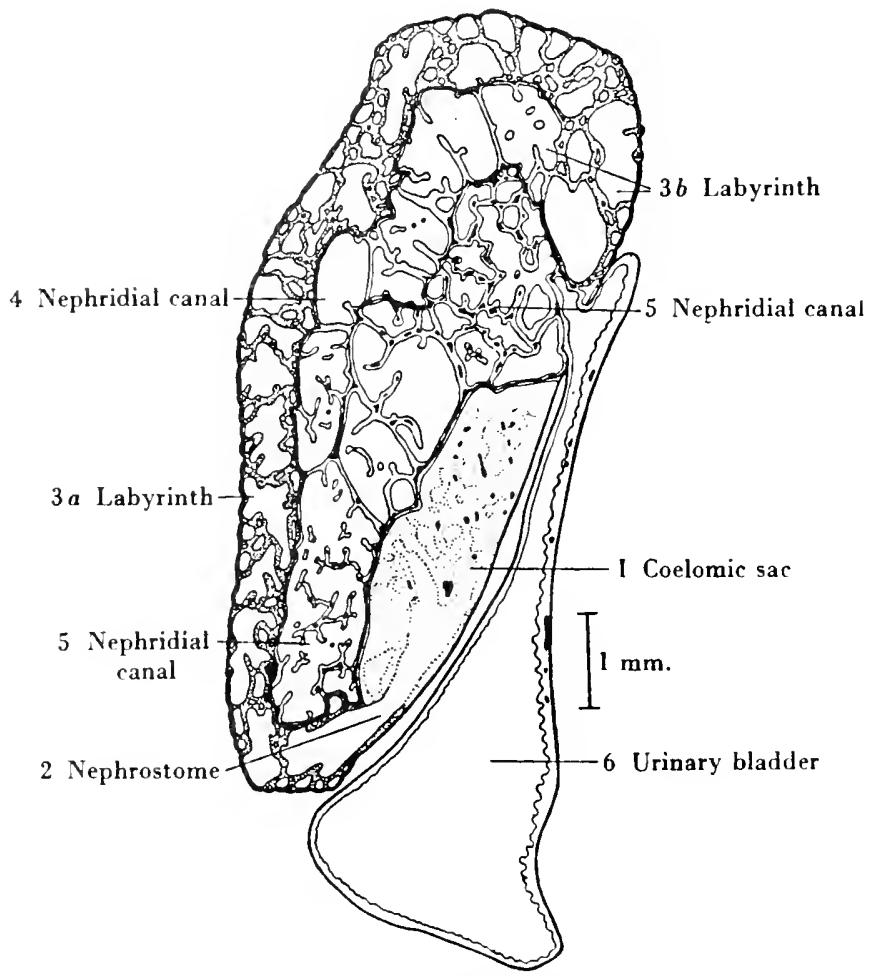

Fig. 16. Cruss section of the kidney of a crayfish, consisting of a much coiled but continuous lube with coelomic sac, labyrinth, nephridial canal, and bladder. From Peters."'n

such as magnesium are much less concentrated in the urine than in the blood, but chloride content is similar in each fluid, whereas in the crayfish all of the urine salts are lower in concentration than are the blood salts.

'I he crayfish kidney consists tirst of a coelomic sac penetrated by blood vessels and sinuses and lined by a single epithelial layer (Fig. 16). Then follows the green tubular labyrinth, a sponge-like structure, and a long $(3 \mathrm{~cm}$.) nephridial canal. The canal empties into the urinary bladder. The blood supply is rich to all parts except the bladder. The blood vessels open into hemococte spaces which surround the kidney tubules. In the lobster, which excretes an isotonic urine, the labyrinth tubule is larger, but the nephridial canal is not well diflerentiated. ${ }^{195}$ The nephridial tubule in the fresh-water 
Gammarus pulex is much longer than in the marine (:. lecmba, but there is little or no difference in kidney size in fresh-water and salt watter varjetices of Palaemonetes varians. "2.2 Thus the entire crustacein hilncy is, in principle, like one unit of the vertebrate kidney. By means of a capillary pipette Perese removed samples of fluid from each of the regions of the cors fish hidnes, and then measured their ehloride content. I lis results in m. 1 l. recollculdted by Krogh) are as follows:

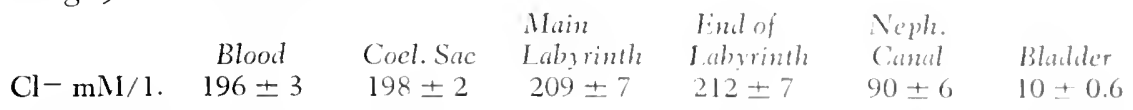

The fluid from the coelomic sae and labsrinth may be a blosel filtrate, essen tially isotonic with the blood, and in the nephridial canal chloricle min be reabsorbed, leaving a dilute urine to enter the blaklder.

Evidence for filtration was given by Picken, ${ }^{193}$ who measured a hedro static pressure of $20 \mathrm{~cm}$. $\mathrm{H}_{2} \mathrm{O}$ in the first leg of a cray fish. The collericl osmotic pressure of the blood was found to be $15 \mathrm{~cm}$. IyO and that of the urine, 2 $\mathrm{cm}$. $\mathrm{H}_{2} \mathrm{O}$. Hence filtration might occur from blood vessels to tubules.

The histological picture is not compatible with filtration, however. 16i. ni. The coelomic sac and tubules lie in hemocoelic spaces, so that the pressure must be similar on all sides, and it is difficult to see how unidirectional pressure could exist as it does in a vertebrate kidney glomerulus. The epithelium of the coelomosac may show vacuoles; the nephridial tubute cells of a cran fish from fresh water contain many vacuoles which can be seen being extruded into the lumen. Crayfish kept in saline for several days lose these vacuoles and regain them when returned to fresh water. ${ }^{16 i l .}{ }^{16.5}$ The laby rinth colls can accumulate and secrete dyes such as cyanol, phenol-red, and indigo carmine. This evidence argues against filtration and reabsorption of salt but demands secre tion of water.

In no condition has a crayfish been observed to exerete totally salt free urine. A crayfish weighing $50 \mathrm{gm}$. loses $600 \mathrm{mAl}$ of Cl daily. 11 ! Some salt is normal ly obtained from food, but crayfish can be kept for wats without food. 'The chloride content of the urine of starved crayfish is less than that of $\mathrm{fed}$ ones. ${ }^{220}$ In 8 days in distilled water the chloride content of the blood fell from $6.8 \mathrm{mg}$. $/ \mathrm{gm}$. to $4.5 \mathrm{mg}$. $/ \mathrm{gm}$., i.e., a loss of about one third, and the cras fish eventually died. ${ }^{11+}$ When cray fish were remored from distilled water to fresh water before they died, recovery was complete. Krogh 11.3 washed out crayfish by leaving them in distilled water for 3 days and found that when they were then put into 0.02 Ringer solution they took up chloride at the rate of 2.3 $\mu \mathrm{M} / \mathrm{hr}$. over 3 hours, even though the external concentration was $2 \mathrm{~m} M \mathrm{l}$ and the internal concentration was $100 \mathrm{mM}$. From pure sodium chloride solution of the same concentration the uptake was initially greater $(6.0 \mu \mathrm{N}$ I. hr. $)$; the rate of uptake later slowed, but at all times the active aborertion was at al lower rate than in Eriocheir. Astacus took up bromide at about the same rate at it did chloride. The gills take up silver salts, and washed out erastisls tathe up $\mathrm{Cl}^{-}$from KCl, Na+ and $\mathrm{Cl}^{-}$from $\mathrm{NaCl}$, and $\mathrm{Nal}_{\mathrm{i}}$ from $\mathrm{Nal}_{2} \mathrm{SO}_{4}$.

By its low permeability and by two energy recuniring processes-salt realh sorption or water secretion in the kidney, and active salt absorption by the gills -the crayfish is able to maintain a high intemal concentration while living in fresh water. 
It has frequently been reported that fresh-water animals consume more oxygen per unit weight than do brackish and marine forms. The following figures ${ }^{153}$ showing the cubic millimeters of oxygen consumed per gram of weight per hour for three species of Gammarus support this:

$\begin{array}{lccc} & \text { Fresh water } & \text { Brackish water } & \text { Sea water } \\ & \text { G. pulex } & \text { G. chevreuxi } & \text { G. marinus } \\ \text { Unanesthetized } & 1098 & 648 & 562 \\ \text { Anesthetized } & 443 & 529 & 300\end{array}$

Further, the oxygen consumption in unanesthetized G. chevreuxi was 516 $\mathrm{mm} .{ }^{3} / \mathrm{gm} . / \mathrm{hr}$. in 100 per cent sea water and $648 \mathrm{~mm} .{ }^{3} / \mathrm{gm} . / \mathrm{hr}$. in 25 per cent sea water. The oxygen consumption of crayfish is 30 to 40 per cent higher in fresh water than in isotonic sea water. ${ }^{198,}$, w.2 Part of the extra energy made available in fresh water must provide for the salt and water secretory movements against concentration gradients; part may be concerned with maintaining low permeability to salt and water.

Krogh suggests that the capacity to absorb salt actively is of wide occurrence, especially among fresh-water animals. He gives evidence from Fritsche indicating that Daphnia absorbs salt. The fairy shrimp, Chirocephalus diaphanus, loses salt rapidly in distilled water but recovers it in a very dilute salt solution. ${ }^{191}$ Branchipus and Apus (Lepidurus) are unable to remain alive long in distilled water, fresh water, or dilute Ringer solution without food. Krogh suggests that these animals lack the power of active salt absorption.

In contrast to those animals which are dependent on salt absorption, Gammarus pulex remained healthy in glass-distilled water which was changed twice daily and lost chloride for only the first 2 to 5 days. ${ }^{2 s}$ Also Asellus and larvae of the mosquito Aedes live well in distilled water. In these animals salt retention is important and survival is possible, at least over periods of days, without salt absorption. However, adjustment to low tonicity of the environment does require some initial loss of chloride. ${ }^{2}$

Regulation Permitting Life in Salt Lakes: Brine Shrimps. The fresh-water Crustacea have specialized in hypertonicity in dilute media. A few Crustacea have gone to the other extreme and maintain hypotonicity in concentrated brine. Artemia, the brine shrimp, lives in Great Salt Lake in a salt concentration of 22 per cent and in salt lakes of even higher concentration in other parts of the world. Artemia is normally hypotonic but varies its internal concentration with the medium. ${ }^{17 \tau}$ In a lake of 8.4 per cent salinity the blood concentration was equivalent to 1.3 per cent sodium chloride. In an external salinity of 17.4 per cent the blood concentration was equivalent to 2.4 per cent $\mathrm{NaCl}$, hence blood concentration did not increase in proportion to concentration of the medium. ${ }^{1 / 18}$ Weight is gained or lost when Artemia is transferred to hypotonic or hypertonic media. The permeability of Artenia to heavy water is low compared with that of Daphnia (Ussing, from Krogh ${ }^{143}$ ). Artemia takes up dyes, which enter most Crustacea through the gills, only by mouth. 204 There must be other mechanisms, besides the low permeability, which enable the brine shrimp to have a blood concentration as low as 10 per cent of that of the medium. 'There is some evidence for water storage in the intestine in a dilute medium. ${ }^{1.1 s}$ 'The gills of Artemia are larger in media of high salinity than in media of low salinity, an effect probably related to lower 
oxygen content in the media of high salinity, but prossibly also reluted to salt excretion. 216

\section{ADAPTATION TO FRESH WATER}

All fresh-water invertebrate animals are hypertonic to the medium. None (except some eggs) are impermeable to water. I Hence probalbly all ficsh water animals have a mechanism for excretion of urine hypotenic to the blexd. Some separation of water from salt is made by the excretory organs. The body surface may retain salt but no fresh-water animal, so har is is known, excereter a totally salt-free urine. The salt necessarily lost in the urine must be replaced by food or by active absorption.

Aquatic Insects. Insects are essentially terrestrial animals but in many orders some members have invaded fresh water, at least for larvall life. There are some larvae in salt lakes, and a few venture into brackish water. There are marine chironomids. A brackish water species of Corixidae or water boatman (Sigura lugubris) has been compared with two fresh-water species S. distincta and $S$. fossarmm). ${ }^{53}$ The brackish-water insect is found over at salinity range of 0.5 to 1.8 per cent. The curves relating internal and external concentrations resemble similar curves for the regulating fresh-water Crustaced. The blood concentration of Sigura lugubris is constant in media ranging from 0.1 to about 1.5 per cent salinity; in media below 0.17 per cent salinity the blood concentration declines, and in media above 1.5 per cent salinity it rises. 'The fresh-water species does not regulate so well, but it increases its blood concen tration by only 30 per cent in going from 0.1 to 1.8 per cent salinity. There is no information regarding the mechanism of this regulation.

The blood and coelomic fluid of various aquatic insects are similar in osmotic concentration to those of other fresh-water animals. Insects differ from other animals in the small fraction of their osmotic pressure due to chlorides and the large fraction due to organic substances, particularly amino acids. The chlorides in body fluids of Culex and Aedes account for only 35 to 40 per cent of the total osmotic concentration. $" * 49$

The only aquatic insects which have been examined carefully as to their osmoregulation are dipteran larvae, particularly mosquitoes and midges. The details of their adjustments are discussed by Krogh $1: \%$ and by IViggles. worth. 250 The so-called anal papillae of gills which had been thought to be

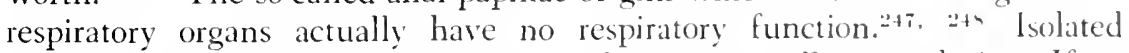
papillae, however, swell in tap water and constrict in Ringer solution. If an Aedes argenteus larva is ligated ahead of the opening of the malpighian tubes into the gut and then put into hypertonic glucose the hind part shrinks, whereas without the ligature the whole larva shrinks. If the animal is ligated just behind the opening of the malpighian tubes and placed in fresh water the posterior region becomes swollen, whereas the anterior part does not. B! several such ligation experiments Wigglesworth found that normal larrae swallow very little water, that the anal papillac are the part of the bod! most permeable to water, and that water is excreted by the malpighian tubes (lig. 17).

When Aedes larvae are transferred to various media a constant osmotic pressure is maintained in an external concentration lower than or equivalent to 0.65 per cent sodium chloride; ${ }^{2 * \pi}, 248,: \pm ! !$ in media of higher concentration 
the body fluid adapts to the medium (Fig. 26). The tolerance of salinity depends on the medium in which the larvae are reared. Larvae of Aedes argentens reared in fresh water are killed when they are transferred directly to 1.1 per cent sodium chloride or to sea water diluted to the equivalent of 1.3-1.t per cent $\mathrm{NaCl}$, whereas if the concentration is increased gradually the larvae are able to live in these media. The anal papillae of Culex pipiens larvae reared in 0.65 per cent $\mathrm{NaCl}$ are very small; when they are reared in 0.006 per cent $\mathrm{NaCl}$, the anal papillae are larger; when'the larvae are reared in distilled water the anal papillae are very big and the epithelial cells may become racuolated. The anal papillae of Aedes detritus larvae in Algerian ponds of a salinity equivalent to $1.2-10$ per cent $\mathrm{NaCl}$ are smaller than those of larvae of A. aegy pti from fresh water: 26 The larva of Chironomus thummi,

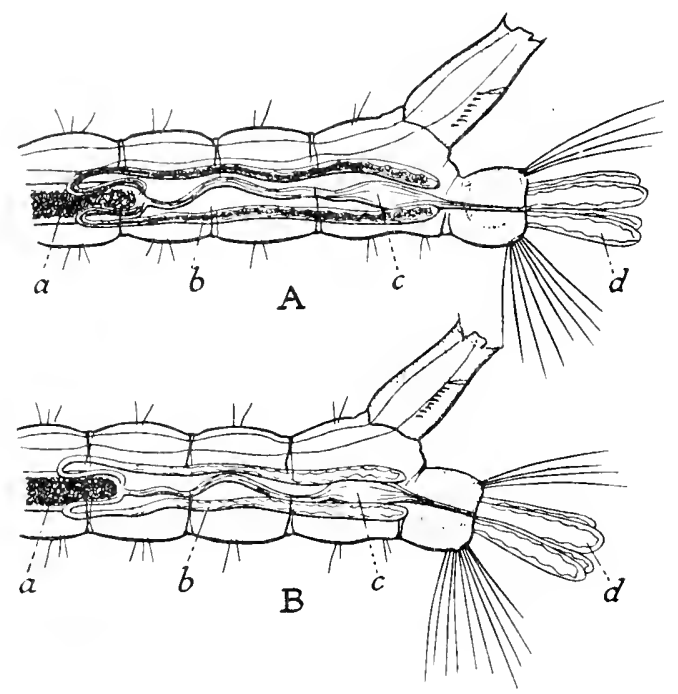

Fig. 17. A, Hind end of larva of the mosquito Acdes aegypti, reared in salt water, showing solid uric acid in lumen of malpighian tubes. B. The same larva, a few minutes after transfer to fresh water; the malpighian tubes have been flushed out by water entering through the anal papillae. $a$, mid-gut; $b$, malpighian tubes; $c$, hind-gut; $d$, anal papillae. From Wigglesworth.

unlike the larvae of Aedes and Culex, takes in water over its entire body surface. $: x$

The anal papillae of Chironomus larvae avidly take up silver from dilute solutions of silver nitrate, ${ }^{13}$ and the anal papillae of Aedes aegyti take up electronegative dyes. ${ }^{\mathrm{x} s}$. After a period of salt deprivation the anal papillae actively absorb salt from dilute solutions (0.01 Ringer solution). 138, 1+2, 249

The lunction of the anal papillac is clear. They are not respiratory but are salt albsorling mechanisms. Their permeability to water is a necessary condition to salt absorption. The body surface is more permeable, especially to water, in (hironomus larvac than in Culex and Aedes, but in the larvae of both groups the anal papillac become enlarged and actively absorb salt when in a dilute medium. The urine as formed in the malpighian tubes is similar 


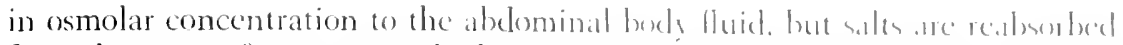

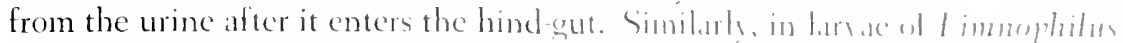

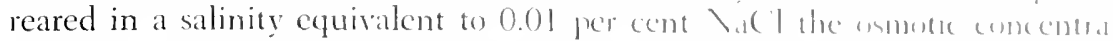

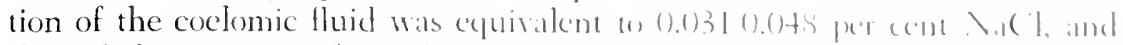
that of the secretion from the malphighian tules was similat $(8.1) 31(0 .(), 6)$

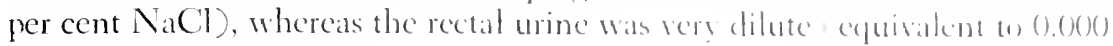
0.009 per cent $\mathrm{NaCl}$ ). 1

Sponges, Coelenterates, Flatworms, and Molluscs. I Iow fresh wilter sponges

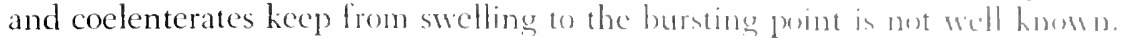
Fresh-rvater sponges appear to climimate water hy contractile vicuofes in amoebocytes and choanocytes. Mirine spenges lack these contractile valu oles. 119 The osmotic concentration of cells of the fresh water sponge, Spongilla, in summer is low $(25.30 \mathrm{~m} M \mathrm{MaCl})$ but at the time of eremmulat tion the concentration rises $(110 \mathrm{mMl} \mathrm{NaCl})$. $2 \cdots$

Two fresh-water coelenterates (Pelmatolivira oligactis and chlorohidra viridissima) have been maintained in brackish water of 0.25 per cent salinity, where the cells were shrunken; in distilled water they were swollen.

The endoderm cells of Hydra contain vacuoles which resemble the vacuoles in the cells lining the gut of many turbellarians. These vacuoles in /hatra are not seen to contract. When Hydra is placed in pyrex-clistilled water these vacuoles greatly enlarge: in a few hours the body cells separate and the animal disintegrates. Addition of small amounts of salts (calcium and other ions to the distilled water does not prevent swelling and disintegration. Some sub stance must be present in pond water which keeps permalibity to water low and prevents separation of the cells.

Free-living flatworms have an excretory systom of protonephrielia which probably function in getting rid of osmotic water. In a culture of Stemostomm oedematus distended individuals were lound to have damaged protoneph ridia. ${ }^{103}$ A turbellarian, Gratrix hermaphroditus, collected from fresh water showed a well developed protonephridial system, parts of which took up vital dyes. ${ }^{147}$ In brackish-water specimens ampullac and parancplirocytes are absent; in marine specimens the tubules also are lacking. Plinalria t: and Dendrocoelum ${ }^{7}$ gain wejght in distilled water and lose weight in physiolo gical saline, hence they must normally excrete a hypotonic urine.

Fresh-water bivalve molluses have a rery low blood concentration. 'The blood concentration goes up with increasing extermal concentration, but the animals are unable to survive in concentrations which aproath the concen tration of the blood of frogs and other fresly-water animals. "i: Anownta, adapted first to distilled water and then transferred to dilutions of sea water. showed no weight change in hypotonic solutions (less than $\triangle_{1}-0_{-1} .1$ but lost weight in more concentrated solutions. As Anesthetized specimens, howerer, gained weight in a hypotonic medium. in inolonta is, therefore. permeahle to water. Fluid from the pericardial carity passes through the nephrostome into the kidney. The blood and pericardial ftuid are equisalent osmotically to 0.1 per cent $\mathrm{NaCl}$ and the urine to 0.06 per ecent $\mathrm{VaCl} 20$ the blood is under a hydrostatic pressure of about $6 \mathrm{~cm}$. 11.2 , and the colloid osmotic pressure of the blood is some $3.8 \mathrm{~mm}$. I l.O. This would allow the pericilrdial fluid to be formed by filtration through the wall of the heart. and Picken and actually drained pericardial fluid for a short time at the rate of $250-300 \mathrm{ml}$. 24 
hr. in an animal weighing 50 grams (without shell). Fluid collected from the kidney (organ of Bojanus) is lower in chloride and calcium but higher in protein and nonprotein nitrogen than the blood. ${ }^{70}$ Hence the kidney reabsorbs some salt but still much is lost in the urine. The salt intake by food could supply only a small fraction of this amount and, furthermore, freshwater mussels will live well for many months in running tap water without food, with little change in blood concentration. "6: To compensate for the salt loss it is likely that salt is actively absorbed. Arrodonta and Unio can actively absorb chloride and sodium from approximately millimolar solutions, but they cannot reduce the concentration below about $0.1 \mathrm{mM} .{ }^{1+3}$ Krogh suggests that when salt absorption by the body surface is prevented, as in distilled water, the nephridial salt reabsorption may be increased so that the urine concentration may approach zero.

The kidneys of most gastropods and pelecypods are connected to the pericardial cavity and may receive pericardial fluid directly into the kidney lumen. In addition, the kidneys receive a rich supply of blood returning from visceral sinuses, and the epithelium in some regions is highly vacuolated and secretory. The hydrostatic pressure in the postrenal sinus may even exceed pressure in the pericardium (see Chapter 15). Urine volume cannot, therefore, be calculated from pericardial filtrate or urine composition be inferred from the fluid from the interior of the kidney. A histophysiological study of the molluscan kidney would be useful.

Snails show wide fluctuations in osmotic concentration according to activity and water supply. ${ }^{110 a}$ The snail Limnaea has a blood concentration equivalent to 0.43 per cent $\mathrm{NaCl}$ and urine concentration of 0.3 per cent $\mathrm{NaCl} .{ }^{201}$ Limnaea is able to absorb salt actively from 0.01 Ringer solution. ${ }^{143}$ The land snail, Helix, has blood equivalent to 0.69 per cent $\mathrm{NaCl}$ when hibernating and to 0.5 per cent $\mathrm{NaCl}$ when active. ${ }^{12.5}$ The blood concentration of a land snail dropped from a normal value of $\triangle_{i}=0.47$ to $\triangle_{i}=0.2$ after a rain. ${ }^{12}$

Earthworms and Leeches. There are many fresh-water annelids, specifically Hirudinea and Oligochaeta; the earthworms are adapted to life in moist soil where osmotic stress is intermediate between that of fresh water and that of air. Soil-dwelling earthworms can live indefinitely in aerated fresh water or in moist air. Adaptive mechanisms of water balance in earthworms have been examined in detail. $3,7,16,164,205 \mathrm{a}, 253$

The blood of freshly collected earthworms is similar in osmotic concentration to the blood of aquatic vertebrates; $\triangle_{\text {f.p. }}$. of Lumbricus body juice is $0.31^{\circ}, 3$ of blood plus coelomic fluid $0.59^{\circ},{ }^{164}$ of coelomic fluid of worms in distilled water $0.3^{\circ} ; 20$ ina the $\triangle_{\mathrm{f} .1}$. of Pheretima blood is $0.4^{\circ}-0.5^{\circ} .{ }^{16}$ There is much variation in osmolar concentration according to the state of hydration.

When an earthworm is transferred to tap water it absorbs water equivalent to as much as 15 per cent of its initial weight in about 5 hours. Water-adapted worms removed from aerated fresh water to moist air or soil lose water. .52 .253 Hence under natural conditions an earthworm is alwars in a semi-desiccated state. $^{3}$. 15 In dry air carthworms can survive a loss of 70 to 80 per cent of their body water.6. 217 Water goes out of the worm after excess hydration more quickly than it is taken in after dehydration. ${ }^{7}$ Earthworms orient toward a moist and away from a dry surface. ${ }^{2 \overline{5} 2}$ 
In air the body surfare is kept moist by cxcreted watter. and upen irruatum

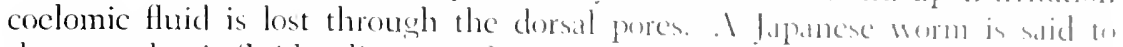

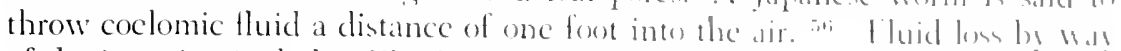
of the intestine is slight. Fuid collected trom the anterion end if the intestine has a higher osmotic concentration than the blexel. Wil Whorme tied at both ends gain weight in water no faster than womm met tied. Prensure of 4.5 cm. H.2O measured by a cannula in the gut correyponds to an intertinal excretion of 0.25 per cent of the hasal weight per hour.

Nephridial excetion by werms in witer is 2.2 .5 per cent of the hal al weight per hour. ${ }^{15}$. 16. 25 Analysis of carthworm urine. collected by an ingenious device, is shown in Table l. The urine is hypotonic ublond and conclonic

TABLE 1. COMPOSITION OF BODY FLLIDS OF PHEPETHM POSTHUMA*

\begin{tabular}{l|c|c|c}
\hline \hline & Blood & Coelomic Fluid & Urine \\
\hline \multicolumn{1}{c|}{ f.p. } & $0.40-0.50$ & $0.285 \cdot 0.31$ & $0.050 \cdot 0.065$ \\
urea (mg. \%) & 2.638 & 2.52 & 3.24 \\
protein (mg. \%) & 3643 & 479 & 30 \\
glucose (mg. \%) & 100 & nil & nil \\
Cl- (mg. \%) & 50 & 80 & 3.7 \\
\hline
\end{tabular}

${ }^{*}$ From Bahl. ${ }^{1 \bar{n}}$, in See also Table 9 in Chapter 3.

fluid and contains much less salts but more urca. In Lumbricus terrestris in tap water the chloride concentration in urinc is $20 \mathrm{mg}$. 100 ce. and that in coelomic fluid is $270 \mathrm{mg}$. $100 \mathrm{cc}$. The osmotic concentration of coclomic fluid is equivalent to 0.53 per cent $\mathrm{NaCl}$ and that of blood is slighty lower. anis

Earthworms show a wide rariety of types of nephridis reviewed ly bahliti. Some are large, occurring as one pair per segment; others are small and rery numerous in each segment. The best known nephridia open into the cuetom by a funnel, but there are many which are chesed intemally. Some carthworme (e.g., Pheretina posthuma in India) have some nephridia which empt! into the intestine, i.e., are enteronephric; whereas others (c.g. Lumbricus, Eut? phoeus) have all of the nephridia opening extemally, i.c., are comephric." The castings of enteronephric worms are drier and these worms live in a drier environment than exonephric worms.

Coelomic fluid enters the nephridial funnels nephrostomes under the force of ciliary beat; particles such as Chinese ink are seen to enter the nephridia from the coelom only when the distal portion or bladeler is empty. at Dos and particulate matter mas be found inside nephridial epithelial cells; these substances are either absorbed from the tubuld 2.51 or are phagecytized by migratory chloragogue cells and deposited in the tubules. Breatidown products of hemoglobin are transferred from blood to cortain neploridial cells where they are stored. ${ }^{1.5}$ Fluid collected in the ampulla and blateler is markedly hypotonic to the coelomic fluid, hence capacity to form hypotonic urine is greatest in the wide tube of the ampulla. "an Ciliary propulsion, active tramport across the tubule cells, and phagocytosis combine to produce the urine from coelomic fluid and hlood. Cytological comparison of nephridid from worms in water and in air might provide evidence regarding secretory function. 
The salts which are lost in the hypotonic urine of worms in water must be replaced. Some salts come from ingested food. Earthworms actively absorb chloride from very dilute salt solutions. ${ }^{162}$ Leeches which have lost some of their body salts by living in distilled water for about two weeks absorb from 0.01 Ringer solution as much as $0.48 \mathrm{mM}$ salt per gm. body weight during the first half hour of immersion. ${ }^{143}$

\section{ADAPTATION TO MARINE, FRESH-WATER, AND PARASITIC LIFE (PROTOZOA)}

Protozoa are a diverse group of animals which are so small that direct measurement of the osmotic concentration of their cytoplasm and their excretion is difficult. Indirect evidence indicates, however, that many marine and parasitic Protozoa have no osmotic problem, that is, they are isotonic with their medium. All fresh-water Protozoa have a distinct osmotic problem in that they are hypertonic to their medium. Some species can readily withstand transfer to extreme concentrations of the medium, from distilled water to concentrated sea water; others are extremely limited in their osmotic tolerance. It has been variously suggested that the contractile vacuoles of Protozoa function in excretion of water, nitrogenous wastes, $\mathrm{CO}_{2}$, and salts.

Structure of Contractile Vacuoles. Some Protozoa have one contractile vacuole, some have two, and others have several. In some marine species contractile vacuoles are absent in sea water but they appear when the animals are transferred to fresh water. Usually a contractile vacuole is formed when numerous small vacuoles or vesicles fuse; these may arise in a given area or in various parts of the cell. In some species, particularly among ciliates, there are canals of various shapes which fill and empty into the contractile vacuole. During the period of filling, or diastole, the viscosity of the cytoplasm in the vicinity of the contractile vacuole is low, while at the time of emptying, or systole, viscosity is high.

The contractile vacuole membrane has many of the properties of cell membranes. Several investigators ${ }^{10 s}$. 111 have succeeded in keeping vacuoles intact after removal from the cell. The membrane resembles the plasma membrane in holding back solutes such as some dyes. The vacuole wall stains with neutral red injected into an Amoeba and when first punctured the vacuole wall shows a wrinkled surface. ${ }^{111}$ Morita and Chambers ${ }^{151}$ stained Amoeba dubia yellow with methyl red; injected hydrochloric acid then caused the cytoplasm to turn red while the vacuole remained yellow.

The emptying of contractile vacuoles is apparently a gelation-solation phenomenon. A gelated region is needed around the vacuole before discharge occurs in Amoeba dubia. ${ }^{112}$ In Euplotes local solation seems to occur at the point of discharge, although behind this point there may be gel. $2: 31$

Volume Regulation. Volume regulation bas been examined in only a few species and these show extreme differences. The volumes of Amoeba proteus ${ }^{17.1}$ and of Pelomya carolinensis "? have been measured by putting the specimens into special capillary tubes. The volumes of Amoeba mira and of Amoeba lacerta have been calculated from their diameters, since they assume a spherical form when agitated. ${ }^{10 x}$, 17: Volunes of three species of peritrichous ciliates, Rhabdostyla brevipes, Zoothamnium marinum, and Cothurnia curvula, have been calculated from measurements of several dimen- 
sions. ${ }^{134,}{ }^{135}$ Of the four rhizopods, $A$. wira and $\Lambda$. lacerta adjust rapidly (1-2 hours) after transfer to a changed medium, so that their wolume in similar at all concentrations. Pelomixa is less tolerant of cotreme changen and requires 6 to 12 hours to adjust its rolume after transfor from fresh water culture to distilled water or to a solution of nenclectrolyce. lmacha protens transferred to a solution of sugar (lactose) above $0.005 \mathrm{~N}$ showed a steads decline in volume for 6 to 7 days, after which there was sometimes a slight rise, but survival was rare after that time in the nonelectrolate. Starration complicated the late reactions, but it seems certain that Amoelua prosens has very little volume regulation. The fresh water ciliate Rhaldustola brevipes and the marine Zoothamminm marinum also failed to show any tendency to return to their original volume, at least for 3 hours of measurement in al solu tion of changed tonicity, whereas the brackish water Cothuruic currula when transferred to a dilute medium swefled initially but after 23 hours showed : slight decline in volume. Three species of Euplotes which ocer: in 1 . water, in 2.5 per cent, and in + per cent salinity are of diminishung sie, in that order. "- If the fresh-water species becomes aclapted over a period of a month or more to 1 per cent salinity it decreases in size.

Membrane Permeability. The preceding observations indicate a consider able permeability to water but an extremcly how permeability to salts in Pelonyxa, A. proteus, Phabdostyla, and Zoothamminum, and a high salt permeability in $A$. mira and $A$. lacerta. Even in the latter two species, volume adjustment is much slower than in marine eggs. Permeability constants for incoming water ealculated on the basis of swelling give, in $\mu^{3} \mu^{2} \mu^{2}$ atm., min., values between 0.026 and 0.031 for A. proteus, ${ }^{17} 0.023$ for Pelomixa, 31 and between 0.125 and 0.25 for fresh-water peritrichs. $13 . .5$ These values are low compared with permeability constants of 3 for human erythrocytes and 0.4 for fertilized Arbacia eggs. It appears that there is no close correlation between the permeability of the different species for water and volume regulation. Lower permeability to salt than to water was indicated for marine peritrichs by their tendency to swell when vacuolar output was reduced hy cyanide. ${ }^{136}$ A high outward permeability to salt in Amoeba mira is shown by the observation 17. that when the animal wals transferred from 100 per ecent to 5 per cent sea water the volume increased fivefold in 12 mimutes and then after 90 minutes decreased to the original size. During this decrease in size of $3000 \mu^{3}$, only $700 \mu^{3}$ of water went out by way of the racuoles. The rest must have gone out across the body surface.

It is probable that Protozoa, like most other animal cells, are more permealble to $\mathrm{CO}_{2}$ and urea than to other nonclectrolytes. Considerathle shrinkiage of marine peritrichs (Cothurnia) in isotonic solutions of sucrose, shlucerol, and even urea, indicates a very low inward permeability for these nonclectro lytes. ${ }^{13.5}$ Several parasitic ciliates failed to take up vital dyes when the eyto pharynx was closed, but accumulated dyes readily when it was open. ${ }^{1}$ is le would be of interest to study the permeability of those parasitic species which lack oral openings. There is much variation, but Protozoil in general are colls of relatively low permeability.

Osmolar Concentration of Cytoplasm. The osmolar concentration of the cytoplasm of marine and parasitic Protozoa is probably similar to the concen tration of the medium in which they live. Mlinimal shrinkage of Amoeba 
protens occurrel in $0.005 \mathrm{M}$ lactose ${ }^{17 t}$ and that of I'orticella in $0.0125 \mathrm{M}$ lactose, $17: 2$ hence the osmotic concentration of the cytoplasm in the respective organisms must be slightly less than these valucs. Kamada ${ }^{12 \tau}$ reasoned from contractile vacuole responses that in a very dilute medium (approaching distilled water) the osmotic concentration of the cytoplasm of Paranecinm is equivalent to $0.025 \mathrm{M} \mathrm{NaCl}$. Measurements of the vapor pressure of Spirostomum cytoplasm $2 m$ gave a value corresponding to $0.025 \mathrm{M} \mathrm{NaCl}$. It is of interest that the oxygen consumption of Paramecimm is minimal in 4 per cent sea water $(-0.02+\mathrm{M} \mathrm{NaCl})$. Hoptopliry'a, a ciliate from the gut of salamanders, is rounded and turgid in salines more dilute, and flat and wrinkled in salines more concentrated, than 0.5 Ringer solution $(0.06 \mathrm{NI})$. $^{15} \mathrm{~s}$ Conductivity measurements are not strictly comparable, but conductivities corresponding to the following concentrations of $\mathrm{KCl}$ s.i were found: Amoeba proteus $0.01 \mathrm{~N} \mathrm{KCl}$, Paramecinm $0.06 \mathrm{~N} \mathrm{KCl}$, and Spirostomm $0.0385 \mathrm{~N}$ $\mathrm{KCl}$. It seems certain from the preceding observations that the osmotic concentration of fresh-water Protozoa in their natural medium is 5 to 10 times lower than that of most other fresh-water animals, and that the concentration of Amoeba proteus cytoplasm is less than that of the cytoplasm of Paranecium or Spirostomintm.

Function of the Contractile Vacuole. Contractile (c.) vacuoles eliminate water and help to maintain a constant volume. In marine and parasitic protozoa the c. vacuole eliminates water of a nonosmotic source, i.e., water from food and metabolic water. The low permeability of the cell surface to salts mentioned previously raises the possibility that the c. vacuole may also function in the elimination of salt. Nothing is known of specific ionic regulation in marine Protozoa, and the c. vacuoles may well eliminate certain ions differentially.

Evidence that the c. vacuole functions in osmotic regulation comes from the distribution of c. vacuoles among protozoa in different habitats as given in the following tabulation. ${ }^{137}$

\begin{tabular}{lc}
\multicolumn{1}{c}{ Class } & Fresh water \\
Rhizopoda & present \\
Flagellata & present \\
Ciliata & present
\end{tabular}

Marine present in few present in many present in most

Sporozoa

This table shows that in all fresh-water Protozoa a contractile vacuole is present. It is absent from most endoparasites except among ciliates. It is present in many marine Protozoil. Kitching ${ }^{1: 2 t}$ suggested that the much higher occurrence of vacuoles among marine ciliates than among rhizopods may be a holdover from previous fresh-water habitat. It seems more likely that it is correlated with the lower salt permeability and poorer volume adjustment in marine ciliates than in rhizopods, as indicated above. It is also possible that the c. vacuole functions in ionic regulation in marine Protozoa; the ionic compesition of marine protogans is unknown.

Further ericlence for osmotic function of the c. vacuole comes from its output of fluid under different natural conditions. Beth the maximum volume and the pulsition frequency of the c. vicuoles must be considered, to get a measure of the total water excretion. Further, measurements must be made 
in a hanging drop or in such al chamber that coverglass pressure and other experimental conditions do not influenec the rate. Neasurements must lxe made on nonfeeding individuals. Ithen these precautions are tahen, there is little doubt that fresh-water Protozoa climinate mone fludel by their c. vacu oles than do marine or endoprarasitic forms in notural media. A summary of all available data on vacuoliar output was made by Kitching. 1:at The the required to eliminate a quantity of water equal in volume to the body of the animal, for fifteen fresh-water species (except one), ranged between 4.1 and 53 minutes, whereas for four marine species it was $23+6$ to $t^{3}+4$ hours, and fon

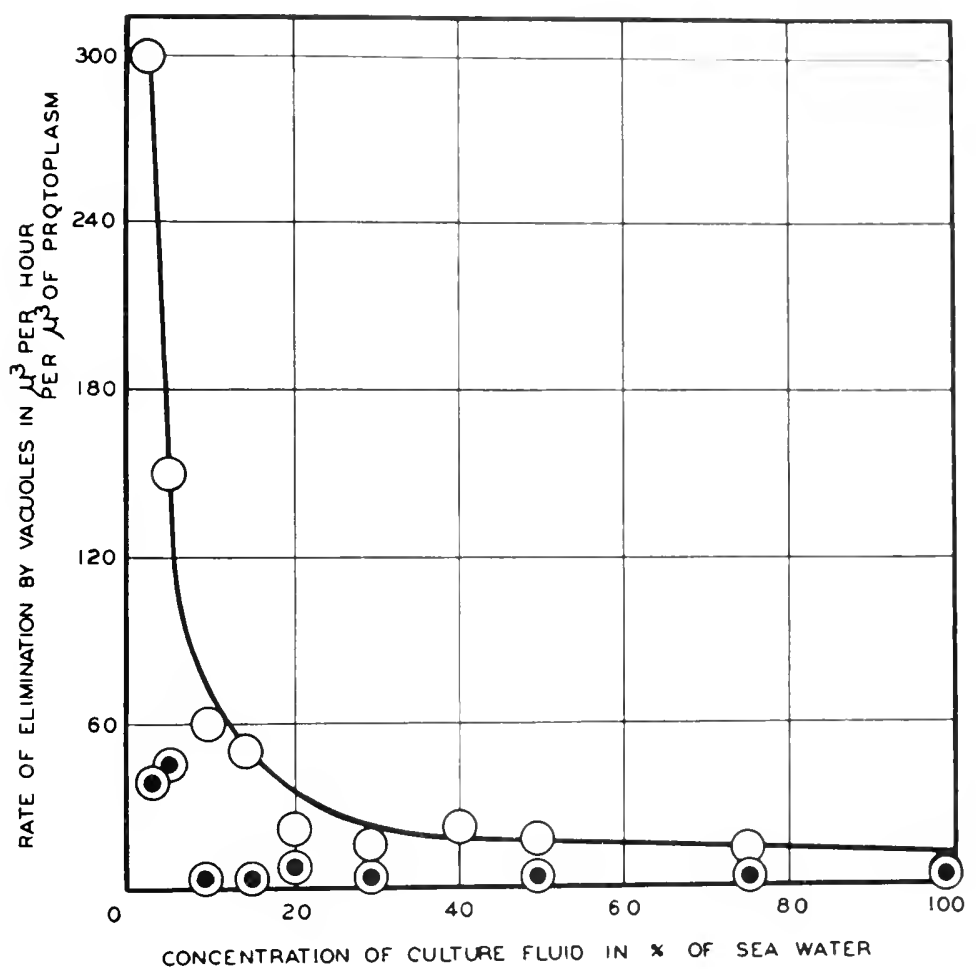

Fig. 18. Rate of excretion in cubic micra of fluid per hour per cubic micron of protoplasm in Amoeba mira adapted to different dilutions of sea water: $\bigcirc$ actively feeding specimens; 0 nonfeeding specimens. From Mast and Hopkins. ${ }^{175}$

one endoparasitic ciliate (measured in fresh watter) it was 4 hours. More recent data on marine and fresh-water forms are in agrement.

Further evidence for the osmoregulating function of c. vacuotes comes from data on Protozoa transferred experimentally to solutions of various osmotic concentrations. A few examples will be selected from the voluminous literature.

Numerous marine and parasitic Protozoa can be cultured in media of different tonicities. Amoeba mira can live in distilled water or in sea water concentrated to one-tenth volume by boiling. The arerage size of the amocbae 
is not significantly different once they are adapted in media of different tonicities. Figure 18 gives the rate of fluid elimination as a function of external concentration. In actively feeding specimens "the rate of elimination is inversely lincarly proportional to the concentration of the culture fluid." 175

Vahlkampfia calkensi, an amoeba parasitic in the oyster digestive tract, was kept growing and multiplying in sea water on agar. ${ }^{107}$ Under these conditions it had no contractile vacuole. When it was transferred to a similar agar culture made up with tap water or distilled water, one or more vacuoles developed and pulsated regularly. The more active the amoebae were, the more frequent the pulsations.

Several species of marine Peritricha survive in dilute sea water. In a dilute medium the volume rises slightly; the vacuolar output initially rises, then decreases to a new steady value higher than that occurring in 100 per cent sea water (Fig. 19). It was possible to balance exactly the increased vacuolar output by adding a nonelectrolyte of proper concentration. Kalmus ${ }^{124}$ made

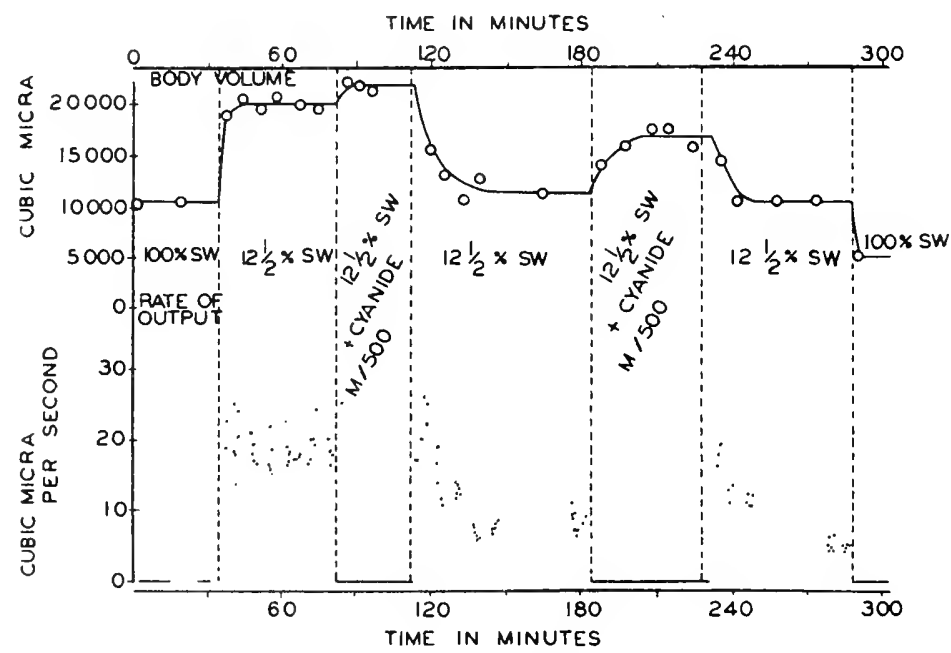

Fig. 19. Body volume in cubic micra (upper half of figure) and excretory output from contractile vacuole (lower half of figure) in marine peritrich Cothurnia successively in normal sea water, in $12 \frac{1}{2}$ per cent sea water, in $12 \frac{1}{2}$ per cent sea water containing $\mathrm{m} / 500$ cyanicle, in $12 \frac{1}{2}$ per cent sea water, in $12 \frac{1}{2}$ per cent sea water containing $\mathrm{m} / 500$ cyanide, in $12 \frac{1}{2}$ per cent sea water, and in normal sea water. From Kitching. ${ }^{135}$

similar observations on the marine ciliate Amphileptus gutta. After a week in 70 per cent sea water the vacuolar rate was 21 per cent higher than in normal sea water; in 60 per cent sea water and lower concentrations, hypervacuolization was noticed.

Numerous tresh-water P'rotozoa show effects the reverse of those shown by marine Protozoa. The fresh-water Amoeba verrucosa has been cultured in 10 per cent sea water. ${ }^{20 \text { ta }}$ When the sea water concentration was increased gradually the pulsation slowed and the contractile vacuole finally disappeared. No vacuole was seen when the animals were in 50 per cent sca water. When fresh water was added, the vacuole reappeared.

Similarly fresh-water peritrichous ciliates showed considerable slowing of 
pulsation of the c. vacuoles in very dilute sca water. In concentrations higher than 12 per cent sea water all pulsations stopped.134 Euplotes putella hept in 65 per cent sea water often contained a large vacuole which did not con tract. 254

More adaptation experiments have been carried out with Paramecium caudatum than with any other species. When Parrmecium is first put into at hypertonic solution the body becomes flattened as water is withdrawn but later recovers its normal shape. ${ }^{7 \pi}, 1: 65$ In increased external concentrations the amount of fluid expelled by the contractile vacuoles decreases. ${ }^{65}, 4.8,1046$ In Paranecium caudatum ${ }^{101}$ the vacuolar excretion of ffuid decreased in salt solutions of increasing concentrations as follows:

Vacuolar output of

fluid in equivalents

of body volume per hour

4.8

1.38

1.08

0.16
Per cent $\mathrm{NaCl}$ solution

0

0.5

0.75

1.0

Cultures of Paramecium woodruffi and Parameciun calkinsi were established in several dilutions of sea water. ix After 5 months the average intervals between pulsations of the c. vacuoles in $P$. woodruffi were 13 seconds in fresh water, 22 seconds in 25 per cent sea water, 32 seconds in 50 per cent sea water, 47 seconds in 75 per cent sea water, and 65 seconds in 100 per cent sea water. $P$. calkinsi showed an average interval of 23 seconds at all concentrations. Paranecinn candatum acclimated much less readily to sea water, although Frisch ${ }^{i 7}$ did culture it in 5 per cent sea water and allowed the water to evaporate so that in 24 to 42 days the culture medium was equivalent to 40 per cent sea water. The intervals between pulsations of the c. vacuoles lengthened from 11 seconds to 89 seconds, although some recovery of rate occurred later in the 40 per cent sea water. There may be specific salt effects in addition to tonicity. For example, in Paramecinm candatum 6.5 in glucose solution of $\Delta_{\text {f.p. }}=0.075$ the c. vacuole pulsated at $2.4-4.1 / \mathrm{min}$., whereas in Ringer solution of the same osmotic concentration the rate was $3.8-4.7 / 1 \mathrm{~min}$. and in $\mathrm{CaCl}_{2}$ of the same osmotic concentration it was $4.2-7.2 / \mathrm{min}$. Differences between pulsation rate in isosmotic $\mathrm{NaCl}$ and glycerol are reported for Glancoma colpoda (Degen, quoted by Metzner ${ }^{179}$ ). It appears, therefore, that the $\mathrm{c}$. vacuoles of Protozoa have an osmoregulating function but that other factors besides tonicity of the external medium may affect the amount of fluid excreted. In marine Protozoa c. vacuoles eliminate water taken in with food and may eliminate ions.

Avenues of Water Entrance. Three routes of entrance for water have been described: (1) across the body surface, (2) with food, and (3) through the cytopharynx membrane. All water must enter through the body surface in nonfeeding rhizopods and in mouthless parasitic ciliates (some of the Opatinidae). The values given previously for vacuolar output in different media are largely nonfeeding determinations. The permeability to water as given above is great enough that if the cells in fresh water had no vacuoles they could not survive long. For example, Pelomyxa carolinensis kept without food decreases about 8 per cent in volume every 24 hours for 3 days 
and loses by its contractile vacuoles 3.8 per cent of its body volume per hour.

Much of the water given off by c. vacuoles has entered with food. If marine Protozoa are isotonic, no water can enter by osmotic gradient. In Amoeba mira, for example, clear vacuoles appear while food is being digested; later the vacuoles expel their contents. ${ }^{175}$ No large vacuoles appear when the amoeba is not feeding (Fig. 18). In fresh-water Peritricha ${ }^{135}$ the rate of uptake of water by the food vacuoles is 8 to 20 per cent of the rate of fluid output by the c. vacuoles. In Paramecium the uptake by the food vacnoles may account for 30 per cent of the excreted water. of food vacuole formation and rate of c. vacuole elimination. ${ }^{11,} 76$

A third route of water intake, utilized by ciliates, is through the pharynx surface. Many parasitic ciliates which have no mouths also have no c. vacuoles, although this is not a universal rule. ${ }^{101}$ In Paranecium c. vacuoles are most active while the animals are at rest and pumping water toward the mouth, as judged by currents of India ink particles. ${ }^{76,77}$ The vacuoles are less active while the Protozoa are swimming and the peristome is partly closed. A group of parasitic ciliates, the Ophryoscolecidae, from the stomach of cattle are able to close the oral passage; when this is done the pulsation of their $\mathrm{c}$. vacuoles is very greatly slowed. ${ }^{157}$

Protozoa differ in the way they take in water. All the water to be excreted must have entered through the pellicle in mouthless and nonfeeding animals. All of it must enter through the food vacuoles in animals like Anoeba mira, which have no vacuoles when not feeding. Much water enters ciliates by way of the pharynx.

Mechanism of Filling of the Contractile Vacuole. There are three theories of the filling of c. vacuoles: (1) hydrostatic pressure, (2) secretion of water, and (3) secretion of solute with subsequent diffusion of water. These could be resolved if a method were available for removing the contents of a vacuole and measuring its osmotic concentration.

A hydrostatic filtration theory has been proposed. 200 The colloid osmotic pressure of the cytoplasm of Spirostomm is $2 \mathrm{~cm}$. $\mathrm{H}_{2} \mathrm{O}$ and the hydrostatic pressure is $4 \mathrm{~cm}$. $\mathrm{H}_{2} \mathrm{O}$. That protozoan cells have some turgidity is indicated by the varicty of their shapes. Hence the filtration pressure across a membrane which would not pass colloids would be the difference between the hydrostatic and the colloid osmotic pressure. However, as Kitching points out, the hydrostatic pressure would be no less if water is squeezed into a vacuole contained inside the cell. Also, vacuoles are often not spherical. This theory, therefore, cannot apply.

Secretory work is done in c. vacuole production. Evidence for this comes from the use of respiratory inhibitors. ${ }^{134,155,136}$ When the marine peritrich Cothurnia was transferred to 12.5 per cent sea water the volume increased and vacuolar output also rose; when cyanide was added the vacuolar output declined nearly to zero and the cell volume increased (Fig. 19). A fresh-water species reacted to cyanide by similar near-cessation of vacuolar pulsation and increase of volume, but when $0.05 \mathrm{M}$ sucrose was added, in addition to the cyanide, the volume remained constant eren though no vacuolar pulsations occurred. Other respiratory inhibitors such as $1 \mathrm{I}_{2} \mathrm{~S}$ and urethane had similar effects.

Secretion is further evidenced by the association of granules with vacuole 
formation. The granules in the vacuoles of Amoeha mira stain with Janus green B; similar granules (mitochondria or mitochondrialike oxeur aromel incipient contractile vacuoles in Amoeba moterus, and there is a reugh correld tion between vacuole frequency and number of granules. ${ }^{173}$ () smim-stain ing granules, presumably Golgi bodies, accur near the vacuokes of many cili

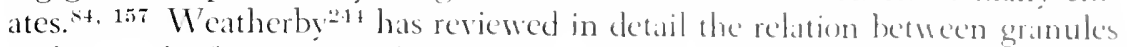
and vacuole formation. Frequently a small vacuole or resicle contains at granule which appears to dissolve as the resicle grows.

There is little doubt, then, that oxidative secretion is inveluced in filling the contractile vacuole in Protozoa, but whether solute or water or both are secreted remains to be demonstrated.

Kitching 1:it favors the hypothesis that water or very dilute sulution is secreted. Nonfecding Protozoa often continue to put out large quantities of water for days in a very dilute medium. When the baling out of water is stopped, the cells swell.

An alternative theory is that solute is secreted first and that water thenenters the small vacuole by diffusion. 10 , 17 In Amoebr mira the small vacuoles often originate in association with food vacuoles. The time required for a given increase in size of vacuoles in this species is less in 2.5 per cent than in 5 per cent sea water, hence Mast and I lophins concluck that in this marine species the cytoplasm is isotonic with the medium, that the vacuoles alle hypertonic when they start and are isotonic when fully grown. In nonfeceding specimens of Anoeba mira many small vacuoles are seen to contain granules and not to discharge water. When active feeding starts, these granules go into solution, becoming osmotically active, and the racuoles swell. When an Amoeba lacerta is transferred from 5 per cent sea water to 100 per cent sea water the size of the vacuole as well as the size of the amoeba decreases during a period of $1 / 2$ to 3 hours. ${ }^{10}$ By crushing under a corerslip free vacuotes are obtained; these swell in more dilute or shrink in higher concentrations of sea water. The vacuole of this species, then, appears to contain osmotically active material.

The rate of growth of racuoles has been said to be lincar; .12 .3 however, these measurements were taken at wide intervals. When very frequent measurements were made ${ }^{15 !}$ on the vacuole of Amoeha proteus it was found that the $\mathrm{c}$. vacuole grows in a stepwise fashion as the small vacuoles fusc with the c. vacuole. No change in size of the c. vacuole occurred during quiescent periods of as long as 120 seconds. This indicates that, unless the racuolar membrane is impermeable to water, the contents must be equimolar with the surrounding cytoplasm.

The principal argument against the theory of secretion of solute has been the supposed amount of solute needed. ${ }^{1: 3}$ Calculations based on the assump. tion that the final concentration of the vacuolar fluid is the same as that of the cytoplasm fail to support this objection. If the osmotically active material is assumed to have a molecular weight and density of one of the smaller amino acids (M.W. =100), for Amoeba proteus, which has a cytoplasmic concentra tion of about $0.05 \mathrm{M}$ and which excretes 110 cubic micra per second $39>$ $10^{-8} \mathrm{~cm}^{3} / \mathrm{hr}$.), the amount of such solute needed would be $1.1 \times 10$ : $\mathrm{cm} .{ }^{3} / \mathrm{hr}$. , whereas the volume of the animal is $2.5 \times 10^{-6} \mathrm{~cm} .{ }^{3}$ Nonfeeding
Pelomyxa carolinensis decrease about 8 per cent in volume every 24 hours. It 
is entirely possible, then, that sufficient solute could be secreted to permit the osmotic filling of the c. vacuoles. Similar calculations for marine and brackish protozoans are reasonable if it is assumed that the vacuolar fluid contains salt equivalent in concentration to the medium.

Efficiency of Contractile Vacuoles. The efficiency of c. vacuoles in volume and concentration regulation is not perfect. In cells such as marine eggs the product of the osmotically active volume times the concentration is a constant, $\mathrm{K}$. In Amoeba mira the volume increases initially in dilute medium but in an hour and a half adjustment has occurred so that the equilibrium volume is constant in all dilutions. In Amoeba mira the product of the vacuolar output $\left(\mu^{3} / \mathrm{sec}\right.$.) at equilibrium times the external concentration is exceedingly constant; this indicates that the osmotic concentration of $A$. mira and its water permeability are similar in sea water and in fresh water. ${ }^{175}$

When marine peritrichous ciliates are transferred to dilute sea water the vacuolar output is initially high, then declines to a little above the output in 100 per cent sea water while the body volume remains elevated (Fig. 19). When vacuolar output is multiplied by the osmolar concentration, $\mathrm{K}$ increases by several times in going from 100 per cent to 20 per cent sea water; $\mathrm{K}$ also increases, although by a smaller amount, when the body volume increase is considered. ${ }^{13 .},{ }^{135}$ The vacuolar output increases out of proportion to the decrease in external concentration. Other data ${ }^{18: 3}$ agree in showing an increasing $\mathrm{K}$ with decreasing concentration down to approximately 30 per cent sea water, but below this $\mathrm{K}$ decreased, probably because equilibrium was not established. Also in the fresh-water Rhabdostyla brevipes ${ }^{134}, 135 \mathrm{~K}$ is greater in dilute than in concentrated media. In these ciliates, the relative increase in water output in dilute solutions indicates an increase in rate of entrance of water. In the ciliates volume changes persist and the relation between excretion and concentration fails to be linear, whereas in Amoeba mira volume regulation occurs and the relation between excretion and tonicity is regular.

The species differences in water and salt permeability, the striking differences in adaptability among the three species of Paramecium mentioned above, the irregularity of pulsations of vacuoles of many marine forms in dilute media and the differences in reactions in different solutes indicate that contractile vacuoles are complex mechanisms. The process of filling may not be the same in all Protozoa in all media. Mlany questions will be answered when direct osmotic measurements of the vacuolar contents and of the cytoplasm are possible.

\section{AN INVASION OF THE OCEANS FROM FRESH WATER; FISHES}

The history of fishes represents a migration from fresh to salt water. Fishes as a group seem to have arisen in fresh water. During the Silurian and Devonian periods numerous groups of fish-ostracoderms, elasmobranchs, dipnoans, and ganoids-inhabited fresh water. Fossil records show that in the late Devonian and carly Carboniferous periods there were marine elasmobranchs but these died out and new migrations occurred in the Jurassic and Cretaceous periods. Bony fish have been in the ocean at most since the Cretaceous period. Some modern fish (teleosts) have reinvaded fresh water, whereas others (holostcans) have lived continuously in fresh water. Numerous migrations 
have occurred and certain marine fish are not independent of ficels water; some,

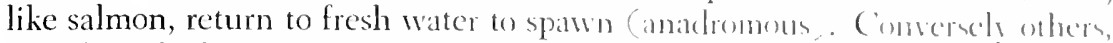
like the eels, breed in the ocean and reach maturity an alduls in tresh waller

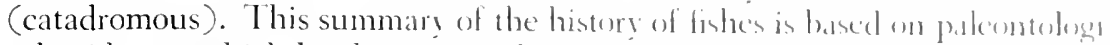
cal evidence, which has been strongly supported by observitions on onmorecus lation.2:4, 22:7 Much of the following account is from Sintt] ; mere recent work fully supports his hypotheses. The kidney arose in fresh water penente brates as an organ which gets rid of water, and in mo fisls is the hidnes of primary importance in excretion of nitrogenous wastes.

Fresh-Water Fish. In fresh water the bleod of fishes hils an osmolum (on) centration of 130-170 $\mathrm{mM}$, and the urine is copious but dilute. The gar pike, for example, in water of $\triangle_{n}=0.03$ has a blood concentration of $C_{2 i}-.19 .57 \mathrm{and}$ a urine concentration of $\Delta_{u}=0.08$. Volume of urine is high 200 for) $\mathrm{ml}$. kg./day). ${ }^{22+}$ Fresh-water fish are reported to drink little witter, althoughts measurements of the accumulation in the gut of colloidial material suspended in the medium indicate that groldfish do drink some water. "1" 'The shin is relatively impermeable to water, 62 but much water enters through gill and oral membranes.

Some fresh-water fish can withstand transfer to dilute sea watcr. Figure 2193 shows that when a carp is gradually introduced into water of increasing salinity the blood concentration increases only slightly at first, then in concen trations above $\triangle_{0}=0.6$ it adjusts to the medium. "On direct transfer to the salt medium the blood does not adjust readily when the concentration of the medium is greater than $\triangle_{n}=0.68$, and the condition of the fish is bad in ans concentrations higher than this.

The inward stream of water through the gill and oral membranes provides a water load, and the glomeruli of the kidneys normally filter a considerable volume while the tubules reabsorb most of the salts, kaving a dilute urine. However, the urine is not as dilute as the externat medium, lience salt loss must be compensated. Part of the salt comes from food. A second part of the needed salt is absorbed by special secretory cells located on the gills, as shown by the use of a chamber which separates the water bathing the anterior part from that bathing the posterior part of the fish. Krogh 1t1:i remored some of the salts from fresh-water fish of numcrous specics by kecping them in running dis tilled water. Salt loss was gradual, but occurred more rapidly if any shin injury had occurred. Much of the salt was lost by way of the kidneys, but with a divided chamber it was shown that some goes out through the gills. Krogh then put the fish into very dilute salt solutions and they absorbed chloride against a gradient. A catfish (Ameimrus), for cxample, which had previously lost considerable salt, took up salt from $1 \mathrm{mM} \mathrm{NaCl}$ or $\backslash \mathrm{albr}$. The roach (Leuciscus rutilus) in a medium of $\mathrm{Cl}^{-}$concentration of $0.0+2 \mathrm{mM} / \mathrm{h}$ egan to absorb chloride after considerable initial loss, and one fish took up chloride from as dilute a medium as $0.02 \mathrm{mMl}$. 'The goldfish (curassius auratus) conld reduce the $\mathrm{Cl}^{-}$concentration from $1 \mathrm{mM}$ to $0.02 \mathrm{mM}$. Ordinary chlorinated tap water contains about $0.3 \mathrm{mN}$ of $\mathrm{Cl}$ per liter, and pond water usually contains less. Acerina cermua, the ruffle, and the perch. Perea fluriatilis, con. tinued to lose salt in all dilute solutions tested and died after 10 to $1+$ dass without food, although there was some evidence of $\mathrm{Cl}$ alsorption from con centrations of $1 \mathrm{mMl}$ and higher. These fish must depend largely upon food to replace lost salt. 
Marine Fish. In the ocean the problem is to conserve water and exclude salts, and the water-excreting glomerular kidney is a liability. The blood of marine teleosts is not much more concentrated than that of fresh-water fish. In other words, a mechanism for maintaining a high degree of hypotonicity is necessary. The urine is scanty, 2.5 to $4 \mathrm{cc} . / \mathrm{kg} . / 24$ hours in sculpin and toadfish. "The urine is always hypotonic to the blood, and there is no relation between urine flow and $\mathrm{Cl}^{-}$content. Hence there must be some other route for getting rid of salt which enters osmotically. Smith":4 found, by the use of dyes and a divided chamber, that marine fish, unlike fresh-water ones, swallow large quantities of water and that both water and salt are absorbed from the intestine. The intestinal concentration decreases down the gut and approaches that of the blood, hence there is no significant separation of salt

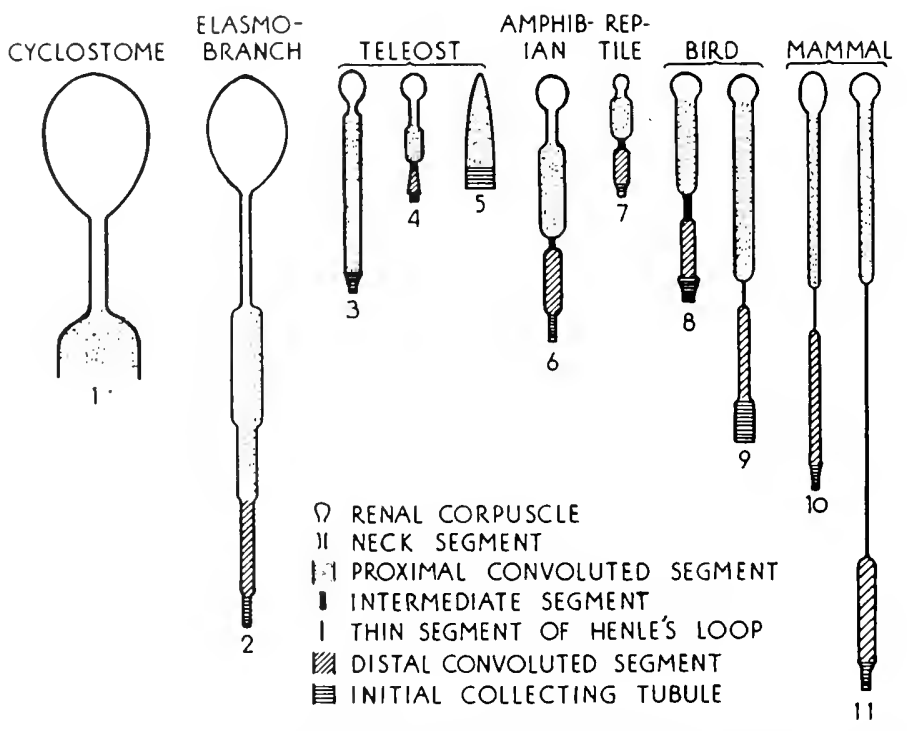

Fig. 20. Schematic representation of the kidney unit (nephron) of different vertebrates. (Elasmobranch modified from Kempton, ${ }^{124}$ others from Marshall..$^{170}$ )

from water and elimination of salt in the digestive tract. Nitrogenous wastes are excreted by way of the gills. When the pyloric end of the stomach is tied, urine formation falls off, body weight declines, and death results. ${ }^{s}$ The urine salts are largely $\mathrm{Mg}++, \mathrm{Ca}^{++}, \mathrm{SO}_{4}=$, and phosphate, and most of the $\mathrm{Na}^{+}, \mathrm{K}^{+}$, and $\mathrm{Cl}^{-}$absorbed must be excreted extrarenally. Also only part of the water drunk is exereted in urine. An eel in sea water excretes only 20 per cent of its fluich by the kidney. The sculpin, daddy sculpin, goosefish, haddock, and others all show zero or low $\mathrm{Cl}^{-}$in their urine, but with handling the fish become diuretic and $\mathrm{Cl}^{-}, \mathrm{Mg}^{++}, \mathrm{SO}_{4}=$, and phosphate in the urine are increased. 2 (2:2

In view of the reduced urine output it is not surprising to find less well developed kichneys in marine teleosts than in fresh-water fish. As shown in Figure 20, some of them lack glomeruli and the tubules are relatively short. ${ }^{171}$ In Opsanus tau, the toadfish, and Lophins, the goosefish, for example, glomeru- 
li are absent in adults, although there may be pseudoglomeruli in the voung. In the toadfish sts the urine flow is only $2.5 \mathrm{ml}$. heg. clat als comparced with $300 \mathrm{ml} . / \mathrm{kg}$. day in fresh-water caltish. Apparently the glomeruli degenerate with age and become nonfunctional. The upical marine telessean kidne? lacks the distal convoluted segment in the tubules. It is as if the ghmerulus. an adaptation for filtration of water, and the distal comvoluted tubule, which reabsorbs salt in fresh-water forms, have been lost with disuse. (inafllin ${ }^{-7}$ described a Siamese pipefish (Microphis hoaja) which has reinvaded fresh water and lacks both glomerulus and distal comvoluted segment; hence glom eruli are not a sine qua non for life in fresh water.

Excretion by an aglomerular kidney is essentially tubular secretion. Phenol red and numerous acid dyes are concentrated hy the aglomerular toadfisls kidney. ${ }^{105}$ A normal sculpin can excrete such a supersaturated $\mathrm{MgH}_{\mathrm{gP}} \mathrm{O}_{4}$ that the urine is turbid. ${ }^{202}$

That the gills are the route of extrarenal cxcretion was clearly shown in the eel by perfusion of a heart-gill preparation. ${ }^{131}$ Chloride is secreted outward into sea water containing nearly three times as much $\mathrm{Cl}$ as the blood. When the perfusion fluid in the blood ressels was increased in osmotic concentration, the chloride transported outward was increased. When eels are in fresh water the blood freezes at about $-0.11^{\circ}$ and at this internal concentration Kers found little or no outward secretion of $\mathrm{Cl}^{-}$. Thus the active chloride transport by the gills is an adaptation to marine and brackish-water life. It is calculated that the gill activity costs $0.1-0.3 \mathrm{cal} / \mathrm{gm}$. of gill tissue hr." confirmed Keys' experiments and showed that water and $\mathrm{Cl}^{-}$are transported independently by the gills. Secretion of $\mathrm{NaCl}$ could be stimulated by $\mathrm{NaCl}$, $\mathrm{Na}_{2} \mathrm{SO}_{4}$, glucose, or saccharose. In the epithelium of the gills of Fundulus are secretory cells which appear to eliminate chloride when the fish is in sea water and to take in chloride when the fish is in fresh water. Wh When the fish are in sea water or when fresh-water fish are injected with sodium chloride, these cells contain a distal vesicle which gives a histochemical test for chloride.

Marine teleosts, therefore, maintain blood concentrations similar to those of fresh-water teleosts; their kidneys still excrete urine hypotonic to the blood. The kidneys are, then, a liability, and the distal convoluted tubule and, in some fish, the glomeruli are lost. To rid themsclves of excess salt the fish drink much sea water and excrete by way of the gills (and possibly oral membranes, considerable salt and nitrogenous wastes. It may be that Amphibia have not successfully invaded the ocean because of the lack of a mechanism of extrarenal salt elimination.

Anadromous and Catadromous Fish. Many fish can live in either fresh or salt water. In general, their curves relating intemal to extemal concentration are relatively flat. The blood of salmon in Nonterey Bay freezes at -0.762 , blood of the salmon from the head of the tidal water on the Sacramento River freezes at $-0.737^{\circ}$, while blood from fish at their fresh-water spawning grounds freezes at $-0.668^{\circ}$. 91 Thus the king salmon blood is diluted by about 12 per cent on going to its breeding territory. Comparable values were found for Atlantic salmon in brackish and fresh water. ${ }^{32}$

The killifish, Fundulus heteroclitus, lives well in either fresh or sea water. When it is transferred from one medium to the other there is a trunsitor initial gain (F.W.) or loss (S.W.) of weight. Acclimatization as based on body 
density measurement is accomplished in 6 hours on going to sea water or in 24 hours on going to fresh water. The body density decline in fresh water is due partly to chloride loss and partly to increase in gas volume in the swim bladder. Reverse changes occur on going to sea water. Thus marked changes in density can occur with little change in osmotic concentration. ${ }^{40}$

Both Ámerican and European eels breed in the Sargasso Sea of the midAtlantic; then the young fish migrate to fresh water where they mature. Osmotic adaptation in the European eel, Anguilla vulgaris, has been carefully studied. Figure 21 shows that the blood is hypotonic in sea water and hypertonic in fresh water. ${ }^{62}$ It changes in concentration in going from the ocean to fresh water by about the same amount as that of the king salmon. The osmoconcentration of blood of the eel is as follows: in sea water $\triangle_{i}=.73$, in fresh water $\triangle_{i}=.61$ (data from Keys ${ }^{133}$ ); in sea water $\triangle_{i}=.69$, in fresh water $\triangle_{i}=.62$ (data from Duval ${ }^{62}$ ) (Fig. 21).

A starving Anguilla in either fresh or sea water lost 0.2 to 0.5 per cent of its weight per day. ${ }^{133}$ When the esophagus was blocked with a balloon the eel died in sea water after a 12 per cent loss of weight in 3 days, whereas in fresh water it lived well but lost 0.7 per cent of its weight per day. Thus the eel does not drink fresh water but does drink sea water. When transferred from fresh to sea water there is a weight loss and a new equilibrium is reached in about 48 hours if drinking is permitted. Conversely, if the eel is transferred from sea to fresh water there is an initial weight increase. According to Duval about 75 per cent of the osmotic pressure of the blood is due to $\mathrm{NaCl}$, and the $\mathrm{NaCl}$ concentration of the blood is higher in sea water than in fresh water; the serum protein also is higher in sea water. ${ }^{133}$

The kidney of Anguilla has glomeruli, also a distal convoluted segment, and in fresh water its kidneys behave as do the kidneys in typical fresh-water fish. In addition, Anguilla has a very low skin and gill permeability to both chloride and water. Eels in distilled water lose chloride at a rate about onetwentieth the rate of loss from Salmo irideus. ${ }^{1+1 a}, 1+3$ Krogh was unable to obtain evidence for chloride absorption from dilute solutions, and unfed eels died even in dilute Ringer solution for lack of salt before they starved for organic material. It may be that the secretory epithelium can concentrate chloride outward against a gradient to sea water, but not inward. Duval and others maintained that the mucus which surrounds an eel is a protective barrier which decreases permeability to water and to salt, but Krogh believes that the increased permeability after mucus removal is due to skin injury in the removal. Anguilla, then, in the ocean is hypotonic, swallows sea water, and gets rid of salt by the gills. In fresh water it excretes a dilute urine with its glomerular kidneys, replaces salt by leeding, and shows rery low skin permeability to salt and water.

Elasmobranchs and Cyclostomes. Elasmobranch fishes are today largely marine, although paleontelegical cridence indicates that they were once more abundant in l'resh water.

Osmoregulation in clasmolum ths is resicwad by Smith. 2:- As shown in 'Table 2, the blood is alwe he pertonic to the medium.

The salt concentration rif the blood of both marine and fresh-water elasmobranchs is of the same oreter as that in fresh-water teleosts, but the osmotic pressure of the blood due to $\mathrm{NaCl}$ is only 41 to 47 per ent of the total. ${ }^{6: 2}$ 


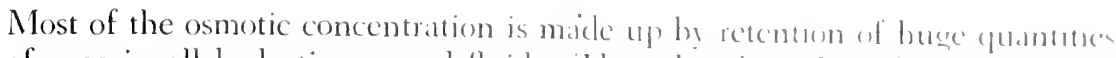

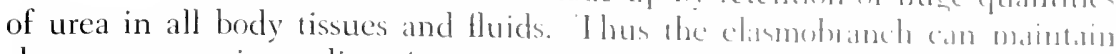
the same osmotic gradient in scal water ats in fresle 11 ater, and continumes on excrete a hypotonic urine. It was formerly believed that dhe hidnes unit hat

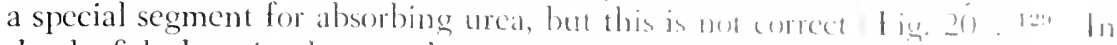
the dogfish there is a long neck segment, a wide prosimal tubule with scattered cilia, then a narrow proximal tubule followed hy a long distal mbule which winds around the neck segment. Smith estimates that an anerane daily filtrit

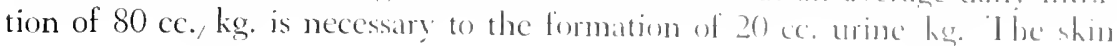

\section{TABLE 2. OSMOTIC CONCENTRATIONAND URIIINBOH IJ (II)S IN ELASMOBRANCHS HTOM S.IITHI}

\begin{tabular}{|c|c|c|c|c|c|}
\hline \multirow[b]{2}{*}{ Species } & \multirow[b]{2}{*}{$\triangle_{0}$} & \multirow[b]{2}{*}{$\triangle$ serum } & \multicolumn{2}{|c|}{ Blood } & \multirow[b]{2}{*}{$\Delta u$} \\
\hline & & & $\begin{array}{c}\mathrm{Cl} \\
\mathrm{m} .1 \mathrm{l} / \mathrm{li}\end{array}$ & $\begin{array}{l}\text { Urea } \\
\text { mg./li }\end{array}$ & \\
\hline $\begin{array}{l}\text { Raja stabuliforis } \\
\text { Raja diaphenes } \\
\text { Squalus acanthias } \\
\text { Raja sp. } \\
\text { Pristis microdon } \\
\text { Carcharlinus melanopterus } \\
\text { Torpedo ocellata } \\
\text { Torpedo marmorata } \\
\text { Scyllium canicula }\end{array}$ & $\begin{array}{l}1.85 \\
1.85 \\
1.33 \\
1.484 \\
0.0 \\
0.0\end{array}$ & $\begin{array}{l}1.93 \\
1.924 \\
1.622 \\
1.617 \\
1.02 \\
0.90\end{array}$ & $\begin{array}{l}273 \\
272 \\
234 \\
224 \\
170 \\
158\end{array}$ & $\begin{array}{c}2010 \\
2143 \\
1490 \\
1855 \\
780 \\
018 \\
1550-2000 \\
1450-18+0 \\
2080-26+0\end{array}$ & $\begin{array}{l}0.781 .06 \\
0.10\end{array}$ \\
\hline
\end{tabular}

and gills are relatively impermeable to urea and the young are provided with a store of urea, either by viviparity or by placement in an impermeable ege case containing urea until they develop their own renal mechanism for comsers ing it.

Smith studied elasmobranchs which live in fresh and brackish water in southeastern Asia. Table 2 shows that for a 100 per cent increase in osmotic pressure of the blood on going from fresh-water species to marine species the urea increases by nearly 100 per cent and the serum $\mathrm{Cl}$ by only 60 per cent. The urine output is low in marine elasmobranchs, and the urine is concentrated but always remains hypotonic to the blood. Magnesium, phosphate, and sulfate are excreted by the kidneys, other salts extrarenally.

The ability of individual rays and dogfish to adapt to changed tonicity of medium is not great. The dogtish Scyllitum increased in weight hy 10 per cent in 4 hours after transfer to 80 per cent sea water. ${ }^{11 .}$. The limits for Scyllimm are said to be 46 to 102 per cent sea water. ${ }^{62}$

Osmotic regulation in cyclostomes is not understood. Blood of the marine Myxine glutinosa is reported to be hypertonic "2-2t or slightly hypotonic to sca water. ${ }^{45}$ Another marine cyclostome Polistotrema stouti may lo slightll! hypertonic. ${ }^{224}$ The chloride concentration of the blood is lover than that of sea water, ${ }^{43}$ and the urea concentration is between that of clasmobranchs and that of the teleosts. ${ }^{4 .}$ Petromyzon fluriatilis from fresh water shows a serum freezing point of $-0.48^{\circ}$ (Dekhurzen, from Smith:" W's). When P. fluviatilis was put into 33 per cent sea water, $i:$ the blood concentrations rose from $\triangle_{i}=0.46$ to $\triangle_{i}=0.52$ after 22 hours, but irregular respiration indicated 
a weak condition. It is certain that marine cyclostomes do not maintain hypertonicity by urea retention, nor are they as dilute as teleosts.

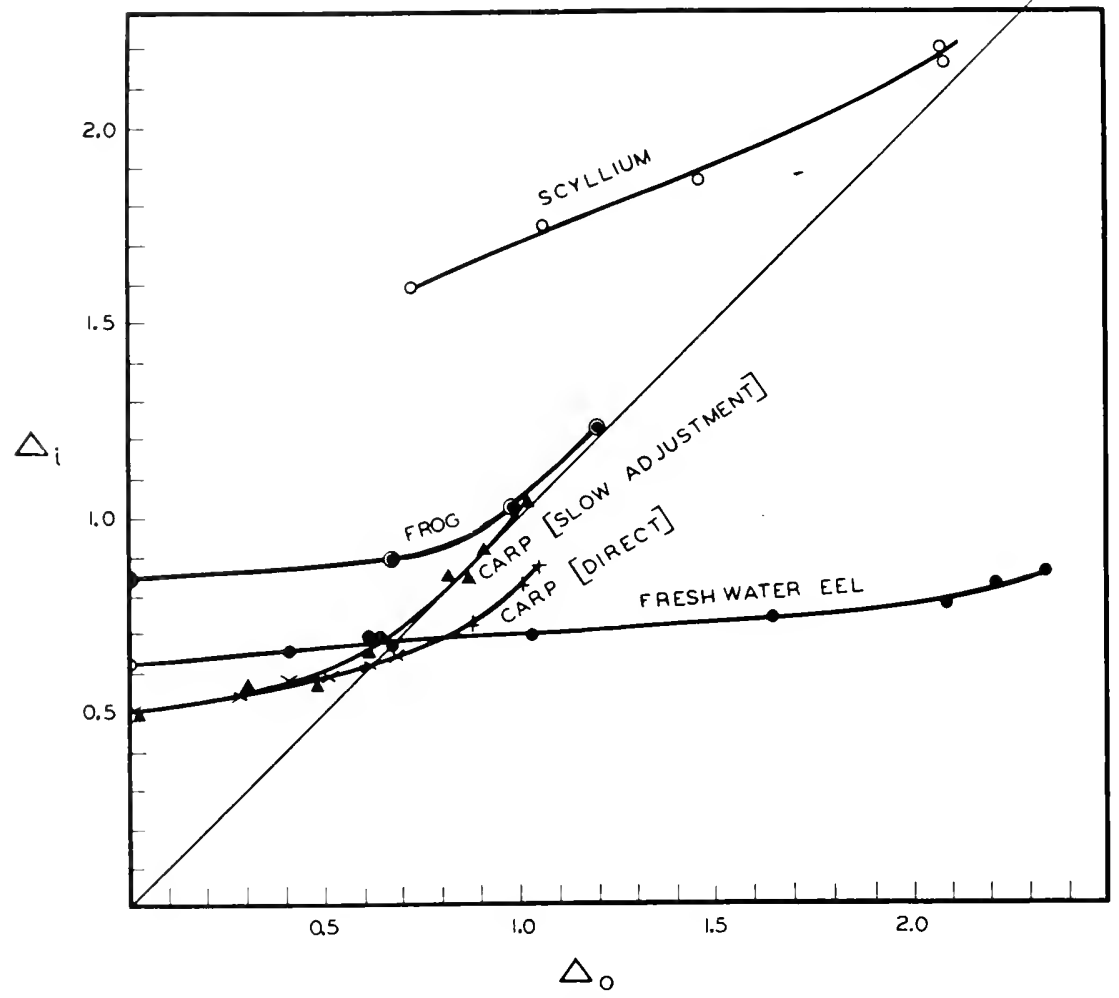

Fig. 21. Concentration of blood $\left(\triangle_{i}\right)$ as a function of external concentration $\left(\triangle_{0}\right)$ : $\checkmark$ the elasmobranch Scyllium; - in a fresh-water eel; in a carp $\times$ when transferred directly, and $\boldsymbol{\Delta}$ when adapted gradually to dilute sea water; and $\odot$ in a frog (Rana). Data from Duval. ${ }^{82}$

\section{A PREDOMINANTLY FRESH-WATER GROUP; AMPHIBIA}

The amphibia are a group of fresh-water animals. Some are permanently' aquatic; others spend at least a part of their life on land. Eggs are laid either in water or in a very moist environment.

The blood of a frog is hypertonic to pond water (Rana blood $\triangle_{\mathrm{i}}=0.563$, Bufo serum $\left.\triangle_{\mathrm{i}}=0.761\right)$. ${ }^{4 i}$ The urine is hypotonic to the blood and is more copious in a dilute than in a concentrated medium. Bottazzi adapted frogs to salt solutions, resulting in the following values:

$\begin{array}{ccc}\triangle . & \triangle i & \triangle u \\ 0.07 & 0.44 & 0.17 \\ 0.70 & 0.74 & 0.70\end{array}$

The osmotic behavior of frogs has been extensively studied. Kroght*3 summarizes Overton's observations, and Adolpht, 7 review's the work by himself and his students. If the cloaca of a frog is tied shut and the frog is left in pond or tap water, the body weight increases; when the cloaca is later 
untied, urine is voided and the weight falls to the original value. When the concentration of the medium is increased, cither by salt or sugar, the rate of gain of weight is decreased and in hypertonic solutions weight is lost. Adolph calculated that at $20^{\circ}$, water equivalent to 31 per cent of the body weight can enter and be excreted every 24 hours. This is only a small fraction of what would enter a true osmometer of similar concentration and surface. $A$ skinless frog gains weight faster than a normal frog. If the brain is destroyed or lesions are made in the midbrain or anterior medulla, but not in the spinal cord, the rate of water intake increases at once by as much as 4 to 5 times. Slime secre. tion may be important in decreasing skin permeability to water. Adolph found that a normal frog with cloaca open will gain weight for 25-50 hours when placed in a very dilute solution of $\mathrm{NaCl}$, but not in sucrose. Dilute $\mathrm{NaCl}$ seems to stimulate water uptake above water excretion, even though in distilled water the same frog may maintain a constant weight.

No amphibian has become totally independent of a moist medium, although many do not lay their eggs directly in pond water, and some toads live as adults in relatively dry air. Needham ${ }^{i n t}$, ist lists the moisture-conserving adaptations used by various Amphibia which do not lay their soft-coated eggs in pond water; they deposit eggs in leaf mold, in foam in holes in the ground, or between leaves which form a cup to collect moisture. The rate of water uptake by tadpoles during the first day after hatching is three times the rate in adults. Some desert Amphibia develop very rapidly and the adults burrow underground. ${ }^{50}$ One burrowing form, Chiroleptus platycephalus, "can store so much water in its urinary bladder, subcutaneous tissues and peritoneal cavity ... that it is occasionally used by the Australian aborigine as a source of drinking water." A few frogs are known to breed in brackish water, ${ }^{1: 8}$ but none are truly marine.

The water content is similar in terrestrial and aquatic anurans, but the terrestrial species survive greater loss of body water, as shown in Table 3. ${ }^{232}$

There have been numerous indications that the inward permeability of the amphibian skin for water is greater than its outward permeability, but experiments with heavy water on frogs ${ }^{104}$ and on isolated frog skin "11" led Krogh to conclude "that an irreciprocal permeability for water does not exist in normal frogs," although some water may be carried along with salt which is actively transported.

In fresh water or in hypotonic solutions frogs, like most fresh-water fish, do not drink water, but they absorb water through the skin; in hypertonic solutions $(140 \mathrm{mM} \mathrm{NaCl}$ ), they do drink and produce a concentrated (but blood-hypotonic) urine. Adolpht showed that the skin is limited protection against loss of water by evaporation in air. At 100 per cent humidity at $20^{\circ} \mathrm{C}$. one fifth of the heat produced in the metabolism of the frog is lost as latent heat of evaporation, hence the frog is continually at a higher temperature than the air and could never take up water from saturated air. However, water loss by way of the skin is reduced by high humidity. Lesions to the midbrain and anterior medulla increase skin permeability to water, possibly via the hypophysis. "

The posterior lobe of the pituitary (neurohypophysis) acts to increase the water content of amphibians. Injections of the active pituitary principle caused increase in weight in amphibia according to species in the following 
order: Necturus maculatus < Rana clamitans < Rana pipiens <Bufo americanus. "2!? This order is the same as the order of decreasing dependence on a water medium. Toads are unaffected by pituitary injections until the end of metamorphosis, when they become terrestrial. ${ }^{110}$ The hormone increases skin permeability to water in amphibians but probably not in other classes of vertebrates; it is found, however, in the pituitary of other vertebrates. ${ }^{100}$ The amphibian water balance principle has not been proved to decrease urine output and is different from the antidiuretic hormone of mammals. Permeability of the skin to water is greatly increased by the principle.

A frog, like a fish, can never excrete a salt-free urine. The salt is partly made good by food, but the ability for selective salt absorption is as well developed as in fresh-water lish. Isolated frog skin bathed by Ringer solution on each side transports chloride from the outside in. ${ }^{113}$ Oxidative energy is necessary for this process. Frogs kept in distilled water which is changed daily lose chloride by way of both skin and kidneys. The loss in

TABLE 3. VITAL LIMIT OF W'ATER LOSS

\begin{tabular}{l|c|c|c|l}
\hline \multicolumn{1}{c|}{ Species } & $\begin{array}{c}\text { \% Body Wt. } \\
\text { Lost }\end{array}$ & $\begin{array}{c}\text { \% Water } \\
\text { Lost }\end{array}$ & $\begin{array}{c}\text { Watcr } \\
\text { Content }\end{array}$ & \multicolumn{1}{|c}{ Habitat } \\
\hline Scaphiopus holbrookiii & +7.9 & 60.2 & 79.5 & terrestro-fossorial \\
Bufo borcas & 44.6 & 55.8 & 79.8 & terrestrial \\
Hyla regilla & 40.0 & 50.3 & 79.4 & terrestrial \\
Hyla cinerea & 39.3 & 49.0 & 80. I & terr.-arboreal \\
Rana pipiens & 35.5 & $4+.9$ & 78.9 & terr. semiaquatic \\
Rand aurora & 34.3 & +3.0 & 79.7 & semiaquatic \\
Rana grylio & 29.5 & 38 & 77.5 & aquatic \\
\hline
\end{tabular}

urine is relatively constant $\left(\mathrm{Cl}^{-}\right.$in urine 0.5 to 1 millimolar), whereas loss from the skin starts at a high rate and declines after 3 days to a very low level. ${ }^{1+11},{ }^{1+3}$ When the frogs are replaced in tap water or dilute Ringer solution an active uptake of salt occurs. In tap water or 0.01 Ringer solution, chloride was taken up at $0.05 \mu \mathrm{M} / \mathrm{hr} . / \mathrm{cm} .-2$ of body surface. ${ }^{2+1 \mathrm{~b}, 1+2,1+3}$ This ability actively to absorb sodium and chloride from dilute solutions must be inportant in replenishing the body salts during periods when food is not taken. Axolotls absorbed salts similarly.

In addition to salt replacement by food and selective absorption, Amphibia have a well developed ability to retain salt by their kidneys. The functioning of the amphibian (frog and Necturus) kidney has been extensively studicel by Richards $75,239,2+0,243$ and his associates, and by Höber (see summary in Chapter 35 of Physical Chemistry of Cells and Tissue ${ }^{160}$ ). Necturus is very suitable because of its small number of large (308 $\mu$ cliancter units. The glomeruli lie in a tayer close to the mesial border of the hicher. Beyond each glomerulus is a ciliated neck, then the thick-ivalled proximal tufule, then a narrow intermediate tubule, and finally the distal tubule which cmpties into a collecting tube (Fig. 20). Richards' group collected fluid from various regions of a kidney unit by means of a mercury- 
filled micropipette and analyzed each flund sample. In both frog and Necturus the glomerular fluid has essentially the same sugar and chloride concentration as the blood plasma; it is free of the normal hlosel proseims, although some small protein molecules can puss.i.n. 2:- $21: 3$ These lindings support the view that the glomerular fluid is an ulerafiltrate from the plasma, i.e., contains the blood solutes except for protein, and that the filtration pres sure is the difference between the bleod pressure and the colloid protein osmotic pressure of the bleod. The analyses by Richards and his ansociates of fluid collected at different regions of the tubules shenced that the dheriele content decreases along the distal tubule, hence chloride realosurption must occur in that region. Glucose diminishes in concentration earls in the proximal tubule. After injection of the drug phlorizin the glucese is mot reaborbed and actually increases in concentration so that the reducing perser of theid at the distal end of the proximal tubule is 25 per cent above that of the plasme in Necturus and to per cent above that of frog plisma, while at the distal end of the distal tubule reducing porver of the Huid is $2 \frac{1}{2}$ times that of the plisma

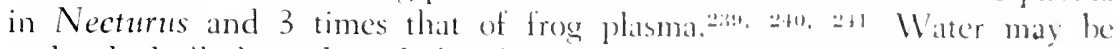
reabsorbed all along the tubule, about twice as much in the distal an in the proximal tubule. Acidification of the urine occurs in the distal tubules. The water reabsorption in Necturus is sufficient to account for the doubling of the concentration of urea. In the frog, however, the water reabsorption is insul ficient, and some urea is probably secreted by tubule cells. ${ }^{16: 2.2+1}$ Ureat excretion by frogs with renal arteries ligated is due to filtration from collateral circulation. "1:-

The mechanisms of osmoregulation in alquatic Amphibial can be summar ized as follows. Water enters osmotically but alt a retarded ratte, owing to low permeability of the normal skin. The water which does enter is excreted als a copious dilute urine. Salt is lost in the urine, and to a lesser degree by the skin. This salt loss is made good by food and by active absorption from pond or tap water. Also salt less is minimized by active reabsorption in the distal tubule of the chloride which filters through the glemeru!i. There is also some water reabsorption, which accounts for increased urine consecuntration of waste products that are not reabsorbed; under some conditions tubular secretion of specific substances may occur. Among these mechanisms at least four require expenditure of energy: maintenance of low shin permeability, active salt absorption by the skin, tubular reabsorption angainst a concentration gradient, and tubular secretion of specific wastes.

\section{ADAPTATIONS TO LAND, WITH SOME DIVERSIONS TO FRESH AND SALT WATER}

In two large groups of animals, certain land arthropods and the amniotes, adaptation away from an aquatic medium is complete or nearly so: their prob. $\mathrm{lem}$ is to retain water rather than to exclude it, and to aroid desiccation.

Terrestrial Arthropods. Of the land arthropords such as insects, spiders, scorpions, onychophorans, and isopods, the inscets are the animals in which the mechanisms of water retention and respenses to bumidity have been best

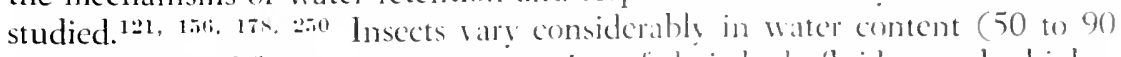
per cent). $2-5$ The osmotic concentration of their bod! fluids maly be bigher than that in any other group of animals. Osmotic concentrations viry with the 
stage of development, diet, humidity, season of year, and other factors, but the order of magnitude is illustrated in Table 4 , showing the freezing points. ${ }^{209}$

The principal loss of water from insects is by evaporation, and the most important mechanisms of water regulation are those limiting evaporative loss. Insects with a soft cuticle, such as many larvae, undoubtedly lose considerable water directly through the body surface. The chitin of most insects is nearly water impermeable, except at high temperatures at which changes in the cuticular wax occur. ${ }^{205}$ The greatest water loss is through the spiracles. When the spiracles are plugged by wax the water loss is reduced by about two-thirds. Most of the remaining loss occurs at the intersegmental furrows

TABLE 4

\begin{tabular}{l|l|l}
\hline \hline Group & \multicolumn{1}{|c|}{ Species } & $\Delta$ r.p. \\
\hline Insects & Carabus intricatus & 0.943 \\
& Tenebrio molitor (larva) & 1.165 \\
& Mantis religiosa & 0.885 \\
Scorpion & Ephestia elatella (larva) & 1.122 \\
Spiders & & 1.125 \\
Earthworm & & 0.894 \\
Snails & Helix pomatia & $0.3-0.75$ \\
& Limax maximus & 0.421 \\
& & 0.248 \\
\hline
\end{tabular}

(Bombyx mori; Gastrinargus ${ }^{250}$ ). Whenever spiracles are kept open, as by exposure to $\mathrm{CO}_{2}$ (in Rhodnint ${ }^{250}$ ) or by increase in metabolism by activity (in Blatta $^{205}$ ), the water loss is increased many times. Those insects which lack mechanisms for closing their spiracles lose more water than those which have spiracular control. Most insects excrete their nitrogenous wastes and either eliminate or retain feces in semisolid form, hence they lose little water as solvent for wastes and in air have no problem of salt loss or retention. It is probable ${ }^{24 s}$ that dilute urine is formed in the malpighian tubules and water reabsorbed in the rectal glands.

Since most water loss is by evaporation, the effects of relative humidity, air movement, and temperature are important. For example, Mellanby ${ }^{178}$ found that adult bedbugs (Cimex lectularius) at $8^{\circ}$ lose approximately 33 per cent of their body weight by evaporation in + hours at 0 per cent humidity and about 12 per cent in the same time at 90 per cent humidity. Grasshopper nymphs, potato beetles, and meal worms, but not lepidopteran caterpillars, may actually take up water at humidity above 82 per cent. ${ }^{1.5 \pi}$, 156 This hygroscopic property of some insects deserres more study. If water loss is normally low, death occurs at the same temperatures at all humidities; if water loss is normally high, the insect (Pediculus, Lucilia, and others) can tolerate a higher temperature at high humidity than at low humidity ${ }^{156},{ }^{178}$ (Figs. 88 and 89). The water content of meal moth larvae but not of pupae or adults varies with the humidity. ${ }^{13}$ In cockroaches, fleas, and butterflies, surface evaporation is proportional to saturation deficiency of the air (difference in $\mathrm{mm} . \mathrm{Hg}$ vapor pressure between saturation and actual humidity) up to about $30^{\circ}, 1: 1,: 05$ and survival time is inversely related to water loss. At higher temperatures water loss in Blatta with spiracles plugged increases very sharply owing to 
changes in cuticular wax, whereas the tracheal los increases gradually and very slowly with temperature. In other insects there is less correlation between water loss and saturation deficiency. ${ }^{2} 1,171$ The ability of inseets (n) survive starvation depends on both temperature and desiccation; these two factor probably act on different mechanisms and can be separated. At the sume rela tive humidity the absolute vapor pressure of the atmosphere increases with higher temperature, hence absolute humidity is more important than relative humidity. ${ }^{72,} 149$

Lost water is replaced by drinking in land insects and most insects take water in the food. However, some insects, such as clothes moths, wax moth larvae, and meal worms, get most of their required water metabolically. 'The complete oxidation of 100 grams of carbohydrate (glucose) yields 60 grams of water. Larvae of the flour moth (Ephestia) and of the beetles Tribolinm and Dermestes, and also Tenebrio, eat more and yet grow more slowly at low humidity. ${ }^{2} 149$

Another adaptive mechanism in osmotic regulation is the hehavioral tend. ency to select a region of optimal humidity. The optimal humidities for development and for survival are not necessarity the same, and they differ for various species. ${ }^{15}$ Insects taken from a moist environment appear more sensitive to dry air than do insects from a dry environment; grasshoppers range widely from hygrophilic to extremety xerophilic. Meal worm beetles, Tenebrio molitor, choose the drier side of a humidity gradient and distinguish smaller differences of humidity in the higher moisture range than in drier air. 45 Specific humidity receptors, probably pit and peg organs, are located on the antennae. Other insects which avoid moist air are the mosquito Culex fatigans, the cockroach Blatta orientalis, and the louse Pediculus humanus var. corporis. Wireworms (Agriotes), on the other hand, avoid the lower humidity in a gradient. They are able to distinguish differences as small as 0.5 per cent relative humidity by hygroreceptors located on the head. ${ }^{150}$ The grain beetle Ptinus also collects in drier regions of a humidity gradient. $3: 3$ The sign of the response may depend on the state of hydration (Blatta, Ptinus), the insect going to high humidity when desiccated or to a dry region when hydrated. 60

The onychophoran, Peripatopsis, lacks mechanisms for restraint of water loss by the trachea and actually loses water twice as fast as an earthworm, 40 times as fast as a smooth-skinned caterpillar, and 80 times as fast as a cockroach. ${ }^{167}$ Peripatus loses water half as fast as an earthworm, but twice as fast as a centipede, and 20 times as fast as a millepede. ${ }^{15:-2}$ Restriction to a damp environment may account for the lack of successful radiation of the Onychophora as a group.

Isopods occur in sea water, in fresh water, and on land. Their respiratory organs present extensive moist surfaces. The nephridial canals of a freshwater species are larger than those of a marine species, and in both the canals are larger than the canals in a land form. ${ }^{20 .}$. Terrestrial isopods lose water more readily and are more restricted to a moist environment than myriapods. A wood louse (Porcelio scaber) loses 4 per cent of its weight per hour in dry air, compared with 0.05 per cent loss by a meal worm, 0.14 per cent loss by a cockroach, or 1.8 per cent loss by an earthworm. "The wood louse goes to a region of higher humidity in a gradient.

The most important means of prevention of desiccation in insects and 
related animals are low permeability of the body surface, reabsorption of water during excretion, and selection and occupancy of regions of "optimal" humidity.

Reptiles, Birds, and Mammals. The water problem of a land vertebrate and of a land insect is, like that of marine fishes, retention of water, but without the complication of an inward salt gradient. Pearse ${ }^{196,}$, 197 has listed many of the factors in the transition from water to land.

Reptiles are more independent of a moist environment than Amphibia because of protection by horny scales against surface evaporation, and because their eggs, in most groups, are cleidoic (protected) or else the animals are viviparous. An intact newt loses water about as fast as a skinless lizard. ${ }^{89}$ Table 5 gives the blood and urine concentrations of a number of reptiles.

TABLE 5. FREEZING POINT LOWERING IN TURTLES

\begin{tabular}{l|c|c|c}
\hline \hline & $\Delta \circ$ & $\Delta_{i}$ & $\Delta$, \\
\hline Caretta rempi $^{224}$ & 1.85 & .66 & .64 \\
Caretta caretta $^{24}$ & 1.85 & .76 & .70 \\
Emys europea $^{4 i}$ & fresh water & .44 & .10 \\
Testudo graeca $^{224}$ & land & .60 & (from bladder) \\
\hline
\end{tabular}

These figures show that the blood of marine turtles is somewhat more concentrated than that of land and fresh-water turtles, and that the urine of the marine forms is relatively still more concentrated. There is great individual variation in concentration of tortoise blood." In no turtle, however, has the urine been reported as being hypertonic to the blood. The renal corpuseles of most land reptiles are poorly vascularized. ${ }^{171}$ These corpuscles are small and have connective tissue centers with capillaries on the outside only; vascularity is better in kidneys of turtles and crocodiles than in kidneys of snakes and lizards. The amount of urine excreted is small, and in snakes and lizards the urine may be solid or semisolid. In the alligator the urine flow is 0.4-1.2 $\mathrm{ml}$./kg./hr., compared with $1.5-20 \mathrm{ml}$. in Rana, and in the alligator glomerular filtrate is $1.5-3.4 \mathrm{ml}$. $/ \mathrm{kg}$. / hr. compared with $2.8-40.0 \mathrm{ml}$. in the frog. ${ }^{170}$ The loss of water from the skin of a tortoise is estimated to be only $0.1 \mathrm{ml} . / 100$ $\mathrm{cm} .{ }^{2} / \mathrm{hr} .{ }^{143}$ The principal loss of water from land reptiles, then, must be by way of the lungs, and desert reptiles get most of their water from food. Bux$\operatorname{ton}^{50}$ mentions an Australian lizard which can absorb water through the skin after a rain. Numerous reptiles-Chry'semis, Anolis, Phrynosoma, the alligator Cnemidophorus, and the garter snake Thamnophis sirtulis-all drink water (under experimental conditions) to compensate for a deficit. ${ }^{7}$ Amphibia in water deficit take up water only by skin absorption.

Both birds and mammals have the ability to excrete a hypertonic urine. Their kidneys, particularly those of mammals, have the thin loop of Ienle (Fig. 20). Renal corpuscles in birds are poorly vascularized, and filtration is low. The osmotic concentration of eloacal urine of the hen may be slightly higher than that of the blood. ${ }^{17}$ Korr ${ }^{1+0}$ found the osmotic concentration of the blood of a hen to be equivalent to 0.925-0.940 per cent $\mathrm{NaCl}$, and that of ureteral urine to be the equivalent of 0.95-1.1 per cent $\mathrm{NaCl}$, while normal 
glomerular filtration was $1.22 \mathrm{ml}, \mathrm{kg}$, min. and totil urine output was 25.70 $\mathrm{ml} . / \mathrm{kg}$. day. The urine concentration was higher during water depritation and after injection of pitressin from the posterior pituitary, hence water reab sorption occurs, and antidiuretic hormone increases water reabsorption. Water is also reabsorbed in the cloaca, ${ }^{17 i}$ and the urine may be a semisolid mass of uric acid crystals. Chick mesonephric tubules form cy'sts in tissue culture which accumulate water so that the cyst contents are 10 per cent more dilute than the medium, suggesting a water-secreting capacity. 13:3

The physical properties of yolk make osmotic measurements difficult. I ree ing-point measurements ${ }^{1 n}, 120$ indicate a difference between yolk and white; vapor pressure measurements show a higher osmotic concentration in the center of the yolk than just bencath the vitelline membrane, the mean for the

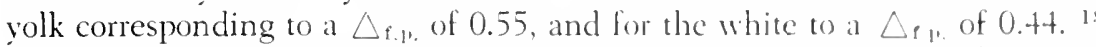
The difference is not maintained by metabolism but by slow diffusion, 2 "2:3n and some authors fail to find any osmotic difference between white and yolk. ${ }^{109}$

Water regulation in mammals has been studied cxtensively and reviewed frequently. The daily water loss of a man weighing $70 \mathrm{~kg}$. is $600-2000 \mathrm{ml}$. by the kidneys, 50-200 ml. by feces, 350-700 $\mathrm{ml}$. by insensible evaporation from the skin, $50-4000 \mathrm{ml}$. by sweat, and 350-400 $\mathrm{ml}$. from the lungs. A lactating mother may lose an additional $900 \mathrm{ml}$. as milk. Thus the total normal daily loss may range from 1 to more than 8 liters. "The total loss varies with temperature and humidity, and with various physiological conditions, and must be made up by water drunk, water in food, and metabolic water. Most common mammals have a blood concentration which is about 0.30 osmolar (equivalent to 0.95 per cent $\mathrm{NaCl}$ ). Dog plasma freezes at $-0.576^{\circ}(0.31$ osmolar). ${ }^{47}$ In man, urine concentration varies but is usually about 0.65 osmolar; the maximum concentration of urine in thirsting man is 1.4 osmolar $\left(\triangle_{u}=2.6\right)$. $\rightarrow$ Much water is reabsorbed atong with salts in the proximal tubule of the kidney (guinea pig), although the fluid distal to the loop of Henle may still be isotonic with the plasma and the site of urine concentration is not definitely known. "-2: The permeability of the skin of mammals to water is extremely low $: 34$ as measured both by transfer of heary water and by evaporative loss. Low permeability is particularly important in aquatic mammals. In man loss of 10 per cent of the body water causes serious illness, and 20 per cent loss results in death; much greater proportions of protein, fat, and glycogen can be lost with little ill effect. "N A water loss of $2+$ to 30 per cent of the body weight is fatal to a mouse.

The water load of the blood is kept delicately balanced by nervous and hormonal factors. When it rises by excess water intake a cliuresis appears after a certain time. This diuresis can be inhibited by emotional disturbance, by
epinephrine or tyramine. 235 Injections of hypertonic solutions of salt or sugar which cause only a 2 per cent or lower rise in osmotic concentration reduce the diuresis by 90 per cent. Osmoreceptors, apparently located in the brain, stimulate the liberation of antidiuretic homone from the posterior lobe of the pituitary which reduces urine production (sce (h. 22).

Water balances were compared in three rodents, Microtus, which inhabits dry prairie, Peromyscus, which lives on the forest floor, and Blarima, which is subterranean. ${ }^{51}$ Blarina shows the highest water turnover; Peromiscus 
vaporizes and drinks less, and Microtus, the least. High water exchange is associated with the greatest water supply and least evaporative forces in the subterranean habitat, while the greatest adaptation for water conservation permits life in the prairie grassland. The evaporative loss of water from the lungs of desert rats is half that from most rodents. ${ }^{219}$

The water relations of mammals, including man, in the 'desert have been examined. ${ }^{8}, 61$ The loss of water by skin evaporation and from the lungs parallels resting metabolism. The sweat glands are under control of the autonomic nervous system, and serve a cooling function. Active sweating in man normally begins when air temperature reaches about $86^{\circ} \mathrm{F}$. If evaporation is prevented at high temperature, as by high humidity or by rubber or oilskin clothing, the heart rate goes up and exhaustion occurs. Loss of water by the skin is necessary in temperature control, but sweating diverts blood to the skin from other areas, and by sweating the body loses salt. Both the salt and water lost must be replaced. A dog suffers more in the desert than does man. The dog loses only water, whereas man loses salt also, hence the dog's blood concentration rises. The dog loses water mostly from its mouth and respiratory passages, hence cooling and oxygenation are associated, and a panting dog may thus develop an alkalosis, whereas in man cooling and respiration are separate. Finally, the dog's fur-coated skin absorbs heat. A burro, on the other hand, has many sweat glands, but its sweat contains little salt, hence the blood chloride rises during work in heat. Buxton ${ }^{50}$ gives an extensive list of desert animals which may dispense entirely with drinking and get their water from food and possibly from dew. Camels apparently can live without water if there is plenty of good grazing, but at hard work Buxton claims that about 8 days is their limit without water. Fat produces more metabolic water per gram oxidized and yields more energy for a given amount of water than does carbohydrate; fat is often stored by desert mammals.

A man walking in the desert at $110^{\circ} \mathrm{F}$. loses one quart of water per hour; sweating increases by $20 \mathrm{gm}$. per hour for each $1^{\circ} \mathrm{F}$. rise in air temperature. ${ }^{8}$ During such exposure an exercising man does not voluntarily drink enough water to restore his water balance. Signs of serious dehydration are diminished blood volume, high concentration of blood cells, nausea, numbness, and inadequate circulation. If water loss exceeds 12 per cent of the body weight in dry heat a heat stroke is suffered. Water can be conserved if man ceases activity, avoids the sun, remains clothed and reduces urine output by reducing intake of protein and salt. In the tropical jungle where humidity is high, cooling cannot be effected by sweating, and dehydration is not significant; water intake is much less in the tropical jungle. ${ }^{180}$

There are striking species differences in the water drunk and the water excreted as a function of water deficit. Figure 22 shows the course of relative water load when drinking was permitted after periods of privation. The dog and burro rapidly drink more than they have lost, whereas man drinks less. ' Recovery from excessive hydration follows a different pattern; the rat recovers more rapidly than the dog. Desert animals (kangaroo rat and pocket mouse) on a dry diet excrete urine with chloride as concentrated as $900 \mathrm{mM}$, compared with maximum chloride concentration of $370 \mathrm{mM}$ in man and of $330 \mathrm{mM}$ in the dog. ${ }^{218}$

Marine mammals have blood only a little more concentrated than that of 
land mammals. The urine of marine mammals can be mene consentratted $1 l_{1,11}$

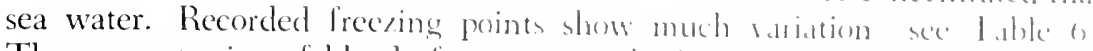

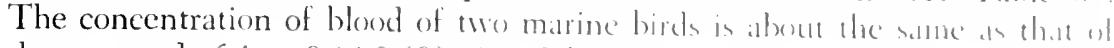

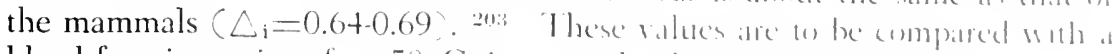

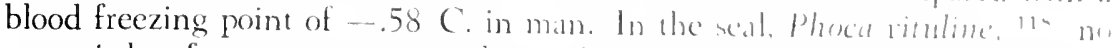

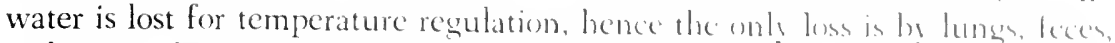
and urine. To saturate the air breathed from the lunge would tahe $160 \mathrm{gm}$. water; the feces require $200 \mathrm{gm}$. Water lor fish forch corresponcling (o) Iox) calories. When seals are feel herring which are so per cent water. the amount yielding 100 calories $(1250 \mathrm{gm}$. fish) would contain $1000 \mathrm{gm}$. Watter. Bs

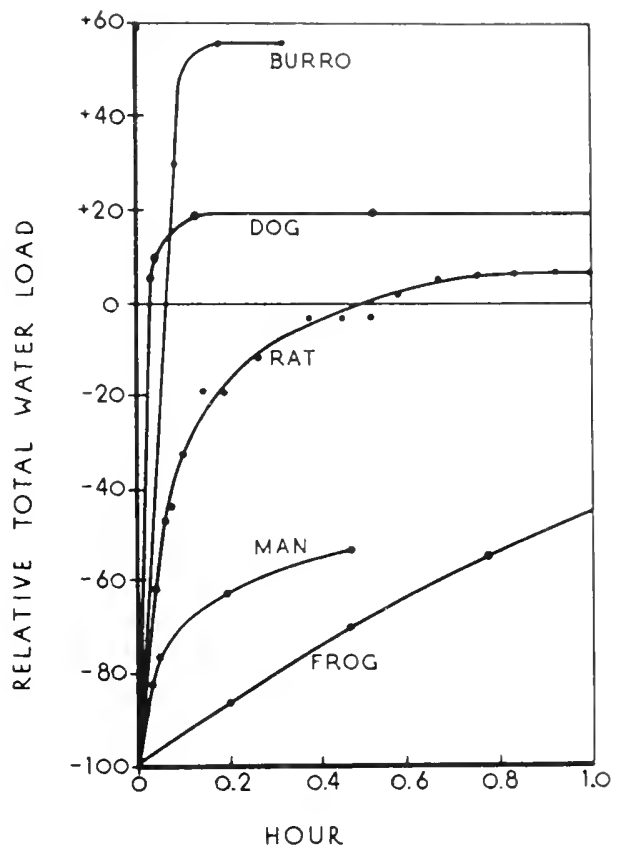

Fig. 22. Recovery of water load in per cent of initial load after access to water followins? periods of privation resulting in deficits equivalent to + to 8 per cent of body weight. From Adolph. ${ }^{\top}$

oxidative brcakdown the fat and protein would vield $121 \mathrm{gm}$. of metabolic water. Thus 1121 minus 306 leaves $815 \mathrm{gm}$. H.O for the urine. The food (1250 gm. of herring) would yield urea and salt sufficient to give in $500 \mathrm{ml}$. of urine a freezing point of -2.7 C. $(6.3$ per cent ureal and 1.4 per cent salt . Homer Smith 2:-1i observed that harbor seals when fasting excrete 0.030.11 $\mathrm{ml} . /$ urine/min. with a $\triangle_{n}$ of 1.948 , whereas after al meal of herring the rate of excretion increases to $0.08-1.28 \mathrm{ml}$. min., and the $\triangle_{11}$ rises to $1.98-3.646$. The magnesium and chloride of the urine and intestinal residue are so low that it is unlikely that any sea water has been swallowed. Thus marine mammals living on fish can get ample water from their food alone to keep their blood more dilute than the ocean. 
Krogh extended the calculation to the walrus and whalebone whales which feed largely on marine invertebrates that are more concentrated than fish and more concentrated cren than the blood of the mammals themselves. Less water would be lost from the lungs in the whale because, owing to the increased pressure under water, it can probably extract more of the oxygen and thus would not need to saturate so much air. Assuming a little less water loss by feces than in the scal, Krogh calculates that the kidney could easily excrete the salt and urea with the water arailable from its food. Freezing point depressions of 1.83-2.49 have been observed in urine of the pollack whale (Morimura, from Smith":-6). Dolphins given hypertonic salt solution by mouth had feces isotonic with blood, and in several hours 53 per cent of the

\section{TABLE 6. CONCENTRATIONS OF BLOOD AND URINE OF VARIOUS MIARINE MIAMMALS}

\begin{tabular}{l|c|c}
\hline \hline & $\Delta \mathrm{u}$ & $\Delta \mathrm{u}$ \\
\hline Whale (Delphinus phocaena) & \\
Whale (Balaenoptera sibbaldii) & 0.74 & \\
Whale (Balaenoptera borealis & 1.26 & \\
$\quad$ and Balaenoptera physalus) & & 2.46 \\
$\quad$ (Schmidt-Nielsen \& Holmsen, from Krogh $\left.{ }^{143}\right)$ & & 0.73 \\
Seal (Phoca barbata) & 0.65 & $0.75-4.5$ \\
Seal (Phoca foetida) & 0.70 & \\
Seal (Phocaena communis) & 0.74 & \\
$\quad$ (Rodier trom Portier & \\
Dolphin (Tursiops tursio) & 0.83 & \\
\hline
\end{tabular}

salt and $8+$ per cent of the water was excreted. "is The blood chloride changed very little, hence there must have been a shift of tissue water.

Mammalian urine can be more concentrated than sea water, hence the body should be able to extract some water from sea water and excrete salts. Indeed, Gamble so has found that, in man, utilization of sea water is not limited by the kidney but by the gastrointestinal system. It would be of interest to know the gastrointestinal tolerance of sea water in marine mammals.

In summary, reptiles, birds, and mammals have very low surface permeability to water. Birds and mammals are able to excrete a urine which is more concentrated than the blood. The primitive water-excreting function of the glomerulus has become diverted to a filtration-reabsorption system to excrete waste products with little loss of water. Hence the animals are able to get enough water by food or by drinking to supply their meager demands. The embryos are essentially aquatic but are protected against evaporative loss, in birds by the shell, and in most mammals by retention in uterine fluids.

\section{CONCLUSIONS}

When animals are arranged according to their osmotic performance (Table 7), certain correlations with their distribution and taxonomic relationships are apparent. Nany of the differences among animals from various habitats are differences in the degree of development of a capacity rather than in the specific kind of capacity.

Success in living in the ocean may accompany either osmotic lability or 
osmotic stability. If ocean water becomes too dilute, as m the upper Baltic, poikilosmotic animals disappear. The only ocean amimals in which a sliall gradient is maintained are marine vertebrates. Elasmohranchs, whub retan

TABIE 7

\begin{tabular}{|c|c|c|}
\hline $\begin{array}{c}\text { Osmotic } \\
\text { Characteristics }\end{array}$ & $\begin{array}{c}\text { Principal } \\
\text { Mechanisms }\end{array}$ & Examples \\
\hline \multirow[t]{2}{*}{ Osmotic adjustment } & No volume regulation & $\begin{array}{l}\text { Marine increbrate eges, } \\
\text { Phascolosoma }\end{array}$ \\
\hline & V'olume regulation & $\begin{array}{l}\text { Narine mulluses, Maja } \\
\text { Nereis pelagica } \\
\text { N. cultrifera }\end{array}$ \\
\hline \multirow[t]{2}{*}{ Limited osmoregulation } & $\begin{array}{l}\text { Low permeability; } \\
\text { salt reabsorption } \\
\text { in neplyridia? }\end{array}$ & N. diversicolor \\
\hline & W'ater storage & Gunda \\
\hline $\begin{array}{l}\text { Fair osmoregulation } \\
\text { in hypotonic media }\end{array}$ & $\begin{array}{l}\text { Selective absorption } \\
\text { of salts from mediun, } \\
\text { kidney reabsorption } \\
\text { or secretion, low } \\
\text { permeability }\end{array}$ & Carcinus \\
\hline $\begin{array}{l}\text { Regulation in hyper- } \\
\text { and in hypotonic media } \\
\text { except at extremes }\end{array}$ & Unknown & $\mathrm{U}_{\mathrm{ca}}$ \\
\hline \multirow[t]{2}{*}{$\begin{array}{l}\text { Unlimited regulation in } \\
\text { hypotonic media }\end{array}$} & $\begin{array}{l}\text { Hypotonic copious } \\
\text { urine, salt reabsorp- } \\
\text { tion or water secretion, } \\
\text { low surface } \\
\text { permeability }\end{array}$ & $\begin{array}{l}\text { Crazfish } \\
\text { Freshwater teleosts } \\
\text { Amphibia }\end{array}$ \\
\hline & Water inpermeability & Freshwater embryos \\
\hline $\begin{array}{l}\text { Maintenance of hyper- } \\
\text { tonicity in all media }\end{array}$ & Urea retention & Elasmobranclss \\
\hline \multirow[t]{2}{*}{$\begin{array}{l}\text { Regulation in hyper- } \\
\text { tonic media }\end{array}$} & $\begin{array}{l}\text { Extrarenal salt ex- } \\
\text { cretion, low water } \\
\text { intake }\end{array}$ & Marine teleosts \\
\hline & Unknown & Artemia \\
\hline $\begin{array}{l}\text { Regulation in moist } \\
\text { air }\end{array}$ & $\begin{array}{l}\text { Luw skin permeability, } \\
\text { salt absorption from } \\
\text { medium, salt reabsorption } \\
\text { in kidney }\end{array}$ & $\begin{array}{l}\text { Earrhworm } \\
\text { Frog }\end{array}$ \\
\hline \multirow[t]{2}{*}{ Regulation in dry air } & $\begin{array}{l}\text { Impermeable cuticle; } \\
\text { hypertonic urine }\end{array}$ & Insects \\
\hline & $\begin{array}{l}\text { Hypertonic urine, water } \\
\text { reabsorption in kidney }\end{array}$ & Birds and nummals \\
\hline
\end{tabular}

urea, have a gradient in the same direction as in fresh water. In other marine vertebrates some method of salt secretion exists and the blood is hypotonic. Marine teleosts excrete salt against a gradient by their gills, marine mammals by their kidneys. One important reason why insects and amphibians have 
not extensively invaded salt water is their lack of mechanisms for secreting salt outward. This is perhaps surprising in view of the ability of some of them actively to absorb salt against a gradient. It must mean that inward and outward permeability are physiologically separate.

Ability to live in salinities greater than that of sea water tequires ability to maintain a gradient of hypotonicity, as in brine shrimps. Some marine crabs such as U/ca are able to do this; a few others are hypotonic in normal sea water where the ability can be of little value. Apparently all land crabs can keep the blood hypotonic. Ability to maintain hypotonicity has developed several times among arthropods.

Little is known of osmotic relations in endoparasites, but some helminths show a limited osmoregulation. Gregarines swell and shrink with changes in the medium.

Success in fresh water requires osmotic regulation of such order that a high concentration of body fluids can be maintained against an extreme osmotic gradient. Such regulation requires active mechanisms for (1) low permeability to water, (2) water elimination, (3) salt retention, and (4) salt replacement. Fresh-water animals differ from marine in degree of development of these regulating mechanisms. Copious water elimination is necessary in all freshwater animals except in very impermeable eggs. Marine protozoans put into fresh water develop vacuoles or increase the output of vacuole systems already present. Marine Crustacea which can live in brackish water increase their urine output in dilute media. Fresh-water fish excrete a copious urine, whereas in some marine fish the kidney becomes almost vestigial. But nowhere is an animal known which can excrete a totally salt-free urine, hence there must be mechanisms for salt retention and salt replacement.

Salt retention can be brought about (1) by very low or negligible outward permeability to body salts, and (2) by salt reabsorption in the excretory organs. Low salt permeability is found in many marine animals. Salt reabsorption occurs in those marine animals whose kidneys function in the conservation of certain useful salts, but the power of salt reabsorption is much more highly developed in fresh-water animals, for example in the long tubules of the kidneys of fresh-water crustaceans and vertebrates.

Salt may be replaced from food or by active absorption from dilute solutions. Some fresh-water animals, for example Eubranchipus and Anguilla, seem to depend exclusisely, or almost so, on salt intake by food. In some insects there are special organs for salt absorption, as the anal papillae in Culex and Chironomus larvae. In many other fresh-water animals the respiratory epithelia contain secretory cells.

Success on land (in air) requires protection against loss of water from the body surface and from the respiratory membranes, and against excessive loss of water by excretion. The only truly successful land animals are higher arthropods (arachnids, myriapods, and insects) and higher vertebrates (reptiles, birds, and mammals). All others are largely restricted to an environment which is at least moist. Life in moist soil is osmotically similar to but less rigorous tham life in fresh water. The land arthropods are well protected against surface loss of water by their chitinous coat. Many aquatic Crustacea also have similat protection, and the entire group would perhaps succeed on land if it solved the respiratory problem. Some crabs do spend hours or days on 
land. (See Pearse ${ }^{197}$ for a discussion of migratens to laned. In the vertedrater, protection against surface drying is afforded by hormy seales. Feathers, and hair. All of the successful land animals also hare protection agdinst excessare water loss from their respiratory surfaces. Lack of such protection heep some groups (Onychophora, land Isopoda, Amphibia: restricted to a moist en vironment.

All of the really successful land dwellers seem to be able to retain water by excreting a concentrated urine. Ihis is functionally comparable to salt secretion by the gills of marine animals, but probably no marine animals except mammals excrete a hypertonic urine. Nany land insects excrete nitrogenous wastes in solid or semisolid form and probably reabsorb water in the posterior digestive tract. The kidney tubules and cloaca of birds and the tubules of mammals reabsorb water. Water reabsorption as a kidney function nccurs in many aquatic animals; water reabsorption accounts for urea concentration by the frog kidney, yet is insufficient to permit the frog to live in a dry environ ment. The importance of quantitative rather than qualitative differences in responses to osmotic stress is illustrated by the animals which live in atypical habitats. In Java there are soil sipunculids and land nereid polychactes."it In Java, Florida, and elsewhere there are land-dwelling hermit crabs. In Italy a typical brachyuran crab, Telphusa, lives in fresh water. Insect larvae and one adult Chironomus live in ocean waters near Samoa. There are desert amphibia, some of them ovoviviparous.in. thi Closely related snails dwell in salt, brackish, and fresh water and on land. The fact that many animal groups have representatives in atypical environments demonstrates the lability of osmotic characters.

No particular osmotic character is exclusively associated with life in any particular habitat. The functional superiority by which one group succeeds in an environment which is difficult osmotically, whereas another group is restricted to an easier environment, is quantitative. Sometimes new structures take over a given function; sometimes old structures become obsolete, but in each major environment some animals exist with the kind of capacities used especially in another environment. Whether a given group will succeed in a new environment depends on its ability to respond quantitatively to environmental stresses in making use of functions which it already possesses, rather than in developing totally new functions.

Within a species it is likely that individuals show certain quantitative differences. Some specimens of Carcinus excrete an isotonic, some a hypertonic, and some a hypotonic urine. Would those individuals of Carcinus which excrete a hypotonic urine be more likely to push up estuaries toward fresh water? Keys ${ }^{132}$ noted that some of a group of marine killifish (Fumdulus parvipinnis) readily become acclimated to fresh water, whereas others in the group perish. Combined field and laboratory observations of animals living in a long estuary showed individual and species differences in tolerance of sea-water dilution. ${ }^{233}$ Gammarus from open sea $\left(\triangle_{0}=1.79\right)$ tolerated dilutions down to 8 per cent sea water, whereas individuals from brackish water $\left(\triangle_{0}=0.978\right.$ ) survived dilutions down to 2 per cent sea water. Hya from the open sea were limited in tolerance to 52 per cent sea water, but Mya from brackish water tolerated sea-water dilution down to 36 per cent. The experi. mental data on osmotic regulation agree well with distributional data. A 
statistical study of the abilities of many individual members of groups which are in transitional stages, e.g., brackish water brachyurans, shore and estuarine snails, or some of the Anura (particularly toads), would provide evidence regarding the role of individual functional differences in invasion of new environments. Individual differences in response to osmotic stress may be cither physiological, representing the normal range of variation, or genetic, providing the means for appearance of new varieties or subspecies which may differ in range of distribution.

Osmotic regulation, then, comprises a group of very labile characters. Evolution of osmotic function has proceeded in many directions by many small changes.

\section{REFERENCES}

1. Арсоск, E. M., J. Exper. Biol. 17:449-463 (1940). Permeability of gregarines.

2. Adolph, E. F., J. Exper. Zool. 44:355-381 (1926). Water turnover by amoeba.

3. Adol ph, E. F., J. Exper. Zool. 47:31-62 (1927). Volume and osmotic regulation in earthworms.

4. Adolph, E. F., Biol. Rev. 8:224-240 (1933). Exchange of water in the frog.

5. Adolph, E. F., J. Cell. E Comp. Physiol. 5:123-139 (1934). Effect of nervous system on water exchange in frog.

6. Adolph, E. F., J. Cell. \& Comp. Physiol. 9:117-135 (1937). Osmotic relations in Phascolosoma.

7. Adolph, E. F., Physiological Regulations (1943). Lancaster, Pa., The Jacques Cattell Press, 502 p.

8. Adolph, E. F., ET AL., Physiology of Man in the Desert (1947). New York, Interscience Pub., 357 p.

9. Aldped, P., J. Exper. Biol. 17:223-226 (1940). Osmotic concentration of blood of various maminals.

10. Allee, W. C., and Frank, P., Physiol. Zool. 21:381-390 (1948). Drinking by fresh-water fish.

11. Andrejewa, C. W., Arch. Protist. 73:346-360 (1931). Functioning of contractile vacuole in Paramecium.

12. Arvanitaki, A., and Cardot, H., J. Physiol. Path. Gén. 30:577-592 (1932). Tonicity of snail blood.

13. Auber, L., and Raymont, J. E., Nature 153:314 (1944). Water content, Ephestia.

14. BAHL, K. N., Quart. J. Micr. Sci. 76:559-572 (1934). Significance of enteronephric nephridia in earthworms.

15. BanL, K. N., Quart. J. Micr. Sci. 85:343-389 (1945). Anatomy and physiology of nephridia of Oligochaeta.

16. BanL, K. N., Biol. Rev. 22:109-147 (1947). Excretion in earthworms.

17. Baldes, E. J., J. Sci. Instruments $11: 223-225$ (1934). Thermo-osmometer.

18. Baldes, E. J., Proc. Roy. Soc. Lond., B. 114:436-440 (1934). Osmotic pressures in hen's egg.

19. Ватемan, J. B., J. Exper. Biol. 9:124-127 (1932). Osmotic properties of medusae.

20. Batianan, J. B., and Keys, A., J. Physiol. 75:226-240 (1932). Chloride and osmotic relations in secretory activity of gills of eel.

21. Baumberger, J. P., and Dill, D. B., Physiol. Zool. 1:545-549 (1928), Changes in water and sugar content of crabs during molt.

22. Baumberger, J. P., and Olmsted, J. M. D., Physiol. Zool. 1:531-544 (1928). ()smotic changes in crabs during molt cycle.

23. Beadize, L. C., J. Exper. Biol. 8:211-227 (1931). Osmotic relations in Nereis.

24. Beadele, L. C., J. Exper. Biol. 11:382-396 (1934). Osmotie regulation in Gunda.

25. Bendele, L. C., J. Exper. Biol. 14:56-70 (1937). Volume and osmotic control in Nereis diversicolor. 
26. Beadle, L. C., J. Exper. Biol. 16:346362 (1939. Osmetic and chloride regulat tion in mosquito larvac from salt ponds.

27. Beadle, L. C., and Cragg, J. B., J. Fxper. Biol. 17:153-163 (19.40. Adaptatum of osmotic concentration and chlorinity in Ganmarus to salnity ol medium.

28. Beade, L. C., and CragG, J. B., Nature 1 $46: 588$ (19+0. (Osmotic and clalorule regulation in Gammarus.

29. Belda, W. H., The Salesianum 37:68-81 (19+2). Volume changes in Pelomyx. in different concentrations of medium.

30. Belda, W. H., The Salesianum 37:125-134 (19+2). Contractile vacuile of Pelomyxa.

31. Belda, W. H., The Salesianum 38:17-24 (1943,. P'ermeability al Pelomyxa tor water.

32. Bendett, E., Morruson, P., and lrving, L., Biol. Bull. 80:429-440 (1941). Osmotic concentration of salmon blood.

33. Bentley, E. W., J. Exper. Biol. 20:152-158 (1944). Reactions of grain bectle Ptinus in humidity gradient.

34. Berger, E., and Betine, A., Pfliig. Arch. ges. Physiol. 228:769-789 (1931). Permeability of body surface of animals for iodinc.

35. Bethe, A., Pfliig. Arch. ges. Physiol. $221: 34+362$ (1929 :. Ion permeability of marine crustaceans and molluscs.

36. Bethe, A., J. Gen. Physiol. 13:437-444 (1930). Permeability of molluscs and crustaceans.

37. Bethe, A., Pfliig. Arch. ges. Physiol. 234:629-64t (1934). P'ermeability of marine animals for salts and water.

38. Bethe, A., Holst, E., and Huf, E., Pfrig. Arch. ges. Plysiol. 235:330-334 (1935). Osmotic regulation, Carcinus.

39. Bialaszewicz, K., Arch. Internat. Physiol. 35:98-124 (1932). Urine formation, Maja.

40. Buack, V. S., Biol. Bull. 95:83-93 (19+8). Changes in density, chluride, weight, and swimbladder gas in Fundulus.

41. Boguck1, M., Arch. Internat. Physiol. 35:197-213 (1932). Water and salt balance in a marine isopod, Mesidotea.

42. Bogucki, M., Arch. Internat. Physiol. 38:172-179 (1934). Ionic and osmotic regulation in crayfish.

43. Bond, R. M., Carr, M. K., and Hutchinson, G. E., J. Exper. Biol. 9:12 14 (1932). Chloride in cyclostome blood.

44. Boné, G., and Koch, H. J., Ann. Soc. Roy. Zool. Belg. 73:73-87 (1942). Urine formation, aquatic insects.

45. BoreI, H., Ark. Zool. 28B:1-5 (1935). Composition of bluod of cyclostome, Myxine.

46. Botтazzi, F., Arch. Ital. Biol. 28:61-72 (1897). Osmotic concentration of blood of marine animals.

47. Borrazzi, F., Ergebn. Physiol. 7:161-402 (1908). Frecling points and conductivities of fluids of many organisms.

48. Brown, A. H., in Adolph's Physiology of Man in the Desert (1947). New Yorh, Interscience Pub., Ch. 17, 271-279. Maximum dehydration of man.

49. Buchanan, J. W., Biol. Bull. 60:309-326 (1931). Volume clanges of Planaria in different salinities.

50. Buxton, P. A., Animal Life in Deserts (1923). London, Arnold Co., 176 p.

51. Chew, R., Doctor's Thesis, Univ. Ill. (1948). The water exchanges of some small mammals.

52. Churney, L., Biol. Bull. 82:52-67 (1942). Osmotic swelling of nuclei, marine eggs.

53. Claus, A., Zool. Juhrb., Abt. allg. Zool. u. Physiol. 58:365-432 (1937). Osmotic responses of the water boatman, Sigura.

54. Conkrin, R., and Krogh, A., Ztschr. vergl. Physiol. 26:239-248 (1938). Osmotic behavior of Eriocheir and Mytilus.

55. Copeland, D. E., J. Morph. 82:201-228 (1948). Cytology of secretury cells in gills of Fundulus.

56. CuÉnot, L., Arch. Zool. 15:79-12+ (1898). Function of nephridia of earthwurms. 
57. Dakin, W., and Edmonds, E., Austral. J. Exper. Biol. \& Med. Sci. 18:169-187 (1931). Osmotic regulation and permeability to water and salts in snails and crustaceans.

58. Das, S. M., Biol. Bull. 95:307-319 (1948). Excretion in Molgula.

59. Dekiluyzen, M. C., Arch. Néerl. Physiol. 5:563-571 (1921). Osmotic relations in Sipunculus.

60. Dethler, V., and Chadwick, L., Physiol. Rev. 28:220-254 (1948). Chemoreception, hygroreception in insects.

61. Dill, D. B., Life, Heat and Altitude (1938). Harvard Univ. Press, 211 p.

62. Duval, M1., Ann. de l'Inst. Oceanogr. Monaco N. S. 2:1-231 (1925). Relation between medium and osmotic concentration in aquatic animals.

63. Duval, M., and Prenant, M., C. R. Acad. Sci. 182:96-98 (1926). Osmotic concentration of an ascidian.

64. Edmonds, E., Proc. Linn. Soc. New So. Wales 60:233-247 (1935). Relation between medium and osmotic concentration in blood of marine crustaceans.

65. Eisenberg, E., Arch. Biol. 35:441-464 (1925). Function of contractile vacuole in Paramecium.

66. Ellis, W. G., J. Exper. Biol. 14:340-350 (1937). Water and electrolyte exchange, Nereis diversicolor.

67. Ellis, W. G., J. Exper. Biol. 16:483-486 (1939). Water and electrolyte exchange in Nereis.

68. Fetcher, E. S., and Fetcher, G. W., J. Cell. \& Comp. Physiol. 19:123-130 $(19+2)$. Osmotic regulation of dolphins.

69. Florkin, M., Bull. Acad. Roy. Belg., Cl. Sci. 24:143-146 (1938). Osmotic concentration in blood of Anodonta.

70. Flonkin, M., and Duchateau, G., Physiol. Comp. e. Oecol. 1:29-45 (1948) Osmotic regulation in Anodonta.

71. Fox, D. L., Biol. Bull. 80:111-129 (1941). Tissue chloride in Mytilus.

72. Fraenkel, G., and Bleivett, M., Bull. Ent. Res. 35: 127-139 (1944). Effect of humidity on larvae of flour moth.

73. FrédéricQ, L., Bull. Acad. Belg., Cl. Sci. 428-454 (1901). Osmotic concentration of blood of marine animals.

74. Frédérice, L., Arch. Biol. 20:709-730 (1904). Osmotic concentration of blood of aquatic animals.

75. Freenian, B., Livingston, A. E., and Richards, A. N., J. Biol. Chem. 87:467-477 (1930). Chloride in frog plasma and glomerular filtrate

76. Frisch, J. A., Arch. Protist. 90: 123-161 (1937). Functioning of contractile vacuole in Paramecium.

77. Friscri, J. A., Arch. Protist. 93:38-71 (1939). Experimental adaption of Paramecium to sea water.

78. Friscil, J. A., Anat. Rec. 89:571 (1944). Vacuolar rates in several species of Paramecium adapted to different salinities.

79. Galloway, T. M., J. Exper. Biol. 10:313-316 (1933). Osmotic concentration of blood of cyclostome Petromyzon.

80. Gamble, J. L., Proc. Am. Phil. Soc. 88:151-158 (1944). Minimum water requirements for men at sea.

81. Garrey, W. E., Biol. Bull. 8:257-270 (1905). Osmotic concentration of blood of marine animals.

82. Gause, G. F., J. Exper. Zool. 87:85-100 (1941). Natural selection in acclimatization of Euplotes to different salinities.

83. Gaw, H. Z., Arch. Protist. 87:185-193 (1936). Effect of medium upon contractile vacuoles of ciliates.

84. Glizel, J. von, Arch. Protist. 64:479-494 (1928). Secretory granules and contractile vacuoles.

85. Gelfan, S., Protoplasma 4:192-200 (1928). Conductivity of protozoan cytoplasm.

86. Ghafflin, A., Am. J. Physiol. 97:602-610 (1931). Urine flow in marine fish.

87. Grafflin, A., J. Cell. \& Comp. Physiol. 9:469-475 (1937). Structure of kidney tubules of teleosts.

88. Graffinin, A., and Ennis, D., J. Cell. E Comp. Physiol. 4: 283-296 (1934). Effect of stomach bluckage on urine production in marine fish. 
89. Gray, J., J. Exper. Biol. 6:26-31 (1928). Water in the evolution of land vertebrates.

90. Gray, J., J. Exper. Biol. 9:277-299 (1932). Osmutic properties, trout egg'.

91. Greene, C. W., Plyysiol. Rev. 6:201.241 (1926). Physiolugy of spawning migration of salmon.

92. Gross, F., Arch. Protist. 83:178-196 (1934.. Acidity of Noctiluca.

93. Gueylard, F., C. R. Soc. Biol. Paris 89:78-80 (1923). Survival ut stickleback in different salinities.

94. Gunn, D. L., J. Exper. Biol. 14:178-186 (1937). Humidity reations of wool louse, Porcellio.

95. Gunn, D. L., and Pielou, D. P., J. Exper. Biol. 17:286-316 (1940,. Hlumidity behavior of mealworm beetle.

96. HaLl, F. G., Biol. Bull. 42:31-51 (1922). Vital linit of desiccation of terrestrial and aquatic animals.

97. Harms, J. W., and DragendolifF, A., Ztschr. wiss. Zool. 1+3:263.322 (1933). Osmotic characteristics of tropical land crabs.

98. Harnisch, O., Ztschr. vergl. Physiol. 21:281-295 (1935). Osmotic regulation in larvae of Chironomus.

99. Hayes, F. J., Ztschr. vergl. Physiol. 13:21+222 (1930). Respiration of Paramecium in different salinities.

100. Heller, H., Biol. Rev. 20:147-158 (1945). Effect of neurohypophysis un water balance in lower vertebrates.

101. Herfs, A., Arch. Protist. 44:227-260 (1922). The role of contractile vacuoles in acclimatization of Protozoa to different salinities.

102. Hermann, F., Ztsclir. vergl. Physiol. 14:479-524 (1931). Osmotic and volume regulation in crayfish.

103. Hess, M., Science 81:363 (1935). "Edema" in flatworms.

104. Hevesy, G., Hofer, E., and Krogh, A., Skand. Arch. Pliysiol. 72: 199-214 (1935). Permeability of skin of frogs to water.

105. Höber, R., J. Cell. \& Comp. Pliysiol. 6:117-128 (1935). Secretion of dyes by tubules of toadfish kidney.

106. Huber, R., Et al., Physical Chemistry of Cells and Tissue (1945). Philadelphia, Blakiston, $676 \mathrm{p}$.

107. Hogue, M. J., ). Elisha Mitchell Sci. Soc. 39:49-55 (1923). Contractile vacuole responses in an amoeba parasitic in oysters.

108. Hopkins, D. L., Biol. Bull. 90:158-176 (1946). Responses of contractile vacuoles to changes in medium (Anoeba).

109. Howard, E., J. Cell. \& Comp. Physiol. 24: $201-220$ (1944). Freczing point of egg yolk.

110. Howes, N. H., J. Exper. Biol. 17:128-138 (1940). Development of waterregulating mechanism in toads.

110a. Howes, N. H., and Wells, G. P., J. Exper. Biol. 11:327-344 (1934). Water relations of snails.

111. Howland, R. B., J. Exper. Zool. 40:251-262 (1924). Membrane of contractile vacuole of amoeba.

112. Howland, R. B., and Pollack, H., J. Exper. Zool. 48:441-458 (1927). Role of cytoplasmic viscosity in contractile vacuole discharge.

113. Huf, E., Pflïg. Arch. ges. Physiol. 237:143-166 (1936). Active transport of water and chloride through frog skin.

114. Huf, E., Pfling. Arch. ges. Physiol. 237:240-250 (1936). Lifiects of hydrustatic pressure on water permeability of body surface of Crustacea.

115. Hukuda, K., J. Exper. Biol. 9:61-68 (1932). Volume changes in marine animals.

116. Hykes, A. V., C. R. Soc. Biol. Paris 103:355-358 (1930). Salinity tolerance of ctenophores.

117. Hyman, L. H., Nature 151:140 (1943). Water content of jellyfish. Also Biol. Bull. 79:282-296 (1940).

118. Irving, L., Fisher, K. C., and McIntosh, F. C., J. Cell. \& Comp. Plissiol. 6: 387-391 (1935). Water balance in the seal.

119. Jeeps, M. W., Proc. Roy. Soc. Lond., B. 134:408-417 (1947). Contractile vacuoles in fresh-water sponges. 
120. Johlin, J. M., J. Gen. Physiol. 18:481-484 (1934). Osmotic relations in hen's egg.

121. Johnson, C. G., Biol. Rev. 17:151-177 (1942). Insect survival and water loss.

122. Jones, L. L., J. Cell. \& Comp. Physiol. 18:79-92 (1941). Osmotic regulation in crabs.

123. Jürgens, O., Zool. Jahrb., Abt. allg. Zool. u. Physiol. 55:1-46 (1935). Respiration, osmoregulation and nephridia of Nereis.

124. Kalmus, H., Arch. Protist. 66:409-420 (1929). Contractile vacuole responses in a marine ciliate.

125. Kamada, T., J. Exper. Biol. 10:75-78 (1933). Osmotic concentration of blood of snail.

126. Kamada, T., J. Fac. Sci. Imp. Univ. Tokyo 4:49-61 (1935). Pesponses of contractile vacuole of Paramecium to osmotic concentration of medium.

127. Kamada, T., J. Fac. Sci. Imp. Univ. Tokyo 4:195-202 (1936). Vacuolar output by Paramecium in different media.

128. Kempton, R. T., Am. J. Physiol. 119:175-185 (1937). Excretion by collateral circulation in frog kidney.

129. Kempton, R. T., J. Morph. 73:247-263 (1943). Structure of renal tubule in Squalus.

130. Keosian, J., J. Cell. E Comp. Physiol. 12:23-37 (1938). Secretion by chick niesonephric tubules in tissue culture.

131. Keys, A. B., Ztschr. vergl. Physiol. 15:352-363, 364-388 (1931). Chloride and water absorption and secretion by gills of eel.

132. Keys, A. B., Bull. Scripps Inst. Oceanog, Tech. Ser. 2:417-490 (1931). Selective action of decreased salinity, and distribution of killifish.

133. Keys, A. B., Proc. Roy. Soc. Lond., B 112:184-197 (1933). Osmotic regulation in the eel.

134. Kitching, J. A., J. Exper. Biol. $11: 364-381$ (1934). Osmotic relations in peritrich ciliates.

135. Kitching, J. A., J. Exper. Biol. 13:11-27 (1936). Volume control in marine peritrichous ciliates.

136. Kirching, J. A., J. Exper. Biol. 15:143-151 (1938). Water balance in freshwater Peritricha.

137. Kitching, J. A., Biol. Rev. 13:403-444 (1938). Contractile vacuoles.

138. Kocif, 11. J., J. Exper. Biol. 15:152-160 (1938). Absorption of chloride by anal papillae of dipteran larvae.

139. Korzuni, T., Sci. Rep. Tohoku Univ., Ser. IV, 7:259-311 (1932). Exchange of water and electrolytes in the holothurian Caudina.

140. Korr, I., J. Cell. \& Comp. Physiol. 13:175-193 (1939). Osmotic function of chicken kidney.

14la. Krogh, A., Ztschr. vergl. Physiol. 24: 656-666 (1937). Absorption of ions by fresh-water fish.

14lb. Krogir, A., Skand. Arch. Physiol. 76:60-73 (1937). Absorption of chloride by frog.

142. Krogr, A., Ztschr. vergl. Physiol. 25:335-350 (1938). Active absorption of ions by fresh-water animals.

143. Krogis, A., Osmotic Regulation in Aquatic Animals (1939). Cambridge Univ. Press, 242 p.

144. Krogil, A., et Al., Skand. Arch. Physiol. 80:214-222 (1938). Permeability of fish eggs.

145. Krogil, A., Schimidt-Nielsen, K., and Zeuthen, E., Ztschr. vergl. Physiol 26: $230-238$ (1938). Osmotic properties of frogs' eggs.

146. Krogri, A., and Ussing, H., J. Exper. Biol. 14:35-37 (1937). Permeability of trout egges to water.

147. Kиомнотт, G. A., J. Morph. 72: 167-177 (1943). Protonephridia of turbellarians.

148. Kulnen, E. J., Arch. Néerl. Zool. 3:365-449 (1939). Osmotic behavior of Artemia.

149. Leclence, J., Arch. Internat. Physiol. 55:366-376, +12-419, 45 l-458 (1948). Eflects of humidity on larvae and adults of Tenebrio.

150. Lisis, A. A., J. Exper. Biol. 20:43-60 (1943). Reactions of wireworms to moisture. 
151. Lienemann, L. J., J. Cell. E Comip. Phyciol. 11:149161 (1938 . ()motic and ionic regulation in crayfish.

152. Loeb, J, and Wasteneys, 11., J. Biol. Chem. 21:223.238 (1915. Permeiluility to water of eggs of Fundulus.

153. Lowenstein, O., J. Exper. Biol. 12:217221 (1935). Effect of salinity on respiration of Gammarus.

154. Lucké, B., and McCutchinon, M., Physol. Re'r. 12:68-140 (1932. (iells as osmotic systems, permeability to water.

155. Lunwig, D., Physiol. Zool. 10:3+2-351 (1937). Ellect of hunidity on grass. hoppers.

156. Ludwig, D., Physiol. Zool. 18:103-135 (1945). Eflects al humulity upon incect.

157. Maclennan, R. F., Univ. Calif. Publ. \%ool. 39:205250 (1933) Cintracule vacuole cycle in ciliates from cattle rumen.

158. Machennan, R. F., Tr. Am. Micr. Soc. 63:187-198 (194t). Contractile vacuole cycle in a parasitic ciliate.

159. MacLennan, R. F., Physiol. Zool. 17: 260-269 (194t. Growth ol contracule vacuole in Amocba proteus.

160. Mlaloeuf, N. S. R., Ztschr. vergl. Physiol. 25:1.28 (1938). Osmotic adjustment in Mytilus and Asterias.

161. Maluf, N. S. R., Zool. Jahuh., Abt. allg. Zool. 2l. Physiol. 59:515-53t (1939). Anatomy of kidney and absorption of sills by crayfish.

162. Maluf, N. S. R., Zool. Jahrb., Abt. allg. Zool. u. Physiol. 59:535-552 (1939). Volume and osmotic regulation in Lumbricus.

163. Maluf, N. S. R., J. Gen. Physiol. 24:151-167 (1940). Uptatie of salts by craty fish.

164. Maluf, N. S. R., J. Cell. E Comp. Plysiol. 16:175-187 (1940). Osmotic and volume regulation in Lumbricus.

165. Maluf, N.S. R., Biol. Bull. 81:134-148 (1941). Urine formation in craylish.

166. Manery, J. F., and Irving, L., J. Cell. \& Comp. Physiol. 5:457-46+ (1935). Water permeability of trout eggs.

167. Manton, S. M., and Ramisay, J. A., J. Exper. Biol. 1t:470-772 (1937). If ater loss from the onychophoran, Peripatopsis.

168. Margaria, R., Proc. Roy. Soc. Lond., B. 107:606-62+ (1931). Osmotic changes in marine animals.

169. Marshall, E. K., J. Cell. E Comp. Pliysiol. 2:349-353 (1932). Secretion of urea in trog.

170. Marshall, E. K., Physiol. Rev. 14:133-159 (1934). Comparative physiology of vertebrate kidney.

171. Marshall, E. K., and Smiti, H. WV., Biol. Bull. 59:135-153 (1930). Development of glomerular kidney in relation to habitat.

172. Mast, S. O., and Bowen, W. J., Biol. Bull. 87:188-222 (19tt). Cytoplasmic concentration in Vorticella.

173. Mast, S. O., and Doyle, W., Arch. Protist. 86:278.306 (1935). Relation of cytoplasmic granules to contractile vacuole in amoeba.

174. Nast, S. O., and Fowser, C., J. Cell. G Comp. Physiol. 6:151-167 (1935). Volume measurement, water permeability, Amoeba proteus.

175. Mast, S. O., and Hopkins, D. L., J. Cell. E Comp. Physiol. 17: 31-48 (1941). Osmotic adaptations in Amoeba mira.

176. Mayrs, E. B., J. Physiol. 58:276-287 (1924). Secretion in bird kidney.

177. Medwedewa, N. B., Ztschr. vergl. Physiol. 5:547-55+ (1927). Osmotic concentration of blood of Artemia.

178. Mellanby, K., Biol. Rev. 10:317-333 (1935). Loss of water from insects.

179. Metzner, P., Tabul. Biol. 4: 790-496 (1927). Contractile vacuoles.

180. Molnare, G. W., Adolpii, E. A., ET Al., Am. J. Hyg. 4t:411-433 (1946). Water, salt, and heat exchange in man in tropics and desert.

181. Morita, Y., and Chambers, R., Biol. Bull. 56: 64-67 (1929). Selective permea. bility of contractile vacuole membrane in Amoeba dubia.

182. Morrison, P. R., Biol. Bull. 91:181-188 (1946). Water loss and oxygen consumption by Peripatus. 
183. Mülller, R., Arch. Protist. 87: 345-382 (1936). Osmoregulation by contractile vacuoles in several Protozoa.

184. Nagel, H., Ztschr. vergl. Physiol. $21: 468-491$ (1934). Osmoregulation in Crustacea.

185. Needhan, J., Chemical Embryology (1931). Cambridge Univ. Press, 3 vols. Sections 5 and 6, pp. 777-945. Permeability of embryos.

186. Needham, J., Biol. Rev. 13:225-251 (1937). Review, evolution of osmotic and excretory function.

187. Neenham, J., Biochemistry and Morphogenesis (1942). Cambridge Univ. Press, pp. 32-63. Osmotic relations of eggs and embryos.

188. PAGAst, F., Zool. Jahrb., Abt. allg. Zool. u. Physiol. 56:183-218 (1936). Absorption of dyes by anal papillae of Aedes larvae.

189. Panikkar, N. K., Nature 144:866-867 (1939). Osmotic behavior of Palaemonetes.

190. Panikkar, N. K., Nature 145:108 (1940). Osmotic properties of the prawn, Leander.

191. Panikkar, N. K., J. Exper. Biol. 18:110-114 (1941). Osmotic behavior of fairy shrimp, Chirocephalus.

192. Panikkar, N. K., and Sproston, N. G., Parasitology 33:214-223 (1941). Osmotic relations of parasites.

193. Pantin, C. F. A., J. Exper. Biol. 8:63-72 (1931). Adaptation of the triclad Gunda to different salinities.

194. Pearse, A. S., Biol. Bull. 54:405-409 (1928). Adaptation of marine animals to brackish water.

195. Peanse, A. S., J. Elisha Mitchell Sci. Soc. 46:16I-166 (1931). Osmotic concentration of blood of crabs.

196. Pearse, A. S., Camegie Inst., Wash., Tortugas Papers 28:93-102 (1934). Osmotic concentration of blood of shore and estuarine animals.

197. Pearse, A. S., The Migrations of Animals from Sea to Land (1936). Durham, N. C., Duke Univ. Press, 176 p. Osmotic adaptations.

198. Peters, H., Ztschr. Morph. Okol. Tiere. 30:355-381 (1935). Structure and function of excretory organs of crayfish and lobster.

199. Picken, L. E. R., J. Exper. Biol. 13:309-328 (1936). Urine formation in arthropods.

200. Picken, L. E. R., J. Exper. Biol. 13: $387-392$ (1936). Osmotic concentration of cytoplasm of Spirostomum.

201. Picken, L. E. R., J. Exper. Biol. 14:20-34 (1937). Urine formation in freshwater molluscs.

202. Pitts, R. F., J. Cell. E Comp. Physiol. 4:389-395 (1934). Urinary composition of marine fish.

203. Pontier, P., J. Physiol. Path. Gén. 12:202-208 (1910). Osmotic concentration of blood of marine birds and mammals.

204. Prosser, C. L., Biol. Bull. 83:145-164 (1942). Uptake of dyes by Artemia.

205. Ranisay, J. A., J. Exper. Biol. 12:373-383 (1935). Evaporation from cockroach.

205a. Ramsay, J. A., J. Exper. Biol. 26:46-56 and 65-75 (1949). Osmotic relations and hypotonic urine formation, Lumbricus.

206. Rathbun, M. J., U. S. Nat. Mus. Bull. 129 (1925). Spider crabs of America. Ibid., 132 (1930). Cancroid crabs.

207. Raven, C. P., and Klomp, H., Proc. Kon. Nederl. Akad. Wtnsch. 49:101-109 (1946). Osmotic properties of eggs of Limnaea.

208. Rocenhofer, A., Arb. Zool. Inst. Wien. 17:139-156 (1909). Size of excretory organs in marine and fresh-water isopods.

209. RouschnL, W., Ztschr. wiss. Zool. 153:196-218 (1940). Osmotic concentration in fluids of terrestrial animals.

210. Rubinstein, D. L., and Miskinowa, T., Protoplasma 25:56-68 (1936). Water permeability of skin of frogs.

211. Sayles, L. P., Biol. Bull. 69:233-244 (1935). Effect of salinity on weight and survival of Nereis.

212. Schilienz, W., Arch. Hydrobiol. 14:429-452 (1923). Distribution of crabs in brackish water at the mouth of the Elbc. 
213. Schlieper, C., Ztschr. vergl. Physiol. 9:478-514 (1929). Effects of medium on osmotic concentration in marine animals.

214. Scillieper, C., Ztschr. vergl. Physiol. 18:682695 (1933). (Omotic function uf eel gill.

215. Schlieper, C., Biol. Rev. 10:334-359 (1935). Osmotic regulation and adjustment in aquatic animals.

216. Schmankewitsch, W., Zischir. wiss. Zool. 29:429.494 (1877). Size of gills and caudal spines in Artemia from different salinities.

217. Scrimid, P., J. Exper. Zool. 27:57-72 (1918). Desiccation of earthworms.

218. Schmidt-Nielsen, B., and Schmidt-Nielsin, K., Fed. Proc. 8:139140 (1949). Evaporative loss from desert animals.

219. Schmidt-Nielsen, K., Schuidt-Nielsin, B., and Schmejderman, H., Am.J. Physiol. 154:163-166 (1948). Salt excretion in desert rodents.

220. Scholles, W., Ztschr. vergl. Physiol. 19:552-554 (1933). Ionic regulation in Astacus and Eriocheir.

221. Schopfer, W. H., Rev. Suisse de Zool. 39:59-184 (1932). Osmotic properties of parasitic worms.

222. Schwabe, E., Ztschr. vergl. Physiol. 19:183-236 (1933). Osmotic regulation in crabs.

223. Shapiro, H., J. Gen. Physiol. 32:43-51 (1948). Osmotically inactive volume in sea urchin eggs.

224. Smith, H. W., Quart. Rev. Biol. 7:1-26 (1932). Evolution of vertebrate kidney.

225. Smith, H. W., Biol. Rev. 11: 19-82 (1936). Osmotic function of retained urea in elasmobranchs.

226. Smith, H. W., J. Cell. E Comp. Physiol. 7:465-474 (1936). Composition of urine of seal.

227. Smith, H. W., Lectures on the Kidney (1943). Lawrence, Kansas, Univ. Ext. Div., Univ. of Kansas, 134 p.

228. Smyth, J. D., J. Exper. Biol. 23:47-70 (1946). Weight changes of tapeworms in different salinities.

229. Steggerda, F. R., Proc. Soc. Exper. Biol. E Med. 36:103-106 (1937). Effect of pituitrin on water uptake by amphibians.

230. Svetrov, P., Arch. Entucklngsmech. I14:771-785 (1929). Osmotic concentration in trout embryos.

231. Taylor, C. V., J. Exper. Zool. 37:259-282 (1923). Relation of viscosity to contractile vacuoles in Euplotes.

232. Thorson, T., and Svinla, A., Ecology 24:374-381 (1943). Survival of amphibians after desiccation.

233. Topping, F. L., and Fuller, J. F., Biol. Bull. 82:372-384 (1942). Osmotic concentration as related to distribution of marine and brackish invertebrates.

234. Trolle, C., Skand. Arch. Physiol. 76:220-224, 225-246 (1937). Insensible water loss from man and dog.

235. Van Cleave, H. J., and Ross, E. L., J. Parasitol. 30:369-372 (1944). Volume responses of an acanthocephalan.

236. Viughan, T. W., Annual Report of the Sinithsonian Institution for 1917 (1919), pp. 189-276. Salinity tolerance by corals.

237. Verney, E. B., Proc. Roy. Soc. Lond., B. 135:25-106 (1947). Mechanisms of release of anticliuretic hormone in inammals.

238. Walker, A. M., J. Biol. Chem. 87:499-522 (1930). Comparisons, molecular concentrations glomerular urine and blood plasma, frog and Necturus.

239. Walker, A. M., and Hudson, C. L., Am. J. Physiol. 118:130-141 (1937). Absorption of glucose from renal tubules in amphibians.

240. Walker, A. M., and Hudson, C. L., Am. J. Physiol. 118:153-166, 167-173 (1937). Kidney function, amphibian.

241. Walker, A. M., Hudson, C. L., et al., Am. J. Physiol. 118:121-129 (1937). Total molar and chloride concentrations of fluid from amphibian renal tubules.

242. Walker, A. M., et Al., Am. J. Physiol. 134:562.579 (1941). Analysis of fluid from single nephrons of mammalian kidney.

243. Wearn, J. T., and Richards, A. N., Am. J. Physiol. 71:209-227 (1924). Composition of glomerular filtrate in frog. 
244. Weatherby, J. 11., in Protozoa in Biological Research, ed., G. N. Calkins (1941). Ch. VII, pp. 404-447. Cytoplasmic granules in relation to contractile vacuoles.

245. Webr, D. A., Proc. Roy. Soc. Lond., B. 129:107-136 (1940). Osmotic regulation, Carcinus.

246. Weil, E., and Pantin, C. F. A., J. Exper. Biol. 8:73-81 (1931). Adaptation of Gunda to salinity.

247. Wigglesworth, V. B., J. Exper. Biol. 10:1-15 (1933). Effects of salts on anal gills of mosquito larvae.

248. Wigglesworth, V. B., J. Exper. Biol. 10:16-26 (1933). Salt absorption as function of anal gills of mosquito larvae.

249. Wigglesworth, V. B., J. Exper. Biol. 15:235-247 (1938). Regulation of osmotic concentration and chloride in blood of mosquito larvae.

250. Wigglesworth, V. B., The Principles of Insect Physiology (1939). London, Methuen. 434 p.

251. Willem, V., and Minne, A., Mem. Acad. Roy. Belg. 58 (1900). Nephridial function, annelids.

252. Wolf, A. V., Ecology 19:233-242 (1938). Water balance in earthworms.

253. Wolf, A. V., Physiol. Zool. 13:294-308 (1940). Paths of water exchange in Lumbricus.

254. Yocom, H. B., Biol. Bull. 67:273-276 (1934). Acclimatization of fresh-water ciliates to sea water.

255. Zeuthen, G., Ztschr. vergl. Physiol. 26:537-547 (1939). Osmotic concentration of sponges in hibernation.

256. Zuelzer, M., Arch. Entwcklngsmech. 29:632-642 (1910). Effect of tonicity on rhizopod contractile vacuoles. 


\section{Inorganic Ions}

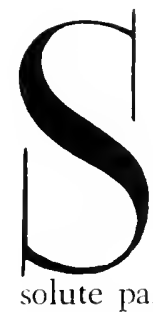

OME ANIMALS MANTAIN their internal osmotic concentration relatively constant in different media; others adjust to the medium. Osmotic pressure is proportional to the total concentration of blood of animals is contributed by inorganic ions. How do animals treat the different elements in the inorganic salts?

In general, living organisms tend to select certain elements and to exclude others. Ninety per cent of protoplasm is composed of carbon, hydrogen, and oxygen. There is little relation between abundance and availability, and only indirect correlation between availability and biological utilization. Nitrogen, although present in far greater amount in the atmosphere than is oxygen or carbon, is much less used. In large part, the chemical properties of an element determine its biological usefulness. Metals have only special uses; marine animals tend to accumulate potassium in relation to sodium and to cxclude magnesium and sulfate. The concentration of hydrogen ions tends to be higher in body fluids than in surrounding aqueous media.

\section{IONS IN BODY FLUIDS, EXTRACELLULAR AND INTRACELLULAR}

All cells appear to have an inorganic composition different from the fluids which bathe them; that is, cell membranes selectively regulate cytoplasmic ion composition. Examples of the differences between tissue and body fluid ions are given in Table 8.* Analytical separation of intracellular and extracellular ions is not always possible. Data for red blood cells (Table 8) show that these cells usually select potassium, although in some species (dog and cat) they have relatively more sodium. The extracellular volume in frog striated muscle has been estimated by histological methods to be 14.5 per cent of the total muscle; if the chloride in muscle were entirely extracellular and at the concentration of plasma chloride, the space it would occupy is 14.7 per cent in frog sartorius, 14.5 per cent in rat, and 12.5 per cent in cat, in remarkable agreement with the histological measurement. ${ }^{40}$ The concentration of potassium, cal. cium, and magnesium inside inuscle fibers is higher than in muscle as a whole (Table 827,119 ). The concentration of potassium is 50 times greater inside the fibers than in the interfibrillar space. The amount of chloride inside muscle fibers is nil 49,50 or is exceedingly small. ${ }^{-7}$ Microincincration datia ${ }^{10}$ agree with the analytical data that most of the calcium and magnesium of muscle

* Inorganic elements are here considered as ions as if in solution of comparable con centrations, although in body fluids unknown and variable amounts are bound to organic molecules. 
is inside the fibers. Chloride in all tissues is mainly extracellular and readily replaceable except in brain. ${ }^{2}$

\section{TABLE 8. CONCENTRATIONS OF IONS IN TISSUESS AND BODY FLUIDS}

\begin{tabular}{|c|c|c|c|c|c|c|c|}
\hline \multirow[b]{2}{*}{ Animal } & \multicolumn{7}{|c|}{ In $\mathrm{mMl} / \mathrm{kg}$. (except where stated as $\mathrm{mM} / \mathrm{l} . \mathrm{H}_{2} \mathrm{O}$ ) } \\
\hline & Tissue & $\mathrm{Na}$ & $\mathrm{K}$ & $\mathrm{Ca}$ & $\mathrm{Mg}$ & $\mathrm{Cl}$ & $\mathrm{SO}_{4}$ \\
\hline \multirow[t]{3}{*}{$\mathrm{cat}^{50}$} & plasma (in & 178.5 & 53 & 279 & 108 & $128 ?$ & \\
\hline & $\begin{array}{l}\mathrm{m}\left(\mathrm{M} / \mathrm{1} \cdot \mathrm{H}_{2} \mathrm{O}\right) \\
\text { whole muscle }\end{array}$ & $\begin{array}{r}1 / 8.5 \\
28.5\end{array}$ & 151. & $\begin{array}{l}2.19 \\
1.2\end{array}$ & $\begin{array}{l}1.08 \\
15.4\end{array}$ & $\begin{array}{l}12.8{ }^{\prime} \\
18.0\end{array}$ & \\
\hline & muscle fibers & 5.7 & 174. & 0.9 & 17.7 & 0 & \\
\hline \multirow[t]{3}{*}{$\operatorname{dog}^{47}$} & serum & 141.4 & 4.6 & 2.47 & 0.95 & 108.8 & \\
\hline & $\begin{array}{l}\text { cerebral } \\
\text { hemispheres }\end{array}$ & 51.0 & 95.6 & 1.07 & 5.63 & 36.7 & \\
\hline & $\begin{array}{c}\text { cerebellum, } \\
\text { brain stem }\end{array}$ & 50.8 & 92.7 & 1.07 & 5.40 & 35.2 & \\
\hline \multirow[t]{3}{*}{$\operatorname{man}^{110}$} & serum & 143 & 5. & 5. & 2.2 & 103 & 1 \\
\hline & $\begin{array}{l}\text { red blood } \\
\text { cells }\end{array}$ & 10 & 105 & 0 & 5.5 & 80 & \\
\hline & $\begin{array}{l}\text { cerebrospinal } \\
\text { fluid }\end{array}$ & 151 & 4 & 3 & 5 & 125 & 1 \\
\hline \multirow[t]{2}{*}{$\operatorname{rat}^{34}$} & plasma & 145 & 6.2 & 3.1 & 1.6 & 116 & \\
\hline & muscle & 26.6 & 101.4 & 1.5 & 11.0 & 16.3 & \\
\hline pig & $\begin{array}{l}\text { plasma } \\
\text { red blood }\end{array}$ & & 6.9 & & & & \\
\hline \multirow[t]{2}{*}{$\operatorname{dog}^{3 \pi, 8 y}$} & $\begin{array}{l}\text { cells } \\
\text { plasma }\end{array}$ & 11 & $\begin{array}{r}128 . \\
6.4\end{array}$ & & & & \\
\hline & $\begin{array}{l}\text { red Drood } \\
\text { cells }\end{array}$ & 107 & 6.9 & & & & \\
\hline $\mathrm{Cat}^{37,8 \mathrm{~B}^{\prime}}$ & $\begin{array}{l}\text { plasma } \\
\text { red blood } \\
\text { cells }\end{array}$ & 104 & $\begin{array}{l}6.67 \\
6.67\end{array}$ & & & & \\
\hline \multirow[t]{3}{*}{$\mathrm{frog}^{34}$} & plasma & 103.8 & 2.5 & 2.0 & 1.2 & 74.3 & 1.9 \\
\hline & muscle & 23.9 & 84.6 & 2.5 & 11.3 & 10.5 & 0.3 \\
\hline & $\begin{array}{l}\text { muscle fiber } \\
\text { water }\end{array}$ & 155 & 126.0 & 3.3 & 16.7 & 1.2 & \\
\hline Peri- & hemolymph & 169 & 27.1 & & & & \\
\hline planeta & serum & 109 & 17.3 & & & & \\
\hline (dog chow & muscle & 45.6 & 112 & & & & \\
\hline diet $)^{122}$ & nerve cord & 83.9 & 140 & & & & \\
\hline Loligo $^{85}$ & $\begin{array}{l}\text { blood } \\
\text { muscle } \\
\text { nerve }\end{array}$ & $\begin{array}{l}354 \\
53.6\end{array}$ & $\begin{array}{l}16.6 \\
113.7 \\
244\end{array}$ & & & $\begin{array}{r}469 \\
71\end{array}$ & \\
\hline sea water ${ }^{\theta}$ & & & 12 & & & 510 & \\
\hline Loligo $^{\theta}$ & $\begin{array}{l}\text { blood } \\
\text { giant nerve } \\
\text { fiber axo- } \\
\text { plasm }\end{array}$ & & $\begin{array}{r}17 \\
310\end{array}$ & & & $\begin{array}{l}530 \\
130\end{array}$ & \\
\hline sea water $^{82}$ & & 476 & 9.92 & 10.7 & 55.3 & 562 & 28.6 \\
\hline \multirow[t]{2}{*}{ Eledone $^{\mathrm{A} 2}$} & blood & 425 & 12.2 & 11.6 & 57.2 & 480 & 43.1 \\
\hline & muscle & 81 & 101. & 3.7 & 12.7 & 93 & \\
\hline \multirow[t]{2}{*}{ Anodonta ${ }^{62}$} & blood & 15.4 & 0.38 & 5.3 & 0.35 & 10.55 & 1.53 \\
\hline & muscle & 5.18 & 10.5 & 5.39 & 2.46 & 10.6 & \\
\hline sea water ${ }^{73}$ & & 465 & 11 & 11 & & 524 & 29 \\
\hline
\end{tabular}


CONCENTRATIONS OF IONS IN TISSULS AND BODY FLUIDS

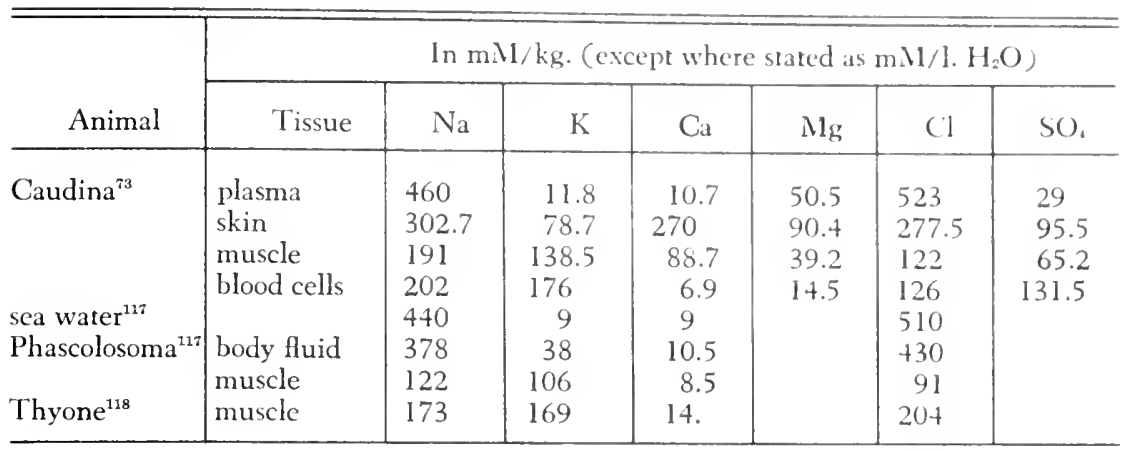

Numerous analyses of body tissues in a variety of animals show the tendency to concentrate potassium and calcium and to limit chloride and sodium (Table 8). In sixteen marine gastropods the concentration of potassium and calcium in the whole snail is uniformly higher than that of these elements in sea water, that of sodium is about the same, and that of magnesium is similar to or lower than that in sea water. ${ }^{49}$ The ability of cells to accumulate certain elements and to exclude others is general. In marine algae the vacuolar sap differs from the protoplasm, and both differ from the sea water. The mechanisms of ionic selection constitute important cellular problems and have been frequently: reviewed. ${ }^{37,}{ }^{9}$ Our present concern, however, is with the ionic correspondence between the body fluids of animals and their medium.

\section{IONS IN BODY FLUIDS AND IN EXTERNAL MEDIA}

Concentration of Ions. Table 9 presents representative analyses of ions in millimols per liter of body fluids of a variety of animals. In a general way the similarities are more striking than the differences among phyla and classes. Usually the total sum of cations exceeds the sum of all anions, the anion deficit being made up by proteins and amino acids.

The concentrations of different ions in the body fluids of an animal in a given medium may vary with the season and temperature, with physiological state, and with sex, age, and other conditions. The blood of crayfish, for example, taken in May and June, has a higher concentration of $\mathrm{Na}^{+}$and a lower concentration of $\mathrm{Ca}^{+}+$than that of erayfish taken in January and February. ${ }^{43}$ The blood of butterflies in the larval stage contains more $\mathrm{Mg}^{++}$ and $\mathrm{Cl}^{-}$but less $\mathrm{K}^{+}$and phosphate than does that in the pupal stage. "*

Insects are remarkable in the high potassium and low sodium in the scrum, the ratio of $\mathrm{Na}$ to $\mathrm{K}$ being consistently lower than that in frogs and mammals. A silkworm has in its body fluids about the same sodium concentration as the mulberry leaves on which it feeds, but on pupation it lises practically all of its sodium and the pupal tissues contain much potassium but negligible sodium. ${ }^{122}$ A series of carnirorous insects showed an $\mathrm{Na}$ to $\mathrm{K}$ ratio greater than 1 , and a series of herbirorous insects showed an Na to $\mathrm{K}$ ratio of less than 1. 24, 25.5 However, diet per se does not determine the $\mathrm{Na} / \mathrm{K}$ ratio, at 
least in the cockroach, $12: 3$ and whether the ratio is less or greater than 1 may be a genetic character.

Many cellular functions vary with the ratio of alkaline metals to alkaline earths. This ratio $[(\mathrm{Na}+\mathrm{K}) /(\mathrm{Ca}+\mathrm{Mg})]$ is higher in vertebrate bloods than in body fluids of invertebrate animals. "

\section{TABLE 9}

CONCENTRATION OF IONS IN SEA WATER AND IN BODY FLUIDS

For sea water, in $\mathrm{mM} / \mathrm{l}$.; in body fluids, in $\mathrm{mMl} / \mathrm{l}$. (except where stated as $\mathrm{mM} / \mathrm{l}$. $\mathrm{H}_{2} \mathrm{O}$ )

\begin{tabular}{|c|c|c|c|c|c|c|}
\hline & $\mathrm{Na}$ & $\mathrm{K}$ & $\mathrm{Ca}$ & $\mathrm{Mg}$ & $\mathrm{Cl}$ & $\mathrm{SO}_{4}$ \\
\hline & \multicolumn{6}{|c|}{ Sea Water } \\
\hline Millport ${ }^{101}$ & 447 & 9.55 & 9.81 & 51.3 & 525 & 34.1 \\
\hline Plymouth & 465 & 9.9 & 10.2 & 53.0 & 542 & 35.0 \\
\hline H lelgoland ${ }^{1.5}$ & 449 & 10.2 & 10.4 & 46.6 & 540 & \\
\hline St. Andrews, N.S. ${ }^{. ”}$ & 429 & 9.04 & 9.4 & 47.0 & 494 & 26.4 \\
\hline $\begin{array}{l}\text { Sal. Cove, Mle. } \\
\text { (Smith, in Cole }{ }^{31} \text { ) }\end{array}$ & 417 & 9.10 & 9.41 & 50.1 & 483 & 30.3 \\
\hline Sal. Cove, Me. ${ }^{31}$ & 457 & 8.5 & 9.68 & 33.8 & 492 & 25.6 \\
\hline Woods Hole & 383 & 10.5 & 10.7 & 53.4 & 524 & 27.2 \\
\hline$\quad 117$ & 440 & 9.0 & 9.0 & & 510 & \\
\hline Japan ${ }^{71}$ & 420 & 9.0 & 9.5 & 50.2 & 500 & 25.0 \\
\hline Marine & \multicolumn{6}{|c|}{ INVERTEBRATES } \\
\hline \multirow{2}{*}{\multicolumn{7}{|c|}{ Aureliast }} \\
\hline & & & & & & \\
\hline St. Andrews & 429 & 12.4 & 9.23 & 43.7 & 485 & 22.1 \\
\hline Casco & 402 & 12.3 & 9.56 & 45.3 & 487 & 20.2 \\
\hline Cyaneail & 420 & 14.9 & 9.6 & 50.2 & 540 & 10.5 \\
\hline Aequorea ${ }^{i t}$ & 430 & 23.0 & 9.5 & 50.2 & 530 & 22.3 \\
\hline Echinoderms & & & & & & \\
\hline $\begin{array}{l}\text { Echinus }^{101} \\
\qquad\left(\mathrm{mM} / \mathrm{l} . \mathrm{H}_{2} \mathrm{O}\right)\end{array}$ & 444 & 9.6 & 9.9 & 50.2 & 522 & 34.0 \\
\hline Echinus $^{15}$ & 530 & 13.3 & 12.8 & 50.3 & 549 & \\
\hline $\begin{array}{l}\text { Strongylocentrotus } \\
\text { (Smith, in Cole }{ }^{31} \text { ) }\end{array}$ & 420 & 9.7 & 3.58 & 47.1 & 488 & 24.0 \\
\hline Strongylocentrotus ${ }^{31}$ & 461 & 9.59 & 8.8 & 31.0 & 510 & 25.4 \\
\hline $\begin{array}{l}\text { Echinarachnius } \\
\text { (Smith, in Cole } e^{31} \text { ) }\end{array}$ & 417 & 9.22 & 9.37 & 49.3 & 488 & 29.9 \\
\hline $\begin{array}{l}\text { Sollaster } \\
\text { (Smith, in Cole }\end{array}$ & 421 & 9.65 & 9.58 & 49.6 & 488 & 30.0 \\
\hline $\begin{array}{l}\text { Chirodotia } \\
\text { (Smith, in Cole }\end{array}$ & 421 & 9.65 & 10.18 & 55.1 & 488 & 30.2 \\
\hline $\begin{array}{l}\text { Asterias v. } \\
\text { (Smith, in Cole }\end{array}$ & 412 & 9.56 & 9.31 & 50.0 & 488 & 30.1 \\
\hline Asterias v. ${ }^{31}$ & 460 & 8.26 & 9.00 & 30.7 & 505 & 25.6 \\
\hline $\begin{array}{l}\text { Cocumarial' } \\
\text { (Smith, in Cole }\end{array}$ & 420 & 9.7 & 9.31 & 50.1 & 487 & 29.9 \\
\hline Annelids and Sipunculids & & & & & & \\
\hline $\begin{array}{l}\text { Plyascolosomana } \\
\text { (Smith, in Cole }{ }^{311} \text { ) }\end{array}$ & 377 & 38.0 & 10.5 & & 430 & \\
\hline Amplitrite b. & 406 & 13.0 & 9.51 & 54.8 & 477 & 30.8 \\
\hline $\begin{array}{l}\text { Echiurus p. } \\
\text { (Sinith, in Colect) }\end{array}$ & 439 & 12.6 & 9.18 & +2.5 & 480 & 30.8 \\
\hline $\begin{array}{l}\text { Glycerat d. } \\
\text { (Sinith, in Colet })\end{array}$ & & 9.6 & 10.0 & 61.2 & 483 & 27.5 \\
\hline
\end{tabular}


TABH F 9 (continued)

CONCENTRATION OF IONS I SHA IIHIRANI)INB()I)Y II (HIOS

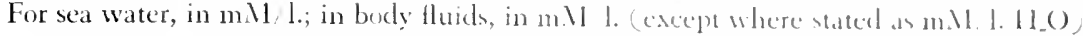

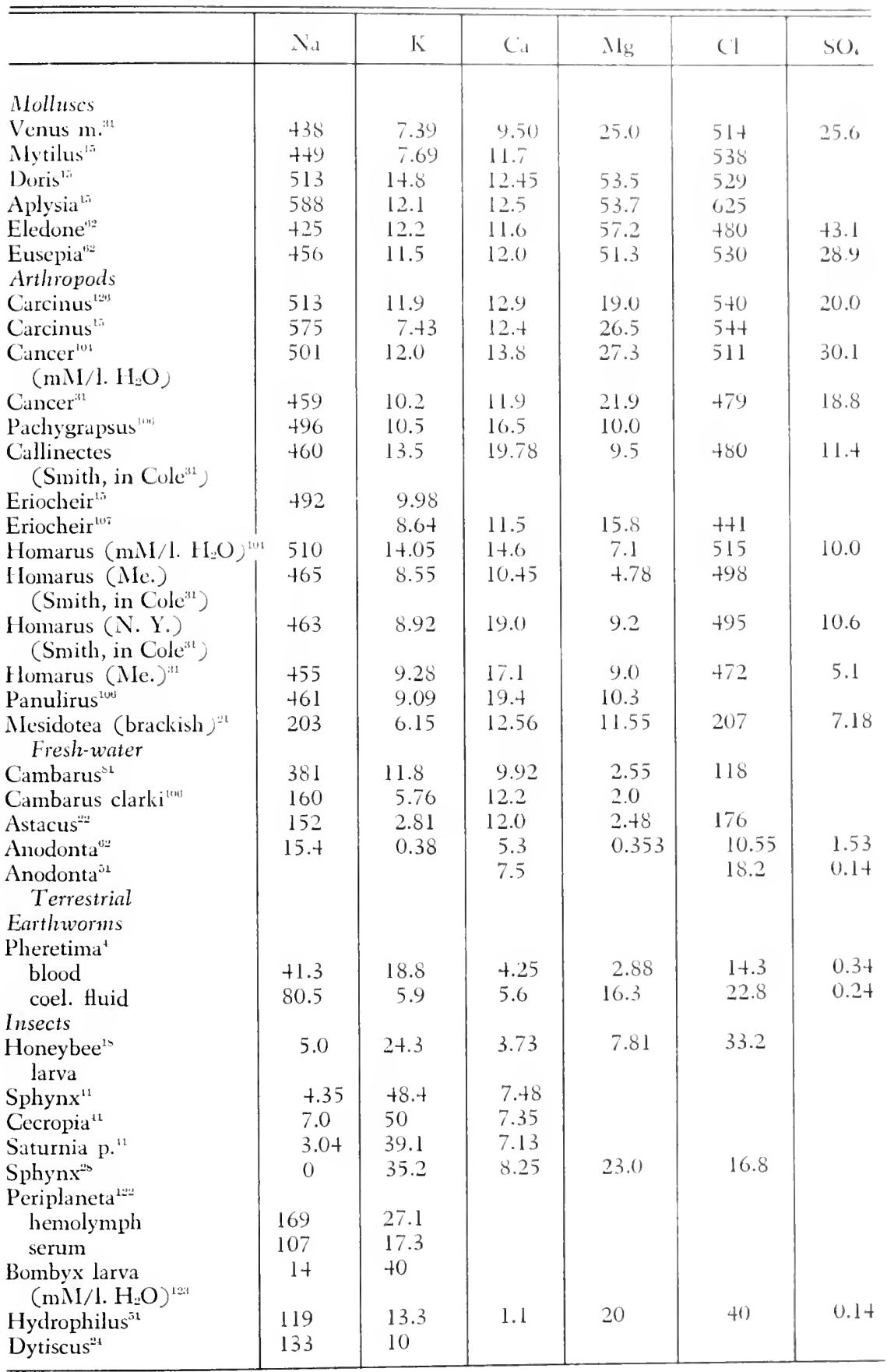




\section{TABLE 9 (continued)}

CONCENTRATION OF IONS IN SEA WATER AND IN BODY FLUIDS For sea water, in $\mathrm{mM} / \mathrm{l}$; in body fluids, in $\mathrm{mM} / \mathrm{l}$. (except where stated as $\mathrm{mM} / \mathrm{l} . \mathrm{H}_{2} \mathrm{O}$ )

\begin{tabular}{|c|c|c|c|c|c|c|}
\hline & $\mathrm{Na}$ & $\mathrm{K}$ & $\mathrm{Ca}$ & $\mathrm{Mg}$ & $\mathrm{Cl}$ & $\mathrm{SO}_{4}$ \\
\hline $\begin{array}{l}\text { Calliphora } \\
\text { Apis }^{24}\end{array}$ & $\begin{array}{r}148 \\
10\end{array}$ & $\begin{array}{l}37 \\
45\end{array}$ & & & & \\
\hline & \multicolumn{6}{|c|}{ VEBTEBRATES } \\
\hline $\begin{array}{l}\text { Marine } \\
\text { Myxine } \\
\left.\text { (Smith, in Cole }{ }^{31}\right)\end{array}$ & 400 & 9.06 & 5.26 & 22.6 & 448 & 6.00 \\
\hline Acanthias ${ }^{54}$ & 257 & 6.91 & & & & \\
\hline Raja stabul. ${ }^{112}$ & $236-267$ & $4.5-5.2$ & & & & \\
\hline Raja diaph. ${ }^{112}$ & $166-237$ & 6.8 & & & & \\
\hline Carcharias lit. ${ }^{112}$ & $258-275$ & 5.45 & & & & \\
\hline Mustelus canis ${ }^{112}$ & 270 & $4.4,5.6$ & & & & \\
\hline Lophius pisc. ${ }^{113}$ & 198 & 7.5 & & & & \\
\hline Lophius ${ }^{46}$ & 228 & 6.4 & 2.3 & 3.7 & 164 & \\
\hline Sygnathus ${ }^{16}$ & 207 & 16.2 & 3.5 & 9.7 & 166 & \\
\hline Muraena"1" & 252 & 24.2 & 3.78 & 2.8 & 179 & \\
\hline Gadus" & 181 & 10.0 & & & & \\
\hline $\begin{array}{l}\text { Caretta car. }{ }^{11 t} \\
\text { Amphibious }\end{array}$ & 151 & 6.7 & & & & \\
\hline Frog $\left(\mathrm{mMl} / \mathrm{l} \cdot \mathrm{H}_{2} \mathrm{O}\right)^{122}$ & 108 & 2.6 & & & & \\
\hline Frog & 103.8 & 2.5 & 2.0 & 1.2 & 74.3 & \\
\hline Chrysemys marg. ${ }^{114}$ & 120,149 & 3.3 & & & & \\
\hline $\begin{array}{l}\text { Pseudemys eleg. }{ }^{11 t} \\
\text { Terrestrial }\end{array}$ & 126 & 3.3 & & & & \\
\hline $\operatorname{Man}^{6+1}$ & 131 & 5.23 & 2.34 & 0.865 & 110 & \\
\hline $\operatorname{Man}^{75}$ & 145 & 5.12 & 2.49 & 1.235 & 103 & $2.5 \mathrm{I}$ \\
\hline $\operatorname{Man}^{16}$ & 109 & 5.0 & 2.52 & 1.0 & 107 & \\
\hline Man ${ }^{110}$ & 157 & 5.4 & & & & \\
\hline $\operatorname{Dog}^{17}$ & 141.4 & 4.66 & 2.47 & 0.95 & 108.8 & \\
\hline $\operatorname{Rat}^{31}$ & 145 & 6.2 & 3.1 & 1.6 & 116.0 & \\
\hline
\end{tabular}

Hydrogen Ion Concentration. Animals maintain a relatively constant hydrogen ion concentration which is usually more acid than the medium (Table 10). Sea water is alkaline $(\mathrm{pH} 8.0$ to 8.1$)$, whereas the blood and body fluids of marine animals have a $\mathrm{pH}$ usually between 7.2 and 7.8. Fresh water is also for the most part alkaline, although in some swamps and bogs the water may be more acid than the blood of inhabitants. Willmer 1:! found the blood of some South American fish to have a similar hydrogen ion concentration, whether the fish came from rivers ( $\mathrm{pH}$ 6.7) or from acid swamps $(\mathrm{pH} 3.8-5.0)$. No data are available on the $\mathrm{pH}$ of gastrotrichs and other invertebrate inhabitants of acid swamps. A few representative $\mathrm{pH}$ values for insect bloods are given in Table 10; most insect bloods are unique in being slightly acid. A tabulation of the $\mathrm{pH}$ values for 72 species of insects gives only fourteen values higher than p 1 7.0. Land vertebrates have a blood $\mathrm{pH}$ usually near 7.4.

Ionic Regulation. The ability of an aquatic animal to adjust to the medium or to regulate the concentration of a particular element is indicated by the ratio of the concentrations inside to the concentrations outside the body (Table 11). 
Departure from unity in this ratio and maintenance of a given ronic compesi tion when the extemal medium is changed reflect ionic regulation. 'The amount of magnesium and sulfate in the marine enviromment is much greater relative to other ions than is the amount in the curvironment of tresh water animals. Animals which adjust osmotically to the medium may regulate ionically.

TABLE 10. AIERAGE I'ALLES OF BLOOD OR OTIHER BODY FLLID pH OF A VARIETY OF ANIMALS, SHI ECTED FROM THE

EXTENSIVE LITERATURE

\begin{tabular}{|c|c|c|c|c|c|}
\hline Animal & $\mathrm{d} \mathrm{pH}$ & Medium & Animal & $\mathrm{d}$ pll & Medium \\
\hline Oyster $^{6 \varkappa}$ & 7.2 & \multirow{3}{*}{8.01} & Myxine glutinosa & \multirow[t]{3}{*}{7.63} & \\
\hline Venus (mantle) $(\text { Del. })^{31}$ & 7.65 & & (Smith, in Cole ${ }^{i 1}$ ) & & \\
\hline Venus (mantle) (Me.) ${ }^{3 t}$ & 7.9 & & Phryganea & & \\
\hline Venus (blood) (Del.) & 7.68 & 8.01 & (Trichoptera) & \multirow[t]{2}{*}{$6.7 \cdot 6.9$} & 7.38 .2 \\
\hline Limulus (Del.) ${ }^{31}$ & 6.98 & 8.01 & Phryganca & & \\
\hline Limulus (Me.) ${ }^{33}$ & 7.47 & 8.1 & (Trichoptera & $6.7-6.8$ & $3.1 \cdot 3.335$ \\
\hline $\begin{array}{l}\text { Limulus (Me.) } \\
\text { (Smith, in Cole }{ }^{31} \text { ) }\end{array}$ & 7.24 & & $\begin{array}{l}\text { Molanna } \\
\text { (Trichoptera) }\end{array}$ & \multicolumn{2}{|c|}{$6.9-7.128$ or 5.36} \\
\hline $\begin{array}{l}\text { Callinectes (Me.) } \\
\quad\left(\text { Smith, in Cole }{ }^{31}\right)\end{array}$ & 7.55 & & $\begin{array}{l}\text { Honeybee } \\
\text { larva }\end{array}$ & \multicolumn{2}{|c|}{6.83} \\
\hline Cancer $^{31}$ & 7.81 & \multirow[t]{4}{*}{8.1} & Several & \multirow{2}{*}{\multicolumn{2}{|c|}{6.79}} \\
\hline Carcinus $^{42}$ & 7.42 & & grasshoppers $^{20}$ & & \\
\hline Homarus (Me.) ${ }^{31}$ & 7.61 & & Bombyx & \multicolumn{2}{|l|}{$6.7-6.8$} \\
\hline Homarus (N.Y.) ${ }^{31}$ & 7.55 & & larvae & \multirow{2}{*}{\multicolumn{2}{|c|}{6.7}} \\
\hline Homarus (Me.) $)^{31}$ & 7.45 & \multirow{2}{*}{$\begin{array}{l}8.1 \\
8.1\end{array}$} & Tenebrio molitor ${ }^{71 a}$ & & \\
\hline $\begin{array}{l}\text { Cucumaria } \\
\quad \text { (coel. f.) }(\text { Me. })^{31}\end{array}$ & 7.8 & & $\begin{array}{l}\text { Lucanus cer. }{ }^{71 a} \\
\text { Carabus canc. }{ }^{71 a}\end{array}$ & \multicolumn{2}{|l|}{$\begin{array}{l}6.7 \\
7.0\end{array}$} \\
\hline $\begin{array}{l}\text { Cucumaria (Me.) } \\
\text { (Smith, in Cole }\end{array}$ & 7.3 & & $\begin{array}{l}\text { Drosophila }{ }^{1:} \\
\text { Chironomus:' }^{1:}\end{array}$ & & 7.49 \\
\hline $\begin{array}{l}\text { Asterias (coel. fl.) (Me.) } \\
\text { (Sinith, in Cole }{ }^{31} \text { ) }\end{array}$ & 7.2 & 8.1 & $\begin{array}{l}\text { Lumbricus (coel. 11. })^{101} \\
\text { Anadonta cygnea }\end{array}$ & \multicolumn{2}{|c|}{\begin{tabular}{l|l}
6.7 & \\
7.29 & Fre
\end{tabular}} \\
\hline Asterias (Me.) $)^{31}$ & 7.54 & 8.1 & Astacus & \multicolumn{2}{|c|}{$7.7-7.8$} \\
\hline $\begin{array}{l}\text { Solaster endica (Me.) } \\
\left.\text { (Smith, in Cole }{ }^{31}\right)\end{array}$ & 6.9 & & $\begin{array}{l}\text { (normal blood) } \\
\text { Astacus } \\
\text { (in calcification })^{35}\end{array}$ & \multicolumn{2}{|l|}{$8.0-8.2$} \\
\hline $\begin{array}{l}\text { Strongylocentrotus (Me.) } \\
\text { (Smith, in Cole }{ }^{31} \text { ) }\end{array}$ & 7.2 & & $\begin{array}{l}\text { haimara (oxyg. blood } \\
\left.\text { at } 0 \mathrm{~mm} . \mathrm{CO}_{2}\right)^{131}\end{array}$ & 6.8 & 6.7 \\
\hline $\begin{array}{l}\text { Strongylocentrotus } \\
(\text { Me. })^{31}\end{array}$ & 7.8 & & $\begin{array}{l}\text { barara (oxyg. blood } \\
\left.\text { at } 0 \mathrm{~mm} . \mathrm{CO}^{1}\right)^{1 \mathrm{nt}}\end{array}$ & 6.5 & 6.7 \\
\hline $\begin{array}{l}\text { Echinus (Me.) } \\
\quad \text { (Smith, in Cole }{ }^{31} \text { ) }\end{array}$ & 7.6 & & $\begin{array}{l}\text { bom-bom (oxyg. blood } \\
\left.\text { at } 0 \mathrm{~mm} . \mathrm{CO}_{2}\right)^{1 \mathrm{kn}}\end{array}$ & 6.7 & $3.8-5.0$ \\
\hline $\begin{array}{l}\text { Amphitrite (Me.) } \\
\text { (Smith, in Cole }{ }^{31} \text { ) }\end{array}$ & 6.8 & & $\begin{array}{l}\text { hassa (oxyg. blood } \\
\left.\text { at } 0 \mathrm{~mm} \cdot \mathrm{CO}_{2}\right)^{120}\end{array}$ & 6.8 & $3.8-5.0$ \\
\hline $\begin{array}{l}\text { Glycera (Me.) } \\
\quad\left(\text { Smith, in Cole }{ }^{31}\right)\end{array}$ & 7.4 & & $\begin{array}{l}\text { electric eel (oxyg. blood } \\
\left.\text { at } 0 \mathrm{~mm} . \mathrm{CO}_{2}\right)^{2 x_{3}}\end{array}$ & 6.2 & $3.8 \cdot 5.0$ \\
\hline
\end{tabular}

Table 11 shows that in echinoderms there is practically no ionic regulation in the inorganic composition of the coelomic fluid except for a slightly higher potassium concentration. The ambulacral fluid is continuous with sea water, except in holothurians, and the coelomic fluid in all echinoderm classes which have been examined is more similar to sea water than that in any other phylum. Despite the similarity of echinoderm body fluid to sea water, the 
TABLE 11. RATIOS OF CONCENTRATION OF IONS IN BODY FLUIDS TO CONCENTRATION IN MEDIUM (i/o).

\begin{tabular}{|c|c|c|c|c|c|c|}
\hline Animal & $\mathrm{Na}$ & $\mathrm{K}$ & $\mathrm{Ca}$ & - Mg & $\mathrm{Cl}$ & $\mathrm{SO}_{4}$ \\
\hline \multicolumn{7}{|l|}{ Coelenterates } \\
\hline Aurelia & 0.99 & 1.22 & 1.08 & 0.99 & 1.04 & 0.65 \\
\hline Cyanea ${ }^{71}$ & 1.0 & 1.67 & 1.0 & 1.0 & 1.08 & 0.421 \\
\hline Aequorea $^{\mathrm{Tl}}$ & 1.04 & 2.55 & 1.0 & 1.0 & 1.06 & 0.894 \\
\hline \multicolumn{7}{|l|}{ Echinoderms (coel. fl.) } \\
\hline Echinus $^{302}$ & 0.995 & 1.005 & 1.01 & 0.98 & 0.98 & 1.00 \\
\hline Echinus ${ }^{1.5}$ & 1.13 & 1.21 & 1.23 & 0.95 & 1.04 & \\
\hline $\begin{array}{l}\text { Strongylocentrotus (Me.) } \\
\text { (Smith, in Cole }{ }^{31} \text { ) }\end{array}$ & 1.02 & 1.065 & 1.015 & 0.942 & 1.02 & 0.80 \\
\hline Strongylocentrotus (Me.) ${ }^{31}$ & 1.015 & 1.13 & 0.92 & 0.93 & 1.03 & 0.99 \\
\hline Echinarachnius (Mc. $)^{m 1}$ & 0.995 & 1.011 & 0.995 & 0.982 & 1.01 & 0.969 \\
\hline $\begin{array}{l}\text { Solaster (Me.) } \\
\quad\left(\text { Smith, in } \text { Cole }^{31}\right)\end{array}$ & 1.01 & 1.06 & 1.016 & 0.992 & 1.01 & 1.00 \\
\hline $\begin{array}{l}\text { Chirodota (Me.) } \\
\text { (Smith, in Cole }{ }^{31} \text { ) }\end{array}$ & 1.01 & 1.06 & 1.085 & 1.00 & 1.01 & 1.007 \\
\hline $\begin{array}{l}\text { Asterias (Me.) } \\
\quad\left(\text { Smith, in Cole }{ }^{31}\right)\end{array}$ & 0.995 & 1.05 & 0.99 & 0.99 & 1.01 & 0.995 \\
\hline Asterias: & 1.01 & 0.975 & 0.93 & 0.901 & 1.02 & 1.00 \\
\hline $\begin{array}{l}\text { Cucumaria } \\
\text { (Smith, in Cole }{ }^{31} \text { ) }\end{array}$ & 0.99 & 1.06 & 0.99 & 1.00 & 1.01 & 0.99 \\
\hline Cucumaria & 1.00 & 0.965 & 0.925 & 0.99 & 1.01 & 1.00 \\
\hline Caudina (Japan) & 0.99 & 1.06 & 0.99 & 0.995 & 0.99 & 1.05 \\
\hline Paracentrotus lividis $^{17}$ & & 1.00 & 1.00 & .84 & .99 & 1.03 \\
\hline Sphacrechinus granularis $^{1 \bar{\tau}}$ & & 1.0 & 1.0 & .86 & .99 & .97 \\
\hline Holothuria tubulosa ${ }^{17}$ & & 1.10 & 1.08 & .97 & 1.06 & 1.01 \\
\hline $\begin{array}{l}\text { Molluscs } \\
\text { Venus (mantle fl.) (Del.) }{ }^{3 x}\end{array}$ & 1.063 & 0.918 & 2.07 & 0.944 & 1.17 & 1.17 \\
\hline Venus (blood) (Del.) $)^{: 11}$ & 1.104 & 0.925 & 2.105 & 0.923 & 1.17 & 1.16 \\
\hline Mytilus ${ }^{1-}$ & 0.96 & 0.70 & 1.12 & & 0.99 & \\
\hline Mytilusii & & & & & 0.99 & \\
\hline Patella $^{i 7}$ & & & & & 0.96 & \\
\hline Doris $^{15}$ & 1.095 & 1.35 & 1.19 & 1.15 & 0.99 & \\
\hline Aplysia $^{1 \overline{3}}$ & 1.258 & 1.08 & 1.27 & 1.15 & 1.17 & \\
\hline Aplysia ${ }^{14}$ & & 1.12 & 1.105 & 0.99 & 0.98 & \\
\hline Astropecten auranticus ${ }^{17}$ & & 1.16 & 1.11 & 0.87 & 1.03 & 1.04 \\
\hline Eledone $^{1 / 2}$ & .89 & 1.23 & 1.08 & 1.03 & .854 & 1.50 \\
\hline Euscpiat:2 & .958 & 1.16 & 1.12 & .93 & .944 & 1.01 \\
\hline Loligo $^{62}$ & & & .91 & & .98 & .98 \\
\hline Annelids and sipnnculids & & & & & & \\
\hline Sipunculus ${ }^{15}$ & & 1.135 & 1.12 & 0.886 & 1.25 & \\
\hline Sipunculus nudus ${ }^{17}$ & & 1.06 & .96 & 1.28 & 1.01 & .54 \\
\hline Phascolosoma & 0.86 & +.22 & 1.16 & & 0.842 & \\
\hline $\begin{array}{l}\text { Amphitrite brunnea } \\
\text { (Smith, in Cole }{ }^{31} \text { ) }\end{array}$ & 0.98 & 1.43 & 1.007 & 1.09 & 0.99 & 1.01 \\
\hline $\begin{array}{l}\text { Glycera dibranchiata } \\
\text { (Smith, in Colc }{ }^{31} \text { ) }\end{array}$ & & 1.05 & 1.06 & 1.22 & 1.00 & 0.908 \\
\hline Aphrodite aculeatat" & & 1.00 & .88 & .93 & .90 & .86 \\
\hline $\begin{array}{l}\text { Arenicola clap. } \\
\text { Arthropods (Marine) }\end{array}$ & & 1.39 & 1.17 & 1.0 & 1.0 & .92 \\
\hline $\begin{array}{l}\text { Limulus (N. Y.) } \\
\left.\quad \text { (Smith, in Cole }{ }^{31}\right)\end{array}$ & 0.933 & 1.346 & 1.039 & 0.522 & 0.882 & 0.644 \\
\hline Limulus (Del.) & 0.99 & 1.112 & 0.949 & 0.828 & 0.972 & 0.824 \\
\hline Limulus: & 1.01 & & & & 0.964 & \\
\hline Cancer ${ }^{102}$ & 1.12 & 1.26 & 1.41 & 0.529 & 0.973 & 0.888 \\
\hline
\end{tabular}




\begin{tabular}{|c|c|c|c|c|c|c|}
\hline Animal & $N_{d}$ & h & $(\mathrm{d}$ & $M_{h r}$ & $(1$ & $\zeta()$. \\
\hline Cancer (Me. $)^{31}$ & $1.00+$ & 1.20 & 1.180 & $0.6+8$ & $0.97 t$ & $0 ., 11$ \\
\hline Maja squinado ${ }^{\text {tz }}$ & & 1.08 & .96 & 1.0 & 1.0 & .83 \\
\hline Carcinus maenas $^{17}$ & & & 1.19 & .79 & 1.03 & .93 \\
\hline Cancer (summer) & 1.09 & 2.78 & 1.29 & $(1) .6,30$ & 1.01 & \\
\hline Cancer (winter) & 1.21 & 0.835 & 0.95 & 0.505 & 0.935 & \\
\hline Maja (ultrafilt. $)^{16}$ & & 1.21 & 0.906 & $0.7+2$ & 0.950 & 0.780 \\
\hline $\begin{array}{l}\text { Callinectes (Me.) } \\
\text { (Smith, in Cole }{ }^{n 1} \text { ) }\end{array}$ & 1.02 & 1.26 & 1.279 & 0.184 & 0.914 & 0.356 \\
\hline Carcinus $^{11}$ & & 1.33 & 1.17 & $0 .+58$ & 0.981 & \\
\hline Carcinus $^{1 . .}$ & 1.238 & 0.668 & $0.8+5$ & 0.570 & 0.975 & \\
\hline Carcinus $^{12}$ & 1.11 & 1.21 & 1.27 & 0.358 & 0.998 & 0.572 \\
\hline $\begin{array}{l}\text { Homarus (Me.) } \\
\text { (Smith, in Cole }{ }^{31} \text { ) }\end{array}$ & 1.12 & 0.94 & 1.11 & $0.09+$ & 1.02 & \\
\hline $\begin{array}{l}\text { Homarus (N. Y.) } \\
\left.\text { (Smith, in Cole }{ }^{: 11}\right)\end{array}$ & 1.028 & 0.838 & 1.23 & 0.178 & $0.9+3$ & 0.300 \\
\hline Homarus (Me. $)^{31}$ & 0.99 & 1.09 & 1.77 & 0.266 & 0.96 & 0.20 \\
\hline Homarus ${ }^{1.5}$ & 1.06 & 1.80 & 1.69 & 0.088 & 0.98 & \\
\hline Eriocheir ${ }^{10 \tau}$ & & 1.11 & 1.51 & 0.396 & 0.898 & \\
\hline $\begin{array}{l}\text { Eriocheir }^{1-} \\
\text { Arthropods (brackish) }\end{array}$ & 1.05 & 0.92 & 1.35 & & 0.82 & \\
\hline $\begin{array}{l}\text { Limulus (N. Y.) } \\
\quad(\text { Smith, in Cole }\end{array}$ & 1.625 & $1 .+77$ & 1.382 & 0.319 & $1 .+97$ & 0.395 \\
\hline Limulus (Del.) & 1.082 & 1.176 & 1.17 & 0.783 & 0.966 & $0.3+3$ \\
\hline $\begin{array}{l}\text { Callinectes (N. Y.) } \\
\left.\text { (Smith, in Cole }{ }^{31}\right)\end{array}$ & 2.531 & 1.879 & +.095 & 0.186 & 2.322 & 0.318 \\
\hline $\begin{array}{l}\text { Homarus (N. Y.) } \\
\quad\left(\text { Smith, in Cole }{ }^{31}\right)\end{array}$ & 1.45 & 1.678 & 3.505 & 0.332 & 1.525 & 0.350 \\
\hline Mesidotea (Baltic) & 2.48 & $3 .+2$ & 4.62 & 1.165 & 2.015 & 1.21 \\
\hline $\begin{array}{l}\text { Arthropods (fresh-water) } \\
\text { Eriocheir }^{10 i}\end{array}$ & & 98.5 & $1+.82$ & 9.2 & 825 & \\
\hline $\begin{array}{l}\text { Eriocheir }^{10 i} \\
\text { Astacus }^{10 \bar{i}}\end{array}$ & & 101. & 16.58 & 7.1 & 575. & \\
\hline Astacus & 152. & 36.4 & 8.1 & 10.0 & 365. & \\
\hline Cambarus"1 & $6+.5$ & $>100$ & 9.1 & 5.63 & 591. & \\
\hline Ascidians & & & & & & \\
\hline Ascidiaa & & & & & 1.05 & \\
\hline Phallusia $^{17}$ & & 1.14 & 1.11 & .97 & 1.09 & .99 \\
\hline Ciona $^{1 \overline{7}}$ & & $1.0+$ & 1.01 & .87 & 1.05 & .73 \\
\hline l'ertebrates (marine) & & & & & & \\
\hline Cyclostomes & & & & & & \\
\hline $\begin{array}{l}\text { Myxine } \\
\qquad\left(\text { Smith, in Cole }{ }^{31}\right)\end{array}$ & 0.968 & 1.0 & $0.56+$ & 0.450 & 0.928 & 0.197 \\
\hline $\begin{array}{l}\text { Polistotrema } \\
\text { Elasmobranchs }\end{array}$ & & & & & 0.89 & \\
\hline $\begin{array}{l}\text { Elasmobranchs } \\
\text { Raja stabuliforis (marine) } \text { ) }^{12:}\end{array}$ & & 0.53 & 0.393 & 0.052 & 0.513 & \\
\hline $\begin{array}{l}\text { Raja stabulitoris (marine) } \\
\text { Raja diaphenes (brackish) }\end{array}$ & $\begin{array}{l}0.00 \\
1.15\end{array}$ & $1 .+7$ & 1.13 & 0.153 & 1.01 & 0.235 \\
\hline Carcharias littoralis (marine) & 0.60 & 0.513 & 0.378 & $0.0+6$ & $0 .+53$ & 0.016 \\
\hline Torpedo ocellata ${ }^{17}$ & & $0.2+6$ & 0.079 & 0.127 & 0.223 & \\
\hline Scyllium canicula ${ }^{17}$ & & $0.96 t$ & 0.352 & 0.108 & 0.495 & \\
\hline Teleost & & & & & & \\
\hline Salmo fontinalis ${ }^{1 \pi}$ & & 0.50 & 0.333 & 0.0151 & 0.185 & \\
\hline
\end{tabular}


ions in muscle, in skin, and in blood cells are all very different (Table 8).*

Coelenterates show limited ionic regulation. Jellyfish, for example (Table 11), have a cardiovascular cavity containing sea water, but the jelly differs from the sea in the proportion of its ions, particularly in higher potassium and lower sulfate. The total osmotic concentration of the jelly is the same as that of sea water. Sea anemones contain less calcium than does sea water. ${ }^{64}$

Ionic regulation in marine molluses, sipunculids, and annelids has not been much studied. The ratio of various ions inside to outside is near 1.0 for Aplysia and Doris, but the blood calcium is high in Venus and the blood potassium low in Mytilus (Table 11). The sipunculid Phascolosoma maintains its body fluid ions at levels differing greatly from those of ions in sea water. ${ }^{117}$ This is the more significant because Phascolosoma has such low salt permeability as to appear semipermeable (Chapter 2, p. 11). ${ }^{1}$ Some marine polychaetes which adjust osmotically to the medium have nephridia which probably are not used for water regulation, but which may well function in ionic regulation.

Ionic regulation in arthropods presents a varied picture. Table 11 shows that in sea water the sodium and chloride content of Limulus, crabs such as Cancer, Callinectes, Maja, and Carcinus, and the lobster Homarus varies by not more than 10 per cent from that of sea water, whereas that of potassium is usually higher. Most of these arthropods also have a calcium concentration a little higher than that of the ocean. The lobster is remarkable in having considerably more calcium than potassium in its blood. All Crustacea show magnesium and sulfate concentrations much lower than those of sea water. Webb ${ }^{126}$ presents a series in order of decreasing degree of ionic regulation: Homarus, Carcinus, Cancer, whereas in osmotic regulation the order is Carcinus, Cancer, Homarus. Arthropods in brackish water maintain the same general proportions among the ions as when in sea water. Callinectes, Mesidotea, and Homarus from brackish water have strikingly high calcium content of the blood (Table 11). Fresh-water Crustacea contain high sodium and chloride, which are responsible for their high osmotic pressure. Potassium content is high, and the ratio of magnesium to calcium is higher in the crayfish than in most marine crustaceans.

Among vertebrates magnesium content is relatively lower than it is among invertebrates. In summary, ionic regulation at the cellular level does not require ionic regulation of body fluids in aquatic animals; some animals that show osmotic adjustment show ionic regulation; all that regulate osmotically also regulate ionically.

\section{RESPONSES TO ALTERATIONS IN THE MEDIUM}

Macallum 8.3, it pointed out that the body fluids of some marine invertebrates are like the ocean of today in ratios of the different salts, except for slightly lower magnesium and sulfate; these invertebrates lack circulatory systems (Aurelia) or have an open type of circulatory system (Limulus). Vertebrates differ most strikingly from invertebrates in lower chloride content and hence lower salinity, virtual absence of sulfate, and very much lower magnesium content. Macallum suggested that the inorganic ions in the bloods of "higher" animals resemble the ionic proportions present in the ocean at the

* Since this chapter was written Robertson ${ }^{103 a}$ has published excellent analyses of dialyzed body fluids of many marine invertebrates. These data show that the potassium concentration of starfish body fluid is significantly higher than that of sea water. 


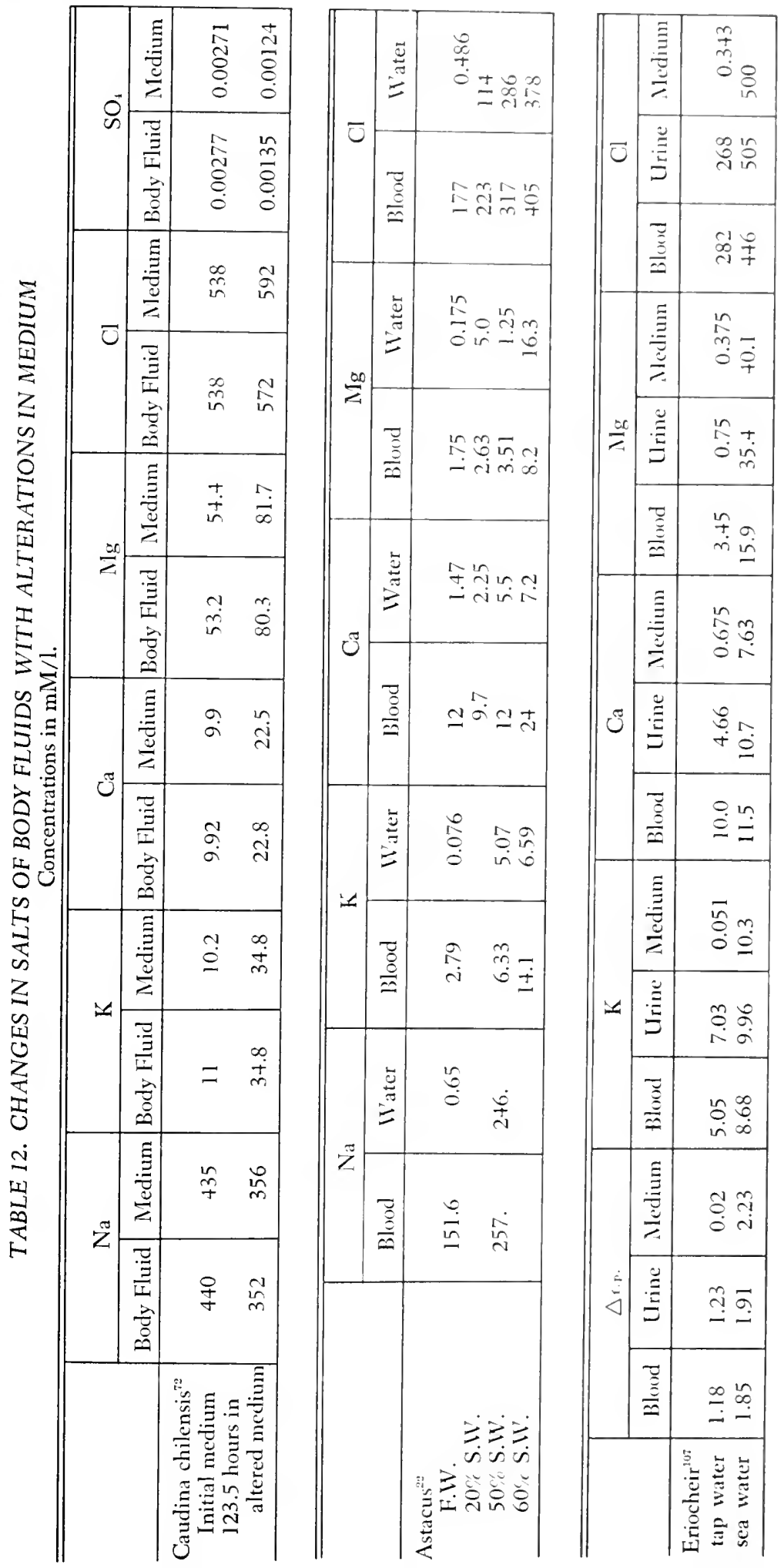


time when their ancestors' blood became separated within a closed circulation, whereas the fluids of animals like Aurelia and Limulus have changed with the ocean, changes in calcium and potassium occurring faster than changes in sodium, magnesium, and especially sulfate. Changes in the environment and in the organisms were thought to be very slow. Macallum suggested that the circulatory system of ancestors of the lobster was cut off from the sea very early, and that possibly the modern lobster came from fresh-water ancestry. Unfortunately for the theory, the circulatory system of the lobster is open, like that of Limulus. Macallum's general theory was well supported, with respect to the vertebrates, by geological evidence regarding the composition of the ocean at different ages.

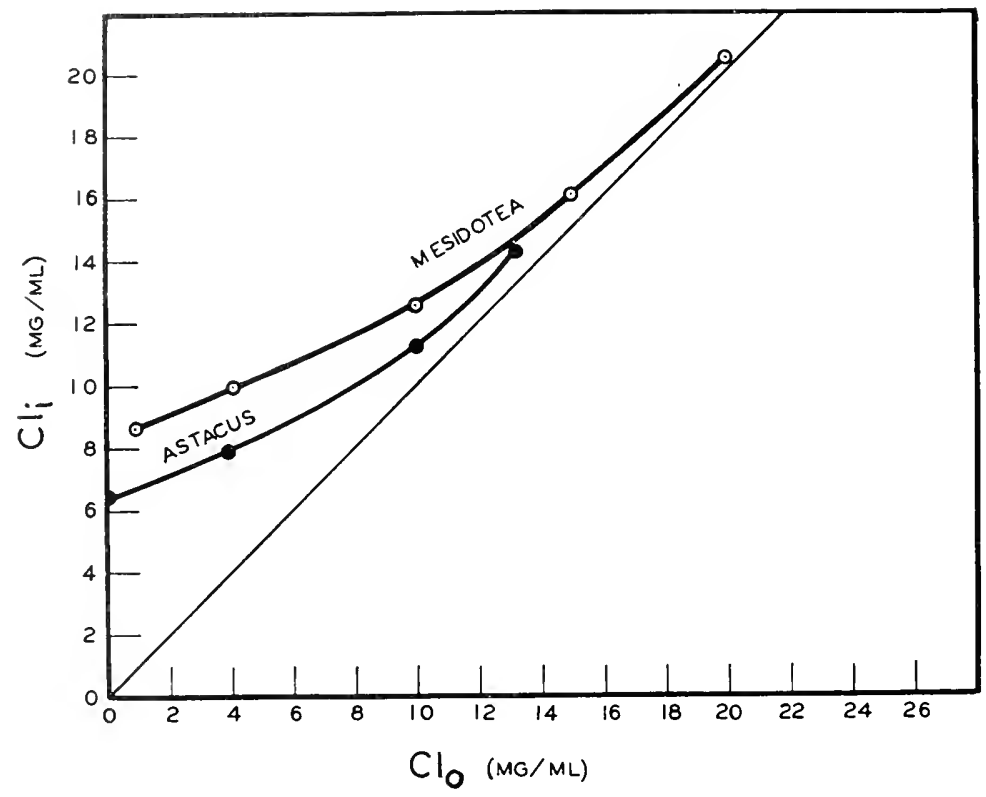

Fig. 23. Concentration of chloride in blood $\left(\mathrm{Cl}_{1}\right)$ in $\mathrm{mg} . / \mathrm{ml}$. as a function of chloride in the medium $\left(\mathrm{Cl}_{0}\right)$, in Mesidotea and Astaclis. From Bogucki. ${ }^{21,}{ }^{22}$

Frédéricq it also emphasized the rapid movement of water and slow changes in ions in the blood of various marine invertebrates on transfer to dilute media.

'Tests of Macallum's hypothesis and of the limitations imposed by salt balance on animal distribution have been made by placing aquatic animals in seal water of diflerent tonicities, or in solutions containing ions in proportions differing from those in the customary media, then measuring the changes in composition of the animals' blood.

The eflects on ionic composition of transfer to different concentrations of sea water are shown for several crustaceans in Table 12 and Figures 23-25. When fresh waller crustaceans such as Astacus or Telplunsa or a fresh-water Eriocheir is transferred to various dilutions of sea water, numerous changes occur. The water content of blood and of muscle may diminish slightly; the 
protein content of blood decreases streatly. 1:3 The various susn increase, hut in different proportions; usually $\mathrm{Xa}$ and $\mathrm{Cl}$ increase mure than $\mathrm{K}$, and all these more: than $\mathrm{Ca}^{++}$. When marine animals such an Mesidoted al and Carcinus $\$ 3$, tot are transferred to dilute sea water. rescrese restions vecur, and the blood constituents are diluted in clifferent proportions. 'I hese ehanges occur rapidly during the first few hours and then more slowly, equilibrum usually being reached in 2 to 7 days. A relatively constant body weight is maintained. The net result is that the ratios among the different ions change

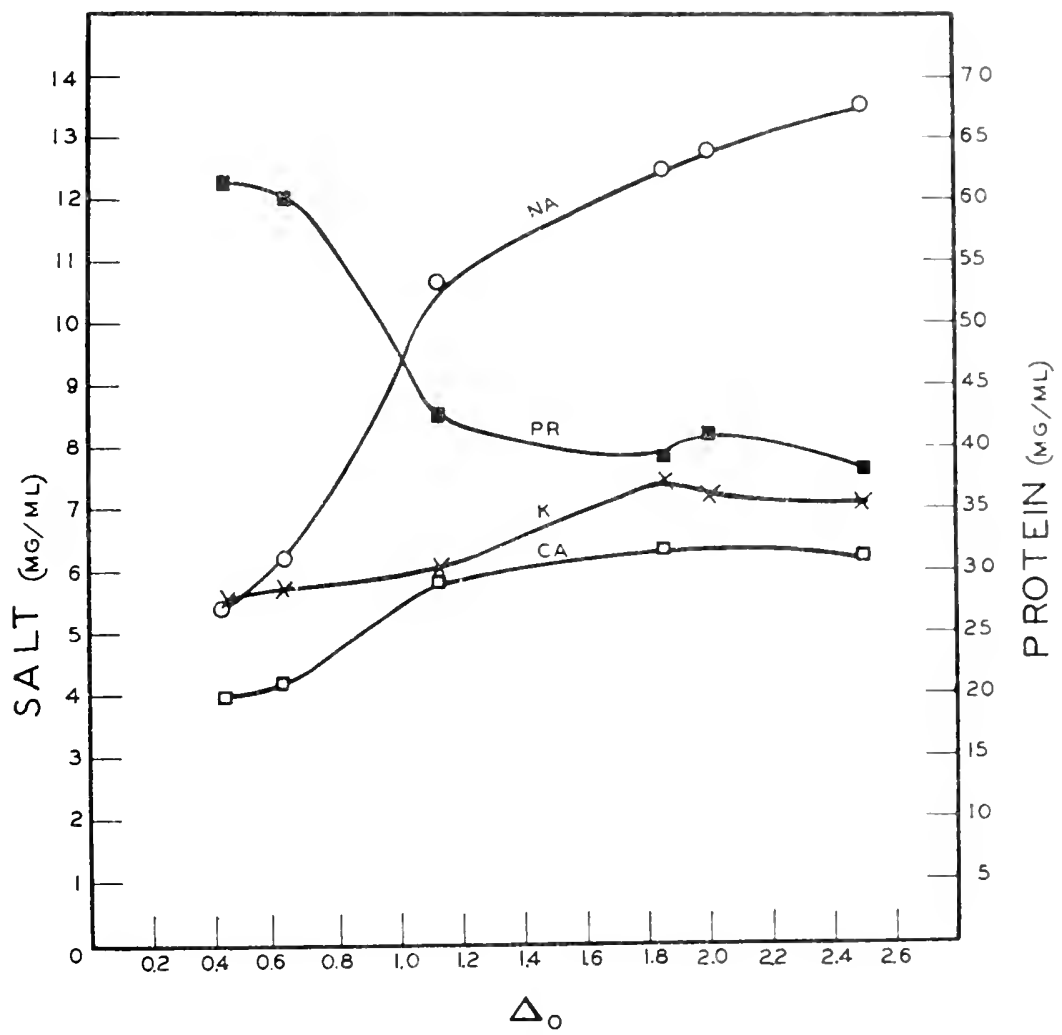

Fig. 24. Concentrations of sodium, potassium, calcium, and of total protein (PR) in the blood of Carcinus as a function of the concentration of the medium (dilute sea water) in freezing point $\left(\triangle_{0}\right)$. From Drilhon Courtois. ${ }^{13}$

with a change in total tonicity. Blood protcins decline with increase in blood salts (Fig. 2t).

Changes in blood chloride have been followed in numerous species. In dilute sea water the blood chloride concentration decreases, but some regulation occurs, and below a certain concentration the blood chloride remains nearly constant (Mesidoted, Fig. 23). In Gammarus, correspondence of blood chloride to chloride in the medium occurs in higher concentrations, and regu lation to a constant blood chloride occurs in very dilute media (Fig. 25). 
Blood chloride appears to be more labile than tissue chloride in crustaceans. When gammarids (fresh-water, brackish, and marine species) were transferred to media of different tonicities, the blood chloride changed more than the muscle chloride did (Fig. 25). Eriocheir, which is inarine-like in excreting a small volume of near-isotonic urine, has a blood-to-tissue $\mathrm{Cl}^{-}$ratio of 280:43, whereas in Astacus, which excretes copious dilute urine, the blood-to-tissue $\mathrm{Cl}^{-}$ratio is 189:378. The ions of Eriocheir and Astacus bloods change disproportionately in different media. ${ }^{10}$ In Mytilus, on the other hand, the blood chloride changes in proportion to the sea water chloride, but changes in muscle chloride are greater. ${ }^{78}$ Changes in blood and tissue chloride occur rapidly in Mytilus, and the animals die if their chloride content is severely depleted. ${ }^{53}$

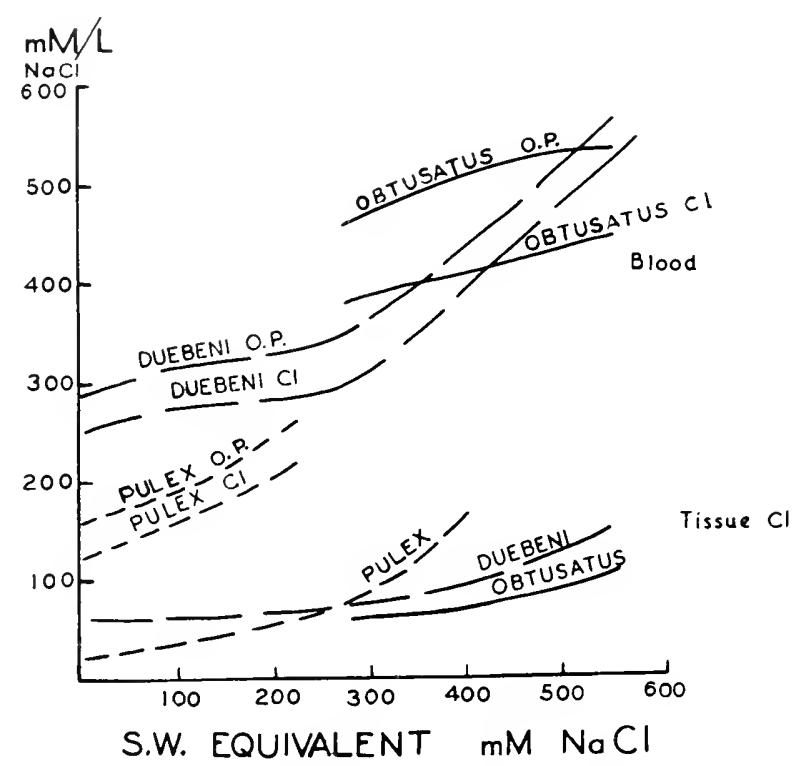

Fig. 25. Blood osmotic pressure (O.P.), blood chloride ( $\mathrm{Cl}$ ) as $\mathrm{mM} \mathrm{NaCl} / \mathrm{l}$, and tissue chloride (Cl) as $\mathrm{mM} \mathrm{NaCl} / \mathrm{kg}$. wet wt. in Gammarus duebeni (brackish), $G$. obtusatus (marine), and G. pulex (fresh-water) in dilutions of sea water corresponding to the concentrations of $\mathrm{NaCl}$ given on the abscissa. From Beadle and Cragg."

When mosquito larvae (Aedes detritus) are in different dilutions of sea water, both total osmotic concentration and chloride concentration are well regulated, but they show progressive changes with external concentration, osmotic concentration and chloride content changing disproportionately. Larvae of Aedes aegy'pti regulate $\mathrm{Cl}^{-}$well in dilute but not in hypertonic media (Fig. 26).

The effects of disproportionate changes in particular elements also indicate selective ionic regulation. Those animals with some ions present in nearly the same concentration as in sea water change their concentration of these ions readily. Sea ancmones ${ }^{64}$ in dilute sea water increase their water and decrease their calcium concentration, whereas in a mixture of sea water and isotonic 
$\mathrm{CaCl}_{2}$ the calcium concentration in the anemones doubles in 48 bours. Table 12 shows in the holothurian, Candina chilensis, the effects of disproportionate increases in $\mathrm{K}^{+}, \mathrm{Ca}^{++}$, and $\mathrm{Na}^{+}$and decrease in $\mathrm{SO}_{4}$ in the medium. Within 5 days the body Huid alters in salt balanee to conform to the altered environment; two-way permeability is indicated. Sea urchins take up Nat by exchange with $\mathrm{NaCl}$ through the body surface. ${ }^{11}$ Echinoderms, which nearly correspond ionically to the sea, lack a closed circulatory sistem and thus conform to Macallum's hypothesis; however, other animals which have an open circulatory system show disproportionate changes in ionic concentrations in a changed medium. The worm, Phascolosoma, shows no osmoregulation but does show good salt regulation; the content of $\mathrm{Cl}^{-}$in the muscles increases more than does that of $\mathrm{K}^{+}$, when these elements are increased proportionately

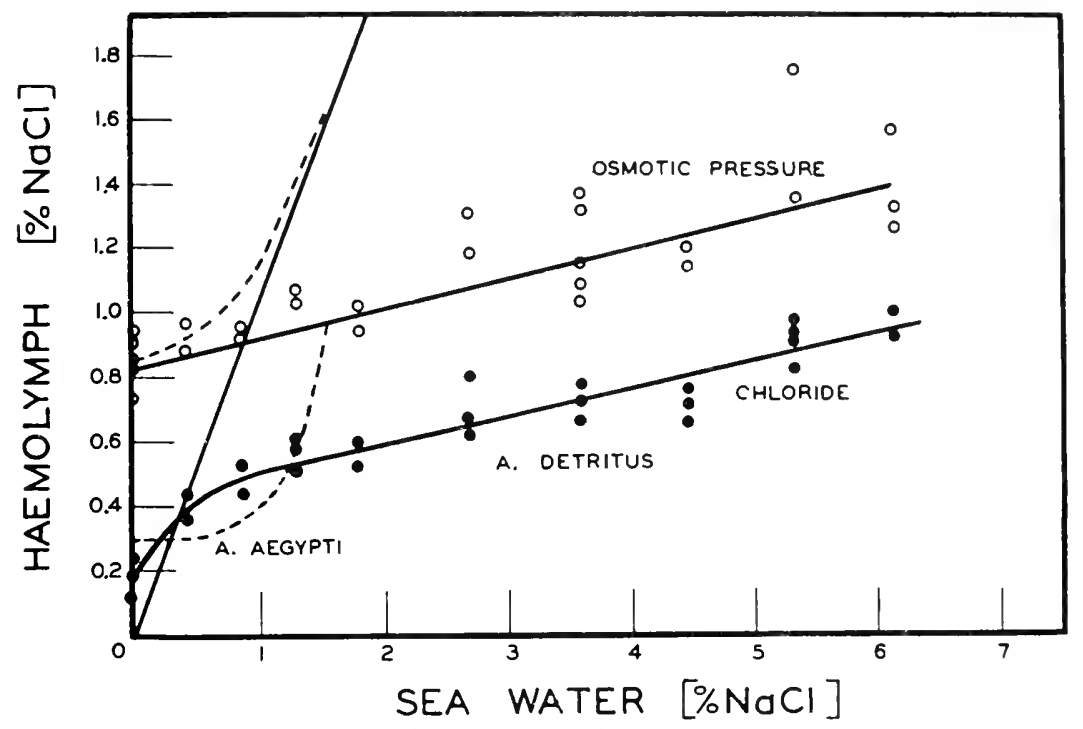

Fig. 26. Relation between total osmotic pressure and chloride in blood of Aedes detritus larvae and those of the medium expressed in equivalent concentrations of $\mathrm{NaCl}$. Broken lines for A. aegypti from Wigglesworth. ${ }^{14}$ Diagonal straight line represents blood and medium as isotonic. From Beadle.

in the medium. ${ }^{117}$ In such animals as the crustacean Carcinus maenas and the molluse Aplysia the ratio of ions can be changed in a few hours by immersion in sea water containing an excess or a deficiency of particular elements. ${ }^{14}$ For example, in sea water lacking calcium the blood $\mathrm{Ca}^{+}+$of Carcinus dropped from 0.6 to $0.3 \mathrm{mg}$./ cc. in 75 hours, whereas the blood $\mathrm{Ca}^{++}$of Aplysia decreased from 0.5 to $0.15 \mathrm{mg}$. cc. in 5 hours. When Carcinus is transferred to dilute sea water there is a net increase in salt excretion and the percentages of $\mathrm{Na}^{+}, \mathrm{K}^{+}, \mathrm{Ca}^{++}$, and $\mathrm{Cl}^{-}$increase relative to the medium, while the percentages of $\mathrm{Mg}^{++}$and $\mathrm{SO}_{4}=$ decrease. ${ }^{2 * 6}$

It is doubtful whether the kind of circulatory system has much relation to salt balance, since some animals with an open circulation show considerable ionic regulation. No studies of jonic regulation in different media have been 
made with aquatic vertebrates, although the concentration of their blood chloride and probably that of other ions can change. The chloride concentration in eels in fresh water is lower than that in eels in sea water by a ratio of about 100 to 117. ${ }^{70}$ Also the ratio of $\mathrm{Cl}^{-}$inside to $\mathrm{Cl}^{-}$outside in hagfish remains relatively constant in different salinities; hence $\mathrm{Cl}-$ must be taken in and given out.": It is probable that the salt ratios in vertebrates tend to be more fixed than the salt ratios in animals with open circulatory systems. In any case, the invertebrates which have been studied show rapid alterations in salt content and may even change their ionic ratios with changes in the environment.

\section{MECHANISMS OF IONIC REGULATION}

Some animals, such as echinoderms, differ very little from sea water in the composition of their body fluids, whereas in others certain elements are selectively concentrated and other elements excluded. The ratios of salts inside to out are not static but vary somewhat according to the medium. How is the unbalance in ionic concentration in body fluids maintained?

Physical Factors. One physical factor which might make for concentration differences is the Donnan equilibrium. That is, some of the ions may normally be bound to ions of opposite sign (largely proteins) which cannot diffuse across body membranes, keeping the concentration of the bound ions higher than on the side lacking the nondiffusible substance. Electrical and osmotic equilibria apply only if some of the protein molecules have more than one jonizing group. The Donnan equilibrium requires that the product of diffusible cations (positive ions) inside and the diffusible anions (negative ions) inside is equal to the product of diffusible cations outside and diffusible anions outside, as follows for sodium, protein, and chlorine:

$$
\begin{aligned}
& \mathrm{Na}_{1} \mathrm{Cl}_{3} \mid \mathrm{Na}_{0} \mathrm{Cl}_{\text {o }} \\
& \mathrm{Na}_{1} \mathrm{Pr}_{1} \\
& \mathrm{Na}_{1} \mathrm{Cl}_{0} \\
& \overline{\mathrm{Na}_{0}}=\frac{}{\mathrm{Cl}_{1}} \\
& \begin{array}{l}
\mathrm{Na}_{1}>\mathrm{Na}_{0} \\
\mathrm{Cl}_{1}<\mathrm{Cl}_{0}
\end{array}
\end{aligned}
$$

In Limulus blood the sodium and chloride ratios agree well with values predicted on the basis of a Domman equilibrium. 38,121

If the concentration gradient of all ions were maintained by protein binding, the Doman ratios should hold for all elements, and on dialysis against sea water (for marine bloods) the ionic differences should nersist. Table 13 gives analyses of blood of several animals before and after dialysis against sea water. This table shows that in Echinus where the protein is low there is no significant difference in ionic composition after dialysis. In the crustaceans, however, with higher protein, some elements, such as $\mathrm{K}^{+}$and $\mathrm{Na}^{+}$, are less concentrated, and others, such as $\mathrm{Mg}+{ }^{+}$and $\mathrm{SO}_{4}^{---}$, are more concentrated after dialysis. The minor differences between the blood and sea water after dialysis across an incrt membrane and the large differences between dialyzed and undialyzed blood show the small part played by protein binding.

$A$ second physical factor is difference in diffusion rates. Most aninals are permeable to salts in varying amounts. Many experiments, for example on Caudina body wall and on the cuticle of the foregut of Homarus, indicate that 
ions cross body membranes, other thinges being equal, in a series correspend ing to their mobilities: $\mathrm{K}>\mathrm{Na}>\mathrm{Ca}>\mathrm{Mlg}_{2} \mathrm{Cl}>\mathrm{SO}_{4}$. Specilie differences in the rate of penetration could, however, ${ }^{302}$ account for concentration differences only if all the ions were lower in concentration inside than out. An im could not become more concentrated inside by fast diffusion alone.

TABLE 13. COMPOSITION OF BODY FLUIDS BFFORE ANI)

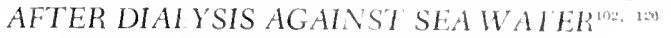

a. Concentration in mg./g. H.O

\begin{tabular}{|c|c|c|c|c|}
\hline Animal & Constituent & Body Fluid & $\begin{array}{l}\text { Dialysed } \\
\text { Body Fhuid }\end{array}$ & Sea Water \\
\hline $\begin{array}{l}\text { Echinus } \\
\quad \text { esculentus }\end{array}$ & $\begin{array}{l}\text { Protein } \\
\mathrm{Na} \\
\mathrm{K} \\
\mathrm{Ca} \\
\mathrm{Mg} \\
\mathrm{Cl} \\
\mathrm{SO}_{1} \\
\mathrm{H}_{2} \mathrm{O}\end{array}$ & $\begin{array}{c}0.3 \\
10.22 \\
0.37+6 \\
0.3994 \\
1.223 \\
18.59 \\
2.601 \\
985.4\end{array}$ & $\begin{array}{c}10.19 \\
0.3687 \\
0.3950 \\
1.220 \\
18.64 \\
2.582 \\
985.9\end{array}$ & $\begin{array}{c}-- \\
10.3 \\
0.37+4 \\
0.3938 \\
1.2+66 \\
18.62 \\
2.609 \\
986.1\end{array}$ \\
\hline $\begin{array}{l}\text { Homarus } \\
\text { vulgaris }\end{array}$ & $\begin{array}{l}\text { Protein } \\
\mathrm{Na} \\
\mathrm{K} \\
\mathrm{Ca} \\
\mathrm{Mg} \\
\mathrm{Cl} \\
\mathrm{SO}_{4}\end{array}$ & $\begin{array}{c}22.55 \\
11.71 \\
0.5503 \\
0.5874 \\
0.1732 \\
18.26 \\
0.7628\end{array}$ & $\begin{array}{c}22.605 \\
10.57 \\
0.3773 \\
0.4618 \\
1.3085 \\
18.375 \\
2.623\end{array}$ & $\begin{array}{c}\cdots \\
10.45 \\
0.3799 \\
0.3995 \\
1.264 \\
18.875 \\
2.646\end{array}$ \\
\hline $\begin{array}{l}\text { Cancer } \\
\text { pagurus }\end{array}$ & $\begin{array}{l}\text { Protein } \\
\mathrm{Na} \\
\mathrm{K} \\
\mathrm{Ca} \\
\mathrm{Mg} \\
\mathrm{Cl} \\
\mathrm{SO}_{4} \\
\mathrm{H}_{2} \mathrm{O}\end{array}$ & $\begin{array}{l}34.94 \\
11.55 \\
0.4684 \\
0.5519 \\
0.6583 \\
18.08 \\
2.306 \\
963\end{array}$ & $\begin{array}{c}34.88 \\
10.73 \\
0.390 \\
0.463 \\
1.301 \\
18.60 \\
2.647 \\
961\end{array}$ & $\begin{array}{c}10.47 \\
0.380 \\
0.4004 \\
1.267 \\
18.93 \\
2.652 \\
986.1\end{array}$ \\
\hline
\end{tabular}

b. Composition of body fluid as per cent of dialysis values

\begin{tabular}{l|c|c|c|c}
\hline \multirow{2}{*}{ Constituent } & \multicolumn{4}{|c}{ Animal } \\
\cline { 2 - 5 } & Echinus & Homarus & Canccr & Carcinus \\
\cline { 2 - 5 } $\mathrm{Na}$ & 100 & 111 & 108 & 109 \\
$\mathrm{~K}$ & 102 & $1+6$ & 120 & 117.5 \\
$\mathrm{Ca}$ & 101 & 127 & 119 & 107.8 \\
$\mathrm{Mg}$ & 100 & 13 & 51 & 37.2 \\
$\mathrm{Cl}$ & 100 & 99 & 97 & 103 \\
$\mathrm{SO}_{4}$ & 101 & 29 & 87 & 60.7 \\
\hline
\end{tabular}

Active Absorption. We are forced, therefore, to seek active forces in ionic regulation; 79 some of these reside in the body membranes and some in excretory organs. Krogh's studies of active absorption of ions have been men- 
tioned in Chapter 2. He found that a wide variety of aquatic animals actively absorb certain ions from their medium. In order that electrical equilibrium be maintained the electrolytes must be absorbed either as neutral molecules or, if as ions, by exchange for another ion of the same sign. $\mathrm{Cl}^{-}$, for example, may be exchanged for $\mathrm{HCO}_{3}{ }^{-}$. Krogh assumed that ammonia and bicarbonate are not taken up by any active processes, and he measured anion absorption from $\mathrm{NH}_{4}+$ salts, and cation absorption from $\mathrm{HCO}_{3}-$ solutions. A freshwater bivalve (Unio pictorum) can concentrate $\mathrm{Cl}^{-}$as $\mathrm{NaCl}$ or can selectively absorb Cl- from $\mathrm{CaCl}_{2}$. A frog also absorbs $\mathrm{Cl}^{-}$with $\mathrm{Na}^{+}$from $\mathrm{NaCl}$ against a concentration gradient of $1: 1000$. The frog can selectively take up $\mathrm{Cl}^{-}$ from $\mathrm{NH}_{4} \mathrm{Cl}, \mathrm{CaCl}_{2}$, and $\mathrm{KCl}$; it also absorbs $\mathrm{Br}^{-}$but takes up $\mathrm{I}^{-}, \mathrm{NO}_{3}{ }^{-}$, and CNS ${ }^{-}$only by diffusion; $\mathrm{Na}^{+}$is actively absorbed, but not $\mathrm{K}^{+}, \mathrm{NH}_{4}{ }^{+}$, or $\mathrm{Ca}^{+}+$. The crab, Eriocheir, actively absorbs $\mathrm{Cl}^{-}, \mathrm{Br}^{-}$, and $\mathrm{CNS}^{-}$, but not $\mathrm{NO}_{3}^{-}, \mathrm{I}^{-}$, or $\mathrm{SO}_{4}=$; it absorbs $\mathrm{Na}^{+}$and $\mathrm{K}^{+}$, but not $\mathrm{Ca}^{++}$. In Crustacea and fish the gills are permeable to salts in both directions; the skin is less so. ${ }^{91}$

Very little is known of the membrane mechanism for selective absorption and exclusion of ions, which is a problem in cellular permeability. Important factors are ion mobility, membrane characteristics, and exchange products of metabolism. An increasing amount of evidence is accumulating, particularly from the use of radioactive tracers, that there occurs continually a two-way flow of salts and water across cell membranes. Measurements of differences in total concentration on the two sides of a membrane, therefore, give no picture of the dynamic exchange. Continuous flow of ions into and out of plant cells occurs; similar two-way passage across the mammalian intestinal wall of $\mathrm{Na}^{+}, \mathrm{Cl}^{-}$, and water, with net balances favoring the blood, has been demonstrated. ${ }^{124}$ Apparently salts cannot move without some accompanying water movement. It is likely that the use of tracers will demonstrate much more lively movement of salts into and out of aquatic animals than has been suspected from chemical analyses of blood, urine, and medium. Active uptake can be most easily demonstrated in fresh-water animals, but active absorption by marine animals occurs (e.g. Carcinus ${ }^{126}$ ). In growing animals the net effect of ionic exchange must favor accumulation.

Undoubtedly salt replacement from food is important in most species. Krogh was unable to demonstrate active absorption of ions by the eel; it must rely entirely on food for its salts.

Excretion. Active selective absorption of ions is necessary in adult animals only because of continuous salt excretion. Conversely, a mechanism for the baling out of unwanted ions is necessary because of their continuous penetration. In the preceding chapter it was shown that in fresh-water animals the kidneys eliminate osmotic water. Yet kidneys certainly evolved in marine animals, which are isotonic with their medium. The coelenterates and echinoderms lack special excretory organs, and they show least ionic regulation. In general, the most successful ion regulators are animals with excretory organs. It is reasonable to assume that in annelids, molluscs, and arthropods, kidneys arose and still function in ionic regulation; on migration to fresh water the kidneys took over water regulation secondarily. In the fishes, on the other hand, kidneys arose in connection with water regulation, and on migration to the sea the kidneys failed to take over ionic regulation; hence extrarenal routes were required. Whether salt is excreted extrarenally by invertebrate animals 
is not known. Possibly the contractile vacuole in marine Protozoa has an ion regulating function.

Evidence for active excretion of selected ions is given by the ratios of urine

TABLE 14. RATIOS OF CONCENTRATIONS OF IONS IN URINETO CONCENTPATIONS IN PLASMA (u/p ratios).

\begin{tabular}{|c|c|c|c|c|c|c|c|c|}
\hline & $\mathrm{Na}$ & $\mathrm{K}$ & $\mathrm{Ca}$ & $M g$ & $\mathrm{Cl}$ & SO & $\begin{array}{l}\because c \mathrm{~b} \\
\mathrm{exc} \\
\mathrm{PO}\end{array}$ & $\begin{array}{l}\text { ody wt. } \\
\text { reted in } \\
24 \text { hrs. }\end{array}$ \\
\hline $\begin{array}{l}\text { Carcinus } \\
\text { Carcinus }^{120}\end{array}$ & 0.95 & 0.78 & 0.94 & 3.90 & 0.98 & 2.24 & & \\
\hline Cancer $^{102}$ & 0.97 & 0.80 & 0.89 & 1.25 & 0.96 & 1.33 & & \\
\hline $\mathrm{Maja}^{18}$ & & 0.83 & 1.33 & 1.44 & 0.98 & 1.47 & & 2.27 \\
\hline Eriocheir (S.W. $)^{11}$ & & 1.15 & 0.93 & 2.17 & 1.135 & & & \\
\hline Eriocheir (F.W.) & & 1.39 & 0.47 & 0.22 & 0.95 & & & 4.2 \\
\hline Astacus (F.W. $)^{10 t}$ & & $<.12 t$ & $0.26 t$ & 0.45 & 0.0525 & & & \\
\hline Cambarus (F.W. $)^{n}$ & 0.465 & 0.140 & 0.087 & $0 .+5$ & 0.082 & & & 5.22 \\
\hline Pheretima (F.W.) & & & & & & & & \\
\hline$(u / \text { coel. fl. })^{4}$ & 0.127 & 0.40 & 0.536 & 0.136 & 0.046 & 0.71 & & +8. \\
\hline Skate $^{87}$ & & & & & 1.0 & 27.0 & & \\
\hline Sygnathus $^{43}$ & 0.407 & $0.2+5$ & 1.85 & 10.75 & 1.70 & & 0.74 & \\
\hline Hippocampus ${ }^{16}$ & 1.01 & & & & 2.08 & & & \\
\hline Lophius $^{16}$ & 0.40 & 0.452 & 5.67 & 19.9 & 0.86 & & 0.229 & \\
\hline Lophius $^{47}$ & 0.58 & 0.23 & 3.4 & 95.4 & 1.18 & 36.5 & 0.23 & \\
\hline Muraena ${ }^{1 B}$ & 0.583 & 0.120 & 3.6 & 26.35 & 1.75 & & 1.4 & \\
\hline $\operatorname{man}^{6}$ & 1.16 & 7.5 & 1.87 & $2 .+1$ & 1.63 & $20-60$ & & 2.0 \\
\hline $\operatorname{man}^{46}$ & 1.0 & 6.85 & 1.2 & 4.8 & 1.33 & & & \\
\hline
\end{tabular}

to plasma concentrations of various elements (Table 14). These ratios are subject to much variation with dietary changes, etc., but they indicate the efficiency of the kidney in salt regulation. In marine crustaceans the concentrations of $\mathrm{Mg}^{++}$and $\mathrm{SO}_{4}=$ are much higher in urine than in blood; the lower concentration of $\mathrm{K}^{+}$in urine indicates some fixation of this element by tissues and also raises the possibility of extrarenal salt excretion. The kidneys favor the retention of $\mathrm{K}^{+}, \mathrm{Na}^{+}$, and $\mathrm{Cl}^{-}$, and the elimination of $\mathrm{Ng}^{+}+$and $\mathrm{SO}_{4}=$. Nagel ${ }^{91}$ injected iodide into Carcinus and $H_{3}$ as and found a concentration in the urine higher than in the blood; the amount of injected iodide excreted was greater in Hyas than in Carcinus. In fresh-water arthropods the urine is hypotonic but not salt-free. It is essential to fresh-water life to have a kidney which either reabsorbs salt or secretes water. Bialaszewicz ${ }^{16}$ injected large amounts of isotonic salt solutions into the crab Maja and measured the amount excreted in urine over a day or two. He found the time required for return to normal level was less for $\mathrm{KCl}\left(1.5 \mathrm{hr}\right.$.) than for $\mathrm{CaCl}_{2}(15 \mathrm{hr}$.), and this was less than for $\mathrm{MgCl}_{2}\left(23 \mathrm{hr}\right.$.) or $\mathrm{Na}_{2} \mathrm{SO}_{4}$ (more than $120 \mathrm{hr}$.). When mixtures were injected, various elements were eliminated at different rates. The amount of the different salts eliminated by the kidner's represented only a small fraction (6 per cent for $\mathrm{Mg}$ ) of what disappeared from the blood over a period of 24 hours. Bialaszewicz concluded that salt is stored in tissues and that the kidneys are not important in salt balance. This conclusion is hardly justified from his nonphysiological experiment, and it becomes important to search for possible extrarenal routes of salt excretion. 
In marine fish the kidneys normally excrete most of the $\mathrm{Mg}^{++}$and $\mathrm{SO}_{4}=$, but the $\mathrm{Na}^{+}, \mathrm{K}^{+}$, and $\mathrm{Cl}^{-}$are excreted extrarenally by way of the gills. In the sculpin, Myoxocephalus, the urine is normally free of or very low in $\mathrm{Cl}^{-}$, 57, 98 but, when the fish are handled, a diuresis ensues and chloride appears in the urine. The sculpin can excrete a urine which is supersaturated in $\mathrm{MgHPO}_{4}$. Smith 113, 115 found no $\mathrm{K}^{+}$in the urine of Lophius or Anguilla. Selective secretion and reabsorption of elements by excretory organs is, therefore, an important factor in ionic regulation.

\section{BALANCED SALT MEDIA}

The preceding sections have considered the relation between animals and their environment with respect to those elements which constitute the bulk of inorganic substances in living systems. The specific functions of the different elements, the antagonisms and interactions among them, comprise a large section of cellular physiology. A proper balance among the different elements is necessary for optimal functioning of organs, and for growth and development; the salt requirements for growth may differ from those for maintenance of a tissue. A proper balance of ions is needed for normal irritability, permeability, contractility, and other functional characters of particular tissues. The effects of salts on heart and other muscles are discussed in later chapters.

In the culture of autotrophic Protozoa the following elements are probably essential: C, H, O, N, P, S, Ca, Cl, K, Na, Fe, and Mg. ${ }^{59}$ The requirements of traces of other elements can be established only by very rigorous chemical controls. The needs of different species vary considerably. Chilomonas paramecitm, for example, fails to grow in the absence of phosphorus and sulfur. ${ }^{88}$ Growth can be accelerated by an element which may not be essential to survival. Manganese accelerates the growth of Euglena anabaens, ${ }^{59}$ and tetravalent vanadium in concentrations below $10^{-5} \mathrm{M}$ markedly increases division in Chilomonas paramecium. ${ }^{26}$ Species of Protozoa which are unable to synthesize organic compounds from simple sources of carbon and nitrogen grow best when their organic food is in a suitable salt solution (see Chapter 5). Numerous culture media have been devised. ${ }^{9 t}$ Failure to culture animals in synthetic media has resulted as often from lack of proper salt balance as from lack of some specific nutritional factor.

In vitro studies of isolated tissues require the bathing of these tissues in solutions which simulate normal body fluids with respect to salts but from which the organic constituents are lacking. The composition of these physiological salt solutions is only rarely based on analysis of body fluids but is more often arrived at empirically. Such physiological salt solutions are not adequate for growth. Table 15 presents some of the balanced solutions found useful for different species.

\section{MINOR AND TRACE ELEMENTS}

In addition to the common elements of animal tissues, certain species require small amounts of other elements for specific purposes. Some examples of the utilization of trace elements are of interest; ${ }^{1: 0}$ in cxcess they may have toxic effects. Sporadic biological distribution of some elements is not readily understood. ${ }^{127}$ 
TABLE 15. SELECTED PHYSIOIOCICAL SOI WTIONS

Quantities in grams unless otherwise indicated.

\begin{tabular}{|c|c|c|c|c|c|c|c|}
\hline Animal & $\mathrm{NaCl}$ & $\mathrm{KCl}$ & $\mathrm{CaCl}_{2}$ & $\mathrm{NaHCO}_{3}$ & Other salts ${ }^{*}$ & Buffer & $\begin{aligned} 110(0) \\
\text { mil. }\end{aligned}$ \\
\hline $\begin{array}{l}\text { Amocba } \\
\text { culture }\end{array}$ & & 0.004 & 0.004 & & $\begin{array}{l}\mathrm{CaHPO}, 0.002 \\
\mathrm{Call}_{3}\left(\mathrm{PO}_{1}\right)_{2} 0.002 \\
\mathrm{MgH}_{1} \mathrm{PO}_{1} 0.002\end{array}$ & pH 16.5 & 1000 \\
\hline Mytilus ${ }^{111}$ & 29.4 & 0.756 & 1.137 & & & $\begin{array}{l}50 \mathrm{ml} \text {. phosphate } \\
\text { butler at pH } 17.2\end{array}$ & 950 \\
\hline Homarus ${ }^{32}$ & 26.42 & 1.12 & 2.78 & & $\begin{array}{l}\mathrm{MgCl}_{2.61 \mathrm{H}} \mathrm{O} 0.82 \\
\mathrm{MgSO}_{3} 3 \mathrm{H}_{2} \mathrm{O} 0.7\end{array}$ & $\begin{array}{l}17.6 \mathrm{ml} .0 .5 \mathrm{M} \\
\mathrm{H}_{3: \mathrm{BO}} ; 0.96 \mathrm{ml} . \\
0.5 \mathrm{MI} \mathrm{NOH}\end{array}$ & 1000 \\
\hline Carcinus & 34.8 & 1.115 & 1.55 & & $\mathrm{MgCl}_{2} 2.66 \mathrm{C}$ & $\mathrm{NaHCO} \mathrm{CH}$ & 1110 \\
\hline Dogfish & 29.6 & 0.994 & 1.475 & & $\begin{array}{l}\mathrm{MgCl}_{2} 0.422 \\
\mathrm{MgSO}_{2} 2.02\end{array}$ & $\begin{array}{l}20 \mathrm{ml} .0 .5 \mathrm{Ml} \\
\mathrm{H}_{3} \mathrm{BO}_{. x ;} 0.96 \mathrm{ml} \\
0.5 \mathrm{MaOH}-\end{array}$ & 1100 \\
\hline $\begin{array}{l}\text { Dogfish, } \\
\text { Skate }^{3 .}\end{array}$ & 16.38 & 0.89 & 1.11 & 0.38 & $\begin{array}{l}\text { urea } 21.6 \\
\text { glucose } 1\end{array}$ & $\mathrm{NaH}_{2} \mathrm{PO}_{4} 0.06$ & 1000 \\
\hline Lophius $^{130}$ & 11.9 & 0.597 & 0.249 & 0.187 & $\begin{array}{c}\mathrm{MgCl}_{2} \\
0.348\end{array}$ & $\mathrm{KH}_{2} \mathrm{PO}, 0.099$ & 1000 \\
\hline Nematode & 9.0 & 0.42 & 0.24 & & & & 1000 \\
\hline Ascaris $^{5}$ & 7.5 & 1.42 & 0.50 & & $\begin{array}{l}\mathrm{MgCl}_{2} \\
0.418\end{array}$ & $\begin{array}{l}\text { phosphate } \\
\text { pHI } 6.7\end{array}$ & 1000 \\
\hline $\begin{array}{l}\text { Fresh-water } \\
\text { mussel }\end{array}$ & 1.2 & 0.15 & 0.15 & & & $\mathrm{pH} 7.8$ & 1000 \\
\hline Craytish $^{(n)}$ & 12.0 & 0.4 & 1.5 & 0.2 & $\mathrm{MgCl}=0.25$ & & 1000 \\
\hline Helix $^{12}$ & 5.87 & 0.73 & 1.99 & 1.87 & $\begin{array}{l}\mathrm{MgCl}_{2.6 \mathrm{H}} \mathrm{OO} \\
5.62\end{array}$ & $\mathrm{KHCO}, 0.22$ & 1000 \\
\hline Earthworm $^{105}$ & 8.0 & 0.2 & 0.2 & & $\begin{array}{c}\mathrm{MgSO}_{3} \cdot 7 \mathrm{H} \ldots \mathrm{O} \\
0.1\end{array}$ & & 1000 \\
\hline Earthworm $^{100}$ & 6.0 & 0.12 & 0.20 & 0.10 & & p117.4 & 1000 \\
\hline Grasshopper ${ }^{28}$ & 7.0 & 0.2 & 0.2 & 0.05 & $\begin{array}{l}\mathrm{NgCl}_{2} 0.1 \\
\text { glucose } 8.0\end{array}$ & $\begin{array}{l}\mathrm{NaH}_{2} \mathrm{PO}_{4} 0.2 \\
\mathrm{pH} 6.5\end{array}$ & 1000 \\
\hline Insect ${ }^{: 0}$ & 9.0 & 0.2 & 0.2 & & $\begin{array}{l}\text { glucose } \\
4.0\end{array}$ & $\frac{\mathrm{pH}}{7.5}$ & 1000 \\
\hline Frog ${ }^{4 \cdot 1}$ & 6.5 & 0.1 & 0.2 & & & $\begin{array}{l}\text { phosphiate } \\
\text { pH } 77.4\end{array}$ & 1000 \\
\hline Frog ${ }^{6 A}$ & 6.5 & $0.1+$ & 0.12 & 0.2 & $\begin{array}{l}\text { glucose } \\
2.0\end{array}$ & $\begin{array}{l}\mathrm{NaH}_{2} \mathrm{PO} \\
0.01 \mathrm{~g}\end{array}$ & 1000 \\
\hline $\begin{array}{l}\text { Mammal } \\
\text { (Ringer } \\
\text { Locke) }^{\infty 3}\end{array}$ & 9.0 & 0.42 & 0.24 & 0.2 & $\begin{array}{l}\text { glucose } \\
1-2.5\end{array}$ & $p 117.4$ & 1000 \\
\hline $\begin{array}{l}\text { Mammal } \\
\text { (Tyrodes) }^{88}\end{array}$ & 8.0 & 0.2 & 0.2 & 1.0 & $\begin{array}{l}\mathrm{MgCl}_{2} 0.1 \\
\text { glucose } 1.0\end{array}$ & $\begin{array}{l}\mathrm{NaHH}_{2} \mathrm{PO}_{4} \\
0.05 \mathrm{~s}\end{array}$ & 1000 \\
\hline $\begin{array}{l}\text { Artificial } \\
\text { Sea Water }\end{array}$ & 23.48 & 0.66 & 1.10 & \multicolumn{3}{|c|}{$\begin{array}{c}0.192 \mathrm{NgCl}_{2} 4.98, \mathrm{NaSO}_{3} 3.92 \mathrm{pH} 3.1 \\
\mathrm{KBr} 0.096, \mathrm{H}_{3} \mathrm{BO}_{3} 0.026 \\
\mathrm{SrCl}_{2} 0.024, \mathrm{NaF} 0.003\end{array}$} & 1000 \\
\hline
\end{tabular}

*If glucose is used, it is added just before saline is to be used.

Chlorine is the common halogen, and is the most widely dispersed and innocuous anion. Bromine can sometimes be substituted for chlorine but appears not to be a normal constituent in animals. Iodinc is essential for thyroxine in the vertebrates. Fluorine occurs in traces in vertebrate bone and 
aids the hardening of dental enamel, but in excess it causes abnormal bone structure. Fluorine is found in some molluscan shells, as in the oyster and in the mantle of the mollusc Archidoris. ${ }^{127}$

Calcium as carbonate or phosphate is a common structural material of endoskeletons and exoskeletons. Because of the low solubility of $\mathrm{CaCO}_{3}$ in alkaline water, much calcium is being continually stored up in masses of coral and of molluscan shell. Magnesium is important in some skeletons-foraminiferans, alcyonarians, echinoderms, and crustaceans. More magnesium is found in skeletons from warm areas, as in crinoids. ${ }^{30}$ Magnesium is an essential component of chlorophyll. Strontium can be substituted experimentally for calcium in bone; it has been reported as essential in a radiolarian skeleton. ${ }^{30}$ Barium is sometimes accumulated and can be deposited in calcareous structures. Silicon is the important material in the skeleton of most radiolarians and diatoms, and in the spicules of siliceous sponges; it is a constituent of radular teeth of certain molluscs.

Heavy metals are essential in the prosthetic groups of numerous enzymes. Iron is an important metal biologically in that it is an essential constituent of heme. The respiratory function of heme compounds is discussed in Chapter 9. The most widely distributed hemochromogen is cytochrome, a link in carbohydrate oxidation. Iron seems essential for the growth of the autotrophic flagellate Chilomonas 26,65 only if thiamine is present. The radular teeth of the marine mollusc, Patella, contain iron as $\mathrm{Fe}_{2} \mathrm{O}_{3}$ to the extent of 50 per cent of the ash weight. Copper also is active in an oxygen-transporting pigment (hemocyanin, Ch. 9) and is present in some respiratory enzymes; it is essential for hemoglobin synthesis in vertebrates. ${ }^{\text {ts }}$ Copper is found in the red pigment of feathers of South African turacos; traces of copper occur in oysters. ${ }^{30}$ Manganese occurs in traces in many animals, particularly in molluscs. The Mn content of oysters is high in gills and ovaries and is highest at the period of sexual activity. ${ }^{55}$ Manganese is accumulated by some hymenopterans and may be stored, as are some other metals, in mid-gut cells. ${ }^{25 a}$ This element is essential for the normal development of mammalian embryos, and lack of manganese causes a bone disease of fowls. ${ }^{120}$ Deficiency studies indicate that in the utilization of iron for hemoglobin there is some interaction between $\mathrm{Mn}, \mathrm{Co}$, and $\mathrm{Cu}$. ${ }^{120}$ Cobalt is more important for ruminant than for nonruminant mammals, and is a constituent of vitamin $\mathrm{B}_{12}$ (Ch. 5).

Zinc has rarely been reported in animal analyses, although it is concentrated by some bivalves, especially Pecten, and it doubtless is essential in traces. ${ }^{52}$ Its only proved function is as an essential constituent of the enzyme carbonic anhydrase (Ch. 9). Vanadium is selectively concentrated by several genera of ascidians (Ch. 9), by Pleurobranchus, and by a holothurian Stichopus. 37, 127 Nickel occurs in feathers; it has been reported in mammalian liver and in two marine molluscs. ${ }^{13}$ Aluminum is widely distributed and may be an essential constituent of the succinic oxidase system. ${ }^{63}$ The study of trace clements is made difficult by the necessity to prepare diets which are totally free of the elements in question; it is quite probable that many elements are required in specific reactions in amounts corresponding to relatively few atoms per cell. ${ }^{66}$ 


\section{SPECIAL FUNCTIONS OF CALCIUMI}

The principal elements of living organisms are widely distributed in all organs; certain elements may be concentrated in particular organs, as iodine in the thyroid. Calcium becomes predominantly localized in skeletal structures and in other organs which are of interest in comparative physiology. An excellent discussion of the function and metabolism of calcium in invertebrates is given by Robertson. ${ }^{103}$ In many arthropods calcium as carbonate and phosphate is deposited in and over the chitin, and no increase in size can occur except at the time of molt. In crabs (e.g. Carcinus) much $\mathrm{Ca}_{3}\left(\mathrm{PO}_{4}\right)_{2}$ is stored in the hepatopancreas before a molt, while in crayfish calcium is stored in gastroliths. Calcium may constitute 16 per cent of the skeleton of a normal crab and only 1 per cent of the skeleton of a freshly molted one. ${ }^{i 1}$ The gastroliths grow rapidly before a molt, and may consist of concentric layers deposited nocturnally. ${ }^{109}$ Secretion of the gastroliths is normally regulated by an inhibitory hormone from the sinus gland ${ }^{* 1}$ (Ch. 22). In insects the chitin is normally not calcified.

In earthworms the esophageal epithelium of the calciferous glands secretes spherules of $\mathrm{CaCO}_{3}$. Four functions of the calciferous glands have been suggested: (1) neutralizing the intestinal contents (this neutralization was shown by Robertson 101 to be by other digestive secretions); (2) fixing metabolic $\mathrm{CO}_{2} ;{ }^{56}$ (3) providing buffer capacity for the blood when in an acid condition; ${ }^{40,101}$ and, principally, (4) excreting excess calcium. ${ }^{101,125}$

In molluscs the calcareous shell is an important source of base in the neutralization of acids produced in anaerobiosis. In Venus kept out of water the total $\mathrm{CO}_{2}$ binding capacity of mantle fluid increases and the calcium concentration also increases. ${ }^{4 t}$ The shell is eroded by the mantle. Some nonvolatile acid (possibly lactic acid HL) increases in the mantle fluid, and this plus the increased $\mathrm{CO}_{2}$ equals the increase in calcium in the mantle fluid, thus forming $\mathrm{Ca}\left(\mathrm{HCO}_{3}\right)_{2}$ and $\mathrm{CaL}_{2}: 2 \mathrm{CaCO}_{3}+2 \mathrm{HL} \rightarrow \mathrm{Ca}_{(}\left(\mathrm{HCO}_{3}\right)_{2}+\mathrm{CaL}_{2}$. In the fresh-water mussel Anodonta cygnea kept in water under oil, a similar anaerobic metabolism produces acid, and the $\mathrm{pH}$ of the blood remains about 7.3. Here the carbonate increases more than does the calcium; hence there must be some additional source of cations. ${ }^{40 a}$

\section{CONCLUSIONS}

The maintenance of suitable ionic balance is an important step in frecing an animal from strict dependence on its environment. In only a few marine groups-particularly in the echinoderms, and to a lesser extent in the marine coelenterates-are the various elements of body fluids in nearly the same proportions as in the ocean. Ionic independence is necessary for life on land or in fresh water. Nothing is known of salt balance in the body fluids of parasites. In general, the farther removed a phylum or class is from the occan, the more divergent are the salt ratios from those of sea water. 'The bloods of vertebrates and of insects are least like the ocean, and each is unlike the other (Table 9). Toleration of changes in environmental salts has not been much investigated, but some animals such as echinoderms readily alter their body 
fluids with the environment, whereas others regulate in varying degrees, the concentrations of different elements changing in different proportions.

The ratios of ions in the body fluids in an individual animal, then, are determined in two ways: (1) by its genetic potentialities of ionic regulation, and (2) by its immediate environment. Macallum's proposal that the ionic ratios of an animal correspond with those of the ocean at the time of the closing of its circulatory system is untenable, although adequate tests with vertebrates have not been made. The principal mechanisms of ionic regulation are selective permeability, protein binding as in the Donnan equilibrium, active ionic absorption from dilute media, and selective excretion.

The availability of particular elements is rarely a limiting factor in arimal distribution. Calcium may determine abundance; fresh-water molluses, crayfish, etc, are more abundant in limestone regions than in areas of low calcium. Animals require sodium chloride, whereas higher plants are said to grow without this salt, but sodium chloride is sufficiently widespread not to limit terrestrial animals. Needham $9: 3$ points out that the eggs of many marine invertebrates are dependent on their medium for minerals. The only groups which can colonize fresh water have the capacity of providing enough ash within the cgg. Dependence on the environment for salts may, then, be more critical in embryonic than in adult life.

Ecological limitation by trace elements has hardly been investigated. Recently certain pporadic diseases of domestic animals have been correlated with lack of specific elements such as iodine, fluorine, manganese, and cobalt. ${ }^{120}$ Conversely, toxic levels of certain elennents may be locally important. It is likely that deficiencies or excesses may operate naturally in limiting fresh-water and terrestrial animals.

\section{REFERENCES}

1. Adolpir, E. F., J. Cell. E Comp. Pinysiol. 9:117-135 (1937). Osmotic relations in Phascolosoma.

2. Amberson, W. R., et al., Am. J. Plysiol. 122:22t-235 (1938). Tissue and plasma chloride.

3. Babkin, B. P., et al., Contr. Canad. Biol. E Fish. N. S. 8:209-219 (1933). Saline for Raja.

4. Bahl, K. N., Biol. Rev. 22: 109-147 (1947). Ionic composition, body fluids, earthworr.ss.

5. Balowin, E., and Moyle, V., J. Exper. Biol. 23:277-291 (1947). Physiological saline tor Ascaris.

6. Ban, P., MacLeod's Physiology in Modern Medicine. Ch. 82. (1941) p. 10931198, the Kidney by M. I. Gregerson.

7. Beadle, L. C., J. Eaper. Biol. 16:346-362 (1939). Chloride regulation, mosquito larvae.

8. Beadle, I. C., and Cragg, J. B., J. Exper. Biol. 17:153-163 (1940). Ionic adaptations, Gammarus.

9. Beali, 12. S., and Scmistr, F. O., J. Cell. \& Comp. Physiol. 14:205-215 (i939). Electrolytes in blood and nerve of squid.

10. Brmger, E., Pfiig. Arch. ges. Physiol. 228:790-807 (1931). Blood salts of freshwater and of brackish water crustaceans.

11. Bergen, E., and Betrie, A., Pflïg. Arch. ges. Physiol. 228:769-789 (1931). Permeability to iodide in crustaceans and sca urchins.

12. Bemnalib, A., and Bonnet, V., C. R. Soc. Biol. Paris 103:1119 (1930). Saline for If lix. 
13. Bertrand, G., and Macieboeun, M., C. R. Acul. Sci. 180:1380.1383 (1925 $\mathrm{Co}$ and $\mathrm{Ni}$ in animals.

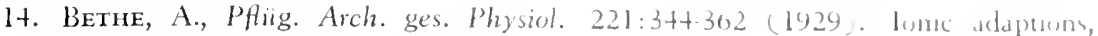
marine crustaceans and molluscs.

15. Bethe, A., and Berger, E., Pflirg. Arch. ges. Physol. 227:571.584 (1931). Innce analysis of bluod of many species.

16. Bialaszewicz, K., Arch. Internat. Physiol. 35:98.124 1932). Ionic regulation in Maja.

17. Bialaszewicz, K., Arch. Internat. Physiol. 36:41-53.(1933). Ionc composition of bloods, marine animais.

18. Bishop, G. H., Briggs, A. P., and Ronzoni, L., J. Biol. Chem. 66:77 $85(1925$. Composition of body thuid of bee larva.

19. Boche, R. 1)., and Buck, J. B., Physiol. Lool. 15:293-303 (1942). Hycloggen iun concentration of insect bluods.

20. Bodine, J. H., Biol. Bull. 51:363-369 (1926). Bloud of Orthoptera, pll.

21. Bogucki, M., Arch. Internat. Physiol. 35:197.213 (1932). Lonic regulation in marine isopod Mesidoter.

22. Boguck1, M., Arch. Internat. Physiol. 38:172-179 (193t). Ionic bulance in craytish.

23. Bond, R. M., Cary, K., and Hutchinson, G. E., J. Exper. Biol. 9:12-14 (1932). Chloride in blood of hagfish.

24. Boné, G. J., Ann. Soc. Roy. Zool. Belg. 75:123-132 (194t). Sodium-potassium ratio in insect hemolymph.

25. Boné, G. J., Nature 157:413 (1946). Sodium-potassium ratio in insects.

25a. Bowen, V. T., Brookhaven Cont. Rep. Biol. Appl. Nucl. Phys. 104-108 (1949). Mineral metabolism of insects.

26. Bowen, W. J., Biol. Bull. 79:114-130 (1940). Eftects of Va, Cu, Mn and le on Chilomonas.

27. Boyle, P. J., Conway, E. J., et al., J. Physiol. 99:401-1+ (19+1). Electrolyte balance in interfiber space and fiber fluid.

28. Brecher, L., Biochem. Ztschr. $211: 40-6+$ (1929 „. Minerals in butterllies.

29. Carlson, J. G., Biol. Bull. 90:109-121 (19+6). Saline for grasslioppers.

30. Clarke, F. W., and Wheeler, IV. C., Prof. Pap. U. S. Gcol. Surv. No. 12t, 1-62 (1922). Minerals in marine invertebrates.

31. Cole, W. H., J. Gen. Physiol. 23:575-58+ (1940), Ionic analysis of blood of marine animals.

32. Cole, W. H., J. Gen. Physiol. 25:1.6 (1941). Saline for Homarus.

33. Cole, W. H., and Kazalski, L. A., Bull. Mit. Desert 1sl. Biol. Lab. +1:40-46 (1939). Ions on lobster heart.

34. Conway, E. J., Biol. Rev. 20:56-72 (1945). Electrolytes in muscle and plarna; frog, rat.

35. Damboviceanu, A., C. R. Soc. Biol. Paris 105:913-91+ (1930). Calcium metabolism, Astacus.

36. Daver, D. G., J. Exper. Biol. 15:217-22+ (1938). Saline for nemattodes.

37. Davison, H., and Danielli, J. F., The Permeability of Natural Menbranes. Cambrictge Univ. Press (1943). 361 p. Sult content uf red bioud cells.

38. Daytey, h. E. Whemont-Smith, F., and (arroil, M. P., Eiul. Chem. $93: 172+(1531)$. Wontan equilibrium between I mulus bluod and seat water.

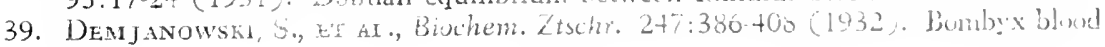
$\mathrm{pH}$.

40. Dotrekweich, H., Pflig. Arch. ges. Physiul. 232:263-286 1935, Calcilerus gland function in eartliworms.

40a. Dotrenweich, H., and ElssNen, E., Biol. Lentralbl. 55:128103 (1935). Hobilization of $\mathrm{Ca}$ by Arodonta.

4I. Drilmon, A., C. R. Sos. Biol. Jaris 115:119+-1195 (1934). Mharals in henulymph, Lepidoptera.

42. Drilhon, A., and Pora, E. A., Tium. Roscoff Smit. Biol. 1t:111.120 (1936, Mineral composition of Carcinus and Sacculinà.

43. Drilhon-Countors, A., Ann. Physiol. 10:377-41+1934, Minerals in crustaceans. 
44. Dugal, L. P., J. Cell. E Comp. Physiol. 13:235-251 (1939). Calcium of shell as buffer in clams.

45. Duval, M., and Prenant, M., C. R. Acad. Sci. 182:96-98 (1926). Electrolytes in ascidians.

46. Edwards, J. G., and Condorell1, L., Am. J. Physiol. 86:383-398 (1928). Electrolytes in blood and urine of fish.

47. Eichelberger, L., and Richter, R. B., J. Biol. Chem. 154:21-29 (1944). Water, electrolytes, in brain of dogs.

48. Elvenjem, C. A., Physiol. Rev. 15: $471-507$ (1935). Review of Cu in animals.

49. Fenn, W. O., Physiol. Rev. 16:450-487 (1936). Electrolytes in cat muscle.

50. Fenn, W. O., Совв, D. M., et al., Am. J. Physiol. 121:595-608 (1938). Electrolytes in cat muscle.

51. Florkin, M., Biochemical Evolution (1949). Ed. and trans. by S. Morgulis. Acad. Press New York. 157 p.

52. Follis, R. H., Day, H. G., and McCollum, E. V., J. Nutrit. 22:223-238 (1941). $\mathrm{Zn}$ requirements of rats.

53. Fox, D. L., Biol. Bull. 80:111-129 (1941). Tissue chloride, Mytilus.

54. Frédérice, L., Arch. Internat. Physiol. 14:309-351 (1922). Effects of minerals upon invertebrates.

55. Galtsoff, P., Physiol. Zool. 15:210-215 (1942). Manganese in oysters.

56. Gieschen, A., Zool. Jahrb., Abt. allg. Zool. u. Physiol. 48:121-168 (1930). Calciferous glands of earthworm.

57. Grafflin, A. L., and Ennis, D., J. Cell. E Comp. Physiol. 4:283-296 (1934). Urine formation in sculpin.

58. Hahnert, W., Biol. Bull. 62:205-211 (1932). Culture fluid for Amoeba proteus.

59. Hall, R. P., in Calkins' Protozoa in Biological Research (1941). New York, Columbia Univ. Press. Ch. IX, pp. 475-516. Food requirements of Protozoa.

60. Harreveld, A. V., Proc. Soc. Exper. Biol. E Med. 34: 428-432 (1936). Physiological saline for crayfish.

61. Harvey, H. W., Recent Advances in the Chemistry and Biology of Sea Water (1945). Cambridge Univ. Press. 164 p.

62. Hayes, F. R., and Pelluet, D., J. Mar. Biol. Ass. U. K. 26:580-589 (1947). Electrolytes in mollusc blood and muscle.

63. Horecker, B. L., Stotz, E., and Hogness, T. R., J. Biol. Chem. 128:251-256 (1939). Stimulation of succinic dehydrogenase-cytochrome system by aluminum.

64. Hosor, K., Sci. Rep. Tohoku Univ., Ser. IV, 10:377-386 (1935). Calcium and water exchange of sea anemones.

65. Hutchens, J. O., J. Cell. E Comp. Physiol. 16:265-267 (1940). Iron requirement, Chilomonas.

66. Hutchinson, G. E., Quart. Rev. Biol. 18:1-29, 331-363 (1943). Biogeochemistry of aluminum, trace elements.

67. Jones, E. I., McCance, R. A., and Shackleton, L. R. B., J. Exper. Biol. 12:59-64 (1935). Fe and Si in mollusc radular teeth.

68. Jullien, A., and Richard, J. G., C. R. Soc. Biol. Paris 122:1108-1110 (1936). Oyster blood $\mathrm{pH}$.

69. Kerr, S. E., J. Biol. Chem. 117:227-235 (1937). Ions in red cells and serum, mammals, birds.

70. Keys, A. B., Proc. Roy. Soc. London, B. 112:184-197 (1933). Chloride regulation in eel.

71. Kleiniolz, L. H., J. Cell. E Comp. Physiol. 18:101-107 (1941). Ca and molting in crabs.

7 la. Kocian, V., and Spacek, M., Zool. Jahrb., Abt. allg. Zool. u. Physiol. 54:180-190 (1934). Blood pH, Coleoptera.

72. Korzumi, T., Sci. Rep. Tohoku Univ., Ser. IV, 7:259-311 (1932). Electrolyte exchange in holothurian Caudina.

73. Korzumi, T., Sci. Rep. Tohoku Univ., Ser. IV, 10:269-275, 277-280, 281-286 (1935). Inorganic composition of tissues in the holothurian Caudina, and effects of changes in the medium.

74. Korzumi, T., and Hosol, K., Sci. Rep. Tohoku Univ., Ser. IV, 10:709-719 (1936). Electrolytes in medusae. 
75. Kramer, B., and Tisdali, F. F., /. Biol. Chem. 47:475+41 (1921. Electrolytes in human serum.

76. Krey, J., Zool. Jahrb., Abt. allg. Zool. u. Physiol. 58:201-224 (1937. Invect blood $\mathrm{pH}$.

77. Krogit, A., Skand. Arch. Physiol. 80:214222 (1938). Chloriele in molluscin tissues.

78. Krogin, A., Osmotic Regulation in Aquatic Animals (1939). Cambridge Unis Press. 242 p.

79. Krogh, A., Proc. Roy. Soc. Lond., B. 133:140-200 (I946). Permeability and active transport of ions.

80. Kyer, L., Biol. Bull. 82:68-78 (1942). Fiffect of sinus gland on gastroliths if crayfish.

81. Lieneriann, L. J., J. Cell. E Comp. Physiol. 11:149-159 (1938). Kidneys in osmotic and ionic regulation in crayfish.

82. Macallum, A. B., J. Physiol. 29:213-241 (1903). Inorganic composition of medusae.

83. Macallum, A. B., Proc. Roy. Soc. Lond., B. 82:602-624 (1910). Inorganic components of bloods and their relation to the sea.

84. Macallum, A. B., Physiol. Rev. 6:316-357 (1926). Paleochemistry of body fluids.

85. Manery, J. F., J. Cell. E Comp. Physiol. 14:365-369 (1939). Electrolytes in squid blood and muscle.

86. Marine Biol. Lab. Formulae and Methods (1936). Ed., O. Richards. Elasmobranch saline.

87. Marshall, E. K., and Graffind, A. L., Bull. Joluns Hopkins Hosp. 43:205-235 (1928). Kidney function, Lophius.

88. Mast, S. O., and Pace, D. M., J. Cell. E Comp. Physiol. 20: 1-9 (1942). Phosphorus requirement of Chilomonas.

89. McCance, R. A., and Shackleton, L. R. B., J. Mar. Biol. Ass. U. K. 22:269-272 (1937). Minerals in marine gastropods.

90. Mothey, H. L., Physiol. Zool. 8:62-84 (1934). Physiological saline for mussels.

91. Nagel, H., Ztschr. vergl. Physiol. 21:468-491 (1934). Ionic balance, Carcinus.

92. Needilan, J., Biol. Rev. 13:225-251 (1937). Osmotic properties of embryos in relation to evolution.

93. Needham, J., Biochemistry and Morphogenesis (1942). Cambridge Univ. Press. $786 \mathrm{p}$.

94. Needhan, J. G., ET. AL., Culture Methods for Invertebrate Animals (1937). Ithaca, N. Y., Comstock Publishing Co. 590 p.

95. PAGE, I. H., Biol. Bull. 52:168-172 (1927). Electrolytes in echinoderm eggs.

96. Pantin, C. F. A., J. Exper. Biol. 11:11-27 (1934). Saline for Carcinus.

97. Phillips, A. H., Am. J. Sci. IV, 46:473 475 (1918). Va in a holothurian.

98. Pitrs, R. F., J. Cell. E Comp. Physiol. 4:389-395 (1934). Urinary chloride, marine teleosts.

99. Pringle, J. W. S., J. Exper. Biol. 15:144-151 (1938). Physiological saline for insects.

100. Prosser, C. L., and Zimimerman, G. L., Physiol. Zool. 16:77.83 (1943). Physiological saline for earthworms.

101. Robertson, J. D., J. Exper. Biol. 13:279-297 (1936). Calciferous glands of earthworms.

102. Robertson, J. D., J. Exper. Biol. 16:387-397 (1939 Ionic composition of bloods of Homarus, Cancer, and Echinus.

103. Robertson, J. D., Biol. Rev. 16:106-133 (1941). Ca in invertebrates.

103a. Robertson, J. D., J. Exper. Biol. 26:182-200 (1949). Ionic regulation in some marine invertebrates.

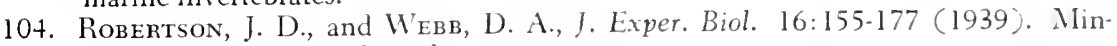
erals in marine animals and in sea water.

105. Rushton, W. A. H., Proc. Roy. Soc. Lond., B. 132:+23-437 (1945). Physi ological saline for earthworms.

106. Schlatter, M. L., J. Cell. E Comp. Physiol. 17:259-261 (1941). Electrolytes in blood of crustaceans. 
107. Scitolles, W., Ztschr. vergl. Physiol. 19:522-554 (1933). Ionic balance, Astacus and Eriocheir.

108. Scotr, G. H., Anat. Rec. 84:91-96 (1942). Distribution of minerals in muscle.

109. Scudamore, H. H., Physiol. Zool. 20:187-208 (1947). Gastroliths and molting in crayfish.

110. Shonl, A. T., Mineral Metabolism (1939). New York, Reinhold. 384 p.

111. Singh, I., J. Physiol. 92:62-90 (1938). Mytilus saline.

112. Smith, H. W., I. Biol. Chem. 81:407-419 (1929). Body fluids of elasmobranchs.

113. Sмгтн, H. W., J. Biol. Chem. 82: $71-75$ (1929). Body fluids of goosefish, Lophius.

114. Smitil, H. W., J. Biol. Chem. 82:651-661 (1929). Body fluids of turtles.

115. Sмiтн, H. W., Am. J. Physiol. 93:480-505 (1930). Absorption and excretion of water and salts by marine teleosts.

116. Smiti, R. I., Biol. Bull. 93: 72-88 (1947). Physiological saline for Cancer.

117. Steinbach, H. B., Biol. Bull. 78:444-453 (1939). Electrolytes in Phascolosoma muscle.

118. Steinbach, H. B., J. Cell. \& Comp. Physiol. 15:1-9 (1940). Electrolytes in Thyone muscle.

119. Steinbach, H. B., Ann. N. Y. Acad. Sci. 47:849-874 (1947). Ions in muscle.

120. Striles, W., Trace Elements in Plants and Animals (1946). New York, Macmillan. 189 p.

121. Thomas, G. W., J. Biol. Chem. 83:71-77 (1929). Donnan ratio of chloride between Limulus blood and sea water.

122. Toв1as, J. M., J. Cell. E Comp. Physiol. $31: 125-142$ (1948). Potassium, sodium, and water in muscle, nerve, and blood of cockroach.

123. Tobras, J. M., J. Cell. E Comp. Physiol. 31:143-148 (1948). Potassium and sodium in tissues of the silkworm.

124. Visscher, M. B., et AL., Am. J. Physiol. 142:550-575 (1944). Water and ion transfer across intestinal wall.

125. Voigite, O., Zool. Jahrb., Abt. allg. Zool. 11. Physiol. 52:677-708 (1933). Calciferous glands of earthworms.

126. Webs, D. A., Proc. Roy. Soc. Lond., B. 129:107-136 (1940). Osmotic and ionic regulation in Carcinus.

127. Webb, D. A., and Fearon, W. R., Sci. Proc. Roy. Dublin Soc. 21:484-504 (1937). Inorganic analyses of invertebrates.

128. Wrggleswonti, V. B., 1. Exper. Biol. 15:235-247 (1938). Chloride regulation, blood of Aedes larvae.

129. Willaier, E. N., J. Exper. Biol. $11: 283-306$ (1934). Hydrogen ion concentration of bloud of South American fish.

130. Young, J. Z., Proc. Roy. Soc. Lond,, B. 120:303-318 (1936). Saline for Lophius. 


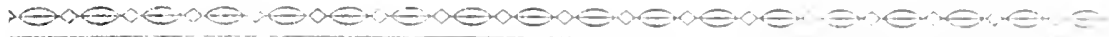

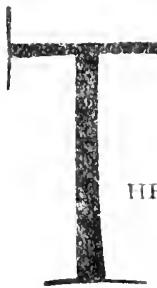

\section{Protein Specificity}

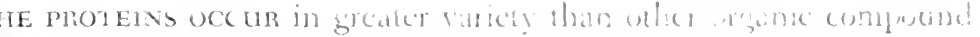

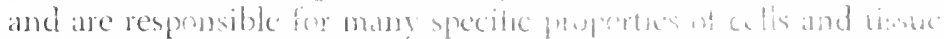
Proteins exist as huge molecules which wry 111 amme wid com position and in their physical form (stereochemistry. Nhen of ife aluctural proteins axe called fibrous-collagen from conncoive tissuce, heratin from law mrosin from muscle, and fibrin from blond cho Nost of the anctabolic pon teins are called globular-hemoglobins, hemexymins, alloumins, and the zho bulins. Fibrous proteins accur in long stender chains which can undergo folding and which show reproducible geoncuric patterns as seen ly x-ray diffraction: they nay be sinuilis amomg different speeses. I lie structure of globular proteins is only slighty hnown; ther viry in moleculan werghts from tens of thousands to a few milliom, and characteristically they are specific for different kinds of animal. Some porteins, such as actin, are reversbly fibrous or globular.

Protein Concentration in Blood. Protein molecules arce for he most part, so large that they cannot diffuse through cell membranes. I lenee in body Huids they are responsible for a "colloid osmotic pressure" which limits the anount of fluid which can diffuse across gill and kidney membranes. lilud proteins also constitute a nitrogen reserve for an animal. In addition, certain proteins have specific chemical functions in the budy Huids.

The absolute concentration of procin in blood is highest in animals with well-developed circulatory systems (1able 10. This maly be related to the higher blood pressures in such amimals. Very lou protein concentrations (about $0.1 \mathrm{gm}$. per cent) are lound in the blood of bivalie molluscs. In annelids coelomic fluid contains much less protein than does the blood. Protein in the blood of arthropods varies from 1 to $6 \mathrm{gm}$. fer cent, and in mammals protein concentration in the blood is usually 5 to 8 gm. per cent. Insects have an unusually high amino acid content in their blool.

Electrophoretic Separation of Proceins. The poteins which have been most studied from the viewpont of comparative phistoles a be buel frotcins. These can he separated by various means. One methud of separting the proteins of blood serum is to observe their migration in an electrophoretic tic ld. In the Tiselius electrophoresis apparatus, protein compurents can be scpirlated visually. In mammalian sera the protcins, in order of decreasme mability, an albumin, the $\alpha$ - and $\beta$-globulins, and linally fibrinogen and $\gamma$ giobulin. The relative proportion of these components diflers among diflerent specics and in various pathological conditions. 1 Dot anly am the various protin fractions be separated visually, but some electrophoresis aclls are so aranged that the particular protein fractions can be remored after separation. In haman 
serum, for example, the $6.03 \mathrm{gm}$. per cent total protein consists of albumin 3.32, $\alpha$-globulin $0.84, \beta$-globulin $0.78, \gamma$-globulin 0.66 , and fibrinogen 0.43 gm. per cent. ${ }^{16}$

TABLE 16. CONCENTRATION OF PROTEINS IN BLOOD OR COELOMIC FLUID

\begin{tabular}{|c|c|c|c|c|}
\hline \multirow{3}{*}{ 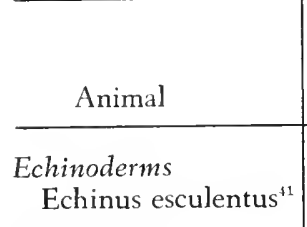 } & $\begin{array}{c}\text { Protein } \\
\text { Concentration } \\
\text { Gm./100 ml. }\end{array}$ & Animal & \multicolumn{2}{|c|}{$\begin{array}{c}\text { Protein } \\
\text { Concentration } \\
\text { Gm./100 ml. }\end{array}$} \\
\hline & & Dixippus & & 1.0 \\
\hline & 0.3 & \multicolumn{2}{|c|}{$\begin{array}{l}\text { silkworm (caterpillar) } \\
\text { (Florkin, in }\end{array}$} & 1.0 \\
\hline Annelids and & & \multirow{2}{*}{\multicolumn{2}{|c|}{$\begin{array}{l}\text { (Florkin, in } \\
\text { Wigglesworth }^{45} \text { ) } \\
\text { (Florkin, in }\end{array}$}} & \\
\hline $\begin{array}{l}\text { sipunculids } \\
\text { Sipunculus }\end{array}$ & 0.005 (coel. fl.) & & & \\
\hline $\begin{array}{l}\text { Sipunculus } 24 \\
\text { Arenicola }^{21}\end{array}$ & $\begin{array}{c}0.005 \text { (coel. fl.) } \\
1.7 \text { (blood) (0.00 coel. fl. })\end{array}$ & \multicolumn{2}{|c|}{ Wigglesworth ${ }^{16}$ ) } & \\
\hline $\begin{array}{l}\text { Arenicola } \\
\text { Pheretima }\end{array}$ & & \multirow{2}{*}{\multicolumn{2}{|c|}{$\begin{array}{l}\text { silkworm (spinner) } \\
\text { (Florkin, in } \\
\text { Wigglesworth"s) }\end{array}$}} & 6.0 \\
\hline$(\text { earthworm })^{3}$ & 3.64 (blood) $(0.48$ coel. fl. $)$ & & & \\
\hline Molluses & & \multicolumn{2}{|c|}{ silkworm (emerging) } & 2.0 \\
\hline Pecten $^{24}$ & 0.075 & \multirow{2}{*}{\multicolumn{2}{|c|}{$\begin{array}{l}\text { (Florkin, in } \\
\text { Wigglesworth }\end{array}$}} & \\
\hline Solen ensis ${ }^{24}$ & 0.12 & & & \\
\hline Mya arenaria ${ }^{24}$ & 0.09 & \multicolumn{2}{|c|}{ Prochordates } & \\
\hline Mytilus edulis ${ }^{2 t}$ & 0.145 & \multicolumn{2}{|l|}{ Phallusia $^{37}$} & 0.11 \\
\hline Aplysia ${ }^{24}$ & 0.28 & \multicolumn{2}{|l|}{ Vertebrates } & \\
\hline Anodonta $^{37}$ & 0.074 & \multicolumn{2}{|l|}{ Petromyzon $^{3 \tau}$} & 2.31 \\
\hline Pinna nobilis ${ }^{37}$ & 0.028 & \multicolumn{2}{|l|}{ Scylliorhinus ${ }^{37}$} & 2.20 \\
\hline Helix $^{37}$ & 2.4 (summer), 3.3 (winter) & \multicolumn{2}{|l|}{$\operatorname{dogfish}^{40}$} & 1.62 \\
\hline Sepia $^{37}$ & 4.978 & \multicolumn{2}{|l|}{ Cyprinus $^{37}$} & 2.59 \\
\hline Crustaceans & & \multicolumn{2}{|l|}{ Barbus $^{37}$} & 3.69 \\
\hline Carcinus maenas ${ }^{21}$ & 4.0 & \multicolumn{2}{|l|}{$\mathrm{Bufo}^{37}$} & 3.42 \\
\hline Carcinus maenas $^{17}$ & 6.0 & \multicolumn{2}{|l|}{$\operatorname{Rana}^{37}$} & 2.40 \\
\hline Cancer pagurus ${ }^{41}$ & 3.49 & \multicolumn{2}{|l|}{$\operatorname{Rana}^{4}$} & 3.27 \\
\hline Cancer pagurus $^{24}$ & 4.37 & \multicolumn{2}{|l|}{ newt $t^{40}$} & 3.7 \\
\hline Eriphia $^{37}$ & 7.38 & \multicolumn{2}{|l|}{ salamander ${ }^{37}$} & 2.16 \\
\hline Homarus vulgaris ${ }^{41}$ & 2.25 & \multicolumn{2}{|l|}{ T'estudo graeca ${ }^{37}$} & 3.76 \\
\hline Homarus ${ }^{24}$ & 4.0 & & 4.57 \\
\hline Pagurus ${ }^{2 !}$ & 4.43 & \multirow{2}{*}{\multicolumn{2}{|c|}{$\begin{array}{l}\text { Anguis }^{37} \\
\text { Trepidonotus }\end{array}$}} & 4.03 \\
\hline Nephrops ${ }^{2+}$ & 4.2 & & & 2.80 \\
\hline Insects & & \multicolumn{2}{|l|}{ Gallus $^{37}$} & 3.18 \\
\hline Hybernia & & chicken $^{4 \theta}$ & & 4.1 \\
\hline (caterpillar) $)^{3 i}$ & 5.86 & goose $^{37}$ & & 3.00 \\
\hline Deilephila (pupa) & 2.79 & & & 5.45 \\
\hline Apis (larva) & 6.6 & $\mathrm{rat}^{37}$ & & 5.9 \\
\hline Bombus (Florkin, in & $\begin{array}{l}0.0 \\
5.0\end{array}$ & $\operatorname{cow}^{37}$ & & 5.02 \\
\hline Wigglesworth ${ }^{48}$ ) & & $\begin{array}{l}\text { horse }^{37} \\
\text { horse }^{40}\end{array}$ & & 7.68 \\
\hline Hydrophilus & $3-4$ & $\begin{array}{l}\text { horse } e^{40} \\
\operatorname{dog}^{37}\end{array}$ & & 7.0 \\
\hline (Florkin, in & & $\begin{array}{l}\operatorname{dog}^{37} \\
\operatorname{dog}^{38}\end{array}$ & & 4.7 \\
\hline Wigglesworth ${ }^{49}$ ) & & & & 5.7 \\
\hline Aeschna nymphs & $2.6-3.3$ & $\operatorname{man}^{37}$ & & $\begin{array}{l}6.0 \\
6.6\end{array}$ \\
\hline (Florkin, in & & $\operatorname{man}^{10}$ & & 7.6 \\
\hline Wigglesworth $^{18}$ ) & & $\operatorname{man}^{5}$ & & 7.1 \\
\hline
\end{tabular}

Few electrophoretic studies have been made on the blood of invertebrates, but in some Crustacea several components of different mobilities have been observed. ${ }^{45,}+6$ The rates of electrophoretic migration of the albumin of eggs 
of various birds and of the hemoglobins of birds and mammals differ by a factor of two at most; this species difference in mobility is mucls less than the immunological differences among protcins. 32

Sedimentation of Proteins. Another method of separating blood proteins is sedimentation by ligh-speed centrifugation. Velocity of sedimentation depends on the weight and shape of the protein molecules. Centrifugal separa tion has been applied particularly well to the respiratory pigment proteins. $4 "$ The chemistry of these pigments is discussed in Chapter 9. From the sedi mentation data it is possible to calculate the molecular size of large proteins. For example, the hemoglobin molecule is similar in size in mammals, birds, and fishes (m.w. about 68,000), is slightly larger in amphibians and reptiles, and is less than half as large in cyclostomes (m.w. about 20,000) (see Table 51 in Chapter 9). Among invertebrate animals the hemoglobin molecules which are free in the plasma are large $(\mathrm{m} . \mathrm{w} .>900,000)$, whereas those in corpuscles are small. The copper-containing pigment hemocyanin always occurs free in the plasma, and its molecular size varies greatly among different species. In general, the molecules of molluscan hemocyanins are very large (m.w. $>2,000,000$ ). Those in arthropods are smaller and often separate into several layers in the centrifuge, each heavier one corresponding to a multiple of a smaller unit (Table 52, in Chapter 9). The blood pigments of invertebrates, both hemoglobins and hemocyanins, show greater specific differences in size than do vertebrate hemoglobins.

Serum proteins of some mammals, particularly of man, have been studied by sedimentation and by a variety of other physical techniques. ${ }^{15}, 17.18$ These globular proteins are shown to be elongate, all being similar in width but differing greatly in length (Table 17).

Crystal Structure: Hemoglobins. Hemoglobins from different species have been separated on the basis of the characteristics of their crystals as obtained by evaporation after lysis by ether. It has been claimed to that the crystals of closely related species are more nearly alike than those of distantly related species. Some exceptions to classical taxonomy are noted. The hemoglobin crystals of the sea lion are similar to those of the bears, and the hemoglobin crystals of seals resemble those of the otter; those of the guinca hen show more resemblance to those of the ostrich than to those of other members of the

TABLE 17. SIZE OF BLOOD PROTEINS ${ }^{1 *}$

\begin{tabular}{c|c|c|c}
\hline \hline Protein & Mol.wt. & length, $\AA$ & width, $\AA$ \\
\hline human serum albumin & 69,000 & 150 & 38 \\
human serum globulin & 156,000 & 320 & 36 \\
human serum fibrinogen & 500,000 & 900 & 33 \\
\hline
\end{tabular}

Gallinae. However, the form of the hemoglobin crystals depends on such factors as the amount of salt present when crystallization occurs, and one species may show one or several crystallographic types. Hence specific differences in hemoglobin crystals have not been much used by systematists in recent years. Specific differences in hemoglobins are also shown by lack of addition of solubilities of the pigment from unrelated animals. 31

Immunological Specificity of Proteins. Far more sensitive than preceding physical methods as indication of differences among serum and tissue proteins 
are immunological reactions. The $\gamma$-globulin fraction of serum proteins contains antibodics of many sorts, natural and acquired, which are highly specific in their reactions with other proteins, with foreign blood celis, and with bacteria.

Most proteins and sone polysaccharides can act as immunizing antigens, in that they can induce the production of antibodies in animals whose circulating fluid does not contain the same protein or polysaccharide. The antibodies produced react with their specific antigens, causing agglutination of cells, lysis of cells, or peccipitation of foreign proteins. Immune reactions of mammals are discussed in textbooks of immunology. ${ }^{7}$ Development of antibodies, such as agglutinins, lysins, precipitins, or others, is important in defense against infectious agents. Inmunity reactions are best known in mammals, but they have been studied in most groups of animals. Among invertebrates many examples of natural immunity are known. Caterpillars and other insects have been successfully immunized against pathogenic organisms, $: 7$ although failure to produce antibodies is reported in echinoderms, annelids, and mol luscs. Phagocytosis is an important immune process in invertebrates and is shown by many types of cell.

Naturally Occurring and Immune Agglutinins. The sera of various vertebrates normally contain agglutinins that act on the red blood cells of certain other species or on the red cells of certain groups of individuals of the same species but of nonhomologous strain. A fraction of a milliliter of serum from a fish injected into a rabbit may cause the death of the rabbit, owing to cell agglutination. Landsteiner $: 3$ has used agglutination to separate the blood types among humans into four groups, O, A, B, and AB. Each individual contains in his serum the natural antibodies for the antigen types which are absent from his erythrocytes. Thus transfused red cells are agglutinated by scrum antibodies of different type from the cells. The chimpanzees, gibbons, and orangutans $2: 30,3.3$ can each be classified under two of the human types, whereas in the old world monkeys, Cercopithecediae, no agglutinogens of human types are found, and in new world monkeys, Platyrrhina, and lemurs there is one blood type which is similar to type $\mathrm{B}$ in man.

In addition, there are two antigens in human erythrocytes, $M$ and $N$, for which there are normally no antibodies; the distribution of $M$ and $N$ antigens is hereditary and independent of the $A$ and $B$ antigens. A third antigen, $R h$, occurs in erythrocytes, and Rh-positive red cells can induce the production of Rh antibodies in a person negative for the Rh factor. This antiserum can then cause agglutination, followed by hemolysis of Rh-positive cells. When the three classes of hemagglutinogens, each independent of the other, are considered, the total number of genetic blook types is large; furthermore, subgroups are known. The three factors are distributed genetically, and certain races display characteristic combinations. Agglutinating factors in the blood of various species of mammals and birds are favorite subjects of genetic studies.

Besides the agglutinins which clump red blood cells, there are agglutinins for other blood cells and for sperm. These have been found not only among vertebrates but also among invertebrates. For example, serum of the spiny lobster, Panulirus interniptus, contains at least ten heteroagglutinins for sperm or blood cells of a varicty of species. ${ }^{45,}, 46$ These agglutinins occur in serum proteins other than hemocyanin. Cross adsorption tests, as used by Land- 
steiner and others, aid in defining a particular agglutinin group; adsonption on cells (blood or sperm) of any one species removes the agshlutinins tor wher species of the same group but not for more distanty related groups. I he seminal thuids and body thuids of various inverebrates contain natural heteroig glutinins for sperm and for vertebrate red blood cells. ${ }_{1}$

Immune Precipitins. The immunological reactions most widely used in indicating degree of difference among specific proteins of different species are precipitin tests. Nuttall :3: applied precipitin tests to seral and egg albumin from many species of vertebrates and indicated the usefulness of the test in taxonomy. A standard procedure at present is to build up antibodies in a rabbit by injecting known amounts of an antigenic protein. 'I he rabbit serum is then mixed with the protein to be tested, and the maximum dilution of this protein giving a precipitate is noted. Homologous serum or egg albumin, that is, protein from the same species as the antigen, gives a maximum precipitate, and more distant taxonomic relations tend to be inclicated by less precipitate. Results express the greatest dilution for heterologous precipitate as percentage of the threshold for the homologous reaction (titer). To be highly signiticant, reciprocal heterologous tests should be performed, that is, test of antiserum $x$ against antigen $y$, as well as test of antiserum y against antigen $x$. Unfortunately, reciprocal tests are not always in close agrecment. Quantitative measure ments of the precipitate are made with a turbidometer, by a "ring test," or by measuring the change in nitrogen concentration after precipitation. Detailed descriptions of methods, and reviews of the literature are given by several authors. ${ }^{8}, 9,11,12,19,51$

Precipitin antibodies can be built up against other tissue proteins besides the blood globulins. Such antibodies show some species and organ speciticity. Proteins of the lens of the eye have antigenic components which are very similar, even in different classes of vertebrates. ${ }^{25}$ Proteins which are indistinguishable chemically can be separated inmunologically.

The degree of similarity of serum proteins among species of mammals as indicated by serological reaction agrees well with taxonomic relationships. Sheep blood is close to that of beef, farther from that of pig and horse, and very far from that of dog. "The superfamilies of rodents are separated readily, families and sub-families less readily. "3; The chicken is close to the turkey, farther from the guinea hen, and still farther from the duck, as inclicated by reactions of either serum proteins ${ }^{1:}$ or egg albumin. "is In the ovalbumin the protein responsible for homologous reaction differs from the protein respon sible for heterologous precipitation. "It In the genus Rana the species cates biana and clamitans are close serologically, whereas pipiens is distant. ${ }^{13}$ Among urodeles the primitive genus Cryptobranchus is far from Amphimma, Siren, and Necturus, and these latter genera are nearly equally separated (Fig. 27).

The relations among prochordates and other invertebrates liave been examined by comparing precipitin titers for several antibodies with proteins from a wide variety of animals. 4!) The percentages of heterologous titer are low between ascidians and balanoglossids, but these titers are higher than between either of these groups and any other phylum. On the basis of such comparison the prochordates are more closely related to echinoderms than to polychactes and to Phascolosoma. Polychaete antisera give low reactions to ascidians and 
echinoderms, medium reactions to Limulus, and high reactions to other annelids. Molluscs (gastropods and pelecypods) are closer serologically to annelids than to arthropods. ${ }^{50}$ The principal antigenic protein in crustacean blood is hemocyanin, ${ }^{45}$ and the antibodies react with proteins of other arthropods but not with those of molluscs. ${ }^{10}$ The crab Pseudocarcinus gigas is related, according to the precipitin test, to four other genera of crabs (Brachyura) but not to Palinurus, the Astacura, or Anomura. ${ }^{15}$ Relationships among orthopteran insects have been investigated by reciprocal serological tests on equivalent amounts of tissue proteins. ${ }^{35}$ The four families are about equally separated, the average interfamily ratio of heterologous to homologous titer being 11.5 per cent, the average intergeneric ratio among grasshoppers (Acrididae) being

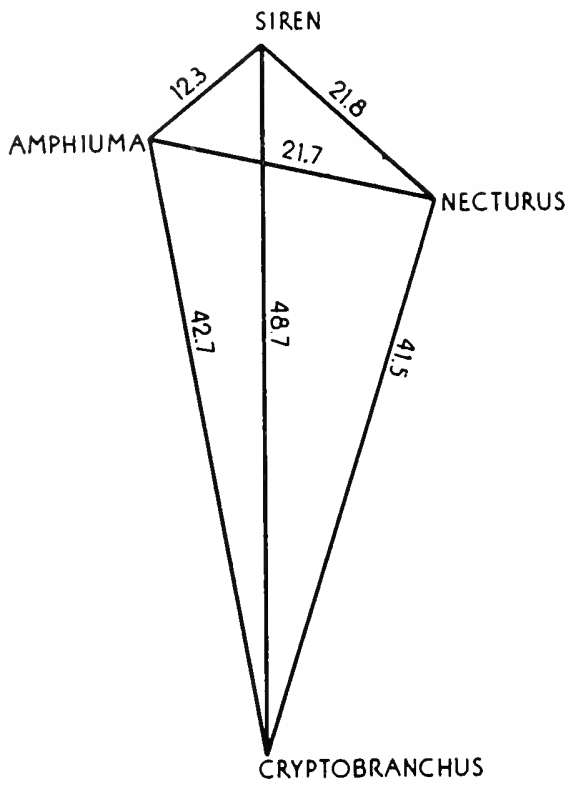

Fig. 27. Relative serologic relationships of four urodele amphibians.

From Boyden and Noble. ${ }^{13}$

28 per cent and the ratio between two species of the genus Melanoplus being 54.7 per cent.

Antisera for cuticular tissue of three species of Ascaris parasitic in mammals react indiscriminately among the three antigens but not with the cuticle of an avian Ascaris. ${ }^{14}$ Acanthocephala appear to be related serologically to the Platylelminthes but not to the Nemathelminthes, although some precipitin reaction occurs between the Platyhelminthes and Nemathelminthes. ${ }^{22}$

The preceding examples are sufficient to indicate the importance of applying immunological reactions to the proteins of comparable tissues from different animals as a test of taxonomic relationship. Why should the antigenic proteins of one species or phylum have such structural similarity that they react reciprocally with their antisera but not with antisera of more distantly related animals? The answer to this question remains one of the unsolved subtleties of protein structure. 
Permeability of Erythrocytes. Another approach to the chemical differences among species is found in the permealbility of red blood cells. The permeability of red cells to various organic molecules has been studied by measuring the swelling and ultimate hemolysis in an isotonic solution of the material to be tested. Table 18 shows the permeability of different mammalian erythroctes for ethylene glycol, glycerol, and erythritol. I his table show's that there are marked species differences in permeability to these substances and that the

\section{TABLE 18. PERMEABILITY OF RED BLOOD CORPUSCIES":}

Time required for 75 per cent hemoly'sis in various media,

in seconds unless otherwise stated.

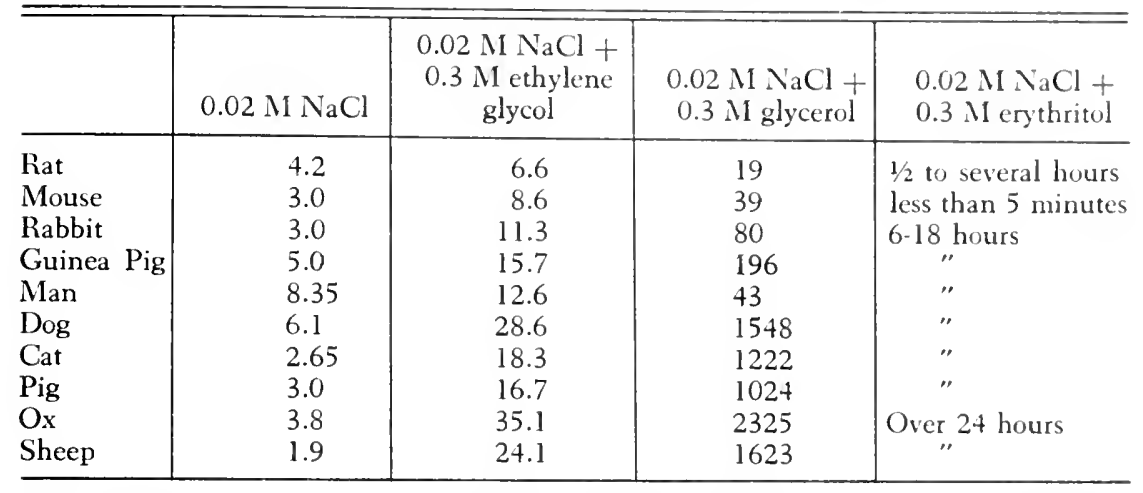

permeability to water, as indicated by swelling of cells from different species in hypotonic $\mathrm{NaCl}$, is unrelated to the permeability to the organic substances. These striking species differences in permeability reflect differences in the composition of the plasma membrane of the erythrocytc.

\section{CONCLUSIONS}

The proteins of an individual animal present a vast array. They can be separated by chemical and physical means as fibrous and globular, primarily structural and metabolic. The globular proteins, particularly those of blood, have been separated according to their electrophoretic mobilities. The proteins of one electrophoretic class, for example the hemocyanins or hemoglobins, exist in different molecular sizes as shown by sedimentation in a high-speed ccntrifuge; they may also differ in crystal form.

The most sensitive tests available for differences anong proteins of similar function and occurrence are immunological. Antibody-antigen reactions probably have the same sort of basis in molecular structure as has enzyme specificity. Antibodies are built up in response to the antigens of a particular species, and will react with those antigenic proteins (by agglutination or precipitation) but not with the proteins of species distantly related to the original one from which the antigen was obtained. One basis of taxonomy may, therefore, be stereochemical. It is possible that equally important specific differences exist among polymerized nucleic acids as among proteins. Antigenic specificity of scrum proteins represents one set of characters which can be used along with other chemical and morphological characters to study relationships among animals. Relations within animal classes and phyla have been corroborated, as have the relations of echinoderms to prochordates, and of acanthocephalans to flatworms. 


\section{REFERENCES}

1. Abramson, H. A., Moyer, L. S., et al., Electrophoresis of Proteins and the Chemistry of Ccll Surfaces (1942). New York, Reinhold. 341 p.

2. Babers, F. H., J. Agr. Res. 57:697-706 (1938). Protein in blood, armyworm.

3. Bahl, K. N., Biol. Rev. 22:109-147 (1947). Pheretima, protein in blood, in coelomic fluid.

4. Beiter, R. N., and Scott, F. H., Am. J. Physiol. 91: 265-274 (1929). Plasma proteins, frog.

5. Best, C. H., and Taylor, N. B., Physiological Basis of Medical Practice (1937). Baltimore, Wood. 1684 p. Ch. I. Blood protein, man.

6. Bishop, G. H., Briggs, A. P., et AL., J. Biol. Chem. 66:77-88 (1925). Blood protein, insects.

7. Boyd, W. C., Textbook of Immunology (1943). New York, Interscience. 503 p.

8. Boyden, A. A., Biol. Bull. 50:73-107 (1926). Precipitin reaction in study of animal relationships.

9. Boyden, A. A., Am. Nat. 68:516-536 (1934). Precipitins and phylogeny.

10. Boyden, A. A., Yearbook Carnegie Inst., Wash. 35:82 (1936), ibid. 38:219-220 (1939). Serological relationships of invertebrate animals.

11. Boyden, A. A., Physiol. Zool. 15:109-145 (1942). Review of systematic serology.

12. Boyden, A. A., Am. Nat. 77:234-255 (1943). Serology in the study of animal relationships.

13. Boyden, A. A., and Noble, G. K., Am. Mus. Novit. 606:1-24 (1933). Serological relations of Amphibia.

14. Canning, G. A., Am. J. Hyg. 9:207-226 (1929). Precipitin reactions, Ascaris and related tissue.

15. Clark, E., and Burnet, F. M., Austral. J. Exper. Biol. \& Med. Sci. 20:89-96 (1942). Serological relations of Crustacea.

16. Conn, E. J., Proc. Am. Phil. Soc. 88:159-173 (1944). Blood derivatives and substitutes.

17. Cohn, E. J., and Edsall, J. T., Proteins, Amıno Acids and Peptides as Ions and Dipolar Ions (1942). New York, Reinhold. 686 p.

18. Cohn, E. J., Oncley, J. L., et Ai., J. Clin. Invest. 23: 417-432. (1944) Protein fractions of human plasma.

19. DeFalco, R. J., J. Exper. Biol. E Med. 46: 500-502 (1941). Comparison of precipitin methods, birds.

20. Dayley, M. E., Fremont-Smith, F., et al., J. Biol. Chem. 93:17-24 (1931). Blood protein, Limulus.

21. Drililon, A., and Pora, E. A., Trav. Roscoff Stat. Biol. 14:111-120 (1936). Protein in Carcinus blood.

22. Eisenbrandt, L. L., Am. J. Hyg. 27:117-141 (1938). Serological relations of helminths.

23. Enhardt, A., Ergebn. Fortschr. Zool. 7:279-377 (1931). Application of serology to phylogeny.

24. Flonkin, M., and Blum, H., Arch. Internat. Physiol. 38: 353-364 (1934). Protein content of invertebrate bloods.

25. Hextoen, L., and Schulhof, H., J. Infect. Dis. 34: 433-439 (1924). Specificity of lens proteins.

26. 1leller, J., Biochem. Ztschr. 255:205-221 (1932). Composition of blood, butterAly pupa.

27. Ilufi, C. G., Physiol. Rev. 20:68-88 (1940). Immunity reactions of invertebrates.

28. Jacons, M., Ergebn. Biol. 7:1-55 (1931). Species differences in red cell permeability.

29. Landsteinen, K., J. Immunol. 15:589-600 (1928). Cell antigens and individual specificity.

30. Landstliner, K., The Specificity of Serological Reactions (1945). Harvard Univ. Press. 310 p.

31. Landstinini, K., and Iemelbercen, M., J. Gen. Physiol. 6:131-135 (1923). Differential solubilities of hemogkbins. 
32. Landsteiner, K., Longswortu, L. G., it al., Science 88:83.85 (1938). Electrophoresis of egg albumins.

33. Iandsteinen, K., and Milleik, (. P., /. Frper. Med. +2:841852, $853.862,81,3$ S72 (1925). Serological studie's of prinates.

34. Landsteiner, K., and Scheer, J. V. D., J. Frper. Med. $71: 415+45+$ (1940), Crm rcactions of $\mathrm{cgg}$ albumins.

35. Leone, C. A., Biol. Bull. 93: 64-71 (1947). Serulugical relation, Ortheptera.

36. Levine, H. P., and Moody, P. A., Physiol. Zool. 12:400411 (1939). Serolugical relations of rodenes.

37. Iustig, B., and ERnst, T., Biochen. Ztschr. 289:365-389 (1937). Bhod fornteins.

38. Muntz, J., Barron, E. S. G., Et. al., u be published, N. N. E. I. S. Serum proteins, dogs.

39. Nutrale, G. H. F., Blood Immunity and Blond Relationship (1904.. Cambridge Univ. Press. $44+$ p.

40. Reichert, E. T., and Browa, A. P., Pub. Carnegie Inst, Wash. 112, 338 p. (1909). Characteristies of hemoglobins from difterent species.

41. Robertson, J. D., J. Exper. Biol. 16:387-397 (1939). Pruteins nn Echinus, Can cer, Homarus.

42. Svedberg, T., and Penerisen, K. O., The Ultracentrifuge (1940). Oxford, Clarenden Press. $478 \mathrm{p}$.

43. Thomas, G. W., J. Biol. Clem. 83:71-77 (i929). Blood protein, Limulus.

44. Tylen, A., Biol. Bull. 90:213-219 (1946). Naturd heteroigglutinns in inverte. brate animals.

45. Tyler, A., and Metz, C. B., I. Exper. Zool. 100:387-406 (1945). Identification of agglutinin proteins in Panulirus blood.

46. Truer, A., and Scheer, B. T., Biol. Bull. 89:193-200 (1945). Natural heterodyglutinins of Panulirus.

47. W'ese, D. A., Proc. Roy. Soc. Lond., B. 129:107-136 (1940). Blonel protein, Carcinus.

48. Wrgglesworth, V. B., Principles of Insect Physiology (1939). I ondon. Methuen. $43+$ p. Ch. X. Data on blood.

49. Whluelm, R. W., Biol. Bull. 82:179-189 (1942). Serology and origin of vertebrates.

50. Wilmenni, R. W., Biol. Bull. 87:96-105 (1944). Serological relations, invertebrates.

51. Wolfe, H. R., and Baier, J. G., Physiol. Zool. 11:63-74 (1938). Cumparison of precipitin techniques. 


\section{Nutrition}

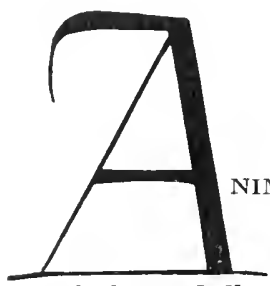

NIMALS DIFFER GREATLY in their capacity to alter their diet, to convert compounds of one class to another, and to synthesize specific compounds which are essential for cellular metabolism. What is a vitamin for one animal may not be a dietary requirement for another. It has frequently been pointed out that the amino acids which constitute proteins are the same in all organisms, although the proportions vary from one protein to another, and also that the coenzymes of glycolysis and cellular respiration, such as the phosphopyridine nucleotides, are similar in all cells. There is variation, however, among animals as to which amino acids and which coenzymes can be synthesized, and which must be taken as food. Also some particular food requirements are associated with physiological specializations of certain animals. Substances needed for specific cellular reactions may be obtained by an animal in two ways: they may be synthesized by the organism, or they may have to be taken in its diet. Some substances may be synthesized by an animal but in insufficient amounts for cellular needs; hence these are required in the diet of the organism. Some of the specific mineral requirements of animals have been mentioned in Chapter 3, page 94. Salt mixtures have been worked out empirically for several groups of animals, particularly for rats. These provide the essential elements which must be added to the diet in nutrition studies.

In this chapter we are concerned principally with the requirements for organic compounds. No attempt will be made to encompass the vast literature regarding food preferences and regarding the biochemical action of specific substances; these are regularly reviewed in the Annual Review of Biochemistry, in Nutrition Reviews, in Vitamins and Hormones, and in other review series; they are also summarized in nutrition texts. ${ }^{127}$ In the following account references are omitted for many statements which are taken from nutrition texts.

\section{ORIGIN OF NUTRITIVE TYPES}

Carbon compounds are stores of energy, and energy is required for their synthesis. How such stores came to be established, their relation to the origin of life, the primitive kinds of energy-yiclding reactions, and the nature of the carbon compounds which ultimately resulted in living organisms are of fundamental importance. Certainly most important biochemical evolution occurred prior to the existence of any organisms such as are now known.

Organisms fall into three classes with respect to the source of energy used for synthetic processes: (1) chemotrophic organisms (largely bacteria) get 
their energy from inorganic reactions such as oxidation of iron or sulfur; (2) phototrophic cells use sunlight; and (3) heterotrophic organisms oxidize organic compounds of varying degrees of complexity. Some heterotrophs can use lower fatty acids such as acetate, but most of them need sugars or higher carbohydrates. Similarly, with respect to nitrogen sources, organisms are autotrophic if they can use inorganic nitrogen, nitrate or ammonia; they are mesotrophic if they can rely on single amino acids (or ammonia and an organic acid); and are metatrophic if they require many amino acids either in organized compounds such as peptones or as simpler mixtures. All animals except green flagellate protozoans are ultimately dependent on photo-autotrophic plants for their carbon sources of energy. Whether the green flagellates are plants or animals is a matter of definition. In evolution autotrophic organisms precede and are required by heterotrophic organisms.

There is no reasonable hypothesis as to how autotrophs originated. It has been argued ${ }^{5 t ;}, 104$ that a vast array of carbon-nitrogen-oxygen compounds were formed by chance combination prior to the appearance of living organisms, and that the first organisms were really heterotrophs, using pre-existing compounds. Then, as these compounds became depleted, synthesizing mechanisms which appeared by mutation had survival value, and thus autotrophic organisms evolved from hetero-metatrophic ones. There are difficulties with

TABLE 19. FORMS OF NINIMAL NITROGEN AND CARBON REQUIREMENT IN PROTOZOANS OF VARIOUS SYNTHESIZING CAPACITIES

Modified from Doyle, ${ }^{24}$ Dusi, ${ }^{, 99}$ Hutner et al.,,"3i Johnson, ${ }^{82}$ Lwoff, ${ }^{83}$ and Needham. ${ }^{99}$

\begin{tabular}{|c|c|c|c|}
\hline Nitrogen Source & Chemotrophic & $\begin{array}{c}\text { Phototrophic } \\
\left(\mathrm{CO}_{2} \text { in light }\right)\end{array}$ & $\begin{array}{l}\text { Heterotrophic } \\
\text { (acetate) }\end{array}$ \\
\hline $\begin{array}{c}\text { Autotrophic } \\
\mathrm{NO}_{3}\end{array}$ & & $\begin{array}{l}\text { Euglena gracilis } \\
\quad \text { (if Mn) } \\
\text { E. stellata } \\
\text { E. klebsii } \\
\text { Chlamydomonas } \\
\text { Chlorogonium }\end{array}$ & Polytoma ocellatum \\
\hline $\mathrm{NH}_{3}$ & $\begin{array}{l}\text { Chilomonas par. } \\
\text { (if } \mathrm{Si} \text { ) }\end{array}$ & E. anabaena & $\begin{array}{l}\text { P. uvella (if } \mathrm{Fe} \text { ) } \\
\text { Chilomonas par. } \\
\text { Poly tomella } \\
\text { E. gracilis (in dark) } \\
\text { Astasia }\end{array}$ \\
\hline $\begin{array}{l}\text { Mesotrophic } \\
\text { (single } \\
\text { amino } \\
\text { acids) }\end{array}$ & & E. deses & $\begin{array}{l}\text { Chlamydomonas } \\
\text { (in dark) } \\
\text { E. gracilis (in dark) }\end{array}$ \\
\hline $\begin{array}{l}\text { Metatrophic } \\
\text { (peptones, etc.) }\end{array}$ & & E. pisciformis & $\begin{array}{l}\text { E. gracilis (in dark) } \\
\text { Glaucoma piriformis } \\
\text { Leptomonas } \\
\text { ctenocephali } \\
\text { Colpidium striatum } \\
\text { Colpidium campylum }\end{array}$ \\
\hline
\end{tabular}

this hypothesis, such as the small amount of atmospheric oxygen present before there were green organisms. Whatever the sequence, certainly the basic cellular needs, particularly for amino acids and coenzymes, were established very 
early, and heterotrophic animals were derived from autotrophs, perhaps from pigmented flagellates (chlorophytes).

The carbon and nitrogen requirements of many bacteria and flagellates have been examined. $2 \times, 8: 3,62, x: 3,121$ In general, the ability to use light or inorganic reactions to provide energy for $\mathrm{CO}_{2}$ reduction goes with the ability to use nitrate or ammonia (rarely atmospheric nitrogen). Conversely, organisms which require fatty acids or sugars as carbon sources also need anino acid nitrogen. However, among flagellates such as the genus Euglena there are exceptions (Table 19). An organism which can use simple nitrogen or carbon sources can also use more complex sources; one which can use nitrate can also use ammonia and amino acids. Only a few phototrophic organisms require amino nitrogen, and only a few protozoans which require organic acids or sugars for carbon can use nitrate or ammonia; many yeasts are in the latter category.

\section{CARBON REQUIREMENTS}

Phototrophic green flagellates (chloroflagellates) reduce $\mathrm{CO}_{2}$ directly in light and use organic acids or sugars for energy in the dark. The rate of photosynthesis is affected by the hydrogen ion concentration and the inorganic elements in the medium. Euglena stellata, for example, requires a high concentration of calcium. ${ }^{2}$ There is some disagreement regarding the forms of nitrogen required by various flagellates, largely because of differences in minerals in culture media, strains of species, and other conditions. ${ }^{.8}$

It has been postulated that colorless flagellates, leucophytes, were derived from chlorophytes by the loss of chlorophyll from their plastids. The leucophytes and the chlorophytes in the dark can use organic acids, particularly acetic to myristic, and some can use alcohols which are first converted to the corresponding acids. Pringsheim 111 described the culture of some twenty different leucophytes on acetate. The effects of various organic acids on growth of flagellates were summarized by Trager. ${ }^{1: 38}$ Polytoma uvella uses acetate or butyrate but not sugar. ${ }^{62}$ The colorless alga Prototheca uses saturated fatty acids up to palmitic, except formic acid; it does not use dicarboxylic acids but can use some alcohols. ${ }^{7}$ Sugars are used by many flagellates. Trypanosoma brucei, for example, ferments sugars in the following series: glucose $>$ mannose $>$ maltose $>$ fructose $>$ galactose, but does not ferment arabinose, xylose, lactose or sucrose. ${ }^{14}$ Differences in sugar utilization may result in part from enzymatic differences and in part from limited permeability.

Chilomonas paramecimm is a colorless flagellate which has remarkable powers of synthesis. It multiplies well, dividing three to four times daily in a medium of acetate or glycine as the only carbon source; it grows better with thiamine and iron than without them. Sulfur stimulates fission, and in the absence of sulfur fat accumulates and the culture eventually dies out. It has been claimed by some authors, ${ }^{17}, 88,89,90,{ }^{105}$ but not by others, ${ }^{51}$ that Chilomonas can also grow in the dark with $\mathrm{CO}_{2}$ as its sole source of carbon; growth under these conditions is less than in acetate and a trace of silicon is necessary. There is an optimal $\mathrm{CO}_{2}$ tension and bacterial contamination has been excluded, also possible oxidation of ammonia.

It is probable that true protozoans were derived from leucophytes. Some Protozoa are unable to survive on organic compounds in solution, but rather 
they require particulate food, dead or alive. The carnivores Stylonychia pustulata and Pleurotricha lanceolata, for example, need live Protozoa as food, in addition to some vitamins. "No Amoeba proteus grows well on Chilomonas but not on Paranecinm as food, whereas Anoeba dubia seems to need chlorophytes. ${ }^{1+i}$ Whether the need for live food represents need for particular compounds as carbon source or for some special growth substances is not known. Holotrichous ciliates of the Colpidium-Tetrahymena group can ob tain their food from solution. Tetrahymena geleii can use some sugars and is stimulated by acetate, whercas some fatty acids are toxic; it can ferment dextrose, levulose, mannose, and maltose, but it cannot ferment some thirteen other sugars and cannot use sucrose. ${ }^{6: 1}$

The salts of fatty acids, together with necessary amino acids, can supply some of the carbon needs of multicellular animals. Certainly the monosaccharide sugars are adequate, and numerous adult insects live exclusively on a diet of sugars. Adult honeybees can survive on any of seven sugars which are sweet to the bee and six which are tasteless, but they cannot use five others which are tasteless. ${ }^{1+4}$ Blowflies can live on $\alpha$-glucosides and $\alpha$-galactosides, but not on other glycosides. 30 The waxmoth larva (Galleria) has no need for carbohydrate but can grow and metamorphose on a diet of beeswax. Fatty acids are formed by the action of the symbiotic bacteria and protozoans in termites and ruminants; these fatty acids are absorbed and used as a principal carbon source (see Chapter 6). Acetate can be substituted for part of the carbohydrates in the diet of a rat. It is difficult to see how an organism which obtains its energy by oxidation via the Krebs tricarboxylic acid cycle (see Fig. 63 , Ch. 8) could survive long without a carbohydrate source of pyruvic acid. However, this minimal carbohydrate can be formed from protein, and rats can survive on a diet of protein and minerals only. Acetone bodies, however, are formed when protein is substituted for carbohydrate. Fatty acids can serve as sole carbon source for some leucophytes, but in all other animals fatty acids are used only along with sugars, although protein can be substituted as a sugar source. Caloric requirements of animals will be considered in Chapter 8 .

\section{NITROGEN REQUIREMENTS}

There is a general relation between utilization of carbon and nitrogen compounds, as shown in Table 19. Any organism which can use an inorganic source of nitrogen to build its own amino acids can also use the more complex nitrogen compounds. The simplest usable nitrogen sources have been examined for several phototrophic and heterotrophic flagellates..?: $:: 3$ Nitrates can be used by Englena gracilis, E. stellata (if much Ca is present), and E. klebsii, whereas ammonia but not nitrate can be used by E. anabaena. Euglena deses requires amino acids, and E. pisciformis is said to grow only if nitrogen is supplied in the form of peptones or polypeptides. It is not known whether this need of E. pisciformis is for some growth substance in the peptones or for a particular combination of amino acids. Some microorganisms require specific polypeptides (e.g., strepogenin). Growth of several species of Euglena in different single amino acids is shown in Table 20, and it appears that some amino acids, such as tyrosine and tryptophane, are not adequate nitrogen sources even for species that can use ammonia or nitrate. It would be of interest to test these mesotrophic and metatrophic species of Euglena in amino acid mix- 
tures. Some other chlorophytes (e.g. Chlamydomonas) can use nitrates in the dark, and some colorless organisms such as Polytoma ocellatum which are restricted to organic acids (acetate) for carbon can use nitrates, whereas some others (Polytoma, Chilomonas) can use ammonia for their nitrogen. The requirements of chlorophytes may be more complex in the dark than in the

TABLE 20. GROWTH OF SEVERAL SPECIES OF EUGLENA

IN SINGLE AMINO ACIDS ${ }^{2 .}$

+ indicates growth; $\bigcirc$ indicates no growth; ++ indicates much growth

\begin{tabular}{l|c|c|c|c|c|c}
\hline \hline \multicolumn{1}{|c|}{ Amino Acid } & $\begin{array}{c}\text { E. } \\
\text { gracilis }\end{array}$ & $\begin{array}{c}\text { E. } \\
\text { stellata }\end{array}$ & $\begin{array}{c}\text { E. } \\
\text { klebsii }\end{array}$ & $\begin{array}{c}\text { E. } \\
\text { anabaena }\end{array}$ & $\begin{array}{c}\text { E. } \\
\text { deses }\end{array}$ & $\begin{array}{c}\text { E. } \\
\text { pisciformis }\end{array}$ \\
\hline glycine & + & + & + & + & 0 & 0 \\
alanine & ++ & ++ & ++ & + & ++ & 0 \\
valine & ++ & + & + & + & 0 & 0 \\
leucine & ++ & ++ & ++ & + & ++ & 0 \\
serine & + & ++ & + & 0 & + & 0 \\
phenylalanine & ++ & + & 0 & ++ & 0 & 0 \\
tyrosine & 0 & 0 & 0 & 0 & 0 & 0 \\
tryptophane & 0 & 0 & 0 & 0 & & 0 \\
histidine & ++ & ++ & ++ & 0 & & 0 \\
arginine & 0 & + & 0 & 0 & & 0 \\
lysine & + & ++ & ++ & 0 & ++ & 0 \\
proline & + & ++ & ++ & + & ++ & 0 \\
glutamic acid & + & + & + & 0 & ++ & 0 \\
aspartic acid & + & + & 0 & + & ++ & 0 \\
asparagine & ++ & ++ & ++ & ++ & 0 \\
\hline
\end{tabular}

light; Chlanydomonas uses nitrate in light, amino acids in the dark. Euglena gracilis, which is photo-autotrophic, can grow in the dark, provided acetate is supplied, and it then can use peptones, ${ }^{60}$ or even ammonia, ${ }^{123}$ while $E$. deses is said not to grow in the dark even with peptones and acetate. ${ }^{\text {tio }}$ There must be some enzymatic connection between the utilization of carbon and that of nitrogen.

All autotrophic organisms can synthesize each of nineteen generally oc- 
TABLE 21.

ESSENTIAL AND DISPENSABLE AMINO ACIDS WHEN AMINO ACID

MIXTURES PROVIDE AN ANIMAL'S NITROGEN SUPPLY

+ indicates essential; - indicates not essential

\begin{tabular}{|c|c|c|c|c|c|c|}
\hline Amino Acid & Tetrahymena"s & $\begin{array}{c}\text { Aedes } \\
\text { aegypti }\end{array}$ & $\begin{array}{c}\text { Attagenas }^{i n} \\
\text { (limited } \\
\text { growth) }\end{array}$ & Chicken $^{3}$ & $\operatorname{Rat}^{114}$ & $\operatorname{Man}^{11 s}$ \\
\hline glycine & $+^{*}$ & + & & $+\S$ & - & - \\
\hline alanine & - & - & & - & - & - \\
\hline valine & + & - & + & + & + & + \\
\hline leucine & + & + & + & + & + & + \\
\hline serine & $+^{*}$ & - & & - & - & - \\
\hline phenylalanine & + & $+t$ & + & + & + & + \\
\hline tryosine & - & $+\dagger$ & & $-\|$ & $-\|$ & $-\|$ \\
\hline tryptophane & + & + & + & + & + & + \\
\hline histidine & + & + & + & + & + & - \\
\hline arginine & + & ++ & + & + & +4 & - \\
\hline lysine & + & + & + & + & + & + \\
\hline proline & - & - & & - & - & - \\
\hline glutamic acid & - & - & & $-\S$ & - & - \\
\hline aspartic acid & - & - & & - & - & - \\
\hline hydroxyproline & - & - & & - & - & - \\
\hline isoleucine & + & + & + & + & + & + \\
\hline methionine & + & + & + & + & + & + \\
\hline threonine & + & + & + & + & + & + \\
\hline cystine & - & - & & & $-^{* *}$ & $-^{* *}$ \\
\hline
\end{tabular}

* Serine can replace glycine for Tetrahymena.

†Phenylalanine and tyrosine not both needed; either one sufficient for Aedes.

†itrulline can substitute for arginine for Aedes.

\$Glycine and glutamic acid needed for maximum growth-chick.

$\|$ Tyrosine needed if phenylalanine low-chick, rat, man.

Arginine needed for growth, not for maintenance-rat.

${ }^{*}$ Cystine needed only if methionine low-rat, man. 
curring amino acids, whereas animals in general (except flagellates) have lost the ability to synthesize some of these. The amino acids that are required in the diet of several kinds of animals are surprisingly similar (Table 21). Different amino acids are apparently needed not specifically for the nitrogen they provide but for their molecular skeletons. For each animal amino acids fall into three groups: (1) the amino acids that are not synthesized and are, therefore, required in the diet; (2) the amino acids that can be synthesized from ammonia and carbohydrate; and (3) those that are synthesized from specific essential amino acid precursors. Using the growing rat as an example, the amino acids required in the diet are:<smiles>CC(C)C(N)C(=O)O</smiles>

valine<smiles>CC(C)CC(N)C(=O)O</smiles>

leucine<smiles>CC(O)C(N)C(=O)O</smiles>

threonine<smiles>CCC(C)C(N)C(=O)O</smiles>

isoleucine<smiles>NCCC(N)C(=O)O</smiles>

lysine<smiles>N=C(N)NCC(N)C(=O)O</smiles>

$\operatorname{arginine}^{*}$<smiles>NC(Cc1ccccc1)C(=O)O</smiles>

phenylalanine<smiles>O=C(O)CCCC1CNC2C=CC=C1C2</smiles>

tryptophane

- Necessary for growth. 
<smiles>CSCCC(N)C(=O)O</smiles>

methionine<smiles>NC(CC1=CN=C[N]1)C(=O)O</smiles>

histidine

The amino acids synthesized by growing rats from carbohydrate and ammonia-yielding compounds such as other amino acids are:<smiles>NCC(=O)O</smiles>

glycine<smiles>NC(CC(=O)O)C(=O)O</smiles>

aspartic acid<smiles>CC(N)C(=O)O</smiles>

alanine<smiles>NC(CO)C(=O)O</smiles>

serine<smiles>O=C(O)C1CCCN1</smiles>

proline

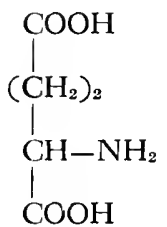

glutamic acid<smiles>O=C(O)C1CC(O)CN1</smiles>

hydroxyproline

Those which are synthesized from specific amino acids are:<smiles>NC(CSSCC(N)C(=O)O)C(=O)O</smiles>

cystine (from methionine)<smiles>NC(Cc1ccc(O)cc1)C(=O)O</smiles>

tyrosine (from phenylalanine)

The interactions among amino acids are important, and reported inhibition of growth by certain amino acids is probably really due to lack of balance among antagonists. An amino acid which is synthesized from another one 
becomes a dietary requirement only when there is a deficiency of its precursor. Several amino acids, such as citrulline and diiodotyrosine, are of limited occurrence and are not building units of protein; hence they differ from the basic nineteen amino acids named above.

The ciliate Tetrahymena geleii strain $W$ has been cultured in mixtures of amino acids and no persistent growth occurs if any of the following is omitted: isoleucine, leucine, lysine, methionine, phenylalanine, tryptophane, valine, histidine, threonine, and arginine (Table 22). Methionine can be replaced by homocystine as a sulfur-containing amino acid. ${ }^{73}$ For most effective culture either serine or glycine is also necessary: these probably are not required for synthesis of the organism's proteins, but rather release the growth inhibitions imposed by some of the other amino acids. The interactions among various amino acids are complex; cystine, glycine, and serine stimulate growth, whereas alanine, aspartic acid, glutamic acid, hydroxyproline, proline, and tyrosine in excess inhibit growth, but the stimulators can release the inhibition.

TABLE 22.

TETRAHYMENA GELEII-MEDIUM USED BY KIDDER AND DEWEY⿱艹, 'T

\begin{tabular}{|c|c|c|c|}
\hline & $\gamma$ per ml. & & $\gamma$ per ml. \\
\hline $\mathrm{L}$-Arginine $\mathrm{HCl}$ & 83 & Sodium acetate & 1000 \\
\hline $\mathrm{L}-$ Histidine $\mathrm{HCl}$ & 36 & Ca pantothenate & 0.10 \\
\hline $\mathrm{DL}-$-1soleucine & 113 & Nicotinamide & 0.10 \\
\hline L-Leucine & 147 & Thiamine $\mathrm{HCl}$ & 1.00 \\
\hline $\mathrm{L}$-Lycine $\mathrm{HCl}$ & 116 & Riboflavin & 0.10 \\
\hline DL-Methionine & 94 & Pteroylglutamic acid & 0.01 \\
\hline L-Phenylalanine & 70 & Pyridoxine $\mathrm{HCl}$ & 2.0 \\
\hline DL-Threonine & 138 & Biotin (free acid) & 0.0005 \\
\hline L-Tryptophane & 28 & Choline $\mathrm{Cl}$ & 1.0 \\
\hline DL-Valine & 76 & Protogen & 0.375 \\
\hline $\mathrm{DL}$-Serine & 157 & Yeast nucleic acid (hydrolyzed) & 100 \\
\hline L-Glutamic acid & 233 & $\mathrm{MgSO}_{4} .7 \mathrm{H}_{2} \mathrm{O}$ & 100 \\
\hline L-Aspartic acid & 61 & $\mathrm{~K}_{2} \mathrm{HPO}_{4}$ & 100 \\
\hline Glycine & 5 & $\mathrm{CaCl}_{2} .2 \mathrm{H}_{2} \mathrm{O}$ & 50 \\
\hline oL-Alanine & 55 & $\mathrm{Fe}\left(\mathrm{NH}_{4}\right)_{2}\left(\mathrm{SO}_{4}\right)_{2 .} 6 \mathrm{H}_{2} \mathrm{O}$ & 25 \\
\hline L-Hydroxyproline & 75 & $\mathrm{CuCl}_{2} 2 \mathrm{H} . \mathrm{O}$ & 5 \\
\hline $\mathrm{L}-\mathrm{T}$ yrosine & 67 & $\mathrm{FeCl}_{3} \cdot 6 \mathrm{H}_{2} \mathrm{O}$ & 1.25 \\
\hline $\mathbf{L}$-Cysteine & 3.5 & $\mathrm{Mn} \mathrm{Cl} .+\mathrm{H}_{2} \mathrm{O}$ & 0.05 \\
\hline \multirow[t]{2}{*}{ Dextrose } & 1000 & $\mathrm{Zn} \mathrm{Cl}$ & 0.05 \\
\hline & & Tween 85 & 500 \\
\hline
\end{tabular}

Also there are strain differences in growth on mixtures of the essential amino acids and strain $E$ appears to require serine. 29a Thus, it appears that, although some flagellates can live well on single amino acids, some ciliates require mixtures comparable to those required to support mammals.

Several insects have been grown on mixtures of amino acids as the nitrogen source. Larvae of the mosquito Aedes aegypti have been maintained with fair but not normal growth in a mixture of eleren amino acids (Table 21); phenylalanine and tyrosine are interchangeable, but if both are omitted no growth occurs; also citrulline can be substituted for arginine. ${ }^{40}$ Growth of Drosophila larvae on an amino acid mixture has been reported. ${ }^{124}$ Carpet bectles have 
been grown very slowly in a mixture of the same ten amino acids required by rats, except for clouble the amount of arginine: however, they grow better in a mixture of twenty amino acids" (Table 21). The cochroach Blatella grows at about half the nomal rate in amino acid mixtures simulating cascin in composition (Noland, unpublished). The roach matures on synthetic diets deficient in both tryptophane and methionine, which are evidently synthesized by intestinal microorganisms at a rate suflicient for slow growth. io: Inability to grow some insects on amino acid mixtures may be due partly to improper mixtures and partly to inadequate salts in the diet. The amount of protein in the diet of growing insects is important; for example, the ants of genus Pheidole on a high protein diet tend to become soldiers, on a low protein diet, workers. $1+0$

The amino acid requirements of mammals hare been carefully investigated by omitting various amino acids from a food mixture 11t. 11. and observing growth (rats) and weight and nitrogen balance (man, dog). Results are given in Table 21. Arginine is synthesized by the rat but not at a rate sufficient for the demands of normal growth. Cystine is also needed and can be synthesized only if adequate methionine is present, the synthesis apparently using the sulfur of methionine. Glycine is also needed but can be synthesized from simpler substances. Observations on dogs 11; indicate that arginine is not needed for maintenance of adults, but that the other nine amino acids as required for growing rats are needed by adults. Man requires eight amino acids, whereas growing rats require ten. The absolute amounts required vary with the caloric intake, being less with high calories. Man requires most of the essential amino acids are supplied, and the conversion does not depend on hence recommended amounts are 0.5 to $2.5 \mathrm{gm}$. per day. In general D-amino acids cannot be substituted for L-amino acids, although some of the phenylalanine and methionine can be in the $\mathrm{D}$-form. ${ }^{11.5}$ The amino acid requirements of the chick differ slightly from those of mammals (Table 2i). Glycine and arginine are required; citrulline but not ornithine can be substituted for arginine; and tyrosine is needed if the ration is low in phenylalanine. $3,4,5$

The nitrogen requirement of young growing animals is much greater than that of adults. In man, an infant requires four to five times as much as an adult on a unit/weight basis. A trout needs $1+$ or more per cent of protein in its diet for normal growth. ":3 Calves fail to grow on a diet containing only t.4 per cent protein, but when urea is added to give nitrogen equivalent to about 16 per cent protein they grow well, presumably by conversion of urea to protein by the symbiotic microorganisms in the rumen. 53,8 Rats can use glycine, urea, and even ammonium salt for nitrogen if minimal amounts of the essential amino acids are supplied, and if the conversion does not depend on intestinal organisms. ${ }^{115}$

Autotrophic organisms can use inorganic sources of nitrogen, such as nitrate, ammonia, and sometimes atmospheric nitrogen, but with metabolic evolution the abilities of synthesis decreased and specific amino acids are required by ciliates and probably by all metazoans. Claims for requirements of peptones and whole proteins cannot be accepted until extensive tests with balanced mixtures of amino acids are made. The specific amino acid requirements differ somewhat for various animal groups, but the ability to use exclusively inorganic sources of nitrogen was lost early in animal evolution. Possibly 
utilization of urea and ammonia may occur in many metatrophic animals when they are supplied with minimal amounts of the essential amino acids.

\section{SPECIFIC FACTORS IN NUTRITION}

A vitamin is an organic compound which must be supplied to an organism in small amounts and which is used in specific ways other than as an energy source or as a basic structural unit, although it may be incorporated as a prosthetic group into a structural protein. Essential amino acids are usually needed in slightly greater amounts than vitamins and are used primarily as structural units in the building of protein; hence they are not vitamins. Some vitamins are synthesized and distributed like hormones, but the vitamins which are synthesized by animals are formed only from closely related substances (sometimes called provitamins), whereas hormones can be formed from the basic foodstuffs. More vitamins are required for growth than for adult maintenance, and what is a vitamin for one animal may not serve for another, or it may be synthesized by another animal. Vitamins are discovered by omitting various fractions from an animal's dietary and observing effects of the deficiencies on growth, blood cell count, and skin appearance, and sometimes pathology at the time of death. These criteria of deficiency are many steps removed from the sites of cellular reaction, and recently a few vitamins of the B complex have been shown to act as coenzymes in certain metabolic reactions. Also the assay of foods for specific vitamins is facilitated by the need of certain microorganisms, bacteria and yeasts particularly, for vitamins. Yeasts and bacteria have a remarkable ability to synthesize most of the water-soluble vitamins, yet certain species and strains are unable to synthesize specific single ones, and thus they can be used for assay of these substances. For purposes of classification, vitamins can be divided into two groups: those which are water soluble, and those which are fat soluble.

Water-Soluble Vitamins: Thiamine (Vitamin $B_{1}$ ). The first B vitamin to be discovered was thiamine, active in preventing beriberi in man and polyneuritis in birds. Thiamine as diphosphothiamine occupies a key position in cellular metabolism as cocarboxylase, the coenzyme in pyruvate oxidation. In all animals thiamine promotes growth, and at least in mammals its lack reduces appetite.

Thiamine is widely distributed in plant and animal tissues, and probably all cells, whether aerobic or anaerobic, require it. Unlike other B vitamins it is readily destroyed by heat. The thiamine molecule consists of two parts, a pyrimidine and a thiazole moiety:

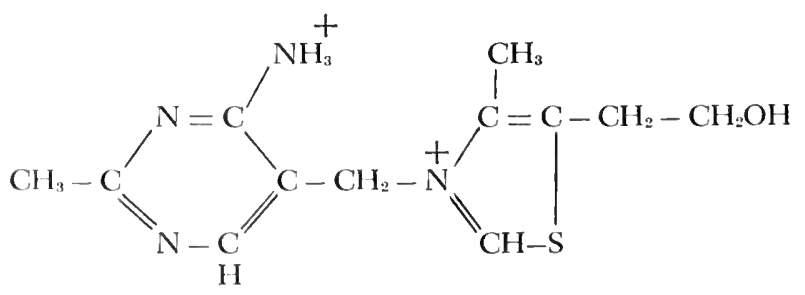

pyrimidine + thiazole $=$ thiamine 
added thiamine may not be necessary for maintenance and for low-level growth. Chilomonas paramecinm, for cxample, grows slowly in an acetateammonia medium; addition of thiamine more than doubles the multiplication rate, and addition of thiamine plus iron increases it by more than five times. in It is, therefore, not indispensable in the diet for Chilomonas, but it is needed for optimal growth. A number of versatile organisms-bacteria, mold, and flagellates-can synthesize both the pyrimidine and the thiazole rings, and from these the intact thiamine molecule (Table 23). Some require either the pyrimidine or the thiazole from outside, others need both the pyrimidine and

\section{TABLE 23. REQUIREMENTS FOR THIAMINE AND ITS COMPONENTS PYRIMIDINE AND THIAZOLE (MODIFIED FROM LWOFF ${ }^{\circ 3}$ )}

- indicates substance synthesized and not required in diet;

+ indicates substance not synthesized and required in diet.

\begin{tabular}{l|c|c|c}
\hline \multicolumn{1}{c|}{ Animal } & Pyrimidine & Thiazole & Thiamine \\
\hline Bacteria & & & - \\
Proteus vulgaris & - & - & - \\
Escherichia coli & - & - & - \\
B. coli & - & - & - \\
Staph. aureus & + & + & - \\
Molds & - & - & - \\
Aspergillus niger & - & + & - \\
Mlucor mamamianus & + & + & - \\
Phycomyces blakesleanas & & + & - \\
Phytophthora cinnamoni & - & - & - \\
Colorless Flagellates & - & - & - \\
Polytoma obtusum & - & + & + \\
Polytoma uvella & - & + & + \\
Polytoma caudatum & + & + & + \\
Polytoma ocellatum & + & & + \\
Polytomella caeia & + & & + \\
Euglena pisciformis & & & + \\
Chilomonas paramecium & & & + \\
Strigomonas sp. & & & + \\
3 trypanosomes & & & + \\
Acanthamoeba castellani & & & + \\
Colpidium striatum & & & + \\
Colpidium campylum & & & + \\
\hline
\end{tabular}

the thiazole, and finally many Protozoa and some molds need the intact thiamine molecule, as do all multicellular animals which have bcen investigated (Table 23). Thiamine stimulates division of Tetraliymena but is not a dietary requirement if $\mathrm{D}$-amino acids are present in the medium. ${ }^{11}, \mathbf{7} \mathbf{7}$ Table 23 shows that with increasing specialization among Protozoa the ability to synthesize and to unite the two components drops out first, then the ability to synthesize one or the other half, and finally the whole molecule is required from outside. $8:, 85$

Thiamine is required by all those insects and vertebrates which have been tested. Mosquito larvae grown on foods in solution need thiamine, 0.0008 
mg./ml. 1+2 Rainbow trout fed largely on canned carp developed malfunction of the nervous system and melanosis, which were cured by intramuscular injections of thiamine. ${ }^{14 \times}$ The dietary need for thiamine by trout is 0.15 $0.186 \mathrm{mg}$. kg./day. Man requires about 1.1-1.5 mg. daily. Many animals have in the digestive tract microorganisms which synthesize thiamine, and the dietary needs may therefore be less than the actual metabolic needs. Much thiamine is synthesized by microorganisms in the rumen of sheep and cattle; hence these animals do not require thiamine in their diet. Calves reared on synthetic milk do not have rumen microorganisms and require dietary thiamine.

Raw fish contains a substance which destroys thiamine, so that when raw fish, particularly viscera, is fed to foxes or chickens ${ }^{131}$ these animals develop nerve and liver symptoms of thiamine deficiency. The active agent in the fish appears to be an enzyme or thiaminase which can destroy thiamine in vitro. ${ }^{7 *}$ How the fish are protected from losing their own thiamine is unknown.

Riboflavin (Vitamin $B_{3}$ ). Ribollavin is needed in the prosthetic group of flavoprotein enzymes which act in cellular respiration between dehydrogenases and either oxygen or cytochrome. Ribollavin is a constituent of Warburg's "yellow enzyme," xanthine oxidase, D-amino acid oxidase and cytochrome reductase. Riboflavin is an alloxazine derivative:

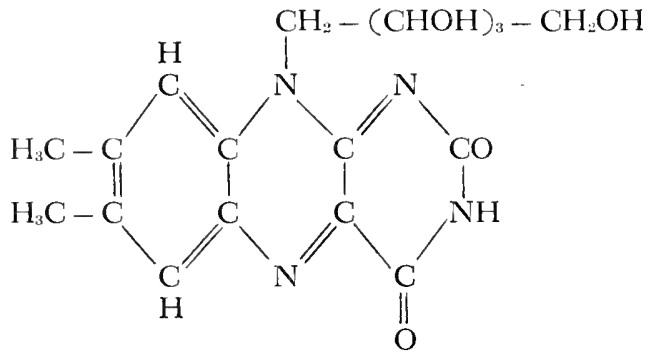

Riboflavin is synthesized by many microorganisms, yeasts, bacteria, and probably flagellates. It stimulates multiplication of Chilomonas but is not a dietary requirement. ${ }^{10 \pi}$ Riboflavin is required by the ciliate Tetrahymena strain $W$. it It is required by insects-Drosophila, ${ }^{13 *}$ mosquito larvae, ${ }^{1+2}$ and numerous beetles (Table 24). Some beetles, e.g., Lasioderma, require riboflavin when sterilized of intestinal flora, but normally the symbionts synthesize sufficient riboflavin for the beetle. In the cockroach Blatella germanica omission of riboflavin from a synthetic diet resulted in either no retardation in growth or at most a 50 per cent retardation (Noland, personal communication). Analyses of roaches reared to maturity on very low riboflavin, of the diet, and of newly-hatched nymphs showed that a 30-fold increase in body riboflavin occurred in insects reared on the deficient diets. There is probably intestinal synthesis of riboflavin in the cockroach.

Trout need $0.44-0.68 \mathrm{mg}$. of riboflavin per $\mathrm{kg}$. body weight per day. ${ }^{9: 3,96}$ The characteristic signs of ribollavin deficiency in mammals are skin lesions. Rats deficient in ribollavin develop skin lesions, some alopecia (shedding of hair), graying of hair, cataracts, and ocular lesions. Pigs require riboflavin for 


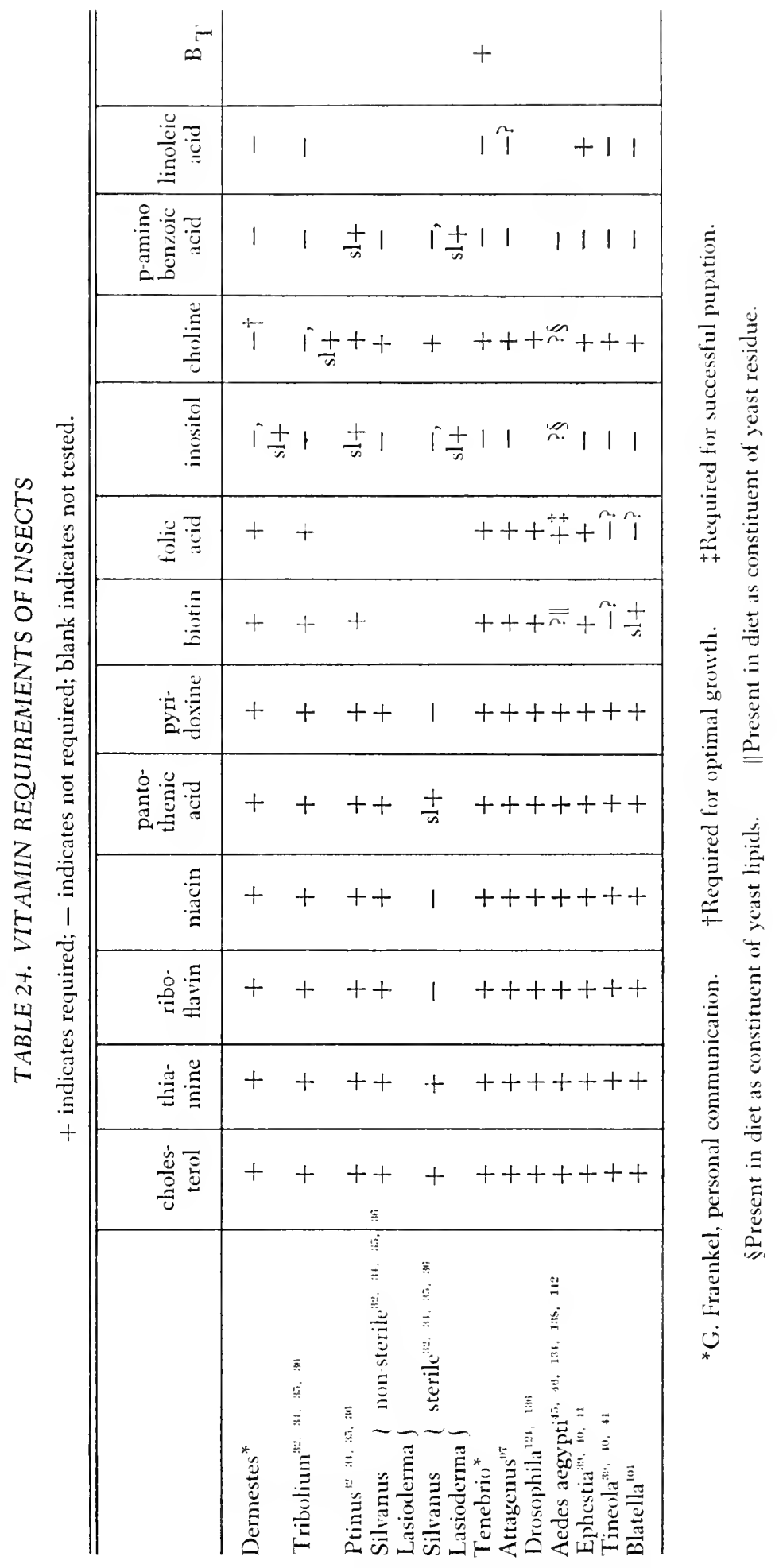


proper growth and for normal skin, hair, and hoofs. ${ }^{106}$ Fur-bearing mammals such as the fox require ribollavin for normal sleekness and color of their fur. $11 \mathrm{~s}, 11:$ Manifestations of deficiency of riboflavin in man appear as lesions of skin, mouth, or eyes, and the recommended intake is $2.7 \mathrm{mg}$./day. Monkeys deficient in riboflavin fail to grow normally, develop an anemia and dermatitis, and on autopsy have fatty livers; they need $25-30 \mu \mathrm{g} . / \mathrm{kg} . /$ day.

It is difficult to know the true body requirement for ribollavin since it is synthesized by digestive microorganisms. When these microorganisms are in the cecum or colon riboflavin may not be absorbed in sufficient quantity and more may be lost in feces than need be taken by mouth. In the rabbit, for example, the total excretion (largely fecal) of ribollavin is 10 to 15 times the intake, and rabbits do well on very low dietary riboflavin, either because of cecal absorption or from eating feces. ${ }^{103}$ Ruminants obtain sufficient riboflavin by the synthetic process of rumen organisms. In sheep the dried rumen contents contained $32 \mu \mathrm{g} . / \mathrm{gm}$. as compared with $0.3 \mu \mathrm{g} . / \mathrm{gm}$. in the food, whereas the milk of cows contained 16-18 mg. per day compared with an intake of $1.8 \mathrm{mg}$. in the food. ${ }^{94}$ Calves lacking ruminant organisms require riboflavin in the diet. ${ }^{14 ;}$ The amount of riboflavin produced by organisms in the digestive tract varies according to the carbohydrates in the diet, and the ribollavin excreted in rats on a high lactose diet is doubled because of stimulation of specific flora. ${ }^{125}$

Niacin (Nicotinic Acid). Niacin is the third vitamin which is known to function in cellular respiration. With adenine, a pentose, and phosphoric acid, nicotinic acid amide (niacinamide) constitutes coenzymes I and II which with specific proteins are dehydrogenases. The structure of niacin is:

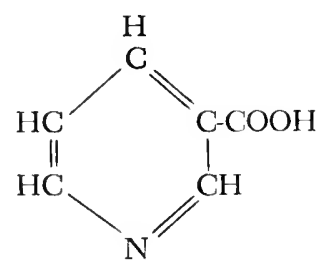

Niacin is șnthesized by many microorganisms, yeasts, bacteria, and flagellates, and it is probably required by all metabolizing cells, hence by all animals. Its synthesis in amounts sufficient for good growth and multiplication has been demonstrated in Chilomonas, sia Niacin is required by the ciliates Tetrahymena and Colpoda duodenaria.

Niacin is required by a varicty of insects, but it is produced by the symbiotic microorganisms in some of them (Table 24). Turkeys deficient in niacin develop a perosis or scaly skin lesions. ${ }^{63}$ In mammals also the microorganisms of the digestive tract synthesize niacin (or nicotinamide), and even in man the body requirement may be partly filled by synthesis from tryptophane. Enough is formed and absorbed in the hamster that none need be added to the diet. In rats, mice, and man tryptophane is converted to niacin, partly by digestive microorganisms but largely in body tissues, particularly the liver, since some additional niacin is still exereted when the flora is poisoned by sulfathiazole or when tryptophane is injected intravenously. Synthesis is increased by adding tryptophane to the diet and favored by the presence of pyridoxine. ${ }^{64 .}{ }^{130}$ 
Further, calves lacking ruminant organisms do not require dietary niacin. Niacin-deficient dogs and foxes"1s, 11 " develop "blacktongue," which is cured by feeding nicotinic acid. In dogs a diet of $0.25 \mathrm{mg}$. kg. day prevents "blacktongue" and permits weight gain; half that anount controls "blacktongue" but does not permit weight increase. 11 In man pellagra results in part from a deficiency of nicotinic acid. There is much evidence that "blacktongue" and pellagra are complex conditions, part of which result from niacin deficiency and part from a diet rich in corn, a grain which is low in tryptophane. 52

Pyridoxine (Vitamin $B_{6}$, Pyridoxal, Pyridoxamine). Pyridoxine is a pyridine derivative:

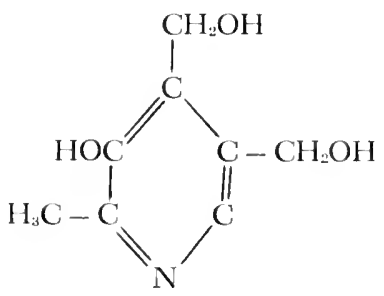

As pyridoxal phosphate, it is a prosthetic group in protein utilization, probably as a co-transaminase, and it may also be concerned in fat metabolism. Pyridoxine is not a dietary requirement of flagellates, although its addition to the medium stimulates the growth of Chilomonas. ${ }^{105}$ It must be supplied in relatively large amounts for optimal growth of Tetrahymena ${ }^{7 ;}$ and Colpoda. 137 Pyridoxine is a general requirement for insects except when synthesized by symbiotic flora (Table 24). Ruminant mammals obtain sufficicnt pyridoxine from their rumen bacteria, the rumen contents of sheep containing ten times as much as the food, and milk containing similar amounts whether the cows are on high or low pyridoxine diet." Birds and nonruminant mammals require pyridoxine in their diet. Pyridoxine-deficient ducklings show retarded growth and anemia. Convulsions appear in extremely deficient rats and pigs, dermatitis in rats, and anemia in dogs, foxes, and pigs. The existence of pathological conditions from pyridoxine deficiency in man is not established, and there is evidence for its synthesis by man.

Pantothenic Acid. Pantothenic acid has the following structure:<smiles>CC(C)(CO)C(O)C(=O)NCCC(=O)O</smiles>

Pantothenic acid acts as a coenzyme in acetylation processes, for example, in acetylation of choline to form acetylcholine. It is needed for optimal growth of Tetrahymena, ${ }^{7 i}$ and Colpidium, $29:$ but added pantothenate does not stimulate the Hagellate Chilomonas. ${ }^{10 i}$ Pantothenic acid favors survival of the malaria organism, Plasmodinm lophurae, in vitro. ${ }^{13:}$ Several insects of different groups have been shown to require pantothenic acid, even when not depleted of microorganisms, and more severe consequences result from 
deficiency of pantothenate than from that of any other B vitamin. Royal jelly of honey bees contains three times more pantothenic acid than yeast or liver. ${ }^{10 \%}$ Ruminants obtain an adequate amount from their symbiotic flora, pantothenic acid increasing by 6 to 25 times in the rumen, 14,15 and rabbits excrete as much as $265 \mu \mathrm{g}$. daily on an intake of $17 \mu \mathrm{g}$. ${ }^{103}$ Even in man pantothenic acid is synthesized by colon bacteria and some may be absorbed. Numerous pathological lesions have been reported in pantothenic acid deficiency. There is initial stimulation and ultimate exhaustion and even atrophy of the adrenal cortex. ${ }^{27}$

Trout need approximately 1.1 to $1.4 \mathrm{mg}$. of pantothenic acid per kilogram per day to prevent a characteristic disease of the gills. ${ }^{109}$ In pantothenic acid deficiency fur-bearing animals, such as the fox, show pathological symptoms in liver and kidney, 119 and monkeys show lack of growth, thin fur, and anemia. "11 Growing chicks and ducklings require much pantothenic acid and when deficient show dermatitis and nerve degeneration; if laying hens are on a deficient diet their eggs are fertile, but embryonic mortality is high.

Biotin. Biotin is:

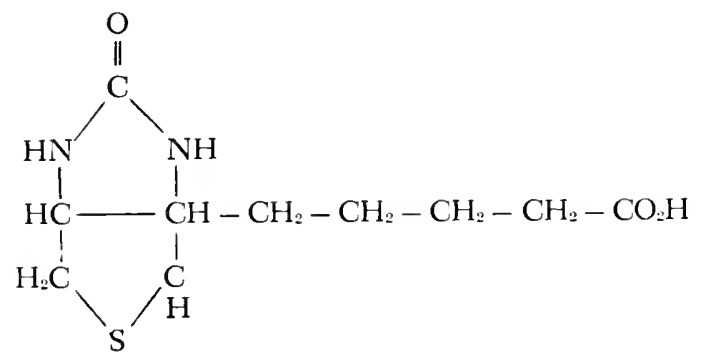

Biotin was discovered independently as needed by certain yeasts, by nitrogenfixing bacteria, and by mammals. Biotin may act in the conversion of pyruvic acid to oxaloacetic acid by $\mathrm{CO}_{2}$ fixation. ${ }^{79} \mathrm{~A}$ diet rich in raw egg white causes a characteristic dermatitis in chicks, rats, rabbits, dogs, and monkeys; this is cured by addition of biotin to the diet.

Biotin is not essential in the diet for the growth of the ciliate Tetrahymena. ${ }^{i}$ It has a pronounced stimulating action on the growth of flour beetles and may be a component of the unkown yeast factors required by some other insects. Mlosquitoes fail to metamorphose without biotin in their diet. ${ }^{111}$ Biotin appears to be synthesized in the intestine by different bacteria from those synthesizing some of the other $\mathrm{B}$ vitamins, and biotin synthesis is favored by dextrin but not by lactose. Not enough is synthesized to satisfy the needs of the hamster, ${ }^{\prime 1}$ but probably enough is produced by symbionts in rabbits. ${ }^{103}$ A deficiency in hens results in high embryonic mortality and perosis or skeletal deformity in young chicks, which can be prevented by injection of biotin into the incubating eggs.?:-24, 25

Folic Acid (Pteroylglutanic Acid). Folic acid is a B vitamin which has spectacular effects in the treatment of certain types for anemia. It is needed by Tetrahymena strain $W$ but not strain E. $29.9 .65,666.77$ In a few insects such as Tribolium, Ephestia, and especially Tenebrio larvae (Table 24), folic acid is essential for growth; it is particularly important in the third instar of Aedes 
larvae and for pupation and pigmentation. ${ }^{47}$ Young turkeys on a diet deficient in folic acid show retarded growth and anemia, whereas in mice hematopoiesis in the bone marrow is arrested, with a hyperplasia of immature cell types. $^{14 .}$ Folic acid is essential for mink, ${ }^{118}$ and in monkeys a folic acid deficiency results in lack of growth, anemia, and reversed lymphocyte/neutrophile ratio. ${ }^{20}$ Further investigation will probably indicate a wide need for folic acid, particularly in growth and cell division.

Folic acid has the following structure:

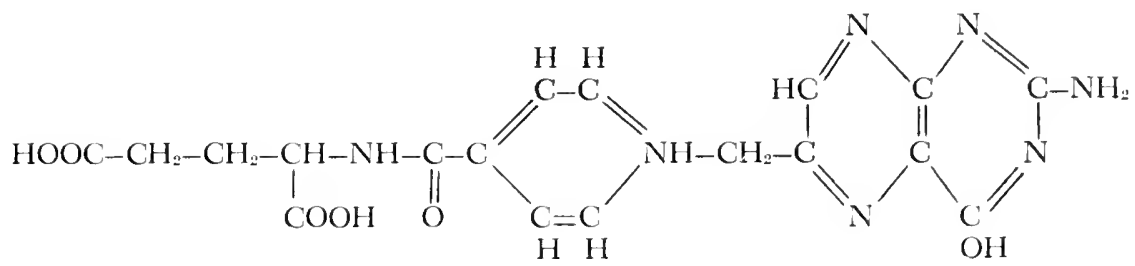

Vitanin $B_{12}$. The anemias which develop in animals deficient in riboflavin, pyridoxine, pantothenic acid, or niacin are microcytic, that is, characterized by the presence of undersized red cells in the blood. Macrocytic anemia, with small numbers of large red cells, occurs in deficiency of folic acid and of vitamin $B_{12}$. Vitamin $B_{12}$ has been crystallized and shown to contain cobalt, ${ }^{113}$ but its chemical identity is not yet known; it is derived from liver and is effective against macrocytic anemia, eren with central nervous system involvement. It is a specific anti-pernicious anemia factor. Vitamin $B_{12}$ not only stimulates hematopoiesis but also promotes growth in chickens, rats, and pigs, and it may be identical with some of the unknown factors found for various animals. There is evidence for interaction between $B_{12}$ and pteroylglutamic acid, and in certain human macrocytic anemias pteroylglutamic acid is effective when vitamin $B_{12}$ is ineffective. In some bacteria, vitamin $B_{12}$ is concerned with the ability to synthesize nucleosides, particularly thymidine. 127 ia Vitamin $B_{12}$ is required by Englena gracilis for maximum growth in and also by Astasia klebsii (Thaver and Kidder, unpublished), whereas it is synthesized by Tetrahymena.

Miscellaneous B V'itanins. Choline and inositol are two substances which are widely distributed in animal tissues and are probably synthesized by most animals, but the rate of synthesis may not be aclequate for the cellular needs; hence they are sometimes considered as vitamins. Choline is a constituent of lecithin and other lipids and is a precursor of acetylcholine, a substance important in nerve function (Ch. 23). Choline serves, in addition, as a source of labile methyl groups needed in transmethylation processes; it is synthesized from methionine and ethanolamine in the rate; methionine also can function as a methyl donator. If a diet is low in protein, particularly in methionine, and high in fat, choline is then a dietary essential, and in such choline deficiency: rats show hemorrhages, increase in liver fat, and ultimately renal degeneration. Choline is essential in fatty acid metabolism. It has a farorable action on growth in numerous insects. It is the most essential vitamin for the cockroach, 101 but apparently many other insects synthesize it in adequate amounts (Table $24^{+1}$ ). Unlike the rat, the cockroach evidently does not make choline from ethanolamine and methionine; however, betaine (trimethyl glycine) can 
substitute quantitatively for choline in the diet of the roach. ${ }^{101}$ Choline is not required in the diet of the ciliate Tetrahymena. ${ }^{77}$

Inositol also is a constituent of many lipids, occurs in nucleic acids, and is widely sinthesized. Certain strains of microorganisms require it, but no insects have been found to need it in the diet. Inadequate amounts may be synthesized by growing chickens and turkeys and by mammals if the diet contains the cereal product phytin; ${ }^{1: 3}, 11 !$, under these conditions it is a dietary essential. Early reports of specific effects of inositol on some types of alopecia (hair loss) appear not to have been confirmed.

Para-aminobenzoic acid is apparently a precursor of folic acid and may not be a true dietary factor for animals if there is an adequate supply of folic acid. Additions of $p$-aminobenzoic acid favor survival in vitro of Plasmodium knowlesii, ${ }^{13: 9}$ but it apparently is not needed by insects or higher animals.

Ascorbic Acid (Vitamin C). Ascorbic acid has the following structure:

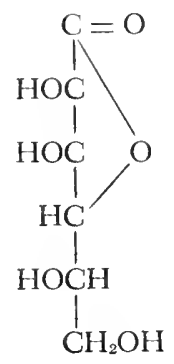

This is a substance which is readily oxidized, and therefore it may be important in maintaining a proper oxidation-reduction balance in many cells. It is widely distributed in animal tissues and in many plant sources and appears to decrease in the adrenal cortex under various stress conditions, as indicated for temperature extremes in Chapter 10, p. 349. Ascorbic acid was early discovered as the anti-scurvy vitamin in man. Its lack results in small scattered hemorrhages and lesions, particularly of the gums. Ascorbic acid is needed in the production of intercellular cement, particularly of capillaries. The human requirement is 1.6 to $1.7 \mathrm{mg}$. $/ \mathrm{kg}$./ day. ${ }^{127}$ Ascorbic acid is not required in the diet by all mammals. It is synthesized in sufficient amounts by the rat and hamster, but must be fed to guinea pigs, monkeys, and men. Ascorbic acid appears not to be essential in the nutrition of insects, but those animals may synthesize jt. ${ }^{140}$ The larvae of honeybees contain more ascorbic acid than do the adults. ${ }^{54}$ It is said to be needed by cultures of the higher trypanosomes (mammalian blood parasites) such as Leishmania and Schizotrypanum, but not by free-living protozoans and primitive trypanosomes such as Strigomonas and Leptomonas. ${ }^{83}$

Vitamin P. This term is applied to a group of products found in green plants and citrus fruits whose action may be more pharmacological than nutritional. These substances, flavone glycosides, rutin, and others, protect against capillary hemorrhages and regulate the capillary cement. They differ from aseorbic acid and may not be true vitamins.

Purines and Pyrimidines. In some animals purines and pyrimidines are dietary essentials. In culturing the ciliate Tetrahymena Kidder and Dewey 
isolated three unkown essential factors. One of these proved to be a purine, and guanine is specifie, although others are additive; another of these factors is a pyrimidine, and in cultures eytidy lic acid or uracil is used; the third is still unknown (see p. 135).

A malaria organism, Plasmodium knowlesii, needs purines and pyrimidines for best survival in vitro. 1::" Drosophila larvae have been grown on a syn. thetic medium which includes ribonucleic acid, 121 and it is possible to substi tute nucleotide or the purine adenine for this requirement. ${ }^{1+3}$ Other insects seem not to require dictary purines. In no mammals have purines and pyrimidins been shown to be dietary essentials, but since all eells contain them in nucleic acids, the enzymes for purine and pyrimidine synthesis must be very widespread. Numerous animals, such as Tetrahymena, lack the enzrmes present in mammals for synthesis of guanine and uracil.

Hematin. Probably all acrobic organisms have oxidative enzymes and carriers (cytochromes) which contain iron in a porphyrin ring similar to that in hematin. The hemochromogen of cytochrome is more primitive in an evolutionary sense than hemoglobin and is synthesized by many animals and plants which do not make hemoglobin. A few blood parasites, the hemoflagellates, have lost the ability to synthesize the hematin which they need and therefore rely on their host. Closely related flagellates which are parasitic on insects and plants (e.g., Strigomonas) do not require hematin when cultured in vitro but presumably synthesize it. Several species of Leishmania and Trypanosoma which parasitize birds and mammals require hematin, protohematin, or protoporphyrin (but cannot use other related substances) when cultured in vitro. ${ }^{\star 3}$ This is an interesting instance of loss of a synthetic function by a parasite with increasing dependence on the host. I Hematin is, then, a vitamin for these trypanosomes and for Leeishmania, but not for other kinds of animals.

Fat-Soluble Essential Substances. The fat-soluble vitamins differ from the water-soluble ones in being much more restricted as dietary requirements. Vitamins $A, D, E$, and $\mathrm{K}$ are known only for vertebrates.

Vitamin A. One form of this is:

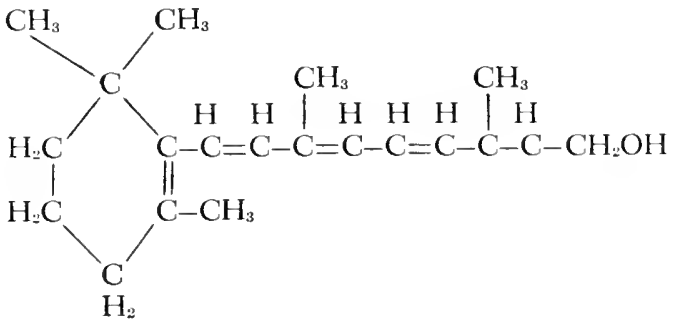

Vitamin A is synthesized by animals from plant carotenes and is a part of several pigment systems. The best known of these is the visual purple pigment of the vertebrate eve. This visual pigment is formed from vitamin $A$, which occurs in two forms $-A_{1}$ found in the retina of higher vertebrates and marine fish, and A.2 found predominantly in fresh-water fish (see Fig. 120, Ch. 11). Vitamin $A$ is stored in the liver, and a deficiency may result in xerophthalmia. Vitamin $A_{2}$ of fresh-water fish can be used by mammals, but less well than $A_{1}$. 
The rat converts carotene to vitamin $A$, probably in the small intestine. Carotene is converted slowly and is stored in the liver as $A_{1}$ in codfish ${ }^{10}$. ${ }^{126}$ and lobster, ${ }^{100}$ and as $A_{2}$ in perch and clace. ${ }^{n}$ Vitamin $A$ is not required in the diet of the cockroach, and analyses indicate that this animal does not sinthesize it. ${ }^{13}$ Vitamin $A$ has not been found necessary for growth or maintenance of any invertebrate animals, although it is suggested for the snail Helix pomatia. ${ }^{5 T}$ Since visual purple occurs in the pigment system of some arthropod and cephalopod eves (see Chapter ll) the possibility of a carotene or vitamin $A$ requirement for visual function in these animals should be tested.

Vitamin D. This is a class of substances, the most important of which are: (1) D.2 or calciferol, which is obtained by irradiation of ergosterol, and (2) $D_{3}$ or activated 7-dehydrocholesterol, which occurs in fish liver oil and is synthesized in the skin of higher animals under action of sunlight. Vitamin D.2 is only one-thousandth as active for chickens as is $D_{3,}$, whereas the two forms are nearly equally active for rat and inan. Vitamin D increases the absorption of calcium and phosphorus, and its lack results in low bone phosphatase and abnormal bone production, one kind of rickets. It is also needed for proper dentin formation in tecth. Unlike cholesterol, no form of vitamin D has been shown to be essential for any nonvertebrate, but the relation of specific sterols to phosphatase activity in shell deposition in crustaceans and molluses seems not to have been investigated.

Vitanin $E\left(\alpha^{-}, \beta\right.$, and $\gamma$-Tocopherols $)$. The most important form of vita$\min \mathrm{E}$ is:

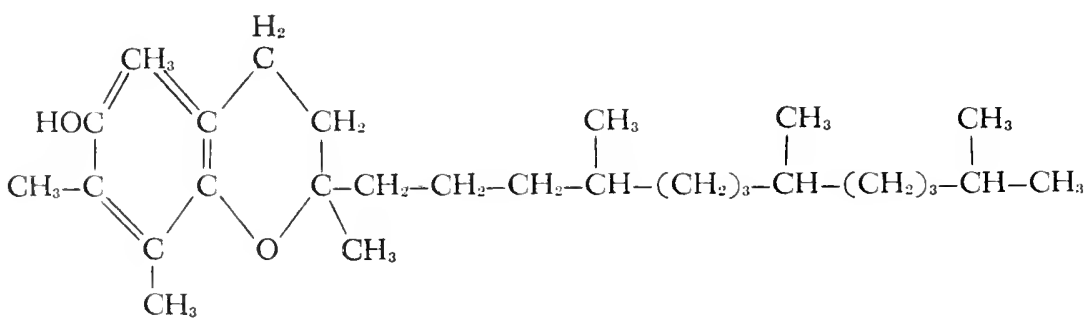

Vitamin E, usually supplied as $\alpha$-tocopherol, is necessary for normal development of vertebrates. Deficiency results in degeneration of germinal epithelium in the male and resorption of embryos from female mammals. Local hemorrhages, blood vessel impairment, brain and liver lesions, and particularly lesions of the testis have been observed in deficient chickens, ${ }^{1}$ tadpoles, ${ }^{110}$ and fish (guppies'is). It is likely, therefore, that vitamin $\mathrm{E}$ is essential for all classes of rertebrates. It is required for the proper use of vitamin A, probably because of its anti-oxidant properties. Among invertebrates, vitamin $\mathrm{E}$ is probably not essential, but in the moth Ephestia $\alpha$-tocopherol protects linoleic acid from oxidation, although other antioxidants, for example, ascorbic acid, can do as well.

Vitamin $K$ (Metlyyl Plytyl Naphthoquinone). Vitamin $\mathrm{K}$ is an antihemorrhagic factor essential for prothrombin formation. Its needs has been demonstrated in several mammals and birds, and it is obtained as $K_{1}$ from green leaves and as $K$. from putrefying fish meal. Supplements of vitamin $K$ 
are particularly useful to prevent hemorrhages in voung chickens, in human infants, and in adults with obstructive jaundice. Vitamin $K_{1}$ is:

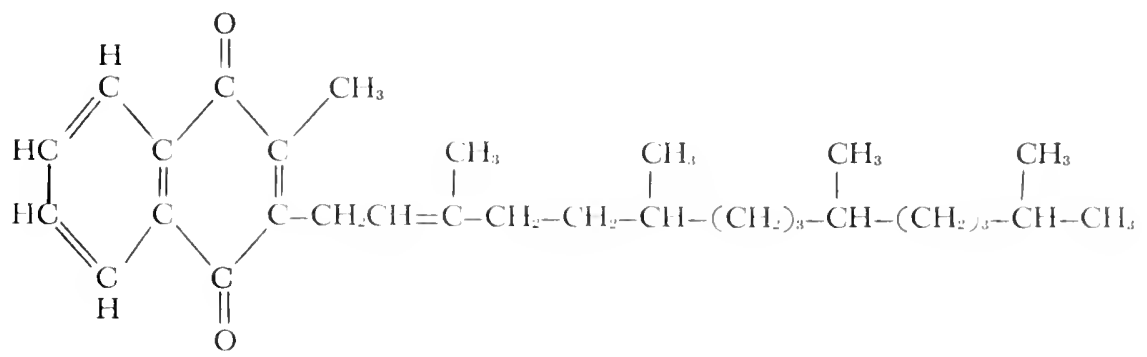

Vitamin $\mathrm{K}$ is not a dietary essential for the cow-it is synthesized in the rumen; also the rat, dog, and probably man obtain enough from bacteria in the in testine. This is not true for chickens, however, and in mammals shortly after birth the prothrombin level of the blood crops unless the vitamin $\mathrm{K}$ level in the maternal blood has been high. The entire molecule is not necessary, for the naphthoquinone is as effective in maintaining blood prothrombin as is the vitamin $\mathrm{K}$ with its side chain.

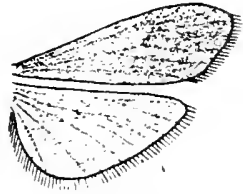

1

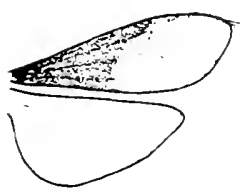

j

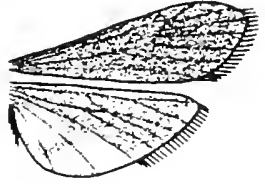

2

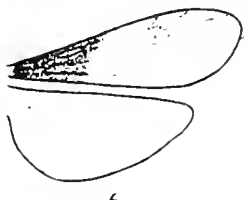

6

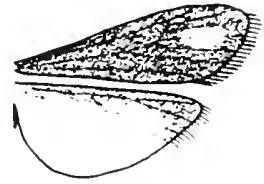

3

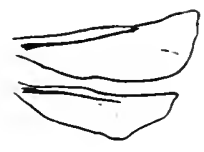

7

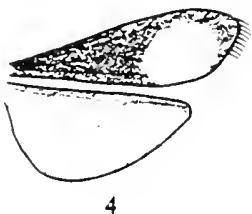

No cmergence

8

Fig. 28. Wings of Ephestia in increasing deficiency of linoleic acid from I no deficiency) 10 8. From Fracnkel and Blewett. ${ }^{0}$

Specific Fatty Acids. Most animals grow and live well with little or no dietary fat, but, since fat is the most favorable storage substance, it can be interconverted to and from carbohydrate and under some conditions to and from proteins; hence fat may be deposited on a high caloric intake even when little fat is fed. This is well shown in the flagellate Chilomonas which accumulates fat droplets when fed acetate, particularly in the absence of sulfur. In higher animals the deposited fat may differ from the food fat in respect to melting point or degree of saturation. The total fat intake can be reduced to very low levels in birds and mammals. Apparently fats, per se, are not necessary in animal diets; however, at least one fatty acid is required by certain animals. Mloths of the genus Ephestia require linoleic acid for successful 
emergence and normal appearance of the scales, and lack of linoleic acid causes an appearance similar to that of certain mutants; possibly a given gene controls linoleic acid metabolism, and hence scale production (Fig. 28). Linoleic acid synthesis occurs in Tenebrio but not in Ephestia, as shown by the following figures:

\section{LINOLEIC ACID CONTENT OF FAT'}

Tenebrio

with linoleic acid in diet without linoleic acid in diet
20 per cent

10 per cent
Ephestia

15 per cent

1 per cent

Rats on a low fat diet develop hematuria, loss in weight, and scaly feet and tail, all of which symptoms are then cured by feeding linoleic acid as sodium linoleate, about $30 \mathrm{mg}$. per day. Linolenic acid cannot be substituted, and vitamin $\mathrm{E}$ or some other antioxidant must be present for proper utilization of the linoleate. ${ }^{40,86,87}$

Men have lived well for six months on a low fat diet which would have been insufficient for rats; however, their blood concentration of linoleic acid declined; hence man needs linoleic acid in metabolism, but can synthesize relatively more of it than the rat can. ${ }^{15}$ Chickens also grow well on a diet containing less than 0.1 per cent of fat, and the depot fat is more saturated under this condition than on a normal fat ration. ${ }^{117}$ Apparently, then, the ability to synthesize fatty acids is universal among animals, but linoleic acid is not synthesized in sufficient amounts by some species and hence is a dietary essential for them.

Cholesterol. Sterols are commonly associated with fatty acids in a variety of lipoidal compounds, and by far the most common animal sterol is cholesterol. As with the fatty acids, there is much variation in ability to synthesize the needed cholesterol, and some species require it in their diet. For example, Lwolf $\cdots: .3 .3$ states that cholesterol is not needed by free-living Protozoa or by primitive trypanosomes (Strigomonas, Leptomonas), but it is needed by specialized trypanosomes (Trichomonas and Eutrichomastix). 1s Probably all insects, beetles (Lucilia, Tribolium), flies (Drosophila), and roaches (Blatella) require dietary cholesterol (Table 24 ). Dermestes, normally a wool feeder, uses only animal sterols, not ergosterol or sitosterol, whereas flour beetles do well on the plant sterols; none require calciferol (vitamin D). It Drosophila can use crgosterol or cholesterol but not calciferol. $\overline{~ L a r v a e ~ o f ~ A e d e s ~ a e g y ' p t i ~}$ fail to metamorphose without some lipid in the diet, a need which is satisfied by cholesterol or lecithin but not by fatty acils. ${ }^{4 t}$ Vertebrates can synthesize the cholesterol which they need.

It was formerly believed that the sterol components of invertebrate animals depended largely on the diet, herbivorous animals having predominantly higher sterols $\left(C_{29}\right)$ and camivorous animals having lower sterols like cholesterol $\left(C_{-7}\right)$. This dictary correlation now appears not to hold, but the sterols of different animals are often complex and may be useful in taxonomy of some groups. ", For example, gastropod and ecphalopod molluses contain mainly cholesterol, whereas pelecypods liave mixtures of higher melting point sterols, mostly with 28 carbons. Sponges contain a mixture including $\mathrm{C}_{27}, \mathrm{C}_{25}$, and C.as sterols, some of which are unique and may be useful in tracing taxonomic 
relations within the Porifera. The principal sterol of insects and vertebrates is cholesterol.

Unknown Factors. The discovery of vitamins often procecds by a tedious process of dietary elimination; an animal is fed a complex diet on which it thrives, then this diet is gradually fractionated into identifiable components which can be eliminated one by one. Sometimes the searches for an essential component for two or more organisms lead to discovery of the same substance, as when folic acid was identified as a factor for certain yeasts, molds, and mammals. A few examples of dietary factors which have not been identified chemically can be cited.

The ciliate, Tetrahymena geleii, has been cultured in a medium of amino acids, dextrose or acetate, inorganic salts, and B vitanins as described above. In addition, these organisms require a purine and a pyrimidine and a third factor which is obtained best from a Norit filtrate of liver extract. This unknown liver factor has been separated into two components (factors II A and II B). ${ }^{2 \pi,} \pi t i .1: 3$, In turn factor II B is found to be replaceable by large amounts of pyridoxine together with copper and iron salts. Factor II $A$ is not replaceable by any of a variety of compounds, including vitamin $B_{12}$; it is stable to hydrolysis in weak acid and alkali, and is precipitated with lead and extracted with butanol. Its identity is not known, but it has been named protogen.

Trout which are fed on a diet of devitaminized cascin, sucrose, salts, known vitamins, and dry liver, which is adequate for rats and chickens, do not grow well. In addition to thiamine, ribollavin, pyridoxine, pantothenic acid, and choline, trout require some unknown substance which is present in fresh liver and yeast. " It will be of interest to test vitamin $B_{12}$ on trout.

Planaria grow rapidly in a sterile medium if fed on animal tissues which provide a complete supply of needed factors. Liver is the most favorable food; the mucosa of such regions as cecum is better than that of other regions such as duodenum; egg white alone is deleterious, whereas egg yolk permits slow growth; and kidney cortex is superior to kidney medulla. 1.1. 1.: The liver from guinea pigs fed on a balanced diet containing grass or kale permits good growth of planaria, but if the green vegetable is replaced by orange or tomato juice, the planaria cease normal growth. ${ }^{10.2}$ Guinea pigs on such a deficient diet ultimately show various deficiency symptoms such as muscle degeneration. ${ }^{153}$ This guinea pig liver factor for planaria is somewhat similar to the unknown factor occurring in liver which is required by trout. There are several reports of unknown factors which can be supplied from liver as essentials for normal growth and maintenance of guinea pigs. ${ }^{1.0}$

Knowledge of the physiology of parasitic worms has long been retarded by inability to culture them in vitro. Many attempts at culture have been made, but the best that ean be done at present is to prolong survival. Some tapeworms and nematodes can be kept for days in salt solutions. The most success ful cultures to date are those of Smyth, 12k, 12! who kept larval tapeworms in a medium of peptone broth, salts, and sugar for 300 dals, during which there was some maturation of the worms and regular utilization of sugar. Whether parasitic worms can absorb essential factors from host tissues is not well cstablished. However, tapeworms grow well in rats which are receiving a diet low in protein or in vitamins $A, D, E$, and $B_{1}$, but not in rats fed a diet deficient in carbohydrate or ribollavin (in female but not in male hosts); hence these 
two latter substances must be supplied in the diet, whereas the protein and other vitamins can be taken from the host mucosa. $\ddot{2}, 19$

Larvae of bark beetles, Synchroa and Dendroides, grow on sterile bark but do not pupate unless the rhizomes of a fungus or some nonsterile bark is added. Apparently the fungus provides some unknown compound essential for pupation. ${ }^{107}$ Several beetles have been shown to require the identified B vitamins, except para-aminobenzoic acid and possibly inositol; in addition, Tenebrio requires an unknown called $\mathrm{B}_{\mathrm{T}}$, and Tribolimm needs two different unknown factors found in yeast. ":1 Roaches fed a synthetic diet equal to the best crude diet for growth nevertheless did not reproduce normally; the necessary factors are unknown (Noland, unpublished).

The diets of many animals under natural conditions are very restricted. Sometimes dietary restrictions are related to feeding mechanisms (Ch. 6); sometimes they depend on chemical sense and orientation with respect to chemical stimuli. In other animals there appear to be unknown nutritional factors which determine food preference. In no group are there so many examples of restricted diets as among insects, particularly the herbivorous ones, which may be attracted chemically to single kinds of plant ${ }^{16}$ and which may eat only certain kinds of leaves. The chemical which attracts an insect to a plant is not necessarily a nutrient. For example, silkworms can be forced to eat some leaves other than mulberry, but they do not grow properly on the substitutes. ${ }^{1+0}$ Female mosquitoes of most species require a blood meal for normal egg development. In several insect groups polymorphism depends on the diet, ${ }^{1+0}$ as the transformations of Hymenoptera. The identification of the active essential components in the food of specialized insects and the study of the ways these components cause morphological changes have scarcely been started.

To ascertain the true nutritional needs of an animal it is necessary to measure not only growth rate but also the ability to reproduce or, better still, to survive normally for many generations on an experimental diet. The discovery of new vitamins for common laboratory animals is becoming more difficult and chemical identification is becoming more prompt than in the early period of vitamin research. It is likely, however, that as more kinds of animals are studied as thoroughly as the rat has been, new essential substances will be found for particular species.

\section{CONCLUSIONS}

It is possible that the first "organisms" were heterotrophic, but certainly animals as we now know them evolved from autotrophic organisms. The history of nutrition shows increasing dependence of animals on autotrophs and a progressive loss of capacity to synthesize. Loss of synthesizing enzymes and increasing elependence on external sources for needed organic compounds is shown particularly well in parasites. For example, the more primitive trypanosomes, parasitic in insects and plants, synthesize their needed hematin, whereas those which parasitize mammals require hematin from outside if they are to grow in vitro. A comparison of the capacities of free-living and of parasitic helninths to synthesize $B$ vitamins would be of interest. Flagellate protozoans (leucophytes) which can use acetates as carbon source form an interesting series with respect to ability to use inorganic nitrogen, nitrate, 
ammonia, single amino acids, and special mistures as of peptones. Similarly some of them can synthesize either pyrimidine of thiazole (or both), and from these thiamine; others can synthesize thiamine only, while the highly special. ized flagellates and free-living ciliates must be supplied with thiamine in the diet.

The loss of ability to synthesize needed compounds is not always complete, since animals in deficiency can be shown to synthesize some needed compounds. When flagellates are transterred from one medium to a simpler one, growth is often poor initially and good later, indicating metabolic adaptation which may be either a change in enzyme pattem or selection of those individuals which initially had the greater synthesizing capacity. 122 Mammals normally obtain their nitrogen as protein, but, if minimal amounts of the essential amino acids are supplied, mammals can use urea or ammonium salts from which to synthesize amino acids and thus protein. The amount of protein normally consumed by man in our western civilization is much greater than the amount required to supply the necessary amino acids, and protein is an uneconomical source of energy. Certain amino acids act in specific processes (e.g., arginine in growth), but are needed by man in gram amounts daily, as compared with milligrams or micrograms of $\mathrm{B}$ vitamins. The amino acid and vitamin requirements for growth are greater than those for maintenance of adults. It is striking that the amino acids which are "dietary essentials" are similar for the ciliate Tetrahymena and for the rat.

It appears that the essential amino acids and $B$ vitamins are similar in all animals and that each one has its particular role in cellular metabolism. Some of the $B$ vitamins act as coenzymes for vital reactions in all aerobic cells; this has been established for thiamine, riboflavin, niacin, pyridoxine, and pantothenic acid. Animals must either synthesize these essentials, get them from a dietary source, or obtain them from symbiotic microorganisms which can synthesize them. Insects and mammals with gastrointestinal tracts sterilized of microorganisms have much higher vitamin requirements than those whose gastrointestinal tracts contain the normal symbionts. It is difficult to state the true metabolic requirement for a substance such as a water-soluble vitamin or amino acid in a higher animal, even when microorganisms are eliminated, since some of the substance is often synthesized, although not in sufficient quantity. It is probable that higher animals can be stimulated to greater synthesis of $\mathrm{B}$ vitamins by a lack of these and with an excess of the precursors in their diet. Mlost of the B vitamins, then, because of their cellular functions, are metabolic requirements of all animals and are dietary requirements of some.

There are also many substances which are required by specific groups of animals. The ciliate Tetralimmena requires guanine, whereas mammals can synthesize it from adenine, and Tetrahymena requires a primidine uracil, which is not metabolized when it is fed to mammals but which is synthesized by them. Apparently Protozoa and many insects and mammals do not need fats, whereas a few insects and mammals need only one fatty acid, linoleic acid. All insects require cholesterol; mammals and birds synthesize it. The fatsoluble vitamins, vitamins $A, D, E$, and $K$, are strictly rertebrate requirements, well known only for birds and mammals. Insects grow well in the absence of these vitamins. Investigation of specific functions of fat-soluble vitamins, such as that of vitamin $\mathrm{A}$ in visual purple synthesis, of vitamin $\mathrm{D}$ in calcium- 
phosphorus utilization, and of others in reproduction, might yicld evidence of their need in some invertebrate animals. In any case, these substances are associated with specialized chemical reactions rather than with the general cellular reactions in which many of the $\mathrm{B}$ vitamins participate.

Several erolutionary trends are shown in nutrition. First, the methods of obtaining carbon and nitrogen from inorganic and simple organic sources for the synthesis of protoplasm evolved early, probably among flagellates, and within that same group the methods of synthesis from simple sources were lost so that complex organic sources of carbon and nitrogen are needed by all other groups of animals. In a general way the kind of carbon source is related to the nitrogen source, and heterotrophic animals (but not yeasts) lack the synthetic enzymes of autotrophic organisms. The total caloric requirements depend on levels of metabolism, which will be discussed in Chapter 8. Second, there are many substances, amino acids and coenzymes, which are universal cellular components, which can be synthesized in adequate amounts by some unicellular organisms but not by the specialized Protozoa. Higher animals also are often unable to synthesize sufficient supplies of these substances and depend on food or symbiotic microorganisms for them. Finally, with increased chemical specialization there appear needs for particular compounds which are not universal requirements. The best examples of these are in fat metabolism, in the deposition of skeletal calcium, and in production of blood cells. The fact that there are still unknown requirements prevents the culture in solution of various animals, such as intracellular blood parasites, parasitic helminths, planaria, fish, and others, which have so far been cultured only on highly organized food, such as that found in mammalian liver. The comparative approach to nutrition can be expected to lead to the discovery of many specialized dietary requirements and may ultimately provide a partial description of the sequence of enzyme evolution. Such a goal will be attained only when the cellular functions of the specific required compounds are known.

\section{REFERENCES}

1. Adamstone, F. B., J. Morph. 52:47-89 (1931), also Arch. Path. 31: 603-612, 613-621 (19+1). Vitamin E deficiency in chicks.

2. Adpis, C. J., and Chandler, A. C., J. Parasitol. 30:229-236 (1944). Vitamin requirements of tapeworms.

3. Almquist, H. J., Fed. Proc. 1:269-273 (1942). Amino acid requirements of chickens.

4. Almouist, H. J., and Grau, C. R., J. Nutrit. 28:325-33I (1944). Amino acid requirements of chicks.

5. Almouist, H. J., and Mecchi, E., J. Biol. Chem. 135:355-356 (1940). Amino acids in chicken diet.

6. Bains, A. M., and Wulzen, R., Physiol. Zool. 5:198-206 (1932). Kidney as food for planaria.

7. Barker, H. A., J. Cell. E Comp. Physiol. 7:73-93 (1935). Metabolism of alga Prototheca.

8. Bergmann, W., and Low, E. M., J. Org. Chem. 12:67-75 (1947). Sterols in marine molluscs.

9. Bergmann, W., Mclean, M. J., and Lester, D., J. Org. Chem. 8:271-282 $(19+3)$. Sterols of marine invertebrates.

10. Bieri, J. R., Fed. Proc. 8:184 (1949). Conversion of carotene to vitamin A in rat.

11. Brrci1, T. W., J. Nutrit. 17:281-292 (1939). Nicotinic acid requirement of dog and rat. 
12. Blewett, M., and Fraenkel, G., Proc. Roy. Soc. Iond., B. 132:212-221 (1943). Symbionts and vitamin requirements of beetles.

13. Bowers, R. E., and McKar, C. M., Science 92:291 (1940). Cockroach growth without vitamin $\mathrm{A}$.

14. Brand, T. r., Ztschr. vergl. Physiol. 19:587-614 (1933). Sugar recuirements of trypanosomes.

15. Brown, W. R., Hanson, A. E., ET al., I. Nutrit. 16:511-524 (1937). Effect of low-fat diet on man.

16. Brues, C. T., Insect Dietary (1946). Harvard Univ. Press. 466 p.

17. Burrows, A., Protoplasma $31: 20-26$ (1938). Nutrition of Chilomonas.

18. Callleau, R., Ann. Inst. Pasteur 59:137-181, 293-328 (1937). Nutrition of flagellates.

19. Chandeer, A. C., Am. J. Hyg. 37:121-130 (1943). Nutrition of tapeworms.

20. Cooperman, J. M.. Elveijuent, C. A., et Al., Proc. Soc. Exper. Biol. E Med. 61: 92-97 (1946). Folic acid requirement, monkey.

21. Cooperman, J. M., Waisman, 11. A., and Elveiljem, C. A., Proc. Soc. Exper. Biol. E Med. 52:250-254 (1943). Vitamin requirements of hamster.

22. Cooperman, J. M., Waisman, H. A., Et. AL., J. Nutrit. 30:45-57 (1945). B vitamin requirements of monkey.

23. Couch, J. R., Cravens, W'. W., et al., Anut. Rec. 100:29-48 (1948). Biotin deficiency in chicks.

24. Couch, J. R., Cravens, W. W., et al., J. Nutrit. 35:57-72 (1948). Intestinal synthesis of biotin in fowl.

25. Couch, J. R., Cravens, W. W., et Al., Arch. Biochem. 21: 77-86 (1949). Function of biotin in fowl.

26. Cummings, H. W., Anat. Rec. (suppl.) 84:499 (1942). Vitamin E deficiency in guppies.

27. Deane, H. W. and Shaw, J. H., J. Nutrit. 34:1-15 (1947). Response of adrenal cortex to $B$ vitamin deficiencies.

28. Doyle, IV., Biol. Rev. 18:119-136 (1943). Nutritional evolution, Protozoa.

29. Dusi, H., Ann. Inst. Pasteur 50: 550-597, 840-890 (1933). Nutrition of euglenoids.

29a. Elliot, A. M., Physiol. Zool. 12:363-373 (1939). Vitamin B complex and growth of Colpidium.

29b. Elliot, A. M., Physiol. Zool. 22:337-345 (1949). Amino acid requirements of Tetrahymena geleii (E).

30. Fraenkel, G., J. Exper. Biol. 17:18-29 (1940). Utilization of sugars by blowHies.

31. Fraenkel, G., Fed. Proc. 8: 382 (1949). Unknown vitamins for Tenebrio and Tribolium.

32. Fraenkel, B., and Blewett, M., Nature 150:177 (1942),ibid. 151:703 (1943). $B$ vitamin requirements of insects.

33. Fraenkel, G., and Blewett, M., J. Exper. Biol. 20:28-34 (1943). Basic food requirements of several Lepidopera and Coleoptera.

34. Fraenkel, G., and Blewett, M., Biochem. J. 37:686-692 (1943). B vitamin requirements of several insects.

35. Fraenkel, G., and Bleivett, M., Biochem. J. 37:692-695 (1943). Sterol requirements of several insects.

36. Fraenhel, G., and Blewett, M., Nature 152:506 (1943). Synthesis of B vitamins by symbionts in insects.

37. Fraenkel, G., and Blewett, M., J. Exper. Biol. 20:28-34 (1943). Basic food requirements of flour-eating insects.

38. Fraenkel, G., and Blewett, M., J. Exper. Biol. 22:156-161 (1946). Nutrition of clothes moth.

39. Fraenkel, G., and Blewett, M., J. Exper. Biol. 22:162-171 (1946). Nutrition of moth, Ephestia.

40. Fraenkel, G., and Blewett, M., J. Exper. Biol. 22:172-190 (1946). Requirements for fat-soluble substances by Ephestia.

41. Fraenkel, G., and Blewett, M., Biochem. J. 41:469-475 (1947). Folic acid and other requirements of insects. 
42. Fraenkel, G., and Blewett, M., Biochem. J. 41:475-478 (1947). Linoleic acid in nutrition of Ephestia and Tenebrio.

43. Fraenkel, G., Blewett, M., and Coles, M., Nature 161:981 (1948). Vitamin $B_{T}$ in insect nutrition.

44. Fraenkel, G., Reid, J. A., and Blewett, M., Biochem. J. 35:712-720 (1941). Sterol requirements of larva of bectle Dermestes.

45. Goldberg, L., and DeMlejllon, B., Biochetu. J. 43:372-379 (1948). Lipoid requirements of Aedes larvae.

46. Goldberg, L., and DeMeillon, B., J. Biol. Chem. 43:379-387 (1948). Protein and amino acid requirements of Aedes larvae.

47. Goldberg, L., DeMeilion, B., and Lavolpierre, M., J. Exper. Biol. $21: 84-96$ (1975). B vitamin requirements of Aedes larvae.

48. Green, R. G., Carison, U. E., and Evans, C. A., J. Nutrit. $21: 243-256$ (1941). Anti-thiamine action of fresh fish fed to foxes.

49. Hall, R. P., Quart. Rev. Biol. 14:1-12 (1939). Synthetic capacity of flagellates.

50. Hall, R. P., Physiol. Zool. 17:200-209 (1944). Effects of vitamins on ciliate, Glaucoma.

51. Hall, R. P., and Loefer, J. B., Protoplasma 26:321-330 (1936). Failure of Chilomonas to grow without organic nitrogen.

52. Handler, P., Ztschr. Vitaninforsch. 19:393-451 (1948). Role of nicotinic acid in pellagra.

53. Harris, L. E., and Mitcheld, H. H., J. Nutrit. 22:167-182 (1941). Utilization of urea nitrogen by ruminants.

54. HAYDEK, M. H., and Vivino, A. E., Arch. Biocheni. 2:201-207 (1943). B vitamin content of honey-bees.

55. Hoog, E. G. v., Ztschr. Vitaminforsch. 4:300-324 (1935), ibid. 5:118-126 (1936). Aseptic culture of Drosophila.

56. Honowitz, N. H., Proc. Nat. Acad. Sci. 31:153-157 (1945). Evolution of biochemical synthesis.

57. Howes, N. H., Biochem. J. $31: 1489-1498$ (1937). Culture of Helix.

58. Hutchens, J. O., J. Cell. E Comp. Physiol. 16:265-267 (1941). Need of Chilomonas for iron and thiamine.

58a. Hutchens, J. A., Jandorf, B. J., and Hastings, A. B., J. Biol. Chem. 138:321325 (1941). Synthesis of nicotinic acid by Chilomonas.

59. Hutner, S. H., Provasoli, L., et al., Proc. Soc. Exper. Biol. E Med. 70:118-120 (1949). Need of Euglena for "anti-pernicious anemia factor."

60. JaIn, T. L., Arch. Protist. 86:251-262 (1935). Nutrition of Euglena gracilis on synthetic medium.

61. Johnson, B. C., Wiese, A. C., et Al., J. Biol. Chem. 167:729-736 (1947). Growth of calves without nicotinic acid.

62. Johnson, W. H., Quart. Rev. Biol. 16:336-348 (1941). Nutrition of the Protozoa.

63. Jukes, T. H., Stokstad, E. L. R., and Belt, M., J. Nutrit. 33:1-12 (1947). Vitamin requirements of young turkeys.

64. Jungulira, P. B., and Schweigert, B. S., J. Biol. Chem. 175:535-546 (1948). Synthesis of nicotinic acid by rats.

65. Kidder, G. W., Arch. Biochem. 9:51-55 (1946). Folic acid requirement of Tetrahymena.

66. Kidder, G. W., and Dewey, V. C., Growth 6:405-418 (1942). Thiamine synthesis by microorganisms.

67. Kidder, G. W., and Dewey, V. C., Biol. Bull. 87:121-133 (1944). Thiamine and growth of Tetrahymena.

68. Kinder, G. W., and Dewey, V. C., Arch. Biochen. 6:425-432 (1945). Amino acid requirement of Tetrahymena.

69. Kinder, G. W', and Dewey, V. C., Physiol. Zool. 18:136-157 (1945). Strain differences in nutrition of 'Tetrahymena.

70. Kinder, G. IV., and Dewey, V. C., Arch. Piochem. 8:293-301 (1945). Properties of unknown factors for 'letrahymena.

71. Kidder, G. W., and Dewey, V. C., Biol. Bull. 89:229-241 (1945). B vitamin requirements of Tetrahymena. 
72. Kidder, G. W., and Dewer, V. C., Proc. Nat. Acad. Sci. 33:347-356 (1947). Growth responses of Tetrahymena to amino acids.

73. Kidper, G. W', and Dewey, V. C., Proc. Nat. Acad. Sci. 34:81-88 (1948). Utilization of homecystine by Tetrahymena.

74. Kinder, G. M'., and Dewey, I. C., Proc. Nat. Acad. Sci. 34:566-57+ (1948). Purines and pyrimidines in nutrition of Tetrahymena.

75. Kidder, G. W', and Dewey, V. C., Arch. Biochem. 20:433-443 (19+9). Components of "factor II" for Tetrahymena.

76. Kidder, G. $11^{\dagger}$., and Dewey, V. C., Arch. Biochem. 21:58.65 (1949). Pyridoxine nutrition in Tetrahymena.

77. Kidder, G. W', and Dewer, V. C., Arch. Biochem. 21:66-73 (1949). B vitamin requirements of letrahymena.

78. Krampitz, L. O., and Wooley, J. Bioi. Chem. 152:9-17 (194t). Inactivation of thiamine by fish tissue.

79. Lardy, 11. A., Potter, R. L., and Elverlyem, C. A., J. Biol. Chem. 169:451-452 (1947). Function of biotin in bacterial metabolism.

80. Lilly, D. M., Physiol. Zool. 15:1+6-167 (1942). Nutrition of camivorous ciliates.

81. LoosLI, J. K., and MCCAr, C. M., J. Nutrit. 25:197-202 (19+3). Utilization of urea by calves.

82. Livoff, A., Ann. Inst. Pasteur 61:580-617 (1938). Growth factors for microorganisms.

83. Lwoff, A., L'Evolution Physiologique: Etude des Pertes de Fonctions chez les Microorganismes (1943). Paris, Hermann. 308 p.

84. Lwoff, A., and Dusi, 11., C. R. Acad. Sci. 205:630-632, 756-758, 882-884 (1937). Vitamins and growth of flagellates.

85. Lwoff, A., and Lwoff, M., C. R. Soc. Biol. Paris 126:6+4-6+6 (1937). Thiamine and growth of Glaucoma.

86. Mackenzie, C. G., Mackenzie, J. B., and McCollum, E. V., Biochem. J. 33:935-943 (1939). Growth of rats on luw fat diet.

87. Martin, G. J., J. Nutrit. 17:127-141 (1938). Linoleic acid requirement of rats.

88. Mast, S. O., and Pace, D. M., Protoplasma 20:326-358 (1933). Growth of Chilomonas on simple synthetic medium.

89. Mast, S. O., and Pace, D. M1., Protoplasma 23:297-325 (1936). Role of sulfur in protoplasmic synthesis by Chilomonas.

90. Mast, S. O., and Pace, D. M., J. Cell. E Comp. Physiol. 10:1-13 (1937). Effect of silicon on growth of Chilomonas.

91. McCall, K. B., Waisman, H. A., et al., J. Nutrit. 31:685-697 (1946). Pyridoxine and pantothenic acid deficiency in monkey.

92. McCay, C. M., Physiol. Zool. 11:89-103 (1938). Nutrition of roach, Blatella.

93. McCay, C. Mi., Tunison, A., et al., Tr. Am. Fish. Soc. 61:58-79 (1931). Nutritional requirements of trout.

94. MicElrov, L. W., and Goss, H., J. Biol. Chem. 130:437-438 (1939). Synthesis of $B$ vitamins in sheep rumen.

95. NicElroy, L. W., and Goss, H., J. Nutrit. 20: 527-540, 541-550 (1940). V'itamin synthesis in rumen of shecp and cows.

96. Mclaren, B. A., Herman, E. F., and Elvenjen, C. A., Arch. Biochem. 10:433$+41(19+6)$. Growth requirements of trout.

97. Moore, IV., Anu. Ent. Soc. Amer. 39:513-521 (1946). Nutrition of carpet beetle, Attagcnus.

98. Monton, R. A., and Creed, R. H., Biochem. J. 33:318-324 (1939). Conversion of carotene to vitamin $A$ by fresh-water fish.

99. Needham, J., Biol. Rev. 13:225-25 I (1938). Evolutionary factors of nutrition.

100. Nellands, J. B., Arch. Biochem. 13:415+19 (19+7). Conversion of carotene to vitamin $A$ in cod and lobster.

101. Noland, J. L., and Baunann, C. A., Proc. Soc. Exper. Biol. E Med. 70:198-201 (1949). Choline requirements of cockroach.

102. Noland, J. L., and Baumann, C. A., Fed. Proc. 8: 237 (1949). Synthesis of tryptophane and methionine by cockroach.

103. Olcese, O., Pearson, P. B., and Schweigert, B. S., J. Nutrit. 35:577-590 (1948). Synthesis of B vitamins in rabbits. 
104. Oparin, A. I., The Origin of Life (1938), transl. by S. Morgulis. New York, Macmillan. $270 \mathrm{p}$

105. Pace, D. M., Exper. Med. E Surg. 5:236-250 (1947). Effects of vitamins on growth of Chilomonas.

106. Patels, A. J., Post, J., and Victor, I., Am. J. Physiol. 133:47-55 (1941). Ributlavin deficiency in pig.

107. Payne, N. M., Ent. News 43:13-15 (1931). Factors for pupation in bark beetles.

108. Pearson, P. B., and Burgin, C. J., Proc. Soc. Exper. Biol. E Med. 48:415-417 (19+1). Pantothenic acid content of bee royal jelly.

109. Pilllips, A. M., Tr. Am. Fish. Soc. $76: 34-45$ (1946). B vitamin requirements of trout. Also: Phillips, A. M., and Tunnison, A. V. Ibid. 74:81-87, 1947.

110. Pвatт, E. M., Anat. Rec. (suppl.) $75: 148$ (1939). Vitamin E deficiency in tadpoles.

111. Pringsiein, E. G., Biol. Rev. 16:191-204 (1941). Evolution and nutritional interrelations among flagellates.

112. Provasoli, L., Hutner, S., and Schatz, A., Proc. Soc. Exper. Biol. E Med. 69:279-282 (1948). Streptomycin-induced chlorophyll-less races of Euglena gracilis.

113. Rickes, E. L., Brink, N. G., et. aL., Science 108:134 (1948). Bı, a cobalt complex.

114. Rose, W'. C., Physiol. Rev. 18:100-136 (1938). Amino acid requirements in rat.

115. Rose, W. C., Fed. Proc. 8:546-552 (1949). Amino acid requirements, man.

116. Rose, W. C., and Rice, E. E., Science 90:186-187 (1939). Amino acid requirements, dog.

117. Russell, W. C., Taylor, M. W., and Polskin, L. J., I. Nutrit. 19:555-562 (1940). Fat requirements, growing chick.

118. Schaeffer, A. E., Whiteinalr, C. K., and Elvehjem, C. A., Proc. Soc. Exper. Biol. E Med. 62:169-174 (1946). Vitamin needs of mink.

119. Schaefer, A. E., Whitehair, C. K., and Elvehjem, C. A., J. Nutrit. 34:131-139 (1947). B vitamin in mutrition of foxes.

120. Schaefer, A. E., Whiteilair, C. K., and Elvehjem, C. A., Arch. Biochem. 12:349-357 (1947). Purified ration, folic acid requirement, fox.

121. Scheer, B. T., Comparative Physiology (1948). New York, Wiley. pp. 57-74, Protozoa; pp. 311-317, insects; pp. 362-367, vertebrates.

122. Schoenborn, H. W., Ann. N. Y. Acad. Sci. 40:1-30 (1940). Use of inorganic nitrogen by Astasia.

123. Schoenborn, H. V., Physiol. Zool. 15:325-332 (1942). Nutrition of Euglena gracilis in dark.

124. Schultz, J., St. Lauretce, P., and Newmeyer, D., Anat. Rec. 96:540 (1946). Basic diet for Drosophila.

125. Schweigert, B. S., McIntine, J. M., et al., Arch. Biochem. 6:403-410 (1945). Synthesis of B vitamins in rat.

126. Sexton, E. L., Mehl, J. W., and Deuel, H. J., J. Nutrit. 31:299-319 (1946). Locus of conversion of carotene to vitamin $\mathrm{A}$ in rat.

127. Silerman, H. C., The Chemistry of Food and Nutrition (1946). New York, Macmillan. 675 p.

127a. Sinve, A., Ravel, J. M., and Eakin, R. E., J. Am. Chem. Soc. 70:2614-2615 (1948). Function of vitamin $B_{12}$ in certain bacteria.

128. Sмyтil, J. D., J. Exper. Biol. 23:47-70 (1946). In vitro culture of tapeworms.

129. Smytil, J. 1)., Biol. Rev. 22:214-238 (1947). Maintenance of tapeworms outside host.

130. Snyderman, S. E., Ketron, K. C., et al., Proc. Soc. Exper. Biol. \& Med. 70 : 569-571 (1949). Nicotinic acid synthesis in man.

131. Sprtzen, E. H., Coombes, A. I., Ex. Al., Proc. Sac. Exper. Biol. E Med. 48:376379 (19+1). Thiamine deficiency in foxes and chickens on fish diet.

132. Spitzlis, R. R., Maruyama, G., et al., J. Nutrit. 35:185-193 (1948). Role of vitamin D) in utilization of phytin phosphorus.

133. Stoksiad, E. L. R., Hoffman, C. E., Et Al., Arch. Biochem. 20:75-82 (1949). Protogen as essential factor for Tetrahymena. 
134. Subbarow, Y., and Trager, W., I. Gen. Physiol. 23:561-568 (1940). Pantothenic acid and pyridoxime requircments of mosquito larvac.

135. Tatum, E. L., Proc. Nat. Acal. Sci. 25:490-497 (1939). Nutritional repuire ments of Drosophila.

136. Tатum, E. L., Proc. Nat. Acal. Sci. $25: 490497$ (1939). B vitamin require. ments of Drosophila.

137. Tatum, E. L., Garnjobst, L., and Taylon, C. V., J. Cell. E Comp. Physiol. 20:211-224 (1942). Vitamin requirements, cilitte Colpoda.

138. Trager, W., Physiol. Rev. 21:1-35 (1941). Nutrition of lnvertebrates.

139. 'Trager, W., J. Parasitol. 33:345-350 (1947). Culture of malaria parasite, Plas modium, in vitro.

140. Гrager, W., Biol. Rev. 22:148-177 (1947). Insect nutrition.

141. Trager, W., J. Biol. Chem. 176:1211-I223 (1948). Biotin and nutrition of mosquito larvae.

142. Trager, W., and Subbarow, Y., Biol. Bull. 75:75-84 (1938). Riboflavin and thiamine in nutrition of mosquito larvae.

143. Villee, C. A., and Bissell, H. B., J. Biol. Chem. 172:59-66 (1948). Nucleic acid requirement in Drosophila.

144. Vogel, B., Ztschr. vergl. Physiol. 14:273-347 (1931). Sugar utilization by honeybee.

145. Weir, D. R., Heinle, R. W., and Welch, A. D., Proc. Soc. Exper. Biol. E Med. 69:211-215 (1948). Folic acid deficiency, mice.

146. Wiese, A. C., Johnson, B. C., ET AL., J. Nitrit. 33:263-270 (1947). Riboflavin deficiency in calves.

147. Williamson, J. W., Phy'siol. Zool. 17:209-228 (194t). Nutrition of amoeba.

148. Woodsury, L. A., Tr. Am. Fish. Soc. 72: 30-3+ (19+3). Thiamine deficiency, rainbow trout.

149. Woolley, D. W., J. Nutrit. 28:305-3I+ (1944). Nutritional significance of inositol.

150. Woolley, D. W., and Sprince, H., J. Biol. Chem. 153:687-688 (1944). Folic acid requirement for guinea pig.

151. Wulzen, R., Univ. Calif. Publ. Plyysiol. 5:175-187 (1923), ibid. 7: 1-7 (1926). Feeding Planaria.

152. Wulzen, R., and Bafirs, A. M., Physiol. Zool. t:20+213 (1931). Balanced diet for Planaria.

153. Wulzen, R., and Bahrs, A., Physiol. Zool. 8:457-473 (1935), ibid. 9:508-529 (1936). Dietary factors making guinea pig tissuc suitable for Planaria.

154. Wulzen, R., and Bairs, A. M., Am. J. Physiol. 133:500 (1941). Effect of milk diet on guinea pig. 


\section{Feeding and Digestion}

\section{FEEDING MECHANISMS}

N THE ANINAL KINGDON as a whole there have evolved mechanisms enabling various animals to deal with almost every conceivable source of food materials. For the most part there is no correlation between the types of food materials utilized and the major groups in the natural scheme of classification. Practically all of the major divisions in the animal kingdom have species feeding on a wide variety of foods and using correspondingly diversified feeding mechanisms. The nature of feeding mechanisms appears to have been determined largely by the habitat and the food materials available to a particular organism. The form and function of the digestive tract, as well, are in most cases definitely correlated with the types of food used and the feeding mechanisms.

Soluble Food. All natural waters have organic material dissolved in them in concentrations of the general order of magnitude of $10 \mathrm{mg}$. per liter, the amounts being greater in regions of much organic detritus. There are proteins, some carbohydrates which are principally pentosans, some amino acids and fatty acids, all of which have arisen from dead organisms or as waste products of living ones. Pütter ${ }^{137}$ was the first to consider the significance of these dissolved substances as food for animals. He believed that he demonstrated them to be important as food, but this conclusion was seriously questioned by many later investigators. The subject has been ably reviewed by Krogh.97 Only a few free-living animals, particularly the protozoans and possibly sponges, appear capable of relying in nature on simple dissolved substances such as amino acids, sugar, and fatty acids as an important portion of their food supply. In the protozoans the substances appear to be absorbed directly through the cell membrane; in the sponges the feeding cells or choanocytes are probably responsible for uptake of the dissolved foods. Direct absorption of simple foodstuffs must be the principal "feeding mechanism" in helminth parasites, such as restodes and acanthocephalans, which possess no digestive tract, and in parasitic protozoans. Where simple compounds in solution are an important food source there must usually be mechanisms for active transport across cell membranes since the materials are carried from a low to a higher concentration. The utilization of complex dissolved foodstuffs such as proteins and polysaccharides probably requires digestion either in food racuoles or in a functional digestive tract.

There is some evidence that higher animals, such as lamellibranch molluscs, and even fishes and frog tadpoles, are able to absorb and utilize dissolved nutrients from their aqueous environment when these substances are present in rather high concentrations. I fowever, even when high concentrations are present the amounts absorbed are insufficient for normal metabolic require- 
ments. There is good evidence that in these higher animals the absorption occurs by way of the gut, into which the material must first pass. It has been generally held that fresh-water fishes and amphibians do not drink significant quantities of water, but the ability of these animals to absorb dissolved substances by way of the gut renders this questionable, as do recent observations on the concentration of colloidal material in the digestive tract of the goldfish. ${ }^{2}$

Particulate Food. For the vast majority of animals, other organisms and particulate organic detritus constitute the principal sources of food. The best classification of the innumerable mechanisms used by animals in dealing with such foods is that of Yonge, ${ }^{1 s i}$ who separates feeding mechanisms into three large categories: (1) mechanisms for dealing with small particles; (2) mechanisms for dealing with large particles or masses; (3) mechanisms for taking in fluids or soft tissues.

Mechanisms for Dealing with Small Particles. Such mechanisms are found only in aquatic species; most multicellular animals possessing these mechanisms are sessile or quite sluggish. A viscous secretion is usually produced, and in this the particles are trapped prior to ingestion. Yonge subdivides these mechanisms into six groups:

PSEUDOPODiaL. These are exemplified by the reticulate pseudopodia of the radiolarians and foraminiferans, upon which particles are caught and swept inward toward the main portion of the body for ingestion.

CILIARY. These are very widespread among animals. Special patterns of distribution and activity of cilia are virtually as numerous as the number of species which possess such mechanisms. Generally speaking, feeding currents are produced which bring large volumes of the medium into close association with the feeding parts; the food particles are carried along definite tracts where they are entangled in a secreted viscid material and finally are carried into the mouth. Most fine-particle feeders appear to show little or no chemical but only mechanical selection of food particles, accepting or eliminating the particles on the basis of size; ciliate Protozoa may show chemical selection. Ciliary mechanisms for fine-particle feeding are found among the ciliate protozoans, sponges, many tubicolous annelids, echiuroids, rotifers, entoprocts, ectoprocts, phoronids, brachiopods, some gastropods, most lamellibranchs, and many tunicates and cephalochords. In some of these the mucous filter is not used.

TENTacular. Some holothuroids possess tentacles about the mouth on which small particles fall and then become entrapped in mucus. The tentacles are then pushed into the mouth and the material removed.

Mucoid. The sessile gastropod Vermetus extrudes from its mouth a film of mucus which is retracted at intervals, bringing into the mouth the particles entrapped upon it.

muscular. The scyphozoan jellyfish has, instead of a single mouth, numerous fine pores leading to the coelenteron. Particle-laden water is drawn in and the water is expelled by rhythmic muscular contractions of the bell. The particles are filtered out in the digestive cavity. The polychaete Chaetopterus and the echiuroid Urechis secrete mucous bags which filter out food particles and are eventually swallowed. Currents which fill the bags are set up by parapodial movement in Chaetopterus and by body peristalsis in Urechis. ${ }^{109}$ By comparing the filtration of different proteins MacGinitie 
estimated the pores of the sacs of Chaetopterus to be about $40 \AA$ in diameter. Certain vertebrates, such as the baleen whale, many fishes, and even a few birds which capture insects on the wing, may be considered filter feeders, in which locomotor and sometimes pharyngeal muscular activity serves as the force involved in filtration.

SETOSE. This type of mechanism is characteristic of numerous aquatic crustaceans such as Daphnia, copepods, cirripedes, ostracods, and larvae of certain insects such as mosquitoes and mayflies. Movements of certain appendages with their fringes of setae sweep particle-laden water into certain areas among the appendages where setae strain out the particulate matter. The particles are then bound into larger masses by a viscid secretion and carried to the mouth.

Mechanisms for Dealing with Large Particles or Masses. Mechanisms of this type are subdivided by Yonge into three groups:

FOR SWALLOWING INACTIVE FOOD. Organisms possessing mechanisms for swallowing inactive food, such as mud, are typically burrowing forms, rather sluggish in habit. Included in this category are certain holothuroids which use their tentacles to force mud into their digestive tracts. Annelids such as Lumbricus and Arenicola possess eversible pharynges for this purpose; echinoderms such as spatangids use their tube feet; and burrowing crustaceans such as Upogebia and Callianassa use their mouthparts. The organic content of the mud is utilized, and the residue is ejected in the feces.

FOR SCRAPING AND BORING. These mechanisms are found in animals characterized by more or less continuous feeding. They usually have organs for attachment to permit the special apparatus for scraping or boring to be used effectively. Examples of this type of mechanism are the Aristotle's lanterns of echinoids, the radular apparatus of many molluscs, the special boring valves of Teredo, and the heavy mouthparts of termites.

FOR SEIZING PREY. Mechanisms for seizing prey are further subdivided by Yonge into three groups: (1) For seizing only. These would include the pseudopodia of amoebae, the impaling proboscis of Didinium, nematocysts of coelenterate tentacles, lasso-cells of ctenophore tentacles, the turbellarian pharynx, the jawed pharynges of many polychaetes, the chitinous hooks of chaetognaths, the teeth and radula of some gastropods, and the suction mechanism of feeding of septibranchs. (2) For seizing and masticating. Examples of these are the jaws and radulae of many molluscs; the jaws and other mouthparts of crustaceans, insects, arachnids, and myriapods; and the toothed jaws of vertebrates.

In the jawed vertebrates or gnathostomes the anterior teeth often bite or gnaw, and posterior teeth masticate; however, there is much variety, ranging from amphibians and billed birds which chew very little, to the ungulates with heavy chewing molars.

(3) For seizing followed by external digestion. This type is illustrated by the sarcodinian, Vampyrella, which digests a hole through the walls of the algae on which it feeds; by Asterias, whose everted stomach kills and digests its prey; by many carnivorous gastropods; by Sepia; and by many insects such as Dytiscus larvae.

Mechanisms for Taking in Fluids and Soft Tissues. Such mechanisms usually include a pumping muscular pharynx. There may be chitinous jaws or 
stylets for piercing. If blood is the material ingested as food, the presence of an anticoagulant is not uncommon. Yonge further subdivides this class into three groups:

FOR PIERCING IND SUCKING. Included in this group are the sucking mechanisms of hookworms, leeches, parasitic copepods, tardigrades, prcnogonids, many Diptera, mites, and ticks, and many cyclostomes.

FOR SUCKING ONLY. Examples of this category are the feeding mechanisms of the suctorians, trematodes, most nematodes, lepidopterans, Mluscidae, pentastomids, and suckling mammals.

FOR ABSORPTION THROUGH THE GENERAL BOdy SUIFFACE. This mechanism was discussed earlier (see p. 144). Species possessing this means of feeding have no digestive organs or mechanisms and depend wholly on absorption of nutritive materials from their surroundings. Many parasites are included in this category.

\section{MECHANICAL FACTORS IN DIGESTION}

The digestive tracts in animals, like the feeding mechanisms, show striking adaptations to the character of the foods and the feeding habits of the organism. The general problem of the evolution and adaptation of the digestive systems of animals has been ably discussed by Yonge. 1:0 The digestive tract in several animals is diagrammatically shown in Figure 29. Yonge divides the tract into five functional regions.

Functional Regions of Alimentary Tract. Reception. This includes the mouth and mouth cavity. This region, except in fine-particle feeders, usually possesses glands which have received various names, largely depending on their particular position, such as labial, buccal, or sublingual. These glands are primarily lubricating, although special functions have been secondarily assumed by them. These glands in many blood-sucking species, such as leeches, ticks, and some insects, are the sources of anticoagulants. Many insects and the snail Helix secrete carbohydrases from such glands. Some snails produce acid which is used in dissolution of food. Some nematodes and carnivorous gastropods produce proteases in the oral glands. Toxins for the paralysis or killing of prey are found in a wide range of species from many groups.

Conduction and Storage. Following the region of reception is one of conduction and storage. This includes the esophagus and crop of many species. The crops of leeches, of some insects, and of herbivorous birds are simply dilatations of the esophageal region. Digestive glands may be present in this region, or digestive enzymes may be regurgitated from more distal regions.

Digestion and Internal Trituration. The third general region is for digestion and internal trituration. In this region one often finds the differentiation of gizzards or gastric mills. Such special grinding mechanisms are seen in rotifers, some clams, certain annelids, crustaceans, some insects such as the cockroach (Fig. 29, A), and birds. It is not uncommon to find associated with the grinding mechanism a filtering mechanism which maintains the food particles in the region of the triturating mechanism until they have been reduced to a particular degree of fineness, as in the crayfish. In this region also the greater portion of the digestive enzymes are liberated and the lining may be rich in unicellular glands, distributed uniformly through the region or distributed in a characteristic pattern. This region also often possesses conspicu- 
ous diverticula, the functions of which vary greatly. They may simply increase surface for intracellular digestion, as in most clams, brachiopods, and starfish. In arachnids and many snails they function in both intracellular and extra-

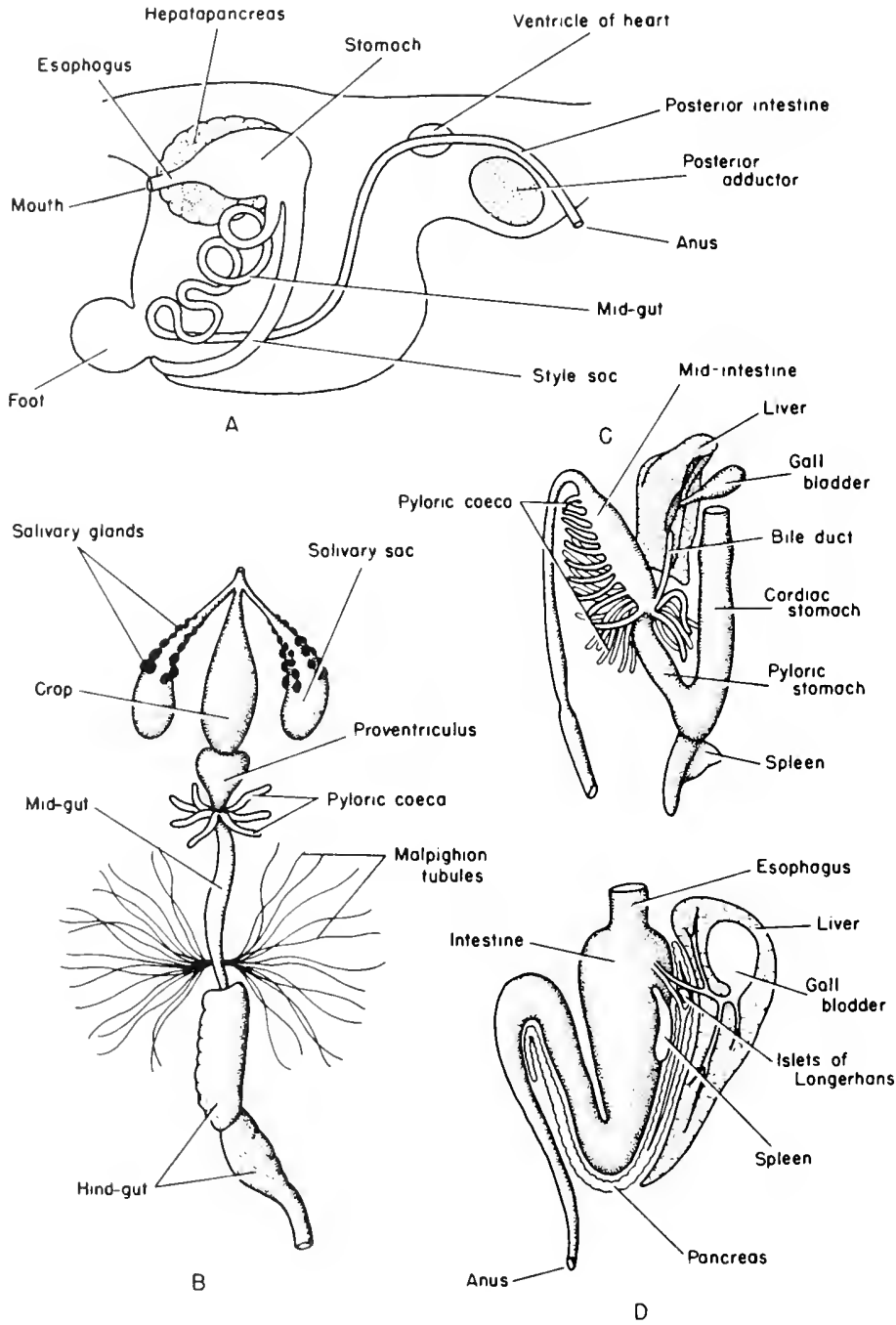

Fig. 29. Diagrammatic representation of digestive tract in various animals: $A$, in clam, Mya; B, in cockroach, Periplaneta; $C$, in lake trout, Trutta lacastris; D, in Fundulus, a stonachless fish. (Modified drawings: A from Yonge, ${ }^{144} \mathrm{~B}$ from Schlottke, ${ }^{1, \pi} \mathrm{C}$ and D from Vonk. ${ }^{171}$ )

cellular digestion. In crustaceans such as crayfish they liberate digestive enzymes and are also areas of active absorption. Finally, in such animals as the cephalopods and the chordates, the diverticula are concerned almost exclusively with secretion. 
Absorption. The fourth region is one of absorption. This region usually overlaps the preceding one, and in many animals, such as the clams, starfishes, and crustaceans, it is indistinguishable from the region of storage and internal trituration. In the inajority of species, including the sea urchins, sea cucumbers, insects, annelids, and vertebrates, the region of absorption is more or less distinct.

Conduction and Feces Formation. The fifth and final region is one of conduction and of formation of feces. This region is very conspicuous in such animals as the terrestrial insects and vertebrates, in which an important function is the absorption of water from the fecal material, as in the insect hindgut and the vertebrate colon. The whole intestine of crustaceans and of most molluscs comprises this region.

Movement of Food Mass. Food material is propelled along the digestive tract at a rate which permits effective digestion and absorption of the utilizable constituents of the food. This propulsion is by one or both of two methods: (1) ciliary activity, and (2) muscular activity. Ciliary activity appears to be the exclusive means of movement through the digestive tract in such organisms as the ectoprocts, entoprocts, and pelecypod molluscs. Muscular activity is involved to a greater or less extent in food propulsion in other animals. Sometimes movement of somatic musculature associated with locomotion serves as an effective force in the propulsion, supplementing the action of poorly developed visceral musculature, for example in worms and holothurians. IVell developed visceral musculature operates effectively by itself in arthropods, vertebrates, and probably cephalopods. In addition to propulsion the muscular activity serves to triturate further and to $\mathrm{mix}$ the food with digestive fluids. There may be peristaltic waves, standing waves, and pendular movement. The action of emulsifying agents on fats is aided by the mechanical action of the digestive organs.

The coordination of the mechanical activity of the digestive tract has been studied significantly only in the vertebrates, in which both endocrine and nervous factors operate. Epinephrine inhibits gastrointestinal activity, whereas acetylcholine increases intestinal motility, antagonized by atropine. Sympathetic nerves increase and parasympathetic nerves decrease motility of the gut (see Chapter 16).

The time of retention of food in the digestive tract is inversely related to the efficiency of the digestive processes. The rate of movement of food is dependent on temperature in a fashion parallel to the effect of temperature on the digestive reactions. A blood meal may be retained by a leech for several months. ${ }^{60}$ One blood meal lasts the bug Rhodnius for one instar, whereas food passes rapidly through caterpillars and grasshoppers. In fish several days may be.required for digestion (three days in dogfish ${ }^{166}$ ). In warm-blooded animals digestion is more rapid. About 70 per cent of an indigestible meal is passed in feces in 24 hours in a rabbit, in 72 hours in man, in 18 to 20 hours in a chicken. ${ }^{47}$ Food remains in the human stomach 3 to $41 / 2$ hours, carbohydrates remaining a shorter time than proteins; in cattle, food remains in the rumen an average of 61 hours, in the omasum 8 hours, and in the abomasum 3 hours. ${ }^{47}$ A delicate balance exists between the kind of food, the rate of digestion, and the rate of propulsion along the digestive tract; part of the regulation may be local and much of it reflex in nature. 


\section{SITE OF DIGESTION : INTRACELLULAR OR EXTRACELLULAR}

In evolution, digestion inside the cells which use the food products metabolically preceded extracellular digestion. In most animals there is both intracellular and extracellular digestion, but certain animals have specialized in one or the other.

Unicellular organisms show much variation in the site of action of digestive enzymes. Autotrophic organisms (some bacteria, yeasts, and flagellates) absorb only simple organic molecules, building them into complex ones. Some bacteria secrete digestive enzymes, such as proteases which liquefy gelatin, absorb the products of intermediate size, and complete the digestion intracellularly. Extracellular digestion by Protozoa occurs occasionally. The amoeboid cell Vampyrella extrudes a cellulose-digesting enzyme onto a filament of Spirogyra, digests a hole in the cell wall, then consumes the cell contents in 1 to 5 minutes. ${ }^{106}$ It is probable that Protozoa which are intracellular parasites, such as the malaria organism, destroy portions of cell membranes before penetrating the host cell. There are few convincing observations of enzymes freed into a culture medium by Protozoa. Glancoma is said to secrete an enzyme which attacks peptone in the medium. ${ }^{108}$ Most Protozoa take in food and digest it in food vacuoles or in the cytoplasm. A food vacuole is extracellular in the sense that the contents are separated from the cytoplasm by a membrane which has very low permeability to many substances such as organic acids. Enzymes are secreted into the food vacuole; moreover, enzymes contained within live ingested food may act autolytically in the vacuole. Protozoa may take in indigestible food and later pass the particles out; certain Protozoa may show some selection of food. ${ }^{123}$

Among metazoa, intracellular digestion appears to be correlated with sluggish habits, or with feeding on small particles. Extracellular digestion is associated with the appearance of mechanisms for internal trituration and the chemical breakdown of small particles or molecular aggregates. Extracellular digestion appears to have evolved first as an adaptation for dealing with large particles, to reduce them to such size that intracellular digestion could take over; it is a sort of chemical trituration. The evolutionary trend has been toward replacement of intracellular by extracellular digestion. Excellent accounts of the site of digestion are given by Yonge. ${ }^{190}$

In sponges digestion is exclusively intracellular. Among triclad turbellarians digestion is predominantly intracellular; the large cells lining the gut are phagocytic and contain digestive vacuoles in which the breakdown of meat can be followed. ${ }^{* 9,}$, is In the rhabdocoel worms digestion occurs in the copious lumen of the gut. In some of the Acoela, the cells lining the gut form a syncytium in which digestion occurs; this is possibly an intermediate condition between extracellular and intracellular digestion. ${ }^{175}$ Intracellular digestion is also predominant among coelenterates, although their gastrovascular cavity contains a protease which permits extracellular breakdown of protein (Metridium,26, $46 \mathrm{H} 1 \mathrm{Hdr} \mathrm{a}^{22}$ ). All other digestion is carried on inside the cells of the cndoderm (Hydra' $)$, of the mesenteric filaments (Metridium, $\left.26,{ }^{2} i\right)$, and of the lining of the manubrium and stomach of medusae. ${ }^{79}$ Similarly in madrepore corals the mesenteric filaments secrete proteases into the coelenteron, then absorb polypepticles and complete the digestion in vacuoles. ${ }^{18 x}$ In carnivorous coelenterates some digestion probably also occurs by 
autolysis before the food is absorbed into the endodermal cells. In Tardigrada and to a less extent in Rotifera and Brachiopoda, intracellular digestion predominates. ${ }^{190}$

Molluses show wide variety in the relative importance of extracellular and intracellular digestion. ${ }^{190}$ Lamellibranchs digest protein, fat, and disaccharides inside the cells of the digestive diverticula and in the wandering amoebocytes which are abundant throughout the gut. ${ }^{1 \text { tht }}$ The amoebocytes migrate into the wall and lumen of the gut in clams, charge their bodies with food particles, digest them, and make their way again into the tissue spaces of the animal. Starch is digested extracellularly by the action of amylase from the crystalline style. Among gastropods the prosobranchs resemble lamellibranchs in having only one extracellular enzyme, amylase, whereas carnivorous species digest also protein and sugars extracellularly. ${ }^{190}$ In snails such as Helix, lacking amoebocytes in the gut, the hepatopancreas or foregut diverticula secrete several enzymes and the initial phases of digestion are extracellular; the final phases occur inside the cells of the diverticula. Intracellular digestion is prominent among nudibranchs. ${ }^{12 t}$

Concurrent intracellular and exiracellular digestion also occurs in echinoderms which secrete enzymes upon food while it is still outside the body. In addition, amoebocytes are abundant in the lumen of the echinoderm digestive system, and cells of the pyloric and intestinal cacca are phagocytic. The arachnoid Limulus carries out preliminary digestion of its prey extracellularly but completes digestion in the primitive way in the cells of the digestive diverticula.

Digestion is predominantly extracellular among the Annelida, Crustacea, Insecta, Cephalopoda, and Chordata. ${ }^{190}$ Digestion is extracellular in all classes of vertebrates, but many organs such as liver, spleen, and kidney contain intracellular enzymes which are capable of hydrolizing foods. Vertebrate cathepsins are intracellular proteolytic enzymes. Inside the cells these enzymes may function in protein synthesis. It is possible that terminal digestion, even in mammals, may be intracellular. From observations on intestinal loops in dogs the secreted enzymes are evidently insufficient to account for the breakdown of peptone, sucrose, and lactose; hence it is possible that these substances may be digested inside the mucosal cells where peptidases and sucrase are found. ${ }^{31,1 \times 3}$ Similarly in the silkworm, Bombyx, starch digestion is extracellular, and subsequent sugar breakdown takes place inside the gut cells. ${ }^{154}$

The animal groups with extracellular digestion are the most active and by many standards the most successful of animals. They have mechanisms for pulverizing their food and can consume, digest, and eliminate residues more rapidly than do animals with intracellular digestion. Further, the surface required for extracellular digestion is much less extensive, compared with the ramified chambers of digestive diverticula in molluses and flatworms. Although extracellular digestion has evolved several times, it has not always developed simultaneously for different foods in the same animal.

\section{HYDROGEN ION CONCENTRATION OF DIGESTIVE FLUIDS}

There are two general patterns of secretion and locus of action of the extracellular digestive enzymes. Among most invertebrate animals with extracellular digestion the various enzymes are secreted together into one chamber where most digestion occurs. A sequence of chambers into which specific 


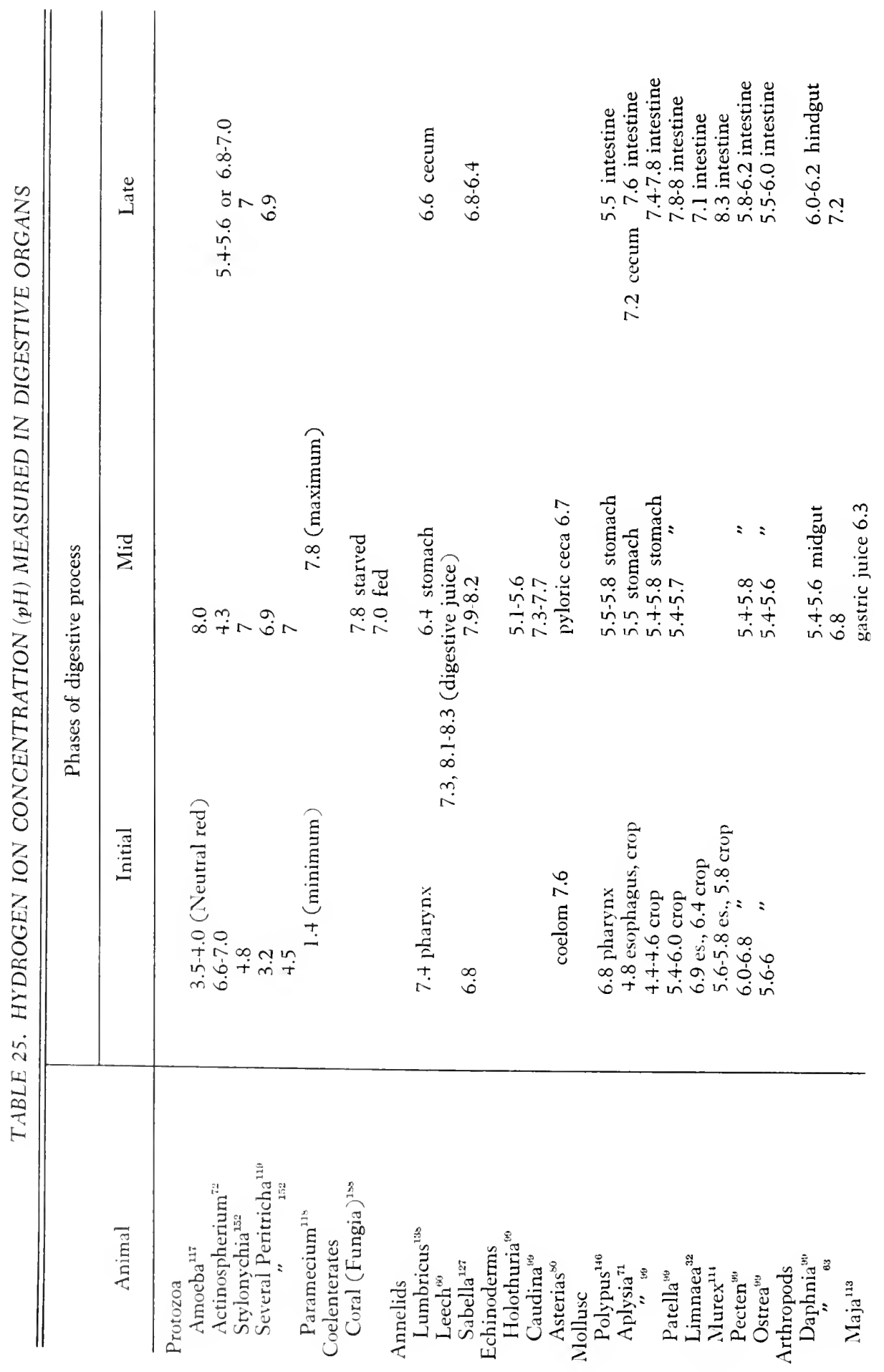




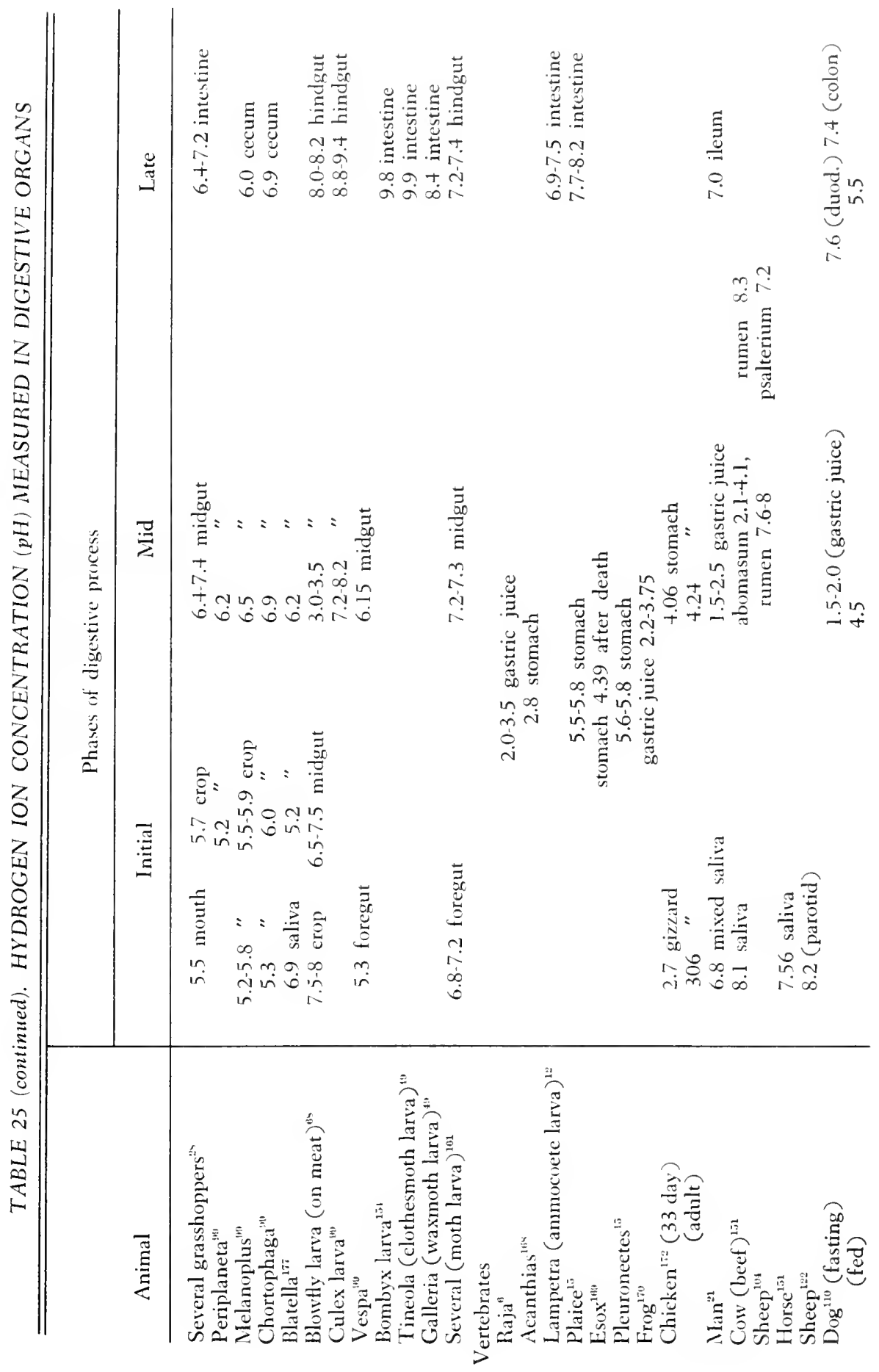


enzymes are secreted characterizes vertebrates. Mammals show a definite sequence of secretion in both protein and carbohydrate digestion; fish show sequential secretion for protein only.

During the course of digestion, food is subjected to successive changes in the medium. This is true even in an intracellular digestive cycle in which, as in Protozoa, the food vacuole may change in acidity, water content, osmotic concentration, and enzyme content. In extracellular digestion, the various glands secrete a variety of acids, salts, and other substances besides enzymes. In most animals the hydrogen ion concentration of the digestion mixture changes during the course of digestion or during passage along the alimentary tract. Specific enzymes function only over a limited $\mathrm{pH}$ range and are inactivated by extremes of acidity or alkalinity. However, the $\mathrm{pH}$ optimum of an enzyme is not necessarily the same as the $\mathrm{pH}$ in the digestive organ in which the enzyme normally functions.

Table 25 gives some selected values of the $\mathrm{pH}$ of digestive fluid or of the contents of different digestive chambers in representatives of several animal groups. A more detailed table is given by Kruger. ${ }^{93}$ Food in a digestive organ affects the acidity, and the acidity of secreted fluids differs in resting and in actively digesting states. A general pattern of acidity changes is indicated, however, in Table 25.

In mammals food passes through a series of changes in $\mathrm{pH}$. The mouth is nearly neutral in reaction. In man under basal conditions parotid saliva has an average $\mathrm{pH}$ of 5.8 , and mandibular (mixed) saliva a $\mathrm{pH}$ of 6.4 , although the $\mathrm{pH}$ may rise as much as two units in response to chemical and other stimuli. ${ }^{150 \mathrm{a}}$ The saliva of other mammals is reported to be alkaline, although methods of collection have not always excluded loss of $\mathrm{CO}_{2}$ to the air. Saliva of the cow is more alkaline $(\mathrm{pH} 8.8)$ than the regurgitate from the rumen $\left(\mathrm{pH} \mathrm{7.9)}{ }^{34}\right.$ The copious liquid and the mucin content of mammalian saliva make it a good food lubricant. Man secretes 0.5 to 1 liter of saliva per day, and animals on dry diets sccrete much more; a horse may secrete 40 liters, and a cow 60 liters daily; ${ }^{104}$ each parotid gland of a sheep secretes 930 to $1840 \mathrm{ml} . / 24 \mathrm{hr}$. ${ }^{12: 2}$

In the mammalian stomach the parietal cells secrete $\mathrm{HCl}$ to the extent of 0.4 to 0.5 per cent ( $\mathrm{pH} \mathrm{0.91).} \mathrm{When} \mathrm{diluted} \mathrm{with} \mathrm{food,} \mathrm{the} \mathrm{contents} \mathrm{of} \mathrm{the}$ stomach range from $\mathrm{pH} 1.5$ to $2.5 \mathrm{in}$ normal humans. Part of the acid is free, more is combined. The acid of the stomach is bactericidal, kills cells of food which may be alive when swallowed, and may aid in decalcification of food. ${ }^{14}$ Stomach acid also activates the principal gastric enzyme, pepsin, and provides an optimal $\mathrm{pH}$ for its action. Monotremes lack stomach glands. In ruminants the storage portions of the stomach are alkaline, and only the true stomach or abomasum is acid. In young chickens the gizzard is more acid than it is in adult hens. The stomach contents in frogs are nearly as acid as those in mammals. In the bony fish which have a stomach the gastric $\mathrm{pH}$ is not as low as it is in mammals, but elasmobranchs have extremely acid gastric contents. Stomachless fish swallow food directly into the alkaline intestine (Fig. 29, D).

Among invertebrate animals the relation of acidity of the digestive tract to diet and to the presence or absence of food is even more marked than it is among vertebrates. In general the anterior regions of the digestive system are nearly neutral or weakly acid (Table 25). In molluscs and some insects they are acid, but in the earthworm the region of the pharynx is alkaline. The crop 
of a blowfly larva feeding on meat is alkaline. The mid-gut and stomach of many animals is a region of acid secretion ( $\mathrm{pH} 5-6$ ), although in no group of invertebrates is the mid-gut as acid as it is in mammals. Farther back in the digestive tube the pH rises, sometimes to alkaline levels (Table 25). In many molluscs the entire gut is acid, the stomach most so, and the gastric acidity is controlled in part by the crystalline style. The mid-gut fluid is alkaline in Trichoptera, Lepidoptera, and some Coleoptera, : although the mid-region of the mid-gut of blowfly larvae may have a $\mathrm{pH}$ of 3.2. Digestive fluids of the silkworm are as alkaline as $\mathrm{pH}$ I 9.5. ${ }^{1 . i 4}$ In corals living in sea water of $\mathrm{pH}$ I 8.2 the coelenteric fluid had a $\mathrm{pH} I$ in starvation of 7.78 ; two hours after feeding the $\mathrm{pH}$ was $7.05 .1 \mathrm{sm}$

In free-living Protozoa the $\mathrm{pH}$ of food vacuoles can be followed if food or fluid medium is stained with appropriate indicators. Greenwood in 1894 pointed out that in Carchesitm the food vacuole is first acid and then alkaline, and that most digestion occurs in the alkaline phase. This sequence holds for numerous ciliates; ${ }^{3.5}$ Paramecium reaches the highest acidity. ${ }^{12 n}$ Mast followed individual stained food vacuoles in Amoeba protens, 117 scveral peritrichs, ${ }^{119}$ and Paramecium caudatum, $11 \mathrm{~s}$ and in each the food vacuole becomes acid soon after it is formed; while it is acid its volume decreases. After a few minutes a food vacuole becomes more alkaline and enlarges rapidly. It is postulated that products of hydrolysis during the acid phase increase the osmotic concentration in the vacuole; then alkaline fluid enters from the cytoplasm. The source of the acid is not entirely certain, but there is good evidence that the vacuolar membrane does not permit loss of acid during the initial period. Some of the acid-perhaps all of it in Amoeba, where the lowest $\mathrm{pH}$ is 3.5 to 4.0 -comes from food organisms contained in the vacuole, and death of prey occurs in the acid phase. In the ciliates-such as Paramecium, where the pH may go as low as 1.4-some acid is probably secreted by the pharynx. As the vacuole swells in the alkaline phase the $\mathrm{pH}$ may rise above that of the cytoplasm; enzymatic digestion then proceeds.

The digestive secretions of most animals are acid in the early and alkaline in the late phases of digestion. This general pattern holds whether the enzymes are secreted together into one chamber or follow in sequence. Variations may be correlated with food habits and enzyme complement of different animals.

\section{STIMULATION OF SECRETION OF DIGESTIVE FLUIDS}

When a digestive organ does not contain food, there is usually a low-level continuous secretion of fluid which may have a lubricating function, and which differs in composition from the secretion during active digestion. Also in animals which feed most of the time, as in pelecypods by continuous ciliary propulsion of food or as in continually browsing scavengers, the flow of digestive fluid must be continuous. However, in most animals feeding is periodic, and secretion is elicited to correspond to the presence of food. Gland cells can be stimulated by chemical agents in tissue fluids which bathe them, by direct mechanical stimulation, by nerve impulses, or by combination of all three methods of stimulation.

The control of digestive secretion has been examined very extensively in mammals, particularly in the dog, and hardly at all in lower animals, and the mammalian mechanisms are well described in medical physiology texts. In 
mammals the submaxillary and sublingual glands receive parasympathetic stimulation by the chorda tympani nerve, and the parotid gland by the auriculo-temporal nerve; in addition the salivary glands are innervated from the cervical sympathetics. Both parasympathetic and sympathetic nerves stimulate secretion, although the nature of the fluid secreted differs according to the nerves stimulated and the glands concerned. Normally there is some continuous secretion, but salivary secretion can be stimulated reflexly by the presence of food or other substances in the mouth or by stimulation of other sense organs which are associated with food by conditioning. Control of salivary secretion is, therefore, entirely nervous. The stimulating action of parasympathetic nerves is prevented by atropine, which probably blocks the action of acetylcholine liberated at the nerve endings. Pilocarpine stimulates the glands to continuous secretion.

Secretion of gastric juice is stimulated by the vagus nerve and is antagonized by atropine. The sympathetic nerve to the stomach may antagonize the vagus and may elicit a slight mucous secretion. Reflex stimulation of gastric secretion can be elicited by odor, taste, or sight of food; in addition, mechanical stimulation of the gastric mucosa elicits secretion. More important is the fact that certain foods, particularly meat, initiate the liberation into the blood of a substance which stimulates the gastric glands. This hormone, gastrin, has the properties of and may be identical with histamine. Stimulation of the vagus nerve causes limited secretion of pancreatic juice, particularly rich in enzymes. When the acid chyme from the stomach enters the duodenum, a hormone is liberated from the intestinal mucosa; this substance, sccretin, carried in the blood, strongly stimulates pancreatic secretion. Nervous influence upon intestinal gland secretion is slight; the sympathetic are said to be inhibitory, and the vagus is said to cause secretion in the absence of influence of the sympathetics. The intestinal glands respond readily to mechanical stimulation of the mucosa. A hormone from the intestinal wall, enterocrinin, apparently stimulates the intestinal glands to sccrete. Secretion of bile can be elicited by impulses in the vagus nerve; certain foods and secretin also stimulate secretion of bile from the liver. In some mammals bile is retained and concentrated in a gall bladder; liberation of bile from the gall bladder is elicited by a hormone, cholecystokinin, which is produced by the duodenal mucosa under the influence of the acid food mass. The three hormones from the intestinal mucosa, secretin, enterocrinin, and cholecystokinin, differ in their effects, although all three have many chemical properties in common (see Chapter 22). A progressive series from nervous to hormonal control is evident. Salivary secretion is entirely nervous; gastric and pancreatic secretion are ncrvous and hormonal; and secretion of intestinal fluids and that of bile are predominantly hormonal.

The control of secretion in non-mammalian vertebrates is known only for the stomach and pancreas. Secretion of gastric juice by the proventriculus of bircls, as by the mammalian stomach, is stimulated by the vagus nerve, also by secretin. "s ln the frog, mechanical stimulation by food or by objects such as beads in the stomach causes gastric secretion by a reflex via the sympathetic nervous system. on Similar sympathetic stimulation occurs if various sensory nerves are stimulated in frogs with the central nervous system intact. The vagus and vagomimetic drugs are ineffective. There is uncertainty whether any 
hormones influence normal gastrie secretion in amphibians, although histamine and mammalian gastrin are effective when injected into frogs. is, wh

In elasmobranch lish, as in amphibians, the vagus nerve does not affect gastric secretion." $4: 3$ In fasting skates and dogfish there is a continuous secretion of small amounts of gastric juice; this secretion is inhibited by adrenalin or by sympathetic nerve impulses but is not altered by stimulating or cutting the vagus. If the spinal cord is pithed, removing simpathetic inhihition, a copious "paralytic" secretion appears, possibly due to vasodilatation. "In perfusion experiments both acetylcholine and histamine increase gastric secretion in elasmobranchs. ${ }^{16 i .}$ Gastric secretion is under nervous control of the vagus in mammals and birds; in amphibians the sympathetics stimulate, and in elasmobranchs the sympathetics inhibit gastric secretion. No fixed pattern exists among vertebrates, and a search for gastric hormones in lower vertebrates is desirable. Secretin has been obtained from the small intestine of birds, turtles, frogs, salamanders, $9: 3$ and teleosts and elasmobranchs. ${ }^{16}$

In the earthworm Lumbricus, stimulation of segmental nerves elicits the secretion by the anterior intestine of a protease which clots milk. ${ }^{12-i}$ In no other invertebrate animal is there evidence for either nervous or hormonal secretion of digestive fluids. I lowerer, histological studies have demonstrated waves of secretory activity in the buccal glands and digestive diverticula of snails, ${ }^{4}$ and in the digestive diverticula of the crayfish. "it $\operatorname{In}$ the snail Helix the cytological changes include elaboration of granules that may be stored or may form vacuoles which are liberated into the lumen of the gland. In starved snails the buccal glands show a small amount of asinchronous activity; the digestre diverticula show rhythms in which all the cells work synchronously. When the snails are fed, both glands become rhythmic and show a faster sequence. Feeding is the stimulus to the buccal gland, and food in the crop stimulates the digestive diverticula. In Astacus the rhythm of the digestive diverticula is paced by formation of new gland cells by mitotic division. The rhythm is slow during starvation but is accelerated markedly when food enters the stomach. In several coelenterates, focd in the coelenteron or in contact with mesenteric filaments stimulates digestive secretion. 1:0 Food, then, is the ultimate stimulus for secretion of digestive fluids in all animals. The manner in which the stimulus is transmitted is known well only for mammals.

\section{DIGESTION OF PROTEINS}

Proteins during digestion are broken down to their component amino acids which may then be absorbed and built into specific new proteins. Protein digestion is essentially a series of hydrolyses of peptide linkages:

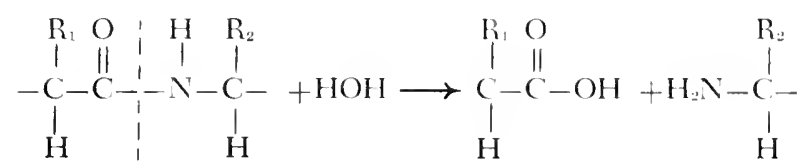

Classification of Proteases. The classification of proteolytic enzymes has changed radically during the past fifteen years with better understanding of the composition of proteins. In the following account, some features both of 
the older schemes and of the modern classification of Bergman is are combined in a general plan based on that of Baldwin." Proteases differ according to the substrates attacked, the optimum $\mathrm{pH}$, and the effects of activating and inhibiting agents.

Endopeptidases. These enzimes attack central peptide bonds, and may also act on smaller molecules than proteins, for example peptones.

CARBONYL-PROTEINASES.

(1) Pepsin. Pepsin attacks a peptide linkage between a dicarboxylic and an aromatic amino acid if the second carbonyl of the dicarboxylic acid is free and if there is no free amino group near the peptide link. That is, pepsin requires amino acids such as phenylalanine or tyrosine in the backbone of the molecule, attached as in the following example:

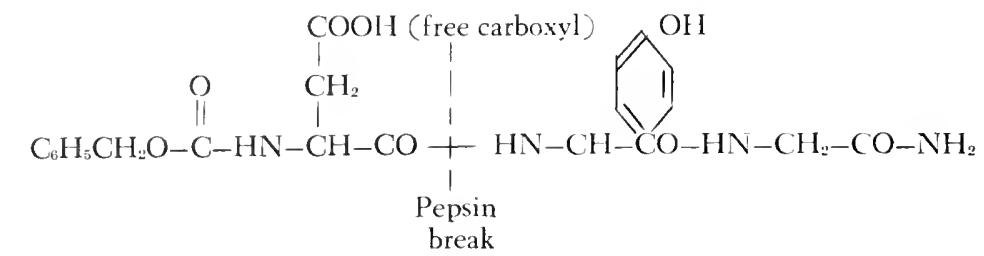

carbobenzoxy

glutamyl

tyrosy]

glycine amide

(dicarboxylic)

(aromatic)

Pepsin is usually characterized physiologically by working in an acid medium and by being inactivated in an alkaline one. According to Northrup, pepsin acts on positively charged proteins. Pepsin is secreted as pepsinogen which is activated autocatalytically in an acid medium $(\mathrm{HCl})$.

(2) Cathepsin I. Cathepsin I. isolated from mammalian liver and spleen, resembles pepsin in the specificity of its substrate requirements. However, it acts in a meclium which is neutral or slightly acid and is activated by reducing agents such as IICN 11.2 , cysteine, and glutathione.

(3) Trypsin. Trypsin acts at a pepticle link adjacent to arginine or lysine. It may attack some other peptide links as well but is identified by use of a substrate such as the following:
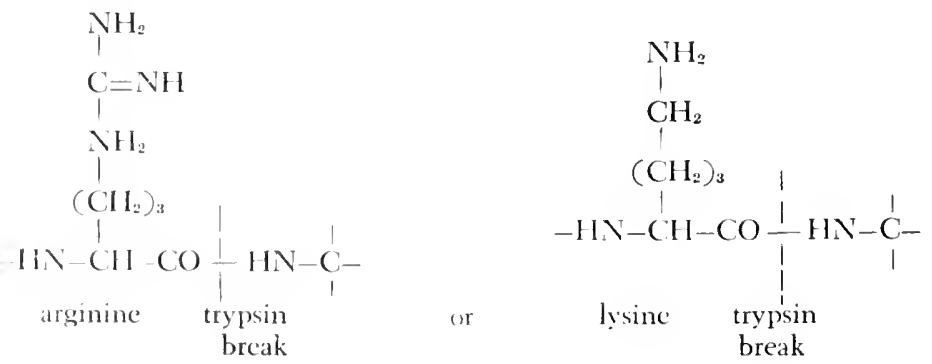

Trypsin is characterized physiologically by acting in an alkatine medium (pll 7 (t) 9), in which most proteins are negatively charged. It is secreted as trypsinogen, which is activated by some specilic enzeme (zookinase), such as the intestinal enterokinase; once started, trypsin activation may occur autocatalytically: 
(4) Cathepsin II. Cathepsin II is similar in its substrate reguirements to trypsin but acts in a weakly acid medium ( $\mathrm{pl}$ I 4.9). It is activated by I ICN, $\mathrm{H}_{2} \mathrm{~S}$, cysteine, and glutathione.

IMIDO-PROTEINASE.

(1) Chymotrypsin. Chymotrypsin is a separate pancreatic enzume from trypsin. It attacks a peptide link with an aromatic amino acid on the carboxyl side of the link.

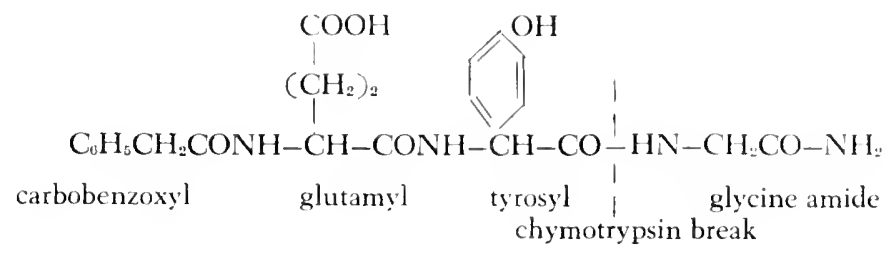

Chrymotrypsin acts in an alkaline medium, $\mathrm{pH} 7$ to 9 , and is activated by trypsin or by enterokinase in the presence of trypsin.

Exopeptidases. These enzymes attack terminal peptide links and act largely on products of proteinase action, particularly polypeptides. Their $\mathrm{pl} I$ requirements are not strict; they usually show a broad pll optimum in the neutral or weakly alkaline range.

CARBOXYPEPTIDASES. A carboxypeptidase removes a terminal amino acid with a tree carboxyl group. It is inhibited by a free amino group nearby. A favorite substrate used to test for carboxypeptidase is chloracetyltyrosine. The enzyme is formed in the pancreas of mammals.

aninopeptidases. An aminopeptidase acts on a peptide bond next to a terminal amino acid with a free amino group. It is inhibited by a free carboxy] nearby. Leucyldiglycine is often used as substrate to test for aminopeptidase. The difference in specificity of carboxypeptidases and aminopeptidases is shown in the following tripeptide substrate:

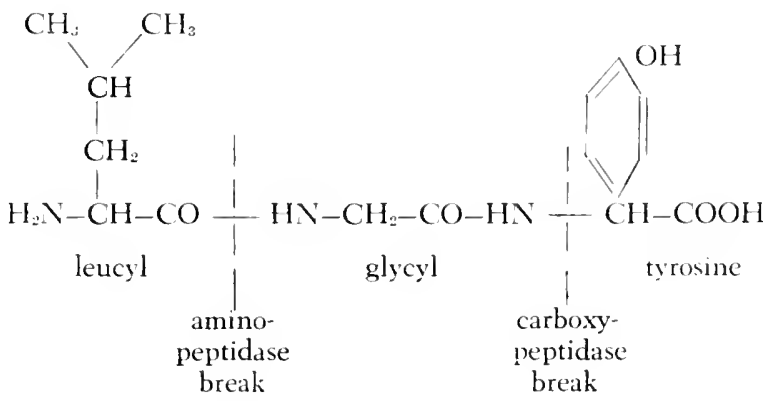

The substrate requirement of eathepsin III is similar to that of aminopeptidase.

DIPEPTIDases. There may be numerous dipeptidases. Like all other proteolytic enzymes, they act on compounds of the L-series (lace configuration). Dipeptidases break dipeptides into their component amino acids. Glycylglycin and leucylglycin are commonly used substrates. Some dipeptidases are activated by magnesium and inhibited by HCN. 
TABLE 26

\section{CHARACTERISTICS OF PROTEINASES}

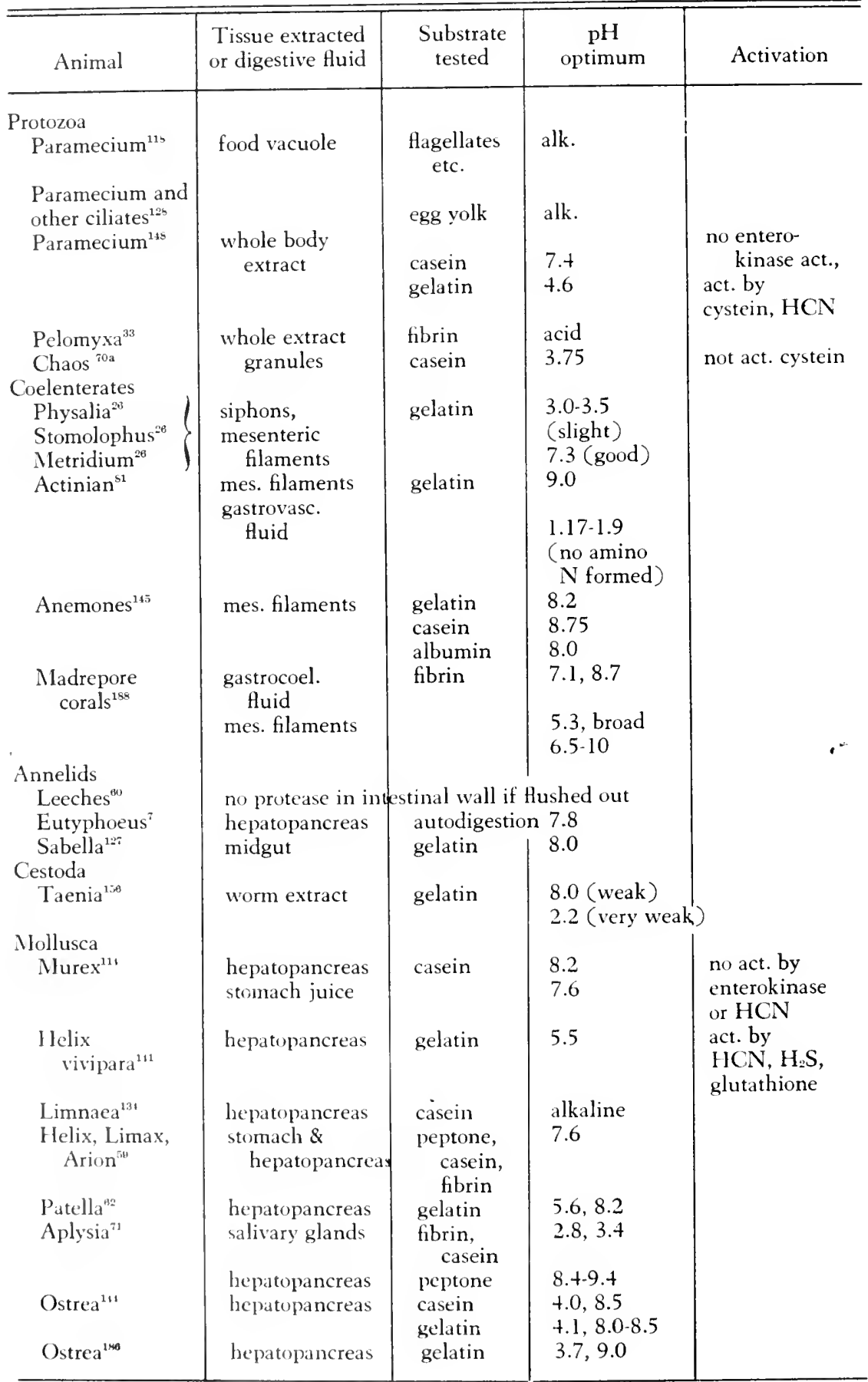


TABLE 26 (continued)

CHARACTERISTICS OF PROTEINASES

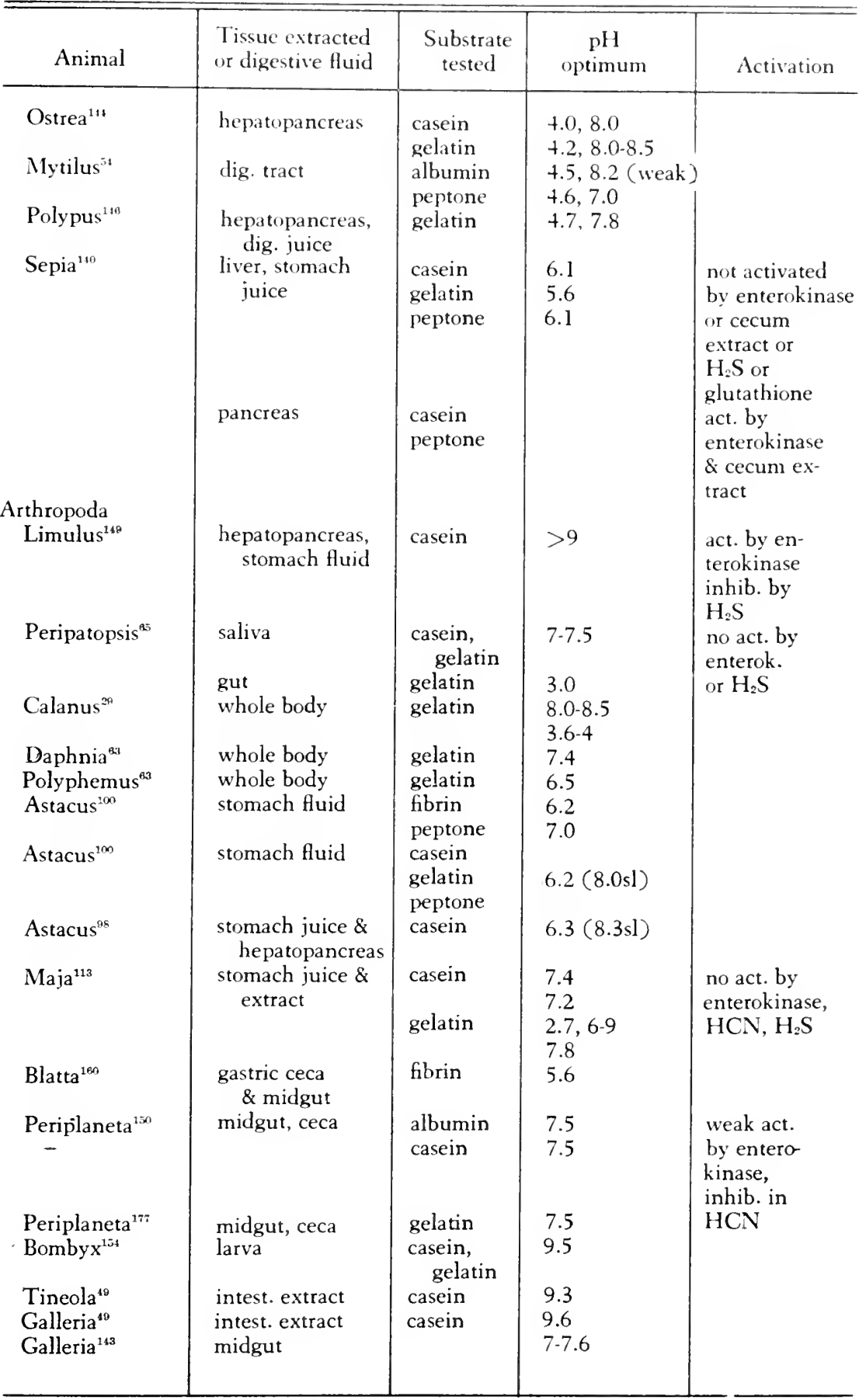


TABLE 26 (continued)

CHARACTERISTICS OF PROTEINASES

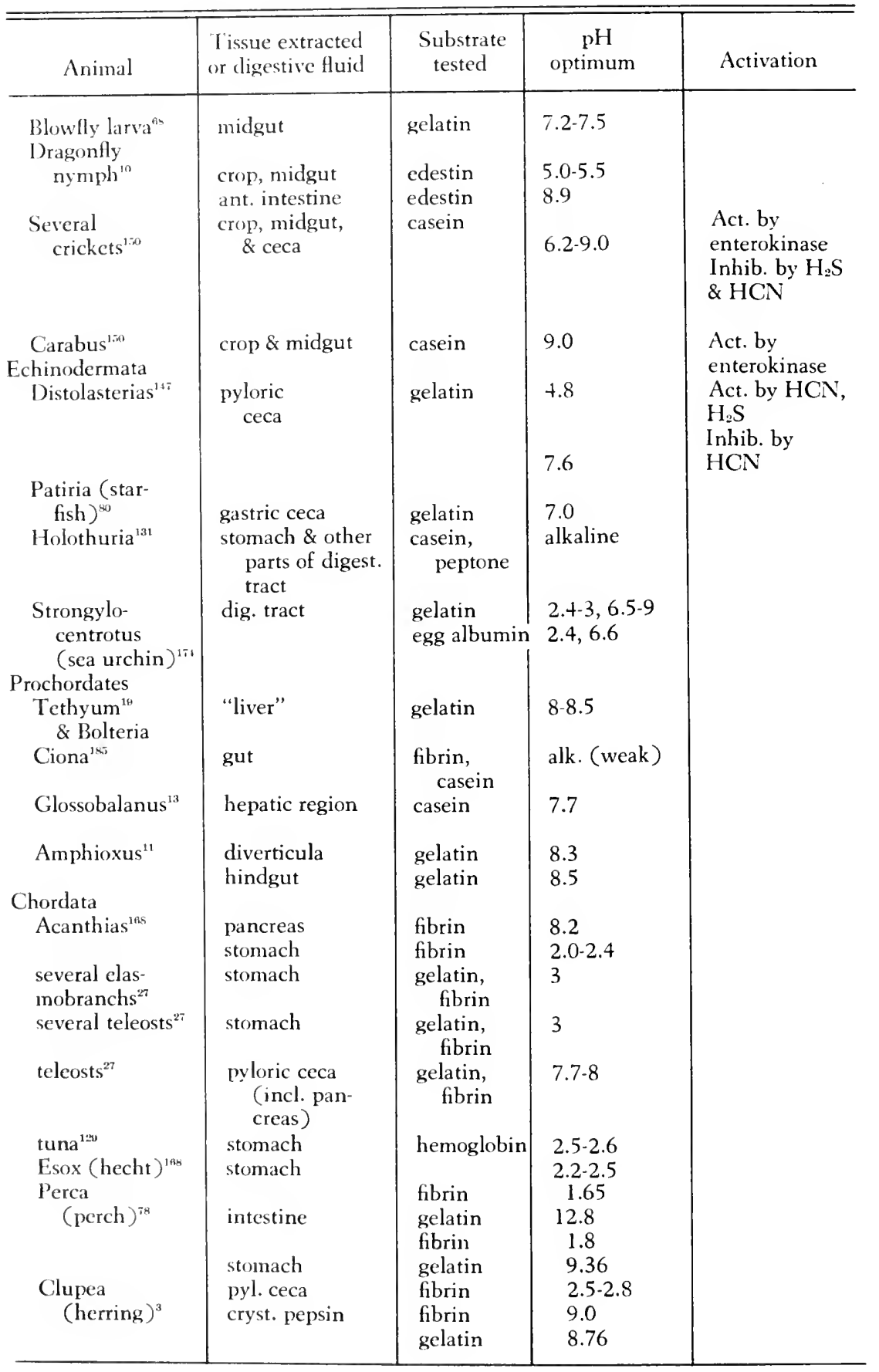


TABLE 26 (continued)

CHARACTERISTICS OF PROTEINASES

\begin{tabular}{|c|c|c|c|c|}
\hline Animal & $\begin{array}{l}\text { Tissue extracted } \\
\text { or digestive fluid }\end{array}$ & $\begin{array}{c}\text { Substrate } \\
\text { tested }\end{array}$ & $\underset{\text { optimum }}{\mathrm{pH}}$ & Activation \\
\hline $\begin{array}{l}\text { Cyprinus } \\
\quad \quad \text { (carp) } \\
\text { Plaice }^{165} \\
\text { Frog }^{16 t} \\
\text { Testudo }^{16 s} \\
\text { Pig }^{16 s} \\
\text { Mammal }\end{array}$ & $\begin{array}{l}\text { pancreas } \\
\text { stomach } \\
\text { stomach } \\
\text { pancreas } \\
\text { stomach } \\
\text { stomach } \\
\text { purified pepsin }\end{array}$ & $\begin{array}{l}\text { fibrin } \\
\text { casein } \\
\text { fibrin } \\
\text { fibrin } \\
\text { fibrin } \\
\text { fibrin } \\
\text { casein, } \\
\text { gelatin } \\
\text { hemoglobin }\end{array}$ & $\begin{array}{l}8.75 \\
1.5-2.5 \\
2.2 \\
6-8 \\
2.2 \\
1.7-2.5 \\
1.8 \\
2.2-2.4 \\
4.0 \\
8.2-8.6 \\
7-8 \\
8.6-9.2\end{array}$ & $\begin{array}{l}\text { HCN act. } \\
\text { Entero- } \\
\text { kinase act. }\end{array}$ \\
\hline
\end{tabular}

The proteolytic enzymes of tissues (cathepsins) have not been characterized except for the mammalian liver and spleen. In fact little separation of proteases has been made by substrate. Hence for practical purposes we can distinguish proteases with low $\mathrm{pH}$ optimum ( $\mathrm{pH}$ 1.5-3) as pepsins, those working in an alkaline medium ( $\mathrm{pH} 7-9$ ) as trypsins, and those with a weakly acid optimum ( $\mathrm{pH}$ 4.5-6.5) as cathepsins. This implies that there are both intracellular and extracellular cathepsins; those known from vertebrates are intracellular. The pH optimum has little meaning without a statement of the substrate, since with certain substrates the optimum is lower than with others. ${ }^{130}$

Rennin is an enzyme which clots the milk protein casein (paracasein). Some proteolytic enzymes have a rennin action. However, there are probably specific rennins, as in the stomach of infants, distinct from pepsin.

Distribution of Proteinases. Proteolytic enzymes have been detected in tissue extracts and digestive fluids of many animals. Selected data are summarized in Table 26. Several generalizations are evident from this table.

An enzyme extracted from glandular tissues is essentially the same as the enzyme from the lumen of a digestive organ into which the glands secrete. This has been shown for the hepatopancreas and stomach fluid of Limulus, ${ }^{149}$ Astacus, ${ }^{98}$ and Maja, ${ }^{111}$ and is well substantiated for pepsin and trypsin in mammals.

Cathepsin is an enzyme hydrolyzing proteins in the range of $\mathrm{pH} 4.5$ to 6.5 and activated by $\mathrm{H}_{2} \mathrm{~S}, \mathrm{HCN}$, or cysteine. It occurs in digestive fluids and digestive glands of many invertebrate animals. This type of protease has been demonstrated in Paramecium, ${ }^{14 *}$ several snails, ${ }^{1+1}$ the cephalopod Polypus (Fig. 31), ${ }^{146}$ and in several echinoderms, especially Distolasterias (Fig. 30). ${ }^{147}$ Often two pH optima occur, one in weak acid for a cathepsin and the other in the alkaline range for a trypsin (Figs. 30 and 31). Catheptic 
digestion is indicated by $\mathrm{pH}$ optima in other animals as well (e.g. lamellibranchs). ${ }^{62}, 1^{46}$ Trypsin may be inhibited by agents which activate cathepsin. Cathepsin has not been reported for any insect except from the crop and mid-gut of dragonfly nymphs (Table 26).

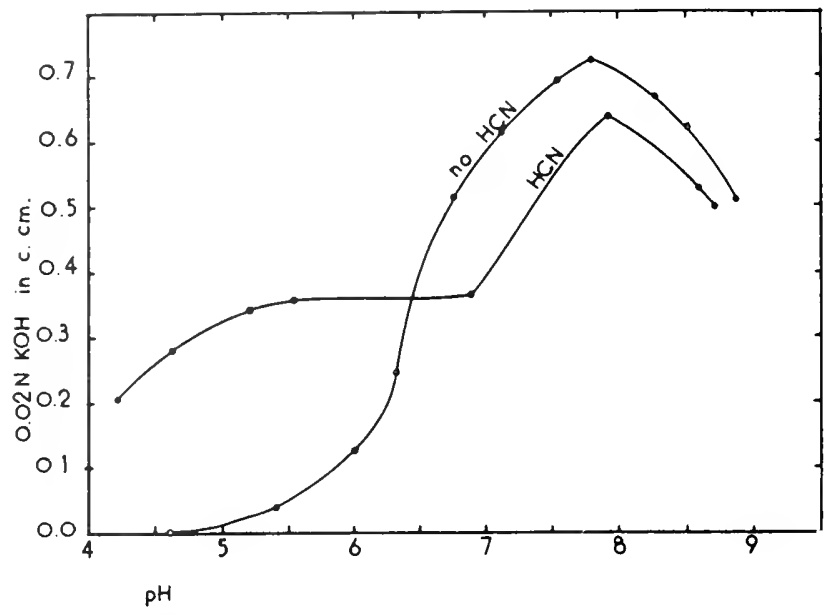

Fig. 30. Digestion of gelatin by pyloric caeca of starfish at different hydrogen ion concentrations, with and without cyanide. From Sawano. ${ }^{147}$

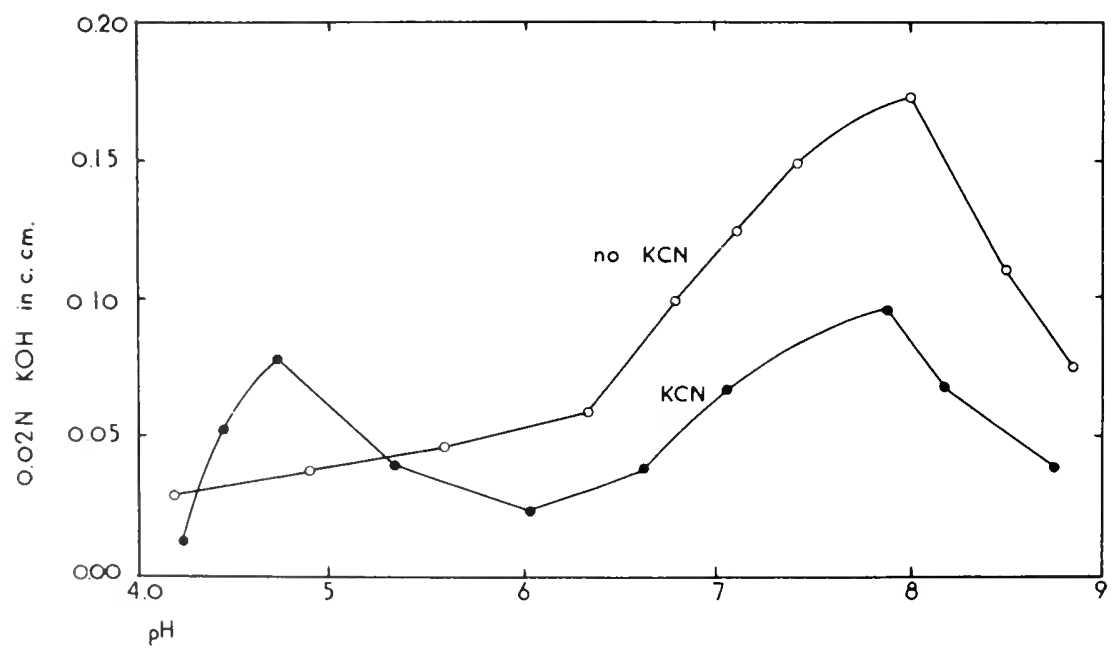

Fig. 31. Digestion of gelatin by extract of liver of cephalopod, Polypus, at different hydrogen ion concentrations, with and without cyanide. From Sawano ${ }^{106}$

It is probable that several trypsins exist. Some, such as those of several insects ${ }^{150}$ and Limulus, ${ }^{14:}$ are activated by mammalian enterokinase. Others, such as the trypsin of Paramecium, ${ }^{1+s}$ Murex, ${ }^{114}$ and Maja, ${ }^{113}$ are not activated by enterokinase. 'The few' attempts to find activating agents 
in tissues of the animals in which the trypsin is produced have not been very successful. The $\mathrm{pH}$ optimum for trypsin is not always 7.5 to 8.0. A protease acting at about $\mathrm{pH} 6.0$ and activated by enterokinase has been reported for Sepia; ${ }^{140}$ this needs confirmation. In two crickets trypsin has a broad optimum with a peak at 6.2. In the silkworm the optimum is pll 9.5, ${ }^{154}$ and in wax moth and clothes moth larvae the optimum is above 9.0. "n A similar high optimum is found for Limulus trypsin. In the elothes moth the gut fluid has a very low oxidation-reduction potential and contains a strong reducing substance without which the trypsin cannot attack the disulfide bonds of keratin. ${ }^{10 .}$ Trypsin reported from pyloric caeca of fish probably comes from attached pancreatic tissue.

The hydrogen ion concentration at which proteolytic enzymes function in an animal is not necessarily identical with the $\mathrm{pH}$ optimum of the enzymes. In Paramecium, for example, proteolytic digestion occurs in the alkaline or near neutral phase of the gastric vacuole. ${ }^{11 \times}$. ${ }^{12 \times}$ Yet the cytoplasm contains a protease with maximum activity at $\mathrm{pH} 4.6,{ }^{14 \times}$ In snails the $\mathrm{pH}$ optimum of the trypsin is much above the $\mathrm{pH}$ of the mid-gut. ${ }^{11:}$ Similar differences are found in Astacus, Limulus, and Polypus. In the fish Esox the pH of the stomach contents varies with the food; it averages 5.2 , whereas the optimum $\mathrm{pH}$ for fish pepsin is 2.2. ${ }^{16 \mathrm{~m}}$ Vonk suggests, however, that at the surface of food particles where the enzyme is acting, the $\mathrm{pH}$ may be lower than the mean $\mathrm{pH}$ of the whole food mass.

Pepsin is characteristic of vertebrates only. Reports of proteolytic action in very acid solutions by tissues of coelenterates 26,41 and Limnaea $3: 2$ are not convincing and probably do not indicate a true pepsin but rather a cathepsin. This also seems likely for proteases acting at $\mathrm{pH} 3$ to 4 as in Peripatopsis ${ }^{65}$ and in a copepod. ${ }^{29}$ The prochordates and mierophagous cyclostomes lack a stomach and have no peptic digestion. ${ }^{14}$ Beginning with the jawed carnivorous fish, elasmobranchs, and bony fish, peptic digestion occurs in the stomach. The pepsin of all vertebrates appears to be similar, ${ }^{171}$ and erystalline trypsin from the tuna ${ }^{130}$ resembles that from mammals. In several groups of teleosts the stomach is lacking (Fig. 29, D), and no pepsin is found, for example, in Fundulus. ${ }^{-}$Acid and pepsinogen are secreted by different cells, and the parietal cells (acid-secreting) may be located in different regions from the chief cells (pepsin-seereting). In the frog pepsin is secreted by the esophagus and upper stomach; hydrochloric acid is secreted by the lower (pylorie) stomach. In birds such as the chicken the glandular stomach or proventriculus lies between the crop and the grinding gizzard and secretes $\mathrm{HCl}$ and pepsinogen. The crop is somewhat acid ( $\mathrm{pH} 5.0$ ), but most peptic digestion occurs in the muscular gizzard. ${ }^{47}$ The gizzard contents are less acid in an adult ( $\mathrm{pH} 3.1$ ) than in a young chicken ( $\mathrm{pH} 2.7$ in 23 day chicken). ${ }^{172}$

It is difficult to compare the amount of proteinase from different animals. In general, proteinase is more active in carnivorous than in herbirorous animals. Proteinases are very weak in lamellibranch and herbivorous gastropod molluscs and in tunicates, ${ }^{1: 10}$ but are powerful in coelenterates, echinoderms, and cephalopods. In carnivorous inseets such as cockroaches proteinases are strong, whereas they are weak in herbivorous insects. ${ }^{150}$

Distribution of Peptidases. Exopeptidases are widely distributed (Table 27). They have a broad $\mathrm{pH}$ optimum in the low alkaline range. In mammals 
TABLE 27

CHARACTERISTICS OF EXOPEPTIDASES (POLY - AND DI-PEPTIDASES)

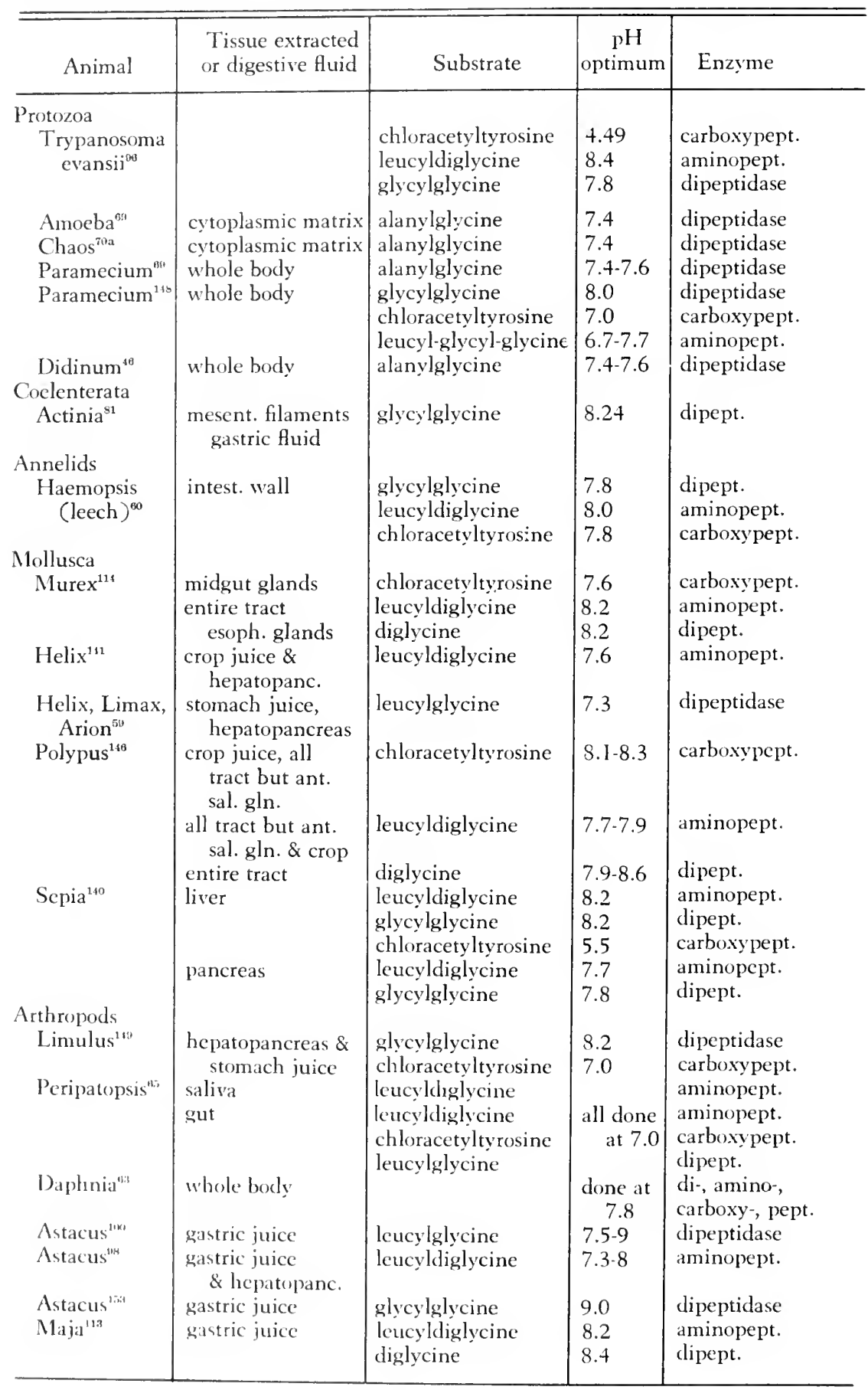


TABLE 27 (continued)

CHARACTERISTICS OF EXOPEPTIDASES (POI Y AND DI-PEPTIDASES)

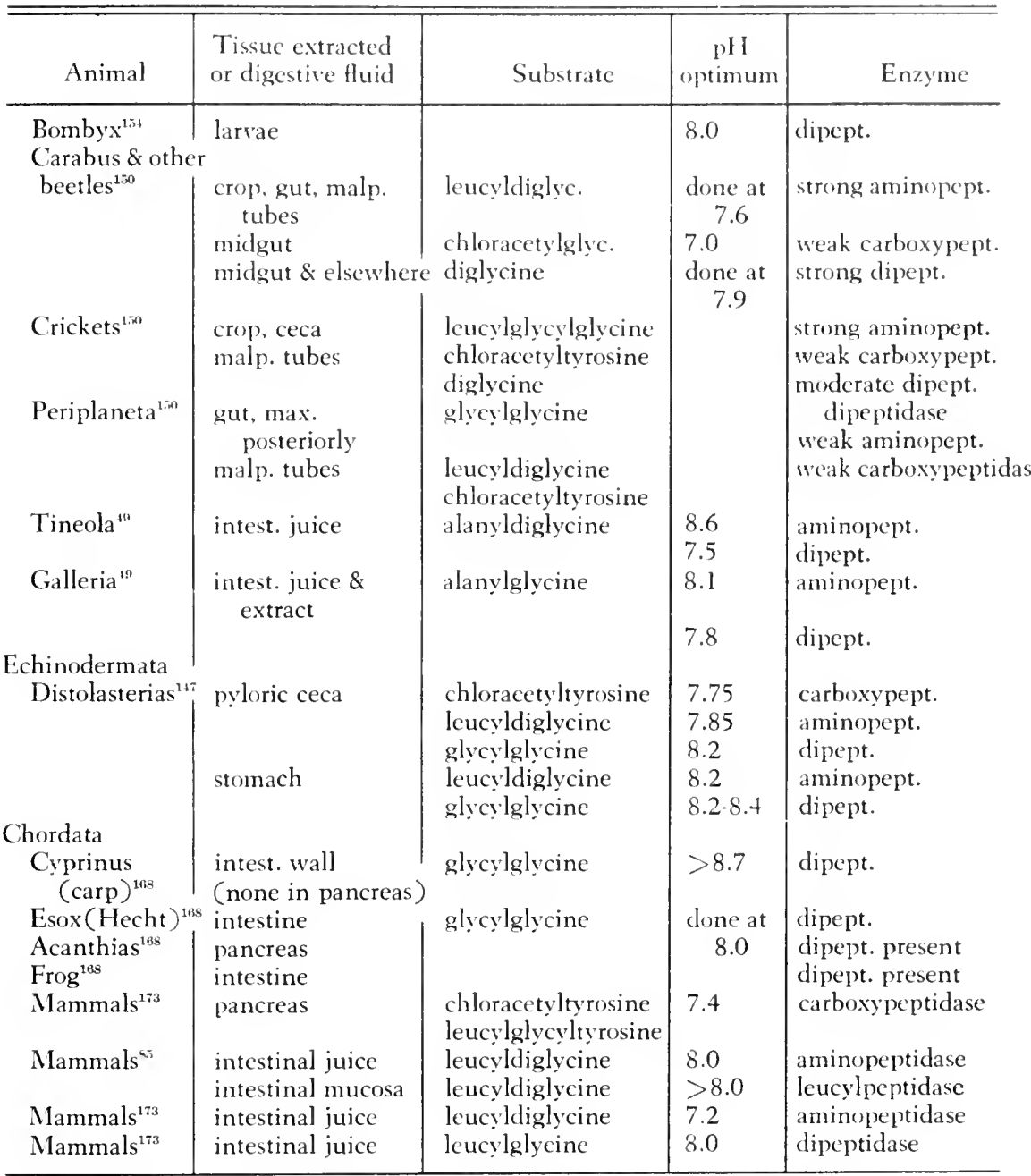

carboxypeptidase occurs along with trypsinogen in the pancreatic juice; aminopeptidase and dipeptidase together constitute the enzyme formerly called erepsin of the intestinal juice and may act intracellularly. There is evidence that mammalian intestinal mucosa elaborates an enzyme leucylpeptidase, in addition to aminopeptidase and dipeptidase. Leucylpeptidase attracks leucyldiglycine but not triglycine, and is activated by magnesium ions. ${ }^{4 t .}$ s. Carboxypeptidase occurs separately from the other peptidases in several animal groups. In Paramecinm extracts the carboxypeptidase is said to be activated by cathepsin activators ( $\mathrm{H}_{2} \mathrm{~S}, \mathrm{HCN}$, cysteine); the aminopeptidase and the dipeptidase are not. ${ }^{1+\mathrm{N}}$ In the onychophoran Peripatopsis the saliva contains a carboxypeptidase alone, and the gut in general contains the three peptidases. ${ }^{15}$ Similar separation of carboxypeptidase occurs in Murex ${ }^{114}$ and 
in the starfish. ${ }^{1+i}$ In some insects the carboxypeptidase is relatively weak, compared with the aminopeptidase and dipeptidase. ${ }^{150}$ The dipeptidase of Amoeba proteus is found in the cytoplasmic matrix. ${ }^{\text {To }}$ The dipeptidase of Didinium is similar to that of its prey Paramecimm, and the amounts are additive when Didinitum is feeding; hence the dipeptidase of this animal is probably derived directly from its food. ${ }^{4 t}$ In some trypanosomes the $\mathrm{pH}$ optimum of carboxypeptidase is lower than the optimum of other peptidases. ${ }^{96}$ The peptidases are relatively similar in all animals, and carboxypeptidases often occur separately from aminopeptidases and dipeptidases.

\section{DIGESTION OF CARBOHYDRATES}

Plants synthesize a vast array of carbohydrates. Animals can break down some of these and can modify a few of them synthetically.

Nature of Carbohydrates and Carbohydrases. Carbohydrates are hydrolized to monosaccharides, mostly hexoses (six-carbon sugars), less often pentoses, and rarely tetroses, trioses, and dioses. Hexoses can be pictured as carbonoxygen rings with side groups attached in a variety of patterns. All the natural sugars used by animals are D-sugars. However, a D-sugar may exist in two isomeric forms, of which the $\alpha$-form is more strongly dextrorotatory than the $\beta$-form. The structure of $\alpha$ - and $\beta$-glucose differs in the position of the $\mathrm{H}$ and $\mathrm{OH}$ on carbon No. $\mathrm{l}$ and is represented as follows:

$\alpha$-glucose (rotation $+111^{\circ}$ )

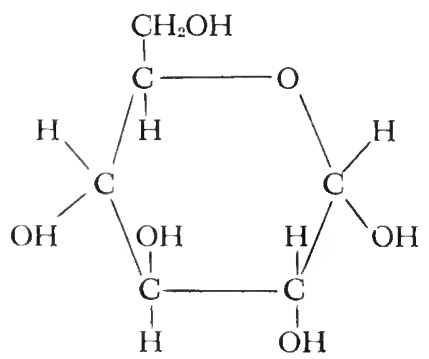

$\beta$-glucose $\left(\right.$ rotation $\left.+19^{\circ}\right)$

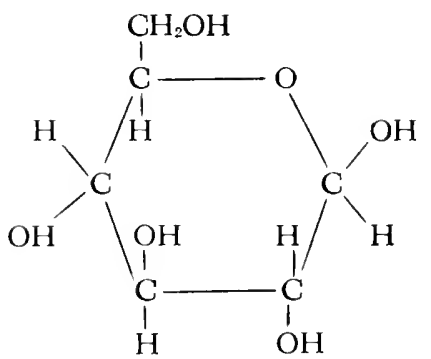

Monosaccharides are combined to form disaccharides and trisaccharides. The structure of sucrose is given as:

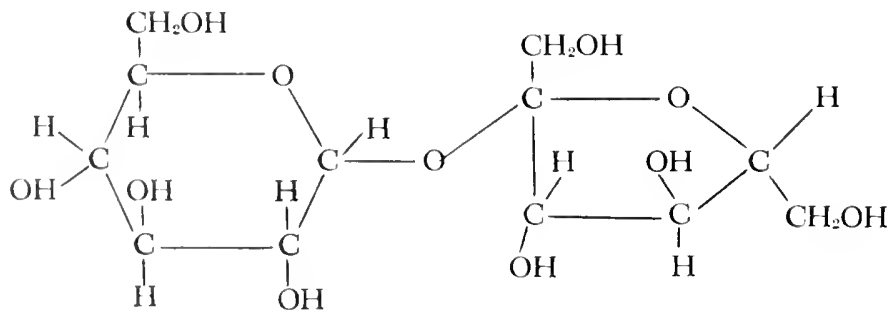


A classification of glycosides commonly used as substrates, that is, natural disaccharides and glucosides with their monosaccharide components, follows: $\alpha-y$ lucosides:

$\beta$-glucosides:

$$
\begin{aligned}
& \text { a maltose } \rightarrow 2 \alpha \text { glucose } \\
& \text { sucrose } \rightarrow \text { a-glucose }+\beta \text {-fructose } \\
& \text { a-methyl glucoside } \rightarrow \text { arglucose }+ \text { methyl alcohol }
\end{aligned}
$$

$$
\begin{aligned}
& \text { cellobiose } \rightarrow \beta \text {-glucose }+\beta \text {-glucose } \\
& \text { salicin } \rightarrow \beta \text {-olucose }+ \text { saligenin } \\
& \beta \text { gentiobiose } \rightarrow 2 \beta \text {-glucose } \\
& \beta \text { methyl glucoside } \rightarrow \beta \text {-glucose }+ \text { methyl alcohol }
\end{aligned}
$$

$\alpha$-galactosides:

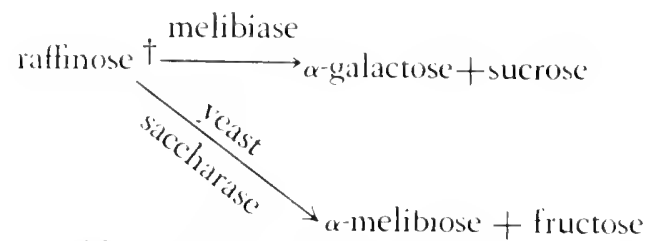

$\alpha$-melibiose $\rightarrow$ galactose + glucose

$$
\alpha \text {-methyl galactoside } \rightarrow \alpha \text {-galactose }+ \text { methỵl alcohol }
$$

$\beta$-galactosides:

$$
\beta \text {-lactose } \rightarrow \beta \text {-galactose }+\alpha \text {-glucose }
$$

$\beta$-fructosides (fructofuranosides):

$$
\beta \text {-methyl galactoside } \rightarrow \beta \text {-galactose }+ \text { methyl alcohol }
$$

$$
\begin{aligned}
& \text { sucrose }^{*} \rightarrow \beta \text {-fructose }+\alpha \text {-glucose } \\
& \text { raffinose }+\rightarrow \beta \text {-fructose }+\alpha \text {-melibiose }
\end{aligned}
$$

Polysaccharides are highly polymerized carbohydrates which occur in greatest variety in plants as stored food and as structural elements. The most important storage carbohydrate is starch and its animal homologue glycogen. Starch and glycogen are complex molecules built of $\alpha$-glucoside units together with small amounts of other compounds. Starch grains are digested by animal amylase (diastase) only if boiled or broken mechanically, or if the coating amylopectin of the starch grains is broken. In the course of digestion a series of dextrins are formed, with maltose split off at each step. Glycogen can be hydrolyzed by the same amylase that splits starch; reports of a separate glycogenase are questionable.

The most important structural polysaccharide of plants is cellulose, which makes up the bulk of plant cell walls. This is built of chains of $\beta$-glucoside units, is much less soluble than starch, and is resistant to boiling and mild acid treatment. Many microorganisms and a few metazoa have enzymes which can attack cellulose. Perhaps the reason most animals are unable to digest cellulose is that their glucosidase and amylase act only on $\alpha$-compounds. A few animals

${ }^{*}$ Sucrose can be attacked from the $\beta$-fructose or the $\alpha$-glucose end. The best known sucrase is from yeast and is a fructofuranosidase, whereas animal sucrase is probably an $\alpha$-glucosidase.

†Raffinose is a trisaccharide which can go to galactose plus sucrose by the enzyme melibiase or to melibiose + fructose by yeast saccharase. There is lack of agreement as to whether animals attack raffinose with an $\alpha$-galactosidase or wirh a $\beta$-fructofuranosidase. 
appear able to synthesize cellulose; the test (coat) of several tunicates (Phallusia and Molgnila) contains a structural carbohydrate which appears to be identical with plant cellulose. ${ }^{66 ;}, 1.57$

Another polysaccharide which is synthesized by some animals and plants is chitin. This is a nitrogen-containing compound which is split by chitinase to acetyl-glucosamine. Very few animals possess chitinase. Several other plant polysaccharides are occasionally digested by animals. These polysaccharides include lichenin, pectin, xylan, inulin, and others.

Carbohydrases illustrate well the various types of specificity of enzymes. ${ }^{15: 4}$ (1) Absolute or type specificity is shown by enzymes attacking different members of a class. Amylase attacks starch, dextrin, or glycogen. Disaccharidases are effective only on small carbohydrate polymers. Glucosidases are probably distinct from galactosidases. (2) Stereochemical specificity is shown by the fact that an enzyme such as maltase attacks many $\alpha$-glucosides, whereas $\beta$-glucosides are attacked only by $\beta$-glucosidases. (3) Relative specificity or specificity within a class is shown by the different rates at which a given $\alpha$ - or $\beta$-glucosidase attacks various substrates within the class specified.

The best known carbohydrases are from plants and especially yeasts, in which these enzymes occur in great variety. Animal biochemists have been concerned more with the enzymes of carbohydrate utilization (intermediary mctabolism) than with those of carbohydrate hydrolysis (digestive metab. olism). In gencral, carbohydrases are not restricted to a very narrow $\mathrm{pH}$ range, but for the most part they function near neutrality.

Distribution of Polysaccharidases. The majority of mammals have a pancreatic amylase which attacks starch and glycogen, and intestinal enzymes acting on maltose, sucrose, and lactose. Probably the same enzyme acts on maltose and sucrose, but at different rates. Most investigations of carbohydrases in digestive fluids and tissue extracts of other animals have been limited to the few substrates which are used by man. However, the number of carbohydrases of many invertebrates, although not so great as the number of carbohydrases of some bacteria, yeasts, and fungi, greatly exceeds the number of enzymes found in mammals. Data on those animals which have been studied with respect to various carbohydrate substrates are given in Tables 28 and 29.

Starch Digestion. Animal amylases are $\alpha$-glucosidases. The amylolytic activity of three protozoans diminishes in the following order: Amoeba $>$ Paramecium $>$ Frontonia. 6! An extract of Pelomyxa yiclds an active amylase. ${ }^{3: 3}$ However, Mast states that Amoeba proteus cannot digest starch if it has not ingested live food recently, and that the amoeba takes over the amylase from such organisms as Chilomonas, on which it preys. In coelenterates amylase acts intracellularly in mesenteric filaments and may be optimal in a slightly acid medium. ${ }^{16 .}$ In annelids an extracellular amylase occurs in stomach and intestine. Sabella digests starch and glycogen best at pH 6.8. 1:27 Starch is said not to be digested by rhabdocoel flatworms. ${ }^{17.5}$ In madrepore corals which contain srmbiotic zooxanthellae in the mesenteric filaments, there is in the symbionts a weak amylase with pl I optimum at 4.75 ; the coral may in addition have a glycogenase with a pll optimum at 6.5. ${ }^{\text {ss }}$

I lerbivorous molluscs have an active amylase, in either the hepatopancreas or style, or in both. Amylase is found in salivary glands of Aplysia ${ }^{71}$ and Limnaea. $3:$ Nlso the amoebocytes of the digestive tract of the oyster can 
digest starch and glycogen ( $\mathrm{pH}$ optımum of extract of the amoebocrtes is 7.0), and several sugars. ${ }^{162}$ All lamellibranchs and most herbivorous but no carnivorous gastropods have a crystalline style which is an allouminoid mass saturated with amylase, sometimes also containing a weah maltase, wh This style is secreted by the style sac (Fig. 29, A); it may be dissolved or reformed in less than an hour, 126 and is lost from clams kept in air. The style is pushed forward by cilia in the style sac, and the tip wears against a gastric shield which protects the gastric mucosa ${ }^{12}$; or aids liberation of amylase. 14, 1n; 'The pll optimum of style amylase is: for (Ostrea 5.96.2, is Pecten 6.2, "62 Mactra and Mya 6.5-7.0. ${ }^{1112}$ The $\mathrm{pH}$ curve for amylase of the style of Mytilus shows a broad optimal range with peaks at 5.9 and 6.9 on starch and at 6.24 on glycogen. ${ }^{5 t}$ The amylase of the style of Ostrea acts best at 6.0, whereas that of the digestive diverticula is optimal at $\mathrm{pH} 6.4$. 1.4 In carnivorous gastropods the style is absent and a weak amylase is present in the hepatopancreas.

Amylase is present in gastric fluid and extracts of the hepatopancreas of Crustacea. $2 \%,[n, 1: n$, It is reported from the saliva of Peripatopsis. is found in secretions of the mid-gut, chiefly from the caleca, of many insects, and in salivary secretions of some such as the cockroach Blatta. ${ }^{16 i 0}$ The tsetse-fly Glossina has as its only carbohydrase a weak amylase in the mid-gut, whereas a non-blood-sucking Hy Callipliora has active amylase in both salivary and mid-gut glands. ${ }^{17 \times}$ Amylase has been found in the mid-gut of honeybees by some but not by all of those who have looked for it. Starch solution is not drunk in quantity by bees unless combined with sweet syrup; boiled soluble starch can be hydrolyzed in the digestive tract, ${ }^{10 T}$ and bees certainly utilize the starch in pollen. The amylase of most insects acts in a slightly acid medium, but an amylase in the silkworm has an optimal $\mathrm{pH}$ of 9.6 or higher. ${ }^{155}$

The caecum of Amphioxus secretes an amylase with $\mathrm{pH}$ optimum of 7.0. 4.. All vertebrates have an active amylase in the pancreatic secretion. Hepatic amylase occurs in the bile of chickens, shcep, and cattle. "i2 Among mammals, a salivary amylase is found in man, apes, elephant, and pig. Its occurrence is doubtful or slight in rodents, dogs. and several ungulates. ${ }^{103}$ Salivary amylase is generally present in birds and a trace occurs in frogs. 171 Human salivary amylase has a $\mathrm{pH}$ optimum of 6.2, contrasted with an optimum of 6.8-7.1 for pancreatic amylase.

It is probable that all animal amylases are similar, despite slight differences in $\mathrm{pH}$ optima. All appear to be $\alpha$-amylases. The animal amylases are activated by $\mathrm{Cl}$ ions (in $\mathrm{NaCl}$ ) and lose their activity on dialysis. 1s; Iydrolytic digestion of glycogen is brought about by amylase and there is probably no specific glycogenase.

Cellulose Digestion. Enzymes which attack cellulose have evolved independently in several diverse groups of organisms. Digestion of cellulose by putrefactive bacteria and fungi is well know'n, but cellulase has not been studied in any free-living Protozoa, except Vampyrella. However, several groups of parasitic Protozoa can digest cellulose.

Cellulases have been found in digestive extracts of earthworms, some snails (Helix and Linmaea), and the wood-boring bivalve Teredo. Extensive studies of the kinetics of Helix cellulase were made by Karrer, ${ }^{x} i$ and the difference from plant cellulase was pointed out. ${ }^{1: 1}$ Wood contains various carbohy. drates, cellulose, hemicellulose, lignin, starch, and sugars, and wood-dwelling 
animals utilize different compounds. In the digestive tract of Teredo wood loses 80 per cent of its cellulose and 15 to 50 per cent of its hemicellulose. ${ }^{30,44}$ The nutrition of wood-boring insects is summarized by Wigglesworth, ${ }^{179}$ and Mansour and Mansour-Bek, ${ }^{111}$ and data for beetles are given by Mansour and Mansour-Bek, ${ }^{112}$ Ripper, ${ }^{1: 3}$ and Parkin, 13:2 and data for termites by Cleveland $3 \times, 41$ and Hungate. ${ }^{5 i}$ Wood insects can be grouped into several categories: (1) In some-Lyctidae, or powder post beetles, and Bostrychidae-only the stored food, starch and sugar in wood is used, and the cellulose is unchanged in the digestive tract; these insects contain no microorganisms which can act on cellulose. (2) The bark beetles (Scolytidae) consume the cell contents of the wood and can also digest hemicelluloses. Other specific compounds are digested by other insects, as xylan by the Cerambycid larva Phymatodes. 111,112 (3) Numerous Cerambycidae and Anobiidae do digest cellulose. These insects, which either lack organisms that can digest cellulose or else have them at early stages and then lose such organisms, have true cellulase in their own digestive secretions and can attack heartwood, which is low in sugar. (4) Several groups of insects harbor in their digestive tracts bacteria and flagellate Protozoa which can digest cellulose. Larvae of lamellicorn beetles have in the proctodaeal chamber of the digestive tube bacteria which can split cellulose, and flagellates which cannot do so. In three families of termites and in the wood-feeding cockroach Cryptocercus the hind-gut is laden with bacteria and Alagellates. ${ }^{3 \times} .39 .40$ A cellulase can be extracted from the hind-gut contents; the insects can live well on pure cellulose. The protozoan fauna can be removed if the host animals are incubated, starved, subjected to high oxygen tension or high temperature, and after defaunation (but while still possessing bacteria) the termites or roaches die in a few days or weeks on a diet of cellulose. Such defaunated termites can survive if fed glucose or cellulose predigested by fungus, or if the Protozoa are restored. The flagellates produce glucose from cellulose in mass culture and live on purified cellulose as the exclusive carbohydrate. ${ }^{163}$ Particles of wood are engulfed and digested inside the protozoan. The flagellates also produce lower fatty acids, particularly acetic acid; their metabolism is anaerobic. ${ }^{77}$ In one family of termites bacteria are the organisms acting on cellulose. There has been some question whether the termites derive their nourishment from the bodies of the flagellates, ${ }^{111}$ from the glucose formed, ${ }^{40}$ or from the lower fatty acids. ${ }^{17}$ Current evidence favors the fatty acids as being the most important. Termites survive but do not mature and reproduce on cellulose without nitrogen; wood contains some nitrogen, and in decaying logs fungi transport soil nitrogen to the wood from which it is taken by the termites. ${ }^{75}$ Proteolytic enzymes of termites have not been investigated; however, numerous wood-boring beetles are known to have proteases. ${ }^{132}$

In ruminant animals symbiotic microorganisms also digest cellulose. The rumen usually contains a mixture of bacteria, yeast, and ciliated Protozoa. Cultures of the Protozoa showed that Entodinium does not digest cellulose, whereas several Diplodinia do digest it. ${ }^{76}$ However, the action of these Protozoa is small, compared with that of the bacteria, and in numerous experiments, ruminants (lambs and goats) have been freed of the Protozoa by starvation and injections of copper sulfate into the paunch. Such defaunated animals shows no nutritional deficiency. ${ }^{117} 121$ Digestion of cellulose is car. 
ried out br the bacteria of the rumen, and the ciliates are commensals rather than symbionts. ":- The backeria proeluce some sugar and large amounts of rolatile fatts aciels, particularly acetic and propionic. in At the peak of digestion the rumen of a sheep maty contain 6 to 9.7 gm. per eent of fitty acid, compared with $0.269 \mathrm{gm}$. per ecent in the elry food; these products of bacterial action can be absorbed from the rumen, as shown by blood analyses. ${ }^{11.5}$ They are also absorbed in lower regions of the digestive tract. In adelition, any organisms, bacteria or Protozoa, which pass into the aloomasum or true stomach are instantly hilled by the acid medium and are then digested by the enzymes

TABII 2S. DIGESTION OF POI YSACCHARIDES

\begin{tabular}{|c|c|c|c|c|c|c|c|c|c|}
\hline Animal & starch & Gly- & $\begin{array}{l}\text { Cellu- } \\
\text { lose }\end{array}$ & $\begin{array}{c}\text { Hemi- } \\
\text { cellulose }\end{array}$ & Chitin & Pecin & Lichenin & Xylan & Inulin \\
\hline 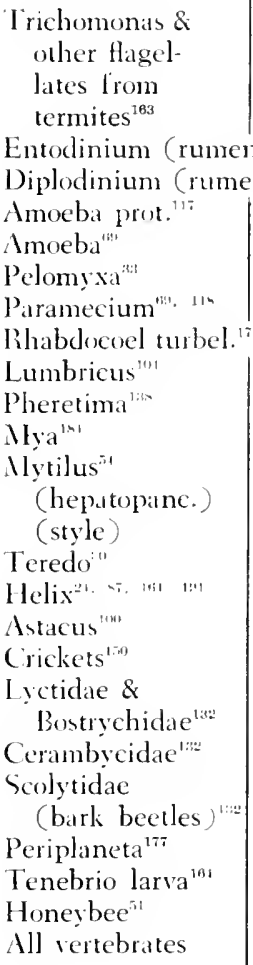 & $\begin{array}{l}\text { (n) } \\
+ \\
+ \\
+ \\
+ \\
- \\
+ \\
+ \\
+ \\
+ \\
+ \\
+ \\
+ \\
+ \\
+ \\
+ \\
+ \\
+ \\
+ \\
+ \\
+ \\
+ \\
+ \\
+ \\
+\end{array}$ & $\begin{array}{l}+ \\
+ \\
+ \\
+ \\
+\end{array}$ & $\begin{array}{r}4+ \\
\\
1+ \\
+ \\
+ \\
1+ \\
- \\
-\end{array}$ & . & - & - & $\begin{array}{l}+ \\
+ \\
-\end{array}$ & + & $\begin{array}{r}\mathrm{sl}+ \\
+ \\
+\end{array}$ \\
\hline
\end{tabular}

of the host animal." In nonruminants, such as the horse, the caecum contains a wealth of bacteria which break down cellulose; absorption from the caccum seems not to have been studied. Even in man some hemicellulose ${ }^{i 3}$ and pectin ":1 are digested by organisms of the large intestine, but it is assumed, although not proved, that the products are not absorbed.

The rare occurrence of cellulases among animals may be correlated with the rarity of $\beta$-glucosidases. As shown in Tables 28 and 29 , each animal which has a cellulase probably has also a $\beta$-glucosidase, and animals which digest 
starch can also digest $\alpha$-glucosides. It is likely that the stereochemical specificity of the enzrme which attacks the polysaccharide is similar to that of the enzyme which attacks the sugir. No vertebrate has evolved a cellulase of its own, and none has a $\beta$-glucosidase. Certainly the discovery of a way to introduce and maintain cellulose digestion in the human digestive tract would go far to circumvent the situation postulated by Malthus.

Other polysaccharides than starch, glycogen, and cellulose are not much used as animal foods. In its carbohydrases the snail Helix is the most versatile animal of those which have been studied (Table 28). Some early reports of widespread digestion of inulin, pectin, and chitin require authentication and are not included in Table 28. It would be interesting to know if all animals which digest inulin also have a $\beta$-fructosidase. Lichenin is digested by many invertebrates-sponges, earthworms, crayfish, tunicates, ${ }^{, 3}$ and many insects, 1.50. ${ }^{164}$ but probably not by any vertebrates.

Distribution of Glycosidases. Disaccharide and trisaccharide sugars must be broken down to their component hexoses before they can be absorbed. The number of animal enzymes is limited, compared with the number present in plants; these enzymes show stereochemical specificity and it is probable that several sugars of one type are digested by the same enzyme. Table 29 shows the distribution of glicosidases, as indicated by the sugars digested or utilized by those animals in which a series has been tested. Maltase ( $\alpha$-glucosidase) is more generally distributed than any other carbohydrase, in combination with amylase or separately. Sometimes a weak maltase occurs with the amylase, as in human saliva or in the crystalline style, and a stronger maltase elsewhere as in the human intestine or in the molluscan diverticula. Maltase, like other hexosidases, has a broad pH optimum, usually weakly acid, pH 6 to 7 . The pll optimum for the maltase of several molluses and crustaceans is lower than for vertebrate maltase. In the silkworm the pll optimum for intestinal maltase is 6.8 , whereas the $\mathrm{pl} I$ of the intestinal contents is very alkaline, $\mathrm{pH}$ I 9.5; hence it is probahle that starch is digested to maltose in the lumen (amylase optimum pl I 9.5), and that digestion by maltase is completed inside the epithelial cells. ${ }^{5.5}$ Mammals have a strong intestinal and an extremely weak pancreatic maltase, whereas in fish and frogs there is a strong pancreatic maltase; reptiles have more active intestinal maltase. ${ }^{182}$

Animal sucrase (saccharase) is an $\alpha$-glucosidase; yeast sucrase (invertase) is a $\beta$-fructosidase. Maltase activity and sucrase activity go together, and the pll optima are identical or closely similar. There may be differences in the rates at which the two substrates are attacked. Separate enzymes attacking maltose and sucrose have not been isolated from any animal, and it is entirely possible that the same enzyme acts on both sugars. ${ }^{159}$ Existence of such activity is rendered more probable by the fact that when one $\alpha$-glucoside is digested by a tissue extract or digestive fluid, other $\alpha$-glucosides are also attacked (Table 29). The few exceptions noted were reported in older observations (c.g., on pulmonates, ${ }^{1: 1}$ on Chrysops, ${ }^{174}$ and on Apis ${ }^{167}$ ), and these should be reexamined. An conzome of the ex-glucosidase class is probably present in all animals.

Other sugals are not so miversally digested. Lactose is digested by a $\beta$ galactosidase in the mammalian intestine but is said not to be digested in the intestinc of turtle or (arp. "on Lactose is not digested by some crustaceans 
Feeding and Digestion

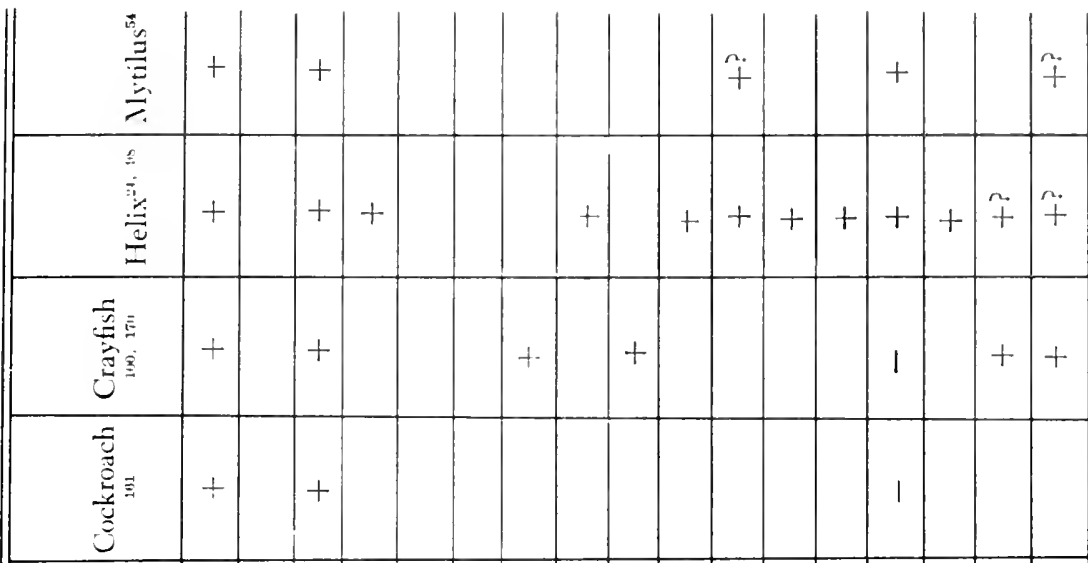

(1)

范

$\stackrel{\bar{*}}{3}++$

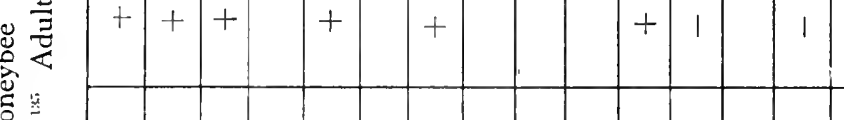

奇

离

$\underset{3}{\stackrel{0}{5}}++$

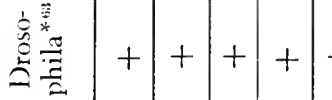

$++1$

จ่

竞突

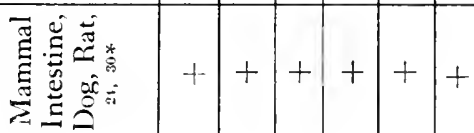

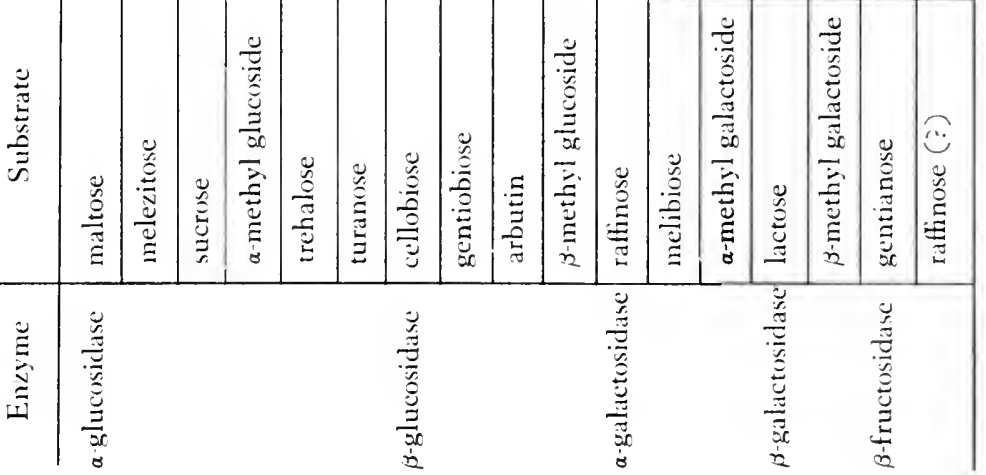

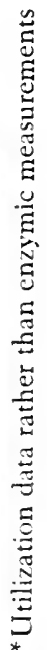


and insects (Table 29). It is not clear whether raffinose is digested in animals by an $\alpha$-galactosidase or by a $\beta$-fructosidase, although observations on blowflies ${ }^{55}$ indicate the enzyme is an $\alpha$-galactosidase. This sugar is digested by some molluscs.

Table 29 shows that the sugar-digesting enzymes of various animals are not the same and that, except for maltose and sucrose (probably all $\alpha$-glucosides), no group of sugars is used by all animals. Present limited data indicate that disaccharidases occur by stereochemical classes and that the utilization of sugars can be accounted for by relatively few enzymes in any one group of animals. For example, the utilization of eight out of twelve sugars by blowfly adults can be accounted for by postulating an $\alpha$-glucosidase and an $\alpha$-galactosidase but no $\beta$-glucosidase or $\beta$-galactosidase. ... In mammals only an $\alpha$-glucosidase and a $\beta$-galactosidase are used.

Sugar digestion may change with age. Honeybee larvae can use lactose,20 adults cannot. ${ }^{13 .}$ Sucrase first appears in the pharyngeal gland of the worker bee when it starts foraging, and it then increases very greatly. "t Larvae of numerous moths and butterflies have sucrase in addition to a lipase and protease in the mid-gut, whereas those adults which suck nectar have salivary and mid-gut sucrase but no other digestive enzymes, while nonfeeding moths have no digestive enzymes whatever. ${ }^{15}, 173$ Sugar digestion is weak in carnivorous animals. For example, the blood-sucking tsetse-fly Chrysops has no salivary carbohydrases and has amylase and sucrase only in the stomach (posterior midgut), whereas the closely related non-blood-sucking Calliphora has amylase in the salivary glands and active amylase, maltase, and sucrase in both anterior and posterior mid-gut. ${ }^{17 x}$

Carbohydrates are absorbed as monosaccharides. Part of the sugars in mammals, at least, must be phosphorylated in the intestinal wall before they are transferred across the mucosa and into capillaries. Sugars then pass to the liver, where they may be converted to glycogen for storage.

\section{DIGESTION OF FATS}

Fats are hydrolyzed by the action of lipases to fatty acids and alcohols. Neutral fats consist of higher fatty acids linked to the trilhydric alcohol glycerol:

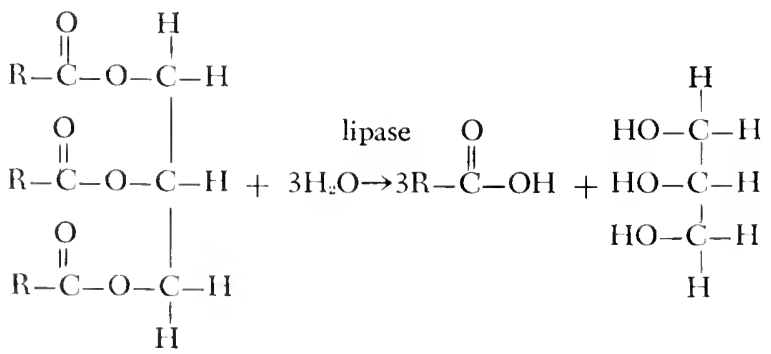

where $\mathrm{R}$ refers to the chain of fatty acid such as palmitic, stearic, or oleic acid. Neutral fat (triglyceride) can be absorbed without hydrolysis in some animals. Lipases, at least the extracts of them which have been prepared, are not highly specific as to substrate. They are generalized esterases and will attack most 
organic esters; the acid can be a short chain like acetic or a long chain like stearic, and the alcohol can be short or long chained, monohydric or polyhydric. Lipases do show some stereochemical specificity, however." In mammals waxes are not usually digested. Lipases can be tested by measuring the acid produced from such substrates as olive oil, tributyrin, or ethyl butyrate. Lipases are inhibited by aldehydes, cupric, mercuric, and ferric ions, and by certain anions, and are activated by some reducing agents. ${ }^{159}$ In vertebrates the action of pancreatic lipase is enhanced by bile salts, sodium glycocholate and sodium taurocholate. Bile salts activate lipase and also facilitate emulsification of fats, thus increasing the total area of oil-water interfaces at which lipases can act. If the bile duct is cut or occluded, fat digestion continues but is retarded.

In Protozoa fat digestion appears not to be very active. Fat from ingested Colpidium is digested by Amoeba protens. ${ }^{116}$ In Pelomyxa ingested or injected fat disappears and stained fatty acids can be seen to pass out from vacuoles into the cytoplasm, where they may be synthesized into neutral fat; hence lipases are present in this animal. ${ }^{1 \times 0}$

Fat digestion does not oceur in the gastrovascular cavity of $H y d r a, 22$ but a lipase is present in this cavity in Actinia. "1, *: H lowever, fat droplets may be absorbed by $\mathrm{Hydra}$, and lipases have been extracted from several coelenterates. (26) Rhabdocoel turbellarians digest fat rapidly. ${ }^{17.5}$

The hepatopancreas of molluses secretes or contains a lipase which is active at a pH slightly above 7.0 in $11 y a,{ }^{1 n+}$ Ostrea, ${ }^{14 t}$ and Mytilus, it and there is an active lipase in Helix $\left(\mathrm{pH}\right.$ 6.0) ${ }^{54},{ }^{15}$ and in Aplysia. ${ }^{71}$ In Sepia both the stomach juice and an extract of the liver contain a lipase with a $\mathrm{pH}$ optimum at 6.0, whereas the pancreatic lipase works best at $6.35 .{ }^{140}$ In annelids the lipases are less important than they are in molluscs. A lipase $(\mathrm{pH}$ optimum 8.2-8.4) is present in the leech Haemopsis, but not in Hirudo. ${ }^{4}$

Arthropods make good use of fats. In the crayfish a lipase is active on methylbutyrate and olive oil over a broad $\mathrm{pH}$ optimum of 5.5 to 7.0, and the stomach juice contains a fat-dispersing agent. ${ }^{100,}{ }^{170}$ Extracts of Daphnia have a weak lipase. ${ }^{63}$ In Limulus the stomach lipase is more active than that from the hepatopancreas ( $\mathrm{pH}$ optimum 7.7). 14: The lipases of the crayfish and of several molluscs (Aplysia, Helix, Octopus) resemble liver esterases of vertebrates in acting better on lower esters, such as tributyrin and methylbutyrate, than on fats such as olive oil. ${ }^{101,170}$

Several insects have been shown to have lipases, particularly in the midgut. ${ }^{173}$ The mid-gut lipase can be regurgitated into the crop of the cockroach. 1 The digestive tract of the silkworm, however, contains no lipase, or if one is present it is very weak. ${ }^{15 t}$ The wax moth larva Galleria uses beeswax as its principal food, nearly half of the wax consumed being digested and assimilated. ${ }^{4 *}$ The mid-gut contains a lipase, ${ }^{1+:}$ but larval extracts are ineffective on myricin, the ester of higher alcohols which composes much of beeswax. Bacteria in the digestive tract probably change the wax so that it can be digested. $42,4 \mathrm{~s}$

The hepatic glands of the prochordate Glossobalanus contain a lipase active on triacetin at $\mathrm{pH} 6-7 .{ }^{13}$

Digestion of fat in vertebrates is primarily intestinal. A gastric lipase has been reported with $\mathrm{pH}$ optima of 6.3 in dog and rabbit, 5.5 in man, 8.6 in 
horse, and $7.9 \mathrm{in} \mathrm{pig} \mathrm{(Waldschmidt-Leitz,} \mathrm{cited} \mathrm{by} \mathrm{Sumner} \mathrm{and} \mathrm{Sommers.}{ }^{159}$ ) This gastric lipase is not physiologically important; at least it cannot be effective at the $\mathrm{pH}$ of the stomach. Pancreatic secretion contains most of the active lipase of vertebrates. In fish it is diflicult to separate pancreatic tissue from the intestinal wall, and extracts of the intestinal wall (plaice ${ }^{15}$ ) or of intestinal slime (carp) yield a lipase which is active in a weakly alkaline medium. In the plaice Pleuronectes fat may be absorbed in the stomach after regurgitation from the intestine. ${ }^{1+.}{ }^{41}$ Pancreatic lipase is more effective in the presence of the emulsifying bile salts.

Bile salts alone can produce neutral fat droplets $2 \mu$ in diameter, but together with monoglycerides they can produce droplets less than $0.5 \mu$ in diameter, which can be absorbed directly. "si In mammals part of the fat is hydrolyzed and the fatty acid absorbed into the mucosa, whence it is carried in the blood to the liver. A large part of the fat $(2 / 3$ to $3 / 4)$ is absorbed into the lacteals and transported in lymph to the thoracic duct; it was formerly thought that all the fat was digested and soaps formed and absorbed, but it seems more probable that finally emulsified neutral fat is absorbed directly into the lacteals. ${ }^{i f}$ It appears, then, that only a small part of ingested fat is hydrolyzed in the digestive tract, and that bile salts are important for absorption of fat. The neutral fat is then broken down elsewhere in the body. Deposited fat to some extent reflects the type of fat ingested; the melting point of subcutaneous fat in hogs is lower when the pigs are fed oils of low melting point than when they are fed harder fats. Absorption of triglycerides has not been extensively examined except in mammals.

\section{CORRELATIONS OF DIGESTIVE ENZYMES WITH FOOD HABITS; CONCLUSIONS}

Numerous examples on the preceding pages have shown that the pattern of digestive enzymes differs greatly, even among closely related animals. Digestion is adapted to the dietary habits in that a given kind of animal may feed on and digest predominantly protein or carbohydrate or even one substance within these classes. The extent to which digestion is labile for individuals if one food is missing and another substituted has hardly been considered, yet this must be the way new food habits arise. It is difficult to obtain quantitative measures of the amount of activity of a given digestive enzyme in different animals, and in only a few groups, such as insects, ${ }^{150}$ has an effort been made to compare amounts of enzymes in different species. Each sort of digestive activity has usually been described as strong or weak, present or absent.

Yonge 1m, 1:n has pointed out that two trends are evident in the relation of feeding beharior and diet to digestive enzymes: (1) selection exclusively of food that can be digested, and (2) indiscriminate feeding. Animals of the first group, food specialists, are of many sorts. The carnivores, or meat eaters, have strong proteases and weak carbohydrases. Animal groups with strong proteolytic activity but in which carbohydrases are weak or absent are coelenterates, Turbetlaria, Asteroidea, cephalopod molluses, stomatopod crustaceans, and some insects. 190 Many coclenterates lack amylase; they digest protein in the gastrovascular cavity and may have very weak carbohydrases intracellularly, as in Hydra, ${ }^{\prime 2}$ in madrepore corals which do not digest sugars, ${ }^{18 \mathrm{~s}}$ 
in actinians," and also in medusae." Blowfly larvac have active proteases but weak carbohydrases in the gut, whereas the adults have good carbohydrases and weak proteases. "in Carnivorous carabid beetles have good protein digestion but weak and restricted amylase activity. 1.n Fat digestion is present in carnirores, but data are inadequate for comparison of fat digestion in different groups of animals.

Herbivores show weak proteolytic power but have active carbohyclrate digestion. Mlany marine and fresh-water molluses (except cephalopods) feed on phytoplankton or larger aquatic plants. In the pelecypods and the gastropods possessing a crystalline style, the onlvextracellular digestion is of starch, as in mussels, it and clams and ovsters. int plant food is triturated in the gizzard of Aplysia ${ }^{i 1}$ and Limnaea. ":2 A style is present in all lamellibranchs, although it is vestigial in the carnivorous septibranch bivalves. A style is absent from carnivorous gastropods and is present in only those herbivorous gastropods which feed by cilia or by slow continuous radular action. wa the tunicate Ciona has practically no protein digestion but has active carbohydrases. 1.. Nectar-feeding and leaf-eating insects may have several glandular sources of amylase and maltase. Herbivorous mammals maintain in a rumen or cecum a microflora which permits utilization of cellulose.

In addition to the carnivores and herbivores, there are many extreme specialists. Most spectacular are the wood-eaters, which digest cellulose by their own enzymes or by those of symbionts. The clothes moth is able to reduce the disulfide linkages of keratin, making possible digestion of wool protein, and the wax moth can utilize the polyglycerides of beeswax. A fly larva lives in oil wells, digesting insects which fall into the oil, and possibly using symbiotic bacteria which break down the hydrocarbons. 1izat The lamellibranchs, a predominantly herbivorous class, have made three attempts at further specialization. ${ }^{190}$ In the Teredinidae or ship-boring clams the valves are modified for boring and a cellulase is present in the digestive diverticula. In the Tridacnidae of the tropical Indo-Pacific, phagocytic amoebocytes retain zooxanthellae "which are farmed in the mantle edges." The Septibranchia, deep-water molluses, have the mechanical adaptations for meat eating in a crushing gizzard but no extracellular protease. Other specialists rely on digestive enzymes within their food. Leeches derive nourishment from a blood meal which slowly autolyzes in the gut. ${ }^{+}$Didininum uses a dipeptidase from Paramecimm. Some parasitic animals appear to have lost most of the essential digestive enzymes and rely on their hosts; for example, trypanosomes "w and parasitic Platyhelminthes, which have fewer enzymes than free-living flatworms.

The animal groups most successful in digesting food are not the specialists, however, but the omnivores with a full complement of enzymes, as some snails (Helix), cephalopods, crustaceans, many insects, and the vertebrates. Proteins, carbohydrates, and fats are all used by these animals. The various enzymes may be mixed in one chamber, as in snails and crustaceans, or may be secreted in successive parts of the alimentary apparatus, as in vertebrates. The tendency toward diversification of enzymes appears even within a limited food habit. Helix, for example, possesses a wider range of carbohydrases than any other animal that has been examined; it has weak protein digestion, but is a very successful genus. Fishes in general have a wide series of enzymes and some are said to digest poly'saccharides, such as lichenin and amydalin, which may 
not be available to higher vertebrates. ${ }^{*}$ Cellulases are restricted to some microorganisms and to a very few invertebrate animals. Pepsin and an acid stomach are distinctly vertebrate characters which have been lost bv several separate groups of bony fish, Dipnoi, some cypridonts, and others. ${ }^{1 t} \mathrm{Am}$ phioxus and the cyclostomes are microphagous and have not acquired a pepsin.

In general, intracellular digestion is associated with the absence of triturating mechanisms, and phagocytic cells, e.g. lamellibranch amoebocytes, may possess a variety of enzymes. Extracellular digestion is more economical of area of cell surfaces.

The duration of retention of food in the digestive tract is inversely proportional to the efficiency, in the sense of rapidity, of the digestive processes, and the speed of extracellular digestion in warm-blooded animals is faster than that at low temperatures. Also the amount of food eaten at a meal depends on storage facilities: a herbivorous ruminant eats much more in bulk than a carnivore. A stomachless fish (roach, for example) can ingest less than 5 per cent of its weight per meal, whereas a trout, which has a stomach, can take in 20 per cent of its body weight at one feeding. ${ }^{1+}$ The rate at which food passes through the digestive tract is correlated with the kind of food and the efficiency of the digestive enzymes.

As shown in the preceding pages, the $\mathrm{pH}$ optimum for a given enzyme is not always the same as the physiological $\mathrm{pH}$ at which it functions. Also the $\mathrm{pH}$ optimum differs slightly according to substrate. However, in a general way, the early phases of digestion are often in an acid medium and later phases occur at a higher $\mathrm{pH}$.

The enzymes of an animal may change with development, as in many butterflies, moths, blowflies, and bees, also as demonstrated in the decrease of rennin in the mammalian stomach.

Animal hydrolytic enzymes are different from corresponding plant enzymes, but given enzymes do not differ greatly from one animal group to another. Animal amylases need to be activated by $\mathrm{NaCl}$; plant amylases do not. ${ }^{171}$ Cathepsin functions extracellularly in invertebrates and intracellularly in vertebrates. There is greater difference in the distribution of carbohydrases than in that of other enzymes. Stereochemical or group specificity is marked, and the existence of a given polysaccharidase is usually associated with the presence of an enzyme for attacking corresponding sugars, as cellulase with $\beta$-glucosidase, amylase with $\alpha$-glucosidase.

Greatest success in the evolution of digestive function appears to be associated with extracellular digestion, enzyme sequences, diversity of enzymes and triturating mechanisms, together with an omnivorous diet.

\section{REFERENCES}

1. Aввотт, R. L., J. Exper. Zool. 4t:219-254 (1926). Digestive enzymes in Periplaneta.

2. Allee, W. C., and Frank, P., Physiol. Zool. 21:381-390 (1948). Drinking by fresh-water fish.

3. Almy, L. H., J. Am. Chem. Soc. 48:2136-2146 (1926). Proteolytic decomposition of herring.

4. Autrum, H., and Graetz, E., Ztschr. vergl. Physiol. $21: 429-439$ (1934). Lipases in leeches. 
5. Babkin, B. P., and Bowie, D. J., Biol. Bull. 54:254-277 (1928). Digestive function in Fundulus.

6. Babkin, B. P., Chaisson, A. F., et al., J. Biol. Board of Canada 1:251-259 (1935). Gastric secretion in elasmobranchs.

7. Bahl, K. N., Quart. J. Micr. Sci. 76:107-126 (1933). Auto-digestion, hepatopancreas of earthworm.

8. Baker, F., Ann. Appl. Biol. Lond. 30:230-239 (1943). Characteristics of fauna of ox rumen.

9. Baluwin, E., Dynamic Aspects of Biochemistry (1947). New York, Macmillan. Ch. III, pp. 56-87. Summary of hydrolytic enzymes.

10. Ballantine, R., Anat. Rec. Suppl. 78 (Abstr. No. I1), p. 44. (1940) Proteases of dragonfly nymphs.

11. Barrington, E. J. W., Philos. Tr. Roy. Soc. Lond., B. 228:269-311 (1937). Digestive system of Amphioxus.

12. Barrington, E. J. W., Proc. Roy. Soc. Lond., B. 121: 221-232 (1937). Protein digestion, ammocoete larva of Lampetra.

13. Barrington, E. J. W., Quart. J. Micr. Sci. 82:227-260 (1940). Feeding and digestion in the prochordate Glossobalanus.

14. Barrington, E. J. W., Biol. Rev. 17:1-27 (1942). Gastric digestion, lower vertebrates.

15. Bayliss, L. E., J. Mar. Biol. Ass. U. K. 20:73-91 (1935). Digestion in plaice, Pleuronectes.

16. Bayliss, W. M., and Starling, E. H., J. Physiol. 29:174-180 (1903). Secretin, vertebrates.

17. Becker, E. R., Schultz, J. A., et Al., Iowa St. Coll. J. Sci. 4:215-251 (1930). Role of stomach infusorians in ruminants.

18. Bergmann, M., Advances in Enzymology 2: $49-68$ (1942); also Bergmann, M., and Fruton, J. S., Ibid. 1:63-98 (1941). Classification and specificity of proteinases.

19. Berrill, N. J., J. Exper. Biol. 6:275-292 (1929). Digestion in ascidians.

20. Bertholf, L. M., J. Agr. Res. 35:429-452 (1927). Carbohydrate use by bee larvae.

21. Best, C. H., and Taylor, N. B., Physiological Basis of Medical Practice (1943). Baltimore, Wood. 1942 p. Ch. 38-44, pp. 695-859. Digestion.

22. Beutler, R., Ztschr. vergl. Physiol. 1:1-56 (1924). Digestion in hydra.

23. Beutler, R., Ztschr. vergl. Physial. 6:473-488 (1927). Acidity of ceolenteron, Hydra.

24. Bierny, H., C. R. Acad. Sci. 152: 465-468, 904-906 (1911), and 156: 265-267 (1913); also Biochem. Ztschr. 44: 402-414, 415-425, 426-445, 446-471 (1912). Carbohydrases of Helix.

25. Bierry, H., C. R. Soc. Biol. Paris 76:710-712 (1914). Disaccharidases of Helix.

26. Bodansky, M., and Rose, W. C., Am. J. Physiol. 62: 473-481 (1922). Digestion by coelenterates.

27. Bodansky, M., and Rose, W. C., Am. J. Physiol. 62:482-487 (1922). Digestion in elasmobranchs and teleosts.

28. Bodine, J. H., Biol. Bull. 48:79-83 (1925). Digestive tract pH, grasshoppers.

29. Bond, R. M., Biol. Bull. 67: 461-465 (1934). Digestive enzymes of copepod, Calanus.

30. Boynton, L. C., and Miller, R. C., J. Biol. Chem. 75:613-618 (1927). Cellulase in shipworm.

31. Cajori, F. A., Am. J. Physiol. 104:659-668 (1933). Intestinal enzymes in dogs.

32. Carriker, M. R., Biol. Bull. 91:88-111 (1946). Digestion in snail, Lymnaea.

33. Cartwright, S. L., Master's thesis, Univ. Ill. (1948). Amylase in Pelomyxa.

34. Chrzaszz, T., and Schechtlowna, Z., Biochem. Ztschr. 219:30-50 (1930). Salivary amylase, cow and horse.

35. Claff, C. L., Dewey, V. C., and Kidder, G. W., Biol. Bull. 81: 221-234 (1941). Feeding and nutrition, ciliates.

36. Clarke, F., Solkot, R., et al., J. Biol. Chem. 131:135-138 (1939). Metabolism of rare sugars by rat. 
37. Claus, C., Grobben, K., and Kuhn, F., Lehrbuch der Zoologie (1937). Ch. 4, pp. 124-136. Berlin, Springer. Anatomy of digestive tracts.

38. Cleveland, L. R., Quart. Rev. Biol. 1:51-59 (1926). Symbiosis of termites and intestinal flagellates.

39. Cleveland, L. R., Biol. Bull. 54:231-237 (1928). Symbiosis between termites and intestinal protozoans.

40. Cleveland, L. R., Hall, S. R., et al., Mem. Acad. Arts \& Sci. 17:185-342 (1934). Symbionts in wood roach, Cryptocercus.

41. Dawes, B., J. Mar. Biol. Ass. U.K. 17:75-102 (1930). Absorption of fats in plaice Pleuronectes.

42. Dickman, A., J. Cell. \& Comp. Physiol. 3:223-246 (1933). Digestion of wax by waxmoth larvae.

43. Dobreff, M., Pfliig. Arch. ges. Physiol. 217:221-234 (1927). Gastric secretion in elasmobranchs.

44. Dore, W. H., and Miller, R. C., Univ. Calif. Publ. Zool. 22:383-399 (1923). Digestion of wood by Teredo.

45. Doyle, W. L., Nature 144:867 (1939). Amylase in Amphioxus.

46. Doyle, W. L., and Patterson, E. K., Science 95:206 (1942). Origin of peptidase in Didinium.

47. Dukes, H. H., The Physiology of Domestic Animals. (1942). Ithaca, N. Y., Comstock Publishing Co., 721 p. pp. 217-226, Digestion.

48. Duspiva, F., Ztschr. vergl. Physiol. 21:632-641 (1934). Utilization of wax by Galleria.

49. Duspiva, F., Ztschr. Physiol. Chemie 241: 168-176, 177-200 (1936). Protein digestion by clothesmoth and waxmoth.

50. Elsden, S. R., J. Exper. Biol. 22: 51-62 (1945). Acid formation in sheep rumen.

51. Evenius, J., Arch. Bienenk. 7:229-244 (1926). Enzymes of digestive tract, honeybee.

52. Farner, D., Biol. Bull. 84:240-243 (1943). Biliary amylase, fowl.

53. Florkin, M., L'Evolution Biochemique (1944). Paris, Masson, 210 p. Comparison of intracellular and extracellular digestion.

54. Fox, D., Marks, G. W., ET aL., Bull. Scripps Inst. Oceanog. 4:I-64 (1936). Digestion by style of Mytilus.

55. Fraenkel, G., J. Exper. Biol. 17: 18-29 (1940), also Nature 137:237-238 (1936). Utilization of sugars by adult blowflies.

56. Frazer, A. C., Physiol. Rev. 20:561-581 (1940). Mechanisms of fat absorption, mammals.

57. Friedman, M. H., J. Cell. E Comp. Physiol. 5:83-95 (1934), ibid. 10:37-50 (1937). Nervous control, drug effects, gastric and esophageal secretion, frog.

58. Fruton, J. S., Irving, G. W., and Bergmann, M., J. Biol. Chem. 141:763-774 (1941). Classification of cathepsins.

59. Graetz, E., Zool. Jahrb., Abt. allg. Zool. u. Physiol. 46:375-412 (1929). Digestive enzymes, pulmonates.

60. Graetz, E., and Autrum, H., Ztschr. vergl. Physiol. 22:273-283 (1935). Protein-digesting enzymes of leeches.

61. Granam, A., Proc. Roy. Soc. Lond., B. 108:84-95 (1931). Digestion in Pecten.

62. Grailam, A., Tr. Roy. Soc. Edin. 57:287-308 (1932). Digestion by limpet, Patella.

63. Hasler, A. D., Biol. Bull. 68: 207-214 (1935), ibid. 72:290-298 (1937). Digestion by cladocerans and copepods.

64. Hassett, C. C., Biol. Bull. 95:114-123 (1948). Utilization of sugars, Drosophila.

65. Heatley, N. G., J. Exper. Biol. 13:329-343 (1936). Digestive enzymes of Peripatopsis (Onychophora).

66. Herzog, R. O., and Gonell, H. W., Ztschr. Physiol. Chem. 141:63-67 (1924). Cellukose in Phallusia and Molgula.

67. Hussch, G. C., and JACOBs, W., Ztschr. vergl. Physiol. 8: 102-144 (1938), ibid. 12: $524-558$ (1930). Secretory cycle and digestive glands, Astacus.

68. Hobson, R. P., J. Exper. Biol. 8:109-123 (1931). Digestion in blowfly larvae.

69. 1loltik, H., and Doyle, W. L., J. Cell. E Comp. Physiol, 12:295-308 (1938). Amylase and peptidase of amoeba and paramecium. 
70. Holter, H., and Kopac, M. J., J. Cell. E Comp. Physiol. 10:423-437 (1937). Localization of peptidase in amueba.

70a. Holter, H., and Lovtrup, S., C. R. Lab. Carlsberg 27:27-62 (1949). Proteolytic enzymes, Chaos.

71. Howells, H. H., Quart. J. Micr. Sci. 83:357-397 (1942). Digestive enzymes, Aplysia.

72. Howland, R. B., Protoplasma 5:127-134 (1938). Acidity of gastric vacuole, Actinosphaerium.

73. Hummell, F. C., Sheperd, M. L., et al., J. Nutrit. 25:59-70 (1943). Disappearance of cellulose from digestive tract of children.

74. Hungate, R. E., Ann. Ent. Soc. Amer. 34:467-489 (1941). Nitrogen requirements, termites.

75. Hungate, R. E., Proc. Tr. Texas Acad. Sci. 27:91-98 (1943). Termite growth and nitrogen utilization.

76. Hungate, R. E., Biol. Bull. 84, 157-163. (1943) Action of rumen Protozoa on cellulose.

77. Hungate, R. E., Ann. Ent. Soc. Amer. 36:730-739 (1944). Cellulose fermentation by termite Protozoa.

78. Hykes, O., and Mazanec, J., C. R. Soc. Biol. Paris 117:166-168 (1934). Digestive enzymes of fish.

79. Hyman, L., The Invertebrata (1940). New York, McGraw-Hill. 726 p. Digestion in coelenterates.

80. Inving, L., J. Gen. Physiol. 10:3+5-358 (1926). Digestive function of caeca, starfish.

81. Ishida, J., Annot. Zool. Japon. 15:285-305 (1935-1936). Digestive enzymes of an actinian.

82. Ismida, J., Annot. Zool. Japon. 15:263-284 (1935-1936). Digestive enzymes in stomachless fish.

83. Jewell, M. E., and Lew1s, H. B., J. Biol. Chem. 33:161-167 (1918). Lichenase in invertebrates.

84. Johnson, M. J., and Berger, J., Adv. in Enzymol. 2:69-92 (1942). Properties of peptidases, review.

85. Johnson, M. J., Johnson, G. H., et AL., J. Biol. Chem. 116:515-526 (1936). Mammalian peptidases.

86. Jordan, H., Pflüg. Arch. ges. Plyssiol. 116:617-624 (1907). Digestion in actinians.

87. Karher, K., Schubert, P., et al., Helv. Chim. Acta 8:792-810 (1925). Cellulase in Helix.

88. Keeton, R. W., Косh, F. C., et Al., Am. J. Physiol. 51:454-468 (1920). Responses to gastrin in various vertebrates.

89. Kelley, E. G., Physiol. Zool. 4:515-541 (1931). Intracellular digestion of nucleoprotein by planaria.

90. Kenyon, W. A., Bull. U. S. Bur. Fish. 41, 181-200 (1925). Digestive enzymes in lower vertebrates.

91. Kertesz, Z. I., J. Nutrit. 20, 289-296 (1940). Fate of pectins in digestive tract, mammals.

92. Kırвy, H., in Calkins' Protozoa in Biological Research (1941). pp. 890-1008. Role of Protozoa in animal digestive tracts.

93. Koschtojanz, C. S., Iwanoff, I., Et al., Ztschr. vergl. Physiol. 18:112-115 (1932). Secretin from lower vertebrates.

94. Kosmin, N. P., and Komarov, P. M., Ztschr. vergl. Physiol. 17: 267-278 (1932). Development of salivary enzymes, honeybees.

95. Krijgsman, B. J., Ztschr. vergl. Physiol. 2:264-296 (1925), ibid. 8:187-280 (1928). Digestive secretion, enzymes and phagocytosis in Helix.

96. Krijgsman, B. J., Ztschr. vergl. Physiol. 23:663-711 (1936). Proteases of trypanosomes.

97. Кrogh, A., Biol. Rev. 6:412-442 (1931). Dissolved substances as food.

98. Knüger, P., Sitzungsb. Preuss. Akad. Wiss. phys. math. Classe 2:548-564 (1929). Enzyme distribution, $\mathrm{pH}$ optima, various invertebrates. 
99. Krüger, P., Ergebn. Physiol. 35:538-631 (1933). Review, pH of digestive fluids, lower animals.

100. Krüger, P., and Graetz, E., Zool. Jahrb., Abt. allg. Zool. u. Physiol. 45:463-514 (1928). Gastric enzymes of crayfish.

101. Kuntara, W., Ztschr. Physiol. Chemie 225:169-188 (1934). Lipase in Helix.

102. Lavine, T. F., J. Cell. \& Comp. Physiol. 28:183-196 (1946). Enzymic function of crystalline style, clams.

103. Lenkeit, W., Ergebn. Physiol. 35:573-631 (1933). Salivary secretion, mammals.

104. Lesser, E. L., and Taschenberg, E. W., Ztschr. Biol. 32:443-455 (1908). Digestive enzymes of earthworms.

105. Linderstrom-Lang, K., and Duspiva, F., Nature 135:1039-1040 (1935), also Ztschr. physiol. Chemie 237:131-158 (1935). Reducing agents and digestion of keratin by clothes-moth.

106. Lloyd, F. E., Papers of the Mich. Acad. Sci., Art. E Lett. 7:395-416 (1926). Digestion of Spirogyra by protozoan, Vampyrella.

107. Lotman, R., Bee World 15:34-36 (1934). Digestion of starch by bees.

108. Lwoff, A. and N. Roukhelman, C. R. Acad. Sci. 183:156-158 (1926). Digestion of peptones by Glaucoma.

109. MacGinitie, C. E., Biol. Bull. 77:115-118 (1939), ibid. 88:107-111 (1945). Feeding methods, Chaetopterus, Urechis.

110. Mann, F. C., and Bollman, J. L., J. Am. Med. Ass. 95:1723-1724 (1930). Acidity of dog digestive fluids.

111. Mansour, K., and Mansour-Bek, J. J., Biol. Rev. 9:363-382 (1934). Review, digestion of wood by insects.

112. Mansour, K., and Mansour-Bek, J. J., J. Exper. Biol. 11:243-256 (1934). Digestion of wood by insects.

113. Mansour-Bek, J. J., Ztschr. vergl. Physiol. 17:154-208 (1932). Proteolytic enzymes, Maja.

114. Mansour-BeK, J. J., Ztschr. vergl. Physiol. 20:343-369 (1934). Protein digestion, Murex.

115. Marshall. R. A., and Phillipson, A. T., Proc. Nutrit. Soc. 3:238-243 (1943). Function of microorganisms in rumen.

116. MASt, S. O., Biol. Bull. 75:389-394 (1938). Digestion of fat in Amoeba proteus.

117. Mast, S. O., Biol. Bull. 83:173-204 (1942). Food vacuoles, pH, Amoeba.

118. Mast, S. O., Biol. Bull. 92:31-72 (1947). Food vacuoles, Paramecium.

119. Mast, S. O., and Bowf.n, W. J., Biol. Bull. 87:188-222 (1944). Food vacuoles, $\mathrm{pH}$, several peritrichs.

120. Mast, S. O., and Doyle, W. L., Arch. Protist. 86:155-180 (1936). Slow utilization of fat by amoeba.

121. McAnally, R. A., and Phillipson, A. T., Biol. Rev. 19:41-5+ (1944). Digestion in ruminant.

122. McDougall, E. 1., Biochem. J. +3:99-109 (1948). Saliva of sheep.

123. Metalnikow, S., Arch. Zool. Exper. Gen. 9:373-499 (1912). Food selection by Protozoa.

124. Millott, N., J. Exper. Biol. 14:405-412 (1937). Digestion in nudibranchs.

125. Millot, N., Proc. Roy. Soc. Lond., B., 132:200-212 (19+t). Nervous control of secretion of protease, earthworm intestine.

126. Nelson, T. C., J. Morpl. 31:53-111 (1918). Origin and function of crystalline style, lamellibranchs.

127. Nicol, E. A. T., Tr. Roy. Soc. Edin. 56:537-598 (1930). Feeding and digestion in Sabella.

128. Nimenstein, E., Zischrr. wiss. Zool. 125:513-518 (1925). Acidity of food vacuoles, ciliates (paramecium).

129. Nomus, E. R., and Mathies, J. C., Fed. Proc. 6:281 (1947). Gastric proteinase of tuna.

130. Nontminup, J. H., J. Gen. Physiol. 5:263-27+ (1923). Differences in pH optima for digestion of different proteins.

131. Oomen, H. A. P. C., Pub. Staz. Zool. Napoli 7:215-291 (1926). Digestion in holothurians. 
132. Parkin, E. A., I. Exper. Biol. 17:36+377 (19+0). Digestive enzymes of wood boring larvae.

133. Pavlovskx, E. N., and Zarin, E. Y., Quart. I. Micr. Sci. 66:509-556 (1922). Anylase in honeybee.

134. Peczenik, O.. Zischr. vergl. Physiol. 2:215.225 (1925). Protease in Limnaea.

135. Phillips, E. F.. I. Agr. Res. 35:385-428 (1927). Carbohydrases in adult honey bee.

136. Pinllipson. A. T., and Mednally, R. A., I. Exper. Biol. 19:199-214 (1942). Acid production in rumen of sheep.

137. Putter, A., Ztschr. allg. Physiol. 7:283.320, 321-368 (1908). Nutrition by dissolved substances.

138. Pun, Y. C., Contr. Biol. I.al. Sci. Soc. China, Zool. Ser. 13:121-133 (1940), (from Chemical Abstracts). Digestive tract pH, earthworm.

139. Ripper, W'., Ztschr. vergl. Physiol. 13:314-333 (1930). Digestion of wood by insect larvae.

140. Ronilj, C., Arch. Néerl. Zool. 1:373-+31 (1935). Digestive enzymes in cephalopods.

141. Rosen, B., Ztschr. vergl. Physiol. 21:176-187 (1934). Protein digestion, Helix.

142. Rosen, B., Ztschr. vergl. Physiol. 24:602-612 (1937). Properties of proteinases of gastropods and crustaceans.

143. Rox, D. N., Ztschr. vergl. Physiol. 24:638-643 (1937). Nutrition of wax-moth.

14t. Sawano, E., Sci. Rep. Tohoku Uni1., Ser. II', 4:327-334 (1929). Digestion in Ostrea.

145. Sawano, E., Anu. Rep. Saito Grat. Found. Sendai 8:149-153 (1931). Enzymes from anthozoan coelenterates.

146. Sawano, E., Tokyo Bunrika Daigaku Sci. Rep. B, 2:101-126 (1935). Protein digestion in cephalopod Polypus.

147. Sawano, E., Tokyo Bumrika Daigaku Sci. Rep. B, 2:179-199 (1936). Protein digestion, starfish.

148. Sawano, E., Tokyo Bunrika Daigaku Sci. Rep. B, 3:221-24l (1938). Protease in paramecium.

149. Sснцоттке, E., Ztschr. vergl. Physiol. 22:359-413 (1935). Digestion in Limulus.

150. Schlottke, E., Zischr. vergl. Physiol. 24:210-247, 422-450, and 463-492 (1937). Distribution and properties of digestive enzymes: carnivorous Carabidae; Tettigonia and Stenobothrus; Periplaneta.

150a. Schmiditielsen, B., Acta Physiol. Skand. $11: 104-110$ (1946). Saliva pH, man.

151. Schwartz, C., et al., Pflïg. Arch. ges. Physiol. 202:475-477 (1924), ibid. 213:592-594 (1926). Acidity of saliva and gastric juices, domestic animals.

152. Shapiro, N. H., Tr. Am. Micr. Soc. 46:45-50 (1927). Food vacuole pH, ciliates.

153. Shinoda, O., Ztschr. vergl. Physiol. 7:323-364 (1928). Proteases in gastric juice of crayfish.

154. Shinoda, O., J. Biochem. 11:345-367 (1930). Iigestive enzymes, silkworm.

155. Shinoda, O., Annot. Zool. Japon. 13:117-125 (1931). Amylase in silkworm.

156. Somorodinzew, I. A., and Debechine, K. V., Bull. Soc. Chim. biol. 18:1097. 1105 (1936). Evidence for weak proteases, tapeworms.

157. Spence, J., and Richards, O. W., Carmegie Inst., Wash., Tortugas Papers 32: 165-167 (1940). Cellulose in tunicates.

158. Stober, W. K., Ztschr. vergl. Physiol. 6:530-565 (1927). Relation between enzymes and diet, Lepidoptera.

159. Sumner, J. B., and Somers, G. F., Chemistry and Methods of Enzymes. (1947). New York, Academic Press, 415 p.

160. Swingle, H. S., Ohio I. Sci. 25:209-218 (1925). Digestive enzymes, Blatta.

161. Swingle, H. S., Ann. Ent. Soc. Amer. $21: 469-475$ (1928). Digestive enzymes of oriental fruit moth.

162. ТАкатsuki, S., Quart. J. Micr. Sci. 76:379-431 (1934). Digestive function of amoebocytes in Ostrea.

162a. Thorpe, W. H., Nature 130:437 (1932). Fly larvae in oil wells.

163. Trager, W., Biol. Bull. 66:182-190 (1934). Cultivation of flagellates on cellulose. 
164. Ullmann, T., Ztschr. vergl. Physiol. 17:520-536 (1932). Digestion of starch, inulin, lichenin, invertebrates.

165. Ungar, G., C. R. Soc. Biol. Paris 119:172-173 (1935). Effects of drugs on gastric secretion, elasmobranchs.

166. Van Slyke, D. D., and White, G. F., J. Biol. Chem. 9:209-217 (1911). Protein digestion in dogfish.

167. Vogel, B., Ztschr. vergl. Physiol. 14:273-347 (1931). Utilization of sugars, honcybee.

168. Vonk, H. J., Ztschr. vergl. Physiol. 5:445-546 (1927). Digestion in fishes.

169. Vonk, H. J., Ztschr. vergl. Physiol. 9:685-702 (1929). Pepsin in different vertebrates.

170 Vonk, H. J., Biol. Rev. 12:245-287 (1937). Review, digestive enzymes in metazoa.

171. Vonk, H. J., Adv. in Enzymol. 1:371-417 (1941). Review, digestion in lower vertebrates.

172. Vonk, H. J., Brink, K., et al., Proc. Kon. Nederl. Akad. Wetensch. 49:972-982 (1946). Gastric digestion, birds.

173. Waldschmidt-Leit'z, E., et AL., Ber. dtsch. chem. Ges. 61:299-306, 640-645, 645-656, 2092-2096 (1928), ibid. 62: 956-963 (1929). Classification of peptidases.

174. Weese, A. O., Pub. Puget Sound Biol. Sta. 5:165-177 (1926). Digestion by sea urchin.

175. Westblad, E., Lunds Arssk. 18:1-102 (1923). Digestion in turbellarians.

176. Wiersma, C. A. G., and van Veen, R., Ztschr. vergl. Physiol. 7:269-278 (1928). Carbohydrases of Astacus.

177. Wigglesworth, V. B., Biochem. J. 21:791-796 (1927). Acidity of digestive canal, carbohydrate digestion, cockroaches.

178. Wigglesworth, V. B., Parasitology 21:288-321 (1929), ibid. 23:73-76 (1931). Digestion in Diptera; tsetsc-fly, Glossina and Chrysops.

179. Wigglesworti, V. B., Principles of Insect Physiology (1939). London, Methuen. Ch. 11, pp. 254-298. Digestion and nutrition.

180. Wilber, C. G., Biol. Bull. 83:320-325 (1942), ibid. $91: 235$ (1946). Digestion of fat in Pelomyxa.

181. Willier, B. H., Hyman, L. H., et al., J. Morph. 40:299-340 (1925). Intracellular digestion in triclads.

182. Wolvekamp, H. P., Ztschr. vergl. Physiol. 7:454-461 (1928). Carbohydrate digestion, turtle.

183. Wright, R. D., et AL., Quart. J. Exper. Physiol. 30:73-120 (1940). Duodenal secretion in dogs.

184. Yonge, C. M., J. Exper. Biol. 1:15-63 (1923). Feeding, digestion and assimilation in clam Mya.

185. Yonge, C. M., J. Exper. Biol. 2:373-388 (1925). Digestion in the ascidian, Ciona.

186. Yonge, C. M., J. Mar. Biol. Ass. U. K. 14:295-385 (1926). Feeding and digestion, Ostrea.

187. Yonge, C. M., Biol. Rev. 3:21-76 (1928). Feeding mechanisms, invertebrates.

188. Yonge, C. M., Sci. Rep. Great Barrier Reef Expedition, Brit. Mus. 1:59-81, 83-91, 135-175 (1930-31). Feeding, digestion and assimilation in corals.

189. Yonge, C. M., Sci. Rep. Great Barrier Reef Expedition, Brit. Mus. 1:259-281 (1932). Occurrence of crystalline style in molluscs.

190. Yonge, C. M., Biol. Rev. 12:87-115 (1937). Review of digestion in Metazoa.

191. Ziese, W., Ztschr. Physiol. Chem. 203:87-116 (1931). Characteristics of cellulase from Helix. 


\section{Nitrogen Excretion}

ATS AND CARBOHYDRATES are oxidized to water and carbon dioxide, which are readily excreted; proteins and nucleic acids contain nitrogen, which is excreted in various compounds according to the physiology of the organism. Nitrogenous wastes may come from proteins (by way of amino acids), from the purine and pyrimidine bases of nucleic acids, and from miscellaneous nitrogenous compounds. 6, $3: 3,34$

\section{CHEMICAL NATURE OF NITROGENOUS PRODUCTS}

Protein Degradation Products. At least six different types of compound may be produced by the degradation of protein.

Amino Acids. Proteins are hydrolyzed to polypeptides and dipeptides, and these are broken down to their constituent amino acids. Amino acids may be excreted without change in some animals.

Ammonia. Amino acids undergo deamination, resulting in the liberation of ammonia as follows:<smiles>NCC(=O)ONC(=O)C(=O)O</smiles>

or<smiles>NCC(=O)O</smiles><smiles>NCC(O)C(=O)O</smiles>

Ammonia is toxic and is low in concentration in the blood of many animals. It is formed rapidly in drawn blood of vertebrates, but the amount present in vivo in mammals is probably not more than $0.001-0.003 \mathrm{mg} . / 100 \mathrm{ml}$. ${ }^{15}$ The amount present is similar (less than $0.1 \mathrm{mg} . / 100 \mathrm{ml}$.) in frogs, reptiles and fishes. ${ }^{32}$ If the blood ammonia reaches $5 \mathrm{mg} . / 100 \mathrm{ml}$. in rabbits death results. ${ }^{6}$ Some ammonia is excreted by the kidneys (about 0.5-1 gm. daily in man). is In mammals most of the urinary ammonia is formed from glutamine by the kidney; the amount which comes from amino acids is much smaller than it is in animals in which ammonia is an important excretory product. ${ }^{91}$ There are invertebrate animals which are more tolerant of ammonia. Blood 
ammonia values have been reported as $0.4-2.4 \mathrm{mg} . / 100 \mathrm{ml}$. in Maja, 2.8-4.8

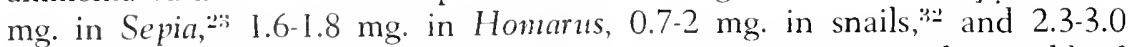
mg. in the earthworm Pheretima. " Ammonia increases on standing in blood of Hydrophilus and Dytiscus, but not in blood of crayfish, lobster, or snail. ${ }^{3 x}$ The blood of Anodonta is low in ammonia ( $0.051 \mathrm{mg}$. $/ 100 \mathrm{ml}$.).:3!

Because of its toxicity ammonia must be excreted rapidly as formed or converted into a less toxic substance. Ammonia is highly soluble in water, is diffusible, and is excreted as ammonia by many animals which have ample water for carrying away excretory products.

Urea. This excretory product is less toxic and slightly more soluble in water than ammonia. Human blood normally contains 18 to $38 \mathrm{mg}$. urea per $100 \mathrm{ml}$.; values above about $40 \mathrm{mg} . / 100 \mathrm{ml}$. indicate uremia, although much higher concentrations are tolerated. The livers of many animals contain an enzyme, arginase, which catalyzes the formation of urea from the amino acid arginine. There is evidence that one method of conversion of $\mathrm{NH}_{3}$ to urea is by the Krebs cycle (Fig. 32). When this cycle operates, urea production by tissue

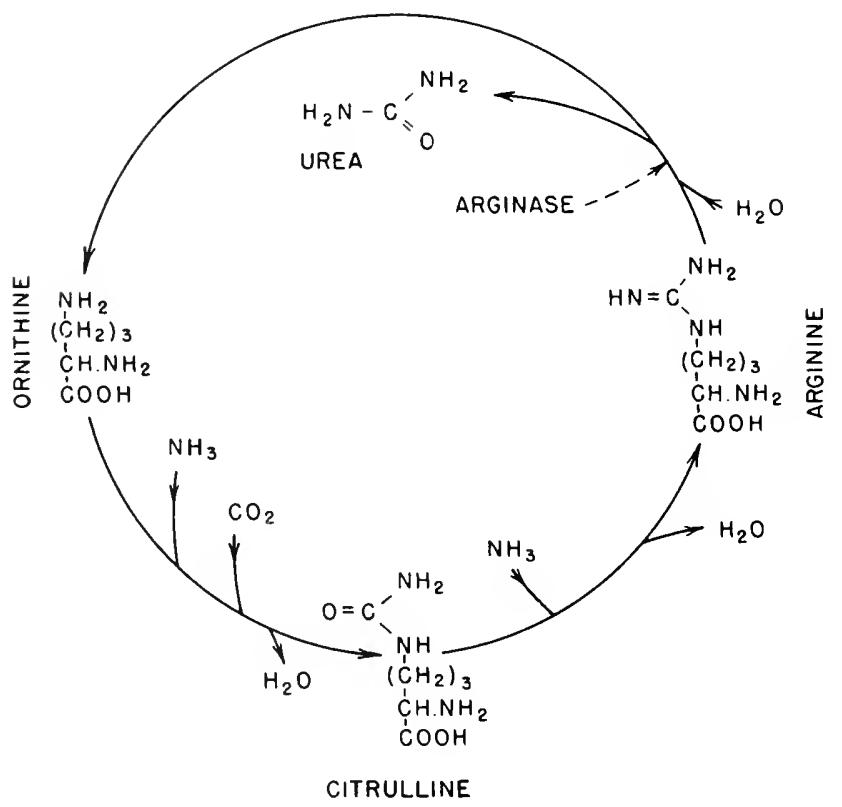

Fig. 32. The ornithine cycle. From Baldwin."

extracts is increased on addition of ornithine and citrulline; urea is formed from dietary arginine by the action of arginase alone. In some animals urea is apparently formed by unknown reactions other than those of the ornithine cycle.

Uric Acid. Some animals, particularly birds, terrestrial reptites, and some snails and insects, convert their ammonia to uric acid. This substance has a low toxicity and is relatively insoluble; it can be stored or excreted in crystalline form. The mechanism of formation of uric acid from ammonia is unknown, 
but apparently hypoxanthine is an intermediate product in the process.

Trimethylamine Oxide. This constitutes a high proportion of excreted nitrogen in certain marine fishes. Possibly ammonia is methylated, and the resulting trimethylamine oxidized.

Guanine (2-amino-6-oxypurine). Spiders excrete most of their nitrogen as the purine, guanine, a substance less soluble than uric acid in water. Its method of synthesis is unknown. "s

Purine Excretion Products. Roughly 5 per cent of excretory nitrogen comes from nucleic acid metabolism, which yields principally the purines adenine and guanine.

Purines. The purines are degraded to different degrees by animals of various groups. Some animals excrete a high proportion as purines without further degradation. In some animals adenine may be oxidized, but not other purines; guanine is accumulated or excreted as such in the pig, but in most mammals it is deaminated. Different enzymes act on the purine adenine and the nucleoside adenosine (adenine-ribose). ${ }^{33}$

Uric Acid. Uric acid is formed as an end-product by oxidative deamination.

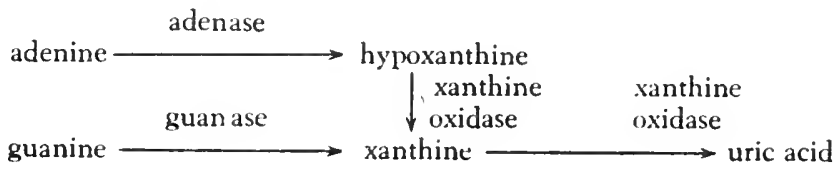<smiles>Nc1nccnc1N</smiles>

Adenine

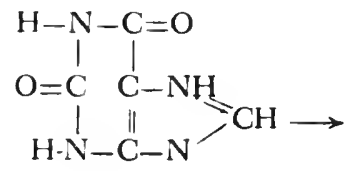

Xanthine<smiles>O=C1CNc2[nH]c(=O)[nH]c2N1</smiles>

Uric acid

Allantoin. The pyrimidine ring of uric acid may be opened by uricase and the substance allantoin formed (Fig. 33).

Allantoic Acid. Further oxidation by allantoinase forms allantoic acid (Fig. 33).

Urea. The molecule of allantoic acid is broken by allantoicase to glyoxylic acid and urea.

Ammonia. A few animals and many plants possess urease, which converts urea to ammonia and carbon dioxide.

The complete sequence of purine breakdown is shown in Figure 33, modified from Florkin. $3: 3$

Miscellaneous Nitrogenous Compounds. Other nitrogenous excretion products include:

Hippuric Acid and Omithuric Acid. Benzoic acid entering in the diet of mammals is removed by combination with glycine to form hippuric acid (benzoylglycine). In birds benzoic acid combines with ornithine to form ornithuric acid.

Creatine and Creatinine. Creatine is important in muscle development (Ch. 16), and its metabolic roles are numerous. Some creatine is apparently 
converted to creatinine and excreted, although creatine may also be excreted unchanged.

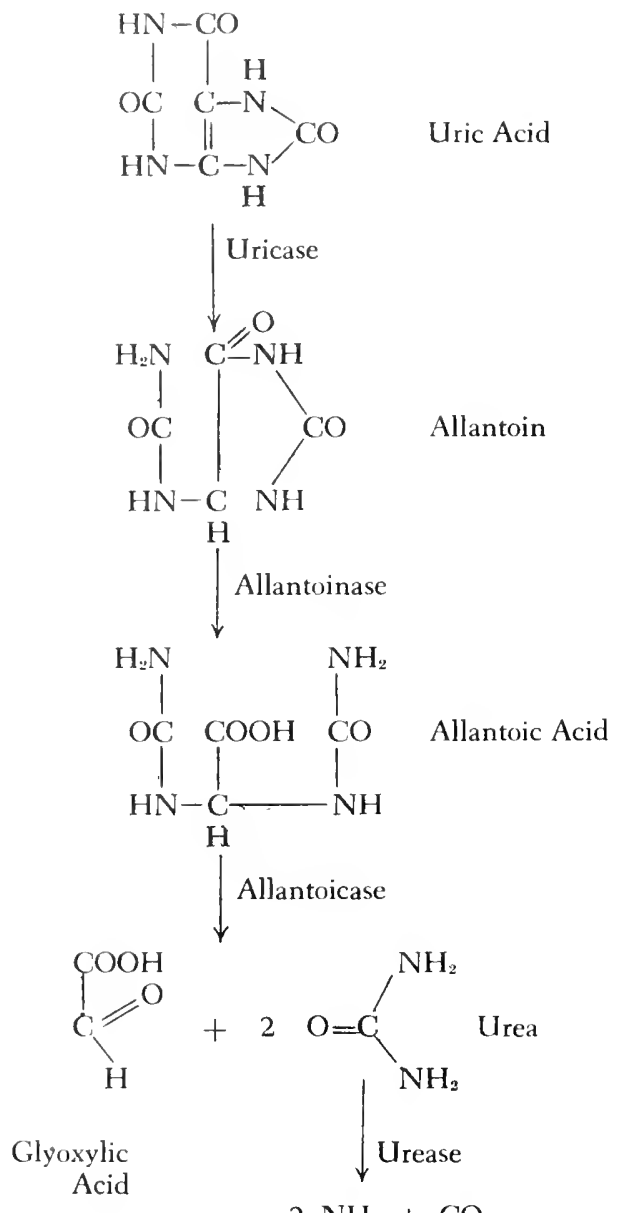

$$
2 \mathrm{NH}_{3}+\mathrm{CO}_{2}
$$

Fig. 33. Sequence of breakdown of uric acid to ammonia. From Florkin..$^{33}$

Nitrogenous Pigments. Some nitrogenous pigments resulting from the metabolism of hemochromogens (e.g., hemoglobin) are excreted, particularly in mammals.

Pterines. These nitrogenous pigments deposited in buttertly wings are sometimes regarded as excretory products.

Pyrimidines. Pyrimidines may he excreted as such, but in mammals, at least, are partly converted to urea.

\section{DISTRIBUTION OF EXCRETORY PRODUCTS OF PROTEIN METABOLISM}

In no group of animals is nitrogenous excretion limited to one product. 
Those animals which excrete significant amounts of amino acids are inefficient, in that they lose potential metabolites. Animals are called ammonotelic when nitrogen (from proteins) is excreted preelominanty in the form of anmonia, ureotelic when urea is the main product, uricotelic when the main product is uric acid. The correlation between excretory products and habitat of adult or embryo is so good that nitrogen excretion appears wo be an important adaptive character. In the series ammonia, urea, uric acid there is decreasing toxicity. Animals whose eggs are protected against water loss (cleidoic eggs) store nontoxic embryonic waste products and the adults tend to excrete the same products, whereas animals whose embryos can excrete freely into surrounding water (noncleidoic eggs) excrete products which mat be more toxic. ${ }^{\text {t: }}$ Embryonic habitat is one but not the only factor detemining nitrogenous excretion.

The percentage nitrogen excretion in various forms is given in Table 30. Data in this table have been selected from what appear to be the more reliable of the many papers. Detailed stmmaries are to be found in Delaunay, $21,23,24$

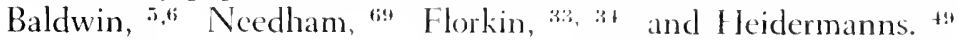

Ammonotelic Animals. Table 30 shows that aquatic invertebrate animals excrete a large percentage of their nitrogen as ammonia. This is a soluble substance which diffuses readily across most body surfaces into water. In Protozoa some early measurements were invalidated by the presence of extraneous organisms, particularly bacteria, and reports of any quantity of urea and uric acid excretion are to be doubted. Also the amount of ammonia varies widely according to diet. In Paramecium, for example, 9l per cent of exereted nitrogen was NH, when the animals had not been fied, 55 per cent was $\mathrm{NH}_{3}$ when they had been fed glycine, and no $\mathrm{NH}_{3}$ was excreted when they had been fed starch; no urea, uric acid, creatinine, or whole protein was found, but some unidentified nitrogenous substance was excreted. ${ }^{17}$ 'These results confirm the earlier work of Emery ${ }^{29}$ and show that Weatherby's observations ${ }^{193}$ of urea were not correct.

Spirostomum ambigumm excretes large amounts of $\mathrm{NH}_{3}$ when the oxygen tension is less than equilibrium with air. "itaucoma eliminates the nitrogen from bacterial food as ammonia about six hours after ingestion. ": Protozoa can utilize amino acids from the culture medium; the extent to which they excrete amino acids from ingested protein has not been shown.

Actinians excrete much ammonia. -t Various echinoderms, starfish, sea urchins, and sea cucumbers, are ammonotelic, but they also give off considerable amounts of amino acids into the water. 20,23 . ammonia predominantly, as does the fresh-water leech. Polychaete worms are ammonotelic, although some urea and traces of uric acid are reported in Arenicola coelomic fluid. $87,: 17$

Among molluscs the cephalopods Sepia and Octopus excrete most of their nitrogen as $\mathrm{NH}_{3}$. The pelecypods which have been examined, both marine and fresh-water (except Mytilus), are also anmonotelic. The gastropod Aplysia is ammonotelic, but excretory products in other gastropods are diverse, as will be shown later.

Crustaceans give off much amino acid nitrogen, but the predominant excretory product of marine and fresh-water crustaceans is ammonia. It has been shown for Eriocleir, and probably it is true for others, that the body surface, 


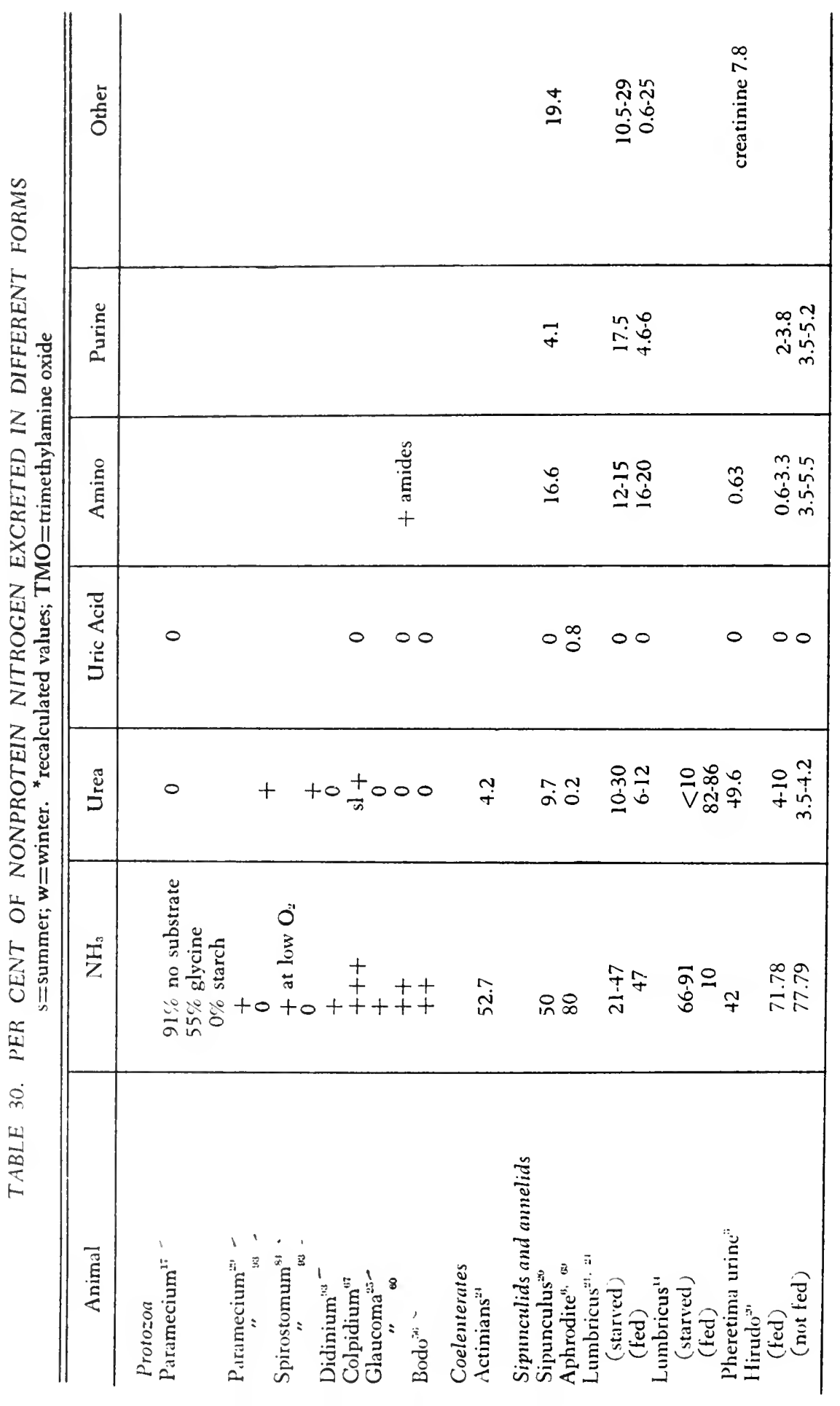




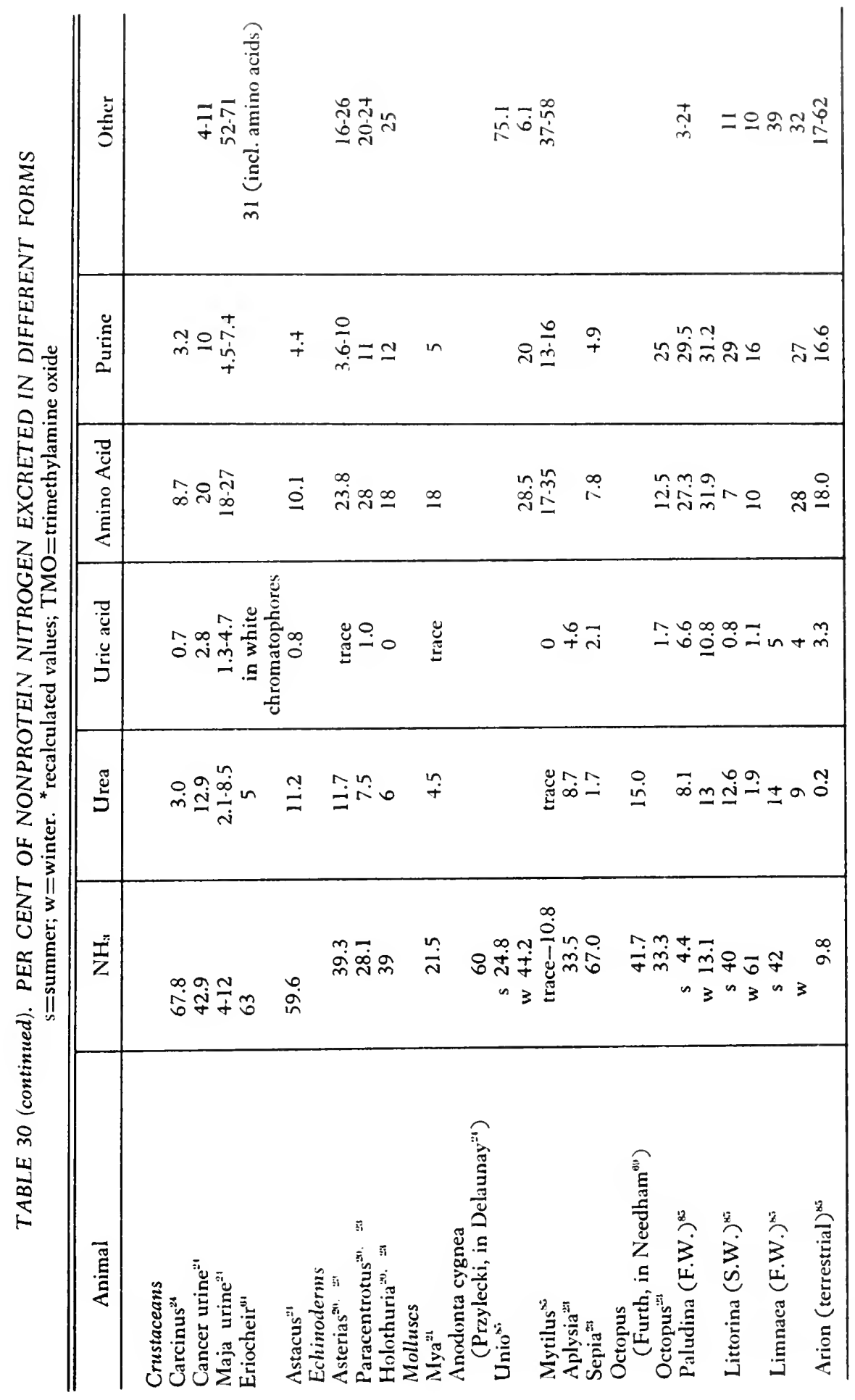




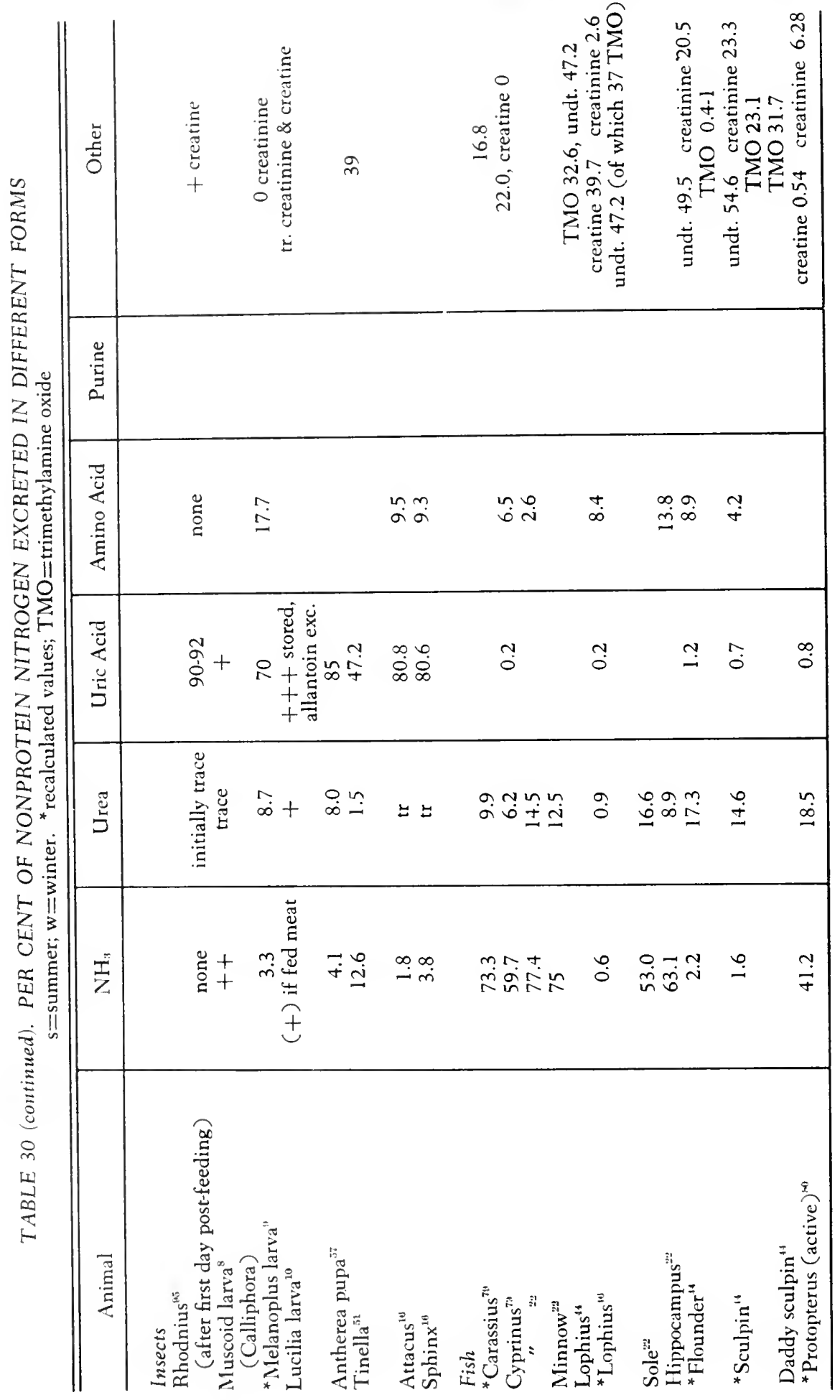


Nitrogen Excretion

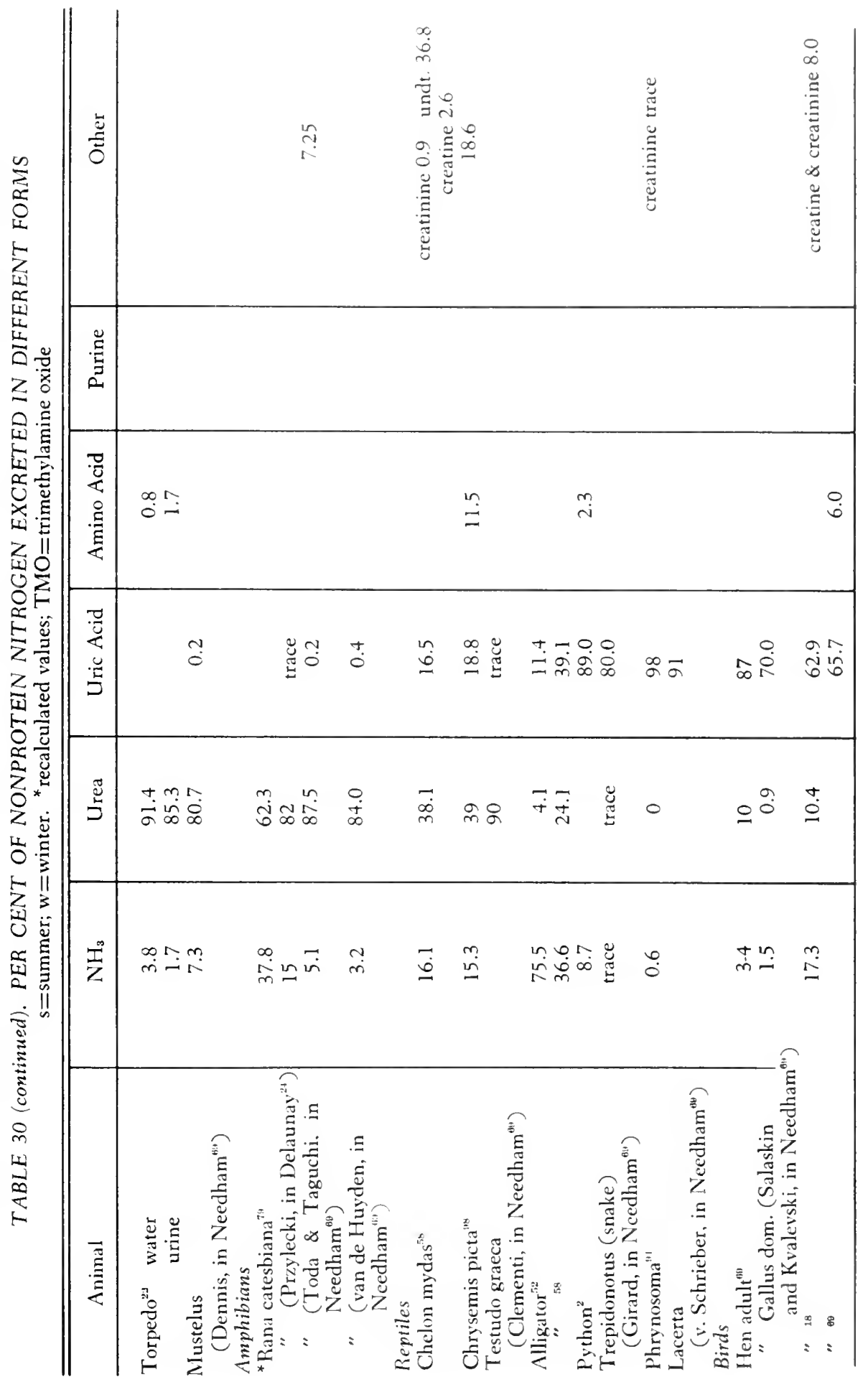




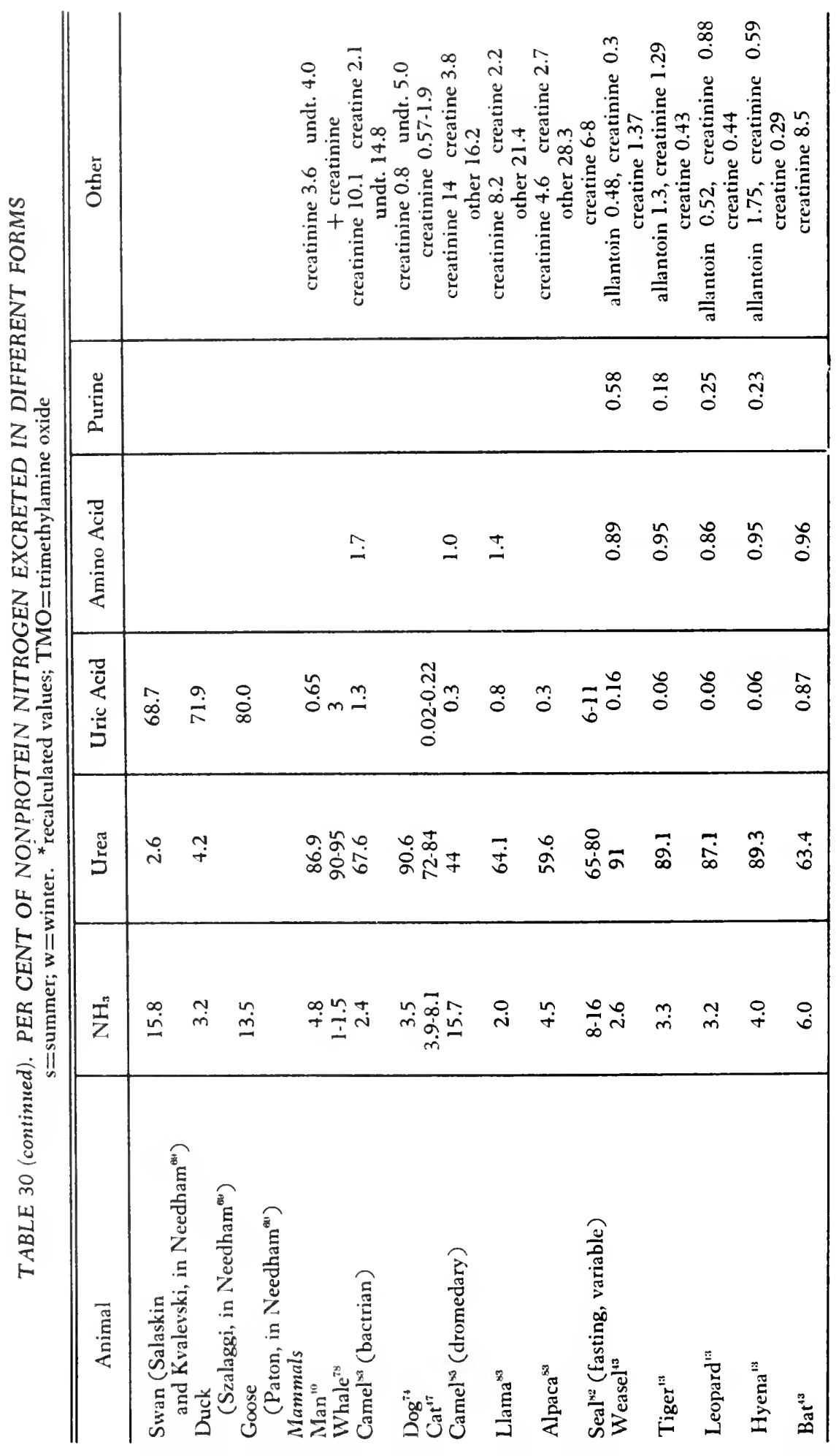


particularly the gills, is permeable to $\mathrm{NH}_{3}$ and urea; excretion of these substances is not altered by plugging the excretory pores. The kidneys excrete dyes and other less diffusible substances, whereas the gills are the chief route for ammonia excretion. "it Analysis of the urine is, therefore, inadequate to measure total nitrogen excretion. Excretory products of terrestrial Crustacea have not been examined, but such investigation would be of interest.

Earthworms are intermediate between aquatic and terrestrial life. The urine collected from worms in moist air contains more urea nitrogen than ammonia nitrogen, ${ }^{3}$ but the excretion from earthworms immersed in water is ammonotelic. ${ }^{2 \neq}$ An explanation of this difference might be that much ammonia is given off from the intestine, not as urine. A better explanation is given by recent observations ${ }^{\text {it }}$ that in normal fed earthworms urea constitutes less than 10 per cent, whereas after starvation urea becomes as much as 86 per cent, of the nitrogenous excretion. It would be of interest to know the effect of water supply on the urea/ammonia ratio.

Fishes, Ammonotelic and Ureotelic; Trimethylamine Oxide Excretion. Ammonia is commonly excreted by animals which have plenty of water to eliminate and which live in an aqueous medium. This holds for fresh-water teleosts, which are ammonotelic, although they also excrete some urea; most of their nitrogenous wastes diffuse out across the gills, and less nitrogen leaves by the urine. ${ }^{i !,} \times 1$ Marine teleosts, on the other hand, are confronted with a serious osmotic problem of retaining sufficient water (Ch. 2). They still excrete considerable ammonia, but they also excrete some urea (Table 30). In addition, some marine teleosts excrete as much as one third of their nitrogen as trimethylamine oxide, a soluble nontoxic substance. This substance is absent from the muscles of several fresh-water fish (eel, carp), but is present in the muscles of all marine fish tested--Pleuronectes, Conger, Gadus, and others (Hoppe-Seyler, quoted by Grafflin and Gould ${ }^{4 t}$ ). Trimethylamine oxide has been reported from cephalopods, but only in marine teleosts where water must be conserved is this substance an important excretory product.

Elasmobranch fish are ureotelic, and marine species retain a concentration of 2-2.5 per cent of urea in the blood. By this high urea concentration they maintain themselves hypertonic to their medium (Ch. 2). All tissues of a dogfish except brain and blood are able to synthesize urea. ${ }^{54}$ "Like marine teleosts, the marine elasmobranchs convert a part of their waste ammonia to trimethylamine oxide. This suggests that they have at some time in the past experienced the same osmotic difficulties as confront the marine teleosts of the present day .... But subsequently, it appears, they evolved the still better trick of making urea, which is cven less toxic than trimethylamine oxide, and, by retaining enough of it in their tissues, managed in the end to turn the osmotic gradient to their advantage instead of their detriment." 6 Excess urea is excreted, largely through the gills. Dogfish eggs contain a large amount of deposited urea; the embryos, however, synthesize quantities of urea, which is stored in the yolk.

Nitrogen excretion in the lungfish, Protopterus aethiopicus, has been studied by Smith. ${ }^{\text {so }}$ When this fish is active in fresh water it excretes nearly three times more nitrogen as $\mathrm{NH}_{3}$ than as urea. When estivating in its mud cocoon, however, it excretes urea which may accumulate to the extent of $1-2$ per cent of the body weight in a year; muscle urea increases by some seven times. When 
the fish returns to water, urea is rapidly excreted and the excretion of ammonia nitrogen increases to its characteristic high proportion. Dipnoans, then, shift their metabolism from ammonotelic to ureotelic and back, according to the available water.

Ureotelic Animals: Amphibia. Nitrogen is excreted predominantly as urea in the frog (Table 30), and the liver of both frog and toad can synthesize urea. ${ }^{66}$ Calculations of glomerular filtration and tubular reabsorption indicate that some urea must be secreted actively by the tubule cells of some amphibian kidneys. ${ }^{2: 2}$, Young tadpoles excrete over 40 per cent of their nitrogen as ammonia, but this declines rapidly to about 12.5 per cent, the adult level (Bialaszewitz, quoted by Delaunay ${ }^{24}$ ).

Mammalia. Mammals, including the monotremes, are ureotelic. Embryonic wastes diffuse readily into the maternal circulation; embryonic life is aquatic. There is need for conservation of water in adults, however, and urea is concentrated by as much as 100 times by the reabsorption of water in the kidney tubules. Low concentrations of ammonia are toxic, but urea is tolerated up to 150-250 mg. per cent-ten times normal concentrations. ${ }^{30}$ Urea concentration in the blood and urine depends on the rate of protein catabolism.

Excretion Associated with Transition from Water to Land: Gastropoda. The gastropods present an interesting series with respect to transition from sea to fresh water to land, and accompanying transition toward uric acid excretion. $^{7}, 4 \overline{5}, 70,71,8 \bar{s}$ Aquatic gastropods, particularly marine ones, excrete much ammonia, terrestrial gastropods excrete little ammonia. The eggs of freshwater and terrestrial gastropods in general have cleidoic characteristics; some gastropods are ovoviviparous. During development of the egg of Limnaea the uric acid content increases from $0.5 \mathrm{mg}$. per cent in the cleavage stages, to 1.0 mg. per cent when the embryo occupies one fourth to one half of the egg case, to $4.5 \mathrm{mg}$. per cent when the egg is about to hatch. ${ }^{4}$ In hibernation the nephridium stores uric acid to as much as three fourths of the organ weight. 70 , ${ }_{85}$ The uric acid content of the nephridium has been correlated with the history of different species and type of egg (Fig. 34). ${ }^{71}$ Needham describes Figure 34 as follows: The uric acid content of the nephridium in marine operculates (lower left) is very low. Terrestrial snails (center top), on the other hand, contain much uric acid, whether they are pulmonates or operculates. The transitional periwinkles (Littorinidae) (left side) are intermediate in uric acid content of the nephridium. A marine pulmonate which is believed to have made a secondary return to the sea (Onchidella celtica) contains practically no uric acid. The fresh-water species (right side) do not provide a clearcut series, but the operculate Hydrobia jenkinsi has little uric acid and has entered fresh water from the sea relatively recently, whereas Bythnia, Paludina, and Linnaea stagnalis contain much uric acid and probably have penetrated fresh water secondarily from land. Other snails are intermediate, for reasons which are not clear. Snails are unable to produce urea by the ornithine cycle and produce it only from the arginine of their food; hence their only choice in protein degradation is ammonia or uric acid. This group of animals merits further study, but the general trend is from ammonia to uric acid excretion as osmotic stress increases.

Chelonia. Turtles, like all vertebrates, can form urea by the ornithine cycle, and they excrete much nitrogen as urea. In addition, their ammonia excretion 


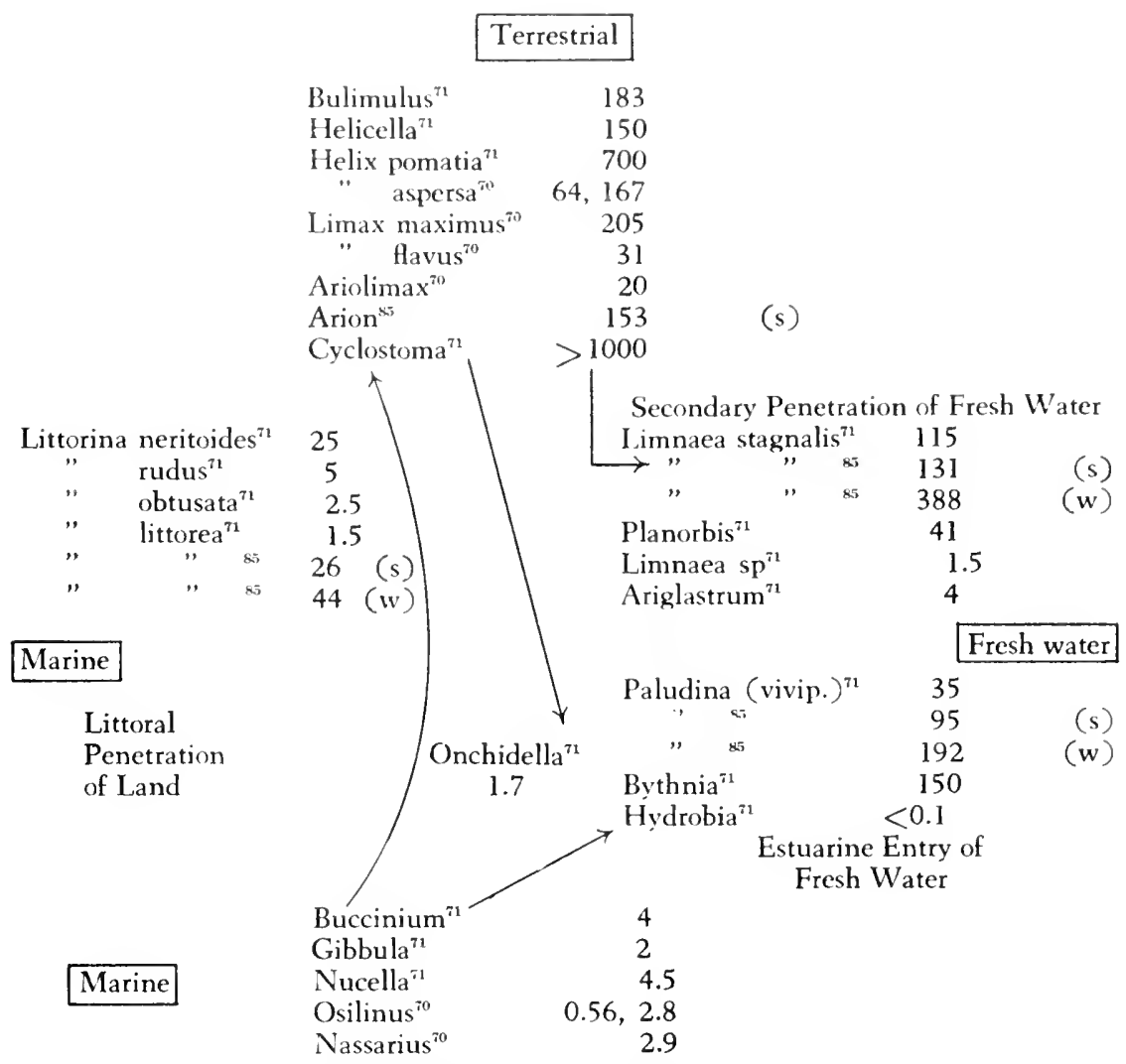

Fig. 34. Uric acid in mg./gm. dry wt. of nephridium of snails. Modified from Needham." (s) summer, (w) winter.

is significant, and two aquatic species excrete some uric acid (Table 30 ). Needham argues that the mode of excretion is related to the water supply in embryonic life; since turtles usually lay their eggs in moist sand where diffusion of $\mathrm{NH}_{3}$ would be too slow to avoid toxicity and yet where storage of uric acid is unnecessary, urea is sufficiently nontoxic. Other reptiles are uricotelic (see below); hence a careful correlation of nitrogen excretion with life history in turtles might throw light on the origin of uricotelism in vertebrates.

Uricotelic Animals: Insects. Most insects are too restricted in their water supply for them to excrete ammonia, or even urea, in quantity. The urine formed in the malpighian tubules of insects is rich in uric acid and may even be a mass of uric acid crystals. 16 Negligible amounts of nitrogen are lost in other forms by adult insects. In the bug Rhodnius, for example, immediately after a blood meal there is considerable urea; later the uric acid is greatly in excess. Blowfly larvae which are eating meat give off much ammonia; this is probably formed in the gut and passed out with feces, and hence is not a true metabolic excretory product. At pupation ammonia excretion stops and uric acid becomes the main product. Some of the uric acid from protein breakdown is converted to allantoin (Table 30). 8,10 Insects rid themselves of much waste material at the time of molting. 
Lizards and Snakes. Water conservation is important for lizards and snakes; also their eggs must retain embryonic nitrogenous excretion until they are hatched. Hence they excrete most of their nitrogen as the relatively insoluble, innocuous uric acid (Table 30). In Phrynosoma, the horned toad, the urine is a solid ball of uric acid.

Birds. Birds convert most of the ammonia from protein to uric acid (Table 30). Their urine is a semisolid mass of uric acid crystals. In the chick embryo the first product is ammonia, which declines rapidly in amount after the fourth day of incubation. Urea increases to a maximum at the ninth day and then declines; the enzyme arginase falls to a minimum at about the twelfth day. The excretion of uric acid on a dry weighe percentage basis is maximal at the eleventh day, and uric acid is the principal adult product (Fig. 35). 68, 6:9. 7:3 If cmbryonic tissues were separated from extraembryonic membranes the absolute amounts excreted by the embryo might differ from those given in Figure 35 , but the general sequence is significant.

Uric acid is found in body fluids of animals in which it is not the principal

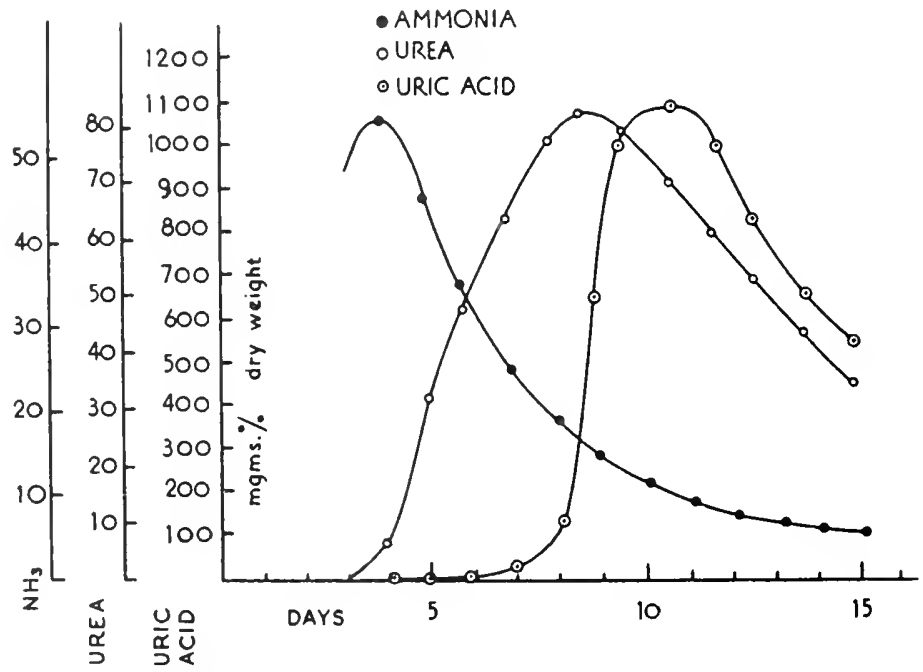

Fig. 35. Excretion in chick embryos. From Needham."

excretory product. ${ }^{4 !}$ For example, in amnelids the uric acid concentration in coelomic Huid is $0.73 \mathrm{mg} . / 100 \mathrm{ml}$. in Chaetopterus 17 and $0.1 \mathrm{mg} . / 100 \mathrm{ml}$. in Arenicola ${ }^{87}$ but negligible in Amphitrite and Nereis. Among Crustacea the blood uric acid may be $2.3 \mathrm{mg} . / 100 \mathrm{ml}$. in Homarus, and $2.1 \mathrm{mg}$. $/ 100 \mathrm{ml}$. in Libinia. ${ }^{6.5}$ In ureotelic mammals blood uric acid is 0.05 to $0.2 \mathrm{mg}$. 100 $\mathrm{ml} .{ }^{75}$ These values are much lower than in true uricotelic animals, as in insects, in which blood uric acid is 5 to $20 \mathrm{mg} . / 100 \mathrm{ml} .^{34}$. $1 \mathrm{mi}$ or in birds, in which it is normally $5 \mathrm{mg} . / 100 \mathrm{ml}$. (chicken). ${ }^{m}$ Hence the distinction between ureotelic and uricotelic metabolism is quantitative, and presence of uric acid in the blood or coelomic fluid does not necessarily indicate predominant uric acid excretion. 


\section{PHYLOGENY OF ENZYMES OF PROTEIN BREAKDOWN PRODUCTS}

Deaminases catalyze the liberation of ammonia from amino acids in all phyla. Urea can be formed by the ornithine cycle (see p. I88), by the action of arginase on dietary arginine independently of the ornithine cycle, and by unknown reactions. Arginase is absent, or present in mere traces, in ammonotelic invertebrates. No arginase was found in the hepatopancreas of a series of marine molluscs; a trace was found in Anodonta and Carcinus and small amounts in several fresh-water snails. ${ }^{+}$In the snail Helix the hepatopancreas contains much arginase, but no urea is formed by the ornithine cycle: possibly arginase has some role in uric acid synthesis in Helix. ${ }^{4}$ The chloragogue tissue of the earthworm intestine can synthesize urea, although the ornithine cycle may not operate as in mammals. Urea production by chloragogue tissue was increased in one set of observations by peptone but not by arginine or ornithine. ${ }^{\mathrm{ts}}$ However, worms fed arginine increased their urea output, and there was a small increase after the feeding of citrulline but not ornithine. ${ }^{1+}$ Teleost fish fall into three groups, having low (cod, salmon, bullhead, trout), intermediate (herring), and high (dogfish) arginase content in their livers,, 3 but the ornithine cycle is said to be absent from teleosts.". 33. . i1 Elasmobranchs contain large amounts of arginase in all tissues except blood and brain; the heart is remarkably rich in arginase. ${ }^{33 .}$. it Whether the ornithine cycle functions in elasmobranchs is not known.

Urea is formed by the ornithine cycle in the livers of ureotelic tetrapods, frogs, turtles, and mammals. In mammals the liver is rich in arginase and the kidney contains a small amount, whereas other tissues are negative for arginase. $3.3 . \quad$ The ornithine cycle is lacking in uricotelic snakes and birds, although bird but not snake kidneys, liver and testes have some arginase. ${ }^{13,}, 2 . .3, \ldots .5,66$ In the animal kingdom urea is formed by several methods, arginase content of liver is correlated with ureotelism, and the ornithine cycle is a specialized development not found among invertebrate animals. The ornithine cycle and most of the usefulness of arginase are lost by birds and higher reptiles.

Conclusions Regarding Excretion of Protein Nitrogen. The form of nitrogenous waste produced by protein catabolism is related to stress of water supply in both embryonic and adult life of an animal. Ammonia diffuses freely out of and away from aquatic invertebrates and fish. Where there is some osmotic stress but ample dilution, urea appears, as in amphibians and mammals. Marine teleosts are subject to osmotic stress, and some of them excrete trimethylamine oxide. Extreme need for water retention, in insects, land gastropods, snakes and lizards, and birds, is satisfied by uric acid excretion; this is associated with cleidoic eggs. Trimethylamine oxide excretion may have arisen only once, but uric acid excretion became dominant in several unrelated groups and represents convergent evolution.

Urea production has arisen by several mcans: urea may be built up from peptones and arginine in Lumbricus, by arginase from dietary arginine in snails and bony fish, by the ornithine cycle from ammonia in amphibians and mammals, and still other routes of urea synthesis probably exist. The ornithine cycle has been lost in reptilian and avian crolution. Embryonic retention of a biochemical trait is shown in nitrogen excretion patterns. Blowfly larvae excrete ammonia before they excrete uric acid, tadpoles produce ammonia 
before they produce urea, and chicks pass through the entire evolutionary series of ammonia, urea, and uric acid.

Nitrogen excretion is a labile character. It appears to have changed during the evolution of several groups according to their habitat. The best example of such phyletic change in form of nitrogenous product is in the gastropods. Also the marine teleosts excrete more of their nitrogen as urea than do their fresh-water relatives, and the earthworm excretes more urea than do aquatic annelids. Even individual animals change their excretion with diet and with osmotic stress. The lungfish stores urea in estivation and excretes ammonia

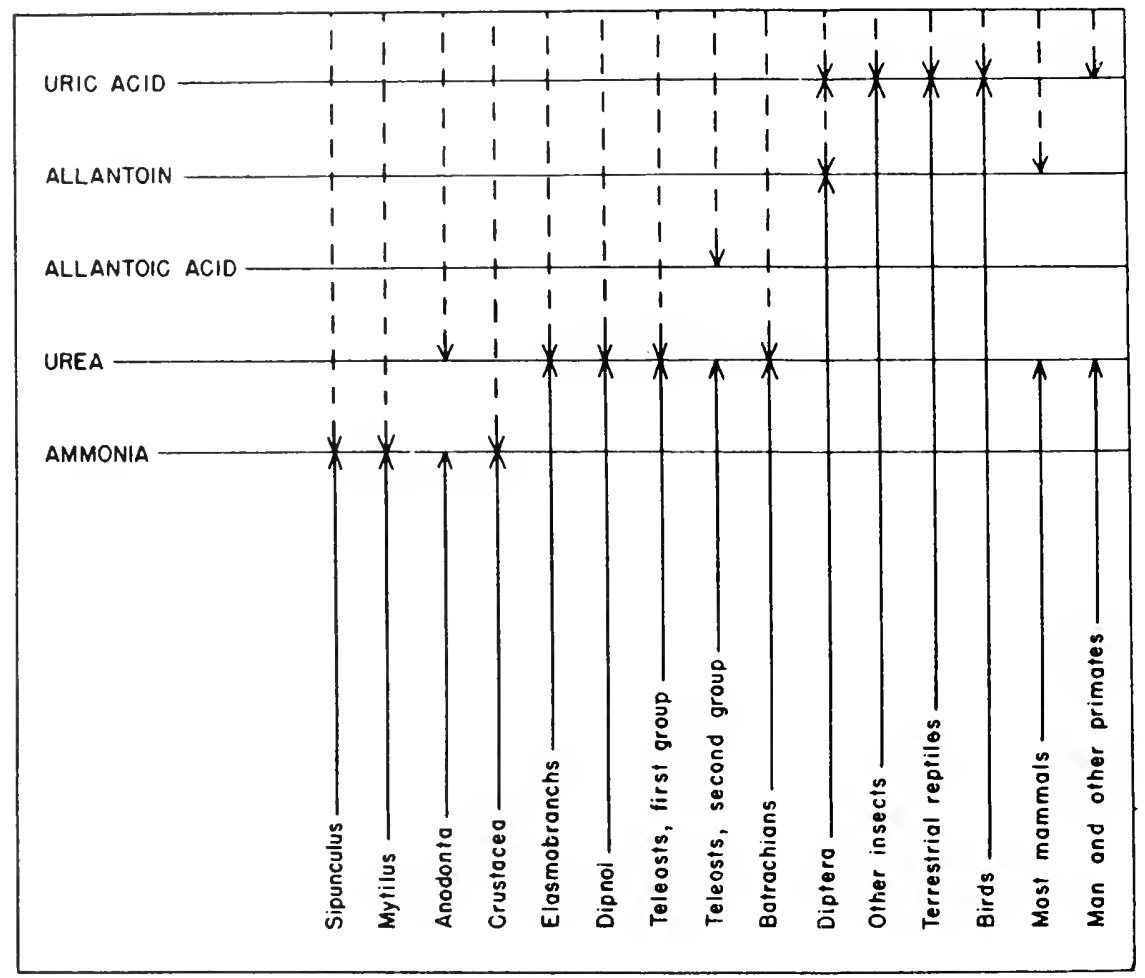

Fig. 36. Comparison of end-products of purine and protein catabolism. From Florkin and Duchateau." Unbroken line, protein breakdown; broken line, purine breakdown.

when active; the bug Rhodnius excretes urea for several hours after a bloodmeal, and then uric acid. Ammonia is the principal nitrogenous product of amino acid breakdown in ammonotelic animals; it comes from glutamine in mammals.

Recent studies on microorganisms indicate their ability to adapt enzymatically to different substrates. Nitrogen excretion in animals is likewise adaptive. Most animals excrete several products, but one predominates. The enzymes for their production may be present in varying degrees, and several enzymatic routes may lead to the same product. Which route and which product become 
dominant in a given species depend more on its immediate osmotic needs than on its ancestry.

\section{DISTRIBUTION OF ENZYMES AND PRODUCTS OF PURINE METABOLISM}

A small amount of the nitrogen excreted by animals comes from nucleic acid metabolism, particularly from purine bases. The amount of nucleic acid eaten varies with the diet, being greater in ineat than in plant food. Enzymes of purine metabolism differ from those of proiein metabolism, even though some of the end-products are the same. An animal may excrete some uric acid from purines, regardless of whether its protein is degraded to ammonia, urea, or uric acid. The various end-products of purine metabolism were listed above (Fig. 33). Purines may be excreted as such, e.g., adenine or guanine, or they may be deaminated to xanthine, which is converted to uric acid by xanthine oxidase. If uricase is present, allantoin is then formed; allantoinase converts this to allantoic acid; allantoicase converts this to urea; urea may be broken down to ammonia by urease. Numerous invertebrates-annnelids, molluses, and arthropods-have deaminases of the free bases (adenase and guanase); many vertebrate livers lack adenase but can deaminate the nucleosides by adenosinase and guanosinase. ${ }^{3 \times,}+1$ The type of purine breakdown product in different animals has been identified partly by analysis of excretory products and excretory organs, and partly by identification of the enzymes in tissues, particularly liver (or hepatopancreas). A detailed summary of purine products

\section{TABLE 31. DISTRIBUTION OF URICOLYTIC ENZYMES}

+ indicates presence; - indicates absence.

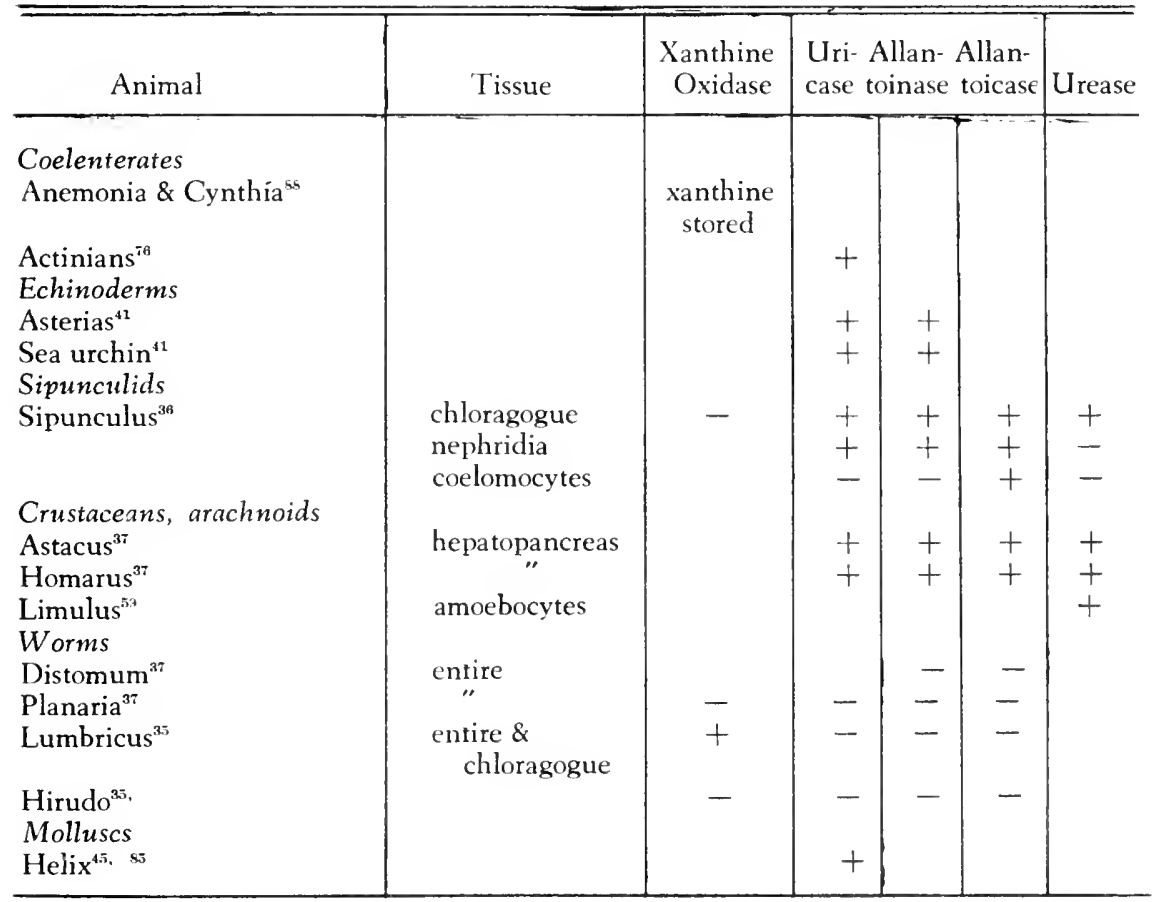


TABLE 31 (continued). DISTRIBUTION OF URICOLYTIC ENZYMES + indicates presence; - indicates absence.

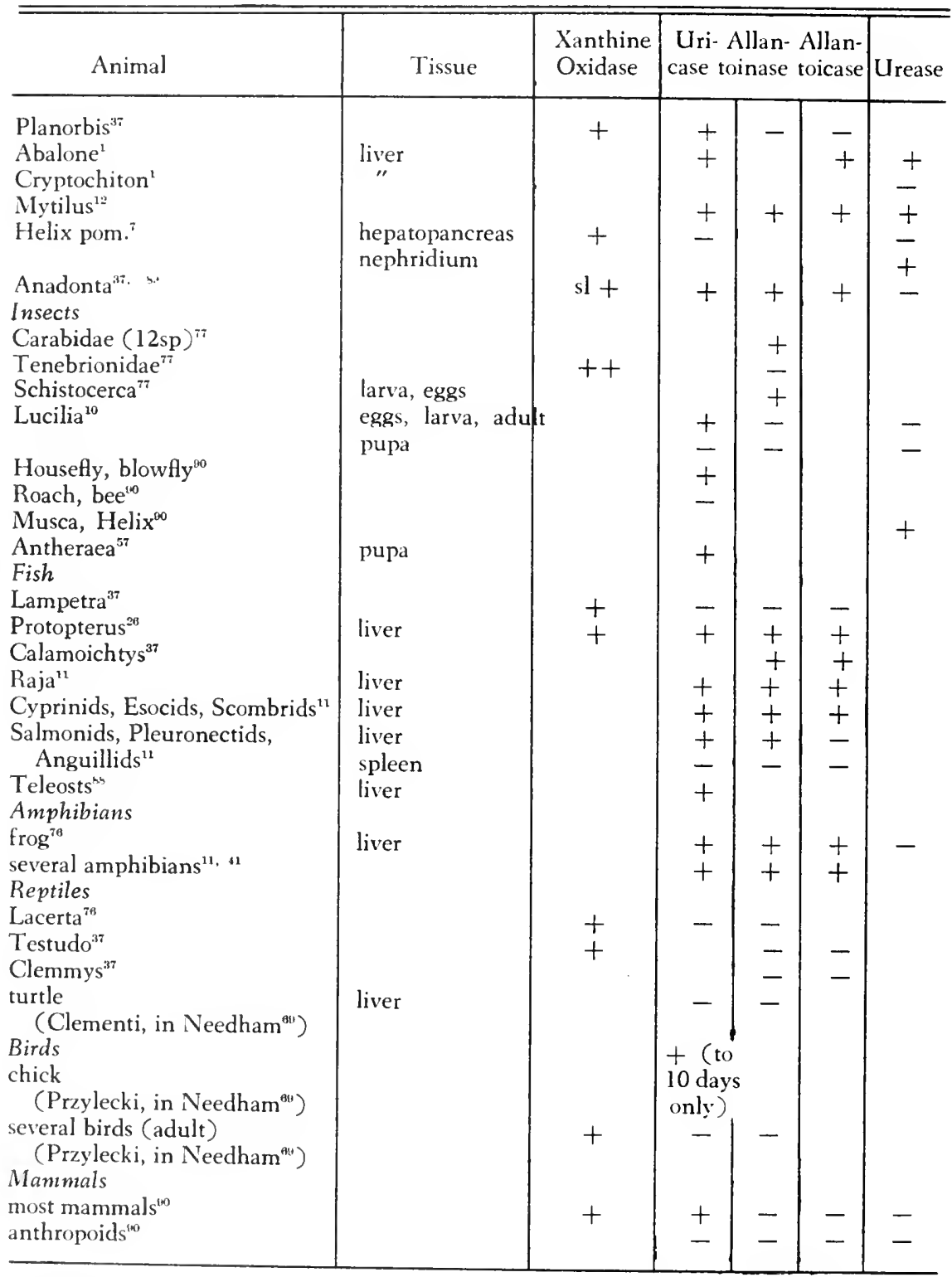

is given by Florkin and Duchateau, ${ }^{36}$ and a general summary by Florkin. ${ }^{33}$ Table 31 and Figure 36 summarize the results.

Some of the ammonotelic invertebrate animals also degrade purines to ammonia. Uricase is indicated in actinians, uricase and allantoinase are indicated in echinoderms; allantoicase and urease have not been looked for in these groups. All four enzymes occur in chloragogue tissue of Sipunculus, and in "liver" of Mytilus, crayfish, and lobster (Table 31). In the flatworms and 
those annelids which have been examined, purines are excreted and enzymes for degrading them are lacking.

Insects are predominant ly uricotelic animals and differ greatly in their uricolytic enzymes. The distribution appears to be random and varies with time in the life cycle (Table 31). Larvae of the blowfly, Lucilia, for example, convert much of the uric acid from protein breakdown to allantoin; uricase is absent from the pupae, however, and reappears in the aclults. ${ }^{10}$

Molluscs, like insects, differ in their uricolytic enzymes. In general, uricase is found in lamellibranchs and in some gastropods. Allintoinase, allantoicase, and urease are present in the marine lamellibranchs (Mytilus), but the urease is lacking in Anodonta, and only uricase is found in gastropods Melix, Planorbis). Comparison of uricolysis in ammonotelic and uricotelic gastropods would be of interest. Apparently, as with nitrogen breakdown, there is some lability in purine degradation among molluses.

One cyclostome fish, Lampetra funviatilis, like the annelids, has no enzyme for degrading purines. The liver of the dipnoan Protopterus anesteus 26 and of the elasmobranch Raja contains uricase, allantoinase, and allantoicase. Among teleost fish those of one group have uricase, allantoinase, and allantoicase, whereas those of another group lack the allantoicase; ${ }^{11}$ their differentiation (Table 31) seems unrelated to habitat.

Amphibians degrade purines to urea but not to ammonia. Reptiles, whether they are ureotelic or uricotelic, lack allantoinase and allantoicase; and some, if not all, of them lack uricase; hence they excrete their purines as uric acid. Likewise in bircls uricolysis is absent; there may be neither uricase nor allantoinase in the liver. Mammals fall into two groups in purine metabolism; most of them have uricase and excrete some allantoin. Man and other anthropoids, however, lack this enzyme and excrete uric acid. Among dogs Dalmatian coach hounds excrete approximately eleven times as much uric acid nitrogen and half as much allantoin nitrogen as other breeds, apparently because the Dalmatians fail to reabsorb uric acid in the kidney as other dogs do for conversion to allantoin in the liver. The necessary enzymes are present in the liver of all breeds. ${ }^{4:}$

Two evolutionary trends have occurred in purine metabolism: (1) Degradation completely to ammonia requires a chain of enzymes and is accomplished only among a few groups of lower invertebrates. (2) Higher groups, both invertebrate and vertebrate, break the chain at various points, and among the most advanced groups (insects, hirds, and most mammals) purines are exereted as uric acid. The evolutionary trend here is elimination of enzymatic steps.

Florkin $:: 3$ has suggested that the end-products of protein and of purine catabolism tend to be the same. This is indicated in Figure 36. Animals, whether ammonotelic, ureotelic, or uricotelic, tend to convert purines to the same end-products as those of proteins. Here is an example of biochemical convergence. Exceptions are found among molluscs and mammals.

\section{REFERENCES}

1. Albrecht, P. G., J. Biol. Chem. 56:483-487 (1923). Urease and uricase in marine molluscs.

2. Bacon, R. F., Philippine J. Sci. 4: 165 (1909). Excretion by python.

3. Bahl, K. N., Quart. J. Micr. Sci. 85, 343-390. (19+5) Excretion in earthworms.

4. Baldwin, E., Biochem. J. 29:252-262 (1935). Arginase in invertebrates. 
5. Baldwin, E., Comparative Biochemistry (1939. Cambridge Univ. Press. 107 p. Ch. IV, pp. 43-61. Nitrogen excretion.

6. Baldwin, E., Dynamic Aspects of Biochemistry (1947). New York, Macmillan. Ch. 10, 12. Nitrogenous products.

7. Baldwin, E., and Needham, J., Biochem. J. 28:1372-1392 (1934). Arginase in snail liver.

8. Brown, A. W. A., J. Exper. Biol. 13:131-139 (1936). Nitrogenous excretion, fly larvae.

9. Brown, A. W. A., J. Exper. Biol. 14:87-94 (1937). Nitrogenous excretion, grasshopper, Melanoplus.

10. Brown, A. W. A., Biochem. J. 32:895-902, 903-912 (1938). Nitrogen metabolism, insect Lucilia.

11. Brunel, A., Bull. Soc. Chem. Roy. Biol. 19:805-826, 1027-1036, 1683-1696 (1937). Purine metabolism, elasmobranchs, teleosts and amphibians.

12. Brunel, A., C. R. Acad. Sci. 206:858-860 (1938). Purine metabolism, Mytilus.

13. Chaudhuri, A. S., J. Exper. Biol. 5:97-101 (1927). Arginase in fowl.

14. Cohen, S., and Lewis, H. B., Fed. Proc. 8:191 (1949). Nitrogen metabolism, earthworm.

15. Conway, E. J., and Cooke, R., Biochem. J. 33:479-492 (1939). Deaminases in rabbit tissues, ammonia in blood.

16. Courtors, A., C. R. Soc. Biol. 101:365-366 (1929). Nitrogenous composition of meconium, Lepidoptera.

17. Cunnningham, B., and Kirk, P. L., J. Cell. E Comp. Physiol. 18:299-316 (1941). Nitrogen excretion of Paramecium.

18. Davis, R. E., J. Biol. Chem. 74:509-513 (1927). Nitrogenous constituents of hen urine.

19. Delaunay, H., Bull. Sta. biol. Arcachon 21:41-84 (1924). Nitrogen excretion, invertebrates.

20. Delaunay, H., C. R. Soc. Biol. 94:1289-1290 (1926). Nitrogen excretion, starfish.

21. Delaunay, H., Bull. Sta. biol. Arcachon 24:95-214 (1927). Nitrogen excretion, mostly invertebrates.

22. Delaunay, H., C. R. Soc. Biol. 101:371-372 (1929). Nitrogen excretion, fish.

23. Delaunay, H., Biol. Rev. 6:265-301 (1931). Review of nitrogen excretion.

24. Delaunay, H., Ann. Physiol. Physicochim. biol. 10:695-724 (1934). Review, nitrogen excretion.

25. Doyle, W. L., and Harding, J. P., J. Exper. Biol. 14:462-469 (1937). $\mathrm{NH}_{3}$ excretion, Glaucoma.

26. Duchateau, G., Florkin, M., and Frappez, G., C. R. Soc. Biol. 134:115-116 (1940). Purine breakdown, Protopterus.

27. Duchateau, B., Florkin, M., and Frappez, G., Bull. Acad. Roy. de Med. de Belg., Cl. Sc. 27:169-173 (1941). Distribution of deaminases of purines and nucleosides.

28. Edlebaciler, S., and Rothler, H., Ztschr. physiol. Chemie 148:264-282 (1925). Arginase distribution, birds and mammals.

29. Emers, F. E., J. Morph. 45: 555-577 (1928). Amino acid utilization, $\mathrm{NH}_{3}$ production, Paramecium.

30. Fearon, W. R., Physiol. Rev. 6:399-439 (1926). Biochemistry of urea.

31. Flonkin, M., C. R. Soc. Biol. 133:274, 433, 436 (1940). Deaminases in crustaceans, lamellibranchs, insects.

32. Flolikin, M., Arch. Int. Physiol. 53:117-120 (1943). Ammonia in blood.

33. Flonkin, M., L'Evolution Biochimique (1944). Paris, Masson, 210 p.

34. ILoikin, M., Biochemical Evolution, transl. and ed. by S. Morgulis. (1949). New York, Academic Press, $157 \mathrm{p}$.

35. llomkin, M., and Duchatian, S., Bull. Acad. Roy. de med. de Belg. Cl. Sc. 27:174-178 (1941). Xanthine oxidase distribution.

36. Flonkin, M., and Duchiateau, G., Arch. Internat. Physiol. 52:261-290. (1942) Nitrogen metabolism of Sipunculus.

37. Flomkin, M., and Duchatenu, G., Arch. Internat. Physiol. 53:267-290 (1943). Evolution of purine metabolism. 
38. Flonkin, M., and Frappez, G., Arch. Internat. Physiol. 50:197-202 (1940). Ammonia in invertebrate bloods.

39. Flonkin, M., and Honet, R., Arch. Internat. Physiol. 47:125-132 (1939). Ammonia in Anodonta blood.

40. Folin, O., Am. J. Physiol. 13:45-65 (1905). Analyses of human urine.

41. Fosse, R., and Brunel, A., C. R. Acad. Sci. 188:1067-1069 (1929). Distribution of allantoinase.

42. Friedman, M., and Byens, S. O., J. Biol. Chem. 175:727-735 (1948). Uric acid excretion, Dalmatian dog.

43. Fuse, N., Jap. J. Med. Sci. II, 1:103-110 (1925). Nitrogen in urine of various mammals.

44. Grafflin, A. L., and Gould, R. G., Biol. Bull. 70:16-27 (1936). Nitrogen excretion, trimethylamine oxide, sculpin, flounder.

45. GraH, H., Zool. Jahrb., Aht. allg. Zool. u. Physiol. 57:355-372 (1937). Uric acid synthesis, Helix.

46. Grollman, A., J. Biol. Chem. 81:267-278 (1929). Nitrogenous excretion by goosefish, Lophius.

47. Hammetт, F. S., J. Biol. Chem. 22:551-558 (1915). Urinary nitrogen, cat.

48. Heidermanns, C., Zool. Jahıb., Aht. allg. Zool. u. Pliysiol. 58:57-68 (1937). Urea synthesis by earthworm.

49. Heidermanns, C., Tabul. Biol. 14:209-273 (1937). Uric acid in invertebrate tissues.

50. Heyde, H. C. v., J. Biol. Chem. 46:421-430 (1921). Nitrogenous constituents of urine and blood of Rana.

51. Hollande, A. C., and Cordebard, H., Bull. Soc. Chim. Biol. 8:631-634 (1926). Nitrogen excretion by clothes-moth.

52. Hopping, A., Am. J. Physiol. 66:145-163 (1923). Nitrogenous excretion by alligator.

53. Hunter, A., Proc. Roy. Soc. Lond., B. 97:227-242 (1924). Arginase in fish.

54. Hunter, A., and Dauphinee, J. A., Proc. Roy. Soc. Lond., B. 97:227-242 (1925). Distribution of arginase.

55. Krebs, H. A., and Henseleit, K., Ztsclir. pliysiol. Chemie 210:33-66 (1932). Arginase in animal tissues.

56. Lawrie, N. R., Biochem. I. 29:2297-2302 (1935). Nitrogenous excretion, ciliate Glaucoma.

57. Leifert, H., Zool. Jahrb., Abt. allg. Zool. u Physiol. 55:131-170, 171-190 (1934). Nitrogen excretion and purine metabolism in Lepidoptera.

58. Lewis, H. B., Science 48:376 (1918). Nitrogen in urine of reptiles.

59. Loeb, L., and Bodansky, O., J. Biol. Chem. 67:79-90 (1926). Urease in Limulus.

60. Lwoff, A., and Roukhelmann, N., C. R. Acad. Sci. 183:156-158 (1926). Nitrogenous excretion, ciliate, Glaucoma.

61. Manderscheid, H., Biochem. Ztschr. 263:245-249 (1933). Ornithine cycle, vertebrates.

62. Marshall, E. K., J. Cell. E Comp. Physiol. 2:349-353 (1933). Urea secretion, frog.

63. Mayrs, E. B., J. Physiol. 58:276-287 (1924). Nitrogen excretion by hen.

64. Mollitor, A., Zool. Jahrb., Abt. allg. Zool. u. Pliysiol. 57:323-354 (1937) Nitrogen excretion, crab Eriocheir.

65. Mongulıs, S., J. Biol. Chem. 50:52-54 (1922). Ulric acid in polychaetes.

66. Munzel, P., Zool. Jahrb.. Abt. allg. Zool. u. Physiol. 59:113-148 (1938). Urea synthesis by vertebrates.

67. Nardone, R. M., Fed. Proc. 8:117 (1949). Nitrogenous excretion, Colpidium.

68. Needham, J., J. Exper. Biol. 4:114-144 (1926). Nitrogen metabolism in avian development.

69. Needian, J., Chemical Embryology (1931). Cambridge Univ. Press. Vol. 2, Section 9, pp. 1055-1145. Nitrogenous waste products of embryos.

70. Needran, J., Biochem. J. 29:238-251 (1935). Uricotelism and habitat of gasteropods.

71. Needham, J., Biol. Rev. 13:22t-251 (1938). Nitrogen excretion, snails, as example of reversibility in evolution. 
72. Nefoham, J., Biochemistry and Morphogenesis. (1942). Cambridge Univ. Press. $787 \mathrm{p}$.

73. Nefdham, J., Brachet, J., and Brown, R., J. Exper. Biol. 12:321-336 (1935). Nitrugen excretion in chick embryos.

74. Osterberg, E., and Wolf, C. G. L., Biochem. Ztschr. 5:30+-343 (1907). Urinary nitrogen, dog.

75. Peters, J. P., and van Slyke, D. D., Quantitative Clinical Chemistry (1946). Baltimore, Williams and Wilkins. Vol. 1. Biochemical interpretations.

76. Pryzlecki, S. J., Arch. Internat. Physiol. 26:33-53 (1926). Purine degradation.

77. Rocco, M. L., C. R. Acad. Sci. 202:1947-1948 (1936). Allantoinase in insects.

78. Schmidt-Nielsen, S., and Holmsen, J., Arch. Internat. Physiol. 18:128-132 (1921). Composition of whale urine.

79. Smith, H. W., J. Biol. Chem. $81: 727-742$ (1929). Nitrogen excretion by gills of fish.

80. Smith, H. W., J. Biol. Chem. 88:97-130 (1930). Nitrogen excretion in lungfish, Protopterus.

81. Smith, H. W., Am. J. Physiol. 93:480-505 (1930). Excretion by marine fish.

82. Sмiтh, H. W., J. Cell. \& Comp. Physiol. 7:465-473 (1936). Nitrogen in seal urine.

83. Smith, H. W., and Silvette, H., J. Biol. Chem. 78:409-411 (1928). Nitrogen excretion in camels.

84. Specht, H., J. Cell. \& Comp. Physiol. 5:319-333 (1934). Ammonia production, Spirostomum.

85. Spitzer, J. M., Zool. Jahrb., Abt. allg. Zool. u. Physiol. 57:457-496 (1937). Nitrogenous excretion and distribution of molluscs.

86. Steudel, H., and Izumi, S., Ztschr. Physiol. Chem. 129:188-194 (1923). Purine nitrogen excretion in rat.

87. Strunk, C., Zool. Jahrb., Abt. allg. Zool. u. Physiol. 52:216-222 (1932). Urea, uric acid in coelomic fluid, Arenicola.

88. Sulima, A., Ztschr. Biol. 63:223-244 (1914). Uric acid formation, invertebrates.

89. Truszkowski, R., C. R. Soc. Biol. 98:1048 (1928). Purine breakdown, leech, mussel.

90. Truszkowski, R., and Chajkinowna, S., Biochem. J. 29:2361-2365 (1935). Uricolysis.

91. Van Slyke, D. D., et al., J. Biol. Chem. 150:481-482 (1943). Glutamine source of urine $\mathrm{NH}_{3}$.

92. Walker, A. M., and Hudson, C. L., Anı. J. Pliysiol. 118:153-166 (1937). Urea secretion by amphibian kidney.

93. Weatherby, J. H., Physiol. Zool. 2:375-394 (1929). Nitrogen excretion by Protozoa.

94. Weese, A. O., Science 46:517-518 (1917). Urinary nitrogen, Phrynosoma.

95. Wigglesworth, V. B., J. Exper. Biol. 8:411-427 (1931). Uric acid in urine of bug, Rhodnius.

96. Wigglesworth, V. B., Principles of Insect Physiology (1939). London, Methuen. Ch. 12. Excretion.

97. Wilber, C. G., J. Cell. E Comp. Physiol. 31:107-109 (1948). Uric acid in body fluids of marine invertebrate animals.

98. Wiley, F. H., and Lewis, H. B., Am. J. Physiol. $81: 692-695$ (1927). Nitrogenous excretion in turtle.

99. Yungherr, I., and Matterson, L. D., Proc. 48th Ann. Meet., U. S. Livestock San. Ass., pp. 185-196 (1944). Chicken uric acid. 


\section{Respiration and Metabolism}

\section{AVAILABILITY OF OXYGEN}

HE DIRECT DEPENDENCE of animals on an adequate oxigen supply has been recognized only since the seventeenth century, when Mayow and Hooke demonstrated the need of animals for air, and later when Priestley and Lavoisier discovered the chemical nature of oxygen and the importance of oxidation as the source of energy within the body.

Wide-spread distribution of oxygen permits life in a great variety of environments under aerobic conditions, exceptions being in deep lakes and in the intestinal tracts of vertebrates, where anacrobic respiratory mechanisms are not uncommon. Oxygen not only is widely distributed but remains fairly constant in amount within a given environment. In some lakes, however, the oxygen supply may be suddenly depleted by plant life and bacteria. Indeed, sudden reductions in numbers of animals have been cited in European waters where, as near the Messina Straits, the paucity of oxygen in the great depths allows hydrogen sulfide from decomposing animal bodies to accumulate to such a degree that, on agitation of the waters during storms, the surface fauna is exposed to toxic concentrations of hydrogen sulfide with disastrous results.

Various animals have evolved different mechanisms for the procurement of oxygen, and, where the supply is particularly limited, emergency adaptations have developed. The responses of organisms to oxygen stress constitute one of the most interesting contributions to adaptive physiology. In swamps of the Paraguayan Chaco, for example, where little or no oxygen can be detected in the subsurface water, a number of adaptations are found in fish for the procurement of oxygen from either the air or the surface water-laver. ${ }^{62}$ Five different types of aerial respiratory systems have been developed to provide the bulk of oxygen for these fish: pharyngeal diverticular "lungs" (Lepidosiren and Symbranchus), gas bladder (Erythrinus), gill lamellae (Hy'popomus), intestinal modifications (Callichthys and Hoplosternumu), and gastric mucosa (Ancistrus).

The adequacy of the oxygen supply is a function of the number of molecules of the gas available to the organisms, whether as molecular oxygen dissolved in water or as molecules of oxygen in air. The amount of oxrgen available to the organism-atmospheric or in solution-depends on the partial pressure exerted by the gas, the value of the partial pressure or oxygen tension being directly proportional to the volume per cent of the gas present. Partial pressures of the important gases of dry air at sea level are given in Table 32.

The amount of gas in solution depends on the absorption coefficient and is proportional to the partial pressure exerted by the gas. The quantity of gas in solution decreases with elevation of temperature and with the addition of solutes to the solvent. Solubility data for oxygen in water, in salt solution 
TABI E 32. PARTIAL PRESSURE OF GASES IN DRY AIR (760 mm. $\mathrm{Hg})$

\begin{tabular}{l|c|c}
\hline \multicolumn{1}{c|}{ Gas } & Vol. per cent ${ }^{210 i}$ & Partial Pressure in mm./Hg \\
\hline Oxygen & 20.95 & 159.22 \\
Carbon dioxide & 0.03 & 0.23 \\
Nitrogen & 78.0 & 592.8 \\
Argon, etc. & 1.0 & 7.6 \\
\hline
\end{tabular}

equivalent to sea water (20 gm. Cl/1.) and in blood, are presented in Table 33.

Although diffusion through a gas phase may be regarded as a simple function of the density and molecular size of the gas, diffusion through a liquid

TABLE 33. SOLUBILITY OF OXYGEN IN BIOLOGICAL FLUIDS

\begin{tabular}{|c|c|c|c|c|c|}
\hline Temp. ${ }^{\circ} \mathrm{C}$. & $\begin{array}{c}\text { Abs. Coeff. (a) } \\
\text { water } \\
760 \mathrm{~mm} \cdot \mathrm{Hg}\end{array}$ & $\begin{array}{c}\mathrm{ml} . \mathrm{O}_{2} / \mathrm{l} \\
\text { water } \\
159 \mathrm{~mm} \cdot \mathrm{Hg}\end{array}$ & $\begin{array}{l}\text { Abs. Coeff. (a) } \\
2 \% \mathrm{Cl} \\
760 \mathrm{~mm} . \mathrm{Hg}\end{array}$ & $\begin{array}{c}\mathrm{ml} . \mathrm{O}_{2} / \mathrm{l} . \\
2 \% \mathrm{Cl}^{* 16} \\
159 \mathrm{~min} . \mathrm{Hg}\end{array}$ & Blood $^{130}$ \\
\hline $\begin{array}{r}0 \\
10 \\
15 \\
20 \\
30 \\
37\end{array}$ & $\begin{array}{l}0.04889 \\
0.03802 \\
0.03415 \\
0.03102 \\
0.02608 \\
0.02386\end{array}$ & $\begin{array}{r}10.27 \\
7.98 \\
7.17 \\
6.51 \\
5.48 \\
5.01\end{array}$ & $\begin{array}{c}0.0380 \\
0.0302 \\
0.0276 \\
0.0253 \\
0.0212 \\
--\end{array}$ & $\begin{array}{l}7.97 \\
6.35 \\
5.79 \\
5.31 \\
4.46 \\
--\end{array}$ & $\begin{array}{l}0.019 \\
0.022\end{array}$ \\
\hline
\end{tabular}

$a=$ the Bunsen Coefficient $=$ the volume of gas (reduced to $0^{\circ} \mathrm{C} ., 760 \mathrm{~mm}$.) which at the tcmperature indicated is dissolved in one volume of the solvent when the partial pressure of the gas is $760 \mathrm{~mm}$. (I.C.T.).

must consider the concentration gradient, area, and time, and may be expressed by the following derivation from Fick's law:

$$
\frac{\mathrm{dn}}{\mathrm{dt}}=-\frac{\mathrm{RT}}{\mathrm{Nf}} \mathrm{a} \frac{\mathrm{dc}}{\mathrm{d} \mathrm{x}}
$$

$d n$ representing the amount of substance diffusing across an area, $a$, in time $d t$, with a concentration gradient dc. $R$ is the gas constant, $T$ absolute tempera$\overline{\mathrm{dx}}$

ture, $N$ the Avogadro constant, and $f$ the "frictional resistance" upon the diffusing molecules. Simple diffusion processes can account for gas exchange

TABLE 34. DIFFUSION CONSTANTS OF OXYGEN AT $20^{\circ} \mathrm{C}$.

\begin{tabular}{ll||lc}
\hline \hline Substance & Diff. Const. & Substance & Diff. Const. \\
\hline Muscle $^{21.5}$ & 0.14 & Water $^{159}$ & 0.34 \\
Connective tissue $^{215}$ & 0.115 & Gelatin $^{215}$ & 0.28 \\
Chitin $^{215}$ & 0.013 & & \\
\hline
\end{tabular}

through tissues for short distances, although exceptional cases of gas secretion against a gradient have been demonstrated in a number of fish and in some invertebrates."21: Diffusion rates of oxygen in water and tissues are presented 
in Table 34. ${ }^{15}$ The value for the diffusion constant ${ }^{*}$ increases with rising temperature, about 1 per cent per degree, taking the rate at $20^{\circ}$ C. as unity. In spite of its larger molecular size, carbon dioxide diffuses through tissues 20 to 30 times faster than oxygen does, owing to the higher solubility of $\mathrm{CO}_{2}$.

Members of the more primitive phyla (Protozoa, Rotatoria, Porifera, Coelenterata, Platyhelminthes, Nemertea, and some Annelida), as well as eggs and embryos of all groups, can procure their oxygen entirely by diffusion without recourse to special respiratory mechanisms or circulatory systems. The relationship between adequate oxygen pressure and oxygen consumption for small spherical organisms is summarized by the equation:

$$
\mathrm{C}_{0}=\frac{\mathrm{Ar}^{2}}{6 \mathrm{D}}
$$

in which $C_{0}$ is the required oxygen pressure, $A$ the oxygen consumption of the tissue in cc. oxygen per gm. per minute, $r$ the radius of the sphere, and $D$ the diffusion coefficient of oxygen through the tissues. ${ }^{14}$ In organisms with fairly high metabolic activities diffusion will suffice if the tissue is no more than 1 $\mathrm{mm}$. in diameter. ${ }^{210}$ However, in animals with tissues more than $1 \mathrm{~mm}$. distant from an oxygen supply some supplementary transporting system is necessary. With an adequate oxrgen source rapid utilization establishes a high gradient, and diffusion over short distances may be very rapid. Bronk"i- has shown by the microelectrode technique that oxygen diffusion in brain tissues is sufficiently rapid to demonstrate fluctuations in oxygen tension concordant with the respiratory rhythm, when the partial pressure is measured at a distance of some $25 \mu$ from an arteriolar oxygen supply.

\section{TYPES OF RESPIRATORY MECHANISMS}

The striking adaptability of animals for the procurement of oxygen is exemplified by the many modifications which have evolved to alleviate limitations imposed by simple diffusion. Organisms have met similar respiratory problems in different ways. The elaboration of respiratory mechanisms has developed along with the evolution of efficient vascular ("convection") systems. Krogh's admirable surver"1fi of respiratory adaptations should be consulted in this connection, as well as the extensive review of respiratory systems by Guieysse. Pellissier. ${ }^{\text {I: }}$ Four main types of respiratory mechanisms are considered here: (1) integument, (2) gills, (3) lungs, and (4) tracheae.

Integument. Cutaneous respiration plays a significant role in the respiratory economy of many invertebrates, and it is safe to say that it occurs to some extent in all animals. A circulating mechanism is required in aquatic animals, either cilia or movement of the organ as a whole, to move the water over the respiratory surfaces. In air-breathing forms a moist integument is essential for significant gas exchange through the skin, and mucous glands are generally found in cutaneous air breathers, as the terrestrial isopods, molluses, and amphibians. Cutaneous respiration is more common than generally recognized

* The diftusion constant $\left(10^{\prime}\right.$ times the diffusion coefficient) is defined as the number of cc. of oxygen (reduced to $0^{\circ} \mathrm{C} ., 760 \mathrm{~mm}$. $\mathrm{Hg}$ ) passing through a distance of $1 \mu$ over an area of 1 sq. $\mathrm{cm}$. with a pressure difference of 1 atmosphere. 
because it will occur by necessity through all permeable membranes when the partial pressure of the gas establishes a sufficient gradient.

Special respiratory mechanisms have evolved in some annelids (Nereis and Chaetopterus), but many members of this phylum respire through the general body surface. In the oligochaete, Drilocritus, a specialized "dorsal groove" in the epithelium permits the drawing of air bubbles below the water surface and the absorption of oxygen through this portion of the skin. ${ }^{6: 3}$ The pulmonate gastropods (Limnaea and Helicosoma) can depend on their integuments when submerged for long periods of time, particularly at lower temperatures, although usually they take in aerial oxygen through the lungs. ${ }^{66}$ Fraenkel and Herford ${ }^{119}$ showed in blowfly larvae (Calliphora) that under experimental conditions 10 per cent of the total oxygen intake could be accounted for by cutaneous channels. Embryos, young larvae, and some "transparent" arthropods also respire through the body surface. The fish Acara (Chromidae) undergoes caudal differentiation with great increase in vascularity so that the tail becomes a specialized respiratory structure.

From the time of Spallanzani, numerous investigators ${ }^{96,210,}, 11$ have studied the problem of gas exchange through the integument of vertebrates, many of which respire through the skin, particularly in eliminating carbon dioxide (Table 35). According to Krogh the eel's skin can provide 60 per cent as much

TABLE 35. CUTANEOUS RESPIRATION OF SEVERAL VERTEBRATES ${ }^{21}$ (Respiratory Exchange expressed as cc./sq. dcm./hour)

\begin{tabular}{l|c|c|c|c|c}
\hline \hline \multirow{2}{*}{ Animal } & \multicolumn{2}{|c|}{ Oxygen } & \multicolumn{2}{c|}{ Carbon dioxide } & $\begin{array}{c}\text { Calculated from } \\
\text { Experiments of }\end{array}$ \\
\cline { 2 - 5 } & Max. & Mean & Max. & Mean & Man \\
Man & -- & 0.50 & - & 1.18 & Gerlach $^{124}$ \\
Pigeon & -- & -- & 3.1 & 0.94 & Schierbeck $^{312}$ \\
Tortoise & 0.92 & 0.47 & 1.1 & 0.60 & Krogh $^{211}$ \\
Frog (R. fusca) & 0.1 & -- & 0.15 & -- & $" \prime$ \\
Frog (R. esculenta) & 1.9 & 1.51 & 5.3 & 3.0 & $"$ \\
Eel & 2.1 & 1.62 & 4.4 & 3.1 & $"$ \\
\hline
\end{tabular}

TABLE 36. COMPARISON OF CUT ANEOUS AND PULMONARY RESPIRATION IN FROGS AT $20^{\circ}$ C. (AFTER KROGH ${ }^{210}$ )

(Respiratory exchange expressed as cc./kg./hr. ${ }^{*}$ )

\begin{tabular}{l|c|c|c|c|c|c}
\hline \hline & \multicolumn{3}{|c|}{ Cutaneous Respiration } & \multicolumn{3}{|c}{ Puhmonary Respiration } \\
\hline \multicolumn{1}{c|}{ Animal } & $\mathrm{O} .2$ & $\mathrm{CO}$ & $\frac{\mathrm{CO}_{2}}{\mathrm{O}_{2}}$ & $\mathrm{O}_{2}$ & $\mathrm{CO}_{2}$ & $\frac{\mathrm{CO}_{2}}{\mathrm{O}_{2}}$ \\
\hline Rana esculenta & 62 & 119 & 1.92 & 59 & 19 & 0.32 \\
Rana fusca & 52 & 129 & 2.48 & 105 & 45 & 0.43 \\
\hline
\end{tabular}

oxygen as is provided when the gills and skin both function in normal respiration. This is sufficient to support the fish for an indefinite period at low' temperatures but is inadequate above $15^{\circ}$ C.. ${ }^{\prime 1} \ln$ man and pigeons less than 1 per cent of the oxygen intake is through the integument.

Studies have demonstrated species differences in the proportion of cutaneous to pulmonary respiration in frogs (Table 36) and have disclosed the relative

${ }^{*}$ Morrison ${ }^{2 a 4}$ has utilized the les; ambiguous mathematical expression, cc./kg.hr. 
importance of skin and lungs in oxygen and carbon clioxide exchange (Fig. 37). Oxigen uptake by the lungs and carbon dioxide elimination by both lungs and skin reach a peat in April and a minimum in December. ()xigen uptake through the skin remains almost constant throughout the vear. I hus the cutancously derived oxygen, entering by diffusion, can supply two thirds of the oxygen requirement during the winter months under laboratery condi tions, but only a small fraction of the spring oxyeren demand. Presumably during hibernation atl of the oxrgen consumed enters through the cutancous route.

Gills. Gills are respiratory appendages, gencrally well rascularied, and usually ciliated and motile, or located in the current of water llow. Thes are usually aquatic but may be aerial, and they are sometimes both. The respira.

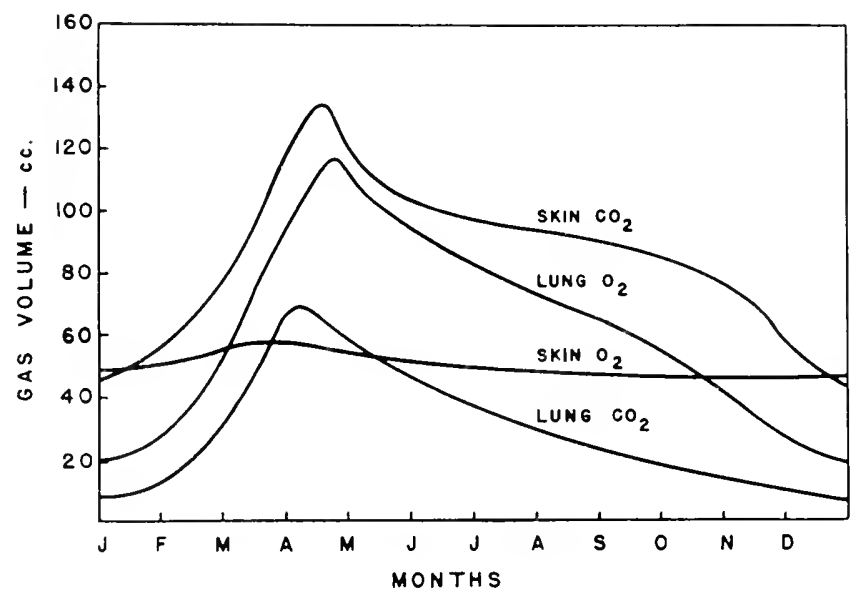

Fig. 37. Oxygen and carbon dioxide exchange shrough the skin and lungs of the frog, Rana temporaria, throughout the year. After Dolk and Postma."

tory functions in some cases have been combined or confused with other processes, such as salt absorption in the so-called "anal gills" of Culex and Chironomus (see Ch. 2):-0t Novement of water orer the surface of alquatic gills is mandatory to insure eflicient respirators exchange. The countercurrent principle of operation in fish, the water outsicle the gill surface and the blood of the adjacent capillaries inside flowing in opposite directions, provides for rapid oxyen uptake and almost complete saturation as the blood leaves the gill filaments. ${ }^{-1 ;}$

Dermal branchiac, the so-called papulace, are found in many of the echinoderms supplementing respiratory exchange through the tube feet. The papulae are evaginations of the body wall ("extensions of the coelom"), bringing the gently moving coelomic fluid in close association with the cilia-stirred external sea water. In Asterias a specialized recion of the ambulacral sistem and a portion of the madreporite plate have been regarded as respiratory in nature. $25.2=$

Polychactes have evolved some claborate gill structures, such as parapodia (Nereis), gills (Arenicola), and branchial tufts (Dasybranchus). Bispara voluti- 
cornis has been shown by extirpation experiments to take up through the filaments of the branchial tufts approximately 37 per cent of the total oxygen consumed, ${ }^{3 \times 6}$ the rest entering through the integument.

Molluscs show a great range of respiratory mechanisms with a marked tendency toward the development of aerial gills. The primitive Chiton has six to eight aquatic gills in each pallial groove. Bivalves generally have two pairs of gills or ctenidia, each with two lamellae, situated in a water supply flowing through the mantle cavity. Gastropods are gill-bearing except for the pulmonate land snails, in which the "lung" is actually modified from the lining of the mantle cavity. Ancula, an opisthobranch snail, with both aquatic and aerial tendencies, possesses on its back a peculiar system of rigid gills which can extract oxygen from both air and water.

Crustacean gills are generally enclosed within a chamber and require water circulation for adequatic respiratory exchange. In the fresh-water crab, Potamonantes, for instance, the paddle-like movement of the scaphognathite is indispensable, and without it respiratory exchange in the gills is inadequate to support life in water. ${ }^{3 \times 7}$ This animal can survive in relatively dry air so long as the branchial chamber remains moist.

Pearse 274,275 has made an extensive study of the respiratory apparatus of crustaceans and has correlated gill reduction with migration from marine to terrestrial habitats. The hermit crab, Coenobita, can get along in air with the gills removed. The ghost crab, Ocypode, has lost many gills but in their place has developed branchial tufts-vascularized membranous projections of the branchial cavity (Table 37).

\section{TABLE 37. REDUCTION OF GILL STRUCTURE IN CRUSTACEA WITH TRANSITION FROM MARINE TO TERRESTRIAL HABITATS (AFTER PEARSE ${ }^{274}$ )}

\begin{tabular}{|c|c|c|c|c|}
\hline & $\begin{array}{r}\text { No. of } \\
\text { Gills }\end{array}$ & $\begin{array}{c}\text { Ratio } \\
\text { between } \\
\text { Volume* } \\
\text { of Body } \\
\text { \& Gills }\end{array}$ & $\begin{array}{l}\text { Bran- } \\
\text { chial } \\
\text { Tufts }\end{array}$ & Habitat \\
\hline Paguristes punticeps & 26 & & 0 & Below low tide \\
\hline Calcinus sulcatus & 26 & & 0 & Low-tide mark \\
\hline Clibanarious tricolor & 18 & & 0 & Near high-tide mark \\
\hline Coenobita diogens & 14 & & 0 & Land \\
\hline Callinectes marginatus & 16 & $21: 1$ & 0 & $\begin{array}{l}\text { Pelagic and } \\
\text { between tides }\end{array}$ \\
\hline Mithrax sculptus & 18 & $36: 1$ & 0 & Coral reefs \\
\hline Leptodius floridanus & 18 & $38: 1$ & 0 & Reefs and along shore \\
\hline Plagusia depressa & 18 & $42: 1$ & 0 & $\begin{array}{l}\text { Rock-walls, low-tide } \\
\text { marks }\end{array}$ \\
\hline Grapsus grapsus & 18 & $35: 1$ & 0 & $\begin{array}{l}\text { Rock-ivalls, along } \\
\text { shore }\end{array}$ \\
\hline Pachygrapsus transversus & 18 & $40: 1$ & 0 & $\begin{array}{l}\text { Loose rocks and } \\
\text { between tides }\end{array}$ \\
\hline Geograpsus lividus & 18 & $57: 1$ & 0 & $\begin{array}{l}\text { Loose rocks, } \\
\text { high-tide mark }\end{array}$ \\
\hline Gecarcinus lateralis & 18 & $48: 1$ & 0 & Land \\
\hline Ocypode albicans & 12 & $61: 1$ & 5 rows & Sand, beaches \& land \\
\hline
\end{tabular}

${ }^{*}$ Measured by displacement of water after hardening in l per cent chromic acid. 
Specialized epithelial modifications are not uncommon in supplementing the activity of the gills. The hermit crab, Coenobita, has such a region on the ventral side of the anterior part of the body which must carry on much of the respiratory exchange in the absence of a well developed gill system. Long ago Birgus littro, a terrestrial robber crab, was shown to possess a modified gill chamber in which vascularized epithelial appendages on the roof of the cavity supplement respiratory exchange by the reduced gills.

The woodlice, Isopoda, represent a seriation from aquatic to terrestrial existence, and the gill-like structures on the endopodites of the pleopods show increasing adaptations from aquatic to aerial respiratory structures in, for example, the three forms: Ligia, which is essentially amphibious and hygric; Oniscus, which dwells in damp places; and the more xeric Porcellio, which prefers drier conditions." ${ }^{\text {"II, }}: 3 t !$ The endopodites of aquatic isopods have thinner walls than do those of terrestrial species. The latter may develop systems of capillary grooves which permit moisture to drain either toward the rectum for absorption or toward the pleopods to moisten the respiratory structures.

In Asellus and Porcellio the "white body" or "tracheal organ" functions as a respiratory structure particularly concerned with the elimination of carbon dioxide. ${ }^{15 x}$ Extirpation of these structures from the pleopods causes death within a few hours to several days.

Certain groups of insects possess tracheal gills, either aquatic or aerial, in which a tracheal system replaces the blood vascular system (Ephemeridae, Odonata, Trichoptera, Coleoptera). Experimental procedures applied to mayfly nymphs indicate that the caudal gills play an important part in the respiratory exchange. ${ }^{256 .} 372$ If the gills are removed from Hexagenia recurvata, the oxygen uptake is decreased 50 per cent. The damsel fly naiad (Enallagma) has been shown by extirpation experiments to take up oxygen normally through the caudal gills and secondarily through the body surface. $27 x$ In normal animals oxygen is removed from the medium down to 2.4 per cent saturation, whereas in gill-less individuals oxygen is removed from the medium only down to 14.5 per cent saturation. In the caddis fly, Macronema, on the other hand, gill removal results in little or no reduction in oxygen consumption. ${ }^{257}$

The pharyngeal gill structure of prochordates is elaborate, but efficiency seems to have yielded to size. Amphioxus has well over 100 functional ciliated branchial bars. The multi-slotted branchial sac of the tunicate is also well equipped with cilia to provide for the circulation of water through the gill slits and over the vascularized gill filaments.

In the vertebrates the gills are either external, as in embryonic lungfish and Amphibia, or enclosed within a chamber as in the teleosts. The adult lamprey with its peculiar jawless sucking mouth has covered gills, and these are ventilated by water which passes both in and out of the several gill openings. The male lungfish, Lepidosiren, develops temporary pelvic gills during the breeding season when he assumes custodianship of the eggs and young and remains below surface in oxygen-deficient water." It has been claimed that the oxygen content increases in the water around the eggs, a result of actual oxygen secretion by the gill filaments. ${ }^{\circ}, 311$ Gills reach their greatest development among the teleosts and, considering the respiratory area, have been regarded as surpassing the lungs in efficiency. ${ }^{\text {tio }}$ Gill efficiency, however, depends on 
the rate at which the water passes over them, and in the mackerel, for instance, respiration is inadequate to support life unless the fish is in constant forward motion. ${ }^{13: 9}$ It is essential that the mackerel keep moving! Teleost gills are capable of independent but coordinated movement, and Bijtel ${ }^{31}$ has observed in windowed specimens a mechanism by which fish gills close the gaps at their tips, thereby impeding water flow during the "coughing" reflexes apparently associated with cleaning out the gill passages. The gills of Amphibia-functional throughout the lifetime of some urodeles and only during the larval

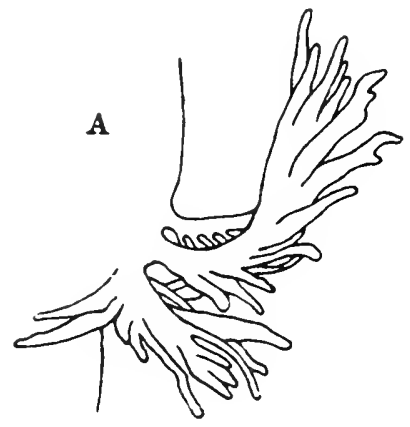

B

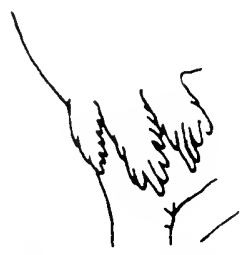

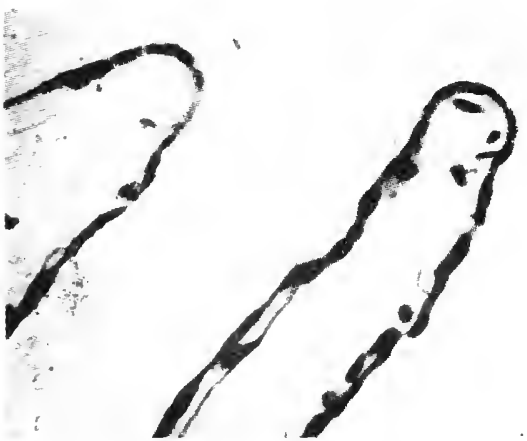

$\mathrm{C}$

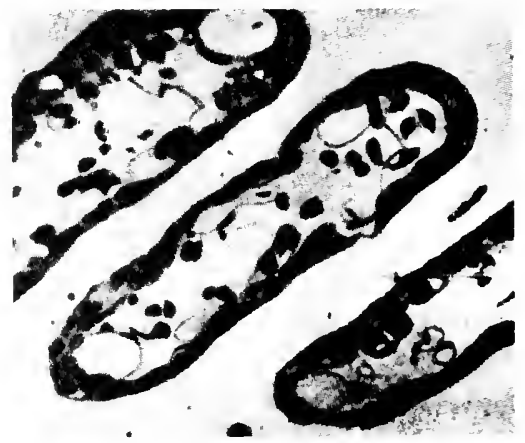

D

Fig. 38. Gill filaments of larval Salamandra. Schematic representation of gill filaments of larvae raised under conditions of (A) low oxygen tension $(80 \mathrm{~mm}$. $\mathrm{Hg}$ ) and (B) high oxygen pressure $(760 \mathrm{~mm} . \mathrm{Hg})$. Transverse sections of filaments of larvae developed in (C) low and (D) high oxygen tensions. From Drastich. ${ }^{88}$

stage of others, but always supplemented functionally by cutaneous or lung -respiration-may show modifications in size and arborization within single individuals in response to changes in the availability of oxygen. The gill filaments of larval Salamandra, for example, are large, branched, and thin-walled on animals raised in low oxygen tensions $(80 \mathrm{~mm}$. $\mathrm{Hg}$ ), but on salamanders developed at high oxygen pressure $(760 \mathrm{~mm} . \mathrm{Hg})$ they are small, stubby, and thick-walled ${ }^{98}$ (Fig. 38).*

Boell (personal communication) reports no decrease in oxygen consumption by Amblystoma deprived of gills. 
A number of accessory respiratory adaptations are found, particularly among fish, to increase the efficiency or to supplement the action of gills. Nany of these adaptations permit air breathing, allowing some olviously aguatic teleosts to become temporary land dwellers. Thus the branchial chamber may become adapted as a gas storage chamber to aerate the gills, as in the "jumping fish" Periopluthalmus.319. 376 By increasing surface area and vascularization the branchial chamber may provicle for some gas exchange itself, as in the electric eel, Electrophorus. ${ }^{61}$ Well developed branchial diverticula, facilitating gals exchange, have been described for many air-breathing fish, as Anabas, Clarias, and Periopthalmus ${ }^{\wedge}$. 244 (Fig. 39). These accessory respiratory structures not only subserve aquatic gills but may become essential in providing adequate oxygen, as in Anabas, which will drown if it is denied access to atmospheric air. Buccal-pharyngeal respiratory exchange among vertebrates is not limited to fish; a considerable body of evidence points toward its occurrence in anurans, ${ }^{3: 69}$ turtles, ${ }^{1 \cdot 3,3,253}$ and lizards. ${ }^{101}$

Gill respiration in a sense represents a stage of cutaneous breathing in which

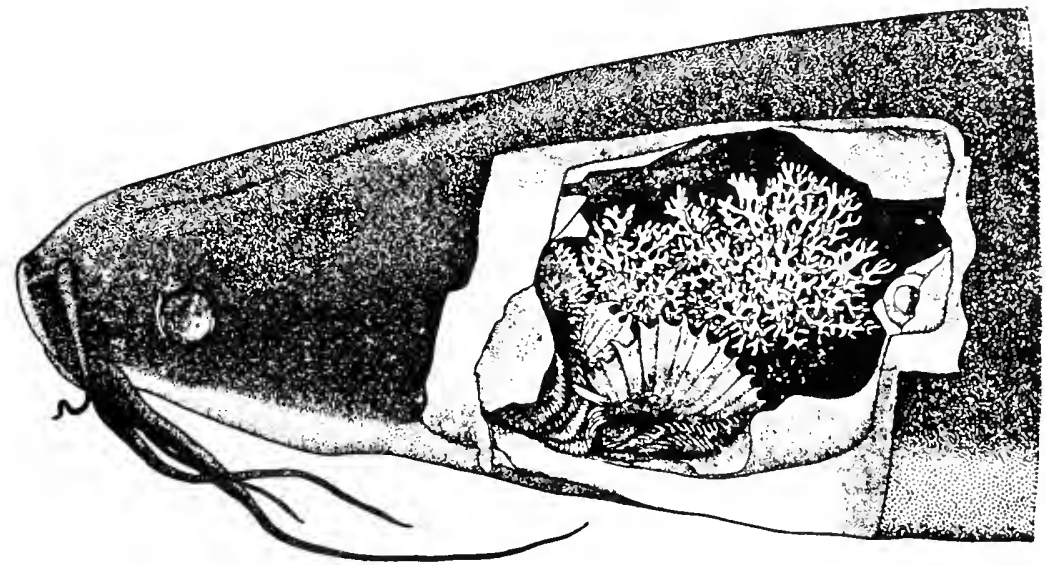

Fig. 39. Branchial diverticula in the air-breathing teleost, Clarias lazera, subserving the gills in aerial respiration. From Marlier. ${ }^{244}$

appendages have become specialized for gas exchange-structures which increase the area and efficiency for gas transport across the respiratory surfaces. They show considerable modification with changes in the oxygen environment and frequently function in combination with other respiratory structures in the exchange of vital gases. Being extemal structures without strong means of support, they are most commonly adapted to the water environment.

Lungs. These are most simply defined physiologically as respiratory surfaces folded into the body, a description that includes a variety of structures."16 They are usually aerial, although in some cases they are water-filled and extract dissolved oxygen. In all cases the lung surfaces must be moist, and gas transport occurs across a thin water film. Lungs are of two general types, diffusion and ventilation, depending on the presence of renewal mechanisms. All the lung-like organs considered here are parts of or outgrowths from the alimentary tract.

Water-Lungs. Water-lungs are found in several invertebrate phyla and 
consist essentially of respiratory cavities filled with water, rhythmically drawn into and expelled from the body. Respiratory trees of holothurians are alternately filled and emptied by means of muscular movements of the body wall, and gas exchange occurs by simple diffusion between the lung water and body fluid. ${ }^{55}$ The entire hind-gut of the gephyrean worm, Urechis caupo, constitutes a respiratory organ, muscular contractions of the cloacal region serving as the pumping mechanism. ${ }^{1+2}$ This thin-walled respiratory surface, adjacent to the coelomic fluid which is constantly agitated by antiperistaltic waves in the hind-gut, provides adequate gas exchange; water expelled from the hindgut contains less oxygen by about 40 per cent and more carbon dioxide than the surrounding medium. ${ }^{298}$ The pulmonate snails, Limnaea and Planorbis, are able to live under water for considerable periods of time, the lungs filling with water and aiding the skin in respiration. Water can be rapidly sucked into the "Endblase" or hindmost gut in the dragonfly larva (Aeschna) and brought in contact with the respiratory surfaces. ${ }^{3+6}$

Alimentary Mucosa. Some vertebrates possess modified gastrointestinal epithelium which permits uptake of oxygen from swallowed air. Gastric respiration is known to occur in such tropical forms as Plecostomus and Ancistruis, ${ }^{61,62}$ and intestinal respiration has been demonstrated in a great many other varieties of fish. In the loach, Cobitus, gas exchange is indicated not only by the histologic nature of the mucosa but also by direct determination of the gas of the intestinal lumen, which shows less oxygen ( 15.7 per cent) and more carbon dioxide ( 3.0 per cent) than does air. ${ }^{5 x}$ Some of the South American tropical fish which inhabit waters low in oxygen are also regarded as intestinal breathers (Doras, Loricaria, Callichthys, and Hoplosternum) ${ }^{87}$

Gas Bladder. The gas bladder, considered by many to be the forerunner of the vertebrate lung, functions in respiratory exchange in many of the physostome (open-duct) teleosts, ganoids, and dipnoans. To be efficient as a respiratory organ, the gas bladder must have some renewal mechanism. Many gas bladders have a respiratory type of epithelium, partitions forming "alveoli," and their own blood supply derived from the pulmonary arch (Fig. 40). The gas bladder can serve merely as an accessory organ when the oxygen tension falls, as in the actinopterygian, Polypterus, or as the main respiratory mechanism in the true lungfishes, Protopterus, Lepidosiren, and Neoceratodus. ${ }^{182}, 273$, 287. 289 (For a stimulating discussion of the adaptability of the African lungfish, see Homer W. Smith's Kamongo. ${ }^{330}$ ) As a respiratory organ the gas bladder must give up oxygen and take on carbon dioxide so that analyses should indicate less oxygen and more carbon dioxide than in the inspired atmospheric air, a situation shown to exist in many physostome fish. ${ }^{137}, 309$ The occurrence of a very high oxygen concentration, up to 87 per cent in some cases, in the gas bladder of physoclyst (closed duct) fish from great depths is of interest in connection with possible gas secretion and hydrostatic function but apparently is of little respiratory consequence. If the bladder is punctured or the fish put under considerable pressure so as to increase the specific gravity of the animal, the oxygen content of the swim bladder increases, indicating gas secretion. This gas exchange is under nervous control.2:0 The physoclyst perch, Perca flavescens, taken from surface water contains oxygen in approximate equilibrium with atmospheric air, but if the fish is subjected suddenly to an oxygen deficiency, as by rapid submergence to a considerable depth, the 
oxygen in the swim bladder is given up to the tissues, indicating a storage function of the structure operating in times of stress (Fig. H1)..$^{137}$ When subjected to asphyxiating conditions in the absence of carbon dioxide the tautog and toadfish use virtually all of the available oxygen in the swim bladder, the scup
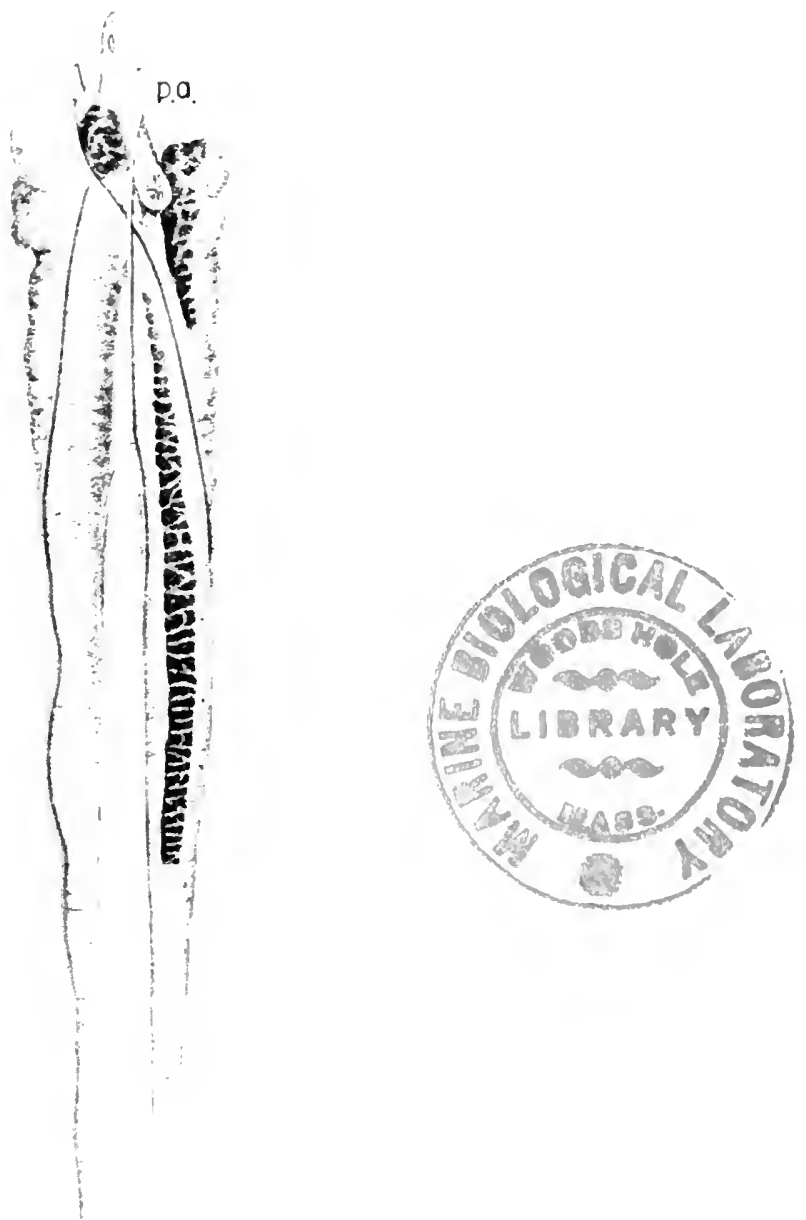

Fig. 40. Lungs of the dipnoan, Protopterus annectens, viewed from the ventral side. The main arterial blood supply (p.a.) is derived from the pulmonary (sixth) arch. From Parker. ${ }^{273}$

and killifish use about two-thirds." water, the oxygen utilized from the swim bladder is diminished.

Diffusion Lungs. In the absence of ventilating mechanisms the lungs quite naturally are supplied with air by diffusion. This system imposes limitations on both the size of the animal and the rate of metabolism, but the mechanism is not uncommon, particularly among the arthropods and molluscs (Scorpioni- 
dae, Pedipalpi, Arancidac, Porcellionidae, Janellidae). Book lungs of the scorpion, Opisthophthalmus capensis, for instance, with no ventilating apparatus, provide for practically all the respiratory exchange of the organism. Removal of the book lungs reduces the gas cxchange to approximately zero. 3 * Adequate diffusion occurs with slight differences in oxygen pressure, owing to the large surface area of the lungs. Calculations of pressure differences across the respiratory epithelium indicate that values as low as $1 \mathrm{~mm}$. $\mathrm{Ilg}$ in the chilopod. Scutigera, ${ }^{216}$ and $2 \mathrm{~mm}$. I Ig in the air-breathing pulmonate. Arion, are suflicient for gas exchange.

Ventilation I ungs. The respiratory efficiency of rentilation lungs is improved as a result of the higher average oxygen gradient maintained across the lung surfaces. Two kinds of rentilating mechanisms are found among vertebrates, a positive-pressure type in which air is forced into the lungs by swallowing (as in the frog), and a negative-pressure system in which air is drawn into the respiratory cavities by increasing the space about the lungs (as in man). During eupnea (normal breathing) in mammals, inspiration is active and expiration largely passive, but in some animals both movements may be effected by active muscular contraction, as in birds $" 234$ and turtles." ${ }^{2 !}$

Increase in metabolic requirements of higher animals is correlated with

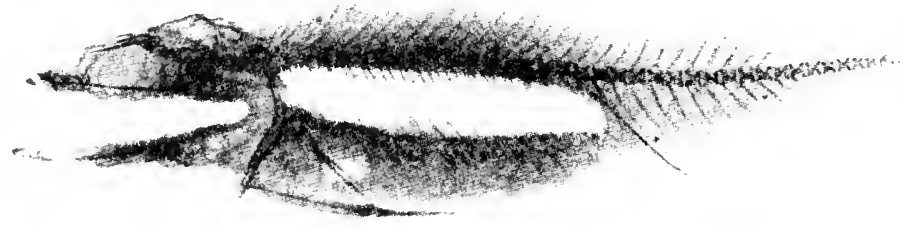

Fig. H. X-radiograph of physoclyst perch, Perca, showing the swim bladder, an emergency oxygen store as well as a hydrostatic organ. From Hall. ${ }^{13 z}$

greater complexity of the lungs and with less dependence on cutaneous and gill respiration. The lungs are characterized by (1) increased surface area and partitioning into alveoli. (2) greater vascularization, and (3) more adequate rentilation.

Nthough the lungs of some mammals have been described as being partiall! devoid of "respiratory epithelium." thereby exposing mesodermal endothelium of the capillaries to the alveolar air, the recent review of Guieysse-Pellissier ${ }^{1: 3+}$ concludes that the eridence is based on inadequate technique and that the respiratory epithelium, although occasionally enucleate, is complete. Muscle fibers can be demonstrated in the walls of reptilian lungs:" and down to the alveolar sacs in those of the mammal. Active contractions of the respiratory musculature correspond to the ventilation rhythm.

Gas exchange across the moist respiratory membranes scems adequately pro- 
vided by forces of physical diffusion and does not require the additional process of secretion as Haldane ${ }^{136}$ contended earlier. ${ }^{17}$, in Mice when administered carbon dioxide containing the radioactive isotope $C^{1.4}$ show a rapid two-way exchange of the gas across the lung surface with a total effective transfer from the blood to the alveoli.i:

In birds the characteristic lung mechanism equipped with air sacs permits a two-way flushing of the lungs and provides for gas exclange during both inspiration and expiration (Fig. 42). Calculations by Krogh"11i indicate that

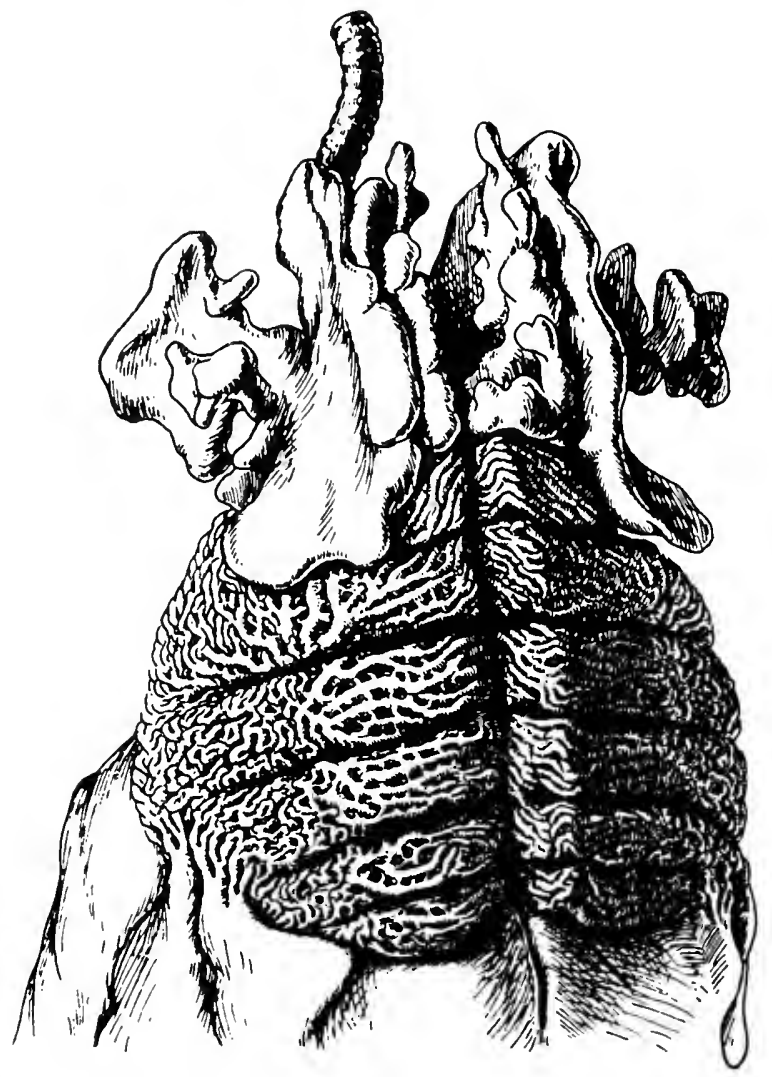

Fig. 42. Lungs and air sacs of pigeon photographed from a metal cast.

From Scharnke. ${ }^{310}$

three fourths of the total inspiration (tidal volume) is taken up by air sacs and one fourth by the lungs themselves. The respiratory exchange is automatically increased during Hight by the symchronization of wing and respiratory movements. Similar coordination between respiration and flight movements occurs in bats. ${ }^{30 .}$

The lung type of respiratory mechanism is advantageous not only by virtue of size and of adaptability to homoiothermic activities, but also by reason of the protection provided against mechanical damage and dehydration, and as further opportunity for efficient rentilation. Lungs, probably derived from the 
gas bladder of primitive bony fish, have very satisfactorily met the respiratory requirements imposed on animals in passing from aquatic to aerial media.

Tracheae. The phylum Arthropoda has monopolized the evolution of the tracheal mechanism, a distinctive system of air tubules, opening to the outside through spiracles and ramifying to all parts of the body to penetrate tissues and even cells. The system is adapted to aquatic as well as terrestrial conditions, permits rapid oxygen uptake, and allows a high degree of animal organization; the main limitation seems to be the size attainable by tracheates. As with lung mechanisms, the tracheal system may be dependent either on simple diffusion for air or, in more active and larger forms, on mechanical ventilation.

Respiratory movements are generally absent from the arthropods Onycho-

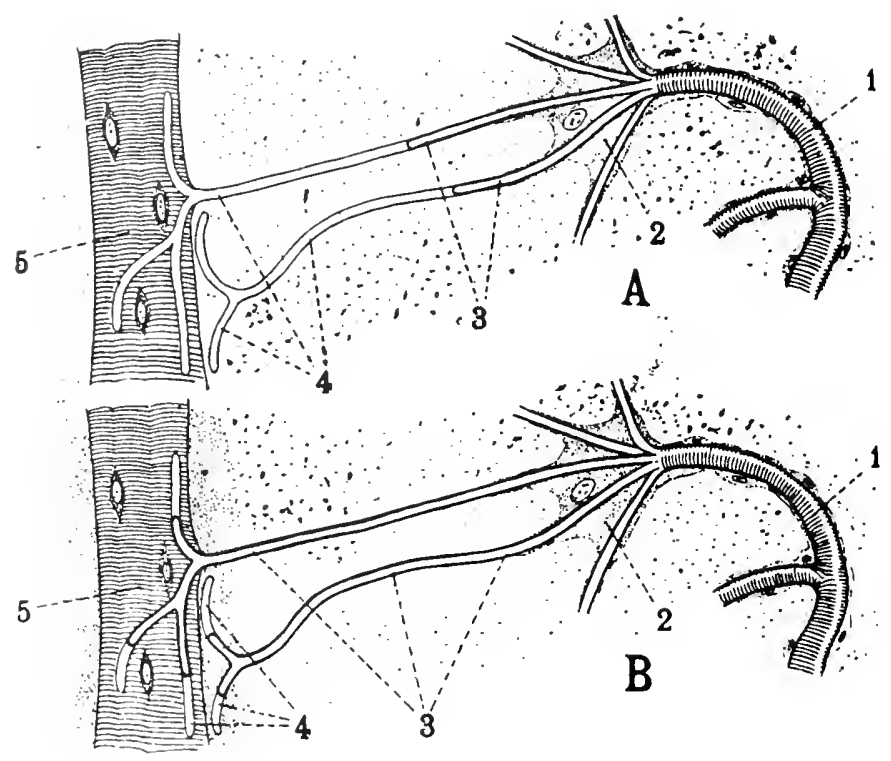

Fig. 43. Tracheal response to osmotic change during rest (A) and after activity (B). 1, trachea; 2, tracheal cell; 3, parts of tracheoles containing air; 4 , parts of tracheoles containing fluid; 5, muscle. From Wigglesworth. ${ }^{36,5}$

phora, Arachnida, Myriapoda, and Chilopoda, from most terrestrial larvae, from all pupae, and from many small winged adult insects."216i Size and metabolic rate are important factors in regard to the adequacy of simple diffusion, and although some insects (Periplaneta. Diaippus) rely on diffusion when at rest, they call upon rentilation movements during higher levels of metabolic activity.

The innermost ends of the tubes, the tracheoles, are fluid-filled in some insects and are certainly permeable to water. They may respond to changes in muscular activity by shifting the fluid reversibly between the tracheoles and the tissue. According to Wigglesworth, ${ }^{363}$ in mosquito (Aedes) and dragontly (Aeschna) larvae water is withdrawn from the tubules by the osmotic effect of increased acid metabolites produced during activity, and air in the tracheoles then moves closer to the cells, the process being reversed 
presumably after the period of exercise (Fig. +3). In Drosophila larvae the entire tracheal system is permeable to respiratory gases and water, and various mechanical stimuli may cause Huid uptake by the tubes. ${ }^{10+}$ In Sciara larvae, however, the tracheoles, even in the terminal regions, never normally contain fluid after their initial filling with gas." ${ }^{201}$ The filling process can begin at al point within a main tracheal trunk, can occur in the near absence of oxygen, but is inhibited by totally anoxic carbon dioxide, carbon monoxide, and nitrogen, and by low temperature $\left(0^{\circ}\right.$ C. $)$. The filling seems to involve an internal source of gas produced metabolically. it

Special tracheal systems permit aquatic respiration in certain insects (see page 215). Many aquatic insects possess hydrofuge faculties which permit them to make contact with air through specially modificd structures of the body. The water-air surface film can thus be penetrated, and unwettable spiracles exposed to the atmosphere, to facilitate gas exchange. Hydrofuge hairs enable some insects (Dytiscus, Notonecta) to trap considerable quantities of air, which is then carried below the surface and used as an oxygen store. ${ }^{365}$ The very great efficiency of such surlace-borne air in the respiration economy of diving insects (e.g., Amphelocheirus, I femiptera) has recently been pointed up in regard to the "plastron" mechanism. ${ }^{343}$ The plastron ("Lufthulle") is essentially an epicuticular hair-mat with its trapped air serving as an oxygen supply' while the insect is submerged. Not only is oxygen which was originally obtained at the surface given up by the air, but, as a result of the invasion coefficients between the gases and water, nitrogen leaves the plastron slowly, while additional oxygen diff uses in from the surrounding water. Such a system was noted by Egetoi as bestowing on the plastron air an oxigen supply about thirteen times as great as that actually afforded by its original volume.

Tracheae may be regarded as the arthropod contribution to respiratory regulation-an efficient mechanism, but restricted by the limits such a system imposes on the size attainable by the organism. No other group of animals has met the respiratory problem in quite this way.

Sumnary. We see then a number of respiratory adaptations which have developed to facilitate gas exchange. Diffusion per se through the body surface is sufficient for the smaller organisms and accounts for gas exchange across special respiratory surfaces. The structural modifications in respiratory mechanisms essentially have been along the lines of increasing surface area, improving ventilation, and bringing the respiratory gases into more inmediate contact with the internal transporting system. Gills are characteristic of aquatic forms; lungs and tracheae have made possible the complex development of land dwellers.

\section{THE LEVELS OF OXYGEN CONSUMPTION}

The metabolic economy of animals shows a wide variation in adjustment to oxygen availability. Most organisms have ptenty of oxygen at their disposal, but some are adjusted to oxygen defieiencies and others to what constitutes essentially an oxigen-free environment. The rate of oxygen consumption reflects the metabolic activity of acrobic organisms and is modified by a number of intrinsic and extrinsic factors. Such physiological changes as hyperthyroidism, aging, hibernation, estivation, the recovery from oxygen debt after exercise, and the like, may alter the oxygen uptake. Such environmental conditions 
as temperature affect the rate of oxygen consumpti . Gas exchange is not the only index of activity or energy production of an organism, but its correlation in a quantitative way with rate of metabolism and heat production has made it the generally accepted standard of the over-all measura of metabolic processes.

The Determination of Oxygen Consumption. It is unnecessary to discuss here in detail the many methods and their uses that have been elaborated by

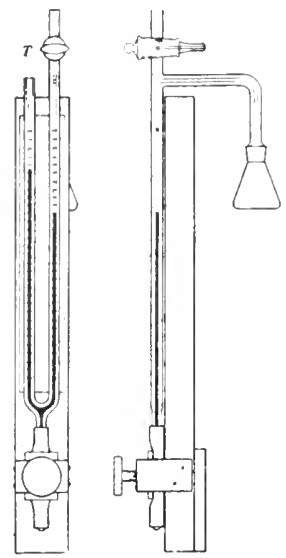

a

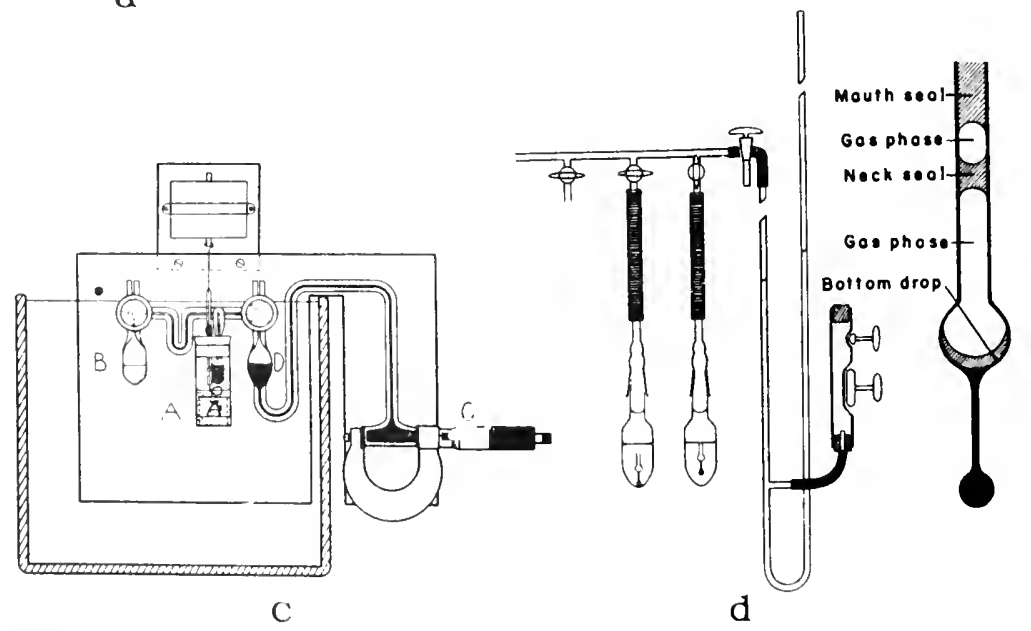

1 ig. 4t. Respirmeters developed by (a) Warburg, and Fdwards," and (d) 1 Holter and Linderstrym-Lang. ${ }^{120}$

a host of investigiters in the field. The monographs of Dixon ${ }^{4 ! 3}$ and Krogh,, 1 t

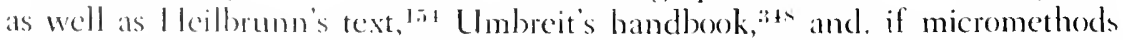
are desired, the important review by Tobias, 3 ta should be consulted for extensive bibliographice an well as discussions of principles, sensitivitues, and limitations involied in the various techniques that have been devised. 'The carly literature was ably summarized by ligerstedt.":t 
The mensuration and interpretation of respiratory gas exchange dates back to the significant efforts of Lavoisier, whose work secms to have inspired the notable group to follow, including Seguin, Liebig, Petrenkofer, Voit, and Rubner. Further comparative physiological investigations were stimulated by the careful determinations of oxygen consumption by Regnault and Reiset during the middle of the past century. Today the literature on respiratory measurement-and the field has moved largely into the detcrmination of cell and tissue respiratory mechanisms-is replete with the works of Warburg, Fenn, Linderstrom-Lang, Needham, and others who have designed and developed various types of apparatus to atack significant respiration problems (Fig. 44).

Determinations of gas exchange and oxygen consumption, particularly, have been made on a great variety of organisms, but owing to the vicissitudes of both animals and techniques the results are sometimes difficult of interpretation. The difference between "standard" and "basal" metabolism,"14 the variations in oxygen consumption due to size and activity, with the attendant difficulty in attaining "normal" conditions, ${ }^{2 \pi 5}$ the apparent shift in anaerobicaerobic respiratory mechanisms induced by experimental procedures ${ }^{2 * 1}-$ these and ther difficulties make the task of presenting representative oxygen consumption values a precarious one. Nevertheless some limited data are tabulated, which bear on currently significant aspects of comparative physiology. Other extensive surveys of oxygen consumption may be found in the rcviews in Tabulae Biologicae by Loew $y^{2: 32}$ and Krebs ${ }^{20 x}$-both of whom consider at length tissue as well as organism metabolism-and particularly in the tables in Heilbrunn's Outline of General Physiology. ${ }^{154}$

The method of expression of the oxygen consumed presents a problem in itselt. A general convention and one that has been followed here is to present the data as cc. oxygen consumed per gm. wet weight of tissuc per hour. Such a figure is of considerable comparative value, easily computed from current gasometric data, and readily converted into standard metabolism units. Oxygen consumption may be expressed in terms of grams rather than cubic centimeters, or as dry (Qo. values) rather than as wet weight of tissue. For some organisms it is convenient to use, in lieu of weight of tissue, numbers of individuals, as the oxygen consumption of Protozoa may be expressed as cc./ million $/ \mathrm{hr} .{ }^{1+1 .}{ }^{270}$ and of sperm as " $\mathrm{Zo}_{2}$ " values-cu.mm./108 sperm/hr. ${ }^{32}$ The recent application of the Cartesian diver principle to the determination of live weight of Amoeba has introduced another standard-the $\mathrm{O}_{2}$ consumed (in microliters) per gamma of "reduced weight" per hour, where reduced weight corresponds to the weight of the live object minus the weight of the water displaced. ${ }^{171}$

Oxygen Consumption in Relation to Size. Small organisms have higher rates of oxygen consumption than larger organisms when determined both for animals within a given species and for those of closely related phylogenctic groups. Since the days of the early metabolism studics on man, fat people have been recognized as having lower oxygen uptake per unit weight than lean individuals. This has been attributed to the "amount of active protoplasmic tissue" as the basis for oxygen consumption. ${ }^{23}$ Recent studies of the metabolism of some of the smallest mammals, including shrews weighing 3 to 4 grams, give additional evidence for the general rule relating gas exchange 
to size (Fig. 45, and Table 38).258, 277 The high oxygen uptake of the longtailed shrew, $13.7 \mathrm{cc} . / \mathrm{gm} . / \mathrm{hr}$., is startling; it may be noted that the food intake too is enormous-something like its own weight in food every 24 hours! As noted recently by Pearson the asymptotic increase in metabolic rate with decreasing size places the lower limit of size for mammals at about $2.5 \mathrm{gm}$.; a smaller mammal would be unable to obtain adequate food for its infinitely rapid metabolism. The metabolic values for these small mammals exceed the oxygen consumption of Protozoa; assuming a specific gravity of about 1, Para. mecium respiration is $6.0 \mathrm{cc} . / \mathrm{gm} . / \mathrm{hr} .,{ }^{269}$ and that of Tetrahymena geleii is $10-12 \mathrm{cc} . / \mathrm{gm} . / \mathrm{hr}$. at $25^{\circ} \mathrm{C} .{ }^{270}$

The rate of oxygen consumption is correlated with body weight as an exponential function and tends to follow the increase in surface area rather than weight per se. For instance, in fish (pike and tench), oxygen uptake

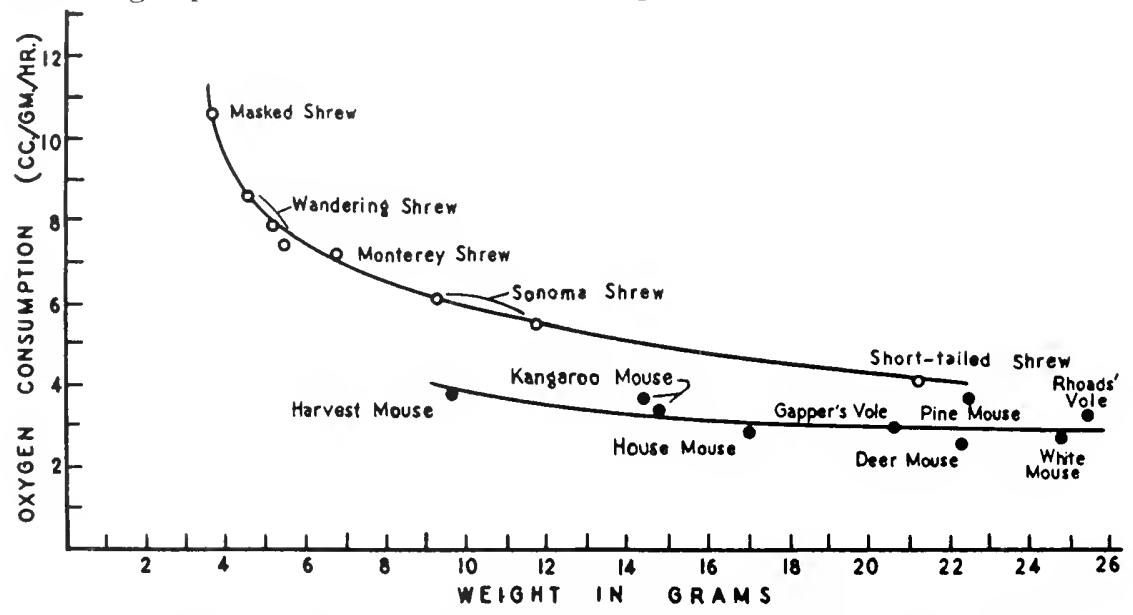

Fig. 45. Oxygen consumption of small mammals plotted as a function of body weight, indicating the surface area rule within this group. From Pearson. ${ }^{277}$

seems to increase in direct proportion to the surface area, i.e., cc. $\mathrm{O}_{2}=\mathrm{kW}^{0.66}$, where $W$ is the body weight, and $k$ is a constant for the organism. ${ }^{304}$ Most determinations yield an exponent in excess of that indicated by the "twothirds" rule. On a series of rodents Morrison ${ }^{2 \pi 8}$ found good agreement with an exponent of 0.73 (adopted by the Conference on Energy Metabolism, 1935) relating weight to basal metabolism, and a slightly higher value of 0.798 was obtained for the kelp crab, Pugettia producta, by Weymouth and his coworkers $^{361}$ (Fig. 46). Results on a number of different species of Crustacea indicate the same general correlation between body surface and oxygen consumption. Failing to establish direct correlation with surface area and body weight in mammals, Brody ${ }^{\overline{5} 0}$ relates basal metabolism to "metabolically effective body weight."

The general relationship of decreasing metabolic rate with increase in body size holds for small marine organisms, although those weighing less than 1 gram frequently do not obey the surface law. ${ }^{384}$ Zeuthen's thorough-going study on the marine microfauna indicates that comparison of organisms on a basis of respiration per nitrogen content reveals considerable agreement among 
such forms as coelenterates with a high ivater content and the more solid forms, and further that the phylogenetically advanced groups show a higher rate of metabolism than the more primitive ones (Fig. 47).

The higher "reaction rate" of smaller animals is associated with increased

TABLE 38. OXYGEN CONSUMPTION OF SMALL ADULT MAMMALS UNDER APPROXIMATELY BASAL CONDITIONS

\begin{tabular}{|c|c|c|c|}
\hline Animal & Weight & $\begin{array}{l}\text { Air } \\
\text { Temp. }\end{array}$ & $\begin{array}{l}\mathrm{O}_{2} \text { Consumption } \\
\text { cc. } / \text { gm. } / \text { hr. }\end{array}$ \\
\hline Long tailed shrew (Sorex c. cinereus) s. $^{254}$ & 3.4 & $2+-28$ & 13.7 \\
\hline Wandering shrew (orex vagrans vagrans) ${ }^{27 t}$ & 4.5 & 24 & 8.6 \\
\hline Monterey shrew (Sorex trombideii montereyensis) $\delta^{2 \pi}$ & 6.7 & 24 & 7.2 \\
\hline Sonoma shrew (Sorex pacificus sonomae) $\delta^{i \pi}$ & 9.2 & 24 & 6.1 \\
\hline Sonoma shrew (Sorex pacificus sonomae) ${ }^{277}$ & 11.2 & 24 & 5.5 \\
\hline $\begin{array}{l}\text { California harvest mouse (Reithrodontomys } \\
\text { megalotis longicaudus) }\end{array}$ & 9.6 & 24 & 3.8 \\
\hline $\begin{array}{l}\text { Kangaroo mouse (Microdipodops megacephalus } \\
\text { nasutus) } \delta^{2 \pi \tau}\end{array}$ & 14.4 & 24 & 3.7 \\
\hline $\begin{array}{l}\text { Kangaroo mouse (Microdipodops megacephalus } \\
\text { nasutus) } q^{277}\end{array}$ & 14.8 & 24 & 3.4 \\
\hline House mouse (Mus musculus) $)^{2 \cdot 5 x}$ & 15.8 & $24-28$ & 1.53 \\
\hline Deer mouse (Peromyscus maniculatus) $)^{2 \pi}$ & 17.1 & $24-28$ & 1.65 \\
\hline White mouse (Mus musculus) $)^{2-5}$ & 21.2 & 28.5 & 1.59 \\
\hline $\begin{array}{l}\text { Red-backed mouse (Clethrionomys gapperi } \\
\text { ochraceous) }\end{array}$ & 22.8 & $24-28$ & 1.50 \\
\hline Deer mouse (Peromyscus leucopus) $)^{25 \lambda}$ & 24.2 & $24-28$ & 1.46 \\
\hline Pine mouse (Pitymys $s p.)^{2 \pi x}$ & 26.3 & $24-28$ & 2.56 \\
\hline Dormouse (Myoxus arbor) ${ }^{200}$ & 43.0 & $24-28$ & 1.75 \\
\hline Hamster (Cricetus auratus) $)^{200}$ & 100.0 & $24-28$ & 1.05 \\
\hline White rat (Rattus rattus) ${ }^{154}$ & 350.0 & 28 & 0.77 \\
\hline
\end{tabular}

need of oxygen, but the matter of heat loss is also important in the metabolic economy of small animals, particularly in that of homoiotherms with inadequate insulation. Mice huddled together apparently for warmth consume less oxygen as a group than they do when separated and measured individually. 276 However, in the absence of data on body temperature and activity, absolute values are not available for a close comparison of these groups. Similar evidence on the brown snake, a poikilotherm, shows this aggregation-oxygen decrease effect. ${ }^{70}$

Oxygen consumption studies by Scholander (personal communication) on arctic, temperate, and tropical mammals indicate that the metabolic regulation may be in part a function of the insulation efficiency of the body surface and in this respect correlated with surface area. Heat production along with oxygen consumption tends to follow roughly the surface rule, the weight raised to the 0.66 or 0.73 power. A linear relationship is obtained when the log of the heat production is plotted against the log of weight (Fig. 48).:-i.

A correlation between oxygen consumption and brain weight was made by Crile, ${ }^{78}$ for a number of invertebrates and vertebrates from various phyla. The correlation apparently reflects the relation of body surface area to brain weight, but represents more significantly the dependence of metabolism on the distribution of active protoplasm. 
In conclusion, although many correlations have been demonstrated between metabolic rate and functions of the body weight approximating surface area, there is scant evidence to indicate the causes for this relationship. The correlation reflects the changing activity of the protoplasm of the body cells. Whether this condition will be demonstrated as due to decrease in rates of chemical reactions, enzyme concentrations, growth substances, or whatever, must depend on future investigations.

Oxygen Consumption in Relation to Age. No simple correlation can be made between oxygen uptake and age; rather, the different phases of develop-

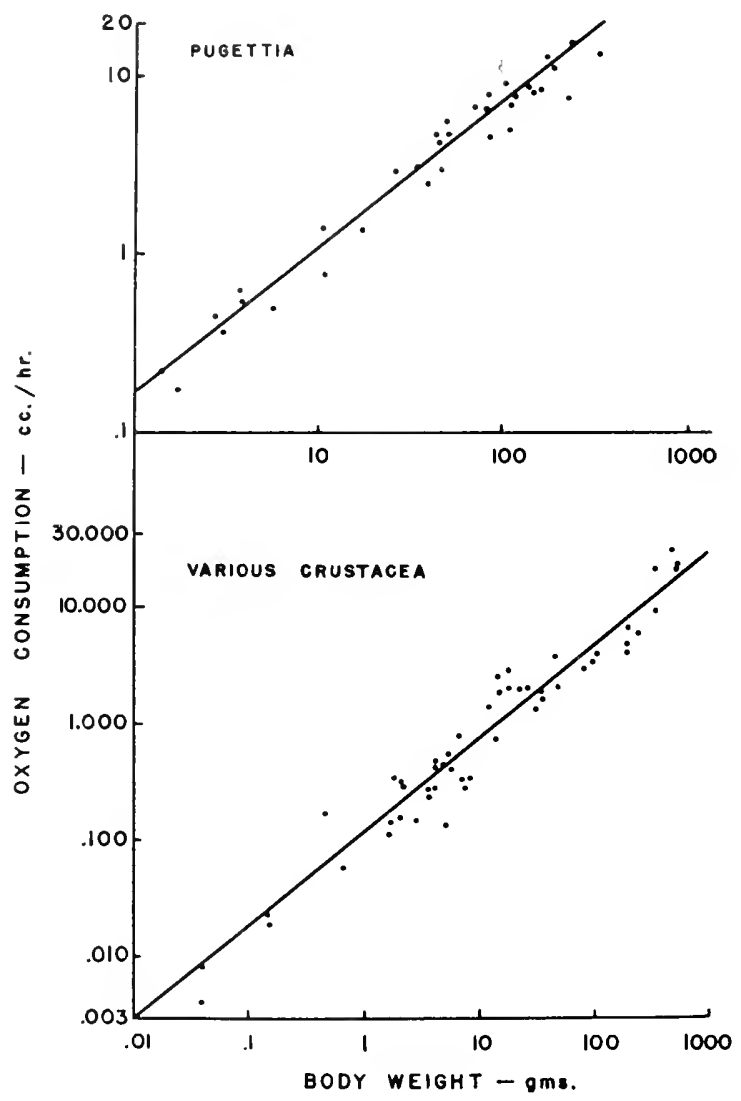

Fig. 46. Double log plots of oxygen consumption and body weight of the kelp crab, Pugettia, and of several related Crustacea. From Weymouth et al. ${ }^{381}$

ment must be considered separately. Oxygen consumption of young animals is higher than that of their older relatives, indicating in some measure the size relationship considered above (Fig. 49). Older organisms tend to "run down" as oxygen-consuming processes go on less rapidly, a phenomenon possibly associated with total enzyme concentrations of the young and older organisms. A peak in metabolic activity is usually well marked a short while after birth or hatching, as, for example, in the chicken (Fig. 50). The period of greatest 
oxygen consumption in a number of domestic animals has been demonstrated by Brody and his co-workers; cattle, for example, reach their maximum metab. olic rate between weaning and puberty, swine at the time of puberty, but horses do not reach it until maturity. ${ }^{51}$ Comparable studies on human metabolism are summarized in the work of Krogh, ${ }^{11+}$ indicating the maximum is reached at two years of age; more recent data, however, would put this somewhat earlier, near the time of weaning. 30.1665 This increasing inetabolic rate in young children is perhaps due to marked development of the musculature during this period rather than to a general development of the body as a whole. Breaks in the growth curves have been demonstrated in lower vertebrates as well, particularly among Amphibia. The break in the growth curve for Rana corresponds to the end of the gastrulation period. ${ }^{\top}$ The break in the otherwise

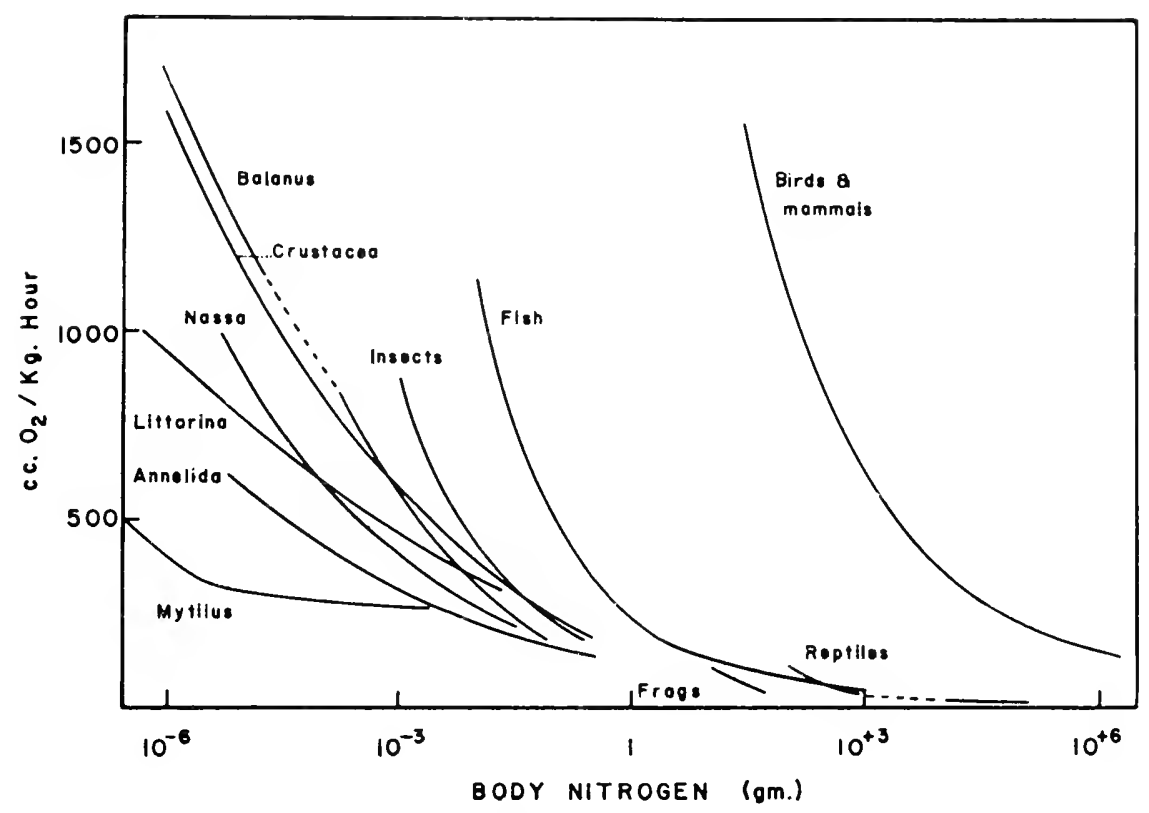

Fig. 47. Comparison of metabolic rate of various animals as a function of body nitrogen. After Zeuthen. ${ }^{354}$

smooth exponential growth curve of Amblystoma is rather prominent and comes at the sixth or seventh day of development (Fig. 51)."34 Amerling's data, ${ }^{6}$ based on the resistance to oxygen lack ("Zeitdauer"), support the concept of increasing dependence on oxygen as the resistance decreases steadily up to about the $12 \mathrm{~mm}$. stage and then levels off more gradually.

A correlation in rate of oxygen consumption with age may be applied to cultures of microscopic organisms. Cultures of the protozoan Chilononas paramecium decrease in oxygen consumption from 0.35 to 0.17 and $0.11 \mathrm{cu}$. $\mathrm{mm}$. per hour per 10,000 individuals in cultures which age 24. 48, and 72 hours respectively. ${ }^{17}$ It would be of interest to know whether subsequent determinations on these cultures might show a further decrease or a leveling 
off of oxygen consumption, thereby giving some indication as to the nature of the mechanism: whether the decrease is due to the utilization of food material, or to accumulation of deleterious metabolites, crowding, etc.

Respiratory exchange of embryonic mammals was regarded by Bohr and Hasselbalch ${ }^{4: 3}$ to be directly proportional to weight rather than surface area. Barcroft's data ${ }^{1 \bar{i}}$ on sheep and goat fetuses, obtained by measuring oxygen tensions in the umbilical artery and veins, indicate furthermore that the oxygen uptake is lower $(0.0026 \mathrm{cc} . / \mathrm{gm} . / \mathrm{min}$.) than would be expected when compared with adult tissues, and may be considered a reflection of the adequate temperature conditions provided by the parent, thereby relieving the offspring of the necessity of using energy for warmth alone. The shift in dissociation

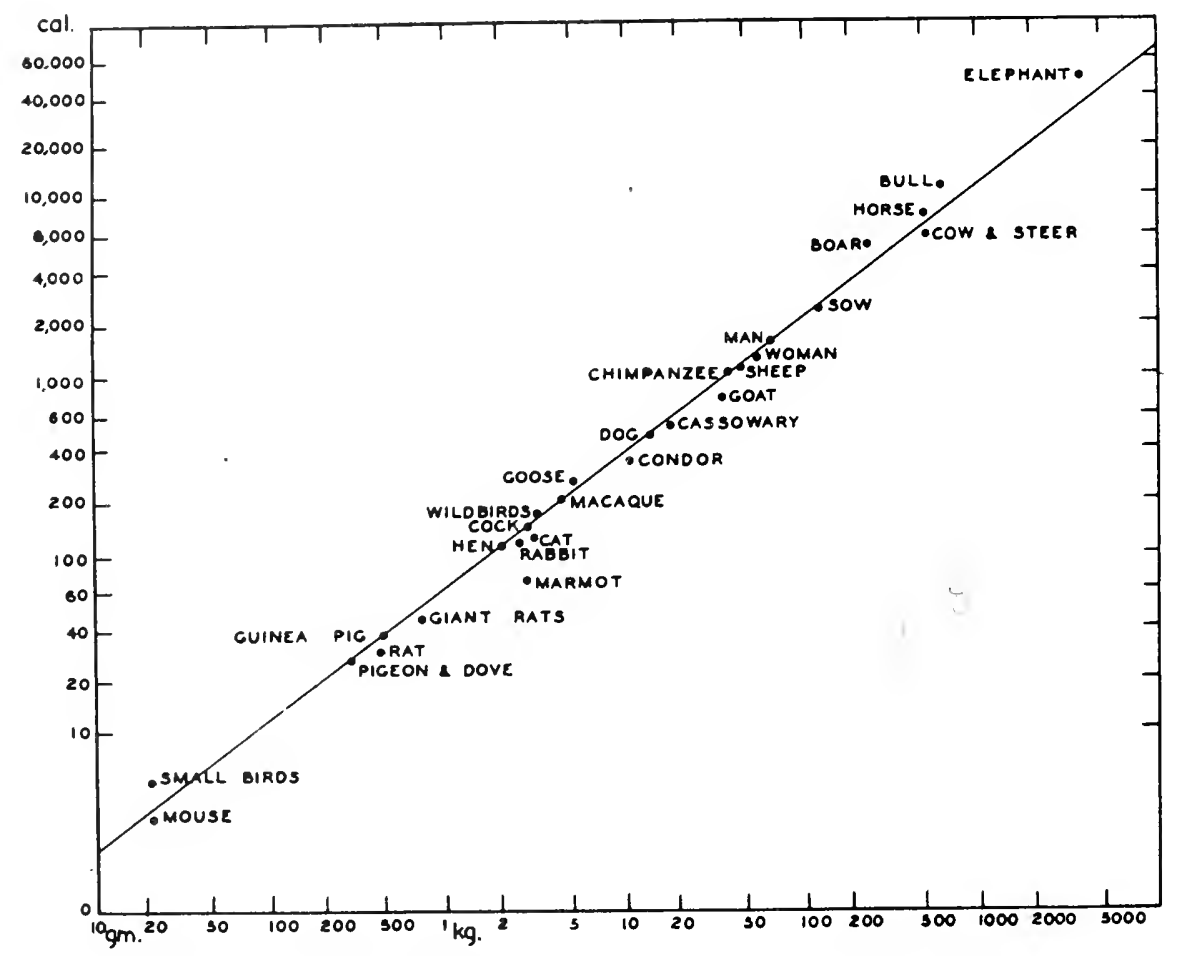

Fig. 48. Double log plot of average total heat production and average body weight. From Benedict. ${ }^{27}$

curves to permit the fetus to pick up, and the parent to give up, oxygen more readily permits adequate gas exchange at these metabolic levels ${ }^{17}$ (see Chapter 9). Bohr $\mathrm{r}^{+1}$ ingeniously controlled the umbilical blood flow in urethanized guinea pigs, at the same time taking tracheal samples, and demonstrated that the gas exchange of the parent and that of the fetus are almost the same. Embryos of snakes ${ }^{+2}$ and turtles $2: 37$ undergo a gradual decrease in oxygen consumption with development; in turtle embryos there is a possibility that a considerable part of the oxygen consumption is associated with the catabolism of quantities of protein. One would expect a new metabolic peak to develop after the young are hatched. 
In the earlier stages of embryonic development there appear abrupt changes in the respiratory exchange, correlated with profound developmental milestones as clearage, gastrulation, diapause, metamorphosis, and fertilization itself. Years ago Warburgiziti demonstrated a seven-fold increase in oxvgen consumption in Arbacia eggs at the time of fertilization, a phenomenon adequately substantiated on a number of other forms. In Fundulus, besides the increase at fertilization, a second marked increase occurs at about the time

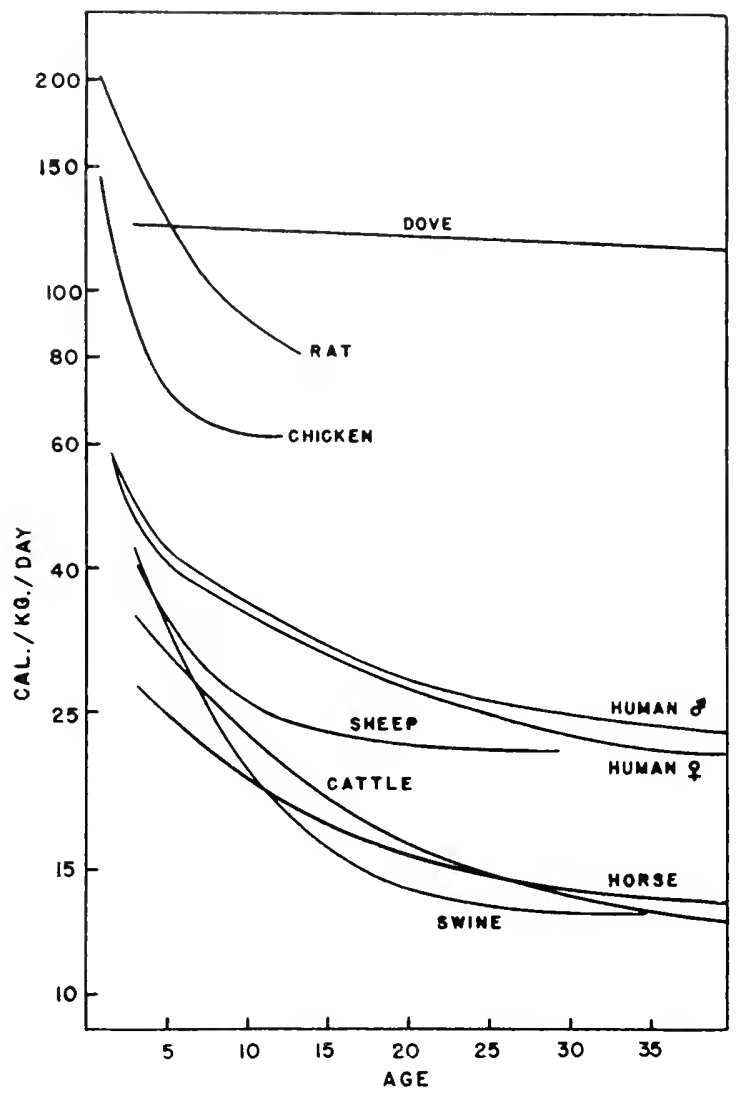

Fig. 49. Decrease in metabolic rate with age of several birds and mammals. Age in months, except for man in years. After Benedict and Talbot. ${ }^{31}$

when the vascular system begins to Hourish. That all embryonic forms do not follow the same respiratory pattern, however, is clear from the work of Tang ${ }^{337}$ on the starfish and that of Brachet ${ }^{47}$ on Amphibia, which show no increase in oxygen consumption at fertilization. In fact, the clam, Cumingia, and the annelid, Chaetopterus, undergo a decrease in oxygen uptake at the time of fertilization. ${ }^{36: 2}, 36: 3$ An increasing metabolic rate of rat eggs during early cleavage is correlated with an increase in volume, in turn attributed to water uptake and probable yolk-protoplasmic conversion. ${ }^{39}$ For an extensive review of the literature concerning embryonic metabolism, the reader is referred to the thorough treatment by Needham ${ }^{259}$ and Boell. ${ }^{38}$ 


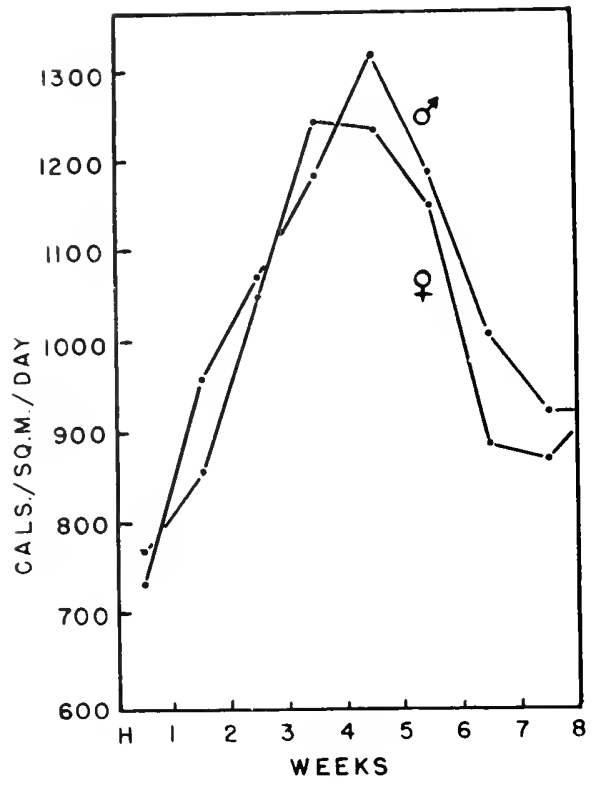

Fig. 50. Netabolic peak in Rhode Island Red chickens at end of first month after hatching. From Kibler and Brody."

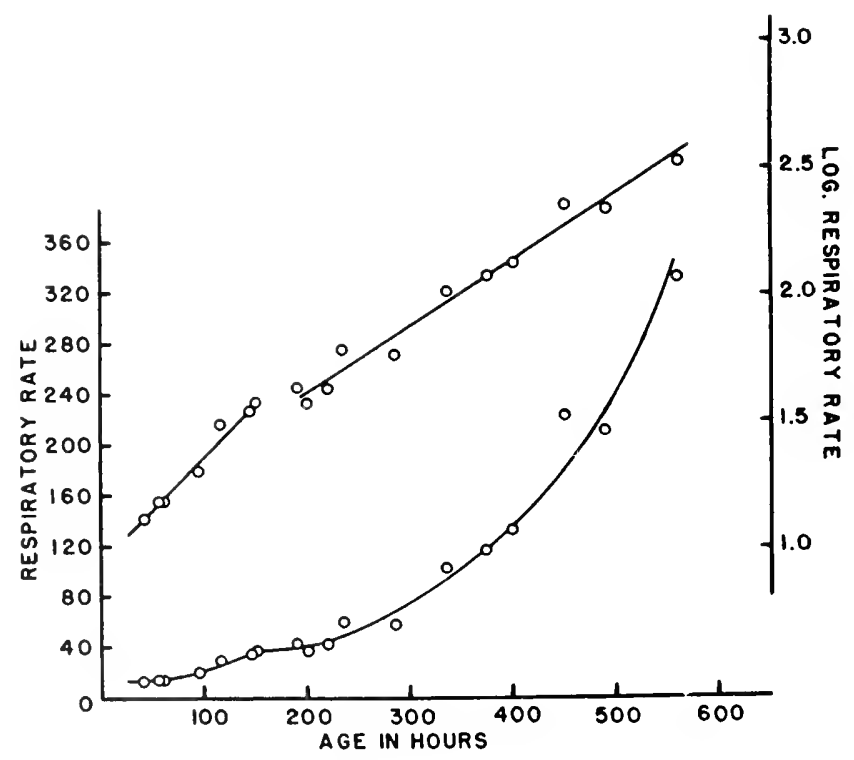

Fig. 51. Respuration of Amblystoma embryos expressed as nul. $0.2 / 100 \mu \mathrm{gm}$. dry wt./ br., showing break in develepmental rate at about seventh day. Arithmetic and logarithmic curves plotted from same data. From Boell."3! 
Rogers ${ }^{302}$ presents an interesting discussion of the decreasing oxigen uptake with age in the small crustacean, Simocephalus, measured immediately after hatching et sequentia, during several larval. 'nd brood mother stages (Table 39).

Pupating insects present a unique case, with general agreement among investigators on the shape of the curves if not on their interpretation when oxygen uptake is expressed as a function of pupation time. A broad U-shaped curve is usually obtained, the minimum conesponding with the middle of the

\section{TABLE 39. OXYGEN CONSUMTTION IN SMMOCEPHALUS EXPRESSED IN}

PER CENT OF UPTAKE IMMEDIATEL YOLLOIVING HATCHING,

ALL ADIUSTHD TO SAME RELATIVE WEIGHT:

\section{Stage}

Hatching $\left(5.6 \times 10^{-5} \mathrm{cc} /\right.$ individual $\left./ \mathrm{min}.\right)$

3rd instar

lst brood mother

3rd

5 th
Relative Oxygen Uptake

100.0

96.4

52.1

50.8

$+1.8$

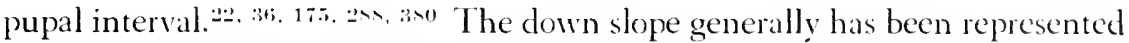
as the period during which histolytic processes predominate, while the up slope, indicating a rise in rate of oxygen consumption, corresponds to the more constructive phase of dedifferentiation. This, however, seems to be an oversimplification and based on inadequate evidence. As Krogh"1t has pointed out, "the expenditure of energy required for tissue formation is quite small," and furthermore the change trom catabolic to anabolic processes for the organism as a whole would not be expected to occur all at once. Thus the oxygen consumption data on the muscid Hies, Calliphora and Lucilia, for example, do not indicate any sudden change, at least on a weight basis, to account for the abrupt change-over from decreasing metabolic rates. seems more likely that the shift in respiratory exchange results from alterations in the respiratory pattern, involving different substrates and the balance between aerobic and anacrobic processes.

Among bees, the larger, better-nourished queen outstrips the workers in regard to metabolic activity and growth (Fig. 52). I ler relative superiority is shown in larval, pupal, and pre-adult stages, calculated on both a weight and an individual basis. $=51$

The use of oxidative poisons and of oxygen deprivation has made it possible to test the relative dependence on oxygen of the rarious stages of development. In many animals a transition from predominanty anacrobic to acrobic cond: tions is indicated. In the squid, Loligo pealei, for instance, an increase in cyanide sensitivity can be shown to parallel the increase in oxyere consumption and carbon dioxide production during derelopment." $=$ - 15 -day cmbryo withstands exposure to $\mathrm{N} 1000 \mathrm{NaCN}$ for 20 hours, hut older embryos shou a gradually increasing cyanide semsitivity until the stage of the newly hatched embryo, which has a tolerance of only $1-2$ hours. A 12 -inch squid and the adult have even less-a tolerance of only 1 minute. Organisms in the same 
class may show considerable variation in cyanide sensitivity; Fundulus eggs are particularly resistant to cyanide and azide in concentrations which are very toxic to the pelagic eggs of the cunnar, mackerel, and scup. $2 \times 2$ With development, however, the Fundulus eggs too become sensitive to the respiratory poisons, indicating a change in respiratory pattern. Frog eggs can cleave under anaerobic conditions to form blastulae, but oxygen is necessary for complete gastrulation and subsequent development. ${ }^{47}$ Cyanide sensitivity in Para-

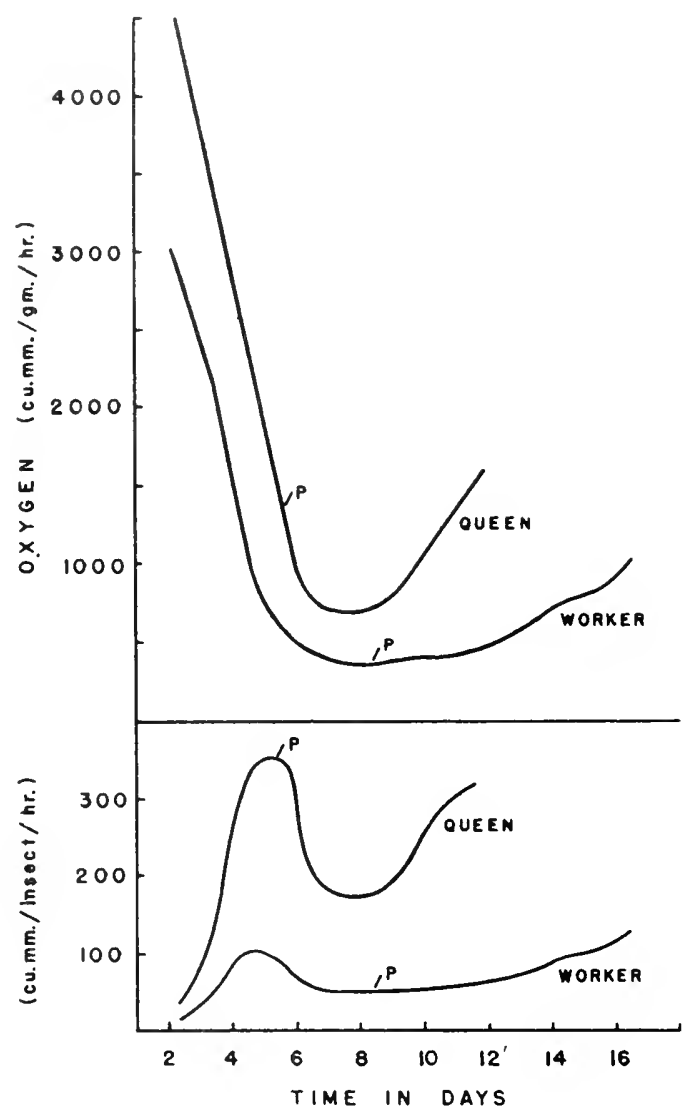

Fig. 52. Oxygen consumption during larval and pupal development in the honeybee, showing the increased rate of the queen compared with that of the workers, on both a weight (upper curves) and an individual (lower curves) basis. $P$, time of pupation. After Melanipy and Willis. ${ }^{2.1}$

mecinm may be correlated with food supply, well-fed (younger) individuals being more sensitive than starved (older) animals. 270

Total metabolism is of course the sum of the individual tissue metabolic activities, and it is not surprising that certain tissues show a variation in oxygen consumption with age. Investigations on the gill tissues of Vemus are consistent with the bulk of the literature in demonstrating that tissues of younger animals respire at a higher rate than do those of older animals. ${ }^{172}$ However, 
in a comparison of newborn and adult dogs the oxygen consumption both of brain tissue and of the intact animal was demonstrated to be significantly lower in the pup. ${ }^{16 i}$ Isolated fibroblasts of the chick heart survive anacrobically and take up little oxygen during the first five days of development, but tissues from older chicks become sensitive to oxygen lack ${ }^{57}$ and increase their rate of oxygen consumption.

In summary, we find that developing organisms tend to show a decrease in oxygen consumption with increasing size, but in very early and embryonic stages reflect profound developmental transitions involving structural changes, tissue formation, and shifts in the types of respiratory mechanisms. A high rate of oxygen consumption at fertilization and cleavage is usually followed by a gradual decrease with embryonic development until birth or hatching, after which the rate ascends to a maximum, to be succeeded by another slump with advancing age.

Oxygen Consumption in Relation to Species. The differences in absolute consumption values between species have in a few instances been shown for "races" within the species, a condition probably representing genetic differentiation within the group. Certainly more such data are needed before any general interpretations regarding their significance may be drawn. Metabolic differences have been claimed for human populations,, 25 but a re-evaluation of the importance of the environmental factors as serious sources of disparity should be made. In comparison with the standard basal metabolism values (B.M.R.) for Caucasian men and women (United States), those of Mayan Indian males are claimed to be 8 per cent higher, those of Miao (West China) males 16 per cent higher, and those of Araucanian Mapuche (Chile) men and women are claimed to be significantly higher than those of comparable age groups in the United States. The metabolism values for Indian women from the southern provinces of Tamil and Malaydis are consistently lower than the figures for Caucasians. ${ }^{25}$

Curiously, adult Drosophila melanogaster of the vestigial strain have been shown to have a slightly higher oxygen uptake than normal wild type flies, and the latter a higher consumption per weight than hybrids. ${ }^{267}$ Dobzhansky and Poulson ${ }^{94}$ showed, in what was then considered Race A and Race B of D. pseudoobscura (now regarded as D. pseudoobscura and D. persimilis), a difference in gas exchange when measured at $25^{\circ}$ and $14^{\circ} \mathrm{C}$., their respective environments. Although the oxygen uptake in the two races was the same at $14^{\circ} \mathrm{C}$., Race A which normally inhabits the warmer climate had a lower oxygen consumption at $25^{\circ} \mathrm{C}$. than did Race $\mathrm{B}$, a form of adaptation to the higher temperature. Specific differences in minimum oxygen requirements for several species of Drosophila have been reported, D. obscura requiring 2.8 per cent oxygen in air, compared with D. melanogaster, which was claimed to need only 1.6 per cent." was shown to have a higher oxygen uptake than the genetically different "sensitive" strain." Owing to the profound if complex genetic control of metabolic activity, it is to be expected that hereditarily distinct varieties of organisms would show different and characteristic physiological behavior patterns, particularly under conditions of metabolic stress, reflecting climatic and other adaptations.

Oxygen Consumption in Relation to Sex. Although sex differences in 
metabolic rates are not easy to detect in lower animals, owing to the difficulty of establishing "basal" or standard conditions, differences in the metabolic rates of men and women have been recognized since the extensive work of Benedict and his co-workers. ${ }^{25}, 26,293$ Human males have a higher rate of oxygen consumption than females, at all ages, and the difference becomes more marked with increasing age. The difference may be attributed to more "active protoplasmic tissue" in males, as was indicated by Benedict for the correlation of oxygen uptake with age; it may also represent a genetically determined endocrine-controlled state of higher metabolism. A method for the determination of the time of ov'ulation in women is based on the abrupt increase in body temperature, presumably accompanied by an increased oxygen consumption, which occurs at about the time of rupture of the ovarian follicle.

When an appreciable difference in size exists between male and female the analysis of sex difference is rendered more difficult, as in the sand crab, Emerita, in which the male is smaller than the female and on a weight basis has a higher gas exchange. ${ }^{105}$ Sex differences have been indicated in insects, although the data are not concordant or easy to evaluate. Oxygen consumption by Drosophila pupae is about 21 per cent higher in females, ${ }^{21 s}$ but the difference decreases in later pupal life $\mathrm{f}^{267}$ and in fact was shown to be counteracted as the males finally develop a higher rate of metabolism than the females, a condition initially reported by Poulson."- In the bee moth, Galleria, the males show a greater initial gas exchange, the difference vanishing with subsequent development. ${ }^{77}, 3+1$ An identical situation occurs in Daphnia.":39 One may conclude with Krogh that these results on immature forms depend on the time of measurement in the period of development, the degree of organized tissue for each sex at that time, and the relative rates of differentiation. A clear-cut case of higher male metabolism has been shown in housefly adults (Musca domestica) measured at $20^{\circ} \mathrm{C} .{ }^{103}$ Another "sex" distinction in oxygen consumption is the significant difference of 12 per cent in two well established mating types of Paranecinm calkinsi demonstrated by means of the Cartesian diver technique. ${ }^{40}$

Oxygen Consumption in Relation to Nutrition. The effect of starvation on animal metabolism depends on ability of the animal to live on stored reserves during prolonged periods. The earlier work on man indicated a decrease in the metabolic rate with prolonged fasting, ${ }^{240}, 303,353$ but the extensive investigations of Lehmann and co-workers ${ }^{2-1}$ and of Benedict ${ }^{23}$ have demonstrated little change before appearance of severe inanition. Comparable results are indicated by the work on other homoiothermic animals. In special cases, however, as during hibernation, the decrease in food intake is correlated with reduced respiratory exchange, as well as with lowering of body temperature and profound cardiovascular changes.

Among invertebrates many correlations have been made between oxygen consumption and nutritive intake in insects ${ }^{33}$ and Protozoa. ${ }^{270}$ The organisms behave as though they ate all they could, and their metabolic levels are determined by the amount of oxygen necessary to utilize the food consumed.

The nature of the organic food consumed determines the amount of oxygen required to oxidize it, a principle on which is based the science of biological calorimetry. Lavoisier first demonstrated that the energy content of the food consumed by an organism very nearly equals the heat produced. The measure- 
ment of metabolic activity as a function of the heat produced (direct calorimetry) is less accurate and involves more care than the determination of the oxygen consumption from which the metabolic rate may be calculated (indirect calorimetry). The oxygen required and the caloric vield clepend on the type and amount of organic food oxidized ( lable 40). "The respiratory quotient

\section{TABLE 40. OXYGEN REQUIRED AND CALORIES PRODUCED IN THE UTII IZATION OF DIFFERENT FOODS}

\begin{tabular}{l|rr|r|c}
\hline \multirow{2}{*}{ Organic Food } & \multicolumn{2}{|c|}{ Oxygen Required } & \multirow{2}{*}{ Calories Produced } & Average R.Q \\
\cline { 2 - 3 } & (Liters) & (Grams) & & \\
\hline 100 gm. protein & 93.3 & 133.4 & 419.9 & 0.79 \\
100 gm. fat & 198.9 & 284.4 & 953.7 & 0.71 \\
100 gm. carbohydrate & 82.9 & 118.5 & 419.3 & 1.00 \\
\hline
\end{tabular}

(R. Q.), the ratio of volume of carbon dioxide released to oxygen consumed, is also shown in the table.

The rapidity with which foods can be metabolized in different organisms is known to vary, even for similar substances in closely related animals. The respiratory exchange for the hexose sugars, fructose, glucose, and galactose, has been studied in several mammals and speed of utilization determined. ${ }^{16 i 5}$ The results for the different animals are indicated in decreasing order as follows:

$\begin{array}{ll}\text { Man } & \text { fructose, galactose, glucose } \\ \text { Monkey } & \text { glucose, fructose, galactose } \\ \text { Goat } & \text { fructose, glucose, galactose } \\ \text { Cow } & \text { glucose, galactose, fructose }\end{array}$

In both adult and larval Drosophila, fructose appears to be the most readily utilizable sugar tested. ${ }^{14 !}$

Oxygen Consumption in Relation to Activity. Every change in activity made possible by aerobic respiratory processes can result in an alteration of oxygen consumption, and it is assumed, in dealing with comparisons of other factors on oxygen uptake, that activity remains constant. Thus "basal" metabolic rates are determined with activity at a minimum." Such a condition as a standard of comparison is not always casily attainable, and some investigators have resorted to the use of anacsthetics. Curare ${ }^{1: 0.310,3 n}$ and urethane $e^{+1 .}$ 21:. 29:2 have been used to suppress general activity with no known specific influence on the respiratory exchinge. Extreme doses must be avoided, owing to suppression of muscular tone.

Oxygen consumption increases proportionally with activity to the limits imposed by the ventilation capacity of the respiratory mechanism and the ability of the transport system to carry the respiratory gases. The maximum metabolic exchange in mammils is some 15 to 20 times greater than the resting rate, although the oxigen consumed during the period of excessive activity

* Not, however, under conditions of sleep, hibernation, or estivalion. For an account of the latter in the lungfish see Smith. 
will not be in this proportion, for ordinarily an oxygen debt develops which must be liquidated after the respiratory stress. Insects, which do not build up much of an oxygen debt, may show respiratory excesses of 100 or more times the resting rate. ${ }^{! 0}$

The greatest metabolic increases with activity are those shown by insects during flight-values as high as 90 liters of oxygen per kilo per hour having been recorded for bees, ${ }^{194,}{ }_{272}$ and for the butterfly, Vanessa (Zeuthen, cited by Krogh ${ }^{216}$ ), an increase of better than 100-fold (Table 4l). Chadwick ${ }^{64}$ ingeniously correlated wing movements with oxygen consumption in individual Drosophila on fights of an hour or more. The oxygen consumption rate

\section{TABLE 41. OXYGEN CONSUMPTION OF SOME INSECTS DURING SIMULATED FLIGHT}

(Expressed in cc./gm./hr.)

\begin{tabular}{|c|c|c|c|}
\hline \multirow{2}{*}{ Insect } & \multirow{2}{*}{ Condition } & \multicolumn{2}{|c|}{ O. Consumption } \\
\hline & & Average & Maximum \\
\hline Blow-Hy (Lucilia sericata)"' & true flight & 95.6 & 187.0 \\
\hline Butterfly (Thais cassandra) & $\begin{array}{l}\text { nicotine } \\
\text { convulsed }\end{array}$ & -- & 216.0 \\
\hline $\begin{array}{l}\text { Butterfly (Vanessa sp.) } \\
\text { (Zeuthen, unpub., in Krogh }{ }^{216} \text { ) }\end{array}$ & true flight & 90.0 & -- \\
\hline Hive bee (Apis mellifera) & true flight & -- & 312.0 \\
\hline Hive bee (Apis mellifera) $)^{1: 4}$ & true flight & 87.0 & 100.2 \\
\hline Fruit fly (Drosoplibla repleta) & true flight & 21.0 & 43.7 \\
\hline Fruit fly (Drosophila anerica) & true flight & 21.8 & -- \\
\hline
\end{tabular}

increases from 28 to $350 \mathrm{cu} . \mathrm{mm}$. $/ \mathrm{gm}$. $/ \mathrm{min}$. to correspond with a wing rate of 11,000 strokes per minute. Only a small oxygen debt develops, and this is paid off within two minutes after cessation of the flight.

Various drugs can be applied to stimulate oxygen consumption in animals, just as anacsthetics may be used to depress metabolic activity. Potent stimulants are the compound dinitrophenol (DNP) and its derivatives, about which a large literature has grown up. As an example of the stimulating capacity of DNP one may cite the 342 per cent increase in rate of oxygen consumption demonstrated by Bodine and Boell ${ }^{35}$ in grasshopper embryos during diapause. Of interest in this connection is the work of Clowes and Krahl ${ }^{72}, 207$ on Arbacia cmbryos, which demonstrates that although the nitrophenols may stimulate metabolism they inhibit cell division, thus emphasizing the polyphasic nature and complexity of growth processes.

Attempts to stimulate oxygen consumption by administration of endocrine products have met with some interestingly conflicting results. It is well recognized that thyroid extract, thyroxin, and thyroglobulin increase metabolic activity in amphibians and mammals. However, this is not true for all vertebrates. Fish do not respond with a clear-cut increase in metabolism after thyroid feeding, $10,2+4 ;$ and lampreys also fail to respond metabolically to thyroidl 173, 21:2 (sec Chapter 22).

The confinement of a number of animals together often stimulates them into increased activity and oxygen consumption, but the opposite effect is recorded for the goldfish, in which the presence of company seems to depress 
metabolism. ${ }^{31:: ~ I f ~ t h i s ~ w o r k ~ o n ~ C a r a s s i n s ~ i s ~ e x p e r i m e n t a l l y ~ s o u n d, ~ t h e n ~ i t ~}$ becomes a hehavioristic problem, because the effect of numbers in reducing metabolism can be prevented by darkening the aquarium or blindfolding the fish. Conversely, according to Schlaifer, the oxigen consumption is decreased if mirrors are added to increase the apparent number of fish seen by the experimental animal. Indeed, even dead fish and fish mounts produce the same effect. A "group effect" also demonstrated in Perca may be attributed to the depressant action of metabolites produced by additional fish. ${ }^{12 \pi}$

The correlation between oxrgen consumption and habitat is perhaps best

\section{TABLE +2. OXYGEN CONSUMPTION OF RELATED ORGANISMS} FROM DIFFERENT HABITATS

(Expressed in cc./gm./hr.)

\begin{tabular}{|c|c|c|c|}
\hline Animal & Habitat or Habit & $\begin{array}{l}\text { IVeight } \\
\text { (gm.) }\end{array}$ & $\frac{\mathrm{O}_{2}}{\text { Consumption }}$ \\
\hline \multicolumn{4}{|l|}{ Isopods* } \\
\hline Asellus aquaticus ${ }^{113}$ & low stream & .0053 & .51 \\
\hline Asellus aquaticus ${ }^{113}$ & swift stream & .0047 & .86 \\
\hline \multicolumn{4}{|l|}{ Trichopterids* } \\
\hline Limnophilus vittatus ${ }^{1 ! 3}$ & pond & .0046 & .51 \\
\hline Hydropsyche sp. ${ }^{113}$ & swift stream & $.005+$ & .77 \\
\hline \multicolumn{4}{|l|}{ Small ephemerid nymphs* } \\
\hline Coenis sp. ${ }^{113}$ & pond & .0011 & .29 \\
\hline Cloëon dipternm ${ }^{1 . s}$ & pond & .0003 & .61 \\
\hline Ephemerella ignata ${ }^{113}$ & stream & .001 & .95 \\
\hline Baetis rhodani & swift stream & .0003 & 2.57 \\
\hline \multicolumn{4}{|l|}{ Large ephemerid nymphs* } \\
\hline Ephemera vulgata ${ }^{113}$ & in mud, in pond & .013 & .28 \\
\hline Ephenera danica ${ }^{113}$ & in sand, in stream & .014 & .37 \\
\hline Ecdyonurus venosus ${ }^{113}$ & swift stream & .006 & .61 \\
\hline \multicolumn{4}{|l|}{ Crayfish $\dagger$} \\
\hline Astacus leptodactyln $\ln ^{3 \varsigma 1}$ & lake & $-\cdots$ & .07 \\
\hline Astacus torrentimmis & swift stream & -- & .10 \\
\hline \multicolumn{4}{|l|}{ Fish } \\
\hline Mackerel (Scomber scombrus) ${ }^{13}$ & active, fast swimmer & 138 & .726 \\
\hline Butterfish (Poronotus tricanthus) ${ }^{13}$ & moderately active & $18+$ (av.) & $.301_{+}^{+}$ \\
\hline Scup (Stenotomus chrysops) ${ }^{13}$ & sluggish & 123 & $.174_{+}^{+}$ \\
\hline Tautog (Tautog onitis) $)^{13}$ & very sluggish & 120 & .062 \\
\hline Puffer (Spheroides maculatus) ${ }^{100}$ & very sluggish & -- & .062 \\
\hline Lungfish (Lepidosiren paradoxa) (n) $^{307}$ & air breather & -- & .042 \\
\hline Pirarucus (Arapaima gigas) ${ }^{307}$ & air breather & -- & .009 \\
\hline
\end{tabular}

considered in regard to oxygen utilization and critical oxygen tensions. Nevertheless, oxygen-rich and oxygen-deficient media are significant and naturally have been investigated widely, although not extensively enough. ${ }^{113,275}$ A number of arthropods and several fish from different habitats and with different habits are listed in Table 42, with oxygen consumption measurements as determined at rest under laboratory conditions. In the aquatic arthropods, the more agitated and oxygenated the medium, the greater the oxygen consump-

* Determined at $10^{\circ} \mathrm{C}$. under mild urethane or chloretone anaesthesia.

+ Determined at $19-21^{\circ} \mathrm{C}$.

$\mp$ Wrong in author's summary and since confused in literature. 
tion. Among fish, too, oxygen uptake is correlated with activity and efficiency of the respiratory mechanism as well as with the availability of oxygen.

Oxygen Consumption in Relation to Seasons. Seasonal variations in oxygen uptake are not easy to distinguish from variations produced by such factors as temperature, nutrition, activity, and the like, with which they are bound. Rather, the seasonal variations represent the balance of these individual factors. along with intrinsic influences of the organism itself, such as endocine control of diurnal and reproductive cycles. Changes in the metabolic rate at oestrus have been noted in birds. Some evidence is available, however, for seasonal oxygen consumption adjustment to temperature changes. Whereas poikilothermic marine animals, for the most part respond to seasonal decreases in temperature by a proportional reduction in oxygen consumption, some regula tion has been demonstrated in the cumner, ${ }^{1,01}$ Fundulus, 360 and the sand crab, Emerita talpoida. ${ }^{10 .}$ The adaptation tends to offset the effect of temperature and to maintain a stable metabolic rate from season to season. Determination of the metabolism of Fundulus at $15^{\circ} \mathrm{C}$. in February gave higher values than measurement at the same temperature in luly. The cumner, Tautogolabrus, when investigated for oxyen consumption in summer and winter at temperatures ranging from $0^{\circ}$ to $30^{\circ} \mathrm{C}$, yielded slightly higher values in winter than in summer for temperatures below 15 . The sand crab shows the best regula tion of all and, according to Edwards, behaves somewhat like homoiotherms, making an internal adjustment in metabolic rate to offset the temperatures experienced during the winter. The oxygen uptake at all temperatures below $20^{\circ} \mathrm{C}$. was greater in winter than in summer, the rate of winter metabolism at 3 being about four times the summer rate at this temperature. It would be of interest to know whether this scasonal temperature regulation might be reproduced in part by short term acclimatization-i.e., would one of these animals acclimatized to low temperature in the summer show increased metabolism?

Oxygen Consumption in Relation to Temperature. The responses of oxygen consumption to temperature changes have been recognized for many years, and valuable survers of the basic literature are available. ${ }^{19 .}$. 19:. 214 i general distinction can be mate between the poikilothermic animals whose oxygen consumption increases with rising temperatures and the homoiothermic animals whose oxygen consumption increases as the temperature decreases. The difference is mainly due to the presence in the latter of a temperature-regu lating mechanism by which external temperature changes are offset to a large extent by metabolic and phisiological reactions, vasomotor control playing a predominant role. The frequently used expression, $Q_{10}$, is a convenient measure of the effect of changing temperature on respiratory processes, but the variation in (2) values orer the biological temperature range makes its use and signilicance somewhat limited (see Chapter 10). Mluch higher $Q_{10}$ values than the expected 2-3 are obtained at low temperatures; Krogh s experiments on poikilothermic vertelorates includes values from $\left(Q_{10}=10.9\right.$. based on measurements over the five degree range from 0 to 5 C., to $Q_{11}=2.2$ at $20-27.5 \quad(-213$

1 more precise expression to describe temperature characteristics is based on the Arrhenius formula and is constant over the biological temperature range for each given set of conditions (Ch. 10). The equation reflects the 
underlying metabolic chemical reactions, and, judging from the constancy of the values obtained, represents fundamental relationships between different biological systems. ${ }^{19.79}$ By this formula the temperature characteristic-energy of activation or "thermal increment"- of the given system may be found. 'This value may vary, but in a wide variety of respiratory processes is constant at 11,500 or 16,500 calories. For practical purposes when the log of the reaction rate is plotted against reciprocal of absolute temperature the thermal increment is given by the slope of the curve. The inference that such thermodynamic constancy between metabolic processes depends on so-called "master reactions" or that comparable enzyme systems are at work remains to be demonstrated.

High basal metabolic rate is a basic condition for homoiothermism and heat regulation. Heat production can be measured by direct calorimetry or may be calculated from $\mathrm{CO}_{2}$ production and $\mathrm{O}_{22}$ consumption. In man with an R.Q. of 0.82 , heat is produced to the extent of 4.8 cal. per liter of $\mathrm{O}_{2}$ con-

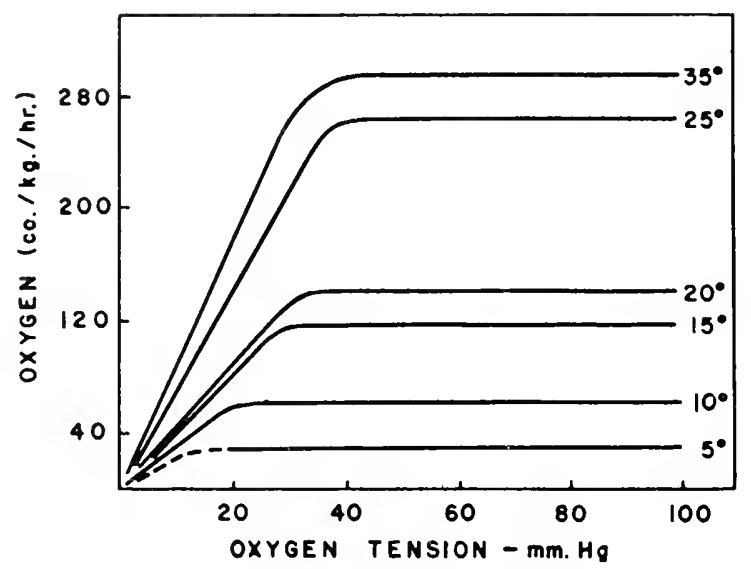

Fig. 53. The interrelation between "maximum steady state" of oxygen consumption, oxygen tension, and temperature in the goldfish, Carassius. The reduction of $\mathrm{O}_{2}$ tension is more effective at higher temperatures. From Fry and Hart. ${ }^{122}$

sumed.".5 The rate of increase in total heat production with body weight varies for different species.

The ability of animals to withstand temperature extremes and their metabolic acclimatization to extreme heat and cold constitute an interesting biological story but one on which there is as yet only scattered information. Some organisms live in environments in which the temperature never exceeds $0^{\circ}$ C. ${ }^{21+}$ Irving ${ }^{1 \times i}$ cites the case of certain arctic animals, notably the ground squirrel, Citellus, which can withstand environmental temperatures down to minus $30^{\circ} \mathrm{C}$. lor six hours, under which conditions the respiratory exchange increases, but the R.Q. decreases, indicating fat utilization. The arctic fox, when exposed to this temperature, shows no metabolic increase (Scholander, personal communication). The gas exchange of poikilothermic animals at higher temperatures increases until a point is reached (at about $35^{\circ} \mathrm{C}$. body temperature) when certain deleterious effects appear, causing the oxygen uptake to drop suddenly. Fry and Hart ${ }^{1} \cdot 2$ have recently shown that the 
"basal" rate of oxygen consumption for the goldfish, Carassius, increases up to a temperature of $35^{\circ} \mathrm{C}$., but during activity the maximum metabolic level is limited to approximately $30^{\circ} \mathrm{C}$. The "maximum steady state," the condition of normal activity, at various temperatures, is definitely related to given rates of oxygen uptake, the higher the temperature maintained the greater the oxygen consumption (Fig. 53). At reduced tensions the oxygen consumption rapidly falls off, being affected at a higher oxygen tension for the higher metabolic rate, indicating the inability of the fish to maintain the specific activity at the designated temperature. One should not lose sight of the fact, moreover, that the solubility of oxygen is much reduced at the higher temperatures, although activity is increased. High temperature tolerance is directly associated with organic composition, particularly that of the unsaturated fatty acids, and this is frequently reflected in the gas exchange and R.Q. The effects of age and size on temperature adjustment are important as seen in recent evidence on sand crabs, click beetles, beach fleas, and killifish, which clearly indicate that smaller animals in a species respond to temperature changes more markedly than do larger ones ${ }^{103,360}$ (Fig. 54).

Metabolic adaptations to temperature differences have been noted in relation to species adjustment in Drosophila (p. 235) and to seasonal acclimatization among crustaceans and fish (p. 240). Likewise adaptations to temperature variations associated with geographic distribution of Crustacea may be demonstrated, as in the comparison of oxygen consumption by prawns from Swedish and from English waters. When measured at the temperatures of their natural environments ( 5 and $15^{\circ}$ C.) Swedish erayfish consume less oxygen than their English relatives. However, if measured at the same temperature $\left(10^{\circ}\right.$ C. $)$, the metabolic rate of the northern forms may exceed by more than twice that of the southern specimens. ${ }^{112}$. 11: As locomotion, breathing movements, and heart rates of the southern species are only slightly increased over those of the northern species (the animals being to this extent somewhat acelimatized to their warmer environment), the higher metabolic rate of the Engtish crayfish may be explained as due to their greater "nonactivity" metabolism (sce Chapter 10).

When measurements were made at the same temperature-16 C.-river lampreys from a $3^{\circ} \mathrm{C}$. environment consumed about 50 per cent more oxygen than those from $16^{\circ} \mathrm{C}$. waters. 311 The same sort of acclimatization has been observed in Drosophila. On the other hand, when both are measured at $25^{\circ} \mathrm{C}$., Amblystoma raised at $20^{\circ} \mathrm{C}$. have a higher rate of oxyen consumption during early development than those reared at $15^{\circ} \mathrm{C}$.

Benedict"- has generalized the situation by stating that, when the temperature of the environment is lowered to $10^{\circ} \mathrm{C}$., the metabolic activity of warmblooded animats will always exeeed that of eolel-blooded animals at that temperature, and, conversely, when the temperature is elevated to the resion of $37^{\circ} \mathrm{C}$., the metabolic heat production of the poikilotherms will never excecel $3 t$ per eent and will average 12.5 per cent of that of the homoiotherms at this temperatture.

Diurnal and seasonal rhythms in the temperature-oxygen eomsumption relationship are important in many organisms-much of which has to do with daily routine on the one land and with seasonal adjustment on the other. An apparenty condegenous diumal variation in oxygen consumption, possibly 
reflecting an intrinsic activity or nutritive cycle, has been claimed for the catfish-measured, although not necessarily maintained, under conditions of total darkness and constant temperature and water flow. $\Lambda$ consistently higher oxygen consumption (about 50 per cent) was demonstrated in the afternoon and evening, compared with that of the forenoon. ${ }^{16 t}$ Depressions in the oxygen consumption correlated with both temperature changes and food shortage have been pointed out in various warm-blooded animals. The chicken, for instance, has a metabolic maximum in the morning ( $78^{\circ} \mathrm{F}$., air temperature) and a low

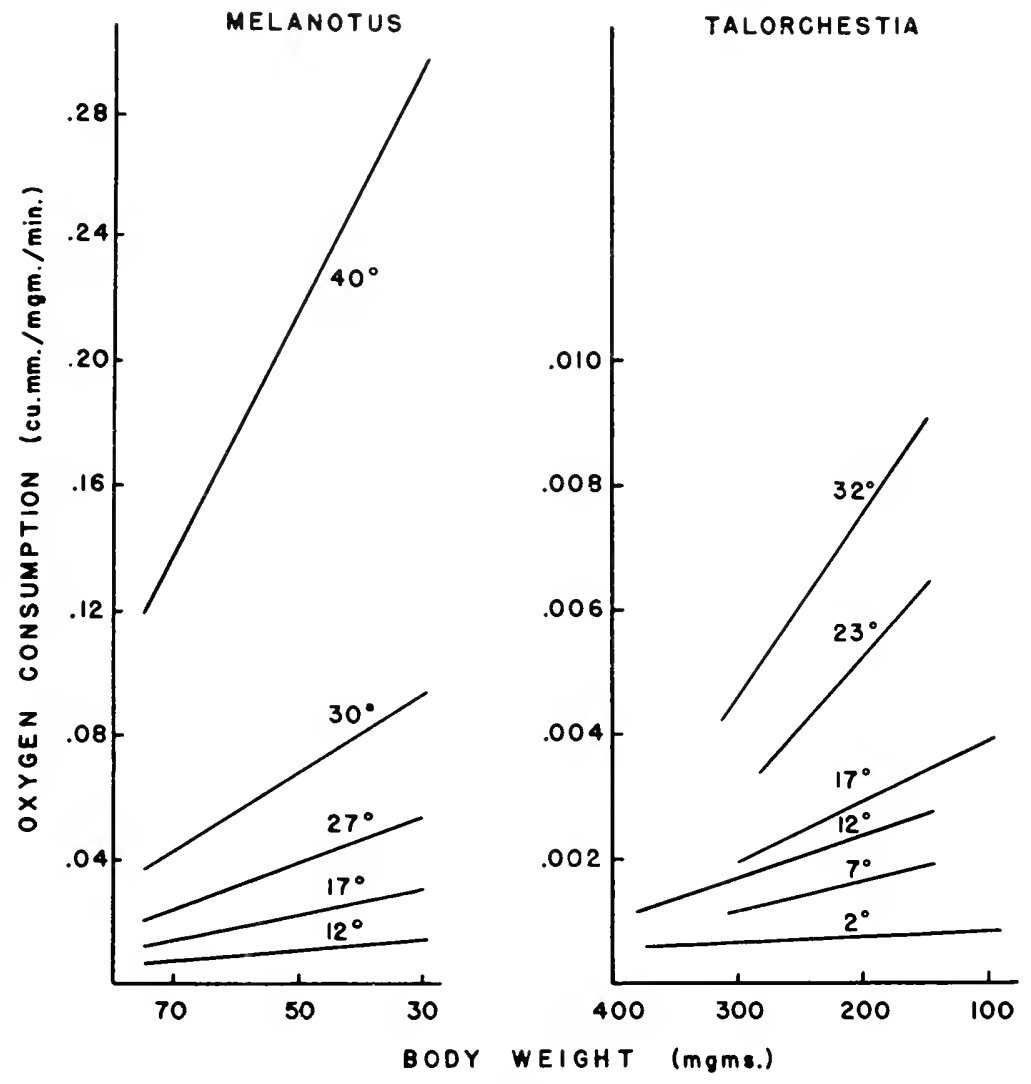

Fig. 54. Oxygen consumption in click beetles, Melanotus, and beach fleas, Talorchestia, of different weights, showing the greater response of the smaller individuals to temperature change. After Edwards. ${ }^{103}$

at evening $\left(61^{\circ}\right.$ F.). ${ }^{20}$ Koskimies ${ }^{205}$ has recently shown that under conditions of partial starvation swifts (Micropus a. apus) become poikilothermic-the body temperature drops in the early morning hours to within $2-3^{\circ} \mathrm{C}$. of the external temperature, respiratory breathing rate falls from about 40 to 10 per minute, and the oxygen consumption decreases proportionately. The sloth ${ }^{15 \%}$ too tends toward poikilothermy as a respiratory increase accompanies a temperature rise from $33^{\circ}$ to $37^{\circ} \mathrm{C}$. All things considered, it becomes increasingly evident that many mammals and birds show marked changes in internal temperature and 
oxygen consumption, and there is no clear-cut distinction between two great groups of animals-poikilothermic and homoiothermic. The initial lack of a temperature-regulating mechanism in rery young animals, followed by an adjustment period during which the temperature control becomes firmly established, is not uncommon in higher rertebrates. The labile respiratory and cardiorascular conditions so pronounced in hibernation among mammals are discussed as a function of temperature in Chapter 10.

Temperature changes thus prove to be intimately interconnceted with the respiratory pattern of animals, in some instances resulting in adjustment to cnvironmental variation and in others, through metabolic regulation, counteracting the external change over a significant temperature range.

The consideration of animal metabolism leads to the conclusion that the rate of oxygen consumption as usually determined under conditions approximating rest may serve as a reference point in defining the respiratory state of an individual, but there are many modifying factors, both within and without the organism, which affect the character of the gas exchange. It is of significance to understand the part each factor plays in the response of the organism to its oxygen cnvironment, for here lies the basis of important physiological adaptation.

\section{INTERRELATIONS BETWEEN OXYGEN TENSION AND OXYGEN CONSUMIPTION}

General Types of Reactions. The rates of oxygen consumption and of breathing movements are variably dependent on the available oxygen, a function of the gas tension. Nany organisms are able to maintain a steady respiratory state and are independent of oxygen tension over a considerable range, as is man down to an oxygen concentration of about 13 per cent ( $100 \mathrm{~mm} . \mathrm{Ig}$ ) or the earthworm to 3 per cent of atmospheric pressure. These animals are capable of respiratory regulation. Many other animals (Nereis, Homarus, etc.) show a direct dependence of oxygen consumption on oxygen tension, and each variation in gas pressure is reflected in a metabolic change in the organism. The different responses to decreased oxygen tensions are in some cases attributed to different activity adjustments as, for example, in the mackerel and the toadfish-the sluggishness of the latter corresponding with its ability to live in oxygen-deficient water, compared with the more active mackerel which an survive only in an oxygen-rich medium. In other metabolic comparisons the lack of dependence of oxrgen consumption on tension is due to sheer efliciency of the respiratory mechanism at low tensions. This is seen in the chironomid insects, for instance, whose plasma hemoglobin permits them to extract oxygen at very low tensions and to survire oxygen depletion much better than their hemoglobinless relatives, a condition which must affiord considerable survival value in oxygen-deficient water. The following paragraphs will consider significant physiological adaptations to oxygen tension and the various ecological consequences involved.

Dependence of Oxygen Consumption on Oxygen Tension. The rate of oxyen consumption varies linearly with tension over a wide pressure range in those animals which show little capacity to regulate, e.g., Nereis, Homarus, Limulus, Callinectes." "The oxygen consumption is thus dependent on tension, indicating little respiratory contiol and the probable importance of 
diffusion in supplying the tissues with oxygen. This proportionality between consumption and tension may apply to pressures above normal atmospheric oxygen tensions in a number of non-regulatory animals, for example, limax and Limulus. Such a phenomenon indicates either an increase in oxidative processes as a result of the added oxygen partial pressure or a normal unsaturation of oxygen in the tissues at atmospheric pressures. Critical oxygen tensions $\left(t_{e}\right)$, gas pressures above which oxygen consumption is constant despite varialtions in the partial pressure, are given by Maloeuf ${ }^{24:}$ as follow's, for four animals, all of which he regards as suffering a state of oxygen unsaturation at normal ambient tensions:

Cambarus clarkii Fundulus heteroclitus $\mathrm{t}_{\mathrm{c}}$ Limulus polyphemus Triturus pyrrhogaster

\begin{tabular}{|c|c|c|c|}
\hline & & $t_{c}$ & \\
\hline $\begin{array}{l}186 \\
190\end{array}$ & & $\mathrm{Hg}_{g}$ & $\mathrm{pO}$ \\
\hline 220 & " & & \\
\hline 760 & $"$ & " & $"$ \\
\hline
\end{tabular}

In contrast to this group of individuals which adjust metabolism according to environmental oxygen tensions stand those organisms with great ability to

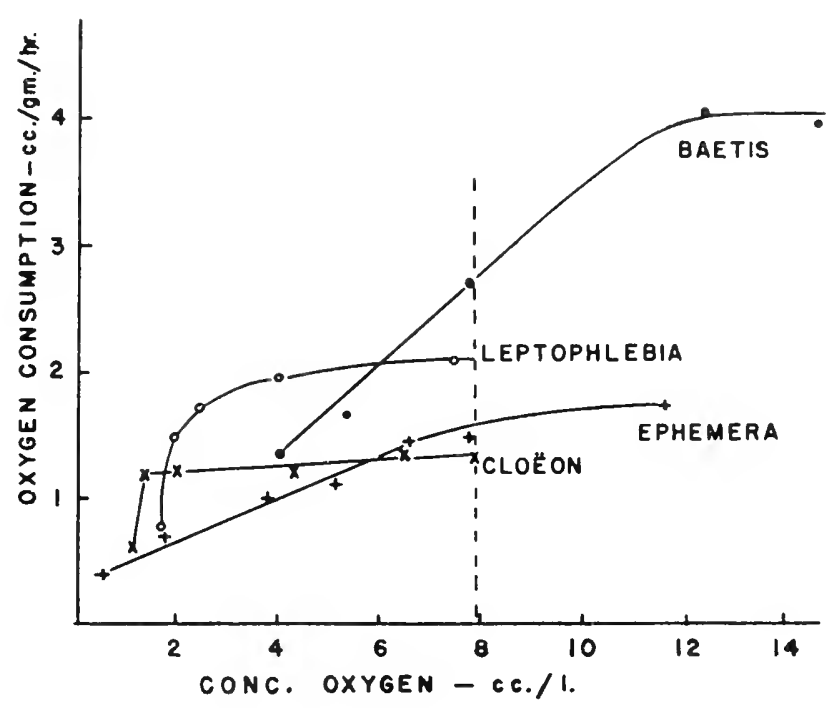

Fig. 55. Oxygen consumption plotted against oxygen tension for maytly nymphs, showing dependence in Baetis, independence in Cloëon, and intermediate conditions in Leptophlebia and Ephemera. From Fox et al. ${ }^{116}$

regulate so as to maintain a constant consumption in the face of changing oxygen pressure. Uptake is therefore independent of oxygen tension, at least over a very wide range--e.g., the crustaceans Astacus and Corcinus, and the molluscs Aplysia and Eledone. In Figure 55 the oxygen consumption of the ephemerid, Cloëon dipterum, is shown constant down to about $1.6 \mathrm{cc}$. (..2/liter of water, corresponding to $32 \mathrm{~mm}$. Hg partial pressure of oxygen. ${ }^{11 \text { if }}$ Such a degree of respiratory independence indicates respiratory control or a state of more than adequate oxygen saturation prevailing in the tissues even at low 
pressures. That such is sometimes the case is known in those hemoglobincontaining invertebrates whose blood pigments are saturated at very low pressures and which show low oxygen unloading tensions, e.g. Chironomis $\left(\mathrm{t}_{1 / 2}\right.$ :a1 $=0.6 \mathrm{~mm}$. $\lg$ at $\left.0 \mathrm{CO}_{2}\right)$ and the snail, Planorbis $\left(\mathrm{t}_{1 / 2}\right.$ sat. $=1.9 \mathrm{~mm}$. IIg at $0 \mathrm{CO}_{22}$ ). The hemocyanin of Busycon blood (Gastropoda) has a $t_{1 / 2}$ sut of $13 \mathrm{~mm}$. I Ig at $0 \mathrm{CO}$, reducible to less than $3 \mathrm{~mm}$. $\mathrm{Hg}$ at $19.6 \mathrm{~mm}$. I lg CO... $19: 7$ This permits survival in oxygen-deficient water in equilibrium with air at less than 5 per cent of an atmosphere.

The relationship of oxygen consumption to oxygen tension for most organisms is summarized in Figure 55, represented by a hyperbolic curve with no clcar break to indicate the critical tension-a plot midway between the extreme non-regulatory Baetis $\left(t_{r}=240 \mathrm{~mm} . \mathrm{Ig}\right)$ and the highly regulatory Cloëon $\left(t_{r}=32 \mathrm{~mm}\right.$. $\left.\mathrm{l} \mathrm{lg}\right)$. In his signilicant review of many such intermediates, Tang $3: 3$ relates ce. per gram hour of $\mathrm{O}_{2}, \Lambda$, to $\mathrm{O}_{2}$ tension, $P$, in the equation:

$$
A=\frac{P}{K_{1}+K_{2} P}
$$

where $K_{1}$ and $K_{2}$ are constants. Different values of $K$ indicate differences in the degree of regulation. In the event $K_{1}$ is very small, $A$ becomes approximately constant; i.e., the oxyen consumption is independent of tension.

The lack of dependence of oxygen consumption on tension may be attributed in some instances to the respiratory stimulating capacity of oxygen want, acting to increase the breathing rate and respiratory activity. Such a control implies the presence of respiratory centers and, ergo, a higher state of respiratory development than for non-regulatory animals. A correlation with phylogenetic position, however, cannot be demonstrated.5. $15.5,1.5 \%$

Critical Tensions. The actual presentation of comparative values of critical tensions is no mean task, owing to the vicissitudes with which these values respond to external conditions, and to the variations in the experimental pro cedures under which the determinations are carried out. It has been shown very clcarly in the flatworm, Planaria agilis, for example, that the oxygen consumption gencrally is constant down to a concentration of $3 \mathrm{cc} . \mathrm{O}_{2} / \mathrm{l}$., but if the ()$_{2}$ deprivation is produced very slowly consumption may continue on down to a concentration of $0.5 \mathrm{cc}$. $/$. The $\mathrm{t}_{\mathrm{c}}$ may be approximately $60 \mathrm{~mm}$. Hg, or 10 , depending on the rate of oxygen decrease. A good deal of caution therefore is urged against too enthusiastic interpretation based on such data as those indicated in Table 43, which includes critical tensions culled from the literature and some recalculated into partial pressures from solubility and saturation data. Absent from this list, of course, are those organisms which show complete

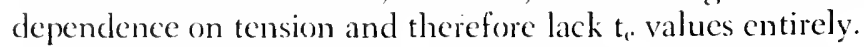

Although a low $t_{\text {r }}$ value undoubtedly connotes higher metabolic efficiency, everything else being equal, other factors are of considerable importance in the matter of low oxygen tolerance-namely, oxygen utilization, metabolic level, and the like. The toadfish is able to extract practically all available oxygen out of his environment, but the mackerel, trout, and similarly active fish require a moderately high oxygen level at all times. The tench can survive indefinitely at ordinary temperatures in water with no more than $0.3 \mathrm{cc}$. $\left(\mathrm{O}_{2} / \mathrm{l}\right.$., but the 


\begin{tabular}{|c|c|c|}
\hline Animal & $\mathrm{t} .(\mathrm{mm} . \mathrm{Hg})$ & lemp. $\left({ }^{\circ} \mathrm{C}.\right)$ \\
\hline Tetralnuena gelei" & 2.5 & \\
\hline Paranecimm' & 50 & $18-20$ \\
\hline Aurelia anrita: & 120 & \\
\hline Pelmatoligdra oligatetis & 60 & \\
\hline Planaria agilis: & 55 & 20 \\
\hline " $\quad 3 \cdots$ & 10 (slow pO-decrease) & 20 \\
\hline Tubifex tubifex ${ }^{\text {tu }}$ & 25 & \\
\hline Lumbricus & 76 & 10 \\
\hline 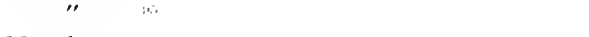 & 19 & 25 \\
\hline Urechis $^{12}$ & 70 & 17 \\
\hline Hirudo & 32 & 25 \\
\hline Ostrea gigas s:ut $^{1}$ & 30 & \\
\hline Helixis & 75 & \\
\hline Loligo & +5 & 20 \\
\hline Arbacia eggs (unfertilized) & 40 & 25 \\
\hline$" \quad$ " (tertilized) & 50 & 25 \\
\hline " " " " & 20 & 18.20 \\
\hline Paracentrotus lividus eggs (lent. & 228 & \\
\hline Paleominetes & 80 & 20 \\
\hline Potamobius astaca ${ }^{1,}$ & 55 & $10 \cdot 11$ \\
\hline Cambarus ${ }^{1::}$ & +0 & \\
\hline Cloëon dipterum $m^{1 . \bar{T}}$ & 32 & 10 \\
\hline Leptophlebia marginat $a^{11 .}$ & 53 & 10 \\
\hline Baetis $^{11 .}$ & $2+0$ & 10 \\
\hline Dixippus morosus ${ }^{\text {iti }}$ & 159 & \\
\hline Calliphora erythrocephala (bluw Hy $)^{129}$ & 57 & 29 \\
\hline Melanoplus differentialis eggs (grasshopper) & 80 & \\
\hline Termopsis navigensis (termite) & 40 & 40 \\
\hline Chironomus thummi (larval lragments) ${ }^{143}$ & 70 & $16-23$ \\
\hline Chironomus plumosus $\mathrm{s}^{\mathrm{tur}}$ & 60 & 17 \\
\hline Limnephilis rhombicus (caddis $\mathrm{H}_{\mathrm{y}}$ ) & 25 & \\
\hline Fundulus heteroclitus & 16 & 20 \\
\hline Tetraodon maculatus (puffer) (::h $^{1}$ & 100 & $20-21$ \\
\hline Stenotomus chrysops (scup) $)^{1,3-}$ & 30 & $20-21$ \\
\hline Cichla ("Tucunare") & 32 & \\
\hline Rana $a^{1: 46}$ & +5 & \\
\hline
\end{tabular}

more active perch require twice this concentration. Conversely, the frog-and this is not indicated in any correlation of critical tensions-has been claimed to get along for 24 hours in the absence of oxygen. $=1$

The response of individual tissues to decreasing oxygen temsions need not be the same as that of the entire organism. Isolated frog muscle, for example. shows a constant oxygen uptake when the partial pressure of oxygen is reduced from $760 \mathrm{~mm}$. to $0.5-2.0 \mathrm{~mm}$. Hg. ${ }^{16.5}$

Blood Pigments and Oxygen Tension. An important factor in the ability of an organism to regulate oxygen consumption and thereby remain physiologically aloof from the depressive effect of reduced tension is the affinity of the blood pigments for oxygen, and particularly the saturation tension $\left(t_{\text {sit }}\right)$ and tension at half-saturation $\left(t_{1 / 2-a t}\right)$, as well as the effects of carbon dioxide, $p^{\mathrm{H}} \mathrm{I}$, and temperature on these blood oxygen levels. This is discussed at length in connection with blood pigments (see Chapter 9). However, some correlations are in order here. 
Hiestand ${ }^{16: 2}$ demonstrated in dragonfly nymphs and crayfish that the $t_{c}$ value is depressed by carbon dioxide, by low $\mathrm{pH}$, and by a combination of both, acting as respiratory stimulants apparently through a control center. On the other hand, in those invertebrates containing hemoglobin, carbon dioxide increase may reduce the oxygen uptake, as Ewer ${ }^{109}$ demonstrated in Chironomus, and so tend to elevate the critical tension. Elimination of the action of hemoglobin in the blood by use of carbon monoxide more effectively accomplishes the same thing. Oxygen consumption in the earthworm is normally relatively independent of tension down to about 3 per cent $(20 \mathrm{~mm}$. $\mathrm{Hg}$.), but in the presence of carbon monoxide it is independent down to only 8 per cent of an atmosphere $(60 \mathrm{~mm} . \mathrm{Hg})$.

Temperature, Tension and Oxygen Consumption. At low temperatures the metabolic activity of poikilotherms is reduced and they are able to withstand oxygen tensions below those required at higher activity levels (Fig. 53).

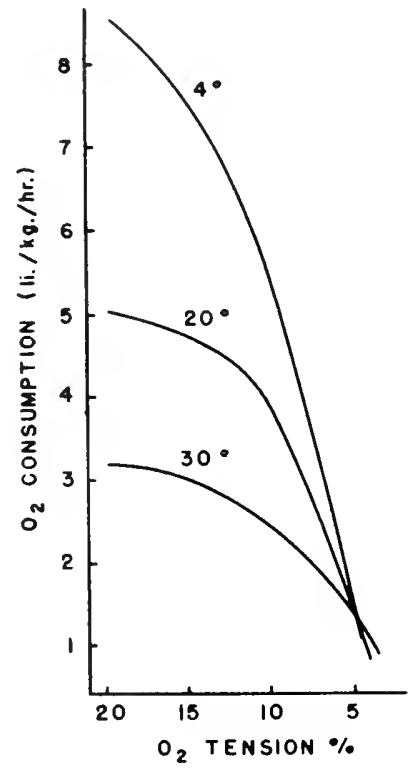

Fig. 56. Relation of oxygen tension to oxygen consumption at different temperatures in white mice. At low tensions the rates of $\mathrm{O}_{2}$ uptake are the same despite temperature differences. After Chevillard et al. ${ }^{\text {s. }}$

In hibernating mammals also the oxvgen requirements may fall to 5 per cent of that of non-hibernating animals. Homoiotherms are faced with another problem at low temperatures, however, and any small change in oxygen tension can have profound effects. Mice maintained at $30^{\circ} \mathrm{C}$. can tolerate oxygen depletion to $38 \mathrm{~mm}$. Hg partial pressure, but those kept at $20^{\circ} \mathrm{C}$. withstand concentration reductions only down to $55-60 \mathrm{~mm}$. Hg when the reduction in oxygen occurs at the same rate. ${ }^{68}$ However, if care is taken to reduce the oxygen concentration slowly enough, those maintained at $20^{\circ} \mathrm{C}$. can also tolcrate oxygen at $38 \mathrm{~mm}$. $\mathrm{Hg}$ pressure. Although the oxygen consumption 
at high tensions is highest for animals maintained at $4^{\circ} \mathrm{C}$. and lowest for those kept at $30^{\circ} \mathrm{C}$, the rate of oxygen consumption is the same for all when measured at $35-40 \mathrm{~mm}$. I Hg oxygen tension (Fig. 56). The oxygen tension necessary to maintain a constant body temperature of $37^{\circ}$ in mice at $30^{\circ}$ C. air temperature is $64 \mathrm{~mm}$. $\mathrm{Ig}$; at $20^{\circ}$ it is $97 \mathrm{~mm}$. Hg, and at 4 it is 155 $\mathrm{mm}$. Hg. At lower tensions the body temperature declines.

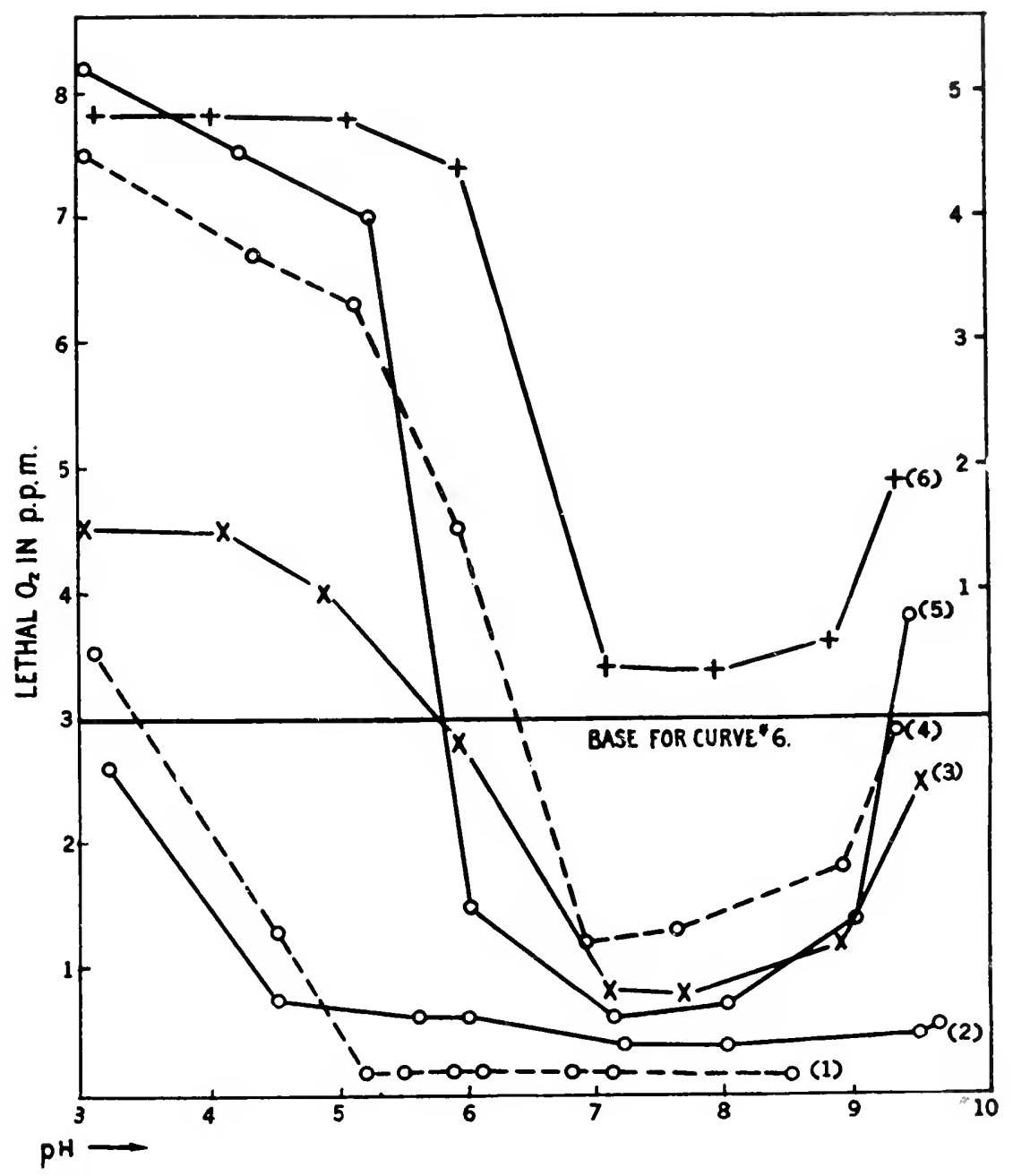

Fig. 57. Curves showing the least tolerable oxygen concentration as a function of $\mathrm{pH}$ for fish: (1) goldfish, (2) yellow perch, (3) and (4) bluntnose minnow, (5) steelcolored minnow, (6) bluegill. Temperatures $18-25^{\circ} \mathrm{C}$. From $\mathrm{W}$ iebe et al. ${ }^{\text {san }}$

Effect of $\mathrm{pH}$ on Minimum Tension. The minimum oxygen concentration which can be tolerated may vary with the $\mathrm{pH}$, but whether this is an effect of its respiratory stimulating powers or of the ability of the organism to extract oxvgen within a limited $\mathrm{pH}$ range is uncertain. Probably the respiratory stimu- 
lation set up by the acidosis, on the one hand, and the Bohr effect, on the other, has a good deal to do with the phenomenon. In a series of fresh-water fish investigated for their least tolerable oxygen tension as a function of $\mathrm{pH}$, the majority reached the minimum oxygen level at or near the neutrality point. ${ }^{\text {:6it }}$ Most of those studied (largemouthed and smallmouthed black bass, white crappie, yellow perch, rainbow trout, sunfish, and goldfish) were able to cxtract oxygen at low tensions over a wide $\mathrm{pH}$ range-pH 5 to 9 in several cases-whereas the bluegill minnow was limited to $\mathrm{pH} 7-9$, and the steelcolored and blunt-nosed minnows to pH 7-8 (Fig. 57).

Relation of Least Tolerable Tension to Size and Age. Size and age may alter the capacity of organisms to utilize oxygen at low tensions and thereby affect the critical tension values. Some discrepancies may appear in Table 45 as a result of such factors. The oxygen consumption rate of large Planaria is limited below $1+-15$ volumes per cent (ca. $110 \mathrm{~mm}$. Hg), well above the $t_{\text {: }}$ values presented in the table. ${ }^{121}$ In the crayfish, Cambarus, higher $t_{\text {. }}$ values were found for larger individuals. ${ }^{15.5}$ Animals weighing 4.3, 9.0, and 17.1 gm. showed critical tensions at 32,48 , and $65 \mathrm{~mm}$. Hg, respectively. The larger (older) the animal, the less the range of respiratory regulation.

Critical Tension and Tissue Oxygen Saturation. The correlation between respiratory regulation and the presence of an oxygen-carrying mechanism, such as the readily saturated hemoglobin transport system in Chironomus, is a relationship which applies directly to interpretations of oxygen consumptionoxygen tension independence. Cloëon (Fig. 55) might be regarded as having a fully saturated oxygen system above $32 \mathrm{~mm}$. Hg tension. This concept has been considered extensively by Maloeuf, $2+2$ who expresses critical tensions as a function of the atmospheric pressure necessary for half saturation of the tissucs. Although some skepticism may be in order concerning the advantages gained by Maloeuf's unorthodox and cumbersome method of handling data, largely recalculated from the work of other investigators, something is to be said for his emphasis on the startling dependence of oxygen consumption on tension-Mytilus, for instance, show's no critical tension at $640 \mathrm{~mm}$. $\mathrm{Hg}$-four times atmospheric pressure of oxygen. The possibility that oxygen saturation of the tissues is of great importance in the problem of respiratory regulation is worthy of consideration. Considerable evidence supports the view that single-celled organisms as well as tracheates without gas transporting systems may be relatively independent of oxygen tension orer a wide oxygen pressure range. Further, animals with transporting systems are on the average about ten times more independent of tension than are those without oxygen transporting mechanisms. Although tissue saturation even at low tensions may be important in the responses of one-celled and tracheate organisms, the absolute rate of metabolism and the adequacy of diffusion at low tension are also important factors. The relative independence of those animals with and without circulating systems probably depends primarily on the presence or absence of blood pigments, rather than of circulation itself.

Oxygen Withdrawal. The ability of animals to withdraw oxygen from their acrial or aquatic media at low pressures governs in part their critical tensions, minimum tolerable oxygen, and indeed their distribution through the ecological adjustment to available oxygen. It is not without some interest to survey the problem of efficiency of oxygen uptake ("utilization" in the sense 
of "Auznutzung" of W' Winterstein" ${ }^{373}$ ) under normal oxygen tensions, in lieu of any wealth of information on measurements at minimum partial pressures (Table Ht). Nthough much variation prevails, certain trends among groups are apparent. W'ithdrawal is low in the sponges, pelecypods, and ascidians, and highest among the molluses and fish. Oxygen extraction here seems to indicate an improved type of respiratory mechanism. Some hesitancy should be shown in accepting these figuses as an over-all measure of respiratory efficiency, however, as the oxygen "utilization" by clefinition is based solely on the percentage removal of oxygen from the water passing across the respiratory surfaces and reveals nothing concerning the important factors of quantity and rate of water pumped. For comparative purposes the withdrawal of several

TABLE H. OXYGEN WITHDRAW AL BY SOME AQUATIC ANIMALS

(Per cent $\mathrm{O}$. removed from incoming water)

\begin{tabular}{|c|c|c|c|}
\hline Animal & $\begin{array}{c}\text { Oxygen } \\
\text { Withdrawal }\end{array}$ & Temp. ${ }^{\circ} \mathrm{C}$. & Conditions \\
\hline \multicolumn{4}{|l|}{ PORIFERA } \\
\hline Sycon raphanus ${ }^{1+2}$ & 39 & 20 & \\
\hline Leucandra aspera & 11 & $16-20$ & \\
\hline Leuconia solida ${ }^{1,52}$ & 7 & $17-18$ & \\
\hline Chondrosia veniformis $\mathrm{s}^{152}$ & 21 & 21 & \\
\hline Suberites domuncula & 15 & 20 & \\
\hline Reniera rosea ${ }^{1,52}$ & 6 & $17-20$ & \\
\hline Hircinia sp. & 20 & $20-21$ & \\
\hline Aplysina aerophoba & 57 & $20-21$ & \\
\hline \multicolumn{4}{|l|}{ ANNELIDA } \\
\hline Nereis virens $\mathrm{s}^{2 \mathrm{~b}}$ & 70 & & \\
\hline Arenicola & $30-50$ & & \\
\hline Ennice gigantea ${ }^{152}$ & 50 & 17 & \\
\hline Aphrodite aculeat ${ }^{1.52}$ & 66 & $21-22$ & \\
\hline Hermione hystrix & 55 & 17 & \\
\hline Chaetopterus sp..$^{1.52}$ & 39 & $17-20$ & \\
\hline Rhynchobolus convolutus ${ }^{1,2}$ & +4 & 18 & \\
\hline Bonella viridis ${ }^{150}$ & 30 & $20-22$ & \\
\hline \multicolumn{4}{|l|}{ CRUSTACEA } \\
\hline Astacus fluviatilis:- & 59 & $13 \cdot 14$ & Low $O:$ tension \\
\hline & 70 & $14-18$ & High $\mathrm{O}$ tension \\
\hline Palinurus vulgaris ${ }^{1,52}$ & +3 & $20-22$ & \\
\hline Scyllarus latus & +3 & 22 & \\
\hline Dronia vulgaris ${ }^{1+2 .}$ & +3 & 20 & \\
\hline Calappa granulata ${ }^{52}$ & 76 & $16-20$ & In sand \\
\hline Maja verrucosa $a^{15 \cdot 2}$ & 45 & 20 & \\
\hline Anilocra mediterranea $a^{152}$ & 45 & 20 & \\
\hline \multicolumn{4}{|l|}{ PELECYPODA } \\
\hline Cardium tuberculatum & 8 & $16-17$ & \\
\hline Solen siliqua & 9 & 18 & ln sand \\
\hline Pinna nobilis ${ }^{1.2}$ & 5 & 20 & \\
\hline \multirow{2}{*}{ Mya arenaria" } & $3-10$ & & \\
\hline & 25 & & After low tide \\
\hline \multicolumn{4}{|l|}{ GASTROPODA } \\
\hline Haliotis tuberculata ${ }^{1,52}$ & 56 & $17-22$ & \\
\hline Murex brandaris ${ }^{1.52}$ & 38 & 20 & In the shell \\
\hline
\end{tabular}


TABLE 44 (continued).

OXYGEN WITHDRAW AL BY SOME AQUATIC ANIMALS

(Per cent $\mathrm{O}$ : removed from incoming water)

\begin{tabular}{|c|c|c|c|}
\hline Animal & $\begin{array}{c}\text { Oxygen } \\
\text { W'ithdrawal }\end{array}$ & Temp. ${ }^{\circ} \mathrm{C}$. & Conditions \\
\hline Tritonizm nodifermm ${ }^{1.52}$ & 79 & 20 & In the shell \\
\hline Doris tuberculata & 67 & 22 & \\
\hline CEPHALOPODA & & & \\
\hline Octopus vulgaris & $\begin{array}{c}63 \\
70-80\end{array}$ & $17-20$ & Low $\mathrm{O}_{2}$ tension \\
\hline ECHINODERMATA & & & \\
\hline Astropecten aurantiacus ${ }^{1,5}$ & 49 & 21 & In sand \\
\hline Echinocardinm sp. & 54 & 20 & In sand \\
\hline Holothuria tubulosa ${ }^{152}$ & 55 & $16-17$ & \\
\hline ASCIDIACEA & & & \\
\hline Ciona intestinalis ${ }^{1.52}$ & 7 & 17 & \\
\hline Phallusia manillatañ & 6 & $17-20$ & \\
\hline Ascidia mentula & + & $17-20$ & \\
\hline PISCES & & & \\
\hline Scyllium caniculal ${ }^{152}$ & 46 & 18 & \\
\hline Scorpaena porcus ${ }^{1-2}$ & 53 & 18 & \\
\hline Trigla corax $x^{1.32}$ & 59 & 18 & \\
\hline Uranoscopus scaber & 82 & 17 & In sand \\
\hline Hippocampus brevirostris & 55 & 18 & \\
\hline Siphonostoma rondelettitis & 65 & 20 & \\
\hline Anguilla sp. & 80 & & \\
\hline Salmo sp."s & 80 & & \\
\hline Leuciscus sp. ${ }^{370}$ & 68 & & Forced $\mathrm{H}_{2} \mathrm{O}$ supply \\
\hline Spheroides $s p .^{1+11}$ & 46 & 20 & \\
\hline
\end{tabular}

air-breathers may be noted: sloth, 2 per cent; man, 4.5 per cent; porpoise, 10 per cent. ${ }^{187,18 s}$

The extraction of oxygen during changes in tension may be variable; in the puffer fish it remains the same over a five-fold decrease in tension, indicating that the gills are equally efficient over the entire range, although they are not able to make up for the lost pressure (Table 45). Furthermore, in this fish withdrawal is not changed through a temperature range of 12 to $22^{\circ} \mathrm{C} . \mathbf{1 4 0}^{140}$ One might expect a really efficient respiratory mechanism to increase utilization at lower tensions, thereby contributing to a constant oxygen consumption and respiratory regulation. Crayfish ${ }^{-1-5}$ react even less favorably to decreased oxygen concentration, the extraction falling from 70 per cent at a tension of $160 \mathrm{~mm}$. I Ig to 59 per cent at $50 \mathrm{~mm}$. I lg. Octopus, on the other hand, as seen in the table, has a higher oxygen withdrawal at the lower tensions.

Adaptations to Low Oxygen Supply. Throughout this chapter we have noted signilicant structural and physiological adaptations which have arisen 10) previck oxygen for the organism, particularly under conditions of stress. Scores of comparable adjustments might be emumerated, ranging from the plastron mechanism of air storage in diving beetles (p. 223) to the hypertrophicel gills in Sulanandra larvac raised in oxygen-deficient water (p. 216).

A scries of andiaptations to oxyen lack may be considered "migratory" in nature, i.c., taxic responses. The annelid worm, Tubifex, forsakes its oozy 
abode, in the absence of oxygen, and comes to the surface for air ("aeroperceptiver Sinn"):" Lumbricus also is claimed to react tropistically to oxygen deficiency.":-1 Likewise the need for oxygen converts the usually sessile protozoan, Vorticella, into a temporary free-swimmer, which takes up its stationary post again in water with adequate oxygen. $25+$ Some telcost fish characteristically "surface" in oxygen-deficient water to gulp air, as for example the catfish and Lenciscus. ${ }^{10,}$, 373 Similar behavior patterns may be concerned in vertical migrations of earthworms and other burrowing forms. The sipunculid worm, Phascolosoma lurco, from the region of the Malay Archipelago, has migrated from its marine beginnings to take up residence in the intertidal zone among the mangrove roots-driven shoreward by the need for oxygen, presumably, but tethered to the sea by osmotic and structural limitations.

\section{TABLE 45. OXYGEN WITHDRAW AL BY THE PUFFER FISH FROM SEA IVATER OF VARYHG OXYGEN TENSHONS ${ }^{10}$ \\ (Temp. $20^{\circ}$ C.)}

\begin{tabular}{c|c|c}
\hline \hline \multicolumn{2}{c|}{ Dissolved Oxygen in cc./1. } & Percentage of Dissolved \\
Oxygen Absorbed
\end{tabular}

Two species of Paranecimm, the chlorophyll-containing P. bursaria and the colorless $P$. caudatum, react differently to oxygen stress in the presence of light and darkness. ${ }^{191}$ Although both avoid regions of oxygen deficiency in the light and seem unaffected by dark when oxygen is plentiful, their behavior is decidedly different when they move from a region of light to one of darkness during exposure to low oxygen tensions. The green infusorian, being able to produce small amounts of oxygen in the light region, is immediately affected by the darkness, which interrupts the photosynthetic production of oxygen; it therefore turns away from the dark region. P. caudatum, on the other hand, being unable to produce oxygen, does not respond to the change from light to dark under comparable anaerobic conditions.

Certain insects resort to a biological supply and obtain oxygen from the underwater stems of aquatic plants. The syrphid fly larvae, Chrysogaster, which lives in oxygen-deficient waters, pierces the roots of aquatic grasses and obtains vital oxygen. ${ }^{3.50}$

Along with these structural and behavioristic adaptations, many types of physiological accommodation have developed to facilitate oxygen uptake at low tensions. Certainly the relative increase in rate of oxygen consumption itself, by way of oxyregulation, as well as hyperventilation, reduction of unloading tension by carbon dioxide (sce Chapter 9), and oxygen secretion, may be considered significant adaptations which become increasingly important at low oxygen pressures. Moreover, in the near or complete absence of oxygen, most animals have found a way of getting along anacrobically, either 
by way of a permanent adjustment to complete anoxia or through the temporary expediency of accumnlating lactic acid and an oxygen debt cluring periods of oxyen deficiency-a problem further claborated below.

In conclusion, the response of oxygen consumption rate to changes in oxygen tension depends on and is characteristic of the individual. Internal factors such as blood pigment, tissue saturation, and basal metabolic rate affect this relationship. Envirommental effects are important, including temperature, pH. and rate of change of the oxyen tension. Two general reaction patterns are found: (1) that in which the oxygen consumption is highly dependent on tension, the non-regulatory type, and (2) that in which consumption is independent of oxygen pressure over a wide range, the regulatory type. Further work is needed to clarify the interrelation of the factors operating in this regulation. It would be of interest to determine, for example, the effects of respiratory inhibitors-carbon monoxide, cyanide, and azide-on the ability to regulate at extremes of the consumption-tension curve. More information is needed, to add to that obtained on lish, pertaining to the effect of $\mathrm{pH}$ on $\mathrm{t}_{1 .}$. Finally, a fruitful line of investigation would appear to be that of determining the degree of oxygen saturation of those tissues which manifest wide regulatory proficiency.

Efficiency of organisms in an adaptive sense requires a compromise between great activity under optimal conditions and wide tolerance under extreme conditions. Consider the active brook trout and the enduring lungfish-each is efficient in his own way.

\section{REGULATION OF BREATHING}

Breathing Movements in Higher Vertebrates. The respiratory movements concened in the breathing of organisms often involve large-scale ventilation, as the pumping of insects or the undulating gyrations of Tubifex during times of low oxygen stress. On the other hand, respiratory movements may consist of small motions, such as the action of spiracular cells to occlude the tracheal tubes in response, for example, to local accumulation of lactic acid. Ventilation movements and respiratory control in the higher vertebrates have been extensively considered in the classic physiological literature and in a number of recent revicws. $126,131,132,160,16 x, 262,263,283,284,255,315,382$ 'T he early comparative literature was ably summarized by Babák in Winterstein's Handbuch der vergleichende Physiologie."

The control of respiration represents a balance between neurologic and chemoreceptive mechanisms acting both directly and reflexly on the respiratory center and in turn on the breathing mechanism itself. Normal inspiration in mammals is an active process stimulated by impulses sent out continuously over vagal and phrenic pathways from the inspiratory subdivision of the medullary respiratory center. ${ }^{2}$ wi The ensuing inspiration results in barrages of sensory impulses, originating in the stretch receptors of the lungs themselves, ${ }^{117}$ and being returned to the respiratory center to inhibit further inspira. tion in aceorelance with the well established Ilering-Breuer reflex. Exhatation is largely a passive process in normal eupneic breathing and does not generally call forth expiration-inhibiting or inspiration-stimulating impulses, as originally put forth as part of the I lering Breuer mechanism. ${ }^{24}$ However, further nervous reflex control of breathing is indicated in hyperpnea in both the inspiratory 
and the expiratory phase, inasmuch as extreme inspiration results in greater excitation of the inspiratory center to provide a short additional respiratory spurt, and extreme expiration causes stimulation of "deflation" receptors by which the suceeding inspiration is augmented more forcibly than it would be otherwise. The cells of the respiratory center show considerable variation in threshokd, thereby permitting a graded response to increased stimulation. Onty a few fibers are active in eupnea, but with the proper respiratory stimulation less exciable neurones react, and the impulse frequency of all the fibers increases. In general the threshold of the expiratory neurones is higher than that of the inspiratory neurones. The medullary respiratory center initiates and coordinates respiration by sending out a constant flow of impulses which are rhythmically interrupted by the llering-Breuer reflex mechanism and, in extreme hyperventilation, by the pontine pneumotaxic center as well.

Wartime studies on pressure breathing, accomplished by means of facial masks to increase the ambient pressure, demonstrate that inspiration becomes passive and expiration is necessarily active, under these conditions, with a respiratory pause following inspiration. ${ }^{16}$

The respiratory center is affected directly by a number of agents, including carbon dioxicle increase in arterial blood (the most important), increased acidity of the blood, oxygen lack, rate of blood flow, and temperature. Response of the center to increases in arterial carbon dioxide is the most delicate mechanism controlling breathing. A decrease in sensitivity of the center to carbon dioxide is frequently a concomitant of abnormal conditions and may result in Cheyne-Stokes breathing or, under severe anesthesia (morphine), in complete cessation of rentilation. ${ }^{10 \prime}$ In man and dogs increased tolerance may be established to carbon dioxide atmospheres of 3 per cent and greater. ${ }^{111}$ On the other hand, an increase in carbon dioxide sensitivity is indicated in cases of acielosis. 29.5

The action of carbon dioxide on the respiratory center is known to be a direct one, but the mechanism is not clear. The older hypothesis of W'interstein $^{3 i t}$ and I laldane $1: 5$ regarded the main action of carbon dioxide to be its acid-producing capacity, the stimulus taking effect through the hydrogen ions resulting from the dissociation of carbonic acid. Carbon dioxide per se has been considered more effective than other hydrogen ion-producing substances and has come to be regarded as having a specific effect on the respiratory center. ${ }^{261}, 316$ However, Gray ${ }^{132}$ finds that alveolar ventilation seems to depend on the sum of the stimulating effects of blood $\mathrm{CO}_{2 .}$ and of hydrogen ion. Gesell12:, 1:26 suggests that carbon dioxide penetrates the cells of the respiratory center, where it gives rise to excitatory hydrogen ions. Considerable evidence favors the action of carbon dioxide itself as a specific stimulant of the center, but the action of hydrogen ions acting under some conditions cannot be disregarded.

The activity of the respiratory center is modified, particularly in times of respiratory stress, by reflexes originating in the chemoreceptors of the aortic and carotid bodies. These end-organs are particularly responsive to decreases in oxygen tension of the blood, and less responsive to increased acidity and carbon dioxide tension. The important role of chemoreception was beautifully demonstrated by the notable work of Heymans and his colleagues. ${ }^{161}$ Largely through the work of the Belgian group, it is recognized that chemoreception 
plays a part in maintaining the mild muscular tone during eupneic breathing, but the mechanism is largely one of emergency control during severe anoxia or deep anesthesia in which the respiratory center becomes insensitive to increased carbon dioxide tensions. Although chemoreceptive reactions are not to be minimized, we would agree with Schmidt ${ }^{31.5}$ that "the reflexes probably do not play an important part in respiratory control under conditions of quiet breathing at sea level but they become much more important under abnormal or emergency conditions in which the blood $\mathrm{PO}_{2}$ or $\mathrm{pH}$ falls or the blood $\mathrm{pCO}_{2}$ rises sufficiently to affect these relatively insensitive but remarkably rugged structures." The oxygen factor as a stimulant may be regarded as negligible at high oxygen tensions and the carbon dioxide negligible at low oxygen pressures.296. Carbon dioxide increase and oxygen lack summate in their stimu-

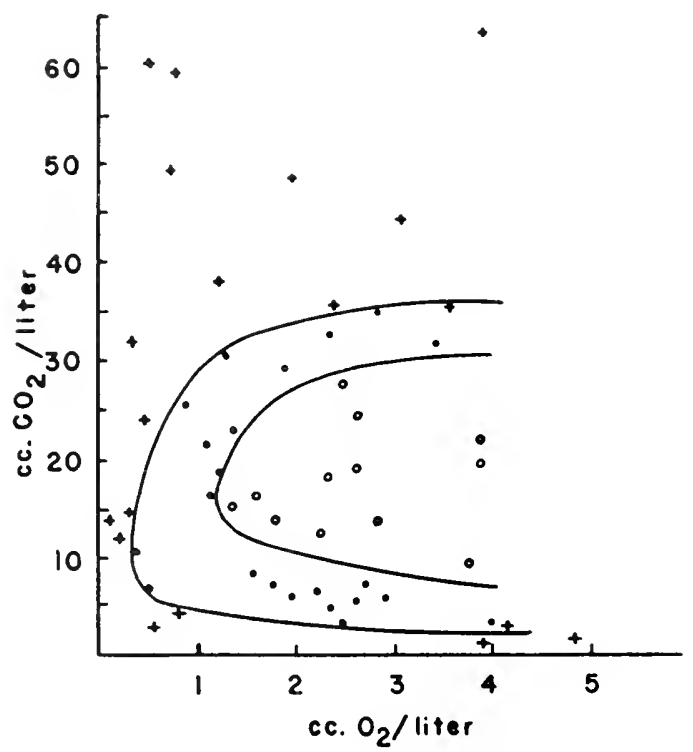

Fig. 58. Influence of $\mathrm{O}_{2}$ and $\mathrm{CO}_{2}$ tensions on type of respiration of the yarrow, a tropical fish. Points designated by circles indicate aquatic respiration, crosses show airbreathing conditions, and solid dots the intermediate stage of transition when either or both may occur. From Willmer.:17t

lating effects as determined by their combined effect on the "breaking point" in breath holding." ${ }^{\text {"tis }}$ Respiratory adaptability to unusual conditions was shown by Schneider, 317 who demonstrited that human breath-holding time could be extended to 1+15 minutes after forced breathing and oxygen ventilation.

In summary, carbon dioxide in the blood is the normal and delicately controlling factor in higher animals and acts primarily on the respiratory center itself; whereas oxygen lack is more of an emergency condition, although acting mainly through the chemoreceptive mechanism of the carotid body after the carbon dioxide stinulation has been decreased. The carbon dioxide mechanism is the more relined and phylogenetically is perhaps the more recently developerl. 
This general account of respiratory control in mammals pertains to both eupneic and abnormal breathing. Certain mammals, however, show profound adaptive differences in their respiratory patterns. These are the relatively poikilothermic "hibernants" (bat, hanster, bear, mamot, ete), whose respiratory eccentricitics are discussed in Chapter 10, and certain lower mammals, for example the armadillo (Edentata), capable of long periods of apnea despite oxygen-deficiency and high blood carbon dioxide. ${ }^{324}$ Diving birds and mam mals also have developed interesting adaptive mechanisms to permit apuea for extended periods while submerged (see below).

A good deal of work has accumulated to indicate well dereloped respiratory control among fish. ${ }^{9}, 178,2.25 .371$ The skate (Selachii) has a well defined and relatively autonomous respiratory center in the medulla. This center, which may be surgically isolated anteriorly and posteriorly without impairing its function, may be regarded as a mechanism functionally intermediate between the segmentally arranged ganglia of the invertebrates and the compact and complex specialized respiratory centers of the higher vertebrates. ${ }^{17 x}$

Certain tropical fish have been extensively studied in their responses to respiratory stimulants. ${ }^{371}$ The electric eel, Electrophorus, a creek-dweller capable of breathing air, is stimulated by oxygen deficiency, carbon clioxide increase, and low $\mathrm{pH}$. The yarrow. Erythrinus, a swamp-dweller in water of about $\mathrm{pH} \mathrm{4}$, can live in either water or air with its gill chamber closed off by the operculum. Its respiratory control responds to oxygen lack, carbon dioxide increase, and blood $\mathrm{pHI}$ decrease down to 6 . It relies on gills and aquatic respiration in water containing $1.3-5 \mathrm{cc}$. $\mathrm{O}_{2} / \mathrm{l}$. and $8-30 \mathrm{cc}$. $\mathrm{CO}_{2} / \mathrm{l}$. At lower oxygen tensions and at very low or verv high carbon dioxide tensions, the yarrow tends to become an air breather, taking air into the gas bladder (Fig. 58).

In fish and amphibians the importance of respiratory center sensitivity to carbon dioxide and its effect on respiratory movements is difficult to evaluate because the $\mathrm{CO} .2$ tension does not ordinarily build up to any great degree, owing to rapid diffusion of the gas through the integument and its solubility in water. Further, considerable oxygen may be cutaneously derived, regardless of breathing movements. In aquatic forms oxygen deficiency appears to be the more profound respiratory stimulant; carbon dioxide is of less significance. This situation is reversed in air breathers.

In the frog, which is responsive to changes in both oxygen and carbon dioxide, the "glandula carotica" may be considered analogous to the carotid bodies of mammals. Carbon dioxide stimulates respiration when those glands are either intact or obliterated (surgically or by painting with phenol). Oxigen lack is effective, however, only when the glands are intact. ${ }^{33: 2}$

Reptilian respiration is influenced by the tension of the blood gases as well as by temperature effects on the respiratory center cells themselves. tortoise has both an inspiratory ("gasping") and an expiratory center. In the diamond-back terrapin, Melaclemy's, a high $\mathrm{CO}_{2}$ tolerance correlates with diving capacity. ${ }^{2-4}$ The reptilian respiratory mechanism may be associated with swallowing and olfaction; in turtles and snakes the development of the motor center of respiratory control parallels physiologically the esophageal peristaltic center involved in swallowing reflexes. ${ }^{5}$ The throat movements of turtles, previously regarded as serving a respiratory pumping function, may be 
regarded as "sniffing" reflexes, and are in reality related to food stimulation and olfaction. ${ }^{249}$ The breathing cycle in the tortoise includes a pronounced compression phase, frequently followed by an apneic interval. Of further interest is the variability in intratracheal pressure developed from respiratory movements with the glottis closed except during actual inspiration and expiration.

Oxygen deficiency serves as a respiratory stimulant in birds, but species differences are found in the response to carbon dioxide. ${ }^{163}$ Carbon dioxide stimulates in the mallard duck, English sparrow, and starling, for instance, but causes apnea in muscovy and pekin ducks. Evidence exists for $\mathrm{CO}_{2}$ inhibitory reflexes originating in chemoreceptors in the nasopharynx.

A review of breathing in vertebrates indicates that central respiratory centers are present among fishes, amphibians, reptiles, birds, and mammals. The development of this control has made possible responses to small changes

TABLE 46. DURATION OF DIVES OF DIVING MAMMALS (AFTER IRVING ${ }^{1 \$ 4}$ )

\begin{tabular}{|c|c|c|}
\hline Animal & Time & Authority \\
\hline Platypus (Ornithorhynchus anatinus) & 10 minutes & $\begin{array}{l}\text { Allport, } 1878 \\
\text { Burrel], } 1927\end{array}$ \\
\hline Sea elephant (Mirounga angustirostris) & 6 minutes, 48 seconds & Harnisch, 1937 \\
\hline Harbor seal (Phoca vitulina) & $15 ",(3-6 \mathrm{~min}$. in zoo $)$ & Millais, 1906 \\
\hline Gray seal (Halichoerus grypus) & $15 "$, in net & " " \\
\hline Muskrat (Ondatra zibethica) & $12 "$ & Irving, 1939 \\
\hline Beaver (Castor canadensis) & $15 "$ & Irving and Orr, 1935 \\
\hline $\begin{array}{l}\text { Hippopotamus (Hippopotamus } \\
\text { amphibius) }\end{array}$ & 50 seconds & Parker, 1932 \\
\hline Florida Manatee (Trichechus latirostris) & 16 minutes, 20 seconds & Parker, 1922 \\
\hline Sperm whale (Physeter macrocephalus) & $1-1 \frac{1 / 2}{2}$ hours & $\begin{array}{l}\text { Millais, } 1906 \\
\text { Scammon, } 1874 \\
\text { Beddard, } 1900\end{array}$ \\
\hline $\begin{array}{l}\text { Bottled-nosed whale (Hyperoodon } \\
\text { rostratus) }\end{array}$ & $\begin{array}{l}2 \text { hours, ( } 1 \mathrm{hr} \text {. } \\
\text { wounded) }\end{array}$ & $\begin{array}{l}\text { Gray, } 1822 \\
\text { Millais, } 1906\end{array}$ \\
\hline Bowhead whale (Balaena mysticetus) & 1 hour, 20 minutes & Scammon, 1874 \\
\hline Greeland whale (Balaena sp.) & 1 hour & Beddard, 1900 \\
\hline Common rorqua] (Balaena musculus) & 49 minutes & Millais, 1906 \\
\hline Blue whale (Balaena sibbaldi) & $50 \quad "$ & Andrew's, 1916 \\
\hline Finback whale (Balaenoptera physalus) & $1 / 2$ hour & Allen, 1916 \\
\hline Fin whale (Balaenoptera plupalus) & 20 ninutes & Ommanney, 1932 \\
\hline $\begin{array}{l}\text { New Zealand humpbacked whale } \\
\text { (Megaptera nodosa) }\end{array}$ & not over $1 / 2$ hour & Lillie, 1910 \\
\hline Man (Homo sapiens) & $21 / 2$ minutes & Irving, 1939 \\
\hline
\end{tabular}

in blood $\mathrm{CO}_{2}$. Chemorceptors, analogous to carotid bodies, have been demonstrated in Amphibia and in more advanced groups, and permit coordinated responses to oxygen lack. Further work is needed to clarify the relative importance of these two types of operating mechanisms, particularly among lower vertebrates, as wetl as the role of hydrogen ions, other than those originating from carbonic acid, als al respiratory stimulant.

Respiratory Control in Diving Birds and Mammals. In diving birds and manmals a number of adaptations in respiration, metabolism, blood, and circulation combine to permit survival under water for long periods (Table 46). 'The major respiratory changes which make such diving possible are not 
the unusual storage of oxygen or increase in lung volume, but rather the more efficient utilization and availability of oxygen for the essential operating processes, the development of anacrobic glycolysis, the high tolerance to lactic acid, and the relative insensitivity to carben dioxicle of the animals while submerged. The rate of oxygen consumption of diving mammals does not differ greatly from that of land mammals, ranging from $5+6 \mathrm{ce}$. O.2 kig. hr. in the seal, down to $180 \mathrm{cc}$. in the manatec, comparable to al value of about 250 cc. for man. The various factors which play a part in the oxygen stores are indicated as follows:

(1) Lungs-the lung volume is only slightly larger in the ctiving mammals than in land animals, although the tidal air is considerably greater in most, as high as 80 per cent of the lung volume in the porpoise. ${ }^{1 n i}$

(2) Blood-in the seal the oxygen capacits of the hemoglobin is 1.78 ce. $\mathrm{O}_{2} / \mathrm{gm}$. H Ib, compared with 1.23 for man, 1 a: and the blood volume of the seal is about 10 per cent of the body weight. ${ }^{\text {lit }}$ the total oxyen calpacity of the blood is slightly higher than that in man in some divers 29.3 rols. per cent in the seal), but not in all (17.7 vols. per eent in the beaver).

(3) Muscle hemoglobin-divers other than the manatee have a rich store of muscle hemoglobin. Seal muscke viclds $7715 \mathrm{mg}$. $1 \mathrm{lb} 100$ gm., conplared with $108+\mathrm{mg}$. from beef: $: 31$

(4) Tissue lluids-thes appear the same as in non-divers.

Scholander ${ }^{3: 20}$ summarizes the utilizable oxygen stores of a bladdemose seal weighing $29 \mathrm{~kg}$. as follows, taking the highest estimates:

$\begin{array}{lr}\text { Air in the lungs: } 350 \mathrm{cc.} \text { 15\% O.2 } & 50 \mathrm{cc} \\ \text { Blood: } 4500 \mathrm{cc} ., 25 \% \mathrm{O} .2 & 1100 \mathrm{cc} \\ \text { Muscle: } 6000 \mathrm{gm} ., 4.5 \% \mathrm{O} .2 & 270 \mathrm{cc} \\ \text { Tissue fluid } & 100 \mathrm{cc} \\ \\ \text { Total oxygen store } & 1520 \mathrm{cc} .\end{array}$

The major part of the store, that of the blood, is used up at practically a constant rate and can last 25 minutes, whereas the muscle oxygen forms an isolated store which is used up during the first 5-10 minutes of the dive. $3: 3.3$ During a 25 minute period at rest this animal consumes about $6250 \mathrm{cc}$ of oxygen, which is four or live times as much as the oxygen stores could possibly supply for the dive. One may conclude therefore that the oxygen consumption by the tissues during a dive is depressed to 20-25 per cent of the resting rate. These findings are in accord with similar observations on penguins and whales,

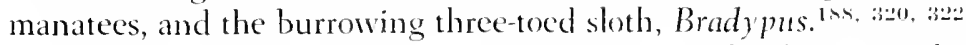

Ruling out oxygen stores as a critical factor in the diving mechanism, other adaptations have been demonstrated to permit submergence for long periods. ${ }^{12}$

(1) Anaerobic glycolysis-although lactic acid does not accumulate in the blood to any great extent during the dive, large quantities literally flood the blood stream immediately after surfacinglet (Fig. 59). Apparently after the oxygen in the muscle is used up in the first few minutes of a dive, anaerobic processes take over entirely and an oxigen debt builds up, counteracted during the dive only to the extent that some small quantities of oxigen may be brought in by the blood, itself pretty well blocked off from the muscle during sub- 
mergence. The initial aerobic metabolism of the muscle during a dive is below the normal resting rate, and the anaerobic rate in the later stages is greater than at rest. ${ }^{2}: 3$ A general lowering of the metabolic activity and the formation of lactic acid probably are not limited to the muscles but occur elsewhere in the diver as well.

(2) Carbon dioxide insensitivity-this is characteristic of diving mammals and reflects an adaptation to unusual conditions where lactic acid accumulates in tissue and would otherwise stimulate the respiratory center to considerable activity. Carbon dioxide insensitivity, due to both decreased circulation of the blood and higher threshold of the respiratory center, has been demonstrated in the beaver, seal, muskrat, and porpoise. ${ }^{67}, 1 \times 3,154,1 \times 7$ The entire resting respiratory mechanism is adjusted to a high carbon dioxide tolerance and relative apnea, with an extremely low breathing rate in these animals, 2-4 inspirations a minute in the porpoisc and 2-3 in the seal. Furthermore, utilization of the inspired oxygen is high; in Tursiops, the porpoise, for instance, an

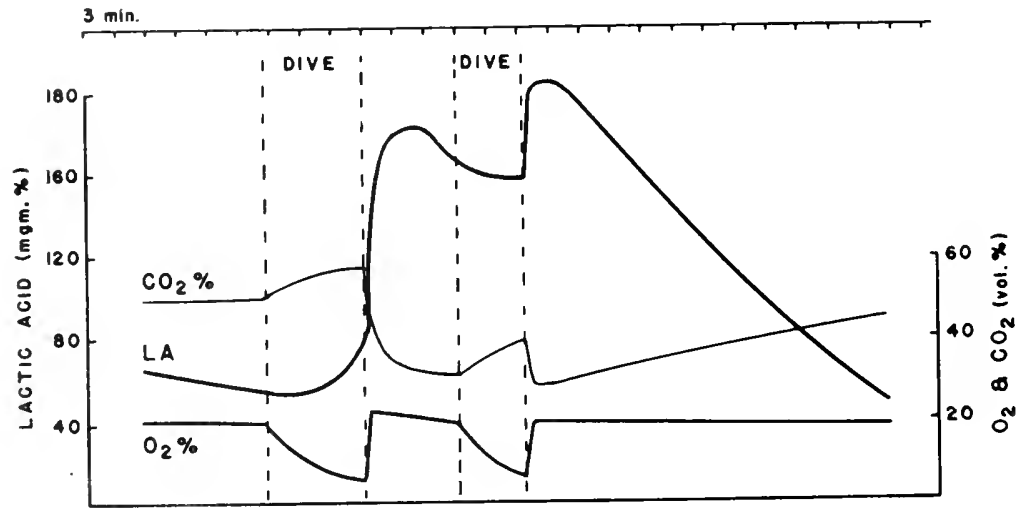

Fig. 59. Arterial bloud changes in the seal, Cystophora, during experimental diving conditions. Af ter Scholander.

animal weighing $170 \mathrm{~kg}$. was found to operate with a resting breathing frequency of once a minute, to inspire 10 liters with each breath, and to comsume about I liter of oxygen a minute-a "utilization" of approximately 10 per cent. two or three times that of man.

(3) Cardiovascular changes-significant alterations in the heart and periphcral ressels tend to reduce the blood flow in most body regions with the exception of the brain. This bradscardia (heart slowing), first described by Paul liert in diving duchs, has been denonstrated in practically all diving mammals, inchuding mant. In the scal the heant slows from a resting value of about 80 per minute to 10 during a dive. This reduction in heart rate is a vagal reflex. Another important reflex is the vasomotor change which effectively shuts the circulation off from the main muscle masses of the body, as manifested by (a) complete reduction of the muscle hemoglobin at a time when the blood is still half saturated with oxigen, and (b) accumulation of large amounts of lactic acid in the muscles and its absence in the blood during the dive. 
The ability of diving mammals to remain submerged is, then, based on several interacting factors, with anacrobic glycolysis and tolerance to high levels of lactic acid and carbon dioxide playing critical roles.

Effect of Temperature on Breathing. In strictly homoiothermic forms there is little change in breathing rate at rest over a fairly wide range of external temperature. I However, at both the high and low limits of normal temperature control respiratory movements may increase severely'. The respiratory center of birds and mammals itself is sensitive to changes in blood temperature, and both the depth and rate of respiration increase with high body temperatures. In the wren. Troglodytes, with a normal body temperature range of $100^{\circ}$ to $107^{\circ} \mathrm{F}$, the breathing rate increases from an average normal of 100 per minute at $105^{\circ} \mathrm{F}$. to 340 per minute at $116^{\circ} \mathrm{F}{ }^{1+}$ Likewise a severe reduction in body temperature in this small bird may produce a ventilation rate of 240 per minute. The respiratory center of the bat is likewise sensitive to thermal stimulation. ${ }^{305}$

Among invertebrates and cold-blooded vertebrates the temperature effects on breathing largely parallel those on oxygen consumption-there is an increase in activity at high temperatures (Fig. 60). 19, 140, 199

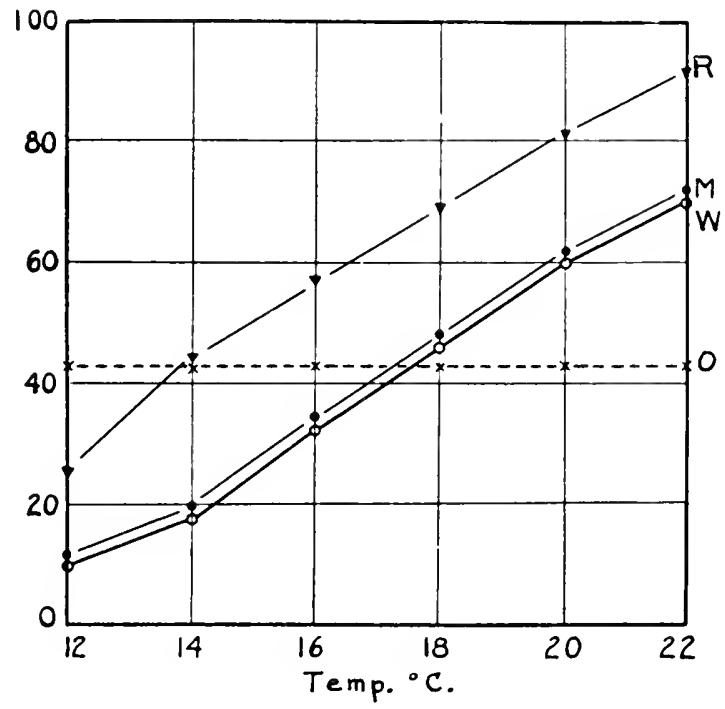

Fig. 60. Effect of temperature on respiration of the puffer fish, Spheroides. $R$, respiration per minute. $M$, cc. $\mathrm{O} / \mathrm{kg} . \mathrm{hr}$. $W, \mathrm{dl} . \mathrm{H}_{2} \mathrm{O} / \mathrm{hr}$. pumped through branchial cavity. $\mathrm{O}$, percentage of $\mathrm{O}$ : utilized. From Hall. ${ }^{40}$

Respiratory Movements During Flight. Wing beat and breathing movements may be synchronized during flight, as in insects, birds, and bats, $216,243.3$, 30.5. 355 but respiratory morements may stop altogether during flight (Fig. 61). ${ }^{117}$ The finch, Fringilla, has a respiratory rhythm of $4-5$ per second both at rest and in flight, and superimposed on this a flight rhythm of 15-20 per second. ${ }^{118}$ The actual respiratory advantage of synchronized movement is not clear, but in those instances in which flight movements greatly augment respiratory rhythms a distinct benefit may be derived. 
Regulation of Breathing in Tubifex. The responses of this small bottomdwelling, fresh-water oligochaete worm constitute an interesting respiratory pattern."3, $n$ These worms have become adapted to an oxygen microstratum just above the layer of mud in which they ordinarily reside. Depletion of the oxygen by bacterial oxidation in the ooze below causes the worms to extend themselves 10 or 12 times their former length to reach a satisfactory oxygen zone. The extension is accompanied by rhythmic corkscrew movements which agitate the water and probably draw down water containing more oxygen. The respiratory movements are stimulated by oxygen lack and in intensity are inversely proportional to the oxygen supply below surface. Carbon dioxide

\section{VESPA}

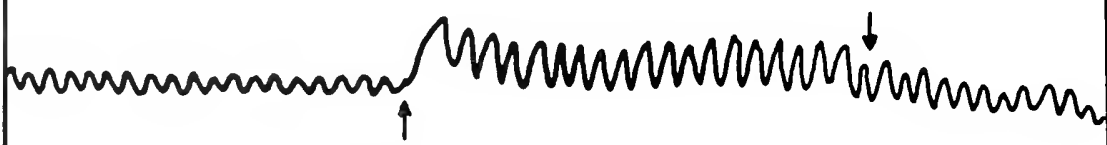

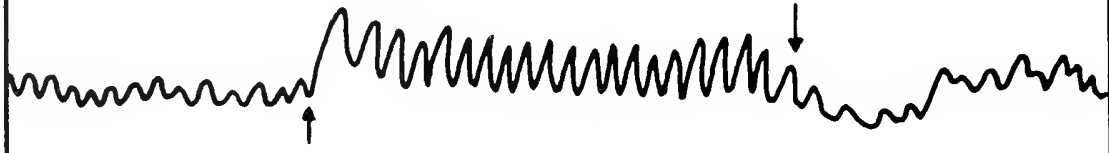

I sec.

\section{SCHISTOCERCA}

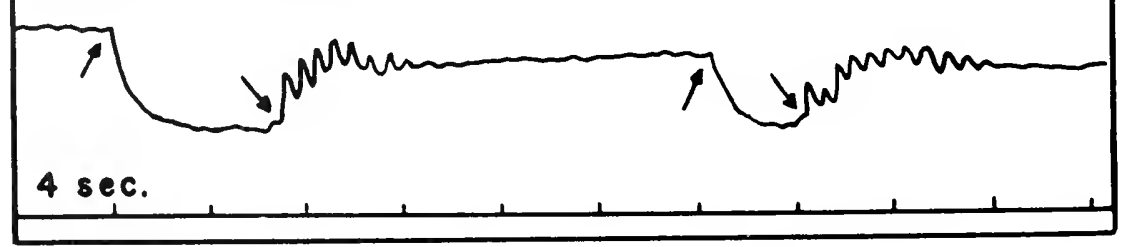

Fig. 61. Breathing movements of the wasp, Vespa orientalis, and the orthopteran, Schistocerca gregaria, during flight. Arrows indicate the beginning $(\uparrow)$ and end $(\downarrow)$ of flight movements. From Fraenkel. ${ }^{17}$

increase does not stimulate the movements but may in fact suspend the rhythm initiated by oxygen lack.

Ventilation Mechanisms in Polychaete Worms. An extensive study of the respiratory mechanisms among polychactes has been made by Lindroth."-?! Methods of ventilation for thirty families of these worms were correlated with the phylogenetic and ecological position of the organisms. Ciliary mechanisms are generally found throughout the group, concentrated most frequently on the head and parapodia. Primitive errant types show undulatory movements; more advanced sedentary groups have compression-type waves. The respiratory currents stream backward in the lower forms and forward in the advanced 
groups. For further information concerning the ecological groupings and the "microstratification" according to the arailability" of oxygen, Lindroth"s paperent? should be consulted.

Respiratory Control in Molluscs. Various hinds of breathing patterns are found in the phylum Mollusca by way of diverse adaptations to oxygen procurement. In the fresh-water pulmonate gastropods, Irmmea, Helicosoma, and Physa, the breathing rhythm, as evidenced by the cluration of the interval between surfacings, is a function of oxigen need.iti In water containing approximately $6 \mathrm{cc}$. O.. l. these molluses remain submerged three times als long as in water with only 2 cc. $\mathrm{O}_{2}$. . The temperature also influences surfacing interval, by its effect on activity and on dissolved oxygen. Individuals at $11^{\circ} \mathrm{C}$. remain submerged 22 times as long as those at $21^{\circ} \mathrm{C}$.

The respiratory morements of air-breathing pulmonates have been investigated, $, 3, \cdots, 3,3,5$ and factors known to affect the ventilation rate are carbon dioxide excess, oxygen lack, temperature, and humidity.3is Two respiratory mechanisms are involved in the breathing regulation of this group-the control of the pneumostome opening and the contraction of the muscle plate contributing to the mantle lung. The stimulating effect of carbon dioxide in low concentrations has been demonstrated in opening of the pneumostome in Limax, Helix, and Arion, and at a tension of 3-5 per cent $\mathrm{CO}_{2}$ the aperture may remain open..3 The effect of oxygen decrease on pneumostome control is questionable, but it is definitely effective in bringing about the contraction of the sides of the mantle carity, thereby increasing rentilation movements. The role of hydrogen ion activity and of carbon dioxide increase have not been elucidated in these pulmonates, although in the common oyster, Ostrea virginica, the effect of acid, administered as $\mathrm{HCl}$, is indicated by increased water passage through the bivalve, subserving both ingestion and ventilation. Oysters exposed for 6 hours to a $\mathrm{pH}$ of 6.75 to 7.0 -somewhat below their normal environmental range $(\mathrm{pH} 8.1)$-continue pumping $95-98$ per cent of the time, considerably more than the normal rate.

The cephalopods have a highly developed state of respiratory control with a nerve action analogous to that of the Hering-Breuer reflex of higher vertebrates. Winterstein studied the effects of respiratory stimulants on the ventilation of Octopus and showed that carbon dioxide is a very potent factor in increasing the frequency and, to a lesser degree, the amplitude of breathing movements. ${ }^{377}$ The volume of water pumped through the mantle cavity of one experimental animal increased from 230 to $1075 \mathrm{cc}$. per minute under the influence of carbon dioxide. Winterstein emphasized the importance of hydrogen ion activity rather than $\mathrm{CO}_{2}$ as the effective respiratory stimulant. ${ }^{376}$

Breathing Movements in Crustacea. Although body motion, abdominal flexion, and leg movement are important for ventilation in Crustacea, the two respiratory mechanisms which have been most thoroughly studied experimentally are scaphognathite beat and pleopod movement. Increase in temperature causes an increase in rate and amplitude of scaphognathite beat. ${ }^{19 .}$ As to the stimulating effect of oxygen deficiency on rate of ventilation there is general affirmative agreement among investigators, most of whom have dealt with the European crayfish, Astacus fluviatilis. However, there is considerable disagreement regarding the effects of carbon dioxide on this species. 11. 197. 314, 325 Some of the difficulty in determining the precise effect of carbon 
dioxide may be due to its absorption by the exoskeleton, as pointed out by Peters, ${ }^{2 \times 11}$ who found it is necessary to coat the animal with collodion to get consistent results; presumably no harmful effects are produced by the sealing. Jordan and Quittart ${ }^{1: 7}$ describe a kind of Cheyne-Stokes respiratory pattern resulting from treatment with excess $\mathrm{CO}_{2.2}$. After an interval of apnea, there ensues a period of discontinuous hyperventilation, followed by eupnea despite continual exposure to 10 per cent carbon dioxide. The evidence indicates a stimulating action of CO. in the crayfish, but, owing both to its possible fixation in the shell and to its solubility in sea water, this stimulant may be less effective than is oxygen lack.

In Eriocheir sinensis both $\mathrm{HCl}$ and $\mathrm{CO}_{2}$ in mild excess inhibit ventilation, but in considerable amounts stimulate respiration. ${ }^{153}$ After removal of the antennal appendages the respiratory inhibition is lost, suggesting the presence of carbon dioxide receptors on the antennae.

The movement of the pleopods on several isopod and amphipod crustaceans, in response to respiratory stimulants, seem to indicate a rather pronounced generic difference in these forms (Table 47). This may be correlated in somc way with habitat; the terrestrial Ligia, for example, responds neither to $\mathrm{CO}_{2}$ excess nor to $\mathrm{O}_{2}$ reduction, but does show a slowing of pleopod rhythm when the oxygen tension is increased. ${ }^{114}$

Breathing in Insects. These organisms have received the major share of attention in studies of invertebrate respiration, largely because of their economic value but also because of the interest in their unique breathing mechanism. A number of excellent reviews are available for consultation of the very extensive literature in this field dating as far back as Vauquelin's stimulating work on the Orthoptera in $1792 .{ }^{174,308,366,368}$

Briefly, two kinds of movement may be distinguished: rhythmic pumping motions which involve the abdomen and part of the thorax, and occasional opening and closing of the spiracles. Expiration is an active process; inspiration is either active or passive. All these movements are coordinated by respiratory centers-"primary" segmental centers and "secondary" higher-order centers in the prothorax: 335.366 A complete elaboration of orthopteran respiration was presented by Lee, $2: 2: 2,2: 3$ who clearly demonstrated the direction of air flow and the correlation of ventilation movements with spiracular patency and occlusion. Further ingenious experiments on grasshoppers by McCutcheon have shown the presence not only of an inspiratory and an expiratory phase, but of a compressatory phase as well, a relatively long interval during which intratracheal pressures may increase from 2 to $30 \mathrm{~mm}$. Hg above ambient pressure." In the grasshopper the anterior spiracles (the two pairs of thoracic and the first two pairs of abdominal) are inspiratory, while the posterior (abdominal) apertures are expiratory. Valvular mechanisms direct the flow, and central reflexes control the synchronization of movement. In abnormal cases, e.g., in blockage of the anterior spiracles, air both enters and leaves by way of the abdominal tracheac. Owing to loss of vital water through open tracheare, the normal preferred condition of the spiracles is a closed one. However, to permit gas exchange the spiracles must either remain partiafly open, as in the fleil, or open and close altemately, as in the grasshopper. That this pattern of directional air flow as described for the grasshopper is only one of many is emphasized by the gas exchange in the larval dytiscid, Cybister, 
which in water inspires through the last pair of spiracles and expires through all the lateral apertures, but in air, inspires and expires through the lateral spiracles.

lngenious experiments have been devised to show the amount of air which can pass through the tracheal system. The locustid, Chortophaga viridifasciata, circulates on the average $0.222 \mathrm{cc} . \mathrm{gm} . / \mathrm{min}$. at $28^{\circ} \mathrm{C}$. of insects is known to be generally high, $60 \mathrm{cu}$. mm. gm. animal weight in Dytiscus." ${ }^{-1 t i}$ In exceptional experimental conditions the air flow may be reversed, passing in through the posterior spiracles and leaving through the anterior ones.

Coordination of the insect respiratory movements lies in segmental nerve centers, responsible for the rentilation pattern in each segment. They in turn receive impulses from central coordinating thoracic centers, which are usually more sensitive to respiratory stimulation from carbon dioxide excess and oxygen lack. Rhythmic potentials corresponding to the breathing cycle have been recorded in ganglia isolated from the water beetle and cockroach. ${ }^{1}, 291$ ln the dragonfly, Acschna, only the secondary (thoracic) centers are very easily stimulated by low oxygen and high carbon dioxide tension, but in the stick insect, Dixippus, both types of centers respond to these stimulants. ${ }^{33 \pi}$ It is well known that isolated abdominal segments and decapitated insects may display respiratory movements for considerable time. There is general agreement that both carbon dioxide increase and oxygen want stimulate ventilation movements in insects (Table 47). McGovran ${ }^{2-50}$ has increased air flow through

TABLE +7. RESPIRATORY RESPONSE TO O. WANT AND CO INCREASE $+=$ positive stimulation. $-=$ no effect. $(\quad)=$ questionable effect.

\begin{tabular}{|c|c|c|c|}
\hline Animal & $\begin{array}{c}\text { Type of Respiratory } \\
\text { Movement }\end{array}$ & O. Want & $\begin{array}{c}\mathrm{CO}_{2} \\
\text { Increase }\end{array}$ \\
\hline \multicolumn{4}{|l|}{ ANNELIDA } \\
\hline Tubifex rivulorum (oligochaete) ${ }^{3}$ & tail undulation & + & - \\
\hline Limnodrilus hoffmeisteri (oligochaete) ${ }^{3}$ & & + & - \\
\hline Arenicola marina (polychaete)" & water pumping & + & + \\
\hline Nereis virens (polychaete) ${ }^{55}$ & & + & $(+)$ \\
\hline \multicolumn{4}{|l|}{ MOLLUSCA } \\
\hline Arion ater (pulmonate) ${ }^{43,33,3}$ & $\begin{array}{l}\text { spiracle }\left(\mathrm{CO}_{2}\right) \text { and lung } \\
\text { wall }\left(\mathrm{O}_{2}\right)\end{array}$ & $(+)$ & + \\
\hline Limax (pulmonate) ${ }^{43,3: 3}$ & $\begin{array}{l}\text { spiracle }\left(\mathrm{CO}_{2}\right) \text { and lung } \\
\text { wall }\left(\mathrm{O}_{2}\right)\end{array}$ & $(+)$ & + \\
\hline Helix (pulmonate) $)^{4,3,3 \times 3}$ & $\begin{array}{l}\text { spiracle }\left(\mathrm{CO}_{22}\right) \text { and lung } \\
\text { wall }\left(\mathrm{O}_{2 .}\right)\end{array}$ & $(t)$ & + \\
\hline Helix (pulmonate) $)^{3 \mathrm{H} 2}$ & $\begin{array}{l}\text { spiracle }\left(\mathrm{CO}_{2}\right) \text { and lung } \\
\text { wall }\left(\mathrm{O}_{2}\right)\end{array}$ & + & + \\
\hline Limnaea stagnalis (pulmonate) & $\begin{array}{l}\text { spiracle }\left(\mathrm{CO}_{2}\right) \text { and nega- } \\
\text { tive geotaxis }\left(\mathrm{O}_{2}\right)\end{array}$ & + & + \\
\hline Octopus (cephalopod) & ventilation movement & + & + \\
\hline \multicolumn{4}{|l|}{ CRUSTACEA } \\
\hline Branchipus (phyllopod) & limb movements & + & + \\
\hline Balanus balanoides (barnacle) ${ }^{114}$ & $\begin{array}{l}\text { morement of terga and } \\
\text { acuta }\end{array}$ & - & - \\
\hline $\begin{array}{l}\text { Cheirocephalus diaphanus } \\
\text { (phyllopod })^{14}\end{array}$ & limb movement & - & - \\
\hline Astacus fluviatilis (crayfish) $: 30$ & scaphognathite movement & + & $t$ \\
\hline
\end{tabular}


TABLE 47 (continued).

RESPIRATORY RESPONSE TO O W W ANT AND CO INCREASE

$+=$ positive stimulation. $-=$ no effect. ()$=$ questionable effect.

\begin{tabular}{|c|c|c|c|}
\hline Animal & $\begin{array}{c}\text { Movement } \\
\text { Type of Respiratory }\end{array}$ & $\mathrm{O}_{2}$ Want & $\begin{array}{c}\text { Increase } \\
\mathrm{CO}_{2}\end{array}$ \\
\hline Homarus i'ulgaris (lobster) & " $"$ & + & + \\
\hline Gammarus pulex (amphipod) ${ }^{114}$ & pleopod movement & + & + \\
\hline Gammarus locusta (amphipod) ${ }^{114}$ & pleopod movement & + & + \\
\hline Pandalus borealis (prawn) & scaphugnathite movement & + & + \\
\hline Eriocheir sinensis (decapod) ${ }^{153}$ & " & + & $\begin{array}{l}+ \text { (in } \\
\text { excess) }\end{array}$ \\
\hline Carcinus maenas (shore crab) ) $^{142}$ & " " & - & - \\
\hline Asellus aquaticus (isopod) $)^{114}$ & pleopod movement & + & - \\
\hline Ligia oceania (isopod) ${ }^{114}$ & $\begin{array}{l}\text { " } \\
\text { (at } \mathrm{O}: \text { tensions above } \\
\text { normal the beat slows) }\end{array}$ & - & - \\
\hline Cirolana borealis (isopod) ${ }^{102}$ & pleopod movement & + & + \\
\hline Idotea neglecta (isopod) ${ }^{102}$ & " " " & + & + \\
\hline Anilocra physodes (isopod) ${ }^{192}$ & $"$ & - & - \\
\hline Nerocila bivittata (isopod) & " & - & - \\
\hline Squilla mantis (stomatopod) $)^{192}$ & & + & + \\
\hline INSECT A & & & \\
\hline Periplaneta americana (cockroach) $)^{165}$ & & $(+)$ & + \\
\hline Corixa geoffroyi (aquatic insect) ${ }^{46}$ & leg movements & $(\rightarrow)$ & + \\
\hline Dixippus (stick insect) ${ }^{58,114}$ & ventilation movements & + & + \\
\hline Dixippus morosus (stick insect) ${ }^{335}$ & $\begin{array}{l}\text { ventilation movements } \\
\text { (pumping) }\end{array}$ & + & + \\
\hline Aeschna grandis (dragonfly nymph) ${ }^{335}$ & " " & + & + \\
\hline Dytiscus (water beetle) ${ }^{8}$ & & - & $\overline{1}$ \\
\hline Chortophaga (grasshopper) & ventilation movements & $?$ & + \\
\hline Dissosteira (grasshopper) ${ }^{250}$ & $\begin{array}{ll}\prime \prime & " \prime\end{array}$ & $?$ & + \\
\hline Arplia (grasshopper) & . & $?$ & + \\
\hline Xeropsylla chepis (flea) & spiracular opening & + & + \\
\hline Melolontha (beetle) ${ }^{n 1}$ & ventilation movements & $t$ & + \\
\hline $\begin{array}{l}\text { ARACHNOIDEA } \\
\text { Spider }\end{array}$ & spiracle opening & $(-)$ & + \\
\hline TUNICATA & wentilation movements & + & \\
\hline Salpa sp. (tunicate) $)^{179}$ & ventilation movements & 十 & $t$ \\
\hline $\begin{array}{l}\text { OSTEICHTHYES } \\
\text { Perca fluviatilis (perch) })^{264}\end{array}$ & ventilation movement & $?$ & + \\
\hline $\begin{array}{l}\text { Perca fluviatilis (perch) } \\
\text { Perca (perch) }\end{array}$ & $\begin{array}{l}\text { ventilation movement } \\
\text { " }\end{array}$ & + & + \\
\hline Perca $(\text { perch })^{302}$ & " $\quad "$ & $?$ & + \\
\hline Phoxinus laevis (minnow) ${ }^{204}$ & " $\quad "$ & + & + \\
\hline Lewciscus (fish) & ventilation movement & + & + \\
\hline Carassius (goldfish) ${ }^{302}$ & $\begin{array}{l}\left(\mathrm{CO}_{2}\right) \\
\text { gulping movements }\left(\mathrm{O}_{2}\right)\end{array}$ & & \\
\hline AMPHIBIA & & & \\
\hline $\operatorname{Rana}(\text { frog })^{332}$ & ventilation movement & + & $t$ \\
\hline Rana esculenta (frog) $)^{354}$ & " & + & + \\
\hline Rana temporaria (frog) & $"$ & + & $\begin{array}{l}t \\
t\end{array}$ \\
\hline Hyla arborea (tree frog) 354 & " " & + & + \\
\hline Bufo marinus (toad) $)^{3 i n}$ & & + & \\
\hline $\begin{array}{l}\text { REPTILIA } \\
\text { Em } \gamma \text { s orbicularis (turtle) }{ }^{3-4}\end{array}$ & $"$ & + & + \\
\hline $\begin{array}{l}\text { Emys orbicularis (turtle) } \\
\text { Chelodina longicallis (turtle) }\end{array}$ & $"$ & + & + \\
\hline $\begin{array}{l}\text { Chelodina longicollis (turtle) } \text { ) }^{334} \\
\text { Tropidonotus natrix (snake) }\end{array}$ & & + & + \\
\hline
\end{tabular}


TABLE +7 (continued).

RESPIRATORY RESPONSE TO O. WANT AND CO INCREASE

$+=$ positive stimulation. $\quad-=$ no effect. $(\quad)=$ questionable effect.

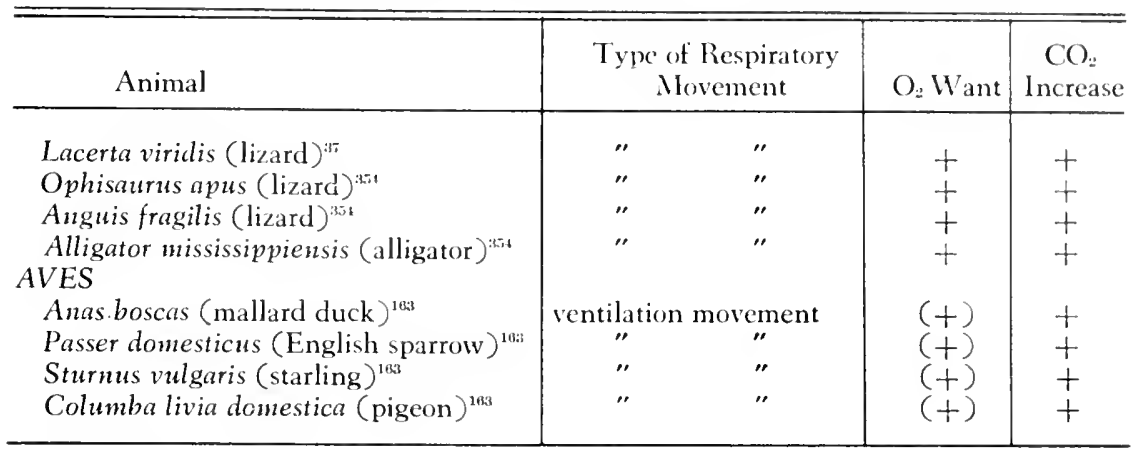

Chortophaga by adding carbon dioxide to the inspired air. In conclusion, the breathing movements of insects and of spiders as well ${ }^{1.51}$ respond to the action of carbon dioxide and oxygen on the respiratory centers in the same gencral way as do the breathing morements of higher vertebrates.

A further type of respiratory control can be evoked in tracheal organisms by action of respiratory stimulants on the cells regulating the size of the aperture of the tracheal ending. These effects have been extensively investigated in the common flea. ${ }^{15 i}$, 36i Tracheal pulsation is accelerated by oxygen deficiency and carbon dioxide increase, as well as by temperature elevation in the region of the spiracle. The effect of carbon dioxide and oxygen has been investigated in a number of "ventilation control" insects. The opening of the spiracle in response to carbon dioxide in the cockroach, Periplaneta, is almost instantaneous and occurs at much lower concentrations (2-3 per cent) of the gas than are necessary to stimulate breathing movements of the abdomen ( 10 per cent) ${ }^{151}$ Exactly what the nature of the spiracle reaction to the respiratory stimulant might be is subject to further investigation and interpretation. Wigglesworth, on the basis of his own extensive investigations and those of other workers, concludes that the action of carbon dioxide is mainly through the acidity produced in the tissue fluid, in other words a hydrogen ion effect. ${ }^{368}$

In this survey of the respiratory control among invertebrates and vertebrates, the importance of carbon dioxide excess and oxygen want is apparent. Many of the findings have been summarized in Table 47, which designates the type of respiratory movement, along with the response to the respiratory gases. In practically all cases in which carbon dioxide increase is a stimulating factor, so also is oxygen want; oxygen deficiency in lower (aquatic) animals appears to be either the only respiratory stimulant or, if carbon dioxide also acts, then the more fundamental one. This implies the absence of carbon dioxide-sensitive respiratory centers in primitive forms and correlates with the fact that carbon dioxide would tend not to build up to such a considerable concentration in aquatic forms as a result of its diffusion through the body surface and away from the organism. Conversely, in higher (generally terrestrial) animals, carbon dioxide tends to play a more important role as a respiratory stimulant and by its action on the highly sensitive respiratory 
centers in low concentration becomes the predominant and delicate controlling factor in the respiratory pattern of the organism, relegating the factor of oxygen want to a minor role except in anoxic and emergency conditions.

\section{METABOLIC ADAPTATIONS}

Oxygen Requirements and Energy Conversion. The significance of oxygen rests on its importance as the ultimate oxidizing agent in the manifold series of energy-releasing metabolic processes in the organism. As such its utilization is a direct measure of the degree of activity, food conversion, heat production, and protoplasmic reorganization occurring within the cells and tissues-a function of those processes dependent on or indicated by free energy release. To deny organisms oxygen, however, is not necessarily to deprive them of the means of survival. Fortunately there are other energy-yielding reactions which do not depend on atmospheric oxygen-in anaerobic environments in which free oxygen is not a characteristic component. These metabolic adaptations bear an important and universal place in the oxidative economy of organisms and permit life under conditions of little or no oxygen, either temporarily or for indefinite periods of time.

Oxidation which depends on the combination of substrate with atmospheric oxygen, forming carbon dioxide and water, is but one of many processes involving redox energy shifts. Oxidation may, for example, be just as truly a process of dehydrogenation or electron loss as one consisting of the addition of oxygen. For reviews of the increasing tide of oxidation-reduction literature a number of important contributions are available. ${ }^{12}, 15,133,169,266$ The breakdown of food substances, the release of pent-up energy, and the electronic shifts characteristic of particular oxidation-reduction systems may occur in the presence of specific substrates and enzymes and often in the absence of oxygen. These anaerobic reactions may be of temporary and topical occurrence, as muscle glycolysis, on which contraction depends, and which in severe exercise or during submergence of diving mammals is accompanied by pronounced oxygen debt. Or they may be more permanently anaerobic and non-localized processes affecting the entire animal, as, for example, in an oxygen-deprived nematode worm, which has yielded the advantages of an oxygen environment in favor of endoparasitic anaerobiosis.

Glycolysis. Oxygen requirements and energy yields from various types of organic food have been cited previously (p. 237). The steps of degradation of these substrates in the animal are important phases in intermediary metabolism, of which carbohydrate breakdown is probably the most common and certainly the best known. Glycolysis, the catabolism of glycogen to lactic acid, occurs under both anacrobic and aerobic conditions; glycolysis under aerobic conditions makes avaitable more energy for the amount of carbohydrate utilized, and may thus be considered more efficient. ${ }^{75}$ Calculated on a basis of energy made available to the system per mol of hexose sugar utilized, the relative efliciencies of the various processes 12 are as follows:

oxidation $\quad \mathrm{CH}_{12} \mathrm{O}_{\mathrm{i}}+6 \mathrm{O} \rightarrow 6 \mathrm{CO}_{2}+6 \mathrm{H} \mathrm{O}+686 \mathrm{kcal} / \mathrm{mol}$

lermentation $\quad\left(\mathrm{II}_{12} \mathrm{O}\right) \rightarrow 2\left(\mathrm{H}_{\mathrm{i}} \mathrm{CH} \mathrm{OH}+2 \mathrm{CO}+50 \mathrm{kcal} . / \mathrm{mol}\right.$

glycolysis $\quad \mathrm{C}_{12} \mathrm{H}_{12} \mathrm{O}_{6} \rightarrow 2 \mathrm{CH}_{3} \mathrm{CH}(\mathrm{OH}) \mathrm{COOH}+36 \mathrm{kcal} . / \mathrm{mol}$ 
The oxidation of lactic acid to carbon dioxide and water occurs only in the presence of free oxygen. Thus the process of anaerobic glycolysis proceeds as far as the formation of lactic acid, and this substance is the common accumulation product of anaerobiosis, piling up in the tissues and flooding the blood stream during excessive activity, incurring an oxygen debt, and awaiting an adequate return of oxygen to balance the oxidation budget.

Glycogen as an energy source is common among invertebrates, according to determinations made by direct analysis and measurement of the R.Q. Some tapeworms consist of as much as 60 per cent dry weight of glycogen; when raised in a glucose-free medium these worms may undergo a decrease in glycogen to 6 per cent of the original level. ${ }^{33: 3}$ Nematodes can synthesize glycogen if glucose is present in the culture medium. In some free-living planarians seasonal changes occur in glycogen content with a minimum in summer, corresponding to a reduced carbohydrate metabolism. Free-living and parasitic forms alike undergo considerable anaerobic glycolysis, with a tendency naturally for extreme anaerobic adaptation in the endoparasitic animals. ${ }^{117}$ Indeed, moderate oxygen tensions are claimed to be poisonous to some anacrobes.

The over-all pattern of anaerobic glycolysis is a complex system of reactions in which phosphorylated esters are formed under enzymatic control with energy shifts of relatively high levels, brought about through phosphate highenergy double bonds. ${ }^{2}: 30,231,334$ Although certain exceptional cases have been cited, ${ }^{260}$ the work at present indicates remarkable consistency and uniformity in the pathway of glycolysis (Fig. 62).12, 17 Phosphorylation appears to be mandatory for glycogen breakdown. It may be noted that all of these reactions are theoretically reversible, but ordinarily move in the direction of degradation. The important thermodynamic aspects of this series of reactions involve the transfer of energy through the adenosine triphosphate (ATP) system between steps four and five, the conversion of fructose-6-phosphate to fructose-1,6diphosphate, and the liberation of energy in the oxidation of the glyceraldehyde diphosphate to diphosphoglyceric acid.

The conversion of pyruvic acid may be through a number of alternate routes: reduction to lactic acid, decarboxylation, or oxidation by way of the familiar tricarboxylic acid cycle (Fig. 63). The latter oxidative transfer system was established in vitro by Krebs and his co-workers, ${ }^{\circ 09}$ and involves the succinate-fumarate-malate-oxaloacetate dehydrogenase systems. Pennoit-de Cooman $^{279}$ demonstrated in the cestode, Cysticercus pisiformis, the presence of succinase, fumarase, and lactic acid and acetic acid dehydrogenases.

This much detail has been considered in relation to glycolysis, inasmuch as glycogen is present in considerable quantities in practically all organisms and is the most readily mobilizable, and conveniently stored, source of energy. Simpler carbohydrates, glucose and fructose, for example, are readily available for glycolysis, but generally are not stored in sizable quantities.

Carbohydrate Conversion. An effective metabolic adaptation which makes possible energy stores at an oxygen saving is the well established conversion of carbohydrate to fat. The synthesis of fat from partially non-lipoidal sources has been experimentally demonstrated, utilizing deuterium as a tracer, feeding it to mice in drinking water. 318 Organisms on lipoid-free diets may synthesize fat, and the "hardening" of fats of livestock by feeding carbohydrate and protein illustrates the conversion that must take place. The general saving in 
Pathway of Glycolysis

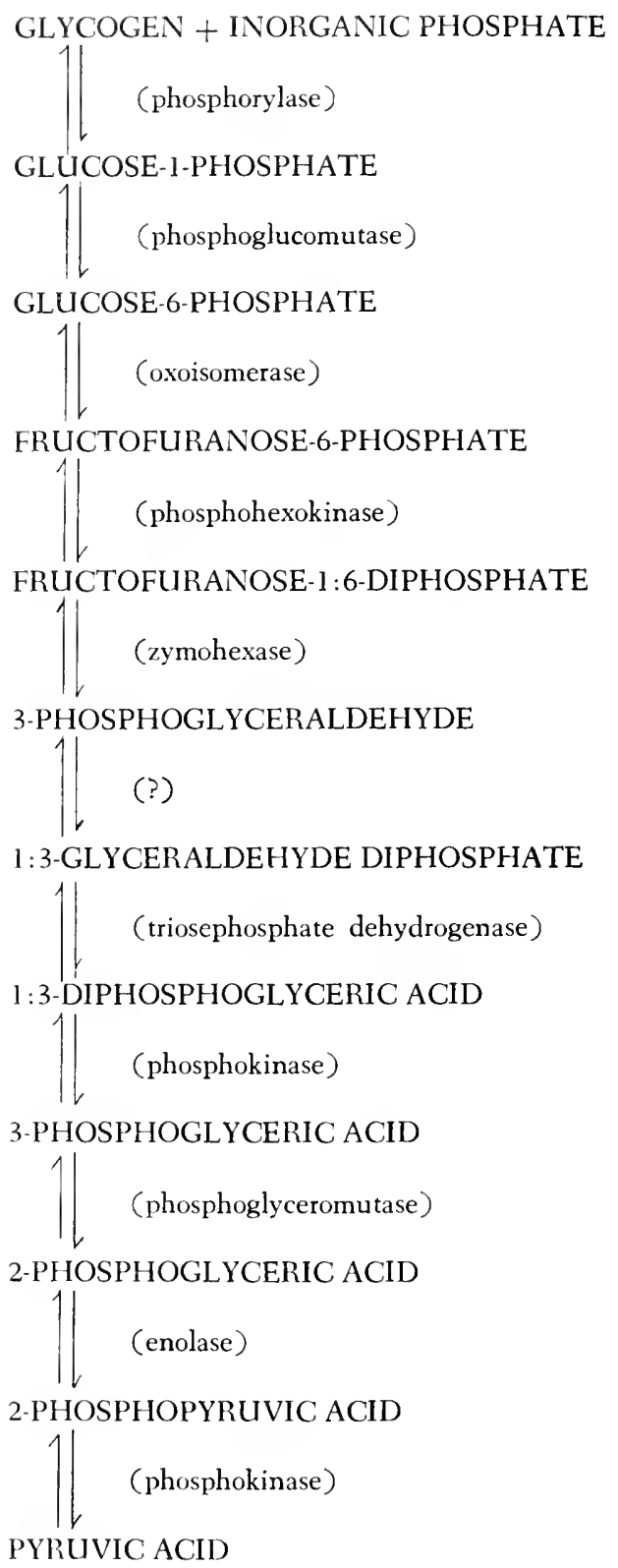

Fig. 62. Pathway of glycolysis involving the phosphorylation of hexose and transfer of energy through the energy-rich phosphate bonds. All these reactions can occur under anacrobic conditions. From Baldwin. ${ }^{22}$ 


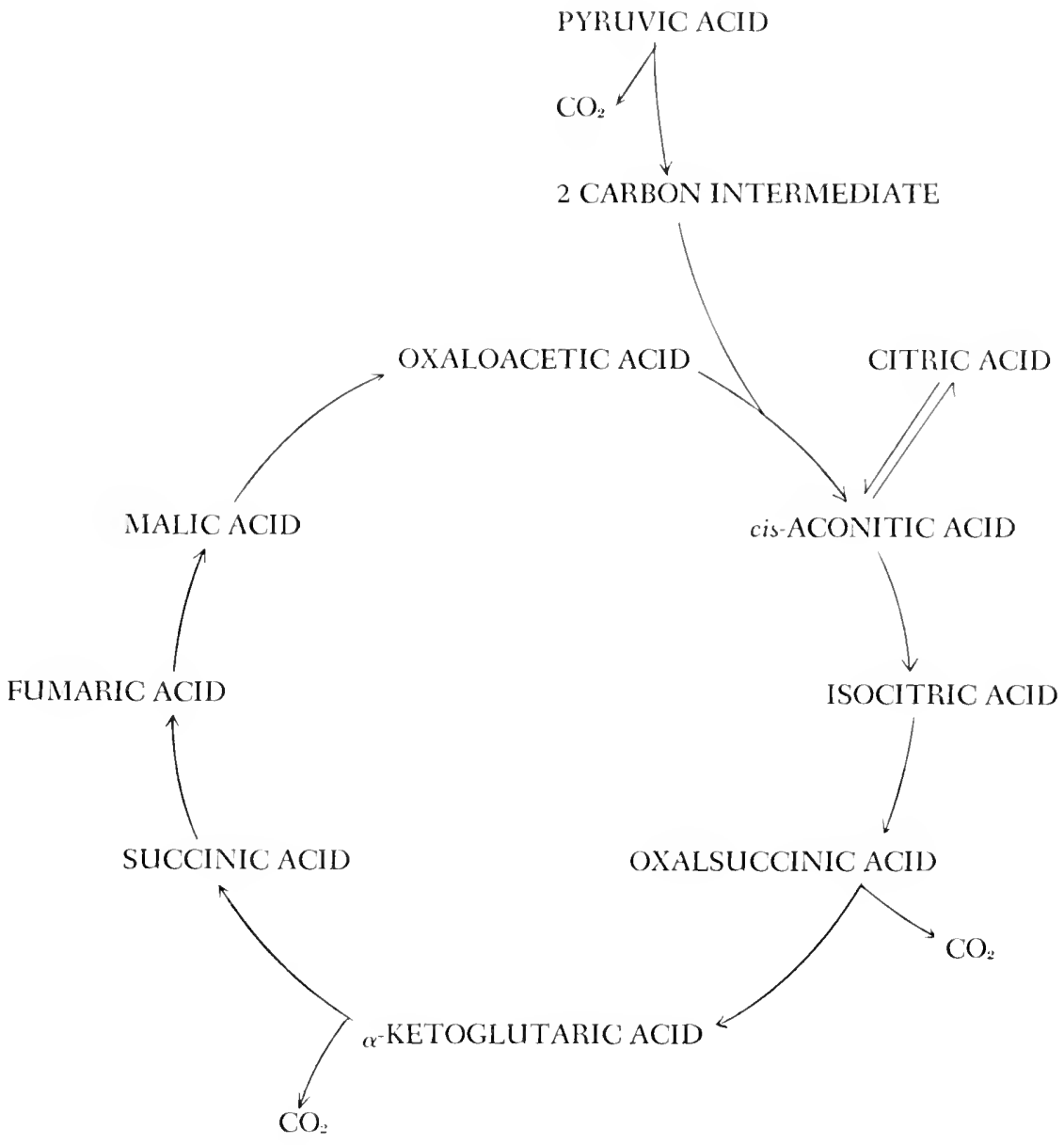

Fig. 63. The tricarboxylic acid cycle involving the oxidation of pyruvic acid. From Gortner, R. A., Outlines of Biochemistry (1949). New York, Wiley.

oxygen in this conversion is derived from the relatively high oxygen content of carbohydrate compared with that of fat. This is further manifested in the R.Q. values. The mechanism of carbohydrate conversion into fat is still not clear, but such conversion probably takes place mainly in the liver and involves some kind of phosphorylation of carbohydrate comparable to that involved in glycolysis. ${ }^{233}$

Anaerobiosis. Relative anacrobiosis is not to be considered an extraordinary metabolic process. Indeed, it is the unique animal which is incapable of some form of anaerobic metabolism, although this may be prescribed by an oxygen-deficient environment rather than an oxygen-free one. The survival period and recovery time for aerobes placed under conditions of enforced 
anaerobiosis vary, of course, from individual to individual. The larva of the helminth, Eustrongylides, for example, can tolerate anaerobiosis for 18 hours and then recover locomotion in 30 minutes. Some worms, molluscs, and crustaceans can survive anaerobic conditions for periods exceeding three months. ${ }^{49}$ Generally speaking, terrestrial organisms are less resistant to anaerobiosis than are aquatic forms. Insects, particularly adults, are sensitive to oxygen deficiency. Likewise, the recovery times of insects are relatively long. An important factor in the survival time is always the degree of activity of the organism in question. ${ }^{\text {sg }}$ The ability of certain organisms to survive complete oxygen lacktrue anacrobiosis--is rather rarely encountered. An even rarer occurrence prevails when organisms are injured by the acidition of oxygen. ${ }^{71}$ We fund, then, animals cxhibiting all degrees of oxygen-deficient metabolism, ranging from (1) those anaerobes which find oxygen loarmful (e.g., intestinal ciliates of termites); (2) those which usually live in an oxygen-free or practically oxygenfree environment but can utilize oxygen if it is supplicd (e.g., tapeworms); (3) those which ordinarily use oxygen but may get along for a while in an environment devoid of oxygen (e.g., frog); to (4) those which may be considered essentially aerobic and depend on oxygen (e.g., man), but in which anaerobic processes such as muscle glycolysis occur, and, during exercise, an oxygen debt is contracted. Anaerobic muscle metabolism is particularly pronounced in the diving birds and manmals (see above).

It is worth noting that under experimental conditions many animals, invertebrates particularly, may be shifted in favor of or against an oxygen-poor environment, causing some organisms to become more, and others less, anaerobic than is their tendency in nature. Such experimental modification is not without significance in consideration of the variations in data that have been reported by some investigators for low oxygen consumers. For convenience as well as for accuracy, experimental anacrobiosis should be clearly distinguished from natural anaerobiosis. Anaerobic demonstrations under experimental conditions in the frog, ${ }^{281}$ cockroach, ${ }^{327}$. 328 earthworm, ${ }^{3: 2 ! 3}$ Paramecinm,, 003 Planaria and Tenebrio ${ }^{147}$ are all valid cases of the adaptive ability of animals to withstand oxygen lack for various periods of time, generally by way of reducing metabolism and enduring considerable oxygen debt. They tell us, however, little else, and it is unlikely that oxygen-free conditions in nature are confronted by any of these animals.

The oxidative accomplishment by which true anaerobiosis is attained in endoparasitic ascarid worms, for instance, rests on two factors: the dependence on anacrobic glycolysis, which, although not a high energy-yielding process, neverthcless is adequate for their needs, and further, the unusual tolerance to acid metabolites, as lactic, valeric, caproic, butyric, and propionic acids, which accumulate to a considerable degrec prior to excretion, rather than being oxidized, as would occur if oxygen were present. $217,247,300,347$

The fact that greater amounts of glycogen may be consumed under anaerobic conditions than during aerobiosis was shown by the work of von Brand ${ }^{49}$ on Spirographis and Halla, and of Dausend ${ }^{*}$ on Tubifex. Concerning the importance of fat and protein as energy sources under anaerobic conditions, von Brand regards fat as of little consequence but suggests that in Hirudo and Ascaris protein catabolism may play a significant role.

The utilization of oxygen when supplied to normal anaerobes has presented 
a good deal of conflicting data. Many obligatory anaerobes are harmed by high oxygen tensions, as in the classic defaunation experiments on termites. ${ }^{71,}{ }^{129} \mathrm{It}$ has been claimed that the period of survival of Ascaris megalocephala in culture is shortened by increased oxygen. ${ }^{347}$ Most ascarids and tapew'orms utilize oxygen whenever it is made available, thereby actually increasing metabolic efficiency. ${ }^{48,89,} 217$

Elimination of carbon dioxide occurs during anaerobiosis, ${ }^{24 i}, 2 \$ 1,359$ but how much of this actually represents a production of carbon dioxide and how much represents its release by the acid metabolites formed is still subject to further experimentation and interpretation. Certainly much of the carbon dioxide, having already been formed prior to the experimental anaerobiosis, is merely released from the tissues. The carbon dioxide recovered post-anaerobically from Termopsis is less than would be expected in oxygen debt payment and may represent a shift in alkali reserve necessary to buffer the large amounts of acid metabolites produced. An interesting buffer safeguard against these acids is found in bivalve molluscs, which utilize calcium carbonate from the shell during long periods of anaerobiosis. ${ }^{102}$

Anaerobiosis among invertebrates has been extensively surveyed by von Brand in a monograph based largely on his own investigations. ${ }^{9}$ Under experimental conditions certain organisms from all major invertebrate groups can survive oxygen lack or its near depletion. The relative resistance to anaerobiosis may vary with activity, nutrition, size, and stage in the life cycle, eggs and cysts, for example, being considerably more tolerant to oxygen lack than freeliving adult stages.

True anaerobiosis is not uncommon among Protozoa, although it is not easy to rule out the possibility of last traces of oxygen. The resistance to anaerobiosis among Protozoa is known to be greater under natural conditions than under experimental oxygen lack, possibly owing to the gradualness with which the organisms in nature become subjected to anaerobiosis, thus allowing for a certain degree of adaptation not seen in the laboratory.

All degrees of oxygen tolerance are found among the intestinal wormsascarids and tapeworms-depending on the worm in question and on the particular site of its location in the intestinal tract. According to von Brand ${ }^{49}$ "the old controversy 'aerobiosis or anaerobiosis,' with respect to intestinal worms, should be abandoned, and ... it should be recognized that one animal may, depending on its organization, live a predominantly anaerobic life in the same surroundings in which another worm is capable of leading a chiefly aerobic life."

Among the molluscs the lamellibranchs show the greatest anaerobic adaptability, cephalopods the least. Anaerobiosis among lamellibranchs must be of considerable importance to those marine forms which live in the tidal zone and are unable to obtain oxygen during intervals of low tide.

Anaerobic conditions have been demonstrated in many crustaceans, including copepods, cladocerans, ostracods, and cirripidians, as well as in certain insect larvae.

In conclusion, anaerobiosis as a physiological adaptation seems to have developed in a number of forms, and is particularly refined to permit certain organisms to survive conditions of oxygen want as endoparasites. The prevailing anaerobic glycolysis throughout the animal kingdom already had pro- 
vided these forms with an energy-yielding mechanism, and it was only necessary to develop an increased tolerance to the acid metabolites produced, and a way of excreting them. Whereas aerobes ordinarily convert the lactic acid back into glycogen or oxidize it to carbon dioxide (and thereby form an oxygen debt when subjected to a nitrogen atmosphere), anaerobes eliminate the lactic acid and its metabolic counterparts (and contract no oxygen debt). The reduction in metabolic activity also operates in behalf of anaerobes, but apparently this presents no hardship to organisms designed to live as endoparasites. There seems little evidence in the work performed so far to indicate that anaerobic mechanisms phylogenetically preceded aerobic ones. Until more information is available, we may assume they both arose early and perhaps have developed together in a parallel manner.

Oxygen Debt. The concept and measurement of the oxygen debt has been applied to both vertebrates and invertebrates. The debt arises during periods of activity when the oxygen supply cannot keep pace with the demand, yet the metabolic processes continue, building up lactic acid and other breakdown products which are oxidized when sufficient oxygen becomes available. The amount of accumulated lactic acid is proportional to the oxygen debt. In man severe exercise may result in a debt of 10 liters or more of oxygen, requiring at least an hour for complete removal of excess lactic acid.

Invertebrates may be grouped according to two main types of reaction to temporary oxygen deficiency: (1) contraction of an oxygen debt-similar to the situation in the vertebrates, or (2) elimination of acid waste products without benefit of complete oxidation-characteristic of anaerobes-thereby avoiding oxygen indebtedness. Among the former group may be noted the earthworm, ${ }^{329}$ cockroach, ${ }^{327}$ grasshopper, ${ }^{33}$ Planaria, ${ }^{234 ;}$ and Planorbis, ${ }^{4+}$ all of which repay the oxygen debt formed under partially anaerobic conditions, and $M y a$, which, interestingly, after low tide utilizes increased quantities of oxygen. ${ }^{x t}$ On the other hand, true anaerobes eliminate lactic and fatty acid metabolites without requiring any oxygen from outside sources. Nereis ${ }^{181}$ and Urechis ${ }^{1: 2}$ react this way. Some organisms characteristically develop only a small oxygen debt and repay it within a very short period of time, e.g., Ascaris, ${ }^{1+5}$ Drosophila, ${ }^{64}$ and Chironomus. ${ }^{355}$ The oxygen debt of Chironomus calculates to about 0.5 per cent of what the value of oxygen consumed would be if aerobic had replaced anaerobic conditions. One assumes the end-products are otherwise eliminated. The small magnitude of the oxygen debt of Drosophila in flight is of interest $-0.18 \mathrm{cu}$. mm./individual. It is paid off within two minutes after the cessation of activity. ${ }^{64}$

Respiratory Quotient. A quantitative determination of the metabolic activity of organisms may be obtained from a measurement either of the amount of oxygen consumed or of the quantity of carbon dioxide produced. By comparison of the two-the ratio of volume of carbon dioxide to volume of oxygen, the respiratory quotient (R.Q.)-an estimation can be made of the types of food materials oxidized. Definite R.Q. values are recognized for the main types of organic food;:14, 300,352 the R.Q. value of pure carbohydrate (e.g., sucrose) is 1.00 , of fat, 0.71 , and of protcin, about 0.79 . For the calculation and interpretation of these values the protein fraction may be determined by means of urinary nitrogen. On an average mixed diet, man has an R.Q. of approximately 0.8 to 0.85 . Herbivores naturally tend to have a high R.Q., carnivores a low one. 
The literature unfortunately abounds with respiratory quotient determinations of doubtful value. Such determinations on animals subjected to partial anaerobiosis are worth little unless carbon dioxide during and alter anaerobiosis is quantitated against post-anacrobic oxygen utilization. An "infinite" R.Q. value reported by various investigators (e.g., Maloeuf"-11) is of little significance except to enunciate the temporary oxyen deficiency or the sudden release of large quantities of stored carbon dioxide. The fixation and release of carbon dioxide from calcium carbonate deposits in or about the body have supplied "false" R.Q. values in some of the earlier investigations. "The lobster, Homarus, for example, gives a normal but "false" R.Q.. of 1.39 when the specimens are untreated, but lower values of 1.00 when the specimens are coated with paraffin, and 0.92 when they are covered with collodion. Subnormal respiratory quotients, as low as 0.4 in the marmot, for instance, are probably to be attributed to the slow release of carbon clioxide from the tissues, at low body temperature. ${ }^{2 \pm}$

Another source of possible error in R.Q. values is pointed up by recent work on carbon dioxide fixation and its synthesis into carbohydratc. ${ }^{73}, 351$ Fasted rats and rabbits, when fed bicarbonate tagged with radioactive carbon, form labelled glycogen in two to three hours after administration. It is obvious that under these conditions the respiratory quotient may be changed, owing to utilization of this inorganic source of carbon and synthesizable carbonate. An interesting use of radioactive carbon in the study of respiratory gases was the recent demonstration of the previously suspected carbon monoxide-carbon dioxide conversion, in which $\mathrm{C}^{14} \mathrm{O}$ administered to turtles and mice appeared in significant quantities as $\mathrm{C}^{14} \mathrm{O}_{2 .} .{ }^{69}$

The respiratory quotient may vary with gas tension or be independent of it. It is apparently independent in Chironomus, perhaps owing to a kind of metabolic control as well as to the presence of blood pigment. ${ }^{1+4}$ The R.Q. of Tubifex is dependent on oxygen tension: 0.70 in 21 per cent oxygen and a high and perhaps misleading 2.75 in 0.8 per cent oxygen. ${ }^{1+i}$ Lumbricus communis gives an R.Q. of 0.75 in 21 per cent oxygen, and 0.99 in 5 per cent oxygen. ${ }^{217}$ The work of Rahn and Otis ${ }^{294}$ on human subjects carried to simulated altitudes of 22,000 feet in a decompression chamber demonstrates a shift in respiratory quotient toward a higher value (1.2), owing to hyperventilation and the blowing off of carbon dioxide, which then decreases while remaining "aloft." On descent, the respiratory quotient is low $(0.55)$ but is shifted toward normality $(0.8)$ as the respiratory balance is regained.

The conversion of food substances within the organism is attended by variations in the respiratory quotient. Thus during the formation of fat from carbohydrate sources a high respiratory quotient prevails as a result of the conservation of oxygen in the organic transformation. The fattening process in livestock in which forced feeding with carbohydrate results in fat formation is accompanied by a high R.Q. This is shown by values as high as 1.49 in overfed geese (Fig. 64).27 Likewise, the utilization of fats and proteins and their possible conversion to carbohydrate is characterized by low respiratory quotients. Low R.Q. values are found in fasting and starved animals, most of which have an R.Q. near or a little below 0.7 .

Changes in the R. Q. occur with activity, inasmuch as carbohydrate stores are first oxidized and with their depletion, as when available glycogen is used up during long sustained moderate exercise, more and more fat and protein 
are utilized. The decrease in R.Q. in hibernating animals is correlated with low body temperature and probably represents a shift in favor of fat utilization. $28,18 \bar{s}$

The R.Q. of insects has been studied extensively before, during, and after flight. Drosophila, removed from culture bottles and measured before flight, proved to have a high R.Q. (1.23) but during flight had values of approximately 1.0. ${ }^{64}$ This is interpreted as possibly due to conversion of carbohydrate

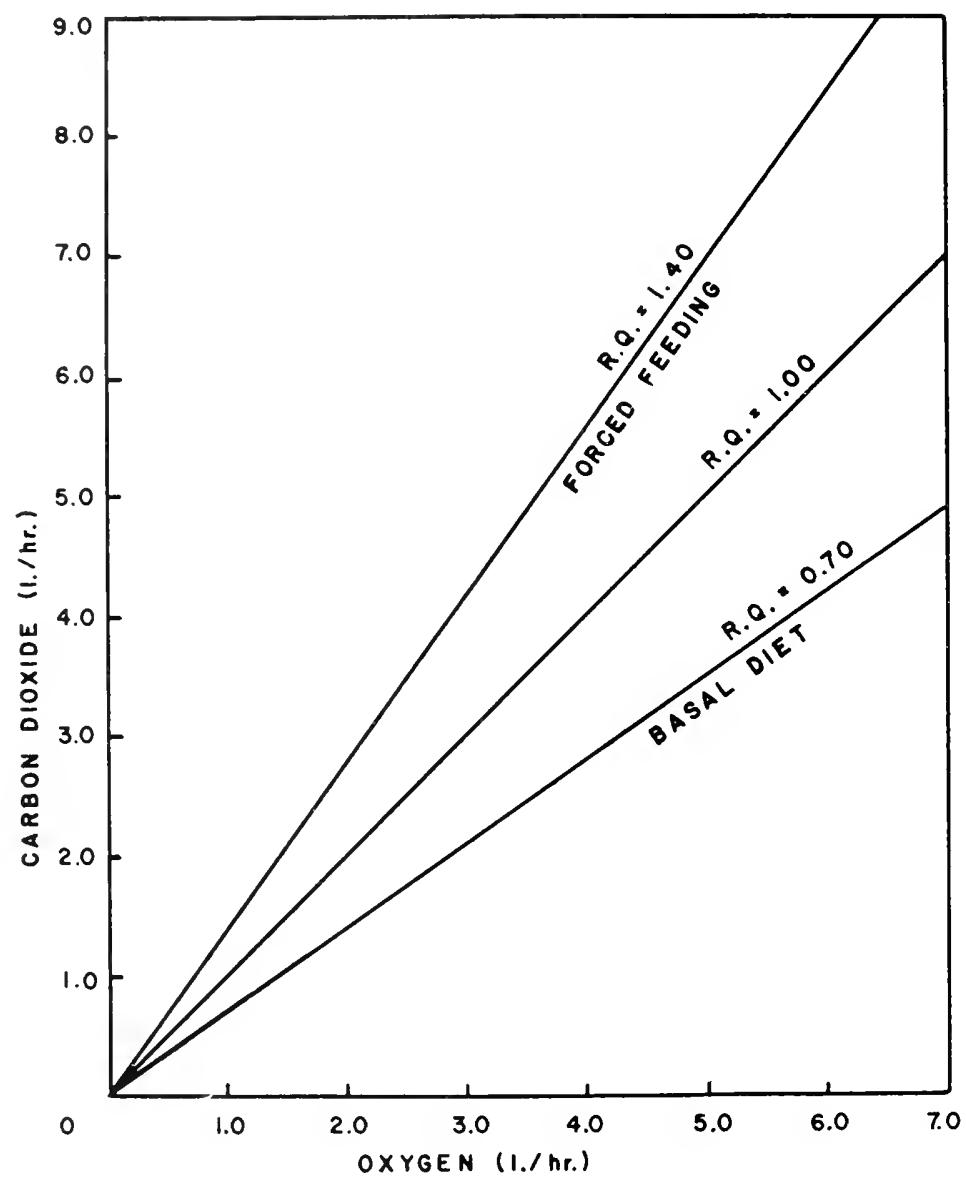

Fig. 64. High respiratory quotient of geese when placed under forced feeding conditions, compared with the R. Q. of those on basal diet. The oxygen saving is due to the conversion of carbohydrate into fat. After Benedict and Lee.:

to fat during rest and indicative of carbohydrate metabolism while in flight. After this activity the R.Q. remains at or falls slightly below 1.0 for a variable period.

Temperature changes in some animals affect the respiratory quotient. I Iall 137 has described a progressive increase in the apparent R.Q. of turtles from 0.52 at $0^{\circ}$ C. to 0.75 at $29^{\circ}$ C. The mound-building ant, Formica ulkei, also is 
claimed to have a very low R.Q. (0.5) at $t$ and a high R.(2. 0.86) at 22 C. ${ }^{17 t}$ A similar increase in R.Q. value with rise in temperature, from 0.70 at $15^{\circ} \mathrm{C}$. to 0.99 at $35^{\circ} \mathrm{C}$., prevails in Paramecinm caudatum.

The R.Q. is not necessarily constant throughout the life cycle of an inclividu al and may reflect changes in diet. In those invertebrates with rather special ized and changeable feeding habits, the R.Q. valriations maly be striking. The R.Q. of the shore crab, Carcinus maenas, falls from a value of 1.0 in the carly stage of development to 0.7 a few hours later, to rise again in successive stanes of ontogeny-i:1 (Fig. 65). The R.Q. of the frog egg increases during derclopment from cleavage to gastrulation, $=1$. 2-: perhaps indicating a cindnge from lipoid to carbohydrate food reserves. The R.Q. of the silkworm embryo, Bombyx mori, is initially 0.79 , drops to $0.6+$ at about the eighth day of incubation, and then increases to 0.75 just before hatehing. ${ }^{17 i}$

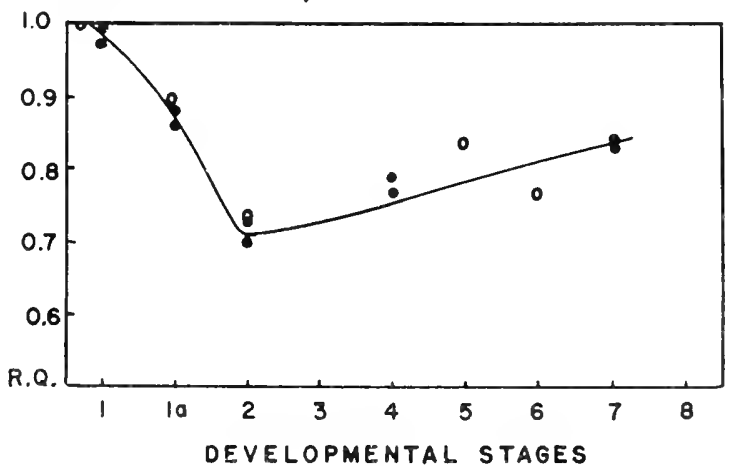

Fig. 65. Respiratory quotient change during embryonic development of the crab, Carcinus, indicating different food sources at various stages of development. After Needham. ${ }^{2.59}$

Despite certain limitations and precautions which must be exercised in interpreting R.Q. data, the respiratory quotient is of considerable value in identifying metabolic adaptations in organisms. Further critical analyses of the sources of variation in R.Q. determinations, of "true" and "false" respiratory quotients, of the fixation and release of CO.2, should extend our concepts rapidly along the route of the actual metabolic processes involved in gas exchange and energy conversion.

\section{CONCLUSION}

The respiratory mechanisms which have evolved to provide the necessary gas exchange for animals are varied and highly adaptive, both morphologically and physiologically. Diffusion of oxygen into the organism proves adequate with very small organisms, and, in fact, cutaneous respiration necessarily occurs if a sufficient oxygen gradient is established across the more or less permeable, usually moist, integument. Gas exchange ahways occurs across a water film. The increase in respiratory surface made possible by the development of gills, lungs, and tracheae, as well as the added efficiency of ventilating mechanisms, permit increase in body size and metabolic activity. All of these specialized mechanisms were adaptable to life on land as well as in water, and aerial 
respiration became common. Lung and tracheal breathers have further progressed to permit some to become secondarily aquatic. Various taxic and migratory movements may be traced to oxygen requirements.

Ventilation movements in invertebrates and vertebrates alike are decidedly influenced by various factors, including temperature, carbon dioxide excess, and oxygen deficiency. Of these, carbon dioxide appears to play the most significant role in respiratory control in the warm-blooded (generally terrestrial) vertebrates but is of lesser relative importance among lower (aquatic) animals.

Metabolic adaptations, including the oxygen-deficiency processes of anaerobic glycolysis, anaerobiosis, accumulation of oxygen debt, carbon dioxide insensitivity, and carbohydrate conversion, have enabled organisms to withstand severe oxygen stress and likewise have played a major role in the ecological distribution and behavior pattern of these animals. The amount of oxygen required, the minimum tolerable gas tension, and the oxygen utilization, although subject to modification by activity, age, size, temperature, season, nutrition, and sex, are characteristic for each organism. A survey of respiratory and metabolic adaptations demonstrates remarkably the many interrelations between the fitness of organisms and their environment.

\section{REFERENCES}

1. Adrian, E. D., J. Physiol. 72:132-151 (1931). Insect ganglionic potentials.

2. Agar, W. E., Anat. Anz. 33: 27-30 (1908). Pectoral gills of lungfish.

3. Alsterberg, G., Lunds Univer. Aarsskr. N. F. Avd. 2, 18:1-176 (1922); ibid. 20:1-77 (192t). Respiration in Tubificidae.

4. Amberson, W. R., Biol. Bull. 55:79-92 (1928). Oxygen consumption and critical tensions.

5. Amberson, W. R., Mayerson, H. S., and Scott, W. J., J. Gen. Physiol. 7:171176 (1924). Oxygen tension and consumption; invertebrates.

6. Amerling, K., Pflïg. Arch. 121:363-369 (1908). Oxygen sensitivity and age.

7. Atlas, M., Physiol. Zool. 11:278-291 (1938). Oxygen consumption of frog embryos.

8. Вава́к, E., in Winterstein's Handbuch der vergleichenden Physiologie (1921). Jena, G. Fischer. Vol. 1, Part 2, pp. 265-1028. Mechanics of breathing.

9. BaвÁk, E., and DẼdek, B., Pflïg. Arch. 119:483-529 (1907). Respiratory control among fish.

10. Baker, C. L., J. Tenn. Acad. Sci. 17:39-51 (19+2). Air breathing teleosts.

11. Baker, E. G. S., and Baumberger, J. P., J. Cell. E Comp. Physiol. 17:285-304 (19+1). Critical oxygen tension; Tetrahymena.

12. Baldwin, E., Dynamic Aspects of Bio-chemistry (1947). Cambridge Univ. Press. Chap. 4, 15.

13. Baldwin, F. M., Proc. lowa Acad. Sci. 30:173-180 (1924). Oxygen consumption in marine animals.

14. Baldwin, S. P., and Kendeigh, S. C., Sci. Publ. Cleveland Mus. Nat. Hist. 3:1-196 (1932). Bird respiration.

15. Ball, E. G., Ann. Rev. Biochem. 11:1-25 (19+2). Biological oxidation and reduction.

16. Barach1, A. L., Fenn, W. O., Ferris, E. B., and Scimidt, C. F., J. Aliation Med 18:73-87 (1947). Pressure breathing.

17. Banchoft, J., Physiol Re1'. 16:103-128 (1935). Fetal respiration.

18. Barchoft, J., and Barcroft, H., Proc. Roy. Soc. Lond. B., 96:28-42 (1924). Pulmonary respiration.

19. Barnes, T. C., Textbook of Physiology (1937). Philadelphia, Blakiston. Chap. 13. 
20. Barott, H. G., and Pringle, E. M., J. Nutrit. 22:273-286 (1941). Chicken metabolism.

21. Bantir, L. G., J. Exper. Zool. 103:463-486 (1946). Netabolism of frog embryos.

22. Bel. , J., Pliysiol. Zool. 13:73-81 (1940). Oxygen consumption; bee moth pupac.

23. Benedict, F. G., J. Biol. Chem. 20:263-299 (1915). Basal metabolism.

24. Benedict, F. G., Carnegic Inst., Wash., Publ. 425, pp. 404-517 (1932). Physiology of reptiles.

25. Benedict, F. G., Carnegic Inst., Wash., Publ. 503, pp. 1-215 (1938). Vital En ergetics. Sex differences in respiration.

26. Benedict, F. G., and Cathcart, E. P., Carnegie Inst., Wash., Publ. 187, pp. 71 96 (1913). Muscular work.

27. Benedict, F. G., and Lee, R. C., Carnegie Inst., Wash., Publ. 489, pp. 153-190 (1937). R.Q. and carbohydrate conversion; goose.

28. Benedict, F. G., and Lee, R. C., Carncgie Inst., Waslr., Publ. 497, pp. 1-239 (1938). Hibernation and nrarmot physiology.

29. Benedict, F. G., and Talbot', F. B., Carnegie Inst., Wash., Publ. 201, pp. $41 \cdot 168$ (1914). Gaseous metabolism of infants.

30. Benedict, F. G., and Tar.bot, F. B., Carnegie Inst., Wash., Publ. 302, pp. 100 186 (1921). Metabolism and growth from birth to puberty.

31. Bijtel, J. H., Experientia 3:158-160 (1947). Fish gill movements.

32. Bishop, D. W., Biol. Bnll. 83:353-362 (1942). Oxygen consumption of sperm.

33. Bonine, J. H., J. Exper. Zool. 32:137-164 (1921). Insect respiration and nutrition.

34. Bodine, J. H., Physiol. Zool. 7:459-463 (1934). Critical tensions; grasshopper.

35. Bodine, J. H., and Boell, E. J., Proc. Soc. Exper. Biol. E Med. 35:504-506 (1936). Respiration stimulation; grasshopper.

36. Bodine, J. H1., and Onr, P. R., Biol. Bull. 48:1-14 (1925). Pupal metabolism.

37. Boelaert, R., Arch. Internat. Physiol. 51:379-437 (1941). Lizard respiratory control.

38. Boell, E. J., Ann. New York Acud. Sci. 49:773-800 (1948). Metabolism and growth; Amphibia.

39. Boell, E. J., and Nicilolas, J. S., J. Exper. Zool. 109:267-282 (1948). Respiration of mammalian egg.

40. Boell, E. J., and Woodruff, L. L., J. Exper. Zool. 87:385-402 (1941). Mletab olism of Paranecium mating ty pes.

41. Borr, C., Skand. Arch. Physiol. 10:413-42t (1900). Foetal and maternal gas exchange.

42. Bour, C., Skand. Arch. Physiol. 15:23-34 (1903). O.: comsumption of snake embryos.

43. Bohr, C., and Hasselbalch, K. A., Skand. Arch. Physiol. 10:1+9-163 (1900). Metabolism in relation to weight.

44. Bonden, M. A., J. Mar. Biol. Ass. Plymonth 17:709-738 (1931). Oxygen debt; Planorbis.

45. Bosworth, M., O’Brien, I., and Amberson, W. R., I. Cell. E Comp. Phy'siol. 9:77-87 (1936). R. Q.; marine animals.

46. Botjes, J. O., Ztschr. vergl. Plysiol. 17:557-564 (1932). Breathing regulation; insect.

47. Brachet, J., Arch. Biol. 45:611-727 (1934). Respiration of frog egg.

48. Brand, T. v., Ztschr. vergl. Physiol. $21: 220-235$ (1934). Gas exchange in Ascaris.

49. Brand, T. v., Biodynamica, Normandy, Mo., pp. 137-278 (19+6). Anaterobiosis in invertebrates.

50. Brody, S., Bioenergetics and Growth (1945). N. Y., Reinhold. Chap. 13, 14.

51. Brody, S., Kibler, H. H., and Trowbridge, E. A., Univ' Mo. Agr. Expt. Sta. Res. Bull. 368, pp. 1-1+ (1943). Oxygen consumption in domestic animals.

52. Bronk, D. W., Am. Sci. 34:55-76 (1946). Fluctuations in tissue $\mathrm{O}_{2}$.

53. Brues, A. M., and Naranjo, A., Fed Proc. 8:18 (1949). Absorption of $\mathrm{C}^{\text {"11 }} \mathrm{O}_{2}$; mice.

54. Buck, J. B., and Keister, M. L., Biol. Bull. 97:323-330 (1949). Tracheal filling in Sciara larvae.

55. Buddenbrock, W. v., Ztschr. vergl. Physiol. 26:303-305 (1938). Holothurian water-lung. 
56. Buddenbrock, W. v., and Rohr, G. v., Ztschr. allg. Physiol. 20:111-160 (1923). Respiration and critical tensions; insects.

57. Burrows, M. T., Proc. Soc. Exper. Biol. \& Med. 18:133-136 (1920). Oxygenlack sensitivity in chick.

58. Calugareanu, D., Arch. ges. Physiol. 120:425-450 (1907). Gas exchange in fish intestine.

59. Carlson, A. J., and Luckhardt, A. B., Am. J. Physiol. 54:55-95, 261-306 (1920). Lung reflexes; amphibians and reptiles.

60. Carter, G. S., Biol. Rev. 6:1-35 (1931). Aquatic and aerial respiration.

61. Carter, G. S., J. Linn. Soc. Zool. 39:219-233 (1935). Respiration in electric eel.

62. Carter, G. S., and Beadle, L. C., J. Linn. Soc. Zool. 37:197-203, 327-368 (1930). Respiratory adaptations in fish.

63. Carter, G. S., and Beadle, L. C., J. Linn. Soc. Zool. 37:379-386 (1931). Respiratory adaptations; oligochaetes.

64. Chadwick, L. E., Biol. Bull. 93:229-239 (1947). R. Q.; Drosophila in flight.

65. Chadwick, L. E., and Gilmour, D., Physiol. Zool. 13:398-410 (1940). Respiration and wing rate; Drosophila.

66. Chattum, E. P., Tr. Am. Micr. Soc. 53:348-407 (1934). Pulmonate respiration.

67. Chapin, J. L., and Rahn, H., Fed. Proc. 7:19-20 (1948). CO. insensitivity; beaver.

68. Chevillard, L., Hamon, F., and Mayer, A., Ann. Physiol. Physiochim. Biol. 13:1145-1163 (1937). Temperature and oxygen consumption; mice.

69. Clark, R. T., Jr., Stannard, J. N., and Fenn, W. O., Fed. Proc. 8:26 (1949). Carbon monoxide conversion; turtles and mice.

70. Clausen, H. J., J. Cell. E Comp. Physiol. 8:367-386 (1936). Metabolism of brown snake.

71. Cleveland, L. R., Biol. Bull. 48:309-325 (1925). O. defaunation; termite.

72. Clowes, G. H. A., and Krahl, M. E., J. Gen. Physiol. 20:145-171 (1936). Metabolic stimulation.

73. Conant, J. B., Cramer, R. D., Hastings, A. B., Kremperer, F. W., Solomon, A. K., and Vennesland, B., J. Biol. Chem. 137:557-566 (1941). CO.2 fixation; mammals.

74. Cook, S. F., and Smith, R. E., J. Cell. E Comp. Physiol. 19:211-219 (1942). Termite metabolism.

75. Cori, C. F., Biol. Symp. 5:131-140 (1941). Phosphorylation mechanisms.

76. Cousin, G., Bull. Biol. France e. Belg. (Suppl.) 15:341 (1932). Pupal metabolism; Diptera.

77. Crescitelli, F., J. Cell. E Comp. Physiol. 6:351-368 (1935). Respiratory metabolism; bee moth.

78. Crile, G. W., Intelligence, Power, and Personality (1941). N. Y., McGraw Hill. Chap. 23.

79. Crozier, W. J., J. Gen. Physiol. 7:189-216 (1924). Thermal increments.

80. Crozier, W. J., and Stier, T. B., J. Gen. Physiol. 7:429-447, 699-704 (1925). Respiratory thermal inerements.

81. Csık, L., Ztscinr. vergl. Physiol. 27:304-310 (1939). Oxygen requirements; Drosophila.

82. Cunningham, J. T., and Reid, D. M., Proc. Roy. Soc. Lond., B. 110:234-248 (1932). Oxygen secretion by lungfish gills.

83. Dahr, E., Lunds. Univer. Aarsskr. N. F. Avd. 2, 20, No. 10:1-19 (1924). Lung movements in pulmonate snails.

84. DAM, L. van, J. Exper. Biol. 12:86-94 (1935). Oxygen utilization; Mya.

85. Dam, L. van, Zool. Anz. 118:122-128 (1937). Respiration in annelids.

86. Dam, L. van, Dissertation (1938). Groningeri. Utilization of oxygen and regulation of breathing; aquatic animals.

87. Das, B. K., Phil. Tr. Roy. Soc. B. 216:183-219 (1927). Air-breathing fish.

88. Dausend, K., Ztschr. vergl. Physiol. 14:557-608 (1931). Tubifex respiration.

89. DaveY, D. G., J. Exper. Biol. 15:217-224 (1938). Nematode respiration.

90. Davis, R. A., and Fraenkel, G., J. Exper. Biol. 17:402-467 (1940). Oxygen consumption of flies during flight. 
91. Demolt, R. v., Ztschr. Biol. 87:8.22 (1928). Respiratory regulation; insect.

92. Dill, D. B., and Edwarns, H. T., Copeia 1:1-3 (1931). Crocorlile inctabolism.

93. Dixon, M., Manometric Methods (19+3). Cambridge Univ. Press.

94. Dobzhansky, 1', and Poulson, D. F., Ztschr. vergl. Physol. 22:473 478 (1935). Oxygen consumption of Dessophila larva.

95. Dolk, H. E., and Panuw, F. v. d., Zischr. vergl. Physiol. 10:32+-343 (1929 Critical tension in earthworm.

96. Dolk, H. E., and Postma, N., 7tsclur. vergl. Physiol. 5:417-44t (1927). Frog respiration.

97. Dorfuan, A., Physiol. Rev. 23:124-138 (1943). Pathways of glycolysis.

98. Drastich, L., Ztschr. vergl. Phy'siol. 2:632-657 (1925). Oxygen tension and gill size; salamander.

99. Drastich, L., C. R. Soc. Biol. 97:1755-1758 (1927). Critical oxygen tension; sea urchin egg.

100. Dripps, R. D., and Dumke, P. R., J. Pharmacol. 77:290-300 (1943). Respiratory center sensitivity to $\mathrm{CO}_{\%}$.

101. Drummond, F. H., Proc. Zool. Soc. Lond. 116:225-229 (1946). Pharyngeoœsophageal respiration; lizard.

102. Dugal, L.-P., and Inving, L., C. R. Soc. Biol. 124:526 (1937). Shell carbonates as buffers; clam.

103. Edwards, G. A., J. Cell. \& Comp. Physiol. 27:53-64 (1946). Sex differences in $\mathrm{O}_{2}$ consumption; arthropods.

104. Edwards, G. A., Quart. J. Florida Acal. Sci. 9:1-5 (1946). Permeability of tracheae.

105. Edwards, G. A., and Irving, L., J. Cell. E Comp. Physiol. 21:169-181 (1943). Temperature and seasonal effects on oxygen consumption; sand crab.

106. Ege, R., Ztschr allg. Plyysiol. 17:81-124 (1915). Oxygen stores in aquatic insects.

107. Elftmann, A. G., Am. J. Anat. 72:1-27 (1943). Lung reflexes; dog.

108. Etrin, W., Root, R. W., and Mofsuin, B. P., Physiol. Zool. 13:415-429 (1940). Effect of thyroid on oxygen consumption; goldfish.

109. Ewer, R. F., J. Exper. Biol. 18:197-205 (1942). Oxygen consumption and tension; Chironomus.

110. Fenn, W. O., Am. J. Physiol. 84:110-118 (1928). Microrespiration technique.

111. Fenn, W. O., Am. Phil. Soc. 92:1+t-15+ (19+8). Accuired carbon dioxide tolerance.

112. Fox, H. M., Proc. Zool. Soc. Lond. Part II, 945-955 (1936). Metabolism of poikilothermic animals.

113. Fox, H. M., and Baldes, E. J., J. Exper. Biol. 12:174-178 (1935). Oxygen consumption and habitat; Arthropoda.

114. Fox, H. M., and Johnson, M. L., J. Exper. Biol. 11:1-10 (1934). Respiratory control; Crustacea.

115. Fox, H. M., and Wingeield, C. A., Proc. Zool. Soc. Lond. 107:275-282 (1937). Activity and metabolism of poikilotherms in different latitudes.

116. Fox, H. M., Wingfeled, C. A., and Simmonds, B. G., J. Exper. Biol. 14:210-218 (1937). Oxygen consumption in relation to habitat.

117. Fraenkel, G., Ztschr. vergl. Physiol. 16:394-417 (1932). Respiration during flight; insect.

118. Fraenkel, G., Biol. Zentralbl. 54:96-101 (1934). Breathing movements during flight; birds.

119. Fraenkel, G., and Herford, G. V. B., J. Exper. Biol. 15:266-280 (1938). Insect cutaneous respiration.

120. Frank, O., and Voit, F., Ztschr. Biol. 42:309-362 (1902). Respiration and curarization.

121. Fraps, M., Pliysiol. Zool. 3:242-270 (1930). Metabolism in Planaria.

122. Fry, F. E. J., and Hart, J. S., Biol. Bull. 94:66-77 (1948). Oxygen consumption and activity; goldfish.

123. Gage, S. H., and Gage, S. P., Am. Nat. 20:233-236 (1886). Turtle respiration.

124. Gerlach, Arch. Anat. Physiol. wiss. Med. 431 (1851). Respiration through human skin. 
125. Gesell, R., Physiol Rev. 5:551-595 (1925). Chemical regulation of respiration.

126. Gesell, R., Ann. Rev. Plyysiol. 1:185-216 (1939). Factors controlling respiration.

127. GEYER, F., and MANN, A., Ztschr. vergl. Physiol. 27:429-433 (1939). "Group effect" depression of oxygen consumption; fish.

128. Gilbert, P. W., and Bond, C. F., Science 109:36-37 (1949). Respiratory regulation and anoxia; ducks.

129. Gilmour, D., J. Cell. \& Conn. Physiol. 15:331-342 (1940). Anaerobiosis, termite.

130. Goddard, D. R., in Höber, R., Physical Chemistry of Cells and Tissues (1945). Philadelphia, Blakiston. Chap. 24. Respiration of cells and tissues.

131. Grar, J. S., Science 103:739-744 (1946). Respiratory regulation.

132. Gray, J. S., Pulmonary Ventilation and Its Physiological Regulations (1950). Springfield, Illinois, Charles C Thomas.

133. Green, D. E., Mechanisms of Biological Oxidations (1940). Cambridge Univ. Press.

134. Guiersse-Pellisier, A., Les appareils respiratoires dans la série animale (1945). Paris, Payot.

135. Haldane, J. S., Respiration (1922). Yale Univ. Press. Chap. 9.

136. Haldane, J. S., Physiol. Rev. 7:363-384 (1927). Oxygen secretion by lungs.

137. Hall, F. G., Biol. Bull. 47:79-126 (1924). Swimbladder of fish.

138. Hall, F. G., Am. J. Physiol. 88:212-218 (1929). Oxygen tensions and oxygen consumption; marine fish.

139. Hall, F. G., Am. J. Physiol. 93:417-421 (1930). Oxygen utilization; mackerel.

140. Hall, F. G., Biol. Bull. 61:457-467 (1931). Respiration of puffer fish.

141. Hall, R. H., Biol. Bull. 75:395-408 (1938). Oxygen consumption; Colpidium.

142. HaLl, V. E., Biol. Bull. 61:400-116 (1931). Cloacal respiration; Urechis.

143. Harnisch, O., Ztschr. vergl. Physiol. 16:76-88 (1932). Critical oxygen tension; Chironomus.

144. Harnisch, O., Ztschr. vergl. Physiol. 16:335-347 (1932). R.Q. of Chironomus.

145. Harnisch, O., Ztschr. vergl. Physiol. 19:310-348 (1933). Oxygen debt in Ascaris.

146. Harnisch, O., Ztschr. vergl. Plyssiol. 22:450-465 (1935). Critical oxygen tension; Tubifex.

147. Harnisch, O., Ztschr. vergl. Physiol. 30:145-166 (19+3). Anaerobiosis and glycolysis.

148. Harvey, E. N., J. Gen. Physiol. $11:+69-475$ (1928). Oxygen diffusion pressure.

149. Hasset, C. C., Biol. Bull. 95:114-122 (19+8). Carbohydrate metabolism; Drosophila.

150. Haugand, N., and Irving, L., J. Cell. E Comp. Physiol. 21:19-26 (1943). Scasonal effects on oxygen consumption; cunner.

151. Hazelhoff, E. H., Dissertation (1926). Utrecht. Spiracular control.

152. Hazelioff, E. H., Ztschr. vergl. Physiol. 26:306-327 (1938). Oxygen utilization; invertebrates.

153. Heerdt, P. F. v., and Krijgsman, B. J., Ztschr. vergl. Physiol. 27:29-40 (1939). Respiratory control; Eriocheir.

154. Heilbrunn, L. V., Outline of General Physiology (1943). Philadelphia, Saunders. Chap. 20, 21.

155. HeLFF, O. M., Physiol. Zool. 1:76-96 (1928). Respiratory regulation; crayfish.

156. Henze, M., Biochem. Ztschr. 26:254-278 (1910). Oxyregulation and phylogeny.

157. Herford, G. M., J. Exper. Biol. 15:327-338 (1938). Tracheal pulsation; Hea.

158. Herold, W., Zool. Morph. Okol. (Berlin) 4:337-415 (1925). Respiratory adaptations; land snails.

159. Hernington, L. P., Heat Production and Thermal Conductance in Small Animals at Various Temperatures (1941). Amer. Inst. Physics, Reinhold Publ. Corp., N. Y.

160. lleymans, C., and Bouckaert, J. J., Ergebn. Physiol. +1:28-55 (1939). Chemoreception of carotid sinus.

161. Hevmans, C., Bouckaert, J. J., and Dautrebande, L., Arch. Internat. Pharmacody'n. 39:400-425 (1930). Chemoreception and respiration.

162. Hiestand, W. A., Physiol. Zool. t:246-270 (1931). Oxygen tension and respiration; Arthropoda. 
163. Haestand, W. A., and Randall, W. C., /. Cell. E Comp. Physiol. 17:333.340 (19+1). Species dilferences in response to $\mathrm{CO}$; birds.

164. Higginbotinan, A. C., Ecology 28:462-46+ (1947). Diurnal oxygen consumption; cattish.

165. Hill, D. K., J. Physiol. 107:479-495 (19+8). Oxygen tension and respiration; frog muscle.

166. Hrwweir, H. E., Anu. Rer'. Physiol. 7:181-200 (19+5). Lnergy metabolism.

167. Hinivich, H. E., and Fаzекаs, J. F., Am. J. Physiol. 132:45+459 (I9+1). Metabolism and age; dogs.

168. Hitchcock, F. A., and Whitehonn, W'. V., Am. Rev. Physiol. 10:277-30t (1948). Factors regulating respiration.

169. Hogness, T. R., Biol. Symp. 5:119-130 (1941). Oxidation catalysts.

170. Holter, H., and Lindenstion-Lang, K., C. R. Trav. Lab. Carlsherg, Ser. Chim. $24: 400-778$ (19+3). Cartesian diver respirometer.

171. Holter, H., and Zeutinen, E., C. R. Trai. Lab. Carlsberg Sér Chim. 26:277296 (1948). Metabolism and weight; Chaos.

172. Hopkins, II. S., J. Exper. Zool. 102:143-158 (1946). Oxygen consumption of tissues; Venus.

173. Honton, F. M., J. Exper. Biol. 11:257-261 (1934). Metabolic response to thyroid; lamprey.

174. Hoskins, W. M., and (raig, R., Physiol. Rev. 15:525-596 (1935). Insect metabolism.

175. Hsuen, T. Y., and TANG, P. S., Physiol. Zool. 17:71-78 (194t). Respiration and growth; bee.

176. Hüfner, G., Ann. d. Physik u. Chem. 60:134-168 (1897). Oxygen diflusion constants.

177. Hutchens, J. O., J. Cell. \& Comp. Physiol. 17:321-332 (1941). Culture age and $\mathrm{O}$. consumption; Chilomonas.

178. Hyde, I. H., Am. J. Physiol. 10:236-256 (1903). Respiratory center of skate.

179. Hykes, O. V., C. R. Soc. Biol. 95:1+52-1+54 (1926). Respiratory movements; Tunicata.

180. Hyman, L., Physiol. Zool. 2:505-534 (1929). Oxygen tension and consumption; Planaria.

181. Hyman, L., J. Exper. Zool. 61:209-221 (1932). Oxygen tension and con sumption; Nereis.

182. Hyrtl, J., Abhand. der k. Böhimschen Gesellsch. der Wissensch., Folge 5, Vol. 3. (1845). Gas bladder mechanism.

183. lrving, L., J. Cell. E Comp. Physiol. 9:+37-451 (1937). Respiratory and cardiovascular clianges during dive; beaver.

184. Inving, L., Physiol. Rev. 19:112-133 (1939). Respiration in diving mammals.

185. Inving, L., Report of Conference on Methodology and Techniques for the study of Animal Soc. (19+8). New York Acad. Sci. Oxygen consumption at low temperatures; mammals.

186. Irving, L., Scholander, P. F., and Grinnell, S. IV., Science 92:483 (1940). Respiratory and cardiovascular characteristics of diving mammals.

187. Irving, L., Scholander, P. F., and Grinnell, S. W., J. Cell. \& Comp. Physiol. 17:145-168 (1941). Porpoise respiration.

188. Irving, L., Scholander, P. F., and Grinnell, S. W., J. Cell. E Comp. Physiol. 20:189-210 (1942). Respiration of sloths.

189. Inving, L., Solandt, O. M., Solandt, D. Y., and Fisiler, K. C., J. Cell. \& Comp. Physiol. 7:137-151 (1935). Metabolic adjustment to diving; scal.

190. Ishida, S., Sci. Rep. Tohoku Imp. Univ. Ser IV, 10:619-638 (1935). Oxygen consumption; uyster.

191. Jennings, H. S., Beharior of the Lower Organisms (1915). Columbia Univ. Press. Chap. 21.

192. Johnson, M. L., J. Exper. Biol. 13:467-475 (1936). Respiratory regulation; Crustacea.

193. Johnson, M. L., I. Exper. Biol. 18:266-277 (19+2). Critical oxygen tension; earthworm. 
194. Jongbloed, J., and Wiersma, C. A. G., Ztschr, vergl. Physiol. 21:519-533 (1934). Oxygen consumption of bees.

195. Jordan, H., Ztschr. vergl. Physiol. 5:179-190 (1927). Respiratory control; insects.

196. Jordan, H. J., Arch Néerl. Sci. Ex. \& Nat., Ser. 3C, 14:305-314 (1929). Oxygen tension and consumption; frog.

197. Jordan, H. J., and Quittart, J., Proc. R. Akad. Net. Amsterdam 41:2-9 (1938). Respiration; crayfish.

198. Kalmus, H., Ztschr. vergl. Physiol. 12:725-759 (1930). Respiration and $\mathrm{O}_{2}$ tension; Crustacea.

199. Kanitz, A., Temperatur und Lebensvorgänge (1915). Berlin, Borntraeger. Parts $8,20$.

200. Kayser, C., Ann. Physiol. 15:1087-1219 (1939). Oxygen consumption; dormouse.

201. Keister, M. L., J. Morph. 83:373-424 (1948). Tracheal development.

202. Kibler, H. H., and Brody, S., J. Nutrit. 28:27-34 (1944). Metabolism of chicks.

203. Krtching, J. A., Biol. Bull. 77:339-353 (1939). Anaerobiosis; Paramecium.

204. Kосн, H. J., J. Exper. Biol. 15:152-160 (1938). Anal "gills"; Diptera.

205. Koskimies, J., Experientia 4:274-276 (1948). Poikilothermy in starved swifts.

206. Kosmin, N. P., Alpatov, W. W., and Resnitschenko, M. S., Ztschr. vergl. Physiol. 17:408-422 (1932). Oxygen consumption of bees in flight.

207. Krahl, M. E., and Clowes, G. H. A., J. Gen. Physiol. 23:413-427 (1940). Metabolic stimulation and mitotic inhibition.

208. Krebs, H. A., Tabul. Biol. 9:209-231 (1934). Oxygen consumption; cells and tissues.

209. Krebs, H. A., Adv. in Enzymol. 3:191-252 (1943). Carbohydrate oxidation.

210. Kroch, A., Skand. Arch. Physiol. 15:328-419 (1904). Frog respiration, cutaneous and pulmonary.

211. Krogh, A., Skand. Arch. Physiol. 16:348-357 (1904). Cutaneous respiration; vertebrates.

212. Krogh, A., Internat. Rev. Hydrobiol. 6:42-47 (1914). Effect of urethane on respiration.

213. Krogh, A., Internat. Ztschr. Physik.-chem. Biol. 1:491-508 (1914). Temperature and standard metabolism.

214. Krogh, A., The Respiratory Exchange of Animals and Man (1916). London, Longmans, Green.

215. Krogh, A., J. Physiol. 52:391-408 (1919). Diffusion of gases through animal tissues.

216. Krogh, A., The Comparative Physiology of Respiratory Mechanisms (1941). Univ. of Pennsylvania Press.

217. KrüGer, F., Zool. Jahrb. 57:1-56 (1936). Anaerobiosis; Ascaris.

218. Kucera, W. G., Physiol. Zool. 7:449-458 (1935). Sex differences in $\mathrm{O}_{2}$ consumption; Drosophila.

219. Leach, W. J., Physiol. Zool. 19:365-374 (1946). Oxygen consumption of lamprey.

220. Ledebur, J. v., Biol. Rev. 12:217-244 (1937). Gas secretion in swim bladder.

221. Ledebur, J. v., Ergebu. Biol. 16:173-261 (1939). Oxygen tropism; earthworm.

222. LeE, M. O., J. Exper. Zool. 41:125-154 (1925). Respiratory control; Orthoptera.

223. Lee, M. O., Quart. Rev. Biol. 4:213-231 (1929). Respiration in insects.

224. Lehmann, C., Mueller, F., Munk, I., Senator, H., and Zuntz, N., Virchow's Arch. f. pathol. Anat. 11. Physiol. 131(suppl.):1-228 (1893). Respiration in man.

225. Leiner, M., Dic Physiologic der Fischatmung (1938). Akademische Verlagsgesellschaft. pp. 1-134. Lcipzig.

226. Liebsch, W., Zool. Jahrb., Abt., allg. Zool. 4. Physiol. d. Tiere 46:161-208 (1928). Respiration in snails.

227. I.indenan, V. F., Phy'siol. Zool. 5:560-565 (1932). Respiratory regulation; leech.

228. LiNDnotil, A., Ark. Zool. 30B:1-7 (1938). Breathing control; crayfish.

229. Linpnotil, A., Ztschr. vergl. Physiol. 28:485-532 (1941). Respiratory regulation in polychaetes.

230. Lipnann, F., Adv. in Enzymol. 1:99-162 (1941). Phosphate binding and energy sources.

231. Lipmann, F., Adv. in Enzymol. 6:231-268 (1946). Acetyl phosphate reactions. 
232. Loewy, Tabul. Biol. 3:461-514 (1926). Oxygen consumption data.

233. Longenecker, H. E., Biol. Symp. 5:99-115 (1941). Carbohydrate-fat conversion.

234. Loofbournow, G. N., and Gesell, R., Am. J. Physiol. 133:365 (1941). Respiratory patterns; birds.

235. Lumsden, T., J. Physiol. 58:259-266 (1924). Respiration of tortoise.

236. Lund, E. J., Biol. Bull. 41:203-220 (1921). Oxygen tension and consumption; Planaria.

237. Lynn, W. G., and Brand, T. v., Biol Bull. 88:112-125 (1945). Oxygen consumption; turtle embryos.

238. MaAs, J. A., Ztschr. vergl. Physiol. 26:605-610 (1939). Respiration of airbreathing snails.

239. MacArthur, J. W., and Baillie, W. H. T., Science 64:229-230 (1926). Sex difference in oxygen consumption; Daphnia.

240. Magnus-Levy, A., Pflïg. Arch. 55:1-126 (1894). Metabolic rate during fasting; man.

241. Maloeuf, N. S. R., J. Exper. Zool. 74:323-351 (1936). R.Q. values; aquatic arthropods.

242. Maloeuf, N. S. R., Ztschr. vergl. Physiol. 25:1-28, 29-42, 43-46 (1937). Critical oxygen tensions in invertebrates.

243. Marey, E. J., Le Vol des Oiseaux (1890). Paris, Masson.

244. Marlier, G., Ann. Soc. Roy. Zool. Belg. 69:163-185 (1938). Air-breathing teleosts.

245. Marvel, R. M., and Fisher, K. C., Biol. Bulll. 94:45-54 (1948). Cyanide sensitivity; squid embryos.

246. Matthews, S. A., and Smith, D. C., Physiol. Zool. 20:161-164 (1947). Metabolic response to thiourea; Fundulus.

247. McCoy, V. R., Physiol. Rev. 15:221-240 (1935). Physiology of helminth parasites.

248. McCutcheon, F. H., Ann. Ent. Soc. Amer. 33:35-55 (1940): Respiratory mechanism in grasshopper.

249. McCutcheon, F. H., Physiol. Zool. 16:255-269 (1943). Respiratory mechanism of turtles.

250. McGovran, E. R., Ann. Ent. Soc. Amer. 24:751-761 (1931). Tracheal ventilation; insects.

251. Melampy, R. M., and Willis, E. R., Physiol. Zool. 12:302-311 (1939). Respiration of larvae and pupae of honeybee.

252. Meyer, H., Zool. Iahrb. 55:349-398 (1935). Starfish respiration.

253. Modlinger, G., Studia Zool. (Budapest) 2:25-51 (1931). Buccal-pharyngeal respiration; turtles.

254. Moldawska Ja, E. A, Arch. Protist. 88:355-365 (1937). Adaptations to oxygen lack; Vorticella.

255. Montuori, A., Arch. Ital. Biol. 59:213-234 (1913). Oxygen consumption; marine animals.

256. Morgan, A. H., and Grierson, M. C., Physiol. Zool. 5:230-245 (1932). Mayfly respiration.

257. Morgan, A. H., and O'Neil, H. D., Physiol. Zool. 4:361-379 (1931). Caddis fly respiration.

258. Morrison, P. R., J. Cell. E Comp. Physiol. $31: 281-292$ (1948). Mammalian oxygen consumption.

259. Needham, J., Biochemistry and Morphogenesis (1942). Cambridge Univ. Press. pp. 562-605.

260. Needham, J., and Nowinski, W. W., Biochem. J. 31:1165-1184 (1937). Embryonic carbohydrate metabolism.

261. Nielsen, M., Skand. Arch. Physiol. 74:87-208 (1936). Carbon dioxide as respiratory stimulant.

262. Nims, L., Ann. Rev. Physiol. 8:99-116 (1946). Respiration.

263. Nims, L., in Howell's Textbook of Physiology (John F. Fulton, ed.) (1949). Philadelphia, Saunders. Chap. 39, 40.

264. Olthof, H. J., Ztschr. vergl. Physiol. $21: 534-544$ (1935). Respiratory regulation; fish. 
265. Olthof, H. J., Proefschrift (Dissertation) (1941). Groningen. Factors regulating respiration.

266. Oppenhelmer, K., and Stern, K. G., Biological Oxidation (1939). The Hague, Junk.

267. Orr, P. R., Physiol. Zool. 10:235-243 (1937). Sex differences in metabolism; Drosophila.

268. Otis, A. B., Rahn, H., and Fenn, W. O., Am. J. Physiol. 152:674-686 (1948). Factors influencing breath-holding time.

269. Pace, D. M., and Kimura, K. K., J. Cell. E Comp. Physiol. 24:173-185 (1944). Respiration in Paramecium.

270. Pace, D. M., and Lyman, E. D., Biol. Bull. 92:210-216 (1947). Oxygen consumption; Tetrahymena.

271. Palmhert, H. W., Zool. Jahrb., Abt. allg. Zool. u. Physiol. d. Tiere 53:212-260 (1933). Respiration and critical oxygen tension; hydroid.

272. Parhon, M., Ann. Sc. Natur. 9:1-58 (1909). Bee metabolism during flight.

273. Parker, W. N., Tr. Roy. Irish Acad. 30:109-230 (1892). Lungfish respiration.

274. Pearse, A. S., Carnegie Inst., Wash., Tortugas Papers, Publ. 391, pp. 205-223 (1929). Crustacean gills and habitat.

275. Pearse, A. S., The Migrations of Animals from Sea to Land (1936). Duke Univ. Press.

276. Pearson, O. P., Ecology 28:127-145 (1947). Mammalian respiration.

277. Pearson, O. P., Science 108:44-46 (1948). Metabolism of small mammals.

278. Pennak, R. W., and McColl, C. M., J. Cell E Comp. Physiol. 23:1-10 (1944). Damselly respiration.

279. Pennoit-de Cooman, E., Ann. Soc. Roy. Zool. Belg. 71:76-80 (1940). Cestode metabolism.

280. Peters, F., Ztschr. vergl. Physiol. 25:591-611 (1938). Respiratory control; crayfish.

281. Pfluger, E., Pfliig. Arch. 10:251-367 (1875). Anaerobiosis; frog.

282. Philips, F. S., Biol. Bull. 78:256-274 (1940). Tolerance to O. lack; fish eggs.

283. Pi-Suner, A., Physiol. Rev., 27:1-38 (1947). Peripheral control of respiration.

284. Pitts, R. F., Physiol. Rev. 26:609-630 (1946). The respiratory center.

285. Pitrs, R. F., in Howell's Textbook of Physiology (John F. Fulton, ed.) (1949). Philadelphia, Saunders. Chap. 41, 42.

286. Pittrs, R. F., Magoun, H. W., and Ranson, S. W., Am. J. Physiol. 126:673-688 (1939). The respiratory center of the cat.

287. Potter, G. E., J. Exper. Zool. 49:45-67 (1927). Swim bladder; lung fish.

288. Poulson, D. F., Ztschrr. vergl. Physiol. 22:466-472 (1935). Oxygen consumption of Drosophila larvac.

289. Powers, E. B., Hopkins, F. G., Hickman, T. A., and Shipe, L. M., Ecol. Monog. 2:385-473 (1932). Respiratory adaptations; fish.

290. Precht, H., Ztschr. vergl. Physiol. 26:696-739 (1939). Respiratory control; pulmonate snails.

291. Prosser, C. L., Physiol. Rev. 26:337-382 (1946). Respiratory rhythm and ganglionic potentials; insect.

292. Raeden, J. C., Biochem. Ztschr. 69:257-288 (1915). Respiration and urethane narcosis.

293. Raffy, A., and Pontier, P., C. R. Soc. Biol. 108:1062-1064 (1931). Butterfly metabolism during flight.

294. Rarn, H., and Otrs, A. B., Am. J. Physiol. 150:202-221 (1947). R.Q. and anoxia.

295. Rahn, H., and Otis, A. B., Fed. Proc. 7:96-97 (1948). Acclimatization to anoxia.

296. Ralin, H., Otis, A. B., and Fenn, W. O., Fed. Proc. 6:183 (1947). CO. and $\mathrm{O}_{2 .}$ as respiratory stimulants.

297. Redrield, A. C., Coolidge, T., and Hurd, A. L., J. Biol. Chem. 69:475-509 (1926). Oxygen transport by haemocyanin; Busycon.

298. Redfield, A. C., and Flonkin, M., Biol. Bull. 61:185-210 (1931). Gas exchange in hind gut; Urechis.

299. Regnault, V., and Reiset', J., Ann. Chim. e. Phys., 3rd Ser. 26:299-519 (1849). Oxygen consumption of vertebrates. 
300. Richardson, H. B., Physiol. Rev' 9:61-125 (1929). Respiratory quotient.

301. Robinson, D., Science 90:276-277 (1939). Oxygen stores during dive; scal.

302. Rogers, C. G., Textbook of Comparative Physiology (1938). New York, McGrawHill. Chap. 16, 17.

303. Rubner, Ml., Ztschr. Biol. 19:536-562 (1883). Metabolic rate during fasting.

304. Rubner, M., Biochem. Ztschir. 148:223-267 (1924). Respiration of fish.

305. SaAlfeld, E. v., Ztschr. vergl. Pliysiol. 26:246-252 (1938). Bat respiration.

306. Safford, V., J. Cell. \& Comp. Physiol. 16:165-173 (1940). Swim bladder and oxygen stores.

307. Sawaya, P., Bol. Fac. Filus. Cièn Letr. Univers., Sao Paulo, 11, Zool. pp. 255-286 (1946). Metabolism of lungfish.

308. SAYLe, M. H., Quart. Rev. Biol. 3:5+2-553 (1928). Metabolism of insects.

309. SChäfer, E. A., Textbook of Physiology (1898). London, Y. J. Pentland. Vol. 1, pp. 692-784, 868-936.

310. Scharnke, H., Ztschr. vergl. Pliysiol. 25:548-583 (1938). Bird respiration.

311. Scherbakov, A. P., Bull. Biol. Med. Exper. 3:268-270 (1937). Oxygen consumption; river lamprey.

312. Schierbeck, Arch. Anat. u. Physiol., Physiol. Abth. Suppl., pp. 116-124 (1893). Cutaneous respiration; man.

313. Schlatfer, A., Physiol. Zool. 12:381-392 (1939). Oxygen consumption; goldfish.

314. Schlieper, C., and Peters, J., Zool. Anz. 120:95-99 (1937). Effect of CO. and $\mathrm{O}_{2} ;$ crayfish.

315. Sснмidt, C. F., Ann. Rev. Plysiol. 7:231-274 (1945). Respiration.

316. Schmidt, C. F., and Comnoe, J. H., Physiol. Rev. 20:115-157 (1940). Stimulating effect of $\mathrm{CO}_{2}$.

317. Schneider, E. C., Am. J. Physiol. 94:464-470 (1930). Breath holding.

318. Schoenheimer, R., and Rittenberg, D., J. Biol. Chem. 114:381-396 (1936). Fat synthesis.

319. Schötтle, E., Ztschr. wiss. Zool. 140:1-14 (1932). Air breathing; "jumping fish."

320. Scholander, P. F., Hrvalraadets Skrifter No. 22 (1940). Det Norske VidenskapsAkademi i. Oslo, Oslo. Respiration in diving birds and mammals.

321. Scholander, P. F., and Edwards, G. A., Rev. Scient. Instr. 13:292-295 (1942). Microrespiration apparatus.

322. Scholander, P. F., and lrving, L., J. Cell. E Comp. Physiol. 17:169-192 (194I). Respiration and diving; manatee.

323. Scholander, P. F., lrving, L., and Grinnell, S. W., J. Biol. Chem. 142:431-440 (1942). Seal muscles during dive.

324. Scholander, P. F., Irving, L., and Grinnell, S. W., J. Cell. E Comp. Phy'siol. 21:53-63 (1943). Armadillo respiration.

325. Segaar, J., Ztschr. vergl. Physiol. 21:492-515 (1934). Respiratory movement; crayfish.

326. Simonsen, D. H., and Wagtendonk, W. J. van, Fed. Proc. 8:250-251 (1949). Oxygen consumption and genetic strains; Paramecium.

327. Slater, W. K., Biochem. J. 21:198 (1927). Anaerobic metabolism; cockroach.

328. Slater, IV. K., Biol. Rev. 3:303-328 (1928). Anaerobiosis.

329. Slater, W. K., and Davis, J. G., Biochem. J. 22:331-338 (1928). Anaerobiosis; earthworm.

330. Smith, H. W., Kamongo (1932). New York, The Viking Press. Lungfish biology.

331. Sмmith, H. W., J. Cell. \& Comp. Physiol. 6:43-67 (1935). Fasting metabolism of lungfish.

332. Sмyтн, D. H., J. Physiol. 95:305-327 (1939). Respiratory control of frog.

333. Sмутн, J. D., Biol. Rev. 23:214-238 (1947). Glycogen content; tapeworm.

334. Soskin, S., and Levine, R., Carbohydrate Metabolism (1946). Univ. of Chicago Press.

335. Stahn, I., Zool. Jahrb., Abt. allg. Zool. 46:1-86 (1928). Insect breathing control.

336. TANG, P.-S., Biol. Bull. 60:242-244 (1931). Oxygen tension and consuinption; unfertilized Arbacia eggs.

337. TANG, P.-S., Biol. Bull. 61:468-471 (1931). Oxygen consumption; Asterias eggs.

338. TANG, P.S., Quart. Rev. Biol. 8:260-274 (1933). Oxygen consumption and oxygen tension. 
339. Tang, P.-S., and Gerard, R. W., J. Cell. E Comp. Physiol. 1:503-513 (1932). Oxygen tension and consumption; fertilized Arbacia eggs.

340. Tangl, F., Biochem. Ztschr. 34:1-40 (1911). Effect of curare on respiration.

341. Taylor, I. R., and Steineach, H. B., Physiol. Zool. 4:604-619 (1931). Pupal metabolism; bee moth.

342. Thill, H., Ztschr. wiss. Zool. 150:51-96 (1937). Critical oxygen tension; Aurelia.

343. Thonpe, W. H., and Crisp, D. J., J. Exper. Biol. 24:227-269, 270-303, 310-328, (1947); 26:219-260 (1949). Oxygen stores in diving insects.

344. Trgerstedt, R., Handbuch der physiologischen Methodik (1911). Leipzig, Hirzel. Vol. 1, pt. 3, pp. 71-149. Respiratory equipinent.

345. Toвias, J. M., Physiol. Rev. 23:51-75 (1943). Microrespiration techniques.

346. Tonner, F., Ztschr. wiss. Zool. 147:433-454 (1936). Respiration in hind gut; dragontly larva.

347. Toryu, Y., Sci. Rep. Tokohu Inp. Univ., Ser. IV, 10:361-376 (1935). Anaerobic glycolysis; Ascaris.

348. Umbreit, W. W., Burris, R. H., and Stauffer, J. F., Manometric Techniques and Related Methods for the Study of Tissue Metabolism (1945). Minneapolis, Burgess.

349. Unwin, E. E., Rep. Brit. A. Adv. Sci. 68:736-737 (1908). Respiration of land isopods.

350. Varley, S. C., Proc. Ent. Soc. London 12:55-60 (1937). Plant O.2 sources for insect larvae.

351. Vennesland, B., in Recent Advances in Biological and Medical Physics (Lawrence and Hamilton, eds.) (1948). New York, Academic Press. Vol. 1, pp. 45-1 16. $\mathrm{CO}$ utilization in carbohydrate metabolism.

352. Vernon, H. M., J. Physiol. 19:18-70 (1899). Respiratory exchange; marine invertebrates.

353. Vort, E., Ztschr. Biol. 41:113-154 (1901). Starvation and metabolic rate.

354. Vos, H. J., Proefschrift (Dissertation) (1936). Groningen. Respiratory control; amphibians and reptiles.

355. Walshe, B. M., J. Exper. Biol. 24:329-342 (1947). Oxygen debt; Chironomus.

356. Warburg, O., Ztschr. physiol. Chemie. 57:1-16 (1908). Oxygen consumption; Arbacia eggs.

357. Warburg, O., Über den Stoffwechsel der Tumoren (1926). Berlin, Springer.

358. Weinland, E., Ztschir. Biol. 47:186-231 (1906). Metabolic rates of insect pupae.

359. Weinland, E., in Oppenheimer's Handbuch der Biochemie (1910). Jena, Fischer. Vol. 4, sect. 2, pp. 446-528. CO.2 elimination during anaerobiosis.

360. Wells, N. A., Physiol. Zool. 8:196-227 (1935). Temperature effects on respiration; killifish.

361. Weymouth, F. W., Crismon, V. E., Hall, H., Belding, S., and Field, J., Physiol Zool. 17:50-70 (1944). Respiration in relation to body weight; Crustacea.

362. Whitaker, D. M., J. Gen. Physiol. 15:183-190 (1931). Oxygen consumption and fertilization; Cumingia.

363. Whitaker, D. M., J. Gen. Physiol. 16:475-494 (1933). Oxygen consumption; Chaetopterus and Arbacia eggs.

364. Wiebe, A. H., McGavock, A. M., Fuller, A. C., and Marcus, H. C., Physiol. Zool. 7:435-448 (1934). Low oxygen tolerance and $\mathrm{pH}$; fish.

365. Wiggleswortil, V. B., Proc. Roy. Soc. Lond., B. 106:229-250 (1930). Tracheal respiration.

366. Wigglesworti, V. B., Biol. Rev. 6:181-220 (1931). Respiration of insects.

367. Wigglesworti, V. B., Proc. Roy. Soc. Lond., B. 118:397-419 (1935). Regulation of respiration; flea.

368. Wiggleswontri, V. B., Principles of Insect Physiology (1939). London, Methuen. Chap. 9, 13.

369. Willem, V., Mem. Acad. Roy. Belg. (Cl. Sci.), Ser. 2, 10:1-194 (1931). Buccalpharyngeal respiration; fish.

370. Willem, V., and Boelaert, R., Bull. Acad. Roy. Belg. (Cl. Sci.), Ser. 5, 23:942-959 (1937). Aerial respiration; fish.

371. Willmer, E. N., J. Exper. Biol. 11:283-306 (1934). Oxygen secretion; tropical fish. 
372. Wingfield, C. A., I. Exper. Biol. 16:363.372 (1939). Caudal gill respiration; maylly nymphs.

373. Winterstein, H., Pflig. Arch. 125:73-98 (1908). Fish respiration.

374. Winterstein, H., Pflig. Arch. 138:167-184 (1911). Respiratory regulation and $\mathrm{pH}$.

375. Winterstein, H., Handbuch der vergleichenden Physiologie (1921). Jena, G. Fischer. Vol. 1, part 2, pp. I-1052.

376. Wintenstein, H., Natumissenschaften 11:625-630 (1923). Hydrogen ion con trol in respiration.

377. Winterstein, H., Ztschr. vergl. Plysiol 2:315-328 (1925). Factors controlling respiration; cephalopods.

378. WITt, F., Ztschr. vergl. Ply'siol. 18:115-124 (1932). Respiratory movements; pulmonate snails.

379. Wolsky, A., Ungarisches biol. forschunginstitutet (Arch.) 7:116-124 (1934). $\mathrm{O}_{2}$ consumption in relation to habitat; crayfish.

380. Wolsky, A., J. Exper. Biol. 15:225-234 (1938). Os consumption of Drosophila pupae.

381. Wolsky, A., and Holmes, B. E., Ungarisches biol. forschunginstitutet (Arch.) 6:123-126 (1933). Crayfish respiration.

382. Wyss, O. A. M., Ann. Rev. Physiol. 11:469-492 (1949). Respiration.

383. Ysseling, M. A., Ztschr. vergl. Physiol. 13:1-60 (1930). Breathing movements; pulmonate snails.

384. Zeuthen, E., C. R. Trav. Lab. Carlsberg, Sér. Chim. 26:17-161 (1947). Relation of body size to metabolic rate.

385. Zimmer, K., Zoologica 33:1-69 (1935). Breathing movements during flight; birds.

386. Zoond, A., J. Exper. Biol. 8:258-262, 263-266 (1931). Respiration in sabellid worms and scorpions.

387. Zoond, A., and Chanles, E, I. Exper. Biol. 8:250-257 (1931). Respiratory mechanism; crab.

388. Zuntz, N., Pfiig. Arch. 12:522-526 (1876). Effect of curare on respiration. 


\section{Respiratory Functions of Body Fluids}

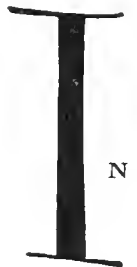

N MOST ANIMALS which have a circulatory system (insects excepted) there is a pigment in the blood or body fluid which is capable of combining with oxygen. In higher mammals, for example, oxygen is dissolved in the plasma to the extent of 0.3 volumes per cent, and in whole blood to the extent of 0.24 volumes per cent. Actually, arterial blood contains 19 volumes per cent of oxygen; 98 per cent of the oxygen in the blood is combined with hemoglobin. In this chapter we shall consider the role of blood pigments in oxygen transport under usual physiological conditions and at times of hypoxic stress. All blood pigments contain some metal in an organic complex. Good summaries of the literature are given by Redfield, ${ }^{120}, 121$ Barcroft, ${ }^{7}$ and Florkin. ${ }^{50,} 52,53$

\section{DistRibution OF PIGMENTS}

Hemoglobins. The most common of blood pigments are iron-containing red pigments, the hemoglobins; their protein components differ considerably, and hemoglobins have arisen in many unrelated animal groups. The molecules of invertebrate hemoglobins are much larger than those of vertebrate hemoglobins, and for this reason invertebrate hemoglobins have been called erythrocruorins. ${ }^{1+7}$ However, the prosthetic groups in all hemoglobins are similar (porphyrins), and the proteins differ even from species to species of vertebrates; hence it seems preferable to apply the name hemoglobin to all of the red, iron-containing porphyrin-proteins.

The distribution of blood hemoglobin and of other pigments is given in Table 48. Hemoglobin is found in all vertebrates, although it is absent from some transparent young fish (e.g., eels), which have colorless erythrocytes. ${ }^{102}$ Hemoglobin is lacking in Amphioxus; it has been reported from one prochordate-Discoglossus. ${ }^{145}$

Among the annelids, hemoglobin (erythrocruorin ${ }^{130}$ ) is found in the plasma of many oligochaetes-Lumbricus and others, in some leeches-as Hirudo, and in many polychaetes-such as Nereis and Arenicola (Tables 48 and 49). In some other polychaetes, Glycera, for example, hemoglobin is contained in nucleated corpuscles. In general, those annelids whose hemoglobin is in solution in the plasma have a closed circulation, whereas those with the pigment in cells have a poorly developed vascular system and the pigmented cells are in coclomic spaces only. ${ }^{1: 2}$ In two genera, Terebella and Travisia, hemoglobin is found both in solution in the blood plasma and in cells in the coelomic fluid.

Hemoglobin occurs in a few molluses, in the plasma of the snail Planorbis, and in corpuscles of the clam Arca (Table 48). Hemoglobin appears sporadi- 
TABLE 48. DISTRIBUTION OF PIGMENTS IN BOIDY FLUIDS

\begin{tabular}{|c|c|c|}
\hline Animal & Examples & Location \\
\hline \multirow{2}{*}{$\begin{array}{l}\text { Chordata } \\
\text { All vertebrata }\end{array}$} & \multicolumn{2}{|c|}{ Hemoglobins (Fe) } \\
\hline & (cxcept leptocephalan & corpuscles \\
\hline Prochordata & $\begin{array}{l}\text { (cel) larvae) })^{102} \\
\text { (absent from Amphioxus) } \\
\text { Discoglossus }{ }^{112,2,11 .}\end{array}$ & $"$ \\
\hline \multicolumn{3}{|l|}{ Echinodermata } \\
\hline \multirow[t]{5}{*}{ Holothuria } & 1 hyone $e^{1+1}$ & $"$ \\
\hline & $\begin{array}{l}\text { Cucumaria frauenfeldi }{ }^{\prime 3} \\
\text { miniata }{ }^{2 . \bar{n}}\end{array}$ & $"$ \\
\hline & Molpadia roretzii"- & $"$ \\
\hline & Anadara" & $"$ \\
\hline & Caudina"t & $"$ \\
\hline \multicolumn{3}{|l|}{ Annelida } \\
\hline Oligochaeta & Lumbricus and many others ${ }^{102}$ & plasma \\
\hline Hirudinea & $\begin{array}{l}\text { Hirudo, Analastoma, } \\
\text { Nephelis and others }{ }^{102}\end{array}$ & \\
\hline Polychaeta & Many-see Table 50 & \\
\hline Echiuroidea & Urechis, Thalassema ${ }^{203,123}$ & corpuscles \\
\hline Phoronidea & Phoronis ${ }^{1+42}$ & corpuscles \\
\hline \multicolumn{3}{|l|}{ Arthropoda } \\
\hline \multirow{3}{*}{ Crustacea } & Daphnia ${ }^{1112}$ & plasma \\
\hline & Cheiroccphalus $^{1112}$ & plasma \\
\hline & Branchipus ${ }^{i t}$ & plasma \\
\hline \multirow[t]{2}{*}{ Insecta } & Chironomus ${ }^{11 \cdot 2:}$ & plasma \\
\hline & Gastrophilus (horsefly) larva" היו & tracheal cells \\
\hline \multicolumn{3}{|l|}{ Mollusca } \\
\hline \multirow[t]{2}{*}{ Pelecypoda } & Solen ${ }^{\prime \prime \prime}$ & \multirow[t]{2}{*}{ corpuscles } \\
\hline & Arca, Pectunculus ${ }^{21 i}$ & \\
\hline \multirow{2}{*}{$\begin{array}{l}\text { Gastropoda } \\
\text { Nemertinea }\end{array}$} & $\begin{array}{l}\text { Planorbis } \\
\text { Polia }{ }^{1, \pm=}\end{array}$ & $\begin{array}{l}\text { plasma } \\
\text { plasma }\end{array}$ \\
\hline & $\begin{array}{l}\text { Drepanophorus } \\
\text { (Hubrecht, in Redfield }{ }^{120} \text { ) }\end{array}$ & \\
\hline \multirow[t]{4}{*}{ Platyhelminthes } & Derostoma & \\
\hline & (Moseley, in Redfield ${ }^{\prime \prime \prime}$ ) & \\
\hline & $\begin{array}{l}\text { Sy'ndesmis } \\
\text { (Prenant, in Redfield }{ }^{120} \text { ) }\end{array}$ & \\
\hline & Telorchis and others ${ }^{15 \pi}$ & \\
\hline \multirow[t]{4}{*}{ Nemathelminthes } & Ascaris, Nippostrongylus"- & perienteric \\
\hline & Camallanus ${ }^{15 \overline{5}}$ & Huid and \\
\hline & Eustrongylides (larvae) & body wall \\
\hline & \multicolumn{2}{|c|}{ Chlorocruorin $(\mathrm{Fe})$} \\
\hline \multirow{4}{*}{$\begin{array}{l}\text { Annelida } \\
\text { Polychaeta }\end{array}$} & Sabellida & plasma \\
\hline & Serpulidae $e^{j 6}$ & plasma \\
\hline & Chlorhaemidae & plasina \\
\hline & \multicolumn{2}{|c|}{ Hemerythrin (Fe) } \\
\hline \multirow[t]{3}{*}{ Sipunculoidea } & Sipunculus & corpuscles \\
\hline & Phascolosoma ${ }^{10:}$ & corpuscles \\
\hline & Phymosoma ${ }^{109}$ & corpuscles \\
\hline \multirow{3}{*}{$\begin{array}{l}\text { Annelida } \\
\text { Polychaeta } \\
\text { Brachiopoda }\end{array}$} & & \\
\hline & Magelona ${ }^{10 \%}$ & corpuscles \\
\hline & Lingula $^{\cdot 3}$ & corpuscles \\
\hline
\end{tabular}


TABLE 48 (continued). DISTRIBUTION OF PIGMENTS IN BODY FLUIDS

\begin{tabular}{|c|c|c|}
\hline Animal & Examples & Location \\
\hline \multirow{2}{*}{$\begin{array}{l}\text { Arthropoda } \\
\text { Arachnoidea } \\
\text { Arachnida } \\
\text { Crustacea } \\
\text { Mollusca } \\
\text { Cephalopoda }\end{array}$} & \multicolumn{2}{|c|}{ Hemocyanin $(\mathrm{Cu})$} \\
\hline & $\begin{array}{l}\text { Limulus }{ }^{31,}, 1 \text { s } \\
\text { Scorpionidae } \\
\text { many Malacostraca }\end{array}$ & $\begin{array}{c}\text { plasma } \\
"\end{array}$ \\
\hline \multirow[t]{2}{*}{ Gastropoda } & $\begin{array}{l}\text { Loligo } \\
\text { Sepia } \\
\text { Octopus } \\
\text { Eledone } \\
\text { Busycon } \\
\text { Buccinium and others }\end{array}$ & $\begin{array}{c}\text { plasma } \\
\text { " } \\
" \\
" \\
"\end{array}$ \\
\hline & \multicolumn{2}{|c|}{ Pinnaglobin $(M \mathbf{n})$} \\
\hline \multirow[t]{2}{*}{$\begin{array}{l}\text { Mollusca } \\
\text { Pelecypoda }\end{array}$} & Pinna squamosa ${ }^{6 \pi}$ & \\
\hline & \multicolumn{2}{|c|}{ Echinochrome (Fe) } \\
\hline \multirow[t]{2}{*}{ Echinodermata } & Echinus and others ${ }^{22}$ & \\
\hline & \multicolumn{2}{|c|}{ Vanadium chromogens } \\
\hline Tunicata & $\begin{array}{l}\text { Rhodosomatidae }{ }^{\mathrm{v}_{1}, 156} \\
\text { Ascidia, Phallusia } \\
\text { Perophoridae } \\
\text { Diazonidae } \\
\text { Cionidae }\end{array}$ & $\begin{array}{c}\text { vanadocytes } \\
\text { ", } \\
\text { plasma } \\
\text { plasma }\end{array}$ \\
\hline
\end{tabular}

cally among the arthropods, e.g., Daphnia, Cheirocephalus, and Branchipus, and in the larvae of certain chironomids. Among the echinoderms hemoglobin is also found in nucleated corpuscles in the sea cucumbers, such as certain species of Thyone and Cucumaria. Cucumaria elongata, a mud-dweller, has hemoglobin, whereas Cucumaria saxicola, which lives among rocks, with greater oxygen availability, lacks hemoglobin. ${ }^{117}$ In a few nemerteans and parasitic flatworms and in several roundworms hemoglobin has been detected. The properties of the hemoglobin in some of these parasites are distinctly different from those of the hemoglobin in the host.

In addition to the blood hemogtobins, there are also many tissue hemoglobins. Muscle hemoglobin or myoglobin is abundant in the "red" muscle of mammals and birds, in the heart muscle of all classes of vertebrates, and in scattered skeletal muscles of cold-blooded vertebrates, as in the dorsal fin muscle of Hippocampus. ${ }^{112}$ In Urechis hemoglobin is present not only in coelomic cells but also in muscke and nerve cells. "Myoglobin occurs in the radular and pharyngeal muscles of certain snails, especially Busycon, ${ }^{\circ}$ in the ganglion cord of Aphrodite, ${ }^{102}$ and in ganglia of certain nemerteans. ${ }^{1.0}$ A similar pigment is found in the "tracheal body" of larvae of the Aly Gastrophilus, which is parasitic in the stomach of horses, and also in the tracheal cells of some other insects. ${ }^{17}$ I lemoglobin has even been reported from Paramecium. ${ }^{140}$ Hemo- 
globin is found with bacteria in the root nodules of certain leguminous plants, where it appears to function in nitrogen tixation."*i

Chlorocruorin. In three families of polvchaete worms, particularly the Sabellidae and Serpulidae, there is a pigment, called chlorocruorin, which contains iron in a different porphyrin from hemoglobin and which is green in color in dilute solution.

Hemerythrin. A third iron-containing pigment, hemerythrin, occurs in the polychaete worm Magelona, in the sipunculoid worms Sipunculus and Phascolosoma, and also in the brachiopod Lingula. Hemerythrin is found in corpuscles and is brown in color. The corpuscles of Magelona are nonnucleated. Hemerythrin does not contain a porphyrin, and hence is very different in chemical properties from hemoglobin and chlorocruorin.

Hemocyanin. The pigment which is next in importance to hemoglobin, judged by its distribution, is hemocyanin. This is a copper-containing protein which occurs in cephalopod molluscs, some gastropods, higher crustaceans, Limulus, and a few arachnids. The concentration of hemocyanin roughly parallels that of copper in the blood. Hemocyanin is a protein without any porphyrin group.

Miscellaneous Pigments. Several other blood pigments should be mentioned, even though their respiratory function has not been demonstrated. In the body fluid of a mussel, Pinna, there is a brown pigment, called pinnaglobin, which contains manganese. ${ }^{67}$ The body fluids of some ascidians contain a pigment which is a vanadium chromogen..$^{s 0,156}$ In certain cells, the eleocytes of the coelomic Huid of sea urchins, there is a red pigment, echinochrome. This contains little iron, has a low oxidation potential, and is probably enzymic in function.":- Crescitelli"s identified by its absorption bands a pigment which he called molpadin in the holothurian Molpadia.

\section{SIZE AND COMPOSITION OF ERYTHROCYTES}

The hemoglobin of vertebrates is contained in corpuscles called erythrocytes. In higher mammals the erythrocytes constitute 40 to 50 per cent of the total blood. Mammalian erythrocytes are circular (except in the Camellidae), nonnucleated, and biconcave. Erythrocytes of most other vertebrates are elliptical, nucleated, and double convex. The number of erythrocytes per cu. min. varies with the size of the cells. Table 50 gives the size and red cell count for a number of representative species. In man, the count is higher in infants $\left(6,000,000 / \mathrm{mm}^{3}\right.$ ) than in adults (males 5,000,000 and females $4,500,000 /$ mm. ${ }^{3}$ ). The number of red cells is higher at high altitudes than ai sea level. Data collected by Talbot in the Andes showed that the normal red cell count of most persons living at altitudes greater than $10,000 \mathrm{ft}$. was over $5,500,000$ per cubic millimeter, with great individual variability.:2 Hemoglobin content of the blood parallels the increase in red cell count at high altitudes.

Table 50 shows that in general the red cells of mammals are smaller and more numerous than the red cells of lower vertebrates. The largest red cells occur in the Amphibia, particularly in such animals as Amphimma. The oxygen-combining capacity of the blood is proportional to the total amount of hemoglobin, rather than to the red cell count. Table 50 gives the hemoglobin content of the blood of a number of animals. The small number of red cells in lower vertebrates is partly compensated by larger cell size. 
Mammalian erythrocytes are approximately 32 per cent hemoglobin, 60-65 per cent water, and about 8 per cent stroma, consisting of lecithin, cholesterol, inorganic salts, and protein. In fetal life the red blood cells are manufactured in liver and spleen; in adults they are made largely in the bone marrow. Red blood cells are subject to severe wear and tear and survive only a few weeks in the open circulation. Measurements made with radioactive hemoglobin indicate the mean life of the red cell in the dog to be about 100 days; ${ }^{77}$ in the chicken the lifetime of the red cell is about 32 days, by measurements made with $\mathrm{P}: 3:$ in the nucleic acid. ${ }^{116}$

\section{TABLE 49. DISTRIBUTION OF RESPIRATORY PIGMENTS IN POLYCHAETE, SIPUNCULOID AND ECHIUROID WORMS}

(Modified and extended from ${ }^{132}$ )

Closed circulatory system

Coelomic Huid pigmented

Hemoglobin in cells of coelomic fluid and in blood plasma

Terebella lapidaria Travisia forbesii

Coelomic Huid not pigmented

Hemoglobin in blood plasma

$\begin{array}{lll}\text { Nereidae } & \text { Arenicola marina } & \text { Amphistenidae } \\ \text { Amphitrite rubra } & \text { Clymene lumbricoides Cirratulidae } \\ \text { Pista cristata } & \text { Eunicidae } & \\ \text { uorin in blood plasma } & \end{array}$

Chlorocruorin in blood plasma

Sabellidae Serpulidae Chlorhaemidae

Hemerythrin in non-nucleated blood corpuscles

Magelona papillicornis

No pigment in either blood or coelomic fluid

$\begin{array}{ll}\text { Syllidae } & \text { Aphroditidae } \\ \text { Phyllodice } & \text { Chaetopteridae }\end{array}$

Lepidonotus squamatus

Circulatory system lacking or degenerate, blood not pigmented

Hemoglobin in corpuscles in coelomic fluid

Capitellidae Polycirrus hematodes Urechis caupo

Glyceridae Polycirrus aurantiacus Thalassema neptuni

Hemerythrin in corpuscles in coelomic fluid

Sipunculus nudus Phascolosoma.

No pigment in coelomic fluid

Polycirrus tenuisetis Polycirrus arenivorus

\section{CHEMISTRY OF PIGMENTS}

Hemoglobin: Chenical Composition. The molecules of hemoglobin are large, and consist of a protein, globin, and pigment, hemin. Hemin is the hydrochloride of heme, a metaloporphyrin. Porphyrins are composed of four pyrrol rings, as shown in Figure 66. Other metals than iron can combine with porphyrin; for example, the pigment in the feathers of the South African turaco is a combination of porphyrin and copper. Heme can combine with various nitrogenous compounds to give hemochromogens. A hemochromogen can be produced by the action of acid or alkali upon hemoglobin. The most widespread of all hemochromogens is cytochrome, a respiratory pigment which occurs in three forms, a, b, and $c$, and is found in most, if not all, aerobic cells.". ()ther hemochromogens are such pigments as actiniohematin, which occurs in scia ancmones, and helicorubin, which is found in the muscles and bile of certain snails.5. $: 0$ The heme in all these hemochromogens is similar; 
TABLE 50. SIZE OF RED BLOOD CORPUISCLES IN MICRA DIAMETER, COUNT IN MILLION CELLS PER CUBIC MILLIMETER, AND

HEMOGLOBIN CONTENT IN GRAMS PER 100 CUBIC CENTIMETERS OF BLOOD

(Except where otherwise indicated)

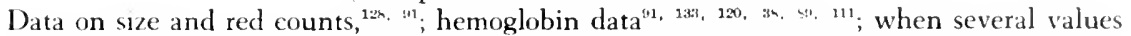
or ranges of values were given, these have been averaged. Individual variability within a species is great, and the values given in this table are reasonable means.

\begin{tabular}{|c|c|c|c|}
\hline Animal & $\begin{array}{c}\text { Red Cell } \\
\text { Diameter in } \mu\end{array}$ & $\begin{array}{l}\text { Red Cell } \\
\text { Count in } \\
\text { Million/mm." }\end{array}$ & $\begin{array}{l}\text { Hemoglobin } \\
\text { gm./100 cc. Blood }\end{array}$ \\
\hline $\begin{array}{l}\text { Mammals } \\
\text { lemur } \\
\text { macaque } \\
\text { man (male) } \\
\text { man (female) } \\
\text { dog } \\
\text { horse } \\
\text { bull } \\
\text { sheep } \\
\text { pig } \\
\text { goat } \\
\text { rat } \\
\text { mouse } \\
\text { guinea pig } \\
\text { woodchuck } \\
\text { rabbit } \\
\text { opossum } \\
\text { camel (dromedarius) } \\
\text { llama (glama) } \\
\text { llama (pocas) } \\
\text { seal }\end{array}$ & $\begin{array}{l}5.7 \\
7.5 \\
7.4 \\
7.0 \\
6.5 \\
8 \times 4.6 \\
7.8 \times+.3 \\
8.0 \times 4.3\end{array}$ & $\begin{array}{r}5.0 \\
5.0 \\
4.5 \\
4.5 \\
5.8 \\
5.0 \\
11.5 \\
6.3 \\
\\
9.0 \\
9.2 \\
5.2 \\
7.3 \\
6.0 \\
5.9 \\
10.8 \\
11.3 \\
19.4 \\
6.01\end{array}$ & $\begin{array}{l}11.2 \\
16 \\
14 \\
14.8 \\
11.0 \\
13.2 \\
13.9 \\
12.8 \\
13.8 \\
14.6 \\
14.1 \\
13.9 \\
13.5 \\
10.0 \\
14.4 \\
13.4 \\
15.9 \\
16.5\end{array}$ \\
\hline $\begin{array}{l}\text { Birds } \\
\text { fowl } \\
\text { mallard duck } \\
\text { pigeon } \\
\text { sparrow } \\
\text { ostrich } \\
\text { goose } \\
\text { canary }\end{array}$ & $\begin{array}{l}13 \\
11.2 \times 6.7 \\
13 \times 6.5 \\
12.5 \times 6.25 \\
18 \times 8\end{array}$ & $\begin{array}{l}3 \\
3 \\
3.6 \\
3.7+ \\
\\
2.71 \\
3.5\end{array}$ & $\begin{array}{l}11.3 \\
15.6 \\
14.85 \\
13.01\end{array}$ \\
\hline $\begin{array}{l}\text { Reptiles } \\
\text { Lacerta agilis } \\
\text { Lacerta muralis } \\
\text { Anguis fragilis } \\
\text { Emys orbicularis } \\
\text { Phrynosoma } \\
\text { alligator } \\
\text { Bothrops (snake) }\end{array}$ & $\begin{array}{l}14.7 \times 6 \\
13.2 \times 6.2 \\
17.8 \times 9.3 \\
12 \times 5 \\
14 \times 25\end{array}$ & $\begin{array}{l}0.94 \\
1.447 \\
1.6 \\
0.503 \\
\\
.85\end{array}$ & $\begin{array}{r}5.7 \\
9.0 \\
11.3 \\
6.6\end{array}$ \\
\hline $\begin{array}{l}\text { Amphibians } \\
\text { Rana esculenta } \\
\text { " temporaria } \\
\text { Hyla arborea } \\
\text { Bufo vulgaris } \\
\text { Necturus maculosus } \\
\text { Triton vulgaris } \\
\text { Triton alpestris } \\
\text { Triturus iridescens }\end{array}$ & $\begin{array}{l}22.8 \times 15.7 \\
19.7 \times 13.3 \\
18.5 \times 11.7 \\
20.5 \times 13.3 \\
32 \times 25 \\
36 \times 13 \\
30 \times 19.6\end{array}$ & $\begin{array}{l}0.53 \\
0.408 \\
0.67 \\
0.38 \\
0.056 \\
0.135 \\
0.11 \\
0.103\end{array}$ & $\begin{array}{r}9.5 \\
10.2 \\
10.2 \\
9.8 \\
6.0\end{array}$ \\
\hline
\end{tabular}


TABLE 50 (continued).

SIZE OF RED BLOOD CORPUSCLES IN MICRA DIAMETER, COUNT IN

MILLION CELLS PER CUBIC MILLIMETER, AND HEMOGLOBIN CONTENT IN GRAMS PER 100 CUBIC CENTIMETERS

\section{OF BLOOD}

(Except where otherwise indicated)

Data on size and red counts, ${ }^{120},{ }^{411}$; hemoglobin data ${ }^{91,} 133,120,30,41,{ }^{111}$; when several values or ranges of values were given, these have been averaged. Individual variability within a species is great, and the values given in this table are reasonable means.

\begin{tabular}{|c|c|c|c|}
\hline Animal & $\begin{array}{c}\text { Red Cell } \\
\text { Diameter in } \mu\end{array}$ & $\begin{array}{c}\text { Red Cell } \\
\text { Count in } \\
\text { Million } / \mathrm{mm} .^{3}\end{array}$ & $\begin{array}{l}\text { Hemoglobin } \\
\text { gm. } / 100 \mathrm{cc} \text {. Blood }\end{array}$ \\
\hline Salamandra maculata & $35.5 \times 20.5$ & 0.057 & 8.0 \\
\hline Salamandra atra & $41.7 \times 19.8$ & 0.054 & 6.8 \\
\hline Proteus anguineus & $60 \times 35$ & 0.036 & \\
\hline Amphiuma & $67 \times 41$ & & \\
\hline Plethodon & $32 \times 13$ & & \\
\hline \multicolumn{4}{|l|}{ Fish } \\
\hline $\begin{array}{l}\text { yellow perch } \\
\text { eel }\end{array}$ & $22.9 \times 13.9$ & 1.10 & 8.7 \\
\hline sole & $12 \times \hat{9}$ & 2.0 & \\
\hline dace & & 2.1 & 17.4 \\
\hline trout & $16.7 \times 10.3$ & & \\
\hline carp & & $\begin{array}{l}1.6 \\
1.98\end{array}$ & $\begin{array}{l}12.2 \\
10.14 \\
26.02^{*}\end{array}$ \\
\hline channel catfish & & 2.4 & 17.6 \\
\hline toadfish & & .585 & $19.5 \dagger$ \\
\hline puffer & & 2.284 & $17.5 \dagger$ \\
\hline scup & & 2.685 & $32.6 \dagger$ \\
\hline sea robin & & 2.536 & $24.0 \dagger$ \\
\hline mackerel & & 3.00 & $37.1 \dagger$ \\
\hline goosefish & & .867 & $15.45 \dagger$ \\
\hline Amia calva & $19 \times 10$ & 1.7 & 9.3 \\
\hline Protopterus ethiopicus & $40 \times 30$ & & \\
\hline torpedo & $27 \times 20$ & 0.14 & \\
\hline Raja & & .2 & \\
\hline Myxine glutinosa & $25 \times 20$ & & \\
\hline Invertebrates & & & \\
\hline Urechis caupo & 25 & & $\begin{array}{l}2.5-4 \\
7.5-12.7^{*}\end{array}$ \\
\hline Sipunculus nudus & $16 \times 12$ & & \\
\hline Glycera convoluta & 50 & & \\
\hline Anadara & & & $1.06-1.64$ \\
\hline
\end{tabular}

the pigments differ, however, in the nitrogenous base. Since heme is so widespread in occurrence, it is not remarkable that it has been utilized in blood pigments in a wide variety of unrelated animals.

In hemoglobin the iron is found to be in the reduced (ferrous) state. The iron content of human hemoglobin is 0.336 per cent (that of horse hemoglobin is 0.38 per cent $^{130}$ ), and the heme content is 4 per cent. Each unit of hemoglobin containing one atom of iron has a molecular weight of 16,400. Hemoglobin in mammalian blood, however, consists of four units, and hence has

${ }^{*}$ Grams per 100 cc. cells.

†Hematocrit index. 
four atons of iron and a molecular weight of about 67,000. Muscle hemoglobin contains two heme units. In all of the vertebrates, with the exception of the cyclostomes (Myxine), the blood hemoglobin contains four atoms of iron; in Myxine there are two. The invertebrate hemoglobins are composed of two or many, but never four, units.

The function of hemoglobin as an oxigen carrier depends on the loose combination of the ferrous iron with oxrgen (oxygenation). The ferrous iron can be oxidized by strong oxidants, resulting in methemoglobin. The resulting ferric iron is incapable of combining further with oxygen. Oxygen combines in the proportion of one molecule per atom of iron.

The rate of dissociation or unloatling of oxygen has been measured photoelectrically.2*, 11. $13:$ The time for 50 per cent dissociation (deoxygenation)

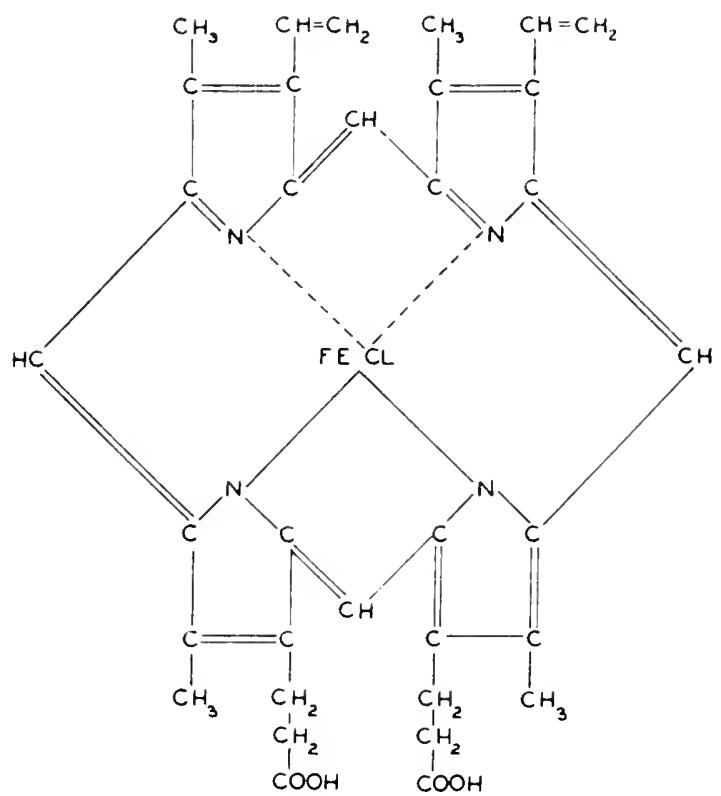

Fig. 66. Structural formula of heme.

is similar in man, sheep, frog, and Glycera, but is longer in Lumbricus (Table 51) and very long in Ascaris. ${ }^{2 \times}$

The protein portion of the hemoglobin molecule differs from species to species and even from embryo to adult in an individual. The protein differences are shown by slight differences in spectral absorption, by differences in oxygen affinity and in the effect of carbon dioxide on oxygen affinity, and by differences in amino acid composition. For example, the amount of methionine is higher in the hemoglobin of man, monkey, and cow than in that of dog and fox, and cystine is higher in the hemoglobin of man, monkey, dog, and fox than in that of the cow and horse, whereas the hemoglobin in all these species contains similar amounts of arginine, lysine, histidine, and tryptophane. ${ }^{13}$

Absorption. Bands. All hemin compounds show characteristic absorption 
spectra; the mid-points of some of these absorption bands are given in Table 52. Reduced (deoxygenated) hemoglobin has a broad band in the yellowgreen, whereas oxyhemoglobin has two principal bands, an alpha band in the yellow, and a beta band in the green. Methemoglobin shows four characteristic absorption bands, of which two are most prominent, $\alpha$ and $\beta$, and the bands of muscle hemoglobin are very close to those of blood hemoglobin. The hemglobins of different vertebrates show qualitatively the same absorption bands; however, it is possible to detect species differences by comparing the extinction coefficients for two maxima. ${ }^{160}$ Also, in the position of the alpha band of oxyhemoglobin there is a difference of as much as $3 \AA$ among several mammals.

TABLE 51 .

DISSOCIATION (UNLOADING OF OXYGEN) RATES OF BLOOD PIGMENTS $\mathrm{t}_{1 / 2 \mathrm{sat}}=$ Time in seconds for unloading 50 per cent of $\mathrm{O}_{2}$.

\begin{tabular}{|c|c|c|c|}
\hline Animal & $\mathrm{t}_{1 / 2 \text { sat }}(\mathrm{sec})$. & Temp. ${ }^{\circ} \mathrm{C}$ & $\mathrm{pH}$ \\
\hline & \multicolumn{3}{|c|}{ Hemoglobin } \\
\hline $\begin{array}{l}\operatorname{Man}^{115} \\
\text { Sheep }^{115} \\
\text { Frog } \\
\text { Lumbricus }^{138} \\
\text { Glycera }^{238} \\
\text { Ascaris } \\
\quad \text { (body fluid) } \\
\text { (body wall) }\end{array}$ & $\begin{array}{l}0.038 \\
0.027 \\
0.028 \\
0.02 \\
0.07 \\
0.027 \\
\\
220 . \\
150 . \\
80 .\end{array}$ & $\begin{array}{l}22 \\
27 \\
22 \\
\prime \prime \\
23 \\
28 \\
16 \\
20.5 \\
3\end{array}$ & $\begin{array}{l}8.6 \\
8.37 \\
8.6 \\
" 1 \\
8.0 \\
8.6 \\
\\
9.0 \\
6.0 \\
6.0\end{array}$ \\
\hline (body wall) & \multicolumn{3}{|c|}{ Hemocyanin } \\
\hline $\begin{array}{l}\text { Maja } \\
\text { Limulus }^{125} \\
\quad \text { (dialyzed) } \\
\quad \text { (non-dialyzed) }\end{array}$ & $\begin{array}{l}0.025 \\
0.08 \\
0.075\end{array}$ & $\begin{array}{l}22 \\
22 \\
22\end{array}$ & $\begin{array}{l}8.6 \\
8.6 \\
8.6\end{array}$ \\
\hline
\end{tabular}

Table 52 indicates also the absorption bands of hemoglobins (erythrocruorins) in invertebrate animals. Usually there are two oxyhemoglobin bands ( $\alpha$ and $\beta$ ) and one deoxygenated band which are very similar to the bands of vertebrate hemoglobin. However, differences have been reported as in the location of the bands of carboxyhemoglobin in Arenicola and Cucumaria, and in some annelids and echinoderms the reduced pigment has two maxima. The striking similarity in different animals, however, indicates that the hemin of the body Huids is essentially similar.

In all of the vertebrate hemoglobins and myoglobins the $\alpha$ band is higher than the $\beta$ band, whereas in the myoglobin of Busycon, of Ascaris, and of legume root nodules there is more absorption at the $\beta$ than at the $\alpha$ wave length. The span between the $\alpha$ and $\beta$ bands for blood hemoglobins is $48-56 \AA$, for vertebrate and snail myoglobin $32-36 \AA$, and for Gastrophilus hemoglobin 95.5. ${ }^{97}$

Molecular Size. Measurements of the molecular weight made by diffusion methods agree well with weights calculated from sedimentation data obtained 
TABLE 52. LOCATION OF ABSORPTION BANDS OF VARIOUS BLOOD PIGMENTS BY THEIR MAXIMA IN MILLIMICRA

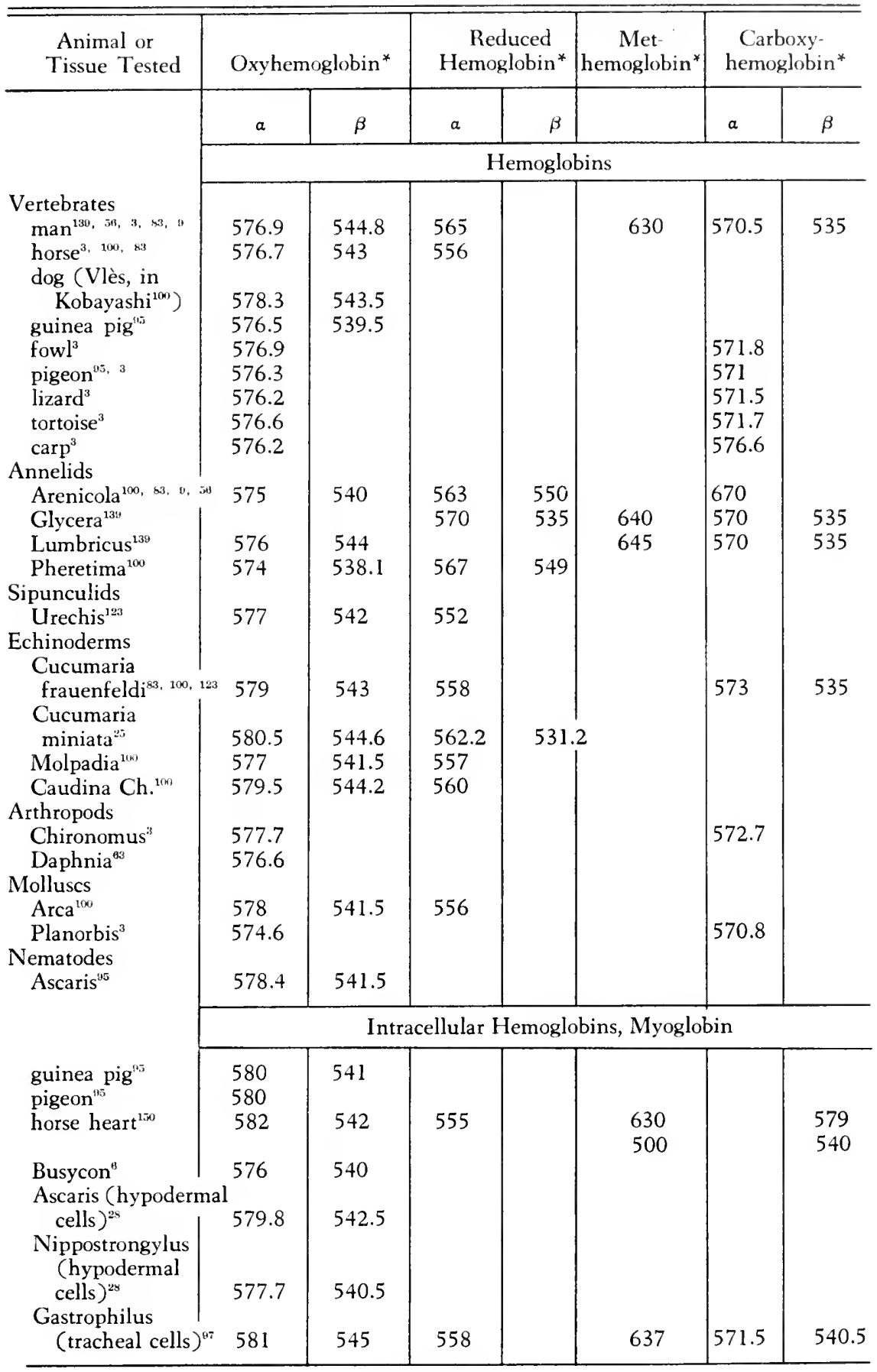

${ }^{*}$ or other pigment. 
TABLE 52 (continued). LOCATION OF ABSORPTION BANDS OF VARIOUS BLOOD PIGMENTS BY THEIR MAXIMA IN MILLIMICRA

\begin{tabular}{|c|c|c|c|c|c|c|c|}
\hline $\begin{array}{l}\text { Animal or } \\
\text { Tissue Tested }\end{array}$ & \multicolumn{2}{|c|}{ Oxyhemoglobin* } & \multicolumn{2}{|c|}{$\begin{array}{c}\text { Reduced } \\
\text { Hemoglobin }\end{array}$} & \multirow[t]{2}{*}{$\begin{array}{c}\text { Met- } \\
\text { hemoglobin }\end{array}$} & \multicolumn{2}{|c|}{$\begin{array}{c}\text { Carboxy- } \\
\text { hemoglobin* }\end{array}$} \\
\hline \multirow{3}{*}{$\begin{array}{l}\text { Legume (root } \\
\text { nodules) } \\
\text { paramecium }^{140}\end{array}$} & $a$ & $\beta$ & $a$ & $\beta$ & & $a$ & $\beta$ \\
\hline & $\begin{array}{l}574 \\
581\end{array}$ & $\begin{array}{l}540 \\
545\end{array}$ & $\begin{array}{l}537 \\
555\end{array}$ & & 633 & 574 & 538 \\
\hline & \multicolumn{7}{|c|}{ Chlorocruorin } \\
\hline \multirow[t]{2}{*}{$\begin{array}{l}\text { Sabella }{ }^{105, ~} \overline{8} \\
\text { Serpula }^{105} \\
\text { Spirographis }^{5 \theta} \\
\text { Endistylia }^{25}\end{array}$} & $\begin{array}{l}604 \\
604 \\
604.8 \\
603.5\end{array}$ & $\begin{array}{l}558 \\
560 \\
554.5\end{array}$ & $\begin{array}{l}604 \\
610 \\
578.5\end{array}$ & $\begin{array}{l}570 \\
\\
574 \\
540\end{array}$ & 525 & & $\begin{array}{l}601 \\
601.8\end{array}$ \\
\hline & \multicolumn{7}{|c|}{ Hemerythrin } \\
\hline \multirow[t]{2}{*}{$\begin{array}{l}\text { Lingula }^{133} \\
\text { Phascolosoma }^{100} \\
\text { Sipunculus }^{129,}, 130\end{array}$} & $\begin{array}{l}470-520 \\
<395 \\
493\end{array}$ & $\begin{array}{l}500-570 \\
425-490\end{array}$ & \multicolumn{2}{|c|}{$\begin{array}{c}<360 \mid>380 \\
\text { none }\end{array}$} & & & \\
\hline & \multicolumn{7}{|c|}{ Molpadin } \\
\hline \multirow[t]{2}{*}{ Molpadia $^{2 \pi}$} & 613 & 569 & 588 & & & & \\
\hline & \multicolumn{7}{|c|}{ Helicorubin } \\
\hline \multirow[t]{2}{*}{ snail bile ${ }^{30}$} & 562.5 & 529.7 & & & & & \\
\hline & \multicolumn{7}{|c|}{ Hemocyanin (diffuse absorption bands) } \\
\hline $\begin{array}{l}\text { Limulus }^{119} \\
\text { Homarus }^{119} \\
\text { Busycon }^{119} \\
\text { Palinurus }^{116}\end{array}$ & $\begin{array}{l}575-580 \\
570 \\
570 \\
558\end{array}$ & $\begin{array}{l}<450 \\
<450 \\
<450 \\
\text { also in ul }\end{array}$ & traviolet & & & & \\
\hline Palinurus ${ }^{116}$ & \multicolumn{7}{|c|}{ Cytochrome } \\
\hline bee muscle ${ }^{45}$ & 604.6 & 566.5 & 550.2 & 521 & & & \\
\hline
\end{tabular}

*or other pigment.

by Svedberg and his associates with the ultracentrifuge. ${ }^{149}$ The sedimentation constant of the protein in $\mathrm{cm}$./ $\mathrm{sec}$./dyne of centrifugal force gives a measure of molccular size. Sedimentation data for many hemoglobins are presented in Table 53, together with such molecular weights as were calculated by Svedberg and Pedersen. The protein is similar in size in mammals, birds, and fishes; it is slightly larger in amphibia and reptiles; and it is reduced by onehalf in cyclostomes, in which the hemoglobin molecule consists of two instead of four units. In those invertebrates in which the hemoglobin (erythrocruorin of Svedberg) is in solution in the plasma (except Chironomus), the molecular weight is greater than $1,000,000$. The large size tends to confine the molecules to the circulatory system. In all those invertebrates in which the hemoglobin occurs in cells, the molecular weight is less than that of vertebrate hemoglobin. Differences in molecular size are due to differences in the proteins and in the number of hemoglobin units per molecule. Svedberg ${ }^{1+7}$ suggested that the 
TABLE 53. SEDIMENT ATION RATE IN CM, $\times 10^{-13} /$ SEC./DYNE REDUCED TO $20^{\circ}$ IN WATER AS DETERMINED BY ULTRACENTRIFUGATION; CALCULATED MOLECULAR WEIGHTS AND ISOELECTRIC POINTS OF BLOOD PIGMENTS

Data from Svedberg and Pedersen ${ }^{19}$ and others.

Figures in parentheses represent secondary and lower sedimentation rates.

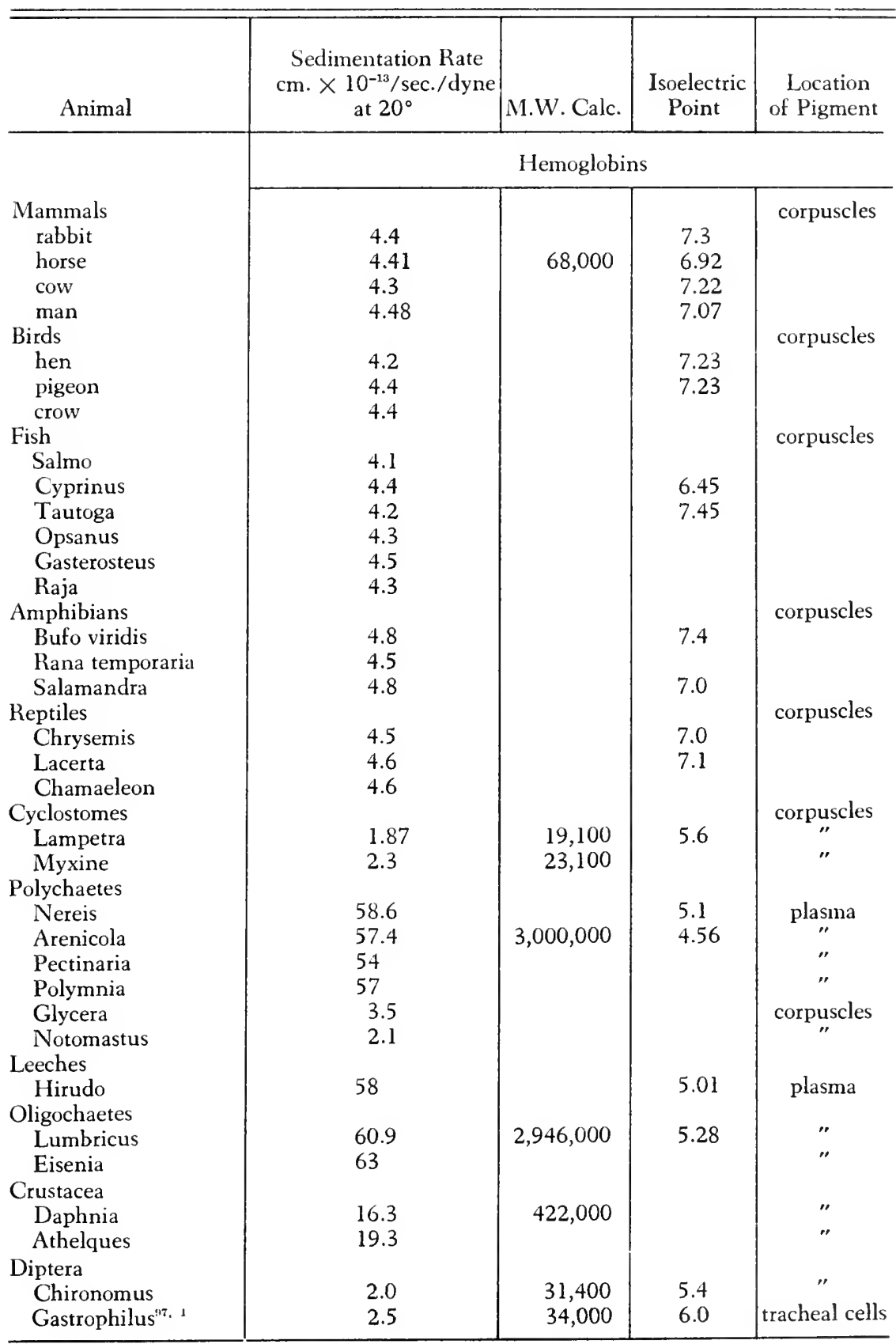


TABLE 53 (continued). SEDIMENTATION RATE IN CM. $\times 10^{-13} /$ SEC./DYNE REDUCED TO $20^{\circ}$ IN WATER AS DETERMINED BY ULTRACENTRIFUGATION; CALCULATED MOLECULAR WEIGHTS AND ISOELECTRIC POINTS OF BLOOD PIGMENTS

Data from Svedberg and Pedersen ${ }^{119}$ and others.

Figures in parentheses represent secondary and lower sedimentation rates.

\begin{tabular}{|c|c|c|c|c|}
\hline Animal & $\begin{array}{c}\text { Sedimentation Rate } \\
\mathrm{cm} . \times 10^{-13} / \mathrm{sec} . / \text { dyne } \\
\text { at } 20^{\circ}\end{array}$ & M.W. Calc. & $\begin{array}{c}\text { Isoelectric } \\
\text { Point }\end{array}$ & $\begin{array}{l}\text { Location } \\
\text { of Pigment }\end{array}$ \\
\hline \multirow[t]{2}{*}{$\begin{array}{l}\text { Gastropods } \\
\text { Planorbis } \\
\text { Lamellibranchs } \\
\text { Arca } \\
\text { Holothuroidea } \\
\text { Thyone }\end{array}$} & $\begin{array}{r}33.7 \\
3.5 \\
2.6\end{array}$ & $\begin{array}{r}1,539,000 \\
33,600\end{array}$ & $\begin{array}{l}4.77 \\
5.8\end{array}$ & $\begin{array}{c}\text { plasma } \\
\text { corpuscles } \\
\text { " }\end{array}$ \\
\hline & \multicolumn{4}{|c|}{ Chlorocruorins } \\
\hline \multirow[t]{2}{*}{$\begin{array}{l}\text { Polychaetes } \\
\text { Sabella } \\
\text { Serpulid }\end{array}$} & $\begin{array}{l}53 \\
59\end{array}$ & sev. million & & $\underset{\Perp}{\text { plasma }}$ \\
\hline & \multicolumn{4}{|c|}{ Hemerythrin } \\
\hline \multirow[t]{2}{*}{$\begin{array}{l}\text { Sipunculids } \\
\text { Sipunculus }\end{array}$} & & 66,000 & 5.85 & corpuscles \\
\hline & \multicolumn{4}{|c|}{ Hemocyanins } \\
\hline $\begin{array}{l}\text { Amphineura } \\
\text { Tonicella } \\
\text { Gastropods } \\
\text { Paludina } \\
\text { Buccinium undatum } \\
\text { Busycon canaliculatum } \\
\text { Helix pomatia } \\
\text { Helix arbustorum } \\
\text { Helix nemoralis } \\
\text { Helix hortensis } \\
\text { Limax maximus } \\
\text { Littorina littorea } \\
\text { Neptunea antiqua } \\
\text { Cephalopods } \\
\text { Loligo vulgaris } \\
\text { Rossia owenii } \\
\text { Sepia officinalis } \\
\text { Octopus vulgaris } \\
\text { Eledone moschata } \\
\text { Eledone cirrosa } \\
\text { Arthropods } \\
\text { Limulus } \\
\text { Euscorpionis } \\
\text { Crustacea } \\
\text { Pandalus } \\
\text { Palinurus }{ }^{11 s} \\
\text { Squilla } \\
\text { Homarus } \\
\text { Nephrops }\end{array}$ & $\begin{array}{c}61 \\
97 \\
102.1 \\
130.4,101.7 \\
98.9(62)(16) \\
91.2(64.1) \\
101(65) \\
100(61.9) \\
136(97.3) \\
99.7 \\
104 \\
56.7 \\
56.2 \\
55.9(18.7) \\
49.3 \\
49.1 \\
48\end{array}$ & $\begin{array}{l}3,316,000 \\
2,785,000 \\
2,791,000\end{array}$ & $\begin{array}{c}4.71 \\
4.61 \\
4.49 \\
5.3,5.0\end{array}$ & $\begin{array}{l}\text { plasma } \\
\text { plasma }\end{array}$ \\
\hline
\end{tabular}


TABLE 53 (continued). SEDIMENTATION RATE IN CM. $\times 10^{-t .1} / \mathrm{SEC} . / \mathrm{DYNE}$ REDUCED TO $20^{\circ}$ IN WATER AS DETERMINED BY ULTRACENTRI-

FUGATION; CALCULATED MOLECULAR WEIGHTS AND ISOELECTRIC POINTS OF BLOOD PIGMENTS

Data from Siedberg and Pedersen ${ }^{119}$ and others.

Figures in parentheses represent secondary and lower sedimentation rates.

\begin{tabular}{l|c|c|c|c}
\hline \multicolumn{1}{c|}{ Animal } & $\begin{array}{c}\text { Sedimentation Rate } \\
\mathrm{cm} \cdot \times 10^{-13} / \mathrm{sec} / \text { dyne } \\
\text { at } 20^{\circ}\end{array}$ & N.W. Calc. & $\begin{array}{c}\text { Isoelectric } \\
\text { Point }\end{array}$ & $\begin{array}{c}\text { Location } \\
\text { of Pigment }\end{array}$ \\
\hline Astacus & $\begin{array}{c}23.3(16.3) \\
27\end{array}$ & & 4.93 & \\
Maja & $23.6(16.4)$ & & 4.65 & \\
Cancer & $23.3(16.7)$ & & \\
Carcinus & 34 & & \\
Calocaris & & & \\
\hline
\end{tabular}

large molecules might be made of units each of a molecular weight of approximately 34,500 .

Table 53 also gives the isoclectric points of different hemoglobins as determined cataphoretically. ${ }^{14: 1}$ In general the invertebrate pigments (erythrocruorins) have a lower isoelectric point than the vertebrate hemoglobins.

Summary. Hemoglobins are derived from widely distributed hemochromogens. The heme is similar in all of them except for slight differences in absorption bands in certain worms and holothurians. Species differences in the proteins are indicated by crystallographic properties (Ch. 4), solubilities, molecular size and isoelectric points.

Chlorocruorin. Chlorocruorin, the green iron-containing blood pigment of the sabellid and serpulid worms, has a porphyrin which differs from heme. Oxychlorocruorin of Spirographis, reduced chlorocruorin, and carboxychloro. cruorin show maximum absorption bands toward the red, compared with analogous hemoglobin bands (Table 52). ${ }^{5 *}$ The iron content of Spirographis chlorocruorin is 1.27 per cent. ${ }^{1: 30}$ The amino acid composition of the protein is different from the composition in hemoglobins.

In molecular size, as judged by sedimentation rate, chlorocruorin is similar to the hemoglobins carried in solution in plasma, with molecular weights of several million (Table 53). The affinity of chlorocruorin for carbon monoxide (some 570 times that for oxygen) exceeds the CO affinity of any hemoglobin tested. $^{62}$ As with hemoglobin, two atoms of oxygen combine per atom of iron. 58

Hemerythrin. Hemerythrin contains iron, but the pigment is not a porphyrin. The iron content of Sipunculus hemerythrin is 1.017 per cent. ${ }^{130} 1 \mathrm{leme}$ rythrin fails to show sharp absorption bands like those of hemoglobin and chlorocruorin. Roche prepared brown-violet crystals from Sipunculus nudus hemerythrin which in oxy-form has an absorption peak at $493 \mathrm{~m} / \mathrm{and}$ absorbs increasingly toward the shorter wave lengths ( 425 to $290 \mathrm{~m} \mu$ ). In Phascolosoma there are broad, indefinite bands with maxima around 350 to $500 \mathrm{~m} \mu$ in oxyhemerythrin and in the violet in reduced hemerythrin; ${ }^{10:}$ different maxima were reported for Lingula:": It is probable, therefore, that the hemerythrins differ considerably among different species. I Hemerythrin is similar in molecular weight to mammalian hemoglobin. ${ }^{131}$ The isoelectric point of 


\section{TABLE 54. CHARACTERISTICS OF BLOODS CONTAINING HEMOCYANIN, AND COPPER CONTENT OF SEA-WATER}

Copper content in mg./ 100 cc. of blood (data from Dhéré, quoted by Elvehjem ${ }^{41}$ ), per cent copper in purified hemocyanin (data from Hernler and Philippi, quoted by Redfield ${ }^{121}$ ), and oxygen capacity (data from Redfield ${ }^{121}$ et al.).

\begin{tabular}{|c|c|c|c|}
\hline & $\begin{array}{l}\mathrm{Cu} \text { in Blood } \\
\mathrm{mg} . / 100 \mathrm{cc} .\end{array}$ & $\underset{\%}{\mathrm{Cu}} \underset{\%}{\mathrm{H}} \mathrm{H}$ & $\begin{array}{l}\text { Total } \mathrm{O}_{2} \\
\text { cap., vols \% }\end{array}$ \\
\hline $\begin{array}{l}\text { Cephalopods } \\
\text { Octopus vulgaris } \\
\text { Sepia officinalis } \\
\text { Loligo pealei }\end{array}$ & $\begin{array}{l}23.5-28.5 \\
23.7 \\
24.9\end{array}$ & $\begin{array}{l}0.25 \\
0.26\end{array}$ & $\begin{array}{l}3.1-4.5 \\
3.8-4.5\end{array}$ \\
\hline $\begin{array}{l}\text { Gastropods } \\
\text { Helix pomatia } \\
\text { Helix aspersa } \\
\text { Busycon canaliculatum }\end{array}$ & $6.5-11.5$ & $\begin{array}{l}0.24 \\
0.245\end{array}$ & $\begin{array}{l}1.15-2.2 \\
1.2 \\
2.1-3.3\end{array}$ \\
\hline $\begin{array}{l}\text { Xiphosura } \\
\text { Limulus polyphemus }\end{array}$ & 8.1 & 0.173 & $0.74-2.7$ \\
\hline $\begin{array}{l}\text { Crustacea } \\
\text { Astacus fluviatilis } \\
\text { Palinurus vulgaris } \\
\text { Homarus vulgaris } \\
\text { Cancer pagurus } \\
\text { Carcinus maenas } \\
\text { Maja squinado } \\
\text { Squilla mantis } \\
\text { Sea water }\end{array}$ & $\begin{array}{c}7.0-8.0 \\
9.5 \\
9-10 \\
\\
5-6 \\
9.0 \\
3.5 \\
6.1 \\
\mathrm{Cu}, \mathrm{mg./100} \mathrm{cc.} \\
0.00001\end{array}$ & $\begin{array}{l}0.148^{118} \\
0.187\end{array}$ & $\begin{array}{l}2.4 \\
1.4-1.8 \\
4.1-14^{146} \\
3.0^{29} \\
1.6-2.3 \\
1.1-1.2 \\
.84-1.75\end{array}$ \\
\hline
\end{tabular}

Sipunculus hemerythrin is $\mathrm{pH}$ 5.85. One molecule of oxygen combines with three atoms of iron in hemerythrin. ${ }^{52}$

Hemocyanin. Hemocyanin is a protein with a prosthetic group containing sulfur and copper; the prosthetic group is probably in part a polypeptide. ${ }^{121}$ Table 54 gives the copper content of several hemocyanin bloods and of purified hemocyanin. The copper content of molluscan hemocyanins is higher than that of crustacean hemocyanins. ${ }^{121,1: 0}$ In general the copper content of the blood, and hence its hemocyanin content in such active animals as Octopus, Sepia, and Loligo, is much higher than that of the more sluggish forms (Table 54). Higher hemocyanin content is correlated with higher oxygen capacity. The concentration of copper by hemocyanin-containing animals is remarkable, since occan water contains only 10 milligrams per cubic meter. ${ }^{41}$

Onc molecule of oxygen combines with a quantity of hemocyanin containing 2 atoms of copper. The copper is normally cuprous, but it can be oxidized by permanganate, and, unlike methemoglobin, methemocyanin can still be oxygenated. Ferricyanide cannot be used to drive off $\mathrm{O}_{2}$ from hemocyanin, as is done with hemoglobin. ${ }^{24}$ The affinity for $\mathrm{CO}$ is one-twentieth as great as the affinity for $\mathrm{O}_{2 .}{ }^{1: 34}$ 'The rate of dissociation of oxyhemocyanin, compared with the rate of dissociation of oxyhemoglobin, is similar in Maia and slower in Limulus (Table 51). ${ }^{11 \pi}$

Hemocyanin does not show sharp absorption bands like those of hemo- 
globin, but rather absorbs broadly. It shows much colloidal scattering (Ty'ndall effect), and when this is subtracted from the total absorption it is seen that maximum absorption for several species lies between 550 and $600 \mathrm{~m} \mu$. In addition there is absorption in the ultraviolet which may be associated with the prosthetic group (see Redfield ${ }^{121}$ for discussion).

Measurements of the sedimentation constants made with the ultracentrifuge indicate that the molecule of hemocyanin may occur in several different sizes. ${ }^{43}$ As shown in Table 53, two genera of the Malacostraca, Pandalus and Palinurus, have a sedimentation constant of 16, whereas two others, Nephrops and Homarus, have a sedimentation constant of 23; Astacus, Cancer, and Carcinus show both of these sedimentation constants; that is, the molecules are of two aggregation sizes. The molecular weight corresponding to the sedimentation constant of 16 is 360,000 , and that corresponding to the constant of 23 (Homarus) is 6t0,000. Hemocyanin from Limulus shows four sedimentation constants, the principal one corresponding to a molecular weight of $1,300,000$. In general, the sedimentation constants of molluscan hemocvanins are much higher than those of hemocyanins of arthropods. For example, the molecular weight of hemocyanin is 2,785,000 in Octopus, 3,316,000 in the squid, and 6,630,000 in a snail (Helix). ${ }^{14 !}$ Hemocyanin, which always occurs free in solution in the blood, has much larger molecular weights than the pig. ments which are contained in corpuscles. There is some evidence (see Redfield ${ }^{121}$ for summary) that the large molecules are aggregates of units each containing 8 atoms of copper ( 4 oxygen equivalents).

Many animals having hemocyanin as the blood pigment nevertheless. have myoglobin in their muscles. The odontophore retractor of Busycon, for example, contains more myoglobin than does dog heart muscle. ${ }^{6}$

Vanadium Chromogens. Many ascidians are rich in a green pigment con. taining vanadium, of which at least 80 per cent is in the blood. ${ }^{00}, 156$ The vanadium content varies from 0.04 per cent of the total dry weight in Ciona intestinalis to 0.186 per cent in Ascidia mentula. This is a remarkably selective concentration of vanadium from sea water, which contains only 0.3 to 0.6 milligrams per cubic meter. Webb ${ }^{1.66}$ found that among the various families and genera of ascidians, vanadium is lacking in the more primitive Larvacea and Thaliacea; it is present in the plasma of the Cionidae and Diazonidae and in special blood cells, vanadocytes, in several families, including Ascidiidae and Perophoridae, and apparently the element has been lost in the higher groups, such as Styelidae, Molgulidae, and Clavelicinidae.

The vanadocytes are about 8 micra in diameter and constitute 1.2 per cent of the total blood volume or 60 per cent of the total blood cells in some Ascidiidae. ${ }^{156}$ The vanadium is present in these cells as a chromogen which apparently consists of a chain of pyrrol rings; it is not a protein and it is not a porphyrin compound. The vanadium is kept in a reduced form by the presence of a high concentration of $\mathrm{H}_{2} \mathrm{SO}_{4}$. Titration of the whole blood showed a total acidity of $0.022 \mathrm{~N}$ and that of cytolyzed corpuscles showed an acidity of $1.83 \mathrm{~N}$ or 9 per cent $\mathrm{H}_{2} \mathrm{SO}_{4} \cdot{ }^{156}$ The oxidation potential of the vanadium chromogen is too low for it to be of use physiologically in respiration. Furthermore, various reducing agents are not oxidized more rapidly by air in the presence of vanadocytes than in their absence. Henze ${ }^{80}$ suggested that the vanadium might be necessary for the synthesis of tunicin, the material of 
the test. However, Webb pointed out that some tunicates which lack vanadium still produce tunicin. It appears certain that the vanadium in tunicate blood has no respiratory function.

\section{OXYGEN TRANSPORT BY BLOOD PIGMENTS}

\section{Function of Hemoglobin}

Oxygen Capacity. The first requisite of an oxygen transporter is ability to combine with enough oxygen to supply the needs of the animal. The oxygen capacity is the measure of the amount of oxygen combined with blood or with blood cells when they are saturated. It is usually expressed as volumes per cent of oxygen in whole blood or cells, and is determined by measuring the amount of oxygen combined after the blood sample is saturated with air. In experimental equilibration of some blood cells, particularly of nucleated erythrocytes, the amount of oxygen consumed by the cells is considerable and must be taken into account. The oxygen capacity depends largely on the amount of hemoglobin or other pigment in the blood or blood cells.

Table 55 shows the oxygen capacity of the blood of a number of animals. The oxygen capacity of the blood of mammals and birds is usually between 15 and 20 volumes per cent. In some but not all of the diving mammals (seal, sea lion, porpoise), the cells have unusually high $\mathrm{O}_{2}$ capacity. Also the llama, even at sea level, has a high $\mathrm{O}_{2}$ capacity. Among cold-blooded vertebrates the $\mathrm{O}_{2}$ capacities are lower, usually between 5 and 12 volumes per cent. In a few fishes which inhabit sluggish, acid ponds and can resort to air breathing (hassa and electric eel), unusually high $\mathrm{O}_{2}$ capacities have been reported. ${ }^{158}$ Among the invertebrates the $\mathrm{O}_{2}$ capacity also corresponds to the amount of respiratory pigment. Individual variation within a species is great. Where there is a significant amount of pigment, as in Arenicola, Urechis, Spirographis, Caudina, Octopus, Loligo, and a few others, the oxygen capacity of the blood is roughly ten times as much as it would be without the pigment. In many other animals the blood at equilibrium with air holds only about 0.5 to 2 volumes per cent. Sea water dissolves 0.538 volumes per cent of oxygen at $20^{\circ}$ when saturated with air. ${ }^{76}$

Oxygen Dissociation Curve. In no animal is the blood exposed directly to atmospheric tensions of oxygen $(150 \mathrm{~mm}$. $\mathrm{Hg}$ ). Most respiratory pigments become saturated at much lower oxygen tensions. The most important differences among the hemoglobins of different animals are in the tensions at which they load and unload oxygen. These tensions determine the range of usefulness of a particular pigment.

In the lungs of man the blood is exposed to oxygen at a partial pressure of approximately $100 \mathrm{~mm}$. Hg. When the blood leaves the lungs it carries 19 volumes per cent of oxygen at $80 \mathrm{~mm}$. Hg and 96 per cent of its hemoglobin is saturated. In the capillaries the blood passes through tissues where the oxygen pressure is low $(5-30 \mathrm{~mm}$. $\mathrm{Hg}$ ). Here 25 to 30 per cent of the oxygen is unloaded, and venous blood returning to the heart carries 14 volumes per cent of oxygen at about $40 \mathrm{~mm}$. Hg pressure.

The relation of the oxygen held by the hemoglobin and the partial pressure of oxygen is best seen by plotting the per cent saturation of the hemoglobin against oxygen tension, the so-called oxygen dissociation curve. This curve is 
TABLE 55. RESPIRATORY CHARACTERISTICS OF BLOOD

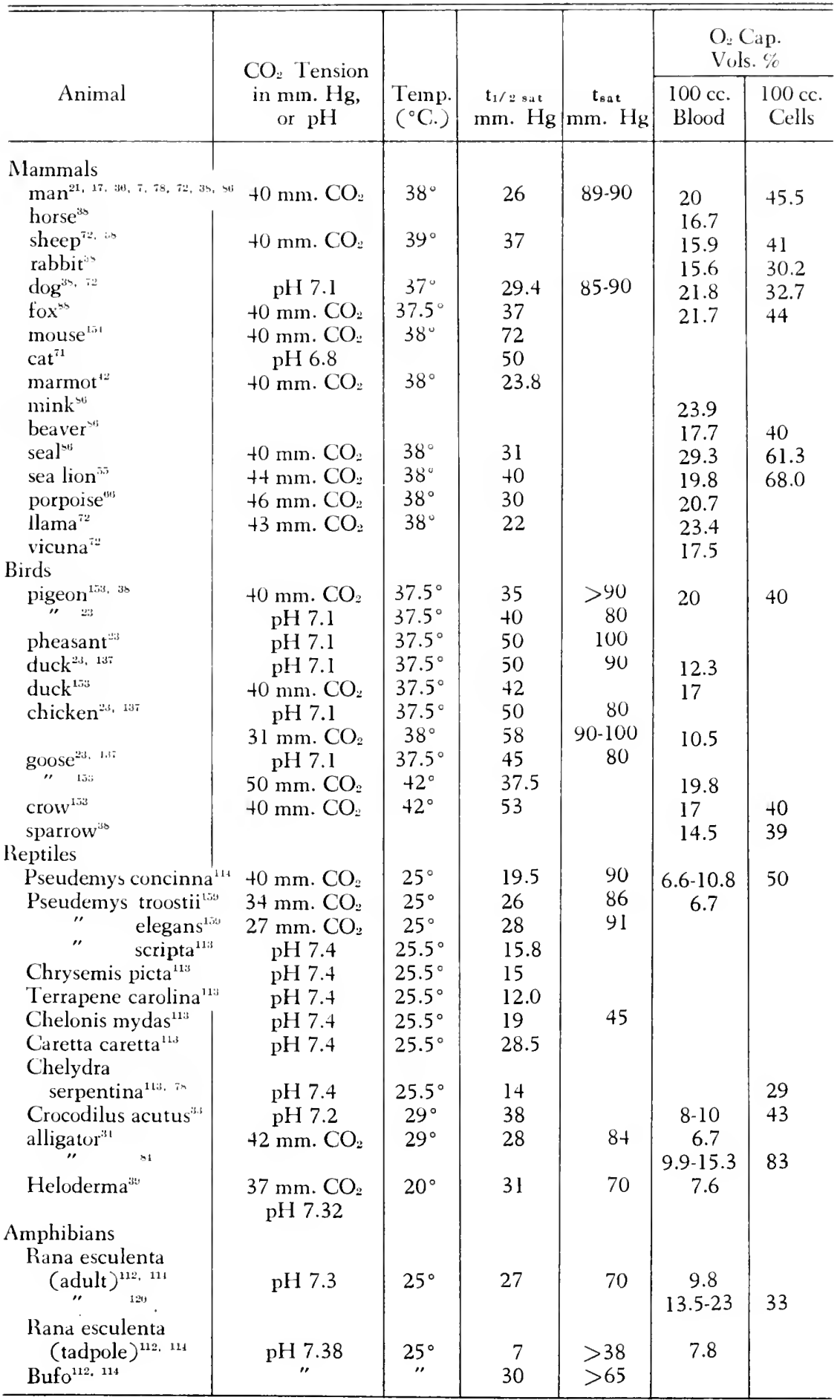


TABIE 55 (continued). RESPIRATORY CHARACTERISTICS OF BLOOD

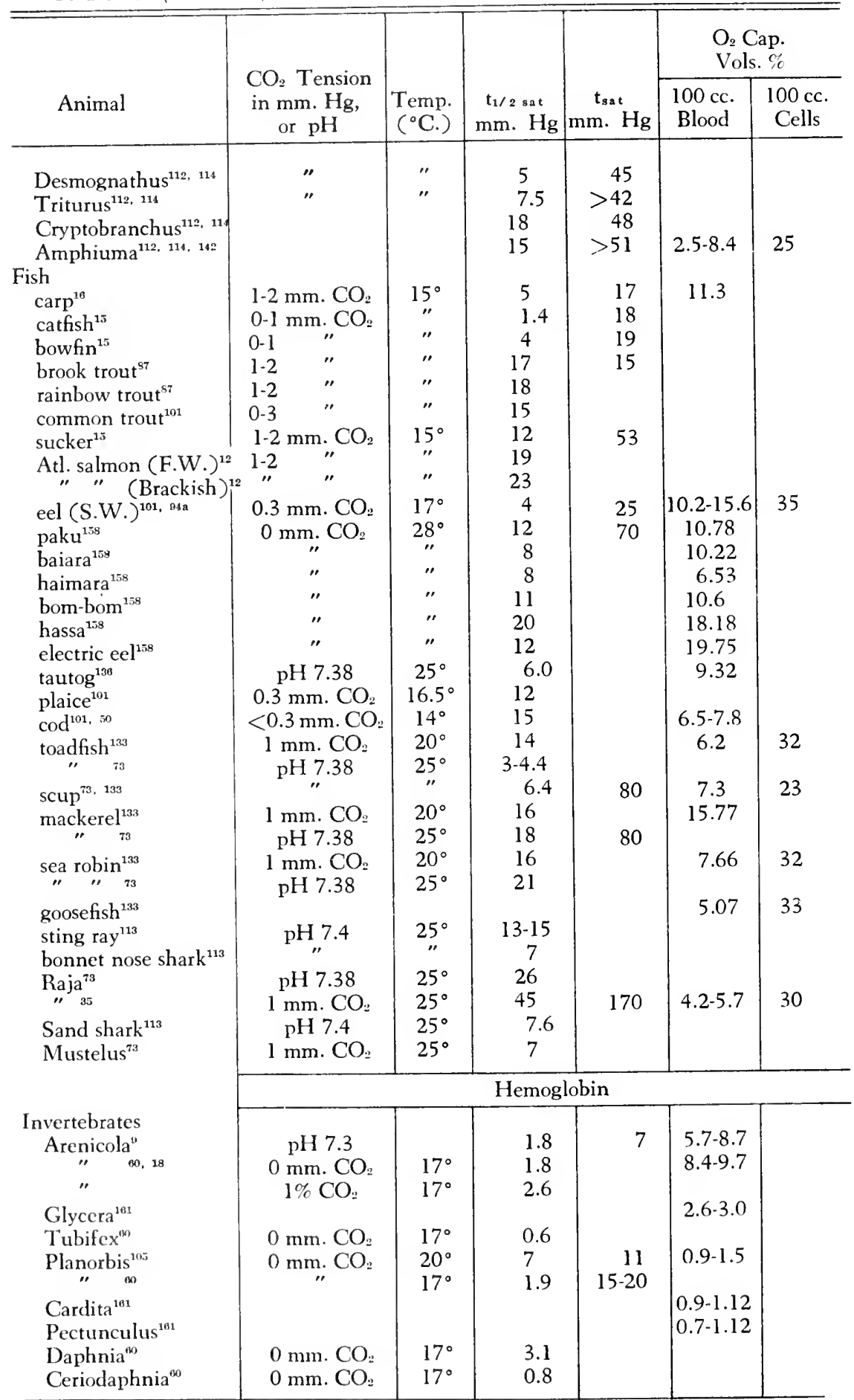


TABLE 55 (continued). RESPIRATORY CHARACTERISTICS OF BLOOD

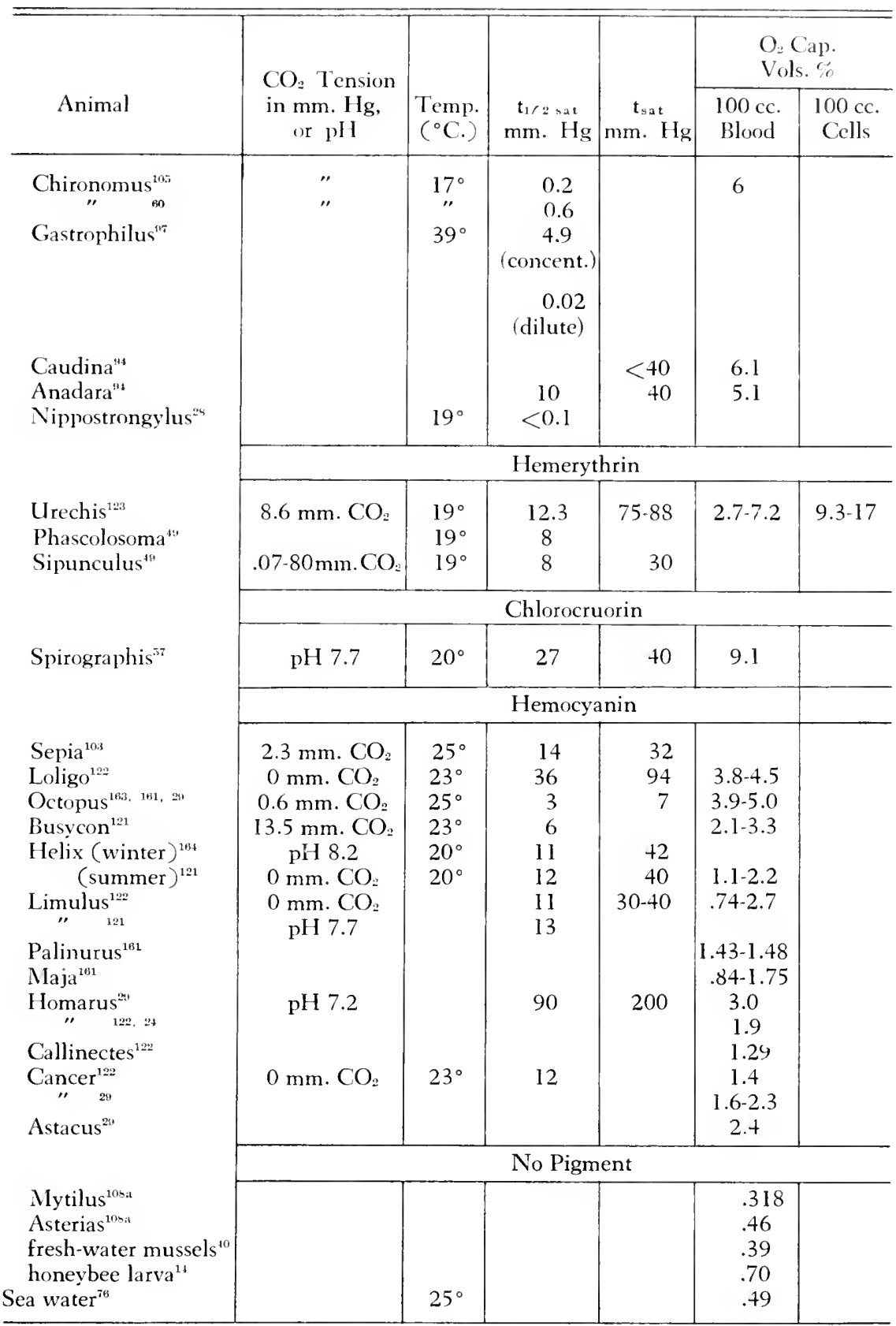

not usually a hyperbola as it would be if one molecule of $\mathrm{O}_{2}$ always combined with a single hemoglobin molecule; actually the four atoms of iron in hemoglobin may combine with four or with less than four molecules of oxygen. The sigmoid shape of the dissociation curve (Fig. 67) is due in part to the 
variable number of iron units combining with oxygen, to the interaction between groups, and in part to the effects on hemoglobin of other components of the blood such as the blood salts. Purified hemoglobin gives a more nearly hyperbolic oxygen dissociation curve than does hemoglobin in blood or in the presence of blood salts. The $\mathrm{O}_{2 .}$ dissociation curve of muscle hemoglobin is more nearly hyperbolic than that of blood hemoglobin, and it lies to the left of the blood curve; that is, myoglobin has a greater oxygen affinity than blood hemoglobin and can take oxygen from it. Dog myoglobin is only 40 per cent dissociated at $5 \mathrm{~mm}$. of $\mathrm{O}_{2}$; the blood hemoglobin is 95 per cent dissociated at this tension. ${ }^{81}$ Except at moments of stress, myoglobin remains saturated.

The oxygen tension at which the pigment is 95 per cent or more saturated is called the loading tension $\left(t_{1}\right)$ or tension of saturation $\left(t_{\text {sat }}\right)$; the oxygen tension at which the pigment is 50 per cent saturated, that is, when Hb equals $\mathrm{HbO}_{2}$, is the unloading tension $\left(t_{\mathrm{u}}\right)$ or tension of half saturation $\left(t_{1 / 2}\right.$ sat $)$.

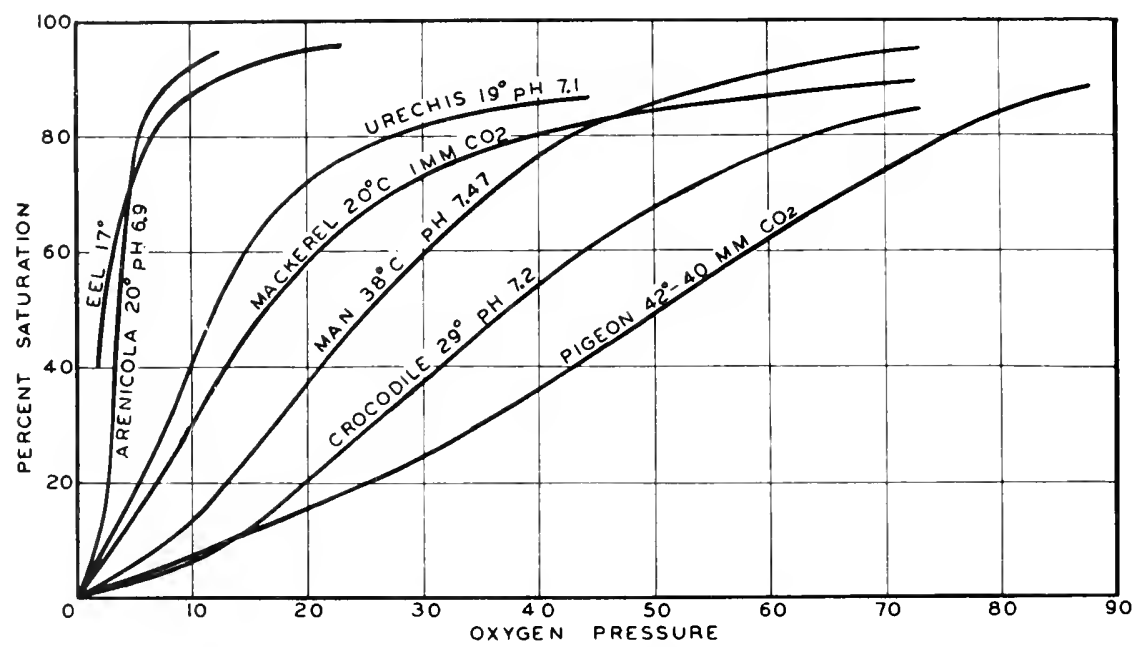

Fig. 67. Oxygen dissociation curves in per cent saturation of hemoglobin as a function of oxygen pressure in $\mathrm{mm}$. $\mathrm{Hg}$ in a varicty of animals. Data assembled by Redfield. ${ }^{121}$

In Table 55 the $t_{1 / 2}$ sat and the $t_{\text {sat }}$ of a number of animals are compared under nearly physiological conditions. In Figure 67 are plotted oxygen dissociation curves of the blood of some animals (pigeon, crocodile) in which the affinity for oxygen is low, i.e., the $\mathrm{t}_{1 / 2}$ sat is high, and of other animals (Arenicola and eel) which have a great affinity for oxygen, i.e., the $\mathrm{t}_{1 / 2}$ sat and $t_{\text {sar }}$ are low. In Figure 68 similar curves are plotted, using the actual amount of oxygen combined in volumes per cent instead of per cent saturation as ordinate. From the data of Table 55 and of Figures 67 and 68, it is apparent that hemoglobins differ greatly in their oxygen affinity and in the amount of oxygen they can carry when saturated. Unloading tensions set the upper limit of tissue oxygen tension and the lower limit of environmental oxygen for function of the hemoglobin.

In the bird bloods which have been studied the affinity for $\mathrm{O}_{2}$ is less than in man, i.c., the $t_{1 / 2}$ sat and $t_{\mathrm{xat}}$ are higher, but the dissociation curves are 
similar in shape. ${ }^{23} .1 .53$ In a duck and pigeon the difference between the oxygen in arterial and venous blood shows a utilization of 60 per cent, compared with 27 per cent in man. In the turtle 744 per cent and in the skate Raja 66 per cent of the oxygen is removed through the circulation. In a general way animals which make quich active movements (mouse, cat) have higher tensions of saturation and half-saturation with oxygen than animals which are slow and steady (dog). Also most cold-blooded aquatic animals have dissocia-

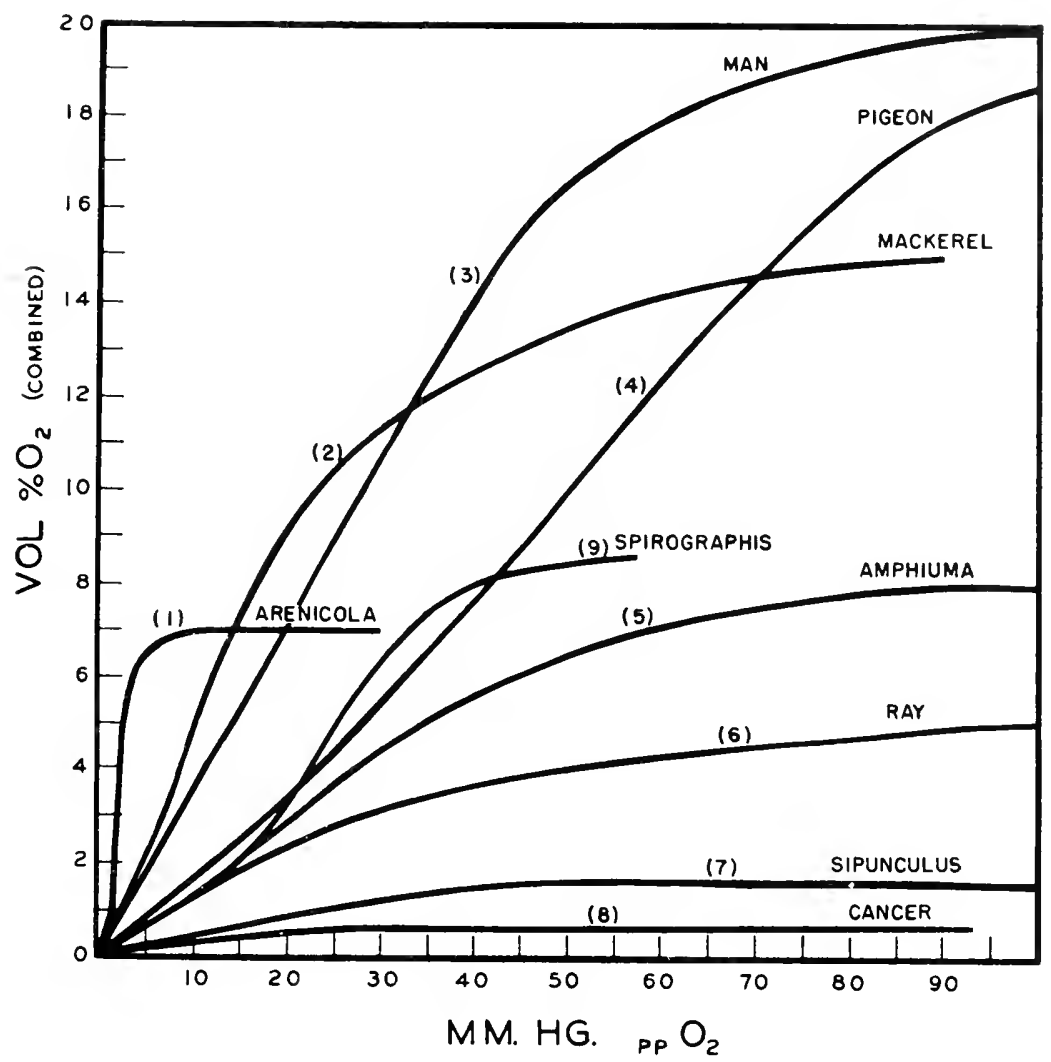

Fig. 68. Oxygen dissociation curves in volumes per cent of oxygen combined as a function of partial pressure of oxygen in $\mathrm{mm}$. Hg. (1) Arenicola $20^{\circ}, \mathrm{pH} \mathrm{4.9;}$; (2) mackerel $20^{\circ}, 1 \mathrm{~mm} . \mathrm{CO}_{3}$; (3) man $38^{\circ}, \mathrm{pH} 7.4$; (4) pigeon $42^{\circ}, 40 \mathrm{~mm} . \mathrm{CO}_{2}$; (5) Amphiuma $20^{\circ}, 43 \mathrm{~mm} . \mathrm{CO}_{2}$; (6) ray $10.4^{\circ}$, pH 7.8; (7) Sipunculus $19^{\circ}, \mathrm{pH} 7.7$; (8) Cancer $23^{\circ}, 1 \mathrm{~mm}$. CO:; (9) Spirographis $20^{\circ}, \mathrm{pH}$ 7.7. Data assembled by Florkin, ${ }^{50}$ except data for Spirographis from Fox. ${ }^{\text {thi }}$

tion curves to the left of the curves of warm-blooded animals; that is, the affinity for oxygen of the hemoglobin of aquatic animals is greater.

Effect of Carbon Dioxide. When carbon dioxide is added to mammalian blood, as it is in the capillaries, the affinity of the hemoglobin for oxygen is reduced and the dissociation curve moves to the right (the Bohr effect). At high $\mathrm{CO}_{2}$ tensions, therefore, the oxygen tension at which hemoglobin becomes saturated with oxygen is higher than it is at low $\mathrm{CO}_{2}$ tensions. In the tissues 
at a given $\mathrm{O}_{2}$ tension addition of $\mathrm{CO}_{2}$ facilitates unloading or reduction, while in the lungs as $\mathrm{CO}_{2}$ is given off the uptake of $\mathrm{O}_{2}$ (oxygenation) is facilitated. The effect of $\mathrm{CO}_{2}$ can be duplicated by lowering the $\mathrm{pH}$ of the blood. In Table 56 and in Figures 69, 70, and 71, the effects of variations in $\mathrm{CO}_{2}$ tension or in $\mathrm{pH}$ on the $\mathrm{t}_{1 / 2}$ sat of the bloods of several animals are compared. In aquatic animals (e.g., fish), the $\mathrm{CO}_{2}$ effect on the dissociation curve is greater than it is in terrestrial animals (particularly mammals). This is related to the

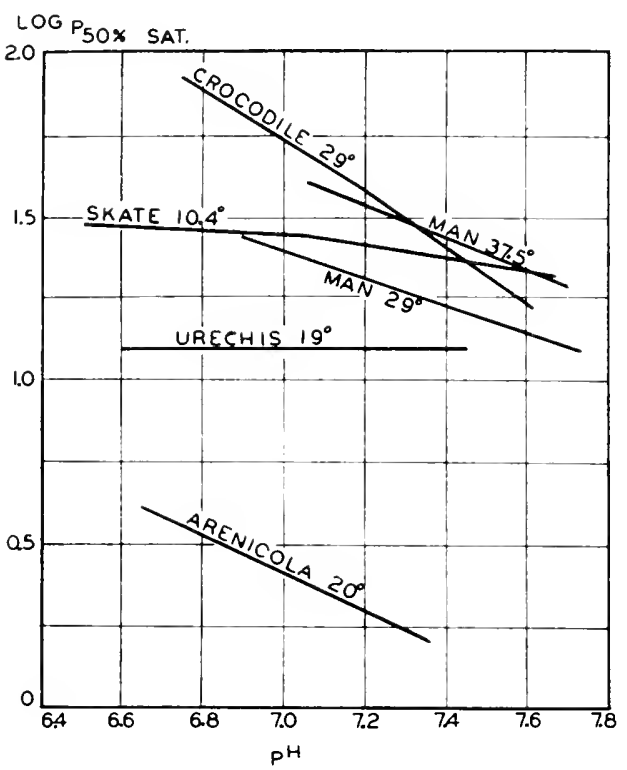

Fig. 69. Effect of $p H$ on $\log t_{1 / 2 \text { sat }}$ of the blood of several animals. Data assembled by Redfield. ${ }^{111}$

fact that the normal $\mathrm{CO}_{2}$ tension in blood of aquatic animals is much lower. Also aquatic animals which live in stagnant water usually have hemoglobin which is less sensitive to $\mathrm{CO} .2$ than is that of animals inhabiting fast-moving waters.

Effect of Temperature Changes. A rise in temperature also shifts the oxygen dissociation curve to the right. If the blood of a frog is warmed to $35^{\circ} \mathrm{C}$. its $\mathrm{O}_{2}$ dissociation curve is far to the right of man's, whereas at $15^{\circ}$ human blood remains saturated at low tensions, hence would be of little use as a carrier. The temperature effect on $\mathrm{t}_{1 / 2 \text { sat }}$ in the frog is about twice as great as it is in man. ${ }^{117}$ The effects of variations in temperature on $t_{1 / 2}$ sat are shown in Figures 72, 73, and 74. Both the $\mathrm{CO}_{2 .}$ effect and the temperature effect are of considerable ecological importance.

Effect of Altitude. At high altitudes the hemoglobin content and the red cell count increase in man so that the oxygen capacity of the blood becomes elevated (Table 57). The tensions for half-saturation of the hemoglobin do not change significantly when measured at a constant $\mathrm{pH}$ in man acclimatized to high altitudes; ${ }^{32}$ i.e., the nature of the hemoglobin is not altered. Physio- 
logically, however, after acclimatization there is a fall in alkaline reserve or acid-binding material so that $\mathrm{CO}_{2 .}$ effects are more marked and the $\mathrm{t}_{1 / 2}$ sat of the hemoglobin may actually increase (up to about 14,000 ft. altitude)!

Table 57 also shows that the red cell count and hematocrit index of acclimatized shcep and rabbits are elevated, but in the native llama and vicuna blood counts are not much different at sea level and in the mountains. ${ }^{7:}$ The oxygen dissociation curves of llama and vicuna lie to the left of those of their sea level relatives, and the bloods of the ostrich and huallata are also saturated at lower tensions than are the bloods of other birds. Hence these native species appear to have hemoglobin of high $\mathrm{O}_{\text {.. }}$ affinity. Dogs reared at 14,890 feet altitude have 60 to $70 \mathrm{per}$ cent more myoglobin in their muscles than do dogs at sea level, ${ }^{5.5}$ although in dogs kept at 18,000 feet for 6 hours daily during several months the hematocrit index rose by 70 per cent, but the myoglobin was unchanged. ${ }^{19}$

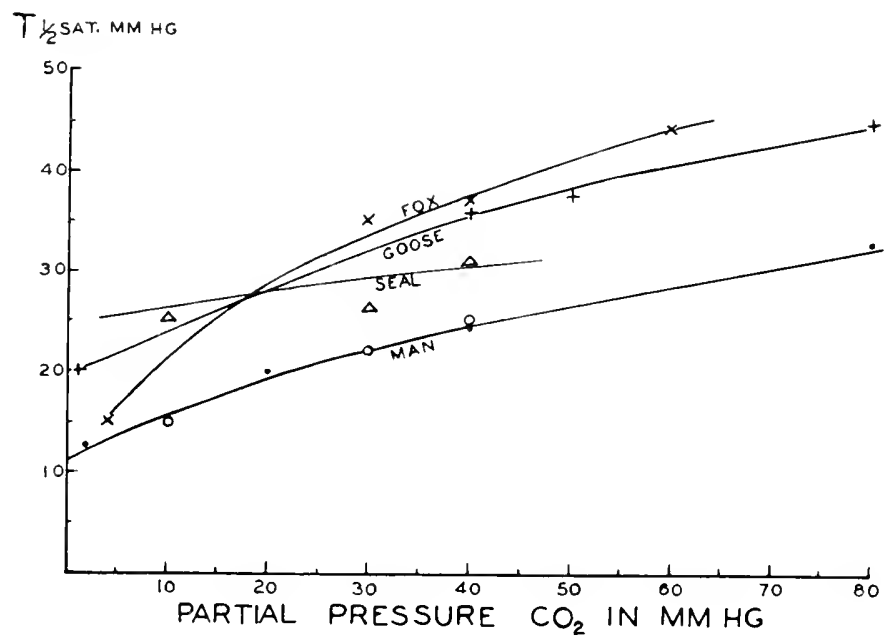

Fig. 70. Effects of $\mathrm{CO}_{z}$ on the $\mathrm{O}_{2}$ tension for half saturation of blood: $\bullet$ man, Bock, Field, and Adair ${ }^{17} ; \bigcirc$ man, $\triangle$ seal, Irving et al. ${ }^{\text {"s; }} ; \times$ fox, Irving et al..$^{\circ s} ;+$ goose, Wast and Leiner. ${ }^{153}$

Hemoglobin of Embryos. Barcroft and his associates found that the oxygen dissociation curve of mammalian embryos is steeper than that of adults and lies to the left of the normal range. The maternal curve, on the other hand, lies to the right, an effect which is largely due to decreased blood pH (Fig. 75). The fetal hemoglobin differs from that of the adult in loading at lower oxygen tensions. $\$$. 10 Thus fetal hemoglobin, which is made largely in liver, resembles the hemoglobin of aquatic animals more than does adult hemoglobin, which is made in marrow. The embryo is adapted by its low $\mathrm{t}_{1 / 2}$ wat to life at lower oxygen tensions.

Similarly in incubating chickens the hemoglobin has a greater affinity for oxygen than it has in the hen. The dissociation curve moves to the right after hatching, and the tension for 50 per cent saturation rises. ${ }^{69}$ ' Young birds are 
more resistant to low oxygen than older birds because of the greater affinity for oxygen. ${ }^{1: 37}$

The blood of the bullfrog tadpole becomes saturated with oxygen at lower tensions than does the blood of the adult frog; the tadpole dissociation curve also is more hyperbolic. Changes in $\mathrm{pH}$ alter the shape as well as the position of the dissociation curves of the blood of the frog and tadpole. ${ }^{112}$ In the tadpole the curve moves slightly to the left when $\mathrm{pH}$ is decreased, whereas in the adult the tensions of loading and unloading are raised as in other animals. I lemoglobin of terrapin embryos also has a higher $\mathrm{O}_{2}$ affinity, that is, a lower $\mathrm{t}_{1 / 2}$ sut than the hemoglobin of adults. ${ }^{11: 3}$ Hemoglobins of embryos in general

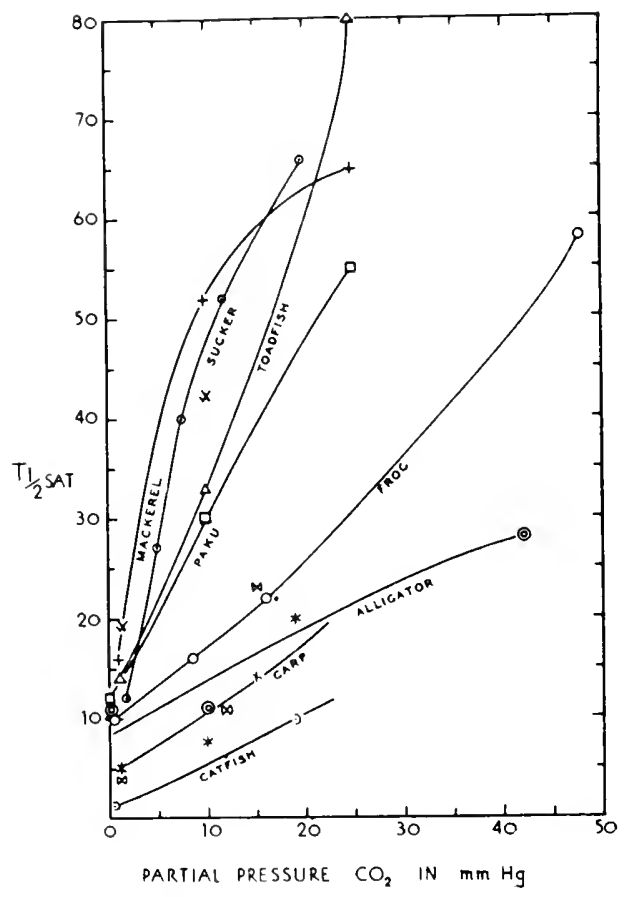

Fig. 71. Fiffects of $\mathrm{CO}_{2}$ on the $\mathrm{O}$, tension for half saturation of blood. (-) alligator, Dill and Edwards": $\bigcirc$ Rana temporaria, Rana esculenta. Wolvekamp ${ }^{145} ; \triangle$ toadfish, +

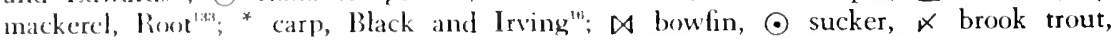
-) cathsts, Black ${ }^{1+i} ; \square$ paku, Willmer. ${ }^{\text {i. }}$

arce, therefore, functional at lower oxigen tensions than are adult hemoglobins.

Relation of Blood Pigments to Distribution of Amphibians and Fishes. ()xygen dissociation curres for a number of amphibia are shown in Figure 76. Howe genera which spend much of their time on land show oxygen disseciation curves to the right of the curves for alyuatic forms; i.e., the loading tensions alce higher with greater availability of oxygen. The curve of Cryptobranchus maly represent al reversion from land to water. ${ }^{1 t}$ Similarly, in a series of seven species of turtes, the terrestrial box turth has the lowest oxygen atlinity $\left(t_{1}, \ldots \ldots t=28.5 \mathrm{~mm}\right.$. $\left.1 \mathrm{lg}\right)$, whereas aquatic turtles have higher $\mathrm{O}_{2}$ 
TABLE 56. EFFECT OF $\mathrm{CO}_{2}$ OR DECREASE IN pH ON THE O. TENSION FOR HALF SATURATION OF BLOOD IN SEYERAL GIROUPS OF ANIMIALS

\begin{tabular}{|c|c|c|c|}
\hline Animal & $\begin{array}{c}\mathrm{t}_{1 / 2} \text { sut at low } \\
\mathrm{CO}_{2}\end{array}$ & $\begin{array}{c}\mathrm{t}_{1 / 2} \text { wut at high } \\
\mathrm{CO}^{2}\end{array}$ & $\begin{array}{c}\text { Per cent } \\
\text { increase in } \\
t_{1 / 2} \text { a at }\end{array}$ \\
\hline \multirow{3}{*}{ 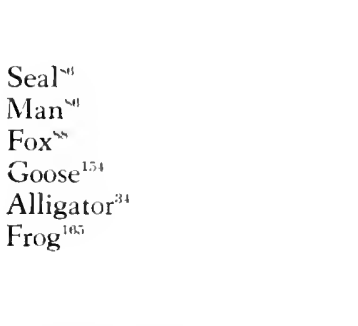 } & \multicolumn{2}{|c|}{$10 \mathrm{~mm} . \mathrm{CO}_{2} \quad 40 \mathrm{~mm} . \mathrm{CO}_{2}$} & \\
\hline & $\begin{array}{l}25 \\
15 \\
21^{*} \\
24^{*} \\
11 \\
17\end{array}$ & $\begin{array}{l}31 \\
25 \\
37 \\
35.7 \\
28 \\
49\end{array}$ & $\begin{array}{r}24 \\
66 \\
76 \\
48 \\
154 \\
188\end{array}$ \\
\hline & \multicolumn{2}{|c|}{$1-2 \mathrm{~mm} . \mathrm{CO}_{2} \quad 10 \mathrm{~mm} \cdot \mathrm{CO}_{2}$} & \\
\hline 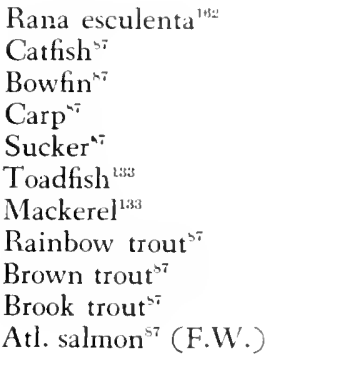 & $\begin{array}{l}11 \\
1.4 \\
4 \\
5 \\
12 \\
14 \\
16 \\
18 \\
17 \\
17 \\
19\end{array}$ & $\begin{array}{r}17 \\
5 \\
9 \\
8 \\
+3 \\
33 \\
52 \\
35 \\
39 \\
42 \\
35\end{array}$ & $\begin{array}{r}54 \\
256 \\
125 \\
60 \\
258 \\
135 \\
225 \\
94 \\
129 \\
147 \\
84\end{array}$ \\
\hline Atl. salmon ${ }^{57}$ (F.W.) & \multicolumn{2}{|c|}{$0 \mathrm{~mm} . \mathrm{CO}_{2}, \quad 25 \mathrm{~mm} \cdot \mathrm{CO}_{2}$} & \\
\hline 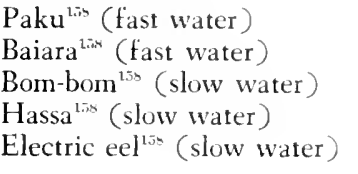 & $\begin{array}{r}12 \\
8 \\
11 \\
11 \\
12 \\
\end{array}$ & $\begin{array}{l}55 \\
22 \\
13 \\
20 \\
18 \\
\end{array}$ & $\begin{array}{r}358 \\
175 \\
18 \\
73 \\
50 \\
\end{array}$ \\
\hline Electric eel ${ }^{15}$ (slow water) & $\mathrm{pH} 7.38$ & $\mathrm{pH} 6.8$ & \\
\hline $\begin{array}{l}\text { Rana esculenta }{ }^{112} \text { (adult) } \\
\quad \text { (tadpole) } \\
\text { Toadfish }^{73} \\
\text { Scup }^{73} \\
\text { Remora }^{73} \\
\text { Mustelus }^{73}\end{array}$ & $\begin{array}{c}26 \\
7 \\
3-3.4 \\
6.4 \\
11 \\
7 \\
\end{array}$ & $\begin{array}{r}32 \\
5 \\
14 \\
39 \\
53 \\
12\end{array}$ & \\
\hline
\end{tabular}

${ }^{*}$ Exirapolated values.

affinities, the $\mathrm{t}_{1 / 2}$ : at for the loggerhead turtle being $12.0 \mathrm{~mm}$. Hg (Table $55) .113$

The properties of the respiratory pigments of fish are important in limiting their distribution. In Tables 55 and 56 are shown loading and unloading tensions ( $t_{\text {xit }}$ and $t_{1 / 2}$ sat $)$ for several different species. Fish such as the trout, which live in regions of high oxygen, require higher oxygen tensions for saturation than do such fish as the catfish and carp, whose hemoglobin loads and unloads at low oxygen tensions. Table 55 shows that the oxygen capacity of these different species is similar, despite marked differences in the position of their $\mathrm{O}_{2.2}$ dissociation curves. ${ }^{1.5}$

The effect of $\mathrm{CO}_{2}$ in decreasing the affinity of hemoglobin for oxygen tends 
to be greater in the fish which have high oxygen tensions of loading and unloading (lable 56, and Fig. 71). Krogh and Leitch ${ }^{101}$ noted a greater $\mathrm{CO}_{2}$ sensitivity in the fresh-water pike and carp than in the marine cod and plaice. The conclusion of more recent investigations ${ }^{1.5,}, \pi, 1,3,3$ is that differences in $\mathrm{CO}_{2}$ sensitivity are not related to marine fresh-water habitat per se, but represent true hemoglobin differences of different species. The amount of oxygen which can be taken up by a trout in the presence of $\mathrm{CO}_{2}$ is less than the amount which can be taken up by a carp. The $\mathrm{CO}_{2}$ tension to which gills of all fish are exposed is much lower than alveolar $\mathrm{CO}_{2}$, and the hemoglobin functions at lower $\mathrm{CO}_{2}$ levels in fish than in terrestrial animals. A trout can be kept in a bottle of water which contains ample oxygen, but as the $\mathrm{CO}_{2}$ in the water increases, the fish is unable to use the oxygen. A catfish, on the other hand, will endure tensions of $\mathrm{CO}_{2}$ at $400-500 \mathrm{~mm}$. I Ig for long periods. Black found

\section{TABLE 57. RELATION BETWEEN ALTITUDE AND RED CELLS, O, CAPACITY, AND $t_{1 / 2}$ sat FOR ACCLIMATIZED ANIMALS Data combined from Dill, ${ }^{* 2}$ Hall, Dill, and Barron, ${ }^{72}$ Hall, ${ }^{\top 0,}{ }^{-1}$ and Keys, Hall, and Barron ${ }^{39}$}

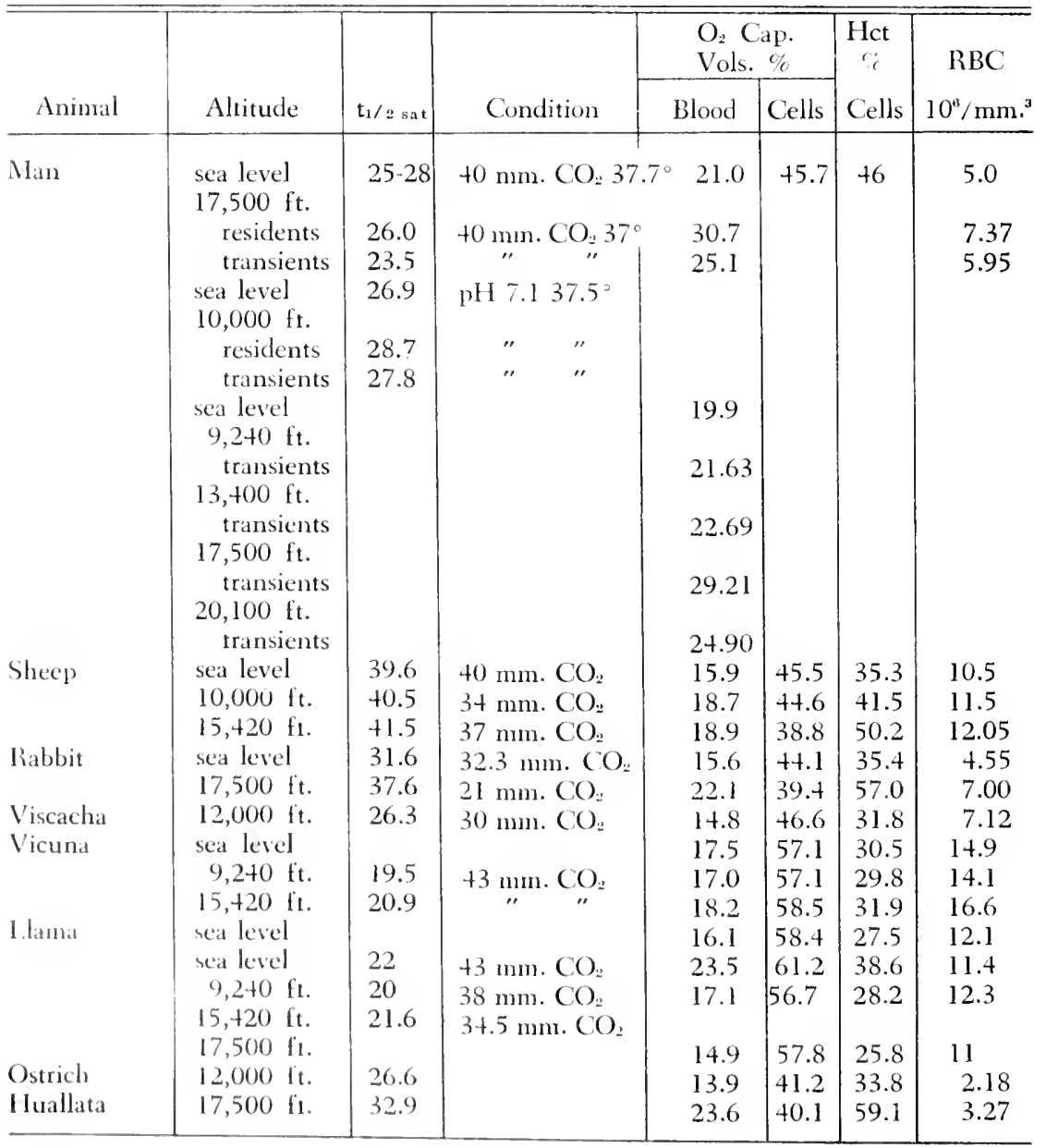


that addition of $\mathrm{CO}_{2}$ to a closed ressel containing trout blood might cause the appearance of a bubble of oxygen." 1 to $11 \mathrm{~mm}$. the $\mathrm{t}_{1} \because 2$ sit of a brook trout rises $\mathrm{lw}_{3} 3 \mathrm{~mm}$., whereas in man an increase of $10 \mathrm{~mm}$. in $\mathrm{CO}$. causes a rise in $\mathrm{t}_{1.2}$.at of about $3.3 \mathrm{~mm}$. $A$ small increase in $\mathrm{CO}_{2}$ may force fish with a fow $\mathrm{O}_{2}$ affunity into water of very high oxygen, whereas in lish with low tensions of loading the $\mathrm{C}(\mathrm{O}$.. eflect is less significant (Fig. 7l, Table 56). Among fresh-water fish the $t_{1} 2$ wat of the catfish, carp, and bowfin is not raised above $10 \mathrm{~mm}$. (O.2 by $10 \mathrm{~mm}$. CO., whereas the $t_{1} 2$ at of the sucker and of each of three species of trout is raised above $35 \mathrm{~mm}$. O... Sensitive marine fish are toadfish, mackerel, and sea robin:

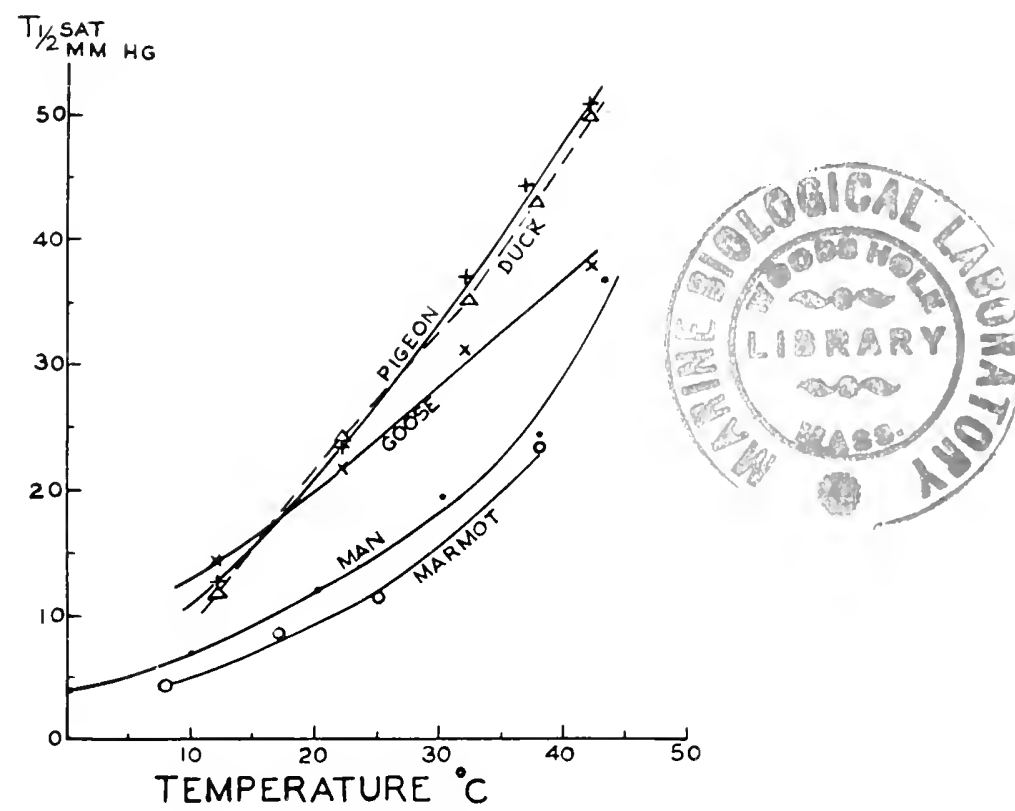

Fig. 72. Effects of temperature on the $\mathrm{O}$. tension for half saturation of blood. $\bullet$ man, Brown and Hill": $\bigcirc$ marmot, Enders" ${ }^{2:} \times$ goose, $t$ pigeon, $\triangle$ duck, Wastl and Leiner. ${ }^{151}$

elasmobranchs are less affected by $\mathrm{CO}_{.2}$, and for the skate (Raja ocellata) the $\mathrm{t}_{1 / 2 \text { sat }}$ is relatively constant over a wide range of $\mathrm{CO}_{22}$ tensions.

The response of the $\mathrm{O}_{2}$ dissociation curve to $\mathrm{CO} .2$ is similar, whether the red cells are intact or hemolyzed, in the trout, sea robin, and Atlantic salmon. In other fish, such as the carp, sucker, tautog, and toadfish, the effect of $\mathrm{CO}_{2}$. is less on hemolvzed blood than on blood with the cells intact. Apparently the hemoglobin of the latter group of fish is made up of several oxygen-combining components which combine with different amounts of oxygen at a given tension while in the cells. ${ }^{136}$ When the blood is hemolyzed the oxygen dissociation curve at a given CO.2 tension lies to the left of that of whole blood.

Willmer ${ }^{158}$ has studied the bloods of several tish in tropical South America. Those which live in active rivers have hemoglobin which is sensitive to $\mathrm{CO}_{2}$ 
(Table 56). In one which is found near waterfalls (the paku), addition of $25 \mathrm{~mm}$. CO. decreased the oxygen saturation by 48 per cent; in three other river fish a similar amount of $\mathrm{CO}_{2}$ reduced the oxygenation by about 25 per cent. In three species inhabiting sluggish marshy ponds, where the water $\mathrm{pH}$ varied from 3.8 to 5.0 , the $\mathrm{CO}_{2}$ effect was much less: $12-13$ per cent reduction in saturation by $25 \mathrm{~mm}$. $\mathrm{CO}_{2}$ in two of them and only 7 per cent reduction in the electric eel. The $t_{1 / 2}$ at in these sluggish-water fish is similar to the $t_{1 / 2}$ sat in those from fast waters, but the blood of the former is tolerant of higher $\mathrm{CO}_{2}$ in the water.

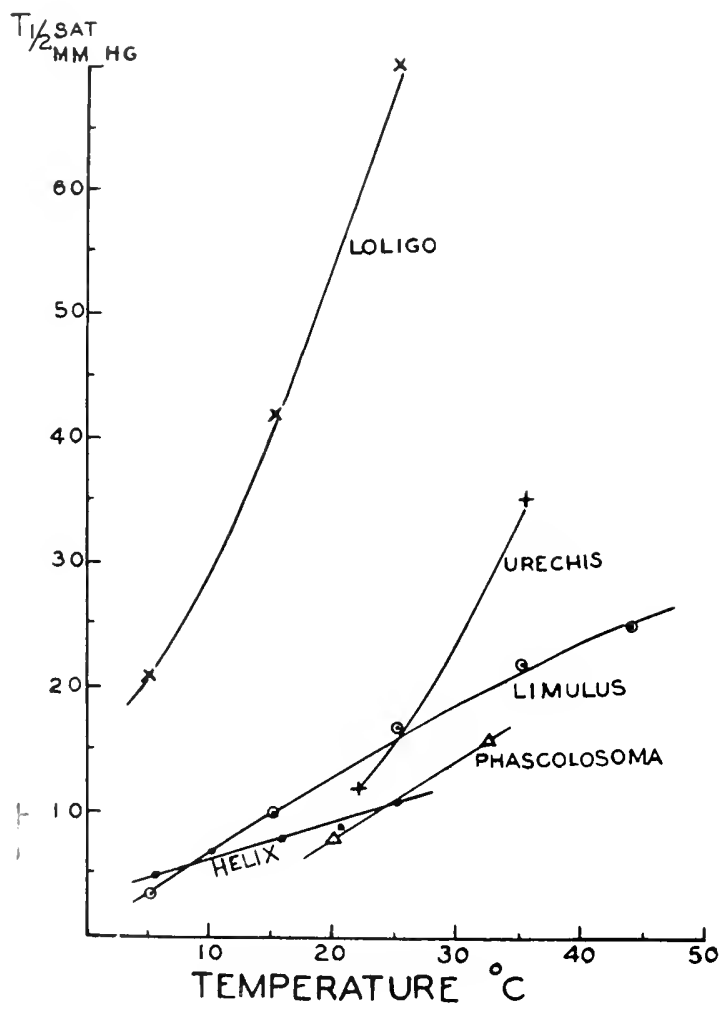

Fig. 73. Effects of temperature on the $\mathrm{O}_{2}$ tension for half saturation of blood. $\bullet$ Helix, Wolvekamp and Kersten ${ }^{1 * 1} ; \bullet$ Limulus, X Loligo, Redfield and Ingalls ${ }^{12 \pi} ; \triangle$ Phascolosoma, Florkin""; + Urechis, Redfield and Florkin. ${ }^{123}$

The blood of some fish is also very sensitive to changes in temperature. A rise in temperature acts like $\mathrm{CO}_{2}$ to lower the $\mathrm{O}_{2 .}$ affinity. Figure 74 shows that in the trout il rise in temperature of a few degrees raises the unloading tension significantly. In three different species of trout the $t_{1 / 2}-$ sat rises about $1 \mathrm{~mm}$. Ilg of oxygen partial pressure per degree centigrade. At higher temperatures the amount of oxygen dissolved in water is also diminished. The effect of temperature on the avalability of oxygen is undoubtedly a factor in the 
distribution of fish. However, in goldfish the effect of $\mathrm{CO}$... on $\mathrm{O}_{2}$ utilization decreases with rising temperature.

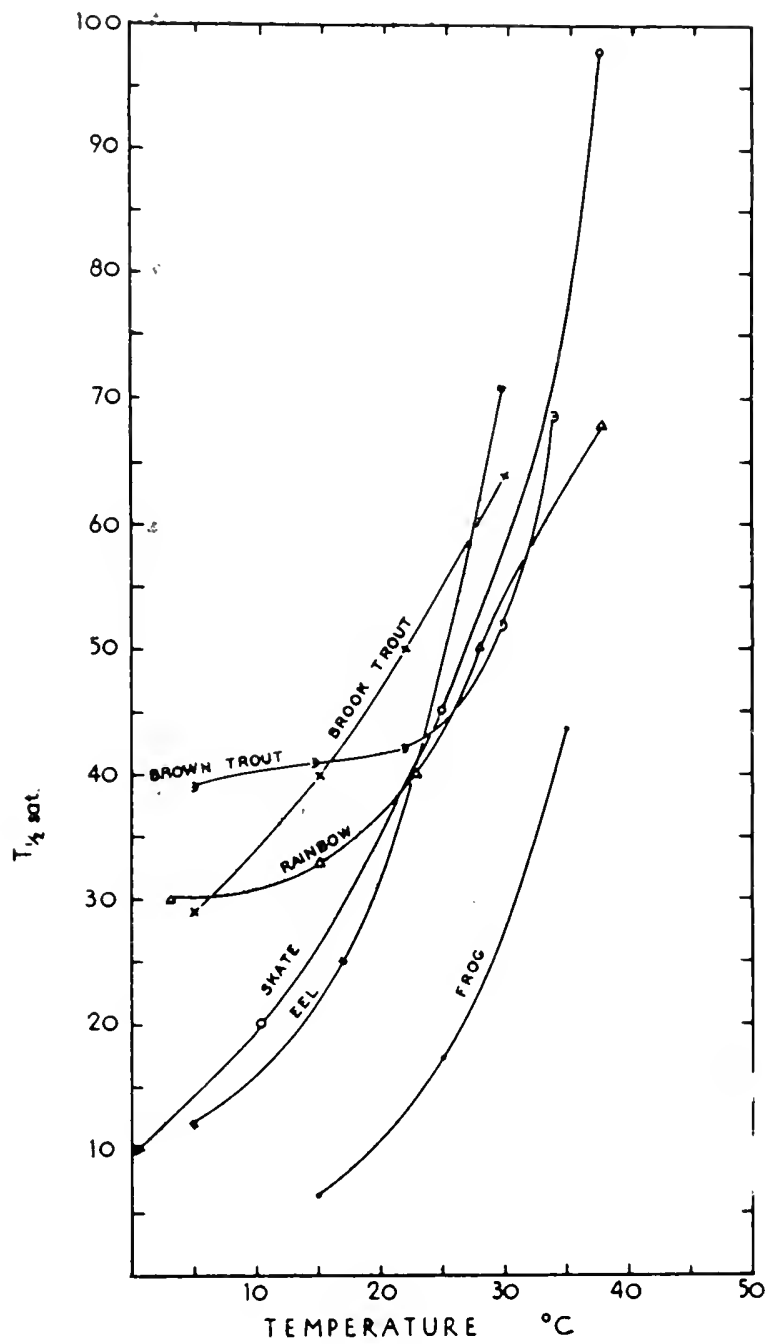

Fig. 74. Effects of temperature on the $\mathrm{O}_{2}$ tension for half saturation of blood of lower vertebrates. - frog, Macela and Seliskar ${ }^{107} ; \bigcirc$ skate, Dill et al. ${ }^{25} ; \times$ brook trout, $\triangle$ rainbow trout, $\odot$ brown trout, Irving, Black, and Safford ${ }^{6 \bar{i}} ;=$ eel, Kawamoto. ${ }^{\text {pas }}$

It has been mentioned previously (Ch. 8) that during periods when the oxygen supply is low some fish may draw on the oxygen in their swim bladders. The oxygen content of swim bladders in physoclystous fish-those with closed bladders, such as sunfish, perch, bass and stickleback-is often higher than that of air. ${ }^{10 t, 138}$ The amount of oxygen secreted into the bladder is related to the $\mathrm{CO}_{2}$ effect on tre dissociation of fish hemoglobin. When $\mathrm{CO}_{2}$ increases 
in the blood, oxygen is forced out in to the swim bladder and the fish may be asphyxiated.

Hemoglobin Function in Invertebrates. The function of blood pigments is in doult for many invertebrates. The following sorts of evidence are useful in judging whether a respiratory pigment functions in oxygen transport: (1) the position of the oxygen dissociation curve, i.e., whether the pigment loads and unloads under phisiological conditions at tensions corresponding to those at the respiratory surface and in the tissues; (2) the effect of $\mathrm{CO}$., i.e., whether

\section{$\%$ SATURATION WITH OXYGEN}

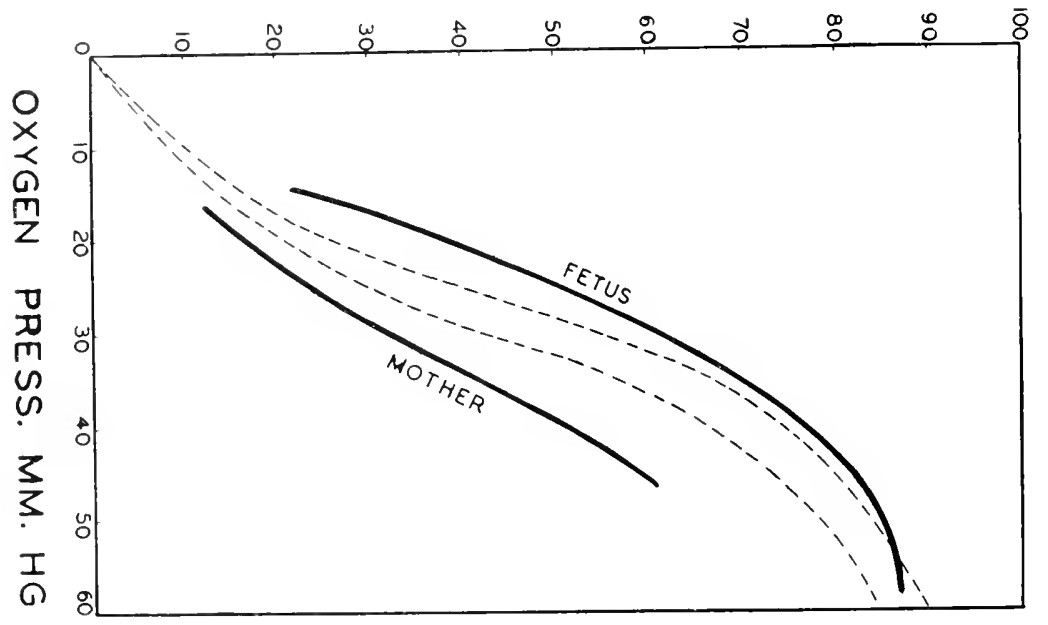

Fig. 75. Oxygen dissociation curves of maternal and fetal blood of goats. Data for grat pregnant 18 weeks, 2 days. Broken lines indicate limits of normal adults. From Barcruft et al. ${ }^{10}$

the addition of $\mathrm{CO}$. facilitates unloading of $\mathrm{O}_{2.2}$ at a given tension and, conversely, whether loss of $\mathrm{CO}_{2}$ aids the loading of $\mathrm{O}_{2}$ at respiratory tensions: (3) the oxygen capacity of the blood and the difference between the $\mathrm{O}_{2}$ combined and uncombined with pigment, as compared with the oxygen requirement of the animal; ( 4 ) differences in $\mathrm{O}_{2}$ content in arterial and venous blood indicating utilization; (5) effect on oxygen consumption of the animal of poisoning or of inactivating the pigment at different oxygen tensions. In invertebrates in which the function of blood pigments is uncertain it is not always possible 
to make all of the aforementioned tests. Evidence is sufficient, however, to show that in some lower animals blood pigment functions in (). transport at all oxygen tensions as in the vertebrates, in some it functions in tramsport only at low tensions, in others it may be an oxygen store for use in periods of hypoxia, and in still others it has no function with respect to oxygen supply.

In Lumbricus, Chironomus, and Tubifex carbon monoxide poisoning dem. onstrates the transport function of hemoglobin. In monoxide experiments it is important that the maintained combination of the hemoglobin with $\mathrm{CO}$ be

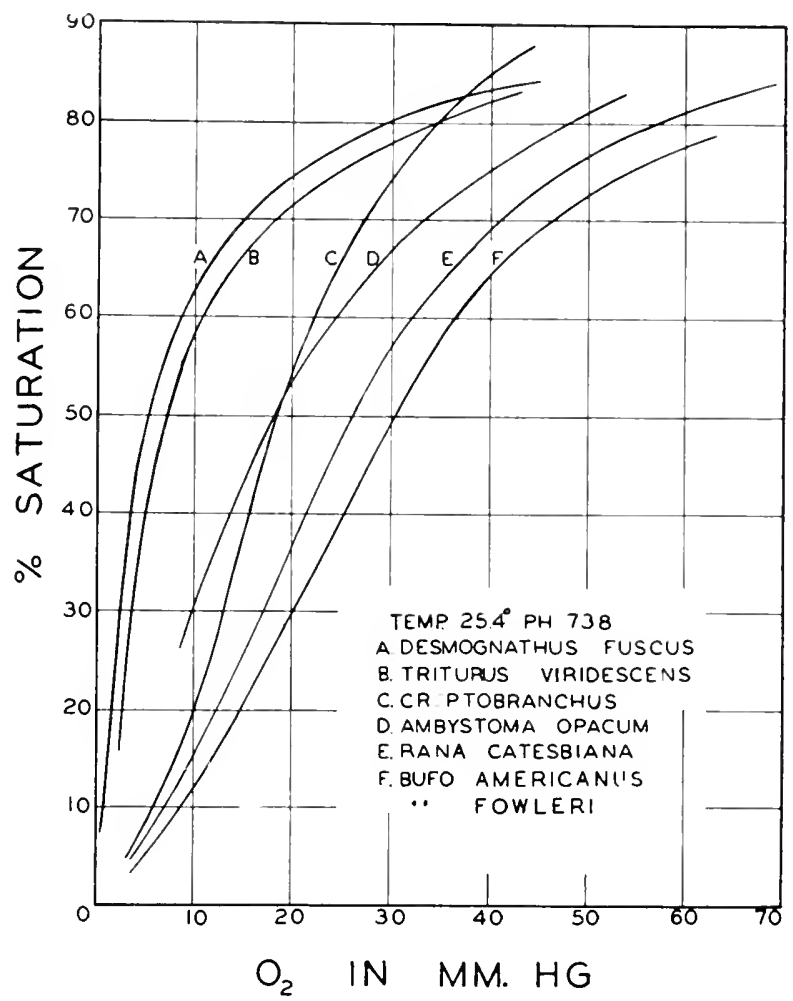

Fig. 76. Oxygen dissociation curves of several species of amphibians.

From McCutcheon and Hall. ${ }^{114}$

checked spectrosepically, and that the amounts of CO used be small enough that there is no interference with tissue respiration. In the earthworm, oxygen consumption has been measured in three investigations at different oxygen tensions in the presence and absence of carbon monoxide. One study ${ }^{151}$ revealed no effect of $\mathrm{CO}$ on respiration. Another:"i showed little effect of $\mathrm{CO}$ in reducing the respiration down to an $\mathrm{O}_{2}$, tension of about $55 \mathrm{~mm}$. ( 7.5 per cent $\mathrm{O}_{2}$ ), but below that value there was some reduction in $\mathrm{O}_{2}$ consumption. In the most careful study, ${ }^{: 0}$ saturation of the hemoglobin by $\mathrm{CO}$ was checked systematically and it was found that the respiration was reduced by $\mathrm{CO}$ at high 
oxygen tensions but that the effect of $\mathrm{CO}$ disappeared in low oxygen (Fig. 77). It was calculated ${ }^{\prime \prime \prime}$ that of the total oxygen used by the worm 23 per cent is carried by hemoglobin at $152 \mathrm{~mm} . \mathrm{O}_{2}, 35$ per cent at $76 . \mathrm{mm} . \mathrm{O}_{2}, 40$ per cent at $35 \mathrm{~mm}$. $\mathrm{O}_{2}, 22$ per cent at $19 \mathrm{~mm}$. $\mathrm{O}_{2}$ and 0 per cent at $8 \mathrm{~mm}$. $\mathrm{O}_{2}$. Dissociation curves for earthworm hemoglobin are not available.

In Nereis diversicolor inactivation of hemoglobin by $\mathrm{CO}$ reduces the oxygen consumption by approximately 50 per cent at high oxygen tensions $(6-7 \mathrm{ml}$. $\mathrm{O}_{2 .}$ 1.), and blocks all $\mathrm{O}_{2 .}$ consumption at $3.3 \mathrm{ml}$. $\mathrm{O}_{2 .} / \mathrm{l}^{9.2}$ Nereis diversicolor,

Fig. 77
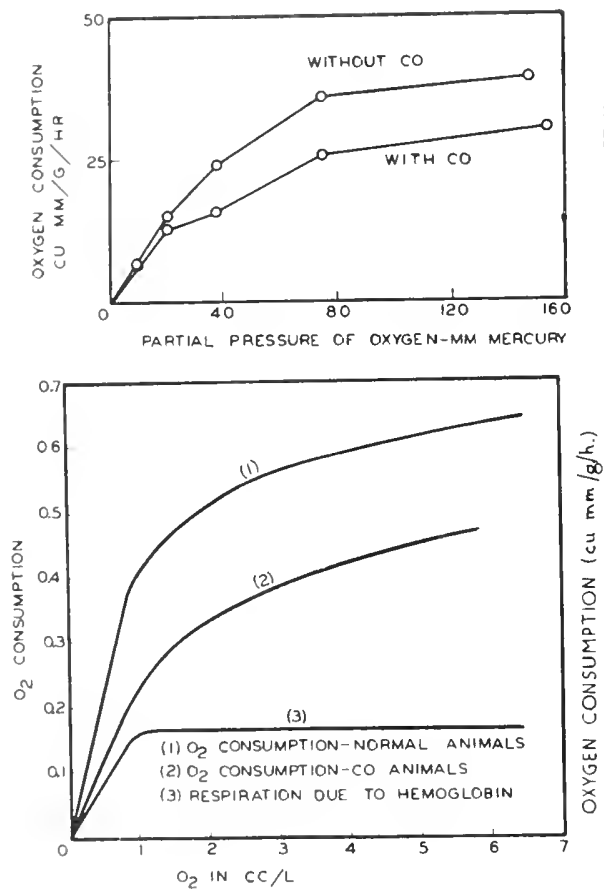

Fig. 79
Fig. 78
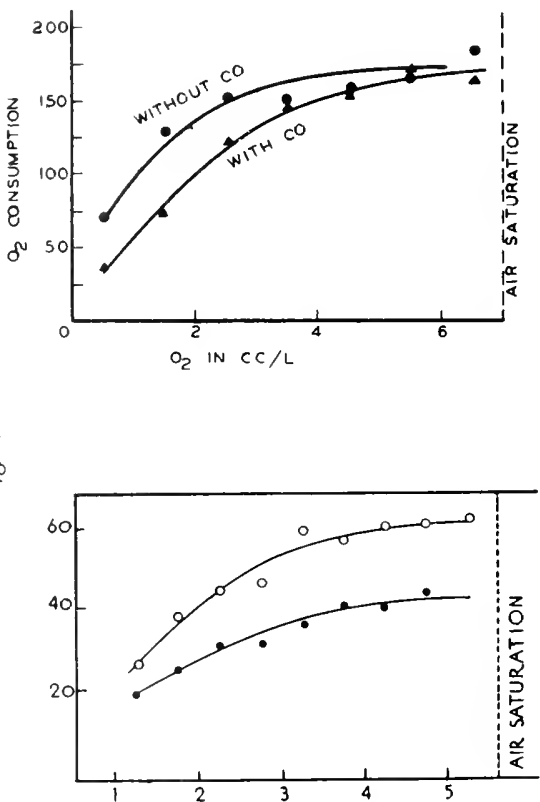

OXYGEN CONCENTRATION (c.c./l)

Fig. 80

Fig. 77. Oxrgen consunption by Lumbricus as a function of oxygen tension with and without carbon monoxide. From Johnson."

Fig. 78. Oxygen consumption by Chironomus as a function of oxygen tension in the presence and absence of CO. From Ewer. ${ }^{+4}$

Fig. 79. Oxygen consumption by Tubifex as a function of oxygen tension in presence and abence of (') ( Curve 3 is the difference between curves 1 and 2. From Dausend."

Fig. 8(). ()xygen comsumption by Sabella paronia as a function of oxygen tension with the chlorscruorin: $\bigcirc$, unpoisoned and $\bullet$, poisoned by CO. From Ewer and Fox.". 
therefore, appears to be more dependent on its hemoglobin than is Lumbricus. Nereis diversicolor consumes approximately ten times as much oxygen as Lumbricus, under comparable conditions.

Chironomus larvae and tubificid worms can live at the bottom of ponds where the oxygen content approaches zero. Those species of Chironomus larvae which lack hemoglobin are not so resistant to low oxygen as those with hemoglobin. Using a microspectrometric method for oxvhemoglobin determination, Leitch ${ }^{10.5}$ found that at $20^{\circ}$ in the absence of $\mathrm{CO}_{2}$ the blood of larvae of Chironomus sp. was half saturated at the exceedingly low oxygen tension of $0.17 \mathrm{~mm}$. Hg. In the presence of 1 per cent $\mathrm{CO}_{2}$, it was 38 per cent saturated at this tension. The very low $\mathrm{t}_{1 / 2}$ at was confirmed $\left(0.6 \mathrm{~mm}\right.$. Hg) by Fox ${ }^{* j 0}$ with Chironomus riparus, although the decreased oxygen affinity with $\mathrm{CO}$ was not confirmed. The oxygen capacity of the blood is 6 volumes per cent, and the total combined oxygen could supply the needs of the larvae for only 12 minutes; hence function as an oxygen store is unimportant. ${ }^{105}$ From the very low $\mathrm{t}_{1 / 2}$ sat it might be concluded that tissues could scarcely make use of oxygen delivered at such low tensions. However, the oxylemoglobin bands disappear in vivo when the oxygen in the water corresponds to $13 \mathrm{~mm} .{ }^{60} \mathrm{In}$ Chironomus plumosus the $\mathrm{O}_{2 .}$ consumption is normal in the presence of $\mathrm{CO}$ down to $\mathrm{O}_{2}$ tensions of approximately $75 \mathrm{~mm}$. (3 cc. $\mathrm{O}_{2 .} / \mathrm{l}$.) but is inhibited below this level (Fig. 78). ${ }^{ \pm t}$ It was also indicated ${ }^{75} \cdot{ }^{152}$ that in payment of an oxygen debt after a period of anoxia the excess oxygen consumption in Chirononus thummi is sensitive to $\mathrm{CO}$ in concentrations which poison the hemoglobin. Here, then, is evidence that the Chironomus hemoglobin unloads at oxygen tension of less than $1 \mathrm{~mm}$. $\mathrm{Hg}$ (0.079 per cent atm., according to Fox 6io), and, at the other extreme, evidence that the hemoglobin functions in oxygen transport at atmospheric tensions outside the animal. The unloading tension of blood in vitro cannot be used to determine the oxygen tension in the medium at which a pigment is functional. It appears certain that in chironomid larvae living in low oxygen the oxygen tension in the tissues is very low and that a steep oxygen gradient exists from water to tissues. The oxidative enzyme pattern in the tissues must differ from that in the vertebrates to make possible functioning at partial oxygen pressures of $0.5 \mathrm{~mm}$. $\mathrm{Hg}$. In a chironomid Tantytarsus, which lives in lakes where the water is never more than half saturated with air, the hemoglobin functions in transport only if the dissolved oxygen is less than 25 per cent air saturation..$^{152}$

In Tubifex also the hemoglobin is half saturated at $0.6 \mathrm{~mm}$. Hg, but in vivo the bands disappear at oxygen concentrations near the limit of the Winkler method of analysis (about $10 \mathrm{~mm}$. $\mathrm{Hg}^{60}$ ). The oxygen consumption as a function of $\mathrm{O}_{2}$ concentration declines steeply below $1-1.5 \mathrm{cc}$. $\mathrm{O}_{2} / \mathrm{l}$. ( $\mathrm{t}_{\mathrm{t}}=$ approximately $37 \mathrm{~mm}$.). In the presence of $\mathrm{CO}$ the respiration is reduced by about one-third at higher oxygen tensions, proportionately less at lower tensions ${ }^{27}$ (Fig. 79). Two thirds of the normal oxygen requirement is supplied by oxygen uncombined in solution in the body fluid. As in Chironomus, the $t_{1 / 2}$ sat is far below the tension in water, where oxygen transport by hemoglobin is important and the gradient from water to tissue is very steep.

Some species of Daphnia contain hemoglobin in varying amounts; the hemoglobin content of the blood increases when the oxygen in the water is low. However, Daphnia can swim actively when the $\mathrm{O}_{2}$ is so low that the 
hemoglobin remains reduced or when it is poisoned by $\mathrm{CO}$ and the hemoglobin can hardly be functional. 61.633

Claims have been made that in the snail Planorbis and the lugworm Arenicola hemoglobin may function as an oxygen store to be used only in times of anoxic stress. In the absence of $\mathrm{CO}_{2}$ the $\mathrm{t}_{1 / 2}$ sat of Planorbis blood is higher than the $\mathrm{t}_{1 / 2}$ sat of Chironomus and Tubifex blood, $7.4 \mathrm{~mm}$. at $20^{\circ 105}$ and 1.9 $\mathrm{mm}$. at $17^{\circ}$.6.0 Carbon dioxide decreases the affinity for oxygen $\left(\mathrm{t}_{1 / 2}\right.$ sat $=8.9$ $\mathrm{mm}$. at $17^{\circ}$ in 1 per cent $\mathrm{CO}_{2 .}$ ). ${ }^{160}$ In vivo the oxyhemoglobin bands disappear at water oxygen of $25 \mathrm{~mm}$. Hg, ${ }^{, 0}$ but the blood remains saturated with oxygen, and skin breathing with the oxygen carried in solution in the blood provides sufficient oxygen for the animal down to a tension of 7.7 per cent $\mathrm{O}_{2}(54 \mathrm{~mm}$. $\mathrm{Hg})^{105}$ In the range 7.2 to 3 per cent $\mathrm{O}_{2}$ the snails frequently came to the surface for lung breathing and the hemoglobin was not completely saturated, whereas below 3 per cent they were at the surface continuously. In the presence of $\mathrm{CO}$ below 3 per cent $\mathrm{O}_{2}$ the snails became very sluggish, and at 1 per cent $\mathrm{O}_{2}$ they floated outstretched on the water. Hence the use of the lung and reduction of hemoglobin go together. From the data of oxygen capacity of the blood (2.4 times that which could be carried in solution), the blood volume (0.58 cc. $/ \mathrm{g}$. body wt.) and the oxygen consumption (0.026 cc./g./hr.) Borden ${ }^{18}$ estimated that the oxygen held by hemoglobin would last 18 minutes of anoxia and Leitch ${ }^{105}$ estimated it would last 3 minutes. It appears that the storage function is negligible, that hemoglobin functions in oxygen transport, particularly at water oxygen of $25 \mathrm{~mm}$. and below, and that the tissue oxygen tension is not so low as in Chironomus and Tubifex.

In Arenicola the hemoglobin is reduced at $1-3 \mathrm{~mm}$. $\mathrm{Hg}\left(1.8 \mathrm{~mm} .{ }^{.00}\right)$ and is completely saturated at $5-10 \mathrm{~mm}$. $\mathrm{O}_{22}$. The oxygen capacity is about one-sixth that in man." $\mathrm{CO}_{2}$ shifts the oxygen dissociation curve to the right.". 60 In the burrows of Arenicola the oxygen becomes depleted at low tide.". is At such times the tension in the tissues must be much reduced. If the hemoglobin were to be completely reduced, enough oxygen would be made available for 21 minutes at the usual rate of $\mathrm{O}_{2}$ consumption. In nature the worms survive periods of about 3 hours when the oxygen in their tubes is negligible. The state of the hemoglobin in living worms is not known, but the hemoglobin must unload oxygen to the tissues when their $\mathrm{O}_{2}$ tension is below $2 \mathrm{~mm}$. If the tension at the gills is greater, oxygen will be taken up. At low tensions the worms may well resort to anaerobic metabolism. In the absence of carbon monoxide experiments and measurements of the saturation of hemoglobin in vivo, judgment must be withheld regarding the function of the pigment at high oxygen tensions.

In study of the worm Urechis Redfield and Florkin ${ }^{123}$ found oxygen capacities of 2.6 to 7.2 volumes per cent in different individuals; there was little effect of (C).2 on the oxygen dissociation curve (Fig. 69). The hemoglobin showed an unloatling tension of $12 \mathrm{~mm}$. O.2 at $8.6 \mathrm{~mm}$. CO.2. The worm lives in mud tubes in the intertidal zone, and at low tide the oxygen in the burrow is greatly reduced. Redfield and Florkin calculated that when the water reached an ().2 tension of $14 \mathrm{~mm}$. the requirements of the animal could be met for only about 15 minutes by the oxygen dissolved in the coelomic fluid and in the water contaned in the respiratory region of the gut, whereas the extra oxygen from the bleod pigment would permit survival for a further 55 minutes. 
It is probable that this reserve is important at extreme low tide; this does not preclude the possibility that some of the hemoglobin molecules are alternately loaded and reduced as they move from the respiratory gut to body tissues.

The intracellular hemoglobins of invertebrates may serve as oxrgen reserves for times of stress, as in parasitic animals living in regions of low oxigen, for example the larva of Castrophilutsit and Ascaris.-- The dissociation curves of both of these are well to the left, with tensions of half saturation about $0.1 \mathrm{~mm}$. Hg partial pressure of oxygen. In Ascaris the hemoghohin holds oxygen avidly, that is, unloads very slowly (Table 51), and when the worms are in anacrobic conditions the body wall pigment, but not that in the perienteric fluid, can be seen to be deoxygenated. Similarly in the nematode Nippostrongy/us, with a $\mathrm{t}_{1 / 2}$ sat of less than $0.1 \mathrm{~mm}$. $1 \mathrm{lg}$, deoxygenation is seen in low oxygen, but the closely related Strongylus dies in low oxygen before the pigment is deoxygenated. 25

It is apparent from the preceding examples that the hemoglobineontaining invertebrates have part of their oxygen needs supplied from the gas in solution in their blood. In Urechis, for example, the worm uses oxygen only to the equivalent of 160 of that held by its hemoglobin per hour. In monoxidetreated animals, usually less than half of the respiration is affected. If the oxygen tension to which hemoglobin is exposed is sufficiently low the pigment will unload oxygen. The reduced molecules may immediately thereafter become oxygenated, or they may wait for hours until oxygen is restored. Thus the question of store versus transport is resolved. In animals like Chironomus and Tubifex, tissue oxygen is very low; in Planorbis and Daphnia it is higher. ${ }^{\text {;") }}$ In an animal like Urechis, with no circulatory system, some molecules will be reduced while others are oxygenated, and they will be continuously mixed by the churning of body movements. The added survival time clue to hemoghobin at oxygen tensions where saturation is no longer possible may be of marginal significance. A number of marine worms carry on an active life with no blood pigment whatever (Table 49). Some of them, like Chaetopterus, are mud dwellers; Daphnia continues active when its hemoglobin is poisoned. Some invertebrate hemoglobins appear to provide a safety factor of oxygen, and they function at low oxygen tensions; they may act across steep oxygen gradients. The observations on Chironomus and Tubifex show the futility of inferring function from saturation values of the pigment. Some parasitic species may unload their hemoglobin only under anoxic stress.

\section{Function of Hemerythrin}

The oxygen dissociation curves of hemerythrins, although in the low oxygen range, show $\mathrm{t}_{1 / 2}$, wat values which might be reached by the body tissues of sluggish worms (Table 55). A rise in temperature shifts the $\mathrm{O}_{2}$ dissociation curve of Phascolosona to the right, but changes in pll do not have much effect on it. ${ }^{10: 4}$ In Sipunculus the $\mathrm{t}_{1 / 2}$ sat Was $8 \mathrm{~mm}$. Hg whether the $\mathrm{CO}_{2}$ was 0.07 or $80 \mathrm{~mm}$. Hg."' The $\mathrm{O} .2_{2}$ tension found in the coelomic fluid of Siptuculus in sea water was $32 \mathrm{~mm}$. I Ig, at which tension its hemerythrin would remain saturated. The tissue tensions are not known. However, in the mud when the tide is out, Sipunculus is exposed to very low oxygen tensions. At such times, like hemoglobin in Urechis and Arenicola, the hemerythrin may give up its oxygen to the tissues. ${ }^{4 !, 50}$ 


\section{Function of Chlorocruorin}

In a series of papers, Fox ${ }^{56}, 57,59$ has reported studies of the function of chlorocruorin in the sabellid worms, particularly in Spirographis. The oxygen dissociation curve of this pigment is shifted to the right, with increase in acidity and with a rise in temperature, just as in vertebrate hemoglobin. The $t_{1 / 2}$ sat is sufficiently high $\left(27 \mathrm{~mm}\right.$. Hg at $\mathrm{pH} 7.7$ and $\left.20^{\circ}\right)$ that the pigment probably is deoxygenated in the tissues and oxygenated at the gills. The oxygen consumption is reduced by $\mathrm{CO}$ over a wide range of oxygen tensions (Fig. 80). ${ }^{45}$ A variable fraction of the needed oxygen is supplied by the blood pigments, the remainder is carried in solution. Chlorocruorin is definitely an oxygen carrier functioning normally at high oxygen tensions.

\section{Function of Hemocyanin}

The blood pigment hemocyanin which occurs in many molluscs and arthropods can combine reversibly with oxygen. The higher the hemocyanin content of the blood as measured by the copper content, the greater the oxygen capacity (Table 54). The physiology and biochemistry of hemocyanin have been well summarized by Redfield. ${ }^{121}$

In the cephalopod molluscs it has long been known that hemocyanin functions as an oxygen carrier. ${ }^{79}$ The blood of Octopus has an $\mathrm{O}_{2}$ capacity of 4.25.0 volumes per cent, ${ }^{161}$ and Redfield and Goodkind ${ }^{12+}$ found the blood of Loligo to have an oxygen capacity of 4.2 volumes per cent. Arterial blood taken from the heart of the squid contained on the average 4.27 volumes per cent of oxygen and 3.82 volumes per cent of $\mathrm{CO}_{2}$, whereas venous blood had 0.37 volumes per cent of $\mathrm{O}_{2}$ and 8.27 volumes per cent of $\mathrm{CO}_{2}$. Thus approximately 92 per cent of the oxygen was removed in the course of circulation, approximately three times as much as in man. This high transfer of oxygen to the tissues fails to provide much reserve against hypoxic stress. Similar data on arterial blood in Octopus were obtained by Winterstein. ${ }^{161}$ The color of the blood can be seen to change as it passes through the gills of a squid, so that the oxygenated blood is distinctly blue. In the cephalopods $\mathrm{CO}_{2}$ and increased acidity shift the oxygen dissociation curve to the right as with hemoglobin (Fig. 81).124, 16: A rise in temperature also raises the tensions of loading and unloading (Fig. 73). The effect of CO.2 upon dissociation of oxyhemocyanin accounts for about one third of the respiratory exchange in the squid. The $t_{8 a t}$ and $t_{1 / 2}$ sat are in a reasonable range for physiological function (Table 56), although they are sufficiently high to make the squid sensitive to asphyxiation. It is well established, then, that hemocyanin serves as an efficient oxygen carrier in the cephalopod molluscs.

In the gastropods, crustaceans, and arachnoids, however, evidence for function of hemocyanin as an oxygen carrier is less convincing. No specific inhibitor, as $\mathrm{CO}$ for hemoglobin, is known for hemoeyanin. In limulus the blood always appears reduced (colorless) when the animals are bled, and it contains only as much (). as sea water does." When saturated with air the blood of Limulus, of sereral crabs, and of Helix takes up only about 2 to 4 times as much oxygen as seal water does (Table 56). The position of the oxygen dissociation curve of Ilelix and Limulus ( $\mathrm{t}_{1 / 2}$ int $=6-12 \mathrm{~mm}$. Hg) is within the usual physiological range, but the curve of Homarus blood is far to the right at blood pl $\left(\mathrm{t}_{1 / 2 \mathrm{sat}}=90 \mathrm{~mm}\right.$. Hg). 
The effect of $\mathrm{CO}_{2}$ or of decreased $\mathrm{p}^{\mathrm{H}} \mathrm{H}$ is shown for Limulus in Figures 81 and 82. As the solution of hemocranin becomes more alkaline the oxygen dissociation curve shifts to the right up to $\mathrm{pl}$ I of 8.6; at pll 9.4 the curve is moved back to the left. In Limulus, Busycon, and Helix the minimum affinity

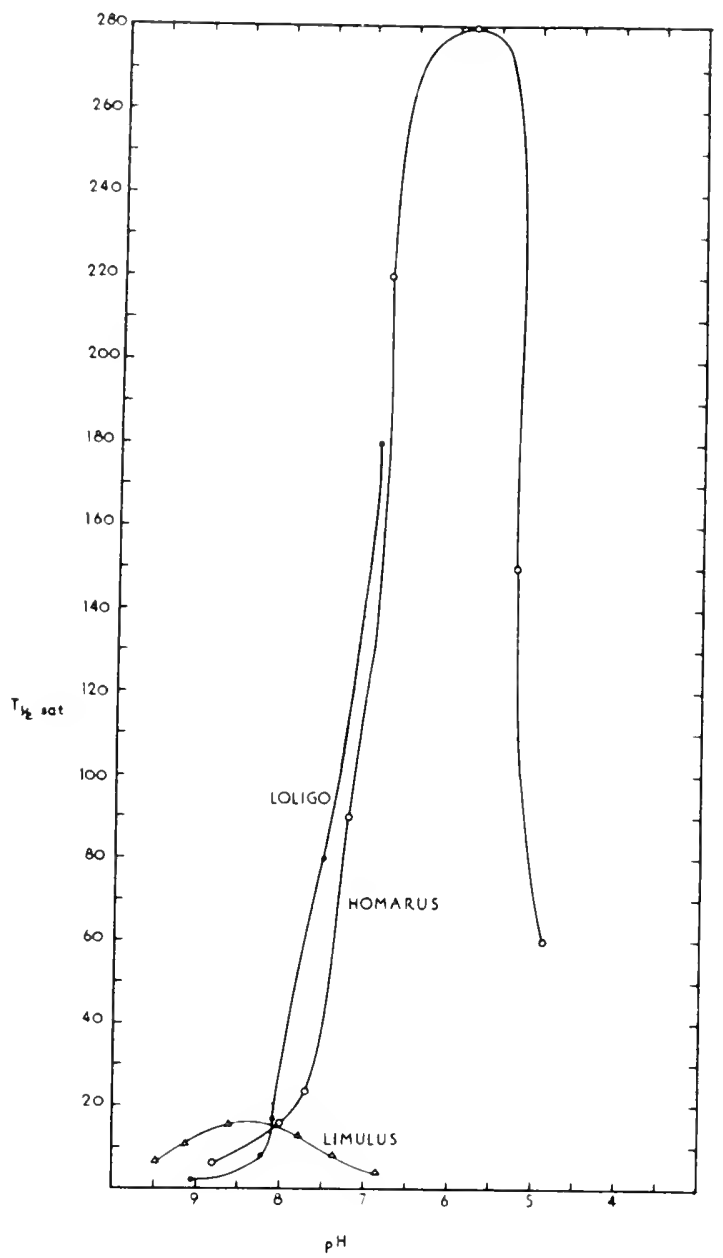

Fig. 81. Effect of $\mathrm{pH}$ on $\mathrm{O}_{2}$ tension for half saturation of blood. Loligo, $\bigcirc$ Homarus, $\triangle$ Limulus. Redfield and Ingalls. ${ }^{1: t}$

for oxygen is in the alkaline range, whereas in Loligo, Homarns, Maja, and Cancer the minimum $\mathrm{O}$. affinity is on the acid side, as it is for hemoglobin (Fig. 81).124 In the snail Helix the affinity for oxyen is minimum at $\mathrm{pH} 7.7$, and the oxygen dissociation curve moves to the left on both the acid and alkaline side of this $\mathrm{pH}^{\mathrm{H}}{ }^{* 2}$ It has been suggested ${ }^{1+1}$ that the increased $\mathrm{O}_{2}$ affinity with added $\mathrm{CO}_{2}$ permits the hemocyanin to load with oxygen when animals like Busycon and Limulus enter stagnant high-CO.- water. Certainly the blood would load at a lower $\mathrm{O}_{2}$ tension in the presence of $\mathrm{CO}_{2}$ than in its 
absence. However, the blood must always be at a higher $\mathrm{CO}_{2}$ tension than the water and the hemocyanin (Hcy) could unload only if the tissue oxygen tensions are unusually low.

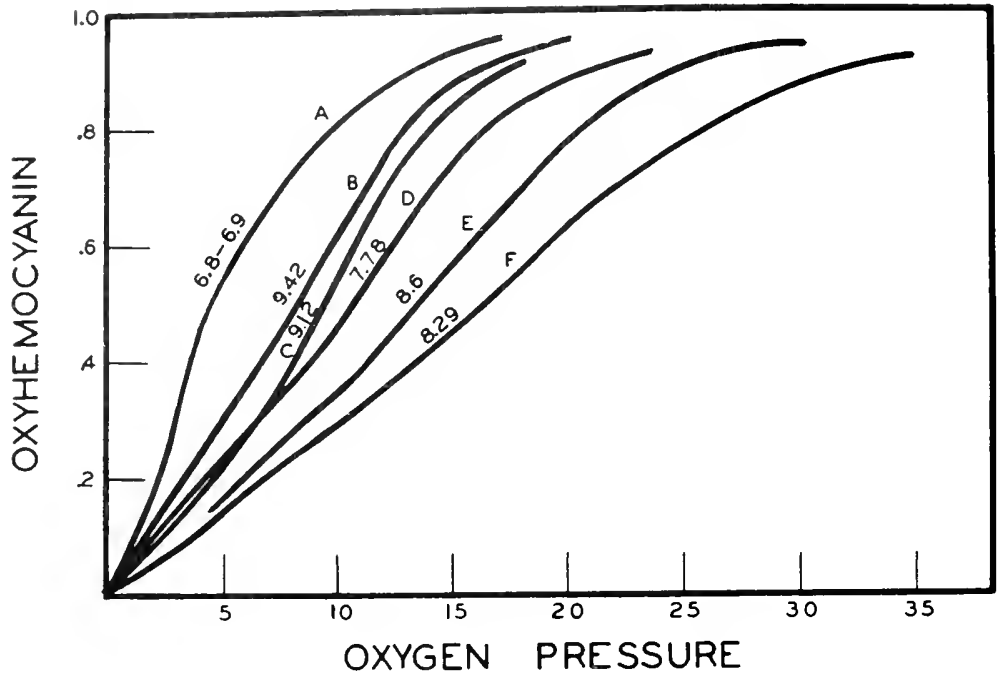

Fig. 82. Oxygen dissociation curves of Limulus hemocyanin at different hydrogen ion concentrations. From Redfield and Ingalls. ${ }^{127}$

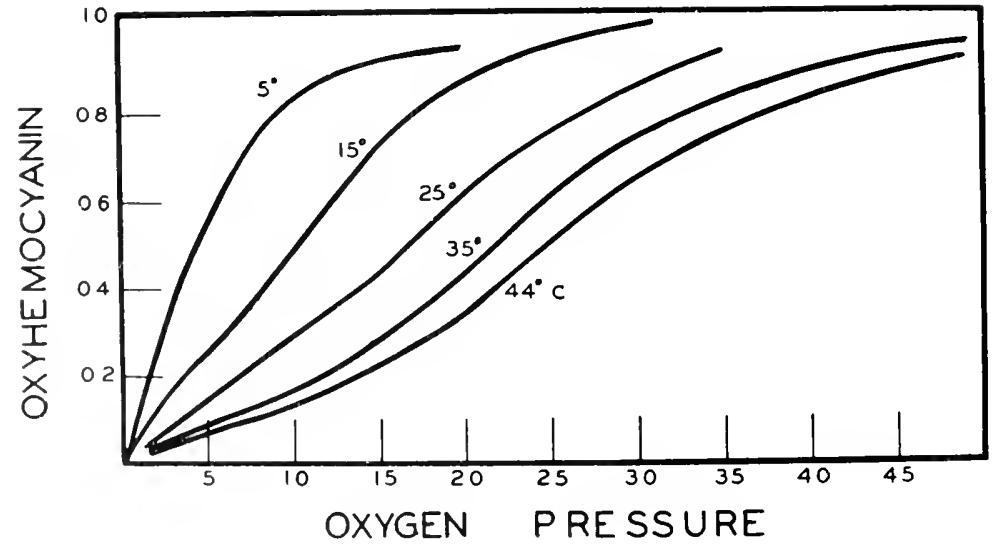

Fig. 83. ()xygen dissociation curves of Limulus hemocyanin at different temperatures. From Ricelficld and Ingalls. ${ }^{197}$

In Butsycon the arterial oxygen is at $36 \mathrm{~mm}$. $1 \mathrm{lg}$ and the venous at $6 \mathrm{~mm}$. IIg, correspending to an unloading to the tissues of 1.7 volumes per cent. Less than 10 per cent of this oxygen is carried in solution.:5: Apparently in Busyeon the tissue oxygen tensions are very low, and the hemocyanin trans- 
ports much of the oxygen. Direct measurements of oxygen tension and Itey saturation in blood entering and leaving the gills in Limulus, Homarns and others would settle the question of whether / lev functions in these animals als an oxygen carrier.

A rise in temperature moses the oxyen disseciation curve to the right in those hemocyanins in which it has been exanined"21 (Fig. 83).

It must be concluded that convincing evidence that hemocyanin functions in oxyen transport is available only for the cephatopod molluses and Busycon. However, measurements of per cent saturation of hemocyanin entering and leaving the gills of Limulus and of similar animals have not been made.

\section{CARBON DIOXIDE TRANSPORT}

CO.2 Dissociation Curve. Just as with oxygen, the amount of carbon dioxide contained in body fluids greatly exceds the amount in solation. The solubitity

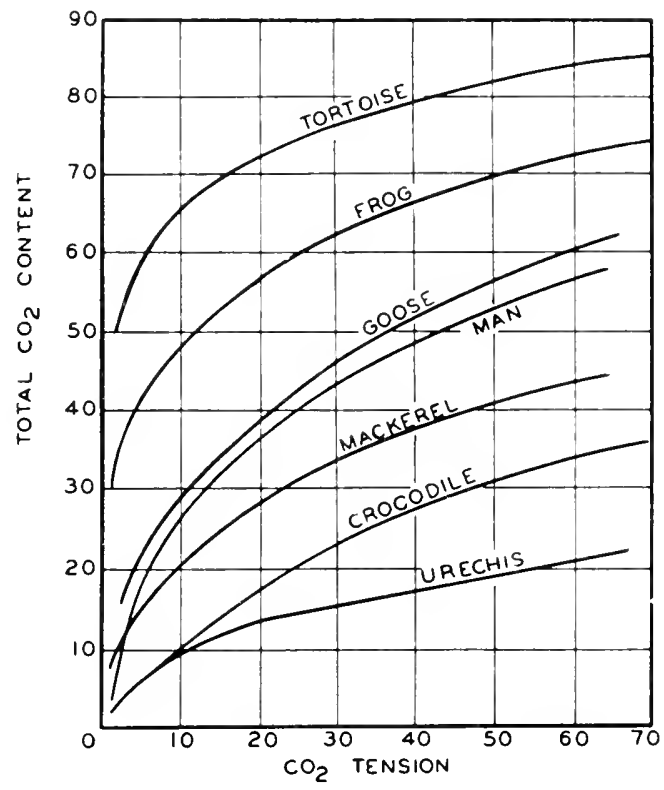

Fig. 84. Carbon dioxide dissociation curves. Volumes per cent of $\mathrm{CO}_{2}$ as a function of $\mathrm{CO}_{2}$ tension in mm. Hg. Data assembled by Redfield. ${ }^{121}$

of $\mathrm{CO}_{2}$ in human blood is 48 volumes per cent at $760 \mathrm{~mm}$. pressure and $37.5^{\circ}$, the $\mathrm{CO}_{2}$ tension in alveolar air is $40 \mathrm{~mm}$., hence the amount of $\mathrm{CO}_{2}$ which might be dissolved in the blood is 2.5 volumes per cent. Actually arterial blood contains 45-50 volumes per cent. Similarly, sea water has a solubility coeffcient at $24^{\circ}$ of 0.71 volumes per cent of $\mathrm{CO}_{2}$ and in equilibrium with air where the $\mathrm{CO}_{2}$ partial pressure is $0.23 \mathrm{~mm}$. I Ig it would dissolve 0.0215 volumes per cent; normally sea water contains about 4.8 volumes per cent of $\mathrm{CO}_{2}$. The difference between the $\mathrm{CO}_{2}$ dissolved and the $\mathrm{CO}_{2}$ actually contained is due to the combination, largely as bicarbonate with cations, from various 
buffers. For each fluid containing buffers a $\mathrm{CO}_{2}$ dissociation curve can be constructed by equilibrating with a known $\mathrm{CO}_{2}$ tension and measuring the volumes per cent taken up. Thus in the two examples above, in human blood 40-50 volumes per cent corresponds to $40 \mathrm{~mm}$. CO.2, whereas in sea water 4.8 volumes per cent corresponds to $0.23 \mathrm{~mm}$. CO.2. Typical $\mathrm{CO}_{2}$. dissociation curves are given in Figure 84 . These indicate differences in the CO.r-combining ability of different species. Aquatic animals with a very

\section{TABLE 58. CO. CONTENT OF DRAWN BLOOD IN VOLUMES PER CENT AND CORRESPONDING CO TENSIONS. VALUES FOR SEA WATER ARE GIVEN FOR PURPOSES OF COMPARISON.}

\begin{tabular}{|c|c|c|c|}
\hline Animal & Type of Blood & Vols. 'c' & $\mathrm{mm} . \mathrm{Hg}$ \\
\hline \multicolumn{4}{|l|}{ Mammal } \\
\hline $\operatorname{Mlan}^{17}$ & $\begin{array}{l}\text { (arterial) } \\
\text { (venous) }\end{array}$ & $\begin{array}{l}45-50 \\
50-53\end{array}$ & $\begin{array}{l}40-42 \\
45-47\end{array}$ \\
\hline $\begin{array}{l}\text { Reptile } \\
\text { alligatorst } \\
\text { Amphibian }\end{array}$ & & $38.6-44$ & \\
\hline Rana catesbiana ${ }^{1-n}$ & $\begin{array}{l}\text { (arterial) } \\
\text { (venous) }\end{array}$ & $\begin{array}{l}58.2 \\
70.1\end{array}$ & $\begin{array}{l}35^{*} \\
65^{*}\end{array}$ \\
\hline \multicolumn{3}{|l|}{ Fish } & $8-10$ \\
\hline sucker ${ }^{11}$ & $(" \prime)$ & $36.3-47.8$ & $7-9$ \\
\hline $\begin{array}{l}\text { carp }^{13} \\
\text { calfish }^{\text {*t }}\end{array}$ & $(" n)$ & $\begin{array}{l}28.6-36.4 \\
21.4\end{array}$ & $\begin{array}{l}5-10 \\
8\end{array}$ \\
\hline lautog ${ }^{1: L b}$ & (venous) & & $10-15$ \\
\hline scup $^{13.3}$ & (arterial) & 9.5 & \\
\hline sea robin ${ }^{133}$ & $(" n)$ & 6.5 & 2 \\
\hline loadfish ${ }^{15: 3}$ & (venous) & 13.3 & 7 \\
\hline goosefish $^{\text {iak }}$ & $\left(\begin{array}{l}\prime \prime \\
(\prime)\end{array}\right.$ & 10.2 & \\
\hline puffer $^{1: 3.5}$ & (") & 14.9 & \\
\hline Raja vocellata & (arterial) & 7.7 & 1.3 \\
\hline Mustelus" & $\begin{array}{l}\text { (venous) } \\
\text { (arterial) } \\
\text { (venous) }\end{array}$ & $\begin{array}{l}10.8 \\
6-12\end{array}$ & $\begin{array}{l}2.6 \\
2-6 \\
4-12\end{array}$ \\
\hline Invertebrates & & & \\
\hline Urechis & & $7-9$ & 9 \\
\hline Limulus $^{12.5}$ & & $10-20$ & \\
\hline Palinurus ${ }^{34 ; i}$ & & $13-17$ & \\
\hline Astacus ${ }^{34 a}$ & & $25-35$ & \\
\hline Planorbis ${ }^{\text {:ii }}$ & & $55-62$ & \\
\hline Sepia & & $11-14$ & \\
\hline Helix & & $45-60$ & \\
\hline $\begin{array}{l}\text { Anodont:i } 1^{3, i} \\
\text { echinoderms }\end{array}$ & & $29-31$ & \\
\hline Sea walerit & & $\begin{array}{l}5-6 \\
4.8\end{array}$ & 0.23 \\
\hline
\end{tabular}

weak buffering supply of cations, such as ascidians, may actually contain less total $(\mathrm{C})$, than the surrounding water. ${ }^{53}$

The $\mathrm{CO}_{2}$ content of circulating blood depends on the total buffering capacity of the blood, the $\mathrm{CO}_{2}$ in body tissues, and the $\mathrm{CO}_{2}$ outside the respiratory surface. In Table 58 are given the CO.2 content in volumes per cent and corresponding $\mathrm{CO}_{2.2}$ tensions for freshly drawn blood from several animals. In terrestrial vertebrates the $\mathrm{CO}_{2}$ tension to which blood is exposed in the lungs 
is high (40 mm. Hg in many mammals), whereas in the water to which the gills of aquatic animals are exposed it is low $(0.23 \mathrm{~mm}$. I lg in equilibrium with air). Actually, the corresponding blood $\mathrm{CO}$. tensions vary nearly in proportion, so that in man there is a gradient of about $6 \mathrm{~mm}$. between venous blood and alveolar air, whereas in fish (fresh-water, ${ }^{+6}$ marine ${ }^{3.5}$ ) the $\mathrm{CO} .2$ gradient be tween venous blood and water is $4-9 \mathrm{~mm}$. In mammals the difference between arterial and venous $\mathrm{CO}_{2}$ indicates an unloading of about 10 per cent of the total in the lungs and loading of a similar amount in body tissues. The total $\mathrm{CO}_{2}$ in the blood is less and the percentage that is gained and lost in tissues and gills is greater in aquatic vertebrates (Table 58 ) than in terrestrial verte. brates.

Reactions to $\mathrm{CO}_{2}$ in Mammalian Blood. The reactions in $\mathrm{CO}_{2 .}$ transport in mammalian blood can be enumerated as follows:

1. CO.2 goes into solution in the blood, much of it diffusing into red cells. The $\mathrm{CO}_{2}$ reacts with water in plasma and in red cells

$$
\mathrm{CO}_{2}+\mathrm{H}_{2} \mathrm{O} \rightleftharpoons \mathrm{H}_{2} \mathrm{CO}_{3}
$$

2. This reaction occurs too slowly to provide for the known rate of loading in the tissues and unloading in the lungs; it is catalyzed by an enzyme, carbonic anhydrase, which is present in red blood cells

$$
\mathrm{CO}_{2}+\mathrm{H}_{2} \mathrm{O} \stackrel{\text { carbonic anhydrase }}{\rightleftharpoons} \mathrm{H}_{2,} \mathrm{CO}_{3}
$$

3. Carbonic acid dissociates according to mass action; it is a weak acid with a pK of 6.1

$$
\mathrm{H}_{2} \mathrm{CO}_{3} \rightleftharpoons \mathrm{H}^{+}+\mathrm{HCO}_{3}-
$$

The dissociation of $\mathrm{HCO}_{3}-$ is negligible at blood $\mathrm{pH}$.

4. Some anions are provided by plasma buffeis which take up excess $\mathrm{H}^{+}$, forming weak acids and leaving cations $\left(\mathrm{B}^{+}\right)$to form salts with $\mathrm{ICO}_{3}{ }^{-}$

$$
\begin{aligned}
& \mathrm{BHCO}_{3}+\mathrm{H}^{+} \rightleftharpoons \mathrm{H}_{2} \mathrm{CO}_{3}+\mathrm{B}^{+} \\
& \text {B proteinate }+\mathrm{H}^{+} \rightleftharpoons \mathrm{H} \text { proteinate }+\mathrm{B}^{+}
\end{aligned}
$$

5. Some $\mathrm{HCO}_{3}{ }^{-}$leaves the red cells in exchange for $\mathrm{Cl}^{-}$from $\mathrm{NaCl}$ of plasma. This provides $\mathrm{Na}^{+}$to form $\mathrm{NaHCO}_{3}$ and results in a shift of $\mathrm{Cl}^{-}$ from plasma to cells in the tissues and back out of the red cells in the lungs.

Tissues

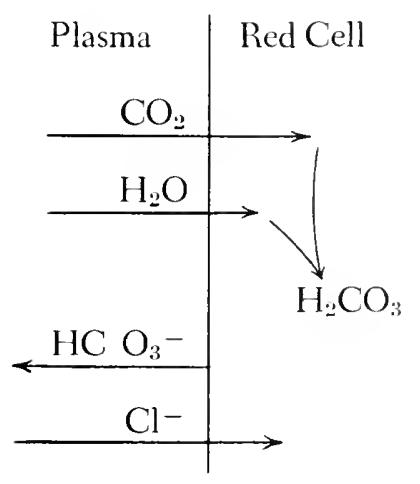

Lungs

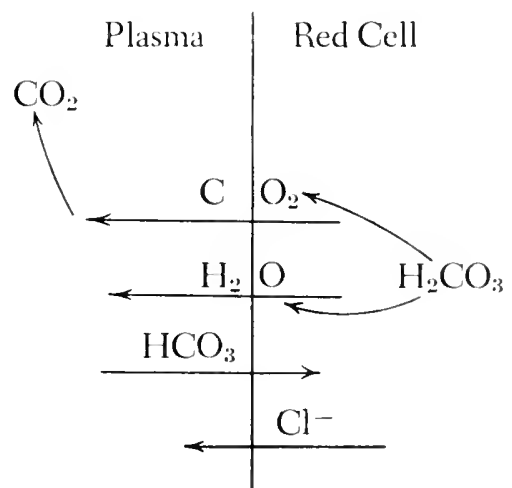


6. In the cells most buffering is provided by phosphate and hemoglobin

$$
\begin{aligned}
& \mathrm{K}_{2} \mathrm{HPO}_{4}+\mathrm{H}^{+} \rightleftharpoons \mathrm{KH}_{2} \mathrm{PO}_{4}+\mathrm{K}^{+} \\
& \mathrm{BH}+\mathrm{H}+\mathrm{H}^{+} \rightleftharpoons \mathrm{HHb}+\mathrm{B}^{+}
\end{aligned}
$$

7. Deoxygenated $\mathrm{Hb}$ is a weaker acid than oxy $\mathrm{Hb}$; hence as $\mathrm{O}_{2}$ is given off in the tissues more cations $\left(\mathrm{B}^{+}\right)$are freed, and in the lungs as $\mathrm{CO}_{2}$ is lost the stronger oxy $\mathrm{Hb}$ attracts more base, thus freeing more $\mathrm{CO}_{2}$.

8. Some $\mathrm{CO}_{2}$ (5-10 per cent of the total in the blood) combines reversibly with hemoglobin as a carbamino compound called carbhemoglobin.

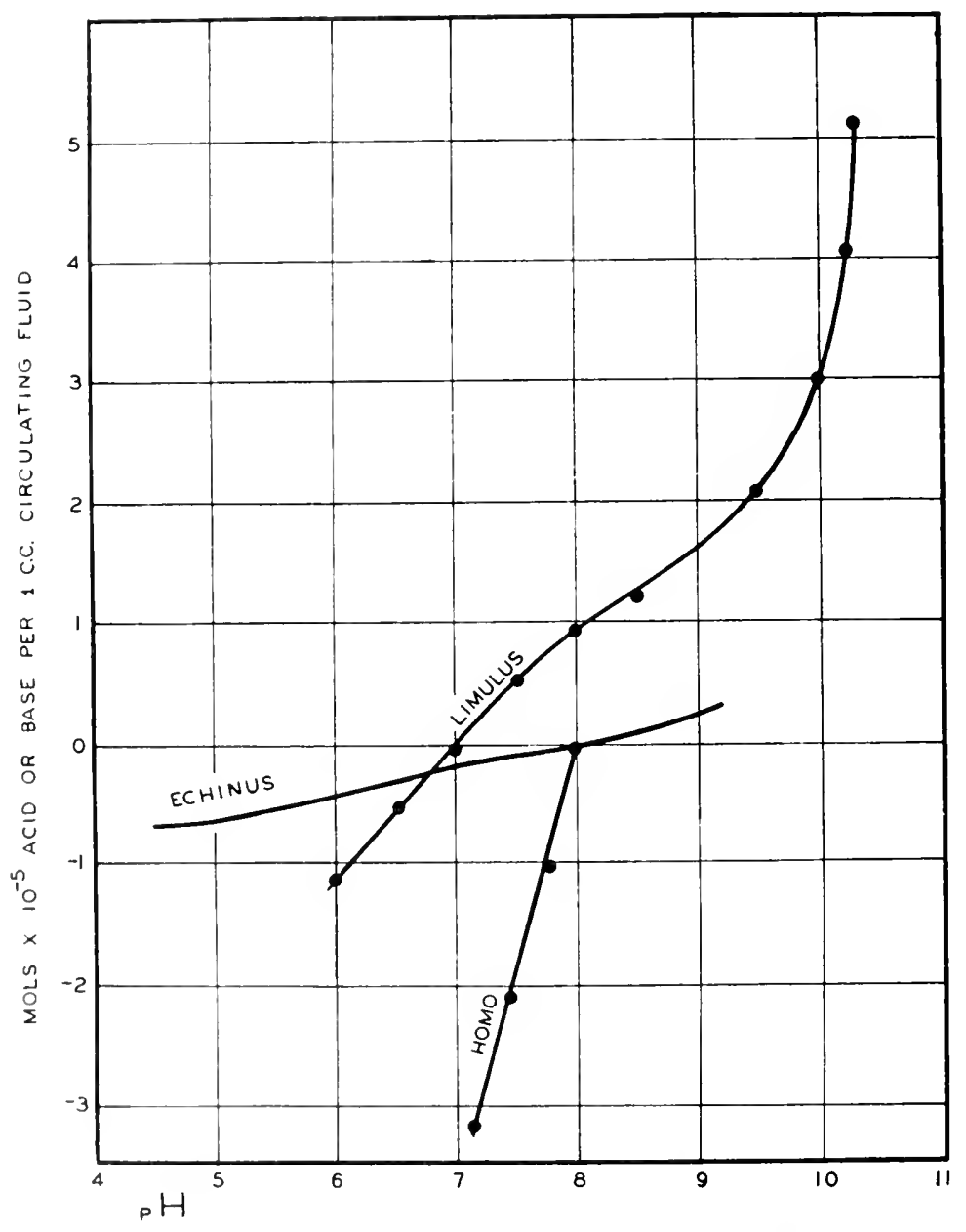

Fig. 85. Thitration curves of blonds of man, Limulus, and Echinus. D)ata assembled by Barcroft. ${ }^{7}$

$\mathrm{CO}_{2}$ Transport in Lower Vertebrates. Buffering capacity of vertebrate blood is previded by the bicarbonates, phosphates, the plasma proteins, and hemoglobin. The relative importance of each kind of buffer varies among 
animal groups. The bulfer value of the blood is given by the ratio BH ICO pll

i.e., the change in pll with $\mathrm{CO}_{2 .}$. It can also be slown by a titration curve of blood (Fig. 85). The buffering power of hemoglobin of different bloods is similar and the hemoglobin bulfering is correlated with the oxygen cilpacity. In reptiles the $\mathrm{CO}$.. content ol the blood is high, $2-3$ times that of mammals (Table 58), but most of it is carried in plasma as $\mathrm{BH} \mathrm{ICO}, 3.3,31^{3}$ The butfering capacity of the trout and mackerel is high. whereas that of the carp, toadtish, and skate is low. The butler value of the serum proteins is greater per gm. of protein in the shate and crocodile than in mammals. ${ }^{120}$

There is much variability in the effect of oxygenation of hemoglobin on the CO.-combining power. In man, at $40 \mathrm{~mm}$. I $\mathrm{g}$ of $\mathrm{CO}_{2,}$, venous blood combines with 52 and arterial blood with 50 volume's per cent of $\mathrm{CO}_{2.2}$. In the crocodile the change in acid strength of hemoglobin when oxygenated is greater than it is in man.": In the skate, however, there is no difference between the $\mathrm{CO}_{2}$ dissociation curve of oxygenated and that of deoxygenated blood:ats ln the tautog oxygenation decreases the $\mathrm{CO}_{2}$-combining power at low $\mathrm{CO}_{2}$ tensions, but above $50 \mathrm{~mm}$. the curves for oxygenated and for deoxigenated blexds are similar. After hemolysis, however, the deoxygenated blood combines with more $\mathrm{CO}_{2}$ at all tensions. ${ }^{1: t ;}$ In general, those animals in which $\mathrm{CO}_{2}$ increases the oxygen affinity are the ones in which oxygenation decreases the $\mathrm{CO}_{2}$ binding power. These effects depend on protein differences which are not understood.

Buffering in Invertebrates. Among invertebrates most of the buffering appears to reside in blood proteins, and the principal proteins are respirator! pigments. In Urechis, for example, the plasma of the coelomic fluid has practically no buffering capacity, but the corpuscles are capable of buffering to about the same degree as the corpuscles of a vertebrate of similar oxygen capacity. ${ }^{1: 3}$ The coelomic Huid of the sea urchin, which contains little protein, has very little buffering power (Fig. 85)..$^{117}$

Redfield and his associates $12 . "$ have shown in a series of papers that in those bloods which contain hemocyanin most of the buffering is due to this pigment. In Limulus, for example, phosphates are negligible, but purified hemocyanin can bind 1.6.10-3 mols of acid per gram of protein, and in the presence of blood salts hemocyanin can bind much more. This buffering capacity is similar to that of hemoglobin. The titration curve of hemocyanin in the presence of a salt mixture like the blood salts is essentially similar to the titration curve of serum of Limnulus. ${ }^{1 \%}$ This titration curve shows the presence of sereral acid and base binding groups. In Helix the blood is less well buffered than in Limulus, but it is buffered better in summer than in the winter. ${ }^{164}$

In Helix, Octopus, and Homarus oxygenated hemocyanin binds more $\mathrm{CO}_{2}$ than does deoxygenated hemocyanin.

In animals with calcium-containing shells, some molluscs and crustaceans, an important source of buffer is the shell. This has been discussed in Chapter 3.

Carbonic Anhydrase. Carbonic anhydrase is widely distributed in the animal kingdom. ${ }^{4 \overline{7}}, 50.51,5 ., 143$ None has been found in the sponges, but some is present in coelenterates, particularly in the tentacles. Carbonic anhydrase is found in coelomic Aluid of Sipunculus and Arenicola but not in that of most other invertebrates."1 A rich supply of this enzyme is present in the 
gills of some polychaetes, where it may facilitate transfer of $\mathrm{CO}_{2},{ }^{143}$ most molluscs show little in blood, although there is much in the gills of the squid and in gills and mantle of pelecypods. ${ }^{64}$

Among arthropods the gills of Limulus contain large amounts; those of Homarus and Libinia contain less, and the gills of Palinurus have none. Similarly, in several fishes, much carbonic anhydrase is found in the gills. In general it appears that carbonic anhydrase is most abundant in aquatic animals in gills, and in higher terrestrial forms in blood cells. Carbonic anhydrase is present in lower concentrations in other tissues.

\section{PIGMENTS AS BLOOD PROTEINS}

It was stated above that among some invertebrates function of the blood pigments in oxygen transport is doubtful. A universal function of these pigments, however, appears to be in buffering; they are particularly effective since each protein molecule may have several acid-binding groups. Another function is to provide blood colloid. ${ }^{121}$ In animals with a heart, and particularly in those with both heart and exoskeleton, the hemolymph (blood) is under hydrostatic pressure. These animals would tend to lose fluid, even though at osmotic equilibrium with the sea, were it not for the proteins of their bloods. Their protein concentrations are considerable-often up to 5 per cent. Experiments correlating hydrostatic pressure with blood proteins in aquatic animals are needed.

\section{CONCLUSIONS}

Certain generalizations can be made regarding the blood pigments. Phylogenetically blood pigments represent a labile set of characters. Chromogens composed of a protein with a prosthetic group containing a metal have appeared many times. Hemochromogens, being universally distributed in aerobic cells, have been used independently by several groups of animals in blood pigments. The hemoglobins may show slight variations in their porphyrins and great variations in their proteins from species to species and at different stages in one species. The molecules of blood pigments differ in size, large ones being free in solution, smaller ones contained in corpuscles. They also differ in function.

The functions of blood pigments in oxygen transport in the vertebrates are clear; among invertebrates, only in the cephalopod molluscs is the pigment, hemocyanin, as important in oxygen transport. In those others where at normal tensions the pigment carries oxygen, the pigment (chlorocruorin in the sabellids; hemoglobin in the earthworm, Nereis, and Tubifex; hemocyanin in Busycon) carries only a fraction of the oxygen required. In others the pigment (hemoglobin in Planorbis, Arenicola, Chironomus, Urechis, and Ascaris; hemerythrin in Sipunculus) functions to supply oxygen primarily at times of physiological stress duc to hypoxia. In still others the pigment (hemocyanin in Limulus and crustaceans) seems to function principally as a buffer and possibly in maintenance of colloid osmotic pressure.

Ecologically, many animals are limited in their range by their blood pigments. Correlations between oxygen-combining power and amount of pigment occur, as in mammals at high altitudes. Striking adaptations with respect to the effects of $\mathrm{CO}_{2}$ and temperature on $\mathrm{O}_{2}$ dissociation are found among fishes and in embryos. 'The decrease of oxygen aftinity with added $\mathrm{CO}_{2}$ is consider- 
able in the squid and in fish from fast waters; it is less in fish from sluggish and acid water and in terrestrial animals. The CO.2 effect is rerersed in some sluggish animals which have hemocyanin.

\section{REFERENCES}

1. Adaik, G. S., Johnston, J. P., and Ogston, A. G., Biochem. J. 40:867-869 (1947). Molecular size, Gastrophilus hemoglobin.

2. Alsberg, C. L., and Clark, W. M., J. Biol. Chem. 19:503-510 (1914). Oxygen in Limulus blood.

3. Anson, M. L., Barcroft, J., et al., Proc. Roy. Soc. Lond., B. 97:61-83 (1924). Absorption spectra of hemoglobins.

4. Anson, M. L., and Mirsky, A. E., J. Physiol. 60:50-68 (1925). Hemochromogens.

5. Anson, M. L., and Mirsky, A. E., J. Physiol. 60:221-228 (1925). Helicorubin.

6. Ball, E. G., and Cooper, O., Biol. Bull. 93:189 (1947). Myglobin in Busycon.

7. Barcroft, I., Features of the Architecture of Physiological Function. (1934). Cambridge Univ. Press. 368 p.

8. Barcroft, J., Researches on Prenatal Life (1946). Oxford, Blackwell. 292 p. Blood of embryos.

9. Barcroft, J., and Barcroft, H., Proc. Roy. Soc. Lond., B. 96:28-42 (1924). Hemoglobin of Arenicola.

10. Barcroft, J., Elliott, R., et al., J. Physiol. 83:192-214 (1934). O. transport by fetal and maternal blood in goat.

11. Baumberger, J. P., and Michaelis, L., Biol. Bull. 61:417-421 (1931). Hemoglobin in Urechis.

12. Bendett, E., Morrison, P., and Irving, L., Biol. Bull. 80:429-440 (1941). Blood of Atlantic salmon.

13. Birkofer, L., and Taurins, A., Ztschr. physiol. Chem. 265:94-101 (1940). Amino acid composition of hemoglobins.

14. Bishop, G. H., J. Biol. Chem. 58:543-565 (1923). Blood of honeybee larva.

15. Black, E. C., Biol. Bull. 79:215-229 (1940). O2 transport by blood of freshwater fish.

16. Black, E. C., and Irving, L., Tr. Roy. Soc. Canada Biol. Sci. $31: 29-32$ (1937). Effect of $\mathrm{CO}_{2}$ on $\mathrm{O}_{2}$ capacity of carp blood.

17. Воск, H. V., et al., J. Biol. Chem. 59:353-378 (1924). $\mathrm{CO}_{2}$ and $\mathrm{O}_{2}$ dissociation curves, blood of man.

18. Borden, M. A., J. Mar. Biol. Ass. U. K. 17:709-738 (1931). Respiration and blood function in Planorbis and Arenicola.

19. Bowen, W. J., Fed. Proc. 8:14 (1949). Hemoglobin and myoglobin, dogs, periodic high altitude.

20. Brand, T. v., J. Parasitol. 23:225 (1937). Hemoglobin in larval nematode.

21. Brown, W. E. L., and Hill, A. V., Proc. Roy. Soc. Lond., B. 94:297-334 (1923). Temperature and dissociation curve of human blood.

22. Cannan, R. K., Biochem. J. 21:184-187 (1927). Echinochrome.

23. Christensen, E. H., and Dill, D. B., J. Biol. Chem. 109:4+3-448 (1935). Dissociation curves of bird blood.

24. Соок, S. F., J. Gen. Physiol. 11:339-348 (1928). Reducing agents on blood pigments.

25. Crescitelli, F., Biol. Bull. 88:30-36 (1945). Absorption spectra of invertebrate blood pigments.

-26. CuÉnot, L., Arch. Zool. Exper. Gen. 9:366-475, 593-670 (1891). Hemoglobin in invertebrates.

27. Dausend, K., Ztschr. vergl. Pliysiol. 14:557-608 (1931). Respiration and hemoglobin function in Tubifex.

28. Davenport, H. E., Proc. Roy. Soc. Lond., B. 136:255-270, 271-280 (1949). Hemoglobin of three nematodes.

29. DHÉrÉ, C., J. Physiol. Path. Gén. 18:221-243 (1919). Oz capacity of hemocyanin bloods. 
30. DHéré, C., J. Physiol. Path. Gén. 18:44-52 (1920). Helicorubin.

31. Dnéré, C., Rev'. Suisse de Zool. 35:277-289 (1928). Respiratory pignents, invertebrates.

32. Dit., D. B., Life, Heat and Altitude (1938). Cambridge Univ. Press. 211 p.

33. Dıt $\iota$, D. B., and Edwards, H. Г., J. Biol. Chem. 90:515-530 (1931). Respiratory properties of crocodile blood.

34. Dill, D. B., and Edwards, H. T., J. Cell. \& Comp. Physiol. 6:243-254 (1935). Respiratory properties of alligator blood.

35. Dill, D. B., Enwards, H. T., and Florkin, M., Biol. Bull. 62:23-36 (1932). Properties of blood of skate, Raja.

36. Dill, D. B., Enwards, H. T., Eт al., J. Biol. Chem. 95:143-152 (1932). Respiratory properties of $\mathrm{dog}$ blood.

37. Dolk. H. E., and van der Paauw, F., Ztschr. vergl. Pliysiol. 10:324-343 (1929). llemoglobin function in earthworm.

38. Drastich, L., Pflïg. Arch. ges. Physiol. 219:227-232 (1928). Comparative hematology.

38a. Duval, M., and Portier, P., C. R. Acad. Sci. 184:1594-1596 (1927). CO2 content of invertebrate bloods.

39. Enwards, H. T., and Dill, D. B., J. Cell. E Comp. Physiol. 6:21-35 (1935). Properties of blood of gila monster (Heloderma).

40. Ellis, M. M., Meirich, A. D., and Ellis, M. D., Bull. U.S. Bur. Fish. 46:509546 (1930). Properties of mussel blood.

41. Elvehjem, C. A., Physiol. Rev. 15:471-507 (1935). Copper content of animals and blood pigments.

42. Endres, G., Proc. Roy. Soc. Lond., B. 107:241-247 (1930). Blood of marmot.

43. Ericksson-Quensel, I., and Svedberg, T., Biol. Bull. 71:498-547 (1936). Hemocyanin molecular weights.

44. Ewer, R. F., J. Exper. Biol. I8:197-205 (1942). Hemoglobin function in Chironomus.

45. Ewer, R. F., and Fox, H. M., Proc. Roy. Soc. Lond., B. 129:137-153 (1940). Chlorocruorin function.

46. Ferguson, J. K. W., and Black, E. C., Biol. Bull. 80:139-152 (1941). CO2 transport in blood of fish.

47. Ferguson, J. K. IV., Lewis, L., and Smith, J., J. Cell. E Comp. Physiol. 10:395-400 (1937). Carbonic anhydrase in invertebrates.

48. Ferguson, J. K. W., et Al., Biol. Bull. 75:381-388 (1938). CO2. dissociation curve, Mustelus blood.

49. Flonkin, M., C. R. Acad. Sci. 195:832-833 (1932). Hemerythrin function in Sipunculus.

50. Florkin, Ml., Ann. Physiol. 10:599-694 (1934). Review of respiratory function of body fluids.

51. Florkin, M., Arch. Internat. Plyysiol. 40:283-290 (1935). Carbonic anhydrase, body lluids, invertebrate animals.

52. Fionkin, Ml., L'évolution biochemique (19+4). Paris, Masson. 210 p.

53. Fонки, M., Biochemical Evolution (19-9) transl. and edited by S. Morgulis. New York, Acadenic Press. 157 p.

54. Florkin, M., and Marcmin, P., Arch. Internat. Physiol. $51: 130-132$ (1941). Carbonic anliydrase in Anodonta.

55. Fronkin, M., and Redfield, A. C., Biol. Bull. 61:421-426 (1931). Respiratory function, blood of sca lion.

56. Fox, 11. M., Proc. Roy. Soc. Lond., B. 99:199-217 (1926). Chlorocruorin.

57. Fox, H. M., Proc. Roy. Soc. Lond., B. $111: 356-363$ (1932). Properties of thlorocruorin.

58. Fox, 11. N1., Proc. Roy. Soc. Lond., B. 115:368-373 (1934). Oxygen:iron ratio, chlersecruorin.

59. liox, II. M., Nature 145:781 (1940). Function of chlorocruorin in Sabella and henoglobin in L_unbricus.

60. Fox, 11. M1., J. Exper. Biol. $21: 161-16+$ (1945). O affinities of invertebrate hemoglobins. 
61. Fox, H. M., Nature 160:+31+32 (1947). Daphuia hemuglobin.

62. Fox, H. M., Nature 162:20 (1948). CO athinity of chlorocruorin.

63. Fox, H. M., Proc. Roy. Soc. Lond., B. 135:195-212 (1948). Daphnia hemoglobin.

64. Freeman, J. A., and W'ilbur, K. M., Biol. Bull. 94:55-59 (1948). Carbonic anhydrase in molluscs.

65. Frr, F. E. J., Black, V., and Black, E. C., Biol. Bull. 92:217-22t (1947). Effect of temperature on $\mathrm{CO}$ zeffect on asphyxiation of fish.

66. Green, A. A., and Redreled, A. C., Biol. Bull. 64:4t52 (1933). Blood of porpoise.

67. Griffitils, A. B., C. R. Acud. Sci. 114:840-844 (1892). Pimnaglobin.

68. Hall, F. G., Biol. Bull. 47:79-126 (1924). Swimbladder gases.

69. Hall, F. G., J. Physiol. 83:222-228 (1934). Hemoglobin function in chick.

70. Hasl, F. G., J. Biol. Chem. 115:485-490 (1936). Altitude and O. aflinity of $\mathrm{Hb}$.

71. Hall, F. G., J. Mammal. 18:+68-772 (1937). Blood of mammals at high altitudes.

72. Hall, F. G., Dill, D. B., and Barron, E. S. G., J. Cell. \& Comp. Physiol. 8:301-313 (1936). Physiology of high altitudes.

73. Hall, F. G., and McCutcheon, F. H., J. Cell. E Comp. Physiol. 11:205-212 (1938). Hemoglubin function in marine fish.

74. Halliburton, W. D., J. Physiol. 6:300-335 (1885). Blood pigments of Crustacea.

75. Harnisch, O., Ztschr. vergl. Physiol. 23:391-+19 (1936). CO effects on respiration in Chironomus larvae.

76. Harver, H. WV., Biolugical Chemistry and Physics of Sea Water (1928). Cambridge Univ. Press, $19+$ p. Oxygen and carbon dioxide in sea water.

77. Hawkins, W. B., and Whipple, G. H., Am. J. Physiol. 122:418-427 (1938). Life of red cell in dog.

78. Henderson, L. J., Blood; a Study in General Physiology (1928). Yale Univ. Press. 397 p.

79. Henze, M., Ztschr. physiol. Chemie 43:290-298 (1904). Henocyanin function in Octopus.

80. Henze, M., Ztschr. physiol. Chemie 213:125-135 (1932). Vanadium pigment in ascidians.

81. Hill, R., Proc. Roy. Soc. Lond., B. 120:472-483 (1936). Dissociation of myoglobin.

82. Hogben, L., and Pinhey, K., J. Exper. Biol. 4:203-21+ (1926). O. dissociation of blood of Helix and crustaceans.

83. Hogben, L. and van der Lingen, J., I. Exper. Biol. 5:292-294 (1928). Hemoglobin in a holothurian, Cucumaria.

84. Hopping, A., Am. J. Physiol. 66:145-163 (1923). Alligator blood.

85. Hurtado, A., Rotta, A., et al., Am. J. Med. Sci. 194:708-713 (1937). Myoglobin at altitudes, dogs.

86. Irving, L., ex al., J. Cell. E Comp. Physiol. 6:393-403 (1935). Properties of seal blood.

87. Irving, L., Black, E. C., and Safford, V., Biol. Bull. 80:1-17 (19+1). Temperature and $\mathrm{O}_{2}$ affinity of fish blood.

88. Irving, L., Safford, V., and Scott, W. J., J. Cell. E Comp. Pliysiol. 13:297-313 (1939). Properties of fox blood.

89. Jacobson, L. O., Personal communication ( $\mathrm{T}_{\mathrm{o}}$ be published in Atomic Encrgy Com., Tech. Ser.) (19+7). Hematology of mammals.

90. Johnson, M. L., J. Exper. Biol. 18:266-277 (1941). Hemoglobin function in Lumbricus.

91. Jordan, H. E., in Downey's Handbook of Hematology. (1938). New York, Hoeber. Vol. 2, pp. 703-862. Comparative hematology.

92. Jürgens, O., Zool. Jahrb. Abt. allg. Zool. w. Physiol. 55:1-46 (1935). Circulation and respiration in polychaetes.

93. Kawagutr, S., Mem. Fac. Sci. Taihoku Imp. Univ. 23:95-98 (1941). Hemeryth- 
64. Kawanoto, N., Sci. Rep. Tohoku Imp. Univ., IV, 3:561-575 (1928). Hemoglobin in holothurians.

94a. Kawamoto, N., Sci. Rep. Tohoku Imp. Univ., IV, 4:643-659 (1929). Temperature and hemoglobin dissociation curve, eel.

95. Keilin, D., Proc. Roy. Soc. Lond., B. 98:312-339 (1925). Cytochrome.

96. Keilin, D., and Wang, Y. L., Nature 155:227-229 (1945). Hemoglobin, root nodules of leguminous plants.

97. Keilin, D., and WANG, Y. L., Biochem. J. 40:855-866 (1947). Hemoglobin in tracheal cells of insect Gastrophilus.

98. Kemnitz, von, Verh. dtsch. Zool. Ges. 24:294-307 (1914). Hemoglobin in larva of Hy, Gastrophilus.

99. Keys, A., Hall, F. G., and Barron, E. S. G., Am. J. Physiol. 115:292-307 (1936). Blood dissociation curve at high altitude.

100. KоваYashi, S., Sci. Rep. Tohoku Univ., Ser. IV, 10:257-267, and 733-751 (1935-36). Spectral absorption of invertebrate bloods.

101. Krogh, A., and Leitch, I., J. Physiol. 52:288-300 (1919). Respiratory function of blood of fish.

102. Lankester, E. R., Proc. Roy. Soc. Lond., B. 21:70-81 (1872). Distribution of hemoglobins.

103. Lankester, E. R., Zool. Anz. 4:350-356 (1881). Hemoglobin in an echiuroid worm.

104. Ledebur, J. F., Biol. Rev. 12:217-244 (1937). Swim bladder gases of fish.

105. Leitch, I., J. Physiol. 50:370-379 (1916). Hemoglobin function in Planorbis and Chironomus larvae.

106. Loewy, O., Tabul. Biol. 3:484-488 (1925). O. capacity of invertebrate and vertebrate bloods.

107. Macela, I., and Seliskar, A., J. Physiol. 60:428-442 (1925). Temperature and $\mathrm{O}_{2}$ dissociation curves.

108. MacMunn, C. A., Quart. J. Micr. Sci. 25:469-490 (1885). Absorption bands, chlorocruorin.

108a. Maloeuf, N. S. R., Ztschr. vergl. Physiol. 25:1-28 (1937). Oxygen capacity, Mytilus, Asterias body fluids.

109. Marrian, G. F., J. Exper. Biol. 4:357-364 (1927). Hemerythrin.

110. McСanthy, E. F., J. Phy'siol. 86:77-82 (1936). $\mathrm{O}_{2}$ dissociation curves of mammalian hemoglobin.

111. McCay, C. M., N.Y. State Cons. Dept. Suppl., 18th Ann. Rep., pp. 140-149 (1928). Hematology of fish.

112. McCutcheon, F. H., J. Cell. \&Comp. Pliysiol. 8:63-81 (1936). Hemoglobin function in tadpole and frog.

113. McCutcheon, F. H., J. Cell. E Comp. Physiol. 29:333-344 (1947). Oz affinity of hemoglobin of turtles and elasmobranchs.

114. McCutcileon, F. H., and Hall, F. G., J. Cell. E Comp. Physiol. 9:191-197 (1937). Hemoglobin function in Amphibia.

115. Mhllikan, G. A., J. Physiol. 79:158-179 (1933). Kinetics of hemocyanin and hemoglobin.

116. Ottesen, J., Nature 162:730-731 (1948). Life span, chicken erythrocytes.

117. Pantin, C. F. A., J. Linn. Soc. Zool. 37:705-711 (1932). Physiological adaptation.

118. Rawbinson, W. A., Austral. J. Exper. Biol. E Med. Sci. I8:131-140 (1940). Crystalline hemocyanin from arthropods.

119. Repfield, A. C., Biol. Bull. 58:150-175 (1930). Absorption spectra of hemocyanin bloods.

120. Ripfield, A. C., Quart. Rev. Biol. 8:31-57 (1933). Evolution of respiratory function of blood.

121. Ri:pfielu, A. C., Biol. Rev. 9:175-212 (1934). The hemocyanins, review.

122. Remield, A. C., Coolidge, T., and Hurd, A. C., J. Biol. Chem. 69:475-509 (1926). O and $\mathrm{CO}_{2}$ transport by If fy bloods.

123. Remileld, A. C., and Flonk1n, M., Biol. Bull. 61:185-210 (1931). Respiratory function ol blood of Ureclis. 
124. Redfield, A. C., and Goomkind, R., J. Exper. Biol. 6:340-349 (1929). Respiratory function of blood of squid.

125. Redfield, A. C., Humpineys, G., and Ingalls, E. N., J. Biol. Chem. 82:759 773 (1929). Hemocyanin, buffer action in Limulus blood.

126. Redfield, A. C., and Ingalls, E. N., J. Cell. E Comp. Physiol. 1:253-275 (1932). $\mathrm{O}_{2}$ dissociation curves of Busycon Hcy.

127. Redfield, A. C., and Ingalls, E. N., J. Cell. E Comp. Physiol. 3:169-202 (1933). O. dissociation curves of bloods containing hemocyanin.

128. Reichert, E. T., and Brown, A. P., Carnegie Inst., Wash., Publ. 112, 338 p. (1909). Characteristics of vertebrate bloods.

129. Rocue, A., and Roche, J., Bull. Soc. Chim. biol. 17:1494-1508 (1935). Molecular weight, hemerythrin.

130. Roche, J., Ann. Pliysiol. 10:583-591 (1934). Comparative chemistry of blood pigments.

131. Roche, J., An. Rev. Biochem. 5:463-484 (1936). Animal pigments, revicw.

132. Romieu, M., Arch. Morph. gén. et expér. 17:1-339 (1923). Blood pigments in polychaetes.

133. Root, R. WV., Biol. Bull. 61:426-456 (1931). Oz transport by blood of marine fish.

134. Root, R. W., J. Biol. Chem. 104:239-244 (1934). Monoxide affinity of hemocyanin.

135. Roor, R. W., and Irving, L., J. Cell. E Comp. Physiol. 16:85-96 (1940). Effect of oxygenation on $\mathrm{CO}_{2}$ transport by tautog blood.

136. Root, R. W., and Inving, L., Biol. Bull. 81:307-323 (1941). Properties of Hb in whole and hemolyzed blood of tautog.

137. Rostorfer, H. H., and Rigdon, R. H., Biol. Bull. 92:23-30 (1947). O transport, resistance to anoxia, chicks and ducklings.

138. SAfFord, V., J. Cell. E Comp. Physiol. 16:165-173 (1940). Swim-bladder of fish.

139. Salamon, K., J. Gen. Physiol. 24:367-375 (1941). Kinetics and absorption bands of invertebrate hemoglobins.

140. Sato, T., and Tamyo, H., Cytologia, Tokyo. Fujii Jubilee Vol., pp. 1133-1138 (1937). Hcmoglobin from Paramecium.

141. Scheer, B. T., Comparative Physiology (1948). New York, Wiley. pp. 180-183. Hemocyanin function.

142. Scort, W. J., Biol. Bull. 61:211-222 (1931). Respiratory function, Amphiuma blood.

143. Sobotka, H., and Kann, S., J. Cell. E Comp. Physiol. 17:341-348 (1941). Carbonic anhydrase in fish and invertebrates.

144. Southworth, F. C., and Redfield, A. C., J. Gen. Physiol. 9:387-403 (1926). Respiratory function of turtle blood.

145. Spillman, J., C. R. Soc. Biol. Paris 131:607-608 (1939). Hemoglobin in Discoglossus.

146. Stedman, E., and Stedman, E., Biochem. J. 19:544-551 (1925), ibid. 20:938948 (1926). O. dissociation curve, crustacean blood.

147. Svedberg, T., J. Biol. Chem. 103:311-325 (1933). Sedimentation constants of respiratory proteins.

148. Svedberg, T., and Hedenius, A., Biol. Bull. 66:191-223 (1934). Sedimentation of blood proteins.

149. Svedberg, T., and Pedersen, K. O., The Ultracentrifuge (1940). Oxford, Clarendon. $478 \mathrm{p}$.

150. Theorell, H., Biochem. Ztschrr. 268:55-63 (1934). Absorption spectrum, purified myoglobin.

151. Thomas, J. B., Ztsehr. vergl. Physiol. 22:284-292 (1935). Hemoglobin function in earthworm.

152. Walshe, B. M., J. Exper. Biol. 24:329-342, 343-351 (1947). Hemoglobin function in chironomid larvae.

153. Wastl, H., and Leiner, G., Pfliig. Arch. ges. Physiol. 227:367-420, 421-459 (1931). Dissociation curves of bird blood. 
154. Wastl, H., and Leiner, G., Pfïg. Arch. ges. Physiol. 227:460-474 (1931). Respiratury properties of bird blood.

155. Wastl, H., and Seliskar, A., J. Physiol. 60:26+-268 (1925). $\mathrm{CO}_{2}$ transport, frog blood.

156. WEBB, D. A., J. Exper. Biol. 16:499-523 (1939). Vanadium in ascidians.

157. Wharton, G. W., J. Parasitol. 27:81-87. (1941). Respiratory pigments of turtle parasites.

158. W'illmer, E. N., J. Exper. Biol. 11:283-306 (1934). Function of blood of tropical fish.

159. Wilson, J. W., J. Cell. E Comp. Physiol. 13:315-326 (1939). Reptilian bloods.

160. Winegarden, H. M., and Borsook, H., J. Cell. \& Comp. Physiol. 3:437-448 (1933). Species absorption bands of $\mathrm{Hb}$.

161. V'interstein, H., Biochem. Ztschr. 19:384-42+ (1909). Respiratory function of blood in marine invertebrates.

162. Wolvekamp, H. P., Ztschr. vergl. Physiol. 16:1-38 (1932). Oxygen transport pigments, Helix, Rana, Planorbis.

163. Wolvekanip, H. P., Ztschr. vergl. Physiol. $25: 541-547$ (1938). O.2 transport by Hcy in Octopus.

164. Wolvekamp, H. P., and Kersten, H. J., Ztschr. vergl. Physiol. 20:702-712 (1934). O. dissociation curve of Helix blood.

165. Wolvekamip, H. P., and Lodewijks, J. M., Ztschr. vergl. Physiol. 20:383-387 (1934). Effect of $\mathrm{CO}_{2}$ on $\mathrm{O}_{2}$ dissociation curve of frog. 


\section{Temperature: Metabolic Aspects and Perception} mat

\section{INTRODUCTION}

ENiperature, perimars mone than other envirommental tactors, has multiple and diverse effects on living organisms. Temperature is a measure of molecular agitation; it limits the rate of chemical reactions; hence it determines growth and metabolism in the widest sense. Temperature limits the survival and the distribution of animals on the earth. The cell temperature limits for active life are from about 0 to about $+45^{\circ} \mathrm{C}$., and many higher animals die within still narrower limits.

Many animals respond to changes in environmental temperature by conforming passively to the environment; these are the cold-blooded or poikilothermic animals. A poikilothermic animal can protect itself against unfavorable temperatures by entering a protective state of dormancy or encystment, or by going to a region of more farorable temperature. Comparatively few animals regulate their body temperature; these are the warm-blooded or homoiothermic animals. Heterothermic animals are those with limited temperature regulation. Warm-blooded animals can react to temperature extremes by protective behavior, and in addition they can alter heat loss by varying their insulation and can alter heat production by varying their metabolism. Sensory mechanisms signal the changes in temperature which evoke the various protective responses.

The temperature of any metabolizing cell is necessarily higher than the temperature of its medium, because oxidation and glycolysis liberate heat. The temperature of an animal depends on the balance of those factors which tend to add heat and those which tend to subtract heat:

Factors Which Cause Addition of Heat

oxidative and other metabolic production of heat

absorption of heat, largely from solar radiation

\section{Factors Which Cause Loss of Heat}

radiation

conduction and convection

vaporization of water

circulation of heat from interior to surface

The measurement of temperature is so eas!, by mercury thermometer or thermocouple, that the literature on temperature relations is vast. Various aspects have been summarized by numerous authors. ${ }^{11}$, 1.., 31, i:

\section{THERMAL. PROPERTIES OF WATER}

The temperature relations of animals to their environment are closely connected with their water relations. The thermal properties of water are 
important in determining the thermal properties of animals in relation to their environment. Animals are 70 to 90 per cent water. The heat conductivity of water is low, compared with that of many materials, being only 0.0014 cal./ $\mathrm{cm} . / \mathrm{sec} .{ }^{\circ} \mathrm{C}$., compared with 0.9 for copper. The heat conductivity of water is higher than that of other liquids, such as ethyl alcohol (0.00042) and olive oil (0.000395). The thermal conductivity of muscle is a little lower than that of water $(0.001 \mathrm{cal} . / \mathrm{cm} . / \mathrm{sec}$. C. $)$. and the conductivity of fat is onethird less. Low heat conductivity results in slow warming or cooling of an aquatic medium, and in limited conduction of heat within an animal. Also, the specific heat of water is high, $1.0 \mathrm{cal} . / \mathrm{gm} .{ }^{\circ} \mathrm{C}$. at $0^{\circ} \mathrm{C}$., compared with $0.09 \mathrm{cal} . / \mathrm{gm} . /{ }^{\circ} \mathrm{C}$. for copper, 0.535 for ethyl alcohol, and 0.389 for benzene. Animal tissues, except compact bone, require 0.7 to 0.9 calories to raise the temperature of one gram of tissue one degree. Hence animals with much tissue mass are slow to warm or cool. Most transfer of heat in animals is by circulating body fluids, and a sluggish circulation makes for slow heat transfer.

The normal vital temperature range is based on the thermal properties of water. Natural waters (hot springs excepted), because of their high specific heat and small heat conduction, rarely have a temperature above the upper limit for most aquatic animals $-35-40^{\circ} \mathrm{C}$. At the cold extreme, the freezing point of aquatic animals (except marine bony fish and brine shrimps) is normally similar to or lower than the freezing point of the medium (Ch. 2). Since ice has a lower specific gravity than water, aquatic animals do not freeze so long as they remain in water. The thermal properties of water make it an ideal solvent as a medium and as the principal cytoplasmic solvent. ${ }^{79}$

Terrestrial animals are subject to a much greater change of temperature than aquatic, but here too body temperature is closely related to water balance. Water has a high heat of fusion $(79.7 \mathrm{cal} / \mathrm{gm}$.), aqueous solutions supercool by several degrees, especially in capillary spaces, and bound water is resistant to freezing. Hence partially dehydrated animals can withstand temperatures well below the freezing point of water without their tissues becoming frozen. In high air temperatures animal body temperatures are limited by the high heat of vaporization of water $\left(586 \mathrm{cal} . / \mathrm{gm}\right.$. evaporated at $0^{\circ}$, compared with 55 $\mathrm{cal} . / \mathrm{gm}$. for $\mathrm{CO}_{2}$ or $302 \mathrm{cal} . / \mathrm{gm}$. for ammonia). Thus water loss by vaporization has a marked cooling effect on any moist surface. An understanding of both water balance in animals and the thermal properties of water is essential to an understanding of the temperature relations of animals.

\section{TEMPERATURE CHARACTERISTICS}

Chemical reactions are accelerated as temperature rises: decrease in a reaction rate as temperature rises in the natural range does not occur in biological sistems. To describe the magnitude of the temperature effect on a variety of processes sereral methods are in use. An approximation to describe the eflect of a narrow specified temperature range is the $\mathcal{Q}_{10}$. which is the factor by which a reaction velocity is increased for a rise in temperature of 10 degrees.

$$
Q_{11}=\left(K_{1} / K_{2}\right) \frac{10}{t_{1}-t_{2}}
$$

where $K_{1}$ and $K_{2}$ are velocity constants corresponding to temperatures $t_{1}$ and 
t. However, the velocity of enzymatic reactions is not a linear function of temperature, and the $Q_{10}$ diminishes at higher temperatures.

A temperature characteristic with a sound theoretical basis is the Arrhenius $\mu$. If velocity constants $K_{1}$ and $K_{2}$ (proportional to measured velocities) at absolute temperatures $T_{1}$ and $T_{2}$ are known, the $\mu$ is obtained from the following relation, where $\mathrm{R}$ is the gas constant 1.98 calories:

$$
\begin{array}{r}
K_{2}=K_{1} e^{R}\left(\frac{1}{t_{1}}-\frac{1}{t_{2}}\right) \\
\mu=4.6 \log \left(K_{2}-K_{1}\right) /\left(\frac{1}{t_{1}}-\frac{1}{t_{2}}\right)
\end{array}
$$

(See discussions by Barnes, ${ }^{11}$ Hoagland, ${ }^{80}$ and Sizer. ${ }^{159}$ ) Definite values of $\mu$ characterize specific catalysts irrespective of the substrate. ${ }^{8 t}$ When the Arrhenius $\mu$ is calculated for a wide variety of biological reactions, certain values appear regularly, and there is reason to associate these with underlying chemical reactions. ${ }^{159}$ The velocity of all reactions in animals varies with the absolute temperature. Animals subject to extreme variations, and particularly to very low body temperatures, are limited metabolically. A frog swimming in ice water cannot possibly go faster than energy is made available in its muscles.

\section{LETHAL EFFECTS OF COOLING}

As the temperature of living cells goes down, many life processes become so slow as to be ineffective. Three low-temperature ranges may be considered: freezing temperatures, vitrifying temperatures, and lethal temperatures above freezing. Protoplasm as an aqueous solution freezes at a few degrees below zero, and freezing of a cell with resultant ice crystals kills. Resistance to frcezing temperature is affected by several factors, the most $\mathrm{im}_{\mathrm{p}}$ ortant of which is water content and the state of contained water; prior desiccation or large content of bound water lowers the lethal temperature. If cooling is slow many organisms become reorganized largely by dehydration and sometimes by acquiring a tough insulating coat. Some Protozoa encyst, and sponges form gemmules. Prolonged subcooling (cooling below freezing point without freezing) also farors survival in the freezing range. Capillarity and existence of body fluids in small spaces favors subcooling and in many insects the free body fluids diminish in winter. ${ }^{135}$ Whether or not an animal freezes at a given low temperature depends on the rate at which it is cooled and the length of time it is kept at the low temperature. The grain weevil Sitophrilus granarius dies in 875 hours at $7.2^{\circ}$, in 100 hours at $-6.6^{\circ}$, and in 2.5 hours at $-17.7^{\circ} .145$ (See also the discussion of supercooling in Wigglesworth. ${ }^{12}$ ) Insects collected in winter freeze at much lower temperatures than do summer specimens. The winter animals are cold-hardened. Blood of wood-boring larvae freezes at a higher temperature than do some other tissues $\left(-22^{\circ}\right.$ : freezing point of fat of coldhardened oak borers), and death is associated with freezing of other tissues rather than of the blood. ${ }^{13+}$ Certain irsects enter a state of dormancy, a reorganized state in which the protoplasm does not freeze at temperatures several degrees below zero (Table 59). Aquatic insects are not subject to temperatures below zero. The water content of terrestrial insects decreases while cold. 
TABLE 59. LETHAL TEMPERATURES OF POIKILOTHERMIC ANIMALS

\begin{tabular}{|c|c|c|c|}
\hline Animal & $\begin{array}{l}\text { Conditions } \\
\text { or Source }\end{array}$ & $\begin{array}{c}\text { Low Lethal } \\
{ }^{\circ} \mathrm{C} .\end{array}$ & $\begin{array}{l}\text { High Lethal } \\
{ }^{\circ} \mathrm{C} \text {. }\end{array}$ \\
\hline 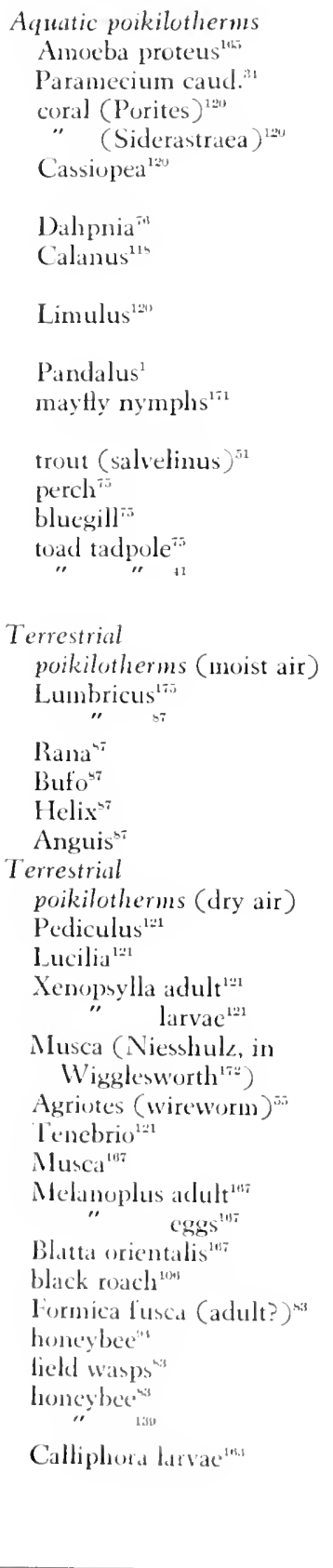 & $\begin{array}{l}\text { Florida } \\
\quad ? \\
\text { Tortugas } \\
\text { Halifax } \\
\text { winter } \\
\text { summer } \\
\text { Florida } \\
\text { Woods Hole } \\
\text { Arctic } \\
\text { stream } 11.6^{\circ} \\
\text { stream } 14.5^{\circ}\end{array}$ & $\begin{array}{c}-3 \\
-8(20 \mathrm{~min} .) \\
-8(48 \mathrm{hr} .) \\
-30(16 \mathrm{hr} .) \\
-5(1 \mathrm{hr} .) \\
0 \\
<0 \\
1-8 \\
0 \\
7 \\
5.5(2 \text { days })\end{array}$ & $\begin{array}{c}35.5-38.3 \text { (l hr.) } \\
40 \text { (9.5 min.) } \\
37.7 \\
38.5 \\
40 \\
26.8-28 \\
35 \text { (28 min.) } \\
24 \\
26 \\
46.25 \\
41.0 \\
11.5 " \\
22.4 " \\
24.7 \\
26.5-28 \text { (few min.) } \\
32 \text { (1 hr.) } \\
34.3 \text { (1 hr.) } \\
37.4(1 \mathrm{hr} .) \\
40.3 \text { (5-10 min.) } \\
43.5 \text { (5-10 min.) }\end{array}$ \\
\hline
\end{tabular}


TABIE 59 (continued).

LETHAL TEMPERATLIRES OF POIKILOTHERMIC ANIMALS

\begin{tabular}{|c|c|c|c|}
\hline Animal & $\begin{array}{l}\text { Conditions } \\
\text { or Source }\end{array}$ & $\begin{array}{c}\text { Low Lethal } \\
{ }^{\circ} \mathrm{C} \text {. }\end{array}$ & $\begin{array}{c}\text { High Lethal } \\
{ }^{\circ} \mathrm{C} \text {. }\end{array}$ \\
\hline 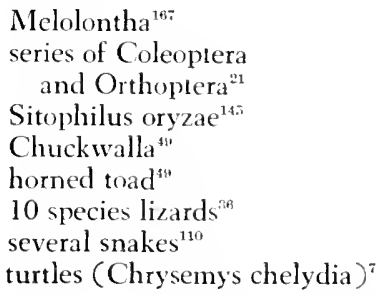 & $\begin{array}{l}\text { Palestine } \\
\text { hardened? } \\
\text { deser! }\end{array}$ & -17.7 (1.5 hr.) & $\begin{array}{c}35-45 \text { ( } 1 \mathrm{hr} .) \\
47 \\
47 \text { (convulsions) } \\
49 \stackrel{4}{"} \\
42 \text { (12 hr.) } \\
39-41 \text { ( } 30 \mathrm{~min} .)\end{array}$ \\
\hline
\end{tabular}

hardiness is developing, ${ }^{12 .}$ and much of the water is in a "bound" state; that is, it is held by protein and is not free solvent. There is no doubt that many insects freeze, in that they become solid in cold winter weather; whether the protoplasm of such cells as neurones contains ice crystals in insects with extracellular fluid frozen is not known but seems unlikely. There are numerous reports of revival of fish from a frozen state; however, the freezing must be fast and superficial; true body temperatures below -0.6 to $-1^{\circ}$ are probably not enclured." $=$ The lower lethal temperature for fish varies within limits according to the temperature to which the fish have been acclimatized. Marine teleosts, which are hypotonic to the medium, and hence have a higher freezing point than sea water, must approach freezing in arctic waters.

If the rate of cooling through the temperature range of freezing is rapid, about 100 degrees centigrade per second, ice crystals do not form and the organism is "vitrified." 11" When vinegar nematodes (Anguillula), slightly dehydrated, are placed directly into liquid air $\left(-197^{\circ}\right.$ C. ), they are solidified in an amorphous state; if they are then warmed rapidly by immersion in water or mercury at $30^{\circ}$ they revive and are active, and many survive. ${ }^{113}$ If freezing occurs, either by slow cooling or by slow warming from liquid air temperature through the freezing temperature range to above-freezing temperature, the animals are killed. Other organisms, including Protozoa and muscle cells, have been vitrified. The cause of death in freezing appears to be not cessation of enzymatic reactions resulting from reduced thermal agitation. but rather disintegration of protoplasmic organization.

Many animals which do not become dormant or encyst die at temperatures well above freezing (Table 59). Metabolism is greatly reduced, and they enter a chill coma in which survival time is limited. It is probable that protoplasmic organization cannot be maintained without a certain rate of energy production from metabolism. Insects go into chill coma at a lower temperature if acclimatized to cold rather than warm air; Blatta orientalis, for example, living at $14-17^{\circ}$, become inactive when cooled to $2.0^{\circ} \mathrm{C}$., whereas cockroaches living at $36^{\circ}$ show no activity below $9.5^{\circ} \mathrm{C} .{ }^{122}$ In birds and mammals, probably also in large cold-blooded animals, circulatory sluggishness results in insufficient oxygen to essential organs, such as the brain, and progressive paralysis occurs. When heat loss exceeds heat production body temperature cannot be maintained. A cat loses consciousness at a body temperature of 
$25-27^{\circ} \mathrm{C}$, but some refleses remain at lower temperatures, and death results in a cat at $16=\mathrm{C}$. In a guinea pig death results at $14^{\circ} \mathrm{C} . * 3$ When men are immersed in water heat production fails to keep up with heat loss below a water temperature of $20^{\circ} \mathrm{C}$. Death occurs after less than an hour's duration

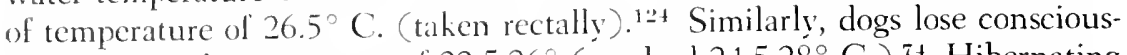
ness at a rectal temperature of $22.5-26^{\circ}$ (cerebral $24.5-28^{\circ}$ C. $) .^{7+}$ Hibernating mammals can survive lower body temperatures than non-hibernating animals (Table 59); hibernating bats die if vital organs are frozen, but opossums die at temperatures a few degrees above that causing freezing. The primary causes of death at low temperatures, but without freezing, are unknown.

\section{LETHAL EFFECTS OF ELEVATED TEMPERATURES}

The highest tolerable body temperature has been ascertained for many animals and some representative values are given in Table 59. Numerous mechanisms of heat death are suggested, and no one mechanism operates for all animals. " Heat may kill by enzyme inactivation. As temperature rises, enzyme activity increases, and destruction of the enzyme is also accelerated; above a certain temperature, the enzyme is so rapidly destroyed that the net effect is reduced activity. ${ }^{15 !}$ Nany enzymes are inactivated above $35-45^{\circ} \mathrm{C}$., although higher temperatures are needed for protein denaturation. Heat may also kill by irreversible protein coagulation. Cellular lipids also change their physical state at elevated temperatures. Blowfly larvae reared at $12-18^{\circ}$ have a lower heat death temperature and have lipoids with more double bonds than have larvae reared at $30-36^{\circ}$, yet two species with the same kind of fat die at different temperatures; hence fat breakdown per se can hardly be the cause of death. ${ }^{633}$ Cell membranes at high temperatures become freely permeable. In higher animals the heated tissues may liberate toxins which cause damage at a distance. ${ }^{77}$ An old theory, that at high temperatures the oxygen supply is inadequate, fails to hold for insects, ${ }^{6: 2}$ although in vertebrates the affinity of hemoglobin for oxygen decreases significantly at high temperatures. Whatever the mechanisms of heat death, they are certainly multiple and they are subject to alteration, as judged by resistance to heat.

The high temperature lethal for an animal depends on duration of exposure to the elevated temperature, and high lethal temperatures as in Table 59 are meaningtul only if exposure times are given. Also, when the temperature is raised gradually the tolerance limits are higher than when it is raised rapidly. For exposures of 15 to 60 minutes (Table 59), there is a wide range of lethal temperatures. In general, the lethal temperatures of terrestrial animals, insects, reptiles, birds, and mammals, are high, around $45^{\circ} \mathrm{C}$. The lethal temperatures for dwellers in moist air (carthworm, frog) are lower than for inhabitants of Ary air. Aquatic animals dic at lower temperatures, some of those normally living in colel waters dying of heat shock even below $30^{\circ} \mathrm{C}$. The minimum lethal temperature depends, then, on the temperature at which an animal has been living and on the rate of change of temperature.

\section{ACCLIMATIZATION}

femperature acclimatization occurs in nature and is of two kinds: (1) genetic, which operates by selection, and (2) physiological, in which individu- 
als alter their temperature resistance within genetic limitations. There are numerous examples of acclimatization to heal and to cold which may have a genetic basis. A frequently-cited but never-repeated experiment was conducted by Dallinger," in which by very gradual increases in temperature flagellate Protozoa were after seven years made to survive and reproduce at temperatures as high as $70^{\circ}$ C., well above the temperature which was lethal at the beginning of the experiment. In hot springs chlorophyll-bearing algae are common at $60^{\circ} \mathrm{C}$., and some non-green algae at $70^{\circ} .2-$ Rhizopods are reported from

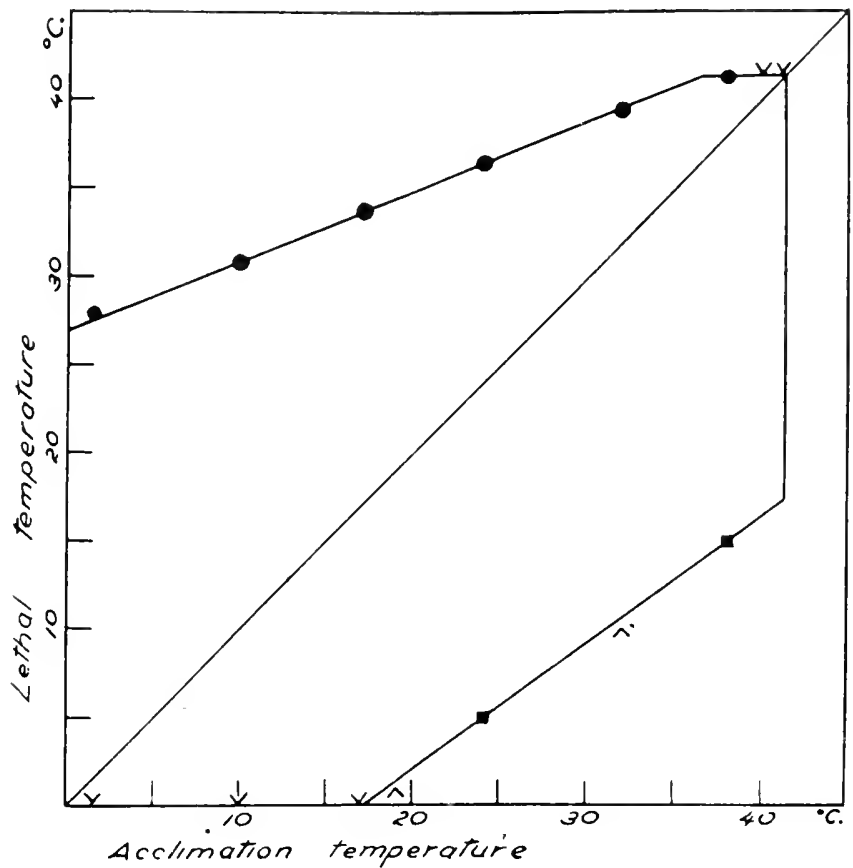

Fig. 86. Relation between acclimatization temperature and the temperature lethal for 50 per cent of goldfish in 14 hours. Arrows indicate death of all of a group of fish. From Fry, Brett, and Clawson. ${ }^{164}$

natural waters at $54.5^{\circ}$, flagellates at $51^{\circ}$, and ciliates at $46^{\circ}$. Brues found numerous insects and some crustaceans and snails in hot springs at around $38^{\circ}$; Chironomus larvae were thriving at $49-51^{\circ} \mathrm{C}$. Fish rarely are found at temperatures above $30^{\circ}$, but frog tadpoles have been collected from water at 40 $41^{\circ} .27$ These natural temperatures are well above lethal temperatures determined on laboratory specimens reared at lower temperatures.

Mayer ${ }^{1: 0}$ reports Aurelia from Halifax dying at $29-30^{\circ}$, those from Tor. tugas at $38.5^{\circ}$; also Limulus from Woods Hole dying at $41.0^{\circ}$, and those from southern Florida at $46.2^{\circ}$. Mayfly nymphs from a $11.6^{\circ}$ stream died at $22.4^{\circ}$, whereas others of the same species from a $14.5^{\circ}$ habitat died at $24.7^{\circ} .171$ Specimens of the prawn Pandalus from waters $5^{\circ}$ to $7^{\circ} \mathrm{C}$. at Kristineberg, Sweden, could not be maintained in aquaria at temperatures above $11^{\circ} \mathrm{C}$., 
whereas prawns of Plymouth, England, from 15 water, survived well at temperatures up to 17 Animals from cold waters have lower lethal temperatures than those from warm waters, and for animals from a given region the temperature causing heat death is higher in summer than in winter.

Acclimatization of individuals (physiological acclimatization) also occurs. Datal for several species of fish reported by Hathaway ${ }^{i j}$ are shown in Table 60. The effects of acclimatization on lethal temperatures of goldfish have been well studied by Fry and his associates (Fig. 86). ${ }^{64}$ The upper lethal tempera-

TABLE 60. TEMPERATURE TOLERATED FOR 1 HOUR ONLY ${ }^{75}$

\begin{tabular}{l|c|c|c}
\hline \hline \multicolumn{1}{|c|}{ Animal } & $\begin{array}{c}4 \text { Days } \\
\text { Acclimatization } \\
\text { at } 10^{\circ} \mathrm{C}\end{array}$ & $\begin{array}{c}\text { Stock Aquarium } \\
22-23^{\circ} \mathrm{C} .\end{array}$ & $\begin{array}{c}4 \text { Days at } \\
30^{\circ} \mathrm{C} .\end{array}$ \\
\hline Perch & 31.3 & 32 & 32 \\
Large mouth bass & 31 & 33.4 & 36.9 \\
Bluegill & 32.7 & 34.3 & 36.3 \\
Sunfish & 31.2 & 35.3 & 38 \\
Toad tadpole & 36.7 & 37.4 & 39.8 \\
\hline
\end{tabular}

ture increased I degree centigrade for every 3 degree rise in acclimatization temperature up to $36.5^{\circ} \mathrm{C}$., when the high lethal was $41^{\circ} \mathrm{C}$.; the lower lethal temperature decreased 2 degrees for every 3 degree fall in acclimatization temperature down to $17^{\circ} \mathrm{C}$, when the low lethal temperature was $0^{\circ} \mathrm{C}$. Apparently the mechanisms causing heat or cold death are labile. Seasonal differences in lethal temperatures are largely due to acclimatization, but other factors, such as endocrine changes, cannot be disregarded.

Some of the numerous alterations which might account for temperature acclimatization in cold-blooded animals are well discussed by Fox. ${ }^{57}, 58$ Animals from a cold environment show increased sensitivity to changes in temperature. The rate of pulsation in the dorsal blood vessel in Perinereis and of the heart in several crustaceans from Plymouth, England, is higher at a given temperature and increases faster with rising temperature than in animals from Tamaris (Mediterranean). At a given temperature the oxygen consumption of animals from cold waters is higher than the oxygen consumption of the same species from warmer waters. For example, the prawn Pandalus montagui from Kristineberg $\left(5^{-}-7^{\circ}\right)$ had a $Q_{0.2}$ nearly twice that of prawns from $15^{\circ}$ water at Plymouth, when both were measured at $10.5^{\circ}, 5:$ and river lampreys kept at 3 consumed 50 per cent more oxygen than lampreys from $16^{\circ}$ water, when both were measured at $16^{\circ} .{ }^{158}$ I lowever, at the temperatures at which they normally live, the metabolism of warm-water poikilotherms is higher than that of aretic species, yet breathing rates and heart rates are not proportionately higher, hence animals from warm water have more "non-activity" metabolism. Individuals of a poikilothermic species reared at low temperature are usually larger than individuals from wamer waters." ${ }^{\circ 2}$ Planaria tested at $30^{\circ}$ for sensitivity to cymicle show greater sensitivity if reared at $20^{\circ}$ than if reared at $30^{\circ} . .^{14}$ Clones of Daphuia with distinctly different temperature optima have been segregalled:"

Non hibernating homoiotherms ean also become acclimatized to temperature extremes. The initial effect of temperature extremes in mammals is stimulation 
of the stress regulating mechanism of the adrend cortex. Ofler endocrines, particularly the theroid, mas also be concerned in acclimats/ation. Rats ate climatized to cold synthesie and store large quantities of ascorbic areid in various tissues, particularly in the adrenals, and guinea pigs are able to condure cold only if fed massive amounts of ascorbic acid. ${ }^{1 /}$ Man shows temperature acclimatization, as when in a hot desent or tropics, but he cannot acelimatie to dehydration. ${ }^{+}$Most arctic birds and mammals hase more insulation than tropical ones, and individuals of the same species vary in fur coat according to the temperature at which they live; the fur is heavier on als and doges maintained at fow temperatures than on those maintained at high temperatures.

The metabolic changes occurring in acelimatization have not been jedenti fied. "is The melting point of fats deposited in animal bodie's at high tempera tures is higher than that of fats depesited at low temperatures. At how tem. peratures predominantly unsaturated lats are formed. Acclimatization may well be associated with lipid changes. Older suggestions of differences in water content seem not to hold. There may also be changes in the temperature response of certain enzymes. The inactivation temperature for amylases, pepsin, and trypsin from fish is lower than lor the sane enzrmes from manmals. ${ }^{2}$. 16i The oxygen consumption (at a given temperature) of brain tissue from a polar cod is higher than that of brain tissue from a black bass, and below $10^{*}$ changes in temperature alter respiration of the bass brain more than they do that of the polar cod brain."is Examination of optimal (critical) temperatures and of temperature characteristics for a variety of enzymes from organisms acclimatized to high or low temperatures might indicate changes in critical enzyme proteins.

\section{TEMPERATURE RELATIONS IN POIKILOTHERMIC ANIMALS}

In poikilothermic animals the factors faroring loss of heat tend to equal the factors producing body heat, and the body temperature approaches environmental temperature. However, small thermal gradients exist from regions of active oxidative metabolism toward the body surface, and mean body tem. perature fluctuates with muscular activity. The temperature relations of poikilothermic animals are different according to whether they live in water, in moist air, or in dry air. In water the heat loss is by conduction and convection; in moist air the evaporative loss is less than in dry air. Water temperatures are more constant than air temperatures; hence aquatic animals are less subject to rapid gain or loss of heat.

Aquatic Poikilotherms: Body' Temperature. Aquatic poikilotherms follow changes in envirommental temperature rapidly and precisely. Loss of heat cannot occur by raporization (except in air breabers), and in most aquatic animals there are no mechanisms for actively reducing the heat loss which normally occurs by conduction and convection. Some older measurements of body temperature of aquatic animals indicate values higher than the environmental temperature, but more recent data show complete conlormance with the environmental temperature."s Cortainly in small aquatic animals heat conduction is rapid, metabolic rate low, and mean body temperature the same as water temperature. In larger animals, such as large fish, it is probable that during active swimming the temperature of the muscles may be appreciably higher than the skin temperature. The metabolism of several species of fish 
at $16.6^{\circ}$ averaged only 3.3 per cent of mammatian heat production. ${ }^{\text {jin }}$ Turtles in water are at the same temperature as the water (data from Baldwin ${ }^{7}$ excepted), and an intact turtle cools or warms faster than a pithed one, because of distribution of heat by the blood. ${ }^{136}$ Frogs have less mass than turtles, and, when dead, frogs cool or warm faster than when alive.

Thermal Reception and Orientation. Aquatic poikilotherms cannot freeze so long as they remain in water. However, certain tempcrature ranges appear to be most favorable for their growth and maintenance, and in a temperature gradient they tend to select or aggregate in a temperature range which is "optimal" or nearly "optimal." A bchavior response implies that the organism senses an environmental difference and that some sort of orientation occurs. The character of the taxic responses (orientation) to thermal stimuli has been discussed by von Buddenbrock" and by Fraenkel and Gunn. ${ }^{61}$

The classic experiments of Jennings" ${ }^{91}$ showed that ciliate protozoans tend to aggregate in a region of thermal neutrality, avoiding extremes of cold and heat. When a ciliate enters a region above or below thermal neutrality, it backs and turns until it arrives in the "preferred" range, where specimens tend to collect. This is well shown by hypotrichs such as Oxytricha, which give a characteristic "shock" reaction when stimulated. There is no direct orientation, but rather selection by trial and error.

Leeches which normally suck warm blood (Hirtto medicinalis) are positive to warm objects, responding to differences of 3 degrees above the surface water temperature; all other leeches fail to show this temperature response. ${ }^{6}$

Water temperature is important in determining the distribution of many fish, although it is often difficult to decide between temperature per se and oxygen and $\mathrm{CO}_{2}$ content as being crucial in natural waters. In a gradient tank many fish, by a combination of locomotor variables, tend to aggregate. Herring are said to show good aggregation reactions in water temperature gradients of $0.5^{\circ} \mathrm{C}$. and to be able to detect gradients as small as $0.2^{\circ} \mathrm{C} . .^{156}$ The temperature selected depends on the acclimatization temperature and the rate of transfer from one temperature to another or the rate of rise or fall of temperature, and it is not necessarily the same as the "normal" temperature; hence the term "optimal temperature" has little meaning in gradient behavior. This is well shown by the following data "for the fish Girella:

Acclimatization
Temperature
$10^{\circ} \mathrm{C}$.
20
30

Selected
Temperature
$18^{\circ} \mathrm{C}$.
23.6
24.3

Sereral kinds of temperature reaction and diverse receptors sensitive to comprature have been described in fish. When the temperature of any of sereral linds of fish is raised rapidly to some specific value $\left(27.6^{\circ}\right.$ for catfish, 26.7 for limudulus), at sudden flutter-type reaction occurs; this reaction is said not to oceur if the lateral line nerves are cut. ${ }^{1 ! 5}$ "The "spontaneous" activity of the neuromasts in the lateral line organ increases very much with rising temperature; hence the fish brain must receive lateral line impulses corresponding " temperature. In clasmobranchs there are large sensory bulbs on the surface of the head, the ampullac of Lorenzini, which have much in common 
with lateral line neuromasts. They show autonomous rhythmic activity which initially increases on cooling and decreases on waming. ${ }^{1-2-2}$ They respond to a difference of half a degree in the range of 10 to $15^{\circ}$; after the initial response to cold or warmth they adlapt, and the equilibrium level of activity varies directly with temperature. The role in beharior of the lateral line responses in bony fish and the ampullar response in elasmobranchs is not clear.

When warm or cold water is squirted against the tlank of a bony fish the tail fin gives a characteristic turning response. This stops after the spinal cord hias been transected between the region of stimulus and response but not after the lateral line nerve has been cut." I Hence the sense organs must be in the skin. Impulses have not been recorded in spinal nerves in response to thermal cutaneous stimulation, ${ }^{n-5}$ but they may well be in such small fibers that asynchronous spikes would be below the sensitivity of an amplifier. Fish of several sorts were trained to give a feeding reaction to warming, and this response persisted after cutting of the lateral line nerves. ${ }^{+2}$ It is probable that assumption of a position in a temperature gradient is also dependent on cutaneous thermal receptors. Such receptors have not been localized in fish.

By a combination of temperature receptors, partly lateral line organs, ampullae of Lorenzini, but principally cutaneous thermal receptors, fish are stimulated by heat and cold to selective orientation reactions. Whether there are any vasomotor reactions as well is not known.

Poikilotherms Inhabiting Moist Air: Body Temperature. In air, loss of heat by conduction is less important than in water, but loss by radiation and convection and loss by vaporization of water are more important. For each gram

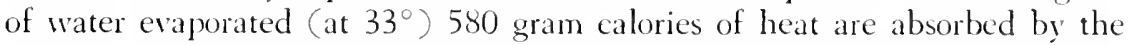
water, and the surface is thereby cooled. In sunlight, absorption of radiant heat from the sun may be important, but this warming mechanism is seldom of significance under water.

Cooling by vaporization depends on the vapor tension and air currents in the vicinity of the organism. The temperature of a slug or of an extended Helix, for example, is well below that of the surrounding air as measured by a dry-bulb thermometer unless the air is fully saturated. However, if the air temperature is measured with a wet-bulb thermometer, the slug is at the air temperature. When Helix withdraws into its shell it is protected against evaporation." The temperature of an earthworm in dry air soon diverges above the wet-bulb temperature, owing to rapid surface drying, ${ }^{, \tau}$ but in water the temperature in the intestine rapidly adjusts to the environmental temperature. ${ }^{10 .} \cdot 1 \mathrm{li}$ It is difficult to compare body temperature with air temperature. Few animals have continuous surface evaporation, as from a wet-bulb thermometer, yet all have some evaporation. Hence, where there is no active temperature regulation, the bod! temperature must be lower than a dry-bulb and may be higher than a wet-hulb temperature. Active physiological regulation can be demonstrated only if phrsical cooling by raporization is eliminated by measuring body temperature against a themometer from which there is equal vaporization, or in a saturated atmosphere. If measurements are made in sunlight, absorption of radiant heat must be considered.

The temperature relations of Amphibia are closely related to their water balance. ${ }^{2}, 3,103.12: 3$ Frogs lose water in proportion to the relative humidity; but they are unable to absorb water even from saturated air, because their 
metabolic heat results in some evaporation. Visceral temperature is never as much as 0.1 C. above surface temperature; it approaches wet-bulb temperature (Fig. 87). In unsaturated air the evaporative heat loss exceeds the metabolie heat gain, and the body is cooled (Fig. 87). In Bufo at $27.6^{\circ} \mathrm{C}$. and relative humidity (R.I.) of 82 per cent, the body temperature was $26.5^{\circ}$; at

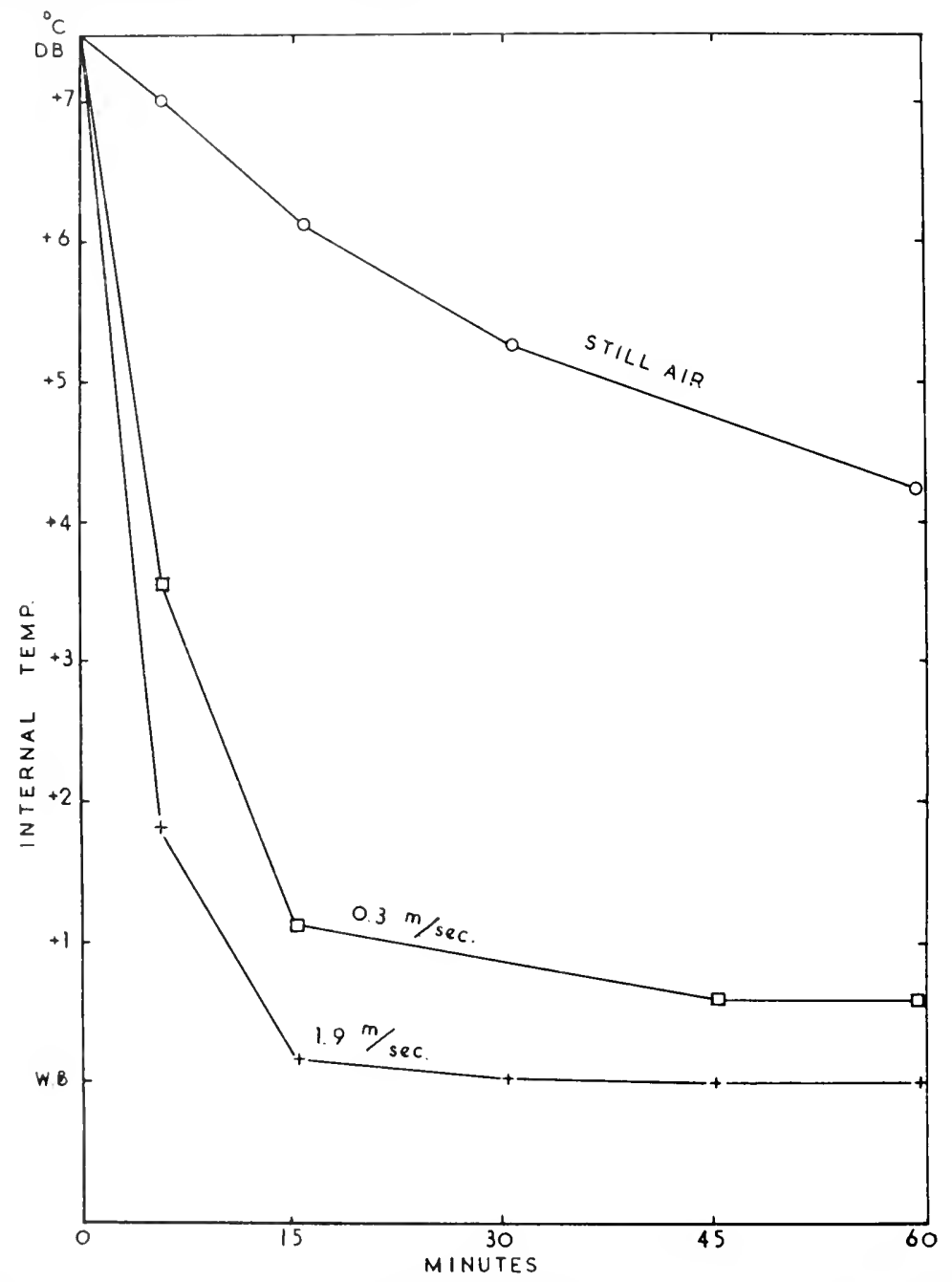

lig. 87. The internal temperature of lrogs exposed to air moving at different speeds. 1) dta are given in relation to the wet-bulb temperature (W. B.); dry-bulb temperature (1). B.). From Mellanby. ${ }^{123}$

the same temperature, with relative humidity of 27 per cent, the body temperature was 17.5 . 1 : In diy air so much heat is lost by vaporization that heat can be gained from the air by conduction." "The metabolism of an amphibian calculated for 37 is only one fifth that of a mammal (mouse) of comparable size. ${ }^{16}$ At 20" a frog produced $\mathrm{CO}_{2}$ equivalent to $6 \mathrm{cal}$./ hr. but hourly lost 
$3.2 \mathrm{gm}$. of water, which absorbed 2000 cal. ${ }^{12: 3}$ I leat hoss by conduction and radiation is low compared with loss by vaporization. Aquatic amphibia are at the same temperature as the water; in air they approach wet-bulb tempera ture, heat loss by vaporization always excecding heat production.

Thermoreception. The skin of the frog contains receptors for both heat and cold, spinal reflexes being elicited when the heat receptors are stimulated at 39 to $43^{\circ}$ and the cold receptors at $10^{\prime} .2^{2}$ These receptors are clearly distinct

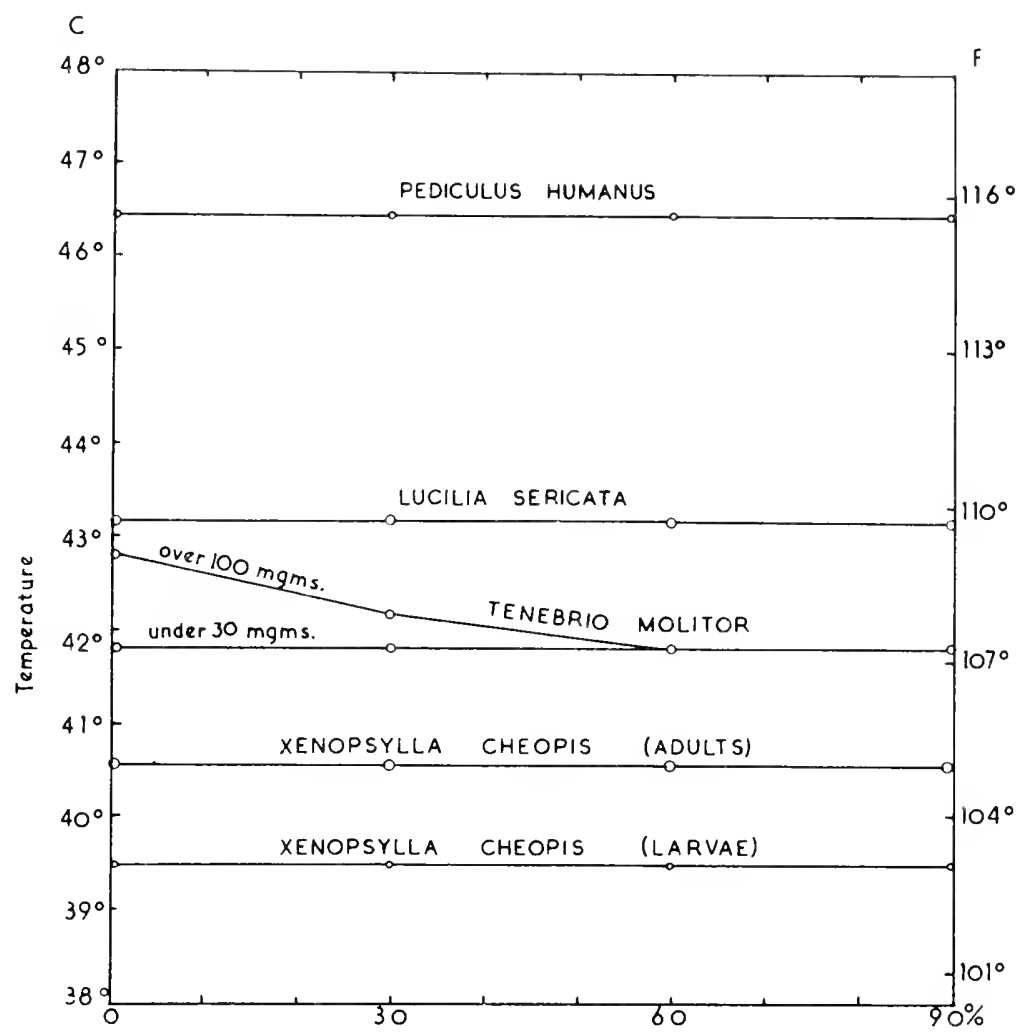

Relative humidity

Fig. 88. The highest temperature at which the insects stated can survive exposures of 1 hour under conditions of controlled atmospheric humidity. From Mellanby. ${ }^{121}$

from touch and pressure receptors, and are probably distinct from pain receptors, although the impulses from both thermal and pain receptors probably travel in very small fibers.

Poikilotherms in Dry Air. Insects and reptiles are poikilotherms which show rudiments of temperature regulation.

Body Temperature of Insects. Insects show wide variability with respect to loss of heat by vaporization and absorption of heat by radiation. Accurate temperature measurements of several large insects (Blatta and Periplaneta, ${ }^{125}$ 
the desert locust Schistocerca, ${ }^{21}$ and the grasshopper Gastrimargus ${ }^{104}$ ) show body temperatures lower than air in warm dry air, but in warm moist air they show temperatures higher than air. At low temperatures differences in humidity have little effect, and the body temperature may be slightly above the air

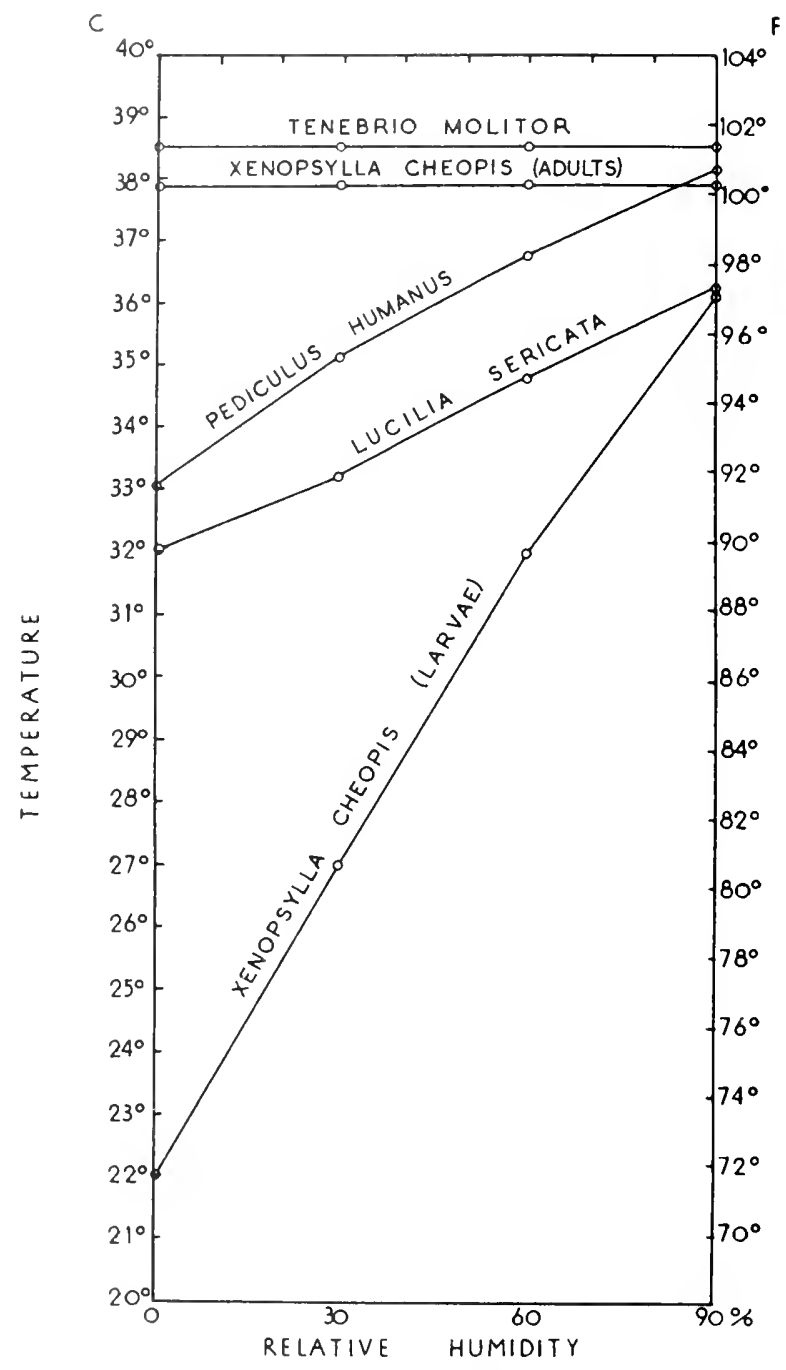

Hig. 89. The highest temperatures at which insects can survive exposures for 24 hours, under conditions of controlled atmospheric humidity. From Mellanby. ${ }^{121}$

temperatture (dry-hulb). Some insects are hygroscopic, that is, gain weight in at high humidity; under such conditions body temperature must rise above air temperature. In the roach, cvaporation increases at temperatures above $30^{\circ}$ because of increased permeability of the waxy layers of the cuticle (see Chapter 2). Most witer loss is by way of the spiracles, and death in warm dry air may 
result from desiccation. The degree of temperature which was lethal within one hour was not affected by humidity for a series of small insects. Specimens of Tenebrio larger than $100 \mathrm{mg}$. survived better in dry air than specimens smaller than $30 \mathrm{mg}$. (Fig. 88). However, for heat death in 24 hours, temperatures effective against those species which lack mechanisms for water retention were much lower in dry air than in moist air (Fig. 89).121 For example, the louse Pediculus survived 24 hours at $38^{\circ}$ at 90 per cent relative humidity, but died at $33^{\circ}$ at 0 per cent humidity. The results indicate that the primary cause of death was dehydration and not high temperature per se. The cooling effect of evaporation in dry warm air is entirely passive and occurs in dead insects. A balance sheet of heat relations of a live grasshopper ${ }^{104}$ shows that essentially no heat is lost by radiation and conduction at low humidities, and that at 90 per cent humidity loss of heat by vaporization still exceeds loss by other channels. The presence of cuticular wax and spiracular closing favors water retention but decreases cooling.

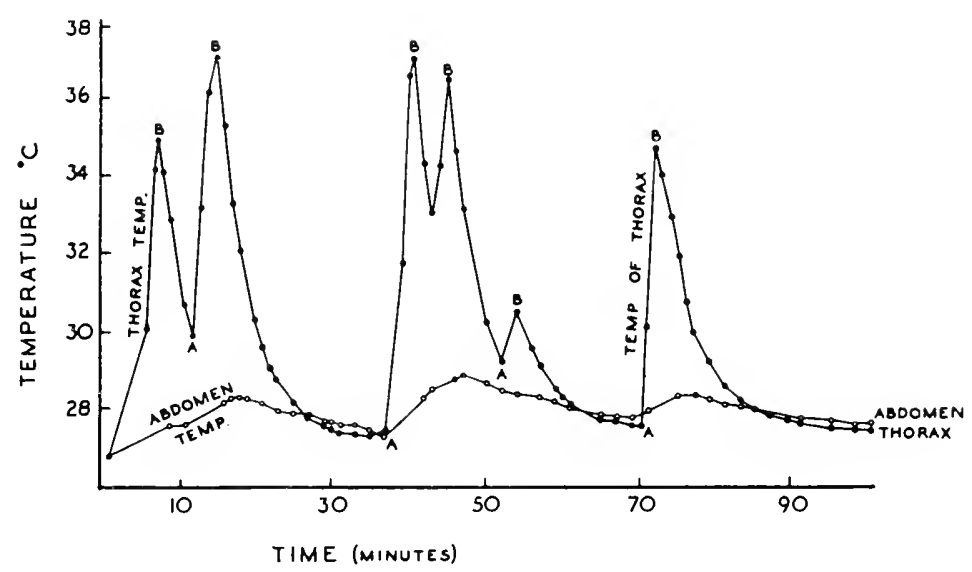

Fig. 90. Influence of muscular activity on the body temperature of a female Cecropia moth, 4 days after emergence. In the diagram $A$ to $B$ denotes periods of wing movement. From Oosthuizen. ${ }^{132}$

Insect heat of metabolism is considerable, especially in flight muscles. Thermocouple measurements of butterflies (Vanessa) and of lamellicorn beetles and some other insects show temperatures similar to that of the environment in resting insects but rapid increase in temperature prior to flight ${ }^{4+10 \times, 1: 3:}$ (Fig. 90). In flight, breathing accelerates, and the temperature rises more in wing muscles than in the rest of the body. Some insects show warming-up movements of the wings, others show muscle action potentials but no visible movement. The duration of warming-up is longer at low than at high air temperature; Vanessa warmed up for over 6 minutes at $11^{\circ}, 1 \frac{1}{2}$ minutes at $23^{\circ}, 18$ seconds at $34^{\circ}$, and not at all at $37^{\circ}$. Muscle temperature in this butterfly is about $35^{\circ}$ at take-off and $37^{\circ}$ during active flight. ${ }^{1+}$ The heat produced corresponds to a metabolism of $30 \mathrm{l} .0 .2 / \mathrm{kg} . / \mathrm{hr}$. before flight. ${ }^{10 \mathrm{~s}}$

Metabolic heat is used in varying amounts in colonial Hymenoptera, and 
the optimal temperature for brood development is high. The optimum for ants (Formica rufa) covers a range of 23 to $29^{\circ}$; the optimum for Vespa is 30 to 32 , whereas for honeybees the brood optimum is $34.5-35^{\circ}$, and brood survival is limited to the range of 32 to $36^{\circ}, 21, \cdots 3$ In the summer when the air temperature rises above the optimum for the brood, the bee workers frequently transport water into the nest, and aid evaporation by fanning. Ants transport brood to cooler chambers in the nest. By these means the brood temperatures are kept lower than the air temperatures.

In winter most colonial Hymenoptera cluster together, and the temperature of the cluster may be kept well above the air temperature (Fig. 91). If the temperature falls to $8-10^{\circ} \mathrm{C}$. bees show great uneasiness, the outer bees being more active than those in the interior of the cluster. Heat is conserved also by the insulating wall of the nest, by closing nest openings (ants), and by other physical means. There is much species variation in the insulating value of the nest covering. Ants also build special hibernating chambers; their body water content is decreased and metabolism reduced in hibernation. Cold rigor
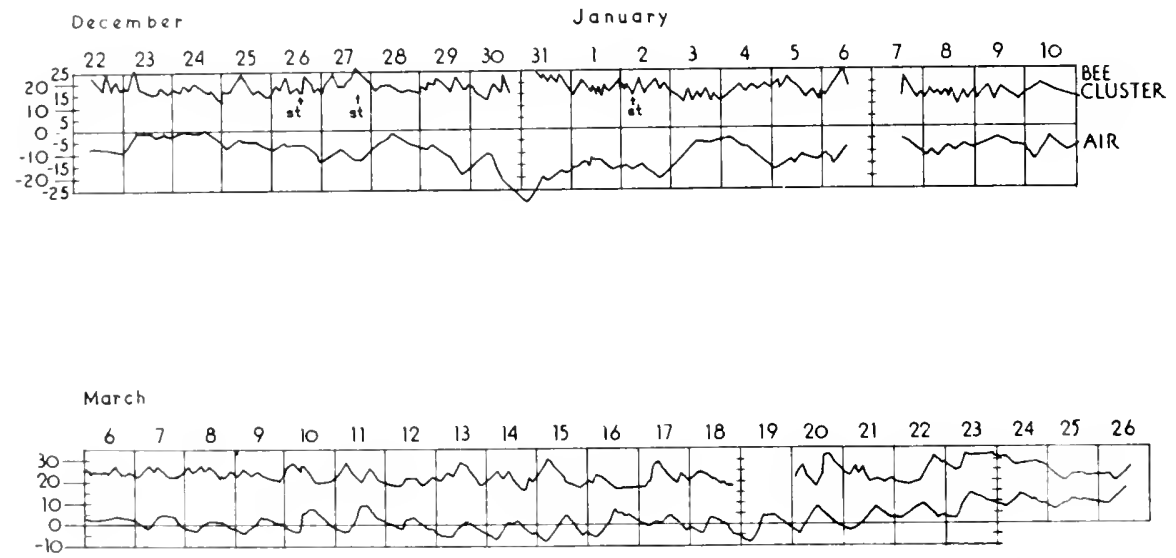

Fig. 91. Temperature of honeybee cluster in winter. From Himmer. ${ }^{83}$

occurs in bees at about $7^{\circ}$, in wasps and ants at about $0^{\circ}$. Apparently food absorption is reduced critically at these temperatures, since bees die at tempera tures above freczing with their stomachs full of sugar. Hymenoptera combine chemical and physical means in maintaining a relatively constant temperature of the nest.

In acddition to producing motabolic heat, insects can absorb radiant heat from the sun and from a radiating substratum such as a hot rock. Summarics of avalable data of absorption and reflection are found in the literature; 69.172 the most complete measurements are by Rucker. ${ }^{151}$ There is little correlation between color as seen in visible light and the transmission by the body wall of inf frared (wave length $1-3 \mu$ ). The absorption of infrared, hence of radiant heat, diflers less from inseet to insect than does absorption of different visible ware lengths. In general, however, dark insects absorb more infrared than lightecolored ones. For example, the dark elytra of the beetle Carabus reflect less than half of the infrared and reflect least at $2.15 \mu$, whereas the white 
wings of the cabbage butterty, Pieris, reflect more of the infrared and reflect least at $3 \mu .^{151}$ A light snout beetle (Compsus niveus) absorbs 26 per cent and a dark carrion beetle (Silpha obscura) absorbs 95 per cent of the infrared impinging on it. A dark brown grasshopper on sunny desert sand remained $4-5^{\circ}$ warmer than a light buff grasshopper.":? Desert locusts (Schistocerca) are very inactive below $17^{\circ}$, begin to move at 17 to $20^{\circ}$, and at this temperature in the morning take a position on an eastem slope with their bodies oriented perpendicularly to the sun's rays, thus receiving maximal radiation. ${ }^{60}$. "il At $28^{\circ}$ they start to migrate, or at higher temperatures (above $+0^{\circ}$ ) they rest on a bush parallel to the sun's rays, thus receiving minimum radiation. In the evening when the temperature falls the locusts again aggregate in a position perpendicular to the sun's rays, this time on a western slope.

Temperature "Sense" in lnsects. Another factor in maintenance of some constancy of temperature in terrestrial poikilotherms, particularly insects, is selection of a "preferred" temperature when subjected to a gradient. ${ }^{172}$ "The selected temperature is not necessarily "optimal," and often varies greatly. with the temperature to which the insects have previously been adapted. The effect of acclimatizing temperature on the selected temperature is shown by the following data:

\begin{tabular}{|c|c|c|c|}
\hline $\operatorname{ants}^{40}$ & $\begin{array}{c}\text { Accimatization } \\
\text { Temperature } \\
3-5^{\circ} \\
25-27^{\circ}\end{array}$ & $\begin{array}{r}\text { Sel } \\
\text { Tenn? } \\
2 \\
3\end{array}$ & $\begin{array}{l}\text { ected } \\
.5^{\circ} \\
.0^{\circ}\end{array}$ \\
\hline ticks ${ }^{36 i n}$ & $\begin{array}{l}18-20^{\circ} \\
1+-16 \\
10-12\end{array}$ & $\begin{array}{l}23^{\circ} \text { imago } \\
19 " \\
15 "\end{array}$ & $\begin{array}{l}19^{\circ} \text { nymph } \\
15 " ~\end{array}$ \\
\hline
\end{tabular}

In some other insects the temperature prior to a gradient test makes little difference.

The type of insect and atso the stage in life cycte are important. For example, some dung maggots while actively feeding select a temperature of $30^{\circ}$ to $37^{\circ}$, which is within the usual temperature range of their medium and near the temperature of maximum growth $\left(34^{\circ}\right)$; when they become ready to leave the dung to enter the ground for pupation the temperature selected falls to $15^{\circ} .{ }^{16 \mathrm{f}}$ In general, diumal bectles select higher temperatures than do nocturnal species, and temperature preferences vary with geographic races of the same species. ${ }^{10: 9}$

Often the selected temperature is higher for insects with a high lethal temperature than for those with a low lethal temperature, as shown by the following data ${ }^{130}$ on flies:

$\begin{array}{lccc}\text { Species } & \text { Temperature of } & \text { Lethal } & \text { Maximum Aggregation } \\ & \text { Heat Paralysis } & \text { Tenperature } & \text { Temperature } \\ \text { Fannia } & 36^{\circ} & 39-41^{\circ} & 20^{\circ}-26^{\circ} \\ \text { Stomoxys } & 43 & & 29-36 \\ \text { Musca } & 43.5 & +5-46 & 40\end{array}$

At low temperatures activity of insects ceases and the temperature at which movement resumes differs with the species. This is particularly evident when one watches the "awakening" of different species in the morning after a cool night. 
The mechanisms of perception of thermal gradients by insects are not well known, and some temperature selection may well depend on the absolute temperature and hence on the activity of the nervous system. There are, however, peripheral thermal receptors in some insects. In crickets the antennae perceive air temperature and the tarsi perceive ground temperature, while mouthparts and cerci also are temperature sensitive. ${ }^{80}$ In grasshoppers which orient in the sun, receptors appear to be scattered over the whole body. ${ }^{60}$ The antennae are more sensitive to thermal stimuli than other regions in most insects. The blood-sucking bug (Rhodnints) is attracted to a mammal partly by smell, but from short range it is attracted by thermal gradient in the air. ${ }^{173}$ The eyes are not used, but the bug waves its antennae about as if comparing the temperature gradient, and, if the antennae are removed, the insects become torpid and unresponsive to warm objects. Bedbugs and phasmids also have warmth receptors on the antennae. A positive reaction to warmth is important also in enabling a mosquito to locate its prey. The physiology of thermoreception in insects has not been studied, and there seems to be no evidence for cold receptors.

The manner of orientation and aggregation in thermal gradients has been discussed. ${ }^{61}$ In no insect is orientation direct, as it may be to light, but rather it is achieved by trial and error. There are random movements, particularly of antennae, so that the response appears to be a "reflex pursuit of the antennae into a zone of optimal stimulation." 173 Stimulation is by heat gradient, not by radiant heat, and only in the parasites of warm-blooded animals can heat act as a token stimulus. Selection then depends more on activity than on direction of movement. The activity of blowflies kept 12 hours at different temperatures was maximal at $30^{\circ}$, which was in the middle of the selected range, whereas if the temperature was raised from $1^{\circ}$ to $45^{\circ}$ during 6 hours, peaks of activity were noted at $20^{\circ}$ and at $42^{\circ}$. Thus, although there might be changes in activity associated with temperature change, the equilibrium activity seemed correlated with temperature selection. ${ }^{129}$ When presented with two sources of heat stimulation, insects such as lice circle between the two sources and then go toward one of them. ${ }^{85}$

In insects in general, body temperature is normally that of the environment, when allowance is made for evaporation, but slight regulation against temperature stress can be brought about by (1) altering water loss or retention; (2) using metabolic heat as in exercise; (3) clustering, fanning, and transporting water, as by colonial species; (4) orientation of position and absorption of radiant heat; and (5) aggregation in regions of selected temperature. Locomotion toward a heat source, best seen in parasites of homoiotherms, is not direct and appears to depend on comparison of thermal gradients by receptors, particularly on the antennae.

Body Temperature of Reptiles. Reptiles are the largest poikilotherms, and from them the homoiothermic animals arose. The change in body temperature of reptiles with change in environmental temperature is slow because of their high specific heat (I.ig. 92), and numerous adaptations anticipate true temperature regulation. The rectal temperature in large pythons increases with activity, and the body temperature of a female python incubating eggs was $33^{\circ}$ in air of $31^{\circ}$, whereas between the coils the temperature was higher $\left(33.5^{\circ}\right) .{ }^{16}$ Reptiles are much less dependent on humidity than are amphibia, 
and when the humidity decreases at a constant air temperature the reptile body fails to cool significantly (Fig. 93).70 'The body coat of horny scales is not sufficiently permeable to water to permit much evaporation, although the temperature of small snakes averaged $0.3^{\circ}$ below air temperature in the range from $10^{\circ}$ to $30^{\circ}$ C. $110 \mathrm{~A}$ series of reptiles, mostly snakes and large turtles, at 20-25 vaporized only $2-6 \mathrm{gm}$. H..O kg. $24 \mathrm{hrs.},{ }^{\prime \prime}$ in contrast to $76 \mathrm{gm}$. by frogs. ${ }^{133}$

Benedict has studied in detail the temperature relations and heat production by reptiles, particularly large snakes. ${ }^{16}$ "The metabolism is relatively constant

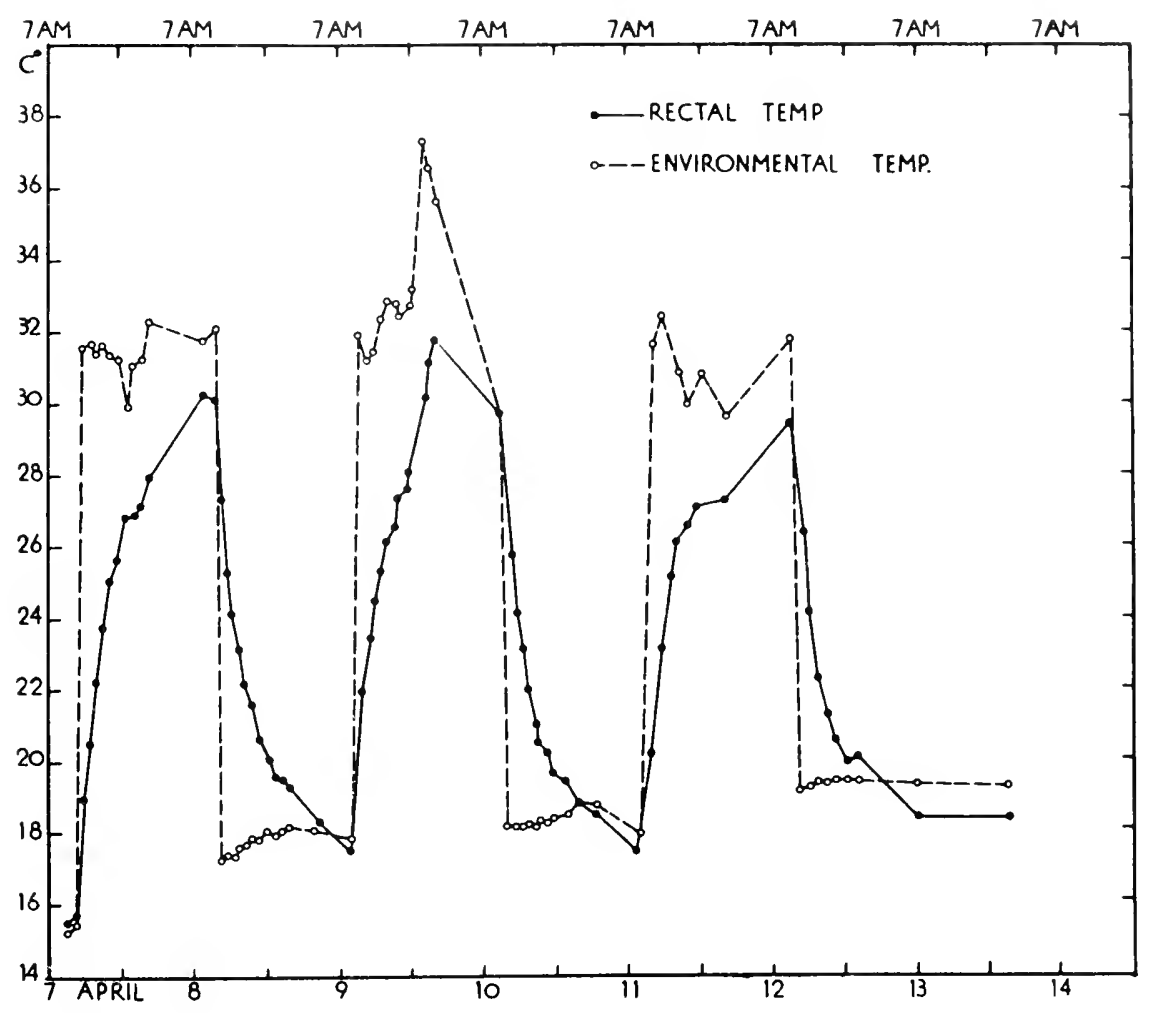

Fig. 92. Influence of changes in environmental temperature on rectal temperature of a python snake. From Benedict. ${ }^{16}$

for various snakes at a given temperature; e.g., at $22^{\circ}$, it is $21-24 \mathrm{cal} . / \mathrm{m} .{ }^{2} / 24$ hrs. The turtles produce more heat than do snakes or alligators, giant tortoises producing several times as much as snakes. In snakes, lizards, and turtles the heat production increases as the temperature rises. At $16^{\circ}$ the level of heat production of reptiles is similar to frog metabolism and may be higher than it is in some fish. ${ }^{150}$

Many snakes and lizards absorb much radiant heat while basking in the sunlight. Lizards in the sun at air temperature of $13^{\circ} \mathrm{C}$. had a cloacal temperature of $38^{\circ} \mathrm{C} .^{38}$ They may orient themselves to receive maximum radiation, and it is reported that the chromatophores of some lizards are expanded during 
the period of warming-up but are constricted once an optimal temperature is reached. Desert animals restrict their foraging to those periods of the day when the temperature is neither too cold nor too hot. When the body temperature of the desert iguana, for example, approaches $43^{\circ}$ it goes into the shade of bushes or undergromend. The homed toad (Phrynosoma) shows discomfort at $+0^{\prime \prime}: i_{\text {and }}$ antrow's at temperatures above $40^{\circ}$ or below $20^{\circ} .{ }^{170}$ An important factor in limiting survival of reptiles at temperatures above $45^{\circ}$ is the decreased atfinity of their hemoglobin for oxygen (Ch. 9, p. 312). At $50^{\circ}$ the blood of a chuchwalla (Samromalus) could not become more than 50 per cent saturated with oxyen at atmespheric tension, ${ }^{+2}$ whereas the tension for 50 per cent saturation of the blood of a gila monster (Heloderma) rises from an $\mathrm{O}_{22}$ tension of $32 \mathrm{~mm}$. H the at $20^{\circ}$ to $60 \mathrm{~mm}$. $1 \mathrm{lg}$ at $37.5^{\circ} .4 .3$

Little is known about thermal sense in reptiles, but there appear to be epidermal receptors, stimulation of which causes snakes to approach warm objects. The pit vipers (Crotalidae) have facial sensory pits and some boas have labial pits, both of which are temperature receptors. These snakes strike

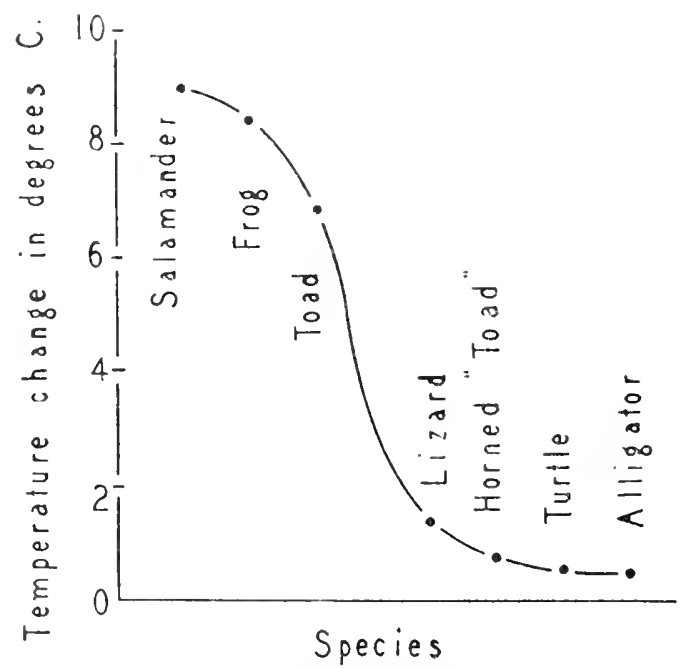

Fig. 93. Comparison of change in buly temperature of several species of amphibians and replites when the relative humidity was lowered from 100 per cent to 7 per cent saturation at $20^{\circ}$. From / Hall and Root."i

at a warm object and distinguish slightly warm from air-temperature objects only il the pits are intact and even when other sensory structures in the head are inactivated. ${ }^{1331 \mathrm{a}}$

Poililotherms lack efficient mechanisms of heat retention; hence in low environmental cemperatures their metabolic activity is limited, and as the temperature rises heat distribution from the body surface depends largely on an active circulatory system. It has been suggested that the giant reptiles were addapted metaldolicilly to high temperatures and that their circulatory systems were sluggish at the kow temperatures of the Pleistocene, so that they could not make sulficient use of radiant heat. Smatler reptiles would be less dependent on their circulation, and their temperature would have less of a lag behind that of the enviromment. 
In turtles and frogs the arterial blood pressure is directly related to blond temperature, and when the brain is pithed the blood pressure is no longer responsive to temperature. ${ }^{116}$ Local warming or cooling in the hypothalamus of the turtle but not in other regions of the brain has an effect on blood pressure. In birds and mammals, also, a drop in body temperature is accom panied by a decrease in bleod pressure. Rodbard ${ }^{1+6}$ has suggested that the temperature-regulating center of homoiotherms evolved from a region of the hypothalamus responsible for circulatory adjustment. Migration of vertebrates from water to land has been accompanied by development of a tolerance for greater and more rapid changes of extemal temperature than occur in water. Reptiles tolerate a wide range of body temperature and a corresponding range of autonomic function; birds and mammals tolerate a narrower range of body temperature. It would be of interest to know whether fish have a temperaturesensitive autonomic center comparable to that in turtles.

\section{TEMPERATURE RELATIONS IN HOMOIOTHERMIC AND HETEROTHERMIC ANIMALS}

Birds and mammals differ from all other animals in having a temperaturesensitive center in the brain which, by altering heat retention and heat production, acts as a thermostat for the body. Cooling mechanisms come into more prominent action in high environmental temperatures, and warming mechanisms in low external temperatures, so that over a limited environmental range body temperature is relatively constint. However, body temperature shows diurnal fluctuations associated particularly with muscular activity, feeding, and digestion. The diurnal temperature variation is less in large birds than in small birds, and nocturnal birds such as the owl have their temperature maximum during the night. ${ }^{15 \pi}$ Similar maxima at night occur in nocturnal mammals, such as the opossum. ${ }^{1 \cdot 2}$ When the activity rhythm is reversed by changing the illumination, the body temperature rhythm is also reversed. Ability to regulate body temperature varies aiso with age, maturity, metabolic state, and other factors. The temperature in the interior of the body is higher than at the body surface. In a nude man at an air temperature of $34^{\circ}$ C. various body regions were within 2.5 degrees of one another in temperature, but at an air temperature of $22^{\circ} \mathrm{C}$. the rectal temperature was $36.5^{\circ} \mathrm{C}$., while that of the hands was $30^{\circ}$, and that of the feet $27^{\circ} .^{16}$ In a dog at $37.2^{\circ}$ C. (per rectum), the temperature of thigh muscle was $36.4^{\circ}$, and of thigh skin 33.4.$^{90}$ At an air temperature of $100^{\circ} \mathrm{F}$. man gains when clothed $200 \mathrm{cal} . / \mathrm{hr}$, and, since the skin absorbs nearly all impinging rays (as a black body), the absorption is twice as great in the sun as in the shade. ${ }^{+}$In exercise the skin temperature first rises, then falls as sweating begins.

Degrees of Homoiothermy. Some representative body temperatures of birds and mammals are given in Table 61. Birds are higher in body temperature than mammals, and "higher" mammals have higher temperature and better regulation than more primitive orders. ${ }^{17 t}$ Extensive tables of body temperatures are given by Kanitz ${ }^{95}$ and by $\mathrm{Wislocki}^{17 i}$ homoiothermism is discussed by Pearse and Hall, ${ }^{136}$ and temperature regulation in man is presented by Burton, ${ }^{31}$ and Dubois. ${ }^{46}$ It has been suggested ${ }^{105}$ that since spermatogenesis is temperature sensitive, the presence of a scrotum keeps the testes at lower than body temperature and favors survival. Sterility occurs in rodents, 
TABLE 61. NORMAL BODY TEMPERATURES AND LETHAL TEMPERATURES OF HOMOIOTHERMIC ANIMALS

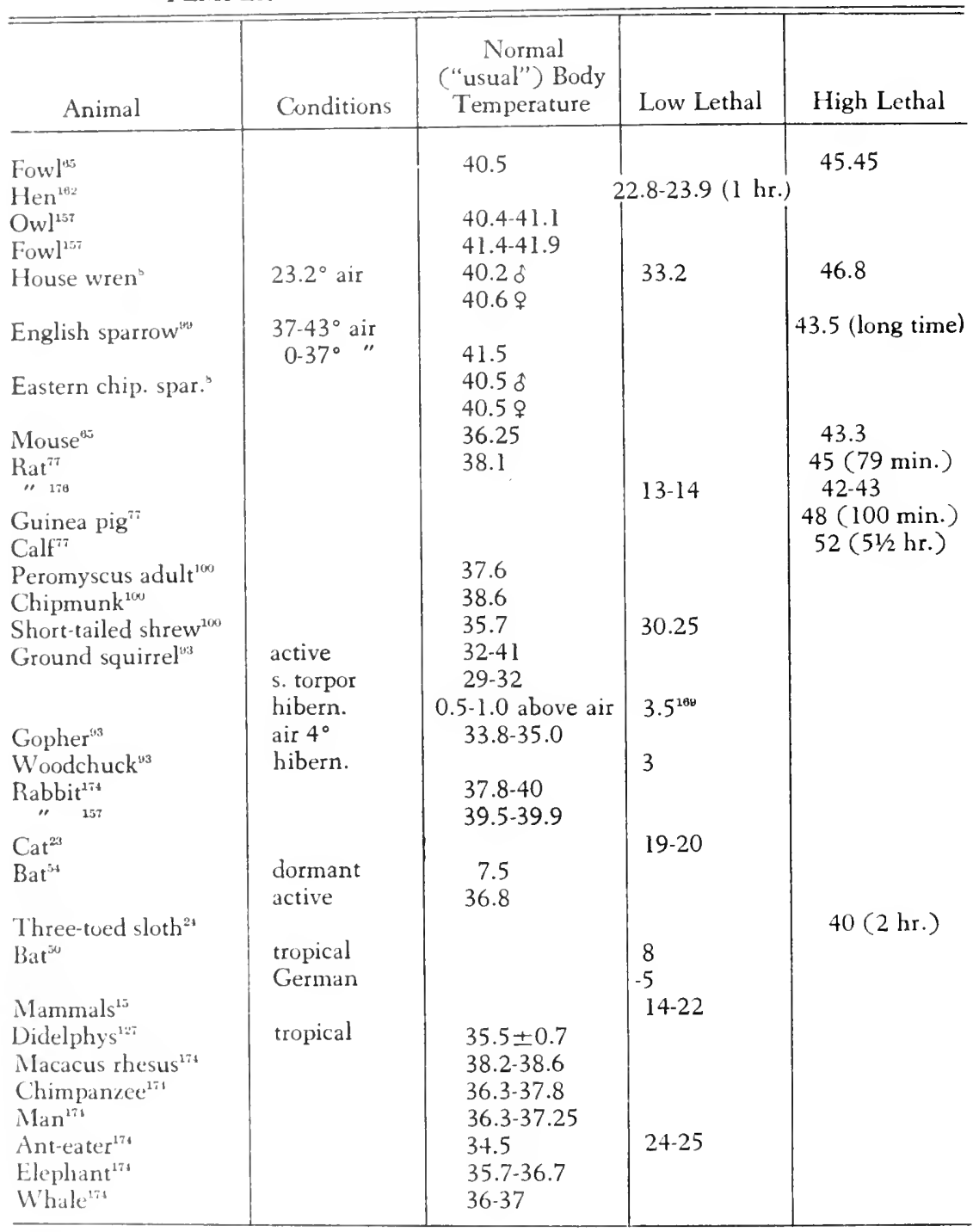

dogs and men when the testes are intra-abdominal or are heated in the scrotum. There are exceptions, however, as in the monotremes and insectivores, the whale, clephant, rhinoceros and seal, all of which have intra-abdominal testes. In birds the testes are intra-abdominal; however, spermatogenesis is said to occur only at night, when body temperature declines, and in some birds the testes migrate into a space between air sacs during the breeding season.

The more primitive mammals have lower body temperatures during normal activity than do higher mammats, and their temperature, although well above that of the environment, fluctuates with air temperature. The poorly regulating lower mammals are heterothermic (Table 62). Temperature regulation 
TABLE 62. BODY TEMPERATURE AS RELATED TO AIR TEMPERATURE IN A FEW SELECTED HETEROTHERMIC MAMMALS

\begin{tabular}{|c|c|c|}
\hline Animal & Air Temperature & $\begin{array}{l}\text { Body Temperature } \\
\text { (Usually Rectal) }{ }^{\circ} \mathrm{C}\end{array}$ \\
\hline Echidna ${ }^{11: 4}$ & 15 & $28-28$ \\
\hline \multirow[t]{4}{*}{ Ornithorhyncus $^{119}$} & 5 & 31.8 \\
\hline & 20 & 32.6 \\
\hline & 32 & 33.6 \\
\hline & 35 & 35.6 \\
\hline Several Australian marsupials ${ }^{114}$ & 15 & 36.5 \\
\hline Didelphis mars. (Eten opossum) ${ }^{127}$ & 26.5 & 37.5 day \\
\hline Metachirus (brown opossum) ${ }^{123}$ & $"$ & 33.8 day \\
\hline \multirow[t]{3}{*}{ Didelphis virg. (Virginia opossum) $)^{17}$} & 14 & $\begin{array}{l}35.7 \text { night } \\
32\end{array}$ \\
\hline & 23 & 34 \\
\hline & 26 & 34.5 \\
\hline \multirow[t]{2}{*}{ Dasypus (armadillo) ${ }^{174}$} & 19 & 32.5 \\
\hline & 24 & 34.5 \\
\hline Eptesicus (bat) ${ }^{5 t}$ & 7.2 & 8.3 dormant \\
\hline \multirow[t]{3}{*}{ Marmota (woodchuck) ${ }^{1 \pi}$} & 16 & $\begin{array}{l}36.5 \text { active } \\
36\end{array}$ \\
\hline & 28 & 37 \\
\hline & $30-35$ & 38.5 \\
\hline Hamster ${ }^{114}$ & 4 & $\begin{array}{l}35-36 \text { active } \\
5 \text { dormant }\end{array}$ \\
\hline \multirow[t]{2}{*}{ Rhinolophus (bat) ${ }^{30}$} & 10.5 & 35.5 \\
\hline & 20 & 40 \\
\hline Bradypus (sloth) $)^{10 \bar{\tau}}$ & 24.4-30 & $27.7-36.8$ \\
\hline \multirow{2}{*}{ Bradipus (sloth) } & 13.4 & 29.2 \\
\hline & 22.1 & 30.3 \\
\hline \multirow[t]{3}{*}{ Citellus (ground squirrel) ${ }^{12}$} & $0-10$ & 35.5 \\
\hline & $25-30$ & 38.4 \\
\hline & $-1.7-10$ & $\begin{array}{ll}.2-5 & \text { above air } \\
& \text { temperature } \\
& \text { (hibernating) }\end{array}$ \\
\hline
\end{tabular}

is poor in Echidna, better in Ornithorhynchus. Sloths, opossums, and bats show some temperature fluctuation with the environment. Figure 94 gives the body temperature of a Central American opossum as a function of air temperature; this is a nocturnal species, and the body temperature while the animal is awake is consistently higher than while it is asleep, although temperature changes are greatest in warm or cold air. ${ }^{126}$ Most, perhaps all, of these heterothermic mammals readily enter a state of cold narcosis at low air temperatures.

Development of Homoiothermy. Newborn mammals or recently hatched birds show poor temperature regulation. A two-day-old mouse, for example, is essentially a poikilothermic animal; ${ }^{161}$ at 10 days a mousc regulates in intermediate air temperatures, and at 20 days in extreme temperatures. A six-day rat has a body temperature 1.3-1.8 degrees above the environment over the range of 24 to $37^{\circ}$, and 0.5 degree or less above the air at temperatures below about $20^{\circ}$; at 12 to 16 days coritrol occurs during activity, and at 25 days the rat approaches the adult condition. ${ }^{68}$ Development of temperature regulation may be correlated with development of myelination in nerve fibers in the 
hypothalamus. ${ }^{2 *}$ In man, development of temperature regulation may take several years. ${ }^{31}$ Temperature regulation is perfected in chickens 4 to 5 days after hatching. ${ }^{1 *}$ In the house wren temperature regulation appears gradually during the two weeks after hatching (Fig. 95). Regulation is accompanied by increasing sensitivity of the heat center and increasing basal metabolism. In the wren, for example, metabolism as measured by $\mathrm{CO} .2$ output increases with age up to 12-15 dars; as regulation appears the $\mathrm{CO}_{2}$ production is highest at low air temperature and is minimal at $37.8^{\circ}$. 'The air temperature at which metabolism is minimal is well below body temperature. Similarly, water loss was increased by 4.6 times from $21.7^{\circ}$ to $37.8^{\circ}$ in wrens 0,3 , and 6 days old, but hardly at all in adults; however, at anr temperature of $40^{\circ}$ the water loss was twice the loss at $37.8^{\circ}$, regardless of age. ${ }^{98}$ Young swifts are able to survive

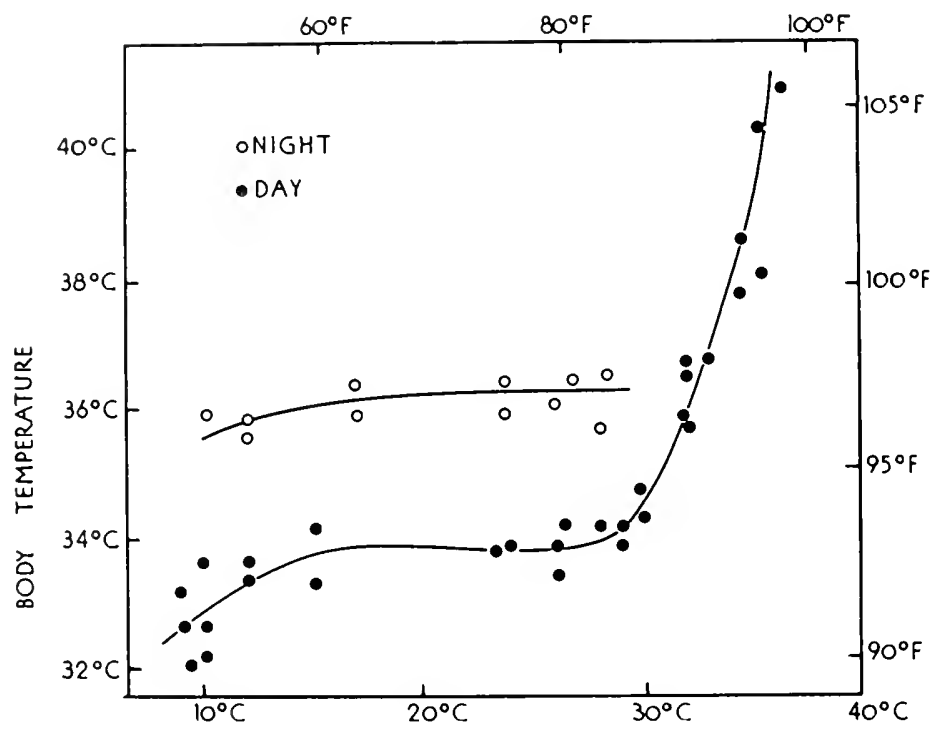

EXTERNAL TEMPERATURE

Fig. 94. Body temperature as a function of air temperature in a nocturnally active Centra! American opossum, Metachirus. From Morrison. ${ }^{127}$

fasting longer than adults, because their metabolism and temperature fall and they enter a hunger coma. ${ }^{10 .}$

Thermal Stimulation. The mechanisms of heat regulation are activated in two ways: by thermal receptors in the skin, and by direct stimulation of the thermoregulator in the brain by changes in blood temperature. The thermal receptors in the skin may elicit vasomotor reflexes even though the individual is not conscious of heat or cold sensation. Observations on man show that the recepters for colt are probably the bulbs of Krause, located at the outer border of the skin layer containing blood vessels, that is, at a depth of about $0.1 \mathrm{~mm}$., whereas the warmth receptors are the Ruffini organs, which lie deeper in the skin $\left(0.3 \mathrm{~mm}\right.$.), where blood vessels are abundant. ${ }^{12},{ }^{13}$ Much sensory 
summation occurs, in that the threshold for stimulation of large areas is less than that for stimulation of single sensory endings, an area of cold receptors being stimulated by a fall of $0.004 / \mathrm{sec}$, and one of warmth receptors by a rise of $0.00 \mathrm{l} / \mathrm{sec}$. for 3 seconds. ${ }^{12}$ Non-penetrating infrared is more effective as a thermal stimulus than are penetrating rays; therefore the thermal sense appears to depend more on the thermal gradient in the skin than on absolute temperature or even rate of change of temperature in the skin. ${ }^{13}$ It is also possible to obtain pain with thermal stimulation, but the skin receptors are not the same,

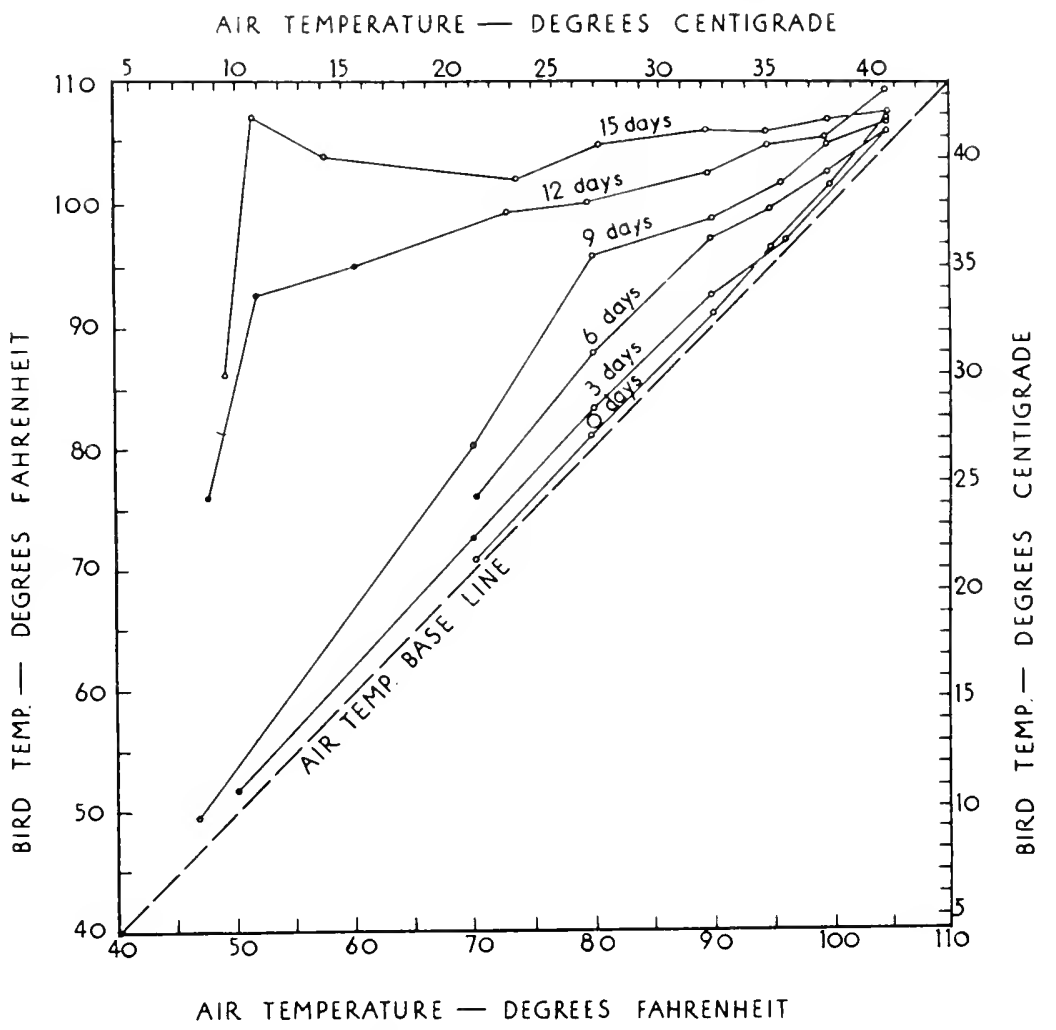

Fig. 95. Body temperature of wrens at different air temperatures at different ages after hatching. From Kendeigh."15

as shown by the following: the pain threshold is raised and the heat threshold lowered by acetylsalicylic acid; after occluding the blood flow to an arm for some minutes only the pain sense remains, and pain shows little summation as compared with heat or cold. ${ }^{73}$ The threshold for pain by a 3-minute thermal stimulation is $0.218 \mathrm{cal} . / \mathrm{cm} .2 / \mathrm{sec}$., compared with the warmth threshold of $0.00015 \mathrm{cal} . / \mathrm{cm} .{ }^{2} / \mathrm{sec}^{12,}{ }^{2} \mathrm{i2}$

The nervous pathways by which reflex responses to thermostimulation are elicited are not well known. There may be local vascular reactions, largely by axon reflexes, also some direct responses of blood vessels to temperature change, but the principal responses of vasodilation, vasoconstriction, and hair 
erection (pilomotor response) may occur in distant regions of the body by reflexes which traverse hypothalamic centers. Whether there are by-pass connections directly to the vasomotor center in the medulla from ascending tracts carrying impulses from thermoreceptors or whether all of these impulses go first to the temperature center in the hypothalamus is not established. In any case the initial reflex reactions have the effect of altering the body insulation, increasing or decreasing heat loss from the skin. Removal of parts of the cerebral cortex from cats and dogs results in abnormal temperature-regulating reflexes, particularly to cold; hence the cortex may be required for proper responses of the temperature center. ${ }^{138}$ Decerebrate and spinal animals are unable to show temperature regulation.

When the environmental changes in temperature are sufficient that the insulating reactions do not adequately maintain body temperature, the blood temperature may change and this stimulates to more drastic reaction the thermoregulating center. This is a region of the caudal hypothalamus consisting of two portions, one heat-sensitive and the other cold-sensitive. ${ }^{116,143}$ These regions evoke many responses in the blood vessels, endocrines such as adrenal medulla, various autonomic effectors, and skeletal muscle. The principal outflow is autonomic, and sympathectomized animals fail to show vasomotor and pilomotor responses to temperature changes.

The mechanism of stimulation of thermoreceptors and the nature of the afferent discharge from them are not known. It is of interest that nerve fibers can be stimulated by local temperature change, the small $\mathrm{C}$ fibers of mammals being excited by heating and large $A$ fibers by cooling. ${ }^{3}$ The cutaneous thermoreceptors are large structures, and a thermal gradient in them may cause a potential from one end of the receptor to the other which is excitant.

Physical Mechanisms of Temperature Regulation. The mechanisms of temperature regulation are sometimes considered as physical (transfer of heat increased or decreased), and chemical (production of heat increased or decreased). The physical factors are well summarized by Dubois. ${ }^{46}$ The initial responses to changes in environmental temperature are local vasomotor reactions which are initiated reflexly from cutaneous thermoreceptors. Skin vessels dilate and blood leaves the viscera when the air temperature is high, and the reverse reactions occur in low air temperatures. Change in temperature of one extremity causes vasomotor responses in the other extremity; the ears of some mammals (rabbit, elephant) are important in vasomotor cooling. Thus the transfer of heat to the body surface by blood regulates loss or retention of heat. Further, the thermal conductivity of skin is increased by vasodilatation. The temperatures inducing dilation and constriction vary with humidity, and an "optimal" temperature exists for an animal at a given humidity. As water is lost in heat the blood volume may increase and water may move from the body tissues out into the blood, whereas in cold the reverse reaction occurs and tissue cells swell. ${ }^{10}$ Thermal conductance can change by 5 or 6 times, and skin blood flow varies much more. ${ }^{32}$ Joss of heat by radiation exceeds loss by vaporization at low temperatures, but above about 30 loss by vaporization predominates (Fig. 96). Water is vaporized over three channels: (1) in the respiratory tract, (2) by the sweat glands, and (3) by insensible loss directly from tissue spaces and out of the skin. The relative contribution of these channels varies with species and conditions of 
the organism. Under basal conditions man loses about 20 per cent of his heat by vaporization, under exercise the loss is $75-80$ per cent. ${ }^{46}$ Sweating begins in man at an average skin temperature of $34-35^{\circ} \mathrm{C} .3^{31}$ The loss by radiation remains relatively constant, its per cent contribution to the total loss is less in exercise. Loss of heat by radiation and conduction is prevented by insulation. Aquatic mammals, many of which are hairless, have an insulating layer of fat. Heat loss by terrestrial mammals-man, dog, and others-in water is twice as great as it is in air. ${ }^{124}$ In air-dwellers the fur coat and feather cover conserve heat and can be erected to encase an insulating layer of air. Permanent bird residents of cold areas have a thicker feather cover in winter than do migrants to warm climates, and mammals have thinner fur coats in summer

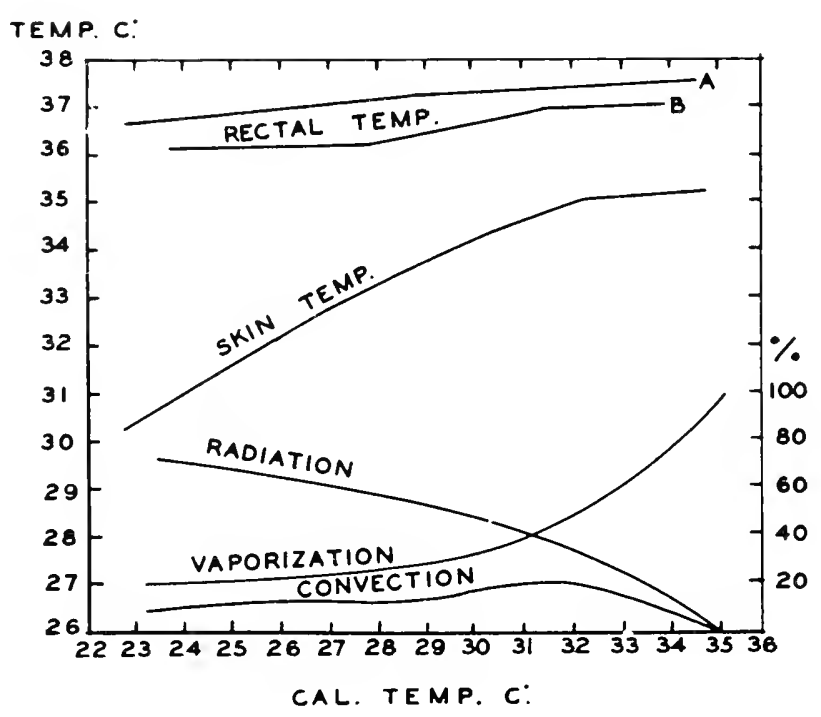

Fig. 96. Relation of rectal temperatures ( 2 subjects), skin temperature, and per cent of heat loss by radiation, vaporization, and convection in man, to environmental (calorimeter, cal.) temperature. From Hardy and DuBois. ${ }^{71}$

than in winter. Not only is the fur thicker, but it is better insulation in arctic mammals (Fig. 97). A guinea pig loses 33 per cent more heat if it is shorn, ${ }^{136}$ and at $20-24^{\circ} \mathrm{C}$. heat loss from a shaved cat was 16 per cent above heat production, indicating that fur-bearing mammals rely on the fur, in contrast to man, who alters heat conductance of the skin. ${ }^{1+1}$ When sleeping, many mammals coil up, and birds bury the head under a wing and fluff out their feathers.

When the body temperature rises above the "optimum" at a given humidity and air temperature, heat loss by vaporization is increased. ${ }^{48}$ Sweat glands are stimulated, and in animals lacking sweat glands and in birds panting appears. Panting begins in dogs at rectal temperatures a few tenths of a degree above the basal rectal temperature. ${ }^{7 \varnothing}$ Breathing and heart rate are accelerated. Man in cool air may lose by evaporation 1 liter of water per day; in hard work 
on the desert he may evaporate 1.51 . per hour. ${ }^{+3}$ Output of sweat increases $20 \mathrm{gm}$. hr. for each $!^{\circ}$ F. rise in air temperature. Nan can withstand very high air temperatures if allowed plenty of water for perspiration. ${ }^{4}$ In secreting sweat man loses much salt, particularly sodium chloride, and in acclimatization to high temperatures the chloride concentration in sweat decreases while that in urine increases. In warm moist air, as in the jungle, evaporation is much less than in the desert. A burro working on the desert does not pant and it secretes a sweat which contains little chloride. A panting dog, on the

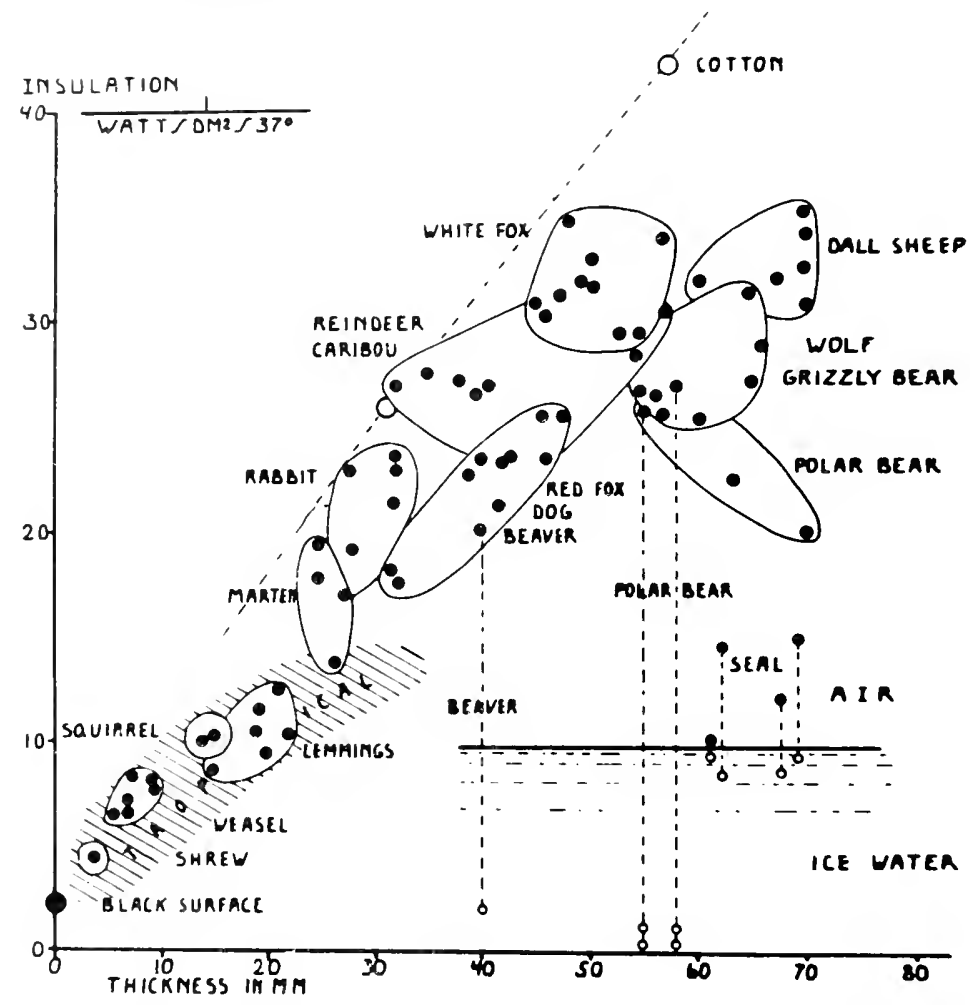

Fig. 97. Maximum insulation of fur of arctic and tropical mammals as a function of fur thichness. Each dot represents one measurement. Values for aquatic animals are given in air (dots) and in ice water (open circles): values for terrestrial animals are given for air only. The insulating value of medium weight cotton is given by the broken line. From Scholander (unpublished).

other hand, evaporates much water lrom its respiratory tract, and this may result in increased chloride concentration in the blood and in the development of some alkialesis clue to the blowing off of $\mathrm{CO}_{2 .}+3$ In bircls, vaporization occurring as air passes through the air sacs may have a cooling effect.

When the bedy temperature is cooled below "optimum," shivering may begin. Ihis is ansociated with or follows rasoconstriction and piloerection. Shivering increases axyen consumption, hence heat production. The abilities to pant and to shiver develop in the young animal during the period when temperature reguldtion appears. 1:31. 16 When the "thermostat" becomes set 
too high, a fever begins by shivering, pilocrection, cutaneous vasoconstriction, and absence of sweating; heat is retained, and the body temperature then rises. If the normal body is cooled, as by drinking ice water, or il it is warmed, the regulatory mechanisms bring the body temperature back to "optimum.":"

Chemical Mechanisms of Temperature Regulation. The chemical or metabolic factors in temperature regulation are important in two respects: responses to temperature change, and batsal metabolic level.

As air temperature becomes lower or higher the metabolism remains constant, while the physical insulating mechanisms, thickness of body coat and vasomotor reactions, compensate for changes in thermal loss. Finally these mechanisms are insufficient, and at elevated blood temperature metabolic rate rises according to tissue temperature, whereas at depressed blood temperature the thermoregulating center is stimulated to activate muscular movement (as shivering) and probably secretion of endocrines which result in increased tissue metabolism (heat production). That is, metabolism of homoiotherms is minimal in a range of thermal neutrality and increases both above and below this range, whereas in poikilotherms metabolism varies directly with temperature.

There is much species variation in the metabolic response to low temperature. In the English sparrow, for example, the body temperature was $41.5^{\circ} \mathrm{C}$. for air temperatures from 0 to $37^{\circ}$, yet the heat produced per gram per hour at $0^{\circ}$ was double the amount produced at $37^{\circ}$; above $37^{\circ}$ the body temperature rose somewhat and heat production increased more. ${ }^{9:}$ When a mammal is chilled there is increased liberation of adrenim, also increased production of adrenal cortical hormone and thyroid hormone, hence increased metabolism. Adrenalectomized or thyroidectomized rats lose ability to compensate for. cold. ${ }^{25}$ The hypophysis may also be involved. Thyroid activity is enhanced at low temperatures. ${ }^{31}$ Nan in water at $20^{\circ} \mathrm{C}$. produces heat at 3 to 6 times the basal rate, and by this means keeps his rectal temperature from going below $35^{\circ} \mathrm{C}$; ; in water at a temperature lower than $20^{\circ} \mathrm{C}$., heat production decreases and the body temperature drops. ${ }^{124}$

Birds and mammals of the aretic have better insulating ability than tropical species. This is indicated by the extent of the temperature range over which metabolism is constant, that is, over which the insulating reactions are sufficient for maintenance of body temperature. In the arctic white fox, for example, air temperature can be lowered to $-30^{\circ} \mathrm{C}$. without any increase in metabolism; cold stress, however, is sufficient to elicit a rise in metabolism in the Alaskan ground squirrel at $-10^{\circ}$, in lemmings at $15^{\circ}$, and in polar bear cubs at $0^{\circ} \mathrm{C}$. In sloths, night monkeys of Panama, or jungle rats, metabolism increases when the air temperature falls to about $25^{\circ} \mathrm{C}$. (Fig. 98)..$^{15 i}$ As the air temperature falls, heat production increases by a small amount in well acclimatized birds and mammals of the arctic and by greater amounts in tropical species. In all of them, however, the slope of the curve of heat production extrapolates back to zero at the normal body temperature (Fig. 98). If an animal is unable to maintain its body temperature by its insulating and metabolic reactions, heat production then falls off with chilling, and long survival is impossible.

The metabolic response to stress of cold can be altered by acclimatization. For example, rats kept for 3 months at $0^{\circ}$ to $2^{\circ}$ maintaincd a constant tempera- 
ture and increased their metabolism when cooled below zero, while others kept for 3 months at 30 to $32^{\circ}$ regulated less well, and when the air temperature dropped to $15^{\circ}$ the increase in metabolism was insufficient to maintain body temperature. ${ }^{66}$ Despite the physiological adaptation of individuals, it is unlikely that tropical mammals could be adapted to live at arctic temperatures. The genetic and physiological limits of temperature tolerance have not been clearly defined in any species.

A basic condition for homoiothermism is high basal metabolic rate. Heat production can be measured by direct calorimetry or may be calculated from $\mathrm{CO}_{2}$ production and $\mathrm{O}_{2}$ consumption. In man, with an R.Q. of 0.82 , heat is produced to the extent of 4.8 calories per liter of oxygen consumed. ${ }^{18}$ In general, at a given body temperature, the metabolic rate is higher for small animals of a kind than for large animals, although metabolic rate is not a simple function of size (see pp. 225-228, Ch. 8).

A small poikilotherm (e.g., frog or fish) has a metabolism at $16^{\circ}$ equal to

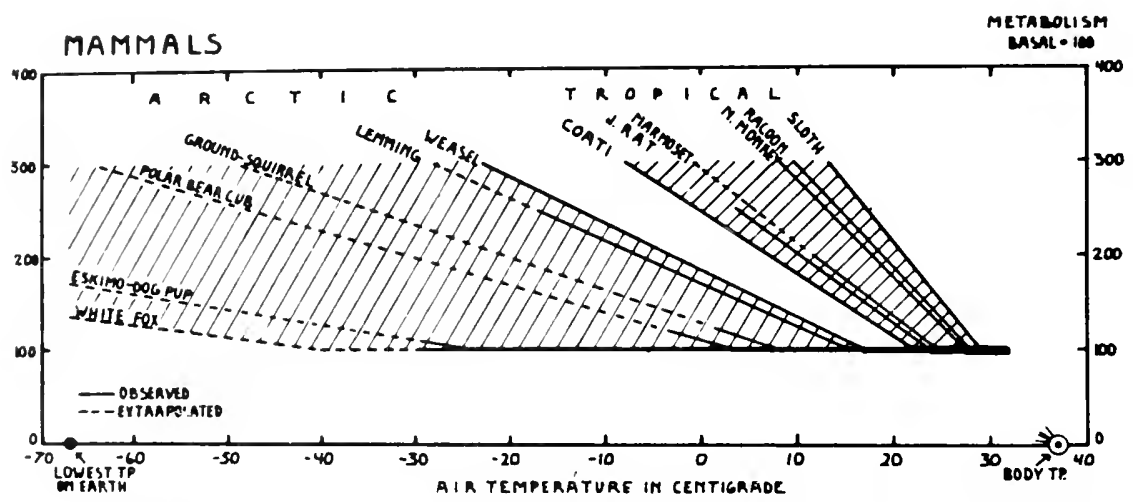

Fig. 98. Metabolism of arctic and tropical animals in resting state as a function of air temperature. Solid lines represent average measurements, broken lines are extrapolations. From Scholander (unpublished).

about 5 per cent of the heat production of a homoiotherm of similar weight at $38^{\circ}$. $\triangle 20 \mathrm{gm}$. mouse at $37^{\circ}$ produces $150 \mathrm{cal} . / \mathrm{kg}$., a $20 \mathrm{gm}$. canary produces $250 \mathrm{cal} . / \mathrm{kg}$., and a $20 \mathrm{gm}$. frog calculated to $37^{\circ}$ produces about $30 \mathrm{cal} . / \mathrm{kg}$. Measurements ${ }^{16}$ of heat production in a poikilotherm, in one good temperature regulator, and in one poor regulator, of identical weight and at the same temperature $\left(2.5 \mathrm{~kg}\right.$ and $\left.37^{\circ}\right)$, are as follows:

$\begin{array}{lcc}\text { Rattlesnake } & 19.35 \text { cal./ kg. } / 2+\text { hours } \\ \text { Woodchuck (Marmota) } & 72 \text { ", ", " " } \\ \text { Rabbit } & 112 \quad ",\end{array}$

there lias been much discussion as to whether weight, body surface areal, total nitrogen, or some other measure of size is the better correlated with metalholism (Ch. 8). Certainly in homoiotherms the body surface is important because for a given degree of insulation the total heat loss for a given amount of heat production depends on the area over which a thermal gradient exists. The basal metabolic rate is higher in individual mammals adapted to a cold cnviromment than in wam-adapted individuals (man, rat). 


\section{MIGRATION AND HIBERNATION}

Some birds migrate with the seasons and some mammals hibernate in winter. Migration is a complex response, initiated by changes in energy balance coordinated with seasonal variations in air temperature and photoperiod. With their high body temperature and high metabolic rate, small birds lose weight rapidly during a few hours without food at low air temperature. 'The length of the daylight period for feeding must be sufficiently long to permit the bird to accumulate enough reserves to last overnight, or the birds are compelled to migrate. During the winter, survival time of English sparrows without food at $-14^{\circ} \mathrm{C}$. is 19 hours; at $+34^{\circ} \mathrm{C}$. it is 61 hours. ${ }^{101}$ During the warmer summer months the resistance of sparrow's to hunger is decreased; at $-1+\mathrm{C}$. survival time is only 11 hours. For the house wren at $-14^{\circ} \mathrm{C}$. during the summer survival time is less than 5 hours, and this is a migratory species. Differences between species in survival time without food depends on the relative amount of utilizable fat stored in the body and on the rate at which it must be metabolized to maintain body temperature. Undoubtedly the factors that control migration may also establish climatic barriers limiting distribution. ${ }^{97,} 101$

When the temperature goes down slowly, some lower invertebrates encyst and their metabolism is reduced essentially to zero; in some insects water content is reduced as they are "hardened" at low temperatures; larger poikilotherms become dormant, surviving as long as their metabolism is not reduced below some critical level. Dehydration is important in initiating estivation, particularly in reptiles.

Certain mammals can reduce the effectiveness of their temperature regulation and enter hibernation. Ilibernation has been well reviewed in the literature. ${ }^{17} .157,93,96,11+$ Hibernation is best known in monotremes, bats, rodents, and shrews. It is doubtful whether carnivores truly hibernate in the sense of becoming virtually poikilothermic, although bears and skunks sleep for long periods in the winter. Numerous heterotherms enter cold narcosis which may be a form of hibernation. The body temperature of bats approaches air temperature and metabolism is reduced by about one-half in their diurnal sleep; in caves in the winter hibernating bats are easily disturbed and frequently active. Sleep can be induced in bats at $10^{\circ} \mathrm{C}$. and they can be kept in a state of cold narcosis in a refrigerator for weeks. The differences between their usual sleep, when they are poikilothermic, and true hibernation are not clear. Tropical bats do not hibernate but become increasingly active in cold air.". 30 If a three-toed sloth is placed in cold air $\left(10^{\circ}-15^{\circ}\right.$ C.) its body temperature falls rapidly to about $20^{\circ}$ and the animal enters cold narcosis; ${ }^{+}+$this may not be true hibernation. The responses of marsupials and eclentates to cold have not been adequately examined. There are probably several kinds of sleep, and several ways of inducing the various kinds of sleep.

There is no simple cause of hibernation. The primary stimulus for the woodchuck is depletion of food supply. ${ }^{17}$ Food reserves, true fat and brown fat, we usually essential and are used during hibernation. Lack of food is also a stimulus for estivation by some desert mammals. The magnesium concentration in blood serum is high, for example, in a hibernating hedgehog, and injection of magnesium chloride may induce hibernal sleep. ${ }^{\text {ti:3 }}$ Blood magnesium is also elevated in hibernating snails. ${ }^{11}$ In many mammals low tem- 
perature is the initiating factor, although in some mammals hibernation can occur without a change in temperature and a hibernating animal can sometimes be awahened by further extreme cold. Hamsters in the cold hibernate in the presence of adequate food, ${ }^{11+}$ and hamsters, ground squirrels, and other animals occasionally wake for brief periods to eat and void.93

The most striking characteristic of hibernation is "turning down the thermostat." The rate of fall of body temperature may be very rapid. The temperature of a ground squirrel may drop 5 per hour." The body temperature of a hibernating mammal fluctuates very much and is usually one to a few degrees above air temperature. I ledgehogs enter hibernal sleep at an air temperature of about 17 , and thereafter the body temperature rises and falls with the air temperature. Below an air temperature of $5.5^{\circ}$ the body temperature sometimes remains at $6^{\circ}$ C., ${ }^{\prime 1}$ or it may go even as low as $2^{\circ}$ for a brief period, although this low temperature is not usually survived. ${ }^{1+0}$ Bats in a cave at $11.8^{\circ}$ areraged $11.9^{\circ}$ body temperature. ${ }^{1+t}$ Ground squirrels become lethargic at body temperature below $30^{\circ} \mathrm{C}$. and show great individual variability in response to environmental conditions which influence hibernation. ${ }^{93} \mathrm{~A}$ woodchuck (Marmota) in hibemation has a rectal temperature several degrees lower than its heart temperature. ${ }^{\text {it }}$

The rate of oxygen consumption declines with body temperature. In the hamster metabolism in 4.5 hours reached a minimum of 3 per cent of the non-hibemating level. ${ }^{114}$ Data for the woodchuck ${ }^{17}$ show the profound changes during hibernation:

\section{Non-hibernating}

Heart rate

Rectal temperalure

Respiratory rate

F.Q. (after 2t-hour fast)

Basal metabolism cal./ $10 w^{2 / 3 / 24 ~ h r . ~}$
$80 / \mathrm{min}$.

$3+-39^{\circ}$ C. (air $\left.10-35^{\circ}\right)$

$25-30 / \mathrm{min}$.

0.71

410

\section{Hibernating}

$4-5 / \mathrm{min}$.

$12-13^{\circ} \mathrm{C}$. (air $\left.10^{\circ}\right)$

$0.2-1 / \mathrm{min}$.

0.7

$17-27$

Ileart rate is slowed and irregular, breathing very slow. Blood sugar in hibernating mammals is little below waking values." A waking dormouse

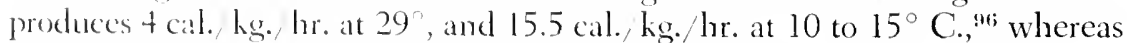
al hibernating dormouse produces only $0.07 \mathrm{cal} . / \mathrm{kg} . / \mathrm{hr}$. at $8.5^{\circ}$. The respiratory quotient in hibernation is low, indicating fat oxidation (Ch. 8).

Recovery from hibernation may be initiated by moving or otherwise stimulating the animal, as well as by increasing the external temperature. Body temperature and oxygen consumption then increase, the latter very rapidly, temporarily to exeed the normal metabolic rate (Fig. 99).114 The respiratory cexcess maly be correlated with unusual activity caused by the "rage" of the hamster cluring the first hours after awakening wather than with any appreciable oxyen debt accumulated during the long hibernation. The temperature of a heclgethog rese 19 in 42 minutes on awaking. ${ }^{137}$

I libernation is a method of self preservation or conservation of body reserves when external conditions of temperature and food supply are unfavorable. In birds and most mammals the heat-regulating center appears to lack the lability to permit adjustment required for hibernation. 


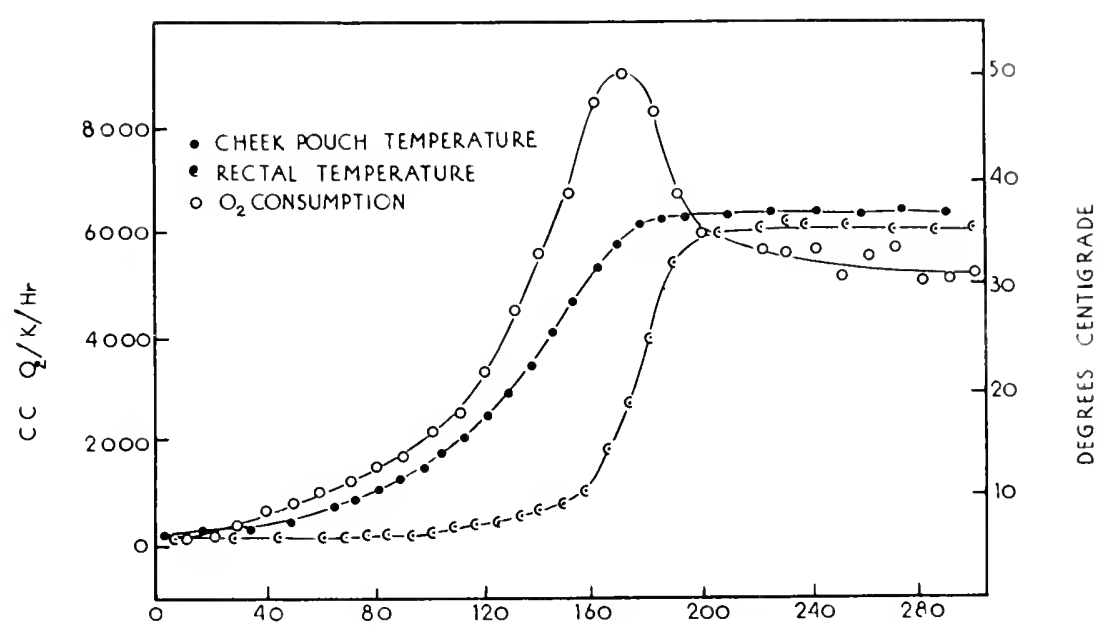

MINUTES

Fig. 99. Average data of waking of three hamsters from hibernation at $+5^{\circ} \mathrm{C}$. oxygen consumption, C rectal temperature, - cheek pouch temperature. From Lyman. ${ }^{11}$

\section{CONCLUSIONS}

The abundance and distribution of animals are influenced by temperature in two general respects: life is possible only within certain rather narrow temperature limits, and metabolic activity varies greatly according to temperature. The thermal relations of animals to their environment are closely related to their osmotic relations.

The normal vital temperature range is based on the thermal properties of water. Natural waters (hot springs excepted), because of their high specific heat and small heat conduction, rarely have a temperature above the upper limits for most aquatic animals -35 to $40^{\circ} \mathrm{C}$. At the cold extreme the freezing point of aquatic animals is similar to that of the medium (in marine animals), or lower (in fresh-water animals). The causes of heat and cold death are multiple. Heat death often occurs at temperatures well below those of protein denaturation, and cold death need not involve freezing. Among aquatic poikilothermic animals the vital limits are lower for animals acclimatized to a low temperature range than for animals acclimatized to higher temperatures. This must mean that chemical changes occur in the organic substances which are critically affected by extremes of temperature. There are few such striking examples of protoplasmic reorganization as in temperature adjustment. The thermal properties of water protect and at the same time fix the temperature limits of aquatic animals.

The over-all end of metabolism is the liberation of energy which can be used in mechanical or chemical work or can be lost as heat. If heat production is excessive, heat is lost; if the body temperature of a homoiotherm falls, metabolism increases. Poikilotherms have a low basal metabolic rate and they lose so much heat by conduction and convection and by vaporization that they 
are largely dependent on the environment for their temperature. The net mechanical efficiency of muscle is about 20 per cent, yet the heat which is lost is not wasted; insofar as it raises body temperature it permits faster enzymatic reactions. The efficiency of a heterothermic animal is good in that elevated temperatures are present when needed and enzymes need not work overtime when the animal is at rest or in hibernal sleep. This efficiency is apparently more than compensated for by slowness of getting started after a period of low body temperature.

Temperature limits the distribution of animals, and success at the limits of environmental temperature is characterized by a variety of physiological adaptations. Among poikilotherms the most important of these are: selection of a favorable temperature, regulation of water loss, variations in water distribution, and variety of metabolic reactions, the extreme being suspension of active life processes at unfavorable temperatures. Among homoiotherms there are differences in degree of insulation (vasomotor responses and thickness of body coat), and in the magnitude of the metabolic response to changes in environmental temperature. Elucidation of the role of temperature in control of the distribution of animals and in the evolution of new species would be promoted by examination of the responses to temperature stress of individuals of a species living at the limits of the temperature range, and ascertainment of whether any observed individual differences are physiological or genetic adaptations.

\section{REFERENCES}

1. Abercrombie, M., and Johnson, M. L., Proc. Zool. Soc. Lond., A. 111:87-99 (1941). High lethal temperature, Arctic Pandalus.

2. Adolph, E. F., Biol. Bull. 62:112-125 (1932). Vapor tensions and temperature relations, frogs.

3. Adolpit, E. F., Physiological Regulation (1942). Lancaster, Pa., Jaques Cattell Press. 502 p. Ch. 14, pp. 301-321. Temperature balance in animals.

4. Adolph, E. F., ET AL., Physiology of Man in the Desert (1947). N. Y., Interscience. $357 \mathrm{p}$.

5. Allen, G. M., Bats (1939). Harvard Univ. Press. 368 p. Ch. 17, pp. 266-279. Hibernation of bats.

6. BaAl, 1. v., Ztschr. vergl. Physiol. 7:436-444 (1928). Temperature sense in lecches.

7. Baldwin, F. M., Biol. Bull. 48:432-445 (1925). Body temperature in turtles.

8. Baldwin, S. P., and Kendeigh, S. C., Sci. Publ. Cleveland Mus. Nat. Hist. 3:1-196 (1932). Temperature relations, birds.

9. Banta, A. M., Carnegie Inst., Wash., Publ. 513, 285 p. (1939). Thermal clone of Daphnia.

10. Banboun, 11. C., in Temperature, Its Measurement and Control in Science and Industry (1941). New York, Reinhold. pp. 436-445. Blood volume changes in temperature regulation.

11. Barnis, T'. C., Textbook of General Physiology (1937). Philadelphia, Blakiston. 554 p. Ch. 13. T'emperature characteristics.

12. BAzET, II. C., in Temperature, Its Measurement and Control in Science and Industry (1941). New York, Reinhold. pp. 489-501. Temperature sense in man.

13. Bazm:1, 11. (.., McGlont:, B., and Brocklemurst, R. J., J. Physiol. 69:88-112 (1930). Stimulation of cutanerus thermoreceptors in man.

14. Pistust, E. IH., Biol. Bull. 35:277-317 (1918). Temperature acclimation in planaria.

15. Bellahadiк, J., Temperature and Living Matter (1935). Berlin, Borntraeger. 277 p. 
16. Benedict, F. G., Carnegie Inst., Wash., Publ. 425, 539 p. (1932). Budy temperature, reptiles.

17. Benedict, F. G., Carnegie 1nst., Wash., Publ. 497, 239 p. (1938). Physiology of hibernating woodchuck.

18. Benedict, F. G., Carnegie Inst., Wash., Publ. 503, 215 p. (1938). Vital energetics; a study in comparative basal metabolism.

19. Benedict, F. G., and Slack, E. P., Carnegie Inst., Wash., Publ. 155, pp. 1.73 (1911). Temperature of different body regions, man.

20. Bernhard, C. G., and Granit, R., J. Gen. Physiol. 29:257-265 (1946). Thermal stimulation of nerve.

21. Bodenheimer, F. S., Zool. Jahrb., Abt. System. 66:113-151 (1934). Temperature relations of grasshoppers, bees and ants.

22. Borodin, N. A., Zool. Jahrb., Abt. Allg. Zool. u. Physiol. 53:313-342 (1934). Survival of fish in freezing temperatures.

23. Britton, S. W., Quart. J. Exper. Physiol.' 13:55-68 (1922). Effect of low temperature on mammals.

24. Britton, S. W., and Atkinson, W. E., J. Mammal. 19:94-99 (1938). Poikilothermism in sloth.

25. Ввовеск, J. R., in Howell's Textbook of Physiology, John F. Fulton, ed. (1946). Philadelphia, Saunders. Ch. 53, pp. 1156-1181. Maintenance of temperature by metabolism.

26. Brody, S., Growth (suppl.) 10:169-178 (1946). Temperature effects on heat production, birds and mammals.

27. Brues, C. T., Quart. Rev. Biol. 2:181-203 (1927). Fauna of hot springs.

28. Buchanan, A. R., and Hill, R. M., Proc. Soc. Exper. Biol. E Med. 66:602-608 (1947). Temperature regulation and hypothalamic myelination, rats.

29. Buddenbrock, W. v., Grundriss der vergleichenden Physiologie (1937). Berlin, Borntraeger. Vol. 1, pp. 228-236. Temperature sense.

30. Burbank, R. C., and Young, J. Z., J. Physiol. 82:459-467 (1934). Body temperature, sleep in bats.

31. Burton, A. C., Ann. Rev. Physiol. 1:109-130 (1939). Temperature regulation, review.

32. Burton, A. C., in Temperature, Its Measurement and Control in Science and Industry (1941). New York, Reinhold. pp. 522-528. Thermorcgulation in man, heat conductivity of skin.

33. Buxton, P. A., Proc. Roy. Soc. Lond., B. 96:123-131 (1924). Temperature and humidity relations, desert insects.

34. Chalklex, H. W., Phy'siol. Zool. 3:425-440 (1930). High lethal temperature, Paramecium.

35. Chesley, L. C., Biol. Bull. 66:330-338 (1934). Temperature relations, amylases.

36. Cole, L. C., Ecology 24:94-108 (19+3). Temperature tolerance, lizards.

37. Cowles, R. B., Science 90:465-466 (1939). Reptilian thermal tolerance.

38. Cowles, R. B., Science 105:282 (1947). Temperature of desert reptiles.

39. Cowles, R. B., Science 105:362-363 (1947); also Cowles, R. B., and Nordstrom, A., Science 104:586-587 (1946). Testicular temperatures, mammals and birds.

40. Dallinger, W. H., J. Roy. Micr. Soc. Lond. 3:1-16 (1880), ibid. 7:185-199 (1887). Acclimatization of flagellates to high temperatures.

41. Davenport, C. B., and Castle, W. E., Arch. Entwcklngsmech. Org. 2:227-249 (1895). Acclimation of tadpoles.

42. Dijkgraaf, S., Ztschr. vergl. Physiol. 27:587-605 (1940), ibid. 30:252 (1943). Temperature sense in fish.

43. Dill, D. B., Life, Heat, and Altitude (1938). Harvard Univ. Press. 211 p.

44. Dotterweich, H., Zool. Jahrb., Abt. allg. Zool. u. Physiol. 44:399-425 (1928). Warming up of butterflies for flight.

45. Doudoroff, P., Biol. Bull. 75:494-509 (1934). Reactions of fish to temperature gradients.

46. Dubors, E. F., Bull. N. Y. Acad. Sci. 15:173-173 (1939). Heat loss, man.

47. Dugal, L. P., and Therien, M., Canad. J. Res. 25:111-136 (1947). Ascorbic acid and acclimatization to cold. 
48. Duthe, J. J. R., and Mackay, R. M., Brain 63:295-319 (1940). Vasomotor reflexes in temperature regulation.

49. Edwards, H. T., and Dill, D. B., 1. Cell. \& Comp. Physiol. 6:21-35, 37-42 (1935). Temperature effect on blood of gila monster and chuckwalla.

50. Eisentraut, M., Sitzungsb. ges. Nat. Freunde Berlin 184:86-90 (1940) (from Biul. Abst.). Temperature regulation of tropical bats.

51. Elson, P. F., J. Fish. Res. Bourl Canad. 5:+61-470 (1942). High lethal temperature, trout.

52. Enders, G.. Mattilews, B. H. C., et al., Proc. Roy. Soc. Lond., B. 107:222-230, $231240,2+1-2+7$ (1930). Physiology of marmot awake and in hibernation.

53. Euler, C. v., Acta Physiol. Skand. (suppl.) 45:1-75 (1947). Differential thermal stimulation of mammalian $\mathrm{A}$ and $\mathrm{C}$ nerve fibers.

54. Evans, C. A., Am. Nat. 72:480-48+(1938). Hibernation, body temperature, bats.

55. Falconer, D. S., J. Exper. Biol. $21: 17-38$ (19+5). Reactions to temperature, wireworms.

56. Filld, J., and Peiss, C. N., Fed. Proc. 8:t+t5 (19+9). Comparison of tissue metabolism at different temperatures in polar cod and black bass.

57. Fox, H. M., Proc. Zool. Soc. Lond., B. 945-955 (1936). Activity, metabolism, puikilutherms from different latitudes.

58. Fox, H. M., Proc. Zool. Soc. Lond., A. 109:1+1-156 (1939). Activity of poikilotherms from different latitudes.

59. Fox, H. M., and Wingfield, C. A., Proc. Zool. Soc. Lond., A. 107:275-282 (1937). Activity, Pandalus from different latitudes.

60. Fraenkel, G., Ztschr. vergl. Physiol. 13:300-313 (1930). Orientation of grasshoppers in sun.

61. Fraenkel, G., and Gunn, D. L., The Orientation of Animals (1940). Oxford, Clarendon. 352 p. Ch. 1t. Temperature reactions.

62. Fraenkel, G., and Herford, G. V. B., I. Exper. Biol. 17:386-395 (1940). Effects of high temperatures on insects.

63. Frienkel, G., and Hopf, H. S., Biochem. /. 34:1085-1092 (1940). Temperature adaptation, degree of phosphatide saturation, blowfly larvae.

64. Fri, F. E., Brett, J. R., et al., Rev. Canad. Biol. 1:50-56 (1942). Temperature acclimatization, fish.

65. Fuller, F. D., and Hiestand, W'. A., Turtox News 25:1+8-150 (1947). High lethal temperatures, birds and mammals.

66. Gelineo, S., Ann. Physiol. Physicochim. biol. 10:1083-1115 (1934). Metabolism of rats adapted to heat and cold.

67. Gohen, P. A., Biol. Rev. 5:213-230 (1930). Plyysiology of hibernation.

68. Gulick, A., Am. I. Pliysiol. 119:322 (1937). Development of temperature contrul in ralt.

69. Guns, D. L., Biol. Rev. 17:293-31+ (1942). Review, body temperature of peikilotherms.

70. IHAL, F. G., and Roor, R. W., Biol. Bull. 58:52-58 (1930). Thermal and water balance, amphibians and reptiles.

71. Hardi, I. D., and Dubors, E. F., Proc. Nat. Acad. Sci. $23: 62+-631$ (1937). Regulation of heat loss from man.

72. Hans, J. D., and Opple, T. W., I. Clin. lunest. 16:533-540 (1937). Sensitivity if themoreceptors, man.

73. Hown, J. D., Woll F, II. G., and Gooderl, H., I. Clin. Inrest. 19:649-657 (1940, Thermal stimulation of pain endings, man.

74. Hetrom, H. O., and Hecisalen, A. H., Fed. Proc. 8:69-70 (1949). Effects if hypothemia, degs.

75. Humawar, E. S., Bull. (1. S. Bur. Fish. +3:169-192 (1927). Acclination of lisher and amphibians to high temperatures.

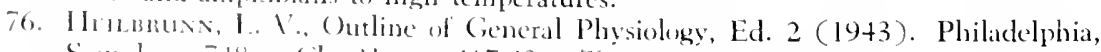

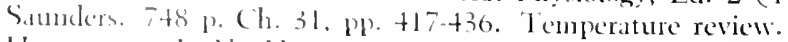

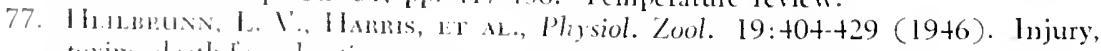
toxim, death from heating.

78. Himinciws, A., Am. !. Plizsiol. 122:511.519 (1938). Rectal temperature for panting, dogs. 
79. Itenderson, L. J., The Litness of the Fnvirmument (1913). New York, Nac. millan. 317 p.

80. Herten, K., Biol. Zentralhl. +3:282285 (1923), alse /tschr. vergl. Physiol. 1:221-288 (I924). Temperature sense, imects.

81. Henten, K., Ztschr. lergl. Phaviol. 20:511-5tt (193t). Temperature and activity of hibernating hedgehog.

82. Hesse, R.. Tiergeographie auf bkologischer Grundlage (1924). Jena, Finder. pp. 16+165. Animal size and temperature.

83. Hommer, A., Biol. Rev. 7:22+253 (1932). Temperature relations, social hymen optera.

84. Hinshelwood, C. N.. The Kinetics of Chemical Change (1940). Oxtord, Clarendon. $27+\mathrm{P}$.

85. Hoagland, H., I. Ge'll. Physiol. 16:715-732 (1933\%. Ellect of temperature on lateral line nerve discharge, eatlish.

86. Hoagland, H., Pacemalers and the Control of Behavior (1935). New York, Macmillan. Ch. 7. Temperature characteristics.

87. Hogben, L., and Kink, R. L., Proc. Roy. Soc. Lond. 132:239-252 (194t.. Temperature relations, snails and earthworms.

88. Holmequst, A. Ni., Physiol. Zool. 1:325-357 (1928). Arthropod hibernation.

89. Honp, R.. Ztschr. iergl. Physiol. 26:1-34 (1938). Thermo-orientation in louse Pediculus.

90. Honvath, S. M., Foltz, E. L., et al., Fel. Proc. 8:77 (1949). Blood tempera ture, dog.

91. Jennings, II. S., Carnegie Inst., Wash., Publ. 16, pp. I-28 (1904). Reactions of ciliates to heat and cold.

92. Jounson, G. E., J. Exper. Zool. 50:15-30 (1928). Hibernation in Citellus.

93. Johnson, G. E., Quart. Rev. Biol. 6:+39.461 (1931). Review, hibernation in mammals.

94. Kalabuchow, N. I., Zool. lahrb., Abt. allg. Zool. u. Physiol. 53:567-602 (1934). Hibernation of bees.

95. Kanitz, O., Tabul. Biol. 2:9-1+ (1925). Body temperatures of mammals.

96. Kayser, C., C. R. Soc. Biol. Paris 128:85-88, 1201-1204, 1205-1208 (1938), ibid. 129:225-226, 1162-1166 (1938). Metabolism of hibernating dormouse.

97. Kendergi, S. C., Ecol. Monog. 4:299-417 (1934). Role of enviromment in life of birds, temperature and migration.

98. Keniejgri, S. (., J. Exper. Zool. 82:+19-438 (1939). Metabolism and derelopment of temperature control, wrens.

99. Kendeigh, S. C., J. Exper. Zool. 96:1-16 (194t). Effect ol air temperature on metabolism, English sparrow.

100. Kendeigh, S. C.. I. Mammal. 26:86-87 (19+5). Body temperature, small mam mals.

101. Kendergu, S. C.. ). Wildl. Managem. 9:217-226 (1945). Survival without food at different temperatures, birds.

102. Kix, H., Sci. Rep. Tohoku Lniv. 5:439+48 (1930). Body temperature, edrthworm.

103. Kirk, R. L., and Hogben, L., I. Exper. Biol. 22:213220 (19+6). Temperature relations, firngs, reptiles.

104. Kondunu, K., Men. Fac. Sci. Agric. Taihokn 12:281-380 (1935). (Quoted from 69.) Temperature relations of grashopper.

105. Koskinies, J., Experientia t:27+-276 (19+8). Develepment of temperature regulation, swift.

106. Kozmantscinkov, I., Zool. Anz. 103:30-35 (1933). Lethal temperatures, cockroach.

107. Kredel, F. E., I. Mammal. 9:48-51 (1928). Temperature of sloth.

108. Krogh, A., and Zeutrien, E.. J. Exper. Biol. 18:1-10 (1941). Pre-flight wam-up, insects.

109. Krumbiegel, 1., Zool. Jahbb., Abt. Systeml. 63:183-280 (1932). Racial and physiolngical diflerences in selected temperatures, insects.

110. Luetr, F. X., Copeia, pp. 125-132 (19+1). 'Temperature relutions, snakes.

111. Lustic, B., Ennst, T., and Reuss, E., Biochem. Ztschr. 290:95-98 (1937). Blood magnesium, hibernating Helix. 
112. Luyet, B. J., and Gehenio, P. M., Life and Death at Low "Temperature (1940). Biodynamica Monograph No. I, Normandy, Mo., 341 p. Survival of "vitrified" animals.

113. Luyet, B. J., and Gehenio, P. M., Biodynamica 6:141-149 (1947). Survival of "vinegar eels" after freezing in liquid air.

114. Lyman, C. P., J. Exper. Zool. 109:55-78 (1948). Metabolism, hibernating hamster.

115. MacArthur, J. W., and Balllie, W. H. I., J. Exper. Zool. 53:221-268 (1929). Effect of temperature on longevity in Daphnia.

116. Magoun, H. W., Harrison, F., Et AL., J. Neurophysiol. 1:100-114 (1938). Localization of heat center in brain.

117. Mansfeld, G., Experientia 3:353-357, 398-404 (1947). Humoral and nervous factors in temperature regulation.

118. Marsilall, S. W., and Nicholls, A. G., J. Mar. Biol. Ass. U. K. 20:1-27 (1935). Lethal temperature, Calanus, marine copepod.

119. Martin, C. J., Phil. Tr. Roy. Soc. Lond., B. 195:1-37 (1902). Temperature and metabolism of monotremes and marsupials.

120. Mayer, H. E., Carnegie Inst., Wash., Tortugas Pcipers 6:1-24 (1914). Temperature tolerance, tropical animals.

121. Mellanby, K., J. Exper. Biol. 9:222-231 (1932). Temperature and humidity relations, insects.

122. Mellanby, K., Proc. Roy. Soc. Lond., B. 127:473-487 (1939). Low temperatures and insect activity.

123. Mellanby, K., J. Exper. Biol. 18:55-61 (1941). Body temperature of frogs.

124. Molnar, G. W., J. Am. Med. Ass. 131:1046-1050 (1946). Survival of hypothermia by men immersed in ocean.

125. Moore, C. R., Quart. Rev. Biol. 1:4-50 (1926). Hypothesis of scrotum as temperature regulator for testes.

126. Morgan, A. H., J. Exper. Zool. 35:83-114 (1922). Temperature senses of frog skin.

127. Morrison, P. B., J. Cell. E Comp. Physiol. 27:125-137 (1946). Temperature regulation, Central American opossums.

128. Necheles, H., Pflüg. Arch. ges. Physiol. 204:72-93 (1924). Temperature relations, cockroaches.

129. Nicholson, A. J., Bull. Ent. Res. 25:85-99 (1934). Influence of temperature on activity of blowflies.

130. Nieschulz, O., Ztschr. angew. Ent. 21:224-238 (1934), also Zool. Anz. 110:225233 (1935). Lethal temperatures and selected temperatures, flies.

130a. Noble, G. K., and Schmidt, A., Proc. Am. Phil. Soc. 77:263-288 (1937). Thermal reception by pits in pit vipers and boas.

131. Onum, E. P., Am. J. Physiol. 136:618-622 (1942). Relation of cold tremors to temperature regulation, birds.

132. Oostiruizen, M. J., J. Ent. Soc. So. Africa 2:63-73 (1939). Muscle temperature before and during flight, insects.

133. Oppel, T. W., and Hardy, J. D., J. Clin. Invest. 16:517-524, 525-531 (1937). Thermal stimulation by different wave length radiations.

134. Payne, N. M., J. Morph. 43:521-546 (1927). Freezing of insects.

135. Payne, N. M., Biol. Bull. 52:449-457 (1927). Cold hardiness in insects.

136. Pearse, A. S., and Hall, F. G., Homoiothermism (1928). New York, J. Wiley. $119 \mathrm{p}$.

137. Pembrey, M. S., J. Physiol. 29:195-212 (1903). Metabolism and temperature of hibernating hedgehog and dormouse.

138. Pinkston, J. O., Bard, P., and Riocii, D. M., Am. J. Physiol. 109:515-531 (1934). Effect of forebrain operations on temperature regulation. 139. Pinscil, G. 13., J. Agr. Res. 24:275-287 (1923). Temperature relations, honey
bee. 140. Procton, E., Nature 163:108-109 (1949). Temperature changes, hibernating
hedgehog. 
141. Prouty, L. R., Fed. Proc. 8:128-129 (1949). Heat balance, cats.

142. Randall, W. C., Am. J. Physiol. 139:56-63 (1943). Temperature regulation in birds.

143. Ranson, S. W., Harvey Lectures (1937), pp. 92-121. Review, temperature center of hypothalamus.

144. Reeder, W. G., J. Mammal. 30:5 I-53 (1949). Body temperature, hibernating bat.

145. Robinson, W., Tech. Bull., Univ. Minn. Agr. Exper. Sta. 4l:1-43 (1926). Cold killing of grain weevils.

146. Rodbard, S., Fed. Proc. 6:19l (1947), and Science 108:413-415 (1948); also Rodbard, S., and Feldman, D., Proc. Soc. Exper. Biol. E Med. 63:43-44 (1946). Body temperature and blood pressure, reptiles.

147. Rogers, C. G., and Lewis, E. M., Biol. Bull. 27:262-269 (1914). Body temperature of earthworm.

148. Romanoff, A. T., Science 94:218-219 (1941). Development of homeothermy, birds.

149. Rubin, M. A., J. Gen. Physiol. 18:643-647 (1935). Role of lateral line in thermal shock reaction, fish.

150. Rubner, M., Biochem. Ztschr. 148:223-267 (1924). Metabolism of cold-blooded animals.

151. Rucker, F., Ztschr. vergl. Physiol. 21:275-280 (1934), also Pflïg. Arch. ges. Physiol. 231:729-741 (1933). Infra-red reflection, insects.

152. Sand, A., Proc. Roy. Soc. Lond., B. 125:524-553 (1938). Temperature stimulation of ampullae of Lorenzini, elasmobranchs.

153. Sawaya, P., Arq. Cirurg. Clin. Exper. 5:235-254 (1941). Temperature fluctuations, three-toed sloth.

154. Scherbakov, A. P., Bull. Biol. Med. Exper. 3:268-270 (1937). Respiration of lamprey at different temperatures.

155. Scholander, P. B., To be published (1950). Metabolic reactions of arctic and tropical animals to temperature stress.

156. Shelford, V. E., and Powers, E. B., Biol. Bull. 28:315-334 (1915). Reactions of fish in thermal gradients.

157. Simpson, S., and Galbraitih, J. J., J. Physiol. 33:225-238 (1905). Diurnal temperature rhythms, birds and mammals.

158. Srouı, H., Zool. Jahrb. Abt. allg. Zool. u. Physiol. 58:284-296 (1937). Thermotaxis and location of thermoreceptors, bedbug.

159. Srzer, I. W., Adv. in Enzymol. 3:35-62 (1943). Temperature effects on enzyme kinetics.

160. Spealman, C. R., Am. J. Physiol. 146:262-266 (1946). Cooling by immersion, rats, rabbits, dogs.

161. Stier, T. J. B., and Pincus, G., J. Gen. Physiol. 11:349-356 (1928). Temperature characteristics of young mammals.

162. Sturkie, P. D., Am. J. Physiol. 147:531-536 (1946). Low lethal temperature, chickens.

163. Suomalainen, P., Nature 1+2:1157 (1938). Magnesium in blood, hibernating hedgehog.

164. Thomsen, E., and Thomsen, M., Ztschr. vergl. Physiol. 24:343-380 (1937). Temperature selection by fly larvae.

165. Thonnton, F. E., Physiol. Zool. 5:246-253 (1932). High lethal temperature, Amoeba proteus.

166. Totze, R., Ztschr. vergl. Physiol. 19:110-161 (1933). Temperature selcction, ticks.

167. Uvarov, B. P., Tr. Ent. Soc. Lond. 79:1-274 (1931). Insects and climate, lethal temperatures.

168. Vonk, H. J., Adv. in Enzymol. 1:371-417 (1941). Temperature of inactivation, enzymes.

169. WADE, O., J. Mammal. 11:160-188 (1930). Temperature, hibernation, ground squirrel.

170. Weese, A. O., Biol. Bull. 32:98-116 (1917). Thermal reactions, Phrynosoma. 
171. WU11NEY, R. J., I. Laper. Biol. 16:37+-385 (1939). Temperature tolerance of matty nymphs.

172. Wicor rowert 1, V. B., Principles of Insect Physiology. (1939). London, Methu(n) 12p. 359 3-2. lieview, temperature relations, insects.

173. Wigrieswortil, V. B., and Gillet, J. D., J. Exper. Biol. 11:120-139 (1934). Temperature sense, Pihodnius.

174. Wisuock1, (i. B., (2uat. Rer. Biol. 8:385-396 (1933). Relation of testes to body temperature.

175. Worf, A. V., Vcology 19:233-242 (1938). High lethal temperature, Lumbricus.

176. Yagi, X., and Simmonzumi, J., Tokyo Bunrika Daigaku Sci. Rep. B, 1:231-242 (1934). Lethal temperatures, rats. 


\section{Photoreception}

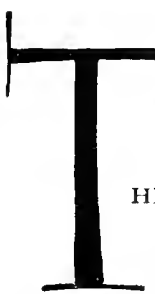

HE ENTIRE GANUT of behavioral adjustments which an organism or its component parts make to changes in the external environment is the direct result of sense organ activity. Of the numerous types of sense organs which regulate the activity of animals in their external cnvironment, those that are sensitive to light, called photoreceptors, serve in a capacity which equals, and perhaps exceeds in importance that of all other sense organs.

The ability to perceive light usually resides in a well defined structural entity, called a photoreceptor, although this is not necessarily true. Some echinoderms, for example, possess light sensitivity in the absence of any known discrete structural photoreceptors, as do many protozoa. Photoreceptors present a great diversity of structure. Despite these morphological differences, there seems to exist a functional similarity in all of the photoreceptors studied.

\section{PHOTORECEPTORS AND ORIENTATION}

Throughout the animal kingdom, as well as in the plant kingdom, light plays an important part in organismic orientation. Utilization of the directional properties of light resides in the morphological and physiological relations which obtain in the photoreceptor proper as well as in the central ramifications of the visual pathway, and in the functional organization of the central nervous system with respect to the visual and effector systems. The mere presence of a photoreceptor does not indicate the nature of the orientation.

Many studies have been performed on the orientation of organisms to light. Much of this material is summarized in the monograph of Fraenkel and Gunn. ${ }^{43}$ According to their definitions, the word tropism is restricted to orientation of sessile animals or plants, taxis to directed orientation of animals which move in their environment, and kinesis to increase in velocity of movement on illumination in those animals which lack a directional orientation.

\section{Diffuse Light Sensitivity}

It has long been known that plants utilize light not only for photosynthesis but also for a type of orientation called phototropism. Many plants bend toward the source of illumination. This phototropism is particularly marked in blue light, which results in relatively little photosynthesis, and is associated with yellow pigments (carotenoids) present in plants ${ }^{170}$ (see page 408.) 
Diffuse sensitivity to light exists in several phyla of animals. Protozoa, such as Amoeba, Stentor, and Peranema, respond to changing intensity of illumination, without possessing any visible light-sensitive structures. Amoeba proteus responds to intense illumination of any part of its body by a cessation of movement resulting from gelation in the illuminated region (Fig. 100). If the whole body is illuminated, locomotion is greatly retarded or stopped, because of gelation at the tips of the pseudopods (Fig. 100). However, if illumination is continued, locomotion gradually increases and becomes normal in 6 to 30 minutes, depending on the intensity. ${ }^{129}$ In the absence of light-sensitive organelles and in view of the periodically fluid condition of amoeba protoplasm, there is probably a direct effect of light on sol-gel reversibility (Ch. 17).

Diffuse sensitivity to light also exists among the echinoderms. In these animals the pigment cells of the integument are believed to be sensitive to
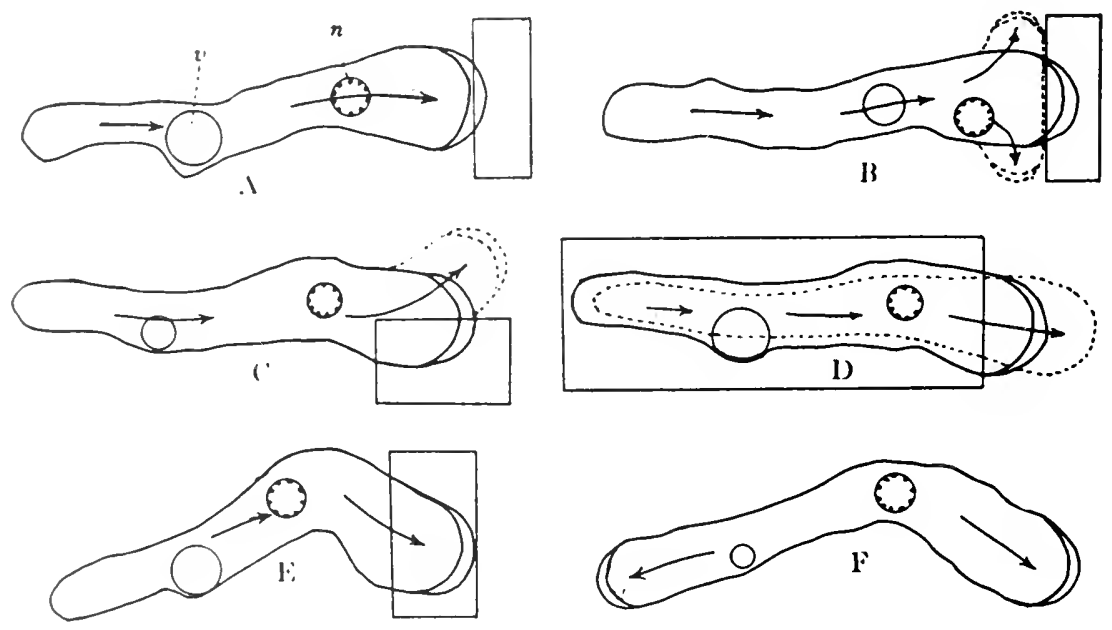

Fig. 100. Responses of Amoeba to localized illumination (rectangular areas). Arrows show the direction of protoplasmic streaming. Broken lines in $\mathrm{B}, \mathrm{C}$, and D indicate position of animal shortly after illumination. F shows the position and direction of streaming assumed by $\mathrm{E}$ after illumination. $v$, Contractile vacuole; $n$, nucleus. From Mast. ${ }^{197}$

light. Von Uexkül157 noted the light sensitivity of the violet spicules around the anal orifice of the sea urchin, Centrostephanus longispinus. He extracted the purple pigment with alcohol, and found that it decomposed when exposed to light. The entire surface of Holothuria is photosensitive, and two pigments are present, one of which is a fluorescent greenish yellow. 25,26 The reaction of three species of Holothuria varies with the amount of this pigment present, and young specimens of $H$. captiva which have little pigment are less reactive than the heavily pigmented adults. Therefore, the greenish yellow fluorescent pigment is believed to be either the photosensitive pigment or a sensitizer.

Jennings ${ }^{111}$ in his monograph on the bchavior of the starfish described reactions to light. In general, if the direction of illumination is such that 
one part of the animal is more intensely illuminated than another, the animal will move in the direction of the less intensely illuminated side. Under uniform but sudden illumination the animal exhibits an increased locomotor activity, which gradually subsides. The brief description accorded the latter type of behavior gives the impression of random movement, which may perhaps be called a kinesis, that is, an increase in random activity.

Many annelids (e.g., Lumbricus) possess no macroscopic photoreceptors, yet in Lumbricus the entire body is sensitive to light. Sensitivity is greatest on the prostomium, next greatest on the dorsal region of the next few anterior segments and a few of the most posterior segments, and least in the middle of the body. The regions of greatest sensitivity coincide with the highest concentration of the so-called epithelial "light cells" of Hess, which are single cell photoreceptors each with a refractile body connected to neurofibrillae. Hess ${ }^{87}$ found that the relative frequency of occurrence of these receptors in certain segments is as follows: prostomium, 57; segment 1, 26; segment 2, 10; segment 3, 7; segment 11, 1; last segment, 14; penultimate, 4; antepenultimate, 2. The distribution of light sensitivity thus parallels the distribution of the light-sensitive cells. In some oligochaetes the photoreceptors are found directly on the nerves of the cerebral ganglia. Earthworms are positively phototaxic at very low intensities of illumination and negatively phototaxic at higher intensities. ${ }^{87}, 139$ The type of orientation which these worms exhibit most often is called klinotaxis (see below).

The molluscs display a great diversity in the mechanism of photoreception, ranging from a diffuse light sensitivity to a photoreceptor as complex as the human eye. The diffuse sensitivity to light in the clam, Mya arenaria, is confined to the siphon. Under constant conditions of illumination, the siphon is extended; when illumination increases, the siphon is withdrawn. The photoreceptors of the siphon are similar to those of the earthworm. ${ }^{118 a}$

\section{Localized Sensitivity to Light}

Localized photoreceptors are present in a great variety of organisms and exhibit a great diversity of structure. For the sake of clarity it seems pertinent to classify the photoreceptors on the basis of their gross function. Thus, certain eyes function only as intensity receptors, while others function in form perception and pattern vision, the latter again forming a large group of great morphological diversity.

Morphology. The simplest type of light-sensitive organs appear among the flagellate Protozoa. The known light-sensitive structures of the Protozoa are stigmas and ocelli, which occur only in flagellates. The stigma of Euglena (Fig. 101) is a mass of red granules, which shade the swelling on the flagellum. The flagellar swelling is the light-sensitive structure, and in any phototaxic organism change of illumination of this swelling causes a change in direction of the beat of the flagellum until the organism becomes properly oriented (Fig. 102). In the Phytomonadida the stigma consists of a refractile structure which serves as a lens and covers the opening of a cup-shaped mass of pigment. The light-sensitive material is between the lens and the inner surface of the cup. The function of the pigment is to make the organelle a directional detector useful in phototaxis. The stigmas of fresh-water Dinoflagellida are similar to those of Phytomonadida. However, those of cer- 
tain marine dinoflagellates are much larger. In general appearance they resemble the ocelli of flatworms, and they are called ocelli.

The ocelli of coclenterates vary considerably in structure, ranging from a simple laver of sensory cells mingled with pigment cells, as in Turris, to cupshaped ocelli in Sarsia (Fig. 103, A) and Tiaropsis.

The ocelli of flatworms are similar in general appearance and in principle to the stigmas of the Phytomonadida and Dinoflagellida. There is a pigment cup and a sensitive layer within the cup (Fig. 103, B), and sometimes a lens. However, the sensitive laver is cellular and is composed of primary neurones, the axons of which go to the brain. The pigment cup may consist of a single cell.

Some annelida (e.g., Nereis) have ocelli. Among the Mollusca there exist several types of photoreceptors which transcend in complexity the simple ncelli heretofore cliscussed. Nlthough the necessary data are not at hand,

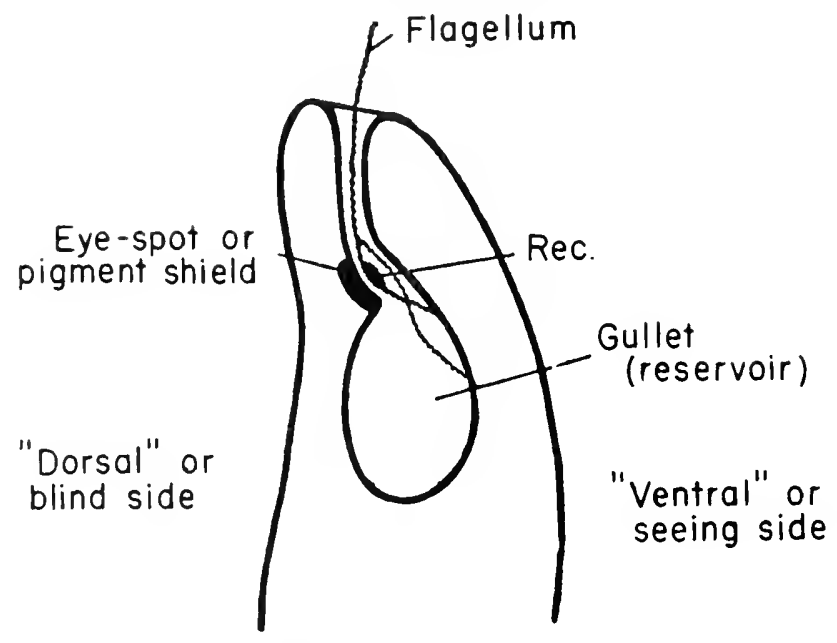

Fig. 101. Side view of Euglena viridis, showing the eye spot or pigmented shield and the enlargement at the base of the flagellum (rec.), believed to be the photoreceptor. From Fraenkel and Gunn. ${ }^{43}$

it is quite possible that the eyes of Nautilus, consisting of an open pit, without a lens, the eres of Helix, consisting of a completely enclosed receptor containing a lens like material, and the eves of Pecten (Fig. 103, D), consisting of a lens and a double layer of retinal cells, backed by a tapetum, are capable of pattern vision as well as intensity perception. The most complicatted type of molluscan eye is represented by that of Sepia (Fig. 103, C). Generally speaking, in structure and development this eve is comparable to the eyes of vertebrates. There is a corneal surface which may be covered by lids; there are an iris and a lens which separate the eye into chambers; the lens is suspended and can be moved by a ciliary muscle; the retina lines the back of the eye; and the eyeball is surrounded by cartilage. During development the eye originates as an ectodermal pit, the lining of which forms the retina. The lens is formed in two parts: the inner part at the point of closure of the pit, and the outer part from a circular fold which forms the 
iris. Secondary and tertiary neurones are located in an optic ganglion im mediately behind the retina, rather than on the fromt of the retina, as in vertebrates. Functionally as well as structurally, the eve of Sepia is similar to that of vertebrates, except that the retinal clements are not inverted.

The sixth abdominal ganglion in the crayfish is photosensitive, but the nature of the photoreceptor cells is unknown. ${ }^{134}$

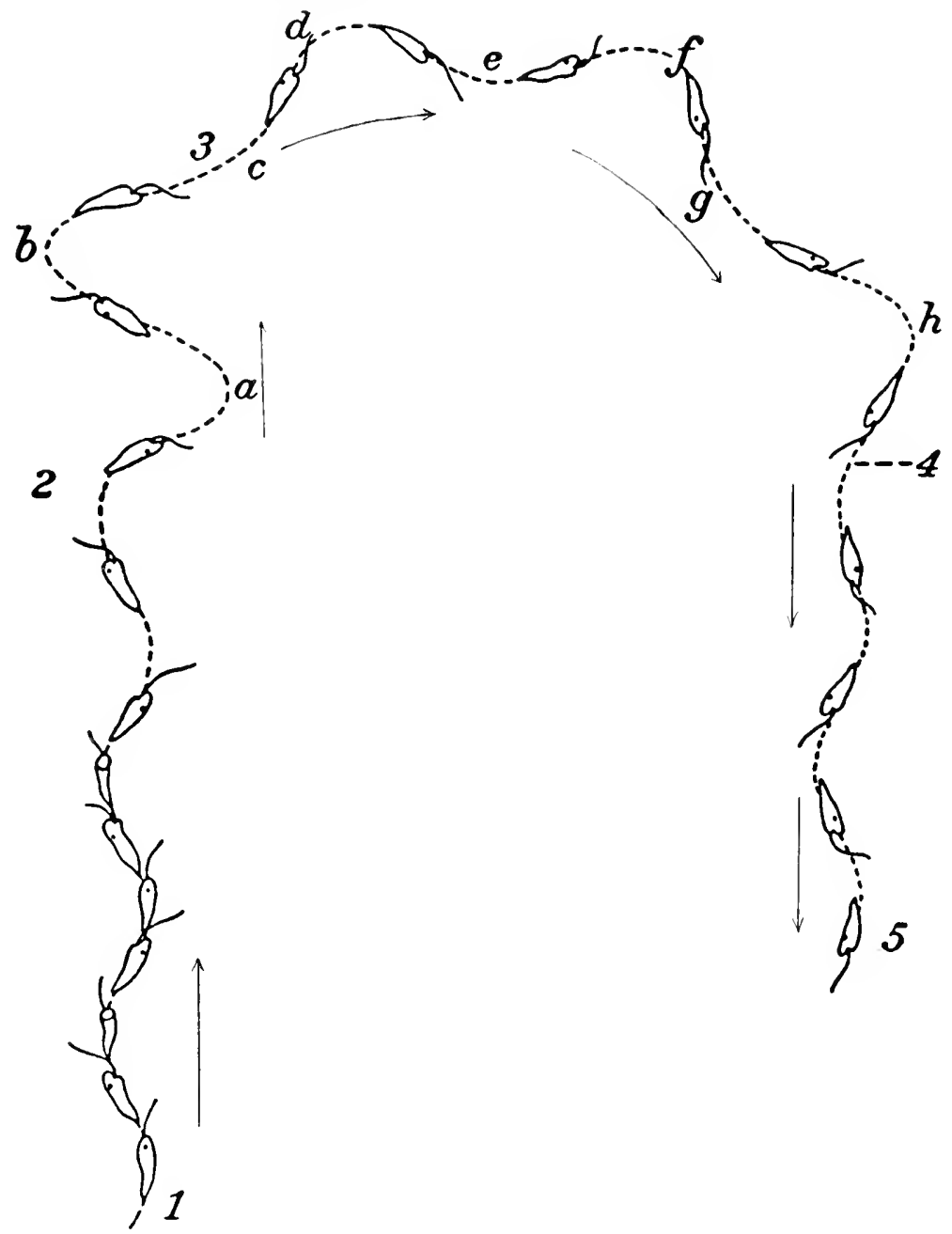

Fig. 102. Orientation of swimming Euglena to light. From 1 to 2, light from direction of top of page; 2 , direction of light reversed. The animal swerves to the dorsal side each time the receptor is shielded from the light by the eve spot. (Jennings, 1906.)

Function in Orientation. The photoreceptor of Euglena and related forms is a directional receptor by virtue of the pigment of the stigma. This orangered pigment mass serves to shade the swelling of the base of the flagellum when illumination is on the "dorsal" surface and does not do so when illumination is from the opposite direction. When exposed to a single light source 
Euglena moves directly toward or away from it, depending on the intensity. When the direction of illumination is changed suddenly, the animal reorients, as shown in Figure 102. This type of orientation is called klinotaxis, a directional orientation made possible by means of regular deviations in movement (pendular or spiral), and involves comparison of intensities at sucessive points in time. The coelenterate Hydra behaves in this way, as do the post-trochophore larva of Arenicola and the tadpole larva of the ascidian

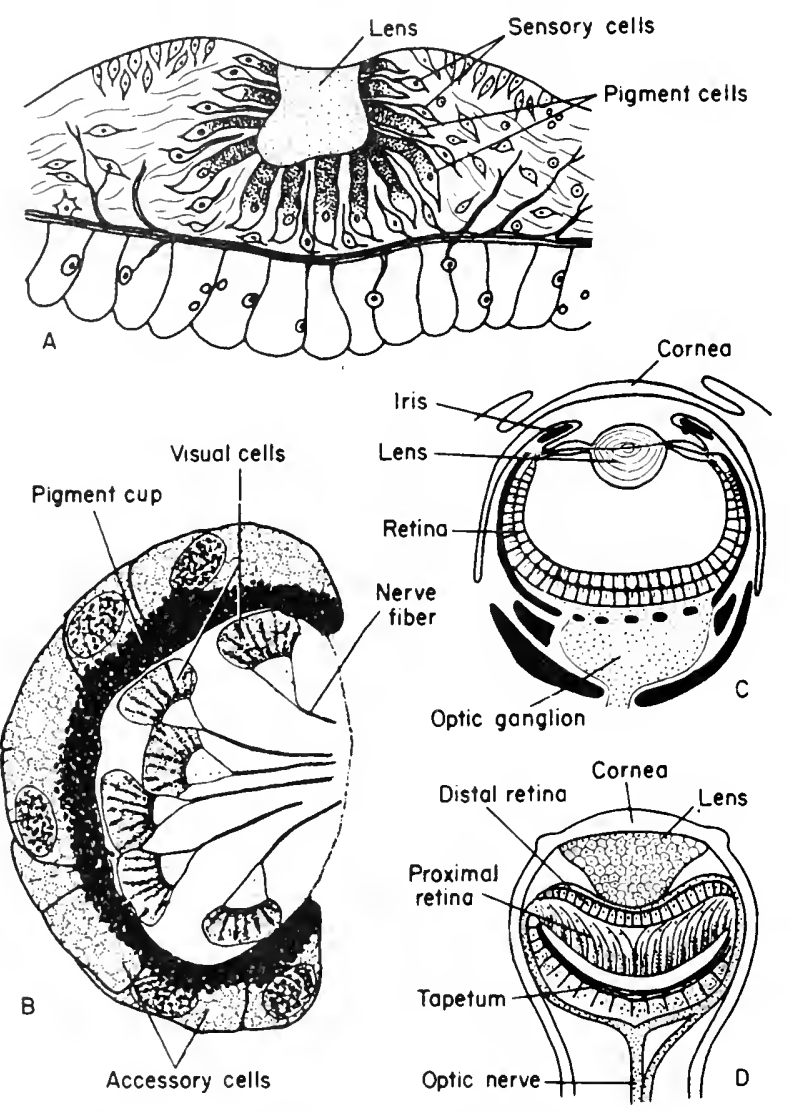

Fig. 103. Diagrammatic representation of photosensitive organs. $A$, Section through the ocellus of Sarsia. B, Transverse section of the eye of Planaria maculata. C, Section through eye of Sepia. D, Section through eye of Pecten. Redrawn from Hyman, ${ }^{93}$ Taliaferro ${ }^{\text {ins }}$ and Borradaile and Potts. ${ }^{1 . i n}$

Amaroucium. Earthworms, under certain conditions, exhibit a pendular movement resulting in orientation, as do the maggot larvae of several species of llies. ${ }^{43}$

With the advent of two or more receptors, a peripheral mechanism exists which may permit direct orientation to light. In Planaria maculata, which possesses two cup-eyes, the orientation of the animal is in that direction which results in equal stimulation of both eyes. This type of orientation is called tropotaxis, and involves the comparison of intensities on two receptors simultancously. Planaria, deprived of their eyes and illuminated, show an 
increased random activity and ultimately aggregate in the darkest region of their environment. ${ }^{153}$

A similar type of behavior was obtained in another planarian, Dendrocoelum, by Ullyott. ${ }^{158}$ Under uniform conditions of illumination without directional components (i.e., from above), the animal lacks a directional response. The perception of light merely increases its random angular motion, which may be expressed as rate of change of direction. Since this rate of change of direction increases with light intensity, it follows that these animals will usually be found in the least intensely illuminated part of their environment, simply because their activity is lower here than elsewhere. This type of orientation is called klinokinesis. ${ }^{43}$

\section{Localized Photoreceptors and Pattern Vision}

Light-sensitive organs capable of pattern vision have evolved several times. The eyes of Nautilus, Helix, and Pecten may possibly function in pattern vision; the complex eye of cephalopods, illustrated by Sepia (Fig. 103, C), resembles in structural and functional complexity the eye of vertebrates and certainly is capable of pattern vision and form perception. In the arthropods the compound eye serves in pattern vision, and in the vertebrates a distinctly different photoreceptor has evolved.

The Compound Eyes of Arthropods. The eyes of arthropods are of two general types: compound eyes and ocelli. All compound eyes are constructed so that an image is formed; some ocelli (e.g., the lateral ocelli or stemmata of insect larvae and pupae) are capable of forming images, but others (e.g., ocelli of adult insects) are primarily simple organs of light detection.

The compound eve is an effective organ of vision which has developed along quite different morphological and optical lines from the eye of vertebrates. The focusing mechanism works on a different optical principle, and the focus is always fixed. In many compound eyes at least, the light-gathering power is far inferior to that of the vertebrate eve. However, the compound eve also has advantages, especially in that the field of view is very great and may encompass well over 200 degrees, as in the stalked eyes of the crayfish.

Structure of the Compound Eye. The compound eye is made of a number of transparent facets in the cuticle of the head or eyestalk, and beneatl each facet is an elongated light-sensitive structure, which is connected to the central nervous system. Each facet, together with the underlying sensitive structure, is called an ommatidium, and the entire group of receptors is called the retina. The distal dioptric part of each ommatidium consists of a facet of the cuticle; all such facets, together, form the cornea. Beneath each facet there is usually a crystalline cone which serves as a lens. Below this is the retinula which usually consists of seven elongated sensory cells, arranged around a central refractile rod, the rhabdom. Each retinula cell is a primary neurone and is continuous with a post-retinal axon. The rhabdom is the collective secretion of the retinula cells, and its function is apparently to conduct light along the length of the retinula cells, which presumably contain the photosensitive pigment.

In some insects the rhabdom is optically continuous with the crystalline cone, and the two form a light-collecting and distributing structure which transmits light entering the facet over the length of the photosensitive retin- 
ula cells. In many nocturnal insects (e.g., Noctuid moths), tracheae are grouped around the retinula cells and especially at the basal end of the rhabdom. These tracheae reflect light distally through the ommatidium and serve the same function as the tapetum lucidum of vertebrates (page 398). In higher Crustacea the retinula cells contain guanin crystals which act as reflecting surfaces just as they do in the tapetum lucidum of rertebrates.

In insects the whole ommatidium is surrounded by a sheath of pigmented cells made up of two sections: the "primary iris cells" around the crystalline cone, and the "secondary iris celts," which" may cover both the primary iris cells and the retinula cells. The proximal ends of the ommatidia rest on a fenestrated membrane through which the axons and tracheae pass. In primitive crustacea (e.g., Gammarms), the retinula cells contain all of the iris pigment and extend distally to surround the crystalline cone. In higher Crustacea (e.g., shrimp, crayfish), the basal iris pigment is in the retinula cells, but the distal pigment is in separate cells.

Diurmal Morements of Eye Pigments. In all compound eyes there is a movement of pigment with light and dark adaptation. ${ }^{132}$ "The arrangement is often such that the eve functions as an appositional eye when light adapted and as a superpositional eye when dark adapted (see page 390). In addition, there are rhythmic diurnal pigment migrations which are independent of light intensity; these persist independently of direct changes in the environmental illumination.

Persistent diurnal rhythm of pigment migration occurs in the eye of Palaemonetes, a shrimp. ${ }^{16 x}$ On the left in Figure 289 (Ch. 22) is shown the position of the pigment in animals kept in constant light, and, on the right, that in animals kept in constant darkness. The upper figures show the position of pigment during the day, and the lower ones during the night. In animals kept in constant darkness the distal pigment moves outward at night and inward during the day; the basal pigment in the retinula cells moves outward cluring the day and inward, largely below the basal membrane, at night. Light adaptation brings about the differences apparent upon comparing the right and left diagrams in the figure. In dark-adapted animals it is evident that the type of image formed is appositional during the day and superpositional during the night. In light-adiated animals the image is certainly appositional during the day and probably also at night. The reflecting pigment $(R)$ is unprotected from light during the night but is covered during the day. This permits the eve to glow at night but not during the day. The effect is readily observed in crayfish, which, if kept in constant darkness, will exhibit an orange eveshine at night but not during the day. This diurnal rhythm of eyeshine mily persist for months under conditions of constant darkiness and temperature. 7 . 170

The persistent diumal rhythm of pigment migration was discovered in the noctuid moth, Plusia, $113^{\prime}$ and hater was seen in numerous crustaceans:

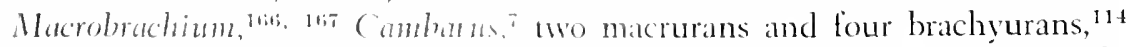

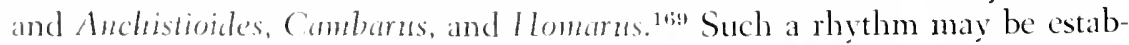
lished by diumal environmental changes. These changes must determine

* In an apposirional eye the retinula cells of an ommatidium are stimulated only by light entering through that ommatidium, whereas in a superpositional cye the retinula cells are stimulated by light eoming through neighboring ommatidia as well. 
some sort of biological timing mechanism which controls the pigment migration. The role of the sinus gland in controlling pigment migration is discussed in Chapter 22.

Image Formation. The crystalline cone is the principal focusing device of the compound eye, and it is not adjustable. Each ommatidium, therefore, has a fixed focus. The crystalline cone is made up of concentric lamellae, the more central layers of which have a higher refractive index than the more peripheral ones. The cone is therefore a virtual lens cylinder. Figure 104 shows light passing through such lenses. If the length of the crlinder is equal to its focal length $(A)$ a beam of parallel light ( $m p n$ ) which enters perpendicular to one end of the cylinder (edge $a b$ ) is focused on the other end (edge $c d$ ), and emerges as a divergent beam $\left(n^{\prime} p^{\prime} m^{\prime}\right)$. Other beams of light $(q)$ entering at an angle to the axis of the cylinder emerge at an angle
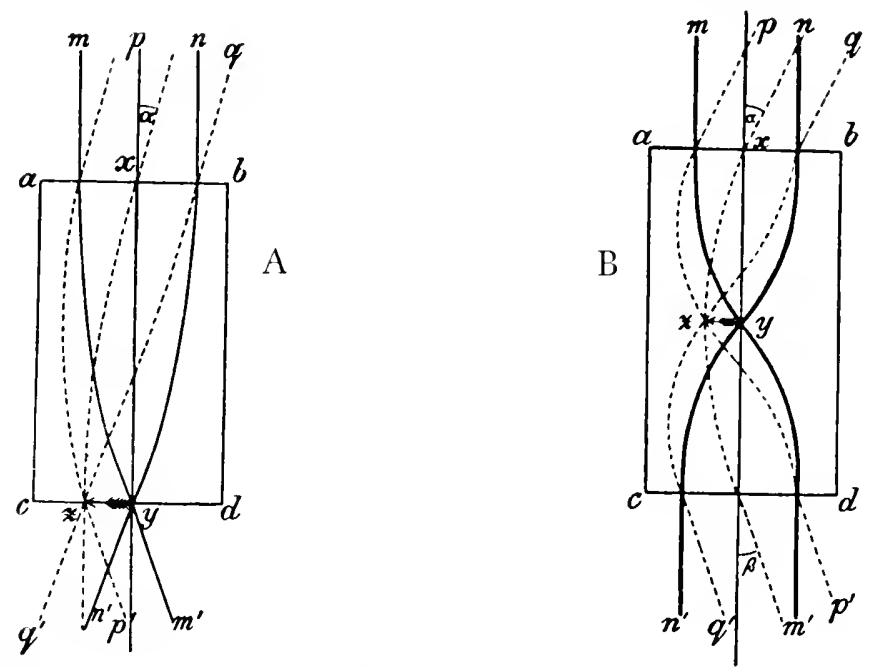

Fig. 10t. Diagram of the path of light rays (A) through a lens cylinder of a length equal to the focal length; (B) through a lens cylinder of a length equal to twice the focal length. After Exner. ${ }^{2}$

$\left(q^{\prime}\right)$. This permits image formation, but since this image is limited to only seven retinula cells and is also subject to considerable distortion by the rhabdom, it is probably useful merely as a spot of light rather than as an image. If the lens cylinder is twice as long as its focal length (B) the beam emerges as a parallel beam whose angle of emergence $(\beta)$ is equal and opposite to the angle of incidence $(\alpha)$. In arthropod eves there are two types of image formation, superpositional and appositional, which correspond to these two possible types of lenses. In both types the light entering a single facet is probably detected as a single spot of light.

There are two chief types of ommatidia as shown in Figure 105. In $A$, the iris pigment completely sheathes the ommatidium and the only light which strikes the rhabdom and retinula cells is that which enters almost parallel to the axis. In $B$, the pigment cells do not completely sheathe the ommatidium and, depending on the angle of incidence, light which passes through the crystalline cone may strike neighboring rhabdoms as well as the 
rhabdum of the crystaline cone through which the light has passed. There fore, there are two possible types of image formation, as shown in Figure 106. In $A$ is shown the so-called apposition image, in which each rhabdom receives light which enters the ommatidium parallel to its axis. In this type the length of the crystalline cone may be equal to its focal length, as in Figure $105, A$. In $B$ is shown an image formed by superposition, in which light from a point (e) may pass through any of a number of crystalline cones and be bent (as in Figure 105, B), so that it is focused on a single

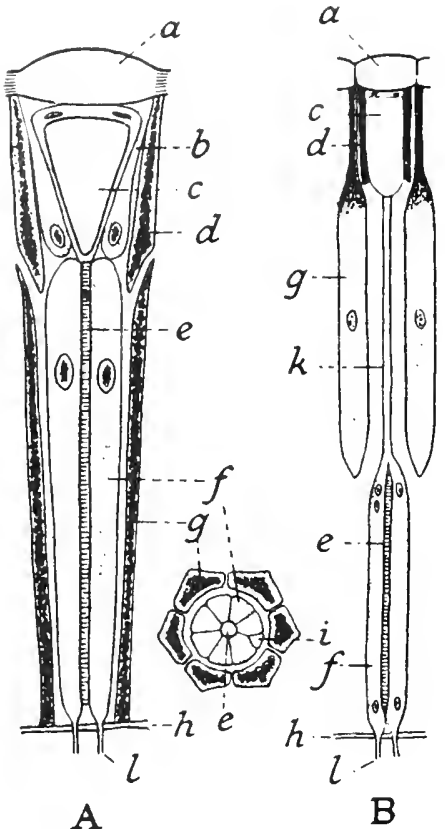

Fig. 105. Diagram of the two types of ommatidium. A, From eye forming apposition image (after Snodgrass); B, From eye forming superposition image (after Weber). a, Corneal lens; $b$, matrix cells of cornea; $c$, crystalline cone; $d$, iris pigment cells; $e$, rhabdom; $f$, retinal cells; $g$, retinal pigment cells; $h$, fenestrated basement membrane; $i$, eccentric retinal cell; $k$, translucent filament connecting crystalline cone with rhabdom; $l$, nerve fibers. From Wigglesworth. ${ }^{171}$

rhabdom. In this type the length of the crystalline cone is twice the focal length. If the secondary iris pigment should migrate, as shown in Figure $105, B$, then the superposition eye may function as an apposition eye.

In general, most diurnal insects have apposition eyes, and nocturnal ones have superposition eyes. Undoubtedly there are many intermediate types. In some insects the cone cells are not refractile and apparently do not serve as lenses. The apposition eye is adapted to function at high light intensity and is really very inefficient as a light-gathering device. The superposition eye is adapted to low light intensities and is much more efficient for gathering light than the apposition eye, but is still much less efficient than most vertebrate eyes.

Because of the fact that the image formed by the apposition eye is made up only of spots of light of different intensities, each of which has entered 
through a single ommatidium, the image is often referred to as a mosaic image. In the superposition eye light which is focused on a single rhabdom (Fig. 106, B) may enter through a number of ommatidia, so that in this case the image is not quite so mosaic. However, in a vertebrate eye, the image that is projected along the optic nerve consists of a record of intensities registered at individual cones or at small groups of rods and/or cones, and thus this image also may be considered as a mosaic of much finer grain. If each retinual cell of an ommatidium is assumed to register different intensities of light and therefore to project a small portion of an image, then the term mosaic takes on a somewhat different meaning, and in the final pathway to the brain this pattern is essentially the same as that in a vertebrate optic nerve.

Ocelli among the Arthropods. A simple type of arthropod eye, found in the larvae of Lepidoptera, Trichoptera, Collembola, and other insects, consists of a structure comparable to a single ommatidium of a compound eye. These simple eyes are known as stemmata, or lateral ocelli, and may occur singly or be scattered on the sides of the animal in small groups. In the larvae of Tenthredinidae and many Coleoptera there is only one ocellus on each side, but it contains many retinulae, each with several sense cells and a rhabdom, perhaps with pigment cells, all beneath a single lens. This more complicated type of lateral ocellus is capable of distinguishing form (Fig. 107) and perhaps color. In the larvae of Cicindela there are six stemmata on each side: two large, two small, and two vestigial. The large ones have 6350 visual cells and are apparently capable of detecting form and, when all of the stemmata are functioning together, of determining distance. ${ }^{44}$

Dorsal ocelli are the simple eyes of insects which usually occur in the frontal region of the head between the compound eyes. In general the structure is similar to that of the complex stemmata described above. The dorsal ocelli of agrionids and libellids have a tapetum and, when the animal is brought from darkness to bright light, there is a rapid movement of brown pigment across the white fundus within a few seconds. The ocelli have a high light-gathering power, with apertures estimated at $\mathrm{f}^{*} 1.8$ for Eristalis and 1.5 for Formica females, whereas the facets of appositional compound eyes have apertures of $\mathrm{f} 2.5$ to 4.5 and therefore a much lower light-gathering power. In sphingids and noctuids the superpositional compound eyes have a high light-gathering power, and ocelli are absent. The ocelli are poorly adapted for image formation for at least two reasons: the angular separation of the rhabdoms is great (3-10 degrees), and the image is usually focused far behind the retina (e.g., in Eristalis the retina is $0.11 \mathrm{~mm}$. behind the lens and the focal plane is $0.29 \mathrm{~mm}$.)..$^{90}$

Most spiders have eight ocelli distributed over the dorsal portion of the cephalothorax. Two of these are known as principal or direct eyes, and the others as lateral or indirect eyes. In the direct eyes the sensory portion of each sense cell is in front of the cell body, and in the indirect ocelli the sensory portion is behind the cell body. The direct eyes are movable by means of radially distributed muscles and permit the animal to fixate its prey without shifting its body. ${ }^{91}$

* Focal length divided by the diameter. 
Other arthropods have various arrangements of ocelli. Many species have ocelli with several retinulae; others (myriopods) have groups of such ocelli placed contiguously so that they may tend to simulate a compound eye; Limulus has a number of ocelli, each with a single retinula, closely spaced to form the lateral eves, which are somewhat similar in function to the true compound eve; and crustacea have true compound eyes similar to those of insects.

The Photic Behavior of Arthropols. The presence of a compound eve, consisting of many ommatidia, supplemented by additional ocelli, provides an organism wit! a great number of individual photoreceptors pointing in different directions.

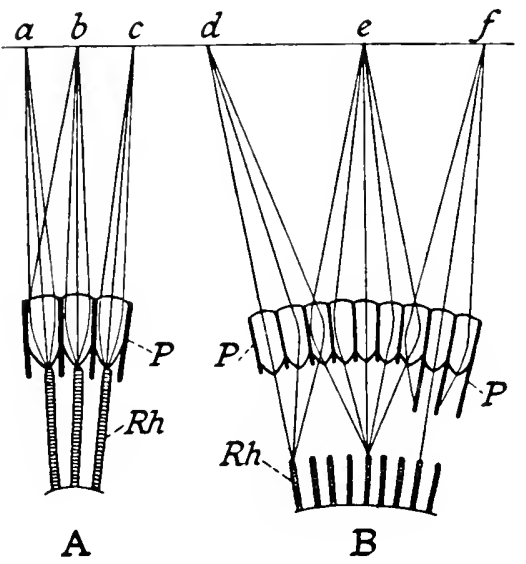

Fig. 106. Diagram to show image formation, by (A) an apposition eye and (B) a superposition eye a-f, I uminous points with the rays emitted by them; $P$, pigment; $R h$, rhabdom. After Kühn, from Wigglesworth. ${ }^{171}$

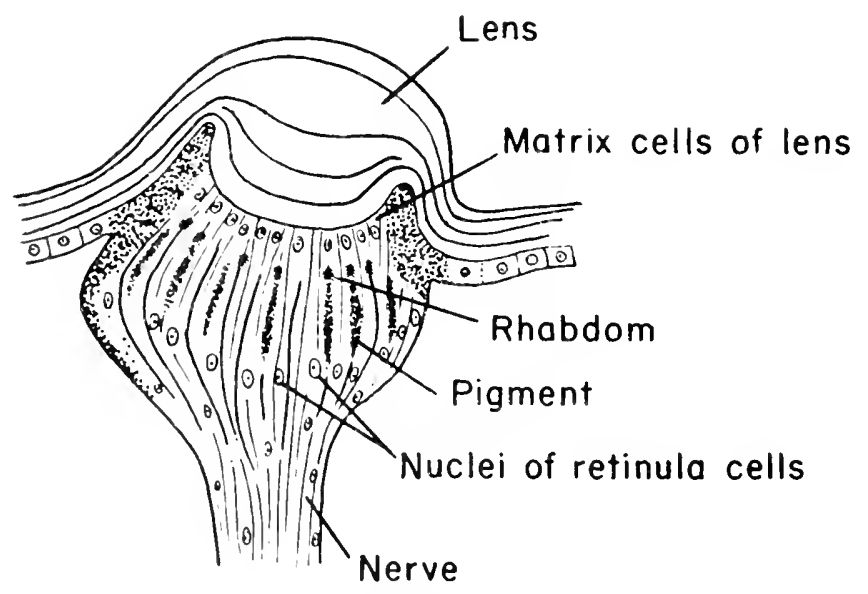

Fig. 107. Ocellus of Aphrophora spumaria (after Imms and Link). From Wigglesworth. ${ }^{171}$ 
It has already been stated that paired intensity receptors may result in a type of orientation called tropotaxis, involving simultaneous comparison of two intensities incident on different end-organs. Among the arthropods there are instances of a type of orientation called telotaxis, in which orientation in the direction of one source of stimulation occurs without comparison. ${ }^{43}$ An organism exhibiting telotaxis orients to one source of stimulation, even though other sources may be present. This implies that, out of a variety of stimuli, one dominates and controls the resultant orientation.

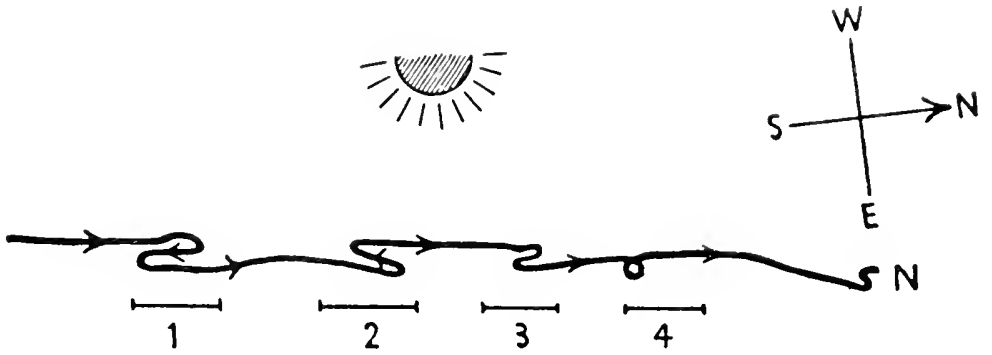

Fig. 108. The itinerary of an ant subjected to sunlight from different directions (After Santschi). The ant was returning 10 its nest with the sun on its left. At 1, 2, 3, and $t$, the ant was shaded from direct sunlight and subjected to the sun's image from a mirror. The animal preserved its position relative to the source of light. From Fraenkel and Gunn..

A second type of orientation among animals possessing compound eyes is the light compass reaction. This reaction is of considerable importance in the "homing" insects, the ants and the bees, and forms the basis of the dominant sensory clue which guides them back to the nest or hive. The structural basis for the light compass reaction is the compound eve, in which a small source of light can stimulate only a few or, perhaps, only one ommatidium at a time. Once a certain angular position relatice to the sun is assumed, the arthropod has merely to retain the sun's image in the same ommatidium in order to remain oriented. The dominant nature of this orientation is shown in Figure 108. ${ }^{43}$

A third type of behavior pattern exhibited by certain aquatic arthropods, although not limited to them, is the dorsal light reaction. The orientation of the body is determined by the direction of illumination and not by gravity. Similar reactions are exhibited by the carp louse, Argulus foliacens; the brine shrimp, Artemia salina, and the prawn, Processa caniculata. In other animals, such as the prawn, Leander xiphias, and the fish, Crenilabris rostatus, the dorsal light reaction is elicited only after removal of the statocysts and the labyrinths.*

The Vertebrate Eye: Morplology. The eve of man is rather generalized in structure and may be taken as representative of the vertebrate group. The structure of the right human eve is shown in horizontal section in Figure 109.

The eve is comparable to a camera in many respects. There is a photosensitive screen (retina) on which the cornea and lens form an inverted image

${ }^{*}$ Recently Waterman (Science 10: 252-25+ (1950)) has confirmed, by recording optic nerve impulses, the earlier evidence from behavior that compound eyes of arthropods can distinguish the plane of polarization of polarized light. 
in accordance with principles of geometric optics. The aperture of the lens is varied by the iris, and the eyelids are used as a lens cover to exclude light and lessen the possibility of mechanical injury to the corneal surface. The photosensitive screen is backed by a layer of black pigment which reduces internal reflection and thereby enhances the clarity of the image. Part of the focusing mechanism (i.e., the lens), is also variable (by contraction of the ciliary muscle), so that objects at very different distances may be seen with equal sharpness if they are not viewed simultaneously.

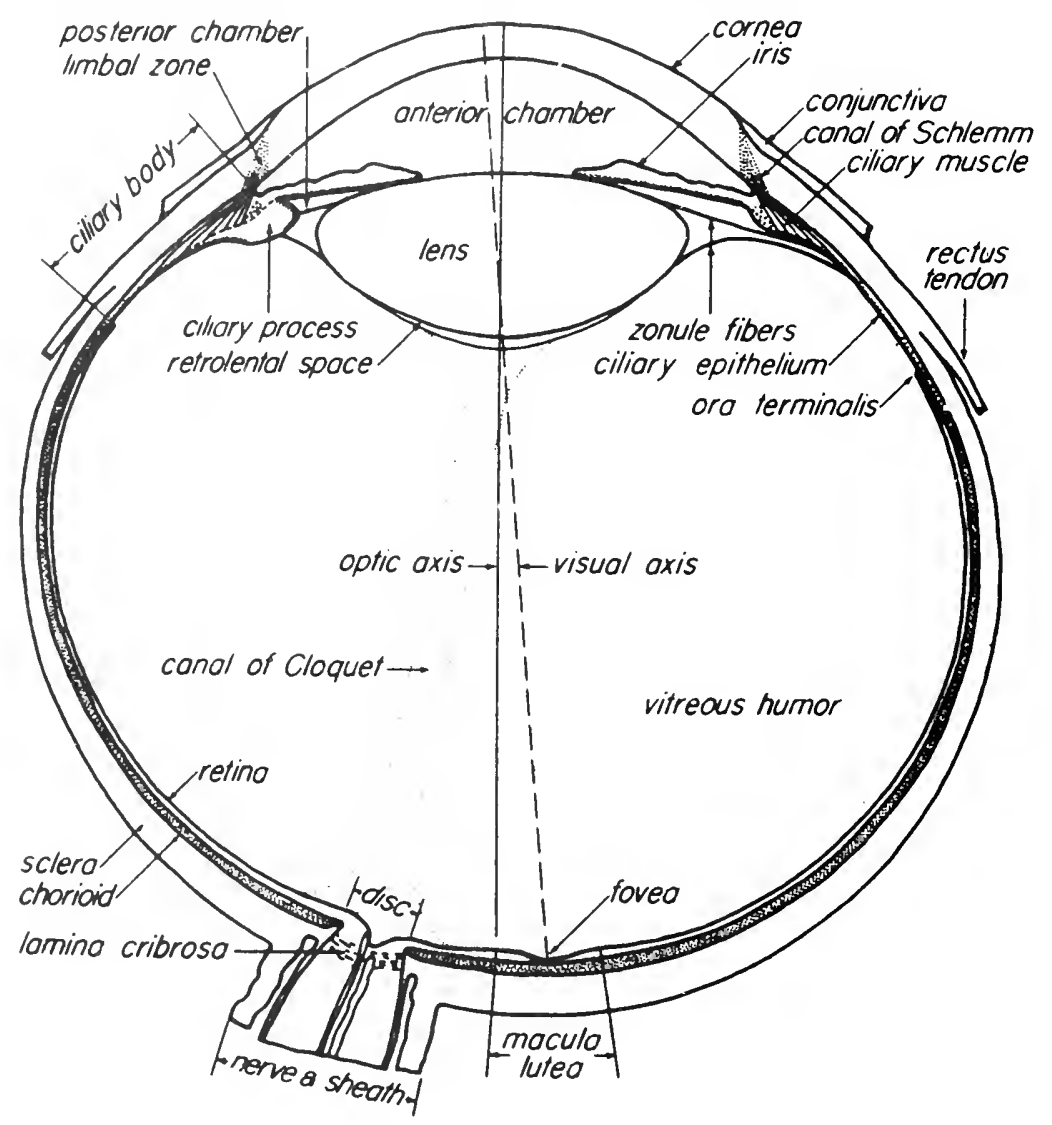

Fig. 109. Horizontal section of the right human eye, $\times 4$. From Walls. ${ }^{103}$ Modified from Salzmann.

Focusing of light on the retina is brought about partly by the curvature of the cornea, which has a refractive index (1.376) much higher than that of air (1.00) and partly by the lens, which has a refractive index (1.42) slightly higher than that of the aqueous and vitreous humors (both 1.33) Since the greatest difference in refractive index, and also the greatest curvature, is found at the air-corneal interface, this is really the most important refracting surface in the formation of the image. The lens serves in a capacity comparable to that of a fine adjustment on a microscope, giving a delicate and accurate control over a short range. The range over which the lens can 
change the focus of the eye is known as the accommodation range, or simply as the accommodation.

The accommodation range is as much as 15 to 20 diopters $^{*}$ in young chil dren and has an average value of about 4 diopters at the age of 42 years. This means that young children can focus objects as close as 2 inches from the eye as well as those at infinity. The decrease in accommodation permits adjustment at age 42 only from about 8 inches to infinity. The relaxed normal eye is focused for infinity, and accommodation is accomplished by the elasticity of the lens when the ciliary muscles are contracted. The decrease in focusing power with age is caused by the hardening of the lens, and its consequent failure to become slightly rounder and thicker on contraction of the muscle. This is the normal condition of farsightedness or presbyopia (meaning "old" sight), which is almost an inevitable result of old age. It can readily be corrected by means of spectacles, and when the power of accommodation is very low, the individual often resorts to the use of two pairs of spectacles or to bifocals, with one lens for near and one for distant objects.

The most common abnormalities of vision which are corrected by spectacles are: (1) elongated eyeball, resulting in nearsightedness, or myopia, in which the image is focused in front of the retina, (2) short eyeball, resulting in farsightedness or hypermetropia, in which the image tends to be formed behind the retina, and (3) non-uniform curvature of the lens or cornea, resulting in astigmatism, the occurrence of different focal points for beams of light in different planes; e.g., vertical lines may appear blurred when horizontal lines at the same distance are not blurred.

Structure of the Retina. The structure of the retina is shown diagrammatically in Figure 110. In this figure the light enters at the bottom, which is the front of the retina, passes through the layer of nerve fibers, a layer of tertiary or ganglionic neurone cell bodies, a layer of synapses, a layer of secondary neurone cell bodies (the inner nuclear layer), another synaptic layer, and then through the cell bodies (outer nuclear layer) of the rods and cones, which are the primary neurones or sense cells. The portion of the rod or cone corresponding to the dendrite of the primary neurone contains the photosensitive material, rhodopsin in the rods and iodopsin in the cones. Very little light is absorbed by the neuronal layers of the retina, except in the photosensitive material. The light which passes the photosensitive material is absorbed by the choroid.

The neuronal connections of the retina are complex. They have been worked out in great detail by Polyak, ${ }^{13 i}$ and some of the types of connections are shown in Figure 110. Some cones are connected indirectly to a nerve fiber (e.g., the far left cone in the diagram), and this fiber receives no impulses from other rods or cones. Such a cone has an "exclusive line" to the brain. Other cones (e.g., the far right specimen) are connected to nerve fibers which may receive impulses which originate in other cones or in rods. Certain cones which are connected to an "exclusive" fiber (e.g., the far left cone) are also connected through secondary neurones (inner nuclear laver) to nerve fibers which receive impulses from several or many rods and cones. These connections are important in the mechanism of color vision. The nerve

* The diopter is defined as the reciprocal of the focal length in meters. 
fibers of the retina pass across the inner surface of the retina and enter the optic nerve. The region of exit of the nerve contains no rods and cones, is thercby insensitive to light, and is known as the "blind spot."

In one region close to the optical axis of the eve the rods are fewer in number and the cones are much more numerous. This area is called the area centralis. In birds, in a few mammals, and in reptiles and fishes the area centralis is thinner than the surrounding retina, because of a decreased thickness of the laver of secondary and tertiary neurones, and is referred to as the forea centralis. In the fovea of certain birds (e.g., hawks, swallows, terns) these neuronal layers are not merely thinner, but their thickness va-

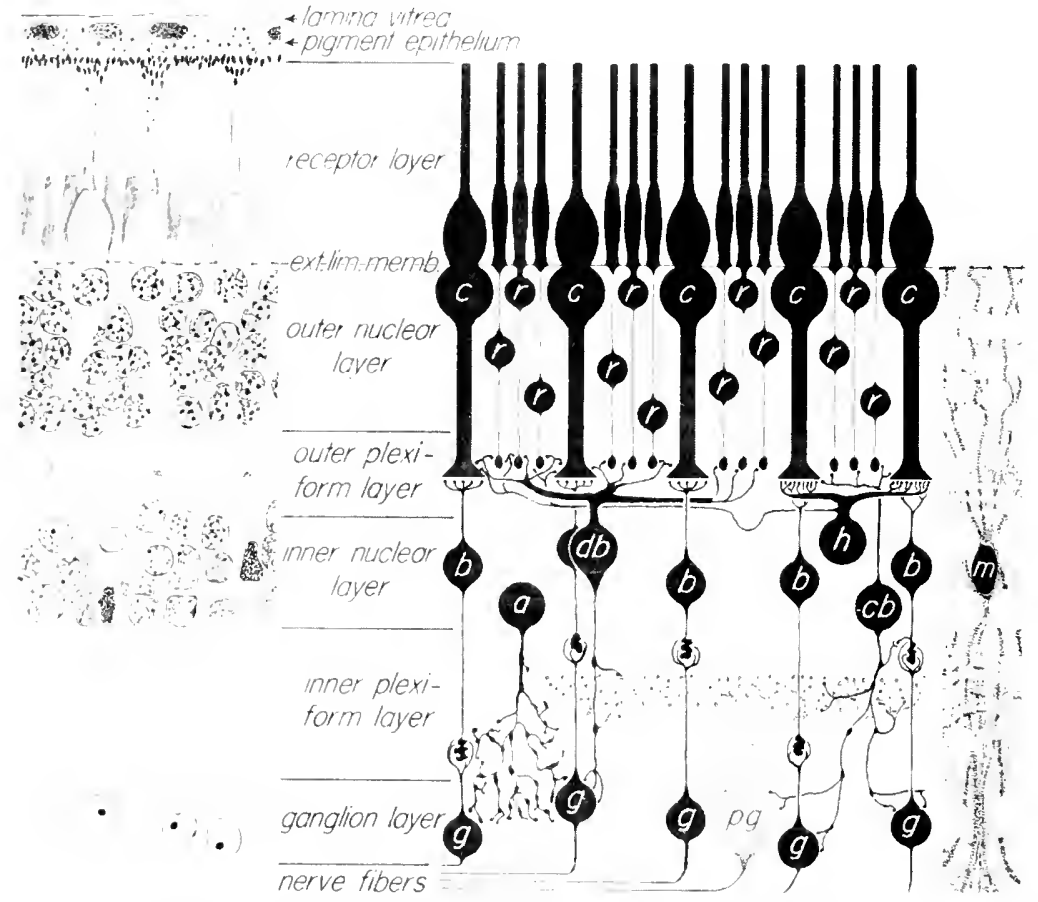

Fig. 110. Histology of the human retina. Left, vertical section through the retina in the nasal fundus; right, neuronal connections in the retina as revealed by silver impregnation methods (after Polyak). $a$, Amacrine cell; $b$, bipolar cells; $c$, cones; $c b$, centrifugal bipolar, believed to conduct outward through the retina; $d b$, diffuse bipolar; g, ganglion cells; $h$, horizontal cells; $m$. Müller fibers, forming limiting membranes; $p g$, parasol ganglion cell; $r$, rods. From $W_{\text {alls. }}{ }^{163}$

ries in such a way as to spread the image over a larger surface, thereby permitting a higher degree of visual acuity. This spreading lens effect is caused by the fact that the refractive index of the retinal tissue is greater than that of the vitreous body. The forea of man is a broad area with very little if any spreading power.

The two types of sense cells, rods and cones, are adapted to function most effectively orer different intensity ranges. The rods are much more sensitive to light than the cones and thereby are adapted to function at very low intensities of light, ranging from moderately bright moonlight downward to 
the lower linit of visibility. At higher light intensities the photescusitive substance (rhodopsin) of the rods is almost completely bleached, and the rods are thereby nonfunctional; in fact they reach maximal sensitivity only after a half hour or more in the dark. The cones contain a photesensitive material (iodopsin) which is not so readily affected by light, and therefore they are able to function at a higher intensity range. The existence of these two types of sense cells gires a combination of sensitivits ranges which accounts for the extremely efficient functioning of the human eve orer an intensity range of at least eight logarithmic units. Other vertebrates and in vertebrates cletect flichering light orer most of this range, despite the lack of such duality of structure, but their ability in terms of other visual functions is probably not so good.

Apart from the fact that the rods and cones in duplex retinas function in different intensity ranges, they differ in another very fundamental manner. The cones are of several types which are differentially sensitive to light of various wave lengths, and this differential sensitivity is the basis for color discrimination (see page +34 ). All of the rods of any given animal seem to have the same spectral sensitivity and probably serve only to detect differences in intensity.

Visual Adaptations of Lower Vertebrates. The structure and function of the eyes of vertebrates show an astouncling range of adaptation to the conditions under which the animal lives. Consequently, rather closely related species which live under different conditions may have quite different vision. This range of adaptation has been described in considerable detail by Walls, ${ }^{14: 3}$ and the outline of the subject presented here is largely adapted from his book.

The general types of adaptations of the vertebrate eve may be listed as follows:

ADAPTATIONS TO ACTIVITY UNDER CONDITIONS OF LOW AND/OR HIGH LIGHT intensity: Adaptation to Arrhythmic Activity, during Both Day and Night. Animals which are equally active by day and night may not sleep at all or may merely take short naps. Such animals may be said to be arrhythmic in behavior and must be capable of good vision over a great range of light intensity. Some of the mechanisms which permit such vision are: (1) a considerable development of both rods and cones; (2) pigment migration from the choroid to the layer of sense cells in order to screen intense light from some of the rods and cones, especially the rods (e.g., Phoximus, a fish); (3) migration of rods and cones into the pigment laser in intense light (Ameiurus, a catfish); (4) high mobility of the pupil, especially in higher vertebrates, where the positional changes of pigment and visual cells (2 and 3 above) are minimal.

Adaptations to Day-Time Activity. Animals which are diurnal, i.e., active chiefly by day, have a high degree of visual acuity, brought about in several ways: (1) A larger image, which may be obtained by increasing the size of the eye and the relative proportions of the lens and eveball, especially by: increasing the lens-retinal distance. Nan, birds, chameleons, and all diurnal animals have a much greater lens-retinal distance than arrhythmic animals such as cougars, dogs, or clromedaries, and wery much greater lens-retinal distances than nocturnal animals such as opossums, house mice, lynx, or frogs. 
(2) A marked increase in the relative number of cones and a decrease in the absolute number of cones which contribute to any one tertiary neurone, i.e., by increasing the concentration of individual nerve channels from the retina to the brain. This results in an actual increase in the resolving power of the retina. (3) Special areas with an especially high concentration of cones and lew, if any, rods, the so-called central areas or foveas. In the flat central area of frogs or in the cup-shaped fovea of man and in the acutely depressed fovea of birds, the concentration of individually connected cones is increased at the expense of group-connected rods. This is merely a local extension of the adaptation mentioned under (2), above. The structure of the fovea is carried to its extreme in certain birds, where the shape of the fovea, together with the difference in refractive index of vitreous body and retina, spreads the image over a larger number of cones, thereby acting as a localized negative lens. (4) Development of intraocular color filters which absorb in the bluc end of the spectrum and thereby reduce chromatic aberration at the retinal surface and consequently increase acuity. These filters consist of (a) red or yellow oil droplets in the cones of turtles and most lizards and birds; (b) a yellowish lens in the lamprey, some lizards, snakes, insectivores, in man, and in most Sciuridae-the lens is almost orange in ground squirrels and prairie dogs; (c) a yellowish cornea in Amia, Esox, Cyprinus, and a few other fishes; $(d)$ a yellow fovea in man; and (e) retinal capillaries in eels and most mammals (but not especially in diurnal animals).

ADAPTATION TO NIGHT-TIME ACTIVITY. The nocturnal animals have a number of adaptations which tend to increase the sensitivity of the eye. Some of these are: (1) Greater sensitivity of the retina to dim light by an increase in the number of rods and also in the number of rods connected to each tertiary neurone, thereby permitting greater summation of sense-cell activity. An increase in sensitivity by this method produces a simultaneous decrease in visual acuity. This fact is largely responsible for the visual acuity differences between diurnal and nocturnal animals shown in Table 63. (2) Presence of a slit pupil instead of a round pupil, as in diurnal animals. The slit pupil is able to close more tightly in bright light and thereby preserve the dark adaptation of the retina. For this purpose the slit pupil can be much more effective than the circular pupil of diurnal animals, and under conditions of dim illumination it can be greatly expanded into a large circular opening. This wide range of adaptability makes it an excellent nocturnal adaptation despite the fact that it does not directly aid dim vision. (3) Presence of a tapetum lucidum, a reflective layer on the choroid. In all animals the choroid consists of a highly absorbing layer which prevents internal reHection. However, in many nocturnal animals there is a highly reflective material (crystalline guanine) in either the choroid or the retina, which is capable of reflecting light after it has passed through the sense cells so that it passes through them again in the opposite direction. This doubles the sensitivity to dim light but also decreases acuity. Some of the reflected light passes out through the pupil, and the animal is said to have "eyeshine," a phenomenon commonly noted in cats. Eveshine occurs in all classes of vertebrates and is a special adaptation to nocturnal habits.

adaptations to space and mónon. Any object in visual space may have a number of perceptible characteristics, such as size, shape, pattern, bright- 
TABLE 63. VISUAL ACUITIES FOR PARALIEL IINES (From various sources)

$\begin{array}{ccc}\text { Visual } & \text { Corresponding distance } & \text { Visual angle corresponding } \\ \text { angle, } & \text { on retina, } & \text { to } 1 \text { mm. distance } \\ \text { minutes } & \text { micra } & \text { along visual cortex }\end{array}$

Diurnal animals

Human adult

$\begin{array}{ll}0.44 & 1.89 \\ 0.48 & 2.06 \\ 0.50 & 2.14 \\ 0.80 & 3.43 \\ 0.82 & 3.52 \\ 0.83 & 3.56 \\ 0.62 & 2.67 \\ 0.47 & 1.86 \\ 0.67 & 2.33\end{array}$

Child

Chimpanzee

Rhesus monkey

Rhesus monkey, along visual axis

Rhesus monkey,

$7^{\circ}$ from visual axis

Cebus monkey

Pigeon

Pigeon, 'homer'

Gamecock (no fovea)

Nocturnal animals

Cat, along visual axis

Cat, $30^{\circ}$ below axis

Alligator

Opossum

Rat, pigmented

Rat, albino

.

...

0.95

2.70

0.38

4.07

5.5

...

11.0

11.0

26.0

52.0

\subsection{9 \\ 2.06 \\ 2.14 \\ 3.43 \\ 3.56 \\ 2.67 \\ 2.33}

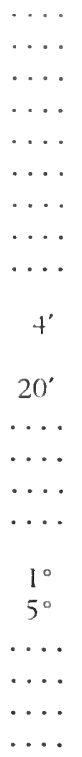

3

4.89

.69

9.58

$\begin{array}{lc}\ldots & 1^{\circ} \\ \ldots & 5^{\circ} \\ \ldots & \ldots \\ \ldots & \ldots \\ 23.8 & \ldots \\ 47.7 & \ldots\end{array}$

ness, color, position, motion, and distance. We are aware of these qualities by virtue of the retinal image which is translated on the basis of experience into what we consider to be the "true" size, shape, and position of the object. The special adaptations which permit enhancement of the perception of some of the above characteristics are as follows:

Accommodation and Its Substitutes. Accommodation, i.e., focusing of the image, may be brought about in various ways. In lampreys, teleosts, and probably holosteans, the active process of accommodation moves the lens backward to permit focusing on distant objects. In elasmobranchs, amphibians, and snakes, the lens is actively moved forward to adjust for near ob. jects; and in mamınals, birds, and reptiles other than snakes the curvature of the lens is increased in order to focus on near objects.

Visual Angles and Fields. The visual angle through which single vertebrate eyes can see is generally about 170 degrees, with a lower extreme of 110 de grees for certain air-living animals (owls) and still less for deep-sea fishes. and a maximum of 200 degrees or more for a cat eye. The visual angle of a human eye is 150 degrees.

The position of the eyes in the head determines the extent of the binocular field, if present. A few vertebrates (lampreys, hammerhead sharks, a few large-headed teleosts, the amphibian Cryptobranchus, penguins of the genus Spheniscus, and the larger whales) have no binocular field of vision; i.e., both eyes can never focus on the same object at the same time. In most fishes 
the binocular fickl is 20-30 degrees horimutally directly ahead, with extremes of $+t a$ to degrees or more. Mlost birds hare binocular lictels (Figs. 111, 112), and these are known to vary from 6-10 degiees in parrots to 60-70 de.

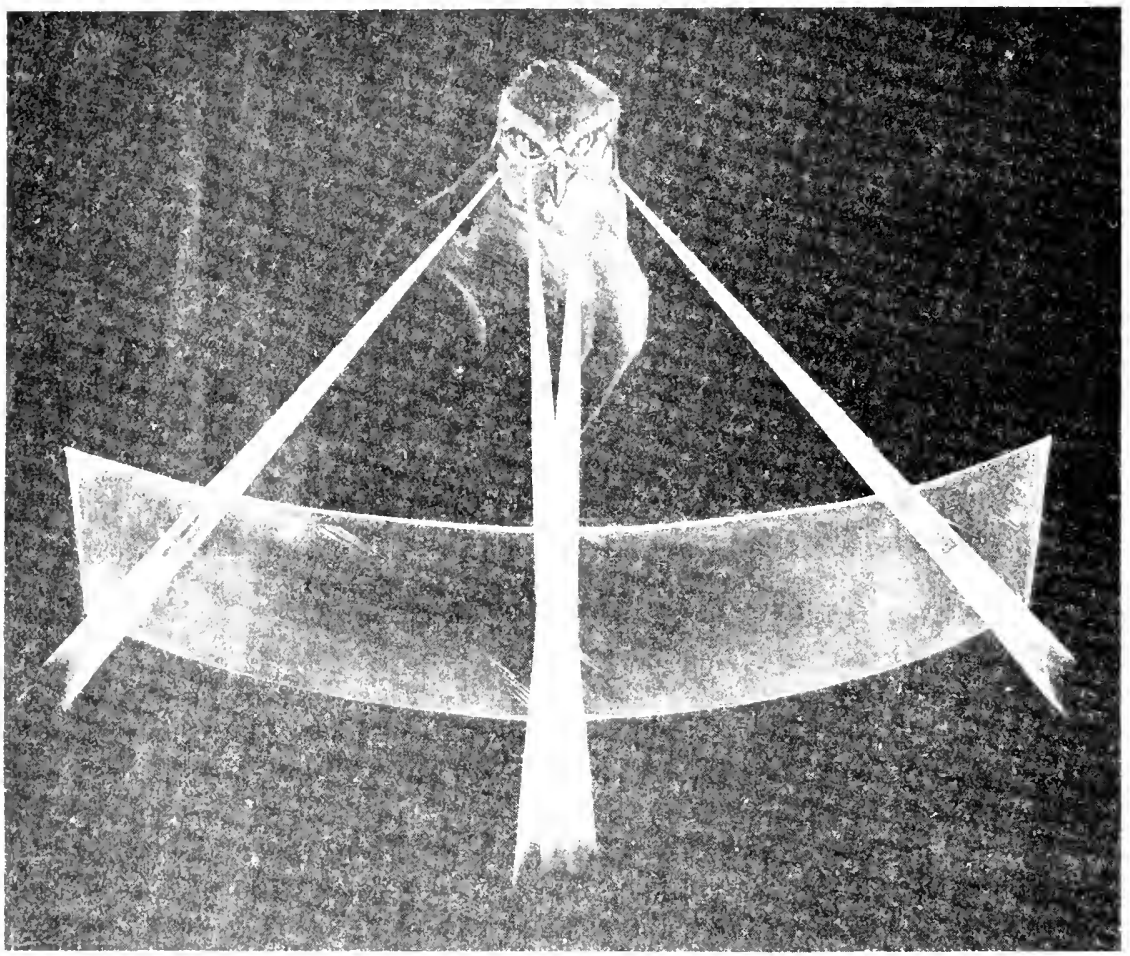

Fig. 111. Projections of the four foveas of a hawk through an imaginary surface at the distance of momentary accommodition and contergenece, forming a "visual trickent" of high resolving perser. (Courtes of Dr. Crordon L. Walls.)

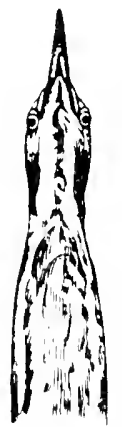

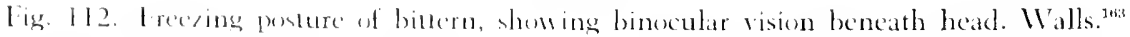
grees in owts. Some birels exen have a binesular field of riew below the bill, "e. a bitfern can assume a "ficering" posture with its bill almost rertical and still hate a himocular liedel rentral wo the raised head, i.e., horizontally (Fig. 112). In simians and in man the binoular lick is about 140 degrees, i.e., the 
risual feeld is almost entirely binocular. I lares have monocular fields of 190 degrees which oserlap to form small binocular ficlds botb anteriorls and posteriorly.

Eye Movements and the lovea. Movement of the eves may be iuroluntan or voluntary. Involuntary movements are those which form automatic reflexes for the purpose of maintaining the visual field comstant during locomotion and during passive jogglings of the head and body. An excellent example of such movement is that which we make when we look another person in the eye and shake our head in the gesture of "no." This eve morement is auto. matic and almost ancontrollable. Moving pictures taken from a moving, jog gling veluicle do not have this automatic adjustment, and the absence is both noticeable and unpleasant to the observer.

During voluntary movements the two eves may more independently with no coordination or may be coordinated to various degrees. In most lizards and in birds the morement is independent and uncoordinated; in some fishes and in chameleons it is independent but coordinated when objects are observed in the binocular field; and in man it is never independent but is always coordinated in such a mamner that the same point of an object is always focused simultaneously on the two forcas.

In birds, moving objects are usually followed br movement of the entire head, and fixation mas be either monocular or binocular. Many birds (ac. cipitrines, swallows, etc., mostly flight feeders) have two foreas in each eve. one of which is used monocularly and one binocularly. Since the eres are directed at an angle of as much as 75 degrees or more from each other, the usual central foreas are useful onty monocularly, but the second forcas are placed on the temporal surfaces of the retinae so that they may be used simultaneously in a binocular manner (Fig. 111). This arrangement is the so-called "visual trident" by means of which a bird may have three objects focused simultaneously on its four foreas, one object on two foreas, and one each on the other two.

Depth and Solidity Perception. Perception of depth and distance is obtained either binocularly or monocularly. Most of the clues to depth are $\mathrm{em}$ ployed in both monocular and binocular vision and may be detected in twodimensional photographs or in good paintings. Perception depends on the following: (1) Retinal image size. The larger the image the closer the object. (2) Perspective. Horizontal lines tend to meet at the horizon, and the amount of taper is an index of size and distance. (3) Overlap and shackow. Objects whose images or shadows overlap other objects are nearer than those objects. Distances with few orerlaps, as over water with no intervening objects, therefore tend to be underestimated. (4) Vertical neamess to the horizon. Our line of sight tilts toward the ground for near objects but is raised for dis tant objects. (5) Aerial perspective. Objects with dim or bluish outlines seem more distant because long atmospheric pathways make them appear so. In very clear air, distances tend to be underestimated; in fog or mist, orerestimated. (6) Parallax, the most important of all monocular clues. This is the change in the apparent angle, at the eve, between near and far objects, produced by lateral movement of the observer's head or body. This is the principal clue which permits one-eyed men to move about crowded rooms as well as they do. 
It is difficult to say which of the six aforementioned clues are used by any given animal. Side-to-side movements of the head are used by some animals as a means of improving parallax, either with monocular or with binocular vision. Some birds may cock their heads several times at a morsel of food before pecking it with complete assurance of its position; many shore birds use vertical bobbing of the head in a similar manner.

Movement Perception. The perception of movement and the factors which influence it are decidedly complex. Under most conditions a small object moving on an otherwise motionless field can be detected more easily than the same object if not moving. The movement of an image on the retina causes a change in the stimulation pattern which is more easily detected than the details of the same pattern if it is unchanging. This is one reason why flicker fusion curves offer a valuable index of vision. An object moving very fast may be seen only as a blur; the same object moving very slowly may not be seen at all. At intermediate speeds, however, the outline may be clearly seen. At speeds which result only in a blur, the details can sometimes be seen if the observer blinks rapidly, e.g., as when viewing a rotating wheel or when a nearby picket fence is seen from a rapidly moving automobile. One possible explanation of this fact is that because of the persistence of visual images the continuous series of images formed by the moving object overlaps to form the blur. By blinking rapidly these may be cut into a discontinuous series of distinct images. Under certain conditions, nystagmus, a fluttering movement of the eveball. may also seem to "stop" linear motion and thereby remove the blur.

One mechanism for the production of discontinuous images for localized areas of the retina of birds is the pecten. Of the thirty or more theories concerning the function of the pecten it seems that the correct one is that of Menner, ${ }^{12}$ who pointed out that the pecten serves as a grating which casts a shadow on the retina. An object which is moving across the field of vision forms an image behind the pecten which is discontinuous for localized areas of the retina. The fusion frequency is higher for light of a given flickering intensity for bircls with a pecten than for animals lacking a pecten, particularly when the light is on for a high proportion of a cycle. ${ }^{30}$

adaptations to media and substrate: Aquatic Vision. The focusing mechanism of fishes is somewhat different from that of non-aquatic forms in that the cornea has almost the same refractive index as the water and is almost uscless as a focusing device. Therefore the curvature may be changed to fit into the streamlining of the animal, and in some fishes the eyes are further streamlined by the addition of an "adipose lid."

One of the most striking differences between aerial and aquatic vision is in the field of view. This is shown in Figure 113. Because of the total internal reflection of light which strikes a water-air interface at an angle of less than 48.8 degrees (fresh water), the only view a fish has with an aerial pathway is through a surface "window" which subtends a 97.6 degree angle. The diameter of the window varies with depth of the fish. Because of refraction at the surface of the water the field of view spreads as it passes through the surface, and distant objects (e.g., the shore and tree in the figure) are compressed around its periphery. Objects on the bottom of the water (e.g., the rock) may appear in the visual mage close to objects which are on dry 
land and are seen through the window. A varicty of devices in deep-sea fishes tend to increase visibility at low intensities. Some of these are relatively larger eves, relatively larger lenses and pupils, a greater concentration of rods, tubular eyes which sacrifice peripheral vision for a more effective central vision, and various patterns of bioluminescence to illuminate the objects tr be seen.

Aerial Vision. The dioptric differences between eves atlapted to rision in air and in water are pronounced and are discussed above. Another group of differences include those pertaining to protection of the cornea, especially from drying. In man and many other animals this is accomplished by blink-

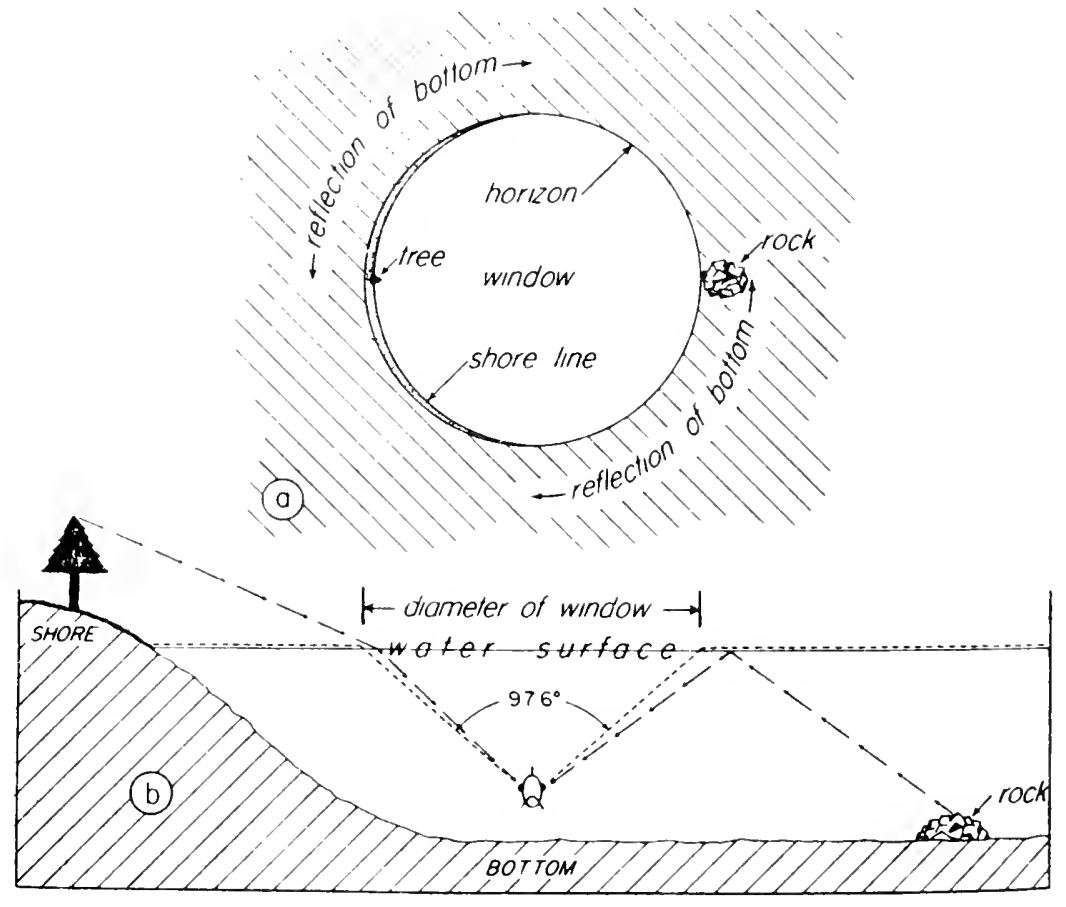

Fig. 113. Diagram showing the upward visual field of a fish. $a$, The aerial window as seen from below; $b$, explanation of the aerial window, assuming calm water. Light rays striking the water surface within the window are refracted, while those striking outside rhe window are reflected. From Walls. ${ }^{163}$

ing. In birds and many mammals, however, there is an additional structure, the nictitating membrane, which probably covers the cornea most of the time during flight and prevents drying by the rapid air stream to which the eye is exposed.

Air- and Water-Vision. Animals which live in both air and water must have an exceptional range of accommodation in order to see well in both media. A fish, when placed in air, is automatically subject to myopia, and any air-living vertebrate, when under water, to hypermetropia. Anableps, the so-called "four-eyed fish," has two pupils for each eye, and two retinas, one 
for use in air and one for water. The curvatures of the respective lens and corneal surfaces are such that proper focusing occurs.

Spectacles. Whenever an eye is free to rotate under a fixed transparent covering through which the light passes, the corering is referred to as a spectacle or goggle. A spectacle may be simply the corneal surface which does not rotate with the ere (lampres, lungfishes, eels, etc.), or it may be a window in a mowable lower lid (some turtles and lizards), or a window in a structure formed by the permanent fusion of both lids (snakes, certain fish, and many lizards). The latter type of spectacle forms a permanent secondary protective covering which is separated from the cornea by a liquid. This type is one commonly seen in snakes and spectacled lizards. In these forms the outer surface is periodically replaced when the skin is shed.

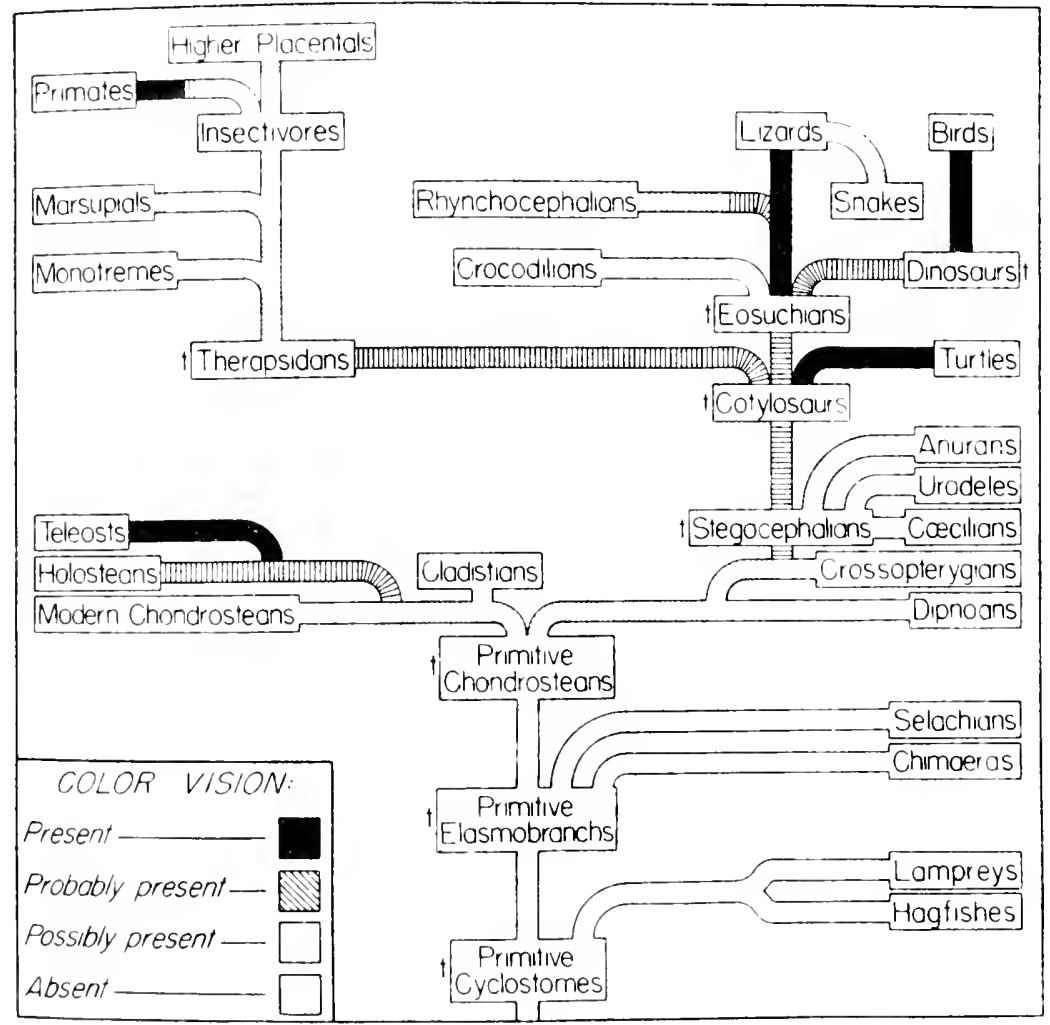

Fig. 11t. The probable phylogenetic tree of vertebrate color vision. From $\mathrm{M}^{\text {alls. }}{ }^{2{ }^{2}}$

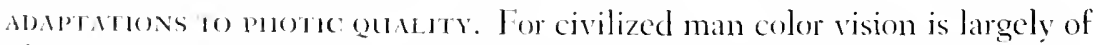
acsthetic value. Howerer, in lower animals it serves a very distinct purpose, in that it increases the visibility of objects and thereby enhanees the ability of the animal to obtain food or to escape enemies. The probable phylogenetic distribution of color vision according to $\mathrm{M}^{7}$ alls is shown in Figure 114 . This chart is based largely on well controlled behavion experiments by numerous authors prior to 19+2. Since that time it has been demonstrated by Granits with electricat methods (c.e. Fig. 1+2), that the neurones of the optic nerve 
are so connected that at least certain fish, tureles, anurans freg and high. er placentals cat have a phosiolegical basis for color ision. Thene data

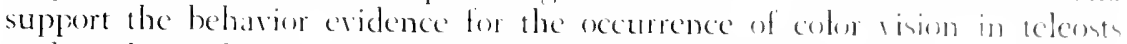
and turtles and extend its pessibiter to frogs and higher placental mammals. Granit found that the optic nerve fibers of the smate have only one type of

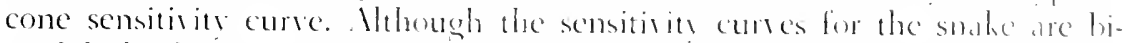
modal, the fact that they are all the sume precludes the existence of a phe in logical basis for contor visions. This is in agreement with the dath on hetrawion

\section{PIIYSIOLOGY OF PHOTORECEPTION}

Sensory endorgans which respond to radiation display great morplolog ical variation. Despite this morphological diversity there exists a common pattern (Fig. 115, . ) of a lightefocusing derice, a photosensitive membrane (retina), postretinal neurones which connect the receptor with the contrat nervous sistem, and the visual center or centers within the central nerrous system. In keeping with this common structural pattem cortain similarities in the basic mechanism of the responsive system are revealed in investigations of the function of light-sensitive end-organs. or phestoreceptors.
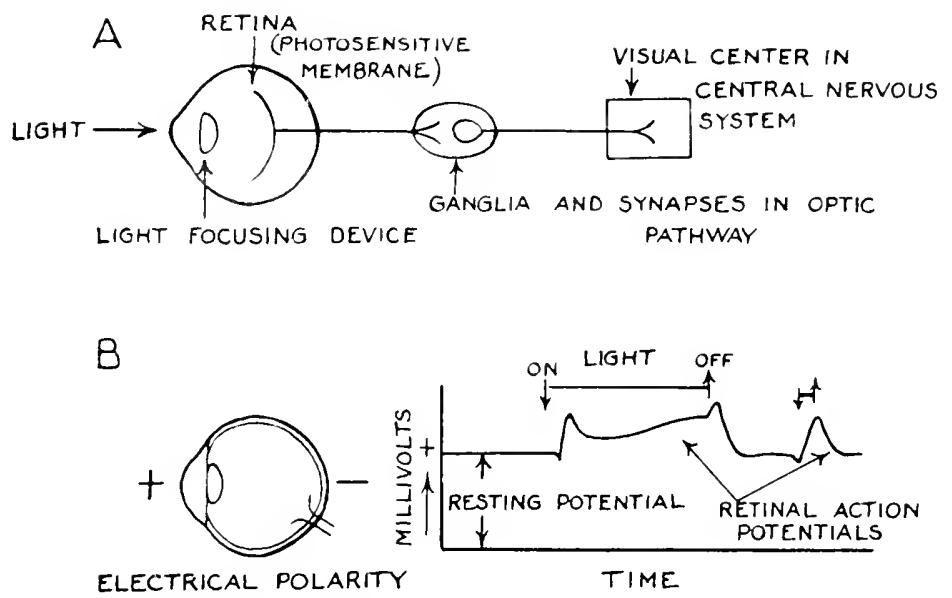

\section{ELECTRICAL POLARITY}

TIME

\section{OF VERTEBRATE EYE}

Fig. 115. Diagrams illustrating $(A)$ the elements in a visual pathway, and $(B)$ the electrical polarity and retinal action potentials of the vertebrate eye.

The phrsiological problems of prime interest center about two parts of the optic pathway (Fig. 115, A): (1) the photosensitive membrane and immediate connecting neurones, i.e., the peripheral structures: and (2) the central nervous system structures which reccive and integrate the activity originating in the retina.

The Peripheral Visual Mechanism: The Photochemical Nature of Photoreception. Today it is an accepted fact that the initial event in the process of vision is a photnchemical reaction. Surprisingly, this fact was only recently established. It was the evidences presented by Hecht, ${ }^{71}$. 75 , it. it. in stemming from his ingenious researches on the marine clam, Mya arenaria, which firmly established the photochemical nature of vision on a factual basis. 
THE RECIPROCITY LAW. The photic response of the clam, Mya arenaria, consists of the withdrawal of the extended siphon. This response is characterized by: (1) a threshold-a certain minimum intensity and duration of illumination is necessary to produce a response; and (2) a variable latent period-the time lapse between the end of the exposure and the beginning of the response varies in a definite manner with the conditions of illumination. The latent period and exposure period are often measured together and referred to as reaction time.

Hecht ${ }^{78}$ determined the intensity of illumination necessary just to produce a response for various durations of illumination in a maximally sensitive animal. The results indicate that the magnitude of the product of intensity and time necessary to elicit a response was a constant equal to 5.62 meter candle seconds. Expressed in mathematical notations,

$$
\mathrm{E}_{\mathrm{t}}=\mathrm{It}_{\mathrm{t}}
$$

where $\mathrm{E}_{\mathrm{t}}$ is the photochemical effect for a threshold response, $I$ is the intensity in meter candles, and $t$ is the time in seconds. This is a form of a fundamental principle in photochemistry, the Bunsen-Roscoe reciprocity law, which states that the photochemical effect is equal to the product of intensity and time. It should be emphasized that the above application of the reciprocity law to the photic response of $M y a$ is valid for only one level of response, namely, the threshold level within the intensity range used. The data do not indicate that a linear relation between photochemical effect and intensity-time product exists for all levels of stimulation.

TEMPERATURE COEFFICIENT OF PHOTOCHEMICAI REACTIONS. Hecht ${ }^{76}$ determined the radiant energy necessary just to produce a response in a maximally sensitive animal equilibrated to different environmental temperatures. Over a temperature range of 15 to $25^{\circ} \mathrm{C}$., the energy required is constant, and the temperature coefficients, $Q_{10}$, ranged from 1.04 to 1.07. Photochemical reactions are known to be thermostable, and these results agree with this well known fact, indicating that the initial and sontrolling reaction in photoreception is a photochemical reaction.

DARK AdAPTATION in the Clam. An increase in sensitivity to light after a sojourn in darkness is common to all photoreceptor systems. This phenomenon is called dark adaptation. Hecht ${ }^{74}$ determined the course of dark adaptation in the clam, Mya. The animal was illuminated with a constant intensity of light for a prolonged period. This illumination was terminated suddenly and the animal was in total darkness. Immediately, the animal was stimulated with a flash of light and the reaction time was obtained. Soon another flash of light (of the same intensity and duration as the previous flash) was admitted and the reaction time again was measured. This proceclure was repeated several times during a 50 minute period in the dark. The reaction time was found to decrease with increasing time in the dark (see Fig. 116, lower curve). Hecht argued that the reaction time was occupied by a reaction whose velocity varied with the magnitude of the photochemical effect; the greater the photochemical eflect, the faster the reaction or the shorter the reaction time. The decrease in reaction time during the stay in the dark implied an increasea photochemical effect elicited by the constant intensity-constant duration test flashes-which in turn implied 
that something which is sensitive to light must have accumulated during the stay in the dark. This sensitivity to light, argued Hecht, resides in a substance which we shall call $\mathrm{S}$.

The shape of the reaction time curve of the dark-adapting clam is significant, for it can be described adequately by the equation for a biomolecular reaction

$$
k=\frac{1}{\text { at }} \times \frac{x}{a-x}
$$

where $k$ is the velocity constant, $t$ is the time, $a$ is the initial concentration of some reactants, and $x$ is the concentration of a reaction product. Hecht concluded that a synthesis of photosensitive substance, S, from two precurs-

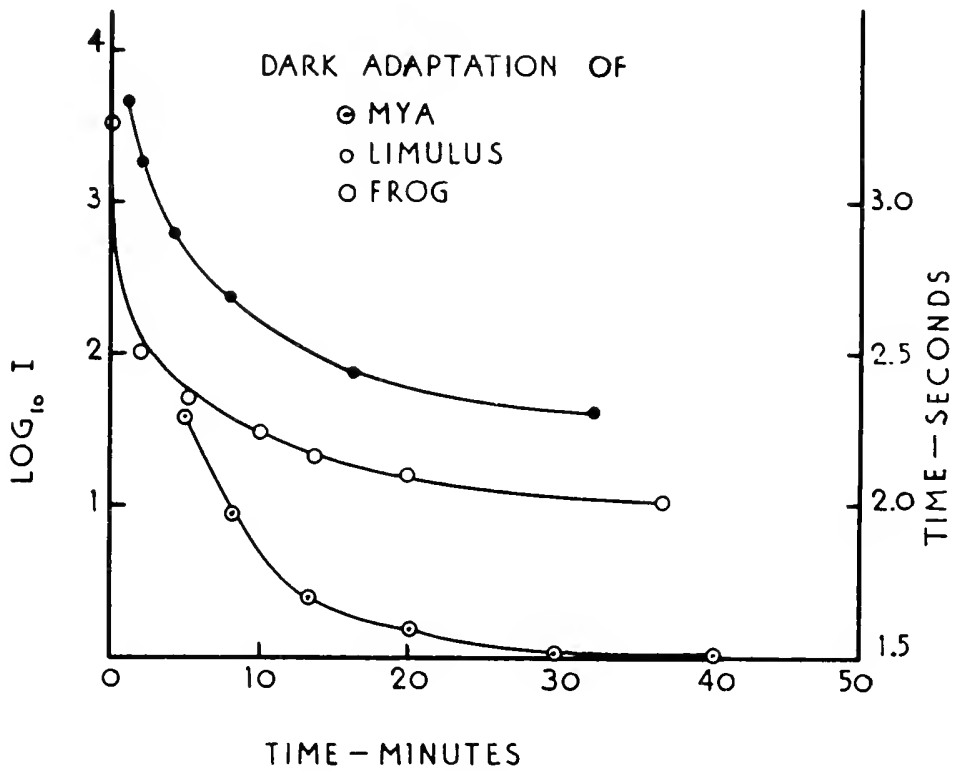

Fig. 116. Curves showing the recovery of sensitivity (dark adaptation) after exposure to white light in the clam, ${ }^{74}$ in Limulus, ${ }^{\text {,5 }}$ and in the frog. ${ }^{1 * 2}$ Ordinate for two upper curves is the logarithm of the threshold intensity; ordinate for lowest curve is the reaction time in seconds; abscissa is time in minutes.

ors ( $\mathrm{P}$ and $\mathrm{A}$ ) took place in the dark-adapting photoreceptor. These precursors, he suggested, originated from the photochemical decomposition of $\mathrm{S}$ during the period of illumination preceding the sojourn in the dark. This gave rise to the three component reversible schemes of Hecht

$$
\mathrm{S} \underset{\text { Dark }}{\stackrel{\text { Light }}{\longrightarrow}} \mathrm{P}+\mathrm{A}
$$

where $S$ is the photolabile pigment, $P$, a precursor, and $A$, an accessory.

RELATION BETWEEN ILLUMINATING INTENSITY AND PHOTOCHEMICAL EFFECT. Realizing that the reaction time was occupied by an event of great importance in the visual process, Hecht believed that here a reaction occurred 
whose velocity varied with the photochemical effect. A measure of the velocity of this reaction could be obtained by measuring the latent period and calculating its reciprocal. Hecht then determined the relation between the reciprocal of the latent period, which he used as a measure of the photochemical effect, and the illuminating intensity, using a constant exposure period. The resulting data, when graphed, were adequately described by a straight line with a slope of 2.2. Hecht concluded that the relation could be expressed by the formulation

$$
\mathrm{E}=\ln \mathrm{I}
$$

where $E$ is the photochemical effect, and $\ln I$ is the natural logarithm of the intensity. This relation is a form of a second fundamental principle in photochemistry expressed in Lambert's and in Beer's law.

REACTIONS FOLLOWING THE INITIAL PHOTOCHEMICAL EVENT. The endresult of the light-induced photochemical reaction is the initiation of activity in the fibers of the optic nerve or, in the absence of a nervous system, the initiation of some response. Hecht was the first to suggest a transitional mechanism between the photochemical event and the response of the organism. This suggestion was based on several characteristics of the latent period of the siphon-withdrawal response. Hecht observed that the reciprocal of the latent period (a measure of velocity) is directly proportional to the duration of exposure, on the basis of which he concluded that the velocity of the latent period reaction is directly proportional to the concentration of photo chemical decomposition products. To Hecht, this linear relation suggested a catalytic reaction, where the decomposition products were the catalyzers. This assumption was supported by: (1) measurement of temperature cuefficients between 2.5 and 3 and a $\mu$ value of 19,680 (calculated from the Arrhenius equation), which agrees well with values for catalytic reactions; (2) the consideration that the photochemical decomposition products $P+A$ are part of a reversible system whose condition of equilibrium must be sustained. This precludes the participation of these preducts directly in a second reaction. On these evidences and reasons Hecht postulated the following scheme

$$
\mathrm{L}\|\mathrm{P}+\mathrm{A}\| \rightarrow \mathrm{T}
$$

where $L$ is some substrate changed to an agent $T$, the reaction being catalyad by the photrehemical decomposition products $P+A$, the parallel vertical lines indicating catalysis. I lecht suggested that $T$ is the agent activating the nervous elements of the ere.

Participation of Carotenoids in Vision. Light incident upon a photoreceptor must be absorbed before it can produce a physical or chemical effect. In a resiew of the researches relating the role of carotenoids to visual func-

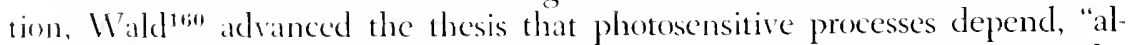
most universally, upon one distinctive and compact group of substances, the carotemoids." Carotenoids form a distinct group of rellow to red pigments. fat soluble and highly unsaturated, which can be identified by their absorption spectra. Carotenoids, either alone or as the prosthetic groups of comjugated proteins, function in absorption of radiant energy.

It is apparent that absorption characteristics of the pigment will be reflected in the response of the photoreceptor or of the organism. Thus, if the 
pigment of a photosensitive system is hight efficient in absorhing hlue light, then the same coler of light will be most effective in preducing a respronse in that system. For eximple, the phototrepic bending of the wat shoot, Arena, is most effectively stimulated by blue light of $+t 0$ p wave length. Other regions of the visible spectrum, adjusted so that the intensity is equal to that of the blue light, are less effective in producing bending. If these data are yuantitated and plotted as in ligure 117 fower curre), the result depicts the action spectrum of the phototropic bending. Fxtraten of the carotenoids of the oat coleoptile (Arena) viekds an absorption spectrum in dicated in the upper pert of Figure 117. The correspondence between the two curves is indeed striking.

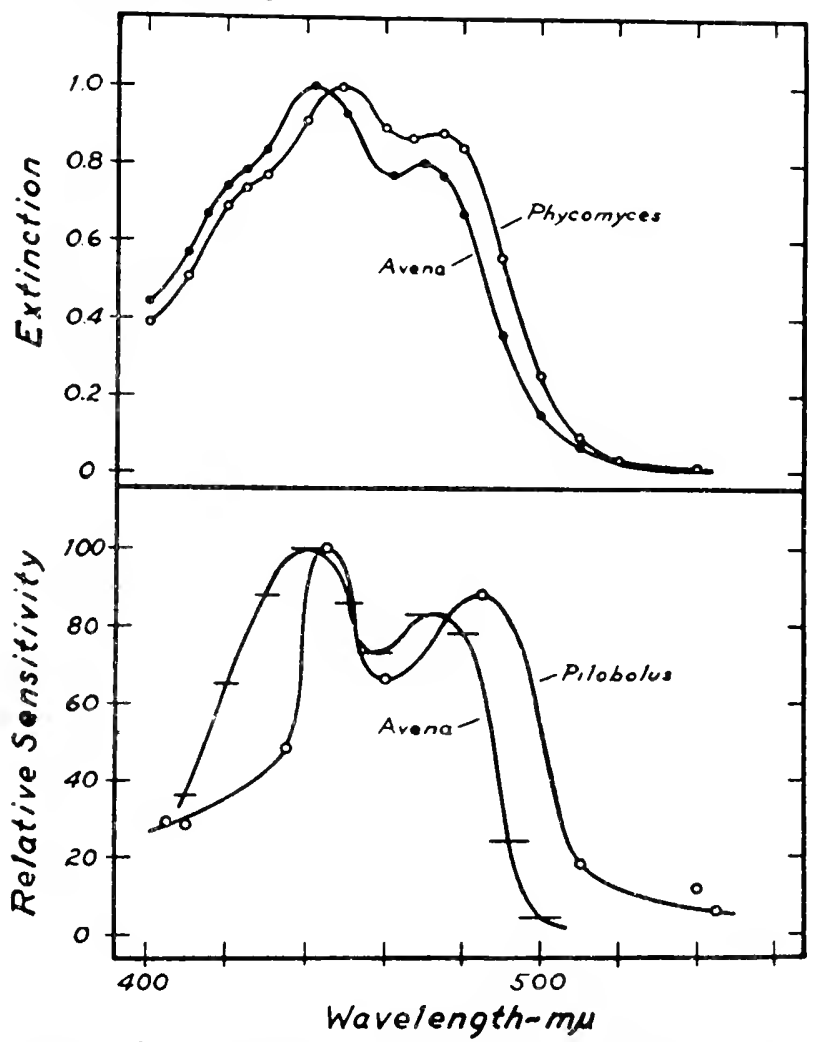

Fig. 117. Upper, the absorption spectra of the total extracted carotenoids of the oat coleoptile, Avena, and of spore-bearer cells of the mold, Phycomyces. Lower, the action spectra of photutropic bending in Aiena and spore bearers of Pilobolus, known to have carotenoid content similar to that of Phycomyces. From Wald. ${ }^{16}$

Similar corespondence exists in the spectral sensitivity of the photic response of certain species of green flagellates and the absorption spectra of an extracted carotenoid, which has been identitied as astixanthin.

For the remainder of the invertebrates there is no conclusive ervidence for the participation of carotenoids in photoreception, but several lines of evidence suggest such carotenoid function. The polyps of cortain colonial co elenterates bend toward light. This effect is sharply maximal in the blue at 
a wave length of $474 \mu$, in a spectrum uncorrected for energy. ${ }^{160}$ Hecht $^{81,83}$ measured the spectral sensitivity of the photic response of the clams $M y a$ and Pholas in relation to broad regions of the spectrum. He found a maximum for $M y a$ at $490 \mu$ and for Pholas at $555 \mu$, with perhaps another maximum in the ultraviolet. The spectral sensitivity of the squid retina suggests that the photosensitive pigment involved is similar to the vertebrate pigment, rhodopsin.

Bliss, ${ }^{12,13,14}$ isolated a pigment from squid retinae which resembles the vertebrate rhodopsin (Fig. 118). The absorption maximum is $495 \mu$, and its absorption spectrum agrees well with the absorption spectrum of visual purple and with the spectral sensitivity of the photoreceptor. There is, how-

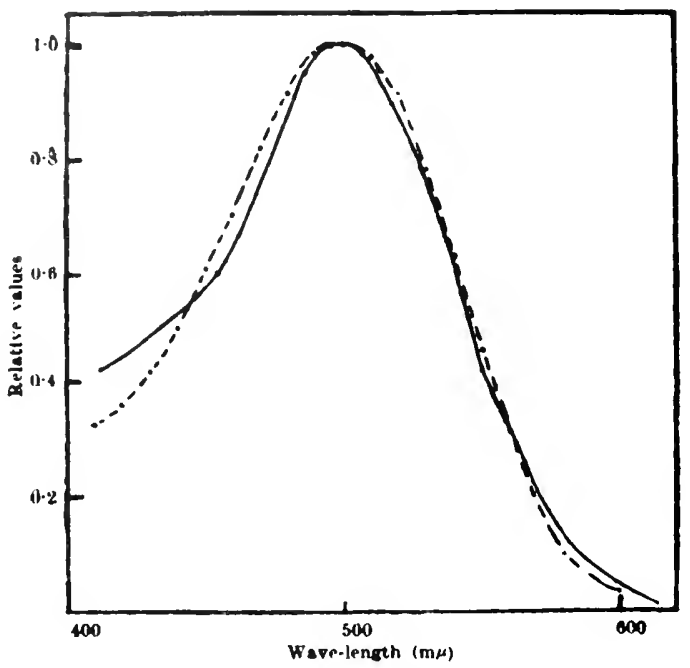

Fig. 118. Action spectrum of the dark-adapted human eye (after Ludvigh, ${ }^{11:}$ ) compared with the spectral absorption curve of visual purple. From Granit.

ever, one difficulty in assigning to this pigment a principal role in the visual process; the squid pigment is light stable. Bleaching in the presence of light occurs only when the extracted pigment is sensitized by a dilute denaturant, such as formalin. The bleaching product is identical with visual yellow of the rhodopsin cycle (page 4lI) and the carotenoid has been identified as retinene $_{\mathrm{I}}$. The spectral sensitivity of several arthropod eyes, ${ }^{49,}, 5 \pi, 45,109 \mathrm{de}$ termined by electrical (see page 422) and behavior methods, ${ }^{165}$ indicates absorption maxima in the blue portion of the visible spectrum, and a second maximum in the ultraviolet, wave length $385 \mu$, for Drosophila and the bee. ${ }^{10.11}$ Iligh concentrations of vitamin $A$ have been isolated from the eyes of several marine Crustacea, the green and fiddler crabs and lobster, and from the eyes of the fresh-water crustacean, Cambarus virilis. The eyes of the latter also contain retinene, in addition to astaxanthin.

In the vertebrate eve the role of carotenoids in photoreception has been clearly set forth. The eyes of marine fishes, frogs, turtles, birds, and mammals contain a rose-colored photolabile pigment associated with rod function in the duplex retina. This pigment has in the past been called visual purple. 
Wald ${ }^{160}$ has revived the name rhodopsin, which will be used because it serves to differentiate this pigment from other photolabile pigments associated with rod vision in fresh-water fishes and tadpoles (see page 412).

Rhodopsin is a conjugated protein. It possesses a characteristic color and other properties by virtue of its carotenoid prosthetic group. Extraction (in the dark) of dark-adapted retinae, rich in rhodopsin, produces a low yield of carotenoid. Rhodopsin exposed to light bleaches rapidly and gives rise to orange or yellow photoproducts. Extraction of retinae shortly after subjection to high illumination yields a stable yellow carotenoid identified as retin. ene $_{1}$, having a characteristic absorption spectrum. Extraction of retinae a long time after onset of illumination results in a high yield of a carotenoid, identified as vitamin $A_{1}$, which also has a characteristic absorption spectrum. These results indicate that retinene ${ }_{1}$ and vitamin $A_{1}$ enter into the photoreception process. Broda and Goodeve ${ }^{16}$ demonstrated that light incident on a solution of rhodopsin in 75 per cent glycerin and at $-73^{\circ} \mathrm{C}$. results in the appearance of an orange decomposition product. At higher temperatures this orange product changes rapidly into the familiar visual yellow. ${ }^{13}, 14$ This material has been called transient orange and is believed to be the immediate result of the bleaching of rhodopsin. At normal temperatures this orange product has only a fleeting existence, which accounts for the infrequency of its discovery. The incorporation of transient orange into the photochemical cycle gives the scheme of Figure 119.

Rhodopsin, extracted in aqueous solution, has an absorption spectrum with a maximum of $500 \mu$. This absorption spectrum agrees well with the

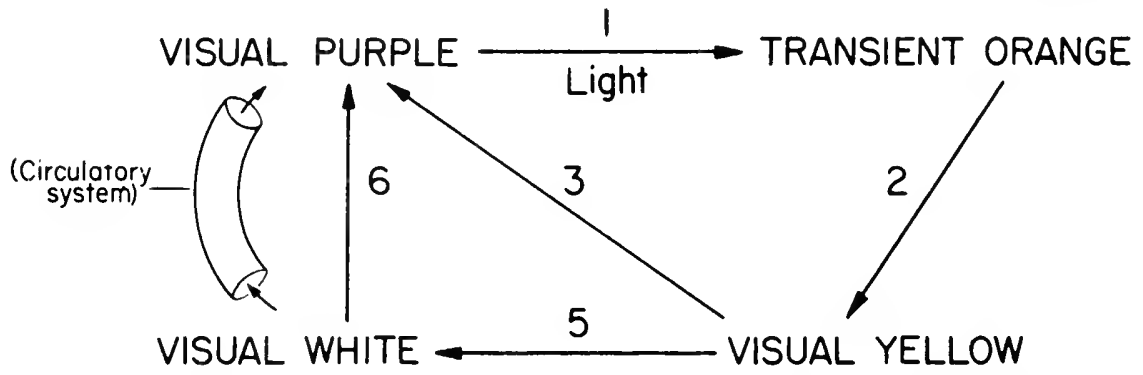

Fig. 119. The photochemical cycle including transient orange and illustrating the relationships of the various reactions. From Jahn."

spectral sensitivity curve of human rod vision (Fig. 118) and with the spectral sensitivity curves of other animals.

A photosensitive pigment associated with cone vision has been successfully extracted from chicken retinae by Wald. ${ }^{159,162}$ This pigment is sensitive to deep red light, and its absorption characteristics are similar to the spectral sensitivity of cone vision in chickens. ${ }^{160}$ Since this pigment is violet in color, Wald suggested the name iodopsin. Although the chemical nature of iodopsin is yet unknown, the similar spectral properties of rhodopsin and of iodopsin imply a chemical similarity.

Despite incompleteness of the data, the indications are that carotenoids are perhaps universally distributed among light-sensitive organisms, and that they are exclusively found in the photoreceptor or photosensitive areas 
or are found there in greater concentration than elsewhere. In those cases in which the action spectrum of a photoreceptive process and the spectral absorption data of carotenoid or conjugated protein pigments are available, the precise correlation between them predicates the conclusion that these pigments are active in absorbing the radiant energy which subsequently initiates other events in the optic pathway.

The Chemical Evolution of Photosensitive Pigments. One of the most interesting stories to emerge from the realm of retinal physiology is that of the phylogenetic origin of two photosensitive pigments, rhodopsin and porphyropsin. The following discussion is adapted largely from Wald, ${ }^{160}$ and earlier publications.

In the years $1879-1880$ Kühne, ${ }^{11 k}$ one of the classic investigators of photochemical systems, observed that the retinae of frogs and higher vertebrates were rose-colored, whereas those of fresh-water fishes were distinctly purple in color. Subsequently, Köttgen and Abelsdorff ${ }^{155}$ demonstrated that solutions of photopigments from eight species of fresh-water fish possessed spect-

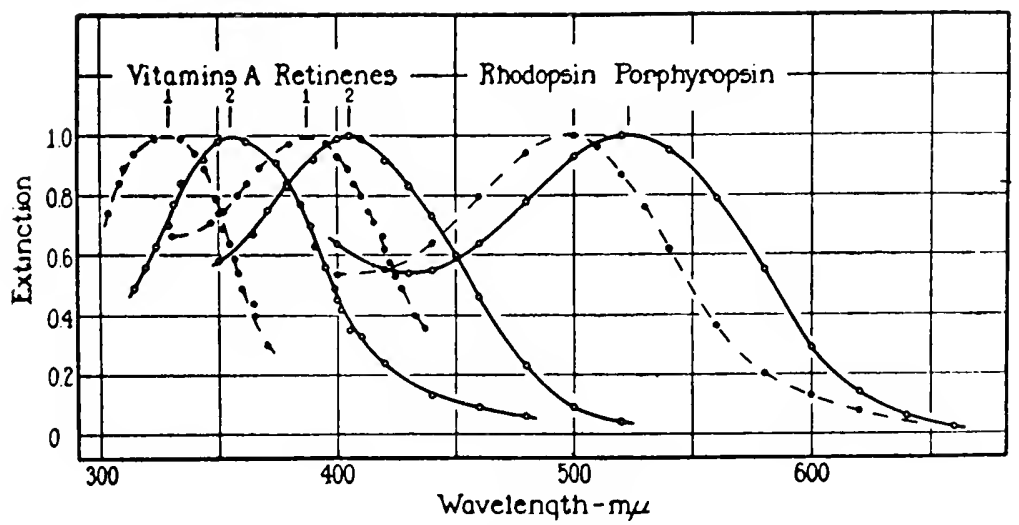

Fig. 120. Absorption spectra of the rhodopsin and porphyropsin systems obtained from crude extracts of retinae of the marine scup (broken lines), and the fresh-water calico bass (solid lines). All maxima have been brought to the same height to facilitate comparison. From $W^{7}$ ald. ${ }^{180}$

ral absorption properties different from the spectral properties of other vertebrate pigments. Many years later Wald ${ }^{160}$ found that the spectral properties of photopigments extracted from retinae of marine fishes resembled those of rhodopsin. The spectral properties of pigments from the eyes of fresh-water and marine fishes were dissimilar. Those of the latter were identical with the spectral properties of rhodopsin; those of the former were not. The pigment from the eyes of fresh-water fishes was called porphyropsin. Figure 120 indicates that the rhodopsin and porphyropsin systems are parallel systems: rhodepsin decomposes into two carotenoids, retinene ${ }_{1}$, vitamin $A_{1}$, and a protein; porphyropsin decomposes into retinene.., ritamin $A_{2,}$ and a protein.

This clear-cut distinction between photopigments of fresh-water and marine lishes (stemohaline) naturally led to the investigation of photopigments in fishes that are transitional between fresh-water and marine environments, or curyhaline fishes. Examination of the salmonids, which spawn in fresh 
water but spend the greatest part of their adult life in the ocean (i.e., anadromous fishes), revealed that they possess both rhodopsin and porphropsin, the latter predominating. This predominance of porphyropsin is further exemplified by the brook trout, which can enter the ocean but rarely does so, and the anadromous white perch, which has only the porphropsin sistem (Figure 121). Examination of the fresh-water eel, which spawns in the ocean but spends the greatest part of its adult life in fresh water (i.e., a catadromous fish), revealed that, although it possesses both pigments, rhodopsin dominates (Fig. 121). The killifish, which spends its entire life in brackish water, is intermediate between the eel and the brook trout. The alewife, an anadromous fish, spends almost all of its life in the sea and yet has only porphyropsin in its retina. In general, it may be said that euryhaline fishes possess predominately or exclusively that photosensitive pigment associated with their spawning environment, i.e., rhodopsin for marine and porphyropsin for fresh-water environment.

Since the fresh-water fishes are believed to have a major place in vertebrate evolution, it was of interest to examine the photopigments of other vertebrates in the evolutionary line. The cyclostomes are the only living primi-

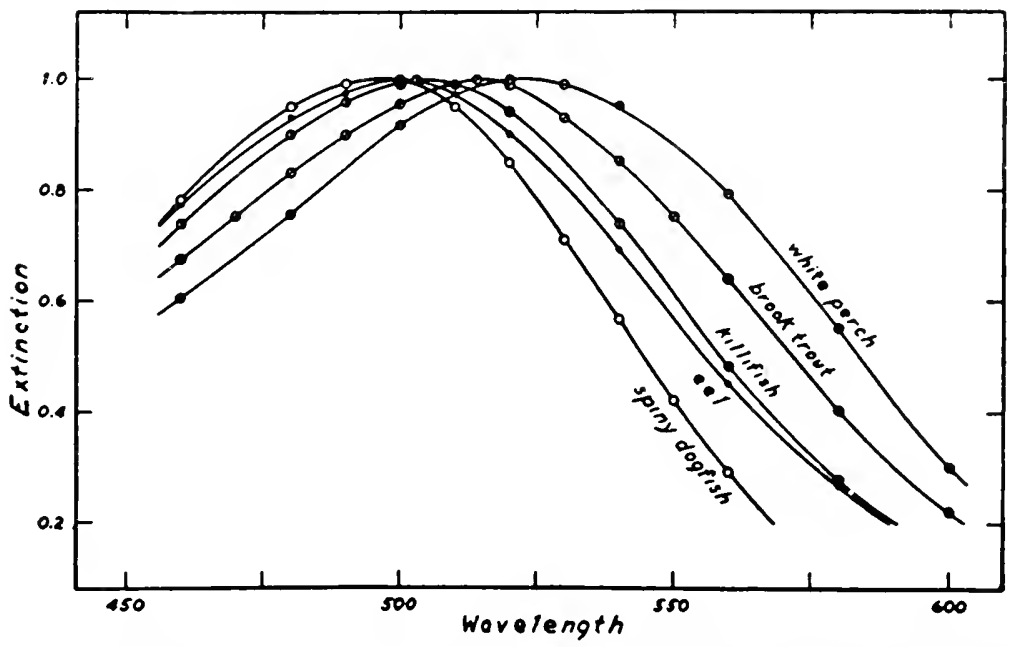

Fig. 121. Absorption spectra of retinal extracts from various fishes, showing the transition from the rhodopsin to the porphyropsin system. The dogfish possesses rhodopsin alone; the anadromous white perch possesses porphyropsin alone, and the other species are intermediate. From Wald. ${ }^{160}$

tive vertebrates which have eves sufficiently well developed to permit analysis of photopigments. The sea lamprey, Petromyzon marinus, an anadromous lamprey, possesses a great preponderance of porphyropsin. Retinas of the lungfish, Protopterus, have been found to contain vitamin $A_{2}$, indicating a porphyropsin system. Examination of the amphibia led to an interesting discovery. The urodele amphibian, Triturus viridescens, utilizes porphyropsin as its visual pigment, which is in accord with its fresh-water spawning habits and its probable ancestry. However, the anuran amphibian, the common frog, uses rhodopsin exclusively as its rod pigment. Yet the frog also 
spawns in fresh water. This dilemma was solved upon examination of tadpoles, just entering metamorphosis (small hind legs). These tadpoles exhibited two pigments, with porphyropsin predominating. Tadpoles further along in metamorphosis (well developed front and hind legs and mouth formation beginning) exhibited equal amounts of vitamins $A_{1}$ and $A_{2}$, and presumably equal concentrations of rhodopsin and porphyropsin. Newly emerged frogs exhibited a preponderance of rhodopsin. Migration from fresh water to land has had the same effect on the photosensitive pigment as migration from fresh water to sea water.

Although the data bearing on the evolution of these photopigments are vet incomplete, the information at hand indicates that the utilization of vitamin $A_{2}$, in a conjugated photosensitive pigment began early in the vertebrate line. Migration from the fresh-ivater environment into sea water and onto land has promoted the utilization of vitamin $A_{1}$.

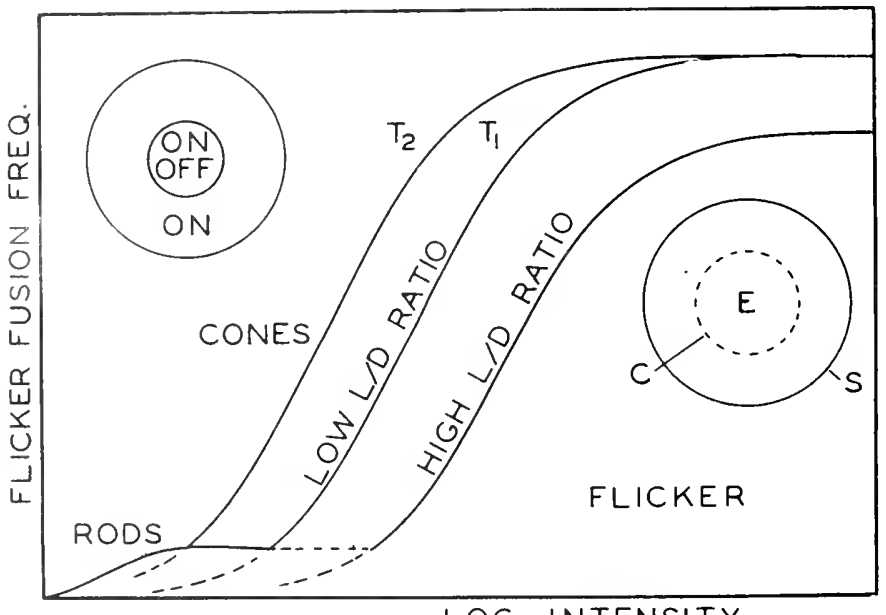

Fig. 122. Diagram showing rod and cone curves for flicker fusion frequency plotted against $\log$ intensity, and the changes produced in the cone curve by changes in temperature $\left(T_{2}\right.$ is higher than $T_{1}$ ), and by changes in the ratio of the light to dark (L/D) periods, during a Hicker cycle. The inserts indicate the type of field (left) and apparatus (right) used in obtaining flicker fusion data. From Jahn. ${ }^{100}$

Measurement of Visual Functions and Their Photochemical Bases. It is possible to design many types of experiments which test the functioning of the visual system of an animal. Such experiments may test numerous items, among which are: (1) the ability of an animal to determine whether or not a light is flickering; (2) the ability of an animal to distinguish varying degrees of brightness; (3) the increase in the ability to deteet light (i.e., sensitivity of the eye) during a prolonged period of darkness; (4) the ability of an animal to detect small objects; (5) the decrease of sensitivity during cither brief or prolonged illumination.

The general types of experiment which are used to measure these abilities are described below. 
FLICKER FUSION FREQUENCY. The flicker fusion frequency is the frepuency at which a flickering light appears to be continuous. This frequency varies with the intensity of the light, as shown in the curves of Figure 122. In c $\mathrm{x}$ periments on man, the subject is usually confronted with a uniformly and continuously illuminated field, and in the center of this field is an area which flickers (as shown in the upper left insert of Fig. 122) with the same average intensity as the surrounding field. The lower right insert shows the arrangement used for animals. The animal is placed at $E, C$ is a rotating glass cylinder with longitudinal opaque black stripes, and $S$ is a circular source of light. The animal is free to look along any radius, and as the cylinder rotates the black stripes give the appearance of Hicker. Many animals (turtles, frogs, fish, isopods, insects) react to the light as long as it appears to flicker and cease to react as soon as it appears to be continuous. If the light to dark ratio of the flicker cycle is altered the curve is shifted as shown (Fig. 122). If the temperature of a cold-blooded animal is raised (c.g., from $T_{1}$ to $T_{2}$ ), the curve is shifted to the left (Fig. 122).

BRIGHTNESS Discrimination. By brightness discrimination we mean the ability to detect a change in brightness. The observer is confronted with a

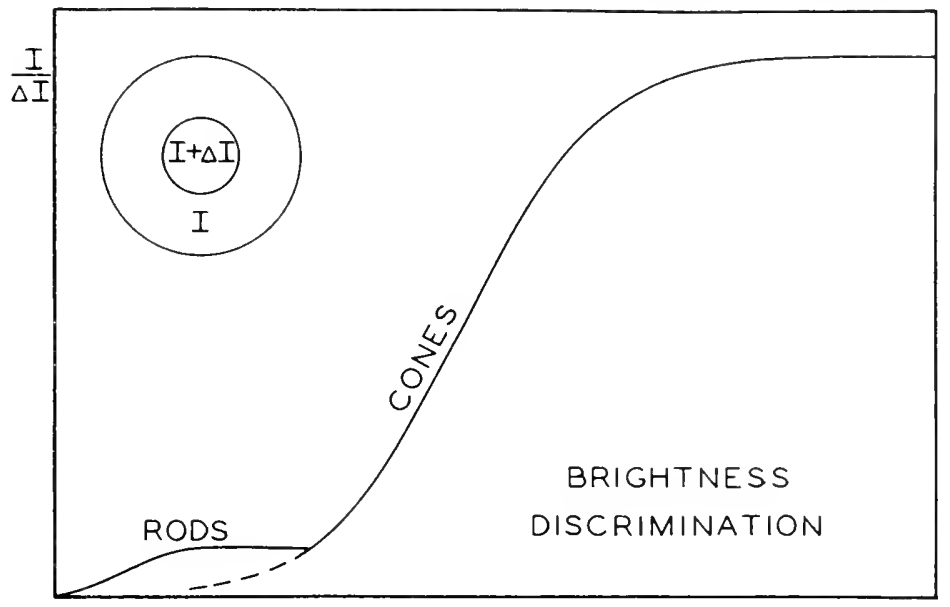

LOG INTENSITY (I)

Fig. 123. Diagram showing the rod and cone curves for brightness discrimination plotted against $\log$ intensity. The insert (upper left) indicates the type of visual field used in these experiments. From Jahn. ${ }^{100}$

uniformly illuminated field of intensity $I$. Then an additional intensity $(\Delta I)$ is briefly superimposed on part of this field, and the observer determines the value of $\Delta I$ which can just barely be detected. A plot of $I / \Delta I$ against $\log I$ is shown in the curve of Figure 123, and one form of the experimental field of view is shown in the insert.

THE KINETICS OF DARK ADAPTATION. Since Hecht measured the time course of the dark reaction in the clam, he and his students and numerous others have determined the time course of the dark reaction of photoreceptors in great numbers of organisms (see page 406, also Jahn"*). These studies have been confirmed by determination of the time course of the resynthesis of bleached rhodopsin in vitro. ${ }^{21}$ 
The several products resulting from the bleaching of rhodopsin, i.e., visual yellow and visual white, indicate that the kinetics of the production of rhodopsin is not as simple as was originally formulated by Hecht (page 408).

Instead of a single reaction, there are now known to be three reactions involved in the synthesis (Fig. 119). This complexity of the reaction is in evidence if a series of dark-adaptation curves obtained from one photoreceptor, as in Figure 124, are compared. The curve of recovery after adaptation to a low intensity of illumination, e.g., 4 millilamberts, is quite different from that of recovery after adaptation to high intensity of illumination, e.g., 4700 millilamberts. Recovery curves obtained after intermediate intensities of illumination form a graded series between the two extremes. Jahn ${ }^{99}$ demonstrated these differences by plotting the data as shown in Figure 124, rather than in the conventional log of threshold intensity-time plot used by most workers.

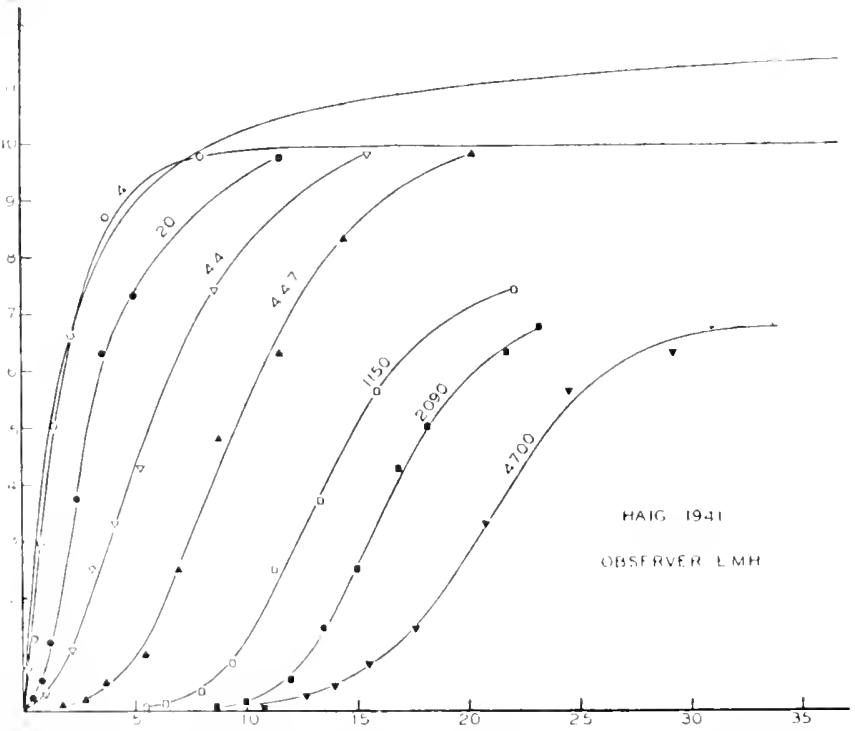

Fig. 124. Curves showing the progress and extent of dark adaptation after exposure to lights of different intensity. Data from a normal human subject. The ordinate represents the reciprocal of threshold intensity, the abscissa represents time (minutes) in the dark. The numbers on the curves indicate the intensity (millilamberts) of the adapting light. From Jahn. ${ }^{90}$

Inspection of this series of curves (Fig. 124) revealed that the data for the lowest adapting intensity could be fitted best by the equation for a monomolecular reaction ( $\mathrm{p} .407)$, whereas the curve for the highest adapting intensity could be fitted with the equation for a monomolecular autocatalytic equation. The intermediate curves could not be fitted by either of these two equations. Jahn ${ }^{99}$ proposed an equation relating the increase of rhodopsin $(Z)$ with respect to time in the dark $(t)$,

$$
Z=1-\frac{1}{t+a}-\frac{t+a}{t+a+l+K e t}
$$

where a denotes the reciprocal of the initial concentration of visual yellow 
(retinene, and protein) at the beginning of dark adaptation. This equation describes quite well all of the recovery curves of the family obtained after different amounts of light adaptation. Dataiz obtained from the photoreceptor of Limulus (p. 426) indicates a family of recovery curves after different degrees of light adaptation rather than the single curve obtained earlier and described (p. 407).

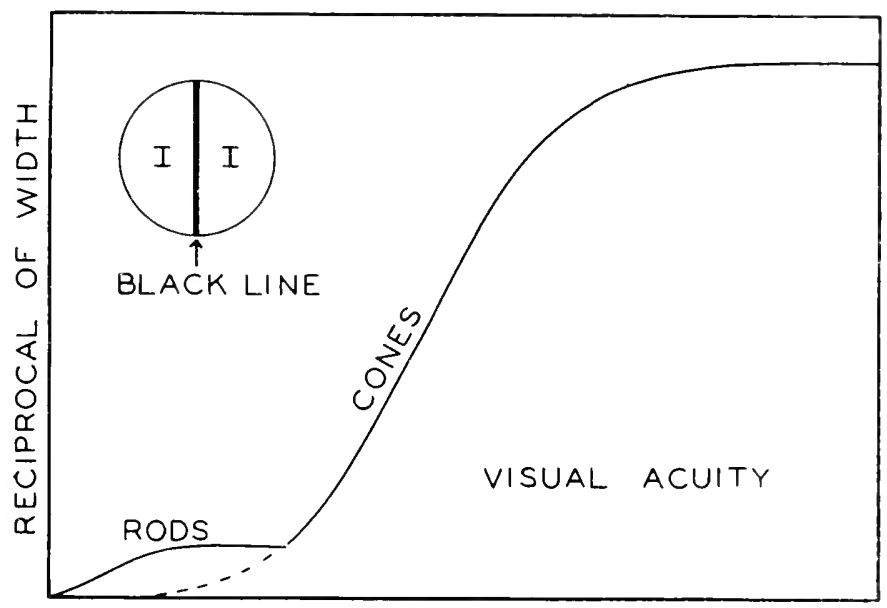

LOG INTENSITY (I)

Fig. 125. Diagram showing the rod and cone curves for visual acuity plotted against $\log$ intensity. The insert (upper left) indicates the type of visual field used in these experiments. From Jahn. ${ }^{100}$

'visual acuity. By visual acuity we mean the ability to see small objects. One simple form of experiment is shown in the insert of Figure 125. The observer is confronted with a field of intensity $I$ and a black line of variable width, and he is allowed to determine the width of the line which can just barely be seen at various intensities. Results of a typical experiment are shown in the curve of Figure 125, where the reciprocal of the width is plotted against intensity. Because of diffraction the image on the retina for the narrowest line that can be seen is not in black and white, but in gray and white. Therefore, this experiment is really one in brightness discrimina tion, and any explanation for brightness discrimination should also explain visual acuity.

Photochemical Explanation. The data for the above types of experiments can be predicted from a knowledge of the photochemical crcle. The theors and equations for that purpose outlined below are those of Jahn $96,97,99$

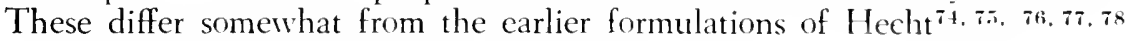
and from those of other investigators. ${ }^{1 \times, 19,130}$

The fundamental assumption is that the threshold (or any other given level) of sensitivity is equivalent to some fixed value for the rate of the $L T$ reaction, i.e., for $d T / d t$. The factors which affect this rate are the concentration of the substrate $L$, the concentration of the enzrme $E$, and a velocity constant which varies with temperature. In the case of flicker it is assumed that the value of the maximal critical frequency is determined not 
by the photochemical cycle but by some other process which is unaffected by temperature. On this basis the phenomena of flicker fusion, brightness discrimination, and visual acuity may be described as functions of intensity. The same assumptions have also been applied to dark adaptation and, when combined with equations for the kinetics of regeneration of visual purple, offer a satisfactory cxplanation of the existing data on dark adaptation. The statement is not meant to imply that factors other than the chemical cycle are not involved or are not important.

The equation for flicker fusion frequency is

$$
F=\frac{k_{4} p}{C^{1}}\left[k_{1} I(a-x)\right]^{a}\left(F_{m a x}-F\right)^{r}
$$

where $F$ is the flicker fusion frequency, $x$ the amount of $\mathrm{P}$ or $\mathrm{A},(a-x)$ the amount of $S, I$ intensity, $P$ the fraction of the flicker cycle during which the light is on, $k_{4}$ is the velocity constant of the LT reaction, $q$ pertains to the mechanism whereby the photoproduct $E$ is utilized by the sense cells, and $r$ denotes a mathematical function of $F .^{96}$

The equation for brightness discrimination and visual acuity is........

$$
\frac{\Delta \mathrm{I}}{\mathrm{I}}=a=\frac{\mathrm{C}_{1}\left(\mathrm{C} / \mathrm{k}_{4} \mathrm{l}\right)^{2} / 4}{\mathrm{k}_{3} \mathrm{x}^{2} \mathrm{t}}-\frac{\mathrm{l}}{\mathrm{k}_{2} \mathrm{t}}
$$

where $\alpha$ denotes visual acuity, $l$ denotes the concentration of $\mathrm{L}, K_{2}$ the velocity constant for $\mathrm{E} \rightarrow \mathrm{P}+\mathrm{A}$ reaction, $k_{3}$ the velocity constant of the $\mathrm{P}+\mathrm{A} \rightarrow \mathrm{S}$ reaction, and the duration of the flash of light. ${ }^{97}$

The validity of these equations for flicker fusion is shown in Figure 126. In this figure the theoretical curves for flicker fusion for the turtle Pseudemys for two temperatures and for man under conditions of rod (on left) and of cone (on right) vision are fitted to experimental data. The data are well fitted, and the direction of the shift in the curve caused by the temperature change is correctly predicted.

Nonphotochemical Theories of Visual Phenomena. In the determination of the sensitivity of the eye to a small spot of light we considered a catenary series of events. Let us call these events the series $A$ to $Z$. If the stimulus is a large spot of light or a geometrically complex light source, then there are many parallel series of such events and a number of $Z$ processes in the brain. As such, the Z's are subject to statistical variation. This would also be true il a single chain ( $A$ to $Z$ ) were repeated time after time; the sensation (event 7) would not alway's, at least under experimental conditions, involve exactly the same neurones in exactly the same way. This would be especially true near the threshold intensity.

I lowerer, the existence of a statistical variation in erent $Z$ has led to an alternative and nonphotochemical explanation of the above visual functions. This alternative theory is based entircly on the existence of statistical variation, and its development was stimulated by the inadequacies of the early photochemical theory.

Acording th the statistical theory proposed by Crozier and his coworkers, $28,31,32,33,34,3,34,37$ the sigmoid curves for flicker fusion, brightness discrimination, and visual acuity as functions of intensity are probability in- 
tegrals (i.e., ogive curves) in $\log I$. The fundamental assumption is that the controlling mechanism in these phenomena is the statistical distribution of thresholds (in $\log I$ ). At low intensities only the most sensitive elements (rods or cones and associated neurones) respond; at high intensities all of them respond. Dark adaptation curves as a function of time are explained as the progressive increase of sensitivity of individual units and may be fitted

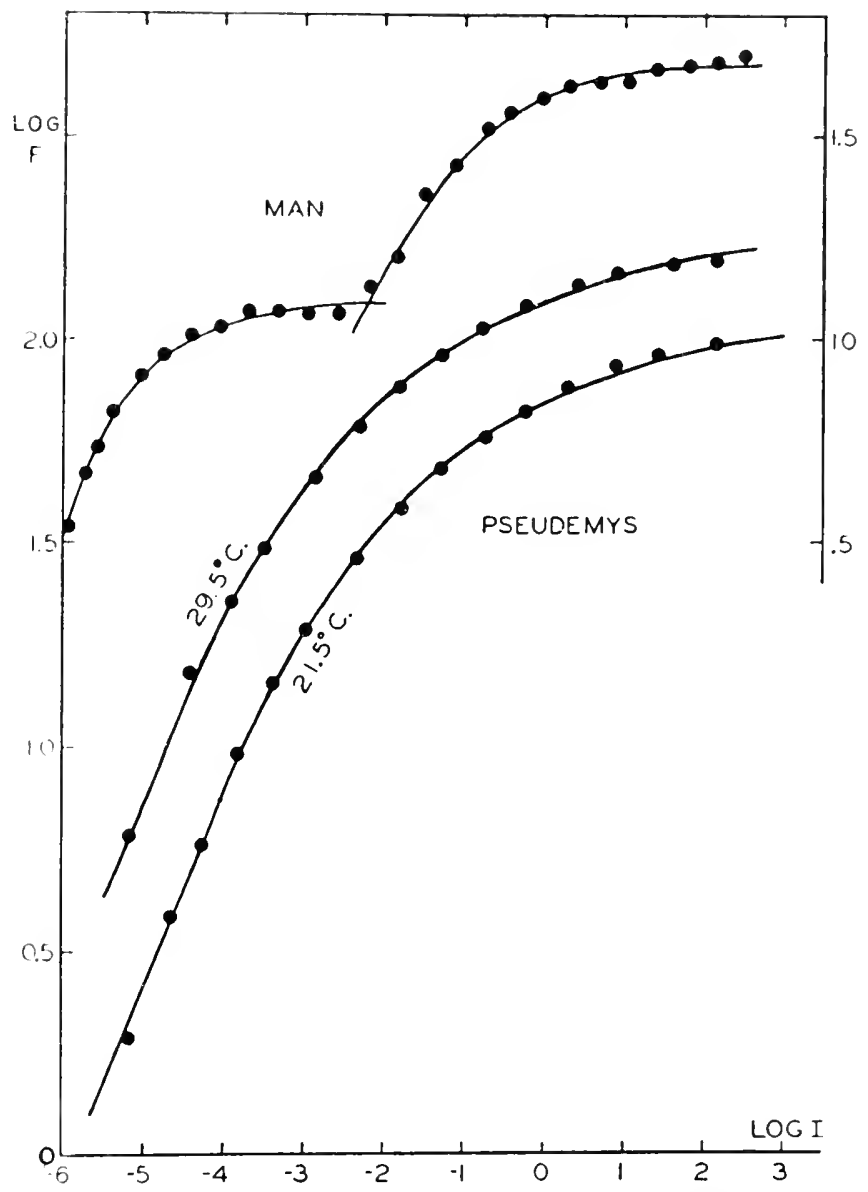

Fig. 126. Data showing the relation of the flicker fusion frequency to the log of the intensity for man (uppermost curve) and the turtle (lower curves). Note the shift to the right in the curve for Psendemys produced by a lower temperature. The curves were calculated from equations. From Jahn. ${ }^{58}$

if the sensitivity is treated as a probability integral in $\log$ of time in the dark. On the basis of this theory the assumed distribution of thresholds covers at least four and sometimes as many as eight $\log$ units. The thresholds are supposed to vary from element to element and from moment to moment. If one accepts these postulates, all of the data can be explained.

This theory, of course, is a source of frustration to investigators who wish to reveal the mechanism of either the sense cells or associated neuronal net- 
works by means of measurements of sensory phenomena. It is generally agreed that the photochemical cycle is responsible for sensation in that it is the mechanism by which the sense cell is activated. However, Crozier's theory holds that in explaining the shape of the curves for various visual phenomena only the statistical distribution of thresholds need be considered.

An additional theory is that of Householder and Landahl. ${ }^{92}$ In this theory the responsibility of determining the shape of curves for visual phenomena is entirely removed from the sense cell and is placed in the central ner. vous system (or possibly in the neurone layers of the retina). On the basis of assumed dynamics of synaptic transmission, Householder and Landahl have derived equations which describe the relationship between critical flicker, light to dark ratio, and intensity, between relative brightness, light to dark ratio, and frequency, and also between brightness discrimination and intensity. Provision is made in the equations for the excitatory and inhibitory processes at the synapses, and it is implied that different intensities of light cause excitation of completely different sets of neurones. As far as they go, the derived equations offer a reasonably good fit for the experimental data.

Evaluation of the Householder and Landahl interpretation in terms of other theories or of known facts is very difficult, principally because the equations have been derived on the basis of a few assumptions concerning synaptic transmission within the central nervous system, without reference to photochemistry or to statistical distribution of thresholds. The equations are applicable to all types of sensory discrimination, and at present there seems to be no way of proving or disproving them or even of estimating their potential value.

The Electrical Activity of Photoreceptors. Consideration of the function of vision reveals that the light, which is the agent activating the visual system, goes no further than the photosensitive membrane upon which it is incident. However, the experience of the light, or its sensation, takes place within the central nervous system. The evidences for the existence of a secondary reaction in the retina, i.e., the catalysis of a substrate by one or several photodecomposition products, into an activating agent $T$, have been presented (p. +08). However, this throws no light on the nature of the agent $T$ or the manner in which it initiates nervous activity in the optic pathway.

The data concerning the electrical activity of photoreceptors reveal certain relationships that indicate its possible importance as a link in the chain of events that constitute the peripheral visual mechanism.

The Nature of Retinal Electrical Activity. The fact that electrical potentials may be measured from the front and back of the vertebrate cye was discovered by Du Bois Revmond in 1849, ${ }^{+1}$ and the fact that this voltage changes with the intensity of illumination was discovered by Holmgren in 1865." Since these pioneer observations were made, it has been established beyond doubt that whenever a photoreceptor is illuminated (assuming a viable photoreceptor and illumination above a minimal intensity), there occurs a fluctuation in the steady potential difference which exists across the eye. This steady potential difference is so oriented (Fig. 115, B, left) that the apical ends of the photosensitive elements (rods and/or cones in vertebrates) are negative to the basal ends. The change in this potential wrought 
by illumination, called the retinal action potential, is such as to cause an in crease in the electrical negativity of the apical ends of the photosensitive elements (Fig. 15, B, right). The magnitude of the retinal action potential is
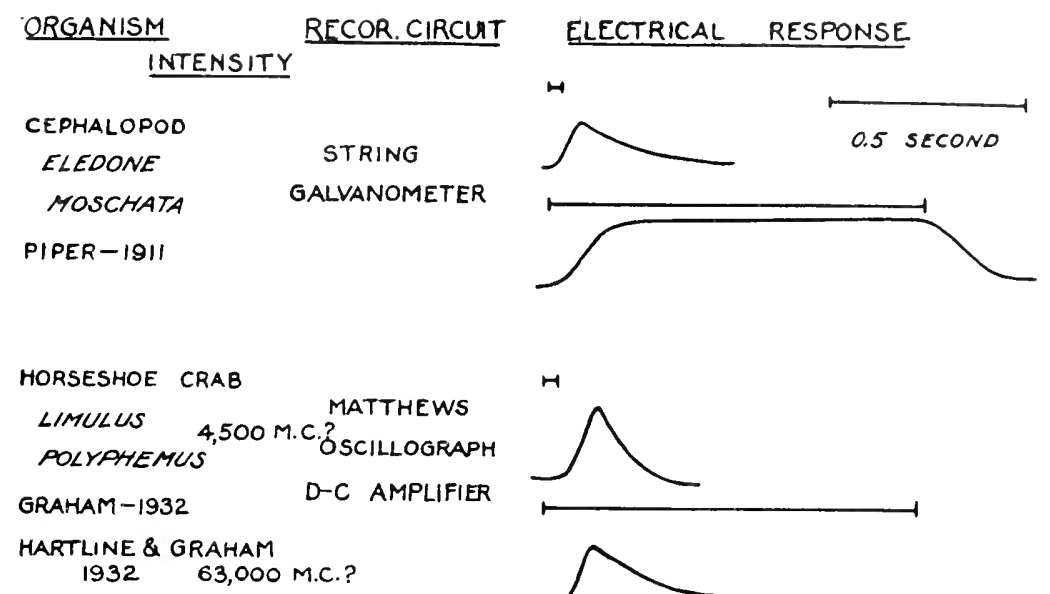

4
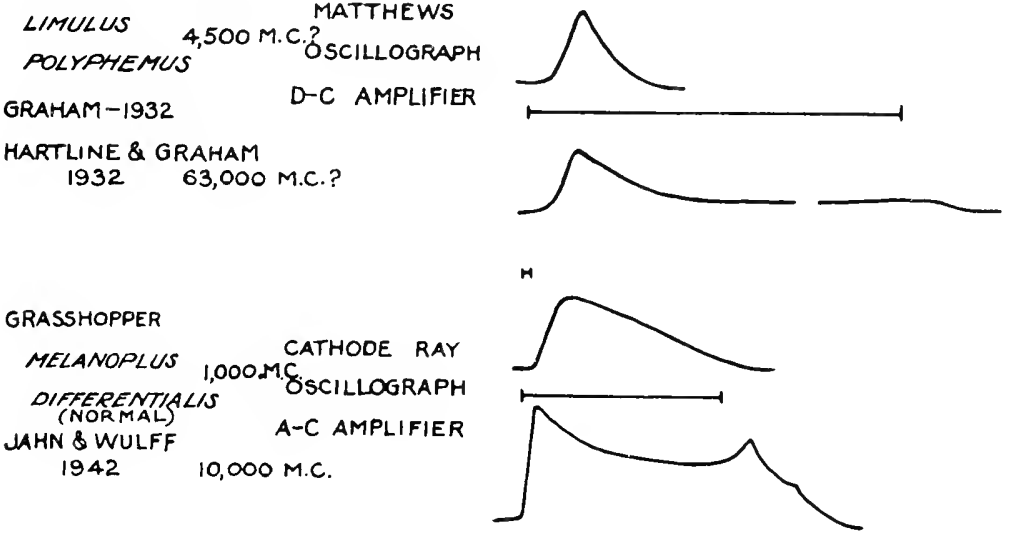

FROG

PIPER - I911
STRING
NIKIFOROWSKI GALVANOMETER
CITED BY KOHLRAUSCH
I931

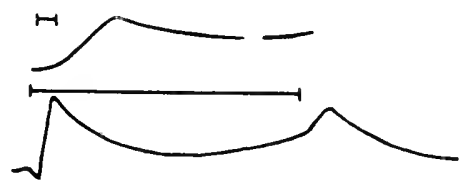

MAN \& WHTEE RAT INK OSCILLOGRAPH ADRIAN-1945 BLUE LIGHT GRAHAM \& RIGGS STRING

\section{GALVANOMETER 29,000 M.C.}

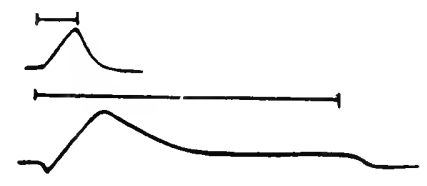

Fig. 127. Retinal action potentials of different animals obtained under somewhat different conditions. Upward deflection in all cases indicates negativity of the electrical lead near the free end of the retinal cells. The magnitude of the responses is not comparable because of differences of amplification.

greatest shortly after the onset of illumination (the b-wave). As illumination continues, the electrical response may decline rapidly to a minimum which is maintained (Fig. 127, Limulus eye), or the electrical response may increase in magnitude again (the c-wave) after a slight decrease (Fig. 127, 
frog's eye, and Fig. 115). In some photoreceptors the cessation of illumination is followed by a further increase in negativity, called the d-wave. In the electrical action potentials of most vertebrate photoreceptors, the b-wave is preceded by a brief low magnitude potential in the positive direction, the a-wave. Although this terminology has been applied to the retinal action potentials of all photoreceptors, a strict homology of the various waves has not been demonstrated.

For the purpose of measuring the action potential of the retina, electrodes may be connected to the front and back of the eye. By means of such connections, either on an isolated eye or on one in situ, the electrical changes are recorded by appropriate instruments. A record of the retinal action potential obtained in this manner is called an electroretinogram (ERG). The wave form of the electroretinogram has been studied as a function of intensity of stimulating light, of the degree of dark- or light-adaptation, of the duration of the flash of light, of the frequency of a flickering light, of temperature, and of the effect of various drugs. ${ }^{52,155}$ As a result of such analyses it is postulated that the ERG represents the algebraic sum of potentials from three sources, which are referred to as the three components, $P$ (meaning process) I, P II, and $P$ III. The exact site of origin of these electrical components has not been determined, but at least one of them exists in the sense cell. ${ }^{57}$ This has been well demonstrated for the sense cells of Limulus, ${ }^{64}$ the compound eye of the grasshopper, Trimerotropis, ${ }^{106}$ and for Dytiscuss, ${ }^{9}$ and it undoubtedly occurs in all photoreceptors.

The simplest electroretinograms obtained are from the eye of Limulus (Fig. 127), an eye lacking lateral connections between sensory neurones. The response to illumination is a simple negative wave, followed by a lower sustained potential as long as stimulation persists. The a-, b-, c-, and d-waves are conspiculously lacking, but the wave form is quite similar to that of $P$ II in the component analysis of the vertebrate retinal action potential. Measurements of retinal electrical activity have usually been made in terms of the magnitude of the b-wave. Measurements of $c$-wave magnitudes ${ }^{115}, 174$ seem to parallel the b-wave measurements, at least in some respects. The former are more difficult to obtain.

Electrical Measurement of Visual Functions. The relation between incident light and electrical response indicates the possible dependence of the retinal electrical event upon the known properties of the photochemical system.

ELECTRICAL MEASUREMENT OF SPECTRAL SENSITIVITY. The magnitude of the electrical action potentials of photoreceptors depends (within limits) rather directly on the intensity and on the duration of the light stimulus. This dependence of magnitude on intensity suggests the possibility of meas. uring the spectral sensitivity of this response, using relatively monochromatic light corrected for equal eners. Techniques for such measurement have been described. ${ }^{45,109}$ The ideal procedure is to select a series of bands throughout the visible spectrum (with a monochromator or with color filters) and adjust the intensity so that it is equal for all the wave length bands. (The bands should be as narrow as possible). The magnitude of the photoreceptor electrical response (b-wave) elicited by constant duration test flashes of the various spectral bands will vary. The resultant plot of magnitude against 
wave length yields a curve which describes the spectral sensitivity of the electrical response (Fig. 128). Numerous such measurements have been made. $50,51,95,134$

The spectral sensitivity curve of the electric response of the frog eve, using intensities below the cone threshold, yiclds a curve which is identical with the spectral absorption curve of rhodopsin, the rod pigment in the frog eye (Fig. 128). Despite the relative abundance of electrical spectral sensitivity measurements on diverse animals, no other direct comparisons with spectral absorption of photolabile pigments can be made because the latter have not been measured in vitro. The action spectrum of the squid eye ${ }^{134}$ resembles the absorption spectrum of rhodopsin, which agrees with the presence of a rhodopsin-like substance in these eyes. ${ }^{13,14}$

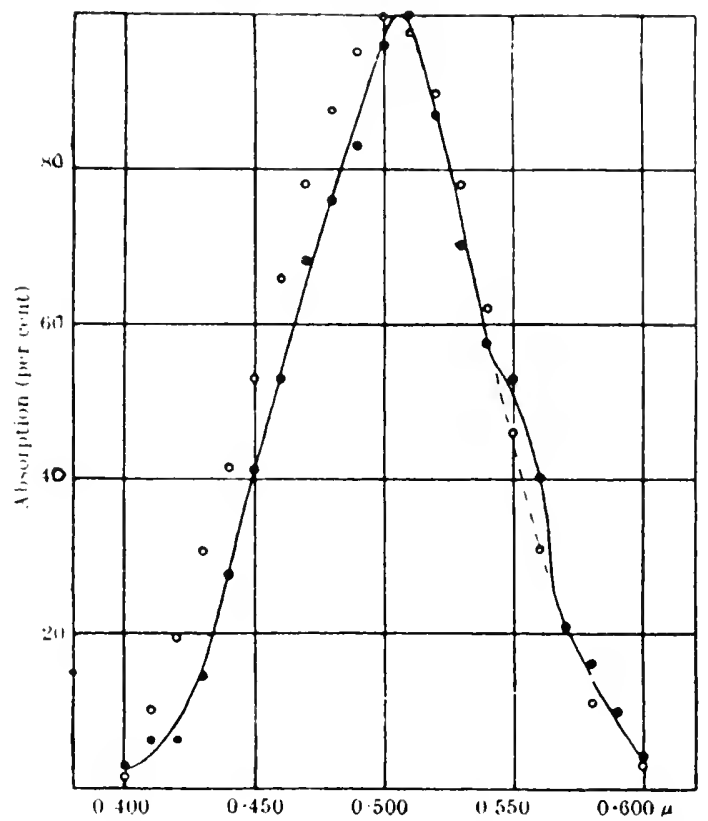

Fig. 128. Spectral absorption curve of visual purple (open circles), and the action spectrum of the dark-adapted frog eye as measured by the size of the b-wave of the retinal action potential (closed circles). From Granit. ${ }^{57}$

Spectral sensitivity curves of photoreceptors obtained by the measurement of different responses (e.g., clectrical and behavioral) are in general agreement. Thus the spectral sensitivity of insects determined by behavior agrees with the electrical measurements. In the human eye the scotopic and photopic (rod and cone) luminosity curves, obtained by verbal response, agree well with similar electrical determinations. ${ }^{57}$

TEMPERATURE EFFECT ON THE RETINAL ACTION POTENTIAL. A second point of comparison between the photochemical process and the electrical event in the retina concerns the effect of temperature on these reactions. It is well known that photochemical bleaching reactions are affected not at all or very little by temperature. It will be remembered that Hecht determined the effect of temperature on the photochemical process in the clam, obtaining 
values of $Q_{10}$ ranging from 1.04 to 1.07 over a temperature range of 15 to $25^{\circ} \mathrm{C}$.

The effect of temperature on the retinal response ${ }^{107}$ was measured in a

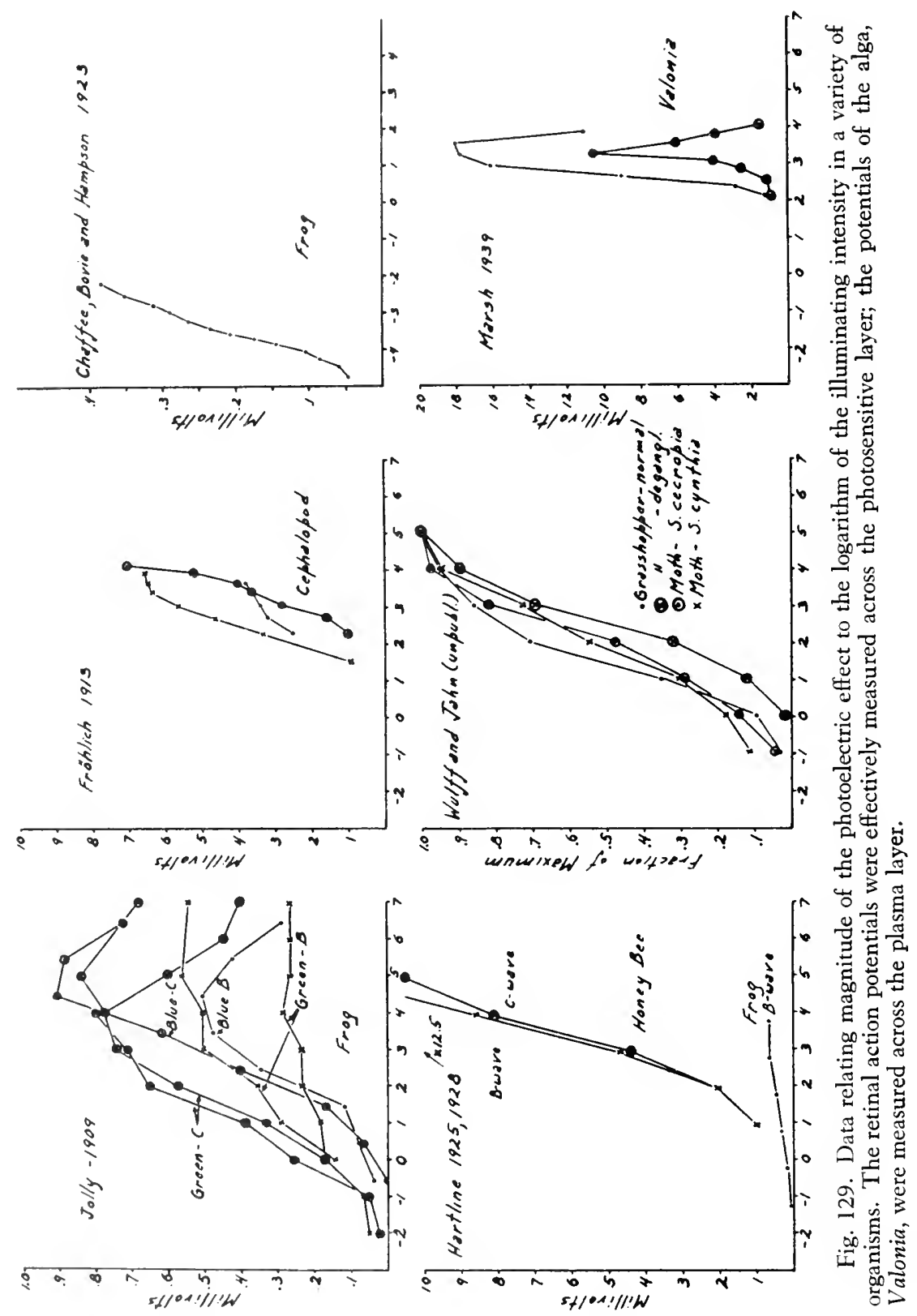

maximally darkadapted animal (the grasshopper). Constant energy test flashes were used and the resulting electrical response magnitude was related to temperature. In the temperature range of 15 to $25^{\circ} \mathrm{C}$., temperature coefficients were computed ranging from 1.10 to 1.38 . The temperature co- 
efficients obtained by Hecht and the above values are in fair agreement with the theoretical value of 1.00 .

RECIPROCITY LAW DETERMINED FROM ELECTRICAL MEASUREMENTS OF

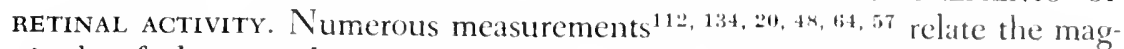
nitude of the retinal action potential to the stimulating intensity, employing a constant flash duration (Fig. 129). The data of I lartline, "it presented in Figure 130, are particularly instructive. He has measured the magnitude of the retinal action potential in relation to intensity of illumination, em. ploying different exposure periods.

These data reveal that over a wide range the intensity and duration of flash to produce a response of constant magnitude are inversely related, i.e., for longer durations the intensity needed is less, and vice versa. Computation of the intensity-time product necessary to elicit a response of constant magnitude is equal to a constant. Here, then, is a duplication of the applic-

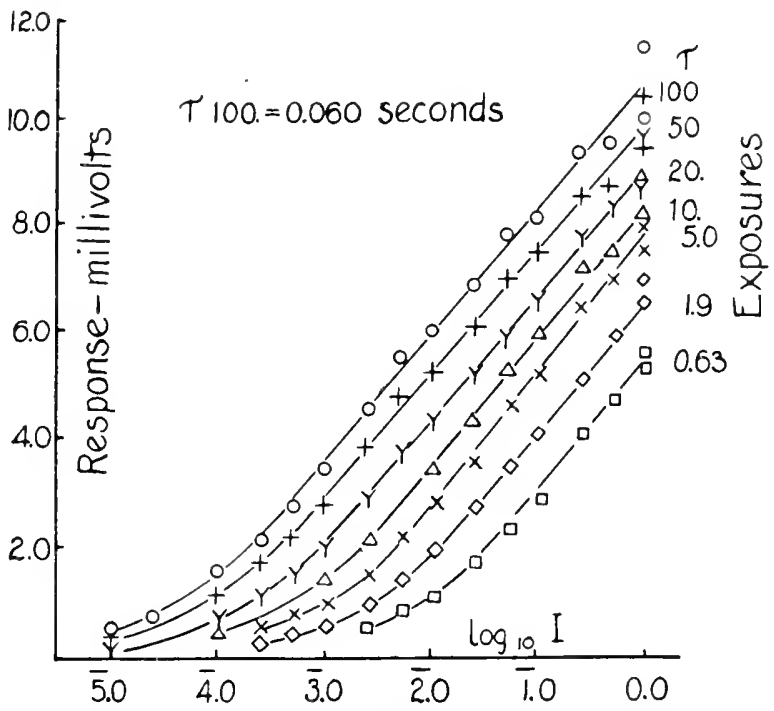

Fig. 130. A family of curves relating magnitude of the retinal action potential to $\log$ intensity for different periods of exposure. The measurements were obtained from the eye of a grasshopper. From Hartline. ${ }^{0 t}$

ability of the reciprocity law to photoreception, an earlier application having been made by Hecht (see page 406). Again it should be pointed out that the above relation is valid only for constant response levels. The over-all relation between $E$ and $I t$ for the photoreceptor of the grasshopper is apparent from inspection of the data in Figure 130, and is the same as the relation between magnitude of the retinal action potential and logarithm of the intensity.

RELATION BETWEEN INTENSITY AND MAGNITUDE OF ELECTRIC RESPONSE. Inspection of the curves in Figure 129 and in Figure 130 indicates that the relation between intensity of the light stimulus and the magnitude of the retinal action potential is a rather comsistent one. The curves are sigmoid, with a plateau at the high and low intensity ends and a rather linear inter- 
mediate region. The data which indicate atypical trends ${ }^{112}$ do so for technical reasons (see $W$ ulff ${ }^{174}$ ). It is apparent that some of those photoreceptors examined give rise to an electrical response which increases with increasing intensity of illumination over a considerable range. In some cases this intensity range is of the order of a million.

Graham ${ }^{ \pm 8}$ has demonstrated that the electrical response (measured as the time integral of the retinal potential) of the Limulus eye increases under the following conditions: (1) when the number of sensory units (ommatidia) illuminated is kept constant and intensity of illumination increases; (2) when the illumination is kept constant and the number of sensory units illuminated is increased (Fig. 131). These results probably indicate that individual sensory units can produce a graded response. It is probable that the intensitymagnitude data of Figures 129 and 130 represent recruitment of increasing numbers of sensory elements as well as the summed effect of graded responses of the sense elements stimulated.

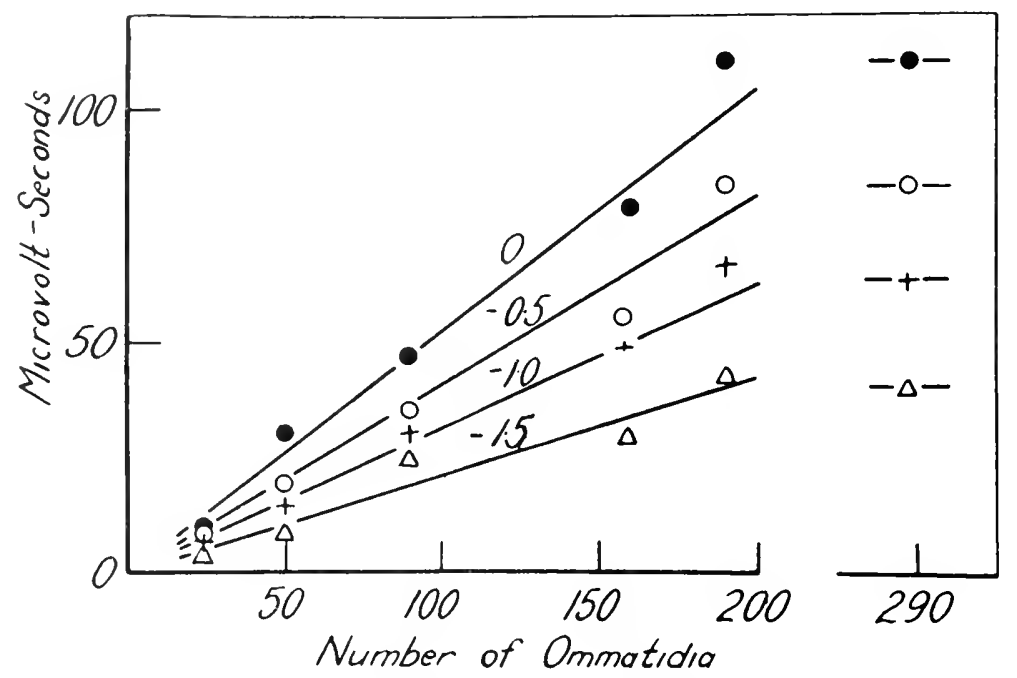

Fig. 131. Data illustrating the relation between response magnitude (time integral of the retinal potential change) and number of ommatidia illuminated at four different intensities. The logs of the intensities are indicated on the curves. From Graham. ${ }^{48}$

electrical measurement of dark adaptation. During dark adaptation a given light stimulus becomes more and more effective, i.e., the photochemical effect becomes greater. This should be reflected also in the electrical response to a given light stimulus during dark adaptation. The time course of clark adaptation has been followed in several species of animals by measurement of the magnitude of the electrical response elicited by a constantintensity, constant-duration light stimulus. The data obtained by Hartline $\mathrm{e}^{6.5}$ from Limulus and by Riggs ${ }^{1+2}$ from the frog ( 2 degree forcal stimulation data), when plotted in terms of $\log I$ necessary to elicit a response of constant magnitude as a function of time in the dark (Fig. 116, middle and upper curves), give curves essentially similar to the curve obtained by Hecht (lower curve, Fig. 116-see page 407). 
There may be some question why these dark-adaptation velocity measurements conform to the bimolecular isotherm, in view of the complexity of the rhodopsin cycle previously discussed (see page 411). As indicated below, it is apparent that these measurements do not reveal the complex relations which actually obtain. In the measurements on frogs, ${ }^{142}$ attention is directed to the fact that the data represent cone adaptation rather than rod adaptation. Electrical measurements of dark adaptation in the albino rat $^{21}$ conform much more closely to the reaction rate of a monomolecular reaction than to a bimolecular reaction rate. In this respect the data of Char pentier agree with the data of Tansley, ${ }^{154}$ who followed dark adaptation in the albino rat by extraction of rhodopsin. Tansley demonstrates that the latter data are fitted best by the velocity equation for a monomolecular re-

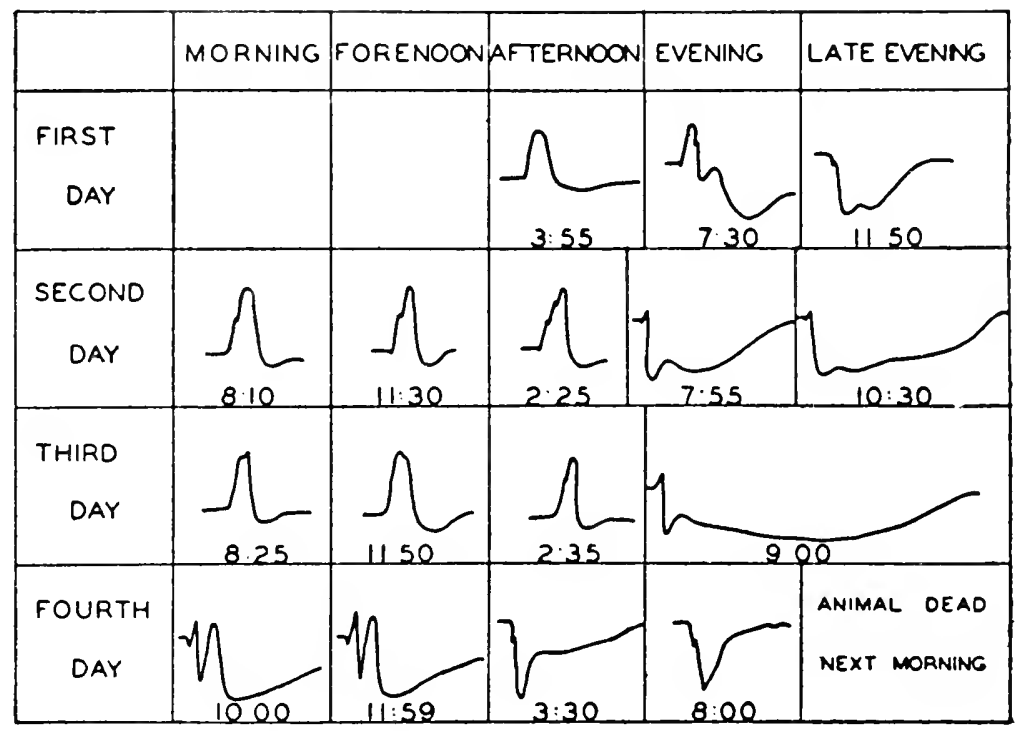

Fig. 132. Retinal action potentials obtained from a beetle, Dytiscus marginatus, during a sojourn in the dark for 4 days. Note the persistent change in the polarity of the response twice every 24 hour period. From Jahn \& Wulff. ${ }^{108}$

action. There is rather good correspondence between the electrical and chemical measurements of dark adaptation in the albino rat. Chase and Smith ${ }^{21}$ measured the time course of regeneration of an in vitro solution of rhodopsin extracted from frog retinac. The data are fitted by the monomolecular equation. Hence, the measurements of dark adaptation velocities as a whole afford considerable inconsistency. More recently the electric response of the human eye has been measured during dark adaptation. ${ }^{145 i}$

OTHER CONTRIBUTIONS FROM MEASURENIENT OF ELECTRICAL ACTIVITY of photoneceptors. At least several genera of beetles (Hydrous, Chlaenius, Harpalus, Dytiscus) exhibit an inherent diurnal rhythm in the type of electrical response of the eye. ${ }^{103,104,105,10 \times}$ Figure 132 shows the types of records obtained from Dytiscus in response to a constant-intensity, constantduration test flash at various times throughout a 4-day period. Until the 
death of the animal, the response changed in character regularly twice daily, even though the animal was maintained in total darkness. It is possible to maintain Chlaenius in complete darkness, except for test flashes, for a week and to record a diurnal variation in the electrical responses.

During the night phase the eye is more sensitive, in that the light in. tensity necessary to produce a minimal detectable electrical response is about $1 / 1000$ of that necessary during the day phase. This diurnal variation in sensitivity is not correlated with iris pigment migration, for in Dytiscus such migration occurs only as a response to changes in the state of light and dark adaptation. ${ }^{10+}$

The electrical activity of the neurones of the optic ganglion of insects may also be detected by measurements made with electrodes on the cornea. The activity of the ganglionic neurones is often rhythmic and appears as ripples superimposed on the electroretinogram. If the optic ganglion is excised the oscillations can no longer be recorded from the eye. Excision of the brain, however, has no obvious effect on these oscillations, ${ }^{24,146}$ The frequency of this oscillatory activity is usually 8 to 40 or more cycles per second and apparently results from the synchronized activity of the ganglionic neurones. Oscillatory activity occurs when the light is on, or for a short period of time just after illumination has ceased. ${ }^{2+}$ The frequency and magnitude depend on the temperature, on the state of adaptation, on the intensity and exposure period of the light, and on the species. In many respects the oscillatory activity of the optic ganglion is very similar to that of the vertebrate cereberal cortex which is recorded in the electroencephalogram.

summary. The measurement of visual functions by means of the retinal action potential agrees with measurements of the same visual functions by other techniques. Moreover, the correlations lead inevitably to the conclusion that the retinal action potential is controlled by a photochemical reaction. The previous discussion does not reveal any glimpse of the transitional mechanism between the photochemical event and the electrical event in the retina; this remains to be demonstrated in the future. Nor does the foregoing discussion prove irrevocably that the retinal action potential is a critical event in the peripheral visual mechanism; it merely indicates the possibility that it is. Consideration of the relation between the retinal action potential and the discharge of nerve impulses in the fibers of the optic nerve is of interest in this connection.

Electrical Activity of the Optic Nerve: Nature of Optic Nerve Activity. One of the most useful techniques in the study of vision is that of recording the impulses from individual fibers of the optic nerve, either from the nerve itself $^{71}$ in invertebrates, or from the fibers on the inner surface of the retina before they form the nerve $e^{68}$ in vertebrates. This method of investigation has yielded much of our knowledge of the action of the sensory cells, both as individual units for the detection of light and as units for the discrimination of wave length (color vision).

Mlost afferent nerves, when stimulated via their end-organs, respond with a train of action potentials rather than with a single action potential. The fibers of the optic nerve are no exception. An example of the response of a single nerve fiber of Limulus to prolonged illumination of the sensory ending is shown in Figure 133. In Limulus each fiber is connected to a single sensory 
ending. The impulses therefore are reflections of the activity of individual sensory units. The first record shows that after a short latent period, there

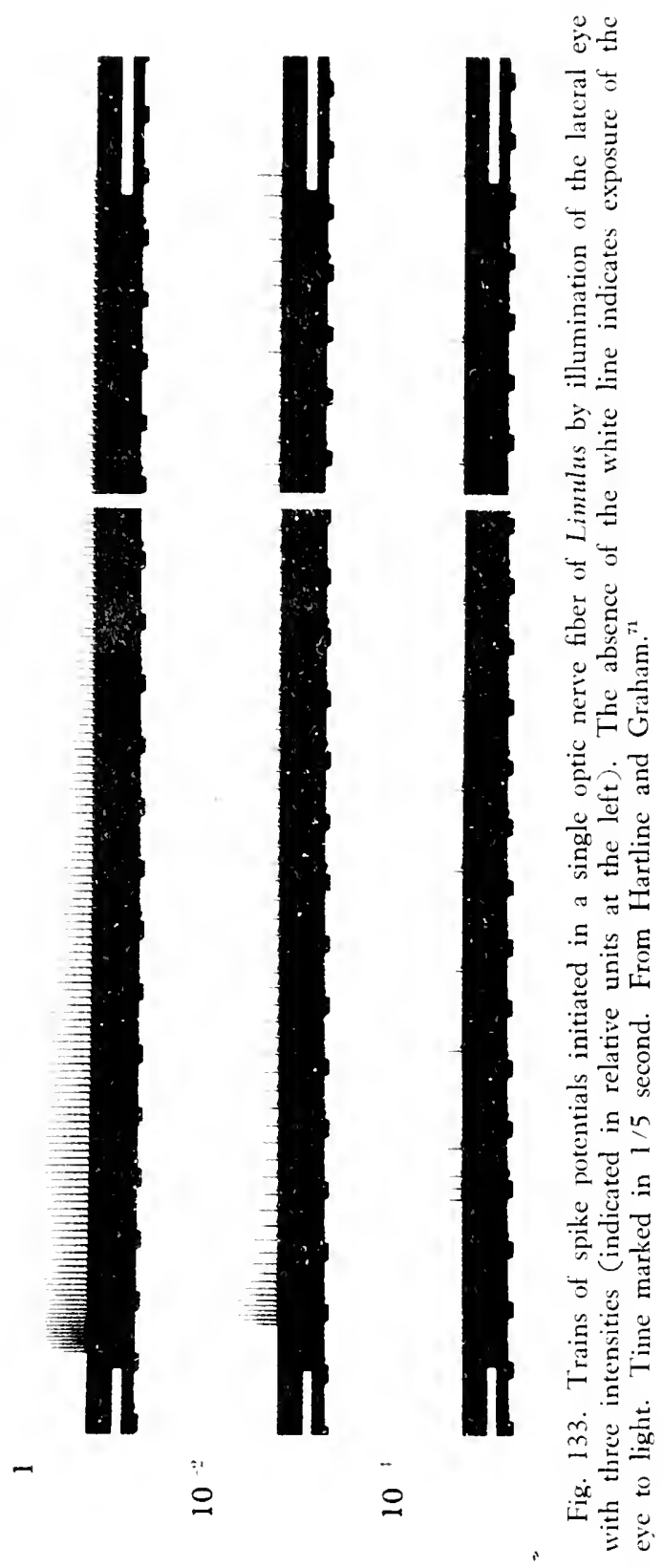

is a prolonged series of nerve impulses of high frequency for slightly more than a fifth of a second and then of a lower but rather constant frequency (Fig. 133). At a lower intensity (second record) the latent period is longer 
and the impulse frequency is lower. At a still lower intensity the latency is further prolonged and the frequency is still lower. In all of these records the impulses stop with cessation of illumination. Impulses in single fibers of the Limulus optic nerve have been recorded for periods of illumination lasting 30 minutes. ${ }^{1+t}$ These impulses occur at a low frequency, and probably continue as long as the eye is illuminated.

The frequency patterns of nerve impulse discharge in all fibers of the Limulus optic nerve are similar. The condition in the vertebrate optic nerve is quite different. ${ }^{6 \times}$ Three functionally different types of fibers have been discovered by Hartline, using the difficult technique of recording from single intraocular fibers in the frog (Fig. 134). Fiber A responds with a volley shortly after illumination begins ("on" burst). The impulse frequency declines, at first rapidly, then more slowly, and is interrupted by a quiet period. The impulse frequency decreases until the end of illumination, at which time the fiber responds with another burst of impulses ("off" burst). These fibers comprise about 20 per cent of the total fiber population. Fiber $\mathrm{B}$ exhibits an "on" burst and an "off" burst of impulses and does not respond to continued illumination. These fibers comprise about 50 per cent of

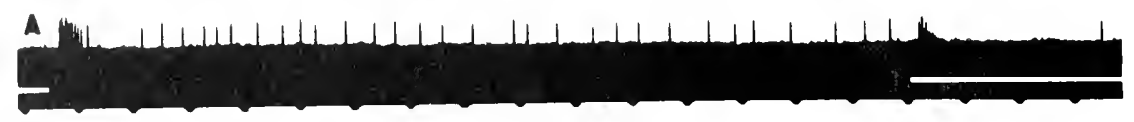

B

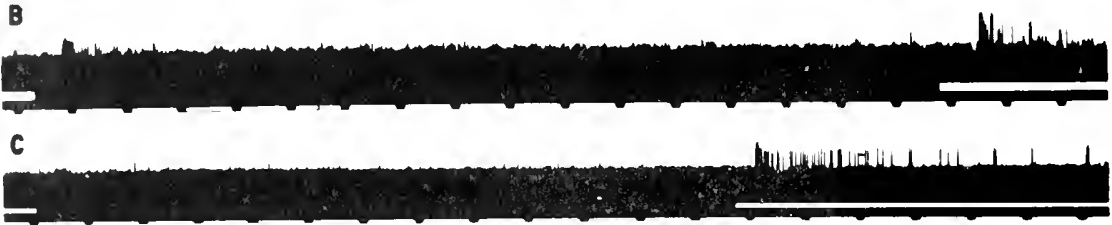

Fig. 134. Trains of spike potentials initiated in three types of frog optic nerve fibers upon onset and cessation of illumination of the eye. The absence of the white line indicates illumination of the eye. A, Fiber responds with initial "on" burst and maintained discharge during period of illumination; B, fiber responds with "on" burst and a burst on cessation of illumination ("off" burst); C, fiber responds only with "off" burst. Time marked in $1 / 5 \mathrm{sec}$. From Hartline. ${ }^{88}$

the total fiber population. Fiber C exhibits only an "off" burst of impulses. This type of fiber comprises about 30 per cent of the total fiber population.

The optic nerve discharge patterns in invertebrates are of considerable interest because these observations cast some light on the complexities of frequency patterns observed in the vertebrate eye. In the segmental nerve of an anterior segment of the earthworm ${ }^{139}$ and in the ventral nerve cord anterior to the sixth abdominal ganglion of the crayfish Prosser ${ }^{138}$ recorded a discharge appearing shortly after illumination which builds up slowly to a maximum. The receptors involved, probably the unicellular receptors of earthworms (page 383) and the sixth abdominal ganglion of the crayfish, are believed to be primitive photoreceptors. Prosser ${ }^{138}$ also recorded a nerve discharge pattern in the pharyngeal commissures of the crayfish on illumination of the stalked compound eyes. The discharge consisted of an "on" burst, a maintained discharge of declining frequency, and an "off" burst. Hartline recorded from single optic nerve fibers of the distal and proximal retinae of 
Pecten; the fibers from the proximal sense cells exhibited a pattern similar to the Limulus pattern (Fig. 133). The fibers from the distal retina exhibited "off" bursts only. The difference in optic nerve discharge must be attributed to the cells of the distal retina. The cells of either area are capable of being excited only by cessation of illumination or-and this is the more plausible view-they serve in the capacity of ganglion cells and receise their excitation from the distal sense cell layer. Hartline and Wilska, ${ }^{73}$ using microclectrodes. found that the fibers in the anterior portion of the optic lobe of Limulus, presumably coming directly from the retina, exhibited the familiar discharge pattern (Fig. 133). The fibers in the posterior portion of the lobe exhibited "off" bursts on cessation of illumination, similar to that shown in the vertebrate picture. These neurones are never active during illumination, and the frequency and duration of the "off" burst depend on the intensity and duration of the preceding illumination. The "off" burst discharge is inhibited by re-illumination, just as it is in the vertebrate eye ${ }^{57}$ and in Pecten. In the vertebrate eye, each third order neurone, from which the frequency patterns

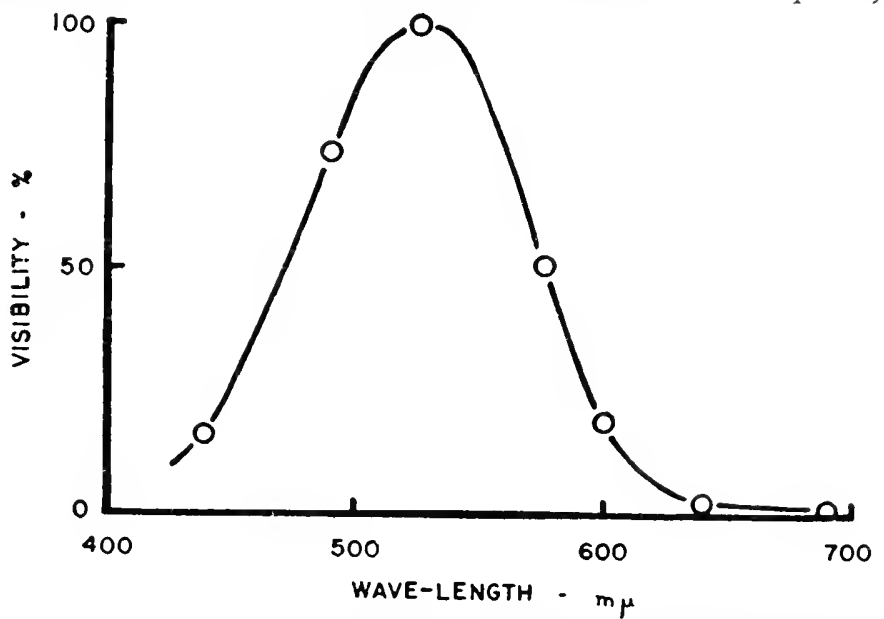

Fig. 135. Visibility curve for a single visual sense cell (Limulus). Visibility of each spectral band is the reciprocal of the relative intensity necessary to produce a specified number of nerve impulses. From Graham and Hartline. ${ }^{49}$

(Fig. 134) were recorded, is separated from the primary neurone, the sense cell, by at least one interspersed neurone, offering considerable complexity of interrelations (see Fig. 110). The complexities of the vertebrate frequency patterns are attributable to this retinal ganglionic layer.

Measurement of Visual Functions by Means of the Optic Nerve Discharge: SPECTRAL SENSITIVITY. Employing different wave length bands of stimulating light, Graham and Hartline ${ }^{4:}$ measured the intensity necessary to produce a constant number of nerve spike potentials in an optic nerve fiber. The reciprocal of the intensity may be taken as a measure of sensitivity. The resultant plot of visibility against wave length gives an action spectrum (Fig. 135) that resembles those obtained by other techniques and from other animals and, indeed, is similar to the spectral absorption curve of rhodopsin.

THE RECIPROCITY LAW. Records of the result of illumination of the Limulus eye with brief flashes of light are shown in Figure 136. ${ }^{66}$ The technique used in obtaining the data of Figure 136 is identical with the technique 
used in obtaining that of Figure 133. The four vertical rows of oscillograms were made from the same dark-adapted fiber with different durations of single flashes of light. The four horizontal rows were taken at different intensities. It is to be noted that at unit intensity (horizontal row 1), as the duration of the flash is increased, the number of resultant impulses in the fiber increases. Also, as the intensity is decreased (horizontal rows 2, 3 and 4), the duration required to produce a single impulse is increaser. The data indicate that the reciprocity law (duration $X$ time $=$ constant) holds for the production of a single impulse.

RELATION BETWEEN ILLUMINATING INTENSITY' AND FREQUENCY OF NERVE impulse Discharge. The discharge patterns of the optic nerve of Limuluis and of fibers $A$ and $B$ of the frog eye exhibit an "on" burst of high frequency.

DURATION

$\begin{array}{llll}0.0001 & 0.001 & 0.01 & 0.1\end{array}$
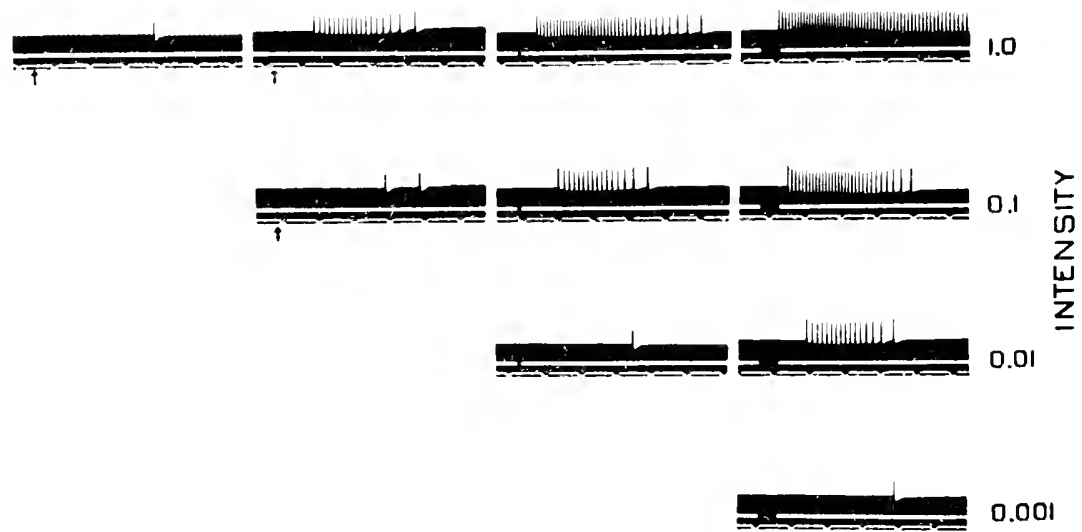

Fig. 136. Spike potentials recorded from single nerve fibers in response to illumination of the eve with different intensities for various periods of time. Duration of exposure to light is indicated at the top of each column in seconds; intensity in relative units is indicated at the right (unit intensity 3,000,000 meter candles). The absence of the white line indicates exposure of the eye. For very short exposures the arrows mark the position of the signal. Time in $1 / 5$ sec. From Hartline.

The relation of this maximal frequency to the intensity of the illumination is interesting (Fig. 137), because there is a rough parallelism between this relation and the relation of the retinal action potential magnitude to the illuminating intensity (see Figs. 129 and 130, page 425). A similar relation seems to hold between other parts of the optic nerve discharge pattern and the illuminating intensity.

Dark adaptation. Employing the Limulus single optic nerve fiber preparation, I lartline and MCDOnald ${ }^{2 *}$ investigated the dark adaptation of single visual receptors. The recovery of a visual receptor after a short period of adaptation to illumination of a moderately high intensity is shown in Figure 138. The nerve spike potentials are elicited by a short flash of light of fixed intensity and duration. The records show that the number of spike 
potentials elicited by the test flash increases rapidly at first and subsequently more slowly.

The relation of this recovery to time may be graphically illustrated in two ways: (1) by determining the intensity of the test flash necessary to elicit a single spike potential cluring the period of dark adaptation, and (2) by measuring the number of impulses elicited by a constant test flash as a function of time. A series of recovery curves plotted according to the second method are illustrated in Figure 139. These curves were obtained after adaptation to lights of different intensities. It is apparent that recovery after

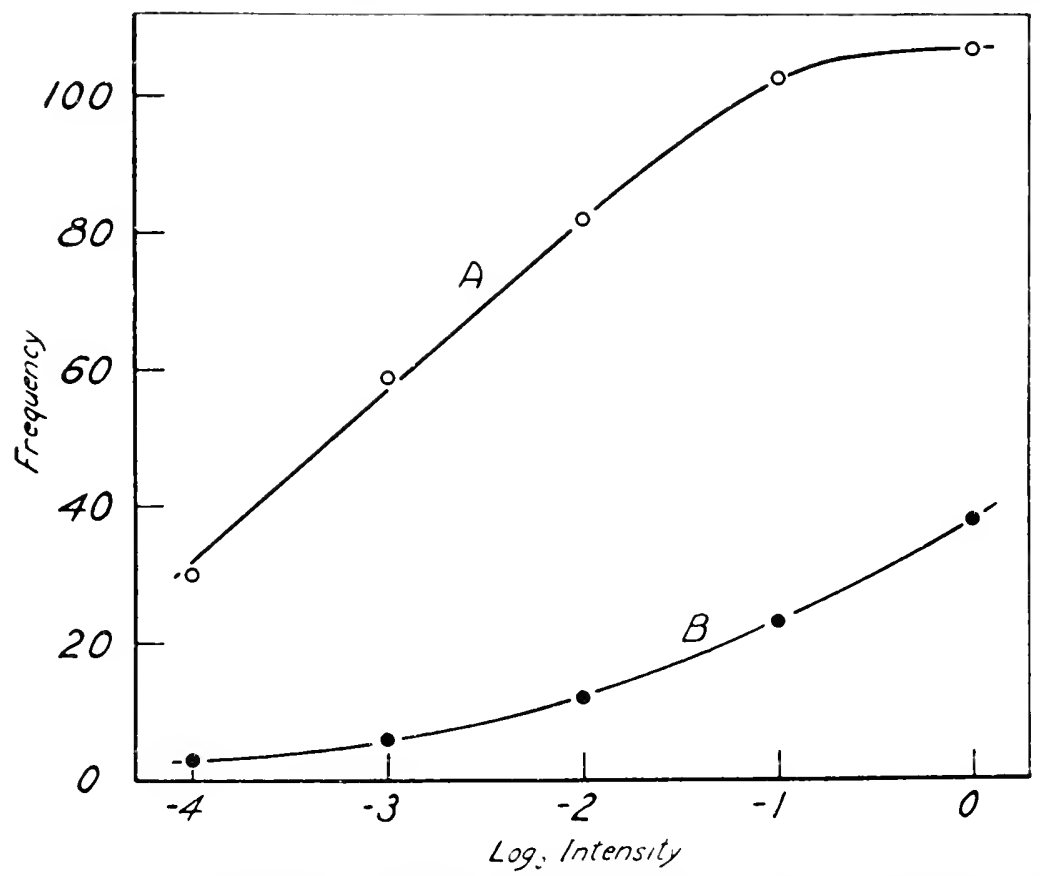

Fig. 137. The relationship between frequency of spike potentials recorded from single optic nerve fibers (Limulus) and the logarithm (base 10) of the illuminating intensity: $A$, Maximal frequency of discharge, shortly after onset of illumination; $B$, frequency of discharge 3.5 seconds after onset of illumination. From Hartline and Graham. ${ }^{.1}$

adaptation to illumination of a low intensity is much more rapid than recovery after adaptation to illumination of a higher intensity. A similar series of recovery curves were obtained after adaptation for varying periods but at constant intensity. These results indicate that the rate of dark adaptation varies with the intensity and duration of light adaptation. In this regard these curres duplicate the complexities of the curves of vertebrate eye rod recovery (Fig. 115, upper curves). ${ }^{171}$ The variations in rate of dark adaptation are explained in terms of the rhodopsin cycle (page 411), and an analogous explanation may possibly be applicd to the Limmulus eye.

Relations between Retinal and Optic Nerve Activity. The foregoing discussion of retinal and optic nerve activity in a variety of photoreceptors indicates that both reflect a dependence on the photochemical nature of photo reception. This follows on consideration of spectral sensitivity measurements 
employing the retinal action potential, optic nerve discharge, or some overt organismic response, and their comparison with the spectral absorption curves of known animal photolabile pigments, such as rhodopsin. Similarly, measurements of the time course of dark adaptation by different techniques agree quite well. Further, the reciprocity law of Bunsen and Roscoe has been demonstrated by a variety of techniques to apply to photoreceptors at given response levels. The conclusion that both retinal and optic nerve activity depends on the absorption of radiant energy by a photolabile pigment seems to be justified.

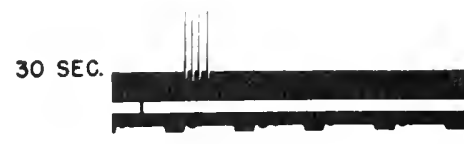

10 MIN.

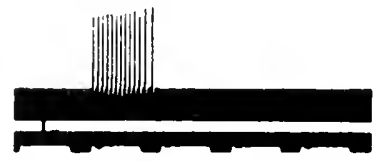

I MIN.

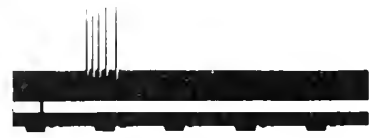

20 MIN.

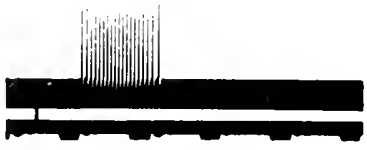

2 MIN.

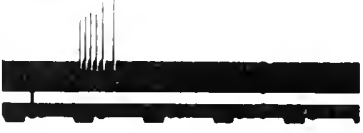

40 MIN.

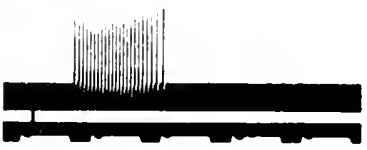

5 MIN.

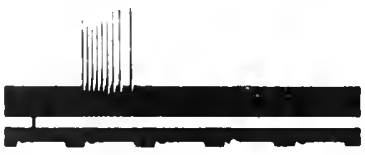

1 HR.

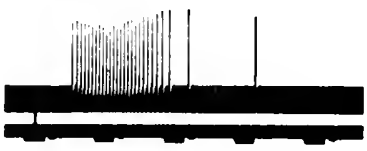

Fig. 138. Spike potentials recorded from a single optic nerve fiber (Limulus) in response to brief and constant illumination of the eye at various times in the dark (indicated at the left of each record), after a period of light adaptation. Time in $1 / 5 \mathrm{sec}$. The light flash is indicated by interruption of the white line. From Hartline and MacDonald. ${ }^{72}$

What then is the relation between the retinal action potential and the activity in the nervous elements of the optic pathway? This question is not easily answered, although it has been raised a number of times. ${ }^{67,57,173}$ It has been suggested, by Hartline ${ }^{67}$ and others, that the retinal action potential produces local action currents which initiate activity in the nervous elements of the optic pathway. This sequence of events, that is, a slow potential followed by nerve discharge, is not unknown in nerve physiology. Such a sequence has been demonstrated in the ganglion of the heart of Limulus, ${ }^{86,}, 14 \mathrm{ma}$ and there is some evidence for a similar sequence of events in the lower motor neurones of the vertebrate spinal cord.

Attractive as this suggestion may be, there is no evidence that affords clearcut proof. It has been shown that the retinal action potential precedes the 
optic nerve discharge ${ }^{67}$ or optic ganglion response ${ }^{176 i a}$ in time; it has also been shown that a nerve impulse may appear on illumination (usually low levels) in the absence of detectable retinal action potential. In response to high intensity flashes of light the train of nerve impulses mas last for sereral seconds, whereas the retinal action potential is over in a fraction of a second (Wulff, unpublished). These observations do not permit an answer; future observations will undoubtedly serve to clarify this problem.

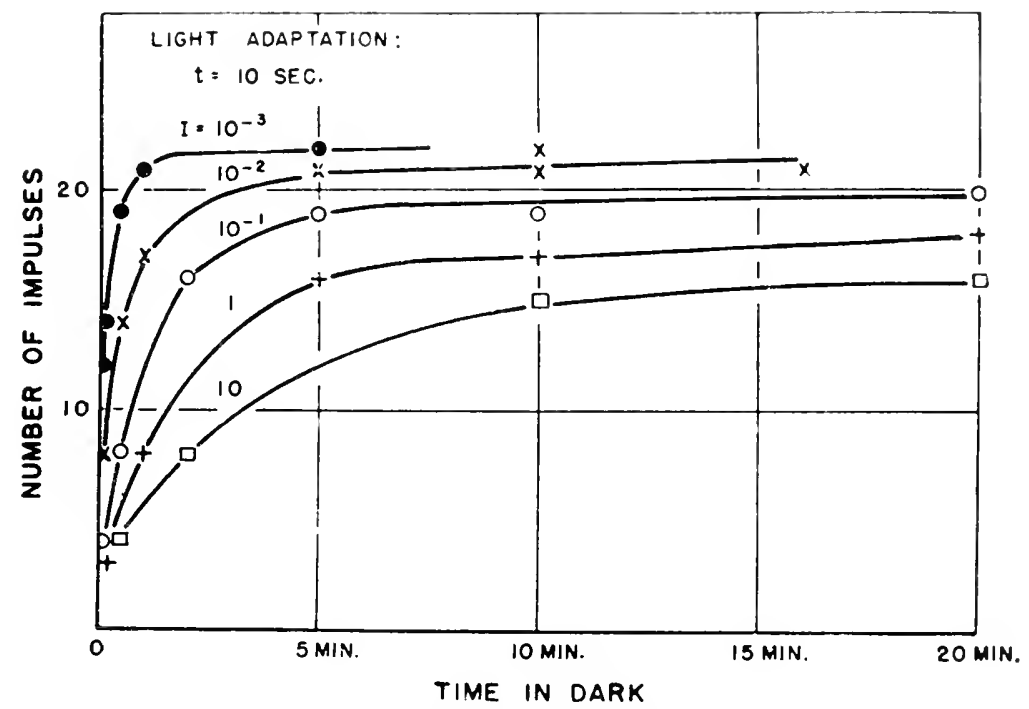

Fig. 139. Dark adaptation of single visual elements of Limulus after exposure to lights of different intensities (indicated on the curves in relative units). The response of the eye is measured by recording spike potentials of single visual elcments in response to a constant intensity, constant duration test flash. Note the depression of the recovery curve after adaptation to more intense illumination. From Hartline and McDonald. ${ }^{\text {?: }}$

\section{The Central Visual Mechanism}

Central Visual Pathways. A vertical line drawn through the center of the fovea of the human retina demarcates the two hemiretinas, the fibers of which follow different pathways to the brain. Fibers from the temporal hemiretina of the left eye pass through the optic chiasma and without crossing pass to the lateral geniculate body of the left side of the brain; those of the temporal side of the right eye enter the right optic tract. Fibers from the nasal half of each retina cross in the optic chiasma, enter the optic tract of the opposite side of the brain, where they join the uncrossed fibers from the temporal half of the other eye, and end in the contralateral geniculate body. In the geniculate bodies the visual fibers enter into synaptic relations with the fourth order neurones which continue on to the oceipital lobe of the cortex. As a result of the regrouping of fibers in the chiasma, the effect of lesions of the chiasma or central to the chiasma causes visual defects which are different from those produced by lesions of the optic nerve.

The Visual Cortex. Knowledge of the manner in which the visual fibers terminate in the optic cortex is obtained by three principal methods: (1) 
correlation of visual field defects with small lesions in the occipital lobe; ${ }^{88,136}$ (2) correlation of degeneration in the geniculate bodies with the site of lesions in the retina or in the cortex (monkey $\left.{ }^{17}, 22\right)$; (3) correlation of electrical action potentials from various parts of the cortex in response to restricted stimulation of the retina. ${ }^{152}$ It has been demonstrated ${ }^{136,137}$ that the retina is projected point-to-point through the geniculate body to the optic cortex. The areas of the striate cortex devoted to impulses from various parts of the retina have been determined by Talbot and Marshall, and are shown in Figure 140. It is evident that the image formed on the retina is projected to the striate cortex and thence to higher association centers.

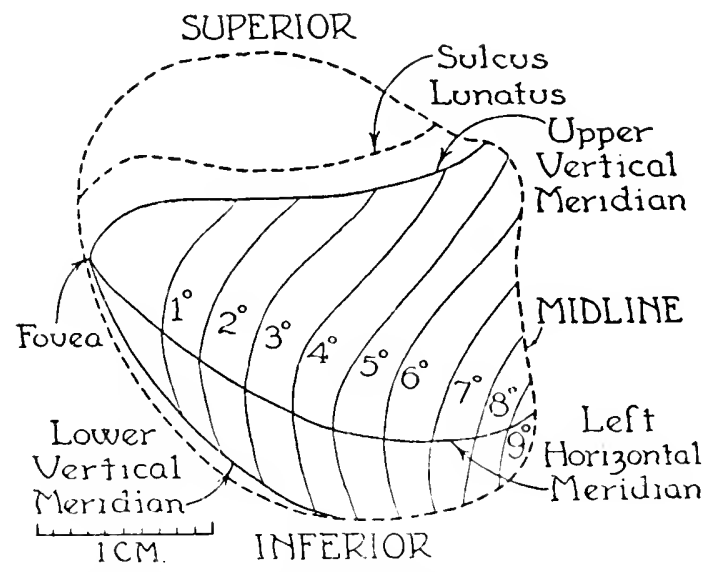

Fig. 140. Projection of the retina on the left occipital lobe of the monkey, determined by electrical methods. After Talbot and Marshall. ${ }^{162}$

\section{The Mechanism of Color Vision}

Normal Color Vision. Color vision is the term ordinarily applied to the ability of an animal to distinguish between light of various wave lengths. Inasmuch as several combinations of wave lengths may appear to have the same color, i.e., to give the same sensation, the discrimination termed color vision is not identical with wave length discrimination. However, the differential effect of wave length on the receptors is the fundamental basis of color vision.

The most obvious fundamental evidence of color discrimination is obtained from color matching. To a normal human observer the spectrum appears as a series of colors varying from dim red through orange, yellow, brilliant yellow-green, green, and blue, to dim violet. The colors seen by a nomal observer can be duplicated with few exceptions by mixtures of three lights, a red, a green, and a blue light, provided each is independently adjustable in intensity. Two lights are insufficient and four are unnecessary for most colors, but when a fourth light is present the matching is more perfect. A normal person is said to be trichromatic, by which is meant that he can duplicate the spectrum with three colored lights.

Abnormal Color Vision. Some individuals with abnormal vision require lights of threc colors to match any given color, but use an intensity adjust- 
ment which is somewhat different from that used by normal individuals. These individuals are said to have an anomalous trichromatism or to have a "color weakness," i.e., a weak sensitivity to certain colors. Other individuals can match the spectrum with only two colored lights; they are said to be dichromatic or color blind. Other individuals are not able to detect any chromatic differences in the spectrum; they are completely color blind.

Observers with dichromatic vision are of several types. One type, the deuteranope, can distinguish yellow from blue but not grecn from red and sees both red and green as shades of gray. Another type, the protanope, is able to distinguish blue from yellow but not green from red, and, in addition, is less sensitive to red than a normal person or a deuteranope, i.e., he sees red not as various shades of gray, as does the deuteranope, but as a very dark gray or black. A third and rare type, the tritanope, is able to distinguish between green and red but not between vellow and blue.

Color blindness of the first two types, deuteranopia and protanopia, and their respective color weaknesses, deuteranomaly and protanomaly, are inherited as sex-linked characters and are therefore about twenty times as common in men as they are in women (total abnormality for men, 8.0 per cent; for women, 0.43 per cent).

The Peripheral Mechanism of Color Vision. It was suggested by Thomas Young in 1807 that the fundamental mechanism of color vision was a dif-

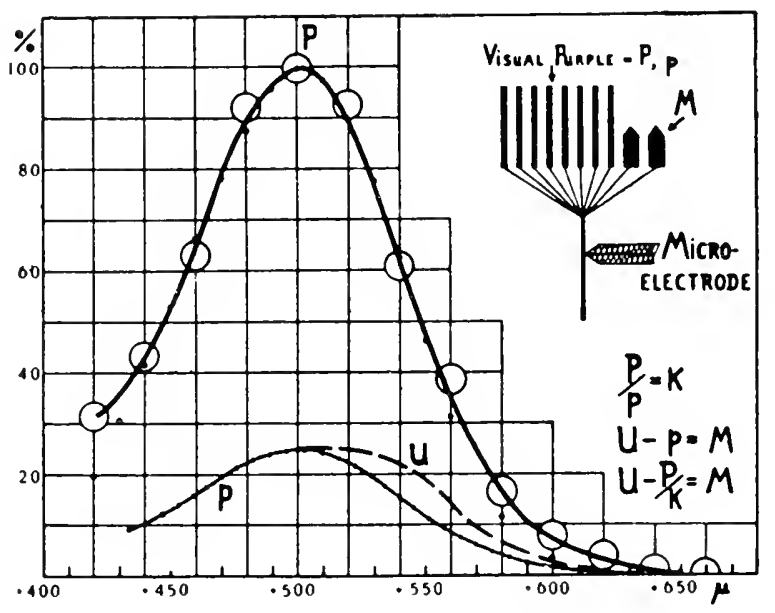

Fig. 141. The sensitivity curve (open circles) of the receptors connected with a single ganglion cell of the dark-adapted retina of a cat. The black dots represent the absorption of light by visual purple. $U$, Experimentally obtained curve from which curve $P$ (in proper magnitude, $\mathrm{p}$ ) is substracted to give the sensitivity curve of specific color receptors. From Granit. ${ }^{57}$

ferential sensitivity of the individual receptors. The theory was expanded by Helmholz in 1852 and finally, after the publication of more than 1200 papers by many investigators during a period of almost a century and a half, the supporting experimental data were supplied for an invertebrate eye by Graham and Hartline ${ }^{49}$ and for the vertebrate eye by Granit. ${ }^{57}$ 
Granit used the technique of placing microelectrodes on single tertiary neurones of the retina before they enter the optic nerve (Fig. 141). Then he stimulated the area of sense cells connected to this fiber with light of known wave length and intensity and varied the intensity at each wave length until he determined the threshold of the fiber for that particular wave length. In this manner he was able to obtain sensitivity curves for individual visual units. Each unit consisted of the tertiary fiber and all sense cells and secondary neurones from which it received impulses. It was found that the units were not all the same. Some gave both the normal scotopic (dark-adapted) and photopic (light-adapted) sensitivity curves as the state of adaptation was appropriately changed, and this indicated that the same tertiary fiber received impulses from both rods and cones. Other tertiary fibers apparently received impulses only from cones. The sensitivity curves from exclusively cone units also seemed to be of several types, some with a narrow range and a peak in

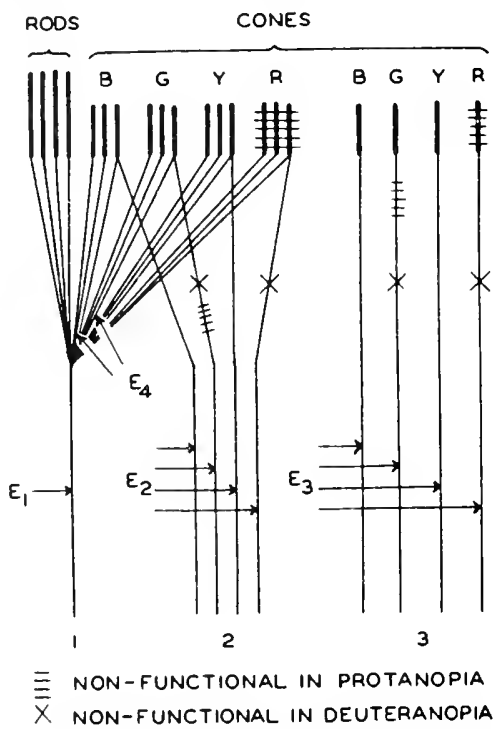

Fig. 142. An explanation of color vision and color blindness based on the findings of Granit and his collaborators and on the histology of the retina. $E_{1}, E_{2}, E_{3}, E_{4}$, recording clectrode positions; $B$, blue-sensitive cones; $G$, green-sensitive cones; $Y$, yellow-sensitive cones; $R$, red-sensitive cones. From Jahn. ${ }^{98}$

the blue, others with a narrow range and a peak in the green, yellow or red and still others with a wide range and a peak which coincided with the normal photopic visibility curve.

The above types of data can be explained by assuming that there are cones with narrow sensitivity curves with peaks in the red, yellow, green, and blue, and that these are connected both singly and in combination to tertiary neurones, as demonstrated morphologically by Polyak. ${ }^{137}$ The connections are shown diagrammatically in Figure 142. The fiber designated as 1 is connected to numerous rods and numerous cones; this fiber has two sensitivity curves, one the normal photopic and one the normal scotopic, depending on the state of adaptation. It is called a "dominator" and is re- 
sponsible for the sensation of brightness but is not responsible for color dis crimination. The fibers designated as 2 and 3 carry impulses from singlc cones which have different spectral sensitivity curves with peaks in the blue, green, yellow, or red $(B, G, Y, R)$. These are responsible for the ability to discriminate between wave lengths and are called "modulators." The theory as outlined here is called the "dominator-modulator" theory of color vision. ": ax "The electrical evidence obtained by Granit was recorded with electrodes at posi tions designated as $\mathrm{E}_{1}$ to $\mathrm{E}_{4}$.

A composite theoretical curve of the tertiary neurones of the human retina, adjusted to fit the data on perception, is shown in Figure 143. For various reasons it seems better to assume four rather than three types of modulators.

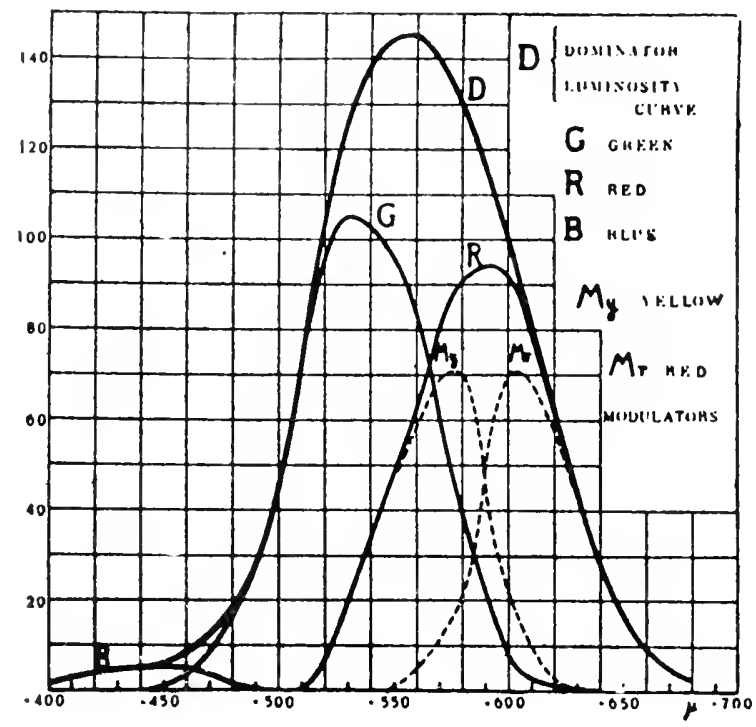

Fig. 143. Synthesis of the human photopic luminosity curve, D, after Coblentz and Emerson (1917). B, G, and $R$ represent blue, green, and red fundamental sensation curves. The $R$ curve is composed of two modulator curves, $M y$ and Mr. From Granit. ${ }^{57}$

Four have been well demonstrated for the frog and three for the cat, and even more may be present. However, four will explain most of the data on human color vision and color blindness. For instance, if, in Figure 142, the red- and green-sensitive modulators are absent or non-functional, the person would be unable to distinguish red from green, both of which would appear gray, but the visibility spectrum determined by the dominator would be normal; this is the condition in deuteranopia. Furthermore, if, in addition, all of the red-sensitive cones were non-functional, the visible spectrum would be shortened; this is the condition in protanopia. Tritanopia is subject to a comparable explanation.

\section{SUMMARY}

Light (visible and ultraviolet) is an important quality of the environment which permits animals to locate food, to escape from predators, and to make 
numerous behavioral adjustments. Light in excess is damaging, and many animals are protected by pigments in outer body layers.

In order that light energy be effective biologically it must be absorbed and a photochemical reaction occur. Light-absorbing compounds of many types have evolved-chlorophylls in plant chloroplasts, melanins and others in chromatophores-but carotenoids scem to be universal in photoreceptors which initiate behavorial responses. From a kinetic analysis of behavior in molluscs, from analysis of pigment extracted from retinas of vertebrates and cephalopods, from action spectra of various visual functions in molluses, arthropods, and vertebrates, it appears that the carotenoid rhodopsin, or compounds very similar to it, is the most widespread and perhaps the most primitive animal photoreceptor carotenoid. Fresh-water fish have used a different substance, porphyropsin, but reversion to rhodopsin occurred on migration to sea and to land. The photosensitive pigment is broken down under the influence of light according to a first order reaction and then is reformed from intermediates from several sources by more complex reactions.

The photochemical breakdown is followed by a series of events which lead ultimately to the reaction of the organism. One such event is the retinal potential which, in simple eyes at least, represents electrical activity of the sensory cells. Next appear impulses in the optic nerve; these may build up gradually, may start at a high frequency and then decline, may show various patterns depending on the synaptic organization of the retina. The events connecting photochemical breakdown with retinal potential and this potential with initiation of nerve impulses are uncertain. The excitatory signal, whether it be transmitted in a nerve or from one part of a cell to another as in a flagellate, elicits a response which depends in part on the complexity of integrative and motor organization.

The nature of the behavioral response is also influenced by the photoreceptor, whether it forms pattern images or merely signals light, whether it permits fine resolution of objects in a pattern, and its manner of response to flicker. To make maximum use of stimulating light a variety of mechanisms have evolved which function in focusing, in varying the range of accommodation, in regulating the sensitivity of the sense cells and the amount of light reaching them in different states of light and dark adaptation. Some animals merely show increased activity on illumination, others orient toward or away from a light by indirect or direct paths and the orientation may vary according to the physiological state of the organism. Some animals go between two sources of light, others go directly to one of two sources. In more complex behavior the response is influenced by past experiences, competing stimuli, and other factors, but even here are direct responses as in pupillary constriction and some eye movements of mammals. The underlying principle in photoreception is the quantitative relation between photochemical breakdown, action potentials, and behavior, even though the type of behavior may vary with type of eye and motor organization.

\section{REFERENCES}

1. Adrian, E. D., J. Physiol. 104:84-104 (1945). Electric response of eye: human.

2. Adrian, E. D., J. Physiol. 105:24-38 (1947). Electrical response of eyes: vertebrate. 
3. Adrian, E. D., and Mattuews, R., J. Physiol. 63:378+1+ (1927). Electrical changes in eye and optic nerve: eel.

4. Adrian, E. D., and Mattuew's, R., J. Physiol. 64:279-301 (1927). Electrical changes in eye and optic nerve: eel.

5. Adrian, E. D., and Matrinews, R., J. Physiol. 65:273.298 (1928). Electrical changes in eye and optic nerve: cel.

6. Barteey, S. H., Vision: A study of its basis (1941). New York, Van Nostrand.

7. Bennitt, R., Physiol. Zool. 5:65-69 (1932). Rhythmic visual pigment migration: crustacea.

8. Bernilard, C. G., Acta Physiol. Scand., vol. 1, Suppl. 1, pp. I-94 (1940). Neurophysiology of optic pathway.

9. Bernhard, C. G., J. Ncurophysiol. 5:32-48 (1942). Electrical response of eye: Dytiscus.

10. Bertholf, L. Ml., I. Agr. Res. 43:703-713 (1931). Ultraviolet sensitivity in bee.

11. Bertholf, L. M., Ztschr. vergl. Physiol. 18:32-64 (1932). Spectral sensitivity of Drosophila.

12. Bliss, A. F., I. Gen. Plysiol. 26:361-367 (1943). Properties of invertebrate photopigment.

13. Buiss, A. F., J. Biol. Chem. 172:165-178 (1948). Retinal vitamin A formation.

14. Busss, A. F., J. Biol. Chem. 176:563-569 (1948). Rhodopsin from the squid retina.

15. Blum, H. F., Cold Spring Harb. Symp. Quant. Biol. 3:210-213 (1935). Orientation of a nimals in light fields.

15a. Borradalle, L. A., and Potrs, F. A., The Invertebrata: A Manual for the Use of Students (1935). New York, Macmillan. 686 p.

16. Broda, E. E. and Goodeve, C. F., Proc. Roy. Soc. Lond., A. 179:151-159 (194I). Bleaching of visual purple at low temperature.

17. Brouwer, B., and ZeEnian, W. P. C., Brain 49:I-35 (1926). Projection of retina in lateral geniculate body.

18. Byram, G. M., J. Opt. Soc. Am. 34:571-591 (1944). Distribution of illumination in retinal images.

19. Byram, G. M., J. Opt. Soc. Am. 34:718-738 (1944). Visual acuity and photochemistry of the retina.

20. Chaffee, E. L., Bovie, W. T., and Hampson, A., J. Opt. Soc. Am. 7:1-40 (1923). Electrical response of eve: frog.

21. Charpentier, G., Acta Opthal., Kbh. Suppl. 9 (1936). Electrical response of eyes: rat.

21a. Chase, A. M., and Smith, E. L., I. Gen. Physiol. 23:21-39 (1939). In vitro regeneration of visual purple.

22. Clark, W. E. LeGros, and Penman, G. G., Proc Roy. Soc. Lond., B. 114:291-313 (1934). Projection of retina in lateral geniculate body.

23. Crescitelli, F., and Jahn, T. L., I. Cell. E Comp. Physiol. 13:105-112 (1939). Electrical response of eyes: grasshopper.

24. Crescitelli, F., and Jahn, T. L., J. Cell. E Comp. Physiol. 19:47-66 (1942). Oscillatory electrical activity of eves: arthropod.

25. Crozier, W. J., Am. J. Physiol. 36:8-20 (1914). Photic orientation of holothuria.

26. Crozier, W. J., Zool. Jahrb., Aht. allg. Zool. u. Physiol. 35:233-297 (1915). Sensory reactions of holothuria.

27. Crozier, W. J., J. Gen. Physiol. 3:57-59 (1920). Role of integumentary pigment in photoreception: Holothuria.

28. Crozier, W. J., J. Gen. Physiol. 19:503-522 (1936). Relation of illumination to flicker fusion and intensity discrimination.

29. Crozier, W. J., and Wolf, E., J. Gen. Physiol. 24:635-654 (1941). Flicker contours vs. flash duration: man.

30. Crozier, W. J., and Wolf, E., J. Gen. Physiol. 27:287-313 (1943). Intluence of pecten on flicker response contour.

31. Crozier, W. J., Wolf, E., and Zerrahn-Wolf, G., J. Gen. Physiol. 20:21 I-228 (1936). Intensity and critical flicker frequency.

32. Crozier, W. J., Wolf, E., and Zerrahn-Wolf, G., Proc. Nat. Acad. Sci. 23 : 516-520 (1937). Specific constants for visual excitation. 
33. Crozier, W. J., Wolf, E., and Zerrahn-Wolf, G., J. Gen. Physiol. 20:393-431 (1937). Temperature, critical illumination and ficker fusion: Anax larvae, sunfish.

34. Crozier, W. J., Wolf, E., and Zerrahn-Wolf, G., J. Gen. Physiol. 21:203-221 (1937). Intensity and critical flicker frequency.

35. Crozier, W. J., Wolf, E., and Zerrahn-Wolf, G., Proc. Nat. Acad. Sci. 24 : 216-221 (1938). Temperature, critical intensity and flicker response.

36. Crozier, W. J., Wolf, E., and Zerrahn-Wolf, G., Proc. Nat. Acad. Sci. 24: 221-224 (1938). Constants for visual excitation.

37. Crozier, W. J., Wolf, E., and Zerrahn-Wolf, G., Proc. Nat. Acad. Sci. 24 : $542-545$ (1938). Constants for visual excitation.

38. Dartnall, H. J. A., Goodeve, C. F., and Lythgoe, R. J., Proc. Roy. Soc. Lond., A. 156:158-170 (1936). Bleaching of visual purple.

39. Dartnall, H. J. A., Goodeve, C. F., and Lythgoe, R. J., Proc. Roy. Soc. Lond., A. 164:216-230 (1938). Bleaching of visual purple at low temperature.

40. Demoll, R., Die Sinnesorgane der Arthropoden: ihr Bau and ihre Function (1917). Braunschweig.

41. DuBois-Reymond, E., Untersuchungen über thierische Elelctrizität (1849). Berlin.

42. Exner, S., Die Physiologie der facettierten Augeh von Krebsen und Insekten (1891). Leipzig.

43. Fraenkel, G. S., and Gunn, D. L., The Orientation of Animals (1940). Oxford Univ. Press.

44. Friederichs, H. F., Ztschr. Morph. Ökol. Tiere. 21:1-172 (1931). Vision in Cicindelidae, Collembola larva and adult.

45. Fröhuıch, F. W., Ztschr. Sinnesphysiol. 48:28-165 (1913). General physiology of sense organs.

46. Fulton, J. F., Howell's Textbook of Physiology (1946). Philadelphia, Saunders.

47. Gernandt, B., and Granit, R., J. Neurophysiol. 19:295-301 (1947). Inhibition in the retina: vertebrate.

48. Graham, C. H., J. Cell. E Comp. Physiol. 2:295-310 (1932). Analysis of retinal electrical potential: Limulus.

49. Graham, C. H., and Hartine, H. K., J. Gen. Physiol. 18:917-931 (1935). Spectral sensitivity of single visual element: Limulus.

50. Graham, C. H., Kemp, E. H., and Riggs, L. A., J. Gen. Psychol. 13:275-294 (1935). Electrical response of eyes: vertebrate.

51. Graham, C. H., and Riggs, L. A., J. Gen. Psychol. 12:279-294 (1935). Visibility curve obtained electrically: rat.

52. Granit, R., J. Physiol. 77:207-239 (1933). Components of retinal action potential, mammal.

53. Granit, R., Nature 1 40:972-973 (1937). Electrical response of the frog's eye.

54. Granit, R., J. Physiol. 103:103-118 (1944). Inhibition in the retina: mammal.

55. Granit, R., J. Neurophy'siol. 8:195-210 (1945). Color receptors of the mammalian retina.

56. Granit, R., J. Physiol. 105:44-54 (1946). Excitation and inhibition in retina: cat.

57. Granit, R., Sensory Mechanisms of the Retina (1947). Oxford Univ. Press.

58. Granit, R., Munsterhjelm, A., and Zewi, M., J. Physiol. 96:31-44 (1939). Visual purple concentration and light sensitivity.

59. Granit, R., and Therman, P. O., J. Physiol. 83:359-381 (1935). Excitation and inhibition in the retina.

60. Granit, R., and Tinerman, P. O., J. Physiol. 91:127-139 (1937). Excitation and inhibition in the retina: vertebrate.

61. Granit, R., Themman, P. O., and Wrede, C. M., Skand. Arch. Physiol. 80:142155 (1938). Electrical spectral sensitivity measurements: frog.

62. Haig, C., J. Gen. Physiol. 24:735-751 (1941). Dark adaptation: human.

63. Hartline, H. K., Am. J. Physiol. 73:600-612 (1925). Electrical response of eyes.

64. Hartine, H. K., Am. J. Physiol. 83:466-483 (1928). Electric response of eyes: arthropods.

65. Hartuine, H. K., J. Gen. Physiol. 13:379-389 (1930). Visual dark adaptation: Limulus. 
66. Hartline, H. K., J. Cell. E Comp. Physiol. 5:229-247 (1934). Excitation studies in single visual clements.

67. Hartine, H. K., Cold Spring Harbor Symp. 3:245-250 (1935). Electrical studies of single visual elements: Limulus.

68. Hartline, H. K., Am. J. Physiol. 121:400-415 (1938). Responses of single optic nerve fibers: frog.

69. Hartline, H. K., J. Opt. Soc. Am. 30:239-47 (1940). liesponse of single visual elements.

70. Hartline, H. K., Harvey Lect. 37:39-68 (1941). Neural mechanisms of vision: review.

71. Hartline, H. K., and Granam, C. H., J. Cell. \& Comp. Physiol. 1:277-295 (1932). Response of single visual receptor: Limulus.

72. Hartune, H. K., and McDonald, P. R., J. Cell. E Comp. Physiol. 39:225-254 (1947). Adaptation in single visual elements: Limulus.

73. Hartline, H. K., and Wilska, A., Am. J. Physiol. 133:491-492 (1941). Origin of "off-response" in optic pathway: Limulus.

74. Hecht, S., J. Gen. Physiol. 1:545-558 (1919). Dark adaptation: Mya.

75. Неснт, S., J. Gen. Physiol. 1:657-666 (1919). Latency of photic response: Mya.

76. Неснт, S., J. Gen. Physiol. 1:667-686 (1919). Temperature effect on photic response: Mya.

77. Hecrit, S., J. Gen. Physiol. 2:229-246 (1920). Photochenical nature of photic response: Mya.

78. Неснт, S., J. Gen. Physiol. 2:337-348 (1920). Intensity effect on photoreception: Mya.

79. Hecht, S., J. Gerr. Physiol. 3:1-13 (1921). Bleaching of visual purple.

80. Неснт, S., J. Gen. Physiol. 3:285-290 (1921). Bleaching of visual purple.

81. Hecht, S., J. Gen. Physiol. 3:375-389 (1921). Action spectra: Mya.

82. Hecht, S., J. Gen. Physiol. 6:731-740 (1924). Bleaching of visual purple.

83. Неснт, S., J. Gen. Pliysiol. 11:657-672 (1928). Action spectra of photosensitive system: Pholas.

84. Hecirt, S., Physiol. Rer. 17:239-290 (1937). Chemical basis of vision.

85. Hecht, S., Chase, A. M., and Shlaer, S., Science 85:567-568 (1937). Molecular size of visual purple.

86. Hecht, S., Chase, A. M., Shlaer, S., and Haig, C., Science 84:331-333 (1936). Regeneration of visual purple.

86a. Heinbecker, P., Am. J. Physiol. 117:686-700 (1936). Electrical activity of Limulus heart ganglion.

87. Hess, W. N., J. Morph. 41:63-93 (1925). Photoreceptors of Lumbricus.

88. Holmes, G., and Lister, W. T., Brain 3:34-73 (1916). Cortical representation of vision.

89. Holmgien, F., Unters. Physiol. Inst. Univ. Heidelb. 2:81-88 (1872-82). Concerning visual purple and retinal currents.

90. Homann, H., Ztschrr. vergl. Physiol. 1:541-578 (1924). Function of ocelli: insects.

91. Honann, H., Ztschr. vergl. Physiol. 20:420-429 (1934). On the physiology of the spider's eye.

92. Housenolder, A. S., and Landahl, H. D., Math. Bioph. Mon. Ser. 1, 124 pp. (1945). Mathematical biophysics of the central nervous system.

93. Hyman, L., The Invertebrates: Protozoa through Ctenophora (1940). New York, McGraw-Hill.

94. Iмms, H. D., Textbook of Entomology (1935). London, Methuen.

95. JAHN, T. L., J. New York Ent. Soc. 54:1-8 (1946). Action spectrum measurements using the electroretinogram.

96. JaHN, T. L., J. Opt. Soc. Am. 36:76-82 (1946). Visual critical flicker frequency as a function of intensity.

97. JAHN, T. L., J. Opt. Soc. Am. 36:83-86 (1946). Brightness discrimination and visual acuity as functions of intensity.

98. Jahn, T. L., J. Opt. Soc. Am. 36:595-597 (1946). Color vision and color blindness. 
99. JahN, T. L., J. Opt. Soc. Am. 36:659-665 (1946). Kinetics of visual dark adaptation.

100. Jahn, T. L., Proc. lowa Acad. Sci. 54:325-343 (1947). Basic concepts in the interpretation of visual phenomena.

101. Jahin, T. L., and Crescitelli, F., J. Cell. \& Comp. Physiol. 12:39-55 (1938). Electrical response of eyes: grasshopper.

102. Jahn, T. L., and Crescitell1, F., J. Cell. E Comp. Physiol. 13:113-119 (1939). Electrical response of eyes: moth.

103. Jahn, T. L., and Crescitelle, F., Biol. Bull. 78:+2-52 (1940). Visual diurnal rhythm: Chlaenius.

107. Jahn, T. L., and Wulff, V. J., Proc. Soc. Exper. Biol. E Med. 48:656-660 (1941). Visual pigment migration: Dytiscus.

105. Jahn, T. L., and Wulff, V. J., Proc. Soc. Exper. Biol. E Med. 48:660-665 $(19+1)$. Flicker response contours: Dytiscus.

106. Jahn, T. L., and WlilfF, V. J., J. Gen. Physiol. 26:75-88 (1942). Origin of electrical response of eyes: grasshopper.

107. Jahn, T. L., and Wulfa, V. J., J. Cell. E Comp. Physiol. 21:41-51 (1943). Temperature effect on electrical response of eyes: grasshopper.

108. Jahn, T. L., and WulfF, V. J., Physiol. Zool. 16:101-109 (19+3). Visual diurnal rhythm: Dytiscus.

109. Jain, T. L., and Wulff, V. J., J. New York Ent. Soc. 56:109-117 (1947). Spectral sensitivity of electrical response of eyes: Dytiscus.

110. Jennings, H. S., Behavior of Lower Organisms (1906). New York, Columbia Univ. Press.

111. Jennings, H. S., Univ. Calif. Publ. Zool. 4:53-185 (1907). Behavior of starfish: Asterias.

112. Jolly, W. A., Quart. J. Exper. Physiol. 2:363-382 (1909). Electrical response of eyes: frog.

113. Kiesel, A., Sitzungsb. kais. Akad. Wiss., Wien 103:97-139 (1894). Physiology of compound eyes.

114. Kleinholz, L. H., Biol. Bull. 72:24-36 (1937). Rhsthmic color changes: Ligia.

115. Köttgen, E., and Abelsdorff, G., Ztschr. Psychol. u. Physiol. Sinnesorg. 12: 161-184 (1896). Retinal photosensitive pigments: fish.

116. Konlrausch, A., Handb. Norm. Path. Physiol. 12:1393-1496 (1931). Electrical responses of eyes: review.

117. KüHn, A., Ztschr. vergl. Physiol. 5:762-800 (1927). Color vision of honey bee.

118. Küнne, W., Chemische Vorgänge in der Netzhaut, in Hermann's Handbuch der Physiologie (1879). Leipzig. F. C. IV. Vogel, Vol. 3, p. 1.

I 18a. Light, V. E., J. Morph. Pliysiol. 49:1-42 (1930). Morphology and distribution of photoreceptors in Mya arenaria.

119. Ludvigh, E., Arch. Opthal. 20:713-725 (1938). The retinal scotopic visibility curve: man.

120. Lythgoe, R. J., J. Physiol. 89:331-358 (1937). Absorption spectra of visual purple and indicator yellow.

121. Lytigoe, R. J., and Quilliam, J. P., J. Physiol. 94:399-410 (1938). Relation of transient orange to visual purple.

122. Mansh, G., Camegie Inst., Wash., Publ. 32, pp. 65-8+ (1939). Electrical response to illumination of Valonia.

123. Mansi1, G., Carnegie Inst., Wash., Publ. 32, pp. 99-120 (1940). Spectral character of electrical response of Valonia.

124. Mast, S. O., Light and the Behavior of Organisms. (1911). New York, John Wiley \& Son.

125. MAst, S. O., Biol. Zentralhl. 34:6+1-674 (1914). Orientation to light: Euglena.

126. Mst, S. O., Biol. Rev. 13:186-244 (1938). Orientation of organisms to light: review.

127. Mast, S. O., in Calkin, Protozna in Biological Research (19+1). New York Columbia Univ. P'cess.

128. Mast, S. O., and Gover, M., Biol. Ball. 43:203-209 (1922). Relation of intensity of light and rate of locomotion: P'hacus and Euglena. 
129. Mast, S. O., and Stabler, N., Biol. Bull. 73:126-133 (1937). Relation between intensity, adaptation and locomotion: amocba.

129a. Menner, E., Zool. Jahrb., Abt. allg. Zool. u. Physiol. 58:481-538 (1938). The significance of the avian pecten.

130. Moon, P., and Spencer, D. E., J. Opt. Soc. Am. 35:43-65 (1935). Modified photochemical theory of vision.

131. Nikifonowsky, P. M., Ztschr. Biol. 57:397-412 (1912). Temperature effect on electrical response of eyes: frog.

132. Parker, G. H., Ergebn. Biol. 9:239-291 (1932). Movements of retinal pigment: review.

133. Peskin, J. C., J. Gen. Physiol. 26:27-47 (19+2). Regeneration of visual purple: frog.

134. Piper, H., Arch. Anat. 1. Physiol. 85-132 (1911). Electrical response of eyes: squid.

135. Pitts, W., and McCulloch, W., Bull. Math. Biophys. 9:127-147 (1947). How we know universals: The perception of auditory and visual form.

136. Polyak, S., Res. Publ. Ass. Nerv. E Ment. Dis. 13:535-557 (1934). Cortical projection of retina: monkey.

137. Polyak, S., The Retina (194I). Univ. of Chicago Press.

138. Prosser, C. L., J. Cell. \& Comp. Physiol. 4:363-377 (1934). Photoreceptor activity in crayfish.

139. Prosser, C. L., J. Comp. Neurol. 59:61-91 (1934). Photic orientation in earthworm, Eisenia.

140. Prosser, C. L., J. Exper. Biol. 12:95-104 (1935). Impulses in segmental nerves, earthworm.

140a. Prosser, C. L., J. Cell. \& Comp. Physiol. 21:295-305 (1943). Electrical activity of the Limulus heart ganglion.

141. Pütter, A., Handb. ges. Angenheibk. 2:1-424 (1912). Organogenesis of eyes.

142. Riggs, L. A., J. Cell. E Comp. Physiol. 9:491-510 (1937). Electrical determination of dark adaptation: frog.

143. Riggs, L. A., J. Cell. E Comp. Physiol. 15:273-283 (1940). Refractoriness in single visual element: Limulus.

144. Riggs, L. A., and Graham, C. H., J. Cell. \& Comp. Physiol, 16:15-23 (1940). Light adaptation in single visual elements: Limulus.

145. Riggs, L. A., and Graham, C. H., J. Cell. E Comp. Physiol. 26:1-13 (1945). Excitability of single visual elements: Limulus.

145a. Riggs, L. A., and Jounson, E. P., J. Exper. Psychol. 39:415-424 (1949). Electrical responses of the human eye.

146. Roeder, K. D., J. Cell. \& Comp. Physiol. 14:299-307 (1939). Electrical oscillations in eyes: grasshopper.

147. Salzmann, M., The Anatomy and Histology of the Human Eyeball in the Normal State (1912). Univ. of Chicago Press. 232 p.

148. Santschi, F, Rev. Suisse de Zool. 19:117-134 (1911). The mechanism of orientation in ants.

149. Schaefer, H., Elektrophysiologie II (1942). Vienna, Franz Deuticke.

150. Schneider-v. Orelli, M., Mitt. natur. Gesellsch. Bern, pp. 87-113 (1908). Investigation of the eye of Anableps tetrophthalmus.

150a. Snodgrass, R. E., Principles of Insect Morphology (1935). New York, McGrawHill.

151. Stephenson, J., The Oligochaeta (1930). Oxford, Clarendon Press. 978 p.

152. Talbot, S. A., and Marshall, W. H., Am. J. Opthal. 24:1255-1264 (1941). Visual localization in the cortex.

153. Taliaferro, W. H., J. Exper. Zool. 31:59-116 (1920). Photic orientation: Planaria.

154. Tansley, K., J. Physiol. 71:442-458 (1931). Regeneration of visual purple.

155. Therman, P. O., Act. Soc. Scient. Fenn. N.S.B., Tom II, No. 1 (1938). Neurophysiology of the retina: frog.

156. Therman, P. O., Am. J. Physiol. 130:239-248 (1940). Electrical response of eyes: squid. 
157. UexкüLl, J. v., Ztschr. Biol. 40:447-476 (1900). Effect of light and shade on the sea urchin.

158. Ullyot, P., J. Exper. Biol. 13:253-278 (1936). Photic behavior of Dendrocoelum: Planaria.

159. WaLD, G., Nature 140:545-546 (1937). Visual pigments of retinae: chicken.

160. WALD, G., Harvey Soc. Lect. 41:117-160 (1945-1946). Chemical evolution of vision.

161. WALD, G., and Clark, A. B., J. Gen. Physiol. $21: 93-105$ (1937). Visual adaptation in the human.

162. Wald, G., and Zussman, H., J. Biol. Chem. 122:449-460 (1938). Carotenoids of chicken retinae.

163. $W_{\text {ALLS }}, \mathrm{G}$. L., The Vertebrate Eye and Its Adaptive Radiation, Cranbrook Inst. of Science, Bull. 19. (1942).

164. W'EBER, H., Ztschr. Morph. Ökol. Tiere 23:575-753 (1931). Biology of Trialeurodes: Hemiptera.

165. WEISs, H. B., Ent. News 54:152-56 (1943). Insect response to colors.

166. Welsh, J. H., J. Exper. Zool. 56:459-494 (1930). Mechanics of visual pigment migration: Palaemonetes.

167. Welsh, J. H., Proc. Nat. Acad. Sci. 16:386-395 (1930). Rhythmic visual pigment migration: Crustacea.

168. Welsh, J. H., Biol. Bull. 70:217-227 (1936). Diurnal eye pigment movement: Anchistioides.

169. Welsh, J. H., Quart. Rev. Biol. 13:123-139 (1938). Diurnal rhythms: arthropods.

170. Welsh, J. H., J. Exper. Zool. 86:35-49 (1941). Retinal pigment migration: crayfish.

171. Wigglesworth, V. B., The Principles of Insect Physiology (1939). London, Methuen. 434 p.

172. Wrede, C. M., Skand. Arch. Physiol. 77:93-94 (1937). Electrical measurement of dark adaptation: frog.

173. Wulff, V. J., J. Cell. \& Comp. Physiol. 21:319-336 (1943). Photochemical and retinal electrical activity: grasshopper.

174. WulfF, V. J., J. Cell. \& Comp. Physiol. 32:31-44 (1948). Electrical response of eyes: frog.

175. WulfF, V. J., and Jahn, T. L., J. Cell. E Comp. Physiol. 22:89-94 (1943). Relation of electrical response of eyes to intensity: Dytiscus, Hydrasius.

176. Wulf, V. J., and Jahn, T. L., J. New York Ent. Soc. 55:65-83 (1947). Electrical response of eyes: Cynomya.

177. Zewi, M., Act. Soc. Scient. Fenn. N.S.B., Tom II, No. 4, 1939. Regeneration of visual purple. 


\section{Chemoreception}

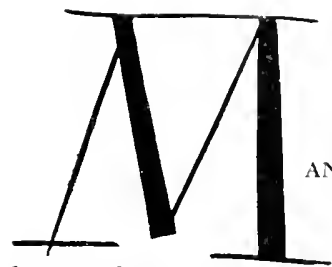

\section{INTRODUCTION}

ANY ORGANISMS utilize sensitivity to the chemical constituents of the environment in the detection of food, initiating the necessary orientation to food, and in regulating the feeding habits and the ingestion of foods, and in the avoidance of chemically unfavorable environments. Among insects and some other animal groups chemoreception plays an important part in reproductive behavior, i.e., attraction of male to female, and in regulating deposition of the fertilized eggs, Chemoreception is, therefore, an important adjunct to the other sensory activities of urganisms, all of which are essential for survival and propagation of the species.

The sense organs responsible for chemoreception have not been completely identified, but all the evidence seems to indicate three types which differ in sensitivity as well as in the role they play in animal orientation. The protozoans and sponges exhibit no specific chemoreceptors and it is generally agreed that, in these phyla, chemosensitivity is a consequence of the general property of irritability inherent in living matter. This general chemical sensitivity has been retained by the higher metazoans, including the vertebrates, although among the latter Parker ${ }^{49}$ has suggested that specific receptors, free nerve endings in the skin or mucous membranes, are involved in general chemical sensitivity. ${ }^{12}$ The outstanding characteristics of this general chemical sense are its low order of sensitivity and the negative avoiding reaction which is typically elicited.

In free living planaria, in insects, and in the vertebrates, there is excellent evidence for the existence of specialized chemoreceptors whose sensitivity is greater and whose role in organismic orientation differs from that of the general chemical sense. Koehler ${ }^{40}$ has published evidence indicating the existence of chemoreceptors located in the region of the auricular organs at the sides of the head of Planaria lugubris which serve to detect food quite remote from the organism, and of chemoreceptors located in the central anterior region of the head which function in feeding when the organism has reached the food. Chemoreceptors similar in function to those of the planarian have been described and in part identified for the insects and for vertebrates, and it is generally agreed that these chemoreceptors fall into two sensitivity groups: those receptors which orient the animal to food at a distance being the most sensitive, and those which initiate and regulate feeding in the proximity of food being less sensitive. Even the latter sense organs, however, are much more acute than those mediating the general chemical sense.

The classification of chemoreceptors into three groups, which is necessary 
for clarity of discussion, poses the problem of names. In man chemoreceptors are classified as olfactory sense organs, gustatory or taste sense organs, and the general chemical sense organs, in the order of their decreasing sensitivity. The application of this terminology to the chemoreceptors of other organisms not only has an anthropomorphic flavor but also leads to certain genuine difficulties. For instance, in man olfactory sense organs are commonly stimulated by air-borne substances, and taste receptors are commonly stimulated by substances in solution, whereas in aquatic organisms both types of receptors are stimulated by substances in solution. One may well ask if aquatic organisms can smell. Olfactory sense organs have also been referred to as distance receptors, as opposed to taste receptors, which are stimulated by contact. Yet the superficial taste buds of fishes may be stimulated by food held near the flank of the animal. Taste receptors, by connotation, are associated with feeding responses, but among the insects there exist chemoreceptors of equivalent sensitivity which function in oviposition. Dethier and Chadwick:00 have partially resolved the difficulty by calling this group (taste and ovipositor receptors) contact chemoreceptors. This name, however, implies the essential condition of contact for this group of chemoreceptors only, which is certainly not the case. The sensory end-organs mediating the general chemical sense are also contact chemoreceptors, and, indeed, even the olfactory sense organs are stimulated only by eventual contact of the stimulating agent with the sense cell.

In view of the fact that new names often serve to increase rather than to dissipate confusion, the human terminology will be adhered to in the following discussion, despite the above difficulties. The terms offactory, gustatory, and general chemical sense should, however, imply chemoreceptors of particular sensitivities rather than the particular sensations associated with these names.

\section{CHEMORECEPTION AND BEHAVIOR}

Chemoreception in the Lower Invertebrates. Protozoa are known to react positively to certain chemicals, negatively to others, and not to react to still others. For example, various solutions applied by means of a capillary pipette to a small portion of the surface of Amoeba proteus produce pronounced changes. Weak alkali causes the formation of a protuberance which develops into a normal pseudopod and results in locomotion toward the alkali. Weak acid or weak sodium chloride solution causes the formation of the protuberance, but not of a pseudopod. Stronger acid or salt solution may cause pseudopods to form on the side opposite the point of application.

In Paramecium the cilia may be reversed by the presence of certain chemicals in apparently the same manner that they are reversed by contact or by rapid changes in temperature or in osmotic pressure, and reversal is usually followed by turning toward the aboral surface and then by forward movement in a different direction. It is the first stage of the avoiding reaction. This results in aggregation in a slightly acid region if surrounding regions are slightly alkaline. Similar effects probably occur in response to changes in gas concentration and may account for many of the aggregations which are often observed when Protozoa are studied on a slide under a cover slip. The 
flagellates Bodo and Chilomonas, and many small ciliates, for instance, often aggregate at a given distance from the edge of the cover slip. Aggregation also occurs in oxygen gradients produced by other causes, e.g., by the removal of $\mathrm{O}_{2}$ from the environment.

The effectiveness of various ions on ciliary reversal of Paramecium has been studied by Mast and Nadler ${ }^{43}$ and by Oliphant. ${ }^{4 i}$ Monovalent cations are effective $\left(\mathrm{K}^{+}>\mathrm{Li}^{+}>\mathrm{Na}^{+}>\mathrm{NH}_{4}{ }^{+}\right)$, bivalent cations are not effective, and anions have little or no effect.

Sponges may react to certain irritant substances (strychnine, cocaine, ether, chloroform) by contraction so that the size of the orifice, and consequently the flow of chemical through to the animal, is reduced. Since there are no special sense cells and no nerve cells it seems that the contractile cells must be stimulated directly.

Coelenterates are quite sensitive to meat juices, which, if atded to the aquarium water containing sea anemones, may cause a great expansion and waving about of the tentacles. If meat juice is placed close to the tentacles the mouth may open. Some anemones, at least, can discriminate between food and inert bodies. Filter paper if soaked in meat juice may be accepted by the tentacles and then rejected either before or after ingestion. However, a hungry anemone may swallow even stones. Nevertheless, there is excellent evidence of chemoreception in the tentacles of most or all anemones and around the mouth of some, but this sensitivity is not present in the remainder of the body. Jellyfish also react to meat juices. It has also been noted ${ }^{38}$ that isolated oral arms of Aurelia and Cyanea give a normal grasping reaction to meat juice but not to sugars, starches, and glycogen.

The orientation to food by a pond planarian, P. lugubris, has been studied in considerable detail by Koehler. "1" The food is placed some distance from the animal and the initial reaction is one of increased random motion on the part of the animal, called orthokinesis (see Chapter 11, pages 386-387). The next phase of the reaction consists of crawling along a very convoluted path, the turning of the animal occurring sooner if it moves into a region of lower concentration of the stimulating agent or agents than if it moves into one of higher concentration. This type of activity, bringing the animal closer to the food in a very indirect manner, is called klinokinesis. When the food is a short distance away, i.e., $8 \mathrm{~cm}$., the planarian follows a path which leads straight to the food but it stops frequently and waves its anterior end from side to side, a type of behavior which indicates the comparison of intensities of stimulation at different times, called klinotaxis. When the animal is but several centimeters from the food, the path may be direct without any turn. ing of the anterior end. This type of behavior indicates the simultaneous comparison of intensities and is called tropotaxis. The olfactory receptors are concentrated in regions at the sides of the head, in the auricular organs, and the taste receptors are located in the center. Chemoreceptors are also distributed generally over the external surface of flatworms.

Most molluscs apparently locate food by means of their chemical senses, and terrestrial snails are reported to ignore food if they are separated from it by glass. The location of specific chemoreceptors has not been well demonstrated in most molluscan groups. The osphradia of most marine molluscs 
are probably sensitive to both chemicals and touch. Taste buds are described in the buccal cavity of some species, and ciliated papillae known as rhino. phores and regarded as olfactory sense organs are found on the cephalic tentacles of many land snails. The cephalopods have chemoreceptors in pitted papillae just below the eye on each side of the head.

Pecten reacts negatively to many substances, particularly to the juice of its natural enemy, the starfish. Chiton also reacts negatively to $\mathrm{N} / 500 \mathrm{HCl}$, $\mathrm{N} / 500 \mathrm{KOl}, \mathrm{N} / 160 \mathrm{KCl}$, and $\mathrm{M} / 1500$ picric acid. Octopus also is sensitive to weak acid, to quinine, and especially to musk.

Starfish have well developed chemoreceptors which are sensitive to meat juices and odors. A hungry starfish can be led about the floor of an aquarium by a piece of meat on the end of a pair of forceps. Asterias will carry small uninjurcel crabs on its back for an hour or more, but if the crab is crushed so that juices escape, the tube feet reach up immediately and pull the crab toward the mouth. Holothurians are sensitive to various chemicals, and the order of decreasing effectiveness for one series is as follows: $\mathrm{HCl}$, atropine, acetic acid, $\mathrm{KOH}, \mathrm{KCl}$, maltose, acetamid, and glycerin.

Annelids have fairly well developed chemical senses, and there also seems to be some differentiation into organs of taste and smell. Darwin demonstrated that earthworms could distinguish between green and red cabbage, onion leaves and those of either cabbage or horseradish, celery leaves and those of cabbage and parsnip, and also between leaves of carrots and those of celery. These experiments, and others, seem to be explicable on a basis of taste, probably localized in the taste sense organs of the buccal wall and pharynx. Darwin believed that selection in certain instances (as between onions and cabbage) might have been aided by odors.

There are also, in the cuticle of the body wall of Lumbricus, sense organs which are very similar in structure to vertebrate taste buds and which seem to serve the same function. The earthworm is sensitive to quinine, and the distribution of susceptibility in various parts of the body parallels the distribution of the sense organs: the anterior end reacts to 0.04 per cent, the posterior end to 0.03 per cent, and the region just behind the clitellum to 0.2 per cent.

The oligochaete Eisenia reacts to acids and is reported to be able to discriminate between $\mathrm{H}_{2} \mathrm{SO}_{4}$ and either $\mathrm{HCl}$ or $\mathrm{HNO}_{3}$. In contrast to the situation in human taste, in Eisenia the cation of salts seems to be more important than the anion. Eisenia also reacts negatively when xylene, turpentine, or ether is held near the anterior end, but not when the substance is held near the posterior end. Therefore, it seems that Eisenia has two types of chemosensitive receptors.

Chemoreception in the Arthropods. The Insects. Among the invertebrate animals chemoreceptors have achieved a great degree of structural and functional differentiation in the insects. In the terrestrial insects and those aquatic species which are occasionally terrestrial, there is good evidence for the existence of (wo types of chemoreceptors: the olfactory receptors, which react to very low concentrations of compounds that are volatile at ordinary temperatures; and the taste receptors, which react on contact with liquids or substances in solution that may or may not be volatile at moderate tempera- 
tures. In the aquatic species of insects the subject of two types of chemoreceptors is controversial, and the problem is well reviewed by Dethier and Chadwick. 20 There is good evidence for the existence in insects of a third type of chemoreceptor which is excited at high concentrations, and the re. sulting response is usually an avoiding reaction. These receptors presumably mediate the general chemical sense previously mentioned (page +47 ).

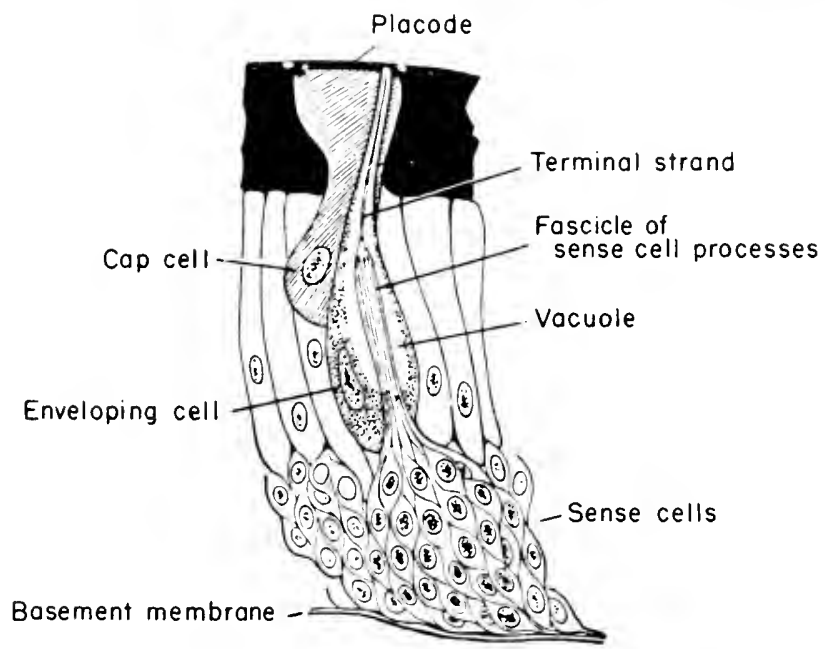

Fig. 1+4. Diagram of a sensillum placodeum of the bee, rertical section. From Snodgrass.:
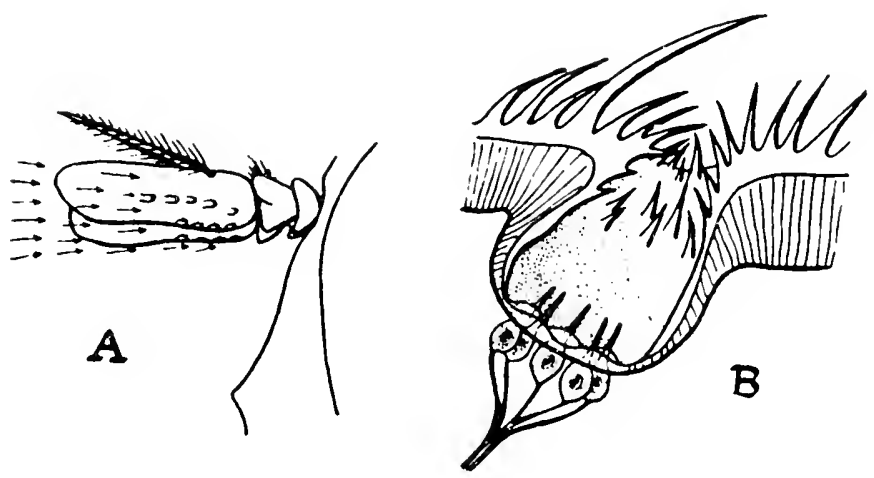

Fig. 145. A, The erected antennae of a Hy during flight, exposing olfactory pits to the air stream. B, Diagram of an olfactory pit showing the rod-like endings of the sense cells. (After Lieberman ${ }^{42}$ from Wigglesworth, ${ }^{6: 2}$ ).

structure. Functionally, the chemoreceptors of the insects tend to simulate the chemoreceptors of the vertebrates, but structurally they are much more complex. Olfaction among insects is mediated by three types of sense organs: pore plates (sensilla placodea), thin-walled pegs or cones (sensilla basiconica), and thin-walled pegs or cones sunken in pits (sensilla coeloconica), illustrated in Figures $1+4$ and 145 . These olfactory receptors all pos- 
sess a covering of cuticle which is partially or completely thin-walled; they are innervated by a group of bipolar sense cells; and each possesses minute refrigerant bodies, situated on the distal process of the neurones (Fig. 145). The olfactory chemoreceptors are commonly found on the antennae, but they also occur on the maxillary and labial palps or their homologues. The receptors on the antennae are generally conceded to be most sensitive, whereas those on the palps are used for the detection of strong odors. ${ }^{20}$ The morphological nature of the chemoreceptors which normally function in feeding and oviposition among the insects is uncertain, and the pertinent data are reviewed by Dethier and Chadwick. ${ }^{2}$ Data advanced by Frings and O'Neal ${ }^{30}$ indicate that chemoreception in the labella and the legs of the horsefly, Tabanus, is mediated by the medium-sized thin-walled hairs or by undiscovered sense organs in the integument beneath these hairs. Dethier ${ }^{16}$ has advanced evidence that long and short thin-walled spines located on the surface of the pre-oral cavity of lepidopteran larvae may function in contact chemoreception. Sense organs functioning in contact chemoreception have been demonstrated on the antennae of bees and ants, the mouthparts or adjacent surfaces of many species, the distal segments of the legs of bees, flies, and adult Lepidoptera, and the ovipositor of ichneumonids and braconids, and of Gryllus. ${ }^{20}$ Regarding the sense organs which mediate the common chemical sense, neither their structure nor their distribution is known.

BEHAVIOR. The role played by the chemoreceptors in the behavior of insects is in itself a fascinating story, which is here only touched upon. Naturally occurring odors assist certain insects in recognizing and locating their mates, in finding the proper oviposition site, in recognizing their fellows, and in finding food. The fabulous story of the ability of certain moths (e.g., the gypsy moth and others) to attract the males of the species from distances greater than two miles by virtue of the liberation of an attractive odor from the scent organs is widely known. That it is an odor which attracts the males is attested by the fact that males deprived of their antennae do not orient to freshly pupated females, and by the fact that males, in the absence of the female, will attempt copulation with the excised scent gland, with a piece of blotting paper previously touched to the scent gland, or even with a male which recently copulated and retained some of the female sex scent. That these odors can orient males over such great distances staggers the imagination. Methyl eugenol is used to collect male oriental fruit flies from at least a quarter mile radius. The effect of odors in attracting gravid females to optimal oviposition sites has been shown for the parasitic I tymenoptera, a variety of Diptera, and the phytophagous insects. Not only do odors attract the females but often they actually induce oviposition. The parasitic 1 tymenoptera are particularly outstanding in the ease with which they locate larval hosts buricel sev. ril centimeters in wood. The female phytoplagous insects in gencral are oriented to lay their eggs on those plants which will serve as food for their larvac. The role of odors in the recognition of nest mates has been demonstrated. Ants which have been washed with water or smeared with body juices of other ants are treated as intruders, and ants which have been deprived of their antennac show little tendency to fight ants of other genera. Social insects like bees live in a workl of odors; they distinguish not 
only individual odors but also caste odor, hive odor, sting odor, and wax odor." The most elemental and important chemical attractants are those which orient insects to foods. These attractants are, for the most part, odors. Often these odors are only sign posts indicating the presence of foods which do not contribute to the odor. Thus the beetle, Creophilus, orients to odors of decaying meat only to feed on the fy maggots developing there; most caterpillars are oriented to their food plants by the odors of essential oils, yet they derive no nutritional benefit from these oils. Once insects are oriented in the vicinity of food, other appropriate stimuli may be necessary to initiate feeding and for the continuation of feeding. Thus, wireworms aggregated in the vicinity of food fail to initiate eating, and larvae of Anosia plexipus, the milk weed feeder, will orient to strange leaves coated with milk weed latex but will spit out the first mouthful and will starve rather than eat the strange food. ${ }^{16}$

Many insects possess a high degree of sensitivity to water vapor, and their behavior is affected by relative humidity (Ch. 2). In many respects this response to water vapor resembles that to olfactory stimulation. The sense organs necessary for orientation to water vapor are the pegs and pits located on the antennae. 20 The acuity of this sense is surprising: the mosquito Culex fatigans can distinguish differences of 1 per cent relative humidity near saturation, and wireworms can distinguish between 100 and 95.5 per cent relative humidity. The response of organisms to water vapor is not always constant and may vary with the water balance of the animal.

Other Arthropods. Crustacea possess a definite chemical sense which in decapods seems to be largely localized in the mouth appendages and the antennules. The external ramus of the antennules is well supplied with basiconical hair organs variously known as aesthetascs, olfactory clubs or tubes, and tubules of Leydig. Other parts of the body are also chemoreceptive, even the hard carapace, which is supplied with numerous pores, at the base of which are sensory cells.

Limulus apparently has contact chemoreceptors in the mandibles and chelae, and distance chemoreceptors in a wartlike bud anterior to the mouth.

There is a considerable amount of controversial literature concerning the regional distribution of chemoreceptors in spiders. Hlowever, evidence indicates that they are widely distributed over the body, especially in the palps, the first two pairs of legs, and possibly in the mouthpirts.

Chemoreception in the Vertebrates. A high degree of structural and func. tional specialization of cell groups to mediate the senses of olfaction, taste, and the common chemical sense has been achieved by many vertebrates. In all vertebrates, with the possible exception of the reptiles, birds, and man, olfaction and taste are probably necessary for survival. The common chemical sense probably serves organisms in a manner analogous to the pain sense, and therefore has survival value.

structure. The morphology of chemoreceptors is much less diversified in vertebrates than in the insects, and the sense organs have been studied in detail in many organisms. The sense cells which mediate olfaction in man are neurones located in the upper part of the nasal carity. The olfactory endorgans cover a small area (2.5 square centimeters for each nostril of man) 
in the superior part of the nasal cavity. In deer the area is much greater. During normal respiration of man most of the air does not pass directly over the olfactory organ, but this is supplied with air (and odors) primarily by eddy currents (Fig. 1+6). The secluded position of the olfactory epithelium seems to be important in preventing desiccation of the protoplasmic processes of the olfactory cells. However, in sniffing, the volume of air passing the olfactory organ is sreatly increased. In dogs more of the air passes directly over the olfactory epithelium. Jacobson's organ or the naso-vomerine organ, located in the nasal septum, is well developed in some fishes and reptiles and is believed to function as an accessory olfactory organ.

l listologically, the olfactory epithelium (Fig. 147) consists of the olfactory receptors (primary neurones) embedded in columnar epithelial cells, some of which contain a yellowish-brown pigment and give the organ a

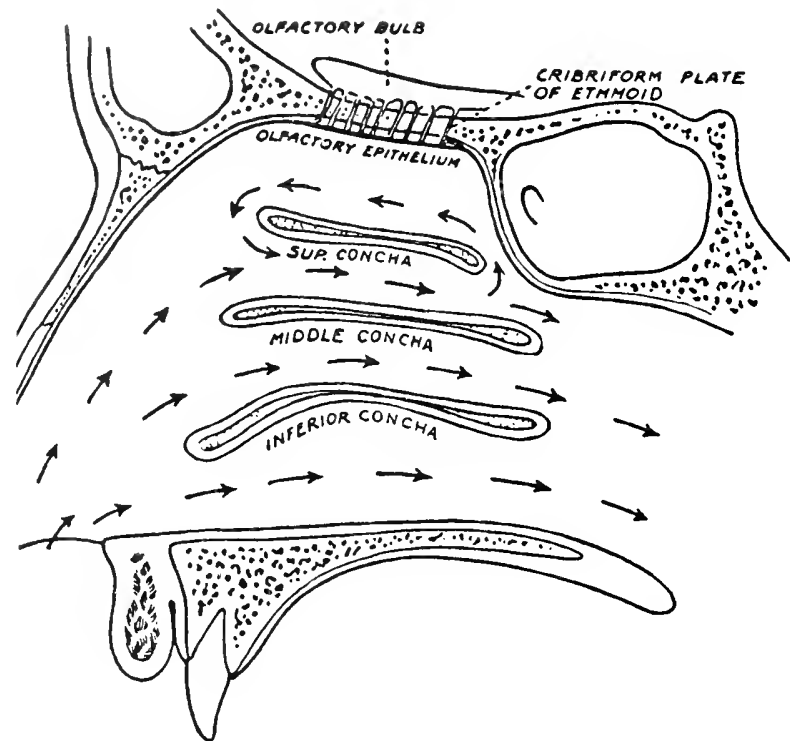

Fig. 146. Diagram of the right nasal cavity of man, showing the direction of air flow. Edely currents around the superior concha are created by sniffing and bring the inspired air inte contact with the olfactory epithelium. After Evans" from Fulton:"

yellowish appearance macroscopically. The primary neurones are bipolar, with the dendrite forming the receptor proper and the cell body performing the function of "ganglion cells" in supplying the fibers (unmyelinated) of the offactory nerve. The olfactory epithelium also contains free nerve endings of the trigeminal nerve which function as part of the common chenical sense receptor. The olfactory organ is the simplest sense organ of the body and is also very sconsitive. Stimulation probably oceurs through the hairlike processes, and the sense cell, serving as both receptor and conductor, transmits this to the brain. Compared with the eve and ear, the olfactory organ is extremely simple.

The sense of taste, or gustation, is mediated by sense organs located in the mouth, although in fishes they are frequently found outside of the mouth 
proper. In adult man the sustators cells are limited to the sides and top of the tongue and to the epiglottis; in children thet are also present in the cheeks. The tongue of man has four types of papillice: I Filitom papillace. which are conical projections corering the whole upper surface and tip and borders of the tongue. These are not concemed in taste but are more highly developed in cats, in which they are used as a rasp for cleaning meat from bones. (2) Fungitorm papillac, which resemble buttom mushrooms in shape,
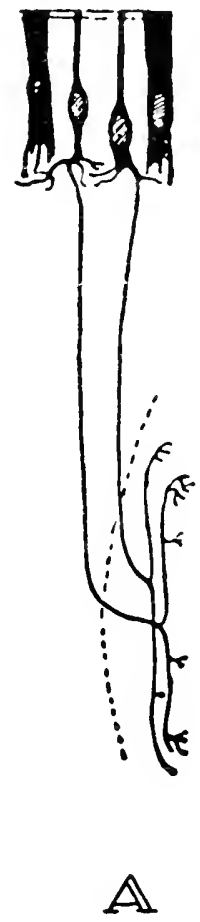

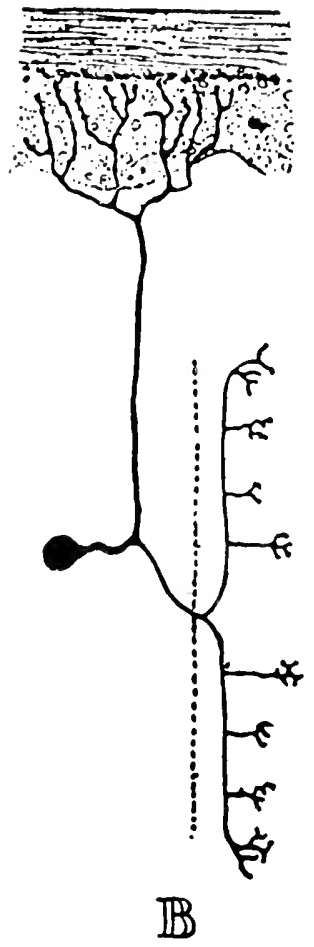

Fig. 147. A, Diagram of a primitice sensory neurone. B, lipical rertebrate sensory neurone. The human ollactory neurones possess a peripheral cell body and resemble the type shown in $A$. Human taste neurones possess a centrally located cell body and resemble the type shown in $B$. After Cajal from Fulton."

are less numerous and are scattered over the front. upper surface. These usually contain taste buds. (3) Circumvallate papillae, which are seren to ten in number in man, are larger than the two previously mentioned, and lie toward the back of the upper surface. Each papilta is a flat mound surrounded by a groove forming a moat, both sides of which contain taste buds. (4) Foliate papillate, restigial in man, which form rertical ridges at the edge of the back of the tongue. Each taste bud consists of a group of a dozen or more elongated cells, some of which have minute hairlike processes. The taste buds are embedded in the sides of the papillae, where they are sunk slightly below the surface, and the opening of the small carity which is thereby formed is known as the gustatory pore. The hairlike processes of the sense cells project through the pore into the cavity between the papillae. 
The general chemical sense in vertebrates is obviously a development of general irritability of all mucous membranes in man and of the entire external surface in aquatic animals. The receptors are probably free nerve endings.

BEHAVIOR. In fishes olfaction is confined to the olfactory pits (only one in the cyclostomes-monorhines), which in some fishes, e.g., the hammerhead shark, are widely separated. In sharks, rays, and dipnoans the nasal openings are on the ventral side of the head, and in some cases the nasal pits also have an opening into the mouth. The teleosts have dorsally located nasal pits which do not open into the mouth, i.e., the water current is into the pit and then back out (Fig. 148). The taste organs of fish are widely distributed. They occur in the palatal organ of the mouth, the soft palate; in a ring around the opening of the pncumatic duct (in some fishes) which leads to the swim blaclder; on the barbels; over the entire surface of the body; and on the pectoral and dorsal fins.": Fishes locate food by means of gustatory
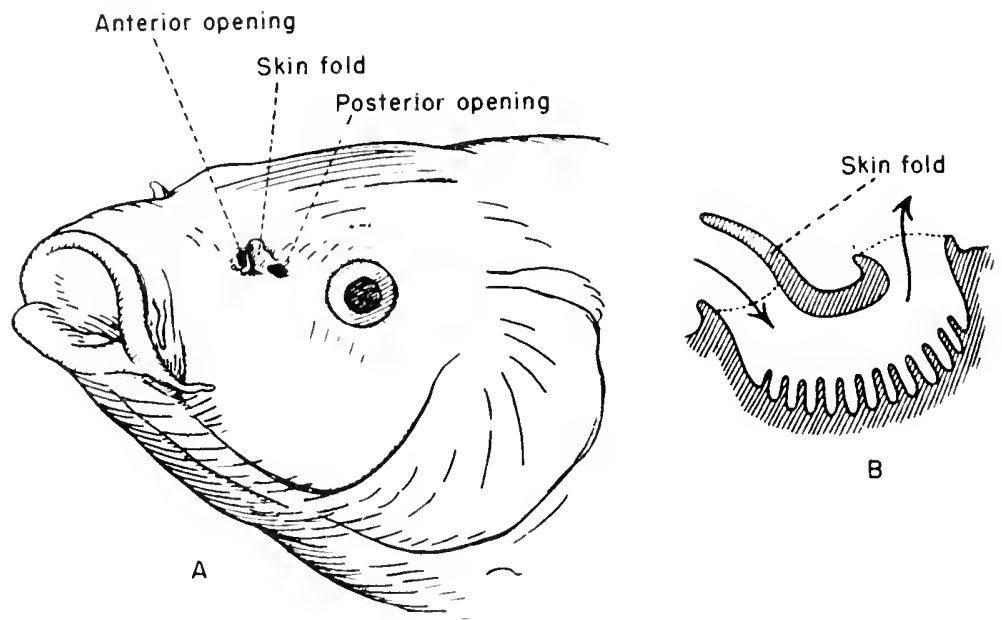

B

Fig. 148. A. The head of a bony fish, showing the nasal pit. $B$, Longitudinal section through the nasal pit, with arrows indicating the direction of water flow.

and/or olfactory sense organs, and it is claimed that some fishes, if eleprived of their sense of taste, would starve to death in the presence of food. Catfishes will snap at food or cotton soahed in meat juice held at their flank, but will not do so if the nerve supplying the taste buds on the flanks is cut.

The amphibia have taste buds in the mouth and olfactory organs in the nasal chambers which open both externally and into the mouth. The senses of taste and smell seem to be at about the same stage of development in ampluibia as in fishes, but they are not nearly so important in feeding in amphibia as they are in fishes. The entire body surface, as in fishes, seems to be quite sensitive to irritants, as seems to be true of all aquatic animals. Among reptiles the sense of smell is not well cleveloped, and the sense of taste seems to be more highly developed in turtles than in snakes and alligators.

Birds, in general, hare relatively poor chemical senses and depend largely on vision for knowledge of their environment. The olfactory apparatus of 
most birds is similar to that of reptiles. The nostrils are narrow, horn encised, and dry. The tongue papillace contain taste-sensitive nerve endings, but they are not well developed. The presence of asafoetida, anise, oil of lavender, or prussic acid has no effect on the selection of food by turkers. Neither do bitter and sweet substances affect selection of food among herring gulls. Canaries do not object to bitter aloes, but they do prefer sugarr solution to plain water and refuse salt or sour solutions at concentrations above the human threshold.

Among mammals the sense of taste seems to be well developed, but there is little definite information available. Nice apparently can taste saccharin, quinine, salt, and acid, and, as far as the evidence inclicates, seem to have about the same preferences as man, i.c., they react most positively to sweet and most negatively to bitter. Olfaction among lower mammals has been studied extensively only in rockents and dogs. Rats and other rodents either have a very poor sense of smell or else they learn with great difficulty when olfactory cues are used in training. All evidence indicates that their olfactory acuity is far bclow that of man.

In dogs, however, there is a vast amount of cxcellent evidence of an olfactory sense far supcrior to man's. Dogs are capable of following human and other animal trails and of selecting objects which have been handled by their master. Dogs can also be trained to detect underground fungi (e.g., truffles) with great accuracy. In trailing there are several possible types of olfactory cues. These are: (1) earth odor from the compression and consequent stronger vaporization; (2) plant odor from destroyed vegetation; (3) odor traces from shoes and shoe polish; (4) odor traces from dccaving animal or other organic matter; and (5) body odor of a specific individual.

\section{THE PHYSIOLOGY OF CHEMORECEPTION}

Sensitivity of Chemoreceptors. Olfactory and gustatory chemoreceptors and those mediating the common chemical scuse can be differentiated on the basis of their sensitivity. Parker and Stabler"in have demonstrated that in man the threshold concentrations of ethanol required for stimulation of the olfactory, gustatory, and common chemical sense organs are $0.000125 \mathrm{M}, 3 \mathrm{MI}$ and 5 to $10 \mathrm{M}$. Comparable values have been determined as rejection thresholds in two species of flies. As a gas ethanol is rejected by the housefly at $0.005 \mathrm{M}$ and, in solution with $0.1 \mathrm{M}$ sucrose in contact with the tarsal receptors of blowflies, it is rejected at $3.2 \mathrm{M}$ concentration. ${ }^{20}$ These results indicate that comparison of the stimulating effectiveness of the same compound on the three types of chemoreceptors of the same or closely related species permits a convenient classification. However, on examination of the relative sensitivities of taste and smell, when the thresholds for the most familiar smell and taste substances are compared and expressed in terms of molecules per cubic millimeter, a continuous spectrum and overlapping stimulating effectiveness is observed. ${ }^{35}$

Olfaction. It is generally recognized that the sense of olfaction is much more acute than the sense of taste. Man can detect the odor of methyl mercaptan at an approximate concentration of $9 \times 10^{-13} \mathrm{M}$. One milligram of skatol, to which man is even more sensitive, would make a hall, 500 meters 
long, 100 meters wide, and 50 meters high, very unpleasant. It is estimated that man, with one sniff of air containing mercaptan at the threshold concentration, would draw in about $1 \times 10^{10}$ molecules. Consideration of the fact that there is only one molecule of mercaptan to every fifty thousand million molecules of air leads to some appreciation of the sensitivity of olfaction. However, it should be noted that the surface activity of mercaptan and of odorous materials in general is such as to enhance the possibilities of increasing the concentration at the receptor surface.

Among insects comparable sensitivity of olfactory sense organs has not been demonstrated, although the fact that the odors (lipoid, protein, or ester) emanating from the body of the female gypsy moth attracts male gypsy moths from distances greater than two miles attests to a great sensitivity. Threshold measurements with other insects show that the dung beetles (genus Geotrupes) respond to the odor of skatol at concentrations of $2.3 \times 10^{-8} \mathrm{M}$ and the tent caterpillar, Malacosoma americana, is repelled by a mixture of benzaldehyde at a concentration of $4.1 \times 10^{-7} \mathrm{M}^{6}{ }^{61}, 1 \times$ These values represent the response threshold level, and it is a safe assumption that the threshold of individual sense organs would be significantly lower. Von Frisch, $31,32,35$ in his classic experiments conditioning the feeding response of bees to the odors of essential oils, demonstrated that the minimum perceptible odor is approximately the same for the bee as for man (Table 64).

Gustation. One of the most effective taste substances for man is quinine hydrochloride, which elicits an extremely bitter taste that can be detected

TABLE 6t. COMPARISON OF OLFACTORY THRESHOLDS OF CERTAIN INSFCTS AND MAN (from Dethier and Chadwick"')

\begin{tabular}{|c|c|c|c|}
\hline Compound & $\begin{array}{c}\text { Man } \\
\text { (Recognition } \\
\text { Threshold, } \\
\text { mgm./l.) }\end{array}$ & \multicolumn{2}{|c|}{$\begin{array}{c}\text { Species of Insect (Threshold of Respons } \\
\text { mgm./l.) }\end{array}$} \\
\hline $\begin{array}{l}\text { Skatol } \\
\text { Indol } \\
\text { Benzene } \\
\text { Cyclohexane } \\
\text { Ethanol } \\
\text { " }\end{array}$ & $\begin{array}{l}0.000,000,4 \\
0.0053 \\
0.25 \\
5.76\end{array}$ & $\begin{array}{l}\text { Geotrupes sylvaticus } \\
\text { G. vernalis } \\
\text { Habrobracon juglandis } \\
\text { " " " } \\
\text { Musca domestica" }\end{array}$ & $\begin{array}{l}0.003-0.009 \\
0.003 \\
0.5-3.0 \\
3-5 \\
5-20 \\
230\end{array}$ \\
\hline $\begin{array}{l}\text { Ammonia } \\
\text { Benzaldehyde }\end{array}$ & 0.035 & $\begin{array}{c}" 1 \\
\text { Pieris rapac }\end{array}$ & $\begin{array}{l}0.04 \\
1 \text { (rejection) } \\
0.058\end{array}$ \\
\hline
\end{tabular}

in concentrations of alpproximately $1.5 \times 10^{\mathrm{7}} \mathrm{M}$. Other substances which most effectively stimulate the other tastes are: hydrochloric acid, $1.25 \times$ $10^{-3} \mathrm{M} ; \mathrm{NaCl}, 9 \times 10^{-3} \mathrm{M}$; and sucrose, $2 \times 10^{-2} \mathrm{M}$. The sensitivity of insect contakt chemereceptors is equal to or greater than those of the human (Table 65). Aquatic beetles may be conditioned to quinine hydrochloride at concentrations of $1.25 \times 10^{-6 i}$ M. Stimulation of the tarsal receptors of some buttertlies with sucrose at $9.8 \times 10^{-6} \mathrm{M}$ and of some flies with sucrose at $3.9 \times 10^{-5} \mathrm{M}$. elicits a response. 
TABLE 65. COMPARISON OF TASTE THRLSHOIDS (from Dethier and Chaduicken)

Compound

Threshold Concentrations

Sucrose man, $0.02 \mathrm{~N}$; bee, 0.06-0.125 M; butterfly (Pyrameis), average ca. 0.01 $\mathrm{M}$, in slarvation as little as $8 \times 10^{-1} \mathrm{M}$, or for Danans, $9.8 \times 10^{-1}$ M; horsefly (Tabanus), 0.005-0.11 M1.

$\mathrm{NaCl}$ man, $0.009 \mathrm{M}$; bee, rejects ca. $0.2+\mathrm{Nl}$ in $0.5 \mathrm{~N}$ sucrose; various cater pillars reject at $0.2 \mathrm{M}$, while others accept over the full range up to and including $5.0 \mathrm{Ml}$.

$\mathrm{HCl} \operatorname{man}, 0.00125 \mathrm{M}$; bee, rejects $0.001 \mathrm{M}$ in $1.0 \mathrm{M}$ sucrose; varjous cater pillars reject at $0.01-0.2 \mathrm{M}$.

Quinine man, $1.5 \times 10^{-7} \mathrm{M}$; bee, rejects at $8 \times 10^{-4} \mathrm{M}$ in $1.0 \mathrm{M}$ sucrose; various eaterpillars reject at $0.002-0.033 \mathrm{M}$; aquatic beelles were conditioned to respond to $1.25 \times 10^{-13} \mathrm{M}$.

Not all gustatory end-organs of insects are equivalent in sensitivity. Thus, in the horse fly, the arerage threshold for sucrose is $0.021 \mathrm{M}$. for the labella, and $0.060 \mathrm{M}$. for the tarsi. The receptors on the antennae of bees are more sensitive than those on the legs, and those on the proboscis are more sensitive than those on the antemae.

Pumphrey, $: "$ using the frog's tongue and the technique of recording electrically from the appropriate nerve, demonstrated a reduction of sensitivity, after intense stimulation which lasted for sereral minutes. Nthough adaptation of gustatory receptors is slow, it is apparently of sufficient magnitude to produce changes in sensitivity. In addition to these variations in sensitivity, it has been reported that the sensitivity of the same sense organs may vary from day to day.

The Common Chemical Sense. The concentration of ethanol necessary to stimulate the sense organs lecated in the mucous membranes of man is 5 to $10 \mathrm{Ml}$. No comparable figures for the inseces are arailable. Numerous experiments show, howeser, that substances which attract insects at one concentätion mas repel at a higher concentration. Indeed, most substances are optimally attractive at a particular concentration and decrease progressively as the concentration increascs (Fig. 149). Substances which are repellent at low concentrations usually elicit a more violent repellent response at high concentrations. These changes in response suggest the participation of a new group of receptors, perhaps those nediating the common chemical sense, at these high concentrations.

Modalities of Chemoreception. Olfactory Receptors. The sense of smell in man not only is the most sensitive of the chemical senses but also gives rise to the greatest variety of sensations. It has been estimated that the odors which a normal person can discriminate number between one thousand and four thousand. All efforts to reduce the olfactory sensations of man to some system, whereby mixtures of certain fundamental odors will reproduce all odors of experience, have met with notable failure. These odors are necessarily classified on a strictly subjective basis. It is hoped that such a classi- 


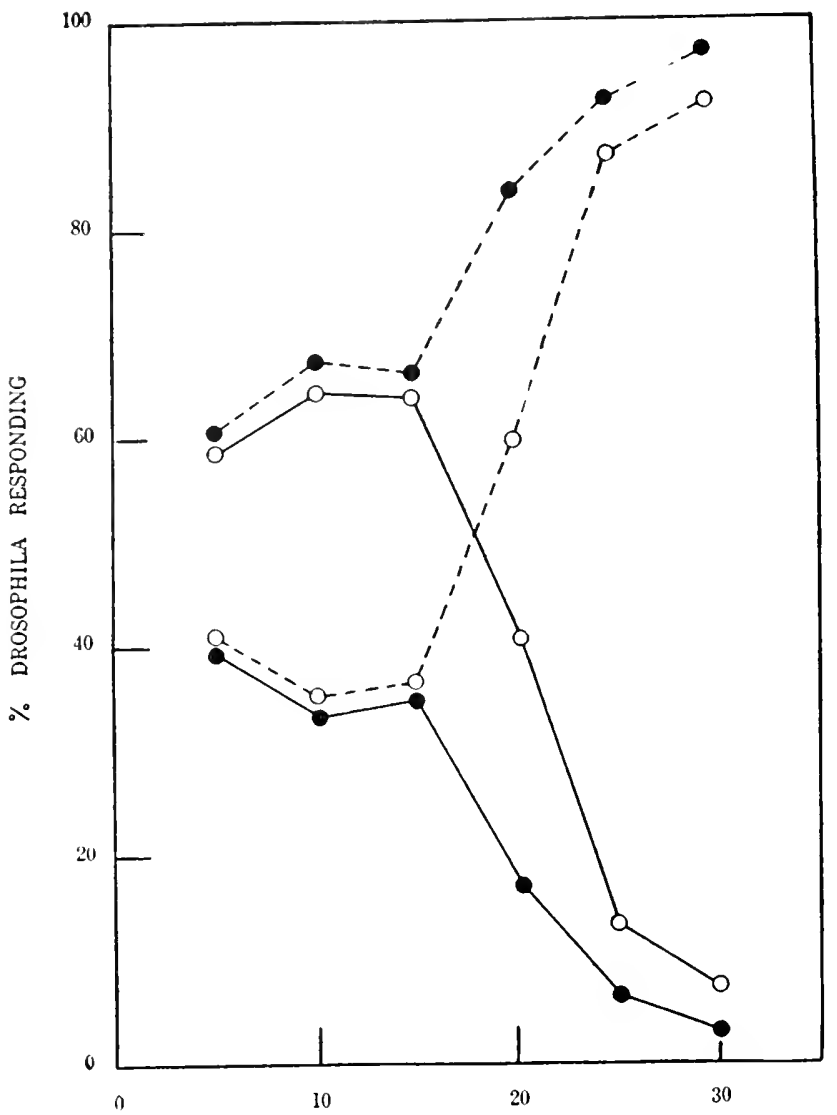

Fig. 149. Curves illustrating the relation between the response of Drosophila and the eoncentration of ethyl alcohol. Solid circles, per cent males responding; open circles, per cent females responding; solid lines, the per eent males and fenales attracted; broken lines, per cent males and fernales repelled. After Reed, from Dethier. ${ }^{\text {is }}$

fication may erentually be replaced by one which is objective and based on some chemical or physical property of the stimulus. One of the earliest and still acceptable subjective classifications of odors is that of Zwaardemaker, who divided odors into nine classes, with two or more subdivisions:

\section{Class}

1. Ethereal

2. Aromatie

(1)

3. Balsomic or Fragrant

4. Anbrosial

5. Alliaccous

6. Empyreunatic

7. Caprilic

8. Repulive .............. (a) Deadly nightshade, (b) Bed-bug

9. Nauseating or Fetid .......... (a) Carrion, (b) Feces

\section{Subdivisions}

(a) Fruit, (b) Beeswax, (c) Ethers

(a) Camphor, (b) Cloves, (c) Lavender,

(d) Lemon, (e) Bitter almonds

(a) Flowers, (b) Violet,

(c) Vanilla and coumarin

(a) Amber, (b) Musk

(a) Sulphuretted hydrogen,

(b) Arsine, (c) Chlorine

(a) Roast collec, (b) Benzene

(a) Chcese, (b) Rancid fat 
It has been suggested that there are nine types of sense cells, cach sensi. tive primarily to one of these classes of ofors. Proof of the existence of these nine types would constitute a great step forward in the study of the theory of olfaction. There is some evidence that during recoven frem anesthesia the ability to smell odors of class 6 (empreumatic) recovers lirst and that for odors of class 8 (repulsive) recosers last. It has also been found that fatigue of the olfactory organ $t$ an octor of one class (or subclass also causes reduced sensitivity to other members of the same class (or sulfelass). 'The best evidence of this nature was obtained by Olmma for the five subclasses of class 2. For instance, be found that camphor, cucalyptel, and eusenol, members of the same subelass. produced perfect fatigue for each other, but only partial fatigue for citrol and safol which are in the same class but in a different subclass.

The olfactory capabilities of the insect chemoreceptors have not been exhaustively explored. Von Frisch $: 3$ trained bees to find food in one con-

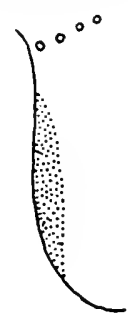

SOUR

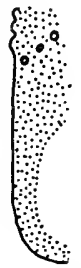

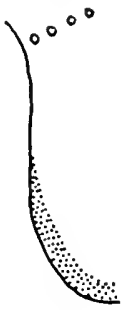

SALT

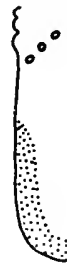

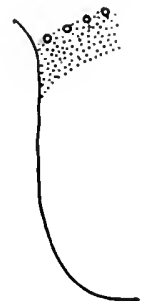

BITTER

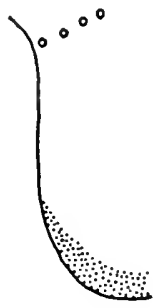

SWEET

Fig. I 50. Diagrams of the right half of the tongue of the human (above) and of the cat (below), indicating the areas of lowest threshold for the four primary tasten, deter mined by punctiform exploration (human) and electrical recording (cat). Afeer tlanig and Pfaffmann," from Fulton."3i

tainer from which emanated the odor of one essential oil and related com pounds. This odor was then presented simultaneousts with other odors in different containers and the frequence of the visits te each was recorded. Of the forty-seven substances tested all crude preparations of essential nils). three in addition to the training odor were attractive. The four essential oils were all distilled from citrus fruit and contained one common substance, limolene, and had similar odors as judeded be man. ('omparison of pure volat tile organic compounds showed that bees react in a manner similar to man. Nitrobenzene and benzaldehrde, although of widely diverse chemical strueture, are confused as one by man, as well as by the bee; methyl cster of anthranilic acid and beta naphthol methyl ether, which are nearly identical 
in structure, are easily differentiated by man and by bees. As far as is known, olfaction among insects is similar to olfaction in man.

Gustatory Receptors. It is generally agreed that there are four fundamental tastes: sweet, sour, salt, and bitter. The taste buds mediating these four tastes are more or less localized in certain regions of the tongue, as shown in Fig. 150. The taste buds sensitive to sour are on the back lateral surfaces, those for sweet and salt near the tip, and those for bitter toward the back. The distribution is somewhat irregular, and there is a considerable overlapping of the areas. However, when this separation of taste buds of different sensitivities is compared with the thorough mixture of the rods and cones of the eyc, or of the olfactory cells, the simplicity of the problem of analyzing taste, compared with that of analyzing color vision or olfaction, is astounding. Here we have four definite sensations which are more or less localized in four different regions of the tongue, differing in position by as much as several centimeters, and which we can stimulate separately by merely placing the stimulating material on different parts of the tongue. This arrangement permits an opportunity for experimentation which has been only partially exploited.

The sour taste which is associated with all acid foods, e. g., vinegar, sour milk, citrus fruits, and apples, is definitely a function of the hydrogen ion concentration. The $\mathrm{pH}$ at which an acid becomes detectable by its sour taste varies. For pure mineral acids the threshold $\mathrm{pH}$ is between 3.4 and 3.5 , and for pure solutions of acetic, lactic, citric, and butyric acids, it is between pH 3.7 and 3.9. If a buffer is added, e. g., if Na-acetate is added to acetic acid, the threshold for sour taste is lowered to $\mathrm{pH}$ 5.6. With concentrated buffers even neutral solutions may have a sour taste. Thus the excitation of receptors mediating the sour taste may not depend simply on the $\mathrm{pH}$ of the solution bathing the receptors.

The typical salt taste is that of sodium chloride, but a number of other salts $\left(\mathrm{KCl}, \mathrm{NH}_{4} \mathrm{Cl}, \mathrm{LiCl}, \mathrm{RbCl}, \mathrm{NaBr}, \mathrm{NH}_{4} \mathrm{Br}, \mathrm{LiBr}, \mathrm{NaI}\right.$ and $\mathrm{LiI}$ ) are also predominantly salty in taste. Other salts are both salty and bitter $\left(\mathrm{KBr}, \mathrm{NH}_{4} \mathrm{I}\right)$, and still others ( $\mathrm{CsCl}, \mathrm{RbBr}, \mathrm{CsBr}, \mathrm{KI}, \mathrm{RbI}$, and $\mathrm{CsI}$ ) are predominantly bitter. ${ }^{3:}$ The sweet taste is elicited by many classes of chemicals. Salts of beryllium, lead formate, acetate, propionate, and isovalerionate elicit a sweet taste attributed to the metallic portion of the molecules. Among the organic compounds which elicit a sweet taste are the sugars, dihydroxy and polyhydroxy alcohols, saccharin, dulcin, amino acids, and many esters. The sweet taste is quite often associated with a bitter taste, as is illustrated in the following listing from Cohn. ${ }^{10}$

Sweet: glycol, glycerin, glycine, sugar, phloroglucinol, saccharin, dulcin

Sweetish: r-dimethyl tartrate

Tasteless then sweet: sodium naphthionate

Sweet and bitter: glyceraldehyde, p-chlorsaccharin, d-valin

Sweet then bitter: urotropin, aloin, p-inethylsaccharin

Sweet then disagreeably bitter: m-chlorphenyl alanin

Sweetish and bitter: guaiacol ester of isovalerianic acid

Sweetish then bitter: magnesium benzoate, butyramide

Sweet then bitterish: methylglyceraldehyde

Sweetish then bitterish: Ethyl butyrate 
The relative sweetness of sugars has been determined by a number of authors, as illustrated in the following list," based on a value of 100 for sucrose:

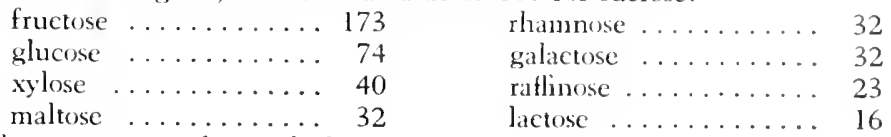

These values vary somewhat with the concentration.

The bitter taste is clicited by a wide variety of chemical compounds. Some salts-e.g., caesium chloride, many iodides, many calcium, ammonium, or magnesium salts $\left(\mathrm{MgSO}_{1}\right.$, or epsom salts)-are bitter, as are picric acid and the alkaloids, the latter being the most bitter of all compounds. A list of some of the substances which elicit a bitter or bitter-sweet taste, compiled by Cohn, " reveals the wicle varicty of their chemical structure:

Bitter: picric acid, phenyl urea, glucose triacelate, strychnine

Bitter then sweet: o-benzoyl benzoic acid, $p$-amino-azobenzene

sulphonic acid, $l$-leucyl-d-tryptophane, phenolphthalein, sodiun-

naphthoyl o-benzoate

Bitter and sweetish: tetrachlorethyl ether, 2, 3-dichlorhexane

Bitterish and sweet: hexenylglycerin

Bitrerish then sweetish: sodium ethyl sulfonate.

Bittersweet, pungent: nitrostyrene

The method of choice in the study of the physiology of chemoreception is the recording of nerve spike potentials in the afferent nerves connected with the peripheral sensory end-organs. Such studies among the insects have met with consistent failure, ${ }^{21}$ which is attributed to the low magnitude of these electrical transients in the small sensory nerve fibers; they are probably below the noise level of electron tube amplifiers (i.e., several microvolts). Among the vertebrates several successful studies have been published. Pumphrey ${ }^{5 * 2}$ recorded from nerve fibers innervating the anterior portion of the tongue of the frog. He obtained records of action potentials (Fig. 151) from fibers which could be excited by applying acid and salt solutions to the tongue. Pfaffman"1 recorded action potentials from single nerve fibers innervating at least three different chemoreceptors, some of which could be stimulated by acid, others by acid and salts, and still others by acid and quinine. Notably absent were the chemoreceptors stimulated by sugar. Only one study has been reported in which sugar produced a response, ${ }^{64}$ and this was observed only once. These results cast some doubt on the discrete nature of the chemoreceptors which mediate the different taste sensations.

The evidence for four specific receptors may be listed as follows: (1) taste qualities are zonally distributed; (2) taste sensations are abolished by cocaine in the following order: bitter, sweet, salt, and finally sour;": (3) gymnemic acid selectively abolishes sweet and bitter but not salt and sour; ${ }^{49}$ (4) papillae can be found that are sensitive to only one of the four tastes; (5) the threshold for electrical stimulation of the taste buds is different for the four different tastes; $;(6)$ in the cat recorded action potentials from single fiber preparations indicate at least three different sensitivities: sour, sour and salt, and sour and bitter. $\overline{1}$

If there are four, and only four, tastes, then it should be possible to duplicate any complex taste by mixture of four types of agent-sodium chloride, 
sucrose, oxalic acid, and quinine hydrochloride. Many complex tastes can be duplicated in this way, and some investigators claim to be able to duplicate all tastes by such mixtures. In experiments of this type the influence of other factors, especially of odors, must be carefully controlled. It can readily be demonstrated that if the nose is held closed boiled turnips, apples, and onions have almost the same taste, ham tastes like lamb, port wine like sugar, and claret wine like weak vinegar.

So far there is no record of a person being completely deficient in any one of the four tastes, i.e., there are no known cases of partial "taste blindness," although complete loss of taste may follow damage to the nervous system. However, some persons are unable to taste certain bitter substances to which others are quite sensitive. Snyder ${ }^{58}$ found that 68.5 per cent of individuals tested could taste p-ethoxyphenyl thiourea, p-HOC $\mathrm{H}_{4}$ NII.CS.$\mathrm{NH}_{2}$, but that 31.5 per cent could not taste the substance. Ability to taste the material is inherited as a non-sex-linked character. Comparable differences in individual tasting ability are known for other materials. 26,54

(a)

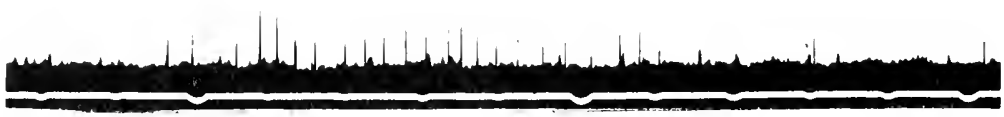

(f)

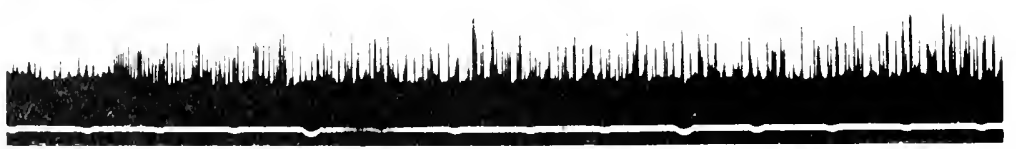

Fig. 151. Action potentials recorded from a single fiber of the lingualis nerve of the frog in response to stimulating the appropriate area of the tongue with 2 per cent $(a)$ and 4 per cent $(b) \mathrm{NaCl}$ solution. Time is marked in $1 / 5 \mathrm{sec}$. From Pumphrey. ${ }^{\text {ta }}$

Many insects respond positively to sugars and it has been demonstrated, using conditioning techniques, that Dytiscus trained to respond positively to sucrose will respond in similar fashion to glucose and 20 other sugars or sugar derivatives." Von Frisch" found that all the sugars which are acceptable to the bee have an additive stimulating effect. There seems to be little doubt that the substances which taste sweet to man also give rise to a distinct sensation in insects.

The situation conceming the other taste modalities in the insects is not at all clear. Dytiscus marginalis, IIydrous picens, and related species learned readily to distinguish between pairs of substances chosen from sucrose. $\mathrm{NaCl}$, $\mathrm{HCC}$, acetic acid, and quinine, and could be trained to accept quinine and avoid $\mathrm{NaCl}^{3}, \pi, \pi$, It was concluded that taste substances could be classified into the same four groups for these beetles as for man. In bees the stimulating effect is additive between $\mathrm{NaCl}$ and $\mathrm{LiBr}, \mathrm{NH}_{4} \mathrm{Br}$ and $\mathrm{HCl}$, but not between guinine and acid. However, acids increased the repellency of bitter substances such as aloin, arbutin, colocynthin, and salicin. It may be said that for bees the taste substances fall into the four groups, but 
there is evidence for considerable interaction among the several modalities. Frings $2: 9$ has advanced the hypothesis that the several taste modalities may merely be points in a continuous spectrum of taste sensation which may be related to penetrating power or some other surface-active property of the chemical agent. The facts that some salts and alkalis at low concentration and all the sugars, which penetrate cells slowly, taste sweet to man and are acceptable to insects, and that there exists a gradual transition from salty to bitter in the series of inorganic salts as their ionic mobilities increase, lend credence to this hypothesis. I lowever, the additive stimulating effects of certain compounds and not of others and the similarity of response of conditioned insects to substances which taste alike to man are observations difficult to reconcile with the theory of Frings.

Although there seems to be some similarity in the taste of insects and man, there appear also to be some differences. Von Frisch ${ }^{3.5}$ tested bees with thirtyfour naturally occurring sweet substances and found they responded to only nine, and that these nine were found in the natural food of bees. Thus many pentoses, sugar alcohols, and true sugars are not accepted by bees. Saccharin is not accepted by bees at low concentrations and is repellent at high concentrations. Acetylsaccharose, which is very bitter to man, is not rejected by the bee.

Intensity Discrimination. Intensity discrimination has not been well worked out in either insects or man. Von Frisch ${ }^{35}$ demonstrated that measurable differences existed in the response of bees to $18 \mathrm{M}$ and to 5/32 M sucrose. Similar experiments show that the American cockroach and the horsefly, Tabanus, can discriminate near threshold between two solutions whose concentrations differ by no more than the above factor. These data do not, of course, establish the lower limit of discriminative ability.

The relation of the intensity of stimulation and the magnitude of the response in chemoreception has received relatively little attention. Krinner, ${ }^{41}$ Crozier, ${ }^{13,}{ }^{14}$ and Hasama ${ }^{37}$ have demonstrated a rough linear relation between magnitude of response and logarithm of the concentration of the stimulating agent, using a variety of different techniques. The technique of recording spike potentials from single nerve fibers innervating chemoreceptors, used by Pumphrey ${ }^{52}$ and Pfaffman, ${ }^{51}$ has yielded results which indicate that the frequency of the nerve spike potential burst (Fig. 151), which begins shortly after application of the stimulating solution, increases with increasing concentration of the stimulating agent. A graph relating the frequency of the nerve spike potential discharge to the concentration of the stimulating agent results in a curve that is roughly logarithmic, although no quantitative expressions have been derived. It appears, therefore, that the response magnitude or intensity is related to the logarithm of the stimulating intensity for chemoreceptors as well as for the other senses (see Chap. 11, p. 415).

Mechanism of Chemoreceptor Activation. Olfaction. Chemoreceptors which mediate the sense of olfaction in the vertebrates and those of insects possess hairlike processes which project from the epithelium. It is assumed that the odorous substance acts on these processes to initiate nerve impulses in the neurones. The manner in which this act is accomplished is not known. Attention has been in the past and is at present concentrated on the chemi- 
cal and physical properties which are directly correlated with the stimulation of the olfactory chemoreceptors, in the hope of arriving at some common denominator which may aid in understanding the mechanism of stimulation.

The relationship between chemical structure and odor is one that has attracted many investigators and is the subject of an enormous quantity of data, which has been adequately reviewed by Moncrieff. ${ }^{46}$ The data relating odor to chemical structure do not submit to any known simple treatment and hence are in a state of chaos. Moncrieff, in an effort to replace chaos by order, has arrived at sixty-two general principles which relate chemical structure to odor. By way of summary it may be stated that whereas substances of similar chemical structure (i.e., a homologous series) may have similar odors, there are numerous examples of isomers and of stereoisomers which have dissimilar odors and, conversely, of substances of quite dissimilar structure which have similar odors. In the final analysis, therefore, the correlation of odor with chemical structure is inadequate.

The relationship between infrared absorption spectra (Raman spectra) and odor has received some attention in the past.," ..." and it has been suggested that heat waves are reflected to the olfactory epithelium according to the absorption spectra of the compounds. Recently Beck and Miles ${ }^{4}$ ts have revived interest in this infrared radiation theory as a direct result of some work on olfaction in bees. Iowever attractive this approach may be, it should be emphasized that certain optical isomers, which have identical Raman spectra, have different odors, and that certain isotopic molecules, such as n-butyl alcohol and the deuterated compound, have similar odors but different Raman spectra. ${ }^{63}$ The correlation of odor with infrared absorption must, therefore, be regarded with some caution.

Examination of odorous substances indicates that they possess in common certain properties which undoubtedly are related to their effectiveness in stimulating the olfactory chemoreceptors. One primary requisite of all odorous materials is that they be volatile, i.e., have a high vapor pressure at moderate temperatures, so that molecules will readily leave the liquid or the solid state. A second common property of odorous materials is that they are readily adsorbed. Activated carbon will adsorb enormous quantities of odorants but very small amounts of substances which are odorless (i.e., hydrogen, nitrogen, oxygen, carbon monoxide, and carbon dioxide). A third common property of alorants is lipoid solubility. It is true that many odorants are also water-soluble, but some of the most effective olfactory chemoreceptor stimulants (synthetic musk, vanillin, indole) are relatively insoluble in water. The conjecture may be introduced at this point that the ready adscrption of odorants on the olfactory sense cells or their processes, coupled with lipoid solubility, may facilitate the concentration on and penetration of these substances into the olfactory neurones.

Relatively recent work on the stimulative effectiveness of organic com. pounds on the olfactory sense organs of insects has yielded an interesting relation between stimulative ability and boiling point. ${ }^{20}$ Cook $^{11}$ determined the optimal concentration (to flies) of a homologous series of alcohols from methyl to amyl, and of esters from acetates to valerates. The optimal concentration proved to be a linear function of the boiling point (Fig. 152), or 
of any other property thermodinamically related to the boiling point. Similar studies indicate that a consistent difference exists between normal and ise compounds, the normal compound being more effective; that ketones stimulate more readily than phenol esters and alcolnols: ${ }^{\overline{1}}$ that esters are more stimulating than their alcohols: ${ }^{17}$ and that increasing the chain length of homologous series of alcolnols or esters results in increased stimulative effectiveness." These results are essentially in agreement with the correlation between boiling point and stimulating effectiveness.

Gustation. In general, it is known that stimulation of the hemoreceptors which mediate taste in man and regulate feeding and ovipusition in other animals reguires the contact of the stimulating substance with the sense organ in question. Iust what hippens after this contact has been established is not known, and indeed, among the insects it is not clear what the sense organs are or precisely where they are located. It has been suggested ${ }^{20}$ that the penetration of the stimulating substances into the sense cells or their adsorption on the cell membranes is an important event in stimulation, and considerable data are in agreement with this suggestion. However, grant-

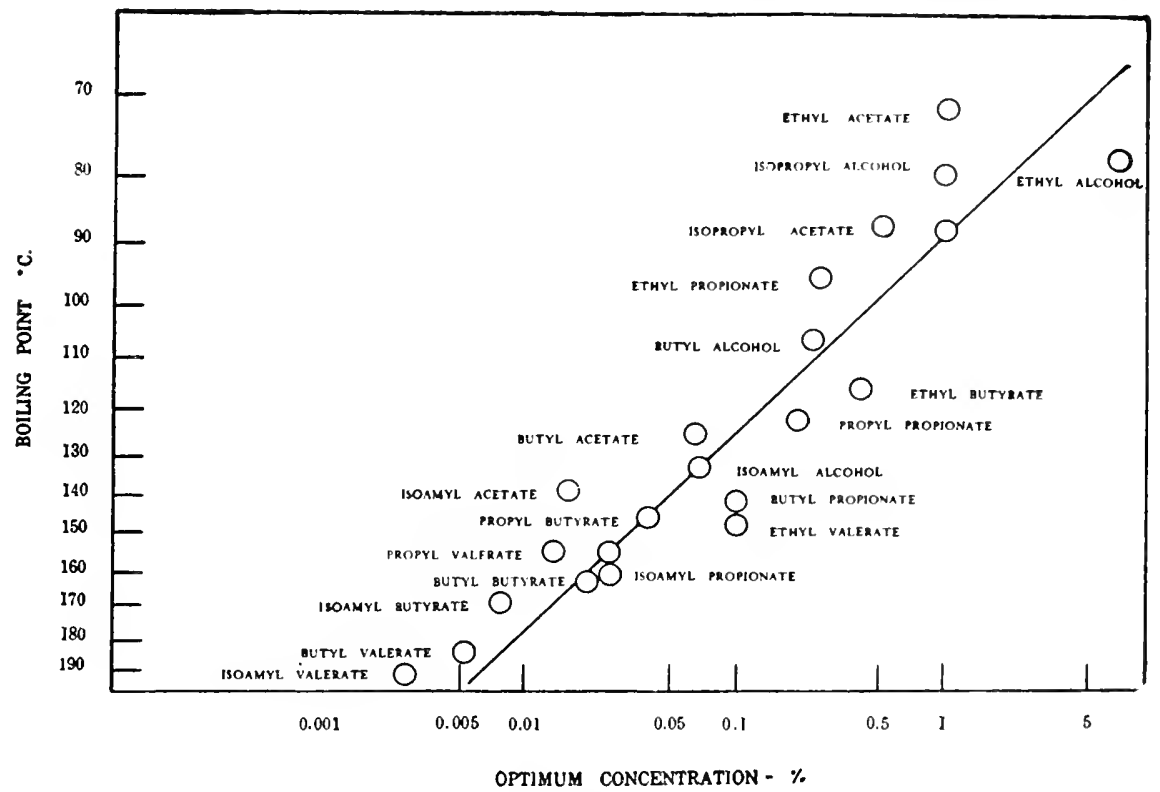

Fig. 152. The relation between boiling point and the most allactive concentration (indicated by the number of houseflies caugh of a variety of paraffin compounds. After Cook," from Dethier. ${ }^{\text {s" }}$

ing the adsorption or penetration of the stimulating agent, there is as set no knowledge of the erents leading to the initiation of the nerve impulses. Consequently, a comprehensive theor! which is in accord with all the known facts of chemoreceptor stimulation is ret lacking.

The role of ehemical structure in stimulation of the gustatory sense organs has received considerable attention. Von Frisch, ats a result of his sugarfeeding experiments with bees, concluded that the factors involved in stimu 
lation are not related to structural formulae. It appears, therefore, that chemical structure as a correlate of stimulating effectiveness may be dismissed for the gustatory chemoreceptors as well as for the olfactory receptors.

The correlation between stimulating effectiveness and lipoid solubility or oil-water clistribution coefficients is in accord with all known facts of gustatory sense organ stimulation. This is illustrated by several groups of data. In man taste of the inorganic acids seems to be a simple matter of $\mathrm{pH}$, but in the case of organic acids or of buffered solution the stimulating efficiency seems to be related also to the presence of undissociated acid. The simplest explanation for these findings is that the $\mathrm{pH}$ of the membrane or the cytoplasm of the sense cell is the determining factor in stimulation. Undissociated molecules penetrate the membrane more rapidly and then may become ionized in the membrane or in the eytoplasm, thus producing an effective change in $\mathrm{pH}^{1 .}{ }^{1 .}$ In this connection it has been demonstrated that the introduction of polar groups $\left(-\mathrm{OH},-\mathrm{Cl},-\mathrm{Br},-\mathrm{COOH}\right.$, or $\left.-\mathrm{NH}_{2}\right)$ into the acid molecule reduces its stimulating effectiveness, whereas the introduction of additional methyl groups or the lengthening of the carbon chain increases the stimulating effectiveness. ${ }^{60}$

Among the insects Frings ${ }^{30}$ has shown that the stimulating effectiveness of several series of inorganic salts with a common anion increased in the following order, $\mathrm{Li}^{+}<\mathrm{Na}^{+}<\mathrm{Mg}^{++}<\mathrm{Ca}^{++}=\mathrm{Sr}^{++}<\mathrm{K}^{+}<\mathrm{Cs}^{+}=$ $\mathrm{Rb}^{+}<\mathrm{NH}_{4}^{+}<<<\mathrm{H}^{+}$, and this scheme is in agreement with most of the reported data on other animals, including man. The order of effectiveness of the salts is in accord with their ionic mobilities and also with their partition coefficients. Dethier and Chadwick ${ }^{1 ! 1}$ measured the rejection thresholds for a series of aliphatic alcohols, and they obtained a high correlation between stimulating effectiveness and boiling point, molecular area, molecular moments, vapor pressures, activity coefficients, and oil-water distribution coefficients. Recently the same authors ${ }^{21}$ determined that the glycols are rejected by blowflies at logarithmically decreasing concentrations as the chain length is increased.

\section{SUMMARY}

The history of chemoreception is studded with man's attempts to explain and rationalize the mechanism involved in the activation of these sense organs. Yet today this is still one huge question mark. True, the work relating physical and chemical properties of compounds to their stimulating effectiveness may soon demonstrate those properties of the stimulus essential for activation of chemoreceptors, but the question of mechanism of activation will still remain unanswered. This answer must be sought through investigation of cellular changes in the sensors end-organ itself, a difficult but challenging problem.

\section{REFERENCES}

1. Amman, E. 1)., and Lunwag, C., J. Physiol. 9-4:441-460 (1938). Nerve discharges from olfaclory organs: fish.

2. Allen, H, and Weinibrg, M., Quart. J. Kixper. Physiol. 15:385-420 (1925). Electrical stimulation of laste buds: man.

3. Ballen, L., Ztschr. vergl. Physiol. 26:107-120 (1938). Physiology of taste in beetles: Hydrous. 
4. Beck, L. II., and Mlurs, Mr. R., Science 106:51I (1947). Infrared absorption and olfactory stimulation: cockroach.

5. Bellows, R. T., and Vay Whaenen, W'. P., Am. 1. Physol. 126:13.19 (1934) Effect of denervation of chenoreceptors on water intake: dogs.

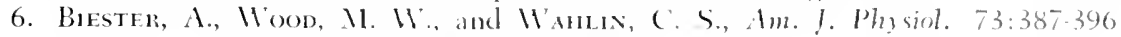
(1925). The relative sweetness of pure sugars.

7. Bunker, (.. W'. O., and IHrschleloer, A. 1). Am. I. Trop. Med. 5:359383 (1925). Mlosquito repetlents.

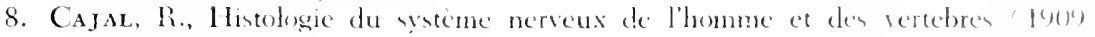
Paris, Maloine.

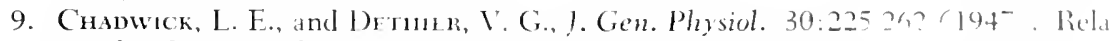
tionship between chenical structure and stimulation: insects.

10. Conn, G., Die organischen Creschmacksstoffe (1914). Berlin, F. Sienrenroth.

11. Соок, W. C., J. Agr. Res. 32:347-358 (1926). Effectiveness of paraffin derivatives as attractants: insects.

12. Crozıer, IV. J., I. Comp. Neurol. 26:1-8 (1916). Regarding the existence of "common chenical sense" in vertebrates.

13. Crozier, II. J., Am. J. Physiol. 45:315-322 (1918). Sensory activation with alkalies: earthworm.

14. Crozier, IV. J., Am. J. Physiol. 45:323-341 (1918). Sensory actisation with acids: earthworms.

15. Crozier. W'. J., in Handbook of General and Experimental Psychology' (C. Murchison, ed.) (1934). Worcester, Mass., Clark Univ. Press. Ch. 19. Chenoreception.

16. Dethiner, V. G., Biol. Bull. 72:7-23 (1937). Gustation and offaction in larvae: Lepidoptera.

17. Dethier, V. G., Am. Nat. 75:61-73 (19+1). Chemical factors in choice of ford: insects.

18. Dethier, V. G., Chemical Insect Attractants and Repellents (1947). Philadelphia, Blakiston.

19. Dethier, V. G., and Ciradwick, L. E., J. Gen. Physiol. 30:2+7-253 (1947). Relation of chemical structure to stimulation: insects.

20. Dethier, V. G, and Chadwick, L. E., Physiol. Rer. 28:220-254 (1948). Chemoreception in insects.

21. Dethier, V. G., and Criadwick, L. E., J. Gen. Physiol. 31:139-151 (1948). Relation of chemical structure to stimulation: insects.

22. Dyson, G. M., Perf. \& Essen. Oil Rec. 28:13-19 (1937). Raman effect and the concept of odor.

23. Drson, G. M., Chemistry \& Industry 57:647-651 (1938). Scientific basis of odor.

24. Evans, C. L., Starling's Principles of Human Physiology (1933). London, J. \& A. Churchill.

25. Evans, H. M., Brain and Body of Fish (1940). A study of brain pattern in relation to hunting and feeding of lish. Philadelphia, Blakiston.

26. Fox, A. L., Proc. Nat. Acad. Sci. 18:115-120 (1932). Relation betreen chemical structure and taste: man.

27. Fraenkel, G. S., and Gunn, D. L., The Orientation of Animals (1940). Oxford, Clarendon Press.

28. Frings, H., Biol. Bull. 88:37-43 (19+5). Gustatory rejection thresholds: moth.

29. Frings, H., J. Exper. Zool. 102:23-50 (1946). Gustatory thresholds for sucrose and electrolytes: cockroach.

30. Frings, H., and O'Neal, B. R., J. Exper. Zool. 103:61-79 (1946). Location and thresholds of chemoreceptors: horsefly.

31. Frisch, K. v., Zool. Jalirb., Abt. allg. Zool. u. Physiol. 37:1-228 (1919). On the taste sense of bees.

32. Frisch, K. v., Zool. lahrb., Abt. allg. Zool. u. Physiol. 38:4+7-508 (1921). The location of olfactory senses in insects.

33. Frisch, K. v., Handh. Norm. Path. Physiol. 2:203-239 (1926). Comparative physiology of olfaction and gustation.

34. Frisch, K. v., Aus dem leben der Bienen (1927). Berlin, Julius Springer.

35. Frisch, K. v., Ztschr. vergl. Physiol. 21:1-156 (1934). On the taste sense in bees. 
36. Fulton, J. F., Howell's Textbook of Physiology (1946). Philadelphia, Saunders.

37. Hasama, B., Pflig. Arch. ges. Physiol. 234:748-755 (1934). Potential changes in central olfactory centers: rabbit.

38. Henschel, J., Wiss. Meeres Untersuch. Kiel, N. F. 22 (1935). Chemical sense of some invertebrates.

39. Kionka, H., and Strätz, F., Arch. Exper. Path. Pharm. 95:241.257 (1922). The manner of gustatory stimulation by sodium chloride.

40. Koenler, O., Ztschr. vergl. Physiol. 16:606-756 (1932). Sense physiology of fresh water planaria.

41. Krinner, M., Ztschir. vergl. Physiol. 21:317-342 (1934). Gustatory sensitivity in fishes: trout.

42. Liebermann, A., Ztschr. Morph. Ökol. Tiere 5:1-97 (1926). Antennal organs of smell in flies.

43. Mast, S. O., and Nadler, J. E., J. Morph. E Physiol. 43:105-117 (1926). Effect of ions on ciliary reversal: paramecium.

44. Mcindoo, N. E., Smithsonian Inst. Pub., Misc. Collections 68:1-78 (1917). Recognition among insects.

45. Miles, W. R., and BeCK, L. H., Science 106:512 (1947). Infrared absorption and olfactory stimulation: bee.

46. Moncrieff, R. W., The Chemical Senses (1944). London, Leonard Hill.

47. Moore, W., J. New York Ent. Soc. 42:185-192 (1934). Repellent effect of esters: fly.

48. Oliphant, J. F., Pliysiol. Zool. 11:19-30 (1938). Effect of ions and temperature on ciliary reversal: paramecium.

49. Parker, G. H., Smell, Taste and Allied Senses in Vertebrates (1922). Philadelphia, Lippincott.

50. Parker, G. H., and Stabler, E. M., Am. J. Physiol. 32:230-240 (1913). Distinctions between taste and smell.

51. Pfaffmann, C., J. Cell. E Comp. Physiol. 17:243-258 (1941). Gustatory afferent impulses: cat.

52. Pumphrey, R. J., J. Cell. E Comp. Physiol. 6:457-467 (1935). Gustatory afferent impulses: frog.

53. ReEd, M. R., Physiol. Zool. 11:317-325 (1938). Response of Drosophila to fermentation products of bananas.

54. Riddell, W. J. B., and Wybar, K. C., Nature 154:669 (1944). Taste of thiouracil and phenylthiocarbamide.

55. Ritten, E., Ztschr. vergl. Physiol. 23:543-570 (1936). Investigation of chemical sense in beetles: Hydrous.

56. Schaller, A., Ztschir. vergl. Pliysiol. 4:370-464 (1926). Investigations of sensory physiology: beetle and fish.

57. Snodgrass, R. E., Principles of Insect Morphology (1935). New York, McGrawHill.

58. SNyder, L. H., Ohio J. Sci. 32:436-440 (1932). Inheritance of taste deficiency in man.

59. Speyer, E. R., Ann. Appl. Biol. 7:124-140 (1920). Chemotropism: housefly.

60. Taylor, N. W., Farthing, F. R., and Berman, R., Protoplasma 10:84-97 (1930). Relation of structure to chemoreceptor stimulation: human.

61. Warnke, G., Ztschr. vergl. Physiol. 14:121-199 (1931). Experiments on olfaction: insects.

62. Wigglesworti, V. B., The Principles of Insect Physiology (1939). London, Methuen.

63. Young, C. W., Pletcher, D. E., and Wraght, N., Science 108:411-412 (1948). Infrared radiation theories of olfaction.

64. Zotterman, Y., Skand. Arch. Physiol. 72:73-77 (1935). Nervous discharges produced by stimulation of chemoreceptors.

65. Zwannemaken, H., Die Physiologie des Geruchs (1895). Leipzig. 


\section{Phonoreception}

\section{INTRODUCTION}

HONORECEPTION IS T11E PROCESS of detecting vibratory motion, usually of frequencies higher than several vibrations per second, in the surrounding medium. Stimulation occurs most commonly by means of sound waves in air (mammals, insects), but may also be produced by sound waves in water (fish, amphibia), or even by vibration of a solid body against the skeletal structure supporting the phonoreceptive organ (bone conduction in man, utilized in certain types of hearing aids).

Sound Waves. Sound waves in any medium consist of rapid vibratory motion of the particles which compose the medium. The motion of one particle tends to disturb a neighboring particle, which in turn disturbs the next one, so that a wave of disturbance passes through the medium. When a tuning fork vibrates in air, the forward movement of the prong compresses the adjacent air, but the elasticity of the air prevents maintenance of this localized region of compression, and expansion occurs at the expense of the adjoining region, so that a wave of excess pressure emanates from the prong. In a similar manner, backward movement of the prong sets up a wave of diminished pressure or rarefaction.

If the tuning fork vibrates with the simplest to-and-fro movement it generates what we call a "pure" tone, and its motion may be expressed quantitatively in two ways: by the frequency of its vibration, and by the amplitude of its excursion. The resulting wave may likewise be described by its frequency and intensity.

The tip of the tuning fork and consequently any given particle in the medium undergoes simple harmonic motion in the principal direction of propagation of the wave, and its position if plotted as a function of time gives a curve that is described as a sine wave (Fig. 153). The pressure at any given point in the medium also varies as a sine wave which leads the displacement wave by 90 degrees. If motion of the fork is not a simple to-andfro movement, then the form of the wave produced is complex, and so is the tonal stimulus. A complex wave may be resolved mathematically into two or more simple waves, which may then be described in terms of frequency and intensity.

If a sinusoidally varying force is applied to an objeci whose resistance to motion does not vary linearly with the displacement, then the object will not move in a simple sinusoidal manner, and if its motion is plotted as a function of time the wave will appear to be a "distorted" sine wave. Such a wave may also be resolved into two or more sinusoidal waves. If the applied frequency is considered to be the "fundamental" frequency of vibra. 
tion, the resulting wave of motion will be found to be the algebraic summation of this fundamental and one or more of its multiples. The multiples of the fundamental are referred to as "harmonics." In this manner improper adjustment of the anatomical components of the ear (e.g., loose coupling of middle ear bones; unequal tension on the ear drum or basilar membrane, or any other elastic structure) could give rise to harmonics when the stimulus consists of a pure tone.

The intensity of sound waves is defined as the rate of flow of energy through a unit area of the medium. Except for a few unusual conditions (especially those in which standing waves occur), the energy of a sound wave is proportional to the square of the root-mean-square value of the alternating pressure. The human ear is responsive to a very wide range in

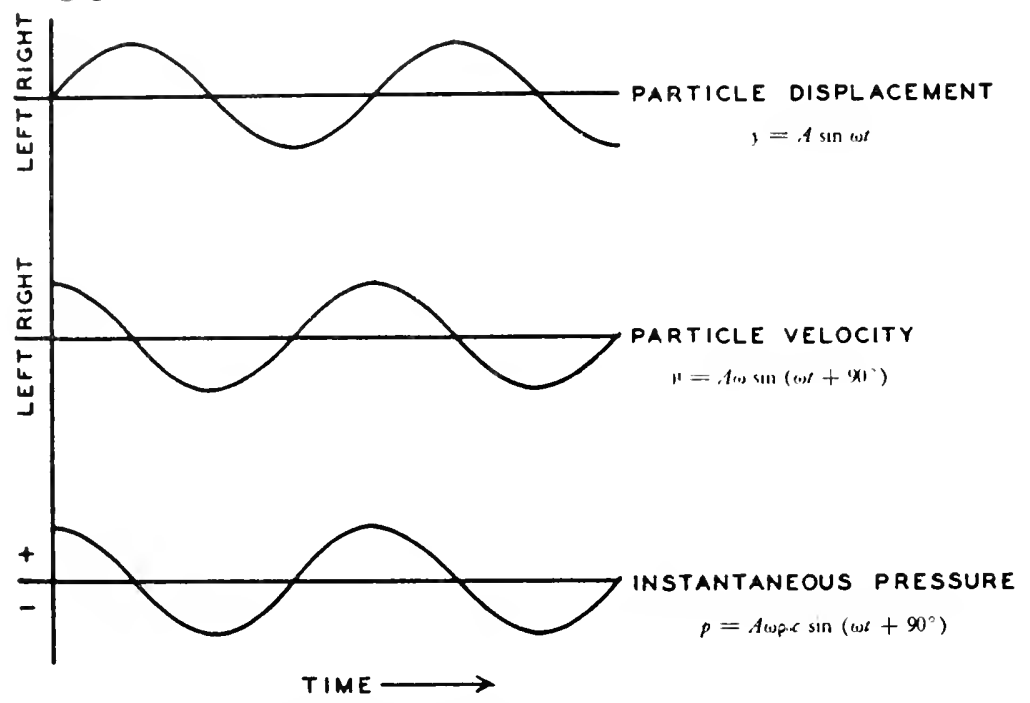

Fig. 153. Curves showing various aspects of plane progressive sound waves, together with their equations, in which $y=$ displacement; $A=$ amplitude; $w=$ angular velocity $=$ $2 \pi \times$ frequency; $t=$ time; $\mu=$ velocity of particle; $p=$ alternating pressure; $P_{0}=$ density of air; $c=$ velocity of sound. From Stevens and Davis. ${ }^{45}$

the energy of stimulation, and for convenience in expression and in plotting of data the scale used is logarithmic. A difference of one log unit (base 10) in energy is called a bel, and a difference of one-tenth log unit a decibel.

The decibel (abbreviated $\mathrm{db}$ ) is thus defined as ten times the logarithm of the ratio of two energies, but it can also be applied to two pressures, velocities, currents, ete., which are related to energy by a square law. The number of decibels in the ratio of two sound pressures thus become 20 times the logarithm of the ratio. The number of decibels (N) relating two energies (E) or two pressures $(\mathrm{P})$ is therefore

$$
\mathrm{N}=10 \log \frac{\mathrm{E}_{1}}{\mathrm{E}_{2}}=20 \log \frac{\mathrm{P}_{1}}{\mathrm{P}_{2}}
$$

On an energy seate a tenfold increase is 10 decibels, but on a pressure scale a tenfold increase is 20 decibels.

The ear can withstand is sound which has a million million times as much 
energy as that which can just barely be detected. This difference may be expressed as $120 \mathrm{db}$ and is just about the ratio of the energy of the sound of very loud thunder to that of a sound just at the threshold of hearing. On the decibel scalc the intensities (i.e., energies) of some common sounds are:

$\begin{array}{llrl}120 \mathrm{db} & \text { Thunder } & 60 & \text { Conversation } \\ 110 & \text { Airplane engine } & 50 & \text { Quiet automobile } \\ 100 & \text { Boiler shop } & +0 & \text { Average office } \\ 90 & \text { Elevated train } & 25 & \text { Average dwelling } \\ 80 & \text { Pneumatic drill } & 15 & \text { Whisper } \\ 70 & \text { Busy street } & 0 & \text { Threshold of hearing }\end{array}$

Since the decibet scale is one of ratios, it is necessary to have some standard for comparison. The threshold of hearing may be used for this purpose, but it is known to vary from person to person and also to vary greatly with frequency. Therefore, an arbitrary standard of 1 microwatt (a watt is a measure of the rate of flow of energy) per square centimeter is adopted for reference, and this approximates the average threshold for a 1000 cycle tone.

Problems of Phonoreception. In the process of hearing the physiological problem of the animal is how to detect sound waves, how to distinguish one frequency from another, how to determine the relative intensity of each frequency, how to determine the direction from which the sound is coming, and, moreover, how to do all of these things simultaneously when the oncoming sound waves are of a high degree of complexity, consisting of many frequencies and intensitics combined together in a complex disturbance of the medium. In some animals, e.g.. in fishes, this problem is only partly solved in that there is a mechanism for detection of sound but almost none for analysis of the sound in terms of frequency. In man the mechanism for analysis is very good. In bats it may be better, in that the frequency range is much wider.

\section{PHONORECEPTION BY MAA'}

Morphology: External Ear. The structure of the hearing mechanism of man is shown diagramatically in Fig. 15t. The external ear, or pinna, leads into an auditory canal (external auditory meatus), which conducts sound waves to the ear drum or tympanic membrane. The external ear of man is too small to act as a concentrating reflector of sound waves and its principal function is that of scattering the waves so that some of the sound passes into the auditory canal. In donkeys and other large-eared animals, concentration by reflection may be important. The tympanic membrane, which separates the auditory canal from the middle ear, is slightly conical or funnel-like in shape, is about $0.1 \mathrm{~mm}$. thick, and is composed of an outside layer of skin, a layer of connective tissue, and an inner layer of mucous membrane.

Middle Ear. The middle ear is filled with air and contains three bones: the malleus, the incus, and the stapes, which are attached to each other. Furthermore, one arm of the malleus is attached close to the center of the tympanic membrane and another projection is attached close to its periphery. Vibration of the membrane causes a rocking of the malleus about the peripherally attached projection. The incus is rather firmly fastened to the malleus so that, at least for motions of small amplitude, the two bones tend 
to vibrate as a unit. This motion causes a rocking of the stapes, which is fastened eccentrically to the inner ear drum covering an oval window in the bony inner surface of the middle ear. There are two small muscles in the middle ear, one attached to the malleus and one to the stapes. Contraction of these muscles reduces the amplitude of the vibrations and thereby decreases the sensitivity of the ear. Contraction is usually under reflex control, but in exceptional cases it may be voluntary. Below the oval window is another opening, the round window, which is also covered by a membrane, the auxiliary ear drum. The bones of the middle ear do not make contact with the membrane of the round window, the function of this membrane being to relieve the pressure exerted on the inner ear by the stapes. When the covering of the oval window is pushed inward by the stapes, the covering of the round window bulges outward. The cavity of the middle ear is connected to the oral cavity by the eustachian tube, the function of which is to equilibrate air pressure of the middle ear with that of the environment and to serve as a drainage canal.

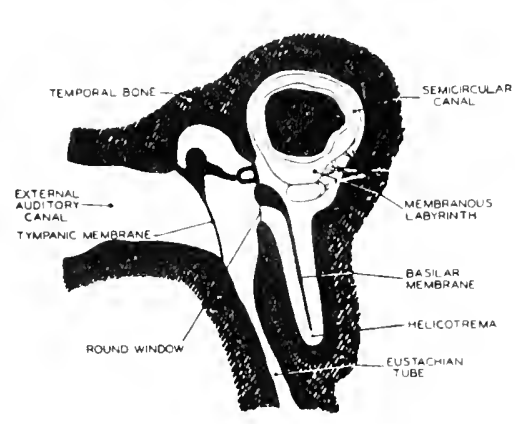

A

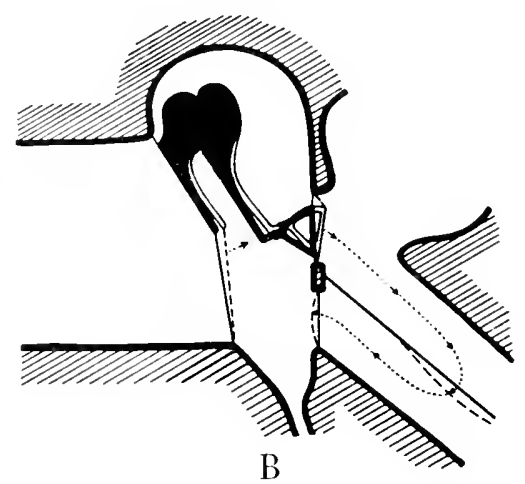

B

Fig. 15t. Diagrammatic representation of the ear. A, Schematic diagram of middle and internal ear. The oval window (unlabeled) lies above the round window and contacts the stapes. $B$, Schematic diagram of the ossicles of the middle ear, showing position of various members at rest and after inward displacement of tympanic membrane. Dotted lines and arrows represent path of sound waves. After Stevens and Davis ${ }^{\text {th }}$ from Fulton. ${ }^{15}$

The principal function of the middle ear is to transmit sound waves from air to licuid. Because of the ratio of the areas of the tympanic membrane and the stapes (90 to 3.2 square millimeters) and the reduction in total amplitude of vibration (by a factor of 2) there exists a very considerable increase (roughly, 50 times) in the force which is available for driving the Huid of the scala vestibuli. This increase in force is of the proper order of magnitude to overcome the increase in the greater cross sectional impedance of the licquid as compared with air. In general, the middle ear seems to be a very efficient system for transmitting vibrations from air to liquid. Lower animils which have no middle ear are at a distinct disadvantage.

Inner Ear. The inner ear is a fluid-filled structure housed in a complex system of cavitics and tunnels known as the osseous labyrinth. The inner ear has two functions: detection of sound and detection of movement. Detection of sound is accomplished by the cochlea, a spiral bony labyrinth 
which is divided internally by membranes to form three long spiral canals. Detection of motion is accomplished within a group of three osseous semicircular canals, more or less at right angles to each other, each of which con tains a membranous canal (Ch. 14, p. 517). These two usseous laburinths (cochlear and semicircular canals) open into a cavity, known as the vestihule, which is separated from the middle ear partly by bone and partly by the oval window.

Within the restibule are two membranous sacs, the sacculus and the utriculus, which open into each other. The utriculus also opens into both ends of the three membranous scmicircular canals, and the sacculus is also connected to the cochlear duct or scala media, the middle of the three spiral canals of the cochlea. The sacculus, utriculus, membranous semicircular canals, and the cochlear duct together form a complete membranous labyrinth, which may be considered as a lonsely fitting lining of the osseous labyrinth. The cavity of the membranous labyrinth is filled with a fluid, the endolymph, and the space between the membranous labyrinth and the bone is filled with another fluid, the perilympl. The two fluids are not in direct contact at any point; however, the space between the membranous labyrinth and the bone is connected by means of the perilymphatic duct with the subarachnoid space of the brain, and the carity of the membranous labyrinth is connected by means of the endolymphatic duct which ends blindly in the subarachonoid space.

STRUCTURE OF THE COCHLEA. In general appearance the cochlea resembles a snail shell with two and three-quarters turns to the spiral. It is about $5 \mathrm{~mm}$. in diameter at the base and measures about $9 \mathrm{~mm}$. from base to apex. There is a central bony structure, the modiolus, around which the spiral cavity is wound. This cavity is separated into three spiral canals by two membranes: (1) the basilar membrane, broadest at the apex and narrowest at the base and held under some tension; and (2) Reissner's membrane, a very thin, delicate structure which spirals on the apical or upper side of the basilar membrane. These two membranes extend throughout most of the length of the spiral but join each other and then stop just short of the apex, thereby leaving the upper and lower spirals joined by a small opening.

A cross section of one of the spirals of the cochlea is shown in Fig. 155, A. The middle canal contains a relatively complex formation of sensory and supportive cells, known as the organ of Corti, attached to the basilar membrane. Above the organ of Corti is the tectorial membrane which, in the living animal but usually not in fixed material, is probably closely applied, and perhaps loosely attached, to hairlike processes of the sense cells which lie just below. There are four rows of sense cellis, one inner row, toward the modiolus, and three outer rows. The organ of Corti consists of the sense cells plus their nerve connections and supportive structure. There is a firm support ing framework above the sense cells, consisting of two pillars lying between the inner and outer rows of hair cells to which is attached a semirigid but porous membrane, the reticular lamina.

Vibrations are transmitted by the stapes to the perilymph of the vestibule through the membrane of the oval window and from there to the perilymph of the lower canal. Reissner's membrane is very thin and delicate and offers 
almost no resistance to the vibrations which pass into the endolymph. Vibration of the basilar membrane causes a movement of the hairlike processes of the sense cell against the tectorial membrane, thereby probably giving rise
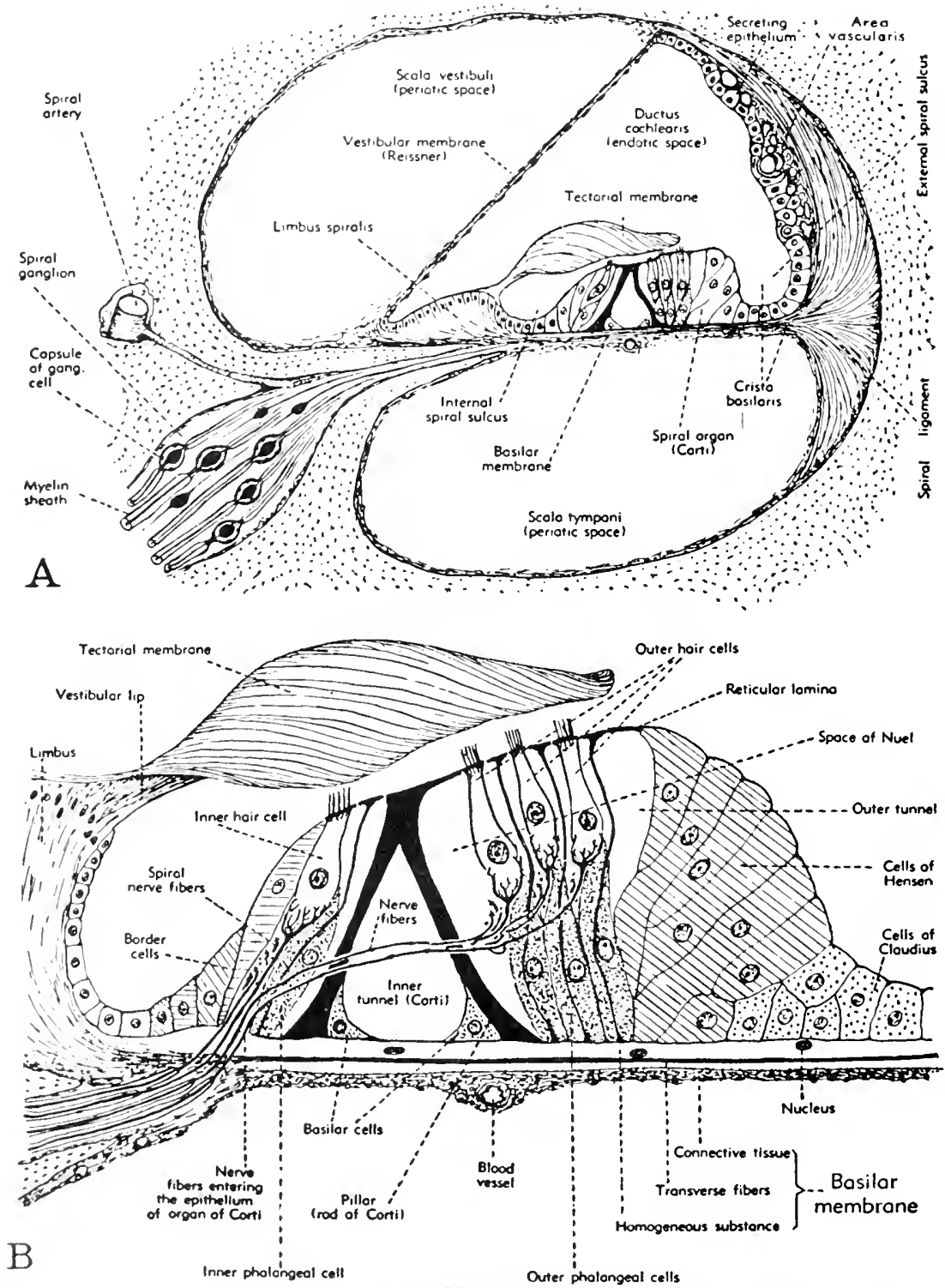

Fig. 155. A, Vertical section of the human cochlea showing the organ of Corti and adjacent structures. B, 'The organ of Corti and the basilar membrane in greater magnification. (After Rasmussen, from Fulton, ${ }^{15}$ ).

to stimulation of the sense cell. The sense cells are supplied with dendrites of ginglion cells of the auditory branch of the eighth cranial nerve, whose cell bodies are contained in a spiral ganglion located in the modiolus. 
Function: The Microphonic Effect. Movement of the hair cells against the tectorial mombrane gives rise to a voltage change across the sense cells. The free ends of the sense cells become negative to the basal ends whenever the basilar membrane is moved upward. This voltage change may be re corded from various parts of the bony structure, including the rounc! and oval windows. This voltage change of the sense cells is referred to as the "microphonic effect" of the cochlea. ${ }^{53}$ The microphonic effect provides a method of determining the frequency sensitivity range of the cochlea of an animal.

The microphonic effect can be distinguished from the nerve impulses by a variety of criteria: a short latency of $0.1 \mathrm{msec}$. as opposed to $0.7 \mathrm{msec}$. for nerve; absence of refractory period; resistance to fatigue, cold, anesthesia, and ischemia; ability to follow notes close to the upper limit of hearing $(16,000$ cycles per second); and ability to follow faithfully the form of the incident sound waves. These characteristics lead to the assumption that the microphonic effect is comparable to that of an electrical transducer which passively converts mechanical energy into electrical energy in very much the same manner as movement of the needle in a phonograph pickup causes distortion of a piezo-electric crystal, thereby producing voltage change.

Stimulation of the Nerve. There are two theories concerning the mechanism by which movement of the hair cells gives rise to impulses in the auditory nerve: (1) through the microphonic effect, and (2) through a chemical mediator. At present, evidence is not conclusive for either theory.

The electrical theory assumes that the voltage of the microphonic effect is the stimulating agent. Evidence for this theory consists of the fact that all nerve fibers can be stimulated electrically, and the voltage of the microphonic effect seems adequate for the purpose. The utter simplicity of this explanation is most appealing. Furthermore, from a study of the timing of the microphonic effect and the nerve impulses produced by a volley of sound waves, it can be demonstrated that the latency of the impulse is constant only if it is assumed that stimulation is associated with the negative phase of the microphonic effect, i.e., when the basilar membrane is moving up and the stapes out.

Evidence that is often cited against the electrical theory is the long latent period, which is usually $0.6 \mathrm{msec}$. or longer. If properties of the auditory nerve are similar to those of other nerves the latency should not exceed 0.1 msec. If we ascribe $0.1 \mathrm{msec}$. to latency of response and the rest of the latent period to conduction time, then the rate of the impulse in the unmyelinated portion of the nerve fiber ( 30 microns long) would be less than 10 meters per second. However, there seems to be no real necessity of limiting the latency to $0.1 \mathrm{msec}$. At synapses, stimulation is delayed in exactly the same manner and for the same approximate times as at the sense cell-neural junction in the ear, and probably for the same reason.

The theory of a chemical mediator assumes that a chemical substance is released by the sense cell under the influence of distortion in shape and that this chemical substance brings about the stimulation of the nerve. This theory permits one to account for the long latent period, but so far there is no proof for the existence of the mediator. If this theory could be proved, then the microphonic effect might be demonstrated to be purely incidental to the process of stimulation. 
In general, it seems that stimulation of the dendrites by sense cells may not differ from stimulation of axons by dendrites of synapses. In both phenomena the principal evidence against the electrical theory is the delay of the impulse, and for this there seems to be an adequate explanation.

The Range of Hearing. The normal human ear is sensitive to vibrations of frequencies between 16 and 20,000 cycles (or 20 kilocycles-20 kc.) per second. At extremely high intensities the range has been reported even as low as 1 cycle per second. Data showing the thresholds at various frequencies as determined by numerous investigators are shown in Fig. 156. The threshold is minimal at 1000 to 1500 cycles; at 100 cycles or at 15,000 cycles it is 40 to $50 \mathrm{db}$ higher on the pressure scale (in which $20 \mathrm{db}$ denotes a tenfold increase in energy).

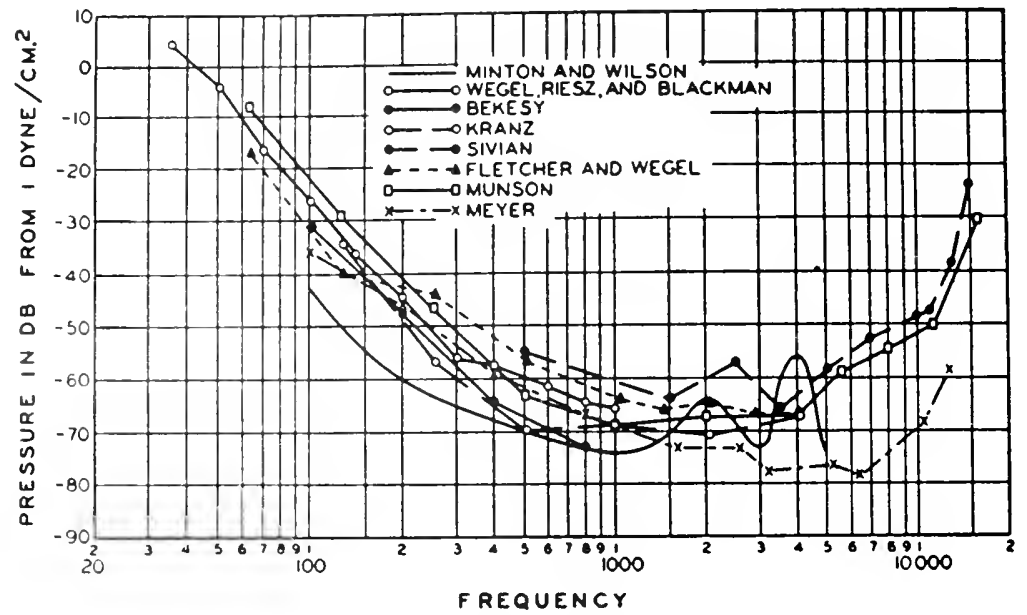

Fig. 156. The minimum audible pressure at the ear drum as a function of frequency, as determined by various investigators. After Sivian and White, ${ }^{43}$ from Stevens and Davis. ${ }^{48}$

At the threshold of hearing the amplitude of vibration of the tympanic membrane (calculations of Wilska, ${ }^{57}$ Fig 157) is less than $10^{-9} \mathrm{~cm}$. at 3000 cycles, and is $10^{-6} \mathrm{~cm}$. at 1000 cycles. This represents an extremely high sensitivity to mechanical movement which is especially apparent when one considers that the wave length of green light is about $5 \times 10^{-7} \mathrm{~cm}$. and the diameter of a hydrogen molecule is only somewhat more than $10^{-8} \mathrm{~cm}$. Since the amplitude of vibration of the basilar membrane is less than that at the ear drum, it appears that the basilar membrane is sensitive to movements of $10^{-10} \mathrm{~cm}$., a distance equal to less than 1 per cent of the diameter of a hydrogen molecule!

The Mechanisu of Frequency Discrimination. The human ear not only is sensitive to the frequency range of 16 to 20,000 cycles, but it is able to distinguish between tones of different frequencies. At an intensity of $40 \mathrm{db}$ there are about 1500 recognizable differences in tone between 20 and 20,000 cycles; at $60 \mathrm{db}$ the number is about 1800 .

It can be demonstrated in a varicty of ways that the base of the cochlea, i.e., the part next to the middle ear, is sensitive to vibration of high frequen- 
cies, that the region toward the apex is sensitive to vibration of low frequencies, and that sensitivity to intermediate frequencies is distributed throughout the spiral. Maps of the cochleas of man and guinea pig are shown in Fig. 158. Men and guinea pigs are sensitive to vibrations of about the same frequency range, but the guinea pig has one additional turn to the spiral.

The allocation of response to particular frequencies to definite positions along the cochlea is usually referred to as the "place theory" of hearing.

Evidence for the place theory consists of the following: (1) Long exposure to loud tones of a given frequency destroys the organ of Corti at the corresponding place on the map in Figure 158. (2) Injections into the cochlea at various points along the spiral of drugs which are destructive to the organ of Corti will decrease the cochlear microphonics produced by the correspond-

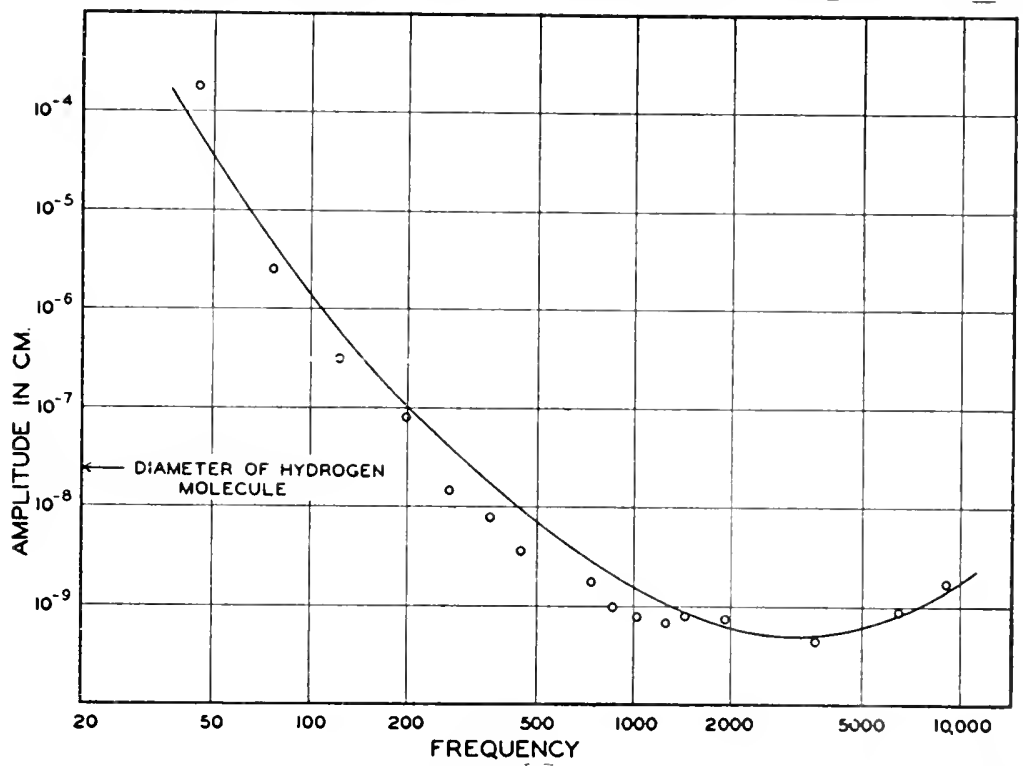

Fig. 157. Curve showing the calculated relation between the amplitude of vibration of air molecules in a sound wave at threshold pressure. The circles show the amplitude of vibration of the ear drum. (After Wilska, "ir from Stevens and Davis." )

ing frequencies to which the particular areas are sensitive. (3) In human high tone deafness there is a degeneration either of the organ of Corti near the base of the cochlea or of the nerve fibers supplying this region. (4) Cochlear microphonics are recorded with greater voltage near the arex in response to low tones and near the base in response to high tones. The region where each frequency produces the highest voltage may also be mapped by moving the recording electrode along the outside of the spiral. Furthermore, destruction or disturbance of certain regions of the organ of Corti by drilling into the cochlea produces a rise in threshold for the cochlear microphonics at the corresponding frequencies. (5) It has been demonstrated ${ }^{1 i}$ that single fibers of the auditory nerve are stimulated most easily by waves of a given frequency, less easily by adjacent frequencies, and very much less easily (threshold $30 \mathrm{db}$ or higher) by frequencies differing by an octave or more 
(Fig. 159). (6) Integration of curves for data on the just-perceptible-difference tones as a function of frequency yields a curve which can be superimposed on a plot of the position of the sensitive region as detected by the drilling experiments mentioned in (4) above as a function of frequency. This should be expected if the minimal detectable difference in frequency corresponds to the minimal detectable distance between two adjacent regions of excitation, and if this distance is constant throughout the length of the cochlea. Since the sense cells are about the same size throughout the length of the cochlea the latter assumption seems reasonable. (7) The mass of vibrating material along the path of a sound wave from oval to round window is much less if the wave passes through the basilar membrane near the base
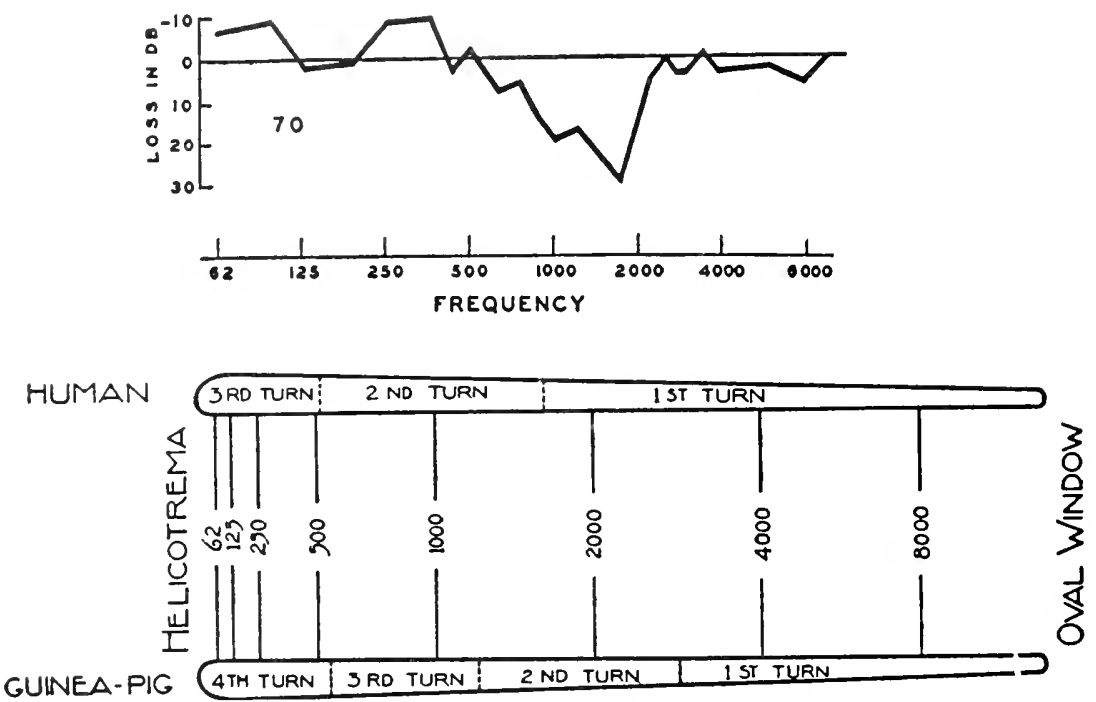

Fig. 158. Upper: Curve of intensity of stimulation necessary to elicit a just-measurable microphonic response from a cochlea which has sustained an injury. Lower: Map of the cochlea of the guinea pig, indicating localization of pitch reception. This map is reconstructed from data of 20 experiments similar to that shown above, together with histological examination of basilar membrane damage. The pitch localization map for man is deduced from the guinea pig data. (After Stevens, Davis and Lurie ${ }^{4 \prime \prime}$ from Fulton. ${ }^{15}$ )

than if it passes near the apex. Since the natural frequency of a vibrating system increases with reductions in mass, the natural frequency of the path through the basal region is higher than that through a region near the apex. In general, there seems to be no reason for doubting the place theory of the hearing mechanism.

Transmission in the Nervcus System. If we assume that each cross row of hair cells (e.g., each such row usually consisting of one inner and three outer hair cells), because of the geometry and physical character of its surroundings, is most sensitive to one frequency, then the next step in an explanation of the hearing mechanism is to show how information concerning stimulation of these cells is transmitted to the brain. There are about 3500 hair cells on the inner row and about 20,000 equally distributed among the 
three outer rows. The neurones of the auditory nerve are bipolar, have their cell bodies in the spiral ganglion which lies within the modiolus, and are between 25,000 and 29,000 in number. Each inner hair cell is innervated by one or two nerve fibers, and each of these nerve fibers is connected to one or two hair cells. The external hair cells, however, have a multiple innervation. A nerve fiber may connect with many external hair cells, which are arranged over as much as onc-half a turn, and each hair cell may be connected to several nerve fibers (Fig. 155). The inner hair cells, because of their connections to only one or two dendrites, may be used for more exact discrimination of frequency than the more numerous outer hair cells.

The axons of the ganglion cells form the cochlear branch of the eighth cranial nerve. The fibers of this nerve are twisted around each other in a very orderly fashion, somewhat like the fibers of a rope. Those from the

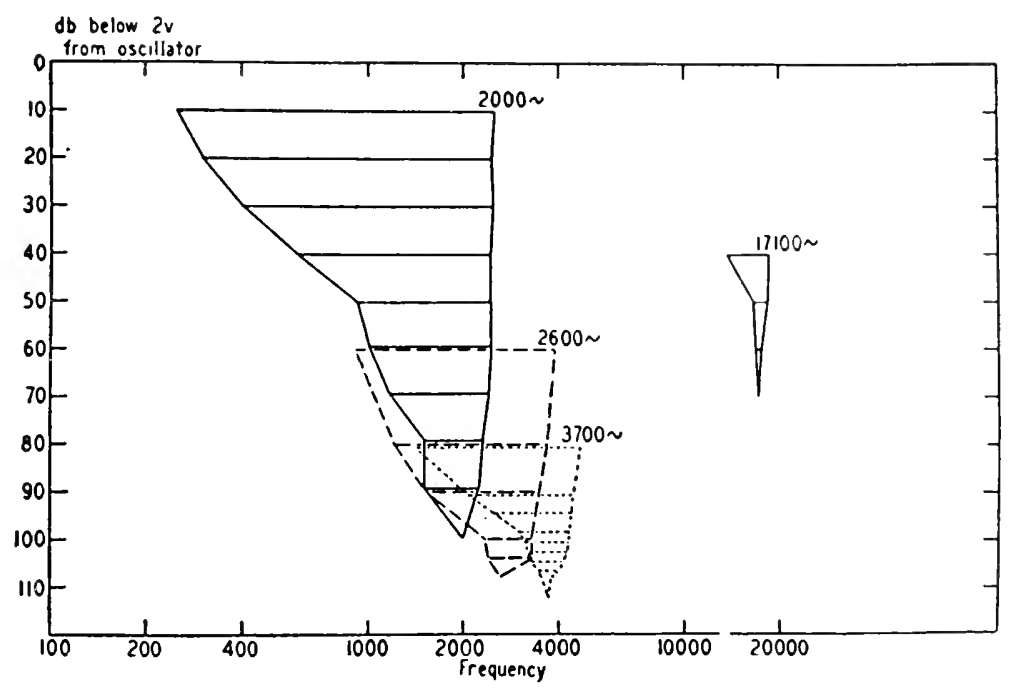

Fig. 159. Curves showing the frequency response of four different auditory nerve fibers as a function of intensity. Note that the range of frequencies capable of exciting a given fiber increases with increasing intensity. After Galambos and Davis, ${ }^{17}$ from Fulton. ${ }^{15}$

region about one fourth of the length from the basilar membrane are not twisted and form the core of the rope. Those coming from the apex are twisted in one direction and those from the base are twisted in the other. The genesis of this arrangement is readily understood from an embryological viewpoint, for the fibers merely follow the organ of Corti as it grows into its final spiral form.

On entering the dorsolateral wall of the pons at its junction with the medulla, the axons of the cochlear nerve pass to the cochlear nuclei. From the dorsal and ventral cochlear nuclei impulses pass by way of the lateral lemniscus to the inferior colliculus of the midbrain and through the medial geniculate body to the temporal lobe of the cerebral cortex. Local exploration of each of the stations of the auditory pathway indicates fairly precise projection of the cochlea. 
Intensity Discrimination. The mechanism for the perception of intensity is apparently dual. ${ }^{17}$ With increasing intensity of a given frequency there is: (1) an increase of the frequency of impulses in single nerve fibers, especially for high frequencies; (2) an increase in the number of fibers which carry impulses. As the vibrations increase in amplitude a wider and wider band of sense cells responds. At low frequencies the impulses of a single fiber have a one-to-one correspondence to the pressure waves of the stimulus. This is true below 500 cycles for the initial waves and at the frequency of 200 cycles with equilibration. However, above these sound frequencies the frequency of impulses varies with intensity. For example, at 7000 cycles (Fig. 160) the frequency of discharge of a single fiber may increase from less than 10 to as much as 135 per second as the intensity is increased from -82 to $-30 \mathrm{db}$ below a given reference level. It can also be seen from Figure

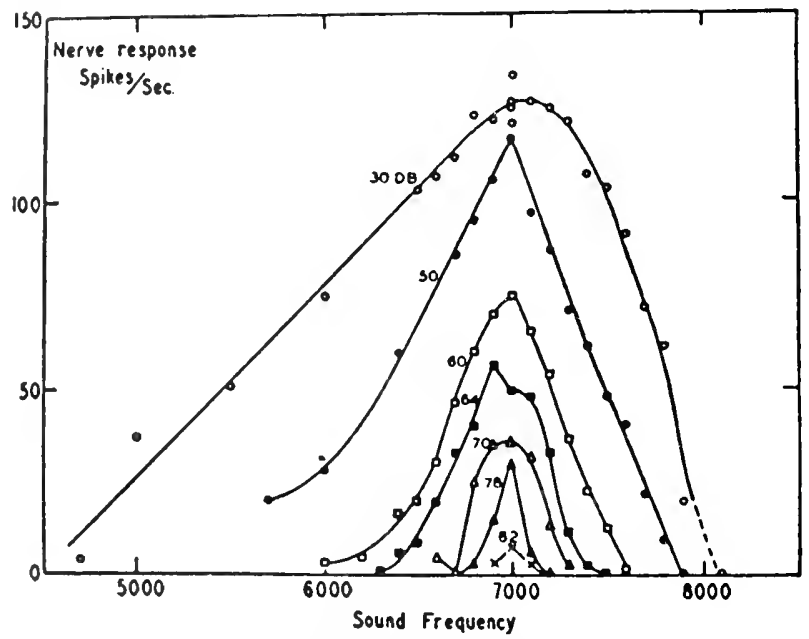

Fig. 160. Curves showing the relation between frequency of spike potential discharge of a single auditory nerve fiber and the intensity and frequency of the sound stimulus. Note that with increasing intensity the fiber responds to a greater frequency band, and, at each frequency, with a greater frequency of spike potential discharge. After Galambos and Davis, ${ }^{17}$ from Fulton. ${ }^{15}$

160 that although this one fiber was responsive only at frequencies very close to 7000 cycles at -78 and $-82 \mathrm{db}$ it was responsive to all frequencies between 5000 and 8000 at $-20 \mathrm{db}$, with, however, a maximal response still at 7000 .

Sound Localization. One important function of the human ear, in addition to discrimination of frequency and intensity, is that of localization of the source of sound. A person with normal hearing is able to locate sounds with a surprising degree of accuracy. This is possible even with very com. plex sounds, such as that of a symphony orchestra, during which even a blind listener, if he is close to the orchestra, is able to locate the various instruments. Such localization is largely a function of the intensity differences and of the phase differences of the sound waves as they strike the two ears. For all tones above 500 cycles and especially for those above 5000 cycles, one ear may be in the "shadow" of the head. For a tone of 10,000 cycles loudness differ- 
ences in the two ears may be as much as $30 \mathrm{db}$, thereby contributing materially to the problem of localization. A phase difference means that the sound pressure reaches a maximum at one ear shortly before it does at the other ear. This should give rise to time differences of nerve impulses in corresponding nerve fibers from the two ears, especially for low frequency sounds, i.e., below 800 cycles. Some individuals are very sensitive to this "phase effect," and others are apparently insensitive. In general, for localization the phase effect seems to be more important at low frequencies, and the intensity effect at high.

\section{PHONORECEPTION BY OTIER VERTEBRATES}

In lower vertebrates the hearing mechanism is not nearly so well developed as it is in mammals. From a very slight development in certain fishes it is possible to trace phylogenetic series showing the development of the auditory organ (Fig. 161). The sacculus and semicircular canals are found in cyclostomes and in all higher chordates. I lagfishes, however, have only one
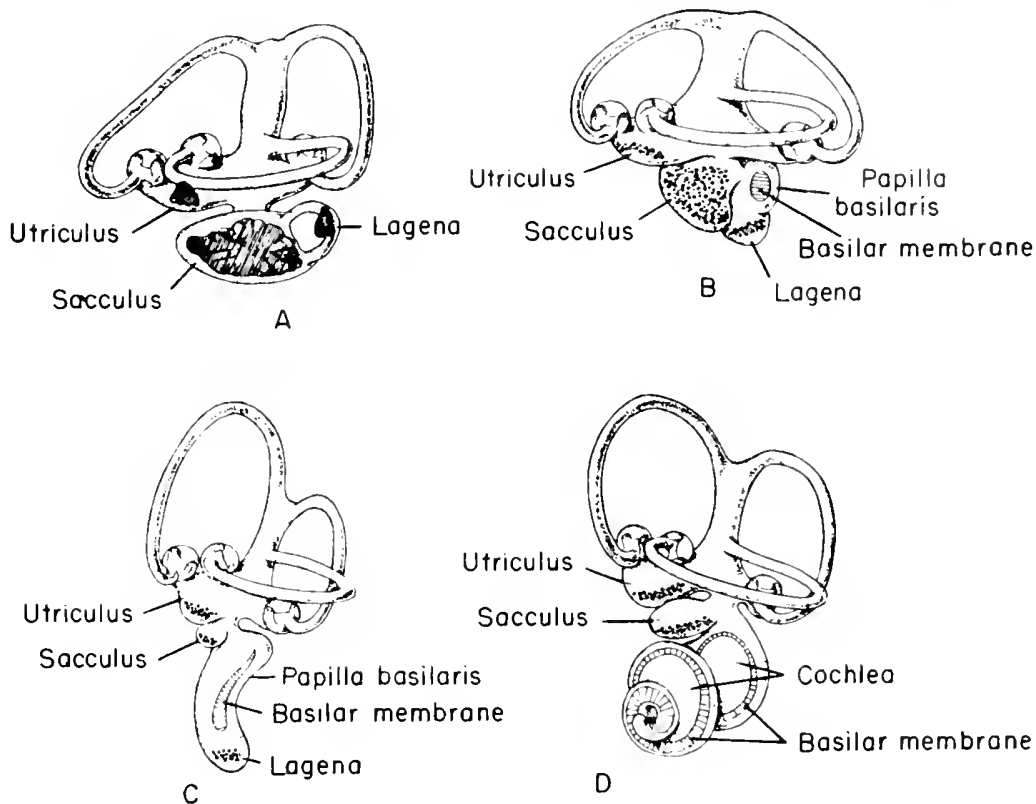

Fig. 161. The labyrinths of various vertebrates. $A$, fish; $B$, turtle; $C$, bird; $D$, mammal. From von Frisch. ${ }^{14}$

canal, and lampreys have two; all higher fishes and higher vertebrates have three. There is little if any differentiation of an auditory mechanism in the cyclostomes. In most fishes the sacculus has a small evagination which is known as the lagena and which is supplied by a separate branch of the eighth cranial nerve. In fishes of the suborder Ostariophysina and in amphibia the lagena is larger, in reptiles, birds, and mammals it has become the cochlear duct. The middle ear containing a single ossicle is first found in amphibians and has developed into the highly efficient structure with three ossicles found 
in mammals. Howerer, the same three bones are found in fishes, in which they are known as the Weberian ossicles and connect the air bladder to the inner ear.

Fishes. In fishes only the vestibule and semicircular labyrinths are present, and they have the dual function of determining equilibrium and of serving as primitive auditory organs. There is no middle ear. The sacculus of the trout is large, and there are three semicircular canals. The sacculus of fishes generally has two small evaginations, the utriculus and the lagena. There are usually statoliths in all three divisions, and these statoliths are especially well developed in fish which produce sounds. The drumfishes, Aplodonitus and Sciaena aquila, have large statoliths, and Sciaena has been reported as being heard six feet above the water when it was swimming sixty feet below the surface. The sounds of the drumfishes are produced by movements of the air bladder, and since they are produced primarily by the males during the mating season it seems very likely that they are perceptible to the females of the same species.

There has been much contradictory evidence concerning whether fish can hear. Most of the negative evidence, however, is based on experiments in which fish did not respond to sounds produced in air. This is often due to the fact that the air-water surface is almost a perfect reflector for sound waves, and therefore sounds produced above water are usually reflected rather than transmitted to the water. A man swimming under water may not be able to hear a pistol shot in air nearby, but he usually can hear sounds made by someone kicking the bottom of a nearby boat, or sounds made by knocking two rocks together under water. Likewise, sounds made by tapping the sides of an aquarium may readily produce a response in fishes.

Zotterman $^{61}$ measured a microphonic effect in the macular region of the sacculus of the pike, Esox lucius, and of the burbot, Lota vulgaris. He stimulated the ear with the tone produced by a 60 cycle tuning fork and recorded a "macula effect" comparable to the microphonic effect in the mammalian ear. He also recorded nerve impulses when the ear was rotated in one direction in the plane of a semicircular canal; with opposite rotation a response occurred only at cessation. The existence of the macula effect demonstrates that there is probably a mechanism for receiving sound of low frequencies.

On the basis of behavior there is considerable evidence that fish have a high ability to hear sounds in water. Parker30, 31 demonstrated that dogfish are responsive to tapping of the aquarium, and that the reaction persists after cutting of nerves to the skin and lateral line. Cutting of the eighth cranial nerve, however, made the fish almost completely insensitive to sound. In the squeteague (Cymoscion) the same phenomenon could be demonstrated." Moreover, in Cynoscion the sacculus is separate from the utriculus and semicircular canals, and Parker was able to show that after destruction of the utriculus and canats the fish still responded to sounds, although it lacked control of its equilibrium. Destruction of the sacculus alone was not possible, but a pin thrust into the large saccular statolith greatly reduced sensitivity to tapping.

Bull, ${ }^{7}$ by means of conditioned reflexes, has been able to demonstrate that cels are sensitive to a submerged 342 cycle buzzer, and that wrasse will re- 
spond either to the buzzer or to a 128 cycle tuning fork. The blenny, however, responds to neither. Stetter ${ }^{46}$ demonstrated that minnows, golden orfe, goldfish, barbels, miller's thumbs, and catfish respond to high notes, the minnow to frequencies as high as 4000 cycles, and the catfish to frequencies as high as 13,000 . With use of the conditioned response method, it was found that two frequencies about an octave apart could be remembered and distinguished, and the best fish learned even to discriminate a minor third. Some

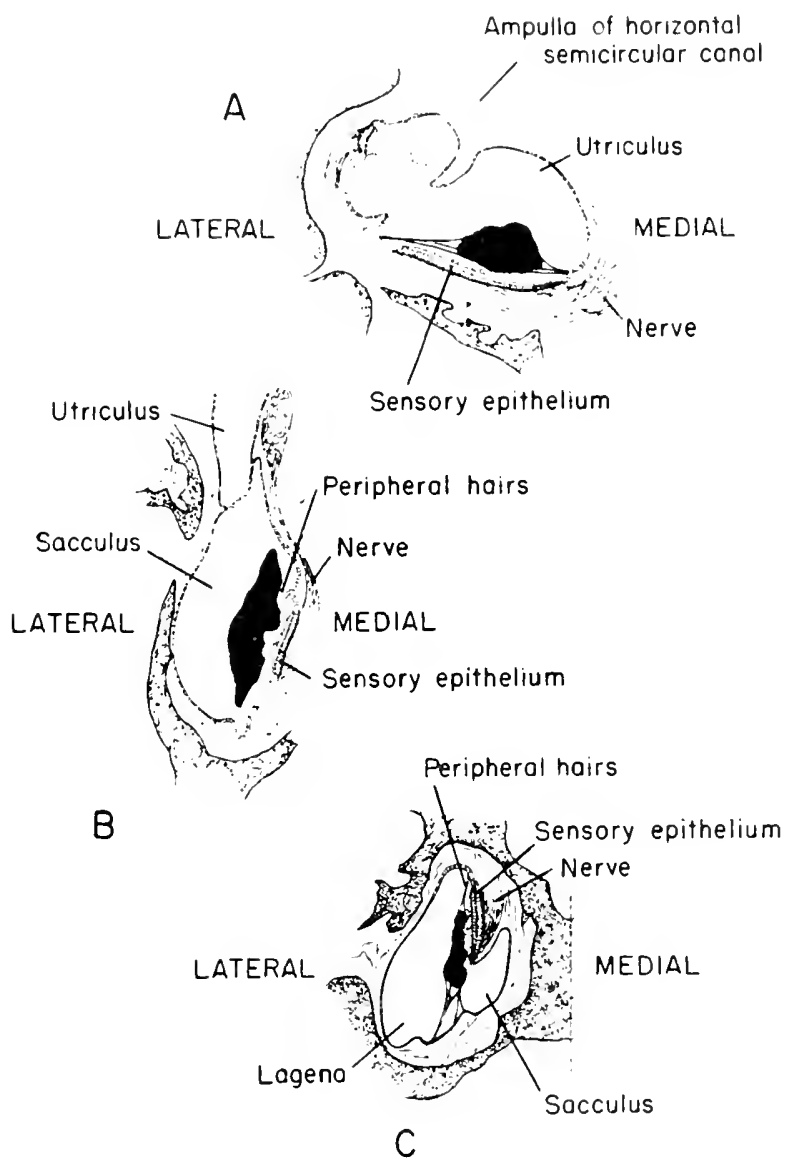

Fig. 162. Sections of labyrinth of the trout. A, Cross sections through the utriculus. $B$, Cross section through the sacculus. $C$, Cross section through the lagena. The statoliths are black. From von Frisch. ${ }^{1 *}$

fish were able to remember as many as five tones. In goldfish Manning ${ }^{26}$ showed that removal of saccule and lagena reduced the upper limit of response from 2752 to 688 cycles and that additional removal of the utriculus reduced the upper limit to $34+$ cycles. Stipetic ${ }^{-11}$ demonstrated that the fresh-water fish Gnathonsemus were sensitive to frequencies of as high as 370 or 392 cycles and could discriminate between intervals of a minor third (frequency ratios of 1.2 to 1.0$)$. 
In fishes of the suborder Ostariophysina (carps, catfishes, characins, and gymnotids) the lagena is large and contains a very large statolith. In the nonOstariophysina both the lagena and the statolith are smaller. The statoliths of the sacculus, utriculus, and lagena of both the Ostariophysina and nonOstariophysina are attached loosely by two marginal membranes to the wall of the vesicle in such a way that they are free to vibrate. As they vibrate they make contact with the sensory epithelium. This relationship is shown in Figure 162.

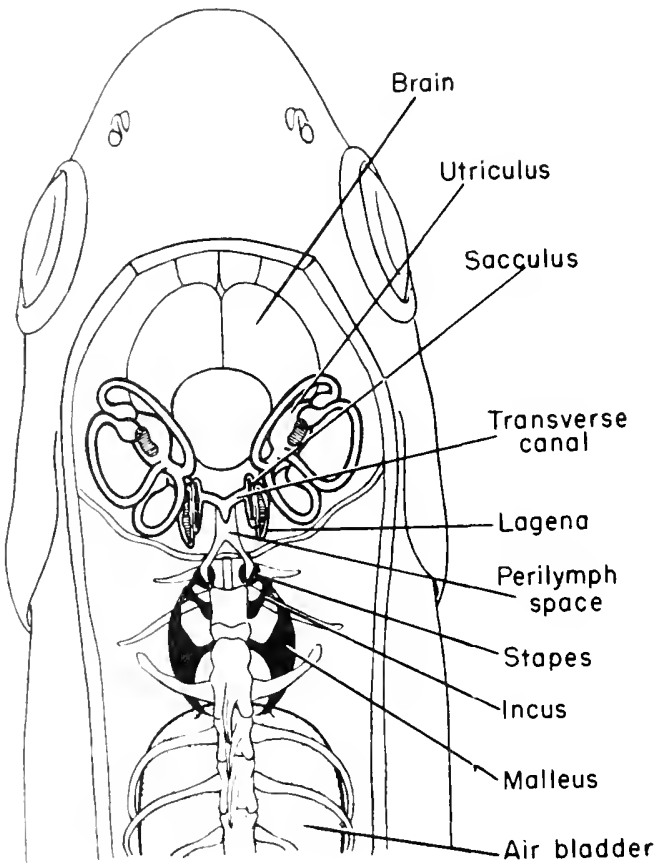

Fig. 103. The coupling between the air bladder and the labyrinth by means of Weber's ussicles in the Ostariophysina. Weber's ossicles, black. Redrawn from v. Frisch. ${ }^{14}$

In the Ostariophysina hearing is very acute, and the air bladder is connected to the auditory organ, particularly to the sacculus, by a chain of bones, the Weberian ossicles (Fig. 163). This bony connection makes it possible for the air bladder to function as a hydrophone which picks up pressure waves over a wide surface of the body wall and transmits them to the ear by means uf the ossicles. These ossicles are homologous to the bones of the mammalian middle calr, but are embedded in tissue rather than suspended in the air, as in mammats. The saccular statolith is especially modified for the reception of sound waves directed toward it by this bony mechanism, which is responsible for the high sensitivity of these fishes.

That the lateral line organs of fishes participate in reception of low-frequency sound waves has been demonstrated by Parker and Van Heusen ${ }^{32}$ and I foigland"'-". Parker and Van I leusen showed that the lateral line organs of catfish are stimulated by sonic vibrations up to 344 cycles per second, as cridenced by absence of the response of the fish to auditory stimuli in the 
absence of lateral line organ participation. I Ioagland $\$ 2$ demonstrated that the typical random discharge of spike potentials in lateral line organ nerves of catfish is synchronized when stimulated with 100, 200, and 250 cretes per second, but that the frequency of synchronization is not related to the free quency of the sound waves cxcept at low frequencies.

It has been demonstrated with operative methods by von Frischent that in minnows the seat of hearing is definitely located in the saceulus and lagenit, which are sensitive to frequencies of 32 to 5000 or 6000 cycles, and the $u f r 1$. culus and labyrinth apparently have no auditory function. Low frequen. cies (below 100-150 cycles) are perceived also by the touch receptors of the skin, and very low frequencies ( 16 cycles) by the touch sense only, as shown:

Lower limit

16-32

\section{Sensitivity of sacculus and lagena}

$\begin{array}{lllllllllll}\text { Tone } & C_{2} & C_{1} & C_{1} & c & c^{1} & c^{2} & c^{3} & c^{1} & c^{5} & C^{6} \\ \text { Frequency } & 16 & 32 & 65 & 129 & 259 & 517 & 1035 & 2069 & 4138 & 8277\end{array}$

Upper limit $5000-6000$

\footnotetext{
Sensitivity

of touch sense

of skin
}

Amphibia. In amphibia the lagena is slightly longer than in fishes (Figs. 164 and 165), but the principal new development is the middle ear, which is found in the Salienta but not in the urodeles. In the frog, for instance, the

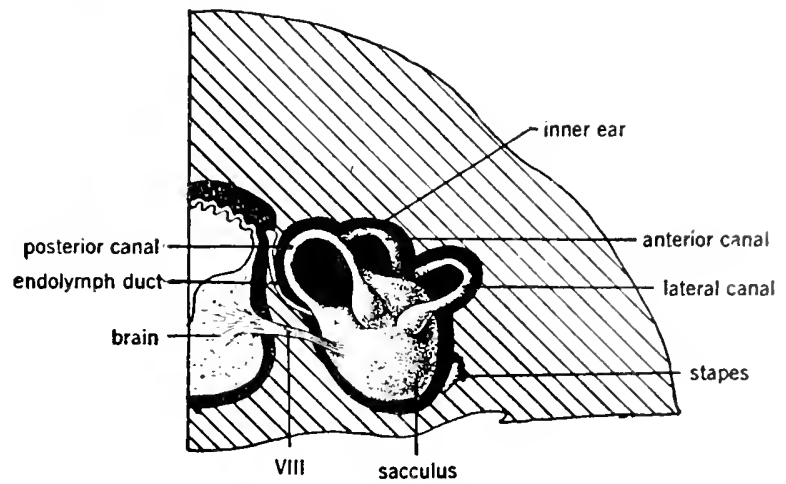

Fig. 164. Diagrammatic presentation of the ear of Amblystoma, as seen from the posterior. From Adams.'

middle ear consists of an ear drum, an air cavity, and a bony rod, the col umella, which connects the drum with the inner ear. This is an adaptation for transmitting the vibrations of air to the car liquid, and therefore serves the same function as the middle ear of man.

Urodeles are commonly considered to be deaf. However, Ferhat-Akat ${ }^{11}$ has demonstrated by means of training experiments that Amblystoma is able to respond to frequencies as high as 194 to 244 cycles. Extirpation of the labyrinth reduced this limit to 97-122 cycles. The lower limit of response of the animal was not determined, but it was shown that the labyrinth did 
not function below 32 cycles. The mechanoreceptors of the skin are apparently as important as the labyrinth. Ferhat-Akat also demonstrated that Amblystoma is able to distinguish between frequencies that differ in pitch as much as a musical interval of a fourth or a fifth (frequency ratios of $1.32: 1$ and $1.50: 1$ ). In a few experiments on Salamander maculosa larvae he showed that the upper limit of reaction was 1035 cycles.

Frogs are relatively unresponsive to sounds, but they may have an acute sense of hearing. Yerkes, ${ }^{60}$ on the basis of a study of conditioned reflexes, reported that frogs can hear sounds between 50 and 10,000 cycles, but he did not obtain evidence of discrimination. Yerkes' frogs continued to respond

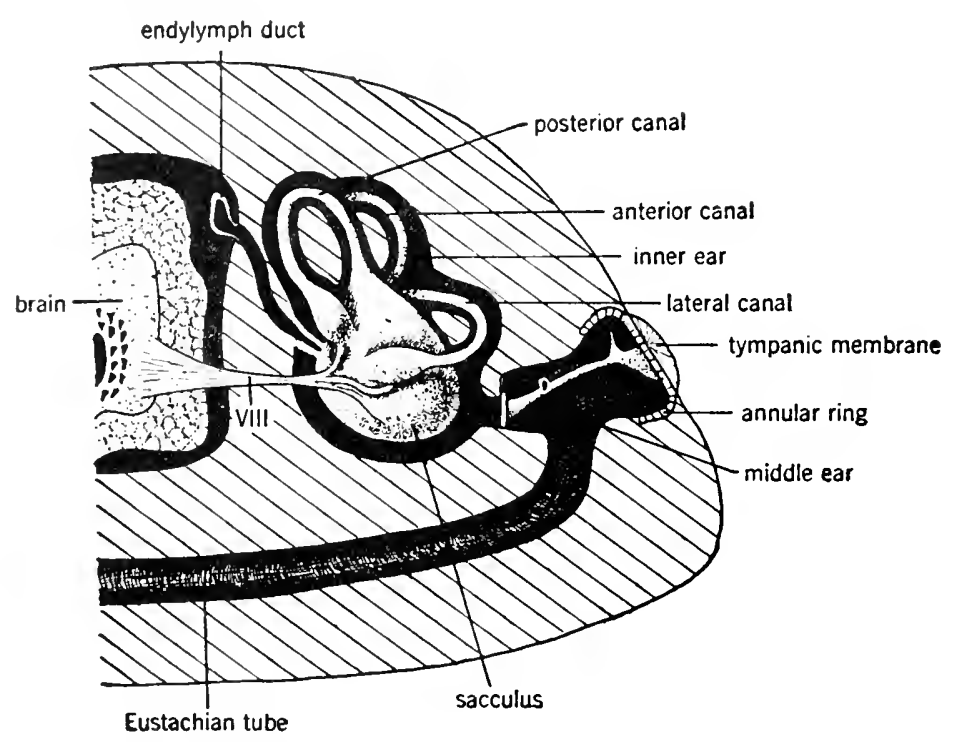

Fig. 165. Diagrammatic presentation of the ear of the frog, as seen from the posterior. From Adams. ${ }^{1}$

after removal of the ear drum and columella but not after cutting of the auditory nerves. Adrian, Craik, and Sturdy ${ }^{3}$ studied the microphonic effect in frogs and obtained responses between 50 and 300 cycles, with the maxi. mum sensitivity between 50 and 200. Ross ${ }^{51}$ in a study of single nerve fiber preparations observed a response to vibrations of about 100 cycles per second but did not investigate the extent of the range.

Witschisx demonstrated that during the early development of tadpoles the round window of the inner ear becomes connected with a bulge of the lung sac by means of a fibrous cord. This cord becomes cartilaginous, picrces the dorsal aorta, and serves as an aortic columella which transmits vibrations to the inner ear. In this respect the aortic columella functions in the same way as the Weberian ossicles of fishes. The oval window serves as a pressure release mechanism. During later development the aortic columella disappears and is replaced by the tympanic columella, which develops in the middle ear and makes contact with the oral window. The round window then serves as a pressure release, as in higher forms. 
Reptiles. It is in certain reptiles that structures characteristic of a true cochlea make their first appearance (Figs. 161, 166). In alligators the lagena is attached to two sides of the surrounding cavity, thereby forming three ductsthe scalae vestibuli, media, and tympani,-and the floor of the lagena forms the basilar membranc. In all reptiles other than snakes there is a middle ear which contains a bony structure similar to the columella of frogs, except that it usually is composed of two bones. The tympanic membrane is usually depressed below the body surface to form an external auditory meatus, and in alligators there is even a skin fold which may serve as an outer ear. Snakes have no middle ear, and the outer end of the columella is attached to the quadrate bone of the skull. This arrangement makes snakes (even the cobra!) insensitive to air-borne sounds but very sensitive to earth-borne vibrations, such as those resulting from the footfalls of approaching animals. There is considerable evidence that rattlesnakes cannot hear their own rattling. ${ }^{25}$

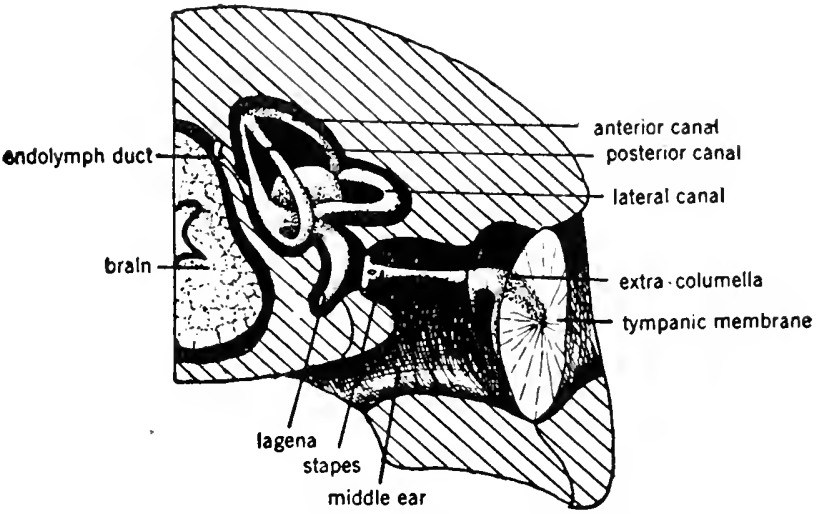

Fig. 166. Diagrammatic presentation of the ear of a reptile, as seen from the posterior. (Adams. ${ }^{\text {) }}$ )

The microphonic effect and the auditory nerve responses of reptiles have been studied by several investigators. Foa and Peroni ${ }^{13}$ recorded impulses from the auditory nerve of Thalassochely's. Wever and Bray.3 found a microphonic effect between 120 and 1000 cycles for Chrysemys picta, with a high response below 500, and Adrian² found that the ear of Cistudo responded only to the narrow band of 80 to 130 cycles, with a maximum at 110 cycles, and the impulse frequency of the nerve was the same as the stimulating frequency. A snake gave an irregular nerve response discharge in response to earth-borne vibrations but none to air-borne sound. Adrian, Craik, and Sturdy ${ }^{3}$ obtained microphonic and nerve responses from tortoises, box turtles, and alligators. The range of the microphonic effect for the tortoise was 50 to 300 cycles with a maximal sensitivity of 50 to 200; that for the box turtle was 50 to 2000 cycles, with a maximum between 200 and 800 ; the range for the alligator was 50 to 4000 , with a maximum between 300 and 2500 . It was found that the upper limit of the nerve responses varied with temperature, and that a rise in temperature from $9^{\circ}$ to $26^{\circ} \mathrm{C}$. raised the maximal frequency of the nerve response from 200 to 500 impulses per sec- 
ond. This variation with temperature makes the frequency of the nerve impulses relatively inconstant for the purpose of frequency discrimination. Therefore, as pointed out by these investigators, an adequate system of temperature regulation seems to be a prime prerequisite for frequency discrimination.

The lizards Lacerta and Tachydromus respond to sounds up to 8000 and 10,000 cricles, but there is no evidence that they are capable of frequency discrimination. 5,24

Birds. The ear of birds is very similar in structure to that of reptiles (Fig. 161 and Fig. 167). In the basilar membrane of birds there are only 3000 fibers (24,000 in man) and there are 20 to 30 hair cells ( 4 in man) in each row across the cochlea. Therefore, one might expect a lower degree of fre-

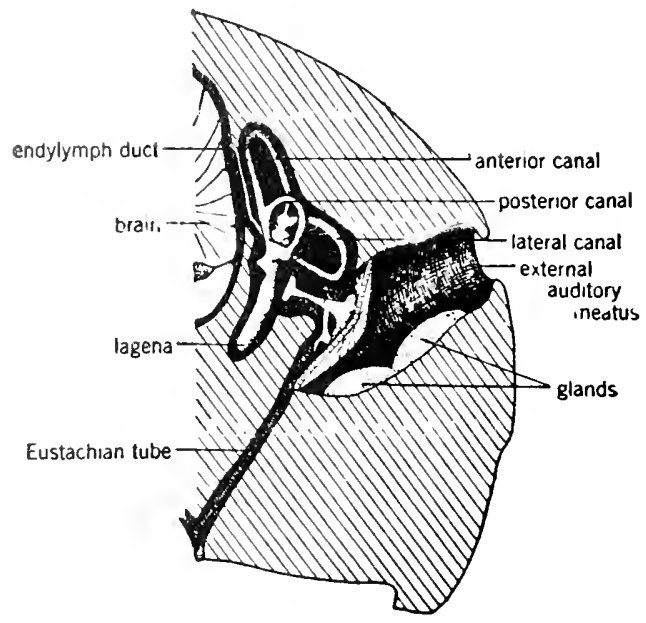

Fig. 167. Diagrammatic presentation of the ear of a bird (Gallus), as seen from the posterior. From Adams. ${ }^{1}$

quency discrimination and perhaps a lower frequency range than that of man. However, there is good evidence ${ }^{23}$ that parrots and crossbills (Loxia curvirustra) have a frequency range of 40 to 14,000 cycles, and a sense of frequency discrimination approximately equal to that of man. Wever and Bray demonstrated a microphonic effect in the pigeon for the range of 100 to 10,000 cycles.

In gencral, the hearing of birds is as well developed as that of mammals. The ability of birds to discriminate frequencies is apparent to anyone who has watched birds respond to the call of their mates or to signals of alarm from bircls of the same or of different species. A walk through any bird nesting ground during the brecting scason provides ample evidence of both hearing and frepuency discrimination. This is also obvious in the mimicry of certain bircts (e.g., mockingbirds and catbirds) which copy the sounds of other bircts, and in the mimicry of parrots which copy human speech. Birds are also capable of accurate localization of sound, provided both ears are normal and uncovered. 
MammaIs. In all mammals the cochlea is highly developed, and in all mammals that are not completely aquatic the middle ear contains three os. sicles similar to those of man and an external ear is usually present (Fig. 168). The cochlea is always coiled, but the number of turns has no apparent rela tion to the size or intelligence of the animal. The duek-billed platypus has a quarter turn; the whale has 1.5 turns, the horse 2 , man about 2.75 , cat 3 , pig and guinea pig nearly 4, and the South American "paca" (Coelogeny's) 5.

Many mammals are sensitive to frequencies above the range of human hearing, i.e., in the so-called "ultrasonic" range. Pavlow:3: demonstrated that dogs have a wide frequency range and a power of frequency discrimination at least equal to that of man. We now know that the upper limit for clogs

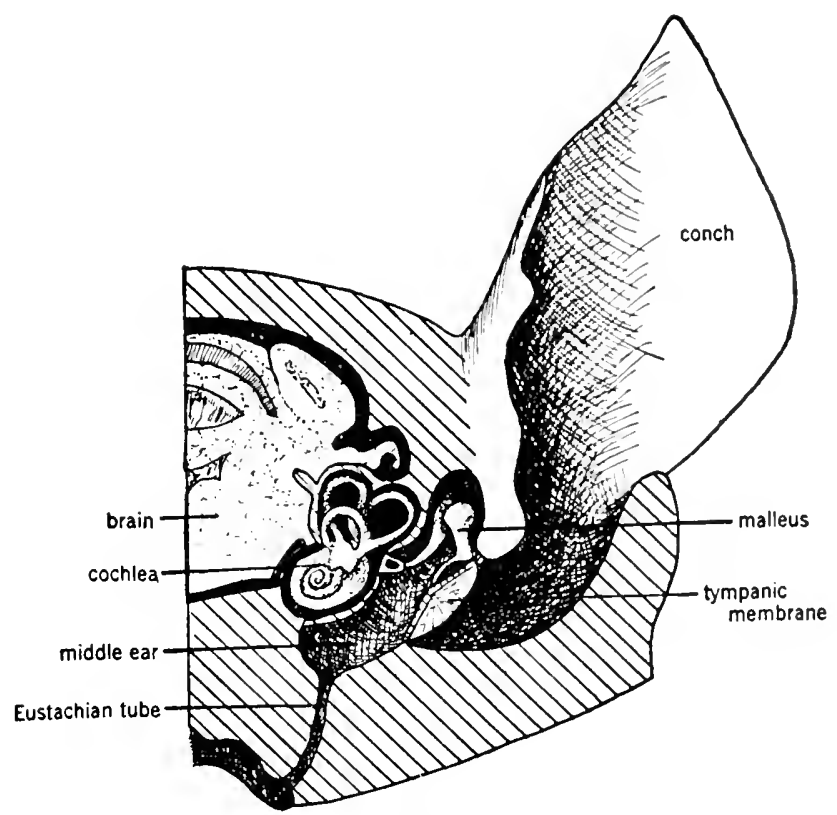

Fig. 168. Diagrammatic presentation of the ear of a mammal (rat) as seen from the anterior. From Adams. ${ }^{1}$

is about $35 \mathrm{kc}$, and "silent" whistles have a frequency well above $20 \mathrm{kc}$. The upper limits for some other mammals are: $50 \mathrm{kc}$. for cats, $40 \mathrm{kc}$. for rats and guinea pigs, $33 \mathrm{kc}$. for chimpanzees, and at least $98 \mathrm{kc}$. for bats.

The hearing of bats is especially interesting in that not only does it serve as a means of warning of the approach of other animals and as a means of communication between individuals, but it also aids flying bats to locate objects in the dark. The vision of bats is poor, but because of their normal cave habitats and their nocturnal habits cven good vision would be of little use in the avoidance of objects while flying. Caves which have sound-absorb. ing walls (e.g., lined with box crystals) are usually not inhabited by bats.

It was originally proposed by Hartridge ${ }^{21}$ that bats might emit ultrasonic cries which could be reflected from obstacles and, if heard by the bat, could thereby serve as a sound echo-ranging device. Through binaural localization 
of the source of the reflecting material a bat might be able to locate an object with considerable accuracy. This method of echo location is directly comparable to that of a radar ranger and localizer which broadcasts pulses of very high frequency radio waves and from the echo permits very accurate determination of both the distance and direction of a reflecting surface. The system of the bat differs from radar primarily in that the waves emitted are high frequency sound rather than high frequency radio waves.

Hartridge's theory of avoidance of objects by bats was proved in a series of papers, $20,1 \times, 16$ and has since been confirmed by other investigators. It is definitely known that during flight bats of the genera Myotis, Eptesicus, and Pipistrellus emit brief cries of a sound frequency between 30 and $70 \mathrm{kc}$. and perhaps also of higher frequencies, with the highest intensity usually at $50 \mathrm{kc}$. Each cry has a duration of 0.001 to 0.002 second, and the cries are emitted about 10 times a second when the bat is hanging on a wall, about 30 times per second when it is flying in unobstructed space, about 50 times per second when it is approaching an object, and usually only 30 times per second just before it flies past an object. The frequency of each cry begins at $70 \mathrm{kc}$. and drops to $30 \mathrm{kc}^{19}$ The emission of cries is facilitated by modification of the pharyngeal ventricles into pockets which act as drumlike acoustic resonators for ultrasonic frequencies. Bats also emit audible sounds of $7 \mathrm{kc}$. and audible "clicks." These sounds are apparently used for communication with each other. The ultrasonic frequencies used are not of unusual occurrence in our daily life (e.g., key jingling, up to $98 \mathrm{kc}$.; air moving through a small opening, up to $80 \mathrm{kc}$.), but bats are the only animals known to make such use of them.

Bats which have one or both ears covered or which have their mouths covered so that their ability to receive or emit sounds is impaired are unable to avoid obstacles as well as normal bats do. In fact, such bats show little inclination to fly at all, whereas normal bats can fly between $1 \mathrm{~mm}$. wires stretched $30 \mathrm{~cm}$. apart, with relatively few collisions. In studies of the microphonic effect in the cochlea Galambos has found that responses are obtained to an upper limit of $98 \mathrm{kc}$, the limit of the apparatus but below that of the bat.

There has been a considerable amount of discussion recently on how a bat is able to locate objects so close that the reflected sound would be heard at almost the same time as the emitted sound; i.e., in the ear the cry and the echo would overlap. For instance, a bat can detect a glass plate at a distance of 2 feet. One suggestion is that bats might use the "trailing edge" rather than the "leading edge" of the series of waves which constitute a cry." Others are that bats (1) may use the Doppter effect, (2) may decrease the duration of the signal as they approach an object, or (3) may have the ears screened from the source, i.e., from the larynx." The two latter possibilities seem most likely.

\section{PIIONORECEPTION BY ARTHROPODS}

\section{Receptors in Insects}

Aside from the vertebrates, the insects comprise the only group of animals in which phonoreception has been intensively studied. Ilowever, although insects probably comprise the only group in which it is highly developed, 
it should not be assumed that phonoreception is completely unimportant in the life of other arthropods.

The phonoreceptors of insects are of two general types: 1) the tympanic organs, and (2) hair sensilla. The tympanie organs are highly spectalized structures which are very sensitive as receptors, especially for high frepuencies. Howerer, it is welf homon that insects which possess no tympanic organs are sensitive to sound, especially to low frequencies, and this sensitivity is localized in hair sensilla.

Morphology of Tympanic Organs. A tympanic organ consists of a thin region of the exoskeleton which acts as a tympanic membrane, an underlying air sac, and the sensory elements known as a chordotonal organ or scolopid. ium. Tympanic organs are paired structures which oceur in the Orthoptera,

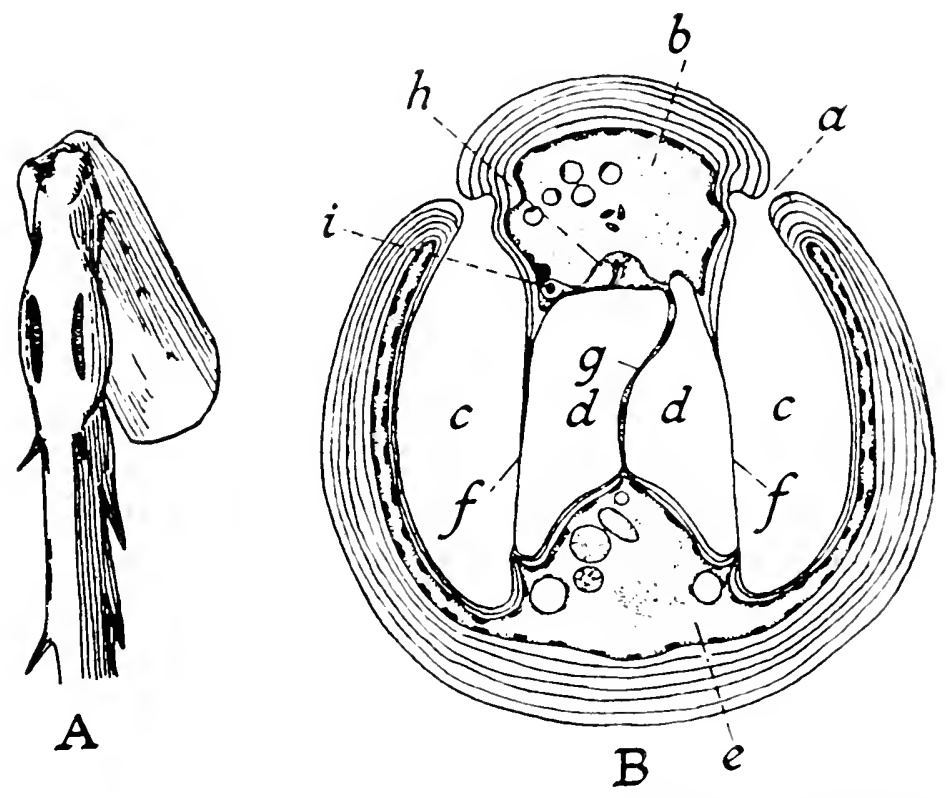

Fig. 169. Tympanal organs in the tibia of Locustids. A, Locusta foreleg, showing the slit openings of the tympanal sacs (after $\mathrm{W}^{\mathrm{T}} \mathrm{eber}^{\bar{\alpha}}$ ). $B$, Transverse section of anterior tibia of Dectycus, through the tympanal organ (after Schwabe $e^{+1}$ ). $a$, opening on surface of tibia; $b$, blood chamel; $c$, tympanal cavities; $a$, rracheae; $e$, blood channel with muscles and nerves; $f$, tympanum; $g$, rigid wall between the enlarged tracheae; $h$, crista acoustica, showing one chordotonal organ; $i$, sense cell. From Wigglesworth.it

Hemiptera, and Lepidoptera. Among the Lepidoptera they occur only in the Noctuoidea, Geometroidea, and Pyralidoidea, and they are found either in the forepart of the abdomen or in the metathorax of the adult. Among Hemiptera they are probably functional only in adult Cicadidae, in which they occur in the abelomen. Among Orthoptera they are found in most species of Acridiidae. Tettigoniidae, and Gryllidae. In grasshoppers they occur in the abdomen, and in katydids and crickets they occur near the proximal end of the tibiae of the first pair of legs. They are present in late nymphal instars but probably are functional only in adults. 
The tympanic membrane, when located on the body, generally lies in a cleft which forms an external meatus (Figs. 169 and 170), and it may be further protected by the folded wings or the femur of the third leg, or both. On the tibia there are usually two tympanic membranes on opposite sides of the limb. In many genera these membranes are protected by heavy folds which almost completely enclose them (Figs. 169, 170). In Gryllus the membrane is completely exposed, but is associated with a very long and prominent hair sensillum, which, on slight contact, evokes an immediate avoiding response of the animal.

The chordotonal organs are the sensory elements of the tympanic organ. On the basis of morphological resemblance the term "chordotonal organ" is also applied to similar structures which are not associated with a tympanic

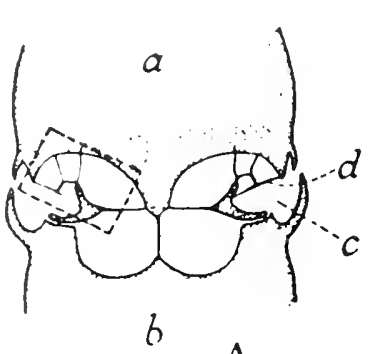

A

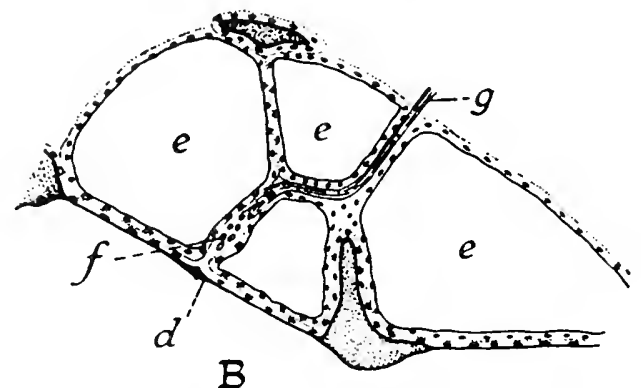

B

Fig. 170. Sections through thoracic tympanal organs of Catocala. A, Horizontal section. B, Detail of organ on left side (after Eggers ${ }^{4}$ ). $a$, thorax; $b$, abdomen; $c$, tympanal pit; $d$, tympanum; $e$, tracheal air sacs; $f$, chordotonal organs; $g$, nerve. From Wigglesworth. ${ }^{56}$

organ and, at least in some cases, do not have an auditory function. The noncommittal term "scolopidia" is also applied to all "chordotonal" organs, regardless of whether or not they are auditory. The non-auditory scolopidia are widespread in the insect body and are found in the legs, antennae, palps, wing bases, and the general body cavity. At least one end is fastened to some pliable region of the cuticle, usually the intersegmental membrane. Any movement, active or passive, of the animal which results in a relative change in the position of two segments is likely to cause stimulation of such scolopidia; they are also sensitive to external pressure, pressure or tension of nearby muscles, and general changes of the internal pressure in the blood or tracheal system. Those attached to tympanic membranes are distinctive only in that they are attached to membranes particularly sensitive to vibration by sound waves in air. Vibration of the substrate which may cause a relative motion of the segments of an insect's body or appendages may cause stimulation of the intersegmental scolopidia. It is also possible that some of the intersegmental scolopidia are auditory in function.

The structure of a typical scolopidium is shown in Fig. 171. Each unit consists of a distal or cap cell which surrounds a scolopale, or sense rod, to which is attached the terminal filament of the sense cell. This terminal filament is surrounded by a sheath cell. The sense cell is a primary neurone with its cell body in the base of the scolopidium. These structures are fundamentally the same in all scolopidia, regardless of whether or not they 
are auditory. In many insects the scolopidia are arranged in a row (the crista acoustica) near the edge of the tympanum in such a way that move. ment of the tympanum produces a relative morement between the sense cell and the scolopale. In other insects the scolopidia are in direct contact with the central portion of the tympanum (Fig. 170), so that the maximal amplitude of vibration is available for stimulation.

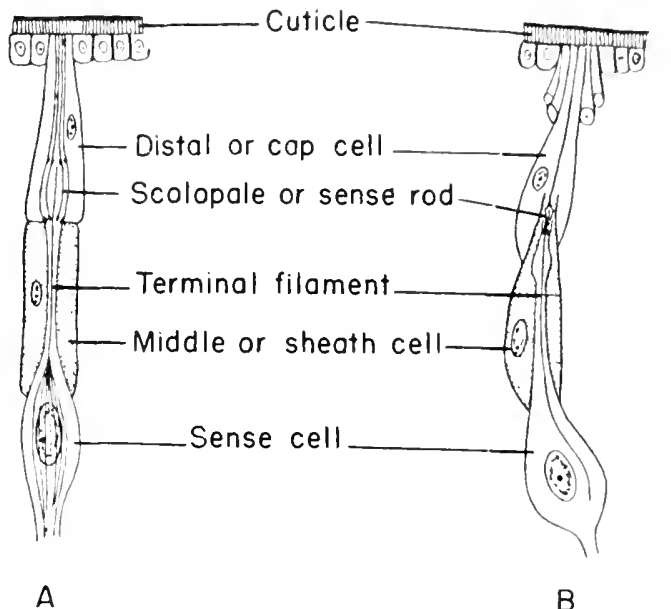

Fig. 171. Schematic diagram of scolopidia. A, Scolopidium from a chordotonal organ (from Debauche, after Snodgrass" ${ }^{15}$ ). B, Scolopidium from Johnston's organ (from Debauche, ${ }^{\star}$ after Eggers"). From Wigglesworth. ${ }^{56}$

Function of Tympanic Organs: Auditory Function. The range of sensitivity of the tympanic organs has been studied by recording electrical changes in the sensory nerves or central nerrous system in response to stimulation by sounds of known frequency and intensity. Typical results are shown in Fig. 172. In one of the Acridiidae a response was obtained for stimuli of between 300 and 20,000 cycles, and the response did not occur after destruction of the abdominal tympanic organ. In the lower part of this range the sensitivity is very much less than that of man, but at 10,000 cycles it is almost equal to the human sensitivity. In two katydids a response was present between 800 and 45,000 cycles; ${ }^{5+}$ in Gryllus from 300 to 800 cycles; ${ }^{5+}$ in Locusta from 300 to above 10,000 crcles. ${ }^{36}$. The threshold as a function of frequency for the tympanum of Locusta is shown in Fig. 172, in which it may be compared with that for the human ear and for the cercus of Gryllus. The response of the tympanal nerve is asynchronous; i.e., the various fibers respond apparently at random, and the character of the response does not change with frequency. These observations afford no evidence that the tympanic organ is capable of frequency discrimination.

Regen ${ }^{3 *}$ demonstrated that a female cricket was attracted to a telephone which was transmitting the chirp of a male cricket. This response was obtained with adult unmated females but did not occur with females which had been mated. ${ }^{37}$ Also two male katydids in the same vicinity tended to sing in concert. ${ }^{37}$ The concerts consisted of introductory and conclusional 
songs, and an intermediate period in which the chirps from the two partners alternated in a regular manner. Regen $\%:$ then tried to substitute an artificial partner. When he used males which had just become adult he was able to get them to alternate with a wide varicty of musical sounds of such a nature as to indicate a sound range of 400 to 28,000 cycles. However, males which had previously sung with other males did not sing with the artificial partner. In both crickets and katydids $3 i$ bilateral extirpation of the tympanic organs abolished the normal responses, but did not abolish all sensitivity to sound.

Quality Discrimination. Regen's experiments demonstrate conclusively that insects are capable of detecting sounds and also that (at least in the two species studied) they are capable of a high degree of quality discrimination. However, he observed that unmated female crickets responded to the male chirp orer the telephone even when it was so distorted as to be unrecognizable to the human ear. If it is assumed that the mechanism of recognition is the same in both crickets and katydids, then it seems that the qualities of sound which facilitate recognition in insects are not the same qualities which facilitate recognition by man.

We recognize sounds primarily on the basis of frequency and to a lesser extent on the basis of amplitude modulation. The structure of the tympanic organ indicates that it functions poorly as a harmonic analyzer, as compared with the human ear. In tettigonids and gryllids the scolopidia of the tympanic organs are arranged in a row and graded in size. In cicadas the

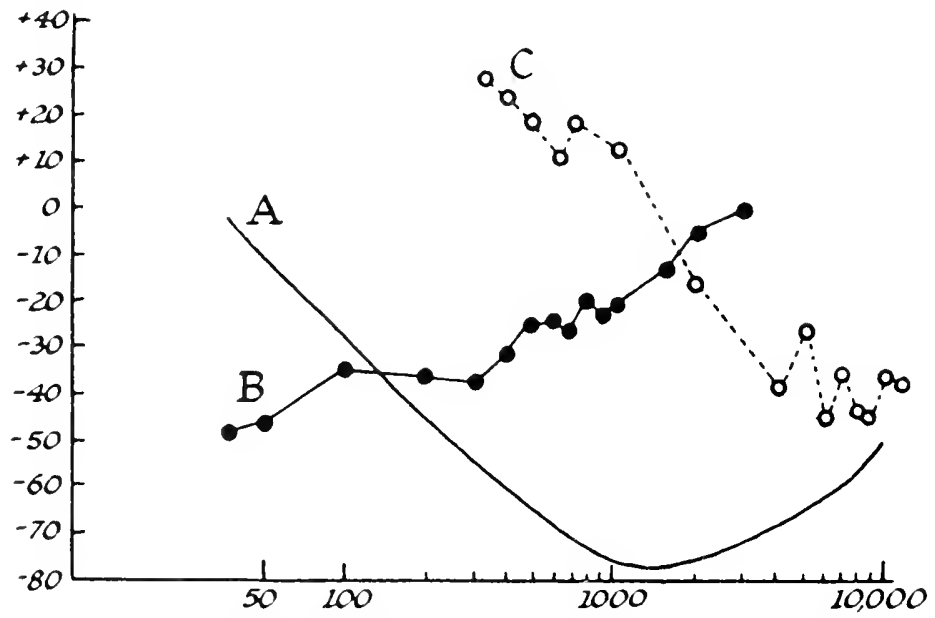

Fig. 172. Frequency response curves for different types of acoustic receptors. (After Pumphrey and Rawdon-Smith ${ }^{35}$ ). Ordinate, sound pressure above or below 10 dynes per sq. cm., the zero point. Abscissa, frequency in cycles per second on log scale. $A$, normal human threshold; $B$, threshold of electrical response in cercal nerve of Gryllus; $C$, threshold as measured electricalty in the tympanal nerve of Locusta. From WVigglesworlh.

scolopidia are in a single mass, and all are about the same size. In acridiids the scolopidia are in three groups on different parts of the tympanum. There is no experimental evidence that these structures are very effective as frequency discriminators, but neither is there much evidence that they are not. 
It merely seems probable, on the basis of present evictence, that frequency discrimination is of a low quality.

The next question is whether or not insects are capable of a high quality of discrimination of amplitude modulation. It has been proposed by Pumphrey and Rawdon-Smith 34.3 .34 that they are capable of such discrimination and that they are also sensitive to small changes in the frequency of the modulating frequency. It was demonstrated experimentally that amplitude modulation of a sound wave could produce a temporal pattern of impulses in the insect auditory nerve. Pumphrey and Rawdon-Smith propose that sound quality discrimination in insects is more a discrimination between amplitude modulation frequencies than between frequencies of the sound waves themselves. The insect tympanic organ would therefore be more nearly comparable to the audio detector in a radio set than to a harmonic analyzer. The human ear is constructed so that it is highly sensitive to changes in the modulated frequency, i.e., it is an excellent harmonic analyzer, but we are relatively insensitive to changes in the modulation frequency.

If Pumphrey and Rawdon-Smith's theory is true, then the scemingly contradictory experiments on insect hearing can be explaincd. For instance, in Regen's experiments the response of the female crickets might be cxplained on an amplitude modulation basis, and so might the unresponsiveness of experienced male katydid concert singers who failed to answer urmodulated artificial partmers. Furthermore, the more common insect sound-producing methods, e.g., the rubbing together of lcgs and wings, are probably more adaptable to the production of amplitude modulated sounds than are the air column devices of vertebrates. This theory of Pumphrey and RawdonSmith is one which warrants considerable investigation.

Sound Localization. Regen ${ }^{3 n}$ observed that unmated female crickets oriented toward a chirping male at a distance of ten meters or more, and that they tended to move toward the male along a straight line. Females deprived of one tympanic organ reached the male over a more devious path. It is evident that the female was capable of locating the sound.

In man, localization is determined by intensity and phase differences in the two ears produced as a result of their separation and of the shadow effect of the head. In insects the influence of these two factors is very much less, and they are probably insignificant. However, the structure of the tympanum indicates that, especially when exposed on the body surface, it may serve directly as a localizer. Pumphrey and Rawdon-Smith ${ }^{34}$ demonstrated this for Locusta. They used a monophasic transient sound wave as a stimulus and determined the intensity necessary to stimulate the isolated tympanic organ as a function of the angle of incidence. The threshold was determined by electrical recording from the nerve. The tympanic organ has very definite directional characteristics which, in general, are comparable to those of a "ribbon" or "velocity" microphone. Since the stimulus used was a monophasic sound wave it was possible to compare the response of the tympanum to a "push" or "pull." No differences were found either in the threshold or in the latent period when the stimulus was reversed. This is in striking contrast to the vertebrate cochlea.

Hair Sensilla as Phonoreceptors. It is well known from the work of 
Minnich 28,29 that caterpillars are sensitive to sound, and that this sensitivity seems to be localized in hair sensilla. In the hairy caterpillar Vanessa antiopa sound waves of frequencies below 1000 cycles cause a contraction of longitudinal dorsal muscles which raise the anterior end of the animal. The lower frequency limit of response was below 32 cycles and was undetermined. The response could be reversibly abolished by application of a water spray or of flour to the hairs. These experiments were repeated with various species, and it was found that relatively hairless caterpillars also responded but at a higher intensity.

Pumphrey and Rawdon-Smith ${ }^{35}$ studied the electrical changes of the nerve from the anal cerci of various adult Orthoptera in response to sound waves. The threshold curve for the cricket is given in Fig. 172; curves for

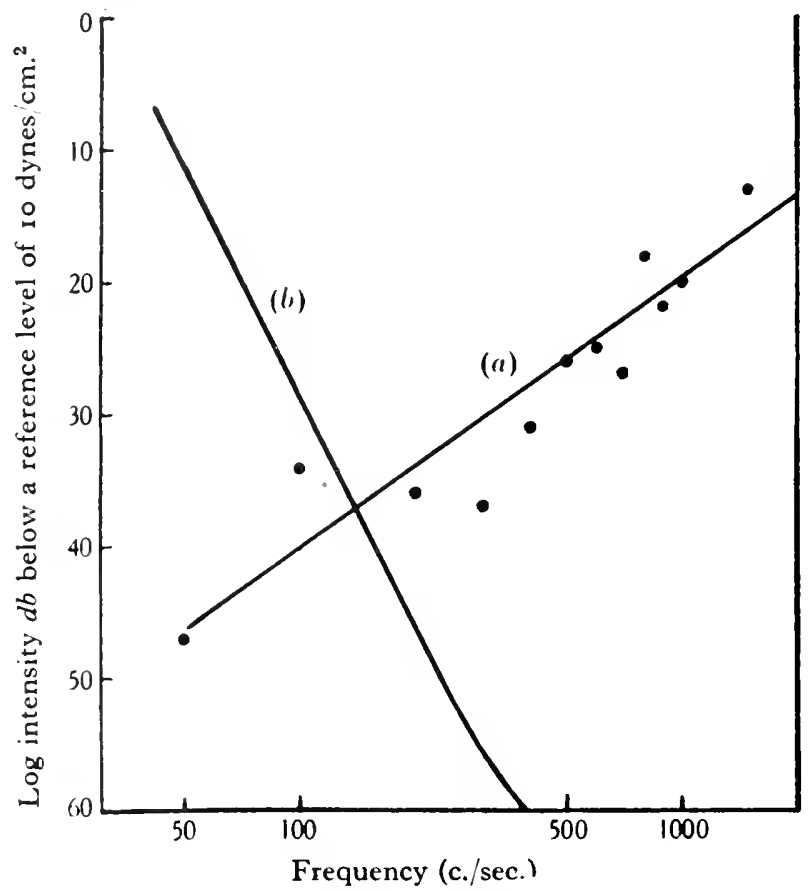

Fig. 173. Curves indicating the frequency response of a cercus preparation of Gryllus (a) and the human ear $(b)$. The points are experimentally determined and the line (a) corresponds to a constant displacement of $56 \mathrm{~m} \mu$ of the distal end of the hair. (Pumphrey."

locusts and cockroaches are similar. The end-organs are the long hair sensilla, and the responses can be inhibited by coating the cerci with dust or petroleum jelly. At low frequencies there was a synchronous response in the nerve, i.e., one impulse for each sound wave. This synchrony was present initially up to 800 cycles, but, as in the mammalian cochlea, "alternation" may be shown at higher frequencies. The lower response limit was not determined, but it probably is well below 50 cycles.

Movement of the hair sensillum produces a displacement of the dendrite of the sense cell. The threshold displacement of the dendrite calculated 
by Pumphrey and Rawdon-Smith is less than $5 \mu$. The threshold rises with frequency (Fig. 173). The sensilla seem to operate as pure displacement receptors over the investigalted frequency range, and there is no evidence of resonance phenomena. Curve (a) of Fig. 173 is drawn on the assumption of a constant displacement of the distal end of the hair of $56 \mathrm{~m} \mu$. This type of mechanism is probably sensitive to very low frequencies and may also function in determining vibrations in the substrate.

Other Orthopteran Receptors. Pumphrey and Rawdon-Smith 3.5 have also recorded a response from the abelominal segmental nerves of the locust in response to sound, especially when the frequency was about 1400 cycles. The threshold curve indicates sharply tuned resonant structures which Pumphrey and Rawdon-Smith assumed were short stiff sensory hairs. However, no such hairs were found. Melanoplus ${ }^{44}$ has segmentally arranged scolopidia which may be auditory in function. If their function is auditory, and if these scolopidia occur in Locusta, then they may be the structures the sensitivity of which was studied by Pumphrey and Rawdon-Smith.

\section{Receptors in Other Arthropods.}

Spiders are sensitive to sound waves in air, and the location of the sense organ is as yet undetermined. However, it seems probable that the hairlike projections on the surfaces of non-insect terrestrial arthropods may serve as acoustic detectors as do those of insects. Furthermore, web-building spiders are capable of very accurate orientation toward a sound that is transmitted through the web or its supports, ${ }^{4}$ and the logical place to look for the responsible receptor seems to be in the hair-like processes.

It is also known that Mysis" and Palaemonetes are sensitive to sound waves in water and are less sensitive after cxtirpation of their statocysts. The statocyst, although primarily statoreceptive, apparently has a dual function, as does the sacculus of fishes.

\section{REFERENCES}

1. Adams, L. A., An Introduction to the Vertebrates (1938). New York, John Wiley \& Sons. 479 p.

2. Adruan, E. D., J. Physiol. 92:9P (1938). The effect of sound on the ear: reptiles.

3. Adrian, E. D., Craik, K. J. II., and Sturdy, R. S., Proc. Roy. Soc. Lond., B. $125: 435-455$ (1938). The electrical response of the ear: vertebrates.

4. Barrows, M. M., Biol. Bull. 29:316-332 (1915). Reactions of spider to vibration of web.

5. Berger, K., Ztschr. vergl. Physiol. 1:517-540 (1924). Sound perception in reptiles.

6. Betre, A., Zool. Jahrb., Abt. Anat. u. Ont. 8:544-564 (1895). The otocyst of Mysis.

7. Bull, H. O., J. Mar. Biol. Ass. 15:485-533 (1928). Conditioned reflexes in fish.

8. Debauche, H., La Cellule 44:45-83 (1935). Antennal chordotonal organs, Trichoptera.

9. Eggers, F., Zool. Anz. 57:22+250 (1923), also Zrschr. Morph. Ökol. Tiere 2:259$3+9$ (192t). Chordotonal and Johnston's organs.

10. Ewer, D. W'., Nature 156:692 (1945). Acoustic control of flight: bats.

il. Ferhat-Aкat, S., Ztschr. vergl. Phy'siol. 26:253-281 (1939). Sound perception in Amphibia.

12. Fletcher, H., Speech and Hearing (1929). New York, Van Nostrand.

13. Foa, C., and Peroni, A., Arch. Fisiol. 28:237-241 (1930). Action currents recorded from the acoustic nerve. 
14. Frisci, K. v., Biol. Rev. 11:210-246 (1936). Hearing in fish.

15. Fulton, J. F., Howell's Textbook of Physiology (1946). Philadelphia, Saunders.

16. Galambos, R., J. Aconst. Soc. Am. 14:41-49 (1942). Cochlear potentials elicited from bats by supersonic sounds.

17. Galambos, R., and Davis, H., J. Neurophysiol. 6:39-57 (1943). Response of auditory nerve fibers to accoustic stimulation.

18. Galamibos, R., and Griffin, D. R., J. Exper. Zool. 89:475-490 (1942). Obstacle avoidance by flying bats.

19. Griffin, D. R., Anat. Rec. 96:519 (1946). The supersonic mechanism: bats.

20. Griffin, D. R., and Galanibos, R., J. Exper. Zool. 86:481-506 (1941). The sensory basis of obstacle avoidance: bats.

21. Hartridge, H., Nature 156:692-693 (1945). Accoustic control in bats.

22. Hoagland, H., Proc. Nat. Acad. Sci. 18:701-705 (1932). Impulses from sensory nerves: fish.

23. Knecitt, S., Ztschr. vergl. Physiol. 27:169-232 (1939). Concerning audition and production of musical notes: birds.

24. Kuroda, R., J. Comp. Psychol. 3:27-36 (1923). Hearing in reptiles.

25. Manning, F. B., J. Comp. Psychol. 3:241-247 (1923). Hearing in rattlesnakes.

26. Manning, F. B., J. Exper. Zool. 41:5-20 (1924). Hearing in goldfish in relation to ear structure.

27. Mliller, D. C., The Science of Musical Sounds (1935). New York, Macmillan.

28. Minnich, D. E., 1. Exper. Zool. 42:443-469 (1925). Reactions of insect larvae to sound.

29. Mlınich, D. E., J. Exper. Zool. 72:439-453 (1936). Response of caterpillars to sound.

30. Parker, G. H., Am. Nat. 37:185-204 (1903). Hearing in fishes.

31. Parker, G. H., Bull. U. S. Bur. Fish. 30:99-104 (1910). Sound directed movements of fish.

32. Parker, G. H., and Van Heusen, A. P., Am. J. Physiol. 4t:463-489 (1917). Mechanical stimulation of skin, lateral line organ and ears: fish.

33. Pavlov, 1. P., Conditioned Reflexes (1927). Oxford Univ. Press.

34. Punpliney, R. 1., Biol. Rev. 15:107-132 (1940). Hearing in insects: review.

35. Pumpirey, R. J., and Rawdon-Smitu, A. F., Proc. Roy. Soc. Lond., B. 121 :18-27 (1936). Electrical responses in nerves from organs of hearing: insects.

36. Pumpiney, R. J., and Rawdon-Smitil, A. F., Nature 143:806-807 (1939). "Frequency discrimination" in insects.

37. Regen, J., Sitzungsb. Akad. Wiss. Math-Nat. Kl. I., Wien 123:853-892 (19l4). Hearing in insects: Thamnotrizon.

38. Regen, J.,. Sitzungsh. Akad. Wiss. Math-Nat. Kl. 1., Wien 132:81-88 (1923) Hearing in Gryllus.

39. Regen, J., Sitzungsh. Akad. Wiss. Math-Nat. Kl. I., Wien 135:329-368 (1926). Tomal influence on stridulation: crickets.

40. Ross, D. A., J. Physiol. 86:117-146 (1936). Electrical studies on the labyrinth: frog.

41. Scinwabe, J., Zoologica, Vol. 20, No.6 (1906). Tympanal sense organs in Orthoptera.

42. Sinlen, 11., Zool. Jahrb., Abt. 2, Anat. E Ontog. 45:519-580 (1924). Sense organs in the cerci: insects.

43. Sivian, L. J., and Winte, S. D)., J. Acoust. Soc. Am. 4:288-321 (1933). Minimum audible sound fields.

44. Stafier, E. II., J. Morph. 58:615-637 (1935). Femoral chordotonal organs: grasshopper.

45. Snobenass, R. E., Principles of Insect Morphology (1935). New York, McGraw I lill.

46. Sтеттек, II., Ztschr. vergl. Physiol. 9:339-477 (1929). Investigations of hearing in fish.

47. Struvins, S. S., J. Acoust. Soc. Am. 6:150-15+ (1935). Relation of pitch to intensity.

48. Stevens, S. S., and Davis, 11., Hearing: Its Psychology and Physiology (1938). New York, John Wiley \& Sons. 
49. Stevens, S. S., Davis, H., and Lurie, M. H., J. Gen. Psychol. 13:297-315 (1935). Localization of pitch perception on the basilar membrane.

50. Stevens, S. S., and Newnian, E. B., Proc. Nat. Acad. Sci. 22:668-673 (1936). The nature of aural harmonics.

51. Stipetic, Elisabetix, Ztschr. vergl. Physiol. 26:740-754 (1939). On the organ of phonoreception: Mormyridae.

52. Weber, H., Lehrbuch der Entomologie (1933). Jena, G. Fisher.

53. Wever, E. G., and Bray, C. W., J. Exper. Psychol. 13:373-387 (1930). Relation between sound and impulse frequency in auditory nerve.

54. Wever, E. G., and Bray, C. W., J. Cell. E Comp. Physiol. 4:79-93 (1933). Method for the study of hearing: insects.

55. Wever, E. G., and Bray, C. WV., J. Comp. Psychol. 22:353-363 (1936). Electric response of inner ear: pigeon.

56. Wiggleswortri, V. B., Principles of Insect Physiology (1939). London, Methuen.

57. Wilska, A., Skand. Arch. Physiol. 72:161-165 (1935). Method for determining amplitude of eardrum vibration.

58. WITschi, E., Anat. Rec. 99:568 (1947). Development and metamorphosis of the auxiliary apparatus of the ear of the frog.

59. Wrighitson, T., and Keith, A., An Inquiry into the Analytical Mechanism of the Internal Ear (1918). London, Macmillan \& Co., Ltd.

60. YeRkes, R. M., J. Comp. Neurol. 15:279-304 (1905). Hearing in frogs.

61. Zotterman, Y., J. Physiol. 102:313-318 (1943). Microphonic effect in teleost labyrinths. 


\section{Mechano- and Equilibrium-Reception}

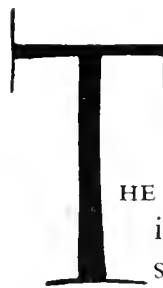

HE POSITION OR ATTITUDE assumed by an animal in its environment is usually the result of a complex of reactions. Several kinds of stimuli-light, temperature, mechanical contact, gravity-may simultaneously affect different sense organs. One sense modality may at times be dominant, or the sum total of sensory stimulation may determine the attitude the animal assumes. The orienting effects of light, heat and cold, and of chemical and auditory stimuli have been discussed in previous chapters. Animals which live on a firm substratum are continually subjected to contact stimuli; motile organs and appendages are regularly stretched or extended and retracted, thus subjecting moving tissues to tension. Most animals orient with respect to gravity, and many have special equilibrium receptors. Thus mechanical stimuli are common, and all animals are sensitive in some way to mechanical stimulation. Sound is high-frequency mechanical stimulation; we are here concerned, however, with stimulation by low-frequency mechanical stimuli and by gravity or equilibrium alteration.

\section{MECHANORECEPTION}

The sensitivity of cells to mechanical deformation of their surface is a more widespread phenomenon than is commonly supposed. Some cells, the phonoreceptors, are specialized in sensitivity to low amplitude vibration; others, tactile receptors, respond to the greater deformation of touch; still others, tension receptors, are specifically sensitive to distortion by stretch. Tactile and tension receptors are more widely distributed than any other type of sensory cells, especially in appendages; they are rarely restricted to particular organs. Changes in equilibrium may be detected by special organs, statocysts, but these are supplemented by scattered tension receptors.

Nature of Mechanical Stimulation. The cellular phenomena associated with mechanical stimulation are poorly known. Nerve axons have been stimulated mechanically with an air jet, and the relation between pressure and duration of stimulus nceded to elicit a response has been shown to resemble a conventional strength-duration curve as obtained with electrical stimulation. ${ }^{\times}$Presumably the mechanical deformation causes local potential changes which initiate impulses electrically. An amoeba stimulated by a weight dropped on the coverglass stops locomotion with a latency which varies inversely with strength of stimulus: after one response a recovery time is required before the amoeba can respond again." It would be of interest to examine quantitatively the responses of many types of cell to mechanical stimulation of various kinds. 
Histological Types of Mechanoreceptors. The differentiation in the course of evolution of certain epithelial cells into mechanoreceptors and chemoreceptors probably occurred before the differentiation of conducting nerve cells. In all animals with nervous systems there are specialized mechanoreceptors of one or more histological types. The simplest are free nerve end. ings; these may lie between epithelial cells, as in the earthworm, or may be in the deeper portion of the skin, as in vertebrates. They usually have a high mechanical threshold and may be stimulated by various noxious agents; that is, they are often pain receptors. Another simple type of tactile receptor is a terminal bulb, as in the frog skin, or Merhel's discs, in mammalian skin. Sensory hairs are widely distributed in many kinds of animals, and at their base there may be free endings, knobs, or specialized spirals. Arthropods have many sensory hairs at joints along various appendages, such as antennae, legs and wings. In insects there are also short sensory spines and more pliable setae with bipolar sense cells. Frequently sense cells

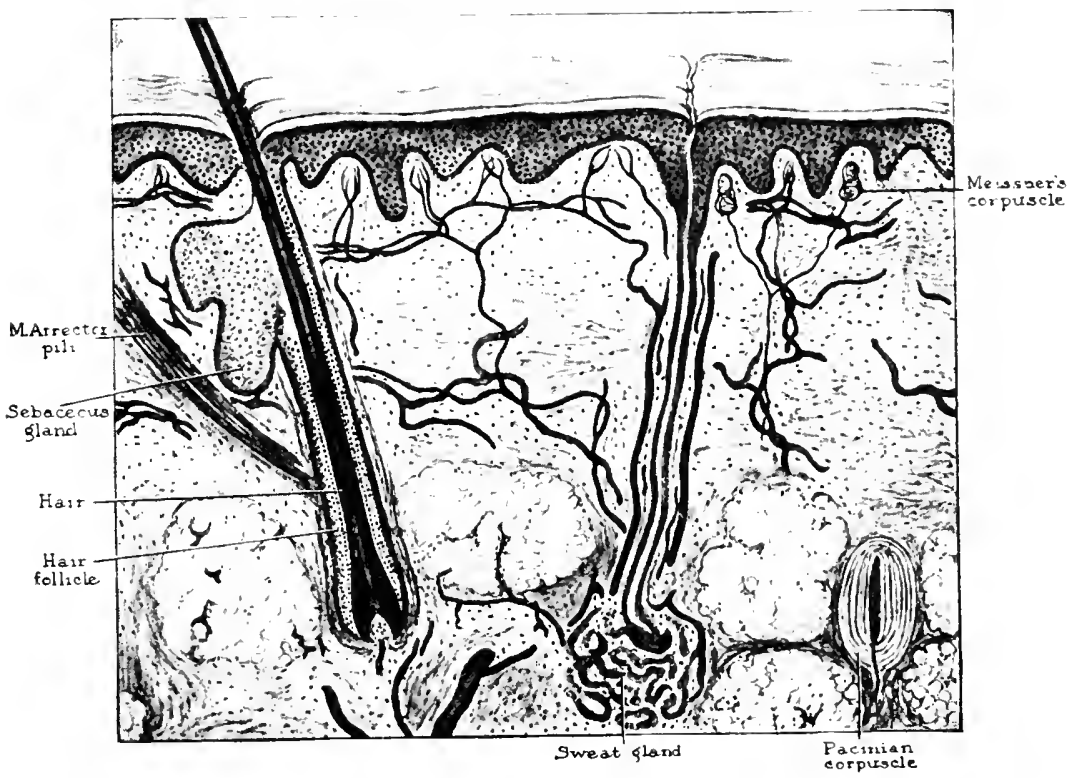

Fig. 174. Schematic drawing of section of skin and its appendages. (Andrews.)

have a short sensory hair and are grouped in organs, as in the earthworm (Fig. 175). Several types of encapsulated endings have been described for mammals, particularly the pacinian corpuscles of tendons and mesentery (Fig. 176), and Meissner's corpuscles of the skin (Fig. 174).

The distribution of proprioceptors is less well known than that of tactile receptors, but in mammals, birds, reptiles, and amphibians there are spiral structures such as the spindles of tendons and intrafusal muscle fibers which respond to a pull (Fig. 177). Muscle spindles are lacking in fish but there are tension receptors in fish muscle. The proprioceptors of vertebrates are usually arranged to respond to tension in small bundles of muscle fibers, whereas in insects they respond more to strains in the cuticle. In the campaniform sensilla of insects a terminal filament ends in a rod under a cuticular 


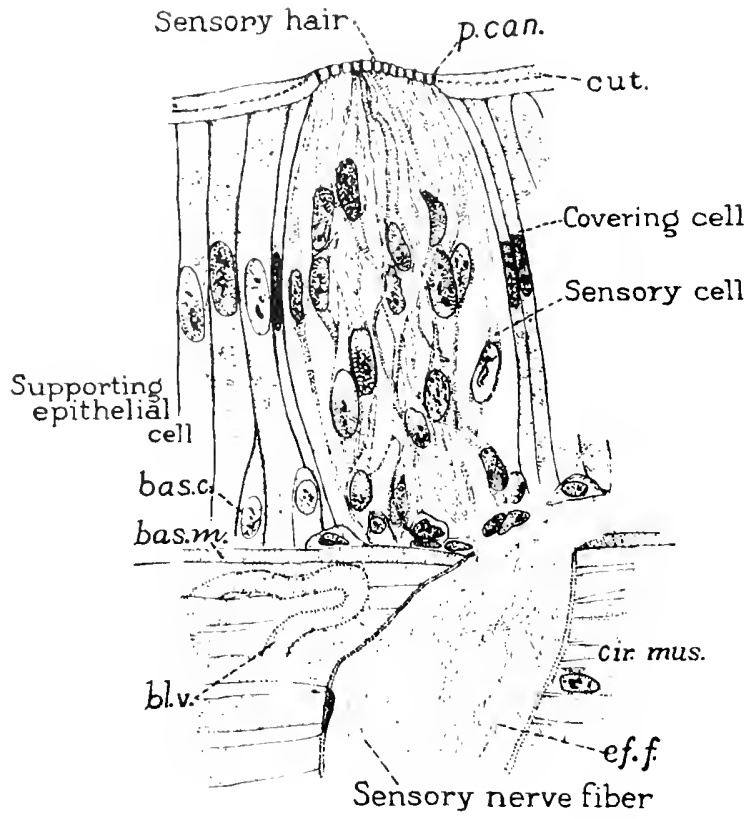

Fig. 175. Epithelial sense organ of earthworm. From Langdon. ${ }^{59}$

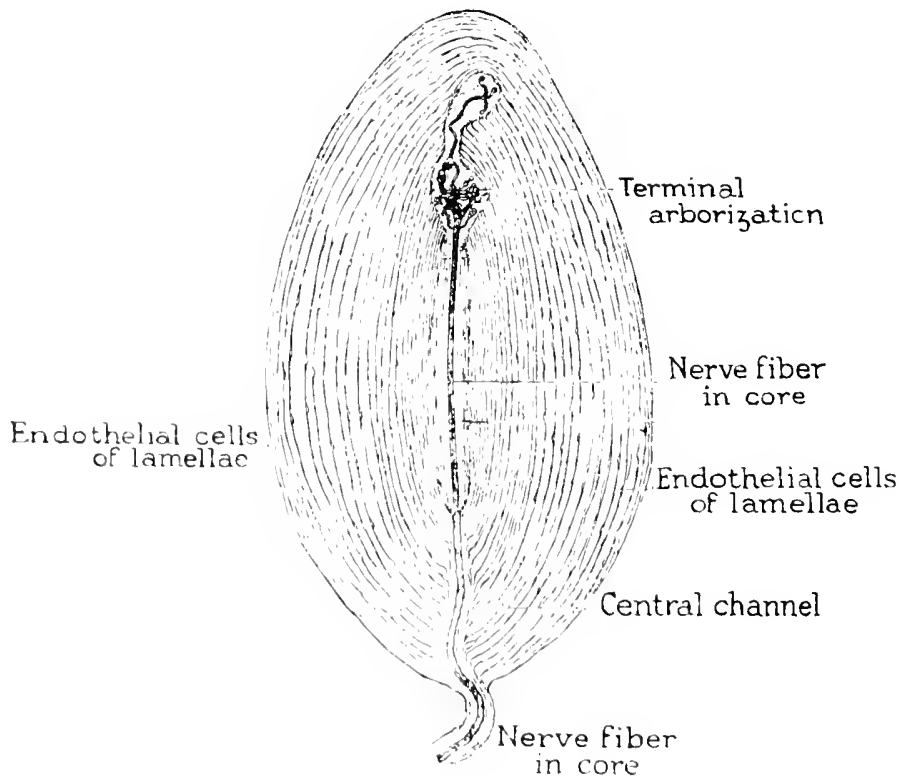

Fig. 176. Section of pacinian corpuscle of cat mesentery. a, Terminal arborization; $n$, nerve fiber in core; $f$, central channel; $c$, $d$, endothelial cells of lamellae. From Schafer. ${ }^{*}$ 


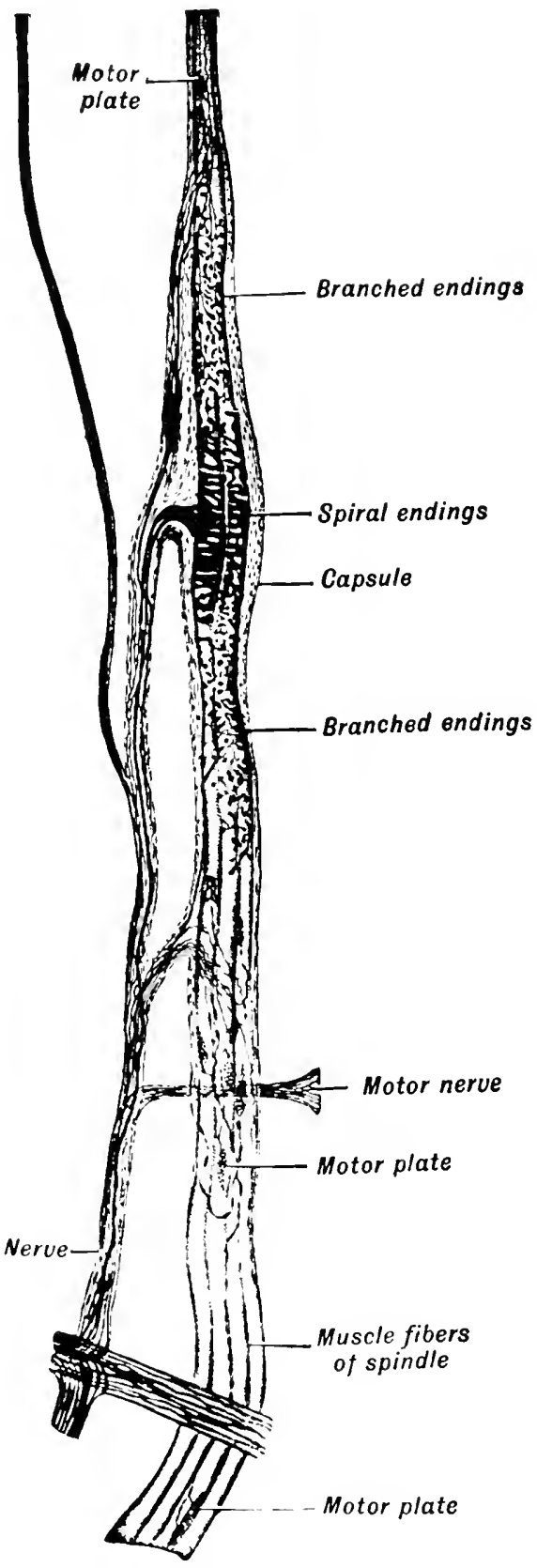

Fig. 177. Diagram of nerve endings in mammalian skeletal muscle. From Maximow and Bloom. ${ }^{67}$ 
dome which magnifies surface strains (Fig. 178); campaniform sensilla are abunclant on the basal joints of extremities, on wings, on the halteres of Diptera, and elsewhere. Chordotonal sensilla have a sensory filament on an elastic strand stretched between two points on the body wall; they of ten occur at the base of wings. Johnston's organs resemble chordotonal sensilla and occur on the second antennal segment (Fig. 171); they are stimulated by movement of the antenna at its base.

There is also wide variation in the location of the nuclei of the sensory neurones. In the earthworm, for example, epidermal sense cells synapse in a peripheral plexus with the afferent neurones, and there are many sense cells per afferent fiber. In Crustacea a single fiber enters a sensory hair and the nucleus lies at its base. The cell bodies of most tactile and proprioceptive endings of vertebrates are located in dorsal spinal ganglia or in sensory centers in the lower brainstem. It is apparent from the differences in form and arrangement that the various histological types of mechanoreceptors differ in the kind of deformation which produces stimulation; they differ also in sensitivity and in the persistence of response to maintained stimulation.

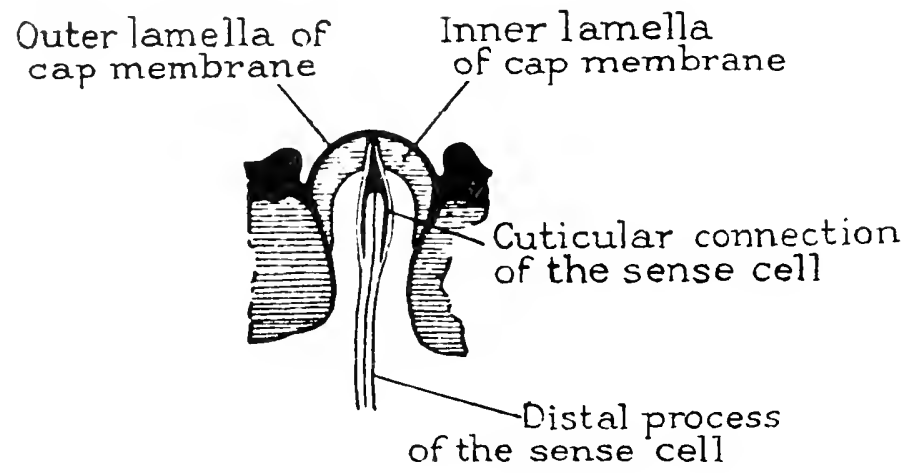

Fig. 178. Diagrammatic campaniform sensilla of an insect. From Pringle. ${ }^{66}$

Behavioral Responses. Distance Mechano- and Vibration-Sense. Mechanoreceptors usually signal either movement of a body region or direct contact with some object; they may signal movement of a distant object if pressure waves are transmitted by the medium. The tactile endings on antennae and vibrissie are, in a sense, distance receptors, and rats lacking both eyes and vibrissac have difficulty in running a maze, whereas either eyes or vibrissie can give adcquate cues. ${ }^{9: 3}$ A distant source of vibration can be detected by various kinds of mechanoreceptors. A spider locates its prey by sight and by vibration of the web, and some spiders use a special signal thread.

The proprioceptors, largely sensilla, of arthropods are exceedingly sensitive to vibrations. The "feeling" of vibration by mammals is due to stretch receptors, particularly those of muscles and tendons; this is shown by the sensory impulses whien a muscle or bone is touched by a tuning fork; the thresholk is lower for a contracted than for a relaxed muscle. $A$ single stretch receptor of a toe muscle of a frog discharged at a maximum freauency of $330 / \mathrm{sec}$. when stimulated by vibration, and $316 / \mathrm{sec}$. when stimulated by 
rapid stretch. Mso, when adapted to strctch, the ending sometimes responded to a tuning fork at one half or one third of the frequency of vibration."-ti

Fish detect pressure waves and ripples from a distant source by means of skin receptors and lateral line organs. Blind fish snap at prey moving 5 to $10 \mathrm{~cm}$. away, and by conditioned reflex techniques it has been shown that minnows locate water jets and moving objects by the lateral line organs. ${ }^{2.5}$ Electrical recording of the nerve impulses from cattish lateral line nerves shows that lateral line organs follow low frequency vibrations well but fail to svnchronize with the stimulus above about 100 eycles per second. $4.2,43$, s6 In Fundulus, the lateral line discharge synchronizes at frequencies as high as 180 per second": (Fig. 179 B, C). There is a continuous background of "spontaneous" activity in lateral line nerres which originates in the neuromasts, and responses to mechanical stimulation are superimposed on this background. The response in the lateral line when another fish swims by is shown in Figure 179. $A$, and the lateral line must be very important in keeping fish properly oriented when they swim in schools. The skin receptors of fish also detect vibrations but have a higher threshold and are more important in

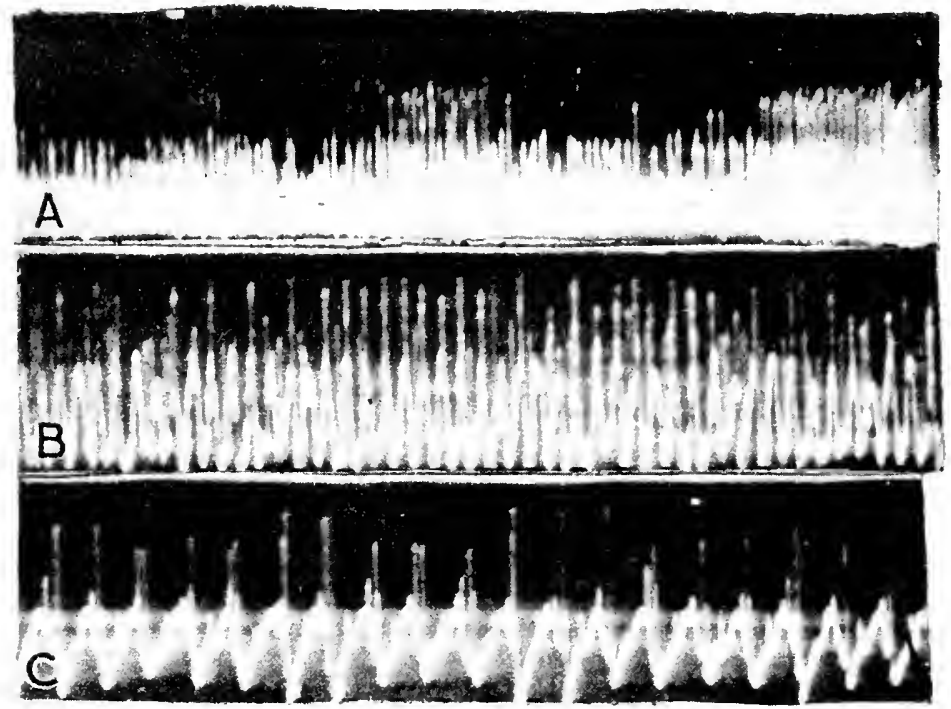

Fig. 179. Responses in lateral line nerve of Fundulus. A, Spontaneous activity with three bursts of impulses as another fish swims by. B. Synchronized impulses at 75 and, $C$, at 45 per second in response to sound. Signal from sound generator on baseline of oscilloscope and impulses superimposed. From Suckling.:11

sensing contact stimuli. lmpulses in nerres from the barbels of cattish show synchronized responses to ripples. ${ }^{43 .}$. sess lateral line organs (Amblystoma, ${ }^{-\overline{-}}$. Xenopus, ${ }^{\text {ini }}$ ) these sensory structures are very efficient in localizing moving objects and in detecting fixed objects by reflected waves.

Orientation to Contact Stimuli. Several kinds of direct responses to mechanical stimulation are known. Many animals, particularly those which crawl, tend to aggregate in crevices and along surfaces, a thigmotaxis. This reaction provides protective shelter and is easily orerbalanced by other types 
of stimulation, particularly by light and chemical stimulation. When such animals crawl along one side of an object they turn toward the stimulating surface when they reach the end of the object (Fig. 180, A), whereas if they crawl in the crack between two simmetrical objects they continue straight ahead at the end (Fig. 180, B). If, after the animal has given this positive response to an edge (or positive stereotactic response), the stimulating object is withdrawn, the crawling animal then straightens the anterior end to be in line with the posterior end, the homostrophic reflex (Fig. 180, A). These contact reactions depend on unequal stimulation of the two sides and have been demonstrated for many animals, including earthworms, diplopods, insect larvae, and young (blind) rats and mice. 17, 1!
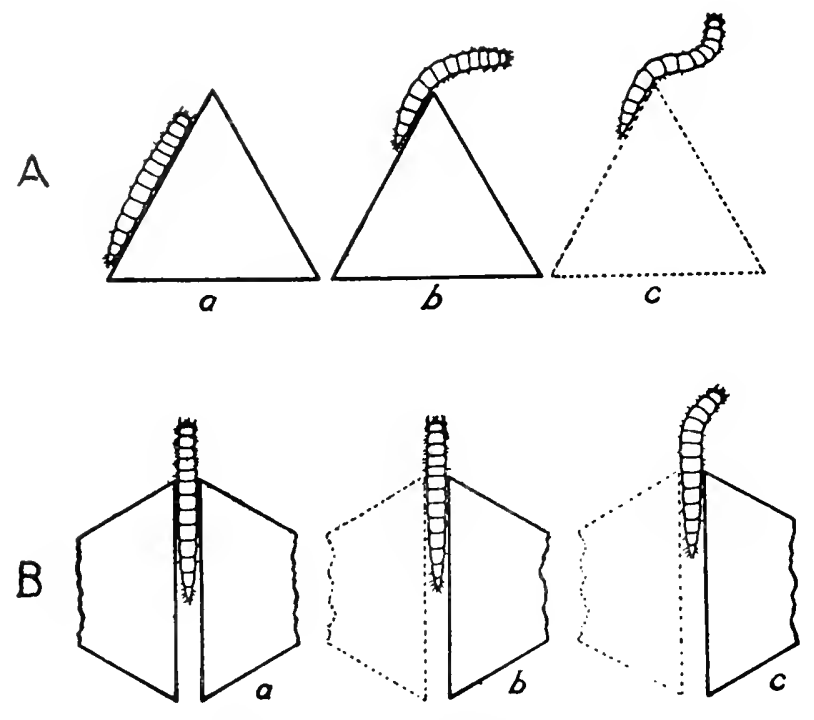

Fig. 180. A, Stereotropic orientation of larval Tenebrio ( $a, b$, successive positions) and reflex homostrophic orientation (c), when source of contact is removed. B, Balanced effect of bilateral contact resulting in straight course; removal of contact plate on one side followed by stereutropic bending. From Crozier. ${ }^{13}$

Many animals show direct reactions to local mechanical stimulation. For eximple, most fish when stimulated on one side near the posterior end turn the caudal fin toward the side of stimulation. Many sessile animals and worms, such as hydroids, scal anemones, loolothurians, sipunculoids, and annelids, withdraw by a strong body contraction when stimulated mechanically at the anterior end.

Anether trpe of contact orientation is rheotaxis or orientation to a stream of water. Planaria often react positively to a stream, depending on receptors scattered wer the bed! surface.":3 Paramecia are positively rheotactic under mest conditions. The orientation of fish inte a strean depends much more on the cres than on mechanoreceptors. Blind fish do not orient in a stream unless they touch the bottom, when they orient by frictional stimulation.":Similarly, vistal cues are important in rheotaxis of the lobster. In the elasmobranch Raja headward flow of thuid in the hyomandibular lateral line canal 
increases the rhythmic activity in the lateral line nerve, whereas tailward flow reduces or stops the spontaneous rhythm. Whether the lateral line is used in rheotaxis in Raja is not known.

Anemotaxis or orientation in air currents is seen in flying animals such as some insects. In flies the antennal sense organ, Johnston's organ, is sensitive to wind velocity and elicits postural changes according to speed of air flow.

When an animal is in contact with a substratum there is continuous asym metric stimulation of mechanoreceptors. Absence of ventral surface stimula tion mar initiate righting reactions as in snails, starfish, and cockroaches, and removal of the substratum initiates flight in many insects. Similarly, lifting of the suclier of a leech initiates swimming. Apparently in the absence of other stimuli, stimulation by contact normally inhibits locomotor reflexes.

Hydrostatic pressure is applied uniformly, and most of the effects of hydrostatic pressure or atmospheric pressure are respiratory rather than sensory One of the familiar effects of rapid changes in hydrostatic pressure is in the amount of nitrogen dissolved in the blood and the bubbles which appear on decompression. Pressures of the order of several hundred atmospheres cause spectacular decrease in protoplasmic viscosity, and many animals arc killed fairly rapidly at about 400 atmospheres. Yet fish and deep-sea invertebrates live at ocean depths of two miles, where the pressure exceeds 300 atmos. pheres. ${ }^{16}$ When deep-sea animals are brought to the surface they die, and, conversely, surface animals cannot survive long at pressures corresponding to the deep sea. The physiological adaptations permitting survival at high pressures are not understood. llowever, fish do tend to remain within a certain pressure range, and they do this in part by varying the amount of gas in the swim bladder. A sudden decrease in pressure by a fraction of an atmosphere results in an increase in gas content in the sivim bladder (guppies ${ }^{1: 3}$ ). In physoclistous fish, that is, in those lacking a duct from gas bladder to esophagus, the gas must be secreted into the bladder from the blood and the effect is to maintain the fish at a constant level. ${ }^{60}$ Similar increases in bladder gas content occur when Fundulus is transferred from sea water to fresh water. ${ }^{7}$ In some physostomes (fish with open swim bladders) certain anterior vertebrate form a chain of bones, the Weberian ossicles, which transmit pressure to the inner ear from the swim bladder (p. 486, Ch. 13), and removal of which prevents normal reactions to a pressure drop. ${ }^{\text {it }}$ An increase in swimbladder pressure in the carp elicits a strong response of all fins, cardiac depression, and initial stimulation, followed by depression of respiration-all effects which cease when the autonomic nerves to the swim bladder are cut.5: The gas bladder of fish is an effector containing sensory mechanisms which aid in maintaining fish at constant hydrostatic pressure.

Tactile Sense. Ability to locate contact stimuli may be very precise. The cleaning habits of most animals are well known-molluscs, insects, and many others remove irritating particles with great precision. Tactile sense in man has been most studied by the use of needles or Hexible prods of different weights. On the skin the sensitivity of hairs is greater than that of cutaneous corpuscles; the sensitivity of shaved skin differs greatly, the sensitivities in various areas decreasing in the following order: forehead, nose, finger tip, back of finger, back of hand, abdomen, back of forearm, loin. The ability to discriminate two points is not the same as the sensitivity series, minimal 
distances for resolution being $2.3 \mathrm{~mm}$. on finger tip, $11.3 \mathrm{~mm}$. on palm of hand, $31.6 \mathrm{~mm}$. on back of hand, and $67 \mathrm{~mm}$. on upper arm, thigh, and middle of back. ${ }^{4}$

A tactile sensory unit appears to be one sensory neurone, together with all those sensory endings whose processes converge on that axon. The most accurate mapping of such an area has been done by recording impulses in single fibers of the long ciliary nerve of the cat; one fiber serves 50 to 200 sq. $\mathrm{mm}$. ( 1 quadrant) of the cornea, together with adjacent sclera and conjunctiva. ${ }^{22}$ In the central portion of such a field the threshold is lowest, impulse frequency highest, and adaptation slowest, hence the endings differ over the same field. Cutaneous sensory endings can be stimulated electrically as well as mechanically. ${ }^{5},{ }^{6}$ Areas of touch and prick (pain) are independent, and as the point stimulus moves over a fingertip the stimulation "feels" as though it went by jumps. Any stimuli within $2-15 \mathrm{~mm}$. of each other on the back of the hand are referred to the same locus, and stimulation of two points in that area can summate. There is some overlap of the unit areas as they are delineated during recovery from anesthesia, and two-point discrimination probably involves several sensory units. Single sensory fibers serve relatively large overlapping areas; the sensitivity of all the endings in such an area is not the same, and much of the discrimination of tactile sense depends on sorting out impulses of different frequencies and in different afferent fibers within the central nervous system. Overlap of areas served by sensory nerves has been observed in mammals, frogs, and earthworms.

Adaptation of Mechanoreceptors. The intensity of any sensory message is given by the frequency of discharge in single units and the number of active units. The frequency increases with increasing tension or pressure on a mechanoreceptor. The characteristic pattern of nerve impulses is an initial burst of high frequency impulses which decline rapidly (adaptation). The maximum frequency is limited by the refractory period of the nerve fiber, but sensory discharge is usually much slower than this, and adaptation is not fatigue in the sense of metabolic failure. Adaptation is the decreasing excitability of sensory endings during maintained stimulation, and the mechanism of adaptation is not well known. Mechanoreceptors differ in rate of adaptation, from those which give only one impulse to those which continue to discharge more or less indefinitely at low frequency during stimulation. In general, tactile receptors adapt rapidly and proprioceptors adapt slowly. ${ }^{1}$

The rapidly adapting endings are best known among tactile hairs and touch receptors of the skin. Each sensory hair along the margin of the telson and uropods of a crayfish gives only one impulsc when flexed; ${ }^{* 0}$ hair receptors in the skin of a rabbit give 1 or 2 impulses (Fig. 181). ${ }^{35}$ Vibration receptors sucl as the cochlea and lateral line organ must also be considered as rapidly adapting, since on synchronization with a sound one impulse appears per wave front. Fine sensory hairs on the leg of a cockroach adapt rapidly. while stout spinous hairs adapt more slowly. ${ }^{\text {.1 }}$

The tactile receptors of vertebrate skin are next in order of decreasing speed of adaptation. The study of these has been aided by the fact that in the dorsal cutancous nerves of the frog there are occasional antidromic fibers, efferent branches of sensory neurones, so that stimulation of the sensory 
area of one nerve initiates impulses in the central end of a fiber of another nerve. ${ }^{2}$ The skin area served by one, such sensory fiber varies from 4 to 100 sq. $\mathrm{mm}$. When the sense endings are stimulated continuously, as by a jet of air, there is an initial burst of 4 to 12 or more impulses lasting 0.1 to 0.2 sec. (Fig. 181, a); when they are stimulated by a puff 5 msec. or less in duration at a rapidly applied pressure one impulse appears (Fig. 181, b). In addition to these fast adapting endings there are some which discharge for as long as $10 \mathrm{sec}$. during continued stimulation. ${ }^{23}$ A different sort of adaptation, secondary adaptation, occurs when the ending is stimulated repetitively,

a
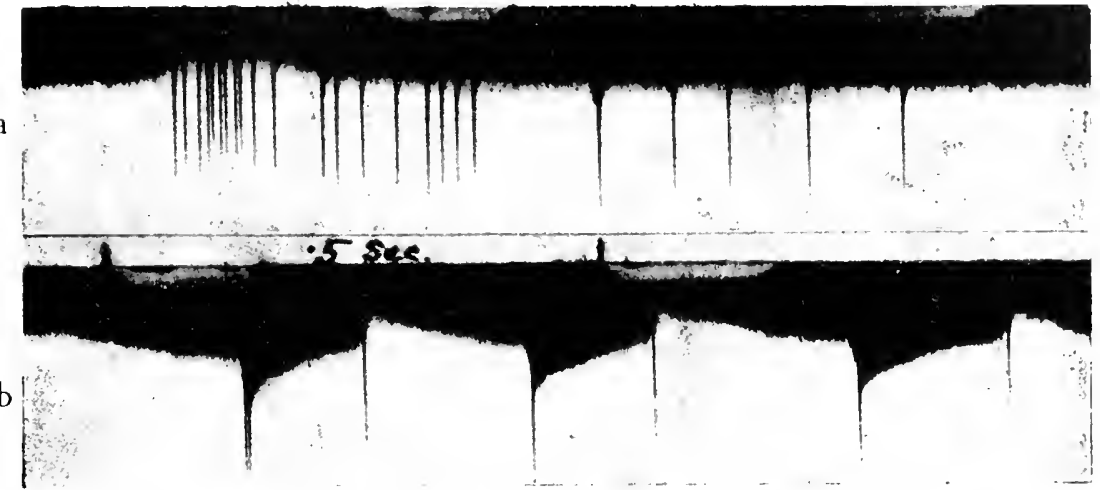

Fig. 181. Responses in single fiber of dorsal cutaneous nerve of frog (a) to pressure suddenly applied and maintained and (b) to pressure applied three times by interrupting air jet (slowly rising baseline indicates duration of sudden blast). From Adrian, Cattell, and Hoagland. ${ }^{2}$

as by an interrupted air jet. The ending may discharge at a rate as high as 250 to 300 per second initially, and may continue at 150 per sec. for 30 to 60 seconds, finally dropping more and more impislses until it discharges only occasionally. Sometimes, when the ending is stimulated at high fre. quency (e.g., 150/sec.), there may be two impulses together for one stimulus, the second one smaller than the first, because it falls in the refractory period of the nerve. Continued repetitive stimulation of the skin keeps the endings adapted, even though there may be no nerve impulses. By sending impulses backward (toward the skin by electrical stimulation), it is found that the adaptation does not reside in the nerve. If the stimulus-to-rest ratio is small, adaptation is slowed, and if the pressure is high adaptation is also delayed. Increase in the potassium content of the saline bathing the nerve ending speeds adaptation reversibly, and it has been shown that repeated mechanical beating of frog skin by an air jet increases the potassium content of bathing saline, hence this secondary adaptation may be due to accumulation of potassium lost. from epithelial cells around sensory endings. ${ }^{44}$ $46,47,48$ Such potassium leakage is probably not the cause of the fast adaptation to a single stimulus.

The encapsulated mechanoreceptors, such as pacinian corpuscles, are intermediate in adaptation speed. When pressure is applied to a mammalian tendon containing a pacinian corpuscle, a discharge starts at an initial frequency of 50-80 per second, adapting in a few seconds to $10-15$ per second. ${ }^{3}$ 
The pacinian corpuscles along blood vessels in the mesentery discharge as the vessels are distended with each pulse. ${ }^{37}$

The campaniform sensilla of insects are also intermediate in speed of adaptation. These sensilla respond to strains in the cuticle produced by straight or lateral bending, the leg sensilla being stimulated while the insect stands. The discharge starts at a high frequency (100-300/sec.) and falls after $1-2$ seconds to low levels. ${ }^{76}$

Tension receptors in muscles adapt very slowly, compared with tactile receptors. The impulses from single sensory spindles in a frog toe muscle under continued stretch or load start at 120-260 per second and decline to a rate of about 20 per second, which is maintained regularly for many min. utes. Responsiveness to a test reload is less as adaptation is prolonged. ${ }^{64,6 \pi}$ Frequency of afferent discharge depends not only on the amount but also on rate of development of tension (Fig. 182). If a muscle is stretched and

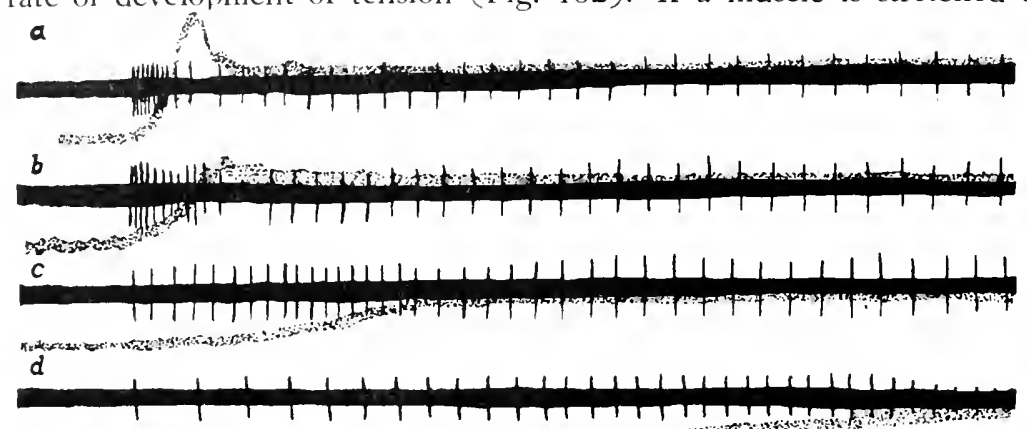

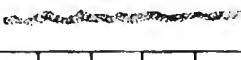

Fig. 182. Response in nerve fiber from a muscle receptor in peroneus longus of cat at different rates of stretching of the muscle to final tension of $25 \mathrm{gm}$. Action potentials, small deflections from central lines, muscle contraction indicated by lower records. From Matthews. ${ }^{8,5}$

the spindle is firing continuously, contraction is accompanied by a brief interruption in the steady sensory discharge. Matthews ${ }^{6 i 6}$ described three types of sensory endings of mammalian muscle: (1) receptors (probably spindles) which are arranged in parallel with the contractile elements, and which respond either on quick stretch or release or in active contraction; (2) receptors of high threshold arranged in series with the contractile elements; and (3) a type occurring in fascia, rapidly adapting and responding only during movement of the muscle.

Stretch receptors in muscles of the elasmobranchs Raja and Scyllium adapt slowly and maintain a steady discharge longer than do the muscle receptors of amphibians and mammals, firing under constant tension for more than an hour. When the tension is suddenly decreased there is a silent period before the discharge resumes at a new level."'?

Virtually no adaptation occurs in the pressure receptors of the carotid sinus of mammals. Some of the sensory elements are normally continually active and lor each pulse wave their frequency increases; other elements fire three or four impulses for each pulse. When the carotid artery is perfused at steady pressures there is little stimulation until the pressure rises above 40-50 
$\mathrm{mm} . \mathrm{Hg}$, when a few impulses appear, and at higher perfusion pressures the impulse frequency in single fibers is directly proportional to the pressure (Fig 183). There may be a slight fall in frequency after the initial discharge on increasing pressure, but the equilibrium frequency is maintained more or less indefinitely. ${ }^{11,12}$

In the preceding account mechanoreceptors are arranged in a serics from fast to slow adaptation. It is evidently useful in behavior that tactile messages be brief and tension reception persistent. There is no clear histological correlation with speed of sensory adaptation, and the cellular mechanism underlying these differences in adaptation remains to be discovered.

Pain. Tactile and proprioceptive impulses travel in relatively large fibers (A fibers of the classification of Erlanger and Gasser, as given in Chapter 23). Free nerve endings of the vertebrate skin are stimulated by

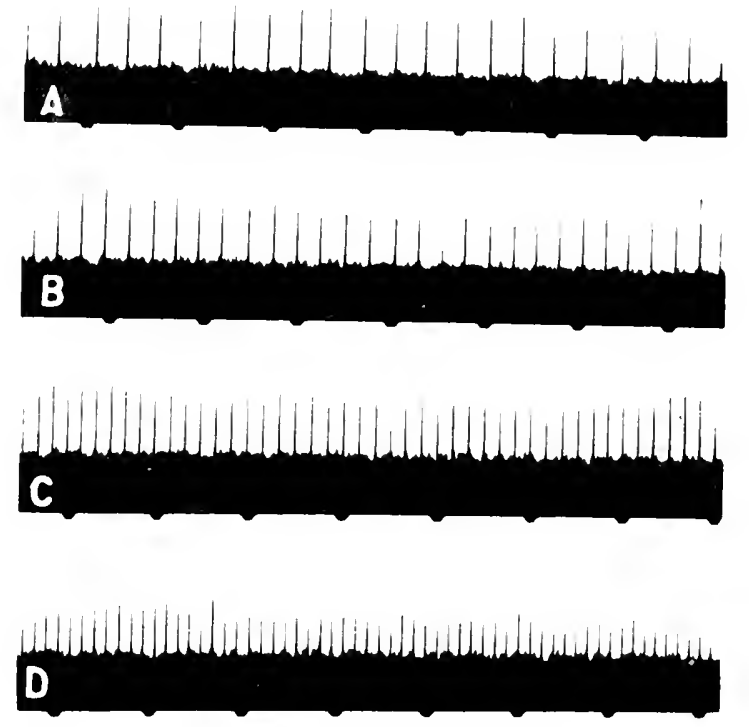

Fig. 183. Steady discharge in single fiber of carotid sinus nerve at the following perfusion pressures: $A, 40 \mathrm{~mm}$. $\mathrm{Hg} ; B, 80 \mathrm{~mm}$. $\mathrm{Hg} ; C, 140 \mathrm{~mm}$. Hg; D, $200 \mathrm{~mm}$. Hg. Time marked in $0.2 \mathrm{sec}$. From Bronk and Stella. ${ }^{12}$

various noxious stimuli, heat, prick, heavy pressure, acid, etc., and give rise to impulses in small fibers. ${ }^{1}$ The pain impulses are of small amplitude and low velocity. In the cutaneous nerves of the frog, for example, narcotics abolish the slow impulses first, $\mathrm{KCl}$ eliminates the fast impulses first; the slow are conducted at 1.5-4.5 M./sec., the fast at 10-12 M. $/ \mathrm{sec}^{+{ }^{+9}}$ and the sensory areas in the skin for light touch and heavy pressure are different. In the cat, impulses from sensory hairs travel at 30-60 M./sec., and pain impulses are conducted at less than $5 \mathrm{M}$./ $/ \mathrm{sec}^{96}$ The maximum frequency of discharge in the small fibers is less than from the tactile receptors; the response gradually builds up and adapts very little.

Similarly, mechanical stimulation of the intestine of a cat elicits impulses in medium-size fibers from the pacinian corpuscles and in small fibers probably from pain endings. ${ }^{38}$ There are also pain endings in the pulp of teeth 
which are stimulated by noxious stimuli and give rise to impulses in small mvelinated fibers. These are to be distinguished from pressure endings of the periodontal membrane, which show much variation in adaptation and some of which follow very high frequency stimulation, even as high as 1200 cycles per second. ${ }^{7.5}$

In the earthworm sensory cells converge at the subepidermal plexus, and the afferent fibers of the segmental nerves are, therefore, secondary neurones. Nevertheless the afferent impulses set up by tactile and proprioceptive stimulation are large and fast compared with the impulses initiated by noxious stimuli, particularly chemical. ${ }^{79}$ It is a general rule that pain impulses are small and slow, in small fibers.

\section{EQUILIBRIUM RECEPTION}

Types of Equilibrium Receptors. One of the more primitive sense organs controlling animal attitude is the gravity receptor or statocyst. In principle a statocyst (Fig. 184) consists of a fluid-filled chamber, the bot-

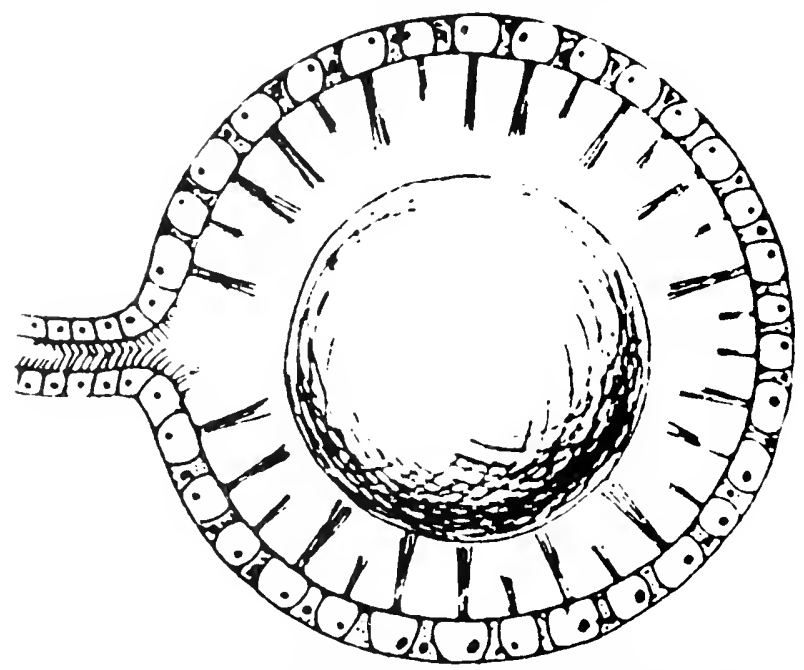

Fig. 184. Apical statocyst of a ctenophore. Note the centrally located structure, the statolith, and the hairlike processes of the sensory epithelium. From Buddenbrock."

tom or top of which contains a sensory epithelium. A solid or semisolid body, the statolith, rests on or hangs from this epithelium and thus presumably activates the sense cells. If these sense cells exhibit moderately slow adaptation, a steady barrage of nerve impulses at rather low frequencies may be passing along the nerve fibers. Any change in position or weight of the body resting on or hanging from the sensory epithelium would either enhance or diminish this barrage of nerve impulses, thus signaling the coordinating centers of the central nervous system of a change in attitude of the organism. The pattern of motor reactions elicited by stimulation of statocysts varies from animal to animal, for it depends on the extent and characteristies of the reflex pathways involved, but many organisms react in such a manner as to bring the statocyst and its contents back to its original resting condition, thus effectively annulling the change in the statocyst 
position. This kind of annulling reaction is widespread throughout the animal kingdom and is the result of a coordinated nervous activity called "negative feedback." 94

The function of statocysts in animal orientation is strikingly illustrated in the experiments of Kreidl. ${ }^{57}$ The statocysts of crabs open to the exterior by slits. At each molt, the statoliths are lost and are replaced de novo from foreign bodics in the environment. Iron or nickel dust in the aquarium forms statoliths with magnetic properties. The introduction of a magnet above a crab immersed in water causes the statoliths to be pulled to the top of the statocyst and the animal promptly turns over on its back.

Orientation to Gravity: Geotaxis. Well differentiated statocysts first make their appearance in the coelenterates, although well defined gravity reactions appear in certain Protozoa, i.e., Paramecium. ${ }^{33}$ Among the coelenterates, the Scyphomedusae have eight statocysts radially arranged around the margin of the mantle. In the normal position the axis of symmetry is kept vertical. When the animal is tilted, the lower portion of the mantle

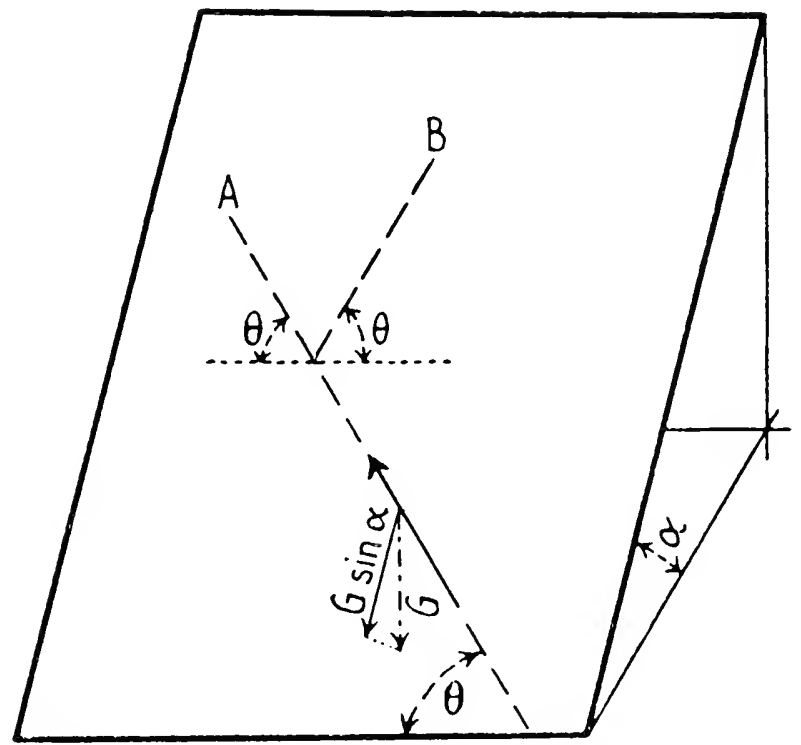

Fig. 185. Diagram showing terms used in the description of orientation of animals on the inclined plane. From Crozier and Pincus. ${ }^{13}$

musculature contracts more strongly than the upper portion, and the animal rights itself.:2 Surgical removal of several of the statocysts completely disorients the animal. Ctenophores likewise are well equipped with functional statocysts.

Many organisms (turbellarians, polychaetes, holothurians, brachipods, isopods, dipteran larvae and branchiate snails) exhibit an orientation toward the source of the force of gravity, called positive geotaxis. Many of these organisms burrow in mud or sand. In some cases this positive geotaxis is statocyst controlled, as evidenced by the lack of this orientation after surgical removal of the statocysts (Arenicola marina, A. grubei, 
Branchiomma resiculosum, Cyathura carinata and Limnophila fuscipennis: for further details see von Buddenbrock ${ }^{14}$ ).

Negative geotaxis occurs less frequently among invertebrates, and is illustrated by the pulmonate snails Limax and Helix. When these animals are placed on an inclined plane in water, they ascend by the steepest path,,$\tilde{x}^{2}$. it regardless of the slope of the inclined plane. These animals have statocysts and, when they are supported by the water, proprioceptive and other kinds of stimulation are minimal. When the snails are placed on an inclined plane in air they choose a direction of ascent which deviates from the steepest path by an angle which is related to the angle of inclination of the plane. Similar responses are obtained from a great variety of animals which exhibit negative geotaxis. The simple and direct control of orientation to gravity by statocysts is certainly not indicated here. Rather, in these animals, a given attitude is probably the result of a complex series of reactions including several sense modalities.

The numerous experiments of Crozier and his co-workers ${ }^{1 \times}$ strikingly illustrate the complexity of the situation. These investigators have tested the responses of numerous animals on the inclined plane (Fig. 185). Their results indicate that for many animals the angle of orientation up the plane varies approximately as the common logarithm of the active component of gravity, $g$ sin $\alpha$. Experiments with the beetle, Tetraopes tetraophthalmos, ${ }^{21}$ proved, interestingly enough, that addition of weight (i.e., wax) to the abdomen resulted in an increase of $\theta$, (the angle of orientation), whereas the addition of weight to the anterior end or the removal of the abdomen resulted in a decrease of $\theta$. Experiments with male fiddler crabs, Uca, ${ }^{58}$ which possess one large claw and one small claw, indicate that angle $\theta$ is greater when the large claw is on the downward side of the animal. These and other experiments have led Crozier and his school to the conclusion that orientation to gravity depends on distribution of the pull of the organism's mass on the supporting musculature; i.e., that the orientation is initiated primarily hy proprioceptive sense organs. That this may not be entirely the situation is illustrated by experiments with ticks.3.3 Unfed ticks will crawl freely in all directions on inclined or vertical planes; ticks gorged with blood, however, always crawl upwards and travel more steeply, the greater the angle of inclination of the plane. Similar results are obtained when inert weights are attached to the abdomen of the tick. Thus, the mechanical alignment of an organism on an inclined plane, due to a posteriorly lecated center of gravity, must be considered. The interpretations of Ilunterin suggest additional complications. A young rat on an inclined plane has its center of gravity above the plane, i.e., there is a tendeney for the animal to roll. This tendency to roll is greatest when the angle $\theta$ is low, or when the angle of in lination, $x$, is increased. Thus, an increase of $\alpha$ may be compensated by an increase of $\theta$. The orientation of a young ratt and of other animals may thus be dependent on a series of postural reflexes imvolving statocysts, proprioceptors, and perhaps cutaneous sense organs.

The Vertebrate Labyrinth and Its Functions. The membranous labyrintl, of the inner car of vertebrates is divided into two parts (see Figure 161, Chapter 13): (1) the lower portion, including the sacculus and lagena or 
cochlea, functions in hearing; (2) the upper portion, consisting of the utriculus and the semicircular canals, functions in oricntation. The utriculus conforms reasonably closely to the basic plan of a statocyst, (page 514). The semicircular canals are bent tubes, both ends of which communicate with the utriculus. The bent tubes bear an enlargement at one end, the ampullat, in which is located the sense organ (Fig. 186), consisting of the crista and the gelatinous cupula, which approximately fills the cavity of the ampulla (p. 475, Ch. 13).

Fibers in the vestibular branch of the eighth nerve are of three types: those activated only by mechanical vibration of low frequency; those activated by tilting movements only; and those activated by angular accelera tion. ${ }^{\times 2}$ McNally and Tait ${ }^{71}$ rendered the utriculus of frogs non-functional by cautery without encroaching on the semicircular canals and determined that the response to tilting and linear acceleration was abolished. It is gen-

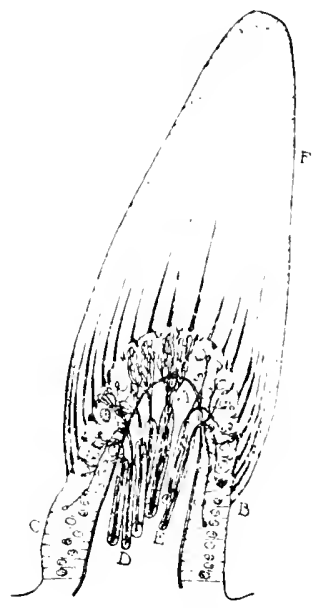

Fig. 186. Diagrammatic sketch of the cupula and crista after Kolmer. A, hair cells whose terminal processes protrude into $F$, the gelatinous cupula; $B$, supporting cells; $D$, large nerve fibers; $E$, small nerve fibers. From Fulton. ${ }^{36}$

erally accepted that the utriculus responds to these movements. The function of the sacculus is less clearly established. In the frog, section of the nerves from the saccular maculae does not produce any disturbance of orientation. ${ }^{71}$ Von Budelenbrock ${ }^{1+}$ states that the sacculus does not function in orientation except in rabbits, in which vertical eve movements are elicited by stimulation of the sacculus. The consensus at present ${ }^{69}$. 3 it is that the sacculus responds to mechanical vibrations of low frequency. Concerning the function of the semicircular canals, there exists a vast litera ture. ${ }^{40 .}$ 14. To The work of Löwenstein and Sand, $": 2$ Steinhausen," 10 and Dohlman":- serves to clarify the function of the semicircular canals.

Löwenstein and Sand ${ }^{6: 2}$ recorded nerve impulses from single nerve fibers from an excised horizontal semicircular canal of the skate, Raja clavata. In all preparations they observed a spontaneous discharge of impulses under stationary conditions. The frequency of this background discharge varied. Rotation of the preparation toward the side of the canal (ipsilateral rotation) 
produced an enhancement of impulse discharge (Figs. 187, 188); rotation in the opposite direction (contralateral rotation) produced a decrease of the spontaneous discharge (Figs. 187, 189). The impulse frequency increases (or decreases) uniformly with constant angular acceleration. Rotation at constant velocity produces an initial increase (or decrease) of impulse frequency, during the acceleratory phase, followed by a gradual return to the spontaneous discharge level in 20 to 30 seconds (Fig. 190). Stein-
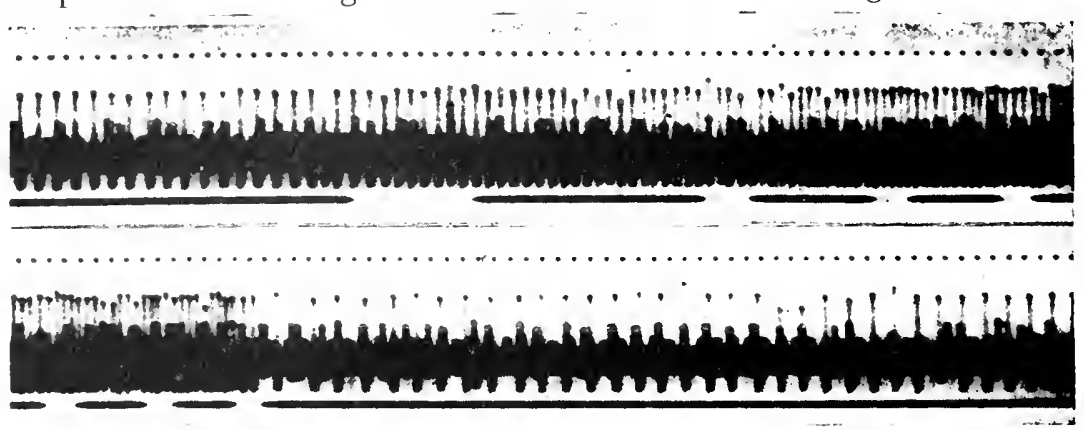

$A$

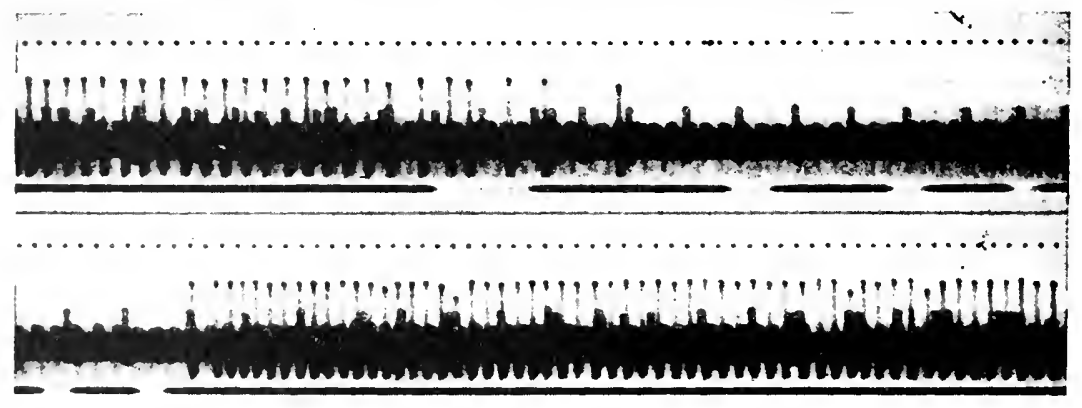

$B$

Fig. 187. Photograph of oscillographically recorded spike potentials obtained from a nerve branch to the left horizontal ampulla of the skate. The uniformity of the large spikes indicates the response of a single fiber. A, Response of a single unit to ipsilateral angular accelcrations. The angular acceleration ( 15 degrees per sec.) is indicated by the broken black lines of unequal lengths. Note the spontaneous activity in other fibers before and after acceleration. $B$, Response of the same unit to contralateral angular accelerations. From Löwenstein and Sand."2

hausen ${ }^{49}$, and Dohlman"2 observed and photographed the cupula in intact semicircular canals of the pike. The results of both investigators show that the cupula nearly fills the lumen of the ampulla and is displaced sharply to one side on rotation of the animal (Fig. 191). Introduction of an oil droplet into the canal (by Dohlman) shows that the displacement of fluid is responsible for the movement of the cupula. Analysis of the time relations indicates that a cupula displaced by sudden acceleration returns to its resting condition in about 20 seconds. (Fig. 192). The cupula thus acts as a highly damped pendulum having a period of 20 seconds.

The results of these investigations indicate that the movement of the 


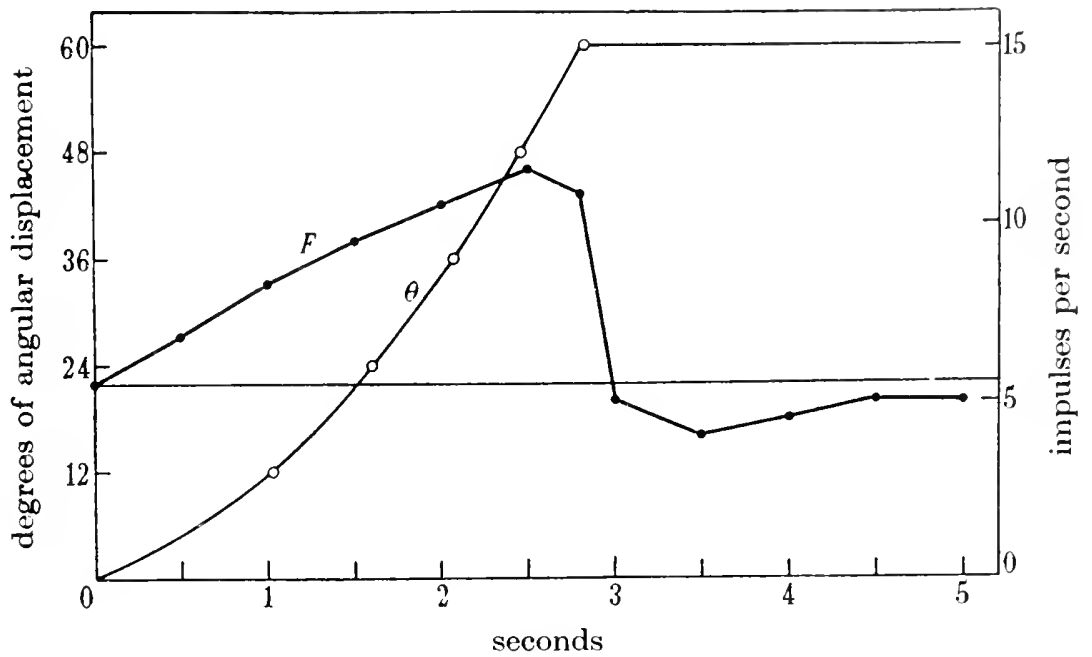

Fig. 188. Relationship between the frequency of the spike potential discharge and ipsilateral angular acceleration. The horizontal line indicates the frequency level of spontaneous discharge. From Löwenstein and Sand. ${ }^{62}$

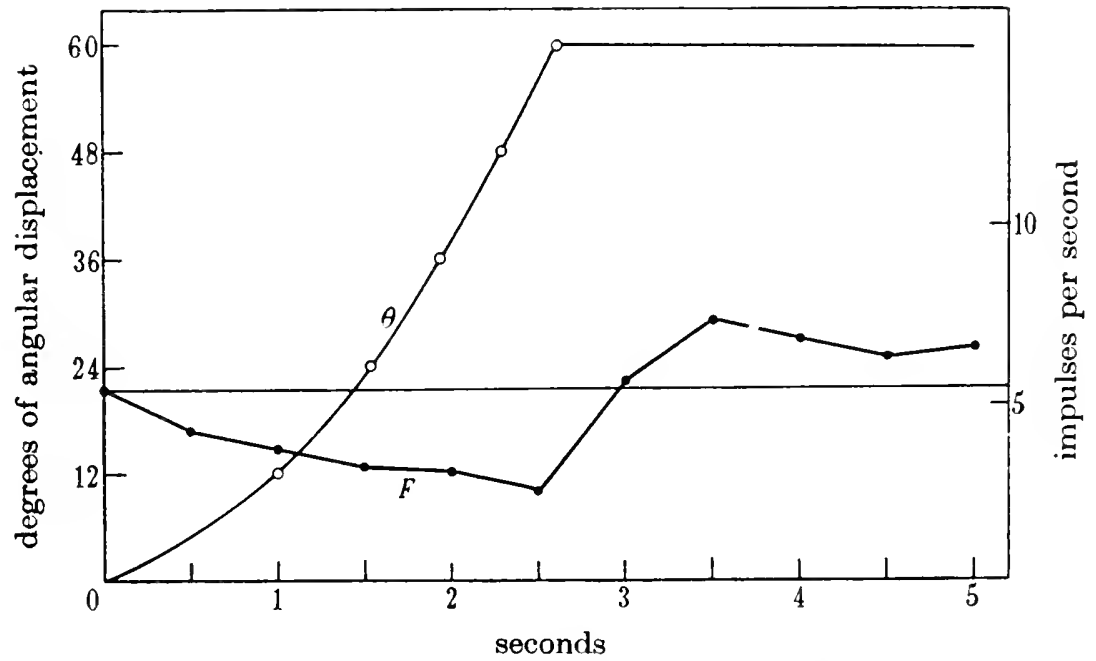

Fig. 189. Relationship between the frequency of spike potential discharge and contralateral angular acceleration. From Löwenstein and Sand. ${ }^{62}$

cupula is the adequate stimulus for changing the frequency of nerve impulses in the afferent nerve fibers. They show further that a given canal will respond to rotation in two directions. The earlier notion, ${ }^{1+}$ that in fishes, amphibia, and reptiles the movement of fluid in the semicircular canals stimulates only in one direction, is not quite correct. What is implied here, probably, is that the functional organization of the nervous systems in these animals does not take cognizance of a reduction in nerve impulse frequency. 
Equilibrium Orientation in Vertebrates. Numerous experiments on the effects of labirinthectomy have been reported on a great variety of ani-

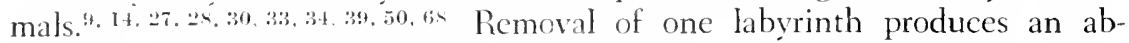
normal posture in which the head and neck are bent toward the operated side. In the absence of other sensory cues (see below), this abnormal reaction is always present and is called a primary symptom. Other symptoms of abnormal posture appear after the operation but gradually subside, until the orientation and response of the organism is normal. These are called secondary symptoms. Most animals with only one labyrinth typically re-

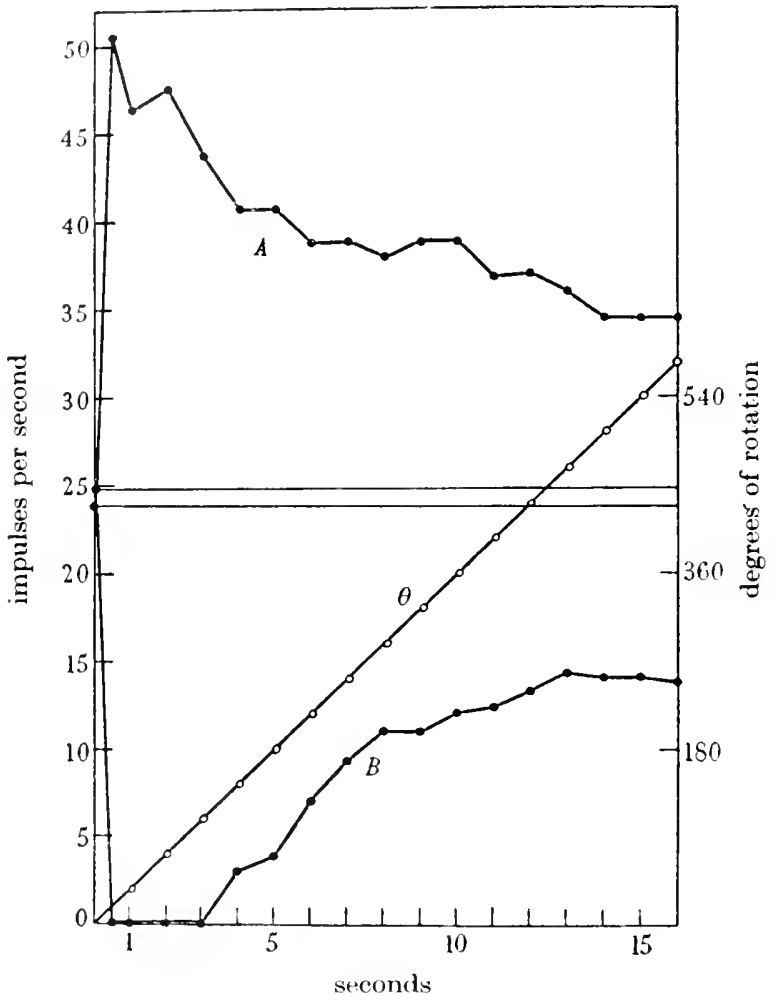

Fig. 190. Relation of spike potential discharge frequency to rotation ( $A$, ipsilateral, and $B$, contralateral) at constant speed of 36 degrees per second, as a function of time. The horizontal lines indicate the frequency level of spontaneous activity at the onset of each experiment. Note the early oceurrence of a maximum change followed by tendency to level off above and below the spontaneous discharge level. From Löwenstein and Sand."

spond to rotation in both directions, suggesting that one labyrinth is capable of scring an organism satisfactorily, a suggestion confirmed by the study of impulses in labirinthine nerves.

The remesal of both labyrinths produces eflects which differ in animals. Frogs thus preparedir maintain any imposed posture of which they are capalble. Mammals, except rabbits and guinca pigs, seem to behave quite normally, provided the eyes are intact, except under two circumstances. When a bilaterally labyrinthectomized animal is placed in water, orienta- 
tion does not take place, and drowning results. The other circumstance is falling through air. A normal cat always lands on its feet regardless of its position at the instant of falling, by executing a series of righting reactions while in the air. A bilaterally labyrinthectomized animal cannot right itself during a fall. ${ }^{2}$, $4: 3$ Bilaterally labyrinthectomized pigeons ${ }^{30}$ eventually regain some ability to fly, only, however, when there is no interference with vision. These observations seem to indicate that higher animals can, under most circumstances, compensate for the loss of both labyrinths.

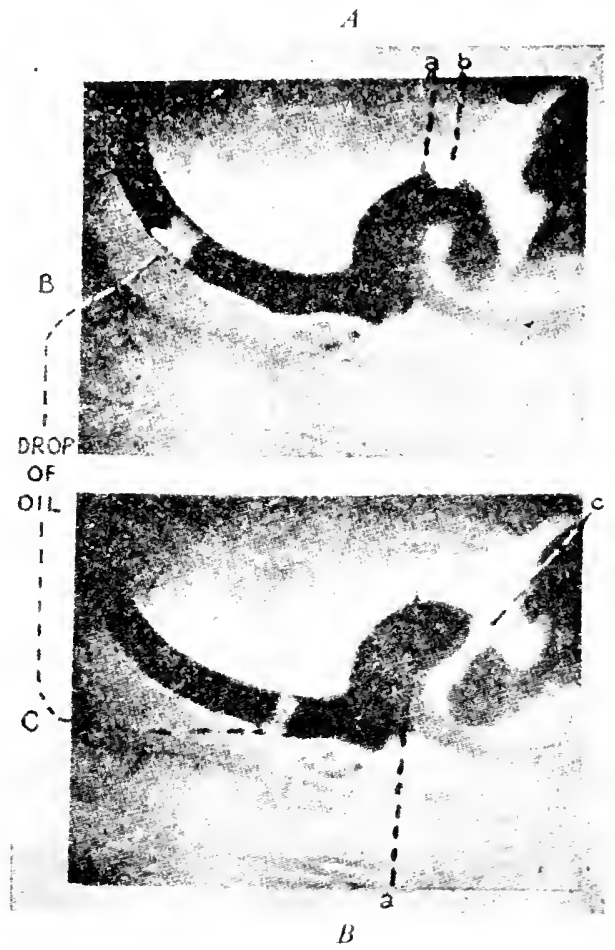

Fig. 191. The ampulla and semicircular canal photographed in the living state (pike) before and during angular acceleration. $B$, oil droplet in canal before acceleration; $C$, oil droplet during acceleration; $b$, position of cupula before acceleration; $c$, position of cupula during acceleration. After Dohlman, from Fulton. ${ }^{38}$

The question naturally arises, "Where does the labyrinthectomized animal obtain the sensory information necessary for orientation?" The answer to this question has been supplied largely through the investigations of Magnus ${ }^{63}$ and has been excellently reviewed by Evans. ${ }^{27}$ Labyrinthectomized dogs and cats orient their heads well when supported in air by the pelvis. However, if these animals are blindfolded under similar circumstances, no orientation of the head takes place. In rabbits and guinea pigs, renowned for their lack of visual righting reflexes, no orientation of the head takes place, even without blindfolding. These experiments indicate that vision is an important adjunct to labyrinthine function in orientation. If a labyrinthectomized rabbit is held in the air by the pelvis, 
the head is not oriented but falls into an abnormal position due to the force of gravity. If this animal is now placed on its side on the table, the head will right itself. If a weighted board is placed on the upper side of the animal, the head is not righted, indicating that unsymmetrical stimulation of cutaneous receptors affords a sensory cue for righting. The righting of the head in such labyrinthectomized rabbits is followed by righting of the body. If the head is held so that it cannot be oriented, the body of the rabbit will right itself, followed by righting of the head, if permitted. This indicates that the neck may also be the source of sensory cues for righting. That this is so can be demonstrated clearly in labyrinthectomized decerebrate (brain stem divided just anterior to entry of the

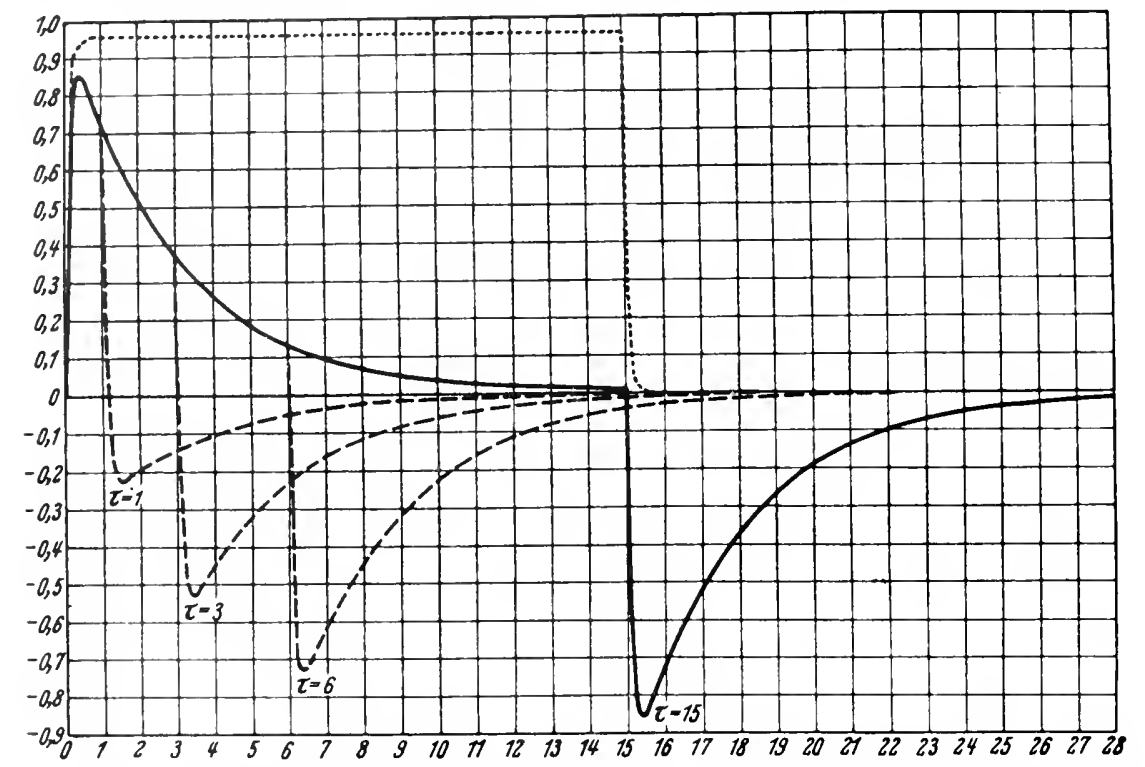

Fig. 192. Relation between deflection of cupula in the intact semicircular canal of the pike and time, during prolonged rotation. Solid lines indicate the position of the cupula (measured in arbitrary units) after the onset, during and after rotation. The lower broken lines indicate the deflection upon stopping the rotation at the times indicated. The upper broken line indicates the theoretical movement of endolymph in the canal. Each division of the time base is equal to $1-1 / 3$ seconds. From Steinhausen. ${ }^{00}$

eighth cranial nerve) animals. Forced rotation of the head in such animals produces an increase of extensor tonus (tonic contractions of extensor muscles are increased) on the side toward which the nose is turned. Section of the cervical dorsal roots abolishes this response, suggesting that sense organs (proprioceptors) in the neck muscles are its source.

In normal vertebrates, distinct reactions to rotation are evident. When an animal is rotated to the right, the eyes tend to remain fixed on an object as long as possible, the head gradually moving to the left as far as possible, then shifting suddenly to the right. This cycle of movement is repeated, and is called head nystagmus. After rotation has been stopped, the nystagmus is in the opposite direction. The eyes exhibit reactions similar to those of the head, and these are referred to as eye nystagmus. Such compensatory 
eye and head movements are absent in bilaterally labyrinthectomized animals, provided optical stimulation is absent. However, bilaterally labyrinthectomized dogs can discriminate higl angular accelerations, as demonstrated by conditioned reflex methods. ${ }^{*-4}$ The sensory cue involved in this discrimination possibly originates in proprioceptors of the neck muscles. Human beings, subjected to constant deceleration, ${ }^{41}$ give markedly different responses when the whole body is tilted and when only the head is tilted during the deceleration. Those differences in response are attributed to the role of the proprioceptors of the neck muscles.

It is well established that, after rapid acceleration and deceleration, eye movements persist for about 20-30 seconds. Heyer, ${ }^{+1}$ working with human subjects under constant conditions of acceleration and deceleration, demonstrated that the subjective sensation of rotation persisted significantly longer on acceleration than on deceleration. This difference in duration of sen sation is not understood. It is safe to say, however, that the duration of eye movements and subjective sensation roughly parallels the time course of cupula deflection.

Orientation in Flying Insects. Statocysts are rare in these animals and, until recently, orientation has been analyzed only in the Nepidae or water bugs. These animals are negatively geotactic, which presumably enables them to reach the surface of the water for breathing purposes. They collect a bubble of air on the under side of their abdomen, which is then utilized during their underwater exploits. Along the lateral edges of the abdomen there is a trough in which are located a series of pits; believed to contain sense organs. ${ }^{1+}$ These pits are filled with air. In the normal attitude of the bug, the bubble shrinks posteriorly as the air is used. This causes the air-water interface to activate the sense organs and signal the organism to ascend.

More recently Pringle 77 demonstrated that the halteres are gyroscopic mechanisms which signal any departure from the normal attitude and presumably initiate reflex adjustment. The halteres are found in all groups of Diptera as dumbbell-shaped organs located behind the wings. These halteres are rather complex (Fig. 193), bearing a rich supply of sense organs, a muscle, and a hinge, and are of a shape suited to their function. These halteres oscillate rapidly in a vertical plane during flight at frequencies of 100 to 200 cycles per second (c.p.s.). Under normal circumstances the wings and halteres are synchronized, but this is not always so. There does, however, seem to be some coupling between the oscillation of the wings and that of the halteres. The oscillation of the haltere begins by mechanical stimulation, i.e., stretching of the muscle. Motor nerve in pulses or electrical stimulation does not produce contractions but merely seems to enhance the excitability of the muscle for mechanical stimulation. ${ }^{74}$ That a reflex of some sort is not involved is indicated by: (1) the persistence of this response under conditions of anesthesia which abolish other reflexes, (2) the persistence of this response when the muscle and its nerve is separated from the thoracic ganglion; (3) the persistence of the response when the proprioceptive sense organs (campaniform sensilla) of the wing base are removed; and (4) the lack of sense organs in the haltere muscle and indirect flight muscles. The fact that electrical stimulation elicits no con- 
traction (except at low frequencies in certain instances: fusion at 10/sec. in haltere muscle), and the fact that there is no synchrony between oscillation of the muscle and incidence of motor nerve impulses or electrical stimuli, seem to indicate that these are not concerned directly with contraction of these insect muscles. ${ }^{\text {in }}$

The natural stimulus for initiating haltere oscillation seems to be the distortion of the thorax caused by the indirect flight muscles. This can be simulated by pressing on the sternum of a slightly anesthetized fly with a needle. Contraction of the haltere muscle causes dorsiflexion of the haltere; relaxation of the muscle is accompanied by ventral movement due

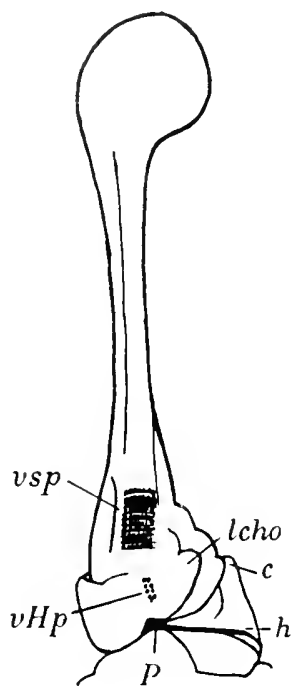

$V$

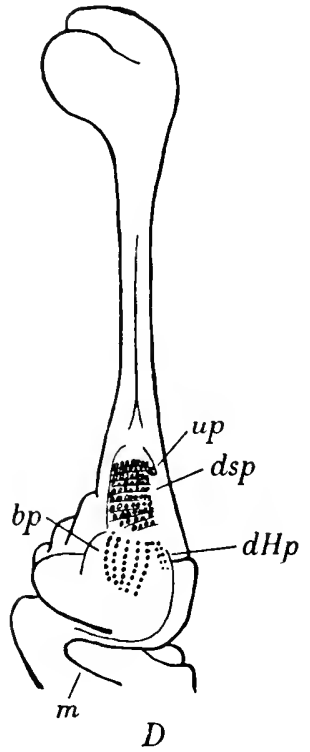

Fig. 193. Ventral (V) and dorsal (D) views of the left haltere of Lucilia sericata. c, condyle of secondary articulation; $h$, main hinge line; $m$, point of muscle attachment; $P$, strong point in the articulation; $b p$, basal plate; $d s p$, dorsal scapal plate; $d H p$, dorsal Hicks papillae; lcho, large chordotonal organ; $u p$, undifferentiated papillae; $v s p$, ventral scapal plate; $v H p$, ventral Hicks papillae, $(\times 100)$. (Pringle. $\left.{ }^{77}\right)$

to the elasticity of the hinge. The ventral movement again acts as a stretch stimulus, and the cycle is repeated, presumably as long as the arrival of nerve impulses to the haltere muscle maintains a heightened excitability.

The haltere, its joint, and the muscle form a mechanical system which oscillates at its resonance frequency. Reducing the mass of the haltere increases its frequency of oscillation.

Remoral of the halteres seriously interferes with the ability to fly (Fig. 194), particularly affecting the ability to maintain orientation in the horizontal plane. The flight abnomalities are not always the same but they always exist, unless some powerful orienting stimulus, as a source of light, is present.

How the balteres regulate orientation during flight is shown in Figure 195. The eenter of gravity of the haltere lies to the rear of the haltere axis. When 
the haltere oscillates about axis $A A$, the line $B B$ will tend to move into the position $Y Y$, due to a torque which develops as a result of the displaced center of gravity. This torque is discontinuous, fluctuating at twice the frequency of the haltere oscillation, but the damping of the fluid contents averages out these oscillations. If the haltere oscillations end abruptly the backward movement of the haltere can be clearly seen: it has a delay of about $1 / 2 \mathrm{sec}$. Thus, during vertical oscillation of the haltere, forces exist which move the haltere forward. It is this movement of the haltere that is significant in orientation, for it is affected by movements of the organism about its transicerse and longitudinal axes (pitch and roll) and its rertical axis (yaw).
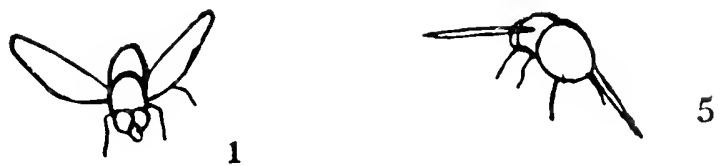

1
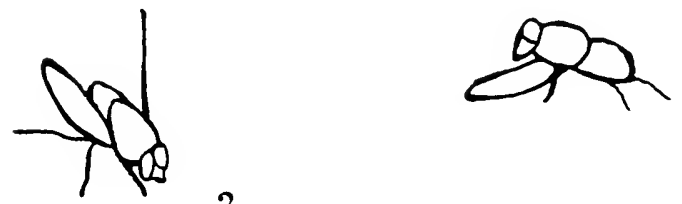

6

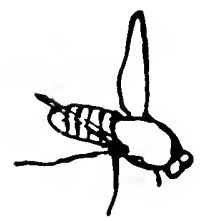

2

3

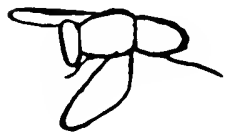

7

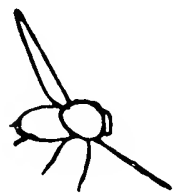

4

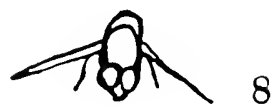

Fig. 194. Tracings from flash photographs of a haltereless fly, Eristalis tenax, in free flight. The interval between exposures is $1 / 8 \mathrm{sec}$. Note the instability in the horizontal or yawing plane. From Pringle. ${ }^{7}$

The electrical recording of action potentials in the nerve innervating the haltere sense organs, under conditions of normal flight, pitching, rolling, and yawing, indicates considerable differences. Oscillations of the halteres with normal attitude result in the appearance of a burst of impulses for each half cycle of oscillation, presumably occurring at the maximum and min. imum of each excursion. There is a period of inactivity between each burst of impulses. During rotation in the rolling plane the nerve activity is augmented, but not to the extent that it is by movement in the yawing plane. Presumably the altered nerve discharge pattern produces reflex 
changes in the wing beat which will restore the normal attitude of the flying insect.

The morphological analysis of the structure and orientation of the sense organs may be summarized as follows:

I. Sensitive to strains produced by vertical oscillation of the haltere

1. Dorsal scapal plate

2. Ventral

3. Dorsal Hicks papillae

4. Ventral

5. Small chordotonal organ

II. Sensitive to strains produced by gyroscopic torque

I. Basal plate

2. Large chordotonal organ

The halteres function as gyroscopic organs of equilibrium sense.

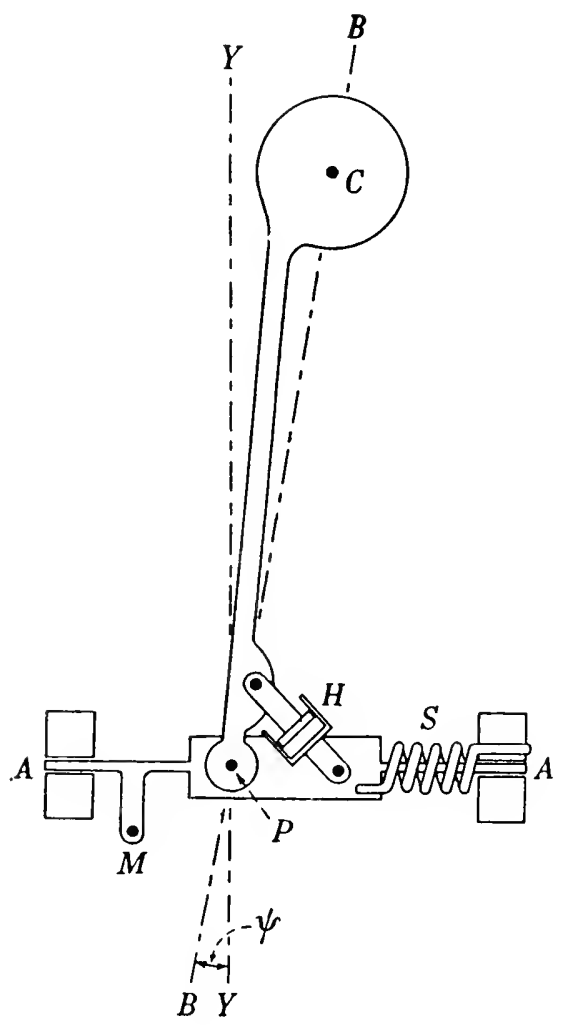

Feg. 195. Didgram to illustrate the mechances of haltere articulations. Pringle."

SUMMALX

Mechanorecepters ase mportant in the positioning of animals. Sometimes the "rest" pesition is one of contict stimulus on one side, as in the orientation of blind lish on a stream bottom or as in the inhibition of insect flight by tarsal contact. Sometimes there is a balance of bilateral 
stimulation, as in a worm crawling in a crevice. In general tactile receptors are most abundant in those regions of an animal where there is most likely to be contact with extraneous objects. Vibration receptors are diverselateral line organs of fish, sensilla attached to the exoskeleton of insects, tendon and muscle receptors of mammals. A change in plane is signaled by equilibrium receptors or by a combination of statocysts, tension receptors, and eyes.

Several questions deserve investigation. What is the mechanism by which deformation excites a cell? A continuous "spontaneous" activity is found in sensory nerves from fish lateral lines, in ampullae of Lorenzini of elasmobranchs (which are said not to be mechanoreceptors), and in vertebrate labyrinths. Are these impulses dependent on some very slight sensory stimulation or do the sense organs contain cells which discharge spontaneously like some central nerrous system neurones? Two kinds of adaptation of mechano receptors are recognized, primary adaptation during continued steady de formation, and secondary adaptation during repeated cleformation of end ings which adapt rapidly to one deformation. What are the mechanisms of these two types of adaptation? There is evidence that secondary adaptation of frog tactile receptors results from an accumulation of potassium around them; does this account for the similar adaptation of vibration receptors? Another question concerns the action of the receptors of hydrostatic pressure in keeping deep-sea and surface fish at the proper ocean depth.

Mechanical stimuli (except for hydrostatic pressure) can hardly be considered as limiting animal distribution, but they are important in liceping animals in position for their normal life activities. Equilibrium receptors are found in many animals and appear to be particularly important for animals which are free swimming, for example, jellyfish, elasmobranchs, and teleosts, and in flying animals, particularly birds and certain fying insects. Equilibrium receptors are also especially important when the center of gravity is well above substratum as in many birds and mammals.

\section{REFERENCES}

1. Adrian, E. D., Mechanism of Nervous Action (1935). Univ. of Pennsylvania Press. 103 p.

2. Adrian, E. D., Cattell, M., and Hoagland, H., J. Physiol. 72:377-391 (1931). Sensory discharges in single cutaneous nerve fibers, frog.

3. Adrian, E. D., and Umratii, K., J. Physiol. 68:139-154 (1929). Sensory impulses from pacinian corpuscles.

4. Best, C. H., and Taylor, N. B., Physiological Basis of Medical Practice (1943). Baltimore, Williams and Wilkins. pp. 1346-1363. Mechanoreception.

5. Bishop, G. H., J. Neurophysiol. 6:361-382 (1943). Responses to electrical stimulation of sensory units of skin.

6. Bisнop, G. H., J. Neurophysiol. 7:71-80 (1944). 'The peripheral unit for pain, man.

7. Black, V. S., Biol. Bull. 95:83-93 (1948). Gas changes in swim bladder of Fundulus in different densities.

8. Blair, H. A., Am. J. Physiol. 114:586-593 (1936). Mechanical stimulation of nerve.

9. Breuer, J., Wien. med. Jahrb. 4:72-124 (1874), ibid. 5:87-156 (1875). Function of the labyrinth, vertebrates.

10. Breuer, J., Pflïg. Arch. ges. Physiol. 48:195-306 (1891). The function of the otoliths, vertebrates. 
11. Bronk, D. W., and Stella, G., J. Cell. E Comp. Physiol. 1:113-130 (1932). Afferent discharge from carotid sinus as related to arterial pressure.

12. Bronk, D. W., and Stella, G., Am. J. Physiol. 110:708-714 (1935). Pressure relations and sensory adaptation in carotid sinus.

13. Brown, F. A., Biol. Bull. 76:48-58 (1939). Responses of swim bladder to pressure decrease, guppy.

14. Buddenbrock, W. v., Grundriss der vergleichenden Physiologie (1937). Berlin, Borntraeger. Vol. 2.

15. Canis, M., The Physiology of the Vestibular Apparatus (1930). Transl. by R. S. Creed. Oxford Univ. Press.

16. Cattell, M., Biol. Rev. 11:441-476 (1936). Physiological effects of hydrostatic pressure.

17. Crozier, W. J., J. Gen. Physiol. 6:531-539 (1924). Stereotropism in Tenebrio larvae.

18. Crozier, W. J., and Hoagland, H., in Handbook of General Experimental Psychology (1934). Worcester, Clark Univ. Press. Chap. 1, pp. 3-108. Tropistic analysis of animal behavior.

19. Crozier, W. J., and Pincus, G., J. Gen. Physiol. 10:195-203 (1926). Stereotropism in rats and mice.

20. Crozier, W. J., and Pincus, G., J. Gen. Physiol. 10:257-269 (1926), ibid. 11: 789-802 (1928). Geotropic reactions, rats.

21. Crozier, W. J., and Stier, T. J. B., J. Gen. Physiol. 12:675-693 (1929). Geotropic orientation in arthropods.

22. Dohlman, G., Proc. Roy. Soc. Med. 28:1371-1380 (1935). Studies in labyrinthology.

23. Dun, F., and Finley, C. B., J. Physio!. 94:170-176 (1938). Rate of adaptation of cutaneous nerve endings, frog.

24. Dusser de Barenne, J. G., in Handbook of General Experimental Psychology (1934). Worcester, Clark Univ. Press. Chap. 4, pp. 204-246. Labyrinthine and postural mechanisms.

25. Dykgraaf, S., Ztschr. vergl. Plyysiol. 20:162-214 (1933), ibid. 27:587-605 (1940). Function of lateral line organs in fish.

26. Echlin, F., and Fessard, A., J. Physiol. 93:312-334 (1938). Vibration sense, frogs, cats, rabbits.

27. Evans, C. L., Recent Advances in Physiology (1930). Philadelphia, Blakiston. \$46 p. Chap. 11. Postural reflexes and function of labyrinth.

28. Ewald, J. R., Physiologische Untersuchungen über das Endorgan des Nervus octarus (1892). Wiesbaden, Bergmann.

29. Fessard, A., and SAnd, A., J. Exper, Biol. 14:383-404 (1937). Stretch receptors in muscles of fish.

30. Flourens, P., Recherches cxpérimentales sur les proprićtés et les fonctions du système nerveux dans les animaux vertebres (1824). Paris, Bailliére.

31. Folger, H. T., J. Morph. 42:359-370 (1926). Mechanical stimulation of amoeba.

32. Fraenkel, G., Ztschr. vergl. Physiol. 2:658-690 (1925). Statocysts in medusae.

33. Fraenkel, G., and Gunn, D. L., The Orientation of Animals (1940). Oxford, Clarendon Press. 352 p. Chs. 15, 16. Gravity reactions, geotaxis; Ch. 17. Mechanical stimulation.

34. Fraenkel, G., and Pringle, J. W. S., Nature 141:919-920 (1938). The halteres of flies as gyroscopic organs of equilibrium.

35. Frankenuaeuser, B., J. Physiol. 107:36P (1948). Slowly-adapting touch receptors in skin of rabbits.

36. Fulton, J. F., in Howell's Textbook of Physiology (John F. Fulton, ed.) (1946). Philadelphia, Saunders. Chap. 9. Labyrinthine reflexes.

37. Gammon, G. D., and Bronk, D. W., Am. J. Physiol. 114:77-84 (1935). Impulses from pacinian corpuscles in mesentery.

38. Gernanit, B., and Zotterman, Y., Acta physiol. Scand. 12:56-72 (1946). Pain impulses in mesenteric nerves.

39. Goltz, F., Pflüg. Arch. ges. Physiol. 3:172-192 (1870). Physiology of semicircular canals. 
40. Griffith, C. R., An Historical Survey of Vestibular Equilibration (1922). Univ. of Illinois Press.

41. Heyer, A. W., Mlaster's thesis, Univ. 1ll. (1947). Effects of vestibular stimulation, man.

42. Hoagland, H., J. Gen. Physiol. 16:685-693 (1933). Impulses from gustatory and tactile receptors, catfish.

43. Hoagland, H., J. Gen. Physiol. 16:695-714 (1933). Responses of lateral line to vibration, catfish.

44. Hoagland, H., J. Gen. Physiol. 16:911-923 (1933). Effect of stimulus/rest ratio on tactile adaptation.

45. Hoagland, H., J. Gen. Plysiol, 17:77-82 (1933). Spontancous activity in lateral line nerve, catfish.

46. Hoagland, H., J. Gen. Physiol. 19:221-228 (1935). Adaptation of cutancous tactile receptors, frog.

47. Hoagland, H., J. Gen. Physiol. 19:943-950 (1936); (with M. A. Rubin) ibid. 19:939-942 (1936). Role of potassium in cutaneous tactile adaptation, frog.

48. Hoagland, H., Cold Spring Harbor Symp. Quant. Biol. 4:347-357 (1936). Mechanism of adaptation of mechanoreceptors.

49. Hogc, B. M., J. Physiol. 84:250-258 (1935). Slow impulses in cutaneous nerves, frog.

49a. Hollick, Q. S. J., Phil. Tr. Roy. Soc. Lond., B, 230:357-390 (1940). Sense organs of flight, flies.

50. Högyes, A., Monatschr. Olirenhk. 46:685-740, 810-840, 1027-1083 (1881). Mechanism of eye movements, vertebrates.

51. Hunter, W. S., J. Gen. Psychol. 5:295-310 (1931). Mechanism of orientation on inclined plane, rats.

52. J̈̈GER, H., Zool. Jahrb. Abt. allg. Zool. u. Physiol. 51:289-320 (1932). Geotactic reactions of some invertebrates.

53. Katz, B., and Young, J. Z., J. Physiol. 107:34P (1948). Regulation of afferent discharge from frog muscle.

54. Koкas, E. v., Zool. Jahrb., Abt. allg. Zool. u. Physiol. 52:179-190 (1932). Function of Weber's apparatus in cyprinoid fish.

55. Koshtojanz, C. S., and Vassilenko, P. Di, J. Exper. Biol. 14:16-19 (1937). Pressure receptors in swim-bladder of fish.

56. Kramer, G., Zool. Jahrb., Abt. allg. Zool. w. Physiol. 52:629-676 (1933). Sensory function of lateral line and skin receptors, Xenopus.

57. Kreidl, A., Sitzungsb. Akad. Wiss. Wien mall-nat. kl. 102:149-174 (1892). Physiology of statocysts, crabs.

58. Kropp, B., and Crozier, W. J., J. Gen. Physiol. 12:111-122 (1928). Geotropic orientation in arthropods, fiddler crab Uca.

59. Langdon, F. E., J. Morph. 11:193-234 (1895). Sense organs of Lumbricus.

60. Ledebur, J. F. v., Biol. Rev. 12:217-244 (1937). Sivim-bladder gases, fish.

61. Löwenstein, O., Biol. Rev. 11:113-145 (1936). Equilibrium function of the vertebrate labyrinth.

62. Löwenstein, O., and Sand, A., Proc. Roy. Soc. Lond., B. 129:256-275 (1940). Single nerve fiber analysis of semicircular canal function.

63. Magnus, R., Körperstellung (1924). Berlin, Springer.

64. Matthews, B. H. C., J. Physiol. 71:64-110 (1931). Responses of single muscle spindle.

65. Matthews, B. H. C., J. Physiol. 72:153-174 (1931). Impulses from muscle spindles, frog.

66. Matthews, B. H. C., J. Physiol. 78:1-53 (1933). Types of sensory endings in mammalian muscle.

67. Maximow, A. A., and Bцоom, W., Textbook of Histology (1942). Philadelphia, Saunders. 695 p. Chap. 9, pp. 197-203. Figures of mechanoreceptors.

68. Maxwell, S. S., Labyrinth and Equilibrium (1923). Philadelphia, Lippincott.

69. MeNally, W. J., Am. Acad. Ophth. \& Otolaryng. St. Louis (1944). Physiology of the vertebrate ear.

70. McNally, W. J., and Stuart, E. A., War Med. 2:683-771 (1942). Physiology of the labyrinth. 
71. McNally, W. J., and Tait, J., Am. J. Physiol. 75:155-179 (1925). Ablation experiments on the labyrinth of the frog.

72. Morson, S., and Pinllips, G., J. Physiol. 88:199-212 (1936). Reflexes elicited by tension receptors in skeletal muscle.

73. Mowrer, O. H., Science 81:180-181 (1935). Electrical responses of vestibular nerve, fish.

74. Pieron, H., Ann. Physiol. Physicochim. Biol. 4:4t-63 (1928). Geotropic reactions in snails.

75. Pfaffalann, J. Physiol. 97:207-219, 220-232 (1939). Afferent impulses from teeth.

76. Pringle, J. W. S., J. Exper. Biol. 15:101-113, 114-131 (1938). Proprioception in insects.

77. Pringle, J. WV. S., Phil. Tr. Roy. Soc. Lond., B. $233: 347-384$ (1948). Gyroscopic mechanism of the halteres of Diptera.

78. Pringle, J. W. S., J. Physiol. 108:226-232 (1949). Excitation and contraction of insect Hight muscles.

79. Prosser, C. L., J. Exper. Biol. 12:95-104 (1935). Sensory impulses in segmental nerves of earthworm.

80. Prosser, C. L., J. Cell. \& Comp. Physiol. 16:25-38 (1940). Sensory responses from single hairs of crayfish.

81. Punphrey, R. J., J. Physiol. 87:6P-7P (1936). Adaptation of tactile receptors in leg of cockroach.

82. Ross, D. A., J. Physiol. 86:117-146 (1936). Electrical studies on the frog's labyrinth.

83. SANd, A., J. Physiol. 89:47-P-49P (1937). Effect of fluid flow on activity of lateral line of Raja.

84. Schafer, E. A., Textbook of Microscopic Anatomy (1912). London, Longmans, Green. 737 p.

85. Scharrer, E., J. Exper. Zool. 61:109-114 (1932). Function of lateral line organs in Amblystoma larvae.

86. Schiriever, H., Pfliig. Arch. ges. Physiol: 235:771-784 (1935). Action potentials in lateral line of various fish.

87. Sherrington, C. S., Integrative Action of the Nervous System (1906). London, Constable.

88. Spiegel, E. A., and Oppenheimer, M. J., Am. J. Physiol. 125:265-275 (1939). Conditioned reactions to position and angular acceleration, mammals.

89. Steinhausen, W., Ztschr. Hals-Nas. Ohrenh. 29:211-216 (1931). Observations on the deflection of the cupula.

90. Steinhausen, W., Pflig. Arch. ges. Physiol. 232:500-512 (1933). Observations on the cupula in living pike.

91. Suckling, E. E., and Suckling, J. A., Personal communication (1949). Action potentials in lateral line nerve, Fundulus.

92. Tower, S. S., J. Neurophysiol. 3:486-500 (1940). Sensory unit for touch in cornea, cat.

93. Vincent, S. B., Behav. Monog. 1:1-81 (1912). Function of vibrissae in behavior of rat.

94. Whener, N., Cybernetics, or Control and Communication in the Animal and the Machine (1948). New York, Wiley. 194 p.

95. Woollard, H. H., Wediell, G., and Harpman, J. A., J. Anat. 74:413-440 (1940). Neurohistological basis for cutaneous pain.

96. Zotterman, Y., J. Physiol. 95:1-28 (1939). Electrophysiological investigation of touch, pain, and tickling. 


\section{Circulation of Body Fluids}

N THE PRECEDING Chapters the physiological and biochemical adapta tions to specific environmental factors have ben. torrithed. We now pass to a series of functions which serve in reactions of the organism to the environment as a wholc. $W_{\mathrm{c}}$ shall consider first the circulation of body fluids, the mechanisms for the transport of food, oxygen, waste products, hormones, phagocytic cells, and other materials from one part of the animal to another.

\section{THE CIRCULATORY SYSTEM AS A WHOLE}

Morphological Types of Transport Mechanisms. A definitive and mutually exclusive morphological classification of transport mechanisms is impossible, because various animals have dissimilar structures serving the same function. A working classification follows, with the recognition that it is incomplete and that many degrees of intergradation exist. ${ }^{39}$

Intracellular Transport. In protozoans there is usually much protoplasmic movement, this streaming supplementing simple diffusion in the exchange of materials between the organism and its environment. Considerable churning and mixing is an accompaniment of amoeboid movement, and in many ciliates food vacuoles follow a definite course through the organism. In metazoans a certain amount of protoplasmic streaming occurs in most if not all cells (see Ch. 17 for discussion of streaming).

Extra-organismic Mechanisms of Transport. In many animals, particularly sponges (Fig. 196, A) and coelenterates (Fig. 196, B), the water in which the animals live provides an effective medium for transport. Exchange between body cells and external medium is as free as it is in the Protozoa. The external medium passes in and through definite channels and may transport food, oxygen, and possible coordinating substances from one region of the animal to another, as in the coelenteron of coelenterates. In the channels the fluid may be propelled by ciliary activity or by muscular movement of the animal. Fluid from outside may enter special cavities and be passed through these cavities in a definite fashion, to serve principally in respiration and digestion.

Transport in Fluid-Filled Body Spaces: Transport in prinary body cavities. A special body fluid is transported, chiefly by muscular movement, through various types of primary body cavities. A pseudocoelom is found in such groups as nematodes (Fig. 196, C), ectoprocts, and rotifers. A hemocoel comprises the principal passages in the "open" circulatory system present in most arthropods and in many molluscs. Here a heart pumps 
blood out through arteries which reach body tissues by way of blood spaces which are derived from the blastocoel or primary body cavity. The blood,

A. SPONGE
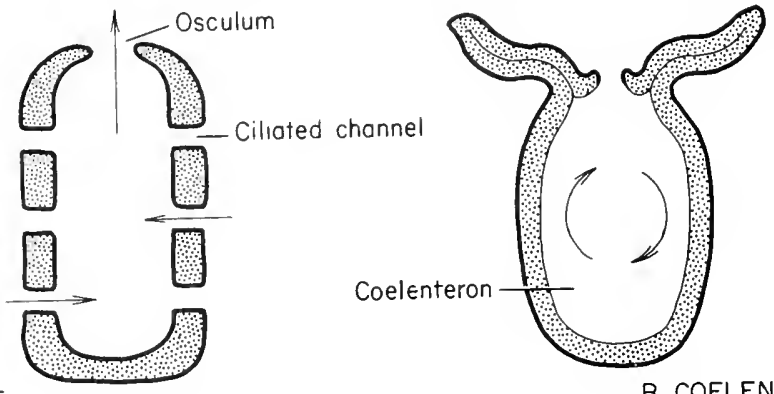

B. COELENTERATE

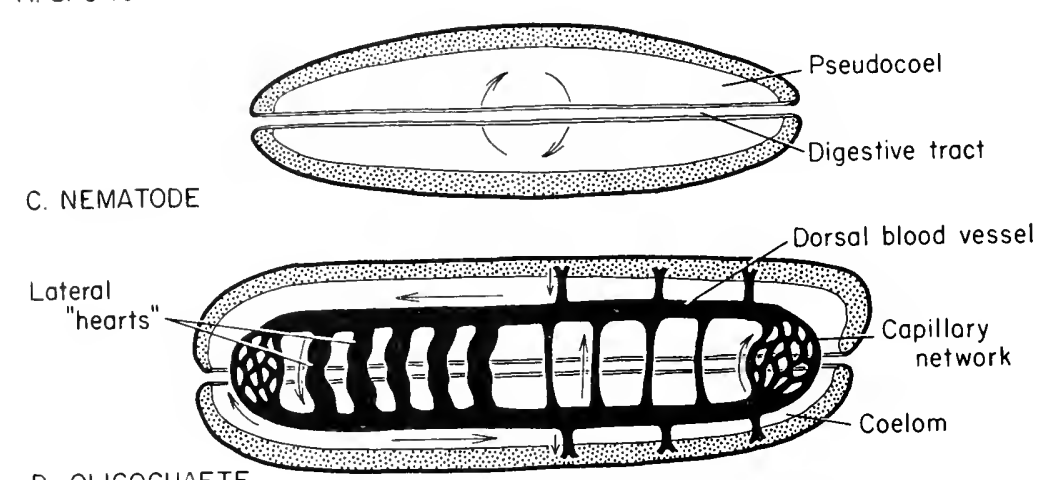

D. OLIGOCHAETE

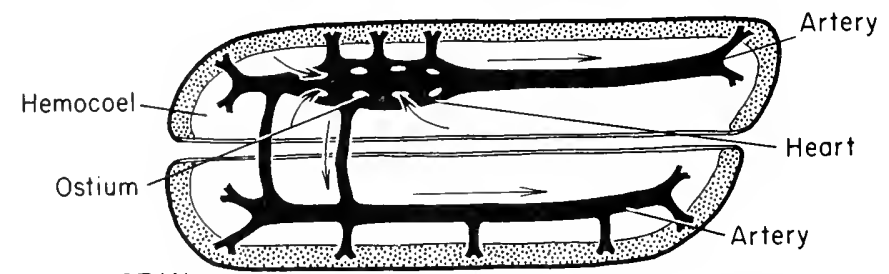

E. CRUSTACEAN

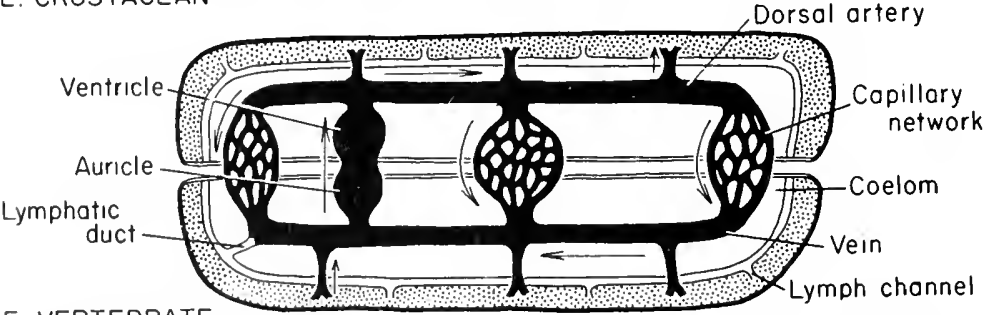

F. VERTEBRATE

Fig. 196. Types of mechanisms for internal transport: A, "Sponge" type with ciliated channels. B, "Coelenterate" type. C, "Nematode" type with pseudocoel primary body cavity. D, "Oligochaete" type with coelom and closed blood system. E, "Crustaceanmollusean" type with blood system opening to hemocoel which is derived from primary body cavity or blastocoel. F, "Vertebrate" type with coelom, closed vascular system, and lymphatic channels.

sometimes called hemolymph, in these animals moves slowly back from the tissue and the intercellular spaces and hemocoelic sinuses to the heart 
(Fig. 196, E). The primary body cavity persists in internal transport in the form of intercellular spaces, in animals with closed circulatory systems.

Coelomic internal transport. The coelom is a special, mesodermally lined organ, more or less extensive in such groups as echinoderms, annelids, sipunculoids, ectoprocts, and chordates. In the blood-sucking (Gnathob. dellid) leeches the coelom is reduced to a system of tubes which contain a pigmented fluid. Also in some polychactes and echinoderms the coelomic fluid contains corpuscles with respiratory pigments. The coelom is reduced, in those molluscs with hemocoelic circulation, to the pericardial cavity and the lumen of gonads and kidneys; the coelom is reduced in arthropods (Crustacea particularly) to cavities of gonads and kidneys. In vertebrates the coelom persists as peritoneal, pericardial, and pleural cavities.

Blood vascular systen. A closed system of tubes, often with one or more pumps, is found in oligochaetes (Fig. 196, D), many polychaetes, rhynchobdellid leeches, phoronids, brachiopods, nemerteans, cephalopod molluscs, holothurians, sipunculoids, and vertebrates. The blood and tissue fluids are separate, although a certain amount of exchange occurs between them. The blood comes into intimate association with the tissues, either by capillaries or by closed sinuses.

Lyaphatic CHANNEls. In the vertebrates (Fig. 196, F) the intercellular space (primary body cavity) is connected with the blood vascular system through lymph channels. These converge on veins and form a lined network which may be as extensive as the capillary bed. In some animals (amphibians and teleosts), there may be lymph hearts.

In the above classification each system builds on the preceding ones. There may be numerous fluid compartments in one animal. For example, in vertebrates the fluid spaces are: cardiovascular system, lymphatics, intercellular spaces, and coelom. The distinction between "open" and "closed" vascular systems is relative rather than absolute. Those animals in which the blood passes from arteries to veins predominantly by way of small lined vessels can be considered to have closed vascular systems. In general, the efficiency of a closed system is greater with respect to velocity of blood flow, economy of blood volume, and maintenance of blood pressure.

Blood Volume. In animals with a closed circulatory system the volume of circulating fluid is relatively fixed. If the fluid volume increases, the pressure in the system rises; if the fluid volume is diminished the pressure falls. Determinations of blood volume are made by measuring the dilution of a known quantity of some material which is added and which remains confined to the blood stream. Early investigators measured the concentration of hemoglobin in a small sample of blood; they then drained out the blood from the animal, extracted the hemoglobin from the viscera, and calculated the blood volume. Another method for calculating blood volume is by using carbon monoxide, which can be given in a small known amount. On the assumptions that circulating cells pick up $\mathrm{CO}$ and that complete mixing occurs rapidly, the total blood volume can be calculated from concentration of carboxyhemoglobin. Dyes are also used: Evans blue (T-1824) combines with plasma albumin in vertebrates and leaves the blood very slowly; a known amount of dye is injected and the dilution of dye in the 
blood is measured. Red blood cells containing radioactive iron or phosphorus, or protein with radio-iodine, have also been utilized in determining blood volume. These various methods consistently give somewhat different values of estimated blood volume, and much has been written explaining these differences. More striking, however, is their general agreement. In Table 66 are given some blood volume measurements made by bleeding and hemoglobin estimation, and published in 1854 by Welcker, and beside them a few values obtained by modern methods.

The blood volume in mammals comprises normally 7 to 10 per cent of the body weight. The lymph volume is not known in any animal, but the total extracellular space as indicated by such substances as thiocyanate and by radioactive sodium, materials which are taken up very little by tissue cells, is 20 to 30 per cent of the body weight of mammals. Birds and amphibians have blood volumes similar to those of mammals. In elasmobranchs the blood volume is less (about 5 per cent of body weight), whereas teleosts have the least blood ( 1.5 to 3 per cent).

In animals with open circulation the blood volume should be essentially the same as the total extracellular volume in animals with a closed circulation. Such data as are available are given in Table 66. In Cambarus both the T-182+ space and the thiocyanate space are 25 per cent of body weight. ${ }^{159 a}$ The blood volume as given by hemoglobin determinations is of the same order ( 30 per cent) in Arenicola and Planorbis; the blood system in Arenicola is closed, and it is difficult to account for this large volume. The blood volume of larval insects is probably much larger than that of adult

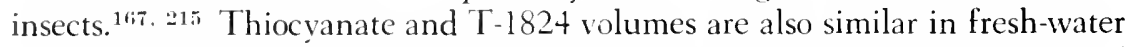
mussels but are low (9-9.5 per cent of body volume), possibly because of by-passing some sinuses. ${ }^{15 \% a}$ In many invertebrate animals the specific gravity of the body differs from that of the blood, hence blood volumes are better expressed as fraction of body volume.

In circulatory systems a small blood volume is more efficient than a large blood volume, in that the same blood is re-used more frequently in transport; on this basis the circulation of bony fish is more efficient than that of crustaceans. Measurements by modern methods of the partition of body fluids in animals with different types of circulatory systems should add materially to our understanding of the maintenance of fluid balance.

Pressure in Closed Circulatory Systems: Measurement and Regulation. In any closed tubular system containing a pump, a head of hydrostatic pressure is developed at the pump and the pressure declines with frictional loss in the tubes, particularly if the tubes are distensible. The principles of blood flow, hemodynamies, have been extensively studied and are discussed in detail in textbooks of mammalian physiology. ${ }^{1: 0}$ In man the pressure in a large artery at the time of heart contraction (systole) is about $120 \mathrm{~mm}$. Hg, and at the time of heart relaxation (diastole) is about $80 \mathrm{~mm}$. $\mathrm{Hg}$, often written 120 80. The difference, or pulse pressure, decreases as the blood proceeds to smaller vessels, and the pressure falls off rapidly in the arterioles. Velocity of flow is slow in the vast capillary bed, then in the veins velocity increases slightly although pressure continues to decrease. Blood pressure is normally determined by the peripheral resistance, the head of pressure 
TABLE 66. BLOOD VOLUME IN MILLILITERS OF BLOOD PER 100 GRAMS OF BODY WEIGHT

Measurements by bleeding and hemoglobin estimation, carbon monoxide, and dye

(T-182t) dilution. Values by Welcker (W) are quoted from Reichert and Brown. ${ }^{192}$ Some experimental data have been averaged.

\begin{tabular}{|c|c|c|c|}
\hline Animal & $\begin{array}{l}\text { Bleeding and } \\
\text { Hb Estimation }\end{array}$ & CO Method & Dye Method \\
\hline $\operatorname{Man}(W)$ & 7.6 & & \\
\hline $\operatorname{Minan}^{171}$ & & 8.21 & 8.4 \\
\hline $\operatorname{Man}^{i+3}$ & & 8.02 & 8.05 \\
\hline $\operatorname{Dog}(W)$ & 7.4 & & \\
\hline $\operatorname{Dog}^{1+3}$ & & 9.54 & 9.52 \\
\hline $\operatorname{Dog}^{162}$ & 7.2 & & \\
\hline $\operatorname{Dog}^{171}$ & & 10.12 & 10.77 \\
\hline $\operatorname{Dog}^{4 b}$ & & & 7.9 \\
\hline $\operatorname{Dog}^{115}$ & & 9.54 & 10.5 (I protein 9.4) \\
\hline Rabbit ${ }^{48}$ & & & 7.0 \\
\hline Rabbit (W) & 5.5 & & \\
\hline Rabbit $^{162}$ & 6.2 & & \\
\hline Bull (W) & 7.7 & & \\
\hline Goat ${ }^{182}$ & 6.2 & & \\
\hline Goat $^{\text {th }}$ & & & 7.0 \\
\hline Horse $^{182}$ & 9.7 & & \\
\hline Horse $^{48}$ & & & 7.2 \\
\hline Mouse $^{162}$ & 8.1 & & \\
\hline Pigeon (W) & 9.2 & & \\
\hline Pigeon $^{162}$ & 7.8 & & \\
\hline Small birds ${ }^{162}$ & 8.0 & & \\
\hline Lacerta agilis $^{\pi}$ & 3.02 & & \\
\hline Chrysemys picta ${ }^{57}$ & 7.56 & & \\
\hline Rana temp. ${ }^{7 \pi}$ & 4.61 & & \\
\hline R. catesb. ${ }^{.57}$ & 6.42 & & \\
\hline Rana pipiens ${ }^{1 \hat{n} ! a}$ & & & $\begin{array}{l}27.3(\mathrm{SCN}) \\
8.0(\mathrm{~T}-1824)\end{array}$ \\
\hline Frog 17 & & & 8.74 \\
\hline Frog $(W)$ & 5.7 & & \\
\hline Salamander (W) & 6.3 & & \\
\hline Bony fish (W) & $1.5-2.0$ & & \\
\hline
\end{tabular}


TABLE 66 (continued)

BLOOD VOLUME IN MILLILITERS OF BLOOD PER 100 GRAMS OF BODY WEIGHT

\begin{tabular}{|c|c|c|c|}
\hline Animal & $\begin{array}{l}\text { Bleeding and } \\
\text { Hb Estimation }\end{array}$ & CO Method & Dye Method \\
\hline Tautoga $a^{57}$ & 1.5 & & \\
\hline Ameiurus ${ }^{15 / a}$ & & & $\begin{array}{l}4.0(\mathrm{SCN}) \\
1.8(\mathrm{~T}-1824)\end{array}$ \\
\hline Ophiodon (ling cod $)^{138}$ & & & $3.1(\mathrm{~T}-1824)$ \\
\hline Sebastodes ${ }^{1335}$ & & & 2.8 (vital red) \\
\hline Cottus $^{130}$ & & & 2.7 (vital red) \\
\hline Raja rhina ${ }^{138}$ & & & $5.3(\mathrm{~T}-1824)$ \\
\hline Raja binoculata ${ }^{138}$ & & & $4.4(\mathrm{~T}-1824)$ \\
\hline Squalus acanth. ${ }^{57}$ & 3.7 & & \\
\hline Squalus sucklii & & & \\
\hline$\delta^{138}$ & & & 5.2 \\
\hline$q$ no young & & & 4.4 \\
\hline q with young & & & 11.2 \\
\hline Lamprey (W) & 5.7 & & \\
\hline Planorbis ${ }^{123}$ & 30.0 & & \\
\hline Planorbis $^{22}$ & 58.1 & & \\
\hline Fresh-water musse $\mathrm{l}^{15 \mathrm{a} / \mathrm{a}}$ & & & $\begin{array}{l}9.5(\mathrm{~T}-1824) \\
9.0(\mathrm{SCN})\end{array}$ \\
\hline Arenicola ${ }^{15}$ & 29.0 & & \\
\hline Arenicola ${ }^{22}$ & 38.2 & & \\
\hline Carcinus $^{145}$ & & & $37(\mathrm{SCN})$ \\
\hline Eriocheir $^{117}$ & & & $33(\mathrm{SCN})$ \\
\hline Cambarus virilis ${ }^{15 \% a}$ & & & $\begin{array}{l}25.6(\mathrm{SCN}) \\
25.1(\mathrm{~T}-1824)\end{array}$ \\
\hline Bombyx larva ${ }^{107}$ & $28.6-31.2$ & & \\
\hline Galleria larva ${ }^{187}$ & 41.0 & & \\
\hline Periplaneta adult ${ }^{215}$ & $4.7-6.9$ & & \\
\hline
\end{tabular}

built up by the heart, and the volume of blood. Peripheral resistance is varied by constriction and dilatation of arterioles and capillaries, constriction of arterioles being by action of smooth muscle and constriction of capillaries largely by endothelial contraction. ${ }^{124,116,52}$ The peripheral responses may be local, as by direct irritation of capillaries or by axon reflexes (i.e., reflexes involving efferent branches of sensory neurones), or the peripheral responses may be truly reflex, or hormonal. Receptors are located in the carotid sinus, 
the aortic bodies of the aortic arch, and in pain endings. Some of the sensory endings of the carotid sinus are stimulated by pressure in the carotid artery. lmpulses go to the vasomotor center in the medulla of the brain, from which vasoconstrictor messages leave over the sympathetics, and vasodilator messages by several pathway's, including certain parasympathetics. A delicate balance exists between vasoconstrictors and vasodilators. Parasympathetic discharge causes vasodilatation and a fall in blood pressure, probably associated with liberation of acetylcholine, whereas sympathetic constriction is associated with liberation of adrenin (sympathin) somewhere in the chain of innervation of vessel smooth muscles. Adrenalin* causes a rise in blood pressure by its vasoconstrictor action. Numerous long-term factors, such as variations in arterial elasticity, presence of the vasoconstrictor, hypertensin, from the kidneys, and the general state of health, contribute to basal blood pressure levels.

In general, the resting blood pressure is higher in large animals than in small ones (Table 67); carotid pressure in the horse ranges up to 190 $\mathrm{mm}$. Hg. Blood pressure at birth depends on the state of development of a particular species; arterial pressure at birth in the rabbit is $21 \mathrm{~mm}$. $\mathrm{Hg}$, in the cat $25-30 \mathrm{~mm}$. $\mathrm{Hg}$, and in the sheep, an animal more mature at birth, $73 \mathrm{~mm} . \mathrm{Hg}^{16}$

The circulation is somewhat more sluggish and pressures are lower in cold-blooded vertebrates than in warm-blooded animals. The systolic arterial pressure in the frog is $30 \mathrm{~mm}$. $\mathrm{Hg}$, in gill arteries of the elasmobranch Scyllium $30 \mathrm{~mm}$. Hg, and in gill arteries of the skate Raja $20 \mathrm{~mm}$. Hg.

The blood pressure is higher in bony fishes than in elasmobranchs. In fishes the blood leaves the heart via the ventral artery, passes through gill capillaries, and then enters the systemic circulation by the dorsal artery. The ratio of pressure in the branchial (rentral) arteries to pressure in the dorsal artery is about 3 to 2 (Table 67). Pressure in the pericardial cavity of fishes is negative. 179

Regulation of blood pressure in fishes contrasts sharply with regulation in tetrapods. The autonomic sustem of fishes, particularly of elasmobranchs, ${ }^{216}$ is not sharply divided into sympathetic and parasympathetic divisions. The heart of fishes receives only what may be called parasympathetic (vagus), ${ }^{25}$ and the peripheral vessels may receive only sympathetic innervation. ${ }^{9}$ The blood pressure in the branchial arteries is closely correlated with heart activity; if water flow over the gills of a skate or shark is stopped, the heart stops and blood pressure falls. If the fish is injected with atropine, which blocks cardiac inhibition due to the vagus nerve, cessation of water flow does not affect the heart and blood pressure. ${ }^{17 t}$ With each expiration the blood pressure rises momentarily. ${ }^{1: 31}$ Afferent impulses in the vagus, hypobranchial, and lateral line nerves result in cardiac and respiratory inhibition. ${ }^{129}$ Increase in pressure in perfused branchial arteries sets up sensory impulses in the branchial nerves, and the sensory discharge occurs when the arteries fill at each heart beat. ${ }^{103}$ The junction of the third branchial vessel and the ventral aorta is the homologue of the carotid sinus in mammals. Injection

*Adrenin (equivalent to epinephrine) is the natural compound produced in the body. Adrenalin is a commercial product. Sympathin is the adrenin-like substance apparently liberated at adrenergic nerve endings. 


\section{TABLE 67. BLOOD PRESSURE IN VARIOUS ANIMALS}

Expressed in $\mathrm{mm}$. Hg; most measurements on cold-blooded animals were made in $\mathrm{cm}$. $\mathrm{H}_{2} \mathrm{O}$ and have been recalculated to $\mathrm{mm}$. Hg. Pressures given are systolic/diastolic (as 120/80), limits of systolic (as 90-100), or mean pressures.

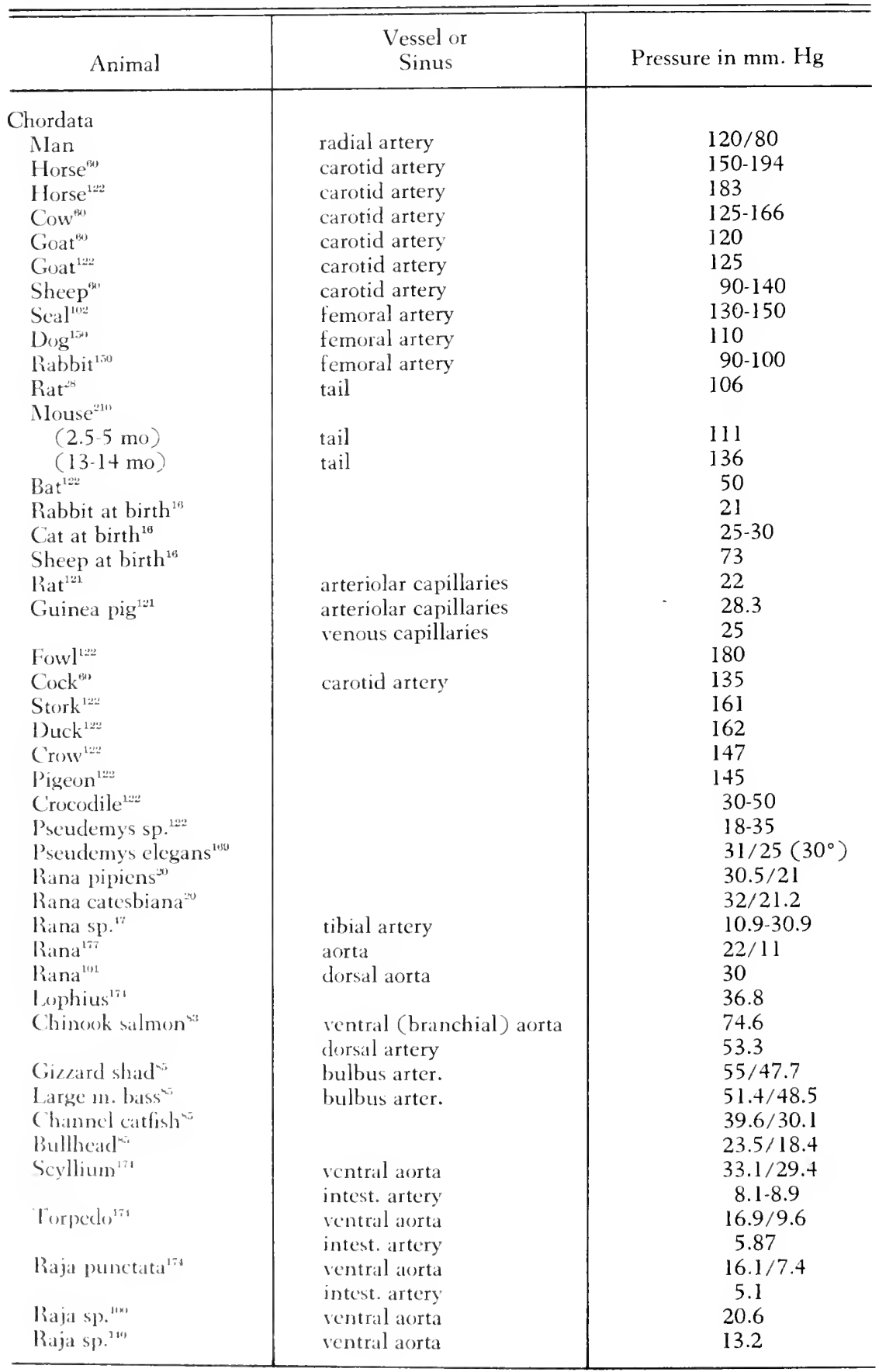


TABLE 67 (continued)

BLOOD PRESSURE IN TARIOUS ANHMAIS

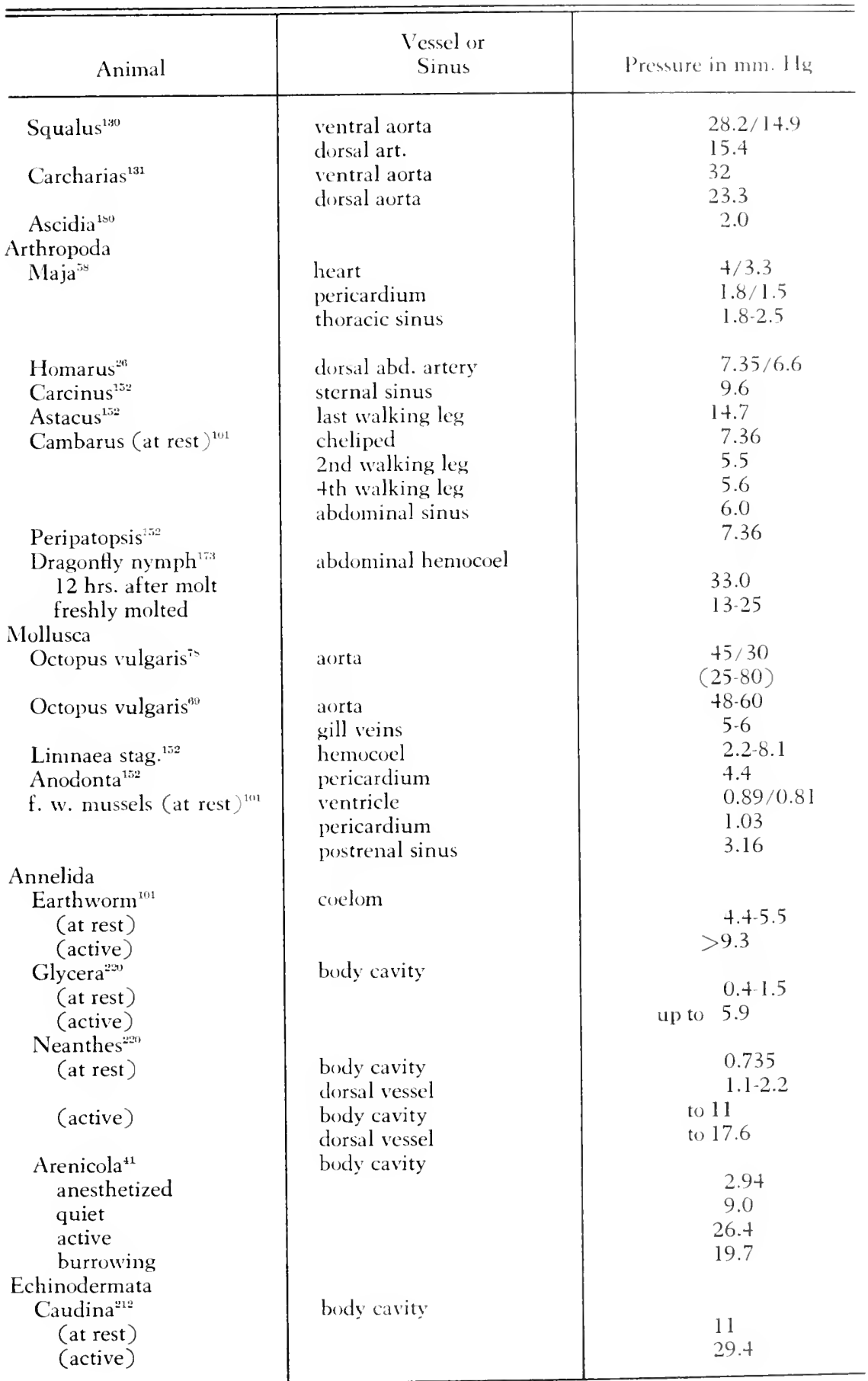


TABLE 67 (continued)

BLOOD PRESSURE IN VARIOUS ANIMALS

\begin{tabular}{c|c|c}
\hline \multicolumn{1}{c|}{ Animal } & $\begin{array}{c}\text { Vessel or } \\
\text { Sinus }\end{array}$ & Pressure in mm. Hg \\
\hline $\begin{array}{c}\text { Thyone } \\
\text { (lightly anesthetized) }\end{array}$ & $\begin{array}{l}\text { radial duct } \\
\text { tentacular duct }\end{array}$ & $2.2-4.0$ \\
(at rest) & body cavity & $7.3-18.3$ \\
(active) & body cavity & $0-1.8$ \\
Sipunculoidea & body cavity & up to 27.1 \\
Pliascolosoma & & $2.2-2.9$ \\
(at rest) & & 14.7 \\
(active) & max. & 79.4 \\
Sipunculus & & 1.5 \\
(quiet in water) & body cavity & 15.8 \\
(active in water) & & 70.5 \\
\hline
\end{tabular}

of adrenalin causes a prolonged pressor effect (rise in blood pressure), which may last as long as 1 to $2 \frac{1}{2}$ hours. ${ }^{104,140}$ However, the branchial vessels dilate to adrenalin, as do mammalian coronaries, in contrast to visceral vessels which constrict in response to adrenalin. ${ }^{113}$ Embryologically the coronaries are derived from the gill arch vessels. Injection of acetylcholine inhibits the heart of skates but causes a rise in blood pressure; atropine antagonizes the cardiac effect but only slightly reduces the pressor action. ${ }^{\mathbf{1 4 0}}$ The pressor action of acetylcholine is not the result of stimulation of sympathetics and adrenals, since direct observations of skate arteries showed that acetylcholine caused them to constrict. ${ }^{9}$ In tetrapods acetylcholine constricts some vessels (e.g., the pulmonaries), but causes dilatation of most vessels; the dilating effect is antagonized by atropine. After atropinization, acetylcholine may raise the blood pressure of mammals by stimulation of the adrenals and of sympathetic ganglia. In fishes, then, the blood pressure falls when the heart is stopped by the vagus; acetylcholine constricts most arteries and raises blood pressure in fish, whereas in mammals it dilates most vessels. Adrenalin constricts somatic vessels in both mammals and fish and dilates coronaries in mammals and branchials in fish. Elucidation of the details of the striking differences in innervation and drug effects in fish and mammals should aid in understanding the site of action of acetylcholine and adrenalin.

In cyclostomes no innervation to the heart has been found, and destruction of the spinal cord failed to alter heart beat in Petromyzon. ${ }^{17:}$ There is probably no distinct autonomic system in cyclostomes.

Invertebrates of large size and high activity, and possessing a closed circulatory system, show blood pressures comparable to pressures in vertebrates. In large cephalopod molluses pressures in the cephalic artery of 47.6-60 $\mathrm{mm}$. I $\mathrm{gg}$ (maximum $80 \mathrm{~mm}$. Hg) have been recorded, and in gill vessels 5.4-6.1 mm. Hg ${ }^{\text {is* }}$ (Table 67). Mechanisms of regulation are unknown.

Pressure in Open Circulatory Systems. One of the most striking differences 
between animals with open and animals with closed circulations is the low and variable blood pressure in the animals with open systems. One of the most active of the large animals with an open circulatory system is the lobster; pressure in its dorsal artery is $5-17$ (average 11.5 ) $\mathrm{cm}$. $\mathrm{H}_{2} \mathrm{O}$, with a pulse pressure of $10 \mathrm{~mm}$. H.O. Pressure in the sternal sinus of Carcinus is $13 \mathrm{~cm}$. $\mathrm{H}_{2} \mathrm{O}{ }^{152}$ In large specimens of Maja the arterial pressure is $45-52 \mathrm{~mm}$. I I.2 $\mathrm{O}$, whereas pressure in thoracic sinuses is $25-34 \mathrm{~mm}$. $\mathrm{I} \mathrm{H}_{2} \mathrm{O} .5 \mathrm{~m}$

Pressure regulation and directions of blood flow in open vascular systems are poorly understond. In all animals in which the blood circulates in sinuses the flow is controlled much more by the activity of somatic musculature than by heart activity and ressel size. Some small crustaceans and insects lack a pulsating heart. Septa separate afferent and efferent channels in gills (crayfish and lobster ${ }^{* 11}$ ) and in legs (dragonfly nymphs ${ }^{1: i}$ ).

In some insects the heart is connected to the dorsal wall by alary muscles, which stretch the heart muscle. It has been suggested that the heart has an aspirator action, and that the pressure in the hemocoel is negative. ${ }^{75,134} \mathrm{~A}$ drop of fluid on a cut appendage is sucked inward in many insects. ${ }^{23,}{ }^{24}$ However, in dragonfly nymphs the pressure in the body cavity is ncgative when the animals are anesthetized but not when they are active. ${ }^{137}$ In a series of measurements on crayfish about 10 per cent of the animals were very lethargic, and, in these, negative pressures were recorded at the heart as well as in leg sinuses. ${ }^{159}$ Pressure may be negative in extreme relaxation but may increase by many times on motor activity. In dragonfly nymphs the pressure in the abdomen rises and falls with movements as in breathing. ${ }^{173,137}$ In the crayfish the pressure in the hemocoel at the distal end of a leg is often higher than at the proximal end, and the pressure in the cheliped (average proximal, 8-6 cm. $\mathrm{H}_{2} \mathrm{O}$; distal, 10-8 cm. $\mathrm{H}_{2} \mathrm{O}$ ) may be higher than in walking legs. ${ }^{101}$ Simultaneous measurements in several regions showed that the pressure increases with activity are not the same throughout the hemocoel. Pressure in the heart evceeds pressure in the legs except in activity. When pressure in an appendage rises because of muscular activity there may be backflow of blood or blood may be confined by valves at the joints of appendages. In Maja thoracic sinus pressure increases with leg activity. ${ }^{5-}$ In fresh-water mussels the pressure in the heart and pericardial sinus is low (Table 67), but when the body contracts during ejection of water through the excurrent siphon, the pressure in the sinus and heart doubles. ${ }^{101}$

In animals with an open circulatory system the blood flow in an organ is more directly a part of "voluntary" activity and pressure changes are many times greater than in animals with closed vessels. In molluses and crustaceans the blood volume is probably comparable to the volume of total extracellular fluid of a vertebrate, yet the blood pressure of a 20 gram crayfish is only about 30 per cent of that of a 20 gram mouse. The velocity of circulatory flow in the crayfish is, therefore, very low; the sluggishness of blood flow may be a limiting factor in the size of animals with an open circulation.

In many tubular animals the pressure in fluids of the body cavities is dependent on tone of the body wall (holothurians, annelids-Table 67). In such animals hydraulic mechanisms are important in other respects than the 
circulation of essential fluids. Burrowing worms, particularly sipunculoids and some annelids, depend on proper changes in the coelomic fluid pressure and turgidity of the body. In Arenicola, for example, ${ }^{41}$ pressure in the anterior body cavity when the animal was at rest averaged $12.2 \mathrm{~cm} . \mathrm{H}_{2} \mathrm{O}$, when active, $36 \mathrm{~cm}$. $\mathrm{H}_{2} \mathrm{O}$, and when burrowing, $27 \mathrm{~cm}$. $\mathrm{H}_{2} \mathrm{O}$; removal of some coelomic fluid delayed burrowing. In Glycera ${ }^{220}$ pressure in the coelomic cavity of the animal at rest was $0.5-2 \mathrm{~cm}$. $\mathrm{H}_{2} \mathrm{O}$ and on activity it was $8 \mathrm{~cm}$. $\mathrm{H}_{2} \mathrm{O}$, whereas in Neanthes the pressure rose from $1-2 \cdot \mathrm{cm}$., resting, to $15 \mathrm{~cm} . \mathrm{H}_{2} \mathrm{O}$ on activity. In Neanthes, the pressure in the dorsal vessel tends to be higher than it is in the coelom, and it tends to increase more with activity.

In sipunculoids high turgidity is essential for burrowing; eversion of the proboscis is accompanied by elevated internal pressure, but the retractor muscles can withdraw the proboscis against a high pressure. In the body cavity of large specimens of Sipunculus, pressures as high as $600 \mathrm{~cm} . \mathrm{H}_{2} \mathrm{O}$ have been recorded, and in small Phascolosoma pressures ranged from 2.8 $\mathrm{cm}$. of body fluid in the relaxed state to $108 \mathrm{~cm}$. when contracted. ${ }^{220}$ Sipunculus is unable to burrow if the posterior part of the body wall is paralyzed by deganglionation, but if a ligature is then placed ahead of the paralyzed posterior half, the anterior portion can disappear rapidly into the sand. ${ }^{192}$ The magnitude and speed of change of pressure are probably greater in sipunculoids than in any other animal group.

Hydraulic mechanisms are also important among echinoderms. In holothurians, for example, there are several fluid compartments, the water-vascular or ambulacral system, the sinus or pseudo-hemal system, the lacunar or blood system, the coelomic cavity, and the respiratory trees. Cilia maintain some fluid flow in the smaller passages; the filling and emptying of the respiratory trees has little effect on pressure in the coelomic fluid. The anterior ambulacral system consists of two portions separated by a valve: the tentacular ducts, which feed fluid into the tentacles; and the Polian complex of radial ducts, ambulacral ring, and Polian vesicles, which is a fluid reservoir for the tentacular system. Pressure can vary independently in these two portions of the system: in Thyone pressure is normally higher in the tentacular duct $\left(10-25 \mathrm{~cm}\right.$. $\left.\mathrm{H}_{2} \mathrm{O}\right)$ than in the Polian complex $\left(3-5.5 \mathrm{~cm} . \mathrm{H}_{2} \mathrm{O}\right.$ in radial duct).20 In the body cavity of a relaxed Thyone pressure is low (0-2.5 $\mathrm{cm}$. $\mathrm{H}_{2} \mathrm{O}$ ), but on muscular activity pressure may rise to as much as $37 \mathrm{~cm}$. $\mathrm{H}_{2} \mathrm{O}$, and chthges occur independently in the Polian vesicle and body cavity. 220

\section{PHYŚIOLOGY OF HEARTS}

Types of Hearts. Any system for circulating a mass of fluid requires a repeating pump. To assure that fluid goes in a constant direction the pump either must be equipped with suitable valves to prevent backward flow or must compress its contained fluid in a continuous progressing wave. Some hearts employ one of these propulsion mechanisms, some use both. Morphologically hearts may be classified as (1) chambered heart, (2) tubular heart, (3) pulsating vesscls, or (4) ampullar accessory heart. ${ }^{43}$

Chambered Hearts. Chambered hearts occur mostly among vertebrates and in some molluscs. Vertebrate hearts are equipped with valves and suc- 
cessive chambers. The sequence of contraction is essentially similar in both the two-sided (4- or 3-chambered) and one-sided (2-chambered) hearts. During ventricular contraction, while the aortal (semilunar) valves are open, the aorta fills and the heart empties; ventricular systole (contraction) stops

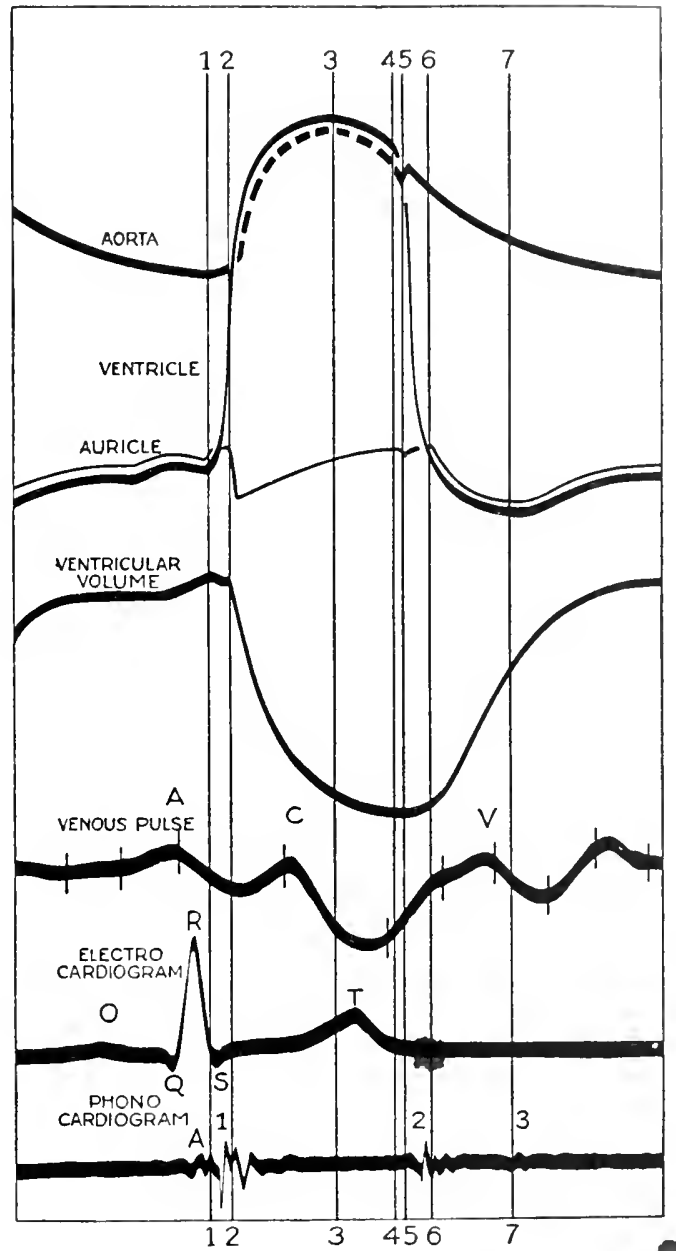

Fig. 197. Correlated events of the cardiac cycle. Venous pulse, elechocardiogram, and heart sounds in man; aortic pressure, auricular pressure, ventricular pressure, and ventricular volume adapted from the dog. 1. Closure of the AV valves and beginning of ventricular contraction; 2 , opening of aortic valves; $2-3$, maximal ejection phase; $3-4$, reduced ejection phase; $4-5$, protodiastolic phase with closure of aortic valves at $5 ; 5-6$, isometric relaxation, opening of $A-V$ valves at $6 ; 6-7$, rapid filling; 7 to auricular systole is phase of diastasis. Time marks at $0.1 \mathrm{sec}$. on venous pulse curve apply to lower three curves. From Fulton. ${ }^{91}$

when ventricular pressure falls below that of the aorta, then the aortal valves close (Fig. 197). During ventricular contraction the auricular pressure is below that in the ventricle and the auriculoventricular valves remain closed; as the auricles fill their pressure gradually rises while the ventricular pressure is falling, the auriculoventricular valves then open, blood enters the 
ventricle, and the auricle contracts until the high pressure in the ventricle closes the auriculoventricular (AV) valves (Fig. 197). In man the ventricles first contract with the AV valves closed (isometric contraction) for 0.05 sec., then during ejection of blood for $0.22 \mathrm{sec}$; ventricular relaxation lasts 0.53 sec., the auricles contract for $0.11 \mathrm{sec}$. and are in diastole $0.69 \mathrm{sec} .11$ Thus the entire sequence in the vertebrate heart consists of successive contractions of auricles and ventricles and the closure of a valve whenever the pressure on the outgoing side exceeds the pressure on the incoming side. In the frog isometric systole lasts 0.06-0.13 sec., and apparently some blowd remains in the ventricle after each ejection. ${ }^{177}$

In the amphibian three-chambered heart oxygenated and non-oxygenated blood are not separated in the ventricle but the blood returns twice to the heart for each cycle. In fishes with a single auricle and ventricle blood passes through the gill vessels before entering the aorta. A two-channel heart not only separates oxygenated from oxygen-depleted blood but also permits higher pressures in systemic arteries than are possible after the blood has passed through gill capillaries. The establishment of a low pressure respiratory blood shunt and consequent high systemic pressure aided reptiles and homoiotherms to withstand the rigors of terrestrial life.

The chambered hearts of the molluses consist of either one or two auricles and one ventricle. The auricles are usually receiving chambers with little musculature, whereas the ventricle is a strong contractile organ. The systemic

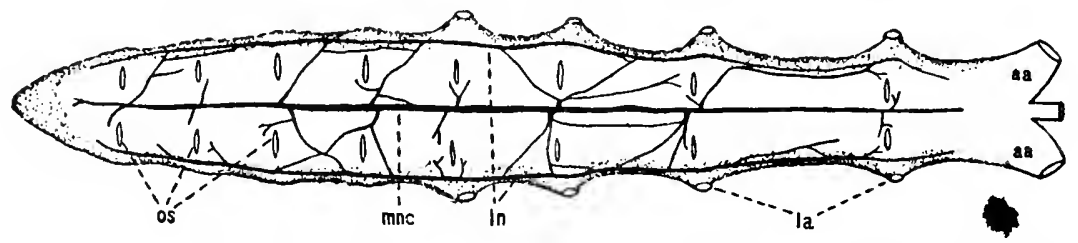

Fig. 198. Diagram of dorsal view of ventricle of the heart of Limulus polyphemus, $a a$, anterior aortae; la, lateral aortae; os, ostia; $m n c$, median nerve cord (pacemaker ganglion ; ln, lateral nerves. From flson. ${ }^{30}$

heart of the cephalopod molluses is particularly well developed; blood returns from the body by veins to the branchial hearts at the base of the gills and passes from the gills in veins to the auricles of the systemic heart. This arrangement gf "booster" hearts proximal to the gills is more efficient than is the plan in rishes, in which the heart has to overcome the resistance of vessels in both gills and body tissues. The muscle of molluscan hearts, particularly of cephalopods and gastropods, is striated. ${ }^{180}$

Tubular Hearts. The systemic hearts of most arthropods consist of contractile tubes. There may be a thin receiving chamber (atrium) surrounding part of the heart, as in Limulus, or the heart may be free in a large sinus. lt is usually anchored at several corners and receives blood through several paired ostia which are valved (Fig. 198). In many insects the heart is suspended by variously arranged alary muscles which maintain tension on the heart (sce Wigglesworth, ${ }^{206}$ for series of types). Blood leaves the heart by one or several arteries, always at the anterior end, sometimes also laterally and posteriorly. Myographic records indicate that the entire tubular heart 
of Limulus contracts nearly simultaneously." A wave of contraction occurs in some insect tubular hearts.

Pulsating ressels. Blood vessels which contract with peristaltic waves are widely distributed. In annelids many blood vessels show rhythmic peristaltic waves. In the earthworm the contraction waves in the dorsal ressel pass from the posterior to the anterior end at a speed of about 20 millimeters per second and waves are repeated at the rate of about $15-20$ per minute. ${ }^{4: 3}$ The lateral vessels of the earthworm, usually referred to as the hearts, contract at rates differing from that of the dorsal vessel and each heart beats at its own rate, although the two hearts of a segment tend to contract together. In the leech, instead of a dorsal vessel, there are two lateral vessels which contract alternately. In Nereis the dorsal vessel contracts rhythmically at a somewhat slower rate than that in the earthworm. The circulation in Arenicola has been extensively studied. ${ }^{79,}, 8,160$ The blood flows forward in the dorsal vessel, passing on each side by the gastric plexus to the lateral gastric vessel, and thence to the lateral heart, which pumps to the ventral vessel. The area connecting the lateral gastric vessel to the heart beats before the heart, and sometimes beats several times during a single contraction of the heart. The dorsal vessel, and the lateral, esophageal, and some nephridial vessels are contractile; the ventral vessel and posterior gastric vessels are not. In general, the contractions of the annelid pulsating vessels are not as regular as the beats of chambered hearts.

The distinction between tubular hearts and contractile vessels breaks down for some insects, particularly dipteran larvae. In Amphioxus many vessels are contractile and the wave of contraction spreads slowly; the heart is little more than "sinus venosus" and "conus arteriosus."1so

Ampullar Hearts. In several animal groups there are accessory organs for propelling blood through peripheral channels. The branchial hearts of the cephalopods consist of a spongy tissue of faintly striated endothelial cells surrounding many small vessels. ${ }^{135}$ These hearts are interposed between the systemic veins and the gill arteries.

Among insects accessory hearts are very common, particularly at the base of antennae, at the attachment of wings, and in legs. ${ }^{134}$ Insect accessory hearts consist of muscle fibers arranged in various patterns and serve to keep the hemolymph moving in the open circulation.

Fishes, amphibians, and reptiles have lymph hearts which are contractile enlargements of lymph vessels and which tend to force lymph into the vcins. Lymph enters veins at many points and not through thoracic ducts as in mammals and birds. The lymph hearts are composed of striated, non-anastomosing fibers and may have valves which prevent a backflow of lymph. In some amphibians destruction of all the lymph hearts is fatal, probably because of accumulation of lymph in tissue spaces. ${ }^{\text {is }}$

The myxinoid cyclostomes have several vein hearts which are ralved but not contractile; a lateral cartilage can be pushed against the vein by muscles controlled by the spinal cord. ${ }^{179}$

Pacemakers. Cardiac rhythms, unlike respiratory rhythms, usually originate within the active organ itself rather than in extrinsic nerve centers. The nature and location of the pacemaker tissues differ in various groups 
of animals. The cellular basis for rhythmic activity may be essentially similar in all those tissues where a continuous energy source (e.g., oxidation) results in repetitive activity - a relaxation oscillator.

Myogenic Hearts. In the vertebrate heart the beat is myogenic; it originates in muscular tissue. In an adult frog or fish the beat can be seen to start in the sinus venosus, and in an adult bird or mammal it can be seen to start in the sino-auricular node and spread to auricle and ventricle. In tissue cultures of embryonic heart muscles the fibers may develop rhythmic contractions, and in a developing chick embryo contractions occur in the myocardium of the bulboventricle after only 29 hours of incubation. ${ }^{1 s}$ With development, the anterior regions of the heart gradually take over control of the rhythm. If the sinus venosus is removed from an adult amphibian heart the normal rhythm stops, but other regions may take over initiation of regular but slower contractions. Similarly in mammals destruction of the sino-auricular (S-A) node causes the heart to stop, at least until some other region initiates slow contractions. Local cooling or warming is most effective in altering rate when applied in the sino-auricular region; electrical stimulation elicits normal electrocardiograms only when applied at the S-A node, and the first trace of the action potential in the heart appears in this region. By warming regions of fish hearts, Skramlik ${ }^{17 s}$ found three pacemakers-the sinus, the auricular floor (canal between sinus and ventricle), and the auriculoventricular junction, and three groups of fishes were described according to the distribution of these centers. Vertebrate heart muscle, then, is apparently capable of spontaneous rhythmicity, but the muscle of the sinus region differs from other regions in rapid recovery of excitability, and this becomes the pacemaker.

The wave of excitation which originates in the sinus is conducted over the atrial myocardium and then spreads in the specialized Purkinje tisssue through the ventricle. The rapidly conducting Purkinje tissue appears to be an adaptation to high body temperature, since it is present only in birds and mammals; in other vertebrates ventricular conduction is in the myocardium. The vertebrate heart contains nerve cells, particularly in the region of the pacemaker, but these are secondary neurones of the vagus system.

Certain drugs act upon pacemaker mechanisms and are useful in distinguishing between neurogenic and myogenic hearts (Table 68). The drug acetylcholine inhibits the adult vertebrate heart with respect to both amplitude and rate. Embryonic vertebrate hearts prior to vagal innervation are either insensitive to ACh, as in Fundulus," or inhibited only by very high concentrations, as in the chick. . $^{\text {sh }}$ Apparently innervation sensitizes the heart to the drug.

Molluscan hearts also are myogenic. Nerve cells occur on or near the beart in cephalopods, but these are secondary neurones of regulating nerves. ${ }^{14 i}$ In certain sastropods nerve celts have been described by some observers and denied by others. ${ }^{15 i}$ Several histologists failed to find any nerve cells in the hearts of clams and mussels. The beat can originate at any point over the heart." A contraction may be local or may be complete.

The action of drugs also supports the hypothesis that the origin of the beat in molluscan hearts is myogenic. Acetylcholine inhibits the hearts of 
molluscs (Gastropdoa, Pelecypoda, Cephalopoda) (Table 68). Molluscan hearts are very sensitive to acetylcholine, and inhibition of the heart of Venus has been obtained with concentrations as low as $10^{-12} 156$. 1:46

In general, the hearts of molluscs are very sensitive to pressure; it has been claimed that some fail to beat unless they are distended. ${ }^{6 \times}, 1 \times 0$ th has also been stated that small beats may occur in a collapsed heart. Certainly, when the internal pressure increases, the beats are stronger and more frequent. In Octopus vulgaris Frédéricq ${ }^{\text {is: }}$ recorded no beats at pressures below 2 $\mathrm{cm}$. $\mathrm{H}_{2} \mathrm{O}$, and the frequency was higher at an internal pressure of $85 \mathrm{~mm}$. $\mathrm{H}_{2} \mathrm{O}(59 / \mathrm{min}$. $)$ than at a pressure of $45 \mathrm{~mm}$. $\mathrm{H}_{2} \mathrm{O}(52 / \mathrm{min}$. $)$. Also in some clams the heart rate is accelerated while water is being pumped through the mantle cavities. ${ }^{11 t}$ The vertebrate heart also adapts to a given load so that as the blood volume in the heart is increased the strength of the beat increases (Starling's law).

Neurogenic Hearts. The hearts of most crustaceans and of Limulus have nerve ganglion cells on the dorsal surface which originate the excitation wave for the heart beat. In crabs and crayfish the number of these large ganglion cells is fixed. ${ }^{3.204}$ The hearts of higher crustaceans are accelerated by acetylcholine, an effect opposite to that in vertebrates and molluscs (Table 68; Fig. 198). Stimulation of the pacemaker ganglion of the crustacean heart by acetylcholine is analogous to stimulation of sympathetic ganglion cells by acetylcholine in vertebrates. In Artemia and Eubranchipus acetylcholine is without any effect, even in high concentrations; these hearts appear to be myogenic and non-innervated. ${ }^{1.57}$

The role of the dorsal ganglion is best shown in Limulus. The Limulus heart has eight pairs of ostia which serve to divide it into eight segments (Fig. 198); in the anterior half are five pairs of arteries plus one anteromedian artery. ${ }^{30}$ Lateral trunks of nerve fibers run along each side and are connected by thin nerve bundles to the median ganglionic trunk. This ganglion contains scattered small multipolar nerve calls and large unipolar nerve cells, the latter located mostly in segments 4 and 5. Local elevation of temperature, as by applying a warm test tube or local electrical stimulation, alters the heart rate most when applied to the fourth and fifth segments of the ganglion. Normal heart activity begins first in the muscle adjacent to the ganglion in the fourth and fifth segments. If the dorsal ganglion is removed. the heart stops beating. If the heart is then placed in $\mathrm{NaCl}$ solution, 31.35 or if tension is applied as by inflating the heart, there may be some contractions; these contractions of the deganglionated heart are slow, and may be local or peristaltic, hence they are not normal beats. In Limulus embryos a heart beat (peristaltic) begins at 22 days, but no nerves are present until the 28th day. ${ }^{3 *}$ Drugs affect the heart differently before and after innervation; ${ }^{157}$ although the adult heart is accelerated by acetylcholine, the beat of the embryonic heart prior to innervation is unaffected. Evidence from action potentials, to be presented below, agrees with the above data, that the large ganglion cells of the mid-segments originate the excitation wave and relay it by way of the smaller neurones out to the muscle. Con duction of excitation is by the median and lateral nerves. When the median nerve cord is cut away, bit by bit, the amplitude of the contractions dimin- 


\begin{tabular}{|c|c|c|c|c|c|}
\hline 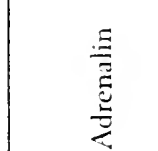 & $+\bar{y}^{\prime \prime}+$ & & & + & + \\
\hline 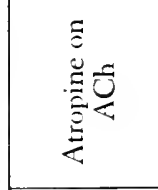 & 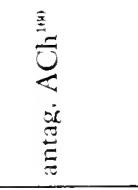 & 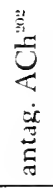 & 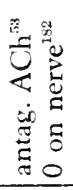 & 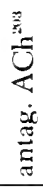 & 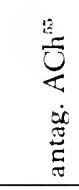 \\
\hline 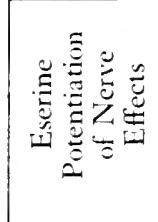 & & & 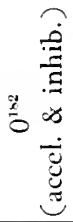 & & 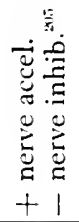 \\
\hline
\end{tabular}

\begin{tabular}{|c|c|c|}
\hline 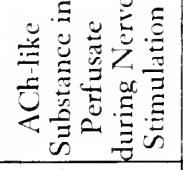 & & \\
\hline 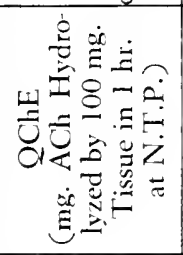 & & \\
\hline 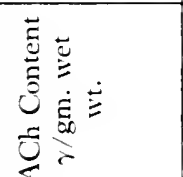 & 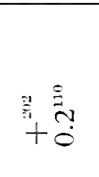 & 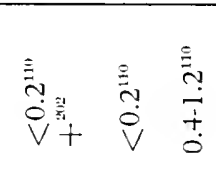 \\
\hline
\end{tabular}

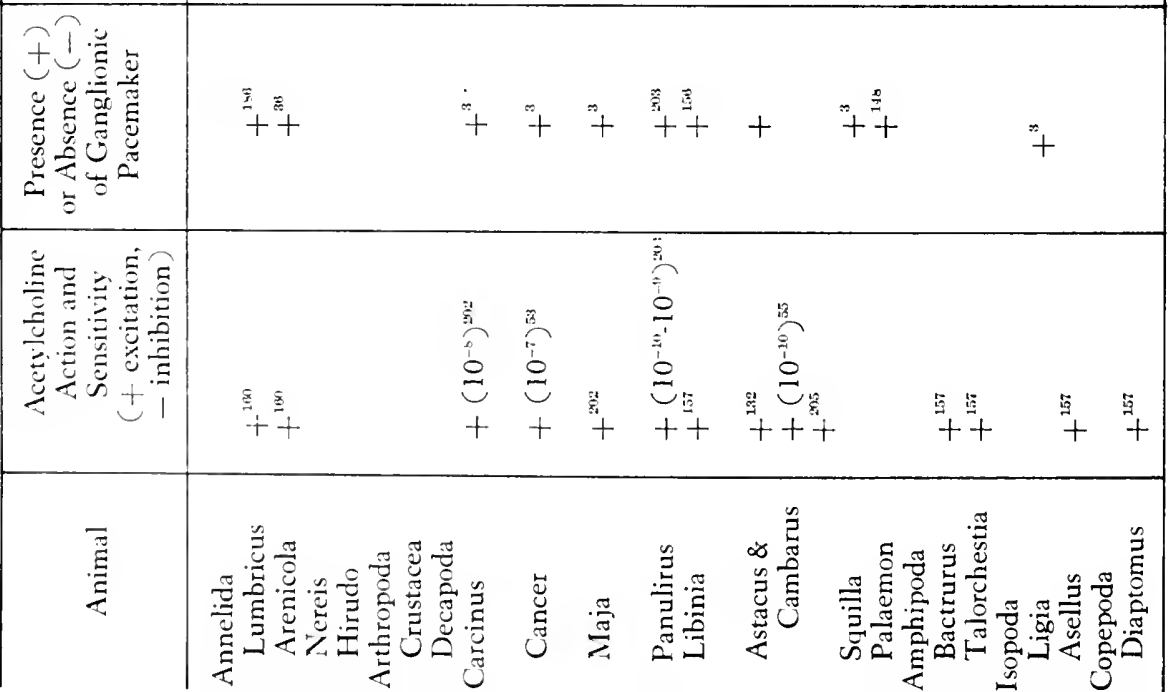


Circulation of Body Fluids

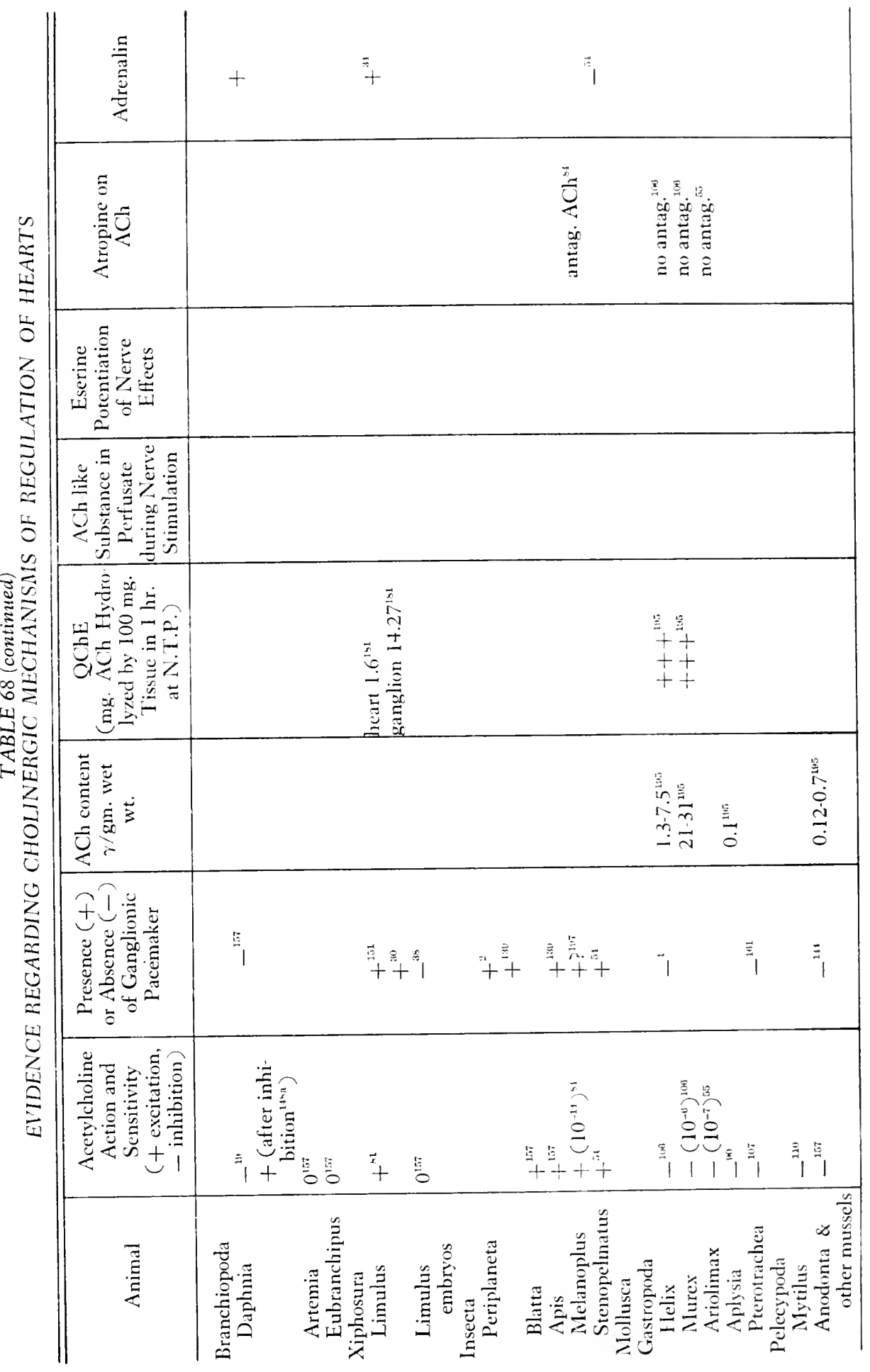




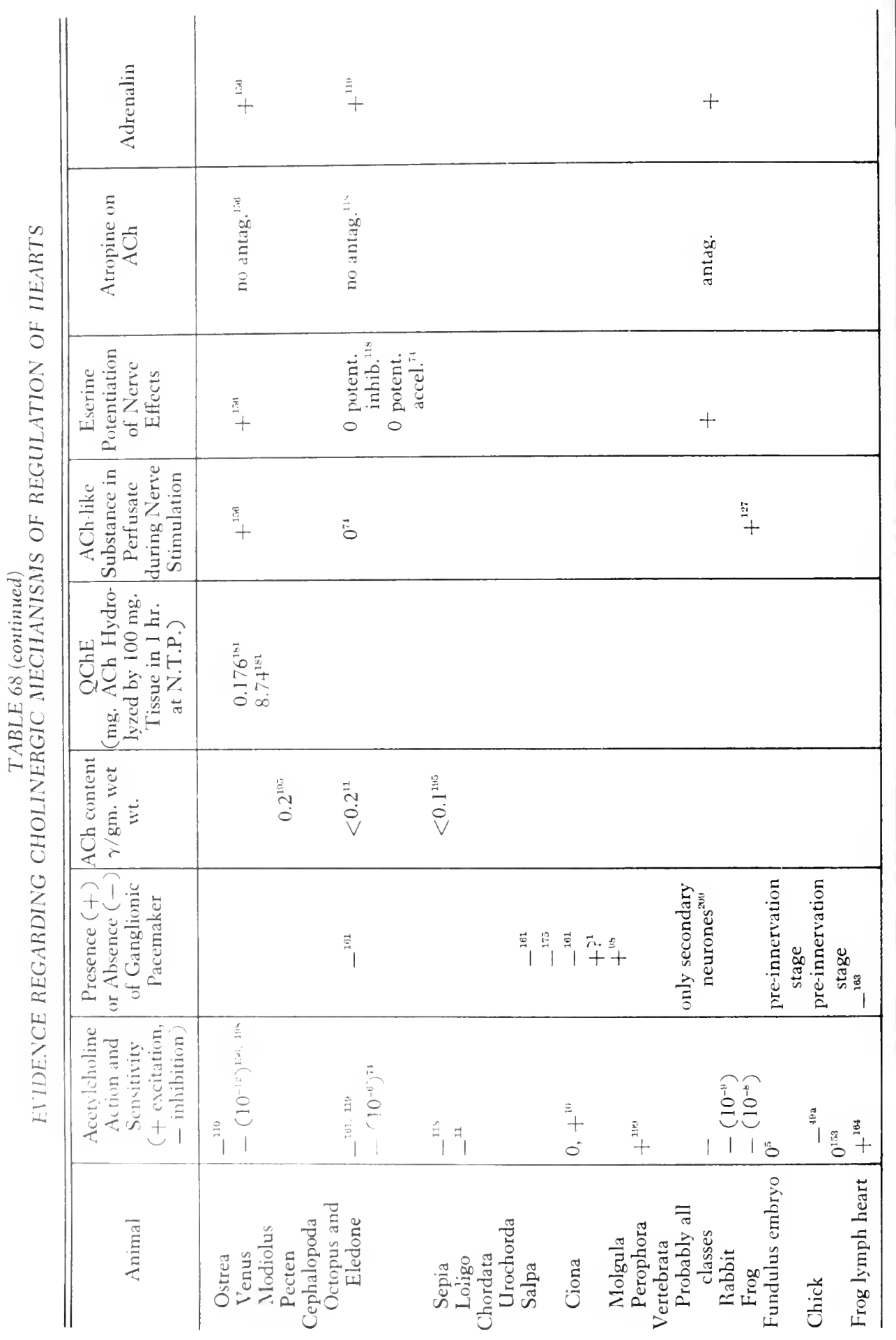


ishes, and when all of the longitudinal nerves are transected coordination between the anterior and posterior ends ccases.

The hearts of some insects are neurogenic. Two chains of nerve cells occur on the heart of the cockroach, ${ }^{1}{ }^{139}$ and these probably are pacemakers. ${ }^{18 t}$ In Aeschna, cells from the cardiac ganglion appear to have migrated to part of the stomatogastric system. ${ }^{218}$ Maloeuf ${ }^{13: 3}$ failed to find nerve cells in preparations of hearts of larval Anax and adult Belostoma, which beat normally so long as dorsal suspensory ligaments connected the heart to the body cuticle. Similarly in Agrion and Hydrophilus cutting of the alary muscles stops the heart, and nerve cells are said to be absent from the heart of Bombyx (Kuvana, in Maloeuf ${ }^{134}$ ). The innervation of insect hearts is summarized by McIndoo; ${ }^{139}$ nerve cells are found in the hearts of several adults and nymphs (bce, cockroach and others) but are absent from hearts of larvae (army worm). Pharmacologically the hearts of the cockroach Blatta, the grasshopper Melanoplus, the cricket Stenopelmatus, and the honeybee behave like the hearts of Crustacea and of Limulus, in that they are accelerated by acetylcholine. ${ }^{157,54}$ The hearts of larval wax moths Galleria are not affected in rate or form of electrocardiogarm by acetylcholine. ${ }^{1 * 1}$ It is probable that insect hearts are unusually sensitive to tension and may not beat unless extended; it is also probable that most adult insect hearts are neurogenic, although the pacemaker cells may have migrated outside, and that some larval hearts may be myogenic. The hearts of spiders are apparently neurogenic. ${ }^{168}$

Among the annelids nerve cells have been described in the hearts of Arenicold $a^{36}$ and Lumbricus; ${ }^{366}$ these hearts contract by a layer of smooth muscle. Nerve cells were not seen in Nereis (Neanthes), in which the contractions are due to endothelium and Rouget-like cells. ${ }^{16 i 6,66}$ The beats of Arenicola and Lumbricus hearts are accelerated by acetylcholine ${ }^{160}$ (Fig. 210); it is probable that they are neurogenic. Distention by blood is important in the establishment of contractions. ${ }^{111}$

In the hearts of ascidians nerve cells have been seen in Ciona ${ }^{1}$ and Molgula, ${ }^{98}$ but not in Salpa. ${ }^{161,175}$ The beat normally originates at the ends of the heart, although Hunter ${ }^{99}$ found that middle pieces in Molgula manhattensis contracted for some time after isolation. In Ascidia atra ${ }^{56 .}{ }^{86}$ and Perophora viridis ${ }^{199}$ the beat is for a time in one direction (abvisceral) and then for a number of beats in the opposite direction (advisceral). Normally the two ends are pacemaker regions. ${ }^{\mathbf{1 8 0}}$ Acetylcholine accelerates in Peropho $r a,{ }^{199}, 157$ although it is relatively ineffective in Ciona. A rise in temperature accelerates the heart by the same amount, irrespective of the end at which the beat originates. ${ }^{20}$ The incomplete evidence available indicates that the hearts of ascidians, at least of Molgula and Perophora, are neurogenic.

Lymph hearts of Amphibia (frogs and toads) and fish (eel) are normally under control of the spinal cord. The pacemaker characteristics in frogs and toads have been well summarized. ${ }^{15 \pi}, 163$ When the connections of the anuran lymph heart to the spinal cord are transected the heart usually stops for a few minutes, and then beats spasmodically, the beat later becoming coordinate and rhythmic. Anterior and posterior homolateral hearts are normally 
synchronized. Lymph hearts transplanted to the superficial lymph sac of the tongue beat strongly although spasmodically. Posterior lymph hearts (Bufo) have several separate chambers, and when they are transplanted the beat of these chambers may not remain coordinated. ${ }^{163}$ Histological examination failed to reveal nerve cells anywhere among the branched striated muscle fibers. In intact animals acetylcholine $\left(10^{-4}-10^{-6}\right)$ may cause reflex inhibition, but transplanted denervated lymph hearts are accelerated by acetylcholine $\left(10^{-7}-10^{-9}\right)$. This is an exception to the rule of inhibition and myogenicity, but lymph hearts are more closely related pharmacologically to skeletal muscle than to systemic hearts. ${ }^{164}$ Normally the lymph hearts are controlled by the spinal cord, but they can readily revert to myogenic beats which are less regular and less coordinated than their neurogenic beats.

From the preceding evidence, cardiac muscle of all animals is potentially capable of spontaneous rhythmic contractions. In embryos of vertebrates and of Limulus the heart beats without a fixed pacemaker. In adults the pacemaker becomes localized-specialized muscle in the vertebrate, and ganglion cells in Crustacea, Limulus, and many insects. After removal of the pacemaker, heart muscle may show some contractions, particularly if the heart is distended. Functional distinction between pacemaker nerve cells and secondary neurones of regulating nerves must be made by deganglionation. Embryonic hearts prior to innervation, whether they are to be myogenic or neurogenic, are insensitive or very slightly sensitive to acetylcholine. Innervated myogenic hearts are inhibited, and innervated neurogenic hearts are accelerated by acetylcholine. Some adult hearts (Artemia and Eubranchipus but not amphibian lymph hearts), are by this test myogenic and non-innervated.

Heart Rates. Statements of absolute heart rates are of little value unless the conditions are stated accurately. Therc are extensive tabulations of heart rates. ${ }^{122,} 170$ In general the heart rate is slower in sluggish animals than in active ones. For example, among molluscs, heart rates in clams range from 0.2 to 22 per minute, whereas in squid and octopus the heart may beat at 40 80 per minute. ${ }^{170}$ Hcart rate in fast-swimming fish exceeds the rate in slug. gish ones. The rate of beat in some hearts, as in Helix, increases many times as the internal pressure increases.

Heart rate varies with size, age, and state of development. The rate of beat in a fish embryo increases as the embryo nears the time of hatching. Body size in a given class has heen stated to be inversely related to heart rate, but it is probable that activity is more important. In Daphnia the heart beats about 150 times per minute, as contrasted with 30-60 beats per minute in a crayfish. The heart rate in large animals such as clephants and horses is 25-40 per minute, compared with rates of 300-500 in rats and mice. Many insect hearts are fast, particularly when the animals are active. In the imago of Sphinx the rate is 40-50 per minute when at rest, 110-140 when active..$^{206}$ The heart rate in birds is very high, 150-180 for domestic fowls, and several hundred per minute for smaller birds.

Temperature is an important variable in determining heart rate. The high rate in birds is in part related to their high body temperature. Heart rate, in general, increases about two to three times for a 10 degree rise in tem- 
perature; the rate of increase is not a linear but rather a logarithmic function of temperature, and temperature characteristics have been obtained for the hearts of many species. ${ }^{17}$ There is no uniformity in temperature characteristics of hearts, but several insects and crustaceans give Arrhenius $\mu$ values of 12,200 calories, whereas several molluscs give values of $11,200 .^{105}$

Heart rates, then, are directly related to rates of metabolism, and any alteration in metabolism is reflected in an alteration in heart rate. In addition, the ability to increase or decrease heart rate, and with it the cardiac output, is necessary for the efficient functioning of any cardiovascular system.

Electrocardiograms. The conduction of the excitation from the pacemaker to and within the muscle of the heart is manifest by an action potential wave. Because of the synchronization of many discharging units the potentials which can be recorded are large, even at some distance from the heart. At the same time the geometrical relations in chambered hearts are such that a complex wave is recorded. The electrocardiogram (ECG) is different in hearts with muscular conduction from the ECG in hearts with nervous conduction.

The electrocardiogram is similar in vertebrates whether ventricular conduction is in Purkinje tissue (mammals and birds) or in muscle (poikilotherms). Typically the ECG consists of a series of slow waves, upward (negative) deflections called $\mathrm{P}, \mathrm{R}$, and $\mathrm{T}$, and downward (positive) deflections $\mathrm{Q}$ and S (Fig. 199). The complex wave form of the ECG cannot be inter-

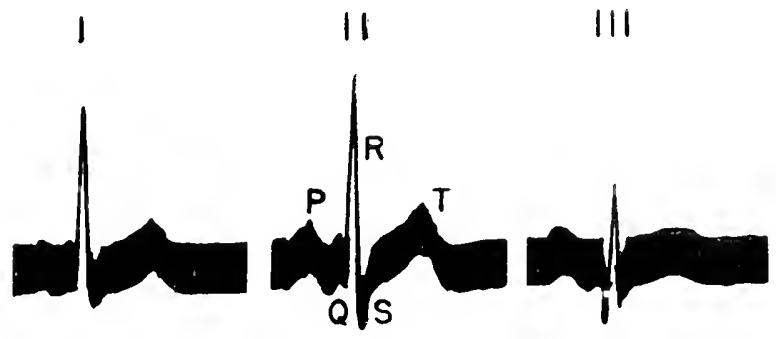

Fig. 199. Electrocardiograms from man, leads I, II and III. From Katz. ${ }^{119}$

preted simply as propagation along a polarized membrane, as in nerve or skeletal muscle conduction. Rather, the ECG appears to represent a wave front spreading over the heart. An isolated sinus from the eel may show rhythmic simple diphasic waves (Fig. 200, B). ${ }^{14}$ In A-V block or isolated auricle and sinus the auricle gives the $\mathrm{P}$ wave, often followed by a recovery wave sometimes called $\mathrm{X}^{63}$ There is no doubt that the $\mathrm{P}$ wave corresponds to conduction in the auricles. The PQ interval is delay at the auriculoventricular junction, and the QRS complex corresponds to conduction in the ventricles. Early theories postulated a spread of excitation in Purkinje tissue down the ventricular septum and then in muscle fibers from endocardium out to epicardium, the different waves representing different times of arrival in the various parts of the ventricles. However, the complex is similar in frogs and fish, which lack a septum. Records from the endocardium and epicardium, and from different electrical leads (across the heart, on one side, etc.) under conditions of local changes in temperature and ex- 
trasystoles led Hoff"1 to the conclusion that the QRST complex represents the algebraic summation of impulses arriving asynchronously at the different surfaces of the heart. The $\mathrm{T}$ wave is said to represent repolarization of the ventricular surface, an upright $\mathrm{T}$ indicating earlier repolarization of the left ventricle, an inverted $T$ earlier repolarization of the right side of the heart (Figs. 199, 200). ${ }^{91}$ That the $T$ wave represents repolarization is indicated by the action potentials of strips of heart muscle..$^{50}$

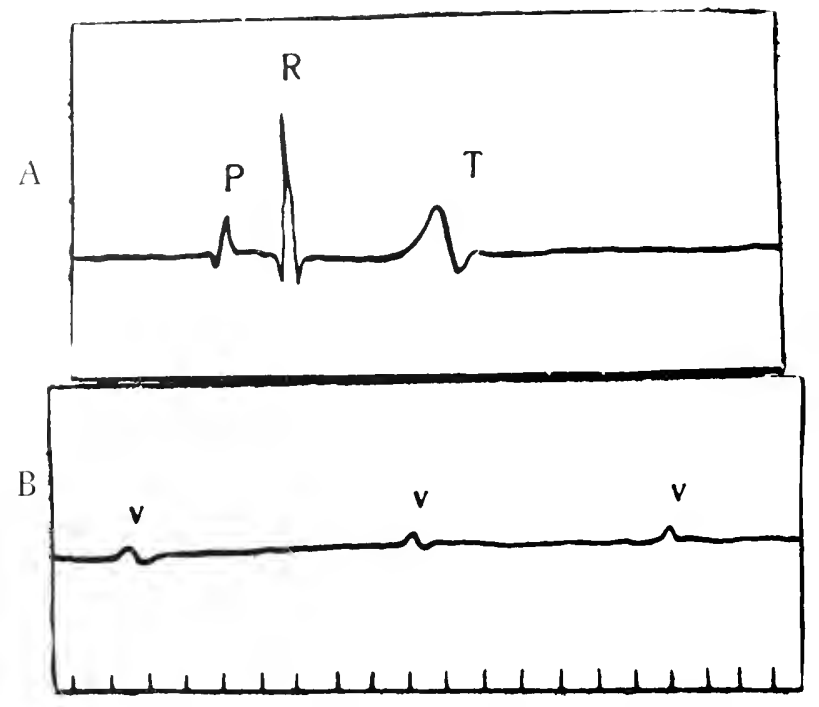

Fig. 200. Electrocardiogram from an eel. A, Normal ECG. B, Activity of isolated sinus venosus. From Bakker. ${ }^{14}$

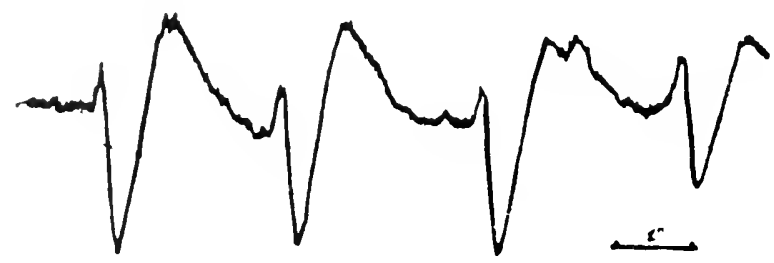

Fig. 201. Electrocardiogram of systemic heart of Octopus. From Luisada. ${ }^{125}$

The potentials of eel lymph hearts may be separated into two phases, a fast and a slow component. ${ }^{1: 1}$ Transplanted lymph hearts of the frog yield a simple diphasic wave. ${ }^{16: 3}$

The electrocardiogram of other myogenic hearts also consists of slow electrical waves. The chambered hearts of Octopus (Fig. 201) show an initial fast deflection, followed by a prolonged wave of negativity.92, 128 In Aplysia the ECG consists of slow waves with irregular deflections superimposed. ${ }^{92}$ In Helix the waves are nearly smoơth and consist of fast systolic 
and slow diastolic portions (Fig. 202). ${ }^{45}$ The electrocardiogram of the oyster $^{1 \times 4}$ and of fresh-water mussels (Fig. 203) consists normally of a diphasic component near the beginning of contraction and a slow wave associated with contraction. The interpretation of the rapid and slow components in the molluscan electrocardiogram is uncertain, but the fast wave probably represents spread of excitation. There is some variability in shape, according to electrode placement.

The neurogenic hearts of arthropods show very different electrocardiograms; there may be fast and slow deflections like those of the myogenic hearts, but in addition the arthropod electrocardiogram is typically oscillatory. The large waves have sometimes been designated by letters, corresponding to the terminology of the waves in the vertebrate ECG. ${ }^{59}$ This practice

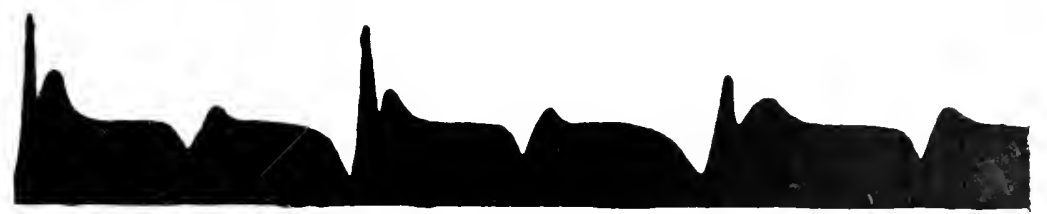

Fig. 202. Electrocardingram from Helix pomatia. From Arvanitaki and Cardot. ${ }^{7}$

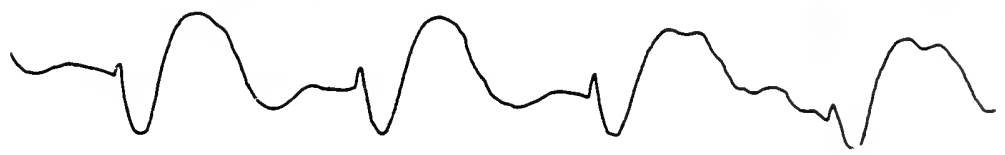

Fig. 203. Electrocardiogram of fresh-water mussel.

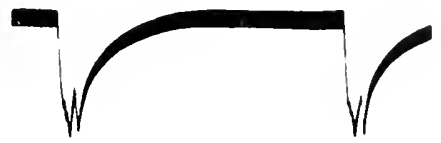

Fig. 204. Electrocardiogram from Astacus. From Hoffman. ${ }^{92}$

is misleading and erroneous, inasmuch as the nature of these deflections. Is very different in the two animal groups. In Figs. 204 and 205 are shown some representative patterns from hearts of arthropods, Astacus ${ }^{59,4: 2}$ and grasshopper. ${ }^{49}$ Simliar oscillatory waves were recorded from hearts of larvae of Galleria ${ }^{1+1}$ and Dytiscus. "i The pattern of the ECG in arthropods varies greatly according to electrode position, temperature, and other factors. In the grasshopper Melanoplus, for example, treatment with cold saline tends to suppress the oscillations and to emphasize the large fast and slow waves (Fig. 205)."'

It is probable that the large deflections of the arthropod ECG represent muscular activity in which repolarization is slow. Such complex action potentials are frequently recorded from sheets of short muscle fibers, as in vertebrate smooth muscle (Ch. 16, p. 591). The oscillations in arthropod elec- 
trocardiograms probably correspond to discharge from the pacemaker ganglion cells. The presence or absence of an oscillatory ECG under "normal" conditions may be an indication of whether a heart is neurogenic or myogenic. The electrocardiogram of the polychaete Arenicola also shows oscillatory waves in addition to slow potentials. ${ }^{15 \pi}$

In Limulus the electrogram of the pacemaker ganglion separated from the heart shows bursts of impulses corresponding to each heart beat. ${ }^{88}, 87$, 158 Each burst of impulses corresponds to a heart beat, and a single neurone

A

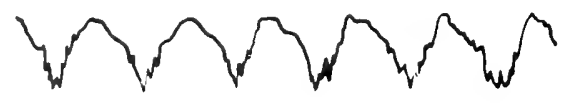

B

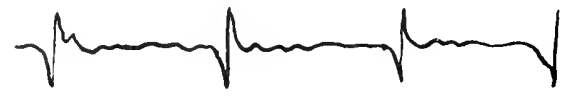

C

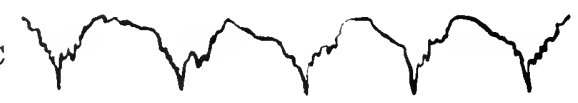

$\mathrm{D}$

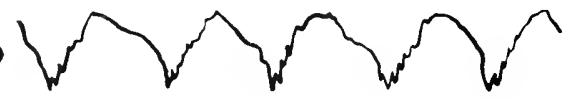

Fig. 205. Electrocardiogram from grasshopper: $A$, at room temperature; $B$, after treatment with cold saline; $C$ and $D$, recovery from cold effect. From Crescitelli and Jahn."'
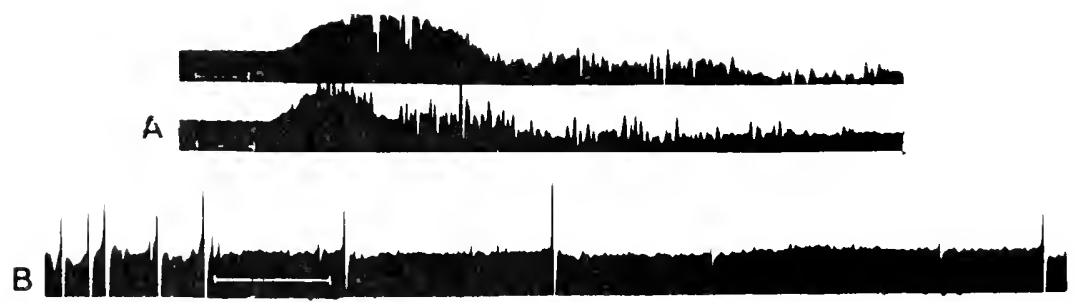

Fig. 206. Electrogram from dorsal ganglion of Limulus heart. A, Beats recorded from fifth and from sixth segments of isolated gangtion, showing slow potentials with superposed axon spikes. B, Discharge in single neurone of pacemaker ganglion during one beat. From Prosser. ${ }^{1 . i s}$

discharges several (2-15) times during a burst lasting approximately a half second (Fig. 206). Each neurone usually discharges initially at a high rate and later at decreasing frequency. In preparations which are no longer fresh, or after treatment with abnomal concentrations of potassium or calcium, asynchrony appears and the individual ganglion cells discharge at random. Records from the midsegments where the large unipolar pacemaker cells are located show slow waves lasting more than 0.1 second, with axon spikes 
superimposed (Fig. 206). It is probable that the excitatory wave originates in these large cells and that their slow potentials electrotonically synchronize the discharge of the smaller multipolar cells.

Electrocardiograms show three components: (1) "fast" waves associated with propagation, (2) "slow" waves probably associated with contraction, and (3), in ncurogenic hearts, oscillations corresponding to the pacemaker discharge.

Nervous Regulation of Hearts: Cholinergic and Adrenergic Systems. The vascular demands for blood vary with activity and stress. The heart accelerates or decelerates reflexly in efficient vascular systems. The higher vertebrate brain (medulla) contains a vasomotor center which receives impulses from: (1) specific vascular receptors by way of the carotid sinus, and depressor and vagus nerves, (2) general somatic and visceral receptors, and (3) higher centers in the brain. The heart rate rises in compensation for a fall in blood pressure, and the heart slows when the blood pressure rises. The vagus nerves slow the heart and reduce the amplitude of contraction; the primary vagus fibers terminate in ganglia located in several regions of the heart, ${ }^{209}$ and secondary vagus neurones end in the heart musculature and nodal tissue. Secondary sympathetic fibers cause cardio-acceleration. In addition, adrenalin added directly to the heart accelerates it, but in the circulation of the intact animal reflex control usually prevents marked acceleration on injection of adrenalin. The embryonic heart beats for some days before innervation; during this early period reflex regulation of heart rate cannot occur."

Activity in the sympathetic fibers to the heart is associated with liberation of sympathin, an adrenalin-like substance. Sympathetic innervation of the heart is lacking in fishes, but vagal fibers are found in all classes of vertebrates, with the possible exception of cyclostomes. ${ }^{25}$. 179 Reflex inhibition of the heart of dogfish is obtained by stimulation of a variety of sensory pathways. Inhibitory vagal reflexes can be elicited in the elasmobranch Scyllium by afferents in the vagus, hypobranchial, and lateral line rerves. ${ }^{129}$ Alteration of water flow over the gills affects both respiration and heart beat reflexly. The inter-renal glands of fish are well developed, and adrenalin increases blood pressure but has no effect or is slightly inhibitory on dogfish hearts. ${ }^{211}$

Acetylcholine $(\mathrm{ACh})$ appears in perfused hearts during vagal inhibition. ${ }^{126,127}$ Atropine blocks the inhibiting action of the vagus, and the drug physostigmine (eserine) enhances the effect. Embryonic hearts beat for some days before innervation; during this early period reflex inhibition of the heart does not occur; the heart is relatively insensitive to ACh. It is probable that the ACh acts at the synapses of the vagus rather than on the heart muscle. 5 Whether the secondary vagal fibers are cholinergic is not known, but, by analogy with postganglionic parasympathetic fibers in the eye, they may liberate ACh. Perfusion of very small amounts $\left(10^{-10}\right)$ of acetylcholine may increase contractile tension of mammalian hearts. ${ }^{13 \%}$

Reflex regulation of hearts is of general occurrence among all phyla of animals possessing hearts. The early literature has been excellently summarized. ${ }^{25}, 37.94$ 
The distribution of inhibitory and accelerator nerves differs among various molluscs. In the pelecypods M ya, Anodonta, ${ }^{31}$ and Venus, ${ }^{27}$ stimulation of the visceral ganglion results in cardiac inhibition. The palliovisceral connectives are accelerators, and no inhibitory fibers have been found in chitons, Aplysia, the prosobranchs Haliotis and Natica, the tectobranchs Bulla and Pleurobranchea, the nudibranch Archidoris, or in Ariolimax. ${ }^{31}$ The heart in Helix, Limax, and the nudibranch Triopha receives both inhibitory and augmentor nerves from the pleural ganglion. In Aplysia, stimulation of the pleurovisceral nerves accelerates and raises the tone of the heart, an effect which is suppressed by caffeine. ${ }^{70}$

In cephalopods, inhibition of both the systemic and branchial hearts by the visceral nerves was first seen by Paul Bert in 1867. In Octopus, ${ }^{78}$ stimulation of a visceral nerve results in slowing of the heart; the two nerves are homolateral with respect to the auricles, but overlap in ventricular action. Synchrony between the two branchial hearts and between the auricles is nervous. ${ }^{65}, 71$ A single inhibitory nerve volley may delay one heart beat, an effect prevented by curare. The visceral fibers terminate in a cardiac ganglion in which secondary neurones originate. ${ }^{161}$

Cephalopod hearts may also have accelerating fibers; ${ }^{i 6}$ in Eledone and Octopus the auricles can be stimulated to high tonus, whereas the ventricle is inhibited, and contraction can be elicited in a quiescent heart by stimulation of a visceral nerve. A positive inotropic (increased amplitude) effect builds up during repetitive stimulation, ${ }^{73}, 7^{74}$ and the threshold is higher for accelerator fibers than for inhibitory ones. ${ }^{11 !}$

Acetylcholine inhibits the heart of all classes of molluses (Table 68, Fig. 206). Eserine fails to potentiate the nervous inhibition in cephalopods, ${ }^{119}$ although it does prolong inhibition by visceral ganglion stimulation in Venus. ${ }^{156}$ Atropine is toxic and does not antagonize the inhibitory effect either of visceral nerve stimulation or of acetylcholine. ${ }^{12.156}$ Evidence for a chemical mediator in Venus was obtained by inhibiting a test heart with fluid from one inhibited by visceral ganglion stimulation. ${ }^{156}$ Perfusion fluid from the heart of a cephalopod (Sepia, Octopus, Eledone) was passed into the heart of another individual; when the excitatory fibers of the visceral nerve of the donor were stimulated, the amplitude of the beat of the perfused isolated heart also increased, apparently because of a stimulating mediator. ${ }^{1 \%}$ Other investigators failed to confirm this experiment; ${ }^{74}$ also tests for $\mathrm{ACh}$ in the perfusate from the hearts of Octopus and Eledone during visceral nerve stimulation were negative. Identification of the substances liberated by inhibitor and accelerator fibers in molluscan hearts would add considerably to the knowledge of nerve function.

The hearts of Crustacea and of Limulus receive from the central nervous system several regulating nerves which are distributed to the pacemaker ganglion and the muscle. Extrinsic cardiac innervation has been described in detail by Alexandrowicz. ${ }^{1,3}$ In 1896 Conant and Clark ${ }^{46}$ proved by cutting and stimulation that the heart of Callinectes receives two pairs of accelerator and one pair of inhibitor nerves. Cerebral ganglion stimulation results in inhibition of the heart in crayfish, crabs, and Limulus. ${ }^{37}, \mathbf{4 6}, \mathbf{1 8 2}$ In Limulus, the inluibitory nerves arise in the posterior part of the brain, in 
Crustacea both inhibitor and accelerator nerves arise from the subesophageal ganglion, the inhibitors always arising anterior to the accelerators. The stomatogastric nerves have no effect on the heart. ${ }^{20 \%}, 1 \times 2$ In Astacus the inhibitor nerve fibers elicit maximal inhibition at a stimulation frequency of $45 / \mathrm{sec}$. and accelerators are most effective at $30 / \mathrm{sec}$. In the grasshopper, stimulation of either brain or ventral ganglia results in augmentation of heart beat.

Acetylcholine accelerates the neurogenic hearts of arthropods, and the effect rescmbles qualitatively stimulation by cardioaccelerators (Fig. 209). Eserine has been said to enhance nervous acceleration but not nervous inhibition in Astacus, ${ }^{20.5}$ and to have no effect on either acceleration or inhibition in the crab Cancer. ${ }^{1.2}$ Atropine antagonizes the effect of acetylcholine but has no effect on nervous control. There is no convincing evidence that either accelerator or inhibitor nerves in Crustacea are cholinergic. In Daphnia mechanical stimulation of the intestine reflexly inhibits the heart and recovery of the beat is hastened by acetylcholine, ${ }^{14 \text { s a }}$ although under other conditions acetylcholine may decrease the heart rate. "1" In the shrimp Palaemonetes a hormone from the sinus gland accelerates the heart. ${ }^{176}$

The only record of extrinsic nervous effects on the hearts of annelids is for Nereis and Arenicola; stimulation of the ventral nerve cord inhibits the lateral hearts in diastole and increases the rate and strength of dorsal vessel contractions. ${ }^{36}$ Earthworm vessels continue to beat after destruction of the ventral nerve cord. ${ }^{1 \times 6 .+4}$

Nervous control of the heart of ascidians has not been clearly demonstrated. Removal of the "brain" from Ciona is said not to affect the heart, ${ }^{10}$ although in Molgula the beat is made irregular. ${ }^{99}$ In Ascidia mentula removal of the "brain" results in faster heart rate for about a day.. "is

Nervous control of cardiac activity is found in most animals. Only in embryos (vertebrate, Limulus) and possibly in certain lower crustaceans, such as Artemia and Eubranchipus, ${ }^{157}$ is there evidence that nervous regulation is absent. In general, chemical mediators associated with activity of cardiac nerves are indicated. Acetylcholine inhibits the myogenic hearts of vertebrates and molluscs; it accelerates the neurogenic hearts of Crustacea, Limulus, and some insects and annelids. Adrenalin accelerates all hearts. Evidence for cholinergic regulating nerves is available only for vertebrates and for Venus. Possibly undiscovered agents are active as mediators in some invertebrates.

\section{DIRECT RESPONSES OF HEART TISSUES}

Effects of Drugs on Hearts. The literature on the pharmacology of circulatory systems is extensive and is useful for comparative physiology in so far as it aiás in an understanding of hearts and peripheral circulation. The inhibiting and accelerating actions of $\mathrm{ACh}$ are illustrated in Figures 207-210; these effects have been discussed in the sections on pacemakers and nervous regulation of hearts. The action of drugs which potentiate or antagonize acetylcholine indicates interesting differences in cardiac mechanisms.

Physostigmine (eserine) and prostigmine prevent hydrolysis of acetylcholine by acetylcholine esterase; in the vertebrates they prolong the inhibiting effect of acetylcholine and of vagal stimulation. Eserine sensitizes hearts to $\mathrm{ACh}$. Potentiation of the inhibiting effect of ACh has been reported in the 


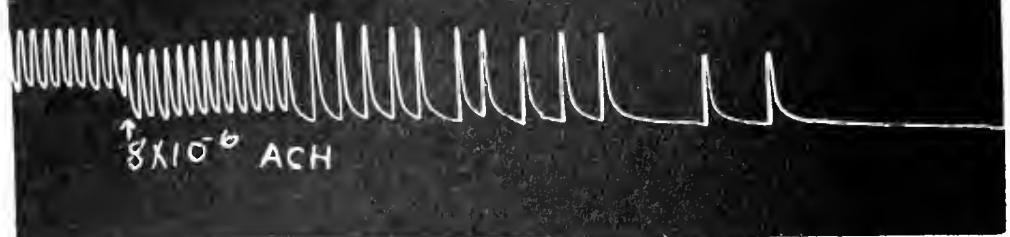

Fig. 207. Effect of acetylcholine ( 8 parts in $10^{5}$ ) on turtle heart.

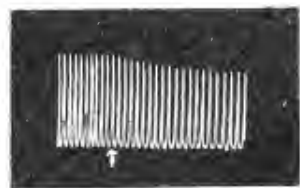

$\mathrm{ACH} 5 \times 10^{-12}$

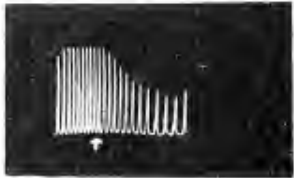

$A C H 7 \times 10^{-11}$

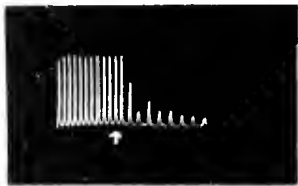

$A C H 10^{-10}$

Fig. 208. Fffect of acetylcholine in three concentrations on heart of l'enus mercenaria. From Wait. ${ }^{1: k}$

ACH CL $10^{-8}$

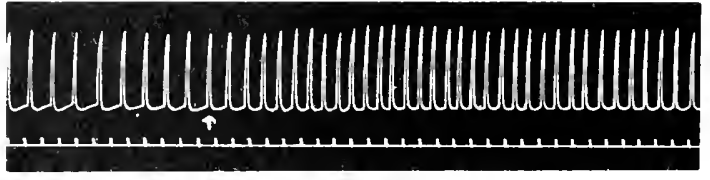

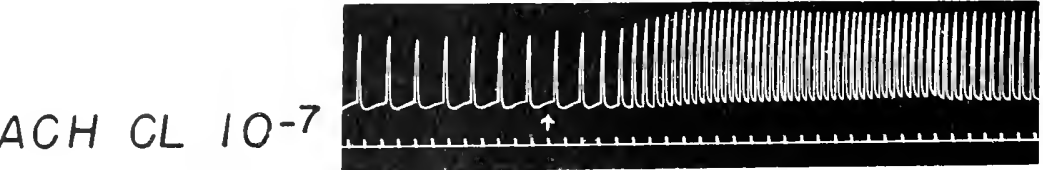

\section{$\mathrm{ACH} \mathrm{CL} 10^{-6}$}

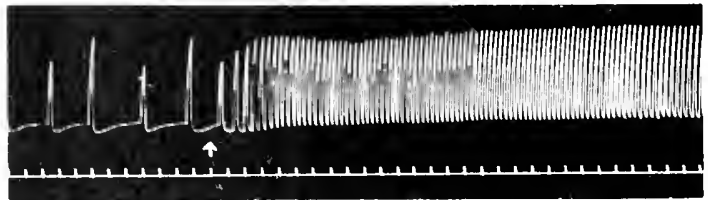

Iig. 209. Effect of three concentrations of acetylcholine on the heart of the lobster.

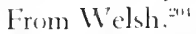
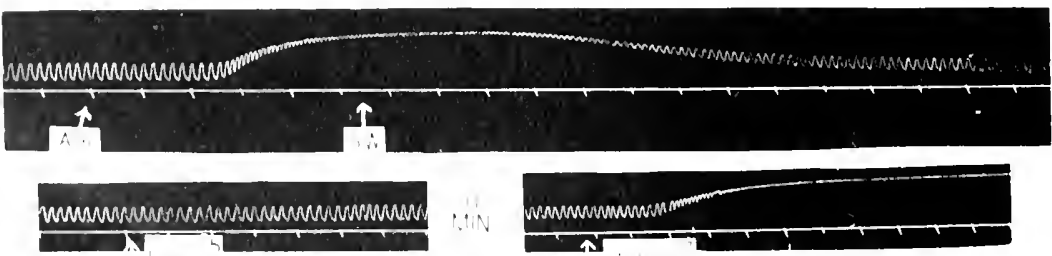

Fig. 210. Itfect of acetrhtholine $\left(1 \mathrm{in} 10^{\circ}\right.$, before and after eserinization of the heart of Arenicola. From Prosser and Zimmeman. ${ }^{\text {tan }}$ 
molluscs Helix, ${ }^{111}$ Venus, ${ }^{15 ;}$ and Ariolimax." Potentiation of the acceler ating effect of $\mathrm{ACh}$ occurs in the arthropods Astacus, $13:$. 5 Panulirus, 20.3 Homarus, ${ }^{204}$ Limulus cardiac ganglion, ${ }^{-1}$ Cancer,, 33 the insect Sienopelma. tus ${ }^{\overline{5}+}$ and in the annelids Arenicola and Lumbricus. ${ }^{160}$ The sensitivity of hearts to acetylcholine is very great. A frog's heart (eserinized) is depressed by l part in $10^{5}$, a lobster's heart is accelerated by 1 part in $10^{4}$, and when eserinized by 1 part in $10^{9} ;{ }^{204}$ a Venus heart is sensitive to ACh 1 part in 1010, and at some seasons to 1 part in 1012 (Fig. 208). ${ }^{15 i 6}$. 1:\%

Atropine antagonizes the action of $\mathrm{ACh}$ and vagal inhibition in the vertebrate heart, not by preventing the liberation of ACh but by blocking its action on the receiving cells. Similarly atropine antagonizes the acceleration of ACh in the arthropods Astacus, ${ }^{132}$ in Melanoplus, ${ }^{x-1}$ Panulirus, ${ }^{203}$ Homarus, ${ }^{204}$ and Cancer. ${ }^{53}$ Atropine has a stimulating effect on the Limulus heart and may not antagonize ACh. ${ }^{\$ 1}$ In molluscs, however, atropine is toxic; its effects are variable, and no antagonism of $\mathrm{ACh}$ has been found in Aplysia, ${ }^{107}$ Ostrea, ${ }^{110}$ Venus, ${ }^{156}$ Sepia, ${ }^{118}$ Loligo, ${ }^{11}$ Helix, ${ }^{109}$ and Ariolimax, ${ }^{5 \pi}$ and no antagonism of muscarine in Anodonta. ${ }^{1:}$ : The receptor cells in which acetylcholine is blocked by atropine appear to be nerve cells in vertebrate hearts (vagus secondary neurones) and in arthropods (pacemaker ganglia) whereas in molluscs the site of $\mathrm{ACh}$ action is muscular.

Pilocarpine depresses the vertebrate heart; it is also inhibitory to the hearts of Anodonta ${ }^{1 \times 9}$ and Anomia. ${ }^{146}$ Pilocarpine accelerates the hearts of Cancer, Maja, and Carcinus, ${ }^{13}$ but has been reported as inhibitory in Limulus. ${ }^{34}$

In the vertebrate heart acetylcholine resembles the drug muscarine in its inhibition of the heart and its atropine antagonism. Muscarine also inhibits hearts of the molluscs Anodonta, ${ }^{1 \times 9} M y a$ (after initial stimulation), ${ }^{217}$ and Octopus. ${ }^{161}$ The heart of Cancer is accelerated by muscarine, ${ }^{53}$ but the Limulus heart has been reported to be inhibited. ${ }^{147}$

Nicotine initially slows and may stop the vertebrate heart, and later and in high concentration it accelerates and blocks the vagus. Inhibition of molluscan hearts, much like ACh inhibition, has been reported for Anodonta, ${ }^{1 \times 9}$ Sepia, ${ }^{118}$ Anomia ${ }^{146}$ Venus, ${ }^{156}$ and Ostrea. ${ }^{149}$

In arthropod hearts, nicotine initially accelerates in Homarus, ${ }^{154}$ Cancer, ${ }^{53}$ Astacus, ${ }^{55}$ Periplaneta, ${ }^{214}$ Melanoplus, ${ }^{34}$ and Limulus. ${ }^{3+}$ After initial stimulation nicotine has a paralyzing effect (Limulus, Periplaneta, Melanoplus), which has been seen in Limulus to consist of a blocking of the pacemaker ganglion..$^{158}$

In its action on hearts of invertebrates acetylcholine cannot be characterized as being like muscarine or nicotine.

Adrenin is a natural cardioaccelerator of the vertebrate heart. Adrenalin is an accelerator of most other hearts: molluscs Aplysia, ${ }^{72}$ Loligo, ${ }^{11}$ freshwater mussels, ${ }^{144}$ Ostrea, ${ }^{1.7,},{ }^{106,}$, 14! Pecten,,${ }^{95}$ Anomia, ${ }^{1+6}$ and Venus, ${ }^{156}$ and arthropods Limulus, ${ }^{34}$ Astacus, ${ }^{55}$ Carcinus, and Maja, ${ }^{13 .}, 202$ Panulirus, ${ }^{203}$ and Homarus, ${ }^{204}$ and annelids Lumbricus and the leech. ${ }^{\times 2}$ Slight species differences exist in the effects on tone and amplitude, but in general adrenalin accelerates hearts, whether they are myogenic or neurogenic. Some hearts are unaffected by adrenalin (Ciona, ${ }^{10}$ Nereis, ${ }^{66}$ and Artemia ${ }^{15 i}$ ).

The effects on hearts of drugs which potentiate and antagonize adrenin 
(or sympathin) have not been much studied. Such substances as tyramine, sparteine, and various animal toxins have been insufficiently studied and may be important as naturally occurring regulating agents.

Effects of Salts on Hearts. Hearts continue to beat for many hours outside the animal body in a solution of properly balanced salts. What specific anions are in the solution seems to matter little unless they are toxic, and most hearts beat actively in a neutral mixture of the chlorides of sodium, potassium, and calcium (see Table 15, Chapter 3); a few hearts, particularly those of some marine molluscs, require magnesium in addition. Deviations from the optimum ratios of these cations have been used in analysis of the pacemaker, and conductile and contractile processes.

The effects of any single element are multiple, depending on the condition and previous history of the heart. The effects of an ion also vary with the concentrations of other ions, e.g., calcium antagonizes some effects of sodium, and potassium antagonizes some sodium action. Ionic ratios such as $\left(\mathrm{Na}^{+}+\mathrm{K}^{+}\right) / \mathrm{Ca}{ }^{+}+$may be more significant than absolute concentrations of each ion. The effects of excess or deficiency of an ion in vivo may differ from the effects of such variations in vitro. ${ }^{91}$ The cellular bases of cardiac action of ions are not clear, but the gross effects show interesting comparative differences.

Table 69 summarizes in simplified manner some of the reports of salt ef. fects on perfused hearts.

Sodium chloride comprises the bulk of the solute in all body fluids and in physiological salt solutions. Effects of a deficit in $\mathrm{Na}^{+}$can be ascertained only by maintaining the osmotic concentration of a solution by adding a nonelectrolyte such as a sugar; effects of excess $\mathrm{Na}^{+}$are difficult to separate from osmotic effects, and in pure $\mathrm{NaCl}$ solution there is a deficiency of $\mathrm{K}^{+}$and $\mathrm{Ca}^{++}$. Table 69 shows that in general sodium has a stimulating action on heart pacemakers and favors contraction of heart muscle. Ringer found in 1882 that pure sodium chloride solution will not maintain the beating of the frog heart; hearts of invertebrate animals also cease to beat in sodium chloride solution. Sodium initiates a fast but irregular rhythm, and when sugar is substituted for sodium the rate of most hearts declines.

Potassium has been said to act oppositely on vertebrate and invertebrate hearts; the explanation is that it may act predominantly on pacemaker, conducting, or contracting mechanisms. In the perfused vertebrate heart, potassium has a striking depressant action on the contracting and conducting systems. In excess potassium the heart relaxes and stops in diastole. Molluscan hearts are not very sensitive to changes in potassium but may be slightly accelerated by increase, and great excesses ( 6 to 7 times) may stop the heart in systole; there is no such effect on contraction and conduction as in vertebrates. In Pecten $\mathrm{K}^{+}$seems not essential for maintaining the heart beat, and in Helix an apparent slowing is really the result of dropping alternate beats." ${ }^{29}$ In arthropods also the pacemaker is stimulated (except in Cambarus); the beat may become fast and weak. The low-potassium effect in Limulus is continuous, high-frequency discharge of many neurones in the pacemaker, and interruption of the normal rhythm; in the absence of $\mathrm{K}^{+}$the ganglionic dis- 
TABLE 69. EFFECTS OF SALTS IN HIGH (SUPRAOPTHMAI AND) IOW (SIBBOPTIMAL) CONCENTRATIONS ON HEARTS IN RISPECT TO RATE OF BEAT, AMPLITUDE, TONE, AND STATE

WHEN ARRESTED.

+ , increase; - , decrease in a function. High concentrations given $\times$ normal.

\begin{tabular}{|c|c|c|c|c|c|c|c|c|}
\hline Animal & Rate & Ampl. & Tonus & Arrest & Rate & Ampl. & Tonus & Arrest \\
\hline & \multicolumn{4}{|c|}{ HIGH SODIUM } & \multicolumn{4}{|c|}{ LOM SODIUN } \\
\hline Frog $^{12, \text { i1 }}$ & $\mathrm{sl}+$ & - & & $\begin{array}{l}\text { tends to } \\
\text { systole }\end{array}$ & - & $+, \quad, \quad c o r$ & $\begin{array}{l}\text { duction } \\
\text { ired }\end{array}$ & y'st. \\
\hline $\begin{array}{c}11{ }^{1 n: 3} \\
\text { Oyster }{ }^{1 \mid M s}\end{array}$ & $+{ }_{\text {arry }}$ & $\begin{array}{l}\mathrm{sl}+ \\
\mathrm{mic}\end{array}$ & $\begin{array}{l}+ \\
+\end{array}$ & tends to & & & & \\
\hline$" \quad 11 m$ & + & & & $\begin{array}{l}\text { systole } \\
\text { systole }\end{array}$ & & & & \\
\hline Pecten $^{143}$ & & & & systole & & & & \\
\hline Aplysia"1 & 0 & & $\begin{array}{c}+ \\
-(\operatorname{diast})\end{array}$ & & - & - & & \\
\hline Homarus"." & $\begin{array}{c}s 1+ \\
+\end{array}$ & $\begin{array}{l}+ \\
+\end{array}$ & - (diast.) & $\begin{array}{c}\text { pure } \mathrm{NaCl} \\
\text { systole } \\
\text { pure } \mathrm{NaCl} \\
\text { systole }\end{array}$ & - & - & & diast. \\
\hline Limulus ${ }^{32,}$ & + & - & & & - & & & \\
\hline
\end{tabular}

HIGH POTASSIUM

LOW POTASSIUM

\begin{tabular}{|c|c|c|c|c|c|c|c|c|}
\hline 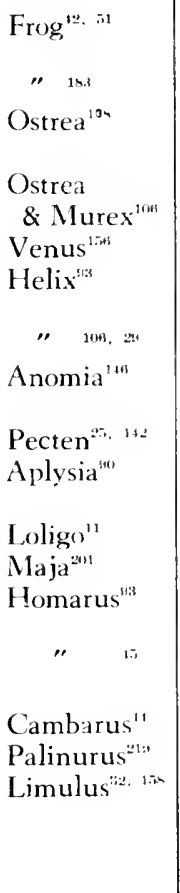 & $\begin{array}{c}+, \\
\text { then- } \\
\text { sl+, } 0 \\
+ \\
\\
+ \\
\text { irreg. } \\
\text { little } \\
\text { effect } \\
\text { irreg. }\end{array}$ & $\begin{array}{c}+ \\
\text { fast, } w \\
\text { in pure }\end{array}$ & $\begin{array}{c}0, \mathrm{sl}- \\
\mathrm{sl}- \\
\frac{+}{+}\end{array}$ & $\begin{array}{l}\text { systole } \\
\text { systole } \\
\text { systole } \\
\text { systole } \\
\text { systole } \\
\text { systole } \\
4 \times \text { diast. } \\
7 \times \text { syst. } \\
\text { systole } \\
\text { I2X semi- } \\
\text { systole } \\
\text { systole in } \\
\text { pure KCl } \\
\text { diastole } \\
\\
\text { systole, } \\
\text { then } \\
\text { relaxes }\end{array}$ & $\begin{array}{l}\mathrm{sl}+ \\
0, \mathrm{sl}+\end{array}$ & $\begin{array}{l}0, \text { sl- } \\
\text { sl- } \\
\text { inhibits } \\
\text { relaxatic }\end{array}$ & beats & $\begin{array}{l}\text { diast. } \\
\text { syst., } \\
0 \mathrm{~K}\end{array}$ \\
\hline
\end{tabular}


TABLE 69 (continued). EFFECTS OF SALTS IN HIGH (SUPRAOPTIMAL) AND LOW (SUBOPTIMAL) CONCENTRATIONS ON HEARTS IN RESPECT

TO RATE OF BEAT, AMPLITUDE, TONE, AND STATE

WHEN ARRESTED.

+ , increase; - , decrease in a function. High concentrations given $\times$ normal.

\begin{tabular}{|c|c|c|c|c|c|c|c|c|}
\hline Animal & Rate & Ampl. & Tonus & Arrest & Rate & Ampl. & Tonus & Arrest \\
\hline & \multicolumn{4}{|c|}{ HIGH CALCIUNI } & \multicolumn{4}{|c|}{ LOW CALCIUM } \\
\hline $\operatorname{Frog}^{1,3.3}$ & sl- & + & + & syst. & $\begin{array}{l}\mathrm{sl}+ \\
-\mathrm{in} \\
0 \mathrm{Ca}\end{array}$ & - & - & diast. \\
\hline $\begin{array}{l}\text { Guinea pigis! } \\
\text { Raja }^{112}\end{array}$ & & + & & & & - & & diast. \\
\hline Ostrea & - & + & + & diast. & + & - & + & $\begin{array}{c}\text { diast., } \\
0 \mathrm{Ca}\end{array}$ \\
\hline $\begin{array}{l}\text { Ostrea } \\
\text { \& Murex }\end{array}$ & - & + & & & & & & \\
\hline Venus ${ }^{1 \text { int }}$ & - & $\begin{array}{c}+, \\
\text { then }-\end{array}$ & & $\begin{array}{l}\text { in } 6 \times \mathrm{Ca} \\
\text { diast. }\end{array}$ & + & sl-- & sen & $\begin{array}{l}\text { ni-syst., } \\
0 \mathrm{Ca}\end{array}$ \\
\hline Pecten $^{112.1: 3}$ & - & & + & $\begin{array}{l}\text { diast., } \\
\text { semi-syst. }\end{array}$ & + & - & + & diast. \\
\hline $\begin{array}{l}\text { Helix"si, } 93 \\
\text { Octopus } \\
\text { tin, } 21: 1\end{array}$ & sl- & - & - & $\begin{array}{l}\text { diast. } \\
\text { diast. }\end{array}$ & $\begin{array}{l}0 \\
+\end{array}$ & & $\mathrm{sl}+$ & $\begin{array}{l}\text { syst. } \\
\text { syst. }\end{array}$ \\
\hline Anomia ${ }^{1+i}$ & & favors & stole & 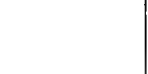 & & - & & $\begin{array}{l}\text { diast., } \\
\text { 0 Ca }\end{array}$ \\
\hline Aplysia:" & - & $\begin{array}{c}+, \\
\text { then }-\end{array}$ & & diast. & & & + & \\
\hline Crayfish $^{1+12 \pi}$ & - & - & + & diast. & + & & & $\begin{array}{l}\text { syst., } \\
0 \mathrm{Ca}\end{array}$ \\
\hline \multirow{3}{*}{ 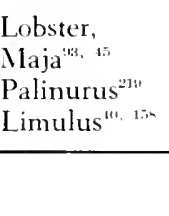 } & - & & + & diast. & + & - & + & \\
\hline & - & & & $\begin{array}{l}\text { diast. } \\
\text { diast. }\end{array}$ & + & & + & $\begin{array}{l}\text { syst. } \\
\text { syst. }\end{array}$ \\
\hline & \multicolumn{4}{|c|}{ HIGH MAGNESIUM } & \multicolumn{4}{|c|}{ LOW' MIAGNESIUM } \\
\hline \multirow{9}{*}{ 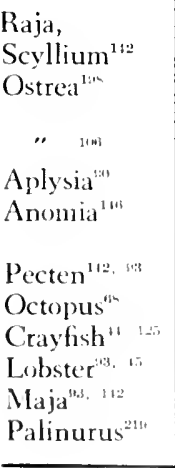 } & $\begin{array}{l}- \\
-\end{array}$ & - & + & $\begin{array}{l}\text { semi- } \\
\text { diast. } \\
\text { diast. }\end{array}$ & + & - & inhibits & syst. \\
\hline & & & & & & $\mathrm{Mg}$ & relax. & \\
\hline & & & & $\begin{array}{l}\text { diast. } \\
\text { in } 3 \times \mathrm{Mg}\end{array}$ & + & & + & syyst. \\
\hline & & & & diast. & & & & \\
\hline & - & & & diast. & $\mathrm{Mg}$ & $\begin{array}{l}\text { ssential } \\
\text { g not ess }\end{array}$ & ${ }_{\text {tial }}^{+}$ & syst. \\
\hline & & & & & beat 2 & hours wi & hout $\mathrm{Mg}$ & \\
\hline & & & - & diast. & & g not ess & ntial & \\
\hline & & & & diast. & & g not ess & ntial & \\
\hline & & & - & diast. & & g not ess & atial & \\
\hline
\end{tabular}


charge gradually stops. Arthropod hearts, with nervous pacemakers and conduction systems, are more sensitive than molluscan hearts.

Calcium antagonizes the effect of potassium and sodium on the pacemaker, and slows heart beats. In the perfused vertebrate heart the predominant calcium action is a marked enhancement of contraction resulting in systolic arrest; in the absence of calcinm the vertebrate heart relaxes and stops in diastole. Calcium is essential for contraction; a heart stopped in zero calcium continues rhythmic action potentials. ${ }^{1+3}$ In molluscs, however, the inhibiting effect of calcium on the pacemaker is predominant, and in excess $\mathrm{Ca}$ the heart stops in diastole by pacemaker inhibition. In the absence of calcium some molluscan hearts stop in diastole (Pecten, Ostrea, Anomia); in nthers (Helix, Octopus) the pacemaker stimulation predominates, and in zero calcium the hearts stop in systole.

In the arthropods the action of calcium on the pacemaker is predominant, and high calcium slows the heart and stops it in diastole, whereas low $\mathrm{Ca}^{++}$ accelerates the heart, and zero calcium stops it in systole. In Limulus the systolic action of low calcium results from an increase in gross frequency of the ganglionic discharge, an increase in nerve unit frequency, and in the number of active units; in zero calcium there is a tendency to asynchronization. ${ }^{5 n}$ The pacemaker-inhibiting action of high calcium predominates over the muscle-contracting action in the arthropods and molluses (except Pecten), but not in the vertebrates. The pacemaker stimulation of low calcium predominates over the muscle-relaxing effect in arthropods and in Helix, Aplysia, and Octopus, but not in Pecten and Ostrea and not in vertebrates. In the oyster the pacemaker effect of high calcium predominates, whereas the muscular effect of low calcium (or high potassium) is predominant.

Magnesium appears essential for rhythmic beating of the hearts of marine pelecypods and gastropods. Magnesium lacks the muscle-contracting effect of $\mathrm{Ca}^{++}$, but like calcium in excess it inhibits the pacemaker, bringing about diastolic arrest. In molluscan hearts which require $\mathrm{Ng}^{++}$the omission of this element results in acceleration and arrest in systole.

Many of the above effects are referable not to single ions but to ionic ratios; e.g., some high potassium effects are prevented by simultaneously increasing calcium. Although the cellular nature of these ionic effects is unknown, salt studies are useful in separating pacemaker, conducting, and contractile systems.

Effects of Electrical Stimulation on Heart Muscle. The muscle of a vertebrate heart is a syncytium of branched striated fibers. Such a heart (adult or embryonic) is absolutely refractory to electrical stimulation during most of systole; it can be excited (is relatively refractory) at the end of systole and in diastole, but an extra contraction elicited during this time is of submaximal height. Recovery is complete by the end of diastole. Any extra contraction is followed by a compensatory pause longer than the normal diastolic pause (Fig. 211). Any contraction, rhythmic or in response to an electrical stimulus, is maximal for the state of the heart, although a distended heart is capable of enhanced contractions and quiescent strips show "staircase" responses to repeated shocks. The vertebrate heart, therefore, contracts in an all-or-none fashion. When tetanized the heart shows spasmodic uncoordinated contrac- 
tions seattered locally over its musculature, the condition of "delirium cordis." Anodal polarization results in relaxation of the heart.

The muscle of molluscan and arthropod hearts is usually striated, sometimes transversely and sharply, sometimes spirally and weakly. ${ }^{13 .}$ In general the muscle fibers of invertebrate hearts are shorter and less branched than those of vertebrate hearts. Molluscan and arthropod hearts differ strikingly from vertebrate hearts in their responses to electrical stimulation. ${ }^{33 .}$ 17: The muscle of these hearts is excitable at all phases of the cycle, but the threshold is high in systole, approaching absolute refractoriness in early systole,

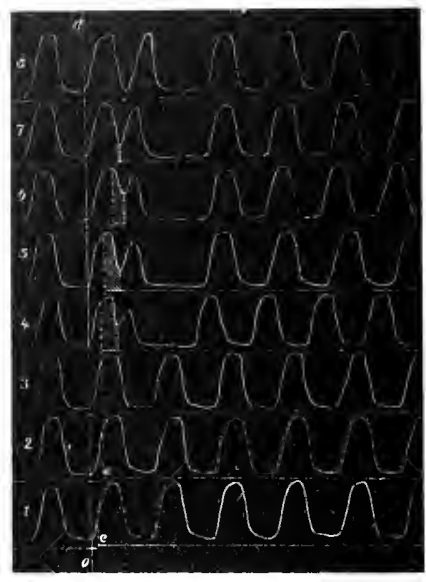

Fig. 211. Electrical stimulation at different times in the cardiac cycle of the frog heart. After Marey from Fulton."

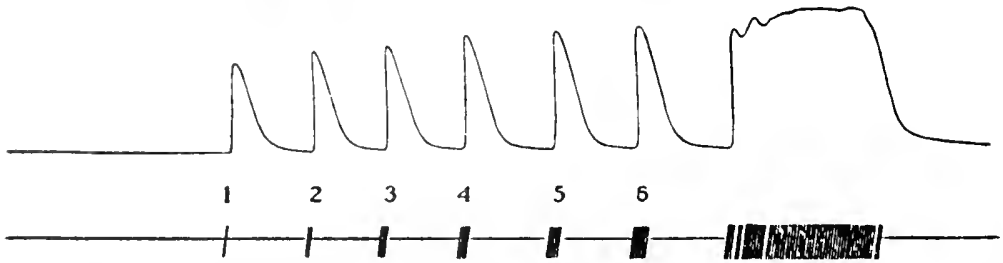

Fig. 212. Responses of Limulus heart to stimulation of lateral nerves. Numbers indicate number of stimuli; note increasing response and final tetanus. From Carlson. ${ }^{3 i}$

and recovery of excitability is gradual during the contraction (Fig. 212). A strong stimulus applied during systole can elicit a contraction of greater amplitude than the normal heart beat. Further, an extra contraction is not followed by a corresponding compensatory pause. Most molluscan and arthropod hearts can be tetanized. These hearts, therefore, do not contract in an allor-none fashion; a normal contraction is never inaximal. Certain intensities of shock applied to mollascan hearts during svstole can result in diminished contraction, $3:$ the explanation of this phenomenon is not clear. Carlson obscrved the above behavior in hearts of Mytilus, Cardinm, Ariolimax, Pali. nurus, Limulus and other species. Similar responses to electrical stimulation have been noted in the lobster, ${ }^{97}$ cockroach, ${ }^{213}$ Limulus, ${ }^{172}$ and Anodonta and 
(Inio. ${ }^{1 \times 9.190}$ In Octopus, ${ }^{16 i}$. "ix also, a shock applied during systole may prolong or heighten the contraction, extrasystoles are not followed by a com pensatory pause, and the heart can be smoothly tetanized. Anodal polarization slows or stops hearts of Leptodora, Daphnia, ${ }^{16 .}$ and Murex ${ }^{16}$ in diastole like the hearts of vertebrates.

In Limulus the cardiac ganglion contains two types of newe fiber, large fibers which show a refractory period of $4-6$ msec. and smaller fibers with a refractory period of 500-600 msec. $^{.7}$

The differences between arthropod-molluse hearts and vertebrates hearts in response to electrical stimulation and in all-or-none properties may be due to differences in the syncytial nature of the heart muscle. The histological correlations with response are poorly known, and research should be extended to additional animal groups.

\section{CONCLUSIONS}

To be of adaptive value a circulatory system must respond to stress; blood must be available where it is needed in adequate amounts when required. Circulatory mechanisms differ greatly in their efficiency of response to stress. Open systems-coelom, pseudocoel, or hemocoel-without hearts are adequate where respiratory and nutritional demands are not great. Such pools of fluid can more adequately distribute oxygen than acquire it. With increase in body size and activity hearts are added to some open systems, as in numerous crustaceans and molluscs. These hearts are incapable of developing high pressures, however, and circulatory flow is largely accomplished by the tone of body muscles. The blood volume is large and the transport of oxygen from gills to muscle is relatively slow. Some annelids have in addition a system of closed blood vessels, particularly for oxygen transfer, but the coelom remains the main circulatory mechanism for excretion and nutrition. The inefficiency of the open system is solved in insects by reduction of the hemocoel to minor functions and by the carrying of air directly in tubes to the muscles; this permits high activity in a compact body. In the cephalopods and vertebrates part of the circulatory system became closed off in vessels, with the result that systemic blood pressure is high, velocity of blood flow is also high, and blood volume is low (not known for cephalopods). The lower vertebrates retained a sort of open system, the lymph system, in which fow is aided by accessory lymph hearts; in higher vertebrates the lymphatics are of much less importance. Among vertebrates there are complex reflex and hormonal systems for the maintenance of blood pressure; the extent of similar regulating systems among invertebrates is unknown.

Heart muscle in all animal groups has some properties of visceral and some properties of somatic muscle. The ability of a heart as a whole to contract in all-or-none fashion may be related to the degree of its syncytial structure. Vertebrate hearts differ strikingly in this respect from mollusc-arthropod hearts. The property of rhythmic activity is inherent in many nerve cells and visceral muscles. It is not surprising, then, that cardiac pacemakers are diverse and it is possible that the causative chemical rhythms are similar in al! pacemakers. Myogenicity, which is more primitive, is retained in adult vertebrates and molluscs but is replaced by neurogenicity in most adult arthro. pods. 
Nervous regulation of heart beat has evolved numerous times, sometimes with augmenting fibers, sometimes with inhibiting fibers, and sometimes with both. There is no evidence for nervous or hormonal control of peripheral blood vessels among any invertebrate groups. Activity in cardiac nerves appears to be associated with liberation of chemical mediators, identification of which is sure only for vertebrates. The effect of acetylcholine on a heart depends on the innervation of the heart.

The evolution of circulatory mechanisms shows many parallel developments, each tending to make a given volume of fluid serve more efficiently the transport needs of the animal. Circulatory mechanisms emphasize the bifurcation of the phyletic tree and the great gap between vertebrates and prochordates (ascidians).

\section{REFERENCES}

1. Alexandrowicz, J. S., Ztschr. allg. Physiol. 14:358-376 (1913). Cardiac ganglia of invertebrates.

2. Alexandrowicz, J. S., J. Comp. Neurol. 41:291-309 (1926). Innervation of the cockroach heart.

3. Alexandrowicz, J. S., Quart. J. Micr. Sci. 75:181-249 (1932). Innervation of heart of decapod Crustacea.

t. Aок1, K., Sci. Rep. Tohoku Univ. 5:717-738 (1930). Pulsation of earthworm vessels.

5. Armstrong, P. B., J. Physiol. 84:20-32 (1935). Acetylcholine action and development of vagus, Fundulus heart.

6. Arvanitaki, A., Cardot, H., and Lee, T., J. Physiol. Path. Gén. 32:36-43 (1934). Electrocardiogram of crabs.

7. Arvanitaki, A., Cardot, H., and Lee, T., J. Physiol. Path. Gén. 32:761-774 (1934). Electrocardiagram of Helix.

8. Asıworth, J. H., Proc. Tr. Liver. Biol. Assoc. 18:209-326 (1904). Morphology of circulatory system of Arenicola.

9. Babkin, B. P., Bowie, D. J., and Niciolls, J. V., Contr. Canad. Biol. E Fish. N. S. 8:209-219 (1933). Circulatory reactions of skate, Raja, to drugs.

10. Bace, Z. M., Bull. Acad. Roy. de Med. de Belg., Cl. Sc. 20:1042-1061 (1934). Physiology of heart of ascidian, Ciona.

11. Bace, Z. Ml., Arch. Internat. Physiol. 38:138-159 (1934). Drug effects on squid heart. lbid. 42:24-42 (1935). Cholinesterase distribution.

12. BaCQ, Z. Ml., Biol. Rer. 22:7391 (1947). Cholinergic and adrenergic systems in invertebrates.

13. Bain, W. A., (uart. J. Exper. Physiol. 19:297-308 (1929). Drug action on crustacean hearts.

14. ВАКкЕR, N. C., Ztschr. Biol. 59:335-365 (1912). Electrocardiogram, eel.

15. Barcroft, J., and Barcroft, H., Proc. Roy. Soc. Lond., B. 96:28-42 (1924). Blood volume, Arenicola.

16. Barchofi, J., and Barron, D. II., J. Exper. Biol. 22:63-74 (1945). Blood pressure in fetal sheep.

17. Bannes, T. C., Textbook of General Physiology (1937). Philadelphia, Blakiston. $55+\mathrm{p}$. $\left(\mathrm{Ch}_{1} .13\right.$, and appendix. Temperature characteristics of hearts.

18. Baику, A., I. Exper. Zool. 91:119-130 (1942). Beat of chick heart fragments.

19. Baýom, E. R., Biol. Bull. 83:165-172 (19+2). Acetylcholine on Daphnia heart.

20. Bein.li, R. N., and Scott, F. I., Am. I. Physiol. 91:265-27+ (1929).

21. Bekinle, J., and Petitfrene, C., Arch. Internat. Physiol. 39:98-111 (1934). Automaticity of heart of Anodonta.

22. Bormen, Mi. A., I. Mar. Biol. Ass. U. K. 17:709-738 (1931). Blood volume, Planorbis.

23. Brocuen, F., Ann. Soc. ent. France 89:209-232 (1920). Circulation in insects.

24. Brochen, F., Arch. Zool. 74:25-32 (1931). Respiration and circulation in insects. 
25. Brücke, E. T. v., in Winterstein's Handbuch (1912). Jena, G. Fischer. Vol. I, pp. 827-1110. Circulation of blood and lymph.

26. Brǘcкe, E. T. v., and Satake, J., Ztschr. vergl. Physiol. 1 t:28-38 (1912). Blood pressure in lobster.

27. Budington, R. A., Biol. Bull. 6:311-312 (1906). Nerve regulation of Venus heart.

28. Brnon, F. B., and Wilson, C., J. Physiol. 93:301-304 (1938:. Blond presure in rat.

29. Cardot, H., C. R. Soc. Biol. 86:1006-1010, ibid. 87:1193 (1922). Ionic effects on Helix heart.

30. Carlson, A. J., Am. J. Physiol. 12:67-74 (1904). Nervous pacemaker in Limulus heart.

31. Carlson, A. J., Am. J. Physiol. 14:16-53 (1905). Nerrous regulation of molluscan hearts.

32. Carlson, A. J., Am. J. Plyysiol. 15:207-234 (1906). Salts on Limulus heart rhythm.

33. Carlson, A. J., Am. J. Physiol. 16:85-99 (1906). Electrical stimulation of in vertebrate hearts.

34. Carlson, A. J., Am. J. Physiol. 17:177-210 (1906). Drug action on Limulus heart.

35. Carlson, A. J., Am. J. Physiol. 18:149-155 (1907). Stimulating action of tension on Limulus heart.

36. Carlson, A. J., Am. J. Physiol. 22:353-356 (1908). Nerve cells in hearts of Nereis and Arenicola.

37. Carlson, A. J., Ergebn. Physiol. 8:371-462 (1909). Review of physiology of invertebrate hearts.

38. Carlson, A. J., and Meek, W. J., Am. J. Physiol. 21:1-10 (1908). Embryonic heart rhythm in Limulus.

39. Carter, G. S., A General Zoology of the Invertebrates (1940). New York, Macmillan. 509 p. Ch. 12, pp. 224-235. Types of transport systems.

40. Criao, I., Biol. Bull. 64:358-382 (1933). Electrolytes on Limulus heart.

41. Chapman, G., and Newell, G. E., Proc. Roy Soc. Lond., B. 134:431-455 (1947). Hydrostatic pressure in Arenicola.

42. Clark, A. J., J. Physiol. 47:66-107 (1913). Ions on frog heart.

43. Clark, A. J., Comparative Physiology of the Heart (1927). Cambridge Univ. Press. $157 \mathrm{p}$.

44. Cole, W'. H., Helfer, R. G., et al., Physiol. Zool. 12:393-399 (1939). Ions on cravfish heart.

45. Cole, W. H., and Kazalski, L. A., Bull. Mt. Desert Isl. Biol. Lab. 40 (1939). Ions on lobster heart.

46. Conant, F. S., and Clark, H. L., J. Exper. Med. 1:341-347 (1896). Regulating nerves to crab's heart.

47. Conklin, R. E., Am. J. Physiol. 95:91-97 (1930). Blood volume and pressure in frog.

48. Courtice, F. C. J. Pliysiol. 102:290-305 (1943). Blood volume in mammals.

49. Crescitelli, F., and Jahn, T. L., J. Cell. \& Comp. Physiol. 11:359-376 (1938). Electrocardiogram, grasshopper.

49a. Cullis, W. C., and Lucas, C. L. T., J. Physiol. 86:53P-55P (1936). Action of acetylcholine on aneural chick heart.

50. Cuntis, H. J., Fed. Proc. 8:30-31 (1949), also Am. I. Physiol. 159:499-504 (1949). Action potential of heart muscle.

51. Daly, I. B., Et al., J. Pliy'siol. 54:367-383 (1921). Action of ions on frog heart.

52. Danielli, J. F., Biol. Rev. 19:81-94 (1944). Structure and permeability of capillaries.

53. Davenport, D., Physiol. Zool. 14:178-185 (1941). Drugs on heart of Cancer.

54. Davenport, D., Physiol. Zool. 22:35-44 (19+9). Drugs on heart of cricket, Stenopelmatus.

55. Davenport, P., et al., Biol. Bull. 79:498-507 (1940). Pharmacology of hearts of Ariolimax and Astacus. 
56. Day, E. C., J. Exper. Zool. 28:307-335 (1919), ibid., 34:45-65 (1921). Relation of central ganglion to heart of ascidian.

57. Derrickson, M. B., and Amberson, W. R., Biol. Bull. 67:329 (1934). Blood volume, lower vertebrates.

58. Dubursson, M., Arch. Biol. 38:9-21 (1928). Pressure in Maja circulation.

59. Dubuisson, M., Arch. Internat. Phy'siol. 40:181-188 (1934). Electrocardiograms, crab, dogfish, frog.

60. Dukes, H. H., The Physiology of Domestic Animals (1942). 1thaca, N. Y., Comstock. pp. 132-150. Blood pressure.

61. Duwez, Y., C. R. Soc. Biol. 122:84-87 (1936). Electrocardiogram, Dytiscus.

62. Edwards, D. J., Am. J. Physiol. 52:276-283 (1920). Contraction of Limulus heart.

63. Elger, M., Pflig. Arch. ges. Physiol. 151:1-51 (1913). Analysis of electrocardiograms.

64. Esser, W., Arch. Biol. 45:377-390 (1934). Histology of Anodonta.

65. Evans, C. L., Ztschr. Biol. 59:397-414 (1912). Electrocardiogram, Helix.

66. Federighi, H., J. Exper. Zool. 50:257-294 (1928). Contraction of Rouget type cells in annelid vessels.

67. Folgia, V. G., Proc. Soc. Exper. Biol. E Med. 46:598-601 (1941). Necessity of lymph hearts for survival of toads.

68. FRÉdérice, H., Arch. Internat. Physiol. 14:126-151 (1913). Physjology of heart of Octopus.

70. Frédérice. H., Arch. Internat. Physiol. 49:299-304 (1939). Nerves and caffein on heart, Aplysia.

71. Frédérice, H., Bull. Acad. Roy. de Med. de Belg., Cl. Sc. 25:611-623 (1939). Nervous regulation of heart, Eledone.

72. Frédérice, H., Biol. Rev. 22:297-314 (1947). Nervous control and drug effects on molluscan hearts.

73. Frédérice, H., and Bace, Z. M., Arch. Internat. Physiol. 49:490-496 (1939). Effects of visceral nerve on cephalopod heart.

74. Frédérice, H., and Bace, Z. M., Arch. Internat. Physiol. 50:169-184 (1940). Acetylcholine and nerves on cephalopod heart.

75. Freudenstein, K., Ztschr. wiss. Zool. 132:404-475 (1928). Heart and circulation of honeybee.

76. Fru, H. J. B., J. Physiol. 39:184-216 (1909). Nervous regulation of heart of cephalopods.

77. Fry, H. K., Quart. J. Exper. Physiol. 7:185-192 (1914). Blood volume, frogs and lizards.

78. Fucris, S., Pflig. Arch. ges. Physiol. 60:173-204 (1895). Circulatory physiology in cephalopods.

79. Gamble, F. W., and Asmwontil, J. H., Quart. J. Micr. Sci. 41:1-42 (1898). Anatomy of Arenicola.

80. Garrey, W. E., J. Cell. E Comp. Physiol. 1:209-223 (1932). Electrocardiogram of Limulus.

81. Garrey, W. E., Am. I. Plysiol. 136:182-193 (1942). Acetylcholine on Limulus heart gangtion.

82. Gaskele, J. F., Phil. Tr. Roy. Soc. Lond., B. 205:153-210 (1914). Pharmacology of blood ressels of leech.

83. Crmenen, C. Wr., Bull. Ul. S. Bur. Fish. 24:429-456 (1904). Blood pressure of salmon.

84. Hantiton, H1. L.. 1. Cell. E Comp. Physiol. 13:91-104 (1939). 1)rugs on grasshopper heart (Melanoplus).

85. Ifant, J. S., Proc. Fla. Acad. Sci. 7:221-246 (1944). Blood pressure and cardiac output of fish.

86. Hecht. S., Am. J. Physiol. 45:157-187 (1918). Heart beat, Ascidia.

87. Hlinвески, P., Am. J. Physiol. 117:686-700 (1936). Discharge of pacemaket ganghon, Limulus.

88. Hernecker, P., and Bhsiop, G. 11., Am. J. Physiol. 114:212.223 (1935). Dis. charge of pacenaker, Limulus. 
89. Herrick, F. H., Bull. U. S. Bur. Fish. 29:149-408 (1909). Norpholugy of cir culatory system, lobster.

90. Heymans, (.., Arch. Internat. Pharm. et Thér. 28:337-377 (1923). Fffects of jons and drugs on Aplysia heart.

91. Hoff, H. E. Howell's Textbonk of Physology (John F. Fulton, ed.) (1946). Philadelphia, Saunders. (h. 35 , pp. 717-780. Cardiac cycle.

92. Hoffinan, P., Arch. Anut. u. Physiol. 135-17+ (1911), also Ztschr. Biol. 59:297313 (1912). Electrocardiograms of invertebrates.

93. Hogben, L. T., Quart. I. Exper. Ply'siol. 15:263-312 (1925). Ions on hearts of Homarus, Aplysia, Helix and Pecten.

94. Hogben, L. T., Comparative Physiology (1926). N. Y., Macmillan. 219 p.

95. Hocbex, L. T., and Homsox, A. D., J. Exper. Biol. 1:487-500 (1924). Drugs on hearts of Maja and Pecten.

96. Hopper, J., ET AL., J. Clin. Invest. 23:628-635 (1944). Blood volume, man, dog.

97. Hunt, R., et Al., Zentralbl. Physiol. 11:274-278 (1897). Response of lobster heart to electrical stimulation.

98. Hunter, G. W'., Anat. Anz. 21:241-246 (1902). Histolugy of heart of Molgula.

99. Hunter, G. W., Am. I. Physiol. 10:1-27 (1903). Heart beat in an ascidian, Nolgula.

100. Hyde, J. H., Am. J. Phyiol. 23:201-215 (1908). Respiration, heart rate and blood pressure in skate.

101. Inama, C., Masters thesis, Univ. of Illinois (1947). Blood pressure in animals with open circulatory systems.

102. Irving, L., Scholander, P. F., Et. Al., Am. J. Physiol. 135:557-566 (19+2). Arterial pressure in seal.

103. Inving, L., Solandi, D. Y., and Solandt, O. M., J. Pliysiol. 84:187-190 (1935). Eranchial pressure receptors in dogfish.

104. Irving, L., Mrman, L. C., and Lutz, B., Biol. Bull. 62:17-22 (1932). Adrenalin in blood pressure of elasmobranch.

105. Jahn, T. L., and Koel, B. S., Ann. Ent. Soc. Amer. 41:258-266 (1948). Temperature on grasshopper heart.

106. Jullien, A., Des réactions comparées des coeurs de vertébrés et d'invertébrés vis a ris des electrolytes et des drogues (1936). Paris, Baillière. $210 \mathrm{p}$.

107. Jullien, A., C. R. Soc. Biol. Paris 12t:650-652 (1937). Drugs on Aplysia heart.

108. Jullien, A., and Marduel, H., C. R. Soc. Biol. Parsi. 127:62 I-623 (1938). Electrical polarization of heart of snail, Murex.

109. Julliex, A., Vincent, D., et. al., C. R. Soc. Biol. Puris. 129:667-670 (1938). Drugs on heart of Helix.

110. Jullien, A., Vincent, D., et. Al., Ann. Physiol.e. Physicochim. Biol. 14:567-574 (1938;. Drugs on molluscan hearts.

111. Jürgens, O., Zool. Jalurb., Abt. allg. Zool. n. Physiol. 55:1-46 (1935). Circulation ir. polychactes.

112. Катz, L. N., Electrocardiography (1947). Philadelphia, Lea \& Febiger. 883 p.

113. Kexs, A., and Batemax, B., Biol. Bull. 63:327-336 (1932). Branchial responses to adrenalin in eel.

114. Koenring, V., Bull. Mt. Desert Isl. Biol. Lab. 25-26 (1937). Heart beat in clams.

15. Knieger, H., Storaasli, J. P., et. al., Proc. Soc. Exper. Biol. E Med. 68:511-515 (1948). Blood volume, several methods, dog.

116. Krocin, A., The Anatomy and Physiology of Capillaries (1927). Yale Unir. Press 422 p.

117. Krogr, A., Osmotic Regulation in Aquatic Animals (1939). Cambridge Univ Press. pp. 65-98. Water distribution crustacea, thiocyanate space in Eriocheir.

118. Kruts, V., C. R. Soc. Biol. Paris 119:608-610 (1935). Drugs on heart Sepia.

119. Kruta, V., C. R. Soc. Biol. Paris 122:582-585 (1936). Cardiac effects of visceral nerves in cephalopods.

120. Lampont, H., in Howell's Textbook of Physiology (John F. Fulton, ed.) (1946) Philadelphia, Saunders. pp. 630-659. Hemodynamics.

121. Landis, E. M., Am. J. Physiol. 93:353-362 (1930). Capillary pressure in mam. mals. 
122. LemmanN, G., Tabul. Biol. 1:107.150 (1925). Summary, circulatory constants, many aninusls.

123. Lfitcil, I., J. Physiol. 50:370-379 (1916). Blood volume of Planorbis.

124. [ewis, T., and Marvin, 11. M., Heart ]t:27-4] (1927). Mechanics of vasodilatation.

125. Lindenas, I. F., Physiol. Zool. 1:576-592 (1928). Ions on crayfish heart.

126. Loswi, O., Pflig. Arch ges. Physiol. 189:239-2+2 (1921), ibid. 206:123-130, 135-1 to (192t). Humoral transmission by vagus in heart.

127. Loewi, O., and Navratil, E., Pflig. Arch. ges. Physiol. 193:201-213 (1922) Liberation of acctyleholine on vagal inhibition of frog heart.

128. Lussida, A., J. Physiol. Path. (ién. 30:593-603 (1932). Electrocardiograms from Octopus.

129. Lutz, B. R., Biol. Bull. 59:170-178, i79-186, 211-216, and 217-221 (1930) Innervation of heart and cardiac reflexes in elasmobranch Scyllium.

130. Lutz, B. R., and Wrana, L. C., Biol. Bull. 62:10-16 (1932). Reflex cardiac inhibition in dogfish, Squalus.

131. Lyon, E. P., J. Gen. Physiol. 8:279-287 (1926). Circulation in sharks.

132. Maclean, M. N., and Beznak, A. B., Arb. Unger. Biol. Forsch. 6:258-263 (1933). Drugs on Astacus heart.

133. Maloeuf, N. S. R., Ann. Ent. Soc. Amer. 28:332-337 (1935). Automatism of insect hearts.

134. Maluf, N. S. R., J. New York Ent. Soc. 47:227-286 (1939). Review of circulatiom in insects.

135. Marceau, F. C. R. Acad. Sci. Paris 138:1177-1179 (1904), ihid. 139:150-152 $(190+)$. Histology of molluscan hearts.

136. Martin, A. W., Fed. Proc. 6:16+ $(19+7)$, also personal communication. Blood volumes of elasmobranchs and teleosis.

137. Nayer, E., Ztschr. Morph. Ökol. Tiere. 22:1-52 (1931). Circulation, pressure, dragonfly nymphs.

138. Mcl)owall, R. J. S., !. Physiol. 104:392+403 (19+6: Acetylcholine stimulation, mammalian heart.

139. Mclndoo, N. E., J. Comp. \eut. 83:1+1-145 (1945). Innervation of insect hearts.

140. McKay, M. E., Contr. Canad. Biul. \& Fish. N.S. 7:17-29 (1931). Drugs on circulation of skate.

1+1. Millatan, N., Mister's thesis, Brown University (1938). Heart physiology of Galleria.

1+2. Mines, G. R., J. Phrsiol. +3:+67.506 (1912). lons on hearts of Peeten and of elasnobranchs.

1+3. Mines, G. R., J. Phisiol. 46:188235 (1913). Ca and Mgeffects on heart.

1+t. Moiley, I1. L., Physiol. Zool. 7:62-84 (1934). Physiology and pharmacology of heart of freshwater mussels.

145. Vagel, H., Ztschr. vergl. Physiol. $21:+68+91$ (1934). Thiocyanate space of Carcinus.

146. Navez, A. E., Mém. Mus. Nat. Mist. Belg. 3:701-737 (1936). Cardiac rhythm in molluse, Anomia.

147. Nukada, S., Mitt. Med. Fak. Kais. Univ. Mokyo 19:1 16+ (1917). Automaticity if lieart of Limulus.

148. Nusbaun, J., Biol. Centr. 19:700711 (1899). I listolegy of circulation in Crus. taceis.

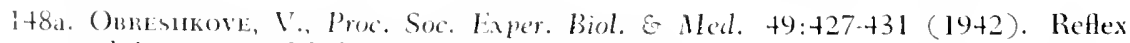
inhibition, acetylcholine action, 1)aphuia heart.

149. ()rs, A. B., Phisiol. Zool. 15:+18+35 (19+2). Drugs and ions on oyster heart.

150. Pavitr, E. E., Prosisen, (. L., ra at., To be published in National Nuelear linergy Serices (19+6). Blond pressure and hlood volume in dog and rabbit.

151. Purlen, W', and Remenibaugil, W. A., J. Morphol. 16:91-200 (1899). Anatomy (1) limulum lecart.

152. P'icken, 1.. F. R., J. Ixper. Biol. 13:309-328 (1936). Blood pressure in Crustacea.

153. Pickeninc; J. W., J. Phrsiol. 18:470 483 (1895). Muscarine on chick cmbryo he'arts. 
154. Plateau, F., Arch. Biol. 1:595-695 (1880). Drugs on lobster heart.

155. Pratt, F. H., and Re1d, M. A., Proc. Soc. Exper. Biol. E Med. 29:1019-1021 (1932). Automatism and synchronism of lymph hearts.

156. Prosser, C. L., Biol. Bull. 78:92-102 (1940). Acetylcholine and nervous inhibition, Venus heart.

157. Prosser, C. L., Biol. Bull. 83:145-16+ (1942). Action of acetylcholine on neurogenic and myogenic hearts.

158. Prosser, C. L., J. Cell. E Comp. Physiol. $21: 295-305$ (1943). Pacemaker discharge, Limulus heart.

158a. Prosser, C. L., Biol. Bull. 98:254-257 (1950). ECG in Arenicola.

159. Prosser, C. L., lnada, C., et al., Anat. Rec. 99:601 (1947). Pressure in open circulatory systems.

159a. Prosser, C. L., and Weinstein, S. J. F., Physiol. Zool. 23:113-124 (1950). Blood volume in frog, cattish, crayfish and mussel.

160. Prosser, C. L., and Zimnerman, G. L., Physiol. Zool. 16:77-83 (1943). Drugs on hearts, Arenicola, Lumbricus.

161. Ransom, W. B., J. Physiol. 5:261-341 (1883). Cardiac rhythm of invertebrates, particularly molluses and tunicates.

162. Reichert, E. T., and Brown, A. P., Carnegie Inst., Wash., Publ. 116, 338 p. (1909). Blood volume data.

163. Reid, M. A., J. Exper. Zool. 76:47-65 (1937). Activity of transplanted lymph hearts.

164. Reid, M. A., Personal communication (1947). Drugs on lymph hearts.

165. Reinmüiller, J., Pflïg. Arch. ges. Physiol. 236:561-567 (1935). Electrical polarization of hearts.

166. Retzius, G., Biol. Unters. 12:75-78 (1905). Nerve cells on blood vessels of polychaetes.

167. Richardson, C., Burdette, A. C., et al., Ann. Ent. Soc. Amer. 24:503-507 (1931). Blood volume, larvae of Bombyx, Galleria.

168. Rijlant, P., C. R. Soc. Biol. Paris 113:917-920 (1933), ibid. 109:38-42 (1932). Automatism of heart, spiders, crayfish.

169. Rodbard, S., and Feldman, D., Proc. Soc. Exper. Biol. E Med. 63:43-44 (1946). Blood pressure, turtle, chicken.

170. Rogers, C. G., Textbook of Comparative Physiology (1938). New York, McGraw Hill. 715 p.

171. Root, W., Gregersen, M., ex al., Am. J. Physiol. 146:739-755 (1946). Blood volume of mammals.

172. Samoiloff, A., Am. J. Physiol. 93:186-189 (1930). Electrical stimulation of Limulus heart.

173. Schafer, G. D., Stanford Univ. Publ. Biol. Sci. 3:307-337 (1923). Pressure in hemocoel of insects.

174. Schoenlein, C., and Willem, V., Bull. Scient. France e. Belg. 26:442-468 (1894), also Schoenlein, C., Ztschr. Biol. 32:511-547 (1895). Circulatory pressure, elasmobranchs.

175. Schultze, L. S., Jen. Ztschr. Natur. 35:221-328 (1901). Heart of Salpa.

176. Scudamore, H. H., Tr. Ill. State Acad. Sci. 34:238-240. (1941). Sinus gland hormone on crayfish heart.

177. Shannon, E. W., and Wiggers, C. J., Am. J. Physiol. 128:709-715 (1939). Dynamics of frog and turtle heart.

178. Sкranilik, E. v., Ztschr. vergl. Physiol. 14:675-681 (1931). Pacemakers of fish hearts.

179. Sкramlik, E. v., Ergebn. Biol. 11:1-130 (1935). Circulation in fishes.

180. Skramlik, E. v., Ergebn. Biol. 18:88-286 (1941). Circulation in molluscs and ascidians.

181. Smith, C., and Glick, D., Biol. Bull. 77:321 (1939). Cholinesterase in hearts.

182. Smith, R., Biol. Bull. 93:72-88 (1947). Action of nerves and drugs on heart of Cancer.

183. Spealman, C. R., Am. J. Physiol. 136:351-354 (1942). Ions on frog heart.

184. Steiner, G., Ztschr. vergl. Physiol. 16:290-304 (1932). Automatism and regulation, heart of Periplaneta. 
185. Straub, W., Pflïg. Arch. ges. Physiol. 103:429-449. (1904). Dynamics of circulation in Aplysia.

186. Stubl, H., Pflitg. Arch. ges. Physiol. 129:1-34 (1909). Rhythm of earthworm pulsating vessels.

187. Tакатsuk1, S., Sci. Rep. Tohoku Univ., Ser. IV, 8:21-29 (1933). Physiology of oyster heart.

188. Taylor, 1. R., and Walzh, E. M., J. Cell. E Comp. Physiol. 18:278-280 (1941). Electrocardiogram of oyster.

189. Ten Cate, J., Arch. Néerl. Phy'siol. 8:43-84 (1923). Physiology and pharmacology of Anodonta heart.

190. Ten CATE, J., Ztschr. vergl. Physiol. 10:309-316 (1929). Electrical stimulation, heart of mussel.

191. Trigt, H. v., Ztschr. Biol. 62:217-242 (1913). Electrocardiogram of eel.

192. Uexкull, J. v., Ztschr. Biol. 44:269-344 (1903). Hydrostatic pressures in Sipunculus.

193. Ungar, G., C. R. Soc. Biol. Paris 123:1065-1067 (1936). Evidence for tyraminic nerves in cephalopods.

194. Ungar, G., and Zerling, M. P., C. R. Soc. Biol. Paris 120:754-756 (1935). Humoral basis of cardiac regulation in cephalopods.

195. Vincent, D., and Jullien, A., C. R. Soc. Biol. Paris 127:631-632 (1938). Cholinesterase in hearts.

196. W'att, R. B., Biol. Bull. 85:79-85 (1945). Acetylcholine on Venus heart.

197. Walling, L. V., Kansas Univ. Sci. Bull. 4:359-367 (1908). Histology of grasshopper heart.

198. Walzl, E. M., Physiol. Zool. 10:125-140 (1937). Ions on oyster heart.

199. Waterman, A. J., Physiol. Zool. 15:61-74 (1942). Drugs on heart of ascidian, Perophora.

201. Wells, G. P., J. Exper. Biol. 5:258-282 (1928.) Electrolytes on Maja heart.

202. Welsh, J. H., J. Exper. Biol. 16:198-219 (1939). Acetylcholine on decapod hearts.

203. Welsh, J. H., Physiol. Zool. 12:231-237 (1939). Acetylcholine and adrenalin on Panulirus heart.

204. Welsh, J. H., J. Cell. \& Comp. Physiol. 19:271-279 (1942). Acetylcholine on heart, Homarus and Carcinides.

205. Wiersma, C. A. G., and Novitski, E., J. Exper. Biol. 19:255-265 (1942). Nervous regulation of crayfish heart.

206. Wigglesworth, V., The Principles of Insect Physiology (1939). London, Methuen. $43+\mathrm{p}$. Ch. X. The circulatory system; types and attachment of insect hearts.

207. Willems, H. P. A., Ztschr. vergl. Physiol. 17:1-100 (1932). Automaticity and electrocardiogram, Helix.

208. Wolf, E., J. Gen. Physiol. 16:89-98 (1932). Temperature on heart of Ciona.

209. Woolard, H. H., J. Anat. 60:345-373 (1926). Innervation of vertebrate heart.

210. Wu, C. H., and Visscher, M. B., Fed. Proc. 6:231 (1947). Blood pressure in mouse.

211. WyMan, L. C., and Lutz, B. R., Biol. Bull. 62:17-22 (1932). Adrenalin on blood pressure of dogfish, Squalus.

212. YazAK1, M., Sci. Rep. Tohoku Univ., Ser. IV, 5:403-414 (1930). Circulation and pressure in holothurian, Caudina.

213. Yeager, J. F., J. Agr. Res. 59:121-137 (1939). Electrical stimulation of cockroach heart.

214. Yeager, J. F., and Gaham, J. B., J. Agr. Res. 55:1-19 (1937). Nicotine on heart, Periplaneta.

215. Yeager, J. F., and Tauber, O. E., Ann. Ent. Soc. Amer. 25:315-327 (1932). Blowel volume, Periplaneta.

216. Young, J. Z., Quart. J. Micr. Sci. 75:571-624 (1933). Autonomic nervous system of selachians.

217. Yung, E., Arch. Exper. Zool. 9:421-444 (1881). Drugs on molluscan hearts. 
218. Zawarzin, A., Ztschr. wiss. Zool. 97:481-510 (1911). Histology of heart of dragontly nymphs.

219. Zoond, A., and Slome, D., J. Exper. Biol. 6:87-95 (1929). Ions on hearts, Palinurus, Octopus.

220. Zuckerkande, E., Master's thesis, Univ. Ill. (1948), also Biol. Bull. 98:161-173 (1950). Blood pressure in some marine animals.

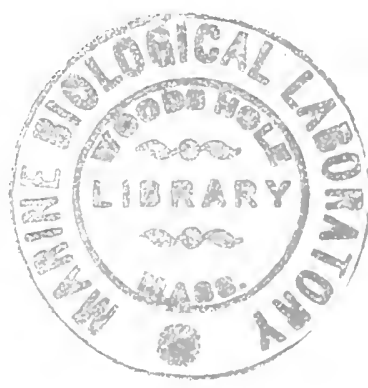




\section{Muscle and Electric Organs}

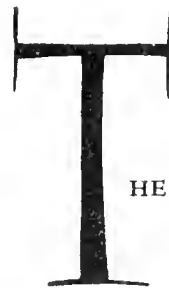

HE SPEED OF LOCOMOTION of an animal, and hence its ability to get food and to escape from predators, is partly limited by the reaction time of its muscles. In addition to locomotion, muscles perform functions associated with digestion, excretion, and reproduction, and most animals have muscles of several characteristic reaction times.

Contractile fibers occur in Protozoa, as, for example, the myonemes of Vorticella and Stentor. In sponges, contractile epidermal cells can close the oscula, while animals of all higher phyla have cells specialized for contraction. The cellular pattern of muscle evolved very early, and even coelenterates have some striated fibers.

The cellular mechanism of contraction and relaxation resides in certain fibrous proteins which can undergo reversible folding. ${ }^{6,}$ 100, 218 How these proteins differ in fast and slow muscles is totally unknown, and a comparative study of the contractile protein, actomyosin, might well give evidence regarding the fundamentals of contraction.

A comparative study of muscles suggests that their properties can be conveniently grouped into five general categories: (1) time relations of contraction-contraction rate, time constants of excitation, conduction rate, refractory period, and frequency of stimulation for fused response; (2) dependence on facilitation (summation of nerve impulses); (3) maintenance of tension, relaxation rate, and tonus; (4) spontaneous rhythmicity; and (5) chemical compounds in activation and liberation of energy.

\section{GROSS FUNCTIONS OF MUSCLE}

Muscles have been classified in many ways and no single classification is entirely satisfactory. We can first put muscles into two groups in terms of their function in the animal:

1. Muscles with origins and insertions on skeletal structures, either endoskeletal or exoskeletal, or on skin. These muscles are generally phasic, or muscles of movement. Examples are the muscles moving body appendages such as legs, wings, and mouth parts, muscles extruding or retracting a proboscis or tentacles, and muscles closing the valves of a pelecypod. Many muscles occur in antagonistic pairs-contraction of one reflexly inhibiting contraction of the other, one causing movement in one direction, the other causing movement in the opposite direction; other muscles pull against an elastic ligament, for example, clam adductors. Usually phasic muscles form part of a lever system and function by shortening (isotonic contraction) or by developing tension while at a constant length (isometric contraction). No 
muscle functions purely isotonically or isometrically, but individual nuscles can approach these conditions.

2. Muscles arranged around hollow structures, lacking strict origins and insertions, one portion of the muscle inserting into and hence pulling on another portion of itself. These are predominantly "holding" muscles. Among the vertebrates the smooth muscles of the bladder, the ureter, the stomach, and the intestine, and striated muscle such as the sphincter ani and esophageal muscle and the heart are circular muscles, as are also the body wall muscles of annelids, holothurians, and other "hohlorganartige Tiere," as Jordan calls them. In general, muscles of hollow structures are slower than phasic inserted muscles; they also occur in pairs, e.g., the circular and longitudinal muscles of the body wall of annelids, producing reciprocal movements. These muscles contract against sacs of fluid rather than against skeletal or cuticular structures.

Muscles are also classified as tetanic (rapidly contracting) and tonic (holding). ${ }^{44}$ Frequently the same muscle can perform both functions. Some muscles with fixed attachments are tonic, as the clam adductors, but in general the tonic properties are more common among the muscles enclosing cavities.

Muscles of both of the above categories are under reflex control, the tetanic more so than the tonic. Within a muscle the motor nerve fibers branch extensively among muscle fibers. A group of muscle fibers together with the motoneurone which innervates it constitutes a motor unit. In mammals several hundred muscle fibers may be served by one motor nerve fiber. In crustaceans and in some molluscs one motor nerve fiber may supply an entire muscle (see p. 596). The extent to which visceral muscles are organized in motor units is not clear. The smooth muscles of vertebrate blood vessels and pilomotors behave as if they were arranged in motor units. ${ }^{49}$ By contrast, in intestinal, uterine, and cardiac muscle, and probably in the molluscan foot, conduction occurs from muscle cell to muscle cell or by a nerve plexus. Many muscles receive several types of innervation, separate motor innervations for fast contractions, for slow contractions, and sometimes for inhibition.

If movement is to be useful not only must it be performed at a suitable rate, but it must be capable of gradation in rate and strength by one or more of the following mechanisms: (1) The grading of morement in a muscle organized in units is regulated from the central nervous system by varying the number of units active. (2) Activity in all types of muscle is graded in part by variation in frequency of motor stimulation of individual units, high frequencies eliciting strong contractions. (3) Another method of gradation is possible wherever multiple innervation occurs; different nerve fibers cause contractions of different rates, or cause either local or all-or-none contractions, and inhibitory fibers diminish the effect of excitatory fibers.

\section{TIME RELATIONS OF MUSCLE}

Histological Correlations: Electron Microscopy of Muscles. Vertebrate muscles have long been classified as striated, cardiac, and smooth, and it has long been recognized that the fastest muscles are striated. Muscles 
consist of separate fibers, and analysis of the ultimate contractile elements of muscle is currently being pushed from microscopic to molecular dimensions. Cross striations per se have no direct importance in contraction; non-striated muscles can contract; extraction of myosin, the principal contractile protein, leaves the striations intact. ${ }^{218}$ Yet cross-striated muscles are in general faster than non-striated, and the material of the striations may have important metabolic effects on the contractile proteins. An electron micrograph of a myofibril (Fig. 213) shows that it consists of myofilaments which pass continuously through the A and I bands and which do not fold in contraction. ${ }^{11 .}$. These myofilaments sometimes show regular spacings $400 \AA$ apart, but these spacings are much less regular and sharp than are the $640 \AA$ spacings of collagen (Fig. 213).

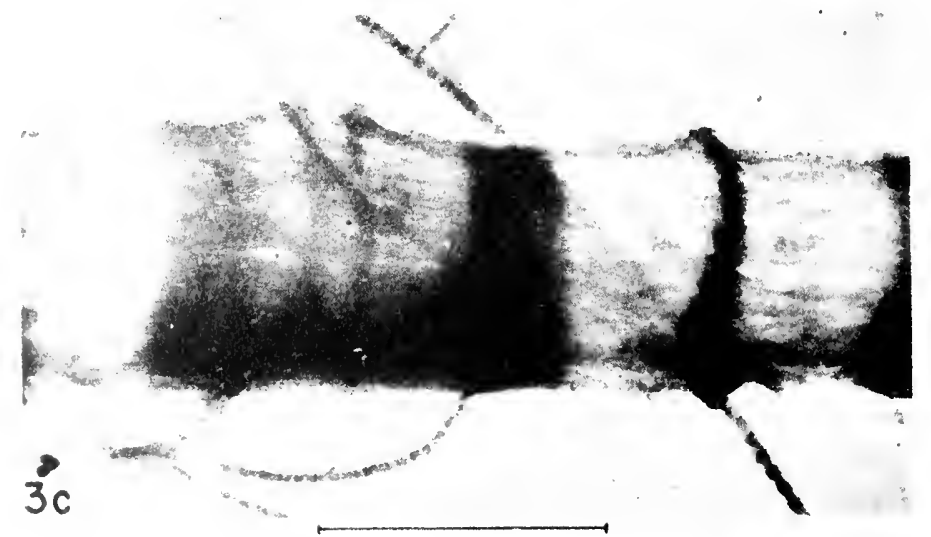

Fig. 213. Electron micrograph of a myofibril of frog sartorius muscle-note filaments in the fibril showing striations. Collagen fibrils cross the muscle fibril. Stained with phosphotungstic acid. Scale 1 micron. From Hall, Jakus, and Schmitt. ${ }^{11.5}$

Chemical extracts of the contractile protein of striated muscle can be divided into two parts, actin and myosin, which combine to form the contractile protein, actomyosin. Discussion of this important field of biophysics is beyond the scope of this book, but molecular threads have been obtained which may be the same as the contractile units of muscle. Figure 214, A shows fibrous actin prepared by polymerization of globular actin and alignment of the threads gives the appearance of striae. Fibrous actin threads (Fig. 214, B) strikingly resemble threads obtained by maceration of muscle in a Waring blendor (Fig. 214, C). Molluscan smooth muscle yields, in addition to "myosin," another protein, paramyosin, with much longer $\mathrm{x}$-ray spatcings ( 1100 \&) than those of "myosin." (Fig. 215.) Paramyosin is present only in "holding" muscles and may not be contractile.

Microscopic Mistology of Muscle. The correlation between the time constants of muscle contraction and muscle histology is striking. Muscles can be arranged in a histological series according to: presence or absence of striations, width and density of striations, fiber length in relation to muscle length, distribution of fibrils and nuclei, proportion of sarcoplasm to fibrils, 

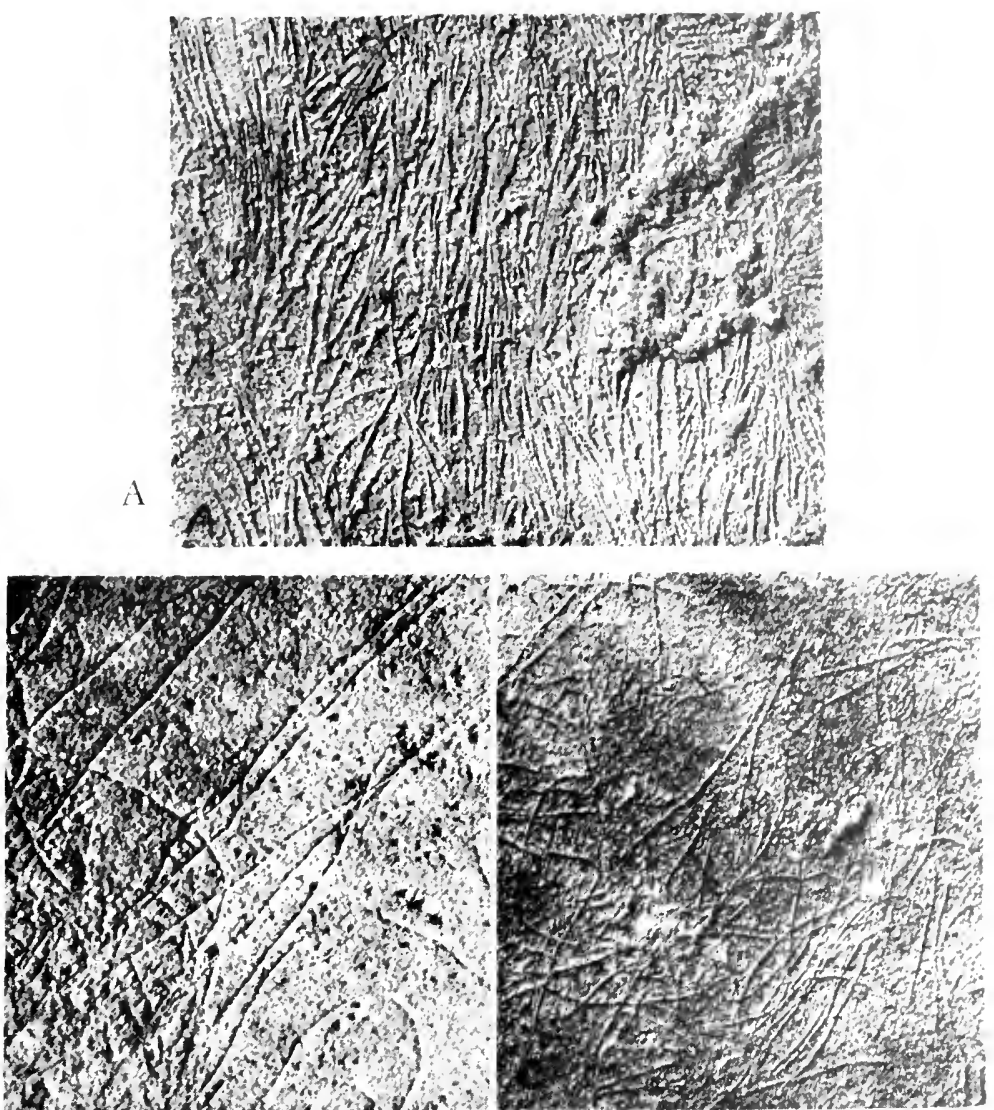

Fig. 217. A, Fibrous actin prepared by polymerization from. G-actin. Note alignment of filaments giving the appearance of striations. 20,000x. B, Fibrous actin. $30,000 \mathrm{x}$. C, Threads prepared by breaking up rabbit muscle in Waring blendor. 20,000x. Note resemblance of actin threads to the filaments obtained from muscle. From Rosza, SzentGyörgi, and Wyckoff. ${ }^{201}$

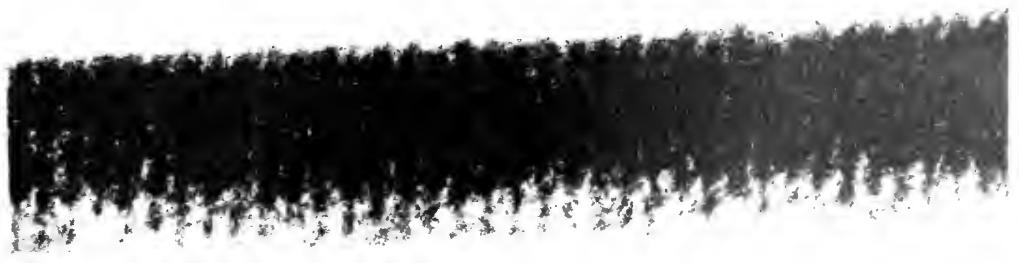

$0.1 \mu$

Fig. 215. Electron micrograph of paramyosin filaments extracted from adductor muscle of Venus, stained with phosphotungstic acid. From Schmitt et al. 
TABLE 70. KEY TO HISTOLOGICAL TYPES OF MUSCLE

\begin{tabular}{|c|c|c|c|c|c|}
\hline Type & Striations & Fibers & Sarcoplasm & Fibrils & Example \\
\hline \multirow[t]{7}{*}{ Striated } & \multirow[t]{6}{*}{$\begin{array}{c}\text { Transverse, } \\
\text { distinct }\end{array}$} & \multirow[t]{4}{*}{$\begin{array}{l}\text { Long, non- } \\
\text { anastomosing }\end{array}$} & \multirow[t]{2}{*}{$\begin{array}{l}\text { Very little, } \\
\quad \text { fibrils } \\
\text { nearly fill } \\
\text { fiber }\end{array}$} & $\begin{array}{l}\text { Distinct, striation } \\
\text { very sharp, } \\
\text { fibers some- } \\
\text { times branched }\end{array}$ & $\begin{array}{l}\text { Arthropod } \\
\text { skeletal } \\
\text { muscle }\end{array}$ \\
\hline & & & & $\begin{array}{l}\text { Abundant, stri- } \\
\text { ations less dense, } \\
\text { nuclei peripheral }\end{array}$ & $\begin{array}{l}\text { Vertebrate } \\
\text { white } \\
\text { muscle }\end{array}$ \\
\hline & & & \multirow[t]{2}{*}{$\begin{array}{l}\text { High sarco- } \\
\text { plasm-fibril } \\
\text { ratio, loosely } \\
\text { packed fibers }\end{array}$} & $\begin{array}{l}\text { Adult muscle: } \\
\text { nuclei usually } \\
\text { peripheral, } \\
\text { sometimes cen- } \\
\text { tral }\end{array}$ & $\begin{array}{l}\text { Vertebrate } \\
\text { red } \\
\text { muscle }\end{array}$ \\
\hline & & & & $\begin{array}{l}\text { Embryonic } \\
\text { muscle: fibrillae } \\
\text { peripheral; } \\
\text { nuclei of ten } \\
\text { central, stria- } \\
\text { tions in process } \\
\text { of differentia- } \\
\text { tion }\end{array}$ & $\begin{array}{l}\text { Vertebrate } \\
\text { embryonic } \\
\text { muscle }\end{array}$ \\
\hline & & \multirow[t]{2}{*}{$\begin{array}{l}\text { Branched, } \\
\text { anastomosing }\end{array}$} & $\begin{array}{l}\text { Intercalated } \\
\text { discs prominent }\end{array}$ & & $\begin{array}{l}\text { Vertebrate } \\
\text { cardiac } \\
\text { muscle }\end{array}$ \\
\hline & & & $\begin{array}{l}\text { No intercalated } \\
\text { discs }\end{array}$ & & $\begin{array}{l}\text { Arthropod } \\
\text { cardiac } \\
\text { muscle }\end{array}$ \\
\hline & \multicolumn{3}{|l|}{$\begin{array}{l}\text { Faint, may } \\
\text { be transverse, } \\
\text { diagonal, zig- } \\
\text { Lag, spiral or } \\
\text { local }\end{array}$} & . & $\begin{array}{l}\text { Molluscan, } \\
\text { annelid, } \\
\text { ascidian } \\
\text { striated } \\
\text { fibers }\end{array}$ \\
\hline \multirow{3}{*}{\multicolumn{2}{|c|}{ Non-striated }} & \multirow{2}{*}{\multicolumn{2}{|c|}{$\begin{array}{l}\text { Long, rarely } \\
\text { branches or } \\
\text { bridges }\end{array}$}} & $\begin{array}{l}\text { Fibrils } \\
\text { readily } \\
\text { seen }\end{array}$ & $\begin{array}{l}\text { Phascolosoma } \\
\text { retractors, } \\
\text { cephalopod } \\
\text { chromatophore } \\
\text { nuscles }\end{array}$ \\
\hline & & & & $\begin{array}{l}\text { Fibrils } \\
\text { not readily } \\
\text { seen }\end{array}$ & $\begin{array}{l}\text { Echinoderm } \\
\text { (Thyone) re- } \\
\text { tractors, } \\
\text { molluscan } \\
\text { adductors } \\
\text { (smooth part) }\end{array}$ \\
\hline & & \multicolumn{2}{|l|}{$\begin{array}{l}\text { Less than } \\
0.5 \mathrm{~mm} \text {. long }\end{array}$} & $\begin{array}{l}\text { Fibrils usually } \\
\text { seen in cross } \\
\text { section, some } \\
\text { interfibrillar } \\
\text { bridges }\end{array}$ & $\begin{array}{l}\text { Vertebrate } \\
\text { smooth } \\
\text { muscle }\end{array}$ \\
\hline
\end{tabular}


and relative amount of connective tissue. Table 70 and Figures 216 and 217 suggest the nature of the histological series.

The cross striations of muscle consist of alternating strongly birefringent and weakly birefringent regions, the anisotropic (A) and the isotropic (I) bands. The $A$ band is normally seen as darker than the 1 band in fresh or fixed tissue. The light I bands are divided by so-called $\mathrm{Z}$ lines, which are transverse boundaries between the structural units, sarcomeres; thus one unit or sarcomere consists of two light areas on either side of a dark one; changes in the width of both bands and in the birefringence occur during contraction (Fig. 216, A). A muscle fiber consists of a bounding sarcolemma, fibrils which are aligned with respect to striations, and a ma-

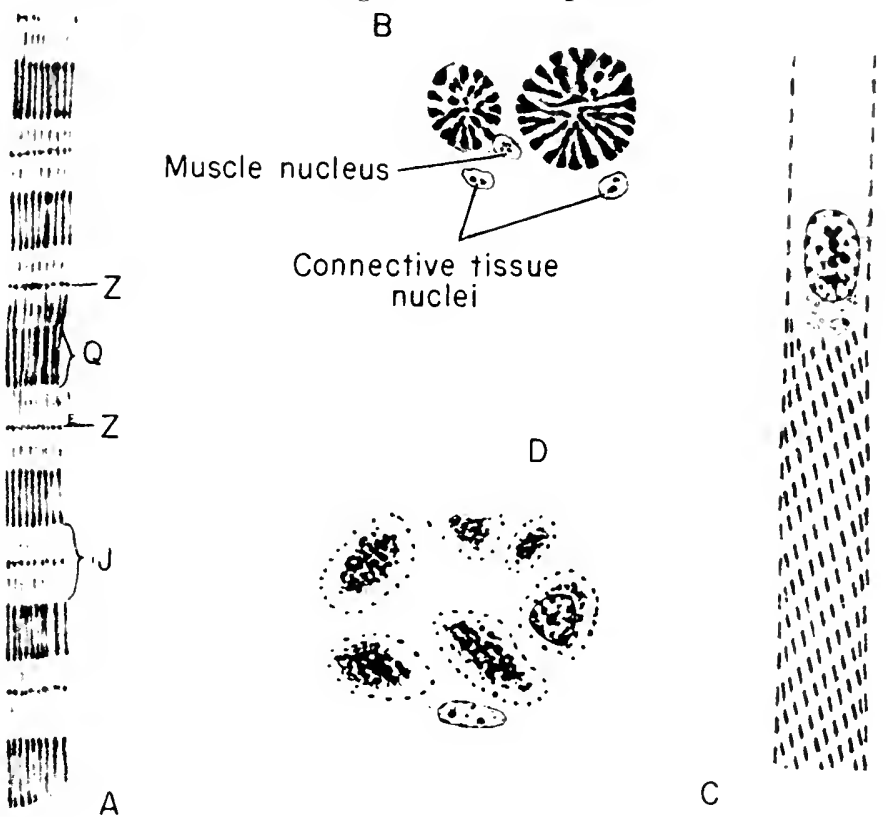

Fig. 216. Views of muscle fibers: $A$, longitudinal, and $B$, cross views of striated fibers from sea-spider (Anoplodactylus). Sharp $Q$ (or A) bands, J (or I) bands with 2 discs. Nuclei peripheral, fibrils nearly fill fibers. After Jordan. ${ }^{131} C$, longitudinal, and $D$, cross views of fibers from heart of Anodonta. Striations diagonal, fibrils peripheral and sparse, sarcoplasm abundant, nuclei central. Marceau. ${ }^{160}$

trix or sarcoplasm. Outside it is a variable amount of connective tissue. The myofibrils are contractile, and the sarcoplasm may also contain some contractile elements. ${ }^{2 \times}$ Smooth muscle fibers of vertebrates contain fibrils which lack striations; optically they are weakly birefringent.

In arthropod limb muscles the striations are sharp and fairly close together, the fibers are long and, in many (e.g., Crustacea) they are branching; the fibrils (sarcostyles) are more separable than in vertebrates (Fig. 216, A). Insect muscles may have striations less than 5-6 $\mu$ apart and very sharp. The fast muscles of flight in a variety of insects exhibit closer striae $(2 \mu)$ than do their slower leg muscles $(+\mu)$; in mandibular muscles striae are still farther apart $(6 \mu) .^{218}$ In other insects the ratio of the 
widths of $\mathrm{A}$ to $\mathrm{I}$ bands approaches $\mathrm{I}$ in faster muscles and is much less than $l$ in slower muscles. ${ }^{53}$ In crabs the striation widths exceed $10 \mu$ but tend to be narrower in the faster muscles of a given individual. ${ }^{130}$ The contractile proteins are distributed throughout the. fiber, hence the material of the striations is not concerned directly with contraction, and there is no clear explanation of why striated muscle is faster than non-striated.
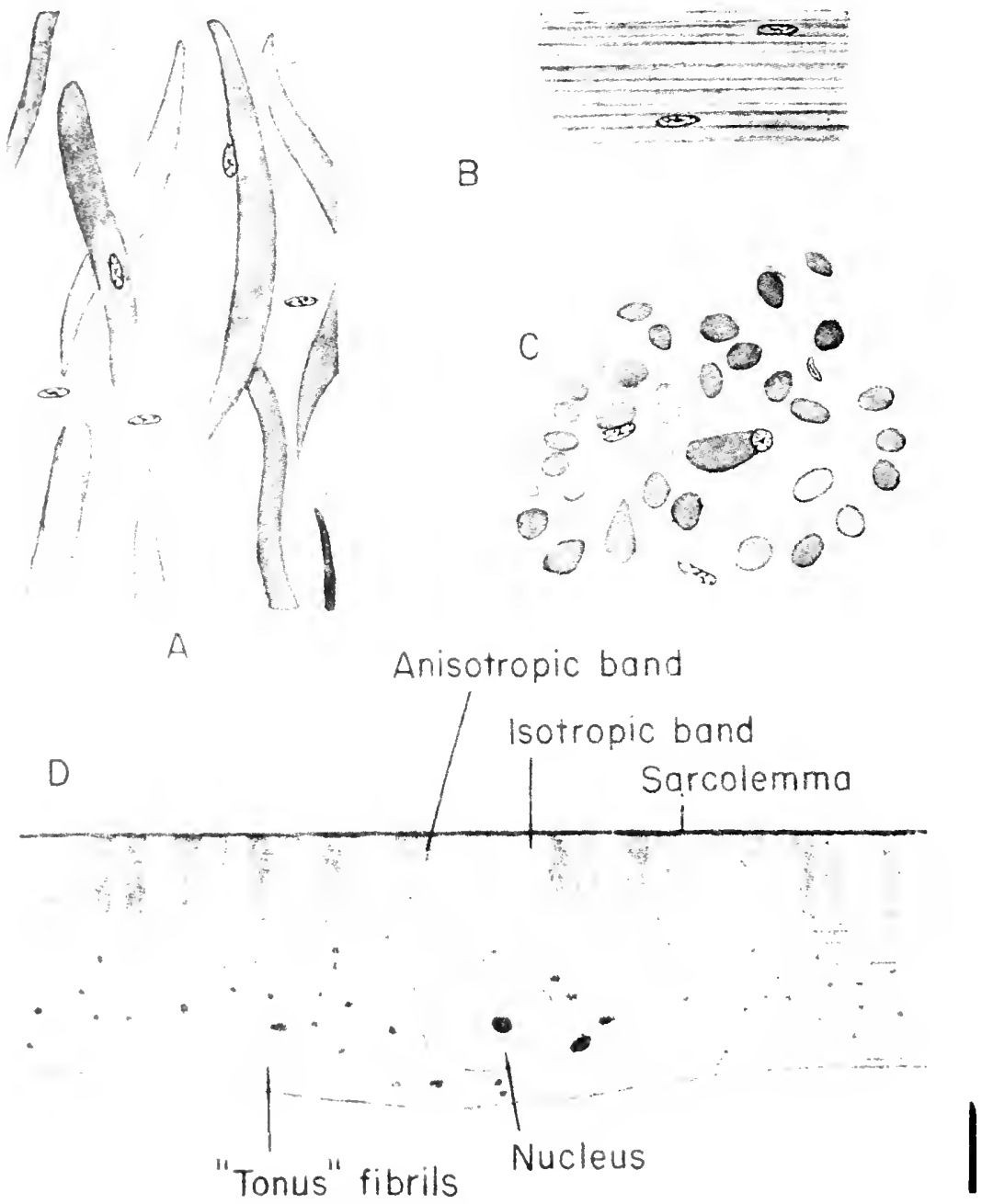

Fig. 217. Views of muscle fibers: fibers of longitudinal retractor muscles of Thyone: longitudinal sections, $A$, in relaxed, and $B$, in contracted state; $C$, cross section in relaxed state. No striations, no fibrils, much connective tissue. Olson. ${ }^{182} D$, Portion of muscle fiber from heart of Ciona. Striated on one side, non-striated on other side. Bozler."

Muscle striations are usually less sharp in vertebrates than in arthropods, sarcomere lengths are usually $2-3 \mu$, and in isotonic contraction they may sometimes be reduced to half this length. In amniotes and in some fish, striated muscle may be white or red. The color is less important than are other characters; in white muscle the fibrils are close together and abun- 
dant; in red muscle there is proportionately much more sarcoplasm, rich in myoglobin (Ch. 9). Muscle nuclei are peripheral in white fibers; some of them are central in red. White muscle is faster and fatigues more rapidly than red muscle. ${ }^{177}, 123$ In the slow-moving sloth all skeletal muscles are of the sarcoplasm-rich, red type (Wislocki, in Hines ${ }^{123}$ ). Vertebrate cardiac muscle consists of long branching and anastomosing red fibers which show striations about $2-3 \mu$ in length and are crossed at intervals by intercalated discs. In vertebrate embryos the muscle fibers are contractile before striations appear, and striations increase gradually in number and in proportion to sarcoplasm. Embryonic muscle shows many of the physiological characteristics of slow muscles ${ }^{208}$. 217 (Table 70).

The muscles of many invertebrates, particularly of annelids, tunicates, molluscs, and coelenterates, are mixed non-striated and striated, and frequently are intermediate in character. Fibers of each type may occur side by side, or a muscle may be mainly striated or smooth. In the heart of Ciona, fibers have been seen which are striated on one side and non-striated on the other (Fig. $217, D^{44}$ ), a condition sometimes seen in embryonic or regenerating amphibian fibers. ${ }^{216}$ In several invertebrate phyla Bozler ${ }^{43}$ distinguished fast (tetanic) fibers with a few large dark fibrils at the periphery, and tonic fibers with smaller fibrils more extensively scattered throughout the fiber. It is probable that fibril distribution may be correlated with many functional properties of muscles. In several animal groups, particularly in annelids, molluscs, and ascidians, spiral striations have been described (Fig. 216, C). The fibrils are peripheral, and the proportionate volume of sarcoplasm to fibrils is high. These muscles are slower than vertebrate red muscle.

The smooth muscles of invertebrates, particularly of molluscs and holothurians (Fig. 217, A), are often long fibered. In the adductors of bivalves or the byssus retractor of Mytilus the non-striated fibers are large and run more than half the length of the muscle. ${ }^{104}$ Fibrils have not been demonstrated histologically in some of these long fibers. The smooth muscles of vertebrates, particularly the visceral smooth muscles, consist of small spindleshaped fibers, often less than a millimeter long and showing fibrils in cross section. In general the short-fibered vertebrate smooth muscles are slower than many invertebrate smooth muscles (Table 70).

Another histological correlation with the course of contraction is the amount of connective tissue in a muscle. The elasticity of a resting frog striated muscle fiber resides partly in the sarcolemma, ${ }^{197,}, 210$ and non-contractile elements such as connective tissue impose a "viscous" resistance to movement. No quantitative data are available, but in many smooth muscles the proportion of connective tissue to sarcoplasm is greater than it is in striated muscle (Fig. 217, A).

More data are needed for an adequate histological-functional correlation in muscle. Table 70 indicates the histological series from fast to slow muscles. The categories in this table are not rigid, and much overlap occurs. Many bizarre types of muscle cells have been described (e.g., in Ascaris ${ }^{44}$ ), about which there is no physiological knowledge. It would be of interest to know more about branched red fibers like those in the tongue of higher ver- 


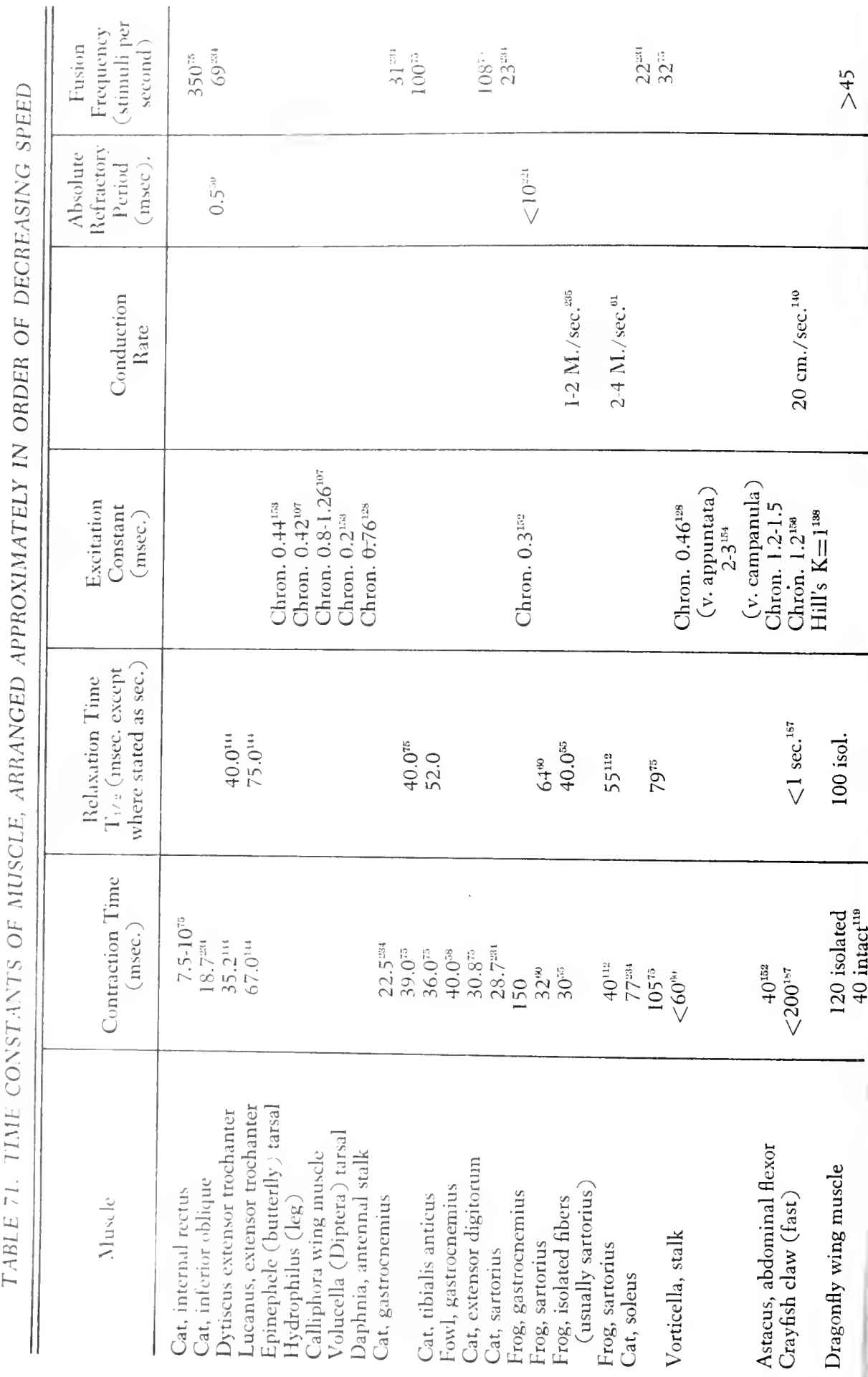


tebrates. Some muscle fibcrs are partly striated and partly smooth (Fig. $217, D)$. In general, sharp, closely spaced striations, many fibrils, a small amount of interfibrillar sarcoplasm, and long fibers are correlated with speed, while absence of striations, abundant sarcoplasm, central nuclei, short fibers, and much connective tissue are correlated with slowness. The size of the sarcomere seems to be less important than the sarcoplasm-fibril ratio.

Time Constants of Contraction and Excitation. The "speed" of a muscle can be measured by several criteria, not all of which are dependent on a common cellular basis. In Table 71 are given representative data on excitation times (e.g., chronaxie), contraction time, relaxation time, conduction rate, fusion frequency, and refractory period in muscles. The values given are subject to certain qualifications. Contraction and relaxation rates of Table 71 are for simple twitches or brief periods of tetanus. Lever systems are not always fast enough to record accurately, hence true contraction rates may be fister than recorded. Since some muscles can contract at several rates, depending on the stimulus (see pp. 596-600), the values in Table 71 are for fast contractions. Relaxation rates also are often a function of the lever system, yet differences in relaxation rates are greater than in

A
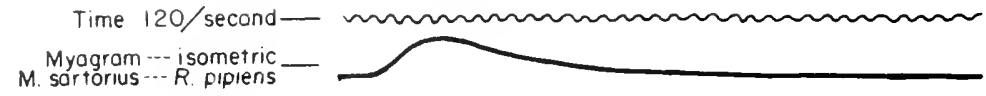

B

Myogram -.. M retractor Penis -- P elegons

Time intervols $0.1 \mathrm{sec}$

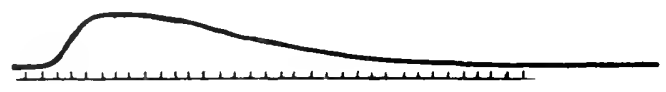

C

Myogram-- circular muscle
Turtle Intestine
Time intervals $6.0 \mathrm{sec}$ -

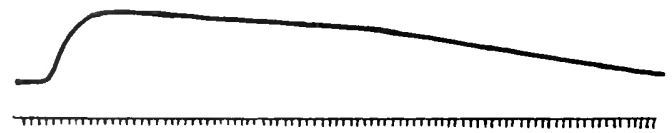

Fig. 218. Contraction curves of three muscles of different rates of contraction and relaxation with adjusted time scales as indicated. From Gilson. ${ }^{112}$

contraction rates of different muscles. Some of the chronaxies are taken with preparations where nerve and muscle excitation could not be distinguished; chronaxies measured with small electrodes are shorter than those measured with large electrodes and tend to approach nerve chronaxies. An attempt is made in Table 71 to select values which are probably muscle chronaxies. Brief contractions of different muscles with adjusted time scales of recording are remarkably similar in shape (Fig. 218).

The contraction rates of some mammalian and bird muscles are faster at $38^{\circ}$ than the recorded rates of other muscles. An eve muscle, e.g., the internal rectus, of a mammal contracts in less than $10 \mathrm{msec}$. A mouse breathes at the rate of 200 times per minute and has a heart rate of 200-300. Bird heart rates are often several hundred per minute, and a humming bird "standing still" in air shows a wing beat 55 times a second. The frequency for smooth tetanic fusion is not known for most of these fast muscles, but for cat internal rectus fusion does not occur below a frequency of 350 per second. 
The flight muscles of insects have very brief twitch durations. Contraction times of $15 \mathrm{msec}$ at room temperature have been recorded, and these are probably longer than the period of contraction in the insect. Wigglesworth"2:32 discusses insect becomotion in detail. Some of the measurements of wing frequency in beats per second given by that authore:3: are: honeybec (Apis) 180-250; bumblebee (Bombus) 130-240; mosquito (culex) 278-307; Ay (Musca) 180-330; butterlly (Pieris) 9, 12; dragonfly (Aeschna) 22, 28; blowfly (Lucilia) 87. In Drosophila repleta the wing beats increase from about 120 sec. at $10^{\circ}$ to 200 sec. at $30^{\circ} .71$ In mosquito flight a complete confraction and relaxation cycle must be completed in about 3 insec. It has been suggested ${ }^{63}$ that in flight the muscles show an incomplete tetanus and that there is alternation among fibers from beat to beat. Leg muscles of insects are somewhat slower (Table 71).

Recent evidence $19: 2$ indicates that in flies the wing muscles are stimu lated by stretch and beat at higher frequencies than are shown by motor impulses from the central nerrous system. The intrinsic rhythm of the wing muscles is modulated by reflexes initiated in part from sense endings in the halteres as discussed in Chapter 14.

Metabolism in rapidly beating flight muscles is very high, and many insects require a warming-up before flight. Most insects and humming birds with fast flight muscles feed on sugar.

Next in speed are the white muscles of vertebrate limbs with contraction times of 25-50 msec., and then the somewhat slower vertebratc "holding muscles," usually red, such as the soleus, with contraction times approaching 100 msec. Other red muscles such as the diaphragm are still slower in contraction. Movements in embryonic muscles, as in a 16-day rat embryo, are slower than they are in adults, thresholds are high, and excitation times are long. Muscle chronaxies of a 5-day chick are reported to be 60 times as long as in the adult fowl. ${ }^{19.5}$

The striated muscles of invertebrates have speeds similar to those of vertebrate red muscle. Crustacean claws, squid mantle, and some coclenterate muscles contract in about $100 \mathrm{msec}$. The adductor muscles of most bivalve molluscs have a striated portion which when isolated contracts in about 100 msec., and a smooth portion which contracts more slowly (Table 71).

It is difficult to get an accurate contraction rate for visceral smooth muscles. Spontaneous or induced contractions are often slow rhythmic waves taking several seconds for contraction. The cat nictitating membrane contraction time is also several seconds. The contraction rate of single vertebrate smooth fibers is not known, and part of the sluggishness of the whole muscle is due to the local nature of contractions.

Conduction at Neuromuscular Junctions and in Muscle. Motor nerve impulses activate muscles by way of a neuromuscular junction. The nature of this junction is not well known except in vertebrate skeletal muscle, in which it is a complex end-plate to which both nerve and muscle contribute (Fig. 236). In smooth muscles the motor nerve fibers branch extensively, surround, and may penetrate the muscle fibers.

An impulse in a motor nerve going to vertebrate striated muscle consists in part of an electrical wave, the action potential, which travels at high 
velocity (Ch. 23). At the end-plate a nerve impulse causes a local depolarization which is manifest as an end-plate potential (e.p.p.). The e.p.p. may be associated with an increase in acetylcholine at the end-plate, either from the nerve endingsiti. ii. $14 i$ or from the muscle sole plate. ${ }^{173 a}$ The local end-plate potential is graded in size and spreads electrotonically for a few

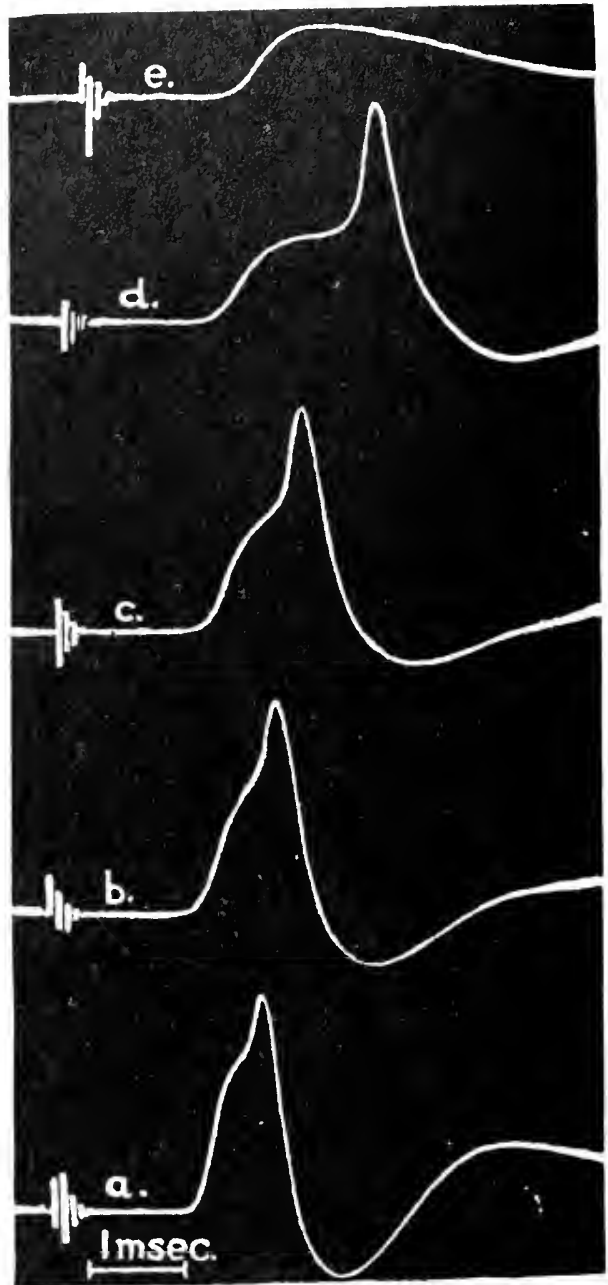

Fig. 219. Action potentials from the region of the end-plate in frog muscle: $a$, before curarization; $b$ to $d$, increasing degrees of curarization; $e$, curarization complete-e.e.p. alone. All or-none propagated spike arises from e.p.p. in a-d. From Kuffler. ${ }^{145}$

millimeters around the end plate, where it sets off the muscle action potential (Fig. 2J9). 'The c.p.p. can be detected only at or near the neuromuscular junction, and under increasing curarization it becomes gradually reduced (Fig. 219). When the endplate potential is reluced by curare to below about 30 per cent of its maximum the muscle impulse can no longer be initiated. ${ }^{1+\pi}$ With two or more nerve impulses the graded e.p.p.s can 
summate. Eserine, which inactivates cholinesterase, enlunces the e.p.p., but has no effect on the muscle impulsc. The e.p.p., then, is graded, is local, can summate, decreases rapidly with distance from the end-plate, and when of sufficient size sets up a muscle impulse which is propagated along the muscle fiber, is all-or-none in height, and is conducted at a measurable velocity. Propagation in frog muscle at room temperature is $1-2 \mathrm{~m}$. $\mathrm{scc}^{2.35}$ (Table 71 ).

In most striated musele fibers there is a marked negative after-poten. tial204, 241 (Fig. 220). Veratrine enhances the negative after-potential; it also may delay relaxation by causing repetitive discharge. ${ }^{14 ;}$, after-potentials can summate and fuse in multifiber preparations, owing to asynchrony. 35

The contractile response to a propagated all-or-none muscle impulse is a twitch. The muscle membrane recovers quickly, more quickly than the muscle can relax, hence the membrane can conduct impulses at high frequencies, kceping the muscle in a contracted state (fused tetanus). In

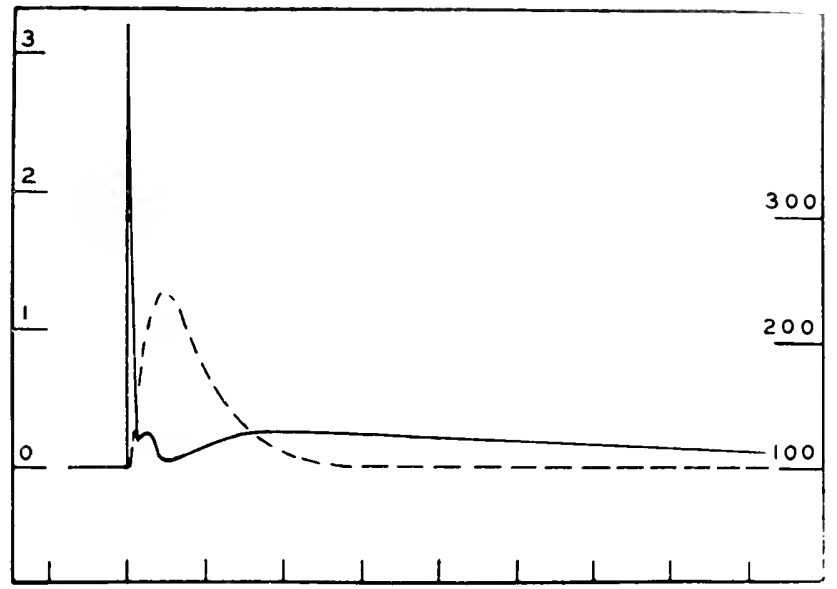

Fig. 220. Action potential (solid line) and myogram (broken line) for sartorius of cat. Rapid propagated spike followed by slow negative after-po+ential. From Rosenblueth and Hoagland. ${ }^{201}$

isolated entire muscle fiber can be stimulated to give all-or-none twitches; ${ }^{197}$ a piece of fiber or intact fibers in poor condition, however, give local contractions which are not propagated. ${ }^{111,5.5}$ In all-or-none twitches the muscle impulse activates the contractile elements over which it passes; in graded local responses the contractile elements are stimulated directly without a muscle impulse. The local contractions are graded, summate, and show no refractory period; hence the all-or-none nature of a muscle contraction resides in the mechanism of conduction rather than in that of contraction. ${ }^{211}$ Under conditions of fatigue, etc., the propagated response may disappear and local contractions occur in the region of a neuromuscular junction ${ }^{99}$ or under the stimulating electrodes. In frog muscles small motor nerve fibers may cause only the graded local type of contraction, large motor nerve fibers the all-or-none propagated twitch (Fig. 229). ${ }^{49}, 150$ How the muscle impulse activates the contractile elements is a major mystery. 
In Crustacea the sraded potentials of the neuromuscular junction contribute largely to the total action potential of the muscle. Their role in contraction will be discussed below.

In long-fibered smonth muscles, such as the anterior retractor of the byssus in Mytilus, impulses appear to be propagated at a rate of $13-20 \mathrm{~cm} . / \mathrm{sec}$. with a simple action potential of duration 2-3 sec. ${ }^{104}$. 10.5 When the muscle is stimulated repetitively the action potentials increase in height (staircase) and at 5-7 per second can fuse. Either there is marked fiber asynchrony or else the action potential, although conducted along the muscle, is not an all-or-none membrane depolarization but resembles more a graded end-plate potential.

In the retractor of the buccal mass of Helix the action potential consists of two wares, fast and slow (Fig. 221). ${ }^{4 \pi}$ When stimulated through the nerve the neuromuscular junction is refractory for $10 \mathrm{msec}$, but thereafter
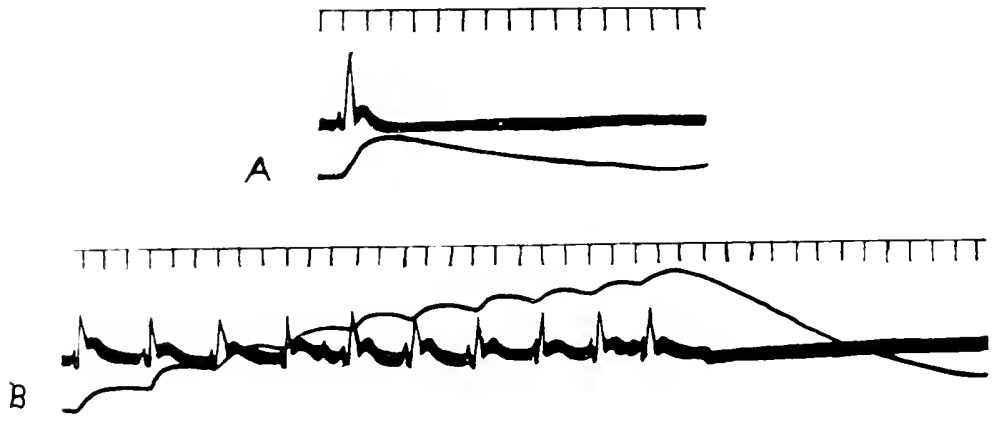

Fig. 221. Action potential of fast and slow waves, and mechanical record from buccal retractor of Helix. A, Response to single stimulus. B, Response to repetitive stimulation at 1 sec., showing summation of contraction. Time in $1 / 5$ second. Bolzer. ${ }^{+5}$

shows supemormal excitability with maximum facilitation at 50 msec.; the muscle potentials in response to stimuli at an interval of about $50 \mathrm{msec}$. summate. W'ith repetitive stimulation at 10 , sec. the muscle potential declines, and at $100 \mathrm{sec}$. there may be continued contraction but no demonstrable action potential. ${ }^{1: 16}$ The retractor muscles of Thyone also show fast and slow clectrical waves:" None of these long-tibered smooth muscles have been examined in regard to conduction in their single fibers.

Short-fibered and branched-fibered muscles present a difficult electrical problem. In the conduction wave of the vertebrate heart there are fast and stow components (Ch. 15). Recent observations ${ }^{\text {-1 }}$ on single cardiac fibers or small bundles show that depolarization persists for tenths of a second, hence the slowness of the muscle may depend on its failure to repolarize guichls. Action potentials have not been analyzed from ans of the shortfibered spiral striated muscles of invertebrates.

Smonth muscles of higher vertebrates are inncrvated by postganglionic autonomic nerses, the ssmpathetics usually liberating an adrenin-like compound, and the parasympathetice liberating acetslcholine. Propagation along a sheet of smootls muscle has been variously claimed to be (1) along each 
muscle cell as in striated muscle, the muscle cells being innervated as motor units (nictitating membrane, blood ressels, pilomotors 1! :21: (2) from muscle cell to muscle cell by protoplasmic bridges (visceral muscles), 49 (3) through the muscle sheet by a nerve plexus ' (4) by diffusion of a mediator (svmpathin) from coll to cell.20.5 Evidence for diffusion comes from the dependence of size of response on number of impulses; maximum tension in striated muscle depends on the number of functioning motor nerve fibers, whereas in smouth muscle the maximum tension when few fibers are stimulated approaches the maximum which obtains when the whole trunk is stimulated. Similar gradation of response occurs with injections of different amounts of adrenalin. ${ }^{20 .}$ Evidence for the first two hypotheses comes from electrical records.

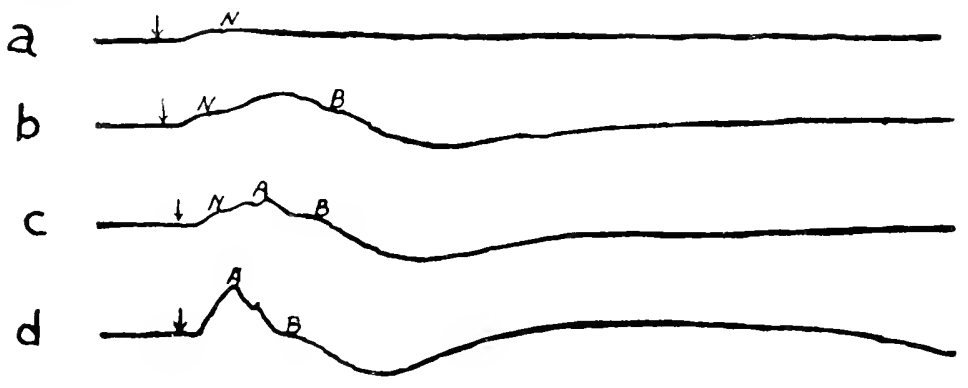

Fig. 222. Action potentials of nictitating membrane of cat: $a$ to $d$, curves resulting from increasing intensities of stimuli. Eccles and Magladerry."

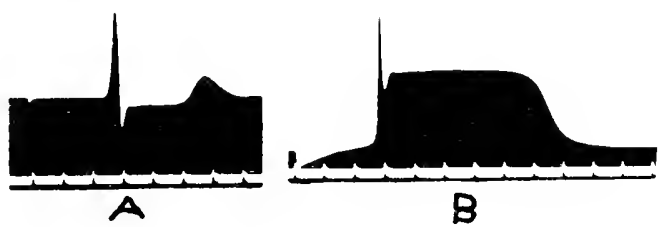

Fig. 223. Action potentials from the ureter of the rat: $A$, dipnasic, and $B$, monophasic record with electrodes $1 \mathrm{~mm}$. apart. Bozler. ${ }^{51}$

In the nictitating membrane of the cat a nerve volley insufficient to elicit a contraction sets up a slow negative wave (N) which may last $10 \mathrm{msec}$. (Fig. 222); threshold volleys elicit larger negative waves which appear to be related causally to contraction ( $A$ and $B$ waves of Eccles and Magladerry, I waves of Rosenblueth et al.:20:3). After these waves a large positive deflection is scen, and thereafter slow rhythmic oscillations may occur. Injected adrenalin elicits rhythmic but less synchronous oscillations than nerve impulses. There is disagreement concerning the parts played by liberation and diffusion of mediator and by muscle cell impulses in this complex electrical response. In visceral smooth muscle records obtained with electrodes close together show monophasic or diphasic spikes resembling the impulses conducted along striated fibers (Fig. 223:1). In addition, a slow non-propagated negative wave may precede a spike, and there may be some residual 
negativity following the impulse (intestine). ${ }^{4 !}$ Under some conditions (e.g., in pendular movement the intestine may show a pre-contraction electrical wave which appears broader when the electrodes are separated; when the electrodes are close together fast spikes conducted in millimeter units are seen.' In the ureter slow waves predominate over fast ones (Fig. 223), but there is much species variation. 50 Rhythmic activity of visceral muscles is accompanied by repetitive discharges, the slow components showing summation. Extension to smooth muscle of the microtechniques used in studying encl-plate potentials in striated muscle should help to elarify the electrical picture.

Striated muscles show graded local mechanical responses which can sum-mate, graded end-plate potentials, propagated all-or-none spikes, and afterpotentials. Just how the "fast" and "slow" electrical waves in smooth museles compare with the striated muscle pattern is not clear. Apparently different components predominate in different muscles and graded potentials may be of widespread importance.

\section{EFFECTS OF REPETITIVE EXCITATION}

In the normal physiology of animals, muscles are rarely stimulated to a response by a single nerve impulse. Repetitive stimulation is the rule. Most muscles develop increased tension with increasing frequency of stimulation, but there is much variation in the magnitude of the frequency effect on tension, and several mechanisms contribute to the effect.

In vertebrate striated muscles a single nerve impulse can elicit an all-ornone contraction. These muscles are non-iterative. In other muscles (e.g., many smooth muscles) repeated stimulation is required to evoke a measurable response; such muscles are iterative. A subthreshold stimulus delivered to any excitable tissue erokes an excitatory process which decays at a rate characteristic of the tissue. The excitatory state from one subthreshold stimulus can summate within a limited time with the excitatory state left from a precerling stimulus. In non-iterative muscles a single nerve impulse sets up sufficient excitatory state at the neuromuscular junction to set off an impulse propagated over the muscle. In iterative muscles facilitation of nerve impulses is usually needed, and the contraction builds up as more impulses are delivered.

When the stimuli applied via the nerves to non-iterative muscles (such as vertebrate striated) are subnaximal, some fibers contract while others are subliminally stimulated, hence repeated stimuli bring into action more and more fibers. When, on the other hand, maximal intensities are used and all the fibers are responding, the tension also increases with increasing freguency but to a less degree than with submaximal shoeks. Possibly the contractile clements do more work, the proteins folding more completely, with repeated excitation. More probably part of the work of contraction is required to orercome certain "viscous" resistance, to align the molecules of sheath and connective tissue; when relaxation is incomplete between contractions. less work is necessary to overcome passive resistance, and when the contractions are smoothly fused maximum energy can go into the development of tension. The tetanus-twitch ratio is higher with sub- 
maximal than with maximal stimulation, because both factors-increased number of fibers and incomplete relaxation-are effective.

Isolated pieces of single frog fibers show summation of the local response, ${ }^{211}$ and isolated entire frog muscle fibers which give all-or-none twitches have tetanus-twitch ratios varying from 1.3 to $6.7 .^{197}$ T'etanustwitch ratios for entire frog muscle with maximal shocks are sometimes greater than 2 (Fig. 224), but in mammalian muscle higher ratios are found, e.g., cat gastrocnemius 3.3 and internal rectus $10.7 .7 .12: 3$ The frequency for fusion is higher in white striated muscle than in red (Table 71). At frequencies 1.5 to 3 times the fusion frequency, the response diminishes.

Not all non-iterative muscles increase in tension with increasing frequency of stimulation. Portions of the squid mantle contract as single motor units. ${ }^{2 \pm 3}$ Mechanical fusion of the response to stimulation through the nerve occurs at 25 per second, but there is no increased tension; as the frequency of stimulation increases the response remains constant (Fig. 225). ${ }^{1933}$

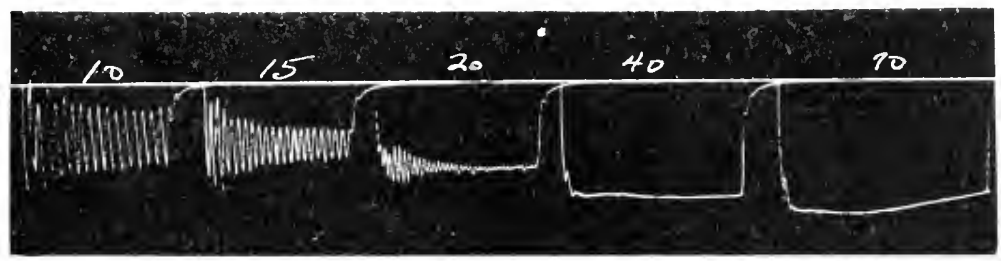

Fig. 224. Isometric contractions of frog sartorius at frequencies given.
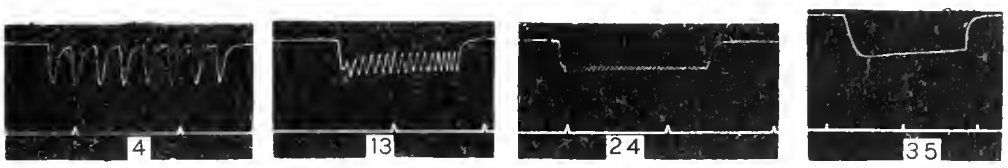

Fig. 225. Contraction of mantle of squid to stimulation of giant fiber of mantle nerve at freouencies stated. Prosser and Young. ${ }^{193}$

Vertebrate cardiac muscle increases in response hardly at all with increasing frequency, and has such a long refractory period that contractions do not fuse (Ch. 15, p. 565). After its refractory period, however, cardiac muscle is hyperexcitable; i.e., its threshold is lower than normal.

Some muscles have properties intermediate between non-iterative and iterative. Many smooth muscles of molluscs, for example, respond to a single stimulus but relax rery slowly, so that contractions build up and tension is held at low frequencies. In the byssus retractor of Mytilus, for example, the tension doubles when the frequency of direct current stimuli increases from one to four per second.

In iterative muscles a single maximal stimulus does not elicit an all-ornone response of the entire muscle; there may be local responses. In addition to the mechanical factor mentioned above for increased responsiveness, two other mechanisms are important, neuromuscular facilitation, and spread of excitation from cell to cell in a sheet of muscle. 
Neuromuseular facilitation is shown by vertebrate striated muscle when it is partially curarized; one end-plate potential is insufficient for a muscle impulse. but by summation an impulse can be started. The muscle is normally non-iterative, but under partial curarization it is iterative. Crustacean muscles, as will be shown below, may respond completely to single impulses in a motor nerve fiber of one trpe but require facilitation when stimulated by another nerve fiber. The claw closer of Limulus responds weakly to single maximal stimuli, but at frequencies of $30-40$ per sec. it develeps 18-28 times as much tension.

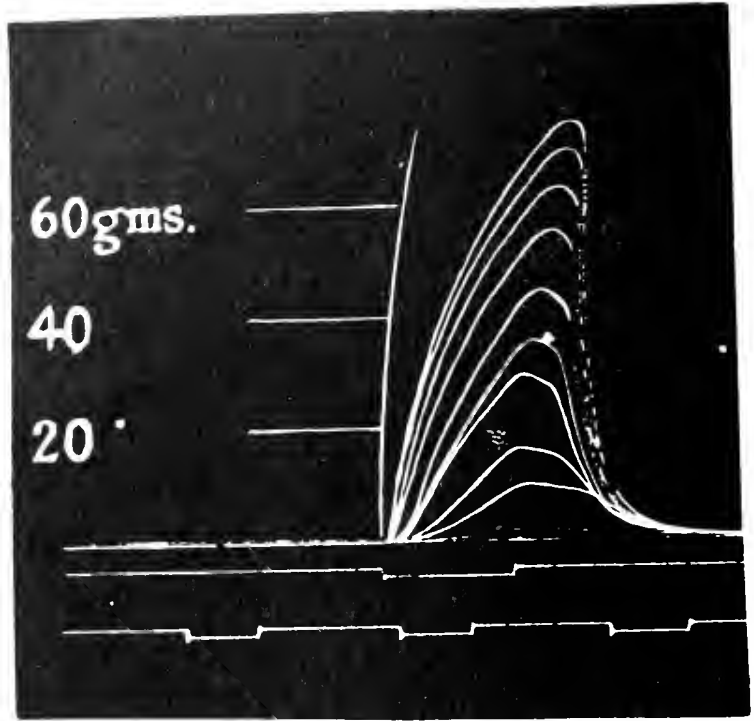

Fig. 226. Responses of flexor muscle of leg dactylopodite of Carcinus to repetitive stimulation. Frequencies, from highest tension, correspond to intervals of $4.0,5.4,7.3$, $9.7,10.6,12.6,15.0,19.7,22.5$, and 30.0 sigma. Pantin. ${ }^{14 \pi}$

092

134

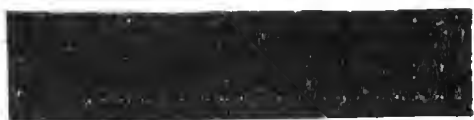

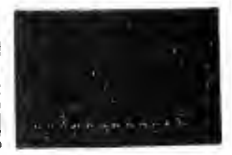
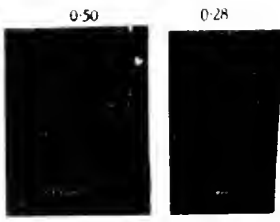

Fig. 227. Response of the sphincter of a sea anemone to repetitive stimuli, intervals between separate stimuli given in seconds. Pantin. ${ }^{1 \text { th }}$

Repetitive stimulation is essential to elicit complete responses of shortfibered muscles where the excitation spreads from fiber to fiber or by a nerve network in the muscle. In vertebrate smooth muscles the meehanical response to single shocks maly be propagated over many fibers (ureter), or it maly be local intestine). The total response increases as more impulses are delivered. In the nictititing membrane of the cat the contraction rate and height of respense increase up to 25-30 per second. ${ }^{1+2}$ In coelenterates 
excitation spreads over a nerve network, and facilitation may be neuro neural as well as neuro-muscular. Relaxation is slow, and as more stimuli are given more of the muscle responds because more junctions are facilitated ${ }^{1 \times 6.66}$ (Figs. 227-228). In both jellyfish and sea anemones a long refractory period in the conducting units prevents any fusion of the mechani-

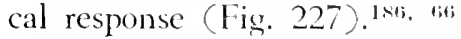

The body wall muscle of the earthworm responds to single shocks but excitation spreads with increasing frequency and fusion occurs at about 14 per second. After a brief tetanus, the responsiveness is enhanced for as long as 30 seconds. ${ }^{+1}$

Long-fibered smooth muscles appear to summate less than short-fibered smooth muscles do. The long retractor of Thyone and the proboscis retractor of Plascolosoma derclop maximum tension (tetanus-twitch ratios of 7 to 10) at low frequencies ( 10 per sec.).

Two general types of mechinism permit increasing contraction with increasing frequency of excitation: the orercoming of mechanical resistance (or greater contractility), and junctional (including interfiber) facilitation. A comparative study of responses to different frequencies would elucidate the basic differences in the importance of these mechanisms.

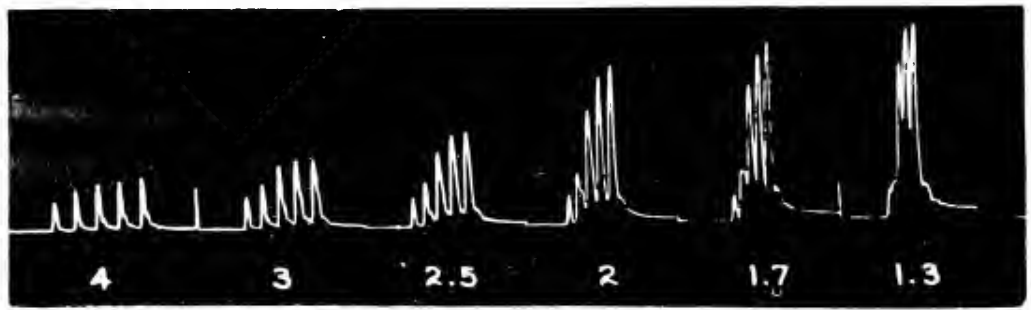

Fig. 228. Responses of jellyfish (Rhopallia) to repetitive stimuli, intervals between separate stimuli given in seconds. Bullock. ${ }^{(t)}$

\section{MULTIPLE INNERVATION; SUMMATION AND INHIBITION}

Many muscles receive dual or multiple innervation. In some the two innervations are antagonistic. The vertebrate heart, blood ressels, and risceral muscles are innervated from the antagonistic sympathetic and parasympathetic systems. Ilere one system is excitatory, the other inhibitory. The intestine is made to contract by parasympathetic and to relax by sympathetic impulses; the systemic arteries are affected oppositely. The retractor penis is contracted by a nerve of sympathetic origin and relaxed by the nerus erigens arising in the sacral cord. A given type of nerve fiber may be excitatory for one muscle and inhibitory for another. The effects may even be reversed by appropriate drugs; hence the state of the effector cells may decide the nature of the nervous effect.

Another type of multiple innervation is found where two excitatory (motor) fibers go to a single muscle fiber. There is evidence ${ }^{44,} 1.50$ indicating that some skeletal muscles of the frog also have, in addition to normal motor innervation, innervation by small nerve fibers which elicit slower, smaller responses. Stimulation of the large-diameter $(15 \mu)$ fibers sets up propagated impulses and fast all-or-none twitches. Stimulation of the 
smaller $(5 \mu)$ nerve fibers elicits local graded potentials, called small-fiber junctional potentials, which summate on repetitive stinulation and may be accompanied by local low-tension contractions, 10-15 per cent of twitch tension in magnitude (Fig. 229). The slow contractions may relax very slowly, but interposition of a twitch response, when repetitive stimulation of the slow nerve fiber has stopped, results in rapid relaxation. The slow contraction develops tension faster with high frequeney than with low frequency stimulation. Whether one muscle fiber receives both types of innerration is not yet certain, but the net effeet of the two types of fiber is peripheral gradation of response, the slow response being "tonic" in character. In mammalian striated muscle, small motor nerve fibers activate intrafusal muscle fibers and so regulate the afferent discharge originating in sensory spindles (Ch. 14).

The most studied examples of multiple motor innervation are in arthropods. Early histological studies ${ }^{15 !}, 126$ showed that whole museles in many crustaceans and insects are innervated by only two (or a few) axons and that each muscle fiber has multiple innervation; i.e., each muscle fiber re-
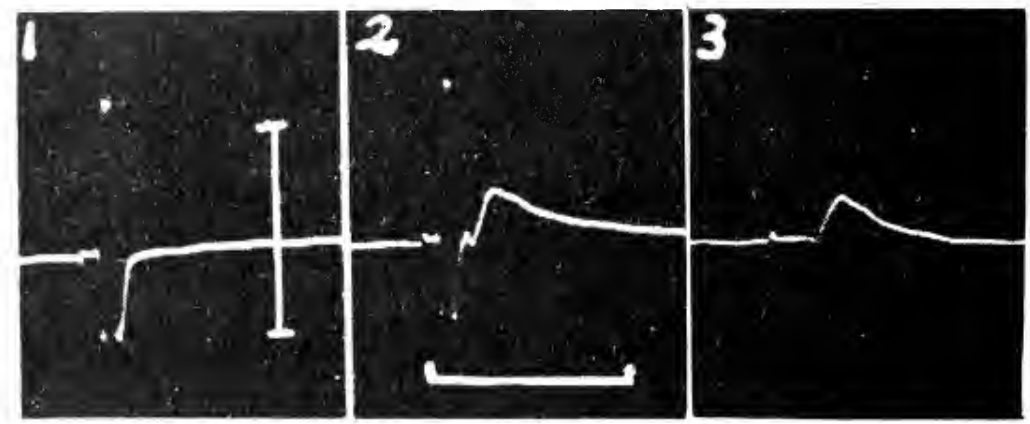

Fig. 229. Response of frog muscle to stimulation of large and small fibers. Action potential from gastrocnemius, progressive pressure block to sciatic: 1, partial block, threshold nerve stimulation, only spike response; 2, increased strength, spike followed by small junction potential; 3 , pressure increased, stimulus as in 2 , only small junction potential remains. From Kuffler and Gerard. ${ }^{1+:}$

ceives branches from two or more nerve libers, often from each of the motor fibers to the muscle. Triple innervation of claw muscles is common anong crustaceans, ${ }^{17}$ and even quintuple innervation has been seen in a muscle of the leg of Panulirus. ${ }^{11}$ It is likely that multiple innervation of single fibers and imnervation of all the fibers of a muscle by the same axon is a general rule among higher crustaceans and insects.

Crustacean neuromuscular systems have several properties which are related to their multiple innervation. First, a single strong shock applied to a crustacean nere sometimes clicits repetitive discharges in the nerve. ${ }^{2}$ This repetitive response depends on the calcium in the medium bathing the nerve. ${ }^{13}$ Second, neuromuscular facilitation is marked with some motor fibers, the contraction increasing in amplitude and rate of rise of tension with increasing frequency (1ig. 226). In addition, stimulation of some motor axons clicits a large fast contraction, whereas other motor axons elicit 
much slower development of tension. Finally, each muscle receives one or more motor (exciting) axons and usually receives one inhibiting axon, the nature of the inhibition differing according to whether the contraction is "fast" or "slow." Thus, in contrast to the vertebrates, which grade movement mostly in the spinal cord, the arthropods show much control of gradation at neuromuscular junctions. A crab "thinks in its claws."

Pantin ${ }^{1 \times 4} .1 \times 7$ analyzed the effects of frequency, duration, and intensity of stimuli to the nerve on responses of walking leg muscles (largely flexors of the dactylopodite) in the crabs Maja and Carcinus. At how frequencies there was little or no response to a single shock, a gream w...nonse alter several shocks (Fig. 226). With intensities just above threshold most units were brought into action at 60-100 shocks per second, and a maximum response occurred at 300 per second. If the frequency or intensity were increased abruptly by 10 to 20 times threshold, or if single strong shocks were inserted in a series of weak ones, the rate and height of contraction increased; i.e., a quick response occurred and the muscle continued to respond at the new height even though the stimuli returned to the original rate. ${ }^{39}$ Pantin contended that such a quick response was due to repetitive nerve impulses started by the interposed stronger stimulus. This would make for economy, as a few extra impulses from the nervous system on a low frequency background could change the response from the slow to the fast type. The order of threshold excitability is as follows: excitor of extensor, inhibitor of extensor and excitor of flexor, and finally inhibitor of flexor.

The functions of the different nerve fibers going to various muscles in many species have been analyzed in detail by Wiersma and his collaborators, particularly van Harreveld and Marmont (summary by Wiersma224). It had been shown earlier ${ }^{15 \%}$. 1+3 that the claw nerve of several crustaceans could be split into two bundles: one containing the large motor fiber caused contraction of the adductor and inhibition of the abductor, the other bundle containing the smaller fibers had the opposite effect. Wiersma and his associates traced the course of individual fibers by methylene blue staining and identified their action by stimulating the separate nerve fibers and recording contractions and muscle action potentials.

The variation in pattern of nerve fiber distribution among different species and in different muscles of one species is remarkable. Some muscles receive one excitatory and one inhibitory fiber; the walking leg muscles studied by Pantin are examples. Other muscles receive two excitor fibers and one inhibitor. ${ }^{117}$ The larger motor fiber causes a contraction of short latency, rapid shortening, and high tension; this response includes an action potential which appears directly with stimulation, and shows little facilita tion. Stimulation of the second or intermediate size fibers causes a contraction which builds up slowly so that there is an apparent long latency, marked facilitation, and low tension; muscle action potentials recorded through holes in the shell are small, may not appear until after several stimuli in a series, and facilitate greatly. Fatigue of the slow system does not at the same time fatigue the fast system. Stimulation of the third, usually smallest, fiber inhibits the response to the motor fibers and is more effective against the slow than against the fast contraction (Fig. 230). During complete 
inhibition no contraction occurs, but facilitation may continue to be built up as shown on release from inhibition.

The inhibitory fiber alone causes no observable electrical response in the muscle. When both slow excitor and inhibitor fibers are being stimulated repetitively (slow excitor as in crayfish abductor) the inhibitor fiber may cause diminution of both contraction and action potential if its impulses arrive less than $10 \mathrm{msec}$. (maximum $2.4 \mathrm{msec}$.) before the excitor impulses (supplemental or electrical inhibition); or the inhibitor may cause reduction of contraction only, if its impulse precedes the excitor impulses by longer times or arrives simultaneously (simple or mechanical inhibition). ${ }^{162}$

The distinction between fast and slow responses is quantitative. The optimum frequency in the slow fiber is lower than in the fast. At low frequencies $(40-50 / \mathrm{sec}$. $)$ the difference in response between the two fibers is slight, but at high frequencies (over 100/sec.) the fast contraction is greater. ${ }^{229}$ The difference between the fast and slow contraction of the same muscle decreases in the genus series: Cambarus $>$ Randallia $>$ Blepharipoda $>$ Cancer, so that in Cancer there is little difference between the two except in

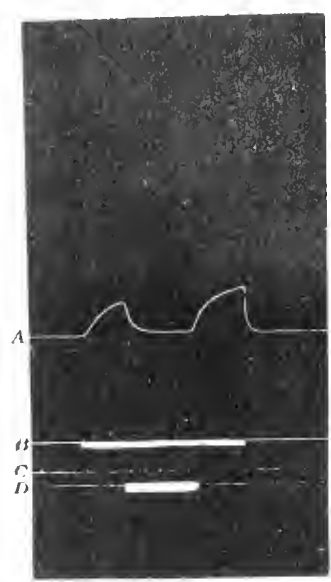

A

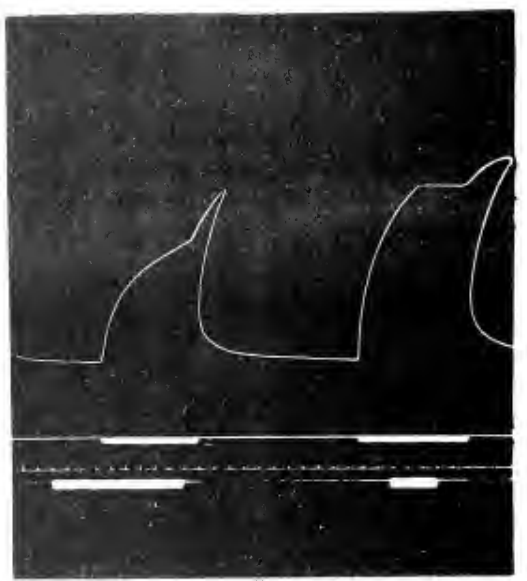

$\mathrm{B}$

Fig. 230. Inhibition of slow contraction (A) and of the fast contraction (B). Records from up downward are myogram, motor stimulus, timer (seconds), and inhibitor stimulus. Nole greater inhibition of slow contraction, postinhibitory facilitation of fast conlraction (from v. Harreveld \& Wiersma ${ }^{12 \pi}$ ).

latency.22, 230 The flexor of the carpopodite of Panulirus has five axons: four excitors and one inhibitor. ${ }^{11 n}$. The amount of facilitation required to elicit a contraction varies with the species. The ratio of optimum inhibitory to excitatory frequencies is fairly constant for a given muscle system. ${ }^{228}$ For eximple, in the slow bender of Pachygrapsus three excitor impulses are suppressed by one inhibitor, in the slow closer response of Cambarus one excitor requires three inhihitor impulses, whereas the fast closers of these species respond to single excitor impulses and are not inhibited. In several instances one nerse axon, either excitor or inhibitor, serves two muscles. Often innervation is not reciprocal, and it is difficult to understand the function of the inhibitors. ${ }^{227}$ 
Wiersma and his associates found that the branching of axon terminations on crustacean muscle is extensive, forming a sort of network. ${ }^{116}$ They postulated that conduction in the muscle is entirely by the nerve elements and that within each muscle fiber there are separate contractile systems each activated by its own nerve fiber. Inhibition is most effective on slow contractions and can act either between nerve impulse and muscle potential or between muscle potential and contraction.

Evidence for a different interpretation of the fast and slow systems has been presented by Katz and Kuffler. ${ }^{140}$, 149 A muscle consisting of many parallel fibers (extensor of carpopodite in crab or crayfish) was exposed and stimulated directly; simple diphasic impulses which were propagated as in

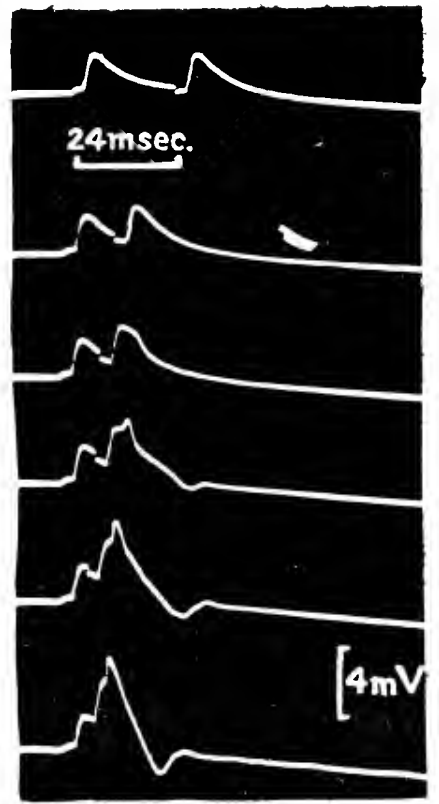

Fig. 231. Electrical responses from extensor of carpopodiıe of crab to paired shocks. Interval between stimuli to fast axon successively from above $24,12,9.6,8,6.4$, and 4.8 sec. End-plate potentials to second shock show summation and propagated spike appears in lower 3 records. From Katz and Kuffler. ${ }^{1+0}$

vertebrate skeletal muscle were recorded. In other preparations some nerve branches were cut, leaving isolated branches intact; when the nerve was then stimulated and a recording electrode moved about on the muscle, local negative potentials with all of the properties of the vertebrate end-plate potentials (e.p.p.'s) were observed (Fig. 231). The fast system has large e.p.p.'s which give rise to conducted muscle spikes; the "fast" e.p.p.'s show little facilitation. The fast system fatigues rapidly, the excitatory process falling to one half in 5 msec. The "slow" system, on the other hand, has small c.p.p.'s which show much facilitation; at frequencies of 50 per sec., for example, the e.p.p. grows to 3 times its initial size, and at 150 per sec. the e.p.p. reaches a plateau 5 to 10 times that at 50 per sec. At low frequencies $(10 / \mathrm{sec}$.), stimulation 
of the slow system elicits no propagated spikes but only e.p.p.'s; although as the frequency increases spikes do appear. When the "fast" system is stimulated, propagated impulses are set up by single shocks and a fast "twitch" occurs. When the "slow" system is stimulated at low frequencies, only local end-plate potentials are recorded and local contractions occur, facilitation is marked; the e.p.p. grows as impulses arrive, some propagated spikes appear at high frequencies and the contraction grows proportionately more than the e.p.p. The e.p.p.'s and associated local contractions are more important than they are in frog muscle because of the much greater number of nerve endings, and the sign and shape of a potential recorded from the muscle s!nface depends on the orientation of the active recording electrode with respect to the loci of the e.p.p.'s. Stimulation of an inhibitory axon ${ }^{149}$ has two effects: (1) reduction in the e.p.p. and consequent suppression of spikes and local contractions, the inhibitory state persisting 20-25 msec., and (2) abolition of the local mechanical response without affecting the e.p.p. Once a propagated impulse is started there can be neither electrical nor mechanical inhibition. The electrical inhibition resembles the action of curare in reducing e.p.p.'s of frog muscle. The mechanical inhibition can summate even at frecpuencies as low as 10 per second.

The motor axons differ in the size of e.p.p. produced and in facilitation. It can be postulated that the "fast" and "slow" fibers liberate different amounts of "exciting" transmitter; however, all attempts to identify chemical mediators in crustacean neuromuscular systems have failed (see p. 614). A modified schematic representation $2=16$, $13 \%$ of the transmission system follows:

Motor Impulse

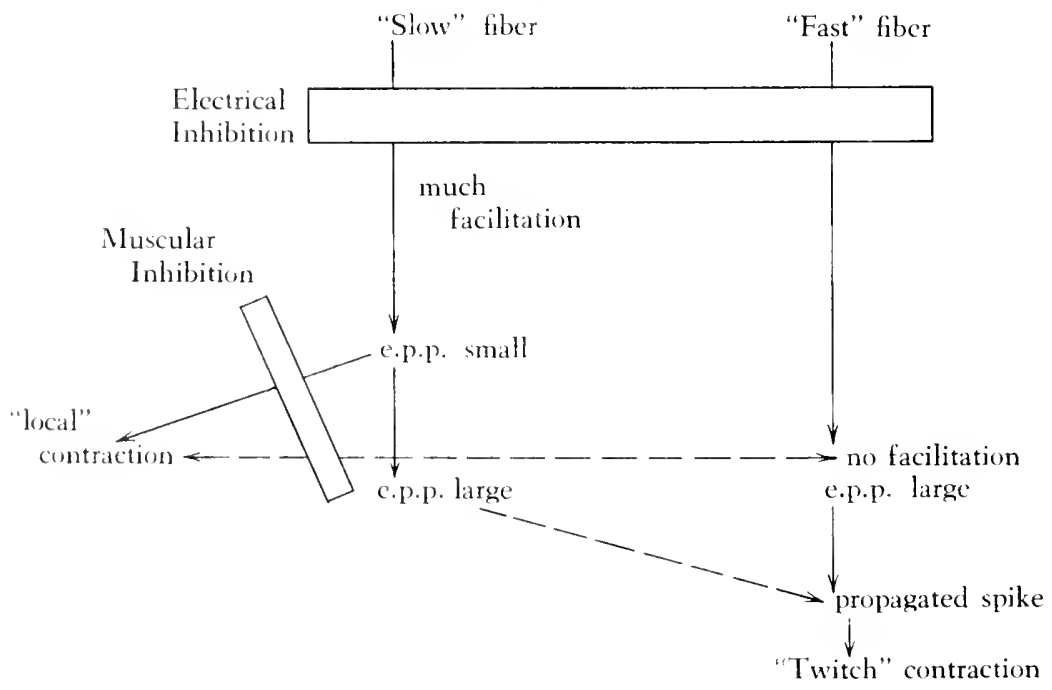

Inbibition is indicated by blocks; broken lines indicate that in many muscles the "fast" and "slow" responses intergrade according to frequency of stimulation. 
The efficiency of this complicated neuromuscular system is high. Bronk ${ }^{54}$ showed that the efficiency in terms of tension developed for heat produced increases as stimulation continues. The slow system is more cfficient than the fast one in that its ratio of heat to mechanical work is lower. ${ }^{141}$ Only a few impulses need leave the nervous system to maintain contraction, and a shift in nerve fiber or a slight change in frequency from the nervous system changes the whole character of the muscular response.

Multiple innervation of muscles occurs also in insects. ${ }^{1.19}$. 1:2: (One of the nerve fibers going to a leg muscle in a cockroach causes a tonic (slow) contraction; it discharges, when attached to the nervous system, at $30 / \mathrm{sec}$, whereas the other shows reflex bursts of large spikes at $75 \mathrm{sec}$ and causes a quick high-level contraction. ${ }^{1: 1}$ The slow system shows much facilitation, the fast system little or none. No inhibitors were found in the cockroach but there is evidence for peripheral inhibition in leg muscles in Dixippus. ${ }^{108}$

In the clam $\mathrm{Hy}$ a arenaria, the adductor muscles and mantle retractor muscles receive two types of nerve fiber. ${ }^{194}$ One type discharges reflexly at high frequency and causes rapid contractions; the other discharges slowly and causes a prolonged tonic type of contraction with low potential electrical response. Such a system is efficient when a low level of contraction must be maintained and occasional quick contractions superposed. The retractor of the buccal mass of Helix appears not to be doubly innervated. ${ }^{196}$ In Pecten the adductor muscle is composed of a striated portion for rapid swimming movements and a smooth portion for maintained contraction. ${ }^{301}$ During swimming the smooth muscle is inhibited and part of this inhibition may be peripheral. ${ }^{34}$ No cvidence is available as to whether multiple innervation exists among annelids or echinoderms.

As comparative studies of neuromuscular junctions are extended, it becomes apparent that these junctions serve many of the functions usually ascribed in vertebrates to the synapses of the central nervous system.

\section{RELAXATION RATES AND TONUS; MECHANICAL PROPERTIES OF MUSCLE}

The preceding sections show that muscles differ in their time constants of contraction and in their dependence on ncuromuscular facilitation and inhibition. Muscles differ also in rates of relaxation and in maintenance of tension. Table 71 shows that relaxation times differ more than do contraction times and that a muscle can contract fairly rapidly and relax extremely slowly (e.g., clam adductor). Contraction and relaxation are active processes, both requiring energy; yet the rates of contraction and relaxation are determined in part by the mechanical properties of the muscle as a wholeconnective tissue elements, sarcolemma, and muscle proteins. These mechanical properties, particularly those of the contractile proteins, change during contraction. To what extent can differences in relaxation and maintenance of tension be accounted for by mechanical properties of muscle?

Tonus is the maintenance of tension under extension, or change in length without change in tension. It has been attributed to: (1) the mechanical "set" of contractile elements, (2) continued nervous bombardment, and (3) continued stimulation by chemical agents. 
The mechanical properties of muscle have been studied by stretching and releasins under different conditions. Nuscles have properties of viscous and of clastic bodies. When vertehrate striated muscle is stretched it develops chastic tension, and when the stretching force is removed the muscle returns rapidly to its initial length; its "visersity" is low. When visceral short-fibered smooth muscle is similarly stretched it lengthens rapidly at first, then more sowl: it shows ...n intial clastic tension which falls off as the stretch continues: smerth muscle behares as a plastic viscous body. According to Winiom ${ }^{2: i t i}$ smonth musele cim be represented by a model of three phases: pure

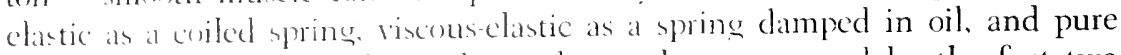
viscous als vanes in oil. Striated muscle can be represented by the first two of these phases. Mhuscles differ in the proportionate effectiveness of their clastic and riscous phases.

V'ertebrate striated muscle develops elastic tension when stretched; in addition it mas derelop comus reflexly, i.e., initiated via the central nervous ststem bi stimulation of stretch receptors within the muscle. Its tone results

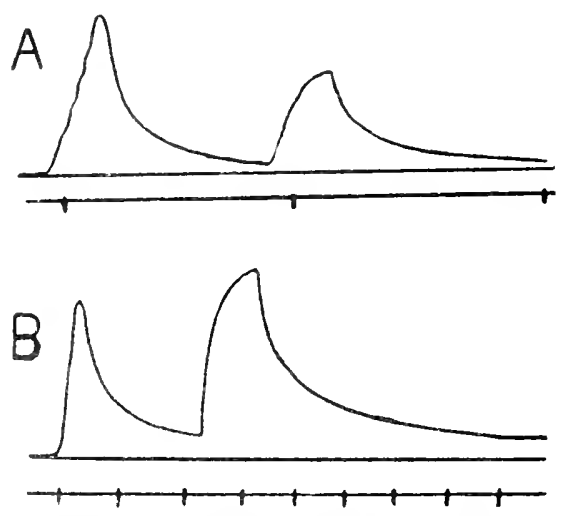

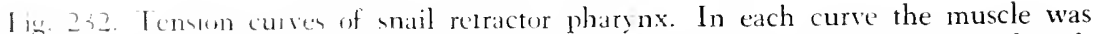
turs simulated, then afeer relixation it was stretched by about 10 per cent of its length and llhen releared. $A$, in air: $B$, in air and 6 per cent $C O$. Time in seconds in each. Note smilarm of relisition and relcase of tension. From Bozler.

fiom continued discharese of motor impulses at low rates in a variable number of motor units. Relaxation is comple:e when such nerrous discharge ceises. In inseces the comtracted state in death leigning results from con-

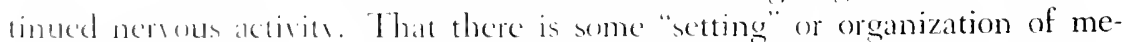
(hanted ckements in siriated muscle is shown by the observation ${ }^{110}$ that, if the muse is suddenls aither rekeased ar stretched during continued isometric contration, ension is again deredoped over a time course corresponding wh its new lemeth. Ihe ension developed by an excited muscle depends on the intitid lensth of that muscte and is maximal at the resting length. 1:4

binen hilered smenth muscle is sometimes said to have no fixed resting

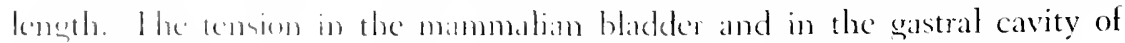
a sea ancmune, in the coctem of Sipunculus, or in the gill cavity of an ascielian, in relanirely comentant, whether the carvity be empty or full. The mus-

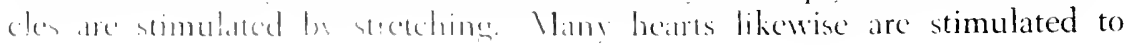


comtract when distended (h. 15, p. 5t7. In ribe hommal anents, par

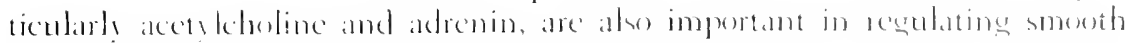

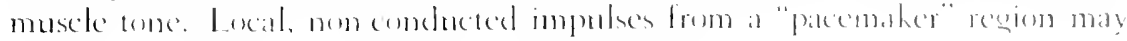
resule in tomus in an organ stech as the toterus.

Between these two extremes of striated shedetal and the sments mascke of hollow oreme are mans muscles in which rapid refles responses mat be

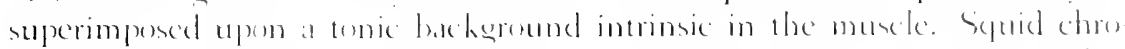

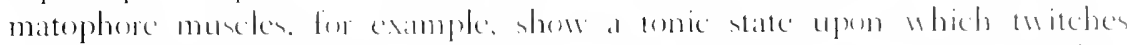

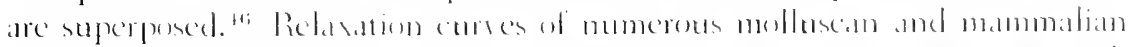

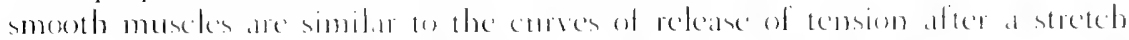

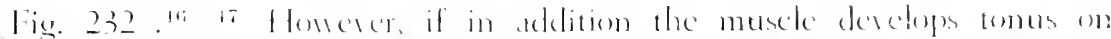

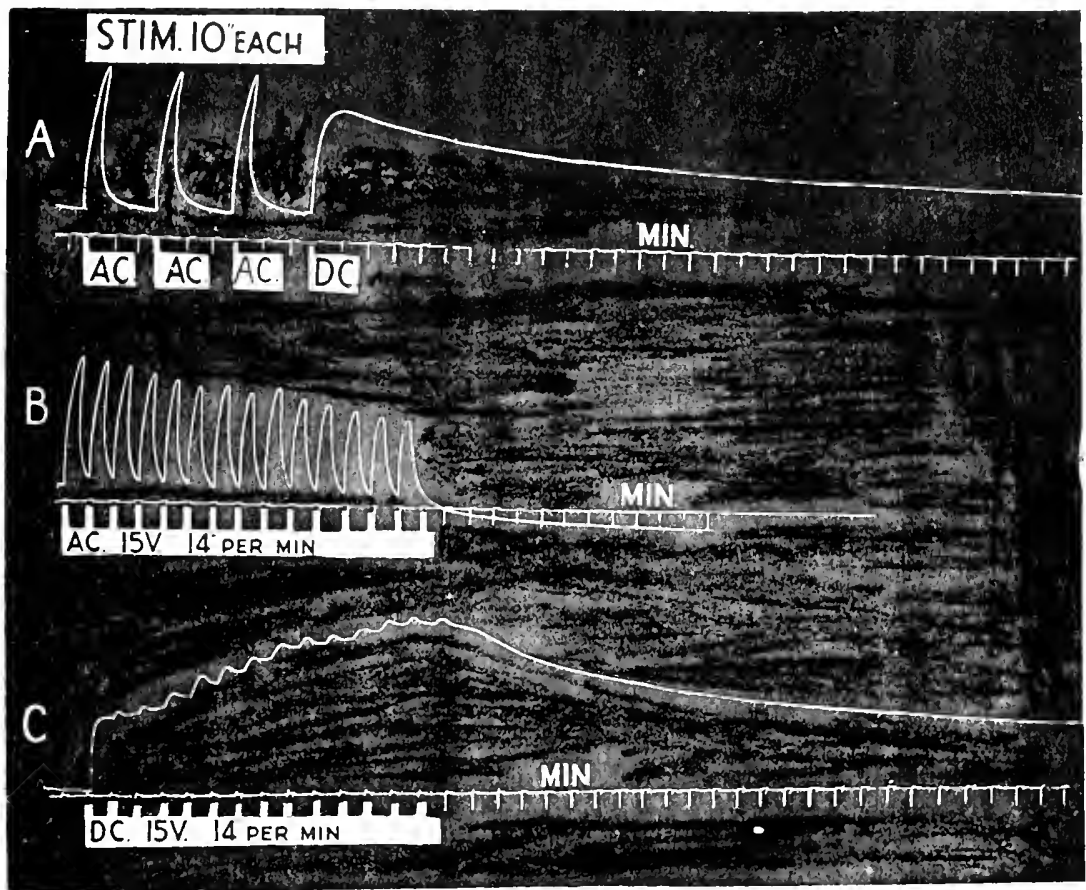

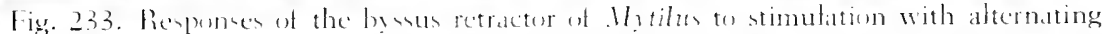
current and direct current. I, I hace contractions in response wo stimulation by AC? (50

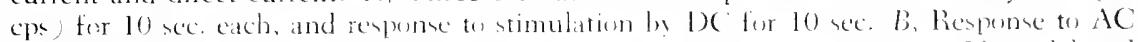

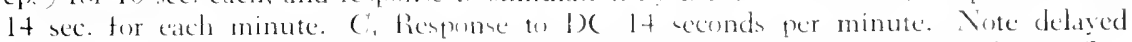
relaxation, summation with 1$)($, tast reladion and fitigue with AC. After llinton.

stretching, the relatition form a contraction mas be faster than release of tension. in, wor fordan and his students in mams papers, summaried by for

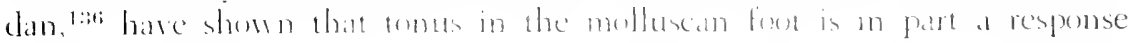

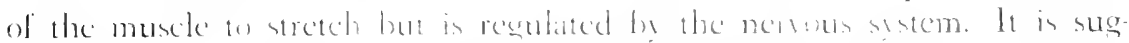
gested that the enic and comtratile me hamisms anc sepalte. Ihe pedal aranglion decreases the resistance (1) stretching but does not affect the contractility of the fon wo an electrical stimulus, whereas the presence of the cerebral gandion is saded w increase the contractility without haring any 
influence on tonus. Similarty in holothurians cutting of the radial nerves decreanes resistance 0 stretch. $1: 34$ At low temperatures resistance to stretch is greater than it is at high temperatures (Anodonta, ${ }^{1: 3}$ Helix $^{1364}$ ). If it is stimulated during the srachual loss of tension while stretched, a Helix foot contracts and then relaises to the level it would have reached had the release of tension continued uninterrupted.

Increase in "viscosity" as judged by resistance to stretch during contraction and during chemically induced tonus has frequently been described. A striking alteration in "viscosity" is found in the anterior byssus retractor of $M y$ -

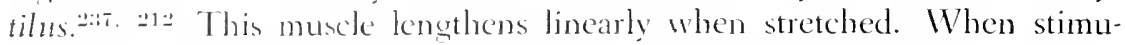
lated with altemating current (AC) the "viscosity" is reduced, whereas after stimulation by direct current (DC) the "viscosity" is raised and may remain high for an hour or two (Fig. 233). Intermittent DC lowers the "viscosity" when the current flows for a small part of a cycle, but the usual DC value is approached as the current flows a larger part of a cycle. AC causes a strong contraction followed by quick relaxation, and brief DC pulses applied every minute or two maintain tension with practically no fatigue (Fig. 233). It is suggested that the maintained tension results from high "viscosity."

In most bivalves the adductor contains a translucent soft striated portion which contracts and relaxes rapidly and fatigues after brief contraction. In Pecten the valve hinge is elastic, and by repeated brief contractions the scallop swims through the water. A smaller portion of the adductor is opaque, tough, smooth muscle which can remain contracted for hours or days with little or no fatigue. This tonus muscle can maintain high tensions, as shown by Marceau ${ }^{161}$ in the following table:

$\begin{array}{lr} & \mathrm{kg} \cdot / \mathrm{cm} \\ \text { Anodonta cygnea } & 5.2 \\ \text { Ostrea edulis } & 12.0 \\ \text { Venus verrucosa } & 35.4 \\ \text { Mytilus edulis } & 11.3 \\ \text { Pecten maximus } & 8.5\end{array}$

It was claimed hy Parnass ${ }^{1 \times n}$ that the adductors could hold great weights for hours without measurable increase in $\mathrm{O}_{2}$ consumption, but Ritchie ${ }^{200}$ calculated that the methods used could not have detected an increase in respiration of this muscle. Lactic acid does increase during 27-84 hours of contraction." If the visceral nerve of Pecten maximus is cut while the adductor is contracted the muscle remains short and cannot readily be stretched:-21. 3n il the nerve is cut while the muscle is relaxed, stimulation of the stow muscle is difficult but causes contraction, after which relaxation mat be more rapid than when the nerve is intact. If Peeten contracts against a bleck of wood between the valves, the animal holds this position when the wool is withelrawn; the muscle resists stretehing although the valves can be pushed wegether. Stimulation of some afferent fibers to the visceral gangtion causes contraction; stimulation of others caluses retaxation. Without nerve stimulation relaxation requires many hours. A stimulus every 30 seconds keeps Pecten contracted, an economy many times greater than that in frog 
muscle. Here in Pecten is a combination of mechanical "cateh" mechanism and effectiveness of occasional motor impulses. The "catch" mechanism is turned off by an inhibitory volley.

Muscles differ greatly in their ability to maintain tension. These differences are due in part to their mechanical properties and in part to the nature of their nervous and hormonal control. Measurements of mechanical properties have been made under such diverse conditions that it is impossible at present to arrange muscles in any logical series even though such an arrangement would be useful. The vague term "viscous-elastic properties" must be replaced by quantitative data regarding the mechanical properties of connective tissue and of contractile proteins. A comparative study of the mechanics of muscle might be a most valuable approach to the basic problems of contraction and relaxation.

\section{RHYTHMICITY AND THE EFFECTS OF DRUGS}

Not only do muscles differ in their rates of contraction and relaxation, but some muscles are capable of maintaining rhythmic activity. In some hearts the rhythm originates in muscular tissue, whereas in others there are nervous pacemakers (Ch. 15). The muscles of neurogenic hearts may beat in embryos before the pacemaker ganglia develop. The origin of spontaneity in rhythmic muscles is sometimes in the muscle cells and sometimes in contained nerve elements; for skcletal muscles the rhythm usually originates in the central nervous system.

Rhythmic movements such as breathing and locomotor reflexes require tetanic trains of nerve impulses from specific nerve centers. Under certain abnormal conditions, as when immersed in pure $\mathrm{NaCl}$ solution or in calcium-precipitating agents such as citrate, striated muscle twitches spontaneously. A deficiency of calcium causes a tendency toward increased excitability and slowed accommodation (delayed rise in threshold during stimulation). Tetany in parathyroid lack is accounted for on this basis.

The body wall of many tubular animals shows spontaneous rhythmic movement. In the earthworm the normal movements of crawling are reflex in origin (Ch. 23). Spontaneous contractions have been seen, however, in strips of earthworm body wall lacking ventral nerve cord.

In holothurians (Tliyone) the cloacal muscles show periods of rhythmic activity initiated from the nervous system. ${ }^{65}$ Similarly in coelenterates general body rhythms are responses to rhythmic nerve centers, such as the "sense organs" of medusae.

Muscles of the visceral type show spontaneous contractions which may occur in the absence of nerve cells. In embryos the amnionic muscles, like the heart muscle, contract rhythmically without innervation. The stomach and intestinal wall of mammals contain inner circular and outer longitudinal muscle fibers; between them is the myenteric plexus of Aucrbach which is connected to the plexus of Meissner in the submucosa. Local rhythmic contractions, segmental and pendular movements, occur in intestinal muscle when the nerve plexuses have been inactivated by nicotine or cocaine ${ }^{31}, 158$ and in circular muscle stripped from the plexuses. ${ }^{11+}$ Part of the electrical activity of intestinal muscle is associated with contraction, but some action 
potentials mat be present esen when muscular activity is negligible.". 3 The rhrthmic contractions are superposed on a background of tonus. Conducted waves, peristaltic contractions, are focal reflexes initiated by distenton and mediated by the nerve plexuses. The activity of the intestine, whether myosenic or menteric reflex, is enhanced by acetylcholine or by ragus stimulation and is diministed by adrenalin or by sympathetic stimulation. Atropine relaxes and pilocarpine excites the gut. Nicotine in low concentrations abolishes the reftex contractions. Potassium stimulates, possibly by acetylcholine release; calcium has the reverse effect." The effects of several drugs on different visceral muscles are compared in Table 72.

\section{TABIE 72. RESPONSES OF VISCERAL MUSCLES TO DRUGS}

+ indicates stimulation (increased amplitude or frequency, or both, of spontaneous contractions); - indicates depression of activity; and 0 indicates no effect.

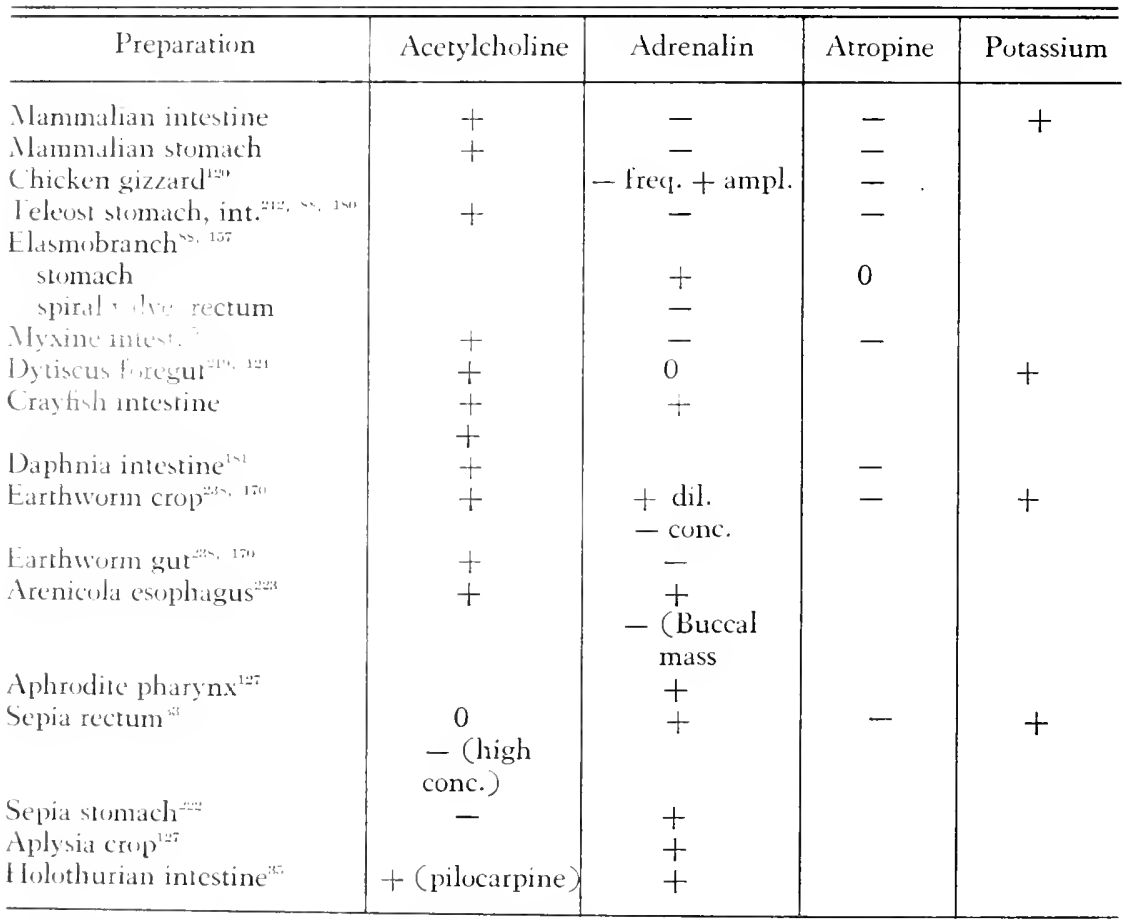

In the uterus, a muscle lacking nerve plexuses, excitability and tonus are low and spontancity is rare during anoestrous, but activity appears in oestrus and after injections of estrogens. Uterine muscle shows waves of electrical activity, usually originating in the uterine homs and possibly conducted from cell to cell by protoplasmic bridges. $1:, 00,5 \pm$ In the ureter similar rhythmic waves originate an the renal end. Peristaltic contractions of the ureter and stomach do not involve nerrous elements and are not abolished by high concentraltions of nicotine and cocaine." Teleostean fish show responses of the intestine similar to lhose of mammals, adrenalin and atropine inhibiting and acetyleholine accelerating contractions." 12 In elasmobranchs, howerer, adrenalin and both sympathetic and vagus impulses stimulate the 
stomach, while adrenalin inhibits the spiral valve and rectum, structures which are stimulated by the splanchnic nerve. ${ }^{1.5 i}$ Similarly in a cyclostome, where there are no sympathetic nerves, the vagus has no effect on intestinal muscle, but atropine, adrenalin, and nicotine inhibit contractions initiated by acetylcholine.5.5 The drug effects are thus not dependent on sympathetic innervation.

The digestive tracts of insects and crustaceans contain circular and longitudinal muscles which are normally active in digestion. Spontaneous rhythms in the cravfish intestine are accelerated by dilute solutions of either acetylcholine or adrenalin (Table 72). Stimulation of the abdominal nerve cord of the lobster elicits rhythmic contractions of the intact intestine and anal muscles. ${ }^{16: 1}$ The gut muscles of the honeybee and probably of many other insects are striated. The cockroach crop and gizzard show much activity in vivo, the gizzard movements stopping when the first thoracic ganglia are removed. ${ }^{40}$ The foregut and intestine of Dytiscus beat well when isolated in saline; acetylcholine, nicotine, ${ }^{21: 1}$ and potassium ${ }^{12 t}$ are excitant, adrenalin is without effect. ${ }^{21: 1}$

In some molluscs adrenalin increases the tone and normal rhythm (crop of Aplysia, ${ }^{12 \pi}$ rectum of Sepia ${ }^{33}$ ). In Helix and Aplysia acetylcholine and potassium increase tonic activity of the intestine, but the Sepia intestine is insensitive to $\mathrm{ACh}$ except at high concentrations which lower tonus and stop automatic beats. ${ }^{3:}$

In the tube-dwelling polychacte Arenicola the proboscis (extrovert) consists of buccal mass, pharynx and esophagus; the stomatogastric nerve plexus extends over the pharynx and esophagus and connects to the circumesophageal ring of the central nervous system. A variety of experiments ${ }^{2: 3}$ show that the esophagus is a pacemaker region from which rhythmic bursts arise at intervals of about 6 minutes. When the extrovert remains attached to a piece of the body wall with the anterior nerve cord intact, the body wall shows rhythmic activity so long as the nerves to the esophagus are intact. The buccal mass can show small-amplitude asynchronous waves when removed from the esophagus; this activity is inhibited by adrenalin. Esophageal contractions and those induced in the buccal mass and body wall from the esophageal pacemaker are greatly stimulated by adrenalin. Acctylcholine also stimulates the esophagus. The stomatogastric nervous system apparently initiates rhythmic movements of the entire proboscis region including body wall: both adrenalin and ACh stimulate this pacemaker. In the free-swimming Glycera, also, waves of activity originate in the stomatogastric system of pharynx and esophagus, but, in addition, impulses from the central nervous system can invade the buccal tube eliciting a different rhythm.:-24

In holothurians the isolated intestine is stimulated by adrenalin, relaxed by atropine. Both pilocarpine and physotigmine cause an increase in tone. ${ }^{3.5}$

Table 72 shows that only in the muscles of gastrointestinal tracts of vertebrates is there strict antagonism between acetylcholine and adrenalin.

Muscle cells in general have an inherent spontaneity of movement. However, adult muscles rarely manifest spontaneity; nervous pacemakers usually gain control. 


\section{CHEMICAL AGENTS IN NEUROMUSCULAR TRANSMISSION}

There are two hypotheses of neuromuscular transmission: one states that a spccific agent is liberated at nerve endings as a muscle excitant; the other postulates that junctional transmission is electrical and that a chemical agent is concerned cqually with the electrical transmission in nerve and muscle.

Activity in vertebrate parasympathetic nerves is associated with liberation of acctrlcholine ( $\mathrm{ACh}$ ) (Ch. 15, p. 537), at their terminations in heart and blood vessels, and activity in most postganglionic sympathetic nerves to these structures is associated with liberation of sympathin (possibly identical with adrenin, Adr). In other animal groups the evidence for cholinergic (acetylcholinc-liberating) and adrenergic (adrenin-liberating) nerves to the heart is not conclusive. There is presumptive evidence that various motor nerves are cholinergic, adrenergic and tyraminic (liberating tyramine). The distinction between cholinergic and adrenergic nerves is not rigorous, and several types of interaction between ACh and Adr may exist (Ch. 23).

Bacq ${ }^{16}$ has stated criteria for identification of a cholinergic system: (1) presence of $\mathrm{ACh}$ in the tissue, ${ }^{*}$ (2) presence of cholinesterase (ChE) (acetylcholinesterase) in the tissue, (3) sensitivity to added ACh, (4) potentiation of $\mathrm{ACh}$ and of nervous action by anticholinesterases such as eserine, (5) blocking of action of $\mathrm{ACh}$ or nerve stimulation by atropine or curare, and (6) identification of ACh in perfusate from the active organ. Sclected data for these criteria in neuromuscular preparations are given in Table 73.

The motor nerves in vertebrate striated muscle are cholinergic. Acetylcholine and acetylcholinesterase (ChE) are present: ChE is low in nervefrec portions of muscle and is maximal in regions of end-plates. The concentration is higher in lizard muscle than in frog muscle, ${ }^{165}$ and in chickens the ChE increases to a maximum at the time of hatching and thereafter declines. $^{171}$ It has been calculated ${ }^{172}$ that enough $\mathrm{ChE}$ is present in the frog sartorius to split $1.6 \times 10^{4}$ molecules of $\mathrm{ACh}$ at each end-plate during the refractory period of the muscle. It is calculated that the output of acetylcholine per nerve impulse per motor end-plate is $10^{-10} \mu \mathrm{g}$, or $1.4 \times 10^{5}$ molecules, which is $1 / 30,000$ of that needed to stimulate. ${ }^{1}$ Immersion in solutions of ACh causes a slow maintained contraction (contracture), and some muscles, such as the frog rectus abdominis, are useful for bioassay of ACh. Close intra-arterial injection of small amounts of ACh causes a twitch-like response, ${ }^{86,}{ }^{8}$ as do local ACh applications in the end-plate region. The sensitivity to ACh is 1000 times greater at the end-plate than along the fibcr, possibly because of permeability differences. ${ }^{1}$ In perfusion experiments ACh has been identified in increasing amounts in the perfusate when the motor nerve to a muscle has been stimulated. Local applications of ACh, like motor impulses, set up a local potential in the region of the end-plate, and muscle impulses arise from this potential; eserine prolongs the e.p.p. Eserine prolongs the summation interval for neuromuscular transmission, and causes repeated contractions in response to a single motor volley, an effect

* Acetylcholine is normally bound to protein in excitable tissues; free acetylcholine appears to increase on stimulation and on standing in excised tissues. Determinations of ACh are not always comparable in that they may not distinguish bound and free ACh. 
Muscle and Electric Organs

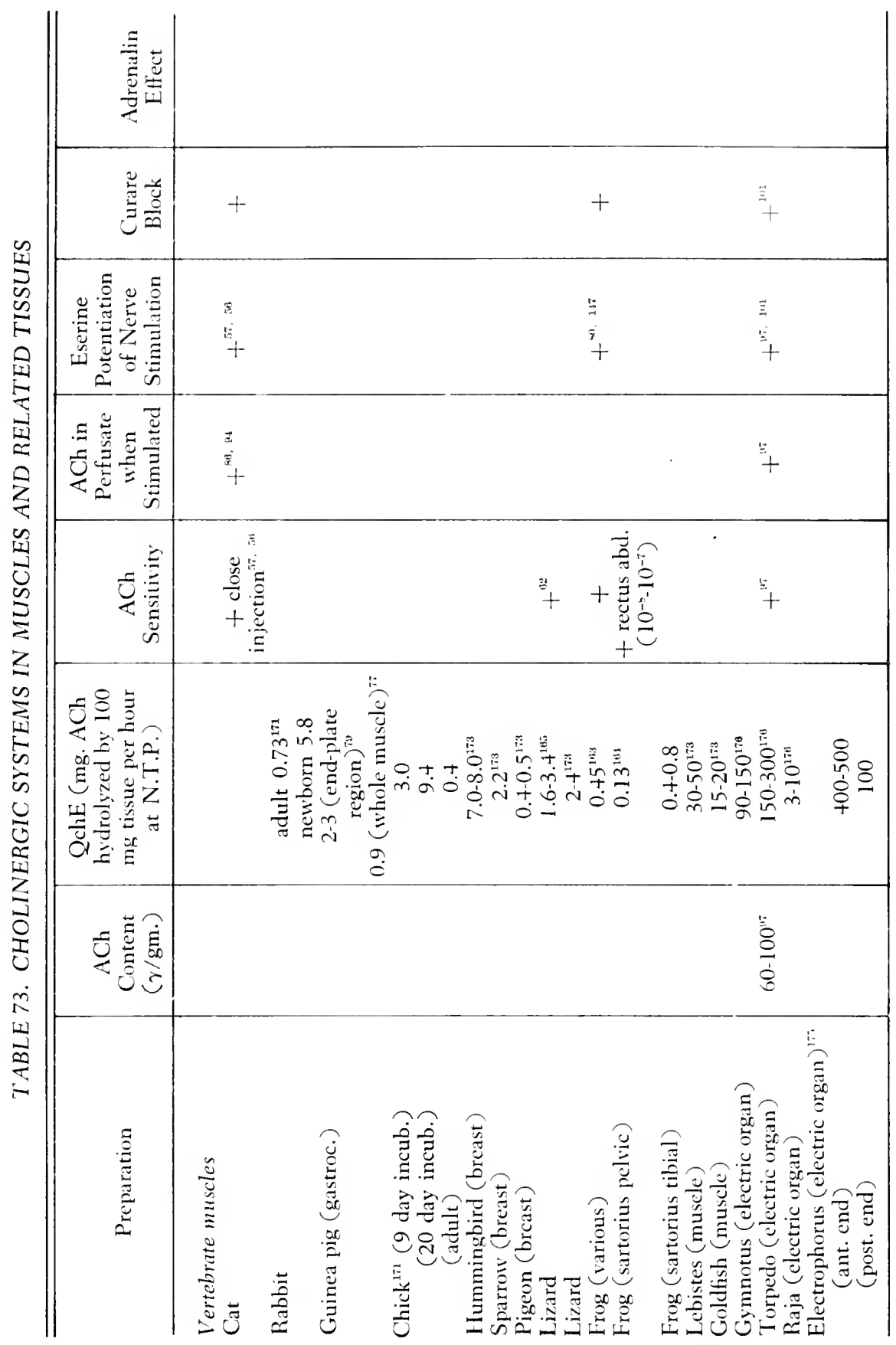




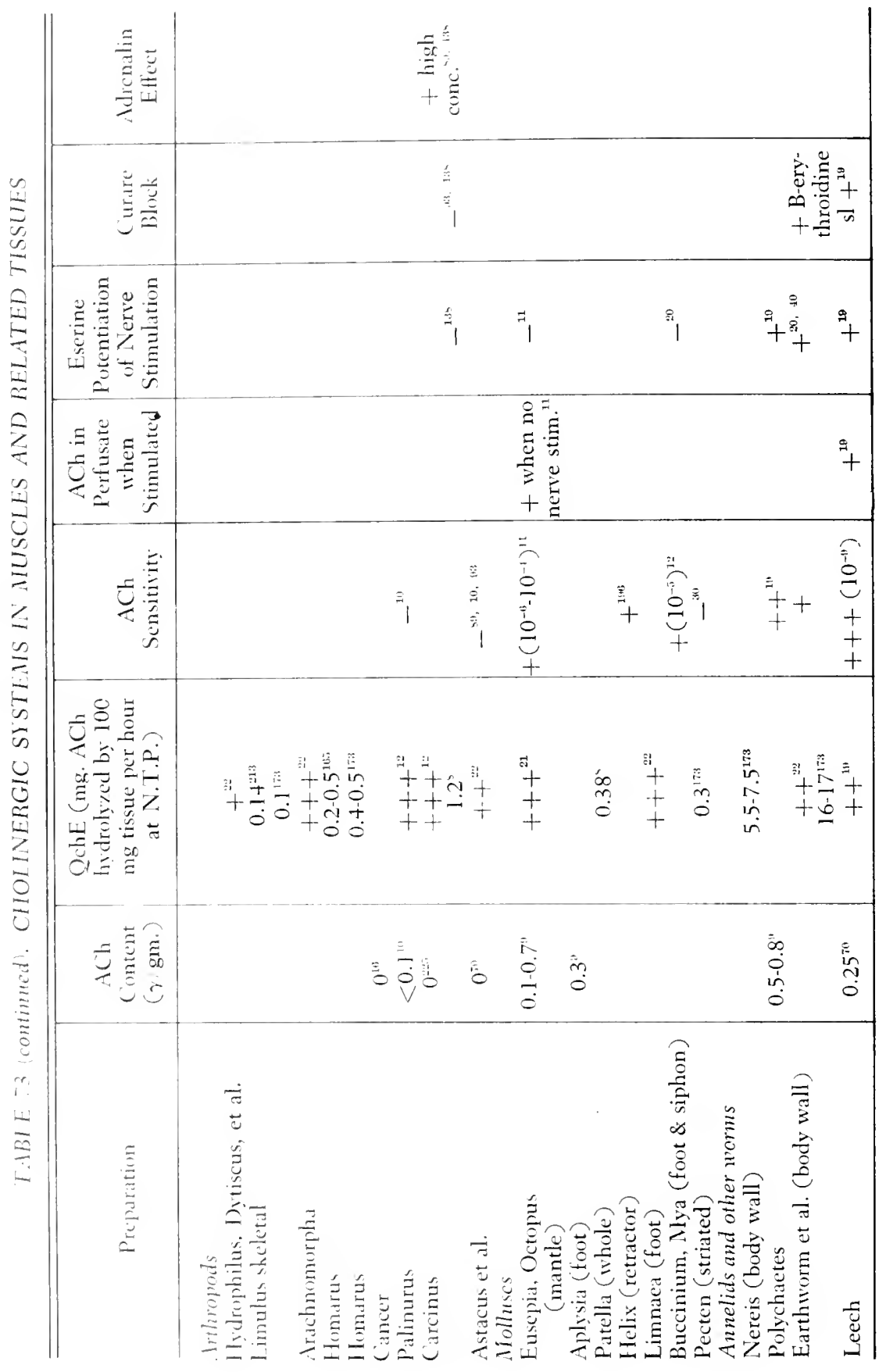




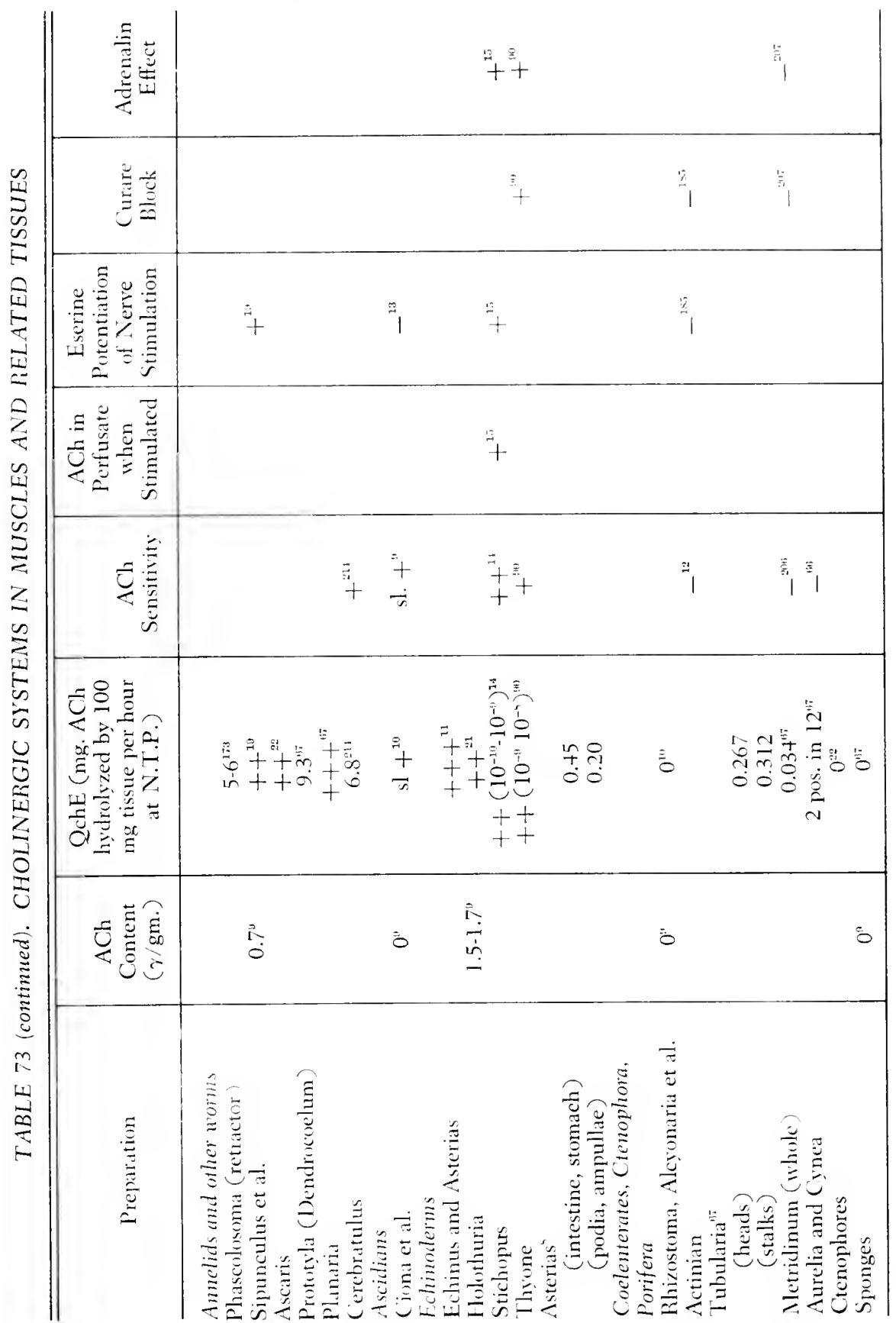


abolished by atropine." Calcium is apparently necessary for ACh liberation. ${ }^{80}$ Curare permits continued liberation of $\mathrm{ACh}$ at the motor nerve endings but prevents its depolarizing action, presumably by combining with the chemical receptor substances at the end plate. ${ }^{1 \text { t. }}$ Depolarization by $\mathrm{ACh}$, nicotine, and caffeine, but not by potassium, can be antagonized by curare. The transmission in vertebrate muscle can be summarized as follows:

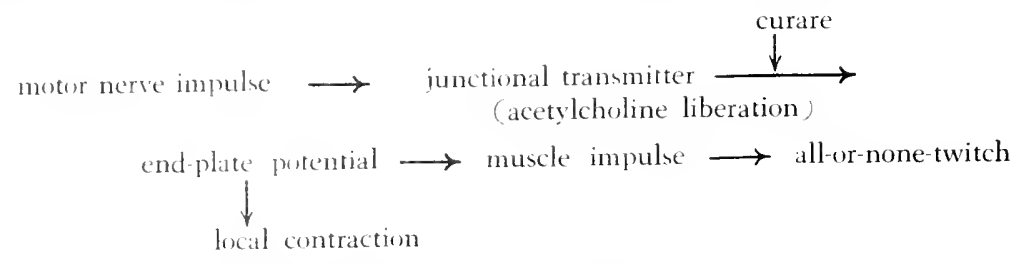

An altemative theory 7 . … $113.17 \%$ is that acetylcholine is concerned in electrical propagation, that the nerve action potential is the junctional transmitter, and that the acetylcholine obtained in perfusion comes from the sole plate of the muscle rather than from the nerve endings. This theory is supported by the distance from nerve ending to sole plate (Fig. 235), by the low perneability of the muscle fiber to acetylcholine except at the region of the cnd-plate, and by evidence that acctylcholine is concerned in nerve conduction (Ch. 23). Nlany of these facts can be accounted for by the electrical theory as well as by the chemical transmitter theory. Whatever its origin in perfusion experiments, there is little doubt that ACh is an important component in the transmission mechanism.

Many vertebrate smooth muscles are innervated by adrenergic postganglionic smpathetic fibers. Adrenalin (or adrenin) causes responses similar to those produced by sympathetic stimulation. The adrenin-like mediator can be identified in the blood, coming from a region of sympathetic discharge. "in The action both of the mediator and of adrenalin is antagonized by ergotoxin. The mediator liberated in structures which are inhibited by the sympathetics differs quantitatively in its effects on test muscles from that liberated in structures which are excited, and the inhibitory agent may be adrenin, the excitatory agent nor-adrenin. ${ }^{17}$ There is good cvidence that the nerves cliciting contraction of pupil constrictors are cholinergic.

The eridence regarding chemical mediation at motor nerve endings in invertebrate animals is inadequate. Acctylcholine liberation in muscle is established only for annelids and sipunculoids, is indicated in molluses and echinoderms, and is unlikely in irthropods and cockenterates. ${ }^{16}$

The most consincing evidence for cholinergie motor nerves is found among annelid and sipunculoid worms. The muscles of many worms of these groups contain $\mathrm{ACh}$ and ChE (Table 73). The body wall muscle is stimulated to contract by acetsleholine, and sensitivity is increased by eserine. Sensitivity is greit (10 " cren without eserine, and body wall muscles of the leches Hirudo and Pontoldella have been used in bioassay. The action of $\mathrm{ACh}$ antidendists should be investigated further. It has been reported that nerve activation is paralyed by curare in Hirude (but not in Lumbricus), by Bcrythroidine in I umblricus, and hy atropine in. Sipunculus and Aphrodite. Nicotine abolishes the direct eflect of $\mathrm{ACh}$ on the eartliwom body wall.239 
Summation of contractile response of the earthworm body wall to repetitive electrical stimulation is increased by eserine. ${ }^{41}$ Eserine increases the muscular responses of Sipunculus, Hirudo, Arenicola, Aphrodite, and Lumbricus to stimulation of the nerve cord. ${ }^{19}, 20$ No such effect was observed when eserine was applied to the nerve cord alone, but in perfusion experiments it appeared that the leech body wall liberated ACh when the nerve cord was stimulated.

The visceral muscles of annelids are spontaneously rhythmic. In earthworm crop-gizzard preparations the wave of contraction usually originates in the crop; this preparation is stimulated and tone increased by acetylcholine $\left(10^{-8}-10^{-10}\right.$ without eserine and $10^{-11}$ with eserine).23s If cooled, spontaneity is abolished, but acetylcholine still causes contraction. ${ }^{5}$ The stimulating action of ACh is antagonized by atropine; adrenalin augments the activity when dilute $\left(10^{-5}\right)$ and depresses at higher concentrations $\left(10^{-5}\right)$. Stimulation of the ventral nerve cord inhibits the crop-gizzard activity, an effect antagonized by ergotoxin; stimulation of the pharyngeal commissures excites, an effect antagonized by atropine. ${ }^{23 *}$ Potassium enhances and calcium inhibits the visceral rhythm. An active crop-gizzard preparation liberates into a perfusate a substance which induces ACh-like contractions in a test preparation. ${ }^{5}$ Antagonistic cholinergic and adrenergic control, as in mammals, is therefore, indicated for the earthworm crop-gizzard.

In the echinoderms as in annelids there is evidence for cholinergic motor systems. The longitudinal retractors of holothurians contain $\mathrm{ACh}$ and are rich in ChE, as are muscles from Echinus and Asterias. "In the holothurian Stichopus the longitudinal muscles are sensitive to ACh, responding to dilutions of $10^{-i}$ without eserine and to dilutions of $10^{-4}$ after eserine, and this muscle has been used in bioassay, ${ }^{13}$ as has also Thyone muscle. ${ }^{90}$ Response to direct stimulation of the holothurian longitudinal retractor muscles, nerve elements included, is potentiated by eserine and prevented by atropine, and stimulation liberates into a perfusion bath a substance which has the properties of $\mathrm{ACh}^{15}$ Curare blocks conducted responses in Thyone retractor muscles and prevents spontaneous contractions." Holothurian muscles are excited by low concentrations of adrenalin and tyramine. ${ }^{14,90}$

In molluscs much acetylcholine is found in nerve and muscle, for example, in Aplysia foot and Sepra mantle (Table 73). ${ }^{1 "}$ Cholinesterase is also present in Sepia mantle. ${ }^{21}$ The muscular responses to ACh differ among various species. The slow adductor muscle of Pecten is not stimulated by $\mathrm{ACh},{ }^{30}$ whereas the retractor muscle of the buccal mass in Helix does respond to the drug. ${ }^{196}$ Eserine fails to potentiate the response of the foot and siphon muscles of Buccinium and $M y a$ and the response of the mantle of Eledone to nerve stimulation. However the cephalopod mantle is sensitive to $\mathrm{ACh}$, and denervation greatly increases the sensitivity as it does in many vertebrate tissues. Bacq ${ }^{11}$ perfused the mantle of Eledone with eserinized saline; the outflowing perfusate contained an ACh-like substance, but stimulation of the mantle nerve did not increase its concentration in the perfusate.

It is possible that some nerves in cephalopods liberate tyramine. The salivary glands contain tyramine and histamine. ${ }^{40}$ The perfused stomach of Sepia shows spontaneous contractions which are inhibited by $\mathrm{ACh} 10^{-9}$ and stimulated by tyramine and histamine $10^{-8}$ and by $\mathrm{Adr} 10^{-7}{ }^{222}$ Cocaine 
increases the effect of adrenalin, has no effect on the action of histamine, and abolishes the action of tyramine. Nervous stimulation augments stomach movement, an effect abolished by cocaine, and when the entire animal is perfused, stimulation of visceral, branchial, and other nerves causes the appearance in the perfusate of something resembling tyramine. ${ }^{222}$ Neuromuscular transmission in cephalopods merits further investigation.

There is inconclusive evidence for cholinergic systems in flatworms ( $\mathrm{Ne}$ merteans and Platyhelminthes); the concentration of ChE in mixed muscle, nerve, and other tissue is strikingly high (Table 73). Strips of Cerebratulus muscle are unresponsive to $\mathrm{ACh}$ alone, but after eserine they respond to $\mathrm{ACh}$ $5 \times 10^{-5.214}$

Evidence points against cholinergic systems in ascidians, crustaceans, and coelenterates. Extraction of the ascidians Ciona and Phallusia failed to show measurable $\mathrm{ACh}$ and showed little ChE (Table 73). The mantle muscle of Cynthia is stimulated by $\mathrm{ACh}$ but the response is not potentiated by eserine. ${ }^{14}$

In Crustacea the nerves, particularly in the central nervous system, are rich in $\mathrm{ACh}$. Muscles in Crustacea have no $\mathrm{ACh}$ but do contain some $\mathrm{ChE}$ (Table 73). Crustacean muscle is insensitive to ACh, eserine, and curare. ${ }^{\mathbf{1 0}}$. $89,138,93$ Careful testing of the effects of many drugs and ions on neuromuscular transmission failed to provide any evidence in Crustacea for humoral transmission. Tyramine is without effect, ${ }^{138}$ although adrenalin in high concentration causes contraction. ${ }^{138}$ Crustacean claws should be well adapted for identifying exciting and inhibiting mediators, but no one has yet succeeded in such identification. Insect muscles are insensitive to acetylcholine, even after soaking in anticholinesterases.

Among coelenterates ChE was found in Tubularia, Metridium, and Sagartia but not in any regions of the medusae Cyanea and Aurelia, and not in the ctenophore Mnemiopsis. ${ }^{67}$ Muscles of the actinians Calliactis and Metriditm were insensitive to $\mathrm{ACh}$, even after eserinization; also no effect was observed with eserine, atropine, curare, and adrenalin. ${ }^{15}$, 206 Contractions of medusae were also unaffected by $\mathrm{ACh}$ or eserine. ${ }^{66}$ In Calliactis, however, tyramine enhances facilitation by 3-4 times in $84-90$ minutes and can even permit a response to single shocks. Extracts of this anemone yield a substance, perhaps tyramine, which promotes facilitation. ${ }^{207}$ Cholinesterase and $\mathrm{ACh}$ are lacking in sponges.

Table 73 indicates that cholinergic neuromuscular systems function in vertebrates, annelids, and echinoderms, are suggested in molluscs and flatworms, and are contraindicated in ascidians, arthropods, and coelenterates. Adreneruic systems have been demonstrated only in vertebraces, and tyraminic systems have been suggested for c' whalopods and coe'znterates.

\section{DISTRBBUTION OF RHOSPHAGENS}

The energy relations and the biochemistry of muscles other than of vertebrate striated muscles are poorly known. The heat liberation by vertebrate striated muscle has been carefully studied and analyzed as heats of contraction, relaxation, and recovery. Except for crustacean muscle, no useful thermal data are available for other muscles. 
The development of our knowledge regarding the chemistry of energyvielding reactions in muscle is one of the most fascinating stories in modern biochemistry and is discussed extensively in most physiology and biochemistry texts. ${ }^{2 * 33}$ It is probable that the underlying reactions by which energy is made availabie to the contractile proteins are similar in all muscles. Three types of energy liberations are used: (1) breakdown of the highenergy phosphate bonds in the specific phosphate donors, adenosine triphosphate and either phosphocreatine or arginine phosphate; (2) glycolysis of carbohydrate; and (3) oxidation of carbohydrate.

The breakdown of adenosine triphosphate (ATP) liberates about 11,000 calories per mol for each phosphate linkage broken. The reactions of the organic phosphates set off the chain of glycolysis whereby glycogen is split, and its component sugars are phosphorylated, going through many intermediaries to form lactic acid.:- 10; Part of the lactic acid is oxidized and a larger part is reconverted to glycogen, usually in the liver. Between the ATP and the glycolytic systems is interposed a carrier of phosphate, phosphagen. This phosphagen in vertebrate muscle is creatine, which is reversibly phosphorylated and dephosphorylated to phosphocreatine.

Phosphocreatine was discovered as muscle phosphagen in 1927. In the following year it was found that the phosphagen of crustacean muscle is arginine phosphate (phosphoarginine). The structural formulas of these two substances are given in Fig. 234. From 1928 to 1937 appeared a series of papers concerning the distribution of phosphocreatine (PC) and phosphoarginine (PA) in the animal kingdom. The results have been summarized sereral times. ${ }^{*}$. dicated in Table 74.

TABLE 74. DISTRIBUTION OF PHOSPHAGENS (References in text)

\begin{tabular}{|c|c|c|}
\hline & Phosphucreatine & $\begin{array}{l}\text { Arginine } \\
\text { phosphate }\end{array}$ \\
\hline \multicolumn{3}{|l|}{ Chordates } \\
\hline all vertebrate classes muscle & + & - \\
\hline electric organs of fish & + & - \\
\hline \multicolumn{3}{|l|}{ Prochordates } \\
\hline Amphioxus & + & - \\
\hline Balanoglossus & + & + \\
\hline Ascidia & - & + \\
\hline \multicolumn{3}{|l|}{ Echinoderms } \\
\hline Asteroidea & - & + \\
\hline Holothuroidea & - & + \\
\hline Crinoidea & - & + \\
\hline Ophiuroidea & + & - \\
\hline Echinoid adult & + & + \\
\hline Echinoid larva & - & + \\
\hline Crustaceans & - & + \\
\hline \multicolumn{3}{|l|}{ Annelids } \\
\hline Sabella, Lumbricus & - & + \\
\hline Errant polychaetes & + in 8 genera & + in 3 of 8 genera \\
\hline Sedentary polychaetes & + in 2 of 8 genera & + in 7 of 8 genera \\
\hline Sipunculoids & - & + \\
\hline Molluscs, all classes & - & + \\
\hline Platyhelminths-Planaria & - & + \\
\hline Nemerteans & - & + \\
\hline Coelenterates (actinians, medusae) & - & + \\
\hline
\end{tabular}


Table $7 t$ shows that phosphocreatine is the only phosphagen in all vertebrate muscles and electric organs. Phosphoarginine is the phosphagen of most invertebrate phyla. The distribution in prochordates and echinoderms is particularly useful in tracing the origin of the chordates. Amphioxus contains PC exclusively, Balanoglossus contains both PC and PA, and Ascidia contains only $\mathrm{PA}$; this supports other lines of evidence that tunicates are far from the main chordate line. Among the echinoderms the muscles of a holothurian, a crinoid, and starfish have PA only, an ophiuroid has PC only, whereas the jaw muscles of two echinoids contain both PC and PA

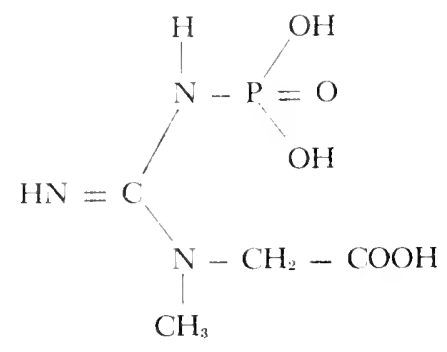

Phosphocreatine

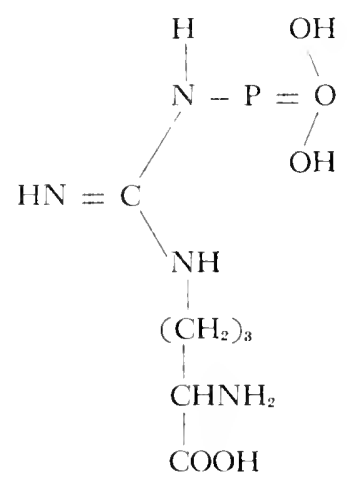

Phosphoarginine phosphate

Fig. 234. Structural formulas of phosphocreatine and phosphoarginine.

in a ratio of about 1 to 2.:6. 17: Larval echinoids have PA only. The enzymes (protein) concerned with phosphorylation and dephosphorylation differ for PC and PA; echinoids contain both enzymes. The presence of both phosphocreatine and phosphoarginine in echinoderms supports the theory of the relation between this phylum and the chordates.

Molluses, arthropods, and coclenterates contain phosphoarginine only. Phosphocreatine has, however, been found in some polychaete worms. ${ }^{27}$ There seems to be a tendency for the more active errant polychactes to possess PC and for the more sluggish tubicolous ones to have PA. A careful examination of many worms-annelids, sipunculoids, flatworms, and othersmay give important cvidence regarding the point of bifurcation of the phyletic tree. 
There was some question regarding the presence of arginine in cephalopods and another nitrogenous base, octopine, was isolated from muscle of squid and scallop. Octopine, however, is a breakdown product formed from arginine post mortem.

\section{ELECTRIC ORGANS}

The first bioelectric potentials observed by man were the discharges from electric fish; recently the end-plate potential, the homologue of the electric organ discharge, has been discovered. An interesting historical account of electric organs is given by Cox. ${ }^{-1}$ The electric catfish, Malopterus, was known to the ancient Egyptians, and the Romans named the "torpedo" which is an elasmobranch or ray. "Electric therapy" with electric fish was recommended by Galen; it was used by Indians of Guiana and by eighteenth century Europeans. The shock from electric fish was experienced for centuries, but what it was remained a mystery. Toward the end of the eighteenth century several observers suggested that the shock from certain fish was similar to lightning or to the electrostatic discharge from a Leyden jar. In 1773 John Walsh related that the shock from a Torpedo is conducted through metals but not through glass or air; two years later Williamson made similar observations on electric eels (Electrophorus, formerly called Gymnotus) brought to North America from the Amazon region. The Cambridge physicist Cavendish built a model of a Torpedo, from which he deduced the distinction between potential difference and quantity of electricity. Faraday ${ }^{96}$ made crucial tests with a galvanometer and spark gap on Electrophorus, thus demonstrating the electrical nature of the shock. He prophetically remarked in his diary: "Oersted showed how we were to convert electric into magnetic forces and I had the delight of adding the other member of the full relation, by reacting back again and converting magnetic into electric forces. So perhaps in these organs where nature has provided the apparatus by means of which the animal can exert and convert nervous into electric force, we may be able, possessing in that point of view a power far beyond that of the fish itself, to reconvert the electric into the nervous force."

All electric fish are sluggish and depend on their electric discharge to stun both their prey and their predators. The low sensitivity of electric fish to their own shocks is unexplained; in an aquarium with numerous other species of fish, electric fish, although obviously stimulated by each other's shocks, may be unharmed while the other aquatic animals are killed. ${ }^{63}$ Stimuli of 220 volts did, however, damage the electric eel.

An electric organ consists of columns of plates, electroplaxes, which run dorsoventrally in the electric rays and longitudinally in the eel. In Torpedo marmorata each organ has about 450 columns of 400 plates per column. Electric tissue comprises one sixth of the body weight in Torpedo and about one half in Electrophorus. The electroplaxes or plates in each column are in series electrically, the various columns in parallel. The long rows of many plates in series in the eel tend to match the external resistance of the freshwater medium and the short rows in the Torpedo tend to match that in sea water, each being well suited for power development in its own medium. ${ }^{83}$ Each electric organ is entirely under nervous control; in Torpedo 
huge branches from the tenth cranial nerve arise in large lobes of the medulla.

The electroplaxes are derived embryologically from electroblasts similar to sarcoblast precursors of muscle.sis The elongate multinucleate cell enlarges laterally, nuclei increase around the cell periphery, and the center becomes filled with mucous protein (Fig. 235). One surface is innervated, usually the electronegative surface, and the other is frequently papilliform. The single electroplax resembles a motor end-plate (Fig. 236) ${ }^{78}$.

When an electric fish discharges, each organ fires repetitively, usually 3 to 5 spikes, sometimes many more. Each spike is of constant height and

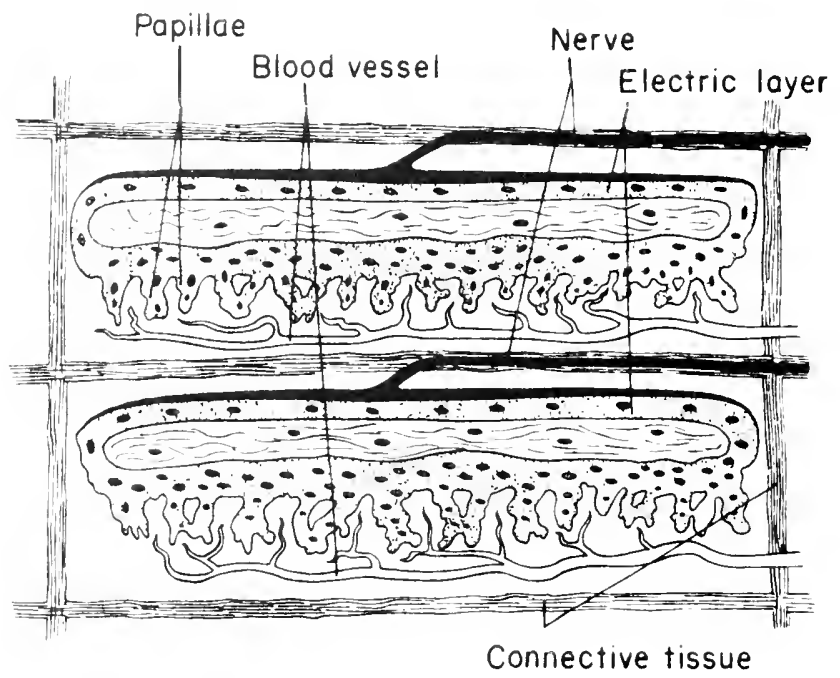

Fig. 235. Diagram of two electroplaxes. Nerve elements in black, electric layer granular and nucleated. From Ihle, Kampen, et al. ${ }^{12:}$

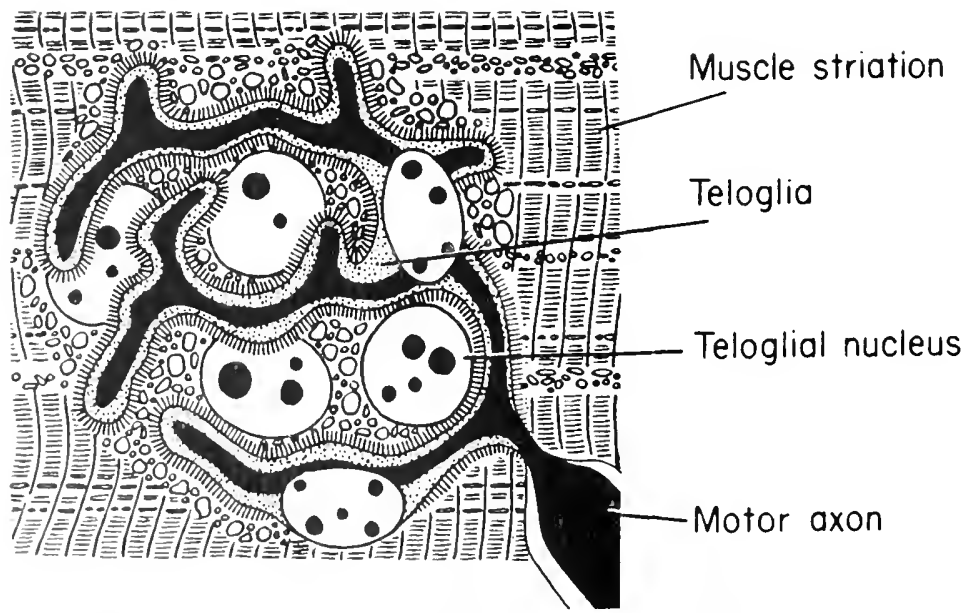

Fig. 236. Diagram of motor end-plate. Axon termination in black; myelin sheath stops before axom branching; zone of reloglia dotted and teloglial nuclei shown. Muscle striations around end-plate. From Couteaux. ${ }^{78}$ 
lasts about 2 msce. ${ }^{73 .} \times 3,{ }^{101}$ (Fig. 237). In Torpedo occidentalis spike duration is $3.5 \mathrm{msec}$. In the electric eel there may be, in addition to the larger spikes, minor discharges either preceding a major discharge or appearing independently. The minor discharge arises in the small organ of Sachs separate from the main electric organ. These minor discharges occur when the fish are cruising about for food, and there is good evidence that prey are loeated by the reflection of electric waves.

The synchrony of the electric discharge is remarkable and is essential for maximum power output. The two organs of Torpedo discharge within 0.1 msec. of each other! In the clectric eel each point in the electric organ is positive with respect to all points behind it, and measurements of time difference between the two ends indicate a wave travelling 450-2500 M./sec. Propagation stops at a cut in the spinal cord. ${ }^{74}$ There is evidence that when Electrophorus is probed with an active lead while a ground lead is placed on the tank, a point about one third of the distance back is electrically neu-
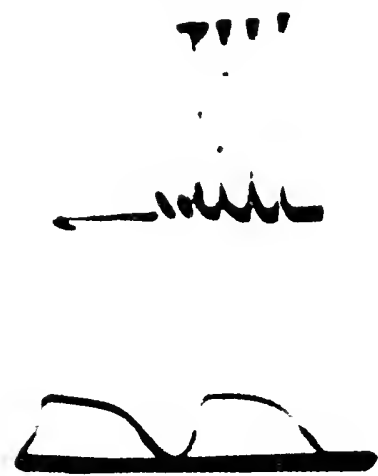
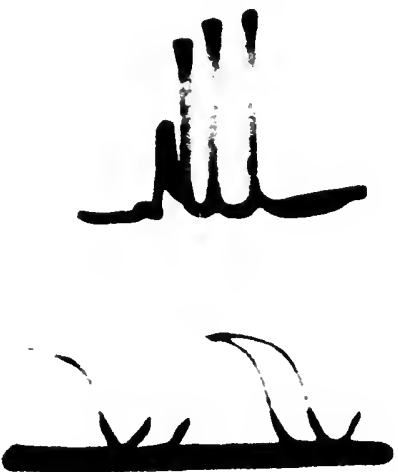

Fig. 237. Electric organ discharge in Electrophorus. Upper, records showing small followed by large pulses; lower, records at higher speed to show shape of discharges. From Coates, Cox, and Granath. ${ }^{72}$

tral. This near synchrony of all parts of the electric organs indicates very precise timing in the central nervous system; the motor impulses going to distant electroplaxes must leave the brain earlier or travel faster than do the impulses to near parts of the organ.

The total peak voltage measured without external resistance is usually 20-30 volts in Torpedo marmorata, 230 volts (sometimes 500-600 v.) in Electrophorus. ${ }^{* 2,} \times$ Older measurements are somewhat lower. In Electrophorus the thickness of the plates increases from the anterior end backward, and the voltage per centimeter length diminishes (Fig. 238). The calculated peak voltage per electroplax is remarkably constant at $0.05-0.15 \mathrm{v}$. per plate for all species investigated.

The voltage has been measured for various shunting external resistances and the current output calculated. Zero current corresponds to the maximum voltage and, as current increases, voltage decreases linearly; the maximum current developed by the electric eel is less than one ampere, whereas from the lower-voltage ray fish higher currents are obtained (2 amperes for Narcine and 60 amperes for Torpedo occidentalis). Power as a function of ex- 
ternal load passes through a maximum at about 3 watts for Electrophorus. One large specimen of Torpedo occidentalisi3 developed a maximum power of $6 \mathrm{kw}$ !

Records made from single electroplaxes or groups agree well in duration and voltage with those calculated from measurements of the total discharge. ${ }^{101}$ Single electroplax discharges are all-or-none, with a rising phase of 0.5-0.7 msec. Each discharge is monophasic because it is not propagated, shows no after-potential, and is all-or-none except after fatiguing, whereupon the voltage diminishes. A single stimulus to the nerve gives one discharge of the electroplax. When the nerve fibers to a portion of an electric organ are allowed to degenerate the organ becomes totally inexcitable. ${ }^{103}$ Injections of curare or of atropine reduce the electric discharge to zero. ${ }^{7}$

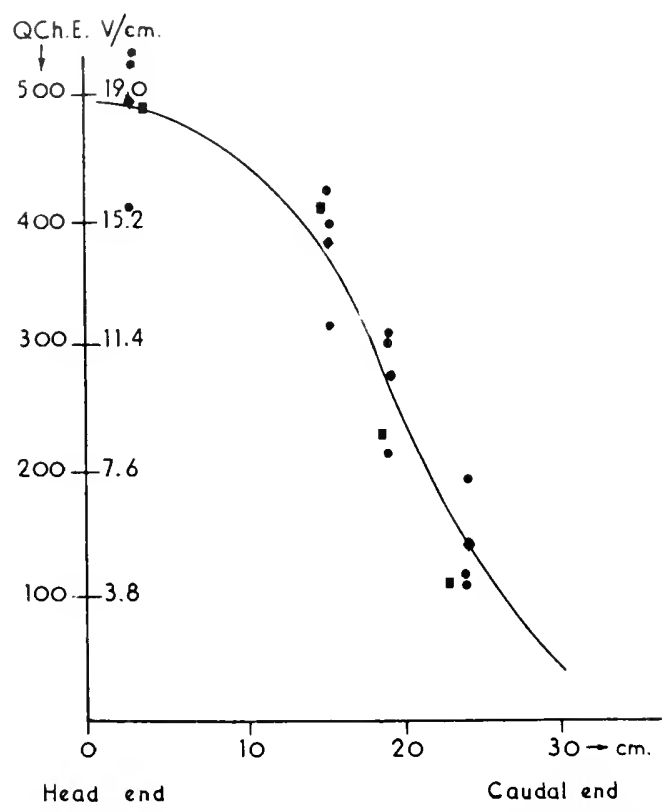

Fig. 238. Relation between potential and cholinesterase in electric organ of Electrophorus. $\bullet$ Average QChE; , volts per cm. From Nachmansohn, Cox, Coates, and Machado. ${ }^{175}$

In addition to being electrically similar to muscle end-plate, the electric organ is chemically similar. The electric organ is the richest tissue yet assayed for acetylcholine, having 40-100 micrograms per gram fresh weight, or nearly 0.1 per cent dry weight. Acetylcholine appears in the eserinized perfusate from an electric organ stimulated through its nerve. 17 Intra-arterial injection of as little as $2.5 \mu \mathrm{g}$. of ACh elicits a brief electrical discharge. Injection of eserine prolongs the spike duration, promotes facilitation of subliminal impulses, and hastens fatigue of the electric tissue. ${ }^{102}$ Cholinesterase is high in concentration in electric organ tissue, and in the electric eel the cholinesterase in centimeter lengths of the organ decreases toward the posterior end along a curve similar to the curve for diminution in voltage; i.e., the cholinesterase per electroplax tends to be constant (Fig. 238).175 
All evidence agrees, therefore, that, like the end-plate potential, the discharge of an electric organ is associated with the liberation of acetylcholine.

Electric tissue contains much phosphocreatine, 2:-26 which diminishes after prolonged activity. An old study $y^{: 37}$ of heat utilization by the electric organ in activity should be repeated with modern themopiles. The voltage output of Malopterus is reported to have a temperature optimum at $19^{\circ} \mathrm{C}$.

The electric organ of tishes has long been an object of wonder and curiosity; it is now most useful in leading to an understanding of the physicochemical processes in the nervous activation of muscle.

\section{CONCLUSIONS}

There are many kinds of muscles, functionally and structurally. Muscles car be arranged in orderly fashion according to physiological and histological properties.

Speed-fast contraction and relaxation, high fusion frequency, short excitation constant-is associated with sharp tramsverse striations and long fibers, with sparse sarcoplasm and abundant fibrils. Concluction is slow and electrical activity complex in sheets of short fibers, particularly when the fibers are branched, or connected by protoplasmic bridges. Local responses, both electrical and mechanical, may be one link in the excitation of fast muscle, but become the dominant response when innervation is multiple. Ability to maintain tension is associated with an abundance of connective tissue or with a "set" in the pattern of contractile elements. Increase in tension with increase in frequency of stimulation reflects facilitation of neuromuscular excitation as well as facilitation of mechanical properties. There are differences among muscles in the agents of neuromuscular transmission and in the phosphagens.

The mechanical properties of muscles make possible their gross function. Correlations of physiological and histological properties of muscle with phylogeny are few, and within an individual animal a number of muscle types exist. Within one muscle there may be fast and slow portions.

Comparison of the forms of phosphagen, one of the principal sources of energy in muscle, supports the echinoderm theory of chordate origin, and suggests that annelids may be farther phylogenetically from arthropods, and tunicates farther from true chordate than is sometimes supposed.

Electric organs are modified neuromuscular junctions, greatly enlarged and altered so that the production of action potentials is their principal function.

Study of the comparative physiology of muscle can be most useful in a correlation of the structure-histological and molecular-with the functional properties of different kinds of muscle; this approach should help to explain the fundamental nature of contractile tissues.

\section{REFERENCES}

1. Acheson, G. H., Fed. Proc. 7:4+7-457 (1948). Chemical aspects of neuromuscular transmission.

2. Alvarez, W. C., and Mahoney, L. J., Am. J. Physiol. 59:42I-430 (1922). Myogenic movements of intestine.

3. Amibache, N., J. Physiol. 104:266-287 (1946). Effect of ions on intestine. 
4. Ambacue, N., 1. Mrsiol. 106:139-153 (1947). Action potentials of intestine.

5. Anbache, N., Dixon, A. D., and Wright, E. A., J. Exper. Biol. 21:46-57 $(19+5)$. Pharmacology of crop and gizzard of earthworm.

6. Astbury, WV. T., Proc. Roy. Soc. Lond., B. 134:303-328 (1947). X-ray diffraction analysis of contractile and structural proteins.

7. Auger, D. and Fessand, A. C. R. Soc. Biol. Paris 135:76-78 (19+1). Effects of drugs on discharge of electric organ.

8. Augustinsson, K. B., Acta. Physiol. Scand. 11:146-150 (19+5). Cholinesterase in marine invertebrates.

9. Bace, Z. M., Arch. Internat. Physiol. 42:24-46 (1935), ibid. 40:357-373 (1935). Acetylcholine senvitivity of tissues of invertebrates.

10. Bace, Z. M., Arch. Internat. Physiol. +2:47-60 (1935). Insensitivity of crustacean muscle to acetylcholine.

11. Bace, Z. M., Arch. Internat. Pliysiol. Ht:17+189 (1937). Sensitivity to ACh, ChE analysis and assay for ACh of perfusate of muscles of holothurians, actinians and cephalopods.

13. Bace, Z. M., Arch. Internat. Physiol. 49:20-2t (1939). Responses of Phascolosoma, Sipunculus and Stichopus to acetylcholine.

14. Bace, Z. M., Arch. Internat. Physiol. 49:25-32 (1939). Action of eserine in ascidians and holothurians.

15. Bace, Z. M., Arch. Internat. Physiol. 49:25-32 (1939). Evidence for cholinergic nerves, holothurians.

16. Bace, Z. M., Biol. Rev. 22:73-91 (1947). Distribution of cholinergic systems in animals.

17. Bace., Z. M., Science 108:135 (1948). Excitatory and inhibitory sympathin as adrenalin and nor-adrenalin.

18. Bace, Z. M., and Brown, G. L., J. Physiol. 89:45-60 (1937). Pharmacology of motor nerve endings.

19. Bace, Z. M., and Coppee, G., Arch. Internat. Plysiol. 45:310-32+ (1937). Evidence for cholinergic nerves in worms.

20. Bace, Z. M., and Coppee, G., C. R. Soc. Biol. Paris 124:1244, ibid. 125:10591060 (1937). Escrine action on body wall of worms.

21. Bace, Z. M., and Nachmanson, D., J. Physiol. 89:368-371 (1937). Cholinesterase in invertebrate rnuscles.

22. Bacq, Z. M., and Oury, A., Bull. Acal. Roy. de Med. de Belg., Cl. Sci. 23:891893 (1937). Distribution of cholinesterase.

23. Baldwin, E., J. Exper. Biol. 10:212-221 (1933). Creatine phosphate in electric organs.

24. Baldwin, E., Comparative Biochemistry (1937). Cambridge Univ. Press. 112 p. Ch. V, pp. 66-72. Distribution of phosphagens.

25. Baldwin, E., Dynamic Aspects of Biochemistry (1947). Cambridge Univ. Press. 457 p. Ch. XI, pp. 282-285. Distribution and function of phosphagens.

26. Baldwin, E., and Neediam, D. M., Proc. Roy. Soc. London, B. 122:197-219 (1937). l'hosphagen in echinoderm múscle, in Torpedo electric organ.

27. Baldwin, E., and Yudkin, W. H., Biol. Bull. 95:273-274 (19+8). Phosphocreatine in annelids.

28. Barer, R., Biol. Rev. 23:159-200 (1948). Structure of striated muscle.

29. Bannes, T. C., Am. J. Ploysiol. 107:4+77-458 (1934). Multiple discharge of crustacean nerve.

30. Bayliss, L. E., Boyland, E., et al., Proc. Roy. Soc. London, B. 106:363-376 (1930). Adductor mechanism of Pecten.

31. Bayliss, M7. M., and Starling, E. H., J. Physiol. 24:99-1+3 (1899). Movements of small intestine.

32. Pilatvallet, M1., C. R. Soc. Biol. Paris 12t:108t-1085 (1936). Ions on movementr of Ildix intestine.

33. Beaumallet, M., C. R. Soc. Biol. Paris 126:1128-1130 (1937). Drugs on digestive mex conents of molluscs.

34. Benson, A. A., Et Al., Proc. Soc. Fxper. Biol. E Med. 49:289-291 (1942). Peripheral inhibition of Pecten slow adductor. 
35. Benson, E. B., and Lutz, B. R., Coll. Net 8:119 (1933). Irug action on holothurian intestine.

36. Bernuaru, C. C., and Funtin, A. S. v., Acta Physiol. Skand. 3:351-360 (19+2). After-potentials in manmalian muscle.

37. Bernstizin, J., and Tscinemak, A., Pflïg. Arch. ges. Plysiol. 112:439-521 (1906). lemperature change's in electric organs.

38. Bethe, A., Ztschr. vergl. Physiol. 24:613-637 (1937). Conduction and contraction of medusac.

39. Blaschiko, H., et AL., I. Physiol. 73:25-35 (1931). Regulation of terision in crustacean muscle.

40. Botazz1, P'., Arch. Internat. Physiol. 18:313-331 (1921). Active products of salivary glands, Octopus.

41. Borstond, F. F., Biol. Bull. 80:299-313 (19+1). Eserine on responses of earth worm body wall.

42. Bouman, H. P., Arch. Nierl. Physiol. 12:403-115 (1928). Chronaxic of fish muscles.

+3. Bozler, E., Ztschr, vergl. Physiol. 7:t07-t35 (1927). Comparative histology of tonus muscle.

44. Bozler, E., Ztschr. vergl. Physiol. 8:371-390 (1928). Tonus muscles.

45. Bozler, E., Ztschr. vergl. Physiol. 12:579-602 (1930). Contraction and con duction in tonus muscles of Helix and Pecten.

46. Bozlen, E., Ztschr. vergl. Physiol. 1+:429-449 (1931). Nechanical properties of muscle.

47. Bozler, E., Cold Spring Harbor Symp. 4:260-266 (1936). Mechanical propertics and activation of smooth muscle.

47a. Bozler, E., Am. J. Plysiol. 122:614-623 (1938). Conduction and excitation of smooth muscle.

48. Bozler, E., J. Cell. \& Comp. Physiol. 18:385-391 (194I). Mechanical properties of smooth muscles.

49. Bozler, E., Biol. Symp. 3:95-110 (1941). Motor units in smooth muscle; conduction in smonth muscle.

50. Bozler, E., Am. J. Physiol. 136:543-552 (1942). Pacemaker activity in ureter.

51. Bozler, E., Am. J. Physiol. 136:553-560 (1942). Action potentials of visceral smooth muscles.

52. Bozler, E., Experientia 4:213-218 (1948). Conduction, automaticity, tonus of visceral muscle.

53. Brenner, H., Ztschr. Zellforsch. u. mikr. Anat. 29:251-276 (1939). Striation widths of insect muscles.

54. Bronk, D. W., J. Cell. E Comp. Physiol. 2:285-294 (1932). Heat production by crustacean muscle.

55. Brown, D. E. S., and Sichel, F. J. M., J. Cell. E Cump. Physiol. 8:315-328 (1936). Contraction of isolated muscle fibers.

56. Brown, G. L., J. Physiol. 89:220-237, 438-461 (1937). Effects of ACh on vertebrate muscle.

57. Brown, G. L., Dale, H., et al, l. Physiol. 87:394-424 (1936). ACh and eserine on mammalian muscle.

58. Brown, G. L., and Harvey, A. M1., J. Physiol. 93:285-300 (1938). Speed of fowl muscles.

59. Brown, G. L., and Harvey, A. M., J. Physiol. 99:379-399 (1941). Speed of mammalian eye muscles.

60. Brücke, E. T., Ergebn. Biol. 6:327-425 (1930). Muscle time constants, review.

61. Buchtal, F., and Knappeis, G. G., Acta Physiol. Skand. 5:256-270 (1943). Propagation in isolated muscle fibers.

62. Buchtal, F., and Lindiand, J., J. Physiol. 95:59P-60P (1939). Acetylcholine on motor end plate.

63. Buddenbnock, W'. v., Grundriss der vergleichenden Physiologie (1939). Berlin, Borntracger. Bd. Il, pp. 991-1067, Comparative properties of muscle. pp. 1099 . 1109 , Electric organs.

64. Budington, R. A., Am. J. Physiol. 7:155-179 (1902). Properties of annelid muscle. 
65. Budington, R. A., Physiol. Zool. 10:141-155 (1937). Spontaneity of cloacal muscles of Thyone.

66. Bullock, T. H., J. Cell. \& Comp. Physiol. 22:251-272 (1943). Neuromuscular facilitation in medusae.

67. Bullock, T. H., and Nachmansohn, D., J. Cell. E Comp. Physiol. 20:239-242 (1942). Cholinesterase in lower invertebrates.

68. Cannon, W. B., and Rosenblueth, A., Am. J. Physiol. 104:557-574 (1933). Simpathin.

69. Cannon, W. B., and Rosenblueth, A., Autonomic Neuro-effector Systems (1937). New York, Macmillan. 229 p.

70. Carayon-Gentil, M., and Gautrelet, J., C. R. Soc. Biol. Paris 127:887-890 (1938). Choline and acecylcholine in invertebrates.

71. Ciladwick, L. E., Physiol. Zool. 12:151-160 (1939). Wing frequency in Drosophila.

72. Coates, C. W., ET Al., Zoologica 22:1-31 (1937). Discharge from Electrophorus.

73. Contes, C. IV., and Cox, R. T., Zoologica 27:25-28 (1942). Discharge and power output, Torpedo.

74. Contes, C. W., Cox, R. T., et al., Zoologica 25:249-256 (1940). Conduction in electric eel.

75. Cooper, S., and Eccles, J. C., J. Physiol. 69:377-385 (1930). Time constants of mammalian skeletal muscle.

76. Coppee, G., Arch. Internat. Physiol. 53:327-507 (1943). Neuromuscular transmission.

77. Couteaux, R., Bull. Biol. France e. Belg. 76:14-57 (1942). Cholinesterase of motor endings.

78. Couteaux, R., Rev. Canad. Biol. 6:563-711 (1947). Structure of motor end plate; neuromuscular transmission.

79. Couteaux, R., and Nachmansonn, D., Proc. Soc. Exper. Biol. \& Med. 43:177181 (1940). Cholinesterase, guinea pig muscle.

80. Cowan, S. L., Proc. Roy. Soc. London, B. 129:356-39l (1940). Eserine-like compounds on neuro-muscular junctions.

81. Cox, R. T., Am. J. Physics 11:13-22 (1943). Review of electric fish.

82. Cox, R. T., and Breder, C. M., Zoologica 28:45-51 (1943). Electric discharge from ray Narcine.

83. Cox, R. T., et AL., Ann. New York Acad. Sci. 47:487-500 (1946). Characteristics of electric tissue.

84. Curtis, H. J., Fed. Proc. 8:30-31 (1949); Am. J. Physiol. 159:499-504 (1949). Action potentials, heart muscle.

85. Dahlgren, U., Carnegie Inst., Wash., Publ. 212, 213 p. (1915). Histology, embryology, and polarity of electric organ.

86. Dale, H., ET AL., I. Physiol. 86:353-380 (1936). Acetylcholine release at motor nerve endings.

87. Davis, 12. A., and Fraenkel, G., J. Exper. Biol. 17:402-407 (1940). Metabolism and wing frequency in flight of insects.

88. Dreyer, N. B., Proc. Tr. Nova Scotia Inst. Sci. 17:199-203 (1927). Pharmacology of fish intestine.

89. DuBuy, H. G., Am. J. Physiol. 114:224-234 (1935). Neuromuscular excitation, crayfish.

90. DuBuy, H., An. J. Physiol. 116:22-23 (1936). Physiology and pharmacology of 'Thyone muscle.

91. Dubuy, H. G., Biol. Bull. 71:408-409 (1936). Contractile properties of long retractors of bolothurian, Thyone.

92. Ecches, J. C., and Magladery, J. W., J. Physiol. 90:31-67 (1937). Action potentials of nictitating membrane.

93. Ellis, C. H., et al., Biol. Bull. 83:334-352 (1942). Effects of drugs on crustacean ncuromuscular transmission.

94. Engelnari, E., Pflïg. Arch. ges. Physiol. 227:220-234 (1931). Acetylcholine from ciliary muscle, mammals.

95. Fange, R., Ark. Zool. 40A, 11:1-9 (1948). Drugs on intestine of cyclostome, Myxine. 
96. Faraday, M1., Phil. Tr. Roy. Soc. Part 1, 1-12. (1839). Experiments with electric fish.

97. Feldebrg, W., and Fessard, J., J. Physiol. 101:200-216 (19+2). Cholinergic nerves to electric organs.

98. Fenc, T. P., Chinese J. Physiol. 13:239-246 (1938). Veratrine fects in muscle.

99: Feng, T. P.. Biol. Symp. 3:121-152 (1941). Neuromuscular trăhsmission and repetitive contraction of muscle fibers.

100. Fenn, W. O., in Höber's Physical Chemistry of Cells and Tissues (19+5). Philadelphia, Blakiston. pp. 445-522. Contractile tissues, review.

101. Fessard, A., Amm. New York Acad. Sci. 47:501-514 (1946). Activity of electroplaxes.

102. Fessard, A., Arch. Internat. Physiol. 55:1-26 (1947). Analysis of discharge of electric organ.

103. Fessard, A., and Pezard, A., C. R. Soc. Biol. Paris 134:525-527 (1940). Decreased excitability of electric organ with nerve degeneration.

104 Fletcher, C. M., J. Physiol. 90:233-253 (1937). Action potentials in smooth muscle of Mytilus.

105. Fletcher, C. M., I. Physiol. 90:+15-428 (1937). Excitation of smooth muscles of Mytilus.

106. Florkin, M., Biochemical Evolution, transl. and ed. by S. Morgulis (19+9). New York, Academic Press. 157 p.

107. Frénérice, H., Arch. Internat. Physiol. 30:300-305 (1928). Chronaxie of insect muscles.

108. Friedrich, H., Ztschr. vergl. Physiol. 18:536-561 (1933). Neuromuscular excitation, Dixippus.

109. Galambos, R., J. Cell. E Comp. Physiol. 17:85-95 (1940). Relaxation patterns of invertebrate smooth muscles.

110. Gasser, H. S., and Hill, A. V., Proc. Roy. Soc. Lond., B. 96:398-137 (1924). Mechanical dynamics of muscle contraction.

111. Gelfan, S., and Bishop, G. H., Am. J. Physiol. 101:678-685 (1932). Local contraction without conduction in single muscle fibers.

112. Gilson, A. S., et Al., Ann. New York Acad. Sci. 47:697-714 (1947). Time course of tension development.

113. Grundfest, H., Ann. Rev. Physiol. 9:477-506 (1947). Theories of nervous and neuromuscular transmission.

114. Gunn, J. A., and Underhill, S. W. F., Quart. J. Exper. Physiol. 8:275-296 (1915). Mlyogenic movement of intestine.

115. Hall, C. E., Jakus, M. A., and Schmitt, F. O., Biol. Bull. 90:32-50 (1946). Electron microscopy of muscle and myosin filaments.

116. Harreveld, H. van, J. Exper. Biol. 16:398-402 (1939). Histology of axon terminations on crustacean muscle.

117. Harreveld, H. van, and Wiersia, C. A. G., J. Exper. Biol. 14:4+8-461 (1937). Triple innervation of crustacean muscle.

118. Harreveld. H. van, and Wiersma, C. A. G., J. Exper. Biol. 16:122-133 (1939). Quintuple innervation of Panulirus muscle.

119. Heipermanns, C., Zool. Jahrb., Abt. allg. Zool. u. Physiol. 50:1-31 (1931). Chronaxies of arthropod muscles.

120. Henry, K. M., MacDonald, A. J., et al., J. Exper. Biol. 10:153-171 (1933). Movement of digestive tract, fowl.

121. Hill, A. V., Proc. Roy. Soc. Lond., B. 100:108-115 (1926). Viscous elastic properties of smooth muscle.

122. Hill, A. V., and Solandt, D. Y., J. Physiol. 83:13P-14P (1934). Contraction of chromatophore muscle, Sepia.

123. Hines, M., Quart. Rev. Biol. 2:149-180 (1927). Red and white muscle.

124. Hobson, A. D., J. Exper. Biol. 5:345-393 (1928). Ions on movements of gut of Dytiscus.

125. Hoffmann, P., Ztschr. Biol. 61:311-325 (1913). Potentials and conduction, turtle retractor penis.

126. Hoffmann, P., Ztschr. Biol. 63:411-442 (1914). Histology of nerve endings in crustacean muscle. 
127. Hogben, L. T., and Hobson, A. D., J. Exper. Biol. 1:487-500 (1924). Drugs on visceral movements in invertebrates.

128. Hon, C. L., and Brucke, E. T., Pfiug. Arch. ges. Physiol. 226:411-417 (1931). Excitation of Vorticella, Daphnia, Hydra.

129. 1hle, J. E., Kampen, P. N. V., ET AL., Vergleichende Anatomie der Wirbeltiere (1927). Berlin, Springer. Ch. IV. Electric organs.

130. Jasper, H. H., and Pezard, A., C. R. Acad. Sci. 198:499-501 (1934). Relation between sarcomere width and speed of contraction.

131. Jordan, H. E., Anat. Rec. 10:493-508 (1916). Histology of leg muscles of sea spider Anoplodactylus.

132. Jordan, 11. E., Carnegie Inst., Wash., Publ. 251, pp. 273-290 (1917). Histology of striated muscle of Limulus.

133. Jordan, H. E., Anat. Rec. 16:217-246 (1919). Histology of striated muscle of Mlantis.

134. Jordan, H. J., Zool. Jahrb. Abt. allg. Zool. w. Physiol. 36:109-156 (1916). Tonus in bolothurian muscles.

135. Jordan, H. J., Allgemeine Vergleichende Physiologie der Tiere (1929). Berlin, Gruyter. 761 p.

136. Jordan, H. J., Ergehn. Physiol. 40:437-533 (1938). Tonus mechanisms of muscle.

137. Jullien, A., and Vincent, D., C. R. Soc. Biol. Paris 129:845-848 (1938). Cholinesterase in Crustacea.

138. Katz, B., J. Physiol. 87:199-221 (1936). Neuromuscular transmission in crabs.

139. Katz, B., Biol. Bull. 24:1-20 (1949). Neuromuscular transmission in invertebrates.

140. Katz, B., and Kuffler, S. W., Proc. Roy. Soc. Lond., B. 133:37+-389 (1946). Neuromuscular excitation in Crustacea.

141. Kejghley, G., and Wiersma, C. A. G., J. Cell. E Comp. Physiol. 17:305-314 (19+1). Heat production by crustacean muscle.

142. Knoefel, P., and Davis, H., Am. J. Physiol. 104:81-89 (1933). Repetition of nerve impulses to nictitating membrane.

143. Knowlton, F. P., and Campelel, C. J., Am. J. Physiol. 91:19-26 (1929). Peripheral inhibition in lobster.

144. Kraemer, F. K., Zool. Jahrb., Abt. allg. Zool. 1. Physiol. 52:86-117. (1932). Temperature on muscle twitch, beetles.

145. Kuffler, S. W., J. Neurophysiol. 5:18-26 (1942). End-plate potentials in muscles.

146. Kuffler, S. W., J. Neurophysiol. 8:113-122 (1945). Veratrine action on nervemuscle preparations.

147. Kuffler, S. W., Fed. Proc. 7:437-4+6 (1948). Electrical evidence for chemical neuromuscular transmitter.

148. Kuffler, S. W., and Gerard, R. W., J. Neurophysiol. 10:383-394 (1947). Small-nerve motor system.

149. Kuffler, S. W., and Katz, B., J. Neurophysiol. 9:337-346 (1946). Neuromuscular inhibition in Crustacea.

150. Kuffler, S. W., et al., J. Newrophysiol. 10:395-408 (1947). Small-nerve motor system.

151. Lambert, E. E., and Rosenblueth, A., Am. J. Physiol. 114:1+7-159 (1935). Nictitating membrane of cat.

152. Lapicque, L., C. R. Soc. Biol. Paris 128:688-692 (1938). Relation between chronaxic and contraction rate of muscles.

153. Laplcque, L., C. R. Soc. Biol. Paris 136:298-300 (1942). Chronaxie of insect muscles.

15t. Lapicque, L., and Faure, F., C. R. Soc. Biol. Paris 74:1194 (1913). Chronaxie of Vorticella.

155. Lapicque, L., and Veil, C., C. R. Soc. Biol. Paris 93:1591 (1925). Chronaxies of earrhworms and leeches.

156. Lucis, K., J. Physiol. 51:1.35 (1917). Double neuromuscular system of crayfish ctaw adductor.

157. LuTz, B. R., Biol. Bull. 61:93-100 (1931). Responses and regulation of visceral muscle of elasmobranchs. 
158. Magnus, R., Pflüg. Arch. ges. Physiol. 102:349-363 (1907). Mvogenic move ments of intestine.

159. MAngold, E., Ztschr. allg. Physiol. 5:135-205 (1905). Nerve endings in arthroporl muscle.

160. Marceau, F., Arch. Anat. Micr. 7:495-589 (1905). Histology of molluscin muscle.

161. Marceau, F., Arch. Zool. Expér. Gén. 2:295-469 (1909). Contraction of molJuscan muscle.

162. Marnont, G., and Winersia, C. A. G., J. Physiol. 93:173-193 (1938). Inhibi tion in crustacean muscles.

163. Mannay, A., and Nichmansonn, D., J. Physiol. 89:359-369 (1937). (holin esterase in striated muscle.

164. Mamnay, A., and Nacumansonn. D., C. R. Soc. Biol. Paris 124:9429+4 (1937) Cholinesterase in striated muscle.

165. Marnay, A., and Nachmansomin, D., J. Physiol. 92:37-47 (1938). Cholinesterase in frog and lizard muscle.

166. MlcSweeney, B. A., and Robson, J. M., J. Physiol. 68:124-131 (1929). Contraction constants, mammalian stomach.

167. Meyer, W. H. F., Arch. Nierl. Physiol. 12:162-187 (1927). Contraction rates in fish muscle.

168. Meyerhof, O., Coll. Net 16:177-181 (1941). Nature and distribution of phosphagens.

169. Mhlen, F. R., J. Physiol. 40:+31-1+1 (1910). Contractions of lobster intestine.

170. Millott, N., Proc. Roy Soc. Lond., B. 131:271-295 (19+3). Properties of visceral muscles, earthworm.

171. Nacumansorn, D., J. Physiol. 95:29-35 (1939). Cholinesterase in muscle of developing chick.

172. Nachmansohn, D., in Currents in Biochemical Research (D. Green, cd.). (1946). New York, Interscience. pp. 335-356. Role of acetylcholine in nerve transmission, review.

173. Nachmanson, D., Personal communication (1948). Cholinesterase in muscles of different animals.

173a. Nachinansonn, D., Bull. Johus Hopkins Hosp. 83:463-493 (1948). Role of acetylcholine in neuromuscular transmission.

174. Nachmansonin, D., et al., J. Gen. Physiol. 25:75-88 (1941). Relation of potential to cholinesterase in Electrophorus.

175. Nachmansohn, D, Cox, R. T., et al., J. Neurophysiol. 5:499-516 (1941). Relation of potential to cholinesterase in Electrophorus.

176. Nachinansonn, D., and Meyerhof, B.. J. Neurophysiol. 4:348-361 (1941). Cholinesterase in electric organ.

177. Neebram, D. M., Physiol. Rev. 6:1-27 (1926). Properties of red and white muscle.

178. Neednam, J., and Needian, 1). M., Science Progress 26:626-642 (1932). Phosphagen distribution as related to origin of vertcbrates, review.

179. Needhan, D. M., Needham, J., et al., Proc. Roy. Soc. Lond., B. 110:260-294 (1932). Distribution of phosphagens in animals.

180. Nicholls, J. V., Contr. Canad. Biol. E Fish. N. S. 7:447-463 (1932). Drugs on gastric motility in elasmobranchs.

181. Obreshikove, V., Biol. Bull. 81:105-113 (1941). Drug action on Daphnia intestine.

182. Olson, M., Biol. Bull. 74:3+2-347 (1938). Histology of retractor muscle, Thyone.

183. Overbeek, J. van, Ztschr. vergl. Physiol. 15:784-797 (1931). Tonus in Anodonta muscle.

184. Pantin, C. F. A., J. Exper. Biol. 11:11-27 (1934). Excitation of crustacean muscle.

185. Pantin, C. F. A., J. Exper. Biol. 12:119-138, 139-155, 156-164, 389-396 (1935). Responses of actinians to drugs; properties of actinian muscle.

186. Pantin, C. F. A., J. Exper. Biol. 12:389-396 (1935). Facilitation in sea anemone. 
187. Pantin, C. F. A., J. Exper. Biol. 13:111-130, 148-158, 159-169 (1936). Excitation of crustaccan muscle: Neuromuscular facilitation (111-130); Quick and slow responses (148-158); Inhibition (159-169).

188. Parnas, J., Pflig. Arch. ges. Physiol. 134:4+1-495 (1910). High efficiency of Pecten smooth muscle.

189. Plenk, H., Ztschir. Mikr. Anzat. Forsch. 33:605-624 (1933). Histology of cephalopod muscle.

190. Postma, N., Proc. Ned. Akad Wtnsch. Amst. 44:1151-1158 (1941). Muscle tone, Helix.

191. Pringle, J. W. S., J. Exper. Biol. 16:220-231 (1939). Slow and fast motor systems in cockroach.

192. Pringle, J. IV. S., J. Exper. Biol. 17:8-17 (1940). Multiple innervation of insect muscle.

192a. Pringle, J. W. S.. J. Physiol. 108:226-232 (1949). Muscle rhythm, flies.

193. Prosser, C. L., and Young, J. Z., Biol. Bull. 73:237-24l (1937). Fepetitive excitation of giant fiber in squid.

194. Pumpitiney, R. J., J. Exper. Biol. 15:500-505 (1938), Double innervation of clam muscle.

195. Quincke, H., and Stein, J., Ergebn. Physiol. 34:907-1064 (1932). Comparative chronaxies.

196. Ramsay, J. A., J. Exper. Biol. 17:96-115 (1940). Contractile properties of buccal retractor of Helix.

197. Ranisay, R., and Street, S. F., J. Cell. E Comp. Physiol. 15:11-34 (1940). Isometric length-tension relations of isolated muscle fibers.

198. Ramsay, R., and Street, S. F., Biol. Symp. 3:9-34 (1941). Mechanical factors in muscular contraction.

199. Riesser, O., Arch. Exper. Path.Pharm. 172:194-212 (1933). Contraction rate, drug effects, holothurian muscles.

200. Rixcuife, A. D., The Comparative Physiology of Muscular Tissue (1928). Cambridge Univ. Press. $111 \mathrm{p}$.

201. Rosza, G., Szent-Grorgr, A., and Wyckoff, R.'W. G., Biochem. et Biophysica Acta 3:561-569 (1949). Electron microscopy of muscle proteins.

202. Rosenblueti, A., Biol. Symp. 3:111-120 (1941). Conduction in smooth muscles.

203. Rosenbluetir, A., Davis, H., and Rempel, B., Am. J. Physiol. 116:387-407 (1936). Action potentials of smosth muscle.

204. Rosenbluetil, A., Hoagland,et al., Am. J. Physiol. 133:724-735 (1941). After-potentials of striated muscle.

205. Roseneluetri, A., and Riocir, D. McK., Am. J. Physiol. 106:365-380 (1933). Diffusion of mediator in smooth muscle.

206. Ross, D. M., J. Exper. Biol. 22:21-31 (1945). Drug effects on facilitation in actinians.

207. Ross, D. M., J. Exper. Biol, 22:32-36 (1945). Active agents (possibly tyramine) from extracts of actinians.

208. Ruckept, W., Arch. exper. Path. 150:221-235 (1930). Tonic properties of fetal muscle.

209. Scinnitit, F. O., et Al., Ann. New York Acad. Sci. 47:799-812 (1947). X-ray diffraction and clectron microscopic study of muscle, review.

210. Sichel, F. J. M., Anı. J. Physiol. 133:446-447 (1941). Elasticity of sarcolemma and whole muscle tiber.

211. Sichiel, F. J. M., and Prossia, C. L., Am. J. Physiol. 128:203-212 (1942). Absence of refracteriness in lical contractions of isolated muscle fibers.

212. Singli, J., J. Physiol. 92:62-90 (1938). Interaction of ions, drugs and electric stimulation of Mytilus muscle.

213. Smiтi, C., and GLick, D., Biol. Bull. $77: 321$ (1939). Cholinesterase in invertebrate tissues.

214. Smitr, C., Eт AL., Biol. Bull. 79:377 (1940). Cholinesterase content, ACh sensitivity of Cercbratulus.

215. Solf, V., Zool. Jahrb., Abt. allg. Zool. u. Physiol. 50:174-264 (1931). Contrac. tion time, wrthopteran muscle. 
216. Speidel, C. C., Am. Jour. Anat. 62:179-235 (1938). Growth and repair of muscle fibors in tadpoles.

217. Straus, WV. L., and Weddell, G., J. Neurophysiol. 3:358.369 (1940). Nature of carly contractions in rat fetuses.

218. Szent-Grongr, A., Chemistry of Muscular Contraction (1947) New York, Academic Press. 150 p.

219. TenCatx, J., Arch. Néerl. Physiol. 9:598-604 (1924). Movements of risceral muscle of Dytiscus.

220. Uexkull, J. von, Ztschr. Biol. 58:305-332 (1912). Adductor mechanisms of Pecten.

221. Umrath, K., Ztschr. Biol. 99:477-483 (1939). Refractory period, frog smooth muscle.

222. Ungan, G., Anu. Physiol. Physiochim. Biol. 13:30t-315 (1936-1937). Drug effects on stomach of Sepia.

223. W'ells, G. P., J. Exper. Biol. 14:117-157 (1937). Movements of oesophagus of Arenicola.

224. Wells, G. P., J. Exper. Biol. 14:290-301 (1937). Movements of proboscis of Glycera.

225. Welsil, J., J. Exper. Biol. 16:198-219 (1937). Assay of crab inuscle for ACh.

226. Wiersma, C. A. G., Biol. Symp. 5:259-289 (1941). Neuromuscular activation in Crustacea, review.

227. Wiersma, C. A. G., J. Comp. Neuról. 7t:63-79 (1941). Distribution of nerves to leg muscles of crustaceans.

228. Wiersia, C. A. G., and Elcis, C. H., J. Exper. Biol. 18:223-236 (1942). Peripheral inhibition in crustaccans.

229. Wiersma, C. A. G., and Harreveld, A. v., J. Exper. Biol. 15:18-31 (1938). Optimal frequencies in crustacean neuromuscular excitation.

230. Wiersia, C. A. G., and Harreveld, A. v., Physiol. Zool. 11:75-81 (1938). Optimal frequencics for fast and slow systems in crustacean muscle.

231. Wiensma, C. A. G., and Wrigit, E. B., J. Exper. Biol. 23:205-212 (1947). Action potentials of crustacean muscles.

232. Wigglesworth, V. B., Principles of Insect Physiology (1938). London, Methuen. $+3+$ p. Ch. 2. Insect muscles and locomotion.

233. Wilhelmi, A. E., in Howell's Textbook of Physiology (John F. Fulton, ed.) (19+6). Philadelphia, Saunders. Ch. 3, pp. 56-96. Muscle chemistry.

234. Wills, I. H., Am. J. Physiol. 136:623-628 (1942). Speed of cat muscles.

235. Wilska, A., Skand. Arch. Physiol. 82:258-26+ (1939). Conduction in single muscle fibers.

236. Wunton, F. R., J. Physiol. 69:393-410 (1930). Tonus in mammalian smooth muscle.

237. W/inton, F. R., J. Physiol. 88:492-511 (1937). Alteinating and direct current stimulation of Mytilus smooth muscle.

238. Wu, K. S., J. Exper. Biol. 16:18+-197 (1939). Physiology and pharmacology of earthworm gut.

239. Wu, K. S., J. Exper. Biol. 16:251-257 (1939). Phamacology of annelid body IIall.

240. Yeager, J. F., Ann. Ent. Soc. Amer. 24:739-745 (1931). Novements of roach crop and gizzard.

241. Young, A. C., Biol. Symp. 3:153-160 (1941). Action potentials in skeletal muscle.

242. Young, J. Z., Proc. Roy. Soc. Lond., B. 120:303-318 (1936). Reactions of visceral muscles of fish.

243. Young, J. Z., J. Exper. Biol. 15:170-185 (I938). Giant fiber control of squid mantle. 


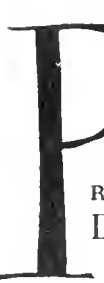

\section{Amoeboid Movement}

ROTOPLASMIC STREAMIING occurs in many living cells, possibly in all.

Diffusion is too slow a process for the transport of solutes from one part of a cell to another, and active cytoplasmic movement provides a rapid transport mechanism. Streaming may proceed in a fixed path and may be fast enough for direct microscopic observation, as the cyclosis around the vacuole of some plant cells, or the transport of food vacuoles and granules about the body of a ciliate protozoan. In other cells streaming may be slow and may be more of a churning than a fixed current; such cytoplasmic activity is best seen by accelerated motion pictures, as of tissue cultures (fibroblasts, etc. ${ }^{23}$ ), or of tips of growing nerve fibers. ${ }^{10,53}$ The cellular mechanism of protoplasmic streaming is unknown. Its dependence on colloidal properties, particularly on viscosity changes, is shown by the effects of hydrostatic pressure; streaming in Elodea is slowed in proportion to a decrease in viscosity. 28

\section{TYPES OF AMOEBOID CELLS AND OF PSEUDOPODS}

Amoeboid movement has a colloidal basis, as does protoplasmic streaming; amoeboid movement is also accompanied by changes in cell shape and often by progressive motion. Amoeboid movement may be directed locomotion, as in the rhizopod Protozoa (Sarcodina), in the plasmodium of myxomycetes, in amoeboid leucocytes (particularly vertebrate lymphocytes), in amoeboid or wandering cells of many kinds of animals (such as the archeocytes of sponges), and in growing nerve fibers; or amoeboid movement may consist in the extension, flexion, and retraction of processes (pseudopods) concerned primarily in feeding, as in most Foraminifera, Heliozoa, Radiolaria, vertebrate macrophages, and various phagocytes such as reticuloendothelial cells (e.g., Kupffer cells of the liver). Locomotory amoeboid movement requires attachment to some substrate; non-polarized amoeboid movement occurs in free pseudopods.

Among the free-living amoeboid animals the manner of locomotion differs slightly according to cell form and type of pseudopod. Pseudopods may be lobopods, broad to cylindrical and round at the tip (Fig. 239, A-E); they may be filopods, slender with pointed tips; they may be reticulopods, threadlike, branching, and anastomosing as in Foraminifera, or they may be axopods, rays with a central axial rod as in Heliozoa and Radiolaria (Fig. 239, G). ${ }^{13}$ Locomotion by lobopods has been much studied; filopods, reticulopods and axopods are primarily freely extended feeding pseudopods; filopods may show streaming without accompanying change in length, and axopods may be very contractile. 
Amoebae differ greatly in cell form; Amoeba limax has a single lobose pseudopod (Fig. 239, D); the stellate form of amoeba has many free pseudopods; A. proteus and A. dubia (Fig. 239, $A$ and $C$ ) are multipodal, i.e., they have several attached pseudopods with streaming in various amounts in each. Amoebae may have surface ridges, as in A. verrucosa (Fig. 239, E), or they may have an irregular "tail" or uroid, as in Pelomyxa palustris (Fig. 239, F). Lymphocytes vary likewise, sometimes having a broad advancing pseudopod and smaller tail, the "looking glass" shape, or they may be worm-

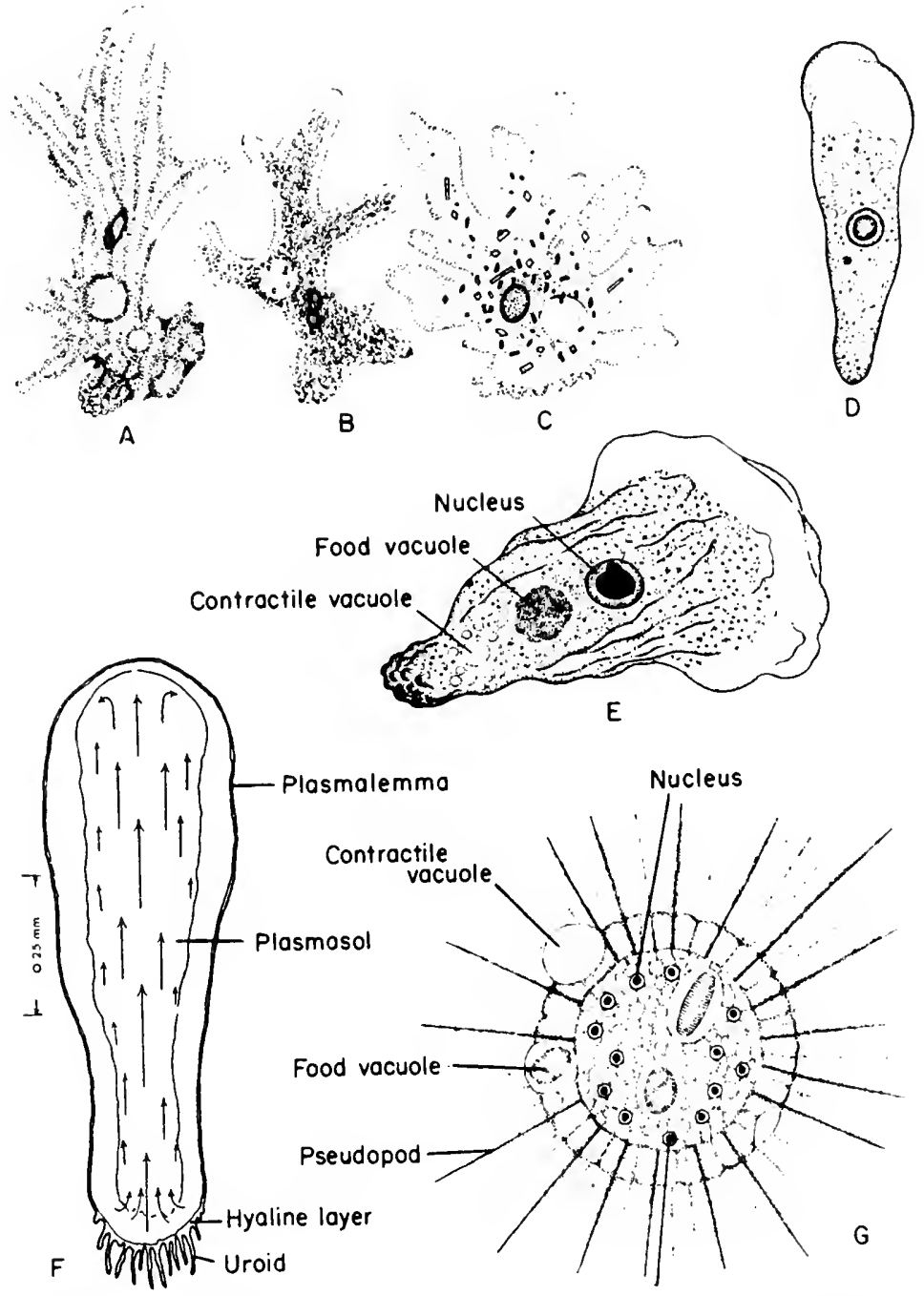

Fig. 239. Drawings showing various types of pseudopods. Typical multipodal amoeboid cells: $A, A$ moeba proteus in locomotion; B, Amoeba discoides in locomotion; C, Amoeba dubia in locomotion. From Schaeffer." Typical monopodal amoeboid cells: D, Amoeba limax; E, Amoeba verrucosa. Note ridges on pellicle. From Kuhn." F, Pelomyxa palustris, showing direction of flow of granules. Note posterior uroid. From Mast. ${ }^{37} \mathrm{G}$, A typical heliozoan, Actinosphaerium eichhorni, showing axopods. From Kuhn. ${ }^{23}$ 
like or may show local constriction rings according to the physical nature of the medium." The various forms of amoeboid cells are not always constant for a species and one individual may take on different forms under various conditions. Amoeba proteus, for example, in distilled water becomes stellate or radiate, whereas it may be active and monopodal in a dilute saline; it is also stellate prior to fission. Amoebae have a central fluid plasmasol and an outer viscous plasmagel. The thickness of the gel varies greatly among different species: there is much gel in A. proteus, A. verrucosa, Pelomyxa carolinensis, and P. palustris, but very thin gel in Amoeba dubia and in the parasitic A. blattae. Anoeba dubia is useful, therefore, for measuring by centrifugation the viscosity of the plasmasol, and $A$. proteus for meas. uring viscosity of the plasmagel. ${ }^{12}$

\section{GENERALIZED PICTURE OF AMOEBOID MOVEMENT}

There are many variations in amoeboid movement in the different cell and pseudopod types, but a basic pattern is found in all. A "typical" amoeba consists of a thin outer layer, the plasmalemma, which has adhesive properties, is not wet by water (is hydrophobic), and slides freely over the next inner layer. Beneath the plasmalemma is a hyaline layer which is fluid as judged by brownian activity when granules enter it. This layer is thin or absent from a region of attachment to substrate; it often thickens as an extensive hyaline cap at the front of an advancing pseudopod. Next is the shell or cylinder of plasmagel which is motionless and rather viscous. In a monopodal amoeba the gel thickens gradually from the anterior to the posterior end. In many species it is extended as a very thin plasmagel sheet beneath the anterior hyaline cap, a sheet which frequently ruptures, allowing granules to enter the hyaline cap. The core of the amoeba is the plasmasol in which granules flow freely forward although the lateral margin of it may be stationary. The nucleus is normally in the plasmasol, the contractile vacuole in the plasmagel. Both regions contain various granules, food vacuoles, and specific crystals contained in little vacuoles. The protoplasm is normally hypertonic (except possibly in marine and parasitic forms), and the plasmagel imparts a turgidity which results in the non-spherical cell forms. In species in which the sol of the pseudopods is continuous to the tip (as in Diffugia) the plasmalemma must oppose free swelling.

Locomotion depends on three basic factors, according to current views. ${ }^{31}$ The first is attachment to the substrate. This is facilitated by traces of salts, particularly calcium, in the medium. Organic solutes such as lactose and glycerin do not facilitate attachment; $\mathrm{Ca}, \mathrm{Mg}$, and $\mathrm{K}$ are additive for attachment but not for locomotion. ${ }^{33}$ In amoebae, as in leucocytes, the firmest attacliment is at the tips, and new pseudopods are more firmly attached than old ones. Second, plasmagel is continuously being converted to plasmasol at the posterior end or at some fixed region, and plasmasol is converted to plasmagel anteriorly or in any extending pseudopod. As plasmasol flows forward granules either are deflected laterally to become plasmagel or break into the hyaline cap and then gelate as a new cap is formed. Attachment and reversible solation-gclation are readily observed. Third is the force that causes forward flow of the plasmasol, which is less apparent. Most current 
theories attribute this to the elastic strength or contractileness of the plasmagel, which is greater posteriorly and squeezes the plasmasol forward.

Lateral examination of granules in an amoeba and of particles adhering to the plasmalemma has shown that, as the cell advances, granules in the gel remain fixed until the posterior end reaches them, when they enter the sol (Fig. 240). Similar stability of the gel is shown in advancing lymphocytes, in which small lateral protuberances remain fixed until the posterior end reaches them. ${ }^{5}$ Particles on the ventral attached portion of the plasmalemma are also fixed, but particles elsewhere on the plasmalemma, both dorsal and ventral, move forward (Fig. 240). Thus the plasmalemma cither is stretched and varies in thickness, or is rapidly constituted at the posterior end and in freely extending pseudopods. ${ }^{31}$ Mid-dorsally a granule may move faster than the rate of advance of the amoeba, indicating active slipping of the plasmalemma over the hyaline layer. ${ }^{47}$ In $A$. verrucosa, rarely in A. proteus or A. limax, there is a "rolling movement," the plasmalemma rolling like a rubber bag of liquid down an incline. ${ }^{20,31}$ When attachment is discontinuous there may be a "walking" movement; the advancing tip

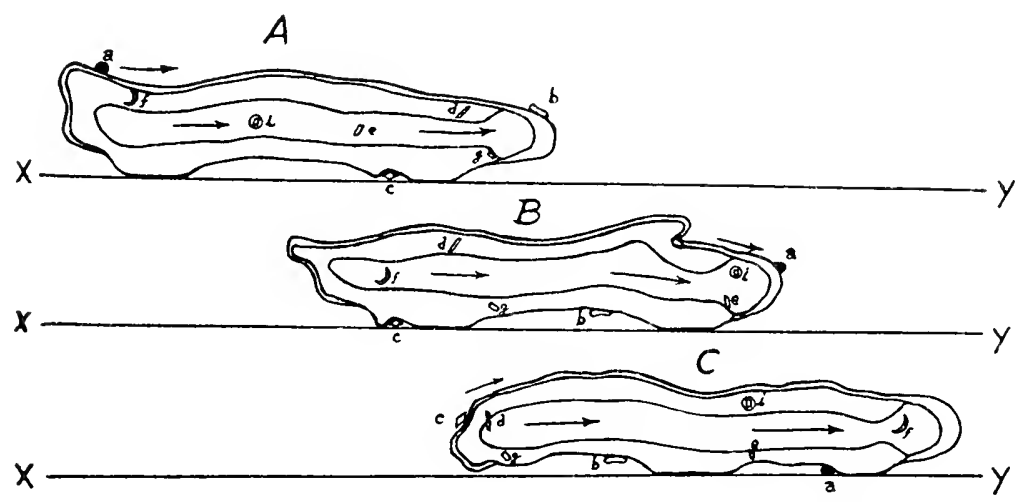

Fig. 240. Series of sketches of Amoeba proteus as seen from the side, representing the movement of different parts of the body during locomotion. $A, B, C$, Three successive positions on the same substratum $x y ; d, e, f, g, i$, particles in the plasmagel and the plasmasol; $a, b, c$, particles attached to the plasmalemma. From Mast. ${ }^{31}$

attaches and by contraction of gel just behind the tip the cell is pulled forward. This is well shown by Difflugia, which has a shell consisting of sand grains cemented together. One pseudopod extends freely, waves about, and finally attaches at its tip, then as the gel of the first pseudopod contracts, a second pseudopod is extended, receiving plasmasol from the attached pseudopods, which thus shortens and pulls the shelled portion forward. ${ }^{7}, 34$

Freely crawling amoebae move at rates of 0.5 to 4.5 micra per second, most of them at the rate of about 1 micron per second. ${ }^{12}$ Monopodal individuals travel faster than multipodal ones; nonopodal specimens of $A$. proteus in $0.001 \mathrm{~N} \mathrm{NaCl}$ averaged 4.6, irregular monopodal 4.3, and multipodal 2.1 micra per second. ${ }^{44}$ Lymphocytes in tissue culture moved an average of 0.55 $\mu / \mathrm{sec}$., whereas the non-polarized macrophiges averaged $0.004 \mu$ or less per second. ${ }^{5}$ 
In feeding, those amoebae which travel by small pseudopods, as A. proteus, form food cups consisting of lateral and dorsal pseudopods flowing around a food mass which may itself be motile. Food particles do not adhere to the plasmalemma. The pseudopods of the cup meet distally, they may or may not touch the food, and the food mass is thus incorporated in a food vacuole. In amoebae with a single broad pseudopod, as in A. limax, there appears to be some adhesion of food masses to the plasmalemma and the cell surface indents, enclosing the food in a vacuole. ${ }^{20}$ Also the surface of reticulopods and axopods are sticky, and food adheres to their surface.

\section{THEORIES OF AMOEBOID MOVEMENT}

Several theories have been proposed to account for amoeboid movement. The fascinating history of this subject has been well reviewed. ${ }^{6}, 31$ The first of these theories was popular with various modifications from 1835 (Dujardin) until 1875 (Schulze). It postulated contraction in elements in various parts of the cell, particularly the pseudopods, which pulled the protoplasmic mass ahead. The second theory involved surface tension forces and was based on the analogy of amoeboid movement with the movement of a mercury drop toward some salt, such as potassium dichromate, or with the currents in a drop of oil in contact with a soap. This theory was developed with many variations by Berthold (1886), Bütschli (1892), and Rhumbler (1898), and still persisted in a paper by Tiegs in $1928 ;^{52}$ it postulates that some organic solute lowers the surface tension at some point on the amoeba, and a pseudopod is there extended. That such action can occur is shown by the modern observation ${ }^{4}, 27$ that a drop of one of various paraffin oils can form a cap, a pseudopod forms beneath it, and fountain streaming, often without forward locomotion, can continue beneath the cap for many hours.

There is abundant evidence against surface tension forces as being normally responsible for amoeboid movement. The plasmalemma is not a liquid surface but an elastic membrane. The currents on the plasmalemma are forward, not backward as on the surface of a drop of mercury or oil moving by a "pseudopod." Also there is no reasonable suggestion regarding the nature and method of secretion of any solute which might lower the surface tension locally.

The most reasonable current theory of amoeboid movement is based on the reversible gel-sol transformation and assigns the propulsive force to contractility of the gel. This notion was actually proposed in 1863 by Wallich and was advanced by Jennings in 1902. The first discussion in terms of colloidal properties of gels and sols was by Hyman; ${ }^{18}$ the theory was advanced by Pantin, ${ }^{22,}+3$ and greatly extended by Mast and his associates. ${ }^{31}$, 3:2. 34 Support has come from pressure experiments by Marsland and Brown, ${ }^{30}$ and from studies of blood cells in tissue culture.5. 26

The plasmagel is truly elastic. Measurements of the extensibility of strands of myxomycete plasmodia gave a value of Young's modulus of $9 \times 10^{4}$ dynes/cm.", which is about one-thirtieth as elastic as muscle. ${ }^{41}$

The tension at the cell periphery, as measured by the centrifugal force to pull an oil droplet out through the surface, is 1 to 3 dynes $/ \mathrm{cm}$. or lowerslightly more than the peripheral tension in marine eggs but much lower than 
the tension of 20 dynes $/ \mathrm{cm}$. at the interface of olive oil and water. ${ }^{10}$ The tensions measured by Harvey and Marsland are not "surface tension" but total restraining tension residing in the cell periphery; there is no rigorous distinction in such a system between contractile and elastic tension. Calculations indicate that tensions of 1 to 3 dynes $/ \mathrm{cm}$. are of the order of magnitude needed to force the sol forward, ${ }^{12}$ but an amoeba can develop much greater tensions, as when it pinches a Paramecium in two. ${ }^{\mathbf{4 0}}$

The contractile tension of the gel presumably resides in long protein molecules whose organization and "contractility" is indicated by measurements

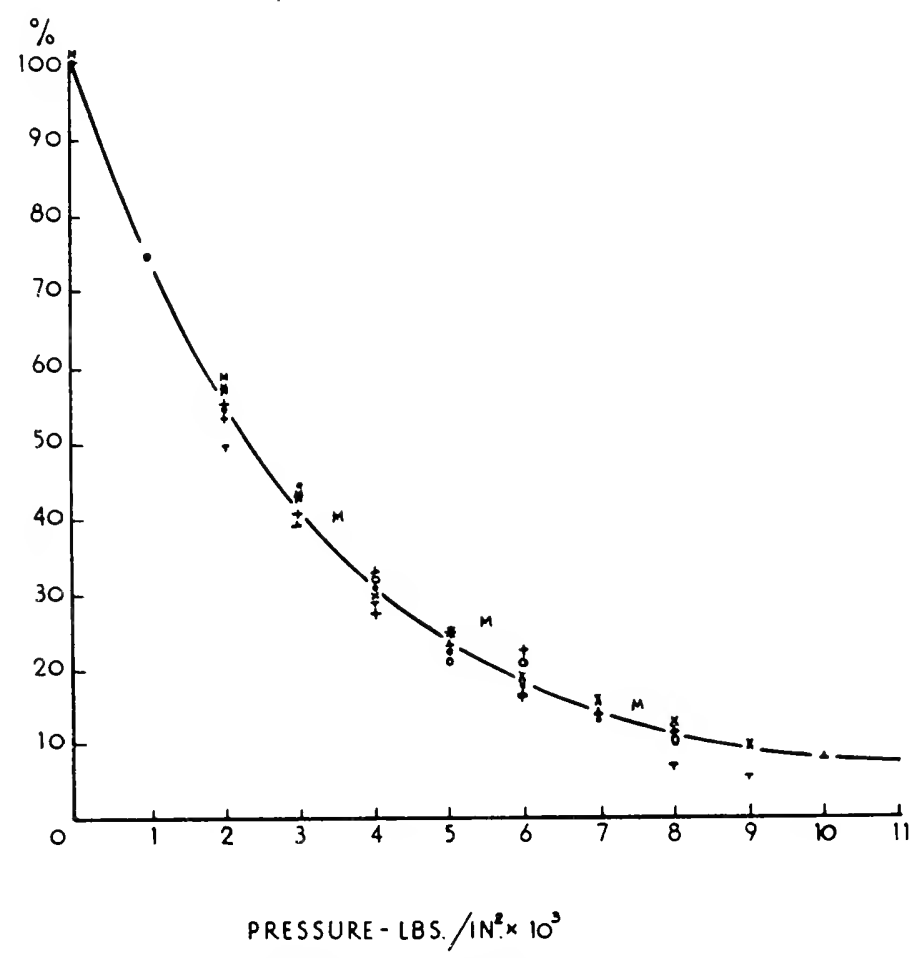

Fig. 241. Proportionality between the effects of hydrostatic pressure on protoplasmic streaming (and cell division) and the degree of solation imposed by pressure in various gel systems. - arbitrary point, all other values relative to this; $T$ gel value, amoeba; $\perp$ gel value, unf'ertilized Arbacia eggs; $\times$ gel value, cleaving Arbacia eggs; + rate of cleavage, Arbacia cggs; - gel value, Elodea cells; $\bigcirc$ rate of streaming, Elodea cells; $M$ gel value, actomyosin gel (rabbit muscle at $\mathrm{pH} 6.5,23^{\circ}-24^{\circ} \mathrm{C}$ ). Redrawn from Marsland 1942 and 1944.",

of resistance to granule movement in a centrifugal field, and by observation of the effects of hydrostatic pressure. The gel of newly formed or advancing pseudopods is less rigid than the gel in posterior regions. When amoebae are subjected to high hydrostatic pressure the "viscosity" falls, the gel solates and locomotion stops. At $2000 \mathrm{lb} / \mathrm{in} . "$ pseudopods are long and cylindrical; above $6000 \mathrm{lb}$./in." no new pseudopods are formed; at about 6500 lb./in." terminal spheres appear on pseudopods and the pseudopods retract as balls of fluid. A series of functions-amoeboid movement, chromatophore 
expansion, cyclosis in Elodea, cleavage in Arbacia eggs-are similarly stopped as the protoplasmic rigidity decreases under high pressures (Fig. 241). These are in contrast to bjoluminescence, ciliary movement, muscle contraction, and nerve propagation, which functions may be enhanced by pressure properly applied.2s, 30

The rate of locomotion is not directly related to the gel-sol ratio, even though the gel is thicker posteriorly. For example, the gel increases with increasing external acidity, it also increases as the temperature is lowered, yet the rate of locomotion show's broad $\mathrm{pH}$ and temperature optima. $39,45,51$ For bricf exposures to different temperatures (not metabolic acclimation) the velocity of locomotion in $A$. proteus rises from zero at about $5^{\circ} \mathrm{C}$, to a maximum at 22.5-24, sometimes a double maximum, then declines to zero at about $33^{\circ}$, 39. 49 whereas in the marine Flabellula the maximum is at $35-40^{3}$. The gel-sol ratio, however, declines with rising temperature, ${ }^{39}$ as does the viscosity (with some irregularity). ${ }^{11}$

In Anoeba protens there are two $\mathrm{pH}$ values at which the rate of locomotion is maximal, pI 6.2 and $\mathrm{pH} 7.5$, in the presence of a salt mixture; but in the presence of single cations, $\mathrm{Na}^{+}, \mathrm{K}^{+}$, or $\mathrm{Ca}^{+}+$, only one $\mathrm{pH}$ maximum occurs. ${ }^{\text {t. }}$ An amoeba which is largely sol, A. dubia, showed an increase in viscosity, as measured by centrifugation, in $\mathrm{NaCl}$ and $\mathrm{KCl}$ and a de. crease in $\mathrm{MgCl}_{2}$ and $\mathrm{CaCl}_{2}$, whereas, in similar concentrations on the gelrich A. proteus, Ca increased and $\mathrm{K}^{+}, \mathrm{Na}^{+}$, or $\mathrm{Mg}^{+}+$decreased viscosity. ${ }^{13}$, It High external calcium tends to increase the gel-sol ratio in a $\mathrm{pH}$ range where $\mathrm{Na}^{+}$or $\mathrm{K}+$ cause a decrease in gel. ${ }^{1+4}{ }^{45}$ The effects of salts depend on external $\mathrm{pH}$, but in no condition is gel-sol ratio or viscosity as measured by centrifugation affected in the same manner as is the rate of locomotion. The frequency of rupture of the plasmagel sheet is, however, related to the gel-sol ratio; those conditions which decrease the amount of gel also increase the frequency of rupture of the sheet."3: Gel-sol ratio and mean viscosity are important in locomotion, but other factors also contribute. It is probable that the rates of gelation and solation are important; also the contractility of the gel need not be proportional to its viscosity or to its amount relative to the sol. Changes in elasticity of the gel as measured by microdissection might be better correlated with rate of locomotion. How external pll is effective on the gel even though the cytoplasm is well buffered is not known.

Locomotion can be altered by local solation or gelation. In an electric field, for example, solation is induced in an amocba on the side toward the cathode and pseudopodia progress in that direction." 36 Intense illumination or mechanical stimulation of a pseudopod tip induces gelation and straming recerses in that pseudopod:3 Pantin ${ }^{42.43}$ suggested that contractility of the posterior gel results from loss of water by syneresis and that the anterior gel or freshly formed gel imbibes water. Ile claimed that in a marine amocha the granules at the anterior end and in active pseudopods were more acid than those in the posterior end. This difference in acidity was not confirmed by Mast.31 I Iowerer, immersion in hypertonic solutions causes gelation and results in a rugose or wrinkled surface. ${ }^{38,42}$ Many amoeboid cells have at times an irregular tail-piece ( $A$. limax, $P$. pa- 
lustris, mammalian lymphocytes), from the gel of which it is likely that water has been removed. If the gel matrix consists of long polypeptides cross-linked by side-chains, shortening or contraction would involve the loss of water. ${ }^{1,} 8$ Another suggested mechanism for solation is that it results from the thixotropic character of the gel, such that the mechanical agitation caused by contraction leads to solation, while gel reforms as soon as movement ceases at the anterior end."

Elastic tension might be developed if there were a decrease in volume of the gel as it sets, as there is in gelatin. However, gels which solate under pressure as does the gel of amocba, increase slightly in volume on setting, , hence the contractile tension can hardly result from a volume decrease. It is more likely that fibrous proteins or polypeptide chains shorten according to their state of hydration.

The contractile elements in the plasmagel may resemble contractile muscle proteins. The "viscosity" of actomyosin decreases with pressure, as does the amoeba gel viscosity. In amoebae two processes must be separated: (I) the continuous solation at the posterior end and gelation at the anterior end, and (2) the persistent contractile tension of the plasmagel. In muscle there are two different processes: (1) the activation of the contractile elements, probably via adenosinetriphosphate (see Ch. 16, p. 605), and (2) the rapid development of tension in the gel. The elements of the gel matrix of amoeboid cells must be much less regularly organized than those in muscles because an amoeba shows no birefringence except for a few granules. However, the axopods of Heliozoa and Radiolaria are very contractile and are birefringent. ${ }^{t s}$ These axopods may well represent an intermediate state of organization between the weakly contractile gel of rhizopods or myxomycetes and true muscle.

The difference in the effect of pressure on muscle and amoebae may be explained as follows: solation in amoebae may result from either blocking the gelation process or reducing gel rigidity or organization; in muscle the enhancement of contraction may be due to the effect of pressure on the process of activation of the contractile proteins. This interpretation is supported by the fact that if pressure is applied after the beginning of contraction in muscle the tension is actually reduced. ${ }^{2}$ It would be of interest to know the effect of pressure on contraction of actomyosin fibers and on the axopods of Heliozoa and Radiolaria.

The energy basis for the gel contractility in amoeboid movement is unknown. Amoebae can move for long periods in the absence of oxygen, but the rate of locomotion gradually declines. ${ }^{17,2.2}$ Newly formed pseudopods are more sensitive to cyanide than are the posterior regions. ${ }^{1 n}$ Enucleated fragments of amoebae round up and cease movement.

The preceding evidence indicates that, although there may be minor variations in amoeboid movement with different cell and pseudopod types, the basic pattern is the same in all amoeboid cells. Velocity of locomotion appears to be more closely related to the elastic tension of the plasmagel than to the amount or viscosity of the gel. The gel may contain polypeptide chains which are in a contracted state but which are less organized than corresponding chains in muscle. Detailed information is lacking regarding 
the adhesiveness of the plasmalemma, the method of interconversion of gel and sol, and the micellar state which gives contractile power to the gel.

\section{MOVEMENT IN GREGARINES}

Gregarines are parasitic in certain invertebrates, particularly in oligochactes and arthropods, and their trophozoites are motile. They have an outer layer of myonemes or contractile fibrils, which cause movement of the body in a variety of directions and are responsible for most of their locomotion. In addition, the cells secrete a mucous film which has been said to have a locomotor function. This film must favor attachment, but evidence that it has a propulsive function is not convincing.

\section{REFERENCES}

1. Astbury, W. T., Ann. Rev. Biochem. 8:113-132 (1939). Theory of folding and dehydration of proteins in muscle contraction.

2. Brown, D. E. S., J. Cell. \& Comp. Physiol. 8:141-157 (1936). Effect of hydrostatic pressure on muscle tension.

3. Brown, D. E. S., and Marsland, D. A., J. Cell. E Comp. Physiol. 8:159-165 (1936). Effect of hydrostatic pressure on viscosity of amoeba.

4. Dawson, J. A., and Belkin, M., Biol. Bull. 56:80-91 (1929). Intake of oils by amocbae.

5. DeBruyn, P. P. H., Anat. Rec. 89:43-63 (1940), 93:295-307 (1944), and 95: 177-187 (1946). Amoeboid movement of lymphocytes, leucocytes, macrophages.

6. DeBruyn, P. P. H., Quart. Rev. Biol. 22:1-24 (1947). Review, history of theories of amocboid movement.

7. Dellinger, O. P., J. Exper. Zool. 3:337-358 (1906). Locomotion in rhizopods.

8. FENN, W. O., in Hober's Physical Chemistry of Cells and Tissues (1945). Philadelphia, Blakiston. Section 7, Ch. 32, 33. Muscular contraction.

9. Hahnert, W., Physiol. Zool. 5:491-526 (1932). Reaction of Amoeba to electricity.

10. Harvey, E. N., and Marsland, D. A., J. Cell. E Comp. Physiol. 2:75-97 (1936). Tension at the surface of Amoeba dubia.

11. Ileilbrunn, L. V., Protoplasma 8:58-64 (1929). Temperature and protoplasmic viscosity.

12. Heirbrunn, L. V., Outline of General Physiology (1939). Philadelphia, Saunders. Ch. 25. Amoeboid movement.

13. Hellbrunn, L. V., and Daugherty, K., Physiol. Zool. 4:635-651 (1931). Action of cations on viscosity of $A$. proteus and $A$. dubia.

14. I leilbrunn, L. V., and Daugherty, K., Physiol. Zool. 5:254-274 (1932). Action of cations on gel viscosity, amoeba.

15. Hopkins, D. L., J. Morph. 45:97-120 (1928). Effects of ions and pH on locomotion, Amoeba proteus.

16. Ilopkins, D. L., Protoplasma 28:161-174 (1937). Relation between temperature and locomotion in Flabellula.

17. Hulpieu, H. R., J. Exper. Zool. 56:321-361 (1930). Effect of oxygen tension on anocboid movement.

18. Hyman, L., J. Exper. Zool. 24:55-99 (1917). Metabolic gradients and amoeboid movement.

19. Hynan, 1., The Invertebrates: Protozoa through Ctenophora (1940). New York, McGraw I lill. 726 p.

20. Jinnings, H. S., Carnegie Inst., Wash., Publ. 16, pp. 129-234 (1904). Movements and reactions of amoeba.

21. Jipps, M. W., Quart. J. Micr. Sci. 70:701-719 (1926). Reticulate pseudopods of foraminilerans.

22. Kitchinc;, J. A., J. Cell. \& Comp. Physiol. 14:227-236 (1939). Effects of anoxia on amocbae. 
23. Kunn, A. R., Morphologie der Tiere in Bildern (1926). Berlin, Borntraeger. Heft 2. Rhizopoden.

24. Leidy, J., Fresh-water Rhizopods of North America (1879). Washington, 1). C., Govt. Printing Office. 324 p.

25. Lewis, W., Bull. Jolnns Hopkins Hosp. 49:29-36 (1931). Locomotion of lymphocytes.

26. Lewis, W. H., in Structure of Protoplasm (Seifriz, ed.) (1942). Ames, Iowa, Iowa State Coll. Press. pp. 163-197. Viscosity and amoeboid novement of tissue culture cells.

27. Marsland, D. A., J. Cell. E Comp. Physiol. 4:9-33 (1933). Oil capping and narcosis in Amoeba dubia.

28. Marsland, 1). A., in Structure of Protoplasm (Scifriz, ed.) (1942). Ames, Iowa, Iowa State Coll. Press. p1). 127-161. Streaming in relation to gel structure.

29. Marsland, D. A., Biol. Bull. 87:252-261 (1944). Pressure effect on gel-sol in chromatophores.

30. Marsland, D. A., and Brown, D. E. S., J. Cell. E Comp. Phystol. 8:167-178 (1936). Effects of hydrostatic pressure on amocboid movenent.

31. Mast, S. C., J. Morph. 41:347-425 (1926). Structure, movement, locomotion and stimulation in Amoeba.

32. Mast, S. O., J. Exper. Zool. 51:97-120 (1928). Factors influencing form in amoebae.

33. Mast, S. O., Protoplasma 8:344-377 (1929). Conditions for attachment, Amoeba.

34. Mast, S. O., Protoplasma 14:321-330 (1931). Locomotion in Amoeba proteus.

35. Mast, S. O., Ztschr. vergl. Physiol. 15:139-147 (1931). Action of light on amoeba.

36. Mast, S. O., Ztsclir. vergl. Physiol. 15:309-328 (1931). Action of electricity on amoeba.

37. Mast, S. O., Physiol. Zool. 7:470-478 (1934). Locomotion in Pelomyxa palustris.

38. Mast, S. O., and Doyle, W. L., Protoplasma 20:555-560 (1934). Intake of fluid by amoeba.

39. Mast, S. O., and Prosser, C. L., J. Cell. E Comp. Physiol. 1:333-354 (1932). Effects of temperature, salts, $\mathrm{pH}$, on locomotion and gel-sol ratio, Amocba.

40. Mast, S. O., and Root, F. M., J. Exper. Zool. 21:33-50 (1916). Surface forces in feeding amoebae.

41. Norris, C. H., J. Cell. E Comp. Physiol. 16:313-322 (1940). Elasticity of myxomycetes.

42. Pantin, C. F. A., J. Mar. Biol. Ass. U. K. 13:24-69 (1923). Locomotion in a marine amoeba.

43. Pantin, C. F. A., J. Exper. Biol. 1:519-538 (1924). Effects of temperature; ibid. 3:275-295 (1926). Effects of calcium; ibid. 3:297-312 (1926). Effects of magnesium on amoeboid movement.

44. Prrts, R. F., Biol. Bull. 64:418-423 (1933). Relation between rate of locomotion and form in Amoeba.

45. Pitts, R. F., and Mast, S. O., J. Cell. E Comp. Physiol. 3:449-462 (1933). Effects of $\mathrm{pH}$; ibid. 4:237-256 (1934). Effects of single salts; ibid. 4:435-455 (1934). Interaction between $\mathrm{pH}$ and salts; Physiology of Amoeba proteus.

46. Rhumbler, L., Ztschr. wiss. Zool. 83:1-52 (1905). Fountain currents in Amoeba blattae and Pelomyxa sp.

47. Schaeffer, A. A., Amoeboid Movement (1920). Princeton Univ. Press. 156 p.

48. Sснмidt, W. J., Die Doppelbrechung von Karyoplasma, Zytoplasma und Metaplasma (1937). Berlin, Borntraeger. pp. 124-128. Birefringence in axcipods, Heliozoa.

49. Schwitalla, A. M., J. Morph. 39:465-509 (1924). Temperature on locomotion, Amoeba proteus.

50. Speidel, C. C., J. Exper. Zool. 61:279-331 (1932). Streaming movenents in nerve fibers.

51. Thornton, F. E., Physiol. Zool. 5:246-253 (1932). Temperature and viscosity of Amoeba.

52. Tiegs, O. W., Protoplasma 4:88-139 (1928). Surface tension theory of amocibuid movement.

53. Werss, Paul, Growth Symposium 3:163-203 (1941). Protoplasmic flow in nerve fibers. 


\section{Cilia}

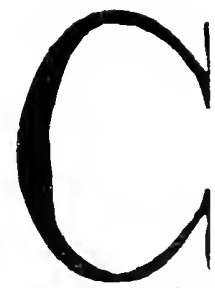

ILIA ARE PERMANENT MOTOR differentiations at the cell surface which are capable of performing work by their rapid and usually rhythmical movement. They are often divided into two types: (1) flagella, which are relatively larger organelles and which are present usually singly or in small numbers upon cells; and (2) cilia proper, which are relatively much smaller and occur characteristically in large numbers upon each cell. Typical Alagella are common among the Mastigophora of the phylum Protozoa, the choanocytes of the Porifera, the gastroderm of many coelenterates, the Hame end-bulbs of certain rotifers, the solenocytes of annelids, and the sperm cells of most groups throughout the animal kingdom. Cilia proper are characteristic of the ciliated protozoans and are found over more or less of the body surface of coelenterates, Turbellaria, and Nemertea. In all other phyla of animals except the Nematoda and the Arthropoda (excluding the Onychophora), they are found at certain locations in or on the body.

Ciliary activity is restricted to an aqueous medium, and hence is found only on surfaces which are submerged, or at least covered by an aqueous film. By typical ciliary movement two types of observed results may be ob. tained, depending on the inertia of the ciliated surface. If the latter is small, then a movement of the ciliated surface through the medium, or locomotion, results; if, on the other hand, the ciliated surface is large, or the ciliated structure is not free to move, the external medium is caused to move over the ciliated surface. It is therefore obvious that ciliated surfaces can become most effective for rapid locomotion only in small organisms such as protozoa, ciliated larvae, etc. In such forms acceleration to maximum speed is very rapid, and the organisms stop very quickly on cessation of their ciliary activity.

Larger organisms may be moved only sluggishly by cilia. Acceleration is generally slow, and the animal fails to come rapidly to a halt on cessation of ciliary beat. Furthermore, unless such larger free-swimming organisms as, for cxample, ctenophores, possess a density very close to that of the surrounding medium, cilia are powerless to serve effectively as locomotory organelles. It is interesting, therefore, that the comb-jelly, Pleurobrachia, with a water content of 94.73 per cent, has a density of 1.02741 , very close indeed to that of the surrounding sea water. 35,36

Cilia are locomotor organelles also in many small worms, in numerous rotilers, and even in certain snails, e.g., in Nassa.

It is perhaps significant that, whereas activity of most ciliated surfaces is continuous throughout the life-time of the animal, cilia whose primary func- 
tion is locomotion commonly show the characteristic of being under the control of the coordinative mechansim of the organism, cnabling the animal to orient its movement, or cease moving. This control of locomotor ciliation appears to be supcrimposed upon a fundamental ciliary automaticity. ${ }^{2}{ }^{2}$

In those instances in which the surrounding medium, rather than the ciliated cells, is caused to nove, cilia may be subserving one or more of numerous functions, such as feeding, circulation, clcansing, respiration, and the movements of materials within ducts. The action of cilia establishes feeding currents for many ciliate protozoans and for sessile or sluggish species in a wide variety of animal groups. The feeding currents of rotifers are casily ob. served. The ciliary activity of the tentacles and oral regions of numerous sea anemones and corals serves to sweep non-nutritious particles away from the mouth and off the tips of the tentacles into the surrounding sea water. ${ }^{5 .}$. $7 !$ However, in the presence of food, the tentacles which are, in response to the food. tipped toward the oral cavity pass these materials into the gastrovascular cavity. In Metridium marginatum, among other species, it has been reported that the direction of the effective beat of the cilia in the stomodaeum is reversed under the stimulus of food. $5 x, . ; 3$ The unstimulated cilia normally beat outward, but in the presence of food such as crab flesh they beat inward. $\mathrm{KCl}$ produces an action similar to that produced by food. ${ }^{57}$ In Actinoloba there are longitudinal grooves in the stomodaeum, the cilia on the ridges beating outward and those in the grooves beating inward. The degree of muscular contraction of this organ would therefore be expected to influence strongly the direction of the dominant currents created. ${ }^{1+}$

Probably nowhere in the animal kingdom are ciliary mechanisms more intricately developed than in the ciliary filter-feeders, principally the lamellibranchs, ${ }^{1,}$ 31, is certain gastropods, ${ }^{5.3}$ and protochordates. ${ }^{34}$ Here the sys tems are so organized that cilia set up feeding currents, filter out suspended particles, collect them into specific ciliary tracts, and convey them to the oral opening of the digestive tract. Along the route special ciliary mechanisms for sorting out the particles on the basis of size, discarding the larger ones, are not uncommon.

Materials are transported within the digestive systems of numerous in vertebrates by ciliary action. Definite courses of circulation through the coelenteron canal systems of Aurelia ${ }^{72}$ and Pleurobrachia ${ }^{17}$ have been lucidly described. Ciliary circulation is an important means of the movement of food in the digestive system of some echinoderms, ${ }^{16}$ numerous molluscs, ${ }^{5}$ and many other organisms.

A cleansing role of cilia appears evident on the basis of the normal direction of the effective beat in sea anemones. In many starfish cilia beat from mouth toward anus over the entire surface, sweeping away debris. The action of the cilia of the epithelium of the frog's mouth and that of the cilia of the respiratory epithelium of mammals appear also to be largely examples of this role.

Clear demonstration that cilia play a role in facilitating respiratory exchange, apart from the functions which have already been described, is usually impossible. Any constant exchange of the medium in intimate contact with the ciliated epithelium would accomplish such a function in at least some degree. 
Cilia are essential to the normal circulation of the body fluids by some annelids such as the pelagic polychaete, Tomopteris, and the sipunculoids and echiuroids, ${ }^{+i}$. th where a true circulatory system is vestigial or absent. The coelomic Huids are caused to circulate in a very effective manner by the action of specifically organized and directed ciliary tracts. The coelomic fluids of starfish are circulated in a similar fashion, the action of cilia even effeclively providing for circulation within the dermal papillae. The cerebrospinal fuid of the vertebrate has been reported to be circulated by action of cilia of "ialls of the rentricles."

In numerous instances among animals, particularly in the tubules of nepliridia and kidneys and in ducts of the genital system, cilia are responsible for facilitating the passage of the materials normally conducted.

The foregoing brief summary of the distribution and general roles of cilia indicates the abundant occurrence and varied functional significances of these organelles. Although they are by no means able to produce movements of the porrer and comspicuousness of those produced by muscle contraction, there are abundant instances where cilia normally are highly essential functional constituents of the organism. And in numerous of these instances they perform functions in a far more efficient and effective fashion than could probabls be done bi an conceivable typical muscular mechanism.

The Structure of Cilia. Cilia in living cells show a great uniformity of structure and organization. The cilium appears, optically, quite homogeneous. In appropriately treated ciliated cells the cilium can be seen to comprise a sheath containing an axial filament. The sheath may be circular or ovoid in cross section. The contained axial filament may pursue a straight course through the sheath or may follow a spiral one. The axial filament not uncommonly extends distally beyond the limits of the sheath.

The Hagcllum of some Aagellates, e.g., Euglena, has attached to the beath a series of diagonally oriented rodlets or mastigonemes which give the Hixgellum a feathery appearance."3. 1., i1. 71 Flagella of this structure are variously called "feather-type," "ciliary," or "stichonematic," in contrast with the simpler whiplike type. The proximal end of the axial filament is invariably associated with a basal granule, which is believed to be derived from the cell centrosome. Many cytologists have described systems of fibrils proceeding from the basal granules to the vicinity of the cell nucleus.

The Hagellimi of Englena has been shown, after treatment with osmic acid, to be resslvable into four longitudinal unbranched fibrils spirally twisted over one another."1: A similar ultrastructure has been disclosed with the aid of electrom micrographs (Fig. 242) for the cilia of Paranecinm. Frontonia, Colpidimm, ${ }^{4}, 30,15$ and the cilia of the clam gill. In the tail of the squid sperm the number of these longitudinal fibrils is 9 or 10 , and this number shows rery little variation among numerous sperm cells from this animal.

The foregoing obseration, together with the fact that cilia show positive form and intrinsic birefringence, ${ }^{\text {it. }}$, all indicate that longitudinally oriented submicroscopic micelles compose the cilium. In this characteristic, therefore, cilia have much in common with contractile fibrils of muscle tissue.

Cilia often appear as compound organelles composed of numerous simpler units. The cirri of Enplotes or the large cilia of Nephthys gills may be re- 
garded as composite bodies comprising numerous cilia arising from a number of basal granules in a circular or ovoid field. "ii The ciliary elements adhere closely to one another in a viscous matrix. The cilia of such a cirrus beat in unison. Membranelles of the adoral region of many peritrichs are small platelets of fused cilia beating synchronously. The undulating membranes of ciliates such as Blepharisma are long rows of cilia, each adhering to its neighbors. The compound character of the membrane may of ten be demonstrated by its fragmentation by appropriate manipulation with a microdissecting needle. ${ }^{7}$ The component cilia, when scparated from one another, beat quite independently; when they have reunited, the characteristic coordinated activity giving rise to the undulatory movement is restored. Such an activity is the result of the metachronic wave of ciliary activity pro ceeding at right angles to the effective and recovery strokes.

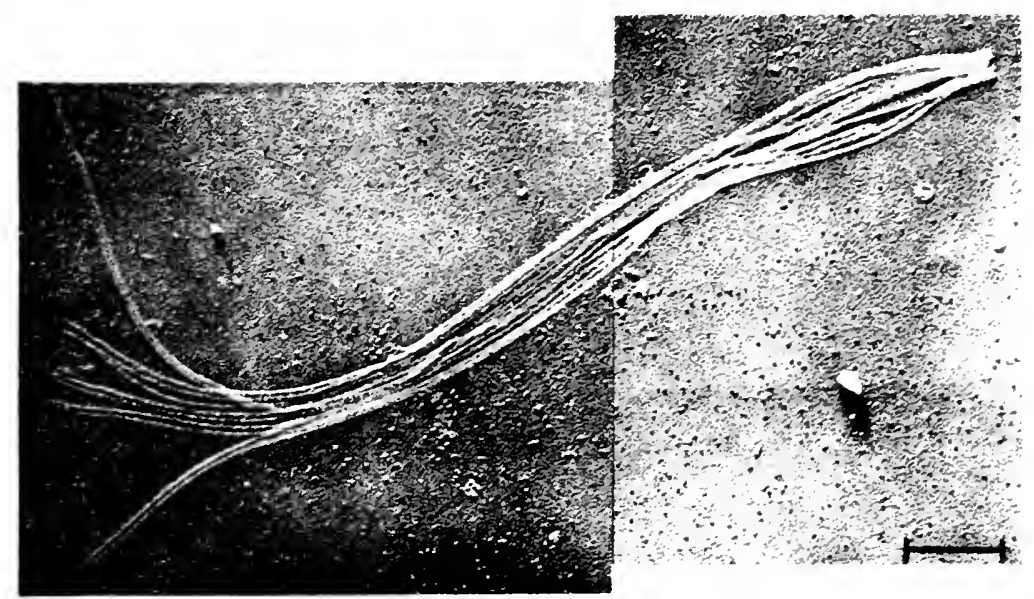

Fig. 242. Electron micrograph of a cilium from Paramecium, shadow-cast with chromium. From Jakus and Hall."

Characteristics of Ciliary Movement. The activity of any cilium may be resolved into one or another, or some combination, of three fundamentally different types of movement. ${ }^{24}$ One of these is pendular movement in which the cilium bends back and forth, Hexing only at its base. No difference in the form of the cilium is observed between the effective and the recovery phases of its stroke, the former simply occurring more rapidly than the latter. This type of movement is seen in the cirri of hypotrichs. A second type of movement is a flexural one. Bending begins first at the tip of the cilium and passes toward its base; in recovery, the cilium progressively straightens from base to tip. Such hooklike bending is observed in the latero-frontal cilia of lamellibranch gills. The third fundamental type of ciliary activity is undulatory movement. This type is characteristic of Alagella. In this type of movement waves pass along the flagellum from base to tip of the organelle, apparently never in the reverse direction.

A type of ciliary activity involving a combination of pendular and flexural activities is observed in the frontal cilia of the gill of $11 y+t i l u s^{-46}$ (Fig. 243). The effective stroke is a rapid, stifly swceping, pendular movement with the 
concavity of the mildly curved cilium in advance. The recovery stroke involves a bending back again of the base of the cilium and then a progressive passage of this flexion to the tip. The effective stroke, therefore, appears to involve movement of a rigid cilium, whercas recovery appears to be concerned with a progessive stiffening from base to tip of an initially limp structure.

Combinations of pendular and undulatory movements are found in the vibratile organs of the mammalian epididymis: ${ }^{102}$ and in a number of flagellates as, for example, in Trypanosoma. ${ }^{\text {in }}$ In such organisms as the latter the undulatory and penclular movements need not occur in a single plane, nor need the two occur simultaneously in the same plane. Thus the tip of the flagellum in the course of its beat may trace out an elliptical orbit, a figure 8 , or a more complicated figure.

The single vibratile element of the flagellate, Monas, has been described to possess the capacity to carry out numerous types and combinations of activities.:" Forward movement is accomplished by a rapid pendular movement of the flagellum from a position clirected forward to one at about right angles to the direction of progression. The recovery stroke is a typical flexural recovery (Fig. 244, A). The effective stroke may, instead, be initiated

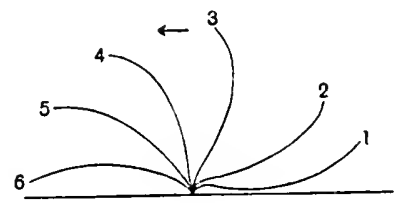

$a$

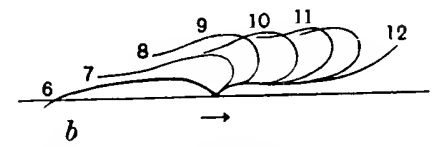

Fig. 243. Movement of a frontal cilium of Mytilus: $a$, effective and $b$, recovery stroke. From Gray."

near the base and rapidly pass toward the tip. In slower forward movement, the total sweep of the flagellum may be reduced to about half the normal amplitude, but with the typical form of beat seen in rapid progression (Fig. $244, B)$. Backward progression is brought about by undulatory activity with the waves passing from base to tip of the organ (Fig. $2+4, C$ ). Lateral movements are the result of undulatory movements of a flagellum flexed ${ }^{34}$ at about 90 desrees (Fig. 24+4, D). Directing the flagellum backward results in forward movement. The undulatory activity may involve only the tip of the Alagellum, or practically its whole course.

The activity of the flagellum has been investigated with the aid of high speed cincma photomicrography.3.,3: Lnclulatory activity of the Hagellum exerts only a pushing action on the medium. Forwarcl locomotion in such common species as Peranema and Englena, in which the flagellum was formerly believed to pull the organism and henee function as a tractellum, appears to result from a bending of the whole flagellum, or at least of its active tip, backward."1. 3: The unchulatory activity typically passes from base to tip 
around the flagellum, as well as along it. Such movement imparts rotational and gyrational components along with a usual backward thrust. The resulting rotation and gyration of the body of the flagellate around an axis which constitutes the direction of locomotion is considered to provide an im portant, if not the chief, force propelling the organism forward. The body proper acts under these circumstances as a serew-propeller. ${ }^{3-.3: ?}$

From the preceding paragraphs we see clearly that ciliary activity varies greatly in its complexity and variability in a single cilium. Some cilia appear to show a simple and uniform activity, others show considerable variability. more or less under the control of the general response mechanism of the body.

Cilia are able to propel an organism through the water, or propel the surrounding medium past a stationary ciliated cell as a result of a directed thrust upon the mediun. More work must be done upon the medium dur ing the effective phase of a stroke than during recovery. In those cases where the effective stroke is of the pendular type and recovery is of the Hexural type, the mechanism is obrious. Simple pendular ciliary acivity would be expected to be quite an inefficient type for directed movement. In undulatory activity the thrust upon the medium is dependent on the progression of a wave along the vibratile organ. A standing wave would obviously exhibit an equalization of all pulls and thrusts. A wave passing from base to

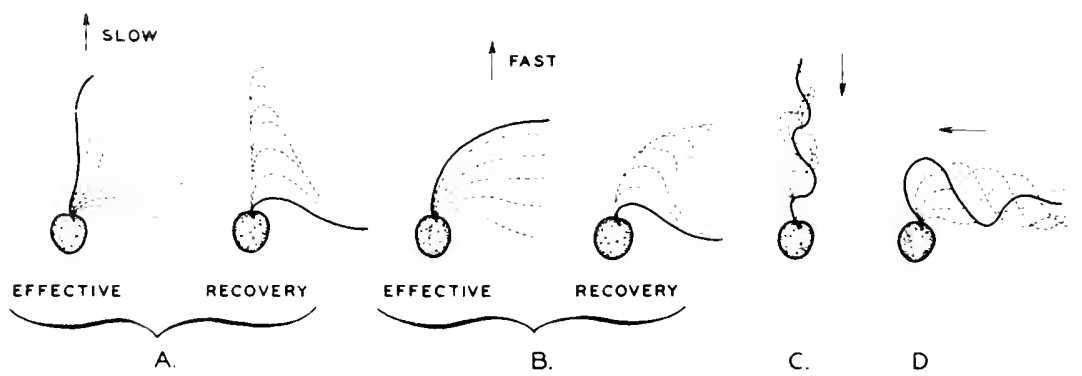

Fig. 244. Some activities of the flagellum of Monas during locomotion. Arrows indicate direction of movement of the organism. (Redrawn from Krijgsman). ${ }^{3:}$

tip would exert a thrust away from the cell upon the medium, proportional to its rate of transmission along the flagellum.

Although cilia appear, on observation, to move through the medium at a very high rate, they are actually moving very slowly, as a simple calculation will show. The angular velocity of the cilia is high. as is seen from the fact that a cilium may show ten or twelve cycles per second, but the tip of a cilium is actually moving through the medium at a maximum rate of only a few feet per hour, a rate quite slow in terms of propulsive instruments of the type of which we ordinarily think. The rate of the effective stroke is usually 2 to 5 times that of the recovery.

The reversal of the direction of effective beat of cilia has often been reported. The most evident instances of this phenomenon lie amone the ciliated protozoans and small turbellarians, such as Stenostomum, : ose reversal of direction of locomotion may reflect this reversal of activ $v$ Some early reported cases of ciliary reversal among metazoans appear to i.nd their 
explanation in terms other than that of a reversal of the direction of beat of individual cilia. The apparent reversal on the labial palps of Ostrea.," and probably also in the gullet of the coelenterate, Actinoloba, ${ }^{1+}$ appears to be explicable in terms of opposite directions of beat of cilia of longitudinal grooves and ridges, together with muscular movements making one or the other of the tracts dominantly influential.

The cilia of the lips and gullet of the sea anemone, Metridiumi, and also other species of anthozoans, whose tentacles are too short for successful transport of food to the mouth, appear capable of reversal of beat in response to direct stimulation by chomical agents. In the normal unstimulated anemone the cilia beat outward. When, however, a piece of food material such as crab flesh is brought into contact with the ciliated epithelium, those cilia in the immediate region of the food reverse their beat, now carrying the food into the gullet. Immediately following passage of the particle the cilia resume their outward beat.

The effect of crab flesh in inducing ciliary reversal in the Metridium can be imitated through the application of $2^{1 / 2}$ per cent $\mathrm{KCl}$ in sea water or the addition of glycogen, ${ }^{58}$ etc. After this treatment the direction both of the effective stroke and of the metachronic wave is reversed. Such treatment does not reverse the direction of beat of the cilia of the tentacle or siphonglyphs.

In Paramecium ciliary reversal is effected by treatment with $\mathrm{KCl}$ and salts of other monovalent cations. ${ }^{51.52}$ When the animal is subjected to an electric field, there is a reversal of beat of cilia on the end of the animal nearer the cathode.

The only seemingly genuine instance of ciliary reversal in higher metazoans appears to be in the ectodermal cilia of amphibian larvae. ${ }^{70}$ Here local ciliary reversal is observed in response to mechanical stimulation. In all other carefully studied ciliated epithelia in higher metazoans, reversal is apparently impossible.

Ciliary Control and Coordination. Cilia appear to show a well developed automaticity. The cilia of ciliated epithelium of even small areas of the tissue continue to beat after removal from the organism. In protozoans the cilia of even small fragments of an individual will continue to beat for some time, even in the absence of any nuclear material. Sperm cells with the head removed continue to show active locomotion. Such observations indicate that the automaticity of ciliary action resides entirely in the cilium and an immediately adjacent region of the cell. A number of observations suggest that an organic connection with the basal granules is essential to the beat. Separating the middle piece from a sperm tail or stripping away the surface of a ciliated epithelium in such a manner as to break the connections between the cilia and the underlying layer of basal granules is reported to result in an immediate cessation of ciliary beat. ${ }^{26}$ It is not yet fully established whether it is the basal granules themselves or some other functional elements in the same general area which are essential for normal activity.

The cilia of any ciliated surface exhibit through their metachronism evidence of the presence of a general coordinating mechanism. Metachronism is a term applied to the characteristic beating of the cilia in any ciliated epithe- 
lium in one direction in a regular sequence (Fig. 245). Each cilium is very slightly out of phase with its neighbor in front of and the one behind it. This fact, together with the fact that the cilia at right angles to this wave of activity are beating in phase with one another, gives the visual impression of waves passing over the epithelium. The crests of the waves include those cilia at the peak of their effective stroke; the troughs include those cilia at the ends of their effective strokes and about to commence their recovery.

The direction of the metachronic wave in a ciliated surface appears to be as fundamental a property of the surface as the direction of ciliary beat. Even a small isolated portion of a ciliated surface continues to show its own inherent direction of metachronism. This is not disturbed by removing and then replacing after rotating through $180^{\circ}$ a portion of the ciliated epithelium of the roof of a frog's mouth. ${ }^{+}$In these circumstances the transplanted portion has its metachronic wave proceeding in a direction opposite to that in the surrounding tissue.

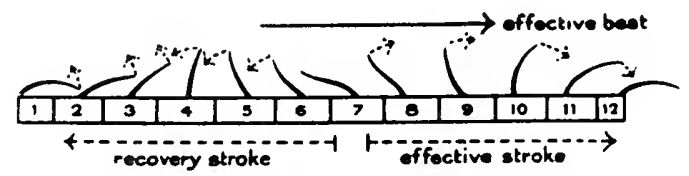

Fig. 245. Diagrammatic representation of the metachronal rhythm.

Cilia 1 to 12 are beating in sequence. From Gray. ${ }^{24}$

Among ciliated tissues from various sources, the direction of the metachronic wave bears no constant relationship to the direction of the effective stroke. In some, as in the frog's mouth, the metachronic wave and ciliary effective strokes are in the same direction. In the rows of ciliated combs of ctenophores the two are in opposite directions. ${ }^{10}$ 56. In the cilia of the gills of the annelid, Nephthys, ${ }^{11}$ and in the lateral ciliated epithelium of Mytilus gills $^{26}$ the metachronic wave is always at right angles to the effective stroke. There is no essential fixed relationship between the two.

Little is known of the mechanisms of the coordination and control of cilia. There is reason to believe that it is more complex among protozoans, in which a number of complex locomotory patterns are exhibited by a single individual, than in the ciliated epithelia of the larger metazoan invertebrates and vertebrates. In these higher animals the pattern of coordination seems for the most part to be a highly invariable one. Among the protozoans there appear to be definite species differences in the pathways of the metachronic wave. This is not interfered with by a transverse cut deep into the animal in Spirostomum and Stentor, but coordination on the two sides of such an incision is lost in Paramecium.75 Transmission of the metachronic wave would appear to be by way of a conducting network in the ectoplasm, permitting passage in all directions through the ectoplasm in the former species and only along ectoplasmic, longitudinal pathways in the latter. In view of the fact that the silver-line systems of ectoplasmic fibrils in the first two species show the form of a network connecting the basal granules, and in the latter species possess the form of only longitudinal fibrils, it has been suggested that this fibrillar system forms the conducting pathway for the metachronic wave in protozoans. 
Studies on the progressive anesthetization of the ciliary mechanism have shown that, in protozoans, the first thing to be lost is the power of ciliary reversal. At a later stage metachronic coordination is lost, leaving the cilia beating independently. Last of all, the cilia entirely cease their activity. This observation has led to the hypothesis that ciliary beat, metachronism, and reversal are controlled by three separate mechanisms. Reversal must be governed by a mechanism of relatively rapid transmission affecting all cilia of the organism nearly simultaneously and passing in all directions over the animal. Transierse cuts passing deeply into those protozoans investigated in this regard do not neasurably interfere with the transmission of excitation in response to stimuli which induce ciliary reversal.

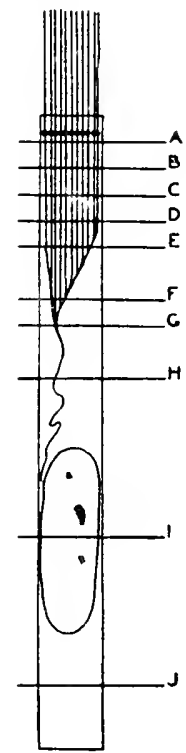

Fig. 246. Diagram of a ciliated epithelial cell of Anodonta showing regions where cuts were made. Cut at $A$ always resulted in cessation of ciliary beat. Cuts at $B$ through $F$ permitted continuation of beat but resulted in more or less loss of normal coordination. Cuts at $\mathrm{G}$ through $\mathrm{J}$ resulted in no apparent disturbance of normal ciliary activity. From Worley. ${ }^{\text {r }}$

Support for the concept of the transmission of excitation in intracellular ciliary coordination by a fibrillar system has come from microdissection studies of the ciliated epithelial cells of clam gills ${ }^{73}, 75,76$ (Fig. 246). Cuts through the distal third of the cell containing the ciliary cone of intracellular fibrils destroy ciliary coordination; cuts through any level of the proximal twothirds have no such effect. Only when transverse cuts are in the immediate region of the basal granules themselves does even the uncoordinated ciliary beat cease. Waves of ciliary metachronism pass smoothly over large sheets of ciliated epithelial cells; the mechanism for this intercellular transmission is unknown.

Although ciliary beat appears in general to be the result of an automaticity of the ciliary mechanism, yet there are numerous bits of evidence that cilia in many cases are controlled in their activities by more basic response mech- 
anisms within the organism. Such hypotrichs as Uronychia and Euplotes show, not one type, but several types of locomotory pattern. ix A role of the neuromotor system in control of locomotion in Euplotes has been indicated from experimental destruction of parts of this system (Ch. 23). In a number of metazoans both direct and indirect evidence has been advanced pointing to nervous modification of ciliary activity. ${ }^{19,}, 40,41,42$

The rows of ciliary combs of ctenophores are controlled by impulses pass ing out over the paddle rows from the aboral sense organs. 2 , 10 The ciliary locomotory systems of the turbellarian, Stenostomum, and of the ciliated larvae of many molluscs and annelids are known to be under the control of the nervous system." The cilia mav be active or motionless or, in the case of Stenostomum, reversed. A freshly isolated piece of ciliated lip of the snail Phy'sa shows no ciliary activity unless a nerve fiber innervating this region is stimulated. ${ }^{46}$ On the other hand, Alectrion, whose locomotion is largely dependent on pedal ciliary activity, can inhibit these cilia. ${ }^{12}$ If the foot is excised, there is first cessation of ciliary beat, which is, however, gradually resumed again a short time after its removal, and then the beat continues unabated for the remainder of the life of the ciliated epithelium.

The cilia of the frog's mouth have been found to be accelerated greatly and the amplitude of the beat increased after electrical stimulation of certain cranial nerve fibers. ${ }^{67}$ There is a latent period of about a second between beginning of stimulation and the response. The influence of the nervous stimulation persists for as long as 10 minutes. It is not yet clearly shown whether these fibers are of sympathetic or parasympathetic character, although the bulk of the evidence appears to suggest their belonging to the facialis group. Acetylcholine has been reported to increase ciliary activity. In further support of normal nervous control of the cilia of the frog's mouth is the observation that these cilia are normally quiescent; ${ }^{44,45}$ they commence to beat in response to such stimuli as the addition of foreign particles. After these particles are swept away the ciliary beat again ceases.

Influence of Environmental Factors on Ciliary Activity. Probably the two most important environmental factors normally influencing ciliary activity are temperature and hydrogen ion concentration. The maximum rate of ciliary activity occurs at about $34^{\circ} \mathrm{C}$. (Mytilus gill) and $35^{\circ} \mathrm{C}$. (ciliated epithelium of the frog's mouth).22, 26, 43 The $Q_{10}$ varies with the temperature range for which it is determined. For Mytilus gill cilia it ranges from about 3 at the lower temperatures to slightly less than 2 at the high end of the physiological range. Above a certain critical temperature there is a rapid drop in rate. It seems reasonable to expect that the maximum temperature for activity of ciliated epithelia would be higher for animals normally found at higher temperatures than for animals living at lower temperatures.

Hydrogen ion concentration, which varies considerably in nature, has a more profound influence on the rate of ciliary activity than concentration of any other ion. $8,27,50,69,77$ Those acids like carbonic acid which penetrate cells more rapidly have a greater effect than those which penetrate less rapidly. ${ }^{21}$ Increase in hydrogen ion concentration can cause cilia to become completely quiescent.

A biological significance of this response of cilia to increased acidity is seen in the fact that in an ordinary bivalve mollusc whose valves have been 
held tightly closed for two or three hours out of water there is a high enough concentration of $\mathrm{CO}_{2}$ to inhibit ciliary activity. Inasmuch as $\mathrm{O}_{2 .}$ consumption of ciliated tissue is a direct function of the rate of ciliary activity, this influence of $\mathrm{CO}_{2}$ is obviously adaptive. The minimum bydrogen ion concentration necessary to produce inhibition of gill cilia varies with different molluscan species. For example, it is lower in $M y a$, which customarily dwells in well-aerated water, than in Ostrea or Mytilus.

Another very striking instance of an apparently adaptive variation in the minimal hydrogen ion concentration essential for ciliary inhibition has been reported for the cilia of the different portions of the digestive tract of $M y a^{77}$ There is a definite correlation between the normal $\mathrm{pH}$ of each region of the digestive tract and the $\mathrm{pH}$ at which the cilia of that region are brought to rest.

Other cations and also anions are also known to influence ciliary activity. $20,21,23,24,25,29,33$ In general, these ions are relatively constant in any given environment in nature. The effects of altering their concentrations and ratios appear to be general physiological ones, quite comparable to their action on all cells; and, as with other cells, cation content of the medium has more influence on ciliary beat than has anion content.

Theories of Ciliary Movement. Little or nothing is known of the actual mcehanism of ciliary motion. Historically, theories have fallen into two general categories: (1) those that assumed the moving force to occur in the cell body proper, with the cilia acting only passively, and (2) those that assumed that the moving force occurred in the cilium itself, the cilia therefore moving as a result of their own contractile powers. The original proponent of the view of cilia behaving as passive bodies was Schäfer, ${ }^{63}$ who considered the cilia to be hollow elastic structures with differences in the degree of elasticity of various portions of their walls. The cilia were believed to beat as a result of a rhythmic surging and ebbing of fluid of the cell body into and out of these cellular extensions.

Much more compatible with our modern theories of the mechanism of act ion of contractile elements in general is the second type of theory, namely, that the cilium itself is an actively contractile element with only one side of the cilium contracting or with the two sides contracting alternately. This view, first clearly advanced by Heidenhain ${ }^{2 \times}$ in 1911, has since acquired many other advocates. Supporting this point of view are numerous observations, including the fact that the waves passing along a flagellum may often show no reduction in amplitude as they pass from base to tip. Such a reduction would be expected were kinetic energy generated only in the cell body proper at the flagellar base. In species such as Peranema, the undulatory activity may be restricted exclusively to the tip region of the flagellum, the remainder meanwhile apparently remaining quiescent. Again, the use of such methods as transmission of polarized light, $X$-ray diffraction, and electron micrography indicates the molecular organization of a cilium to resemble in a striking manner that of muscle fibrils which are definitely known to possess inherent contractility. Both exhibit a fibrillar structure obviously due to an orientation of elongated molecules or micelles.

There is as yet no agreement of opinion as to which portion of the cilium, the axial filament or the sheath, is the actively contractile portion. In sup- 
port of the axial filament as the active portion one can point to the terminal undulation of the flagellum of Peranema. This portion of the organ appears to possess principally the axial fiber.

The ciliary activity normally involves a utilization of oxygen, the rate of $\mathrm{O}_{2}$-consumption varying with ciliary rate. ${ }^{23}$ However, like muscle, cilia appear capable of acting for a time anaerobically. After administration of 0.1 per cent $\mathrm{NaCN}$ to a fragment of Mytilus gill, $\mathrm{O}_{2}$ consumption drops off abruptly, yet ciliary activity diminishes much more slowly. Fragments of Mytilus gill placed in weak hemoglobin solutions in chambers perfused with damp hydrogen show ciliary beat continuing for some time after reduction of the hemoglobin. After ciliary beat has become very slow under the anaerobic conditions, rapid beat is restored quickly on readmission of oxygen and reoxygenation of the hemoglobin. The cilia of the gill of Pecten, on the other hand, are reported to cease beating immediately on removal of oxygen, ${ }^{50}$ as indicated by the reduction of dyestuffs such as janus green and neutral red, and before nile blue is reduced. The cilia of Paramecinum cease activity within a few seconds in an O.--free medium. ${ }^{18}$ It is known, nevertheless, that some ciliates are able to carry on normal ciliary movement in natural environments which are practically oxygen-free. One can not yet conclude whether the need for $\mathrm{O}_{2}$ differs qualitatively or only quantitatively among different species of animals.

\section{REFERENCES}

1. Atkıns, Daphne, Quart. J. Micr. Sci. 80:321-435 (1938). Ciliary mechanisms, Lamellibranchs.

2. Bauer, V., Ztschr. allg. Physiol. 10:231-248 (1910). Control of cilia in ctenophores.

3. Brown, Harley P., Ohio J. Sci. 45:247-301 (1945). Structure of protozoan flagellum.

4. Bruche, E. J. von., Pflïg. Arch. ges. Physiol. 166:45-54 (1916). Metachronism in implanted ciliated epithelia.

5. Carriker, M. R., Biol. Bull. 91:88-111 (1946). Ciliation in gut of Lymnaea.

6. Carter, G. S., Brit. J. Exper. Biol. 4:1-26 (1926). Nerve control of cilia in invertebrate larvae.

7. Chambers, R., and Dawson, J. A., Biol. Bull. 48:240-242 (1925). Structure of undulating membrane.

8. Chisse, A. M., and Glaser, O., J. Gen. Physiol. 13:627-636 (1930). H-ion concentration and ciliary activity.

9. Chu, Hsinng-Yao, Am. J. Physiol. 136:223-228 (1942). Ciliary circulation of anuran cerebrospinal fluid.

10. Coonfield, B. R., Biol. Bull. 66:10-21 (1934). Coordination of swimming combs of ctenophores.

11. Coonfield, B. R., Biol. Bull. 67:399-409 (1934). Ciliary movement on Nephthys gills.

12. Copeland, M., Biol. Bull. 37:126-138 (1919). Nervous inhibition of cilia of snail foot.

13. Dellinger, O. P., J. Morph. 20:171-209 (1909). Fibrils of Euglena flagellum.

14. Elmhinst, 2., Scottish Naturalist, No. 151, pp. 149-152 (1925). Stomodeal ciliary activity, Actinoloba.

15. Foster, E., Baylor, M. B., Meinkotil, N. A., and Clark, G. L., Biol. Bull. 93 : 11+-121 (1947). Structure of Protozoan flagellum.

16. Gemmili, J. F., Proc. Zool. Soc. London 1-19 (1915). Ciliary activities in echinoderms.

17. Genmile, J. F., Proc. Zool. Soc. London 263-265 (1918). Ciliary currents in Pleurobrachia. 
18. Gersch, M., Protoplasma 27:412-441 (1937). O. and ciliary activity in Paramecium.

19. Gormlin, G. F., Skand. Arch. Physiol. 58:11-32 (1930). Influence of nerves on ciliary activity.

20. Gray, J., Proc. Roy. Soc. Lond., B. 93:104-121 (1922). Ciliary beat in Mytilus. Influence of ions on ciliary beat.

21. Gray, J., Proc. Roy. Soc. Lond. B., 93:122-131 (1922). Influence of ions on ciliary activity.

22. Gray, J., Proc. Roy. Soc. Lond., B. 95:6-15 (1923). Temperature and ciliary activity, Mytilus.

23. Gray, J., Proc. Roy. Soc. Lond., B. 96:95-114 (1924). Oz consumption of cilia. Influence of ions on ciliary movement.

24. Gray, J., Brit. J. Exper. Biol. 3:167-187 (1926). Influence of ions on ciliary activity.

25. Gray, J., Proc. Poy. Soc. Lond., B. 99:398-404 (1926). Influence of ions on ciliary activity.

26. Gray, J., Ciliary Movement (1928). Cambridge, University Press. 162 p.

27. Haywood, C., J. Gen. Physiol. 7:693-697 (1925). H-ions and ciliary movement.

28. Heidenhain, M., Plasma und Zelle, Zweite lieferung (1911). Jena, Gustav Fischer, 1110 p. Theory of ciliary movement.

29. Höber, R., Physikalische Chemie der Zelle und Gewebe (1926). Leipzig, Wilhelm Engelmann. 955 p. Influence of ions on ciliary activity.

30. Jakus, M. A., and Hald, C. E., Biol. Bull. 91:141-144 (1946). Fibrillar structure of flagella.

-31. KellogG, J. L., J. Morph. 26:625-70I (1915). Ciliary feeding mechanisms, Lamellibranchs.

32. Krijgsman, B. J., Arch. Protist. 52:478-488 (1925). Flagellar movement in Monas.

33. Lill1e, R. S., Am. J. Physiol. 17:89-1+1 (1906). Influence of ions on ciliary activity.

34. Lowndes, A. G., Proc. Zool. Soc. Lond., A. 111:111-134 (1941). Flagellar movement.

35. Lowndes, A. G., Nature 150:579-580 (1942). Ciliary movement and density, Pleurobrachia.

36. Lowndes, A. G., Proc. Zool. Soc. London 113:28-43 (1943). Density of the ctenophore, Pleurobrachia.

37. Lowndes, A. G., Proc. Zool. Soc. London 113:99-107 (1943). Activity of protozoan flagellum.

38. Lowndes, A. G., Proc. Zool. Soc. London 114:325-338 (1944). Flagellar activity, Monas and Peranema.

39. Lowndes, A. G., School Sci. Rev., No. 100, June, pp. $319-332$ (1945). Locomotion in Euglena.

40. Lucas, A. M., J. Morph. E Physiol. 51:147-193 (1931). Influence of nerves on ciliary activity.

41. Lucas, A. M., J. Morph. E Physiol. 51:195-205 (1931). Influence of nerves on ciliary activity.

+2. Lucas, A. M., J. Morph. 53:243-263 (1932). Influence of nerves on ciliary activity.

43. Lucas, A. M., 1, Morph. 53:265-276 (1932). Influence of temperature on ciliary beat.

44. Lucas, A. M., Proc. Soc. Exper. Biol. E Med. 30:501-506 (1933). Control of cilia of frog mouth.

45. Lucias, A. M., Am. J. Plysiol. 112:468-476 (1935). Control of cilia of frog mouth.

46. Mtrion, 11., Pflig. Arch. ges. Physiol. 198:1-28 (1923). Nerve control of cilia in snaits.

47. Mleyem A., Ztschr. wiss. Zool. 135:495-538 (1929). ('velomic ciliation of Annelids.

48. Meyen, A., Zool. Jahrb., Abt. Anat. u. Ont. Tiere. 64:371-436 (1938). Ciliated coclom in Tomopteris. 
49. Nelson, T. C., Proc. Soc. Exper. Biol. E Med. 21:166-168 (1924). Ciliary movement on molluscan palps.

50. Nomura, S., Protoplasma 20:85-89 (1933). H-ions and ciliary movement. O2 consumption of cilia.

51. Oliphant, J. F., Physiol. Zool. 11:19-30 (1938). Influence of chemicals on ciliary beat in Paramecium.

52. Oliphant, J. F., Physiol. Zool. 15:4+3-452 (1942). Influence of chemieals on eiliary beat in Paramecium.

53. Orton, J. H., 1. Mar. Biol. Ass. 9:444-478 (1912). Ciliary fecding mechanism, Crepidula.

54. Orton, J. H., J. Mar. Biol. Ass. 10:19-49 (1913). Ciliary feeding in protochordates.

55. Parker, G. H., Bull. Mus. Comp. Zool. Harvard 29:107-119 (1896). Ciliary activity in Metridium.

56. Parker, G. H., I. Exper. Zool. 2:407-423 (1905). Activity of swimming combs of ctenophores.

57. Parker, G. H., Am. J. Physiol. 13:1-16 (1905). Ciliary reversal in Metridium.

58. Parker, G. H., Proc. Nat. Acad. Sci. 14:713-71+ (1928). Ciliary reversal by glycogen.

59. Parker, G. H., and Mapks, A. P., J. Exper. Zool. 52:1-7 (1928). Ciliary reversal, Metridium.

60. Perrin, W. S., Arch. Protist. 7:131-156 (1906). Ciliary beat in Trypanosoma.

61. Peterson, J. B., Bot. Tiilskr. 40(5):373-389 (1929). Structure of feathered flagella.

62. Pütrer, A., Ergebu. Physiol., Vol. 2, Abt. 11, pp. 1-102 (1903). Ciliary beat in mammalian epididymis.

63. Schafer. E. A., Proc. Roy. Soc., London 49:193-198 (1891). Theory of ciliary movement.

64. Sснмid, W. J., Protoplasma 28:18-22 (1937). Birefringcnce of cilia.

65. Schmitt, F. O., Phy'siol. Rev. 19:270-302 (1939). Burctringence of cilia.

66. Sсhмitt, F. O., Hall, C. E., and Jakus, M. A., Biol. Symp. 10:261-276 (1943). Fibrillar structure of fiagella.

67. Seo, A., Jap. J. Med. Sci., III Biophysics 2:47-75 (1931). Nervous control of cilia of frog mouth.

68. Taylor, C. V., Univ. Calif. Publ. Zool. 19:403-470 (1920). Structure of cirri of Euplotes. Patterns of ciliary locomotion.

69. Tonnta, G., I. Shanghai Sci. Inst., Vol. 1, Sec. 1V, pp. 69-76, 77-84 (1934). Hions and ciliary movement.

70. Twitry, V. E., J. Exper. Zool. 50:319-344 (1928). Ciliary reversal in amphibia.

71. VLk, W., Arch. Protist. 90:448-488 (1938). Structure of flagella.

72. Widmank, E. M. P., Ztschr. allg. Physiol. 15:33-48 (1913). Ciliary mechanisms in Aurelia.

73. Worley, L. G., Proc. Nat. Acad. Sci. 19:320-322 (1933). Metachronism in ciliated epithelium.

74. Worley, L. G., I. Cell. \& Comp. Physiol. 5:53-72 (1934). Ciliary coordination in Protozoa.

75. Worley, L. G., I. Exper. Zool. 69:105-121 (1934). Metachronism in ciliated epithelium.

76. Wonley, L. G., J. Cell. \& Comp. Physiol. 18:187-197 (19+1). Ciliary coordinition in gill epithelium cells of clams.

77. Yonce, C. M., I. Mar. Biol. Ass. 13:938-952 (1925). H-ions and ciliary movement.

78. Yonge, C. M., I. Mar. Biol. Ass. 1t:295-386 (1926). Ciliary feeding mechanism, oyster.

79. Yonge, C. M., Sci. Rep. Great Barrier Reef Expedition, Brit. Mus. 1:13-58 (1930). Ciliary feeding in corals. 


\section{Trichocysts and Nematocysts}

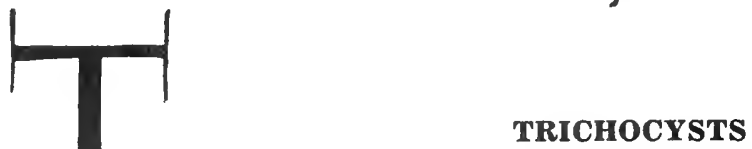

HE ECTOPLASM OF numerous species of ciliates contains extrusible bodies known as trichocysts. 1, 17,7 These are fusiform bodies oriented obliquely or at right angles to the surface. Among various protozoans, they may be uniformly distributed over the surface, as in Paramecium, or restricted to certain regions, as in the proboscis of Dileptus. They may be projected outward on the ends of long tentacles in feeding specimens of Actinobolina.

The trichocysts appear to have their origin within or close to the macronucleus in Frontonia. ${ }^{* 3}$ From here they make their way to their definitive position in the ectoplasm, completing their differentiation as they migrate. In Prorodon, the fully formed trichocyst appears to have the form of a cylindrical sac containing an elongated, coiled filament. In Paramecium the undischarged trichocysts may be seen to possess an oval body proper, about 2-3 $\mu$ long by $2 / 3 \mu$ in diameter, and a cap-covered tip of slightly smaller diameter. On discharge, the tip with its covering cap is separated from the body proper through the elongation of a shaft. ${ }^{15}, 16.9$

The trichocysts can readily be induced to discharge their filaments through chemical (acid or base), mechanical (pressure), or electrical (condenser discharge or induction shock) stimulation. With electrical stimulation there is an increase in the number discharged as the strength of the stimulus is increased." The total discharge is very rapid, occurring in a matter of a few milliseconds. The discharged trichocyst is needle-like in general form, being ten or more times as long as the undischarged body. The discharged trichocysts of Paramecium may be $40 \mu$ long. The trichocysts of Paramecium show birefringence, indicating an orientation of elongated submicroscopic particulates in the long axis of the organelle. ${ }^{21}$ A study of the extended threads of discharged trichocysts of Paramecium with the electron microscope reveals them to be composed of a shaft showing a periodic transverse banding at intervals of 600 to $650 \AA$ (Fig. 247). The characteristics of the shaft indicate it to be a thin cylindrical membrane composed of elongated protein chains showing a periodic structure somewhat resembling that of collagen fibers. The over-all striation of the organelle would then be a consequence of the alignment of these fibers in phase with one another. At the tip of the shaft there is a relatively opaque, thornlike body indicative of the presence of a dense proteinaceous structure or of elements of high atomic mass. ${ }^{9}, 10$

Little or nothing is known of the mechanism of trichocyst discharge or to what extent trichocysts are activated other than in direct response to environmental stimuli. The trichocysts appear connected with the silver-line 
system, which some consider to have a nerve-net type of conductile function. The mechanism of discharge probably involves either a hydration of some protein within the trichocyst or a rapid osmotic inflow of water on excitation of these organelles.

The role of trichocysts in protozoans is still not definitely established. They have been considered by many to possess a protective function. This appears doubtful in such species as Paramecium, which are readily ingested by Didinium even after the Paranecium has extruded many of its trichocysts. More probably the trichocysts function here as organs of attachment. ${ }^{20}$ In ciliates such as Dileptus and Actinobolina, prey coming in contact with a region of the body bearing trichocysts appears to be paralyzed instantly, as if a toxin were associated with the discharged trichocyst.

Arnong the dinoflagellates, Polykrikos and Nematodinium, there are trichocysts that bear a very close resemblance structurally to the nematocysts of coelenterates and are frequently called nematocysts. Trichocysts in various stages of differentiation have been found within these dinoflagellates.

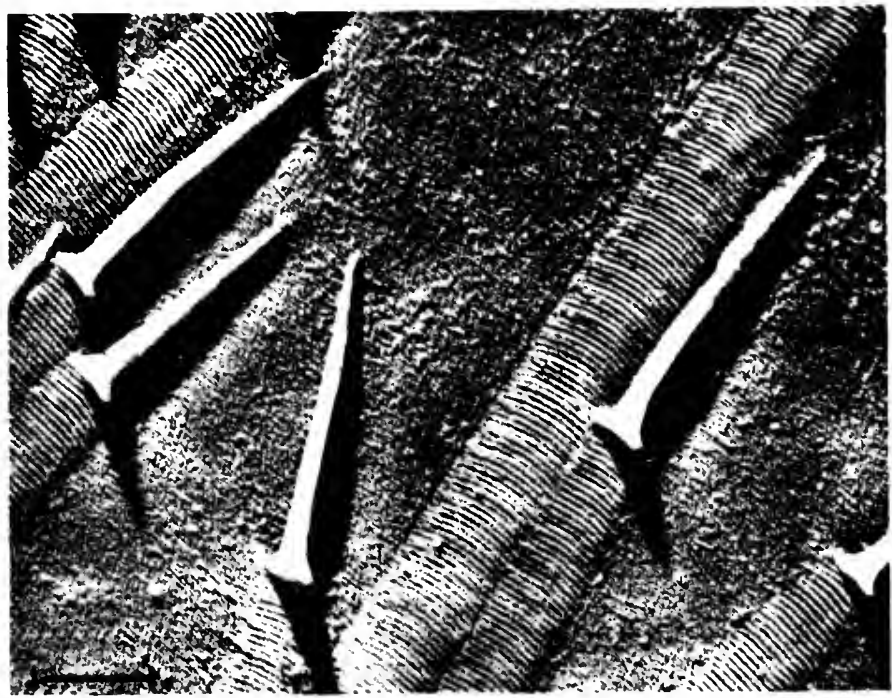

Fig. 247. Electron micrograph of discharged Paramecium trichocysts, shadow-cast with chromium. From Jakus and Hall."

Another type of effector organelle very closely resembling the coelenterate nematocyst is the polar capsule of sporozoans of the groups Myxosporidia and Actinomyxidia. Within the digestive tract of host organisms, the action of the digestive juices induces these capsules to discharge their spirally-coiled hollow thread after the fashion of a nematocyst. The discharged filament serves as a means of temporary anchorage of the parasite to the host tissues.

\section{NEMATOCYSTS}

The nematocysts of coelenterates are extremely small intracellular structures of spherical, oval, or spindle form. They consist of a capsule within which a hollow thread, continuous with an end, is introverted and coiled. ${ }^{7,} 25$ The discharge of the nematocyst involves a rapid expulsion of the thread- 
like process by eversion (Fig. 248). There is usually a cap or operculum covering that region of the undischarged nematocyst through which the thread is ejected. The nematucrsts are protuced within interstitial cells either at their final site or at some distance from this site, to which they move by ameboid activity or passive transportation. A cell containing a nematocyst is known as a cnidoblast or nematocyte. The portion of the cnidoblast containing the nematocyst eventually comes to occupy a superficial position and usually develops at its outer end a bristle-like projection. the cnidocil. The latter is imbedded in a small crater-like elevation on the cell. The cnidoblast also often differentiates trichite-like supporting rods in its peripheral regions and often, too, a fibrillar network associated with the capsule of the nematocrst and extending proximally from it.

The nematecrsts of coclenterates are divisible into two major groups, the spirocysts of the Zoantharia, which are acid-staining bodies possessing peculiar adhesive threads, and the basic-staining nematocysts proper. The latter are widely distributed through the whole phylum. They are of many types in the characters of their discharged threads. In some, e.g., the volvents, the thread is closed at its tip and forms a tightly coiled filament on discharge, wrapping

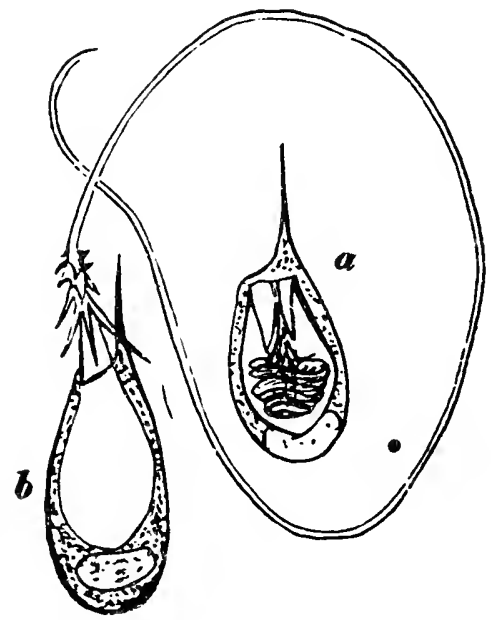

Fig. 248. An undischarged (a) and a discharged (b) nematocyst located in its cnidoblast. The spine-like projection of the cnidoblast is the cnidocil. Redrawn from Sedgwick.

itself about bristles or fibers of organisms in whose presence they are discharged. Most orher types have open tips and are belicved to penetrate prey, injecting into them a toxic substance. The threads of these latter types commonly possess an armature in the form of three spiralling rows of spines which serve effectively to anchor the thread into tissues which have been penetrated. The penetrating capacity of at least some types is so great that even the chitinous cuticles of small organisms can be punctured.

The sting of numerous species gives rise to severe itching and other skin disorders in man. The wexicity is very great in a fow coelenterates such as Physalia, and some barger scyphozoan jellyfish such as Dactylometra, even enough to render them highly dangerous to man. Their sting may in some 
cases produce serious illness or even death. The nature of the toxin associ ated with nematocysts is largely unknown. Various toxic fractions have been prepared from nematocist-containing tissues. ${ }^{2,3}$

The cnidoblasts respond as independent effectors in the discharge of their nematocysts. There is no evidence of control of these by any coordinatory mechanism within the organism. In their normal responses, and in response to highly localized electrical stimuli, there is a complete restriction of the

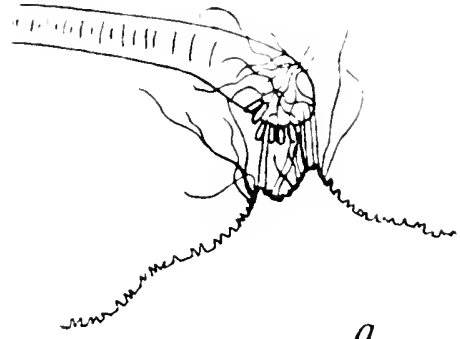

$a$

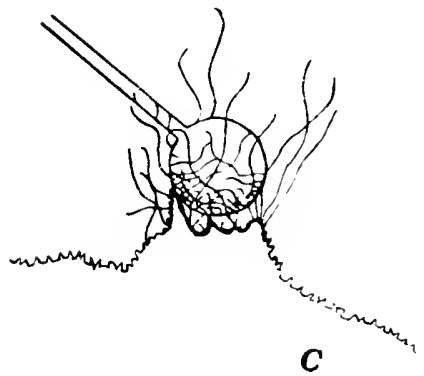

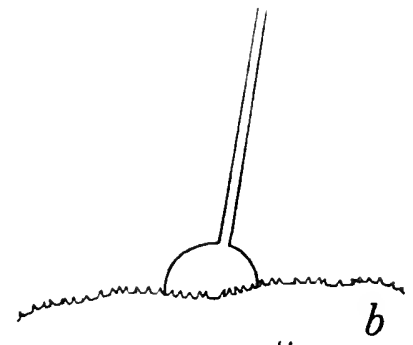

11

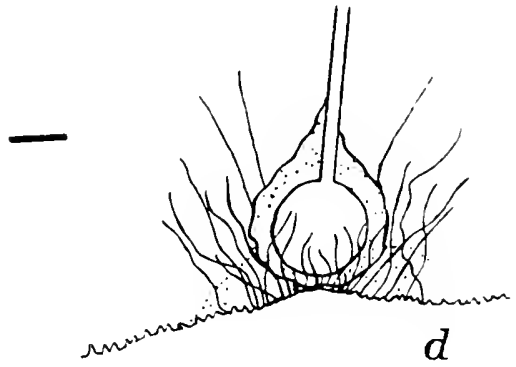

Fig. 249. Discharge of nematocysts of Anemonia to various stimuli. $a$, Touch by a human hair. $b$, Touch by a clear, blunt glass rod fails to cause discharge. $c$, Response to clean glass rod after sensitization of the cnidoblasts with dilute saliva extract. $d$, Response to glass rod smeared with alcohol extract of Pecten gill. From Pantin. ${ }^{18}$

discharge to the specific region excited. ${ }^{19,}$, 8 Intense mechanical stimulation by inert objects will induce only a weak discharge, whereas mild mechanical stimulation by natural foods is sufficient to evoke a strong response (Fig. 249). Submersion of cnidoblasts in a weak extract of a normal food which will typically not itself induce discharge will greatly lower the threshold of these effectors in Anemonia to purely mechanical stimuli. ${ }^{1 \times}$ The specific food factors that are involved in this sensitization are lipoidal substances adsorbed upon proteins. The adsorptive forces are so strong that the factors cannot be removed by ether extraction, but they can be with alcohol. The active chemical substances appear to have properties resembling sterols and phospholipins and to be highly surface-active. In view of the rapid and thorough discharge of nematocysts even to dried foods, it would appear that the normal reaction must be "contact-chemical" in character, chemical sensitization to mechanical stimulation occurring almost instantaneously. 
These highly specialized characteristics of excitation of nematocysts in Anemonia are peculiarly adapted to the normal functional roles of these effectors. The value of having the nematocysts withhold their discharge until the instant of mechanical contact is readily seen in comparing the results of stimulating discharge by a piece of cotton soaked in bile salts with that by a similar piece soaked in an extract of normal food material. In the former case the nematocysts are induced to discharge before contact by the diffusion outward of the bile salts, which are highly potent cnidoblast excitors, and therefore before the discharge can result in any attachment of the discharging filaments to the cotton fibers, as occurs in the case of normal food substances.

The cnidocils, when present, are probably concerned with the excitation process. That they are non-essential, however, is indicated by the fact that they are absent in many of the Anthozoans. It would appear that in their absence the cnidoblast surface comprises the receptive area for the response.

The mechanism of discharge of nematocysts is not well understood. Two types of theories in particular have found favor among students in this field; both theories have in common the postulation that an increased pressure within the capsule forces out the filament. One theory considers the pressure increase following stimulation to be the result of a passage of water into the capsule. Some have believed the nematocyst operates as a simple osmometer. ${ }^{x: 5}$ Others think the water inflow associated with a hydration and swelling of colloids within the nematocyst in response to change in $\mathrm{pH}, 1 "$ and a consequent sivelling of colloidal material contained therein. A second theory attributes the increase in pressure to a contraction of a fibrillar network associated with the capsule. ${ }^{19}$ It is possible that mechanisms fitting both theories are to be found among the numerous nematocyst types of the various coelenterate species. The nematocysts of Metridium appear to discharge as a result of inflow of water; those of Physalia appear to require the activity of contractile fibrils of the nematoblast.

Functional nematocysts have been found in the flatworm, Microstomum, ${ }^{12}$ 13, 14 and in the sea-slug, Aeolis." 4,11 It has been conclusively demonstrated that these nematocysts have been derived from the coelenterates on which these animals feed. It is interesting that in the appropriation of coelenterate nematocysts, these flatworms and molluscs selectively utilize only certain types. For example, Microstomam digests the volvents and utilizes the penetrants. The sea-slugs, feeding on Pennaria, utilize only the highly effective type known as the microbasic mastigophores, to the exclusion of other types. The appropriated nematocysts are quite concentrated in the bodies of their new carriers, and would appear to serve as effective defensive weapons.

\section{REFERENCES}

1. Calkins, G. N., Biology of the Protozoa (1933). Philadelphia, Lea \& Febiger. 607 ก. Trichocysts.

2. Cantacuzene, J., C. R. Soc. Biol. Paris 92:1133-1136 (1925). Nematocyst toxins.

3. Cantacuzene, J., and Damboviceanu, A., C. R. Soc. Biol. Paris 117:136-138, 138-140 (1934). Nematocyst toxins.

4. Glaser, O. C., J. Exper. Zool. 9:117-142 (1910). Nematocysts in Aeolis.

5. Glaser, O. C., and Spanrow, C. M., J. Exper. Zool. 6:361-382 (1909). Discharge of nematocysts. 
6. Grosvenor, G. H., Proc. Roy. Soc. London 72:462-486 (1903). Nematocysts of nudibranchs.

7. Hyman, L. H., The Invertebrates (1940). New York, McGraw-Hill. 726 p. Trichocysts and nematocysts.

8. Iwanoff, N., Anat. Anz. 11:551-556 (1896). Mechanism of nematocyst discharge.

9. Јакus, M. A., J. Exper. Zool. 100:457-485 (1945). Trichocysts; structure and properties.

10. Jakus, M. A., and Hall, C. E., Biol. Bull. 91:141-144 (1946). Electron micrographs of trichocysts.

11. Kepner, W. A., J. Morph. 73:297-311 (1943). Nematocysts in nudibranchs.

12. Kepner, W. A., and Barker, J. F., Biol. Bull. 47:239-252 (1924). Nematocysts of Microstomum.

13. Kepner, W. A., Gregony, W. C., and Porter, R. J., Zool. Anz. 121:114-124 (1938). Nematocysts of Microstomum.

14. Kepner, W. A., and Nurtrycombe, J. W., Biol. Bull. 57:69-80 (1929). Nemato cysts of Microstomum.

15. Krüger, F., Arch. Protist. 74:207-235 (1930). Trichocysts of Paramecium.

16. Krüger, F., Zoologica 34:1-82 (1936). 'Trichocysts.

17. Kudo, R. R., Protozoology (1946). Springfield, Ill., Charles C Thomas. 778 p. Trichocysts.

18. Pantin, C. F. A., J. Exper. Biol. 19:294-310 (1942). Control of nematocyst discharge.

19. Parker, G. H., and Van Alstyne, M. A., J. Exper. Zool. 63:329-344 (1932). Control and mechanism of nematocyst discharge.

20. Saundens, J. T., Proc. Cambridge Phil. Soc. (Biol. Sci.) 1:249-269 (1925). Function of trichocysts of Paramecium.

21. Sснмidt, W. J., Arch. Protist. 92:527-536 (1939). Birefringence of Paramecium trichocysts.

22. Schmitt, F. O., Hall, C. E., and Jakus, M. A., Biol. Symp. 10:261-276 (1943). Trichocysts.

23. Tonniges, C., Arch. Protist. 32:298-378 (1914). Origin of trichocysts in Frontonia.

24. Weill, R., Bull. Biol. France e. Belg. 64:141-152 (1930). Classification of nematocysts.

25. Weill, R., Trav. Stat. Zool. Wimereux. 10 and 11, 701 pp. (1934). Biology of nematocysts. 


\section{Bioluminescence} Protozoa through the Chordata. There appears to be little or no general pattern in the distribution of the capacity among or within the animal groups; its occurrence is quite sporadic. It may occur in one species of a genus and be absent in another. Practically all of the known luminous species are marine or terrestrial. Many luminescent marine species are found among the abyssal and planktonic faunas. Luminescent littoral marine species are also numerous. The only luminescent animal occurring in fresh water is an aquatic glowworm. Luminescent species have never been described for cave faunas.

Luminescence in animals is the result of chemiluminescent reactions in which a substrate is oxidized. The chemiluminescent reaction is usually associated with the presence of special granules in the cytoplasm of the luminous tissue. In many organisms the light-producing reactants are expelled to the exterior, where the actual reactions in production of light occur. This type of light production is known as extracellular luminescence, in contrast with intracellutar luminescence, in which the light-yielding reaction proceeds within cells. In animals with extracellular luminescence the light-producing organs take the form of unicellular or multicellular glands which secrete to the exterior. Sometimes a differentiation of two types of secretory cells are observed in multicellular luminous glands. Both appear to contribute to light production.

In higher animals with intracellular luminescence there is a general tend. ency toward an evolution of specialized photogenic organs. Whereas in the protozoans the luminescing granules are dispersed in the cytoplasm, in many higher animals such as cephalopods, insects, and fishes they are located within light-producing cells, which form only a portion of organs which may possess, in aldition to these cells, light-absorbing and light-reflecting layers, light filters, rcfactive bodies, and nerve supply. Such organs superficially resemble photoreceptors.

It is by no means always an easy matter to determine whether any particular luminescent animal possesses of itself the ability to generate light. Such luminescence may result from the presence of luminescent bacteria in or on the organism in question. One criterion for distinguishing bacterial luminescence from that originating within animal cells is that in the former the light appears to be continuous, whereas in the latter it is usually pro- 
duced intermittently, and commonly only in response to external stimuli. There are numerous exceptions to this, however. Many truly photogenic cells of animal origin exhibit a continuous glow. On the other hand, luminescence of bacterial origin can be made to give the semblance of intermit. tency as, for example, in the fish Photoblepharon, where the symbiotic lumi. nous bacteria are in a light-producing pouch with a lid capable of intermittently screening the light.

Occurrence of Bioluminescence Among Animals. Among the Protozoa numerous luminescent species are observed. These are marine radiolarians and dinoflagellates. The best known example of the latter group is Noctiluca. The light-producing granules are located throughout the organism but particularly in the peripheral regions. ${ }^{5 x}$ The organisms glow briefly in response to stimulation; the glow originates in the region of the oral groove and spreads as a wave over the body. ${ }^{59}$

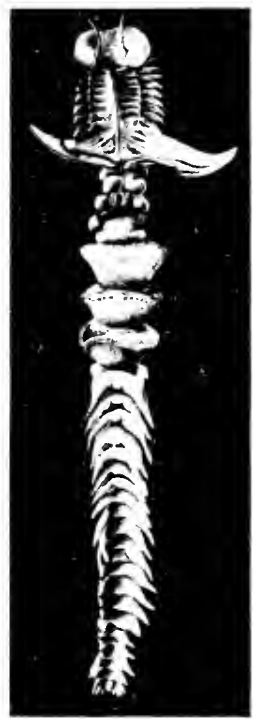

A

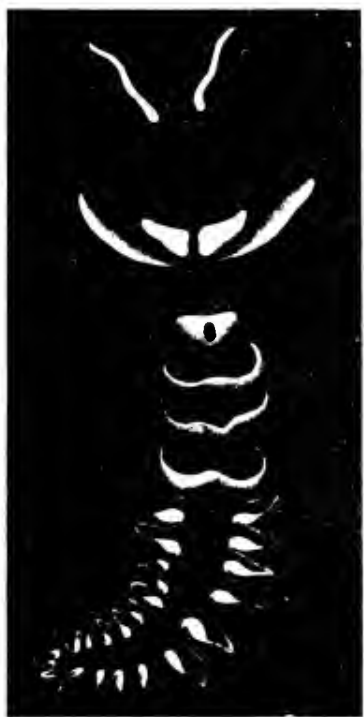

B

Fig. 250 Chaetopterus. A, Dorsal view (after Trojan). B, A luminescent individual in darkness (after Panceri). From Harvey. ${ }^{31}$

Numerous coelenterates are known to be luminous. These include a number of hydroid polyps, jellyfishes, siphonophores, and sea-pens. In this group the region luminescing is usually the whole surface of the organism, but luminescence may be restricted to certain spots. Luminescence occurs only in response to stimuli. The natural stimulus is probably mechanical. The region immediately stimulated is first to respond, and this response is followed by a wave of luminescence proceeding out from that point. ${ }^{5.2} .5$ In the jellyfish, Pelagia, the extent of spread of the luminescence is a direct function of the strength of the stimuius. ${ }^{31}$ Light production in coelenterates appears to be largely of the extracellular type, since luminous mucus can usually be readily rubbed from the surface of the organisms. ${ }^{24}$ 
Numerous Ctenophora, perhaps all, are luminous. Here the light-producing cells are located along the meridional gastrovascular canals in the vicin-
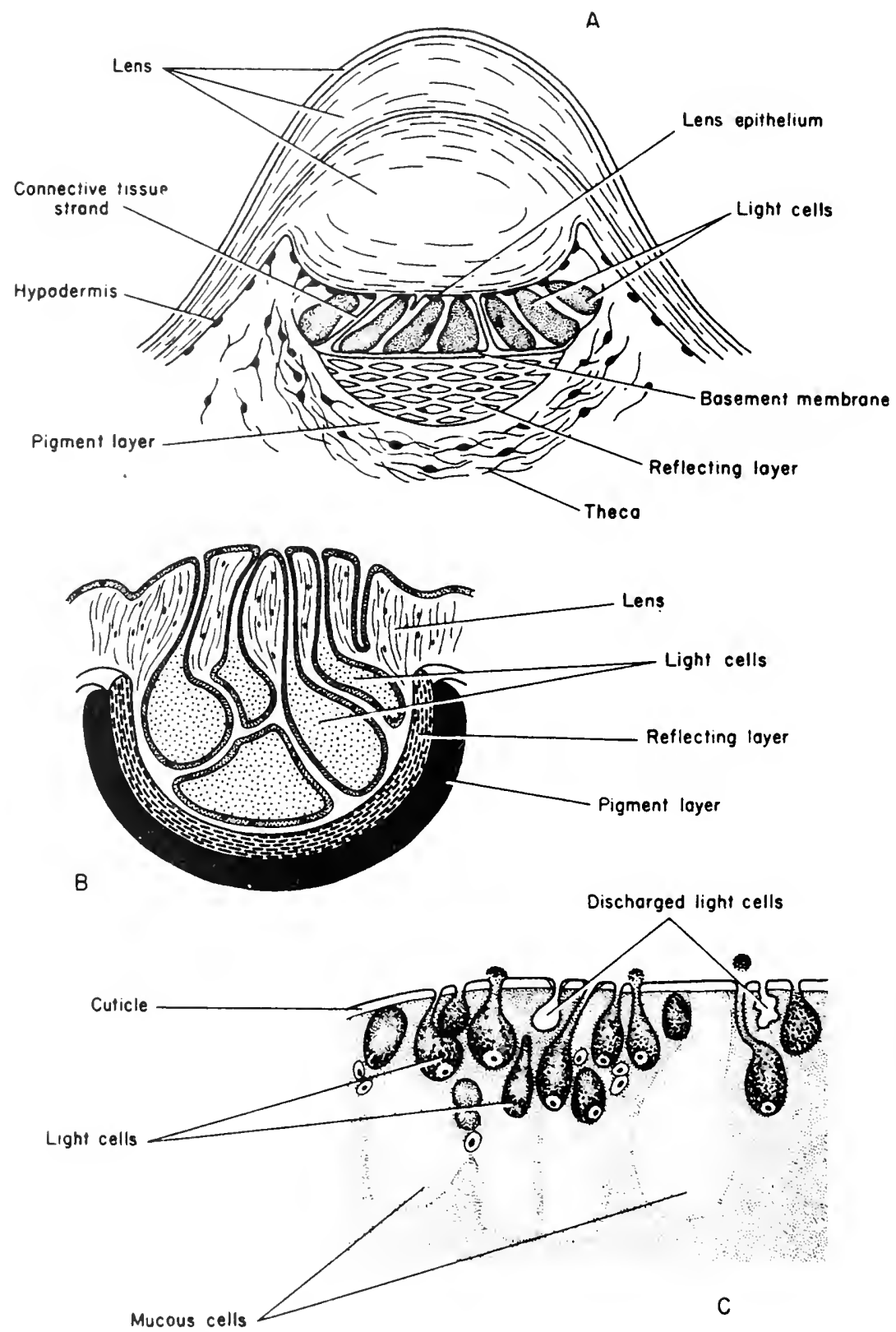

Fig. 251. A, Section through a light organ of the deep sea shrimp, Sergestes. From Harvey: $B$. Section through light organ of the cephalopod, Rondeletia. C, A section of a luminous areal of Chaetopterus epidermis. Fron Harvey, ${ }^{23}$ after Dahlgren.

ity of the germ cells. These organisms luminesce in response to stimuli, but the response is inhibited by bright light. After subjection to daylight the 
animals must be kept for some time in darkness before full responsiveness is regained.

Only a single luminous nemertean, Emplectonema kandai, has been described. ${ }^{* i}$ Photogenic cells, producing light intracellularly, are distributed over the whole worm. A local response to localized tactile stimulation is seen. A generalized response follows stretching of the animal.

Among the Annelida luminescence is restricted to species of terrestrial oligochaetes and marine polychaetes. Many earthworms, on irritation, eject a luminous slime. This may come from the oral or anal opening or from the dorsal pores. ${ }^{1:,}$, ts The polychacte, Chaetopterus, exhibits a striking luminosity, much of the surface becoming luminous whenever the worm is disturbed (Fig. 250). The photogenic cells are located in the hypodermis along with mucus cells, both of which secrete their products to the exterior ${ }^{13}$ (Fig. $25 \mathrm{l}, \mathrm{C})$. Suggestive of nerve control of light production is the fact that stimulation of the anterior end of the worm results in a wave of light-production passing posteriorly. In the transparent pelagic Tomopteris the photogenic organs are specialized nephridial funnels." In luminous scale worms the light originates in certain papillae on the dorsal overlapping scales. Stimulation of any portion of the body results in a wave of light production passing posteriorly from the point of excitation, but not anteriorly, ${ }^{1: 3}$ thus indicating rather clearly an influence of a polarized ncrvous system.

Luminous species of arthropods are numerous. They are largely crustaceans and insects, rarely myriapods and arachnids. In fact, it is from a luminous ostracod, Cypridina, that we have learned much of the fundamental characteristics of the basic photogenic process. The light-producing organ is a large gland located near the mouth. ${ }^{63}$ Two kinds of cells can be scen in the living gland, one containing large yellow granules (luciferin), and the other, small colorless granules (luciferase) (see p. 667 et seq.). The granules are ejected by muscle contraction, and the luminescence is extracellular.

In those copepods showing photogenic capacity the source of the active agents which are expelled into the sea watcr are small groups of greenish secretory cells on various parts of the body.

Many euphausids and shrimp possess rather highly differentiated organs having a reflecting layer and lens associated with the light-producing cells (Fig. 251, A). These organs are distributed widely over the surface of the body. The numerous organs appear coordinated through the nervous system inasmuch as the sequence of their activity may, as in Sergestes, ${ }^{64}$ for example, follow an anteroposterior progression. The decp-sea shrimp, Acanthephyra purpurea, has, in addition to typical photophores (luminous organs) of the general type just described, glands near the mouth from which luminescent substances may be forcefully ejected so as to permit the shrimp to escape from predators in a luminous cloud. ${ }^{30}$

Among the insects, luminescence is restricted to members of very few orders. A few species of Collembola have been described to glow continuously, although varying in intensity with the state of excitation of the individual. ${ }^{39,}$, 61 The larvae of the fungus gnat, Ceratoplanus, and of the tipulid Ay, Bolitophila, ${ }^{42}$ have been described as luminescent, and in the latter species the adults are also. In these latter flies the light appears to be in the malpighian tubules. ${ }^{66}$ 
Perhaps the most striking examples of light production in arthropods are to be found among the Coleoptera, specifically the lampyrids and elaterids,,$\ldots$. . In the "fireflies" or "lightning bugs" the photogenic organ is typ. ically located ventrally in the posterior abdominal region. The organ is composed of two cellular layers, a ventral layer of light-producing cells and a dorsal or internal laver of "reflecting" cells. The reflecting cells contain minute particles of a purine base, probably urates. This layer is said to serve as a diffusing reflector for the photogenic cells, while simultaneously being a dorsal shicld. The photogenic organ is richly provided with tracheal vessels which terminate in numerous tracheal end-cells. Nerves pass into the organ.

Flashing of the firelly is controlled by the nerrous system. One remark able aspect of the activity of these organs, which indicates this, is the synchronous flashing of some tropical fireflies." The fireflies normally flash at random, but mav, when large numbers of individuals are assembled together, flash synchronously, or exhibit some coordination of activity in the assemblage through waves of flashing which proceed out rapidly from some individual of the group.

The elaterid "Cucujo" beetle, Pyrophorns, of the West Indies has a pair of greenish luminescent organs on the dorsolateral aspects of the prothorax and an orange-vellow organ on the ventral aspect of the first abdominal segment. The South American "railway worm," Phrixothrix, possesses a reddish luminescence on the head and greenish luminous spots segmentally ar ranged along the body." A North American species of the related genus, Phengodes (Fig. 252), in its photogenic organs resembles somewhat the South American species, except that it lacks the red head organ. The North American species has been reported to glow continuously, unlike most luminescent animals.

Several species of myriapods secrete a luminous slime in much the same manner as the luminous earthworms. A luminous pycnogonid has been described.

The bivalve mollusc, Pholas, has a number of photogenic glands, which secrete into the siphon. ${ }^{17}$ Some nudibranchs have distributed over the body luminescent cells which flash when the animal is appropriately stimulated. ${ }^{65}$ Of the molluscs, however, the cephalopods as a group show the highest derelopment of this capacity. A large number show luminescence. In many, as for example, Loligo, the luminous organs are open structures and contain luminous bacteria. In others, probably the majority, light is produced by (ells of the animal itself (Fig. 251). In one squid, Heteroteuthis, there is an unpaired luminous organ which opens into the mantle cavity and expels a luminous cloud through the siphon when the animal is disturbed. ${ }^{5+, 60}$ Some species of squids, such as the frefly squid. Watasenia,, in. ti: have complex patterns of luminous organs of the intracellular type over the body, reach. ing a high state of differentiation in such deep-sea forms as Lycoteuthis diadema. In the latter as many as four different colors are produced by the various luminescent organs present.

Only the Ophiuroidea among the cehinoderms contain luminous species. A number of these have unicellular photogenic organs scattered over the body, and luminesce in response to any disturbance of the animal. 
Light production among the chordates is restricted to the protochordates and fishes. I huminous slime is produced, for example, hy species of liatano glossus. Anome the tunicates, the best known example of a fuminescent form is Pyrosmana a colonial speces. The organiom huminesecs on stimulation. and a wave of photogenic activity spreads out over this colonial organism. Light definitely operates as a stimulus in this spread of activity from one luminescing individual of a colony to adjacent ones. Thus the wave of activity has been reported to pass from one colony to another even when glass walls separate the two colonies. Similarly, activity may be induced in a colony by using a light flash as a stimulating agent.

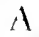

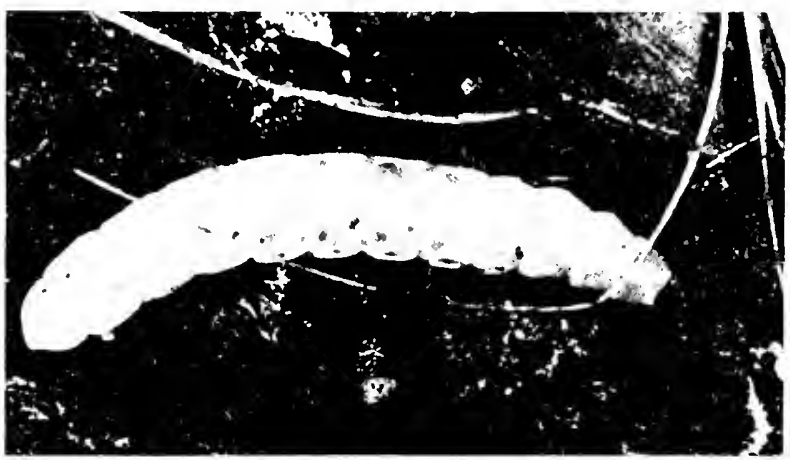

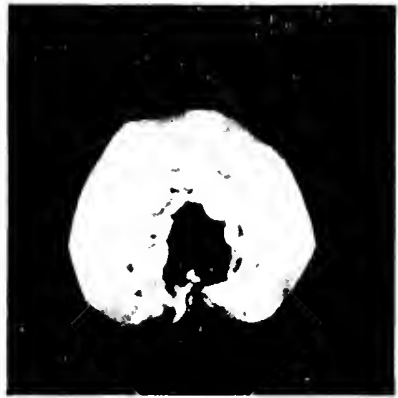

B

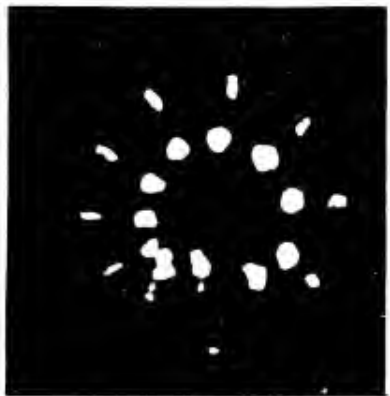

C

Fig. 252. A female larva of the beetle, Phengodes. A, Dorsal view. B, Lateral view. $\mathrm{C}$, A luminous specimen in darkness (From Harvey 1940). ${ }^{31}$

The elasmobranch and especially the teleost fishes have numerous luminescent representatives, particularly among those inhabiting the depths of the seas. In some, like Photoblepharon, Anomalops, Physiculus, Equula, and Monocentris, the light is due to the presence of symbiotic bacteria in special organs found in the cheeks or lower jaw."1 In others, e.g. Malacocephalus. luminescent granules, possibly bacteria, are expelled from ventral sacs onto the ventral surface of the body when the fish is excited. Still others, as Astronesthes and Stomias, possess well-differentiated eye-like organs with lenses and pigmented cups. In some fishes the appearance of light on stimulation is 
Sow. In Porichthes a latent period of 8 to 10 seconds is abserved; then the light lists for about 20 seconds. ${ }^{-1+1}$ Injection of adrenalin will activate the photesenic oreans in this lish and in techiostoma.:"

Physical Characteristics of Animal Light. The intensity of lisht produced by photogenic organs is relatively low. The light of a firelly, Photinus, is the equiralent of 0.0025 to 0.02 candles:" that of the "cucujo" beete, 0.0006 to 0.006 candles.: Such values in candles do not give a gond meas ure of the brightness of the actual luminescing surfaces, since these values vary with the areas of these surfaces. Furthermore, since the surfaces are relitirely small, the values are likely to suggest smaller values of surface brightness than are actually present. Also, since organ-sizes vary considerably from one species to another, the order of brightness of luminescing surfaces need not parallel the order of light intensities. Surface brightness is ex pressed in lamberts or millilamberts. Measurements of the surface brightness of a number of luminescing organs have given in general values ranging, from 0.3 to +5 millilamberts.

The color of the light produced by various photogenic organs of animals vary all the way from blue through red.":3, ; There is not uncommonly a dif ference in the colors of light emitted from different organs in the same animal. It is possible that in some cases these color differences are due to the presence of colored filters covering the luminous areas, but in other cases they appear definitely due to differences in the basic light-producing reactants.

Study of a number of species gives us reason to believe that the light emitted by any single organ shows a continuous spectrum spreading over a restricted region of the visible range of wave lengths. The spectral range for a number of animals is found in the following list modified from Harvey. $=$

\section{Species}

Chaetopterns insignis

Photinus pyralis

Pyrophorus noctilucus

Photuris pennsyluanica

Cypridina hilgendorfii

Phengodes laticollis
Wave Lengths

$550-440 \mathrm{~m} \mu$

670-510 $\mathrm{m} \mu$

640-468 $\mathrm{m} \mu$

$590-510 \mathrm{m \mu}$

610-415 $\mathrm{m} \mu$

650-520 $\mathrm{m} \mu$

When one recalls that the range of wave lengths visible to the human eve extends from 760 to $400 \mathrm{~m} \mu$ it is evident from the foregoing table that in all of the common luminescent species whose spectra have been examined the light produced is entirely within the visible range. Ulltraviolet radiation has never been recorded for any photogenic organ. Furthermore, no significant infrared or heat radiation is produced; hence animal light is said to be a cold light. This fact has led to the popular notion that animal light is nearly 100 per cent eflicient. To determine the actual luminous efficiency of any light source, however, it is necessary to take into consideration the spectral cnergy curve for visibility of the particular organism for which the luminous efliciency is being calculated. If the light were 100 per cent efficient for the human eve, all of the radiation would be at the wave length of maximum sensitivity of the eye. For an eye with the spectral sensitivity characteristics of the human eye, the light of the firefly, Photaris, is about 92 
per cent efficient, that of Photinus about 87 per cent. ${ }^{14}$ For the bluish light of Cypridina and Chaetopterus, the maximum emissions of which lie still further from the region of maximum sensitivity for the human eye, the luminous efficiencies are of the order of 20 per cent or less. However, all of these exhibit strikingly higher luminous efficiencies, of course, than does the carbon incandescent lamp, which approximates 0.5 per cent. We must always bear in mind that the luminous efficiencies would have more real $\mathrm{com}$ parative biological significance if they were calculated in terms of the spectral energy curves of sensitivity of the photoreceptive mechanisms of those species reacting in nature to these light sources.

The Mechanism of Light Production. The actual light-yielding reactions of animals have received considerable study. 'The solution of the problem of their general nature has been very much simplified through the discosery that these reactions will proceed in vitro in a manner superficially indistinguishable from those proceeding in vivo. It has been known for many years that the luminescent cells or tissues of certain animals or their products can be dried with a resultant cessation of light emission. When water is again added the materials will again luminesce. Hence the final reactions are obviously not dependent on the vital organization of cells, although the production of the specific materials which participate in these reactions do have such dependence.

In acldition to an aqueous medium, photogenic reactions of most animals require also the presence of free oxygen. This appears equally true, whether the reactions occur within the living organism or in extracts of dried prep arations of luminous organs. It is true, however, that in some instances the necessary partial pressure of oxygen is extremely small. The only critical experiments which have indicated that luminescence can occur in the complete absence of oxygen have been performed on certain radiolarians, the jellyfish, Pelagia and Aequorea, and certain ctenophores.."- 34. 31 One must conclude that in these animals either the reactions are basically different from those in most other animals or a source of oxygen is available in the ma. terial of the extracts. The latter alternative appears the more probable.

It was clearly demonstrated many years ago that the photogenic reaction in the beetle, Pyrophorns, ${ }^{1+}$ and in the mollusc, Pholas, ${ }^{1.5}$ involved two organic substances. These were separated from one another by utilizing the characteristics (1) that one substance was less heat stable than the other, and (2) that when the luminescent reaction had run its course some of one of the substances, the relatively heat-instable one, remained. The one that was relatively heat stable was named luciferin, the other luciferase. Luciferin solutions could be obtained free from luciferase by extracting the luminous organs in hot water; luciferase could be obtained free from luciferin by extracting the organs in cold water and then permitting the extract to luminesce to exhaustion.

Luciferin and luciferase have also been identified in the polychaete worm, Odontosyllis, in the ostracods, Cypridina and Pyrocypris, in the deep-sea shrimp, Acanthephyra, and in several species of beetles."-2. 2..30 However, careful search has failed to reveal their presence as such in most other $\mathrm{com}$. mon luminous groups. It is reasonable to assume that the functional counterparts of these substances are nonetheless present in these latter groups, 
since there is a conspicuous similarity in the general properties of bioluminescence. wherever it occurs.

The photogenic materials show a certain degree of group-specificity. Luminescence will result only when the luciferin and luciferase from the same or from two rather closcly related species are mixed, as for example with two genera of lireflies, or two genera of ostracols. No light results on the mixing of these substances obtained from two widely separated genera in the same subphylum, as for example, the ostracod. Cypridina, and the decapod, Acanthephyra. Similarly no light results when the two materials come from different phila.

Very litte is known as to the chemical nature of luciferin and luciferase. All studies indicate luciferase to be non-dialyzable and to show a number of properties characteristic of a protein. Such properties include heat lability; precipitation by alcohols, saturated ammonium sulfate, and alkaloidal reagents: destruction by proteinase; and induction of an anti-luciferase on injection into a rabbit: ${ }^{-:}$Furthermore, careful study of the reaction kinetics of Cypriana hiciferase shoss it to have characteristics of a typical enzyme, although it will not oxidize an unlimited quantity of luciferin. The enzyme gradually becomes ctenatured.

luciferin appears definitcly to be non-protein. It is dialyzable, indicating relatively mall moleculur weight. Cypridina iuciferin is readily oxidized by numerous oxidizing agents, but yelds light only when oxidized in the presence of luciferase (sece exception below). Crude luciferin extracts are readily and rapidly oxidized without luminescence in strong light, apparently by a photochemical reacton. This non-luminescent reaction is not observed in solutions of more carefully purified luciferin and luciferase, therefore it probably repuires the presence of photosensitizers for its occurrence.

fust as there has been discovered no oxidizing agent which will, in the presence of C midina luciferin, produce light, so also has no substrate except luciterin ret been found which, on oxidation in the presence of lucjferase, vields light.

The kinctics of the Cypridina luciferin-luciferase reacion have been carefully investigatcd." - The reaction appears monomolecular. There is also a ratler high temperarure coctlicient $\left(Q_{10}\right)$ laving an average value in the iunctional range of $2.7 \mathrm{t}$, inver at the lower end of the temperature range and hisher at the higher end. The relocitr constant of the reaction is proportional to the huciforase concentration. The total light emitted is propor-

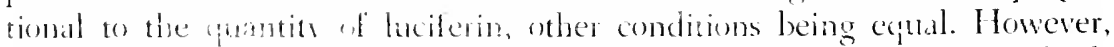
the tonal ameunt of hisht is alse infuenced by the amount and type of salt

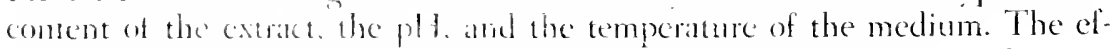
freicncy of light praluetion in toms of units if luciterin decreases as the tem-

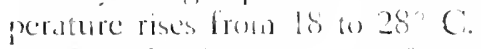

When luciferin and fuciferase are tirst mixed in an oxygenated medium there is an initial flakh of fight, tox intense to fit smoothly with the general curve of decaly of the luminescent raction which follows. The time required for the intensity of the thash to icach a maximum is longer (0.03 sec.) when luseterin and lacif ase are mixed in an oxy genated medium than when oxygen is added to an asyen free and hence non-fuminescing mixed solu. 
tim of the two photogenic substances $(0.008 \mathrm{sec}$.)." This suggests that the reaction between luciferin and luciferase is slow, compared with the rate of the reaction which involves the oxygen.

Cypridina luciferin is apparently oxidized in two ways: (1) through action of luciferase, with light emission, and (2) through other than luciferase activity, without light production." The latter reaction, but not the former, seems to be reversible in the presence of reducing agents such as $\mathrm{Na}_{2} \mathrm{~S}_{22} \mathrm{O}_{4}$ or $\mathrm{H}_{2} \mathrm{~S}$, or in the presence of finely divided platinum and free hydrogen, or at the cathode of an electrolyzed extract, or simply under the reducing action of some cultures of bacteria. This freshly reduced luciferin is able to emit light when oxidized in the presence of luciferase. Luciferin oxidized in the presence of luciferase is not capable of being reduced again by such reducing agents, hence the oxidation in the presence of luciferase must be substantally different from oxidation by other oxidizing agents.

For many years it was the accepted hypothesis that luciferase not only catalyzed the oxidation of luciferin but also was the molecule which actually emitted the light. The lights of the two genera of fireflies, Photuris and Photinus, whose luciferin and luciferase are capable of reciprocal interaction with light production, are of visibly different colors. ${ }^{22}$ In Photinus it is more reddish; in Photurus, more yellowish. When the luciferases and luciferins are interchanged in in vitro experiments, the color of light emitted is characteristic of that species which contributes the luciferase. In view of these and other characteristics of the reaction, the following scheme for the light emitting reaction was proposed:

(Lucifcrase)

Luciterin $+1 / 2 \mathrm{O}_{2} \rightleftharpoons$ Oxidized luciferin ${ }^{*}+\mathrm{H}_{2} \mathrm{O}$

Oxidized luciferin $^{*}+$ Luciferase $\longrightarrow$ Oxidized luciferin + Luciferase $^{*}$

Luciferase $\longrightarrow$ Luciferase $+\mathrm{hv}$

*indicates an activated molecule

These observations, which at one time seemed definitely to indicate luciferase as the actual light-emitter, now appear capable of other intcrpretations. This situation could possibly obtain, for example, if the light enitter were a conjugated protein with luciferin as an emitting prosthetic group, or if luciferase were the specific protcin and luciferin the cocnzyme of a reacting system.

Detailed studies of the spectrographic changes in luciferin during its oxidation both in the absence and in the presence of luciferase have since indicated that the steps are the same in the two instances. " Reduced Cy'pridina luciferin with a maximum absorption at $435 \mathrm{~m} \mu$ becomes oxidized to a form with a maximum absorption at $465 \mathrm{~m} \mu$, and then this latter form is destroyed. The total amount of light produced is proportional to the initial amount of absorption at $435 \mathrm{~m} \mu$. The rate of thesc oxidative changes is about 100 times as rapid in the presence of luciferase as in its absence (Fig. 253).

Such olservations as the foregoing, and further cletailed studies of the kinctics of the bioluminescent reaction, ${ }^{+i}$ together with an observation that luciferin in 95 per cent ethyl alcohol at $.70^{\circ}$ C. will luminesce, ${ }^{--i}$ have given 
strong support to the view that the actual light-emitting molecule is the luciferin.

All our present knowledge of the properties of the bioluminescent process appear capable of fitting into the following scheme of reactions. ${ }^{+5 .}$. t6

Let $A$ stand for luciferase, Lll., for reduced luciferin (max. absorption. $435 \mathrm{~m} \mu$ ) and $\mathrm{L}$ for its oxidation products (max. absorption $465 \mathrm{~m} \mu$ ), L* for excited luciferin, $L_{1}$ for destroved luciferin, and $X$ for an unknown $\mathrm{H}_{2}$ donor, possibly glucose.

$$
\begin{aligned}
& \text { f. } \mathrm{AL}+\mathrm{XH}=\mathrm{ALH}=\mathrm{X} \\
& \text { 2. } \mathrm{A}+\mathrm{LH}=\Longrightarrow \mathrm{ALI}: \\
& \text { 3. } \mathrm{ALH}=\mathrm{O}=\mathrm{OLH}+\mathrm{HH} \text {. } \\
& \text { 4. } \mathrm{ALH}=\mathrm{AL}+\mathrm{H}+ \\
& \text { 5i. } \mathrm{AL}+\mathrm{LH} \longrightarrow \mathrm{AL}^{*}+\mathrm{LH}^{-} \longrightarrow \mathrm{AL}+\mathrm{LH}^{-}+\mathrm{hv} \\
& \text { 5b. } \mathrm{AL}+\mathrm{LH} \longrightarrow \mathrm{AL}+\mathrm{LH} \\
& \text { 5c. } \mathrm{AL}^{-}+\mathrm{LH} \longrightarrow \mathrm{AL}_{1}+\mathrm{LH}^{-} \\
& \text {6. } \mathrm{ALH}+\mathrm{O} \longrightarrow \mathrm{AL}+\mathrm{HOO}_{2} \\
& \text { 7. } \mathrm{AL}+\mathrm{O}_{2} \longrightarrow \mathrm{AL}_{1}
\end{aligned}
$$

Essentially the same reactions, with the exception of reaction 2, also appear to proceed in the absence of $\lambda$, but their rates, especially that of reaction 5 . are such that no light is then produced.

It is readily apparent lrom the foregoing scheme of reactions that only reaction 5 a is the actual lightecmitting one, but that this reaction is de pendent an, or associated with, a number of other reactions which by themselves are dark reactions. The whole problem at the moment appears now to be in such a state that one camnot saly with finality whether it is lucifer-

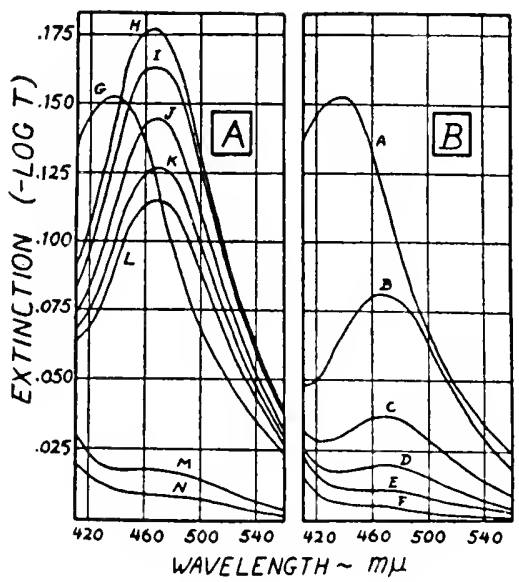

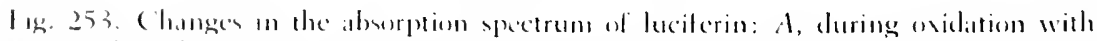
assgen nithout laciferase (nen-lumineseent), and B, in the presence of luciferase (light

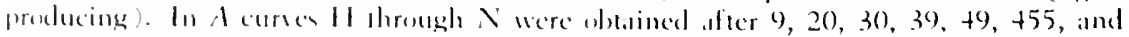

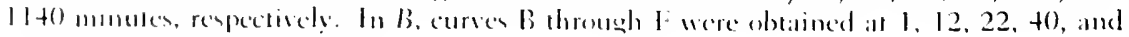
457 mumules. I 10m lohmsum." 
ase, luciferin, or possibly an unidentified third constituent which is the actual light-emitter. Furthermore, some characteristics of the bioluminescent reaction so differ among species, for example between Cypridina and fireflies, that it would appear that a final detailed explanation for one would probably not be a complete explanation of the other.

There is very good evidence that phosphate is liberated during the luminescent reaction." This suggests that energy-rich phosphate bonds are the sources of the energy liberated, as has been demonstrated for muscle metabolism.

Variations in electrical potential in light organs have been described as accompanying light production in some fireflies. ${ }^{36,37.3 \mathrm{~m}}$ In adults, which show a flickering light, there is a rhythmical potential variation; in larvae, lacking a flickering character of light, there is a single, long-duration, potential variation. The former type of response is correlated with the presence of tracheal end-cells; the latter, with their absence.

The Control of Bioluminescence. Among animals the production of light is typically not a continuous process. Light is usually produced intermittently in response to external stimuli. This fact is of such generality that, when an animal appears to have continuous luminescence, it can usually be assumed that this is the result of the presence of symbiotic, pathogenic, or transient luminescent bacteria within or upon that animal.

The means of control of light production in animals are fundamentally of three types. The first two are observed in extracellular luminescence. lin those instances where luminescent materials are expelled from a photogenic sac into the surrounding sea water on stimulation (as in the cephalopod, Heteroteuthis, the ostracod, Cypridina, and the shrimp, Acanthephyra), the control is indirect and operates by means of typical neuromuscular mechanisms. On the other hand, a slightly different situation obtains in those organisms which can secrete a luminescent slime over the surface of the body. Such animals include the luminescent earthworms, Chaetopterus, myriapods, many coelenterates, and the clam, Pholas. In these there is a control of a secretory process by direct nervous, or possibly also, in some cases, endocrine excitation. The third general type of control applies to those numerous animals where the luminescence is an intracellular phenomenon. This is obviously the situation in such well-known animals as Noctiluca, the insects, and certain fishes. Here we have to do with some mechanism whereby excitation of the luminescent cell results in the contained photogenic substances being permitted to interact. The interaction may possibly come about either through rapidly making small quantities of one of the two essential photogenic reactants available to the other within the cell. or through temporarily rendering the milieu favorable for the luminescent reactions. The latter might occur through control of oxygen, water, hydrogen ions, etc. We can at present do little more than speculate on the means by which the animal can, in response to stimuli, give very brief and intense flashes of light which vie in rate of development and decline of intensity with the best of incandescent lamps.

In multicellular organisms the flashing is typically associated reflexly with tactile or photic receptors. The reflex pathways may involve the nervous system alone, or both nerves and endocrines. In the coelenterate, Revilla, the 
spread of luminescence over a stimulated animal proceeds in all directions at a rate characteristic of nerve-net transmission. ${ }^{51 i}$ In the ctenophore, Mnemiopsis, the tactile receptors involved in reflex light production lie along the rows of ciliated combs. ${ }^{51}$

Most studies of the control of luminescence have concerned fireflies. Among other stimuli, risual cues normally excite a responsive flash in these insects. The photogenic organ is innervated, but it is not definitely established whether the nerve supply goes to the photogenic cells proper or to the tracheal endings within the light organ. In the latter case, the nerves might be supposed to innervate a complex valve mechanism located in the tracheal end-cell.

Two general types of theories for the control of Hashing in the firetly have been advanced. The first type presumes that the flash depends on the rapid and transitory admission of oxygen to the photogenic cells. This is considered to be brought about either by direct nervous control of valves in the tracheal end-cells or by means of a stimulated increase in the metabolites in the photogenic tissue and a consequent osmotic withdrawal of water from the terminal portion of the tracheal tubules with the result that oxygen would be brought directly to the glandular elements. The presence of oxygen would then permit an oxidation of the metabolites, again reducing the osmotic pressure of the cell contents, and permit restoration of water to the tubules and exclusion of the oxygen. In support of an oxygen-control mechanism is the fact that microscopic observations of a luminescing gland show's the brightest light to come from the immediate vicinity of the ends of the tracheal tubules. An end-cell valve mechanism is not essential, however, since some insects, especially larvae of fireflies, which show at least some degree of intermittency in light production, even though not true flashing, do not have differentiated tracheal end-cells.

The second general type of mechanism postulated is found in numerous other animals which also flash, although they possess no tracheal mechanism. This is a direct nervous excitation of the light-producing cells through photogenic nerves.

In the fishes, Porichthys and Echiostoma, there is some evidence that the normal reflex pathways of control of the system of photogenic organs includes the secretion and action of the hormone adrenin.

There have been a number of observations indicating that bright light inhibits the production of light by animals, ${ }^{26,11,52}$ although in most cases we know little or nothing of the mechanism of this inhibition. It may result, as some evidence indicates for ctenophores, from a direct destruction of photogenic material within the light-producing cells. In these animals extracts made from specimens exposed to sunlight show practically no capacity to luminesce. Furthermore, after exposure to bright light these organisms have to be kept in darkness often for as long as 45 minutes before they will again luminesce.

There have, on the other hand, been numerous reported observations of the existence of a diurnal rhythmicity in the capacity of certain species of animals to luminesce. Such a rhythm has been described to persist for sevcral days in constant darkness in the firelly Photinus, ${ }^{4}$ in the balanoglossid, Ptychodera, ${ }^{12}$ and questionably in the jellyfish, Pelagia. ${ }^{40}$ Such behavior 
indicates that changes in the responsiveness of the excitatory mechanism are probably also operative, at least to some degree, in the normal influence of light on the photogenic reflex mechanism. The inhibition by light thus appears to act in two ways, with the relative importance of each method probably varying with the species. The direct effect of light on the quantity of photogenic material present may be called a direct effect; and that effect on the excitatory mechanisms for the photogenic cells or organs, an inclirect one.

The luminescent response of ctenophores has been shown to be readily fatigable and to exhibit recovery in much the same fashion as other typical scnsory-neuro-effector mechanisms.

Functional Significance of Bioluminescence. The significance of light production in the lives of the numerous organisms possessing this capacity is often by no means obvious. That some survival value is commonly associated with this function is, however, likely, inasmuch as most higher organisms which luminesce have evolved specialized light cells or organs for the purpose. Morener, the flashing itself may become incorporated into the gen eral response mechanism of the organism.

Three general types of functions have been ascribed to animal luminescence. One of these is the luring of food, but as yet there has been no conclusive evidence for this. A second function ascribed to the organs is that they serve as protective devices. They may operate through warning or frightening predators; by concealing the light-emitting animals, as in the ejec. tion of a luminous cloud by the deep-sea shrimp, Acanthephyra, and the squid. Heteroteuthis; or through distracting predators. The pattern of nervous control of luminescence in such an annelid as the scale-worm, Acholoë, appears to suggest this last role. When the worm is transected, as by an attacking predator, the posterior portion alone luminesces brightly, while luminescence is inhibited in the anterior portion. This behavior could possibly result in a greater chance for the more viable anterior part to escape. Suggestive also of such an emergency protective function of luminescence is the fact that adrenalin induces bright and extensive luminescence in certain fishes. An interesting variation of the protective function of bioluminescence in organisms has been advanced, namely that stimulation to luminescence of one individual in a group might serve as a warning to its fellows and permit their escape.

The function of luminescent organs which appears to have best observational and experimental support up to the present is that of serving as sig. nals for bringing together the two sexes in mating. The fireworm of Bermuda, Odontosyllis, provides a well established illustration of this role. ${ }^{18}$ The females, which exhibit a marked lunar periodicity, appear at the mating periods at the surface of the sea where they swim in small circles, luminescing brilliantly. Males from deeper water swim directly toward the luminescing females and join in the mating "dance"; both sexes then liberate their gametes into the water together. If, perchance, no male joins a luminescing female in a short time, her light gradually fades, but after a briel period of rest her photogenic organs again become active and the luminescent "dance" is repeated. Males moving directly toward a luminescing female but failing to reach her before the end of a luminous period have bcen observed to cease their directed movement and wander aimlessly until the female again 
Other well established eases of the use of light production as mating signals have been described for various species of fireflies." ln many species the females, which mas exen be wingless, remain in the grass while the males Hr about. The females signal in response to the flashing males, which then Hy toward the females. The rarious species differ in characteristics of their flashing, such as in the frequence, total number of Hashes, color, intensity and duration of each flash, etc. The female responds in a characteristic manner to a flashing of the male of the same species. The female of Photimus, for example, alwals flashes about two seconds after the flash of the male. The male continues to respond to the females until the two sexes have met. The attracting light response of the female can be imitated by use of a fashlight which is operated to flash with the temporal characteristics of a typical responding fomale for a species.

Numerous other suggestive examples of a role of luminescence in the mating reactions of animals have been described, but most of them require more convincing descriptions, or experimental studs, before they can be definitely accepted as such. Howerer, inasmuch as it is difficult to imagine any functional significance of bioluminesence in bacteria or fungi, we probably can assume that bioluminescence in many animals, especially lower ones, is fortuitous and of no survival value. In these instances light may have appeared as a by-product associated with oxidative metabolism.

\section{REFERENCES}

1. Amberson, W. R., I. Gen. Physiol. 4:517.558 (1922). Kinetics of Cypridina luciferin-luciferase reaction.

2. Anderson, R. S., J. Cell. E Comp. Phy'siol. 3:45-60 (1933). Kinetics of (ypridina luciferin-luciferase reaction.

3. Anderson, R. S., J. Cell. \& Comp. Physiol. 8:261-276 (1936). Kinetics of luciferin-luciferase reaction.

4. Buck, J. B., Physiol. Zool. 10:45-58 (1937). Diurnal rhythm in Photinus lumin. escent mechanism.

5. Buck, J. B., Physiol. Zool. 10:412-419 (1937). Luminescence in firefly mating.

6. Buck, J. B., Quart. Rev. Biol. 13:301-314 (1938). Synchronous flashing of fireflies.

7. Buck, J. B., Proc. Rochester Acad. Sci. 8:14-21 (19+1). Spectrometric studies of firetly lights.

8. Buck, J. B., Anu. New York Acad. Sci. 49:397-482 (1947). Luninescence in fireHies.

9. Chance, B., Harvey, E. N., Johnson, F., and Millikan, G., J. Cell. E Comp. Plizsiol. 15:195-215 (1940). Kinctics of bioluminescent fashes.

10. Cunse, A. M., /. Cell. \& Comp. Physiol. 15:159-172 (1940). Spectrographic changes in luciferin during oxidation.

11. Coblentz, IV. W., Carnegie Inst., Wash., Publ. 164, 47 p. (1912). Light intensity in Photinus.

12. Carozen, W. J., Anat. Rec. 20:186-187 (1920). Diurnal rhythm in Ptychodera luminescence.

13. 1) Alu GuLN, (1., J. Frunklin Inst. 181:658-696 (1916). Spread of luminescence in polynuid worm. Pholegenic cells of Chactopterus.

14. Dunoss, R., C. R. Soc. Biol. Puris 37:559-562 (1885). Luciferin and luciferase in Pyrophorus.

15. Dubses, R., C. R. Soc. Biol. Paris 39:564-566 (1887). Luciferin and luciferase in Pholas.

16. Dunoss, R., C. R. Acal. Sci. 105:690-692 (1887). Luciferin and luciferase in Pholas. 
17. Dubors, R., C. R. Soc. Biol. Paris 48:995-996 (1896). Luminous glands of Phrolar.

18. Galloway, T. W., and Welch, P. S., Tr. Am. Micr. Soc. 30:13.39 (1911). Luminescence of Odontesyllis during mating.

19. Gates, G. E., Rec. Ind. Mus. 27:471-473 (1925). Luminesecent material from earthworm chorsal pores.

20. Greene, C. W., and Greene, H. H., Am. J. Physiol. 70:500-507 (1924). Luminescence in Poricluthys; action of adrenalin.

21. Hanms, J. W'., Ztschr. Wiss. Zool. 131:157-179 (1928). Bacterial urigin of light in Equula.

22. Harver, E. N., Science +6:2+1-2+3 (1917). Reciprocal interaction of Photinus and Photurus luminescent materials.

23. Harver, E. N., The Nature of Animal Light (1920). Philadelphia, J. B. Lippincott. 182 p.

24. I Harver, E. N., Biol. Bull. +1:280-287 (1921). Luminous slime from Pelagia.

25. Harvey, E. N., J. Gen. Physiol. 4:285-295 (1922). Luciferin and luciferase in animals.

26. Hanvex, E. N., J. Gen. Physiol. 7:679-685 (1925). Light inhibition of lumin. escence.

27. Harvey, E. N., Biol. Bull. 51:89-97 (1926). Luminescence in absence of $\mathrm{O}_{2}$.

28. Harver, E. N., Am. J. Physiol. 77:5+8-55+ (1926). Lucilerin and luciterase in animals.

29. Harvey, E. N., J. Biol. Chem. 78:369-375 (1928). Luminescence by luciferin without lucilcrase.

30. Harvey, E. N., Zoologica 12:70.75 (1931). Luciferin and luciferase in shrimps. Lumious secretion in Acanthephyra.

31. Harver, E. N., Living Light (1940). Princeton Univ. Press. 328 p.

32. Hunver, E. N., I. Cell. E Comp. Physiol. 26:185-187 (1945). Luminencence in Phrixothrix.

33. Hirver, E. N., and Dertrick, J. E., J. Immunol. 18:65-71 (1930). Anti-luciferase furmation in rabbit.

34. Harvex, E. N., and Konn, 1. M., J. Cell. E Comp. Physiol. 12:319-323 (1938). Luminescence in absence of $\mathrm{O}$.

35. Harvey, E. N., and Stevens, K. P., J. Gen. Physiol. 12:269-272 (1928). Light intemsity in Pyrophorus.

36. Hisman, Bunich1, Cytologia 12:366-377 (19+2). Electrical potential variations in plontogenic organs.

37. Hasana, Bun-ron, Cytologia 12:378-388 (1942). Electrical potential variations in photugenic organs.

38. Hasama, Bun-icin, Cytologia 13:155-161 (19+4). Electrical potential variations in photogenic organs.

39. Heidr, K., Biol. Zentralbl. 56:100-109 (1936). Luminous Collembold.

40. Hermass, C., and Moore, A. R., J. Gen. Physiol. 6:273-280 (1923). I)iurnal rhythm in Pelagia luminescence.

+1. Heymans, C.., and Moone, A. R., J. Gen. Physiol. 7:3+5-348 (1925). Inluibition of ctenophore luminescence by light.

42. Hubson, G. V., Anr. Mag. Nat. Hist. 17:228-235 (1926). Luminescence in Bolitophila.

43. I iudson, G. V., Ann. Mag. Nat. Hist. 18:667-670 (1926). Luminescence in Bolitophila.

47. lves, H. E., and Coblentz, Mr. W., Bull. Bur. Staud., Wash. 6:321-336 (1910). Luminous efficiency of firetly.

45. Johnson, F. H., Adv. in Enzymol. 7:215-26+ (19+7). Bioluminescence, review.

46. Johnson, F. H., Erring, H., Steblay, R., Chaplin, H., Huber, C., and CineraRd, G., J. Cen. Physiol. 28:463-537 (1945). Kinetics of biolumineseent reaction.

47. Kanda, S., Biol. Bull. 77:166-173 (1939). Luminescence in Emplectonenua.

48. Komarek, J., Bull. Internat. de l'Acad. Sci. de Boheme Ht:1-10 (1934). Luminescent matcrial from earthworm dorsal pores.

49. McElroy, W. D., and Ballentine, R., Proc. Nat. Acad. Sci. 30:377-382 (19+4). Phosphates in bioluminescence. 
50. MEYER, A., Zool. Anz. 86:12t-133 (1929). Light organs of Tomopteris.

51. Moone, A. R., J. Gen. Plyysiol. 6:403-412 (1924). Tactile receptors in bioluminescence: Mneniopsis.

52. Moone, A. R., Am. J. Physiol. 76:112-115 (1926). Spread of luminescence in Pennatula. Inhibition of luminescence by light.

53. Moone, A. R., J. Gen. Physiol. 9:375-381 (1926). Diurnal rhythm in Pelagia luminescence.

54. Mortara, S., Riv'. Biol. 6:1-22 (1924). Luminescence in cephalopods.

55. Okada, Y. K., Takagl, S., and Sugino, H.. Proc. Imp. Acad. 10:431-434 (1933). Luminescence in $\mathrm{IV}_{\text {atasenia. }}$.

56. Parker, G. I1., J. Exper. Zool. $31: 475.513$ (1920). Spread of luminescence in Renilla.

57. Polmantin, O., Ztschr. Biol. 55:505-529 (1911). Luminescence in Pyrosoma.

58. Pratje, A., Arch. Protist. 42:1-95 (1921). Luminescence in Noctiluca.

59. Quntrefages, A. De, Ann. Sci. Nat. 3 ser. Zool. 14:236-281 (1850). Spread of luminescence over Noctiluca.

60. Skоwron, S., Riv. Biol. 8:236-240 (1926). Luminescence in Heteroteuthis and Sepiolat.

61. Stanmer, H. J., Biol. Zentralbl. 55:178-182 (1935). Luminous Collembola.

62. Takagi, S., Proc. Imp. Acad. 9:651-65+ (1933). Luminescence in Watasenia.

63. Takagl, S., Annot. Zool. Japan. 15:3+4-3+9 (1936). Cypridina light organ.

64. Tenao, A., Annot. Zool. Japan. 9:299-316 (1917). Luminescence in Sergestes.

65. Trojax, E., Arch. Microscop. Anat. 75:473-517 (1910). Luminescence in Phyllirrhoe.

66. Wheller, W'. M., and Williams, F. X., Psyche 22:36-43 (1915). Luminous nephridia in Boletophila. 


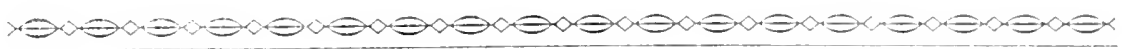

\section{Chromatophores and Color Change}

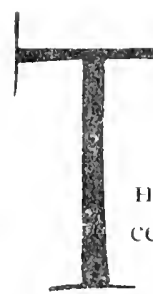

\%

\section{INTRODUCTION}

HE ABIJTY TO CHANGE color through movements of pignents within certain integumentary cells or organs is widely distributed among animals. It has been observed for numerous fishes, amphibians, and reptiles among the vertebrates; among the invertebrates it is exhibited by many higher crustaceans, cephalopods, and leeches, and a fow insects, echioderms, and polychatetes. A comparable activity has been described for a cuglenoid protozoan. The spectacular color changes of the chancleon between black and green and the rapid color changes of the extopus were described as early as the fourth century B.C. by Aristotle, and those of tishes were described somewhat later by Plins, who observed the changes of the ding nullet. The first changes recorded in amplubians were in the frog, and those in crustaceans were in the prawn, Hippolyte. ${ }^{100} 7$ he relatively rapid color changes in the cephalopods were early demonstrated to be due principally to the activity of special organs in the skin, to which the name cromofora was given. Laiter, the movements of pigments in special integu mentary organs were shown clearly a account for color changes in the

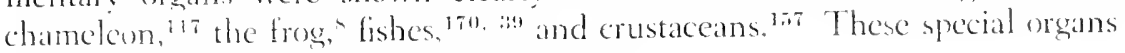
biace come to be known as chromatephores.

Bruche: made studies on the physiolugy of color change in the chameteon;

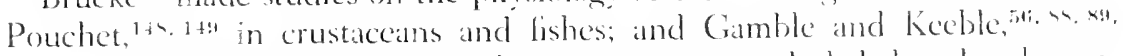
?u, in crustaceans. All these carly investigitors concluded that the chroma tophore systems were under the control of the nersous sistem or respended directly to the action of environmental stimuli on the chromatophores. The possibility of a role of hormones in color changes was sugesested forst hy the disconcry that the injection of adrenalin blanched frogs. ${ }^{10}$ The early work on chromatophores has been thorought revicwed in the extensive accounts of ran hyberk ins and fuchs it Latter erencral summaries include these of

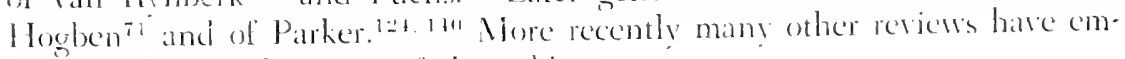
phasized restricted aspects of the subject.

\section{CHROMATOPHORES: STRUCTURE AND METHODS OF ACTION}

Chromatephores are special pignent celts lixated in the shin ere often eren in certain deeper tissuces of the bod! of an animal. Chromatophores persess the ability to bring about redistributions of their pigment in such a manner as to influence the gencral coloration of the animal. I pigment that is concentrated inte a small ball (punctate) contributes little or nothing to the gross coloration of the individual, whereas its dispersion to corer a larger 
unface reviculate results in its imparting its thint to the animal. The fore genge modranism of action concontration and dispersiom of pigment) is

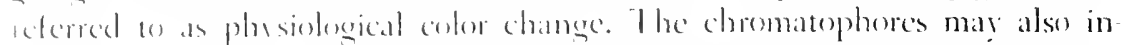
Hucence the coldestion of an animal through their accumulation or production of piement of their loss a destruction of it. This mechanism af action is hmown a morpholenical color chamege.

Physiological Color Changes. Chromatophores are of two major types.

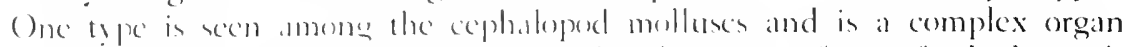

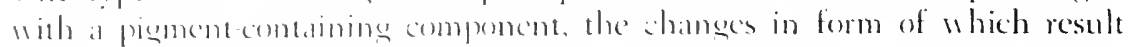

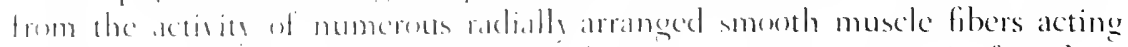

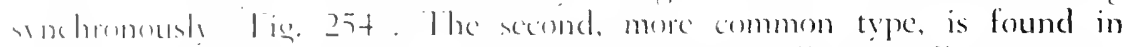

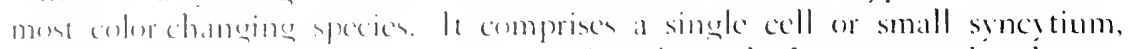
andall, of highl branched outline, and within which pigment distribution

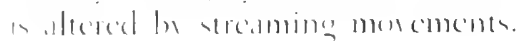
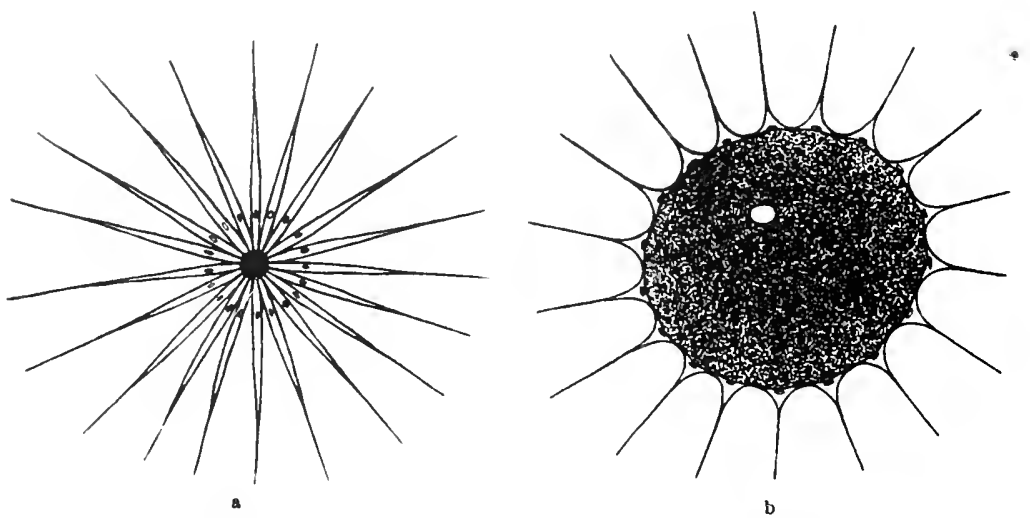

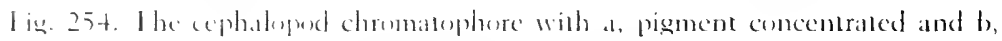
pisment dieperaed. Irom bisker."

Ihe chromentophores of the cephalopects are highly organized groups of

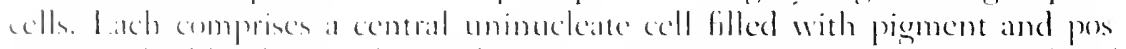
essing a hishl! chatic coll membrane." Radiating out from the central cell in the plane of the skin are from o to 20 or more minucleate smoothmuncle fibers. Ill the tilers of a chromatophere ustally contract simultane

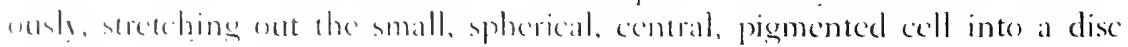

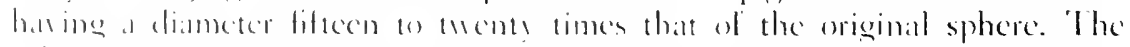
spherical form is restered by the clisticity of the membranc of the central

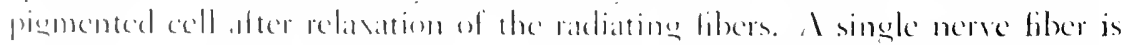

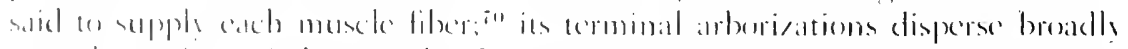

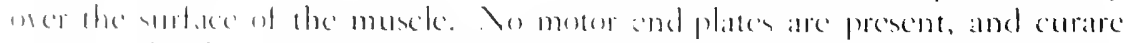

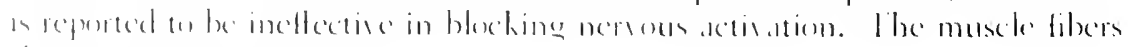

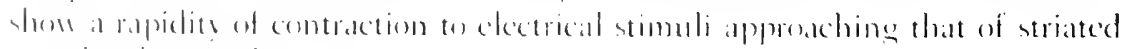

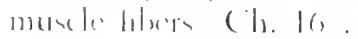

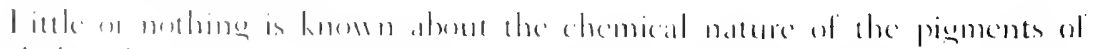

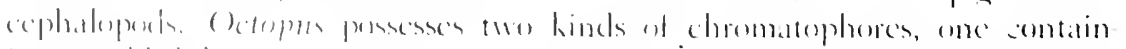

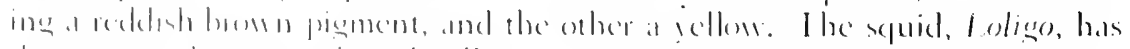

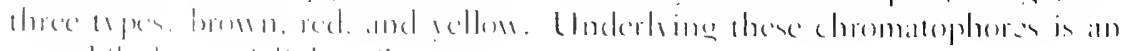

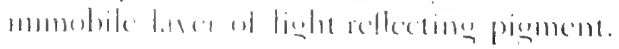


By means of their special type of chromatophores the cephalopods are able to show more rapid color changes than other animils.

In animals other than the cephalopods, the chromatophores are single call (e.g., in most vertebrates) or closely associated groups of cells of $41 \mathrm{~m}$ !ti. (e.g., in crustaceans). These were once thought to be amsdreid c(clls. (on

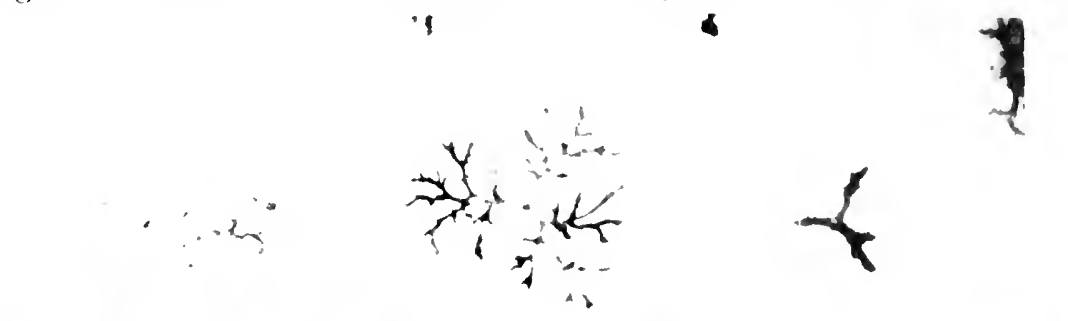

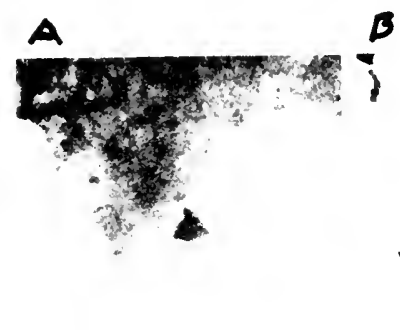

D

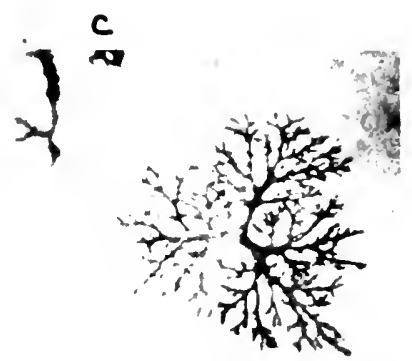

$\boldsymbol{F}$

Fig. 255. A series of photegraphs of a single white chromutophore of Poluemome as a its pigment fully concentrates in response to a black background, and then re dipecter on return of the animal to d white background. From Brown."

traction of whose processes resulted in a concentration of the pigment mas into a small sphere, and whose extensive pseudopodial production resuled in a broad dispersal of the pigments. Nom it is eenerall! comsidered that the chromatophore has a permanently arborizing form, and that the piement granules either become concentrated inter the chromatophore center to form i punctate mass or become dispersed to buring kederes through the in tricately branching structure"1:- to impart color to the macroseppic appear ance of the animal. Matthews and others have reported obering branches of chromatophores whose pigment "as in the punctate condition. Mte up porting this view has been the demomstration of the strihing similatity, coen to the minute teminals of a chromatephore, after piement dispersal an $41 \ldots$ different occasions

In typical details of form. pigments, and seactions, howerer. each ani mal, species, or group has its own chromatopheral peculiartice. (hromate phores are known as monochromatic, dichomatic, or polschromatic; a pending on whether they possess ane, two, ar more hinds of pigment. I he crustaceans commonly possess di-or polychromatic chromatophowes, with cach pigment typically dispersing out into it own prexesses and, 11 hen (an) centrated inte the chromatophore conter. possensing its ann dietime indi

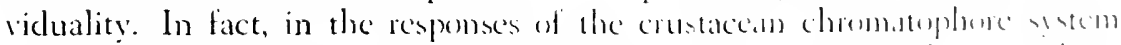

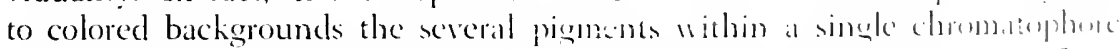
may show a considerable degree of independence of one anther. " " " 
In insects the epidermal cells themselves serve as functional chromatophores. During darkening of the skin the dark brown-black pigment within these cells migrates from small concentrated masses below an evenly dispersed yellow and green pigmented layer to a more superficial position, where it disperses (Fig. 256). The red pigment in skin darkening disperses from small spherical masses to form a continuous sheet of pigment. ${ }^{60}$ Thus the forces which operate in pigment concentration and dispersal in chromatophores in general are apparently of such a nature as can operate within the limits of more conventionally shaped cells. Migration of a red hematochrome pigment in Euglena, from a deeper position in the body internal to the chloroplasts to a dispersed, superficial position, results in a green to red color change in response to elevated light intensity. ${ }^{84,85}$

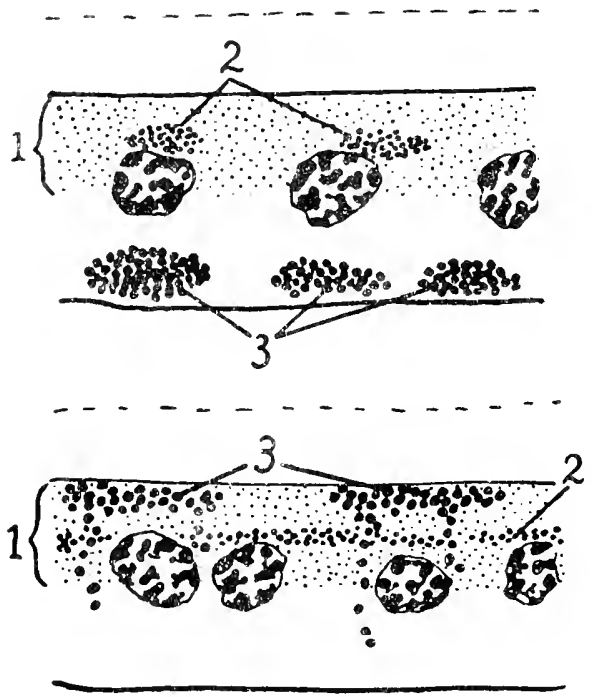

Fig. 256. Diagrammatic sections thru the epidermis of light (upper) and dark (lower) adapted Dixippus. The coarsest stippling (3) indicates brown-black pigment; the intermediate stippling (2), red pigment; and the finest (1), yellow-green pigment. From Giersberg..$^{58}$

In and associated with the chromatophores of crustaceans are a number of different colors of pigments. The kinds which are present vary with the species and even occasionally among individuals within the same species having different histories. Yellow and red fat-soluble pigments appear to be of quite general occurrence within the group. These are carotene derivatives. ${ }^{44 .}{ }^{103}$ Also of quite general presence is a reflecting white pigment which has been described as guanin. The majority of the macruran decapods possess a transparent blue pigment, which is a carotenoid conjugated with a protein (carotenalbumin). ${ }^{1.6 *}$ In fact the application of heat or alcohol to integument containing the blue pigment results in its rapid transformation from a water-soluble blue to a fat-soluble red pigment. Finally, in certain crusta. ceans a black or brownish-black pigment is present. This pigment, a melanin, is found in Crago among the macrurans, in many true crabs, and in many isopods. 
The chromatophores of the vertebrates in general resemble those of crustaceans. Unlike the latter, however, they usually comprise single cells and are for the most part monochromatic. The predominant pigment is melanin, and it is the activity of the melanophores which is principally responsible for the conspicuous color changes in this group. In many vertebrates reflecting white chromatophores, the guanophores or leucophores are also found. Chromatophores known as lipophores, containing fat-soluble red pigment (erythrophores) or yellow pigment (xanthophores), are often present. These pigments are commonly xanthophyll or derivatives of it. ${ }^{47}$ In addition to these more conventional types of pigment cells, there are sometimes glistening bluish-green bodies, the iridocytes, whose color and color changes are structural ones dependent on the form, arrangement, and movements of fine, platelike crystals.

A
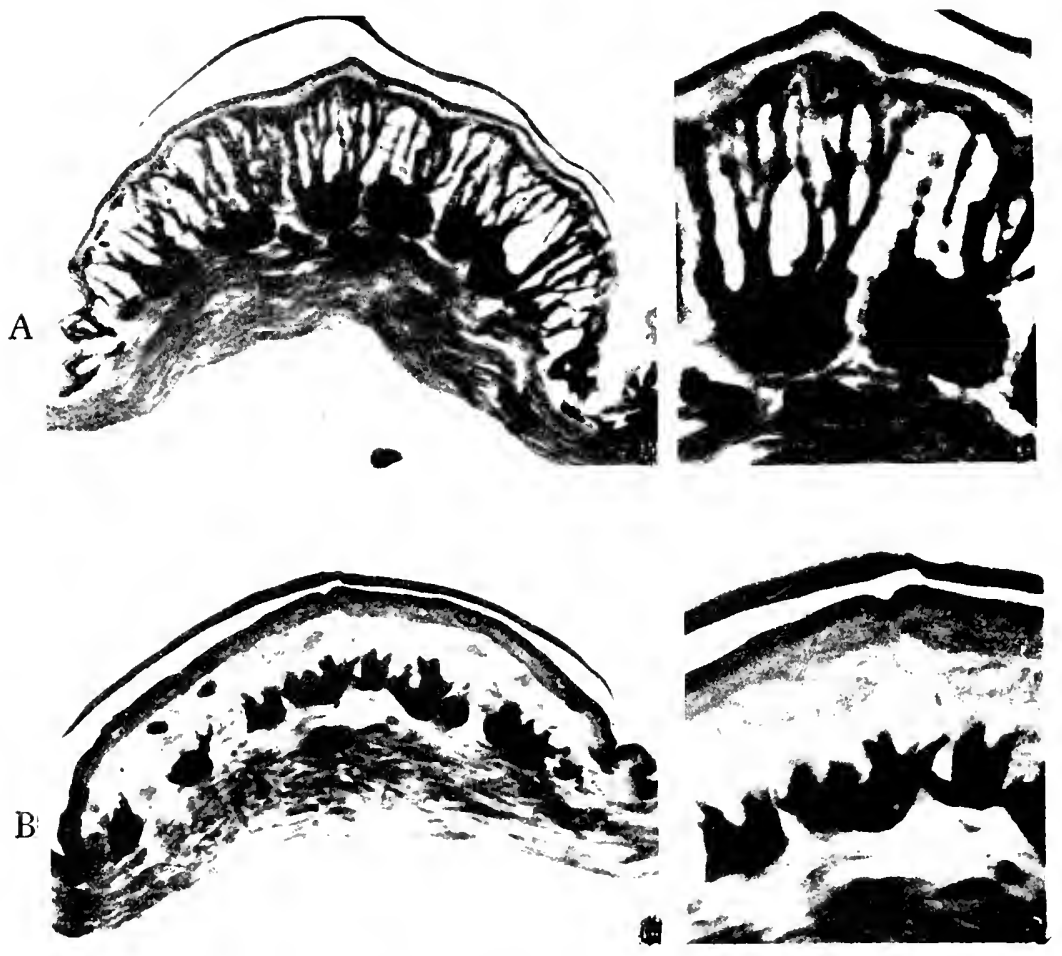

Fig. 257. Sections through scales of Anolis showing the condition of the chromatophores, $A$, in the brown state and $B$, in the green state. From Kleinholz. ${ }^{93}$

Supplementing the contribution of the chromatophores themselves to the coloration of the vertebrate there is often, as in the cephalopods, an immobile layer of whitish or yellowish pigment. This pigment is responsible entircly for the tint of the animal when the active chromatophores contribute little or nothing, or it cooperates with the chromatophores in producing the normal coloration. In species such as the lizard, Anolis, the bodies of the melanophores lie beneath such a passive layer of pigment. As the animal darkens in response to appropriate stimulation the melanin streams within melano- 
phore processes to a position superficial to the inert light-colored laver, thereby concealing the latteris. ${ }^{93}$ (Fig. 257).

The rate of physiological color change is limited by the rates of mechanical response of the effector organ and of its controlling mechanism. There is, however, great variation in such rates among animals. The squid is able to carry out maximum color change in a matter of seconds, as is also the squirrel-fish, Holocentrus. A few minutes suffice for maximum color change in the minnows, Fundulus and Lebistes. From one to several hours are needed by many crustaceans, insects, and the catfish, Ameiurus, and days are required for comparable maximum changes in the Hatishes, the eel, Anguillula, elasmobranchs, and amphibians.

A number of methods have been utilized in the measurements of physiological color changes. These have been critically reviewed by Parker. ${ }^{139}$ None of the methods permit complete differentiation between infuences which are in part the result of the morphological color changes and those which are purely physiological. Instead, the measurements are generally based on

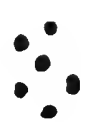

10

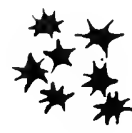

$2 \cdot 0$

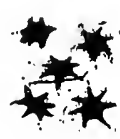

$3 \cdot 0$

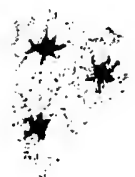

$4 \cdot 0$

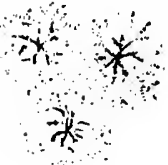

$5 \cdot 0$

Fig. 258. Melanophores showing various degrees of pigment dispersion. 1, Punctate; 2, punctostellate; 3 , stellate; 4 , reticulostellate; 5 , reticulate. From Hogben and Slome. ${ }^{74}$

time intervals of sufficient brevity to assure that morphological changes would not have influenced the results significantly. One group of methods employs simply the gross changes in color of the animal as an index of the extent of dispersion of the dark pigments. This may involve a visual deter mination in which the animal is merely described as being light, dark, or intermediate, or in which subjective grades of variation between known extremes are estimated and expressed numerically ${ }^{28}$ in four or five grades. Some of the subjective aspects have been removed by a method employing photometric determination of the fraction of the incident light reflected from a unit area of skin surface, ${ }^{\text {is }}$ or the relative amounts of light transmitted by isolated fish scales. ${ }^{175}$

$\lambda$ second group of methods has been based, not on the gross light ab sorptive changes in the skin, but rather on the changes in the chromatophores themselves. One of these methods was measurement of the actual cliameter changes in the individual chromatophores. This method was employed originally by Spaeth ${ }^{1 \times 1}$ for fish melanophores and has since also been applied to crustaceans." $: 3$ Another method of this type, and one rather extensively adopted, is one originally proposed by Slome and Hogben ${ }^{172}$ (Fig. 258), in which the chromatophore state was numerically described as folhows:

$1=$ Punctate; $2=$ Punctostellate; $3=$ Stellate; $4=$ Reticulo-stellate; 5 = Reticulate. This sistem has the advantage that quick inspection can, after a little practice, yield a numerical value which is adequate for many comparative purposes. 
In conjunction with both of the preceding groups of methods, technics have often involved photomicrographer or the rapid often heat fir.tin of the animal for more leisurely analy sis hiter, using temperatis or perman cont skin preparations.

Morphological Color Changes. Morpholegical color chances invele the accumulation or chemical production or the destruction of pioneme usihin the chromatophores. In the nomal adaptive color changes of animats. phe siological and morphological coter changes proced simultamenusly. The

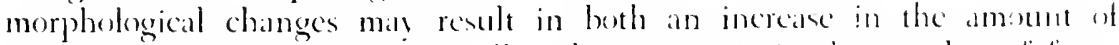
pigment within each pigment ectl and an increase in the number of funce

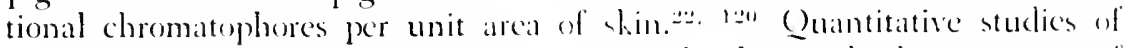
morphological color changes have correspondingly involved two types of technies: (1) the determination of changes in the number of functionil chromatophores per unit area of surface, and (2. the detemination of changes in the total pigment content of the animals by chemical cintrac-
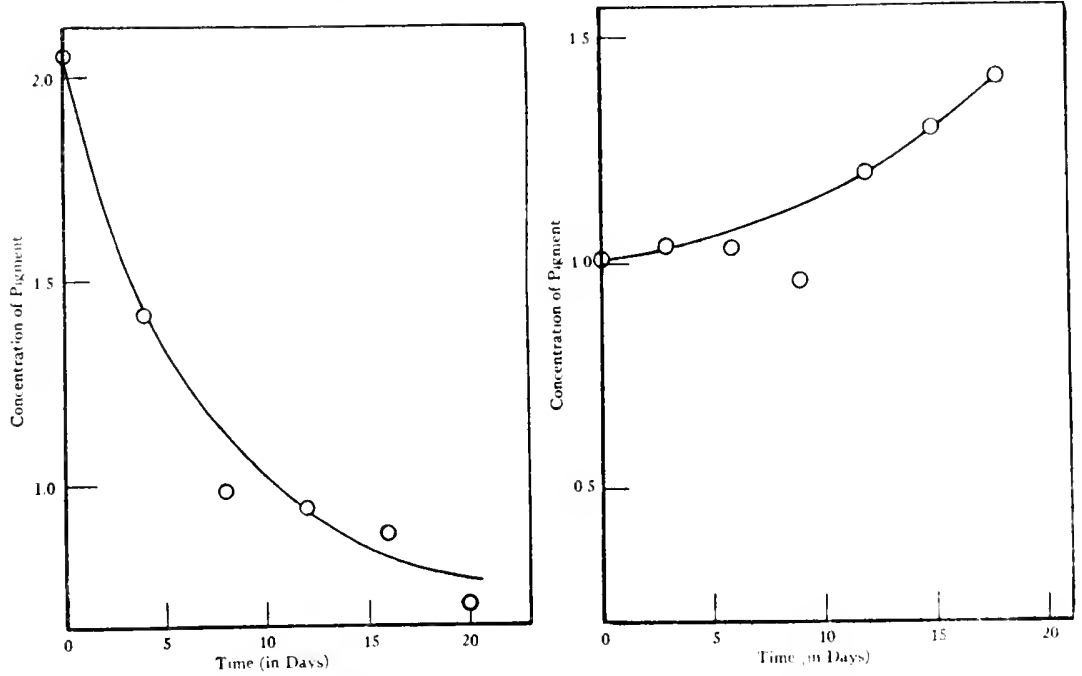

Fig. 259. A, Graph showing rate of loss of red pigment from the bodies of Paluemoneter kept on a white background. B, Graph showing increase in puantity of red pisment in the bodies of animals kept on a black background. From Brown.:-

tion and colorimetric determinations of pigment quantity in the extruts ${ }^{\text {in }}$ Fig. 259). This whole subject has been ably revieved for vertebrater by Sumner, ${ }^{\text {Nit }}$ and in general the situation among invertebrates secms in pre sent a quite similar picture.

There appears to be a close functional relationship between phesiolugical and morpholugical color changes. A corretation between the tus has bee noted by man! investigators."9io. 1" Maintained concentration of a pismest within a chromatophore secms usuatly to be correlated with a relution in quantity of that pigment, and, concersely, piement dispersion uppears asso ciated with the pigment production. The expression of this relationship has sometimes been referred to as Babah's law.': It woukd appear either (1 thit pigment formation or destruction resulted from the state of dispersion of the 
pigment, or (2) that both physiological and morphological color changes were effected in a parallel manner by the same controlling mechanisms. The latter idea seems to be more prevalent, especially in view of the fact that the process may even involve the production of new chromatophores.

There is some reason for postulating that morphological color changes are more primitive than the physiological changes. Color changes by this method appear to occur in numerous species which are known to show no physiological colur changes, as for example in certain spiders ${ }^{55}$ and insect larvae. ${ }^{150}$

\section{COLOR CHANGES IN ANIMALS}

The chromatophore system of animals is influenced by a number of factors. We shall discuss briefly some of the more prominent of these.

Temperature. Temperature appears to have little influence on the coloration of animals. Within wide limits of normal temperatures, the coloration is determined by other factors. Elevation of the temperature of the reptile into the $35-45^{\circ} \mathrm{C}$. range, however, usually results in a contraction of the dark pigment, with consequent lightening of the animal. ${ }^{134}$ Low temperatures, of about 1 to $5^{\circ} \mathrm{C}$., usually induce darkening. Among the invertebrates, on the other hand, the situation is not as uniform. The shrimp, Macrobrachinum, darkens at both high and low temperatures, ${ }^{173}$ as does also the isopod, Idothea. ${ }^{115}$ Hippolyte, on the other hand, has been described as blanching at high and low temperatures. ${ }^{5 t i}$

Humidity. Color changes in the walking-stick Dixiputs are influenced by change in humidity. High humidity induces darkening. ${ }^{58}$

Tactile Stimulation. In general, tactile stimulation seems to have only little influence on the chromatophore system. It was once believed that the color changes of the tree-frog were to a great extent response to the texture of the background to which it was attached, the frog becoming dark on a rough background and green on a smooth one, but it is now generally agreed that this is not the case. Tactile stimulation of the suctorial discs of certain cephalopods has been reported to influence the chromatophores reflexly. ${ }^{183}$ Schlieper ${ }^{161}$ has reported that $H_{y}$ peria galba, a crustacean parasite on jellyfishes, becomes pale when normally attached to its host but darkens when swimming frccly. Attachment to any surface, whether black or white, is said to induce in this parasite the paling response which is obviously adaptive, since it usually becomes attached only to its highly transparent normal host.

Psychical Stimuli. The chromatophores of some animals appear to be influenced by psychic states. An excited squid or cuttlefish shows extraordinary plays of color. Such color plays may be caused by the presence of a predator, such as a large crab. The changes often take the form of waves of change passing smoothly and rapidly over the surface of the body. Color plays also frequently appear to contribute in some manner to mating behavior in these animals.

Reptiles also show characteristic color changes when excited. The hornedtoad, Phrynosoma, on strong excitement exhibits a blanching known as "excitement pallor." Anolis when going into combat with another or when manipulated roughly show's a peculiar change of coloration to a mottled condi. tion. ${ }^{93}$

Light. By far the most important single environmental factor influencing 
the state of the chromatophore system of animals is light, and in the great majority of animals the method of action of the light insolves principally the eves, central uervous system, and various types of efferent pathwals, ner vous, hormonal, or both. The importance of the eyes is ctearly manifested in observing the immediate cessation, or great change in character, of color changes on the blinding of the animal. Color clanges which are controlled by way of the eyes are known as secondary response's. in contrast with primary responses, which are those proceeding under the influence of light through routes other than the eyes. The latter may insole either a direct action of light on the chromatophores or an influence of light operating reflexly on the chromatophores through extra-ocular receptor mechanisms.

Secondary coler changes dominate the situation in most actult animals. Through a wide range of light intensities these changes do not result from changes in the total quantity of light striking the eye, but rather they are determined by the values of the ratios of the amount of light directly striking the exe from above to the amount of light reflected from the background on which the organism resides. On an illuminated black background where

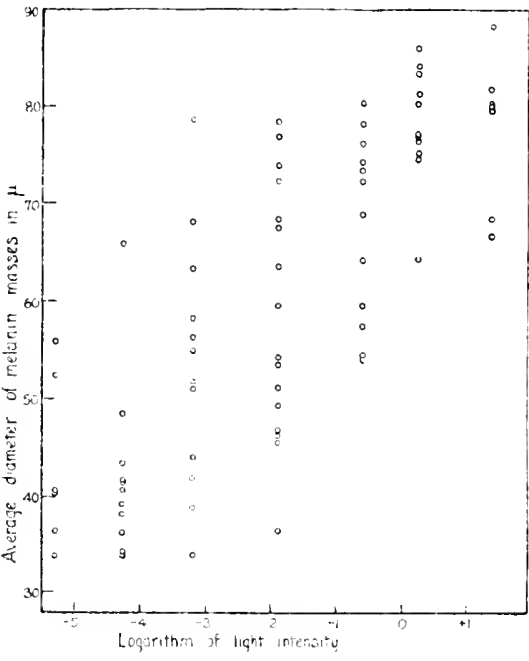

A

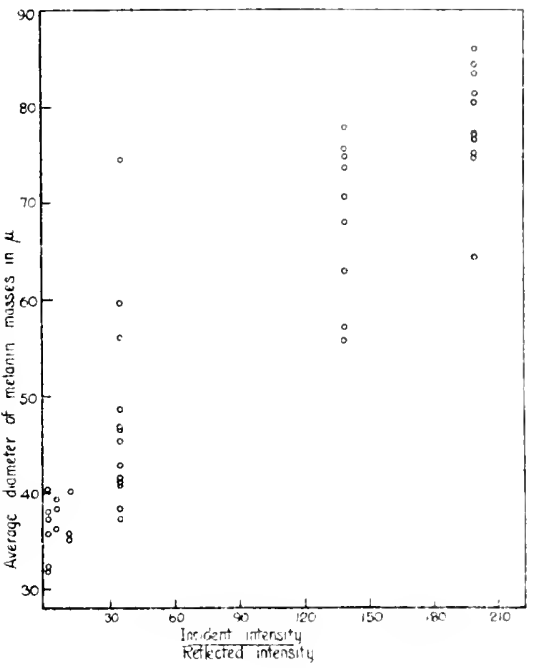

B

Fig. 260. A, Relationship between the $\log$ of the incident light intensity and the average diameter of the melanophores of the minnow, Ericymba. B, Relationship between the ratio of inciden intensity to reflected intensity striking the eye and the arorage melanophore diameter. From Brown. ${ }^{2 .}$

the ratio is large, the animal becomes dark, and on an illuminated white background where the ratio is small, the animal becomes pale, irrespective of the total illumination. It can be shown that there is a good direct correlation between the value of the ratio of incident light to reflected light reaching the eye of the animal and the degree of black pigment dispersion ${ }^{2.5}{ }^{191}$ (Fig. $260, B$ ) or the amount of melanin formed in melanophores, ${ }^{146,}$, 1.96 and, furthermore, that neither of these melanophore responses varies significantly with variation in intensity within a wide range of total illumination. 
Adaptive morphological color changes in response to background have also been reported for the chromatophores of crustaceans ${ }^{1.5}, \cdots 2$ and for melanophores, ${ }^{1 n}$ lipophores, ${ }^{2}$ and guanophores ${ }^{1 \mathrm{~km}}$ of a number of fishes. Functional melanophores have been induced to form on the normally unpigmented ventral surface of flounders either by placing normal fish in black tanks illuminated from below, or by blinding the animals and illuminating them from below. 1 -

The influence of a black background may be simulated in many animals by opaquing the lower half of the eye. This has been demonstrated for the walhing-stick, Dixippus, ${ }^{10}, 1.11$ the shrimps, Palaemonetes ${ }^{6.5}$ and Leander, and teleost fishes. ${ }^{40}$

The character of the influence of light in the secondary color responses of animals is obviously one especially adapted to provide the animal with a certain degree of protective or obliterative coloration with respect to its background. As might be anticipated, the function appears in general better dereloped in animals which are bottom dwellers, or which spend much of their time quietly attached to objects, than in forms which are more freely mobile.

The adaptation to color of background is in many instances by no means restricted to simple blanching and darkening on white and black backgrounds, respectively. Adaptations of the prawn, Hippolyte, to many colors and tints of background were described many years ago. ${ }^{\mathrm{sx}, 11 \times}$ More recently it has been shown that Crago will change its coloration to match red and vellow backgrounds, in addition to black and white."7 Palaemonetes has been shown to change its color within a few days to accord with black, white red, vellow, blue, or green backgrounds.":" The crab, Portunus ordwayi, adapts to black, white, red, and vellow, ${ }^{2}$ as does also Planes." The cephatopod, $\mathrm{Sc}_{c}$. pia, becomes grẹen on a green background and yellow on a yellow one. ${ }^{102}$

Some of the most striking examples of color adaptation in vertebrates are seen in flatfishes. The changes in the flounder Paralichthys albiguttus on red, green, yellow, and blue backgrounds have been described. ${ }^{104}$. 110 This fish, moreover, is able to simulate rather strikingly the color pattern of the background, thus rendering its protective coloration even more effective. Many other teleosts and amphibians are able to show yellow tints in response to vellowish backgrounds.

These adaptations to color of background are the results of appropriate differential movements of the various pigments within the chromatophores, supplemented by morphological color changes to reinforce these physiological ones. In these activities the animal may show the capacity for inducing changes in the distributions or amounts of its various pigment types more or less independently of one another, thus indicating complex mechanisms of chromatophore control. Species may be limited in the colors of background to which they can adapt themselves, through lack of suitably colored pigments. For example, Crago lacks blue pigment and hence is unable to adapt to either blue or green. Palaemonetes, possessing red, yellow, and blue pigments, can, by appropriate pigment manipulation, become almost any color, including black. On the other hand, some species possess pigments of particular colors yet appear to show no ability to adapt to backgrounds 
of those colors. An example of this is seen in Dixippus, which is apparently unable to become reddish, despite the possession of a red pigment in its integument.

It is obvious, from the foregoing descriptions of coloradaptive abilits that the function of the eyes in their association with the chromatic mechan ism is differentiated not only in the dorsal and ientral portions but allos to the extent that lights of different wave lengths are distinguished by that por tion of the retina stimulated by the reflected light to produce specific chroma tophore activities.

The primary color responses of most adult animals are moderatels weil subordinated to the secondary. In the typical adult organism they are best made evident through blinding the animal. Many animals are able to show considerable dispersion and concentration of their pigments in light and darkness respectively, after blinding, but there is no longer any response to the background. Examples of this latter behavior have been described within all the major animal groups which exhibit color changes. The degree of in Huence of the primary responses, relative to the secondary responses, appears to vary from species to species or even among the various pigment type's within a single species.

The most general mechanism of primary response is one of a direct influ ence of light on the chromatophores. In this response the chromatophore acts as an independent effector. Primary responses of this character have been observed in (1) vertebrate chromatophores after complete denerration of the pigment cells resulting from nerve transection and degeneration, (2) localized light responses in species whose chromatophores are normally not innervated, and (3) in young specimens whose chromatophores have not ret come under the control of a typical secondary mechanism.

Color changes in response to light may be reflexly induced in the absence of eves. It has been shown for Phoxinus that the midbrain is a receptive mechanism for this response. ${ }^{1-i x}$ The pineal body has been reported to be a receptor organ in the pigmentary response of Lampetra larvae, 203 with the eyes dominating this response in the adult.

The majority of adult animals showing color change have their coloration correlated within wide ranges of illumination with background color rather than with general intensity of illumination. In the total absence of light there is typically a blanching of the animal, but of ten not to the extent ob served in response to an illuminated white background. Some species, such as Crago and Xenopus, become intermediate in shade through partial dis persion of their melanin. It has been shown for the minnow, Ericymba, that upon a black background there is no influence of amount of illumination on the coloration as long as the illumination is higher than 1.75 foot candles; at illuminations below this value the average diameter of the melanophores is a linear function of the logarithm of the incident light, down to 0.00053 foot candles, which has the same influence as complete darkness ${ }^{2-5}$ (Fig 260, A).

Diurnal Rhythms. Another important factor operating in the control of chromatophores in many animals is a persisting diurnal rhythm. Many species of animals continue to show their characteristic night-day color changes 
even when kept under constant conditions as regards illumination, especially in constant darkness. Such rhythms may persist for considerable lengths of time. Idothea kept in total darkness has shown the rhithm to persist at least 8 or 9 weeks. ${ }^{11 .}$ In $U_{c a}$, the diurnal rhythm whercby the animals pale by night and darken by day. 36 is so strong that only slight color change is induced by variation in background or illumination. 33 Some interesting characteristics of the rhythmical mechanism controlling $U_{c a}$ chromatophores have been reported. 3 tia, 19:a Similar rhythms have been found among vertebrates such as Lampetra, ${ }^{2}: 3$ Phoxinus, salamander larvae, frogs, Plirynosonata, ${ }^{1, i t}$ and the chamcleon. ${ }^{20 .}$

In some instances where the diurnal changes are normally masked by responses to illuminated backgrounds, an underlying influence of the rhythm may be evident in an increased rapidity of those responses which tend to support, and the sluggishness of those which tend to antagonize, the particular phase of the rhythm at that moment. Thus many animals more readily adapt to an illuminated white background during the night phase and to an illuminated black one during the day phase, or show corresponding differing susceptibilities to injections of chromatophorotropins in the night and day phases.

\section{FUNCTIONAL ORGANIZATION OF CHROMATOPHORE SYSTEMS}

Annelids. Certain annelids become pale in darkness and dark when illuminated. This has been observed for the polychacte, Nereis dumerilii, ${ }^{67}$ and the lecches Piscicola geometra, ${ }^{\times 2}$." Protoclepsis tessellata, ${ }^{184}$ Hemiclepsis marginata, ${ }^{\times 2}, \times 3$ Glossiphonio complanata, ${ }^{\times 2}, \times 3,1: 1$, and Placobdella parasitica and P. rugosa. ${ }^{179}$ In no case has there been demonstrated any response to color of the background.

The rhynchobdellid leech, Placohdella parasitica, a common parasite on turtles of the central United States, possesses three types of pigment cells. These pigments contribute to the mottled brownish and white coloration shown by these animals. The amount of white varies considerably from specimen to specimen, ranging from the greater part of the dorsal surface to a few minute lateral papillae and a short median line, both restricted to the anterior half of the animal. One type of pigment cell containing a pale yellowish, granular pigment occupies the characteristic longitudinal mid. dorsal stripe, the numerous light papillae, and segmental blocks along the margin. These cells show no physiological responses. Distributed over all the darker areas of the body are relatively large chromatophores containing dark greenish pigment. This latter pigment participates in physiological color changes. Another pigment, reddish brown, is also located within functional chromatophores. These last chromatophores are much smaller than the greenish oncs and are located more superficially. The green pigment is alcohol-soluble; the reddish brown pigment is alcohol-insoluble. The Europian leceh, Protoclepsis tesselata, possesses three pigments: brown, green, and yellow. The Mediterranean polychaete, Nereis dumerlii, possesses chro. matophores containing yellow and violet pigments.

The lech Placobdella parasitica, like other annelids showing physiological color chinges, blanches in darkness and darkens when illuminated. The time required for the greenish pigments, which are predominant in these 
changes, to complete their concentration in darkness or dispersion in light is approximately one bour.

There are several types of evidence all pointing to nervous control of the green chromatophores. Decapitation or any other transection or injury of the nervous system results in a darkening of pale animals kept in darkness. ${ }^{17: 9}$ This darkening ma! persist for many hours. If a uniformly pale leech in clarkness is stimulated faradically at either the anterior or posterior end the whole anmal darkens. If, however, the experiment is repeated with a specimen whose nerve cord has been transected in the middle, only that half of the animal receiving the stimulus darkens, the body beyond the point of transection remaining light. The experiments lurthermore give little evidence for direct action of light on the chromatophores.

The two exespots at the anterior end appear to play an active but by no means an exclusive role in the responses. Decapitated specimens show the characteristic changes even though responding more sluggishly. When specimens are brought from darkness into light and one half of the body is immediately covered with opaque paper, the covered portion remains largely in the dark-adapted phase while the uncovered portion becomes completely light-adapted. The results are more striking when the posterior end is covered than when the anterior end is. These experiments suggest a role of generally distributed photoreceptors operating through segmental reflexes.

The reddish brown pigment of Placobdella parasitica appears to respond independently of the green and shows no predictable responses to background or to light intensity. Its condition is more or less variable in normal specimens. When dispersed it can usually be made to concentrate by intense stimulation of the animal. In animals with transected cords, the response to electrical stimulation, when it occurs, passes as in the case of the green cells, only to the point of transection. Thus it would appear that the nervous system controls the reddish-brown chromatophores directly, but that excitation induces concentration rather than dispersion, as in the case of the green.

Echinoderms. It was reported many years ago ${ }^{195}$ that the Mediterranean sea-urchins, Arbacia pustulosa and Centrostephanus longispinus, became lighter in color on transfer from light to darkness. These observations have recently been confirmed." Illuminated Arbacia pustulosa are blackish in color whether on a white or on a black background, but in darkness become brown in color. Centrostephanus longispinus, which are dark purple in light, change in darkness to gray. The color changes require about one or two hours for their completion. Microscopic examination of tube feet removed from light-adapted and dark-adapted individuals show numerous reddish brown chromatophores with their pigment dispersed in the light-adapted and concentrated in the dark-adapted individuals. The color changes in these urchins therefore appear to be due to the movements of pigments within definite chromatophores. The chromatophores of isolated tube feet which have been mounted on a microscope slide respond to illumination and darkness in the same manner as when they are present in the intact animal, indicating that the chromatophores are responding to the light either directly or by way of local reflex pathways comparable to those known to function in the locomotor movements of the tube feet. 
The American species of Arbacia. Arbacia punctuiata, appears to show no color changes comparable to those just described. ${ }^{1: 5}$

Cephalopods. Many cephalopods show remarkably rapid color changes as a result of the activity of their peculiar type of chromatophores. These changes may result from many different types of stimuli, but light is one of the most important.

The chromatophores are primarily controlled by the nervous system. Cutting a nerve innervating a particular region of the body results in an immediate cessation of all color changes in that region. It was shown long ago that after a mantle connective is cut ${ }^{t *}$ there is a paralysis of the chromatophores of the corresponding half of the body and a consequent blanched coloration. Since the connectives between mantle ganglion and the chromatophores are still intact it is obvious that the ganglion contributes little to the control by itself, and that the normal control over the responses resides in higher centers of the nervous system. The cerebral ganglion of the brain appears to possess an inhibitory center for the chromatophore system; ; ${ }^{1+6,147.16 i t}$ after its destruction or inactivation there is tonic expansion of the chromatophores. The inhibitory center is believed to operate through control of a color cen$\operatorname{ter}^{16 i}$ located in the central ganglia, which in turn operates through motor centers found in the subesophageal ganglia. The motor centers each control the chromatophores of the corresponding halves of the body.

The eyes are the chief sense organs influencing the central nervous centers. Bilateral blinding does not eliminate changes of color, but the changes which then occur are in no sense adaptive changes. If only one eye is blinded, the responses of the chromatophores on the corresponding side of the body are diminished.

Another significant source of influence on the color-control centers are the suckers on the arms. If these are all extirpated there is a considerable loss of tone in the chromatophores and hence skin lightening occurs. ${ }^{183}$ Removal of both eyes and suckers, however, does not entirely eliminate the chromatophore responses. After such an operation vigorous stimuli will still result in color changes, probably as a result of stimulation of tactile organs, organs of equilibrium, etc.

The chromatophores of the side of the animal that is lowermost, when the animal is in contact with the substrate, always are more contracted than those of the remainder of the body. ${ }^{165}$ This is not a direct influence of illumination as one might first suspect, for it cannot be reversed by illumination from below instead of above. Rather, it appears to be part of a postural response involving stimulation of tactile receptors reflexly through the central nervous system, resulting in the localized chromatophore contraction.

The substances tyramine and betaine are known to be present in the blood of cephalopods. The former, like adrenalin, increases the tonus of the motor centers, resulting in a darker coloration. ${ }^{166}$ Betaine, on the other hand, like pilocarpine or acetylcholine, appears to decrease the chromatophore tone by stimulation of the inhibitory center. If one transfuses blood from a characteristically darker species, such as Eledone or Octopus macropus, into a lighter $O$. vulgaris, the latter darkens. ${ }^{167}$ Interconnection of the circulatory system of the two will yield comparable results. Tyramine is known to be more concentrated in the blood of these darker species than in that of 
the lighter ones. Surgical removal of the posterior salivary gland, known to be the important source of tyramine in the blood of these animals, results in an increase in paleness and a complete loss of tone of the chromatophores. ${ }^{167}$ Darkening may be induced again by injection of tyra. mine solutions. It thus appears evident that tyramine, and probably betaine as well, function as humoral agents operating to modify the general tone of the chromatophores through the central nervous centers. Furthermore, studies of denervated chromatophores ${ }^{16 i, 16: t}$ suggest that both tyramine and betaine also exert a tone-increasing action on the chromatophores themselves, thus functioning directly as well as indirectly on the chromatophores.

Superimposed upon the slower humoral influence is the more conspicuous nervous mechanism responsible for the rapid color changes. Certain observations have led to the hypothesis that the chromatophores have a double innervation. If an isolated piece of the integument of Loligo containing chromatophores is allowed to stand for some time, the chromatophores first contract and later reach a condition of maintained partial expansion. Electrical stimulation of these latter results in chromatophore contraction. Here, therefore, electrical stimulation appears to act to inhibit the tonus of the chromatophore musculature. ${ }^{16,}{ }^{17}$ On the other hand, single electrical shocks to the chromatophore nerve or to the chromatophores themselves give single twitches of the chromatophore muscle. Repeated shocks give tetanus and consequent chromatophore expansion. Bozler therefore concludes that Loligo chromatophore muscles receive double innervatior, an excitatory and an inhibitory element.

Insects. A number of insects can change their coloration in response to external stimuli, usually as the result of morphological color changes only. Among factors having such effects are temperature, humidity, general activity, and illumination. In only a few species are there the relatively rapid physiological color changes.

Many butterfly pupae are darker or lighter in coloration, depending on whether they are reared at lower or higher temperatures, respectively. The effect of temperature operates in Vanessa through the head, the pupa taking on a coloration which is determined by the temperature of that portion of the body when the head and body are maintained at different temperatures.59 A similar influence of temperature on the degree of development of the dark pig. ments has been observed for the wasp, Habrobracon, ${ }^{\times 6 .}{ }^{16: 2}, 16.3$ and the bug Perillus. ${ }^{9:}$

The colors of Pieris brassicae are due to melanin in the cuticle, white pigment in the hypodermal cells, and green pigment in the deeper tissues. The coloration of these pupae is also influenced by the backgrounds. On black or red background the pupae are grayish-white, whereas on green or orange backgrounds they are clear green, the latter background suppressing formation of the black and white pigments. ${ }^{41} .2:$ Exposing the pupae to colored lights gives the same results as the colored backgrounds. The eves or some other head structure is essential for the response, for it ceases and the ani mals behave as in darkness when the eyes are extirpated or the animal is decapitated. ${ }^{18,19.20}$

The migratory locust, Locusta migratoria, shows a limited ability to adapt its coloration to backgrounds. ${ }^{45}$ Through variations in the quantities of yel- 
lowish and black pigments the locusts may become yellowish-white, brown, or black. The quantity of black pigment formed secins to depend on the ratio of incident to reflected light striking the eyes; the amount of the yellowish pigment appears to depend on the predominant wave lengths of the light present, being formed more rapidly at longer wave lengths (550-660 $\mathrm{m} \mu)$ and less rapidly at shorter ones $(450-500 \mathrm{~m} \mu)$. When this species enters its swarming, migratory phase a skin coloration darker than in the solitary phase is produced. There is some evidence that the darker coloration is a result of the more intense metabolic activity. When migrating locusts are returned to solitary conditions they will regain their lighter color phase, but this color change is delayed if the isolated locust is kept in a constantly excited state. ${ }^{4.5}$

A few insects show color change which can be traced to redistribution of pigments within pigment cells or chromatophores. Corethra shows a rapid physiological color change of its air sacs. ${ }^{10:}$ 'This last is due to the presence of special pigment cells. On a black background the pigment becomes dispersed and the pigment cells are scattered uniformly over the sacs. On a

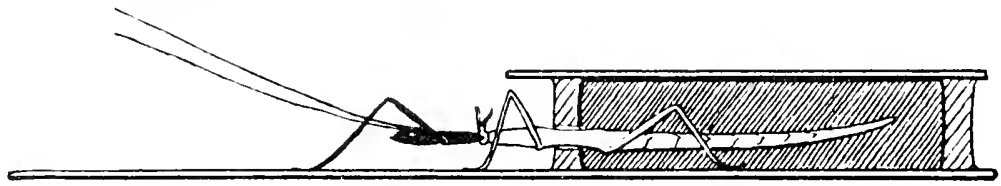

Fig. 261. Diagram illustrating the use of a moist chamber as a stimulus for producing darkening in Dixippus. The darkening in this instance is in response to abdominal stimulation and commences at the head and passes posteriorly only as far as an anterior thoracic ligature. From Giersberg. . $^{\text {th }}$

white background the pigment eoncentrates and the cells appear to wander to one side of the sacs. The eyes are involved in these reactions, as is also the brain.

The color changes of the Phasmid, Dixippus morosus, have been investigated rather extensively. The hypodermal eells of this species contain four pigments, brown (melanin), orange-red and yellow (lipochromes), and green. ${ }^{\circ}, 13: 160$ The brown and orange-red pigments show active concentration and dispersion within the cells in response to external stimuli. The green and yellow pigments show no such activity. Therefore the green varieties found in nature show no physiological color changes, while the brown ones do. Brown specimens are usually dark by night and pale by day, as a result of dispersion and concentration, respectively, of the brown and orangered pigments. A partial independence of a direct influence of light in these changes is indicated by the persistence of typical day-night cycles of color change in animals kept in constant darkness. ${ }^{37,} 159.1600$ It is possible to reverse the rhythm by heeping the animal in illumination by night and in darkness by day, whereupon the newly established rhythm will continue in constant darkness.

Utilizing the fact that high humidity also produces body-darkening, Giersbergin ingeniously proved that the effect of this stimulus on the chromatophores is indirect, operating by way of afferent nervous pathways, the brain, an endocrine source, and finally a bloot-borne agent. When the posterior 
half of a pale Dixippus is inserted into a small moist chamber the whole animal darkens in 30 to 60 minutes (Fig. 261). The darkening commences at the anterior end, which lies outside of the chamber in dry air, and gradu ally spreads backward over the body. Returned to dry air, the animal fightens again in one to two hours. If a ligature is then drawn tightly around the anterior thoracic region and the experiment repeated, the darkening spreads back only as far as the constricted region. If the ventral nerve cord is carefully transected between the subesophageal and the first thoracic ganglia, or between the subesophageal and the supraesophageal ganglia of an otherwise normal pale animal and the experiment repeated, there is no darkening whatsoceer, but if the animal is turned about and the head ininserted into the moist chamber, the head darkens first and the darkening then spreads out over the body in just the same fashion as seen in unoperated specimens. In the ligatured animal the neive pathways were apparently still able to conduct anteriorly the nerve impulses arriving from the nerve endings of the abdominal region which were stimulated by the moisture. A blood-borne hormone was liberated at the anterior end but was unable to diffuse posteriorly past the ligature. After nerve-cord transection the nerve impulses were prevented from reaching the brain and stimulating the liberation of a hormonal substance. Hormone production could still be effected by stimulation of nerve endings anterior to the operated region and then the active principle was free to pass posteriorly in the body fluids.

Further evidence that the physiological color changes in Dixippus are predominantly controlled by hormonal material has come from transplantation of portions of the skin of one animal to another." "The transplanted tissue begins to show color changes entirely paralleling those of the host in 2 or 3 days. It is unlikely that the transplant tissue would have received any in nervation from the host nervous system in such a short period. In fact, normal hypodermal tissue shows no indications of innervation.

The eyes of Dixippus are essential to the normal responses to light." ${ }^{10}$ Section of the optic tracts or blackening of the eye surface stops responses.

Dixippus also shows responses to background when humidity and ilfumination are kept constant. It turns dark-colored on black and red surfaces, and light-colored on white and yellowish ones. The background responses are determined by the ratio of light striking the dorsal and the ventral hatves of the eyes. Painting the lower half of the eves black brings about darkening as on a black background.

Morphological color changes in response to illumination, background, and humidity also occur in Divippus and appear to involve the same mechanism of control as does the physiological change. Such change. however, requires stimulation over a much longer period. ${ }^{10}$, is: lt has been suggested that the substances normally influencing physiological changes so modify the general nutrition and metabolism of the insects as to result in corresponding changes in color by pigment formation or destruction.

Certain mantids also exhibit diumal color changes "is which correspond very closely with the diurnal movements of the retinal pigments in the lateral regions of the compound eye.

Crustaceans. The crustaceans exhibit some of the most remarkable in. stances of color adaptation to be found in the animal kingdom. Most crusta- 
ceans possess within their chromatophores white, red, yellow, and often also black, brown, and blue pigments. By appropriate rearrangements of the individual pigments within the chromatophores many crustaceans are able to approximate rather closely the colors of the backgrounds on which they come to lie.

Although it was believed by all the early investigators that the chromatophores were controlled by nerves, there was never any satisfactory demonstration of nerve terminations at the chromatophores, nor did nerve transection ever appear to interfere directly with the responses of the chromatophores within the animal. Koller, ${ }^{96}, 97$ working with Crago vulgaris, provided the first clear evidence that a blood-borne agent was active in controlling the chromatophores. He found that transfusion of blood from a specimen darkened on a black background into a light animal kept on a white background would cause darkening of the light animal. No evidence for a lightening factor was obtained, however, by the reciprocal transfusion. He also observed that blood from a yellow-adapted specimen would render yellow a white-adapted specimen.

Perkins $^{144}$ discovered that, although denervation of an area of the body of Palaemonetes in no way interfered with the responses of the region when the animal was placed on a black or white background, occlusion of the blood supply to any region resulted in an immediate cessation in the responses of that region. Readmission of blood to the region quickly resulted in a darkening or lightening of the region, to harmonize with the color of the remainder of the body. These results were interpreted to indicate that factors for darkpigment dispersion and concentration were conveyed to the individual chromatophores by way of the blood. Extraction and injection of various parts of the body showed that the eyestalks were found to contain a potent factor for concentrating the predominant red pigment and dispersing the white ${ }^{145}$ in Palaemonetes and hence for blanching the animal. Removal of the eyestalks results in a permanently darkened condition of the animal. These results obtained by Perkins were quickly confirmed in their general principles by Koller, ${ }^{98}$ working with Crago, Leander, and Processa. The hormonal substance involved was shown by reciprocal injection experiments to be neither species-specific nor genus-specific. Since these pioneering efforts, numerous investigators have shown that either the eyestalks or, in a few species, the anterior thoracic region contains the source of a material influencing the state of the chromatophores.

Decapod crustaceans which have been investigated extensively with respect to their eyestalk hormonal activities in color changes appear to fall rather naturally into three groups with respect to roles of the stalks in their chromatics (Fig. 262). Group I contains such genera as Palaemonetes, Penaeus, Hippolyte, Leander, and Cambarus. Their chromatophore systems usually contain red, yellow, blue, and white pigments. Group II includes only the genus Crago, which has a complex pigmentary system with no less than eight differently responding chromatophore types, enabling the shrimp to show not only general shade and tint changes, but also a certain degree of change in color-pattern. The chromatophores contain black, brown, red, yellow, and white pigments. Group III includes all those true crabs (brachyurans) which have been investigated. The best known of these is the fiddler 
crab, Uca, which commonly contains black, red, yellow, and white pigments in the chromatophore system.

After removal of the eyestalks from a member of any one of the three groups, the characteristic type of response for that particular group is observed. In Group I the animals darken rapidly through complete dispersion of their red and yellow pigment and become quite dark (although never as dark as in normal response to a black background) in an hour or two. They remain in this condition indefinitely. The white pigment undergoes a transitory concentration and thereafter exhibits a variable state. Crago, of Group II, most commonly shows a more complex change after eyestalk removal. First there is a transitory darkening of the telson and uropods and a blanch-
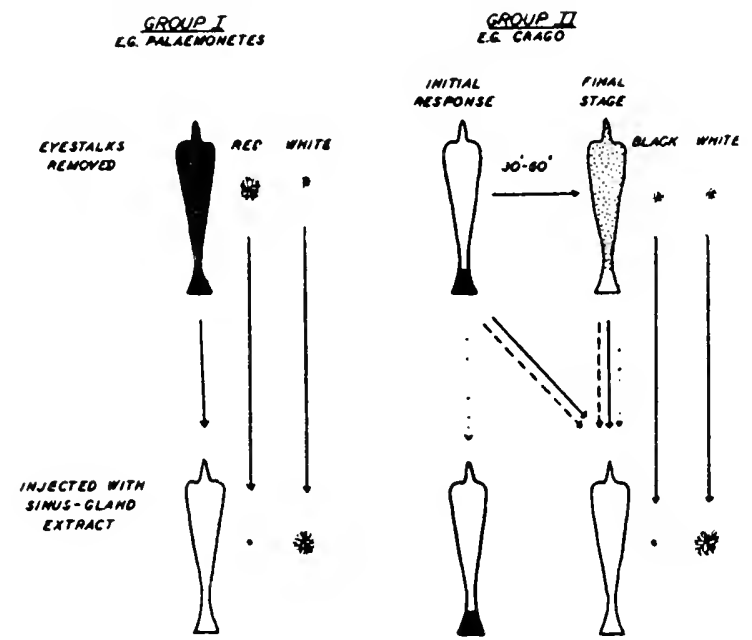

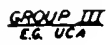

Fig. 262. Schematic representation of the coloration of eyestalkless crustaceans and state of the dominant chromatophores for each of the three differently responding groups, and of the responses of these to injection of eyestalk or sinus gland extract. Solid arrows indicate extract of total water-soluble material, dashed arrows indicate an alcohol-insoluble fraction, and dotted arrows indicate an alcohol-soluble fraction. From Brown, 1948.

ing of the remainder of the body, which lasts from one half to one hour. This is followed by a complete blanching of the telson and uropods and darkening of the body to an intermediate and mottled coloration. The white pigment on the body initially concentrates but then assumes an intermediate condition. The crab, $U_{c a}$, of Group III, blanches rather quickly after removal of its eyestalks, its black chromatophores becoming for the most part punctate, and its white ones commonly broadly reticulate. This condition is maintained without significant change indefinitely. Thus, we see eyestalk removal from various crustaceans resulting in three types of conditions: bodydarkening, adoption of an intermediate coloration, and body-blanching. In all three, the animals lost practically all of their responses to changes in color of background or illumination.

Injection of eyestalk-extract into Group I animals results in a rapid blanching, including dispersal of white pigment. In Group 11 there is complete blanching of both the body and telson and uropods, and also a disper- 
sion of white pigment. In Group IIl, on the other hand, there is a blackening of the whole body, although the white pigment remains broadly dispersed. These strikingly different results observed for the animals of Groups I, II, and III are explained in terms of differences in the responses of the chromatophore systems to the eyestalk material, since reciprocal injections demonstrate that extracts of eyestalks from specimens of other groups produce in the specimen of any given group a response qualitatively the same as that produced by the specimen's own eyestalks. For example, eyestalk extracts prepared from animals of Group III lighten the body of animals of
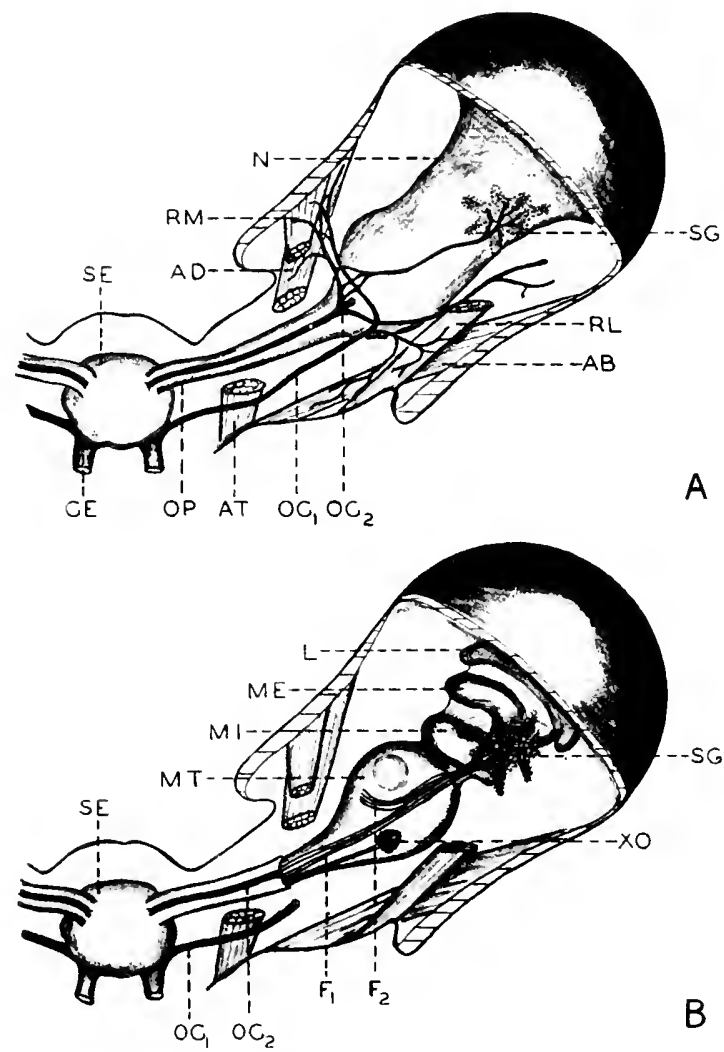

Fig. 263. The sinus gland of Cambarus and its innervation. $A$, Without, and $B$, with sheath of optic ganglia removed. $\mathrm{Sg}$, sinus gland; $N$, Sheath; $S E$, brain; CE, connectives; $O P$, optic nerve; $X O, X$-organ; $O C_{1}$ and $O C_{2}$, oculomotor nerves; $F_{1}$ fiber tract from brain; $F_{2}$, fiber tract from medulla terminalis; $L$, Lamina ganglionaris; $M E$, medulla externa; $M I$, inclulla interna; $M T$, medulla terminalis; $A B, A D, A T, R L$, and $R M$, eye muscles. From Welsh, J. Exp. Zool., 86:35-49 (1941).

Group I from which the eyestalks have been removed, and, conversely, eyestalk extracts prepared from animals of Group I darken eyestalkless specimens of Group) III.

Thu source of the chromatophorotropins from the eyestalk is the sinus gland (Fig. 263). This was postulated and strongly supported by Han- 
ström. ${ }^{64,}$, $: 5$. This gland had been described earlier, ${ }^{63.3 .} 171$ and at that time was called the "blood gland," since it was then believed to be homologous with a blood-forming gland in the evestalks of Crago, which Koller": had crroneously considered to be the source of the active principle. It was later concluded that the glandular source of the active material could not possibly be the "blood gland," and it was renamed the sinus gland. This gland has been found to exist in practically all of numerous malacostracan crustaceans in which it has been sought, hence it is evidently of very general occurrence. I lanström, using evestalkless specimens of $U_{c a}$, Palaemonetes, or Penaeus as animals for bioassay in his numerous expcriments, found that eyestalks of animals whose sinus glands were located in the head near the brain (Gebia and Hippa [Emerita]) showed no chromatophorotropic activity. When the evestalks of other species were sectioned in various ways, the sections possessing the sinus gland always showed activity, other parts were always inactive. By utilizing the species differences in the anatomical arrangement of eycstalk organs, Ilanström was able to get, one by one, all the remaining organs of the eycstalk into portions of the stalk showing inactivity. Furthermore, no other structure in the stalk gave histological evidence of having secretory activity except a glandular organ called the X-organ. Sections containing the $X$-organ, but not the sinus gland, were inactive; and removal of the $X$-organ from eyestalks did not diminish their chromatophorotropic activity. It was thus concluded that the sinus gland was the only eyestalk source of hormones influencing the red or black pigments in the test animals employed.

Hanström's conclusions were confirmed by Brown, ${ }^{27}$ who found that the sinus glands could be removed and extracted by themselves, and that such extracts possessed qualitatively and quantitatively, within experimental error, all the activity of total stalks, despite the fact that their volume was only about 1 per cent of that of the stalk tissue (Fig. 264). Such extracts elicited about 80 per cent of the activity of whole stalks for both Palaemonetes red and U Ua black chromatophores. Thus, for these two widely different chromatophore types, the sinus glands are the sole eyestalk source of hormonal material. Furthermore, implantation of a sinus gland into the ventral abdominal sinus results in a blanching of the animal which lasts about 100 times as long as the effects of an injection of extract that is the equivalent of approximately one gland. It has also been possible to remove the sinus glands from the evestalks of Palaemonetes without substantial damage to other parts of the eyestalk. ${ }^{30}$ Specimens after such removal show broad dispersion of their red pigment irrespective of background color, and extracts later prepared from their eyestalks show no appreciable chromatophorotropic activity.

The sinus glands of the eyestalks provide more than one chromatophorotropin. This has been demonstrated by means of a comparative study of the rclative influences of extracts of the eyestalks of seven genera of crustaceans in concentrating Palaemoneies red pigment and dispersing $U_{C a}$ black pigment. ${ }^{34}$ The ratio of the effect on the two chromatophore types, influence on $U_{\text {ca }}$ Black to influence on Palaemonetes Red, was called the $U / \mathrm{P}$ ratio. The $U / P$ ratios obtained for evestalks or sinus glands from different sources varied from one genus to another. For example, Crago sinus glands showed a 
relatively high value, whereas those of Palaemonetes showed a relatively low value. Uca yielded an intermediate value. The order for the seven species investigated showed no correlation with either the sizes of the animals or the relative potencies as assayed on $U_{c a}$. The hypothesis proposed to explain these data, namely, that sinus glands differed from one another in the proportions of two principles, (1) a factor predominantly darkening Uca (UDH), and (2) a factor predominantly lightening Palaemonetes (PLH), was given strong support by the discovery that the sinus glands of each species yielded two active fractions, one alcohol-soluble and the other alcoholinsoluble. The former gave a very low $\mathrm{U} / \mathrm{P}$ ratio, as if possessing a larger proportion of $\mathrm{PLH}$, and the latter gave a high $\mathrm{U} / \mathrm{P}$ ratio, suggesting a larger proportion of UDH. It was possible to restore the initial $U / \mathrm{P}$ ratio for the gland of a species simply by recombining the two fractions. These results lead to only one possible conclusion, namely, that the eyestalks of all seven species investigated, representing examples of Groups I. II, and III, possess two chromatophorotropins in differing proportions.

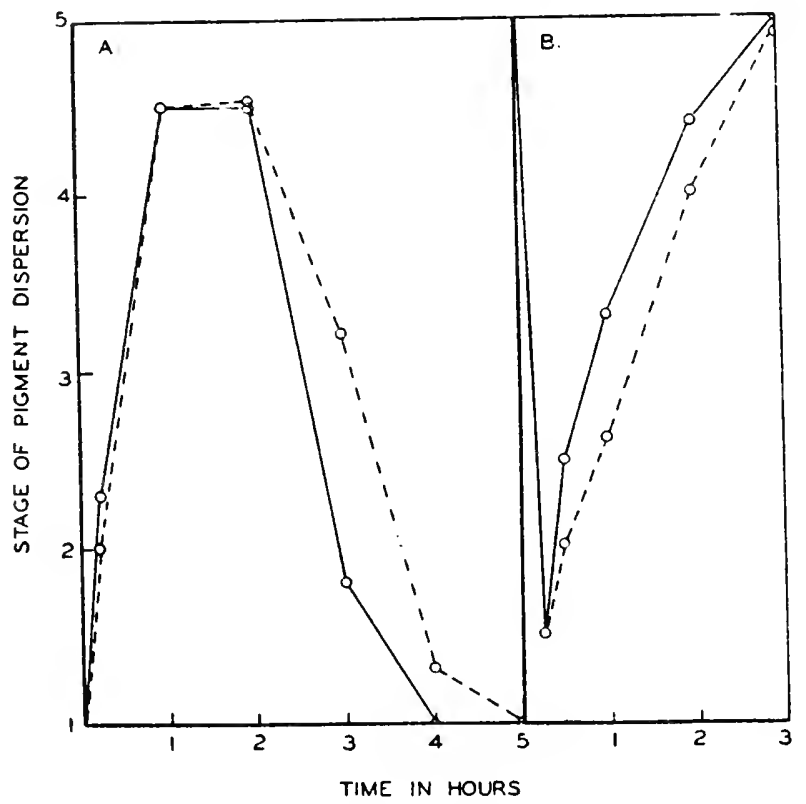

Fig. 264. Change in the average state of black pigment dispersion in eyestalkless $U_{c a}$ (A) and red pigment concentration in eyestalkless Palaemonetes (B), after injection of equal doses of extracts of whole eyestalks (broken line) and extracts of sinus gland (solid line) in equal numbers per unit volume of extracting medium. From Brown ${ }^{27}$

The sinus glands of the crustaceans of Group III so far examined lack one principle present in the glands of species of Groups I and II. This has been established through the discovery that, whereas extracts of the eyestalks or sinus glands of species of Group 1 or Il will lighten the telson and uropods of Crago within three or four minutes after injection, sinus glands of species of group 111 fail to do so. ${ }^{29,37}$ This is so despite the fact that Group III eyestalks are apparently as effective in lightening the bodies of 
Crago as are eyestalks from other groups. This principle which is present in Groups I and II, but absent in Group III, has been called Crago-"tail"lightening hormone (CTLH). The information at hand, although indicating the presence of three chromatophorotropins in crustacean sinus glands, does not permit us to decide between two possibilities: (1) species in Groups I and II possess three principles and those of Group III possess two principles, and (2) all possess only two principles, with one of the two differing in physiological properties between Groups I and II, and Group III.

The sinus glands of the eyestalks are not the sole sites of production of chromatophorotropins in crustaceans. It is well known that undisturbed eyestalkless specimens of Crago not uncommonly exhibit random color changes. Since Koller's original work, it has been found by a number of investigators that eyestalkless crustaceans may be induced to undergo characteristic color changes through the action of blood-borne factors by stimulation with electrical or other means of the cut ends of their optic nerves. Crustaceans of Group I are induced to blanch, but whereas blanching under the influence of sinus gland principles includes white-pigment dispersion, by this means the white concentrates. Members of Group III darken, but here, too, the white concentrates as the black disperses, unlike under sinus gland influence. The responses of Crago of Group II are more obviously complex, and its reactions to electrical stimulation of its optic nerve stubs
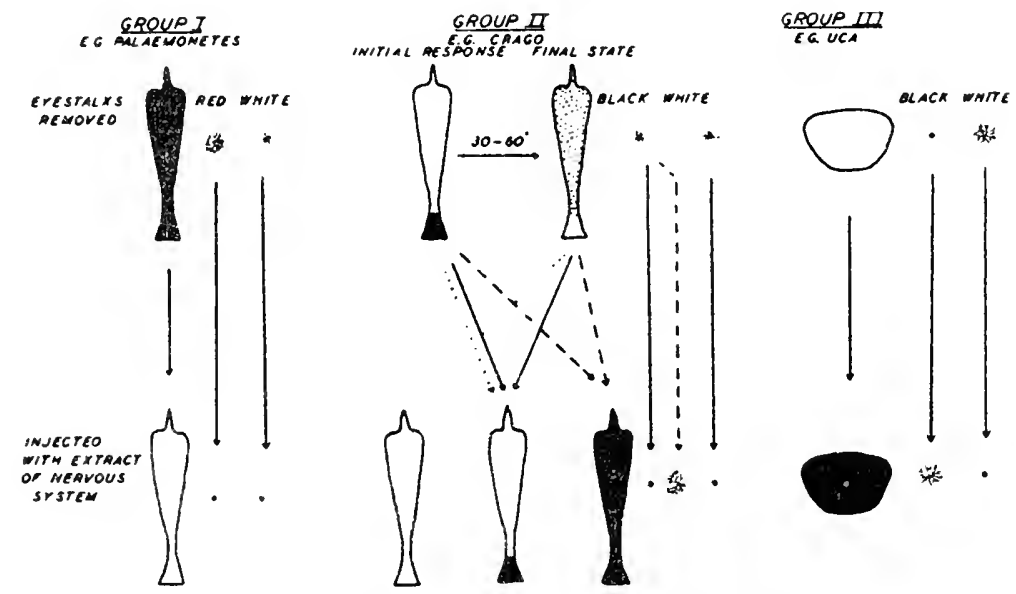

Fig. 265. Schematic representation of the coloration of eyestalkless crustaceans and state of their dominant chromatophores for each of the three differently responding groups, and of the responses of these to injection of extracts of the nervous system. Solid arrows indicate extract of total water-soluble material, dashed arrows indicate an alcoholinsoluble fraction, and dotted arrows indicate an alcohol-soluble fraction. From Brown, 1948.

provided a clue to much of our knowledge of the chromatophorotropins involved in these reactions. If stimulation is mild, the whole animal blackens; if stimulation is intense, only the telson and uropods darken, the remainder of the body blanching. ${ }^{37}$ It is thus evident that some of the chromatophorotropins arising outside of the eystalks obviously antagonize the activity of sinus gland factors and others, at least in part, supplement them. 
The sources of the ehromatophorotropins involved in these reactions of animals lacking the sinus glands are within more or less restricted regions of the central nervous system and its sheaths (Fig. 265). Extracts of central nervous svstems of certain species of Group I, on injection, lighten eyestalkless specimens and concentrate white pigment. ${ }^{21,80}$ Similarly extracts of the central nervous system of $U_{c a}$, of Group III, darken eyestalkless specimens of $U_{c a}$ and simultaneously concentrate white pigment. Extracts of the nervous system of Crago, of Group II, lighten the body and darken the telson and uropods while concentrating white pigment. Therefore, in all three groups, injection of extracts of central nervous system organs produces the same major results as strong stimulation of the eyestalks. No source of ehromatophorotropins needs to be sought outside of the central nervous system.

A careful survey of the central nervous system of Crago show's by far the major portion of the telson-darkening and uropod-darkening action, as well as body lightening, resides in the minute tritocerebral commissure of the head region (Fig. 266). The remainder of the central nervous system, how-

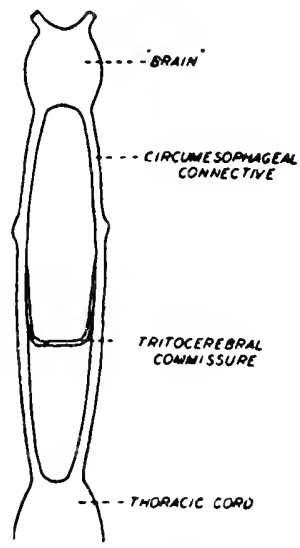

Fig. 266. Diagram of the anterior region of the crustacean central nervous system showing the position of the tritocerebral commissure. From Brown, 1948.

ever, possesses significant body-lightening activity. ${ }^{-4}$ This latter fact, together with the discovery that an alcohol-soluble fraction of the tritocerebral commissures possesses very strong body-lightenung and no "tail"-darkening actwity, while the alcohol-insoluble fraction causes strong "tail"-darkening and simultaneously strong body-darkening, ${ }^{31}$ establishes the presence of two chromatophorotropins in Crago nervous systems (Fig. 267). One lightens the body but not the "tail" of Crago, and hence has been called Crago-bodylightening hormone (CBLH); the other, in the presence of CBLH, darkens only the "tail," but in the absence of CBLIH darkens the whole body and is hence called Crago-darkening homone (CDH). We can now see the probable cause of the striking difference between strong and weak stimulation of the eyestubs in this species. Strong stimulation appears to induce the liberation of both principles, whereas weak stimulation liberates almost exclusively CDH. 
The sheath of the tritocerebral commissure of Crago possesses in its midregion greatly distended cells whose cytoplasm is richly charged with granules that are probably secretory. It is believed that these large cells are the source of both homones.

A survey of the influence on Crago of extracts of the nervous systems of various species of Group I led to the conclusion that all possess both CDH and CBLH, although in none of these other species is it as localized as in Crago.:- It is usually widely distributed along the nervous system (Palaemonetes, Cambarus, Homarus) or restricted to regions other than the tritocerebral commissures (e.g., to the posterior portion of the thoracic cord in Pagurus and other Anomurans). In some nervous sistems and more especially in some portions of the nervous systems the ratio of CDH activity to CBLH activity is so large that the extracts blacken eyestalkless Crago at least as effectively as the alcohol-insoluble fraction of the Crago tritocerebral commissures (e.g., lobster or crayfish abdominal cords). The high CDH activity of lobster abdominal cords can be shown to reside predominantly in

A

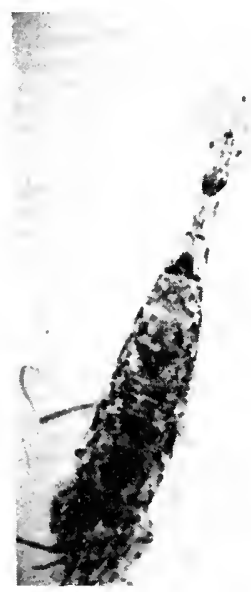

C

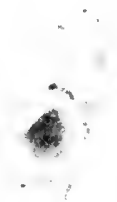

B

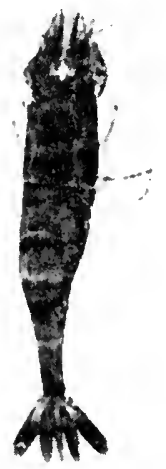

Fig, 267. Appearance of three Crago, closely matched in coloration initially, in response to injection of various solutions: $A$, Sea-water (control, exhibiting no change); $B$, an alcohol-insoluble fraction of the commissures; $C$, total water-soluble fraction of tritocerebral commissures. From Brown and Klotz. ${ }^{31}$

the sheath rather than in ganglionic neurosecretory cells. In contrast to crustaceans of Group I, those of Group III possess no CDII anywhere within their central nervous systems or, for that matter, anywhere in their bodies (Fig. 263). CBLH is present in the nervous systems of all crustaceans examined.

It has been established that $U_{c a}$ of Group III, though possessing no $\mathrm{CDH}$, does actually possess at least two chromatophorotropins in its nervous system. ${ }^{1.5 i a}$ This evidence comes from comparison of the relative influences of extracts of the brain, circumesophageal connectives, and thoracic c d on eyestalkless $U_{c a}$. Extracts of the brain and thoracic cord show 
strong actirity in black-pigment dispersal and simultaneously leave white pigment dispersed or induce its dispersal. Extracts of connectives, on the other hand, produce only a weak black-dispersing action but cause concen-
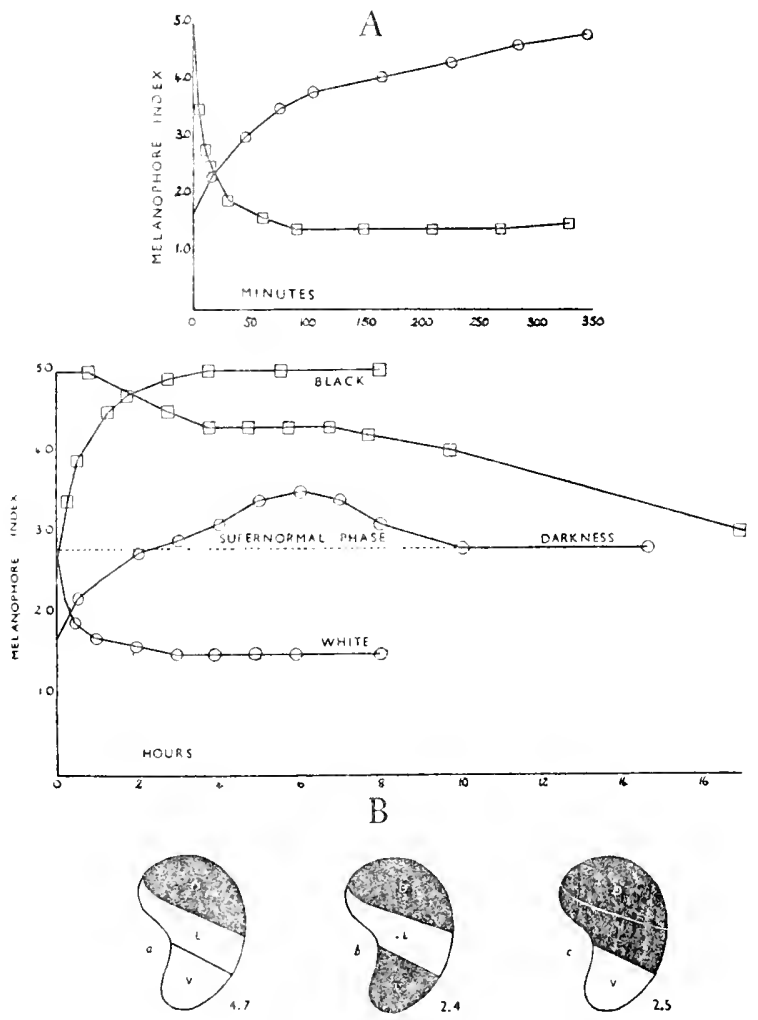

-
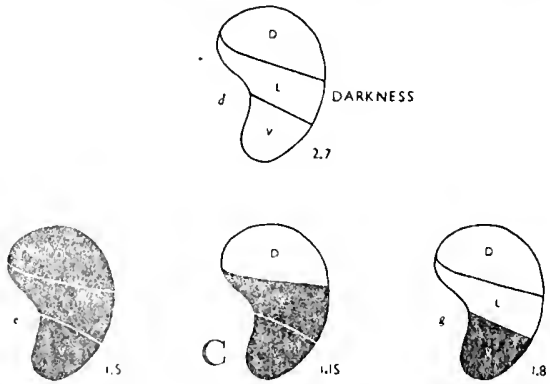

Fig. 268. A, Relationship between time and melanophore state in Ligia after transfer from a black to a white background and vice versa. $B$, Changes in melanophore state after transfer from darkness to black and white backgrounds, and vice versa. C, Relationship between portion of Ligia eye stimulated and the average melanophore state. The illuminated portion of the cye is black. From Smith. ${ }^{177}$

tration of the white pigment. The ciffering results cannot be duplicated simply by varying the concentrations of nervous system extract. Although these observations establish the presence of two chromatophorotropins, they 
do not permit one to decide between two possibilities: (1) all portions have a black-dispersing and a white-concentrating action, with the brain and thoracic cord having in addition an antagonistic white-dispersing agent; and (2) all portions have a white-dispersing and a black-dispersing action, but the connectives alone contain a white-concentrating principle.

Numerous reciprocal-injection experiments among Crago, $U_{c a}$, and Palaemonetes, in which the comparative distributions of Crago-darkening, Crago-body-lightening, $U_{c a}$ black-dispersing, and $U_{c a}$ white-concentrating activities within the various nervous systems are compared, show rathe: clearly that CDH and UDH cannot be the same, nor like CBLH or UWCH. However, there is still a possibility that CBLH and UWCH are identical since there is a qualitative parallel in their distributions within the three nervous systems studied. Certain quantitative differences of activity, howcver, cast doubt on this identity.

In summary, decapod crustacean nervous systems possess at least three or four chromatophorotropins. Their roles in the total chromatic responses of the whole organism have not been worked out for any one species. It seems likely, however, that the nervous-system chromatophorotropins, together with those from the sinus glands, will be shown to go far toward accounting for the intricate control of the crustacean pigmentary systems.

A study of the time relations of melanophore changes in the isopod, Ligia oceanica, has led Smith ${ }^{177}$ to postulate a dual endocrine control of these pigment cells, operation of both a darkening B-substance and a lightening W-substance. Two antagonistic substances were postulated, since if only a $\mathrm{W}$-substance were present then the time of white to black change, wTb, should be greater than white to darkness change, wTd, or darkness to black change dTb, which was not found to be true. On the other hand if only a $\mathrm{W}$-substance were present, then bTw should be greater than bTd or dTw, which is not the case. The data seemed reasonably explained in terms of two factors with the B-substance more slowly eliminated from the blood than the W-substance. This would account for the long bTd and the supernormal phase of the wTd (Fig. 268). On the basis of experiments in which different portions of the eye were opaqued, or differentially stimulated by careful adjustment of the background, support was given for the view that stimulation of dorsal elements of the retina results in production of B-substance, and stimulation of the lateroventral portions in production of $\mathrm{W}$ substance.

Vertebrates. All the vertebrates possessing chromatophores show, in general, a fundamental similarity in their functional organization of this system. It seems profitable to develop the evolutionary trends separately within each of the major divisions of the vertebrates in which functional chromatophores are found.

Amphibians. The amphibians may show in their early development a period in which only primary color responses occur, the animals darkening in light and becoming pale in darkness. This was originally described for very young Axolot by Babak ${ }^{11}$ and has since been observed in very young Rana pipiens ${ }^{79}$ and Amblystoma. ${ }^{105}$ These changes do not involve the eyes. It has been suggested that this is a period during which the eyes are still non-functional. ${ }^{198}$ Other amphibians appear to show the secondary types of 
response involving stimulation through the eye, immediately on hatching. Such species are Bombinator and Hyla ${ }^{11}$ and Xenopus. ${ }^{19 s}$ In the secondary phase primary responses are still operating but are dominated by the secondary ones.

Investigation of the physiology of color changes in amphibians has provided no evidence of any direct nervous control of the integumentary melanophores. The striking discovery was made very early that hypophysectomized tadpoles remain pale indefinitely. ${ }^{17 x}$ This strongly suggested that the pituitary might be the normal source of a melanin-dispersing hormone in amphibians. The relation of the hypophysis to color changes in Rana was carefully investigated by Hogben and Winton. ${ }^{76,77,75}$ Upon hypophysectomy the animals were rendered both pale and refractory to further color changes. Removal of the anterior lobe by itself, however, showed no significant interference with the background responses. Extracts of the posterior lobe showed a tremendous capacity to darken pale frogs. All attempts to produce specific chromatophore responses through nerve stimulation or nerve transection failed. Therefore, these workers concluded that the color changes in Rana could be readily accounted for in terms of the activity of a single hormone

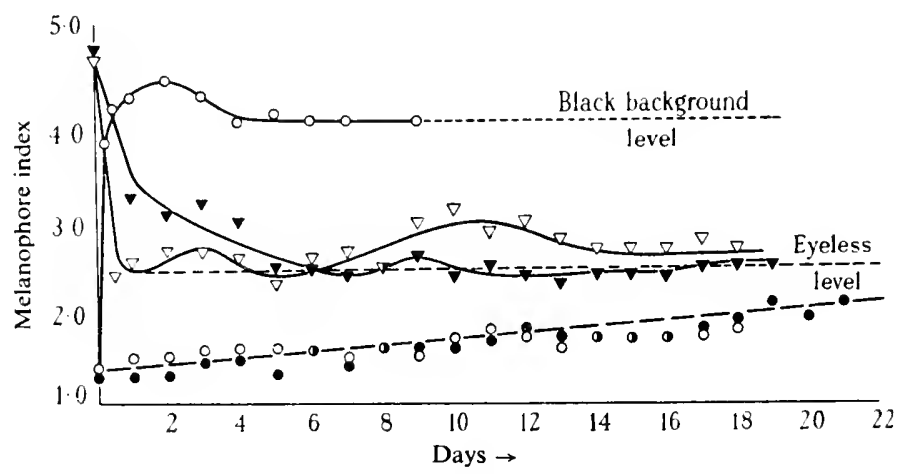

Fig. 269. Changes in melanophore state in Xenopus after transfer from white to black backgrounds and vice versa, and from black background to darkness. From Hogben and Slome. ${ }^{\text {ij }}$

arising in the posterior lobe of the pituitary, whose concentration in the blood was controlled by environmental stimuli operating through the eyes. This conclusion has been confirmed through transfusion of blood from a dark to a light Rana. ${ }^{143}$

More recently much work has been done on Xenopus, ${ }^{74,75}$ in which direct innervation of the melanophores seems also to be lacking. It, too, lightens after hypophysectomy and darkens on injection of extracts of posterior lobe of the pituitary. A critical and detailed examination of the characteristics of change of the melanophores of Xenopus after transfer from a white to a black background and the reverse, from black to darkness and reverse, and from white to darkness and reverse (Fig. 269) fails to reveal an adequate explanation in terms of a single principle and leads to the postulation that the hypophysis produces two principles, one with melanin-dispersing action, referred to as the B-substance (probably intermedin), and the other with 
melanin-concentrating action, called the $\mathrm{W}$-substance. All the data obtained appeared readily interpretable in terms of an excitation of secretion of the W-substance by stimulation of peripheral retinal elements and excitation of secretion of the B-substance by stimulation of basal retinal elements (Fig. 270). Furthermore, the data require the additional assumption that the $W$ substance is added to the blood more slowly than the B-substance and also disappears from the blood more slowly. The B-substance and the $\mathrm{W}$-substance are antagonistic. Thus, according to this hypothesis, in an illuminated environment B-substance is always secreted, but its secretion is reduced in low illumination and in darkness. The responses to black and white illuminated backgrounds involve changes in the amount of the $\mathrm{W}$-substance and consequently changes in the B W ratio. The very slow responses observed in the change from an illuminated white background to darkness and the
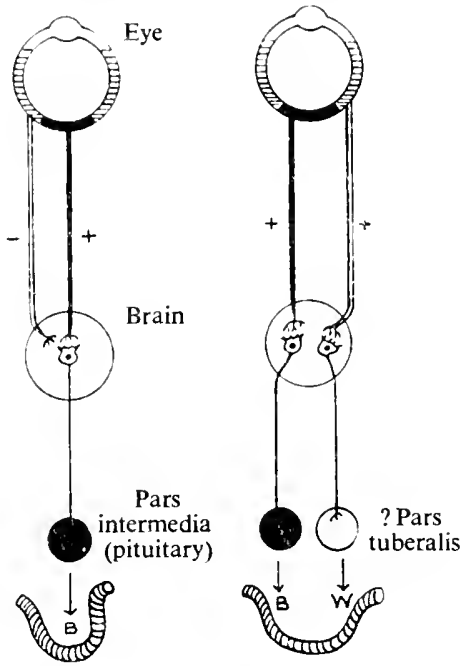

Blood vessel

Fig. 270. Schematic representation of two hypotheses of hormonal control of melanophores in Xenopus, one involving a single hormone, and the nther, two. From Hogben and Slome. ${ }^{75}$

reverse appears explainable in terms of concomitant decreases or increases, respectively, of the two antagonistic substances. In the change from darkness to an illuminated black background the melanophores pass through a supernormal phase (more dispersed than typical for the background), a fact explainable in terms of a more rapid secretion of B-substance than of W-substance. As the latter increases to its full quantity, the melanin reaches its slightly less dispersed final state. Similarly, in passing from an illuminated black background to darkness the melanophore passes through a transitory stage of greater concentration than the ultimate state, apparently due to a more rapid reduction in B-substance than in $\mathrm{W}$-substance.

The only direct evidence for the existence of the W-substance in Xenopus has come from observations on the responses of the animals to environmental stimuli after various types of operative procedures. ${ }^{75}$ When the anterior lobe of the hypophysis is removed the animal responds quite as it does normally. 
When the anterior lobe and the pars tuberalis are extirpated, the melano. phores become maximally dispersed and show no background response. When the posterior lobe is removed, the animals are maximally pale. The source of the darkening hormone appears definitely to reside in the intermediate lobe. ${ }^{9,1}, 12$ Removal of the whole gland leaves the pigment slightly dispersed and non-responsive. All these facts fit the hypothesis of the existence of two factors, with the pars tuberalis responsible for the $\mathrm{W}$-substance and the posterior or the intermediate lobe for the B-substance.

Support for the presence of two factors also comes from the relative effects of injection of B-substance into completely hypophysectomized Xeno. $p_{u} s^{\tau 5}$ as compared with its effects in normal animals or animals with only the posterior lobes removed (Fig. 271). As would be expected, a larger dose

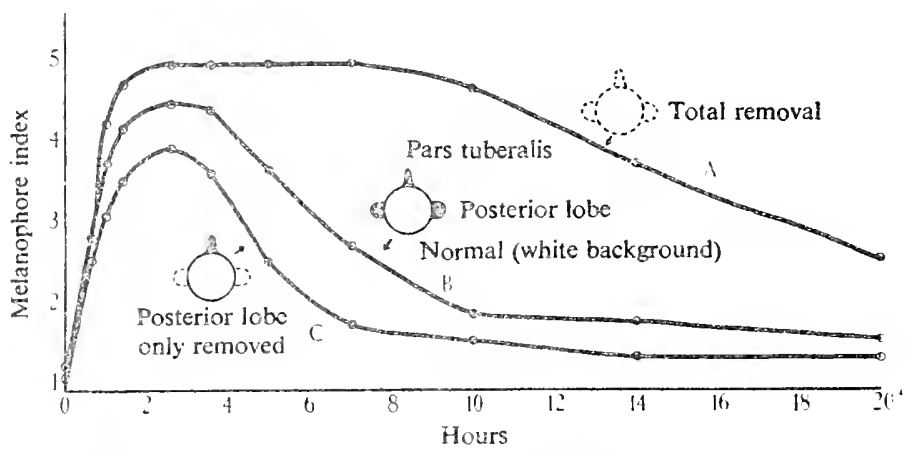

Fig. 271. Responses of the melanophores of Xenopus on a white background to injections of equivalent doses of pituitsry extracts into totally hypophysectomized specimens, specimens with only posterior lobe removed, and normal specimens. From Hogben and Slome. ${ }^{75}$

is required in the last two types of recipients to bring about a given re. sponse, while in those with complete hypophysectomy a much smaller dose has an equivalent action. This latter observation finds a most logical explanation in terms of the resulting absence of an antagonist secreted by the pars tuberalis.

Fishes. The fishes, which have probably been more intensively investigated than any other group, with respect to their chromatophore system and color changes, have several types of chromatophores. The most common and conspicuous type is the melanophore. Other common types contain yellow pigment (xanthophores), red pigment (crythrophores), and white pigment (leucophores). In addition, many fishes possess chromatophores containing small clusters of glistening plate-like crystals that impart a bluish green coloration (iridosomes).

The activities of the melanophores are the principal ones involved in the conspicuous responses to light and darkness and to black and white backgrounds in fishes. The mechanism of response in fishes, as with amphibians, commonly shows a change from primary color responses to secondary during their early development. Young Perca and Salmo, ${ }^{+3,119}$ Macropodus, ${ }^{192}$ and Hoplias ${ }^{114}$ show such a transition in response mechanism. On the other hand, a number of other species appear not to pass through a phase of pri- 
mary responses but to have initially the secondary phase. Species of the latter type include Fundulus, ${ }^{61}, 201$ Lebistes, Xiphophorus, Gambusia, ${ }^{192}$ Scyllium, ${ }^{19 \times}$ and Mustelus. ${ }^{129}$ A number of fishes normally showing secondary color responses will revert to primary responses after blinding. ${ }^{192}$

The secondary color responses of fishes are dependent typically upon the eyes. They involve nervous pathways to the central nervous system, thence either to endocrine glands affecting the chromatophores through blood-borne hormones, or by way of efferent nervous pathways directly to the chromatophores where chemical mediators are liberated by the nerve terminations. Both hormonal and nervous mechanisms may cooperate in many cases. There is considerable variation among fishes as to the normal mochanism of control. Parker, ${ }^{13 \times}$ the leading investigator in the field of animal color changes, divides the fishes into three groups on the basis of the degree to which direct innervation of the melanophores is found. Dineuronic chromatophores possess double innervation with separate dispersing and concentrating fibers. Mononeuronic chromatophores possess single innervation in which the activity is always pigment-concentrating. Aneuronic chromatophores possess no innervation, their responses being due solely to acivity of blood- anc? lymph-borne chemical factors.
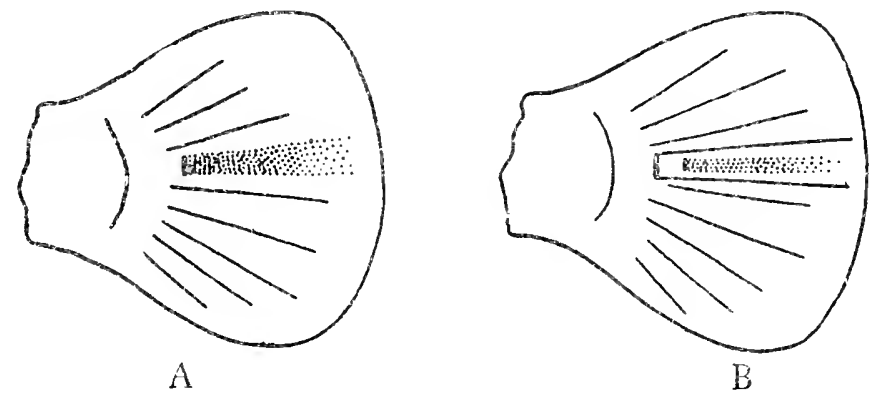

Fig. 272. A, Dark band in caudal fin of white-adapted Fundulus produced by severance of radial nerves. $B$, Redarkening of a faded band following a second, more distal cat. From Parker. ${ }^{126}$

The great majority of the teleost fishes thus far carerully investigated appear to possess dineuronic melanophores. One type of innervating nerve fiber, a pigment-concentrating one, is readily demonstrated by electrical stimulation of appropriate loci in the central nervous system, of central nerve tracts, or of peripheral nerves. The extent of the area of the skin blanching under this stimulation parallels the area of the skin innervated by thesc nervous elements. Furthermore, after denervation of any area of the skin the denervated area no longer exhibits blanching responses to stimulation of the nerves central to the point of denervation, even though responses may continue in the adjacent areas. Therefore the blanching produced by nerve stim. ulation in the animals is a result of localized responses at the region of the nerve terminations and is not due to freely diffusible substances in the blood. The action of the concentrating fibers is believed by Parker ${ }^{-135}$ to be mediated through adrenalin-like material which is liberated by the nerve terminations and which diffuses through the tissues. 
The presence of a second set of fibers, pigment-dispersing ones, has been demonstrated for many teleosts through experimentation with melanophores of the tail fins. If, in a teleost such as Fundulus, kept on an illuminated white background, a group of the radiating caudal nerves is cut by a transverse incision, the band of fin innervated by these fibers darkens quickly and then orer the course of a few days fades again ${ }^{126}$ (Fig. 272). If now a second cut is made parallel to the first cut, and somewhat distal to it, the faded band will repeat the transitory darkening behavior. This latter behavior leads to the hypothesis that the melanophore response observed must be due to a restimulation of the dispersing nerve fibers that had been transected and stimulated by the first incision. It cannot be explained simply in terms of a transection of the concentrating fibers, as possibly the response to the first cut could be. Interpolation of a cold-block between the point of the nerve transection and the melanophores abolishes the response. ${ }^{131}$ Such redarkening of facled bands after a second incision has been observed by a number of investigators for a number of species of teleost fishes. These include Holocentrus, ${ }^{13: 2}$ Parasilurus, ${ }^{111}$ Pterophyllum, ${ }^{1933}, 194$ Ameinurus, ${ }^{1366}$ and Gobins."." An activation of melanin-dispersing fibers in the catfish tail has also been produced by electrical stimulation. ${ }^{1+2}$

Other lines of evidence, both morphological and physiological, have given further support to the hypothesis of a dual innervation of teleost melanophores. Ballowitz ${ }^{1: 3}$ many years ago clearly demonstrated that the melanophores of the perch receive nerve terminations from more than one fiber, thus providing an anatomical basis for the conclusions reached by more recent physiological experimentation. Furthermore, a critical examination has been made of the responses of chromatophores at the edges of denervated caudal bands of Fundulus, ${ }^{116}$ and of those near the regenerating front of nerve fibers in the course of re-innervation of denervated bands, ${ }^{+}$as the animals darken on black backgrounds and lighten on white ones. These observations provide strong evidence that many of the melanophores located in these regenerating fronts possess only one type of fiber, either concentrating or dispersing, but not both as under normal circumstances. Some of these melanophores show rapid pigment concentration and very slow dispersion; others show the reverse. Studies of the influence of drugs on chromatophores of Phoxinus ${ }^{i 0}$ and Fundulus ${ }^{17 t}$ also support the concept of a dual innervation.

It now appears that the dispersing fibers exert their action on the melanophores through the mediation of acetylcholine. Acerylcholine is known to cause dispersion of the melanin of fishes ${ }^{13 .}$ when the latter are eserinized to prevent rapid destruction of the material. In fact, a bioassay of the acetylcholine content of the skin of a dark-adapted eatfish Aneinurus ${ }^{135}$ or snakefish Ophiocephalus showed its presence in a concentration of about 0.078 gamma per gram of skin. This is approximately the concentration of acetylcholine which, when injected into the body fluids of eserinized fish, was in general ron-toxic and at the same time quite effective upon melanophores.

Of the fishes thus far investigated the dogfish Mustelus and Squalus appear to possess mononeuronic melanophores. If a transverse cut is made in the pectoral fin of a dogish of intermediate tint a light band is produced distal to the point of the cut. Such light bands may be revived after they 
have redarkened ${ }^{130}$ or may be produced by electrical excitation ${ }^{12 .}$ of the integumentary nerves. These bands follow the distribution of the cut nerves and not necessarily of the blood ressels. Furthermore, light bands may be produced by a similar transverse cut in a fin from which the blood supply has been cut off. All these facts point to a nerve supply to the melanophore whose function it is to induce pigment concentration. There is no indication whatsoever that pigment-dispersing nerve libers are present in this animal.

Parker ${ }^{137}$ postulated that the concentrating fibers influence the melanophores through the production of a chemical mediator which he called selachine. If skin from a pale animal is extracted with ether or olive oil, but not water, the material mas be extracted. Injections of an olive-oil extract of this substance into a dark-adapted animal will produce temporary lightening, which spreads very slowly from the point of injection. Olive oil by itself produces no such effect.

The other clasmobranch fishes which have been investigated, the skate, Raja, ${ }^{133,3}$ the dogfish, Scyllium, evidence of direct innervation of their melanophores, and hence their melanophores may be said to be aneuronic.

Blood-borne agents typically supplement the nervous system in the secondary responses of the melanophores of fishes to light stimuli. In the more primitive fishes, such as the cyclostomes and many of the elasmobranchs which possess aneuronic color cells, hormones alone are the agents involved. Among these hormones an important substance is a pigment-dispersing principle from the pituitary, the B-substance of Hogben, or intermedin. 00 This substance is produced by the posterior lobe of the pituitary. Lundstrom and Bard" observed that hypophysectomized Mustelus become and remain pale. The lish may be darkened again by injection of extract of the posterior lobe. A similar role of the posterior-lobe principle has since been demonstrated for other elasmobranclis, Raja and Scyllimm, ${ }^{i 2}$ and the cyclostome Lampetra. ${ }^{203}$

There is some evidence that in Scyllium and Raja a second neurohumor from the pituitary acts as a pigment-concentrating agent. ${ }^{2}$. Evidence for a role of such a body-blanching principle has been derived from studies of the characteristics of the melanophore responses to background and lightintensity changes and to the influence of hypophysectomy on the state of the pigiments, and of pigmentary responses to injections of posterior-lobe extract. The general methods of experimentation and logic involved in these experiments are the same as those developed in the studies of amphibian melanophore control. According to the bihumoral concept of Hogben and his associates the state of the melanophores in these fishes is determined by the ratio of $B$-substance to $W$-substance present in the blood at any given instant, and this ratio is in turn controlled by visual stimuli. The visual stimuli, through differential dorso-ventral retinal stimulation, result in different rates of secretion of the two principles by their two respective sources.

The chromatic pituitary hormone involved in melanin dispersion seems also to be present and active in normal color change to a greater or lesser degree in all teleost fishes. The eel, Anguilla, shows sluggish color changes requiring days for completion. ${ }^{11: 3}$ In this fish, despite the apparent presence 
of both concentrating and dispersing nerve fibers, color changes seem predominantly determined by hormones. In its activity in the eel, the B-substance is believed to be assisted by a $W$-substance. ${ }^{197}$ That direct innervation does play some role in color changes in this fish is seen in the limited background response after hypophysectomy.

The B-substance is found to be slightly less important in normal color clianges in the catfish Ameiurus. Hypophysectomized catfishes continue to show color changes in response to black and white backgrounds but show only an intermediate degree of darkening on black. ${ }^{3}$ Injection of posteriorlobe extract will, however, completely blacken these fish. Here we must assume that a blood-borne B-substance supplements the action of dispersing nerve fibers in the normal responses to black backgrounds; there is as yet no evidence for the operation of a $W$-substance in this species.

The killifish, Fundulus, on which a vast amount of research has been done, is a species in which the dominant mechanism of melanophore control is nervous. Color changes are very rapid, only a minute or two being required for nearly maximal color change. These changes continue to occur

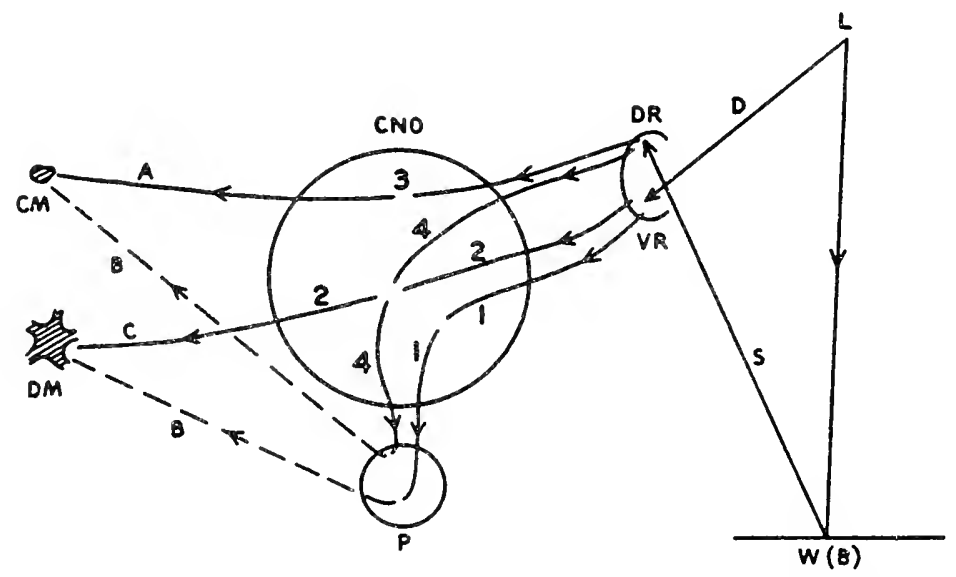

Fig. 273. Diagram of the controlling mechanism of melanophores in the eel, Anguilla. $L$, incident light; $W(B)$, white or black background; $D R$, dorsal retina; $V R$, ventral retina: $C N O$, central nervous system; $P$, pituitary; $A$, adrenergic fibers; $C$, cholinergic fibers; $B$, blood and lymph; $D M$, dispersed melanin; $C M$, concentrated melanin. From Parker. ${ }^{133}$

in hypophysectomized specimens. ${ }^{113}$ Furthermore, injection of extracts of posterior lobe into pale fish on a white background does not produce significant darkening. Since such extracts will induce darkening in denervated areas of the skin we must conclude that the chromatophores are normally influenced to some extent by this substance, which is shown to be present in their pituitaries." 12 However, its normal influence is probably seen only in the production of extreme conditions of dark adaptation maintained over relatively long periods of time.

All teleost fishes thus far investigated appear to fall into a series in the relative influences of direct innervation and blood-borne hormones. A reasonable hypothesis has been advanced that the humoral control is phylogenet- 
ically the older, and that direct nervous control has been superimposed upon it in those fishes of more recent evolutionary origin. ${ }^{198}$ The latter tendency is associated with more rapid response to background, which has become possible through a simultaneous increase in the speed of the melanophore change itself. The typical teleost controlling mechanism for melanophores is diagramıned in Figure 273.

A survey of the character of the response of melanophores of fishes to war gases suggests the presence of two differently responding types, with catfishes possessing one type and scaly fishes the other. ${ }^{152}$

Very much less is known about the control of the erythrophores, xanthophores, leucophores, and iridocytes than about the control of the melanophores. The erythrophores of the squirrel-fish, Holocentrus, are rapidly responding effector organs. ${ }^{132}$ Through their activity the fish can change from red to white in about 5 seconds, and make the reverse change in about 20 seconds. These responses may be induced by change from black to white background, and vice versa. Transection of nerve tracts in a fish on a white background results in dispersion of red pigment. The areas blanch again in a short while and may be darkened again by a second more distal cut, indicating the presence of dispersing nerve fibers. The presence of concentrating nerve fibers can be demonstrated by electrical stimulation of the medulla, resulting in rapid paling of all innervated erythrophores. Experimentally denervated cells fail to give this response. Adrenalin concentrates the pigment. Pituitary extracts from other squirrel-fish produce no effect when injected into normal light-adapted or dark-adapted specimens. It thus appears that the erythrophores of Holocentrus normally are exclusively under nervous control. ${ }^{175}$ The erythrophores of Phoximus, on the other hand, have been shown to be influenced by a principle from the hypophysis. ${ }^{60}$

The xanthophores of Fundulus appear to possess double innervation. comprising concentrating and dispersing fibers. ${ }^{51}$ A concentrating hormone, probably adrenalin, also appears to be responsible for the concentration of the pigment which results from handling of the fish. This latter concentration occurs as rapidly in denervated xanthophores as in innervated ones. On the other hand, intraperitoneal implantation of Fundulus pituitaries into hypophysectomized specimens induces pigment dispersion in denervated xanthophores, regardless of color of background. Such implants also impede the typical pigment-concentration in innervated cells in response to blue or white backgrounds. ${ }^{52}$ It therefore appears that several factors normally inHuence the state of the xanthophores in this species. Melanophores and xanthophores of Fundulus react independently in background responses. ${ }^{5}$

A number of fishes, including Fundulus, possess non-iridescent reflectingwhite chromatophores known as guanophores or leucophores. ${ }^{49}$ These may show physiological changes in the adaptive responses of the fish to background. Little is known of their normal control. They continue to respond to background after hypophysectomy, and after sufficient dosage of the fish with ergotamine to prevent any response of the accompanying melanophores. They disperse their pigment under the influence of adrenalin. ${ }^{121}$

Iridosomes or chromatosomes play only a passive role in adaptive color changes, becoming more or less obscured through activity of the other chromatophore types. Normally these bodies are green or blue. They are highly 
responsive to certain environmental stimuli, changing reversibly through the spectral colors to red on excitation and in the opposite direction on recovery. This response is direct, and does not involve coordinating mechanisms within the animal, either nervous or humoral. ${ }^{4 t j}$

Reptiles. The functional organization of the melanophores of reptiles shows, as in the fishes, a great diversity. It appears to involve, to differing degrees in different reptiles, the activities of hormones and nerves.

The melanophore responses of the iguanid. Anolis, have been investigated very extensively by Kleinholz. ${ }^{9: 3}$ "These lizards show color changes ranging from bright green to dark brown. They typically assume the former color in an illuminated white container, and the latter in an illuminated black one. The response to change from a white to a black background is

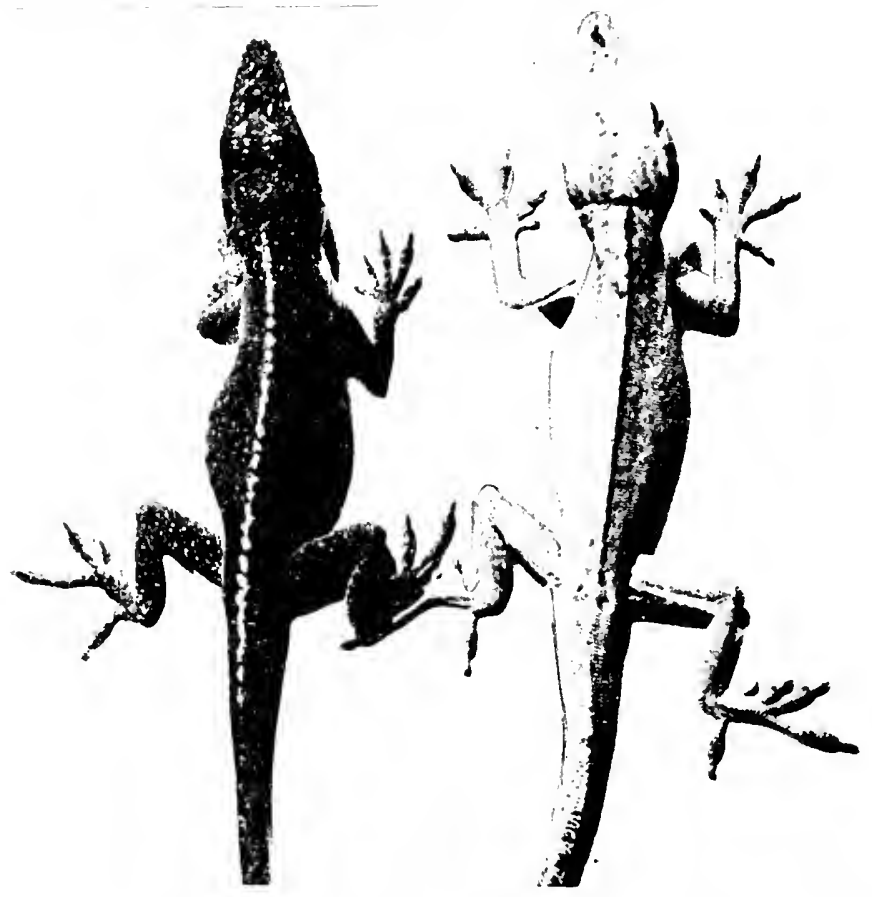

Fig. 274. A normal dark and a hypophysectomized light Anolis. From Kleinholz."

usually completed in 5 to 10 minutes. The reverse change normally requires 20 to 30 minutes. These background-induced responses depend on the eyes; they cease after bilateral blinding. However, such blinded specimens still are capable of color change; they darken in light and become pale in darkness, through primary responses.

For many years after Brücke's" classical studies of color change in the chamelcon, in which he demonstrated nervous control of the melanophores by the sympathetic system, it was considered that reptilian chromatophores gencrally were thus controlled. Studies on Phrynosoma, ${ }^{15}$ however, prorided basis for a strong suspicion that nerves were not the sole method of control. The lizard, Hemidactylus, becomes pale in color after hypophysect- 
omy. Hypophysectomized Anolis remain permanently bright green ${ }^{43}$ (Fig. 274). They no longer darken in response to black background or bright light. They can be darkened readily by injection of extracts of whole pituitary of fishes or the intermediate lobes of frogs or reptiles. The melanophores of the rattlesnake appear also to be normally dispersed by a principle from the pituitary. ${ }^{1.33}$

Transection of nerves, such as section of the spinal cord at various levels, or cutting the sciatic nerve in Anolis, in no way interferes with the normal color responses. In fact, attempts at histologic demonstration of nerve terminations at the melanophores have been uniformly unsuccessful. Skin grafts very soon show color changes which are sunchronous with those of the host. Exclusion of the blood supply from any region, on the other hand, results in a paling of that region in about 15 minutes.

The roles of the animal's own hypophysis and adrenals can be shown by electrical stimulation with one electrode placed in the cloaca, the other in the mouth. Stimulated pale animals kept in darkness become uniformly dark brown. Denervated areas respond just as do the innervated ones. Similar stimulation of hypophysectomized specimens gives, on the other hand, a characteristic mottling of the body. This last is not obtained after both adrenalectomy and hypophysectomy. Furthermore, injection of adrenalin or of extracts in Ringer solution of the animal's own adrenals produces the typical inottling. Adrenalectomized animals lighten in response to a white background. All of these observations, and others, point strongly to the conclusion that the melanin in Anolis normally disperses as a result of the activity of a principle from the intermediate lobe, and that its gradual disappearance from the circulation suffices to account for lightening. Rapid blanching which normally follows electrical stimulation or excitement may perhaps be accounted for by integumentary vasoconstrictor activity and under some circumstances by the production of adrenin.

Light appears to have no significant influence on the melanophore state in intact Anolis, other than through the eves.

The available evidence indicates roles of both nerves and hormones in the melanophore responses of the iguanid, Phrynosoma. The early work of Redfield ${ }^{15 t}$ on this form has been largely confirmed and considerably extended by Parker. ${ }^{134}$ These animals are normally gray with characteristic black patches. The latter patches show no color changes, whereas the intervening area varies with appropriate stimulation from dark gray to pale grayishwhite. These changes are due primarily to melanophore activities. Darken ing of the animal occurs in about 15 minutes, and lightening in approximately twice that time.

Phrynosoma darkens on an illuminated black background, at low temperatures, and in response to very strong illumination. It lightens on an illuminated white background, at high temperatures, and in darkness.

The melanophores are normally under the influence of pigment-concentrating nerve fibers. Stimulation of a sciatic nerve will induce lightening in the corresponding hind leg. Electrical stimulation of the roof of the mouth or of the cloaca results in a paling of the whole animal, which is quickly reversible. Following denervation of a region of the body, leg, or lateral trunk, similar stimulation results in a lightening of all regions except the denerv- 
ated one, despite the fact that the melanophores in the denervated area still show pigment concentration in response to an injection of adrenalin. No indication of pigment-dispersing fibers has been uncovered in this species.

Phrynosoma, like Anolis, after hypophysectomy becomes pale and remains so indefinitely. Injection of pituitrin or of extract of Phrynosoma pituitary induces strong darkening either in normal pale or in hypophysectomized individuals. Furthermore, injection of defibrinated blood from a dark specimen into the leg of a pale one produces darkening in the latter. These results provide strong evidence that a hormone arising in the pituitary is normally concerned in body-darkening. An action of a pigment-concentrating hormone is also seen in that adrenalin or extract of Phrynosoma adrenals strongly blanches dark specimens. The presence of a similarly acting agent in the blood of white-adapted animals is seen in that their defibrinated blood will, on injection into a leg of a dark specimen, lighten the latter. It has been known for a long time that animals held on their back or otherwise stimulated to struggle vigorously will lighten rapidly. By comparison of the influences of denervation and occlusion of blood supply on the production of this type of lightening it can be clearly demonstrated that a blood-borne agent is involved. These observations lead to the obvious conclusion that the paling is brought about by two methods, nervous and hormonal, either one alone capable of producing the response.

Both the dispersing hormone from the pituitary and the concentrating hormone from the adrenals operate directly on the melanophores; each is capable of exhibiting its complete action after nerve transection and degeneration of the nerve fibers.

The influence of temperature and of light and darkness on the melanophores of Phrynosoma is a direct one, in which the melanophores act as independent effectors. The responses may be obtained locally by application of the stimulus to the specific region in completely denervated portions of the body. Since even degeneration of the innervating fibers does not result in termination of the response, axon reflexes cannot be responsible.

The coordination of the melanophores of chameleons, Chamaeleo or Lophosaura, unlike that in the iguanids, appears to be exclusively nervous. ${ }^{73}$ Nerve transection is followed by a darkening of the area normally innervated by the nerves. These nerves are of the autonomic nervous system. The melanophores are readily caused to concentrate their pigment by electrical stimulation of the nerves. The results of nerve transection have been interpreted to be due to the absence of tonic impulses reaching the pigment cells. ${ }^{156,205}$ There is no clear evidence as yet that the pigment is actively dispersed by a second set of nerve fibers, as appears to be true of many teleosts.

There is little or nothing known for the most part as to possible roles of hormones in the color changes in the chameleon, but the fact that color patterns can be produced on the body by alternate light and shadow ${ }^{38}$ argues against any considerable importance of such factors.

The melanophores of intact animals respond to light and darkness by pigment dispersion and concentration, respectively. Dark regions produced by denervation show no such responses. These results were interpreted by Zoond and Eyre ${ }^{205}$ to prove that the responses of the melanophores can occur only by way of reflexes involving the central nervous system. But Park- 
er ${ }^{134}$ has pointed out the possibility of an alternative interpretation, that the pigment cells in these forms may show direct responses when their state is not determined by a dominating mechanism.

In summary we appear to find among the reptiles, as with fishes, varying degrees of evolution of chromatophore control from systems involving a probably primitive hormonal mechanism of coordination of the chromatophores, through those in which both nerves and hormones cooperate, to those largely dominated by direct nervous innervation.

\section{FUNCTIONAL SIGNIFICANCE OF CHROMATOPHORES AND COLOR CHANGE}

Since the responses of the chromatophores are predominantly responses to color or shade of background, one is led to the hypothesis that the color changes contribute significantly to the obliterative coloration of the animal for protection or aggression and hence increase its chances for survival. One of the few experimental demonstrations that physiological color

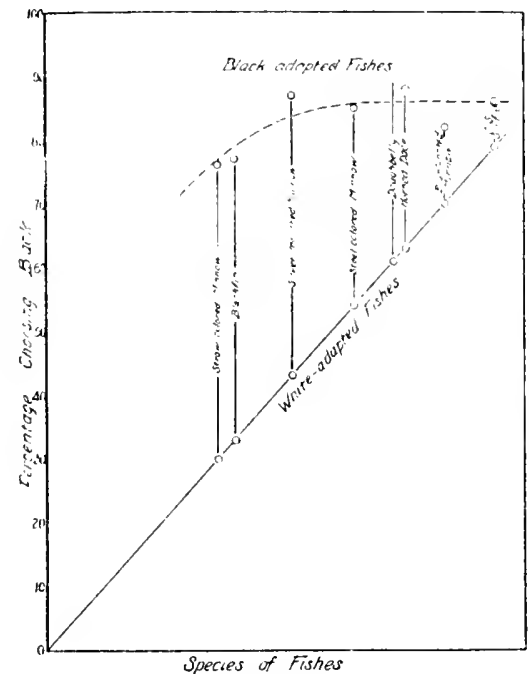

Fig. 275

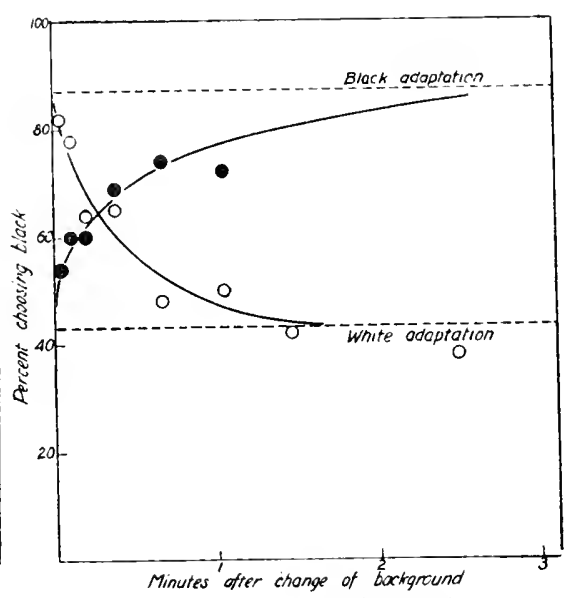

Fig. 276

Fig. 275. Percentage choice of a black over a white background for each of eight species of fishes adapted to black and white backgrounds. From Brown and Thompson. ${ }^{25}$

Fig. 276. The rate of change in percentage choice of a black background following transfer of Ericymba from a black to a white background and vice versa. From Brown and Thompson. ${ }^{35}$

changes do actually increase chances for survival, a view often questioned, is that of Sumner, ${ }^{185}$ in which he found that fishes given time to change their coloration were seized in smaller percentages by a predatory bird than were unadapted ones. It has also been clearly demonstrated that fishes which are black-adapted tend to select black backgrounds, when given a choice of black or white, more commonly than do white-adapted individu$\mathrm{als}^{35}, 110$ (Fig. 275), and that the rate of change of choice with change of background in Ericymba is approximately the same as the change in skin 
coloration (Fig. 276). Fishes with more rapid and striking color changes seem to have their choice more strongly modified along with background adaptation than do ones with less effective changes. The crayfish, Cambarus, also appears to possess an adaptive background selection. ${ }^{26}$

Chromatophores appear also to serve in the protection of animals from bright illumination which may be deleterious. In some animals, e.g., certain leeches and sea-urchins, the chromatophore pigment disperses only in response to bright illumination and with no regard to a possible protective coloration in relation to the background. The black and white pigments in the crab, $U_{c a}$, are normally dispersed in light during the daytime and concentrated in darkness at night. This is due partly to an inherent rhythm and partly to a response to total illumination. It is also noteworthy in this regard that the white chromatophores of such brilliant-light-inhabiting Sargasso-weed crustaceans as Latreutes fucorum, Leander tenuicornis, and Hippolyte acuminata are very abundant and richly charged with white pigment. In bright illumination this pigment disperses broadly, providing a continuous layer of a very effective diffusing reflector. In view of the character of the primary color responses of animals being that of pigment dispersal in light and pigment concentration in darkness, it appears reasonable to suspect that this light-protective function of chromatophores is a most primitive one, with the role of production of obliterative coloration evolving later.

Another function which has been attributed to chromatophores is that of thermoregulation. ${ }^{14}$, 123 The desert lizard Phrynosoma is light at night and during midday, and dark during the early morning and late afternoon ${ }^{101}$ (Ch. 10). These and similar observations have led to the hypothesis that the chromatophores function in thermoregulation in this species. The animal apparently is adaptively controlling heat absorption and radiation at the various times of day by chromatophore activities. Strongly supporting this view are the observations of numerous investigators of reptilian color change that elevation of the body temperature to approximately $40^{\circ} \mathrm{C}$. leads to melanin concentration and that lowering of the temperature to about $5^{\circ} \mathrm{C}$. leads to dispersion. ${ }^{134}$ The black pigment of the crab, $U_{c a}$, also tends to concentrate as the body temperature is elevated above 25 to $30^{\circ} \mathrm{C} .{ }^{33}$ These conditions obviously result in control of the amount of light absorbed by the black pigment in a manner beneficial to the animal.

One additional role of color changes in animals is suggested by the color displays that sometimes accompany mating behavior in such species as Anolis."i2 During pairing the males show a striking change from green to brown. Color displays are also associated with the breeding season in certain teleosts and cephalopods. The importance of these mating color displays is unknown. Special nuptial morphological color changes are not infrequently associated with the breeding season. ${ }^{+11}$

\section{SUMMARY}

Color changes involving movements of pigments, and formation or accumulation and destruction or loss of pigments, within special bodies in the integument, the chromatophores, have been observed among reptiles, amphibians, teleosts, clasmobranchs, cyclostomes, crustaceans, insects, cephalopods, annelids, and echinoderms. These color changes are most characteristically 
ones involving adaptation of the animal to its background through a functional relationship between the eyes and the chromatophores, although total amount of illumination, temperature, humidity, tactile stimuli, endogenous rhythms, and other factors may also influence the process.

The reflex pathways, initiated at the eyes, which are responsible for the control of the color changes in all crustaceans, insects, amphibians, and many fishes and reptiles, involve hormones. Both blood-borne hormones and direct innervation operate importantly in most other species, with the possible exception of the chamcleon among the reptiles and such fishes as Fundulus among the teleosts, and possibly also in leeches where purely nervous reflexes possess dominant or exclusive control. Responses of the chromatophore system to light continue in most animals in the absence of the eves, either through the responses of the chromatophores acting as independent effectors or reflexly through other light receptors, e.g., the pineal complex. These latter responses are responses only to intensity of illumination and bear no relation to color of background. These latter reactions typically operate to render the animal darker in higher illumination and lighter in lower.

Several functions have been ascribed to the chromatophore system, among them being: (1) protective and aggressive coloration: (2) thermoregulation; (3) protection of the body tissues from intense illumination; and (4) mating color displays.

\section{REFERENCES}

1. Aвgamowitz, A. A., Proc. Nat. Acad. Sci. 21:132-137 (1935). Morphological color-change. Fundulus xanthophores.

2. Aвramowitz, A. A., Proc. Nat. Acad. Sci. 21:677-681 (1935). Adaptation to colored backgrounds, Crustacea.

3. Aвramowitz, A. A., Biol. Bull. 71:259-281 (1936). Hypophysis and color change in Ameiunas.

4. Abranowitz, A. A., Proc. Nat. Acad. Sci. 22:233-238 (1936): Dual innervation of teleost melanophores.

5. Arramowitz, A. A., Am. Nat. 70:372-378 (1936). Independence of action of melanophores and xarthophores, Fundulus.

6. Abramowitz, A. A., Biol. Bull. 73:134-142 (1937). Role of hypophysis in Fundulus color change.

7. Ampanowizz, A. A., and Abramowitz, R. K., Biol. Bull. 77:278-296 (1938). Diurnal rhythm and chromatophore state, Crustacea.

8. Ascinerson, Arch. Anat. Physiol. wiss. Med. 7:15-23 (1840). Color change, frog.

9. Atwell, W. J., and Holley, E., J. Exper. Zool. 73:23-42 (1936). Pituitary in amphibian color change.

10. Atzlen, M., Ztschr. vergl. Physiol. 13:505-533 (1930). Relation of eyes to Dixippus color change.

11. BABAK, E., Arch. ges. Physiol. 131:78118 (1910). Color change in young amphibia.

12. BABAK, E., Arch. ges. Physiol. 149:462 470 (1913). Relationship of morpholugical to physiological color change, vertebrates.

13. Ballowitz, E., Ztschr. wiss. Zool. 56:673-706 (1893). Innervation of melanophores of teleosts.

14. Bauer, V., Ztschr. allg. Physiol. 16:191-212 (1914). Melanophores in heat regulation.

15. Bowman, 'T'. E., Am. Nat. 76:332-336 (1942). Morphological color-change, Cambarus.

16. BozLer, E., Ztschr. vergl. Physiol. 7:379-406 (1928). Cephalopud chromatophores and their innervation. 
17. Bozler, E., Ztschr. vergl. Physiol. 8:371-390 (1929). Cephalopod chromatophores and their innervation.

18. Brecher, L., Arch. Entw. Mech. 45:273-322 (1919). Color-change in insect pupae.

19. Brecher, L., Arch. Entw. Mech. 50:40-78, 209-308 (1922). Color-change in insect pupae.

20. Brecher, L., Arch. Mikr. Anat. 102:501-548 (1924). Color change in insect pupae.

21. Brown, F. A., Jr., Proc. Nat. Acad. Sci. 19:327-329 (1933). Chromatophorotropins from the crustacean central nervous system.

22. Brown, F. A., Jn., Biol. Bull. 57:365-380 (1934). Morphological color-change, Palaemionetes.

23. Brown, F. A., JR., J. Morph. 57:317-333 (1935). Adaptation to colored background, Crustacea. Behavior of chromatophores in color change in Palaemonetes.

24. Brown, F. A., Jr., J. Exper. Zool. 71:1-15 (1935). Functional independence of pigments, crustaceans.

25. Brown, F. A., Jr., Biol. Bull. 70:8-15 (1936). Total illumination and chromatophore state, fishes. Ratio of incident to reflected light and color change. Measurement of chromatophore activity.

26. Brown, F. A., Jr., Ecology 20:507-516 (1939). Adaptive background selection by crayfishes.

27. Brown, F. A., Jr., Physiol. Zool. 13:343-355 (1940). Crustacean sinus gland and color change.

28. Brown, F. A., Jr., Physiol. Zool. 19:215-223 (1946). Central nervous sources of crustacean chromatophorotropins. Measurement of chromatophore activity.

29. Brown, F. A., Jr., and Ederstom, H. E., J. Exper. Zool. 85:53-69 (1940). Chromatophorotropins from the crustacean central nervous system.

30. Brown, F. A., Jr., Ederstrom, H. E., and Scudamore, H. H., Anat. Rec. (suppl.) 75:129-130 (1939). Crustacean sinus gland and color change.

31. Brown, F. A., Jr., and Klotz, I. M., Proc. Soc. Exper. Biol. E Med. 64:310-313 (1947). Chemical separation of chromatophorotropins from crustacean central nervous system.

32. Brown, F. A., jr., and SAigh, L. M., Bicl. Bull. 91:170-180 (1946). Central nervous sources of chromatophorotropins, Crustacea.

33. Brown, F. A., Jr., and Sandeen, M. I., Physiol. Zool. 21:361-371 (1948). Light intensity and Uca color change.

34. Brown, F. A., Jr., and Scudamore, H. H., J. Cell. E Comp. Physiol. 15:103-119 (1940). Two chromatophorotropins from crustacean sinus glands.

35. Brown, F. A., JR., and Thompson, D. H., Copeia No. 3, pp. 172-181 (1937). Adaptive background selection by fishes.

36. Brown, F. A., Jr., and Weвв, H. M., Physiol. Zool. 21:371-381 (1948). Diurnal rhythm in Uca color change.

36a. Brown, F. A., JR., and Wевв, H. M., Physiol. Zool. 22:136-148 (1949). Rhythmical Mechanisms in Uca.

37. Brown, F. A., Jr., and Wulff, V. J., I. Cell. \& Comp. Physiol. 18:339-353 (1941). Chromatophorotropins from the crustacean central nervous system.

38. Brücke, E., Dnkschr. Akad. Wiss. Wient. 4:179-210 (1852). Color changes in the chameleon.

39. Bucmiolz, R., Arch. Anat. Physiol. wiss. Med., pp. 71-81 (1863). Color change, fishes.

40. Butcher, E. O., J. Exper. Zool. 79:275-297 (1938). Relation of portions of eyes to color-changes, fishes.

41. DünkeN, B., Ztsclir. wiss. Zool. 116:587-626 (1916). Color change in insect pupae.

42. Dünken, B., Arch. mikr. Anat. 99:222-389 (1923). Color change in insect pupae.

43. Duspiva, F, Sitzungsh. Acad. wiss. Wien. math.-nat. Kl. I, 140:553-596 (1931). Color change in young fishes.

44. Fabre, R., and Lederer, E., Bull. Soc. Chim. biol. 16:105-118 (1934). Chemical nature pigments, crustaceans. 
45. Faure, J. C., Bull. Eut. Res. 23:293-405 (1932). Color-change in Locusts.

46. Fosten, K. W., Proc. Nat. Acad. Sci. 19.535-540 (1933). Control of iridosome changes in Fundulus.

47. Fox, D. L., Ann. Rev. Biochem. 16:443-470 (1947). Chemical nature pigments, review.

48. Fréérice, L., Arch. Zool. Exper. Gen. 7:535-583 (1878). Control color change, cephalopods.

49. Fries, E. F. B., Proc. Nat. Acad. Sci. 28:396-401 (1942). Control of Fundulus leucophores.

50. Fries, E. F. B., Biol. Bull. 82:261-272 (1942). Control of Fundulus xanthophores.

51. Fries, E. F. B., Biol. Bull. 82:273-283 (1942). Dual innervation of teleost melanophores.

52. Fries, E. F. B., Physiol. Zool. 16:199-212 (1943). Control of Fundulus xanthophores.

53. Friza, F., Ztschir. vergl. Physiol. 8:289-336 (1928). Diurnal color changes in Mantids.

54. Fuchs, R. F., Winterstein, Handb. vergl. Physiol., vol. 3, part 1, pp. 1189-1656 (1914). Color change, review.

55. Gabritschevsky, E., 1. Exper. Zool. 47:251-267 (1927). Color changes in spider, Misumena.

56. Gamble, F. W., and Keeble, F. W., Quart. J. Micr. Sci. 43:589-698 (1900). Color changes, crustaceans. Influence of temperature.

57. Geldern, C. E. von, Proc. Calif. Acad. Sci., Ser. 4, 10:77 117 (1921). Chromatophore activity, Reptilia.

58. Giersberg, H., Ztschr. vergl. Physiol. 7:657-695 (1928). Color change in Dixippus. Influence of humidity.

59. Giersberg, H., Ztschr. vergl. Physiol. 9:523-552 (1929). Temperature and morphological color change, insects.

60. Giersenerg, H., Ztschr. vergl. Physiol. 13:258-279 (1930). Control of erythrophores of Phoxinus. Influence of drugs. Dixippus color changes.

61. Gilson, A. S., JR., J. Exper. Zool. 45:415-455 (1926). Color-change in young fishes.

62. Hadley, C. E., Bull. Mus. Comp. Zool. Harvard 69:108-114 (1929). Color changes during mating of lizards.

63. Hanström, B., Zool. Jahrb., Abt. Anat. u. Ont. Tiere. 56:387-520 (1933). The crustacean sinus gland.

64. Hanström, B., Proc. Nat. Acad. Sci. $21: 584-585$ (1935). Sinus gland and color change, Crustacea.

65. Hanström, B., Kungl. Svenska Vetenskap. Handl. 16:1-99 (1937). Relation of portions of eyes to color changes, Crustacea. Crustacean sinus gland and color change.

66. Hanström, B., Kungl. Fysiogr. Sällsk. Handl. N.F. 49:1-10 (1938). Relation of portions of eyes to color changes, Crustacea.

67. HempelmanN, F., Ztschr. wiss. Zool. 152:353-383 (1939). Color change, polychaetes.

68. Hill, A. V., Parkinson, I. L., and Solandt, D. Y., J. Exper. Biol. 12:397-399 (1935). Measurement of chromatophore activity.

69. Hrтснсоск, Н. B., Biol. Bull. 30:26-30 (1941). Adaptation to colored backgrounds, Crustacea.

70. Hofmann, F. B., Arch. Mikr. Anat. 70:361-413 (1907). Innervation, cephalopod chromatophore.

71. Hogben, L. T., The Pigmentary Effector System (1924). Edinburgh, Oliver and Boyd. $152 \mathrm{p}$.

72. Hogben, L. T., Proc. Roy. Soc. Lond., B. 120:1+2-158 (1936). Hypophysis in elasmobranch melanophore control.

73. Hogben, L. T., and Minvish, L., Brit. J. Exper. Biol. 5:295-308 (1928). Control of color changes in chameleons.

74. Hogben, L., and Slome, D., Proc. Roy. Soc. Lond., B. 108:10-53 (1931). Mechanism of chromatophore control, Xenopus. 
75. Hogben, L., and Slome, D., Proc. Roy. Soc. Lond., B. 120:158-173 (1936). Mechanism of chromatophore control, Xenopus.

76. Hocben, L., and Winton, F. R., Proc. Roy. Soc. Lond., B. 93:318-329 (1922). Hypophysis and amphibian color change.

77. Hogben, L., and Winton, F. R., Proc. Roy. Soc. Lond., B. 94:151-162 (1922). Hypophysis and amphibian color change.

78. Hogben, L., and Wrnton, F. R., Proc. Roy. Soc. Lond., B. 95:15-30 (1923). Hypophysis and amphibian color change.

79. Hooker, D., Am. I. Anot. 16:237-250 (1914). Color-change in young Rana.

80. I Hosoi, T., I. Fac. Sci. Imp. Univ. Tokyo 3:265-270 (1934). Chromatophorotropins from the crustacean central nervous system.

81. JANDA, V.. Zool. Anz. 115:177-185 (1936). Color change in skin transplants in Dixippus.

82. Janzen, R., Ztschrt. Morph. Okol. Tiere. 24:327-341 (1932). Color change, leeches.

83. Janzen, R., Zool. Anz. 101:35-40 (1932). Color changes, leeches.

84. Jomnson, L. P., Tr. Am. Micr. Soc. 58:42-48 (1939). Color changes, Euglena.

85. Jofinson, L. P., and Jahn, T., Physiol. Zool. 15:89-94 (19+2). Color changes, Euglena.

86. KaEstner, H., Arch. Entw. Mech. 124:1-16 (1931). Temperature and morphological color change, insects.

87. Kalmus, Hans, Ztschr. vergl. Physiol. 25:494-508 (1938). Diurnal rhythm in Dixippus color change.

88. Keeble, F. W., and Gamble, F. W.. Proc. Roy. Soc. London 64:461-468 (1900). Functional independence of pigments, crustaceans.

89. Keeble, F. IV., and Gamble, F. W., Proc. Roy. Soc. London 71:69-71 (1903). Color changes, crustaceans.

90. Keeble, F. W., and Gamble, F. M., Plit. Tr. Roy. Soc. Lond., B. 196:295-388 (1904). Relationship of morphological to physiological color change, crustaceans.

91. Keeble, F. W., and Gamble, F. M., Proc. Roy. Soc. Lond., B. 198:1-16 (1905). Color changes, crustaceans.

92. Kleiniouz, L. H., Biol. Bull. 69:379-390 (1935). Role of hypophysis in Fundulus color change.

93. Kleinholz, L. H., J. Exper. Biol. 15:474-491, 492-499 (1938). Control of color changes in Anolis.

94. Kleinhorz, L. H., Pub. Staz. Zool. Napoli 17:53-57 (1938). Color change. echinoderm.

95. Knight, H. H., Ann. Ent. Soc. Amer. 17:258-272 (1924). Temperature and morphological color-change, insects.

96. Koller, G., Verh. dtsch. Zool. Ges. 30:128-I32 (1925). Mechanism of chromatophore control.

97. Koller, G.. Ztschr. vergl. Physiol. 5:191-246 (1927). Functional independence of pigments, crustaceans. Color adaptations. Mechanism of chromatophore control.

98. Koller, G., Ztschr. vergl. Physiol. 8:601-612 (1928). Mechanism of chromatophore control, Crustacea.

99. Koller, G., Ztschrr. vergl. Physiol. 12:632-667 (1930). Hormones in crustacean color change.

100. Knös en, H., Kong. Dansk. Videnskap. Selskabet. 9:209-361 (1842). Color changes, Hippolyte.

101. Krüger, P., and Kern, H., Arch. ges. Physiol. 202:119-138 (1924). Melanophores and heat regulation.

102. Kühn, A., and Hғberdey, R. F., Zool. Anz. (suppl.) 4:231 (1929). Adaptation to colored backgrounds, Cephalopoda.

103. Kunn, R., and Lederfr, E., Ber. dtsch. chem. Ges. 66:488-495 (1933). Chemical nature pigments, crustaceans.

104. Kuntr, A., Bull. U. 5. Bur. Fish. 35:1-29 (1916). Adaptation to colored backgrounds, lishes.

105. Laurens, 11., J. Exper. Zool. 16:195-210 (1914). Color change in young Am. blystoma. 
106. Laurens, H., J. Exper. Zool. 18:577-638 (1915). Color change in young Amblystoma.

107. Lieben, S., Zentralhl. Physiol. 20:108-117 (1906). Adrcnalin and color change, frog.

108. Lundstron, H. M., and Bard, P., Biol. Bull. 62:1-9 (1932). Hypophysis in elasmobranch melanophore control.

109. Martini, E., and Achundow, I., Zool. Anz. 81:25-4t (1929). Color changes in Corethra.

110. Mast, S. O., Bull. U. S. Bur. Fish. 34:173-238 (1916). Adaptation to colored background, fishes. Adaptive background selection.

111. Matsushita, K., Sci. Rep. Imp. Univ. Sendai, 4, Biol. 13:171-200 (1938). Dual innervation of teleost melanophores.

112. Matruews, S. A., J. Exper. Zool. 58:471-486 (1931). Mechanism pigment migration in chromatophores, fish.

113. Matrinews, S. A., Biol. Bull. 64:315-320 (1933). Color changes in hypophysectomized Fundulus.

114. Mendes, E. G., Bol. Fac. Filos. Cien. Letr. Univers., Sao Paulo, 15, Zool. 6:285299 (1942). Color change in young fishes.

115. Menke, E., Arch. ges. Physiol. 140:37-91 (1911). Diurnal rhythm and chromatophore state, Crustacea. Influence of temperature on chromatophores.

116. Milus, S. M1., J. Exper. Zool. 64:231-244 (1932). Dual innervation of teleost melanophores.

117. Milne-Edwards, H., Ann. Sci. Nat. ser. 2, Zool. 1:46-54 (1834). Color-change, chameleon.

118. Minkiewicz, R., Bull. Acad. Sci. Cracovie, pp. 918-929 (1908). Adaptation to colored backgrounds, Crustacea.

119. Neill, R. M., J. Exper. Biol. 17:74-94 (1940). Color change in young fishes. Color change in Anguilla.

120. Odionne, J. M., Proc. Nat. Acad. Sci. 19:329-332 (1933). Morphological color change, fishes.

121. Odionne, J. M., Proc. Nat. Acad. Sci. 19:750-754 (1933). Adrenalin and teleost leucophores.

122. Osborn, C. M., Proc. Nat. Acad. Sci. 26:155-161 (1940). Induced pigmentation on ventral surface of flounder.

123. Pakker, G. H., J. Exper. Zool. 3:401-414 (1906). Melanophores and heat regulation.

124. Parker, G. H., Biol. Rev. 5:59-90 (1930). Chromatophores, review.

125. Parker, G. H., Proc. Nat. Acad. Sci. 17:594-596 (1931). Color change, echinoderm.

126. Parker, C. H., Proc. Nat. Acad. Sci. 20:306-310 (1934). Dual innervation of Fundulus melanophores.

127. Parker, G. H., Proc. Am. Phil. Soc. 75:1-10 (1935). Measureinent of chromatophore activity.

128. Parker, G. H., Biol. Bull. 68:1-3 (1935). Innervation of Mustelus melanophores.

129. Parker, G. H., Biol. Bull. 70:1-7 (1936). Color change in young elasmobranchs.

130. Parker, G. H., Biol. Bull. 71:255-258 (1936). Single innervation of Mustelus melanophores.

131. Parker, G. H., Cold Spring Harbor Symp. Quant. Biol. 4:358-370 (1936). Coldblock of chromatophore nerves.

132. Parker, G. H., Proc. Nat. Acad. Sci. 23:206-21l (1937). Control of erythrophores of Holocentrus. Dual innervation of melanophores.

133. Parker, G. H., Proc. Am. Phil. Soc. 77:223-247 (1937). Control of elasmobranch melanophores.

134. Parker, G. H., J. Exper. Biol. 15:48-73 (1938). Control of color changes in Phrynosoma. Influence of temperature.

135. Parker, G. H., Proc. Am. Phil. Soc. 83:379-409 (1940). ACh and fish color change.

136. Parker, G. H., Proc. Am. Phil. Soc. 85:18-24 (1941). Dual innervation of teleost melanophores. 
137. Parker, G. H., J. Exper. Zool. 89:451-473 (1942). Chemical mediator in Mustelus melanophore control.

138. Parker, G. H., Quart. Rev. Biol. 18:205-227 (1943). Vertebrate color changes, review.

139. Parker, G. H., Biol. Bull. 84:273-284 (1943). Methods of measurement of color changes.

140. Parker, G. H., Animal Colour Changes and Their Neurohumors (1948). Cambridge University Press. $377 \mathrm{p}$.

141. Parker, G. H., and Brower, H. P., Biol. Bull. 68:4-6 (1935). Seasonal development of nuptial secondary sex coloration, Fundulus.

142. Parker, G. H., and Rosenbluth, A., Proc. Nat. Acad. Sci. 27:198-204 (1941). Electrical stimulation of melanophore nerves.

143. Parker, G. H., and Scatterty, L. E., J. Cell. E Comp. Physiol. 9:297-314 (1937). Hormonal control, Rana color-change.

144. Perkins, E. B., J. Exper. Zool. 50:71-105 (1928). Pigment migration in chroma tophores, crustacean. Mechanism of chromatophore control.

145. Perkins, E. B., and Snook, T., J. Exper. Zool. 61:115-128 (1932). Mechanism of chromatophore control, Crustacea.

146. Phisalix, C., Arch. Physiol. Norm. et Path., Ser. 5, 4:209-224 (1892). Control color change, cephalopods.

147. Phisalix, C., Arch. Physiol. Norm. et Path., Ser. 5, 6:92-100 (1894). Control color change, cephalopods.

148. Pouchet, G. J. Anat. Physiol. 8:401-407 (1872). Color changes, crustaceans, fishes.

149. Pouchet, G., J. Anat. Physiol. 12:1-90, 113-165 (1876). Color change, crustaceans, fishes.

150. Poucton, E. B., The Colors of Animals (1890). New York, Appleton. 360 p.

151. Priebatsch, Irma, Ztschr. vergl. Physiol. 19:453-488 (1933). Relation of portions of eyes to color changes, insects.

152. Prosser, C. L., Limbach, B. von, and Bennetr, G. W., Physiol. Zool. 20:349354 (1947). Reactions of fish chromatophores to war gases.

153. Rahn, Hermann, Biol. Bull. 80:228-237 (1941). Hypophysis and color change in Rattlesnake.

154. Redfield, A. C., J. Exper. Zool. 26:275-333 (1918). Control of color change in Phrynosoma. Diurnal rhythm of color change.

155. Rynberk, G. van, Ergebn. Physiol. 5:347-571 (1906). Color change, review.

156. SAND, A., Biol. Rev. 10:361-382 (1935). Control of color changes in Chameleons.

156a. Sandeen, M. I., Physiol. Zool. (in press). Chromatophorotropins in Uca.

157. Sars, G., Histoire naturelle des Crustacés d'eau douce de Norvège (1867). Christiania. 145 p. Color changes, crustaceans.

158. Scharrer, E., Ztschr. vergl. Physiol. 7:1-38 (1928). Pineal body and color changes, fishes.

159. Schliep, W., Zool. Jahrb. Physiol. 30:40-132 (1910). Color change in Dixippus. Diurnal rhythm in Dixippus color change.

160. Schliep, W., Zool. Jahrb. Physiol. 35:225-232 (1915). Color change in Dixippus. Diurnal rhythm in Dixippus color change.

161. Schlieper, C., Ztschr. vergl. Physiol. 3:547-557 (1926). Color change in Hyperia galba.

162. Schlottke, E., Ztschr. vergl. Physiol. 3:692-736 (1926). Temperature and morphological color change, insects.

163. Schlottke, E., Ztschr. vergl. Physiol. 20:370-379 (1934). Temperature and morphological color change, insects.

164. Sereni, E., Boll. Soc. Ital. Biol. Sper. 2:377-381 (1927). Control color change, cephalopods.

165. Sereni, E., Ztschr. vergl. Physiol. 8:488-600 (1928). Inhibitory color change center, cephalopods. Postural chromatophore reflexes, cephalopods.

166. Sereni, E., Boll. Soc. Ital. Biol. Sper. 3:707-711 (1928). Betaine and Tyramine in cephalopod color change.

167. Sereni, E., Boll. Soc. Ital. Biol. Sper. 4:749-753 (1929). Salivary gland (tyramine) and cephalopod color change. 
168. Sereni, E., Ztschr. vergl. Physiol. 12:329-503 (1930). Color change mechanism, cephalopods.

169. Serenl, E., Biol. Bull. 59:247-268 (1930). Direct action of betaine and tyramine on cephalopod chromatophores.

170. Siebold, K. T. E. von, Dic Süsswasserfische von Mitteleuropa (1863). Leipzig. 431 p. Color-change, fishes.

171. SJögren, S., Zool. Jahrb., Abt. Anat. u. Ont. Ticre. 58:145-170 (1934). The crustacean sinus gland.

172. Slome, D., and Hogben, L. T., So. Afr. J. Sci. 25:329-335 (1928). Measurement of chromatophore activity.

173. Smith, D. C., Biol. Bull. 58:193-202 (1930). Temperature and color change, Crustacea.

174. Sмiтh, D. C., J. Exper. Zool. 58:423-453 (1931). Autonomic drugs and teleost color change.

175. Smith, D. C., J. Cell. \& Comp. Physiol. 8:83-87 (1936). Measurement of chromatophore activity.

176. Smith, D. C., and Smith, M. T., Biol. Bull. 67:45-58 (1934). Control of erythrophores of Holocentrus.

177. Smith, H. G., Proc. Roy. Soc. Lond., B. 125:250-263 (1938). Dual control of isopod melanophores.

178. Smith, P. E., Anat. Rec. 11:57-64 (1916). Hypophysis and amphibian color change.

179. Smith, R. I., Physiol. Zool. 15:410-417 (1942). Mechanism of color change, leeches.

180. Spaeth, R. A., Anat. Anz. 44:520-524 (1913). Pigment migration in chromatophore, fish.

181. Spaeth, R. A., Am. J. Physiol. 41:597-602 (1916). Measurement of chromatophore activity.

182. Steggerda, F. R., and Soderwall, A. L., J. Cell. \& Comp. Physiol. 13:31-37 (1939). Dual humoral control of melanophores, Rana pipiens.

183. Steinach, E., Pflizg. Arch. ges. Physiol. 87:1-37 (1901). Tactile stimuli and color change, cephalopods.

184. Stschegolew, G. G., Rev. Zool. Russe 7:149-166 (1927). Color change, leeches.

185. Sumner, F. B., Am. Nat. 69:245-266 (1935). Protective value of fish colorchange.

186. Sumner, F. B., Biol. Rev. 15:351-375 (1940). Morphological color change in fishes and amphibians, review.

187. Sumner, F. B., Biol. Bull. 84:195-205 (1943). Morphological color changes; Girella, Fundulus.

188. Sumner, F. B., Proc. Nat. Acad. Sci. 30:285-294 (1944). Morphological colorchange, guanin, fishes.

189. Sumner, F. B., and Doudoroff, P., Proc. Nat. Acad. Sci. 23:211-219 (1937). Ratio of incident to reflected light and morphological color change.

190. Sumner, F. B., and Doudoroff, P., Biol. Bull. 84:187-194 (1943). Assay of melanin in fislses.

191. Sumner, F. B., and Keys, A. B., Physiol. Zool. 2:495-504 (1929). Ratio of incident to reflected light and color change.

192. Tomira, G., J. Shanghai Sci. Inst. IV, 2:237-264 (1936). Color-change in young fishes. Color change in blinded teleosts.

193. Tomita, G., J. Shanghai Sci. Inst. IV, 4:1-8 (1938). Dual innervation of teleost melanophores.

194. Tomita, G., J. Shanghai Sci. Inst. IV, 5:151-178 (1940). Dual innervation of teleost melanophores.

195. Uexküll, J. von, Ztschr. Biol. 34:319-339 (1896). Color change, echinoderm.

196. Verne, J., Arch. Morph. Gén. Expér. Paris (16) 168 pp. (1923). Chemical nature pigments, crustaceans.

197. Waring, H., Proc. Roy Soc. Lond., B. 128:343-353 (1940). Dual hormonal control of Anguilla melanophores.

198. Waring, H., Biol. Rev. 17:120-150 (1942). Vertebrate color changes, review. 198a. WebB, H. M., Physiol. Zool. (in press). Rhythmical mechanisms in Uca. 
199. Wells, G. P., Nature 129:6-687 (1932). Color change, leeches.

200. WYKes, U., J. Exper. Biol. 14:79-86 (1937). Measurement of chromatophore activity.

201. Wranan, L. C., J. Exper. Zool. 40:161-180 (1924). Color change in young fishes.

202. Young, J. Z., Quart. J. Micr. Sci. 75:571-624 (1933). Control of elasmobranch melanophores.

203. Young, J. Z., J. Exper. Biol. 12:25+-270 (1935). Pineal body in color change, Lampetra. Control of Lampetra melanophores.

204. Zondex, B., and Квонn, J., Klin. Wchnschr. 11:405-408 (1932). Intermedin in melanophore responses.

205. Zoond, A., and Eyre, J., Phil. Tr. Roy. Soc. Lond., B. 223:27-55 (1934). Control of color change in chameleons. Diurnal rhythm of color changes. 


\section{Endocrine Mechanisms}

HE PRODUCTION and dispersal within the organism of chemical substances which subserve definite integrating and coordinating roles, and thereby supplement the activity of the nervous elements, are characteristic of all living things. Such substances may be referred to descriptively as chemical coordinators. In the broadest sense, every substance which enters the body fluids from the external environment or from the constituent cells of a higher organism and thus contributes to the normal com. position of the internal medium is a chemical coordinator. $\mathrm{O}_{22}$ and $\mathrm{CO}_{2}$, for example, certainly operate importantly in the coordination of organismic activities. Other coordinatory substances, such as the D vitamins, maly enter the body or may under certain circumstances be synthesized within certain cells of the organism and thence be liberated into the blood. Some chemical substances, e.g., secretin, appear to be more restricted in their region of origin within the body and adaptively participate in a specialized activity within the organism, namely, stimulation of the liberation of pancreatic juice in response to the presence of food in the duodenum. Finally, many groups of higher organisms have differentiated specialized glanclular cells, tissues, or organs which elaborate coordinatory substances for the organism as a whole. This latter development seems to have paralleled the general specialization and restriction within the organism of numerous other organs and organ systems; these developments could come about as soon as an effective mechanism of internal transport was provided in the form of a circulatory system. It is therefore not surprising to find most described instances of the existence of specialized endocrine organs among the annelids, molluses, and arthropods, $37.71,93,98,137,166$ in addition to the vertebrates.

The terms hormones and endocrines are applied to special chemical coordinators which are produced at some more or less restricted region or regions within the organism and which possess specific physiological action at that or other regions within the body. They are usually, but by no means always, produced in well defined glandular organs. No two endrocrinologists may agree on how broadly one should interpret such a definition of a hormone. Obviously, in view of the complete intergradation of all types of chemical coordinators with one another it is impossible to make a sharp natural division between hormones, on the one hand, and other chemical coordinators, on the other. Ascorbic acid may fulfill the definition of a hormone for the rat, for example, and yet fulfill the definition of a vitamin for man.

Our knowledge of the modes of action of chemical coordinators is still far too scanty to permit an attempt to make any distinction in terms of how the substance exerts its infuence, e.g., whether it actively participates in chem- 
ical reactions or acts only as a biocatalyst. In view of the inherent impossibility of arriving at a clear and restricted definition of a hormone, it is proposed in this chapter to deal only with those chemical coordinators which have come to be considered by the majority of investigators in those fields as endocrines.

The important point to emphasize for comparative physiological purposes is that in a number of phyla and classes of animals special chemical substances essential to the integrative activities of the body are produced. The point of origin within the organism, the specific chemical nature of the hormone, and the routes or forces of transport are secondary in importance to the abilities of these hormones to induce certain characteristic, and often differential, effects within the animal. Furthermore, the nature of the effects produced depends as much on the nature of the reacting tissues as on the chemical properties of the circulating hormone. Hormones spreading randomly through the body in the body fluids are obviously powerless to produce tissue and organ differentiation, or induce any directed or organized activities in the absence of an underlying gene-determined differentiation. The activities of hormones are in a sense, therefore, superimposed on the basic pattern of the organism, bringing into functional integration the numerous and complex latent differentiations. In a study of endocrine mechanisms the phylogenetic and ontogenetic development of ability to respond to an endocrine is therefore as important as the appearance of the endocrine itself.

\section{HORMONES AND GENERAL GROWTH AND DIFFERENTIATION}

Vertebrates. Vertebrates are characterized by a growth and differentiation in which the organism increases in size through the accumulation of water, salts, and organic constituents. Not only is there an absolute inerease in the quantities of these substances, but there are also during this period characteristic changes in the ratios of these within the body until growth terminates in the adult. The latter period is one where a balance of constructive and destructive phases of metabolism is reached and a steady state is maintained. Variations from this steady state are largely the results of fat depositions; the proportions of protein, ash, and water remaining strikingly constant. Hormones are known to operate not only in regulating the growth and differentiation processes but also in the maintenance of the final steady state of the adult. We shall first treat the developmental processes, although there is actually no true separation between the hormonal control of growth, on the one hand, and of general metabolism, on the other.

The anterior lobe of the pituitary is essential to normal growth in the mammal, its importance being least in early embryonic development, but gradually increasing thereafter. The subject has been well reviewed by Long. ${ }^{10 t}$ Hypophysectomy results in the rapid cessation of growth in 80-100 gm. rats, and injection of crude alkaline pituitary extracts into such animals restores growth. Such extracts also accelerate growth in normal young mammals or stimulate further growth in those whose growth-curve has reached a plateau. The additional growth that results resembles quite closely normal growth and gigantism. One of the important sites of action of the growthpromoting pituitary extracts is the epiphyseal cartilage. Histological evidence of an activation of this tissue is an early response to the extract injections. 
Furthermore, at least in man and some dogs, an excess of pituitary substance after closure of the epiphyses results in the characteristic bone deformities of acromegaly. Skeletal abnormalities in the skull are also observed in extract-treated or hypophysectomized mammals of other species.

There is also clear evidence that crude alkaline pituitary extracts exert a strong influence on growth of the soft tissues of the body. Protein metabolism is affected in such a way as to increase the ratio of protein synthesis to protein degradation. A number of investigators have observed a significant drop in blood non-protein nitrogen (N.P.N.), urea, and amino acids, and a substantial drop in urinary N.P.N. after the injection. Mice injected with anterior lobe extract daily for 105 days have been shown to have increased water, ash, and protein content. Fat content, on the other hand, may even be reduced below that of controls. Thus the growth induced by the extract resembles closely the growth observed in normal young and rapidly growing mammals. These changes can also be shown to occur in fasting as well as in well-fed animals. These influences of the pituitary on growth are cxerted both through its known influences on other growth-essential endocrine glands, such as the adrenals, thyroid, pancreas, and testes, and directly through the action of a growth hormone arising in the pituitary itself.

The problem of resolving the role of a growth-promoting principle from the pituitary in the face of the numerous trophic influences of this origin on other glands is a difficult one. Anterior lobe extract has been shown to modify protein metabolism, as indicated by the striking drop in N.P.N. of blood even after adrenalectomy, thyroidectomy, or pancreatectomy, although after pancreatectomy the mammal must receive a standard insulin treatment in order to obtain the N.P.N.-reducing action in response to the pituitary extracts. These facts indicate that the action of the pituitary principle in accelerating protein synthesis is exerted directly in the body rather than through activities of other endocrines.

The thyroid gland is essential to growth. Growth virtually ceases after thyroidectomy in the young mammal, a dwarfed condition resulting. Administration of thyroxin to a thyroidectomized rat will restore normal growth. If hypophysectomy has also been performed, the thyroid extract shows no growth-promoting action. In such thyroidectomized and hypophysectomized animals, anterior lobe extract alone will promote growth, but its action is greatly increased by addition of thyroid hormone. In new-born rats, unlike in older ones, thyroidectomy is followed by a failure of response to the pituitary growth hormone. This appears to suggest that thyroxin is essential for some developmental processes which must be completed before the capacity to respond directly to the pituitary is present.

Removal of the adrenal cortex of the mammal leads in a relatively short time to death of the organism. Failure to grow is therefore obviously one of the less significant accompaniments of adrenalectomy. Growth is restored in adrenalectomized animals by administration of cortical extracts; after both adrenalectomy and hypophysectomy the growth response to anterior lobe extract is greatly enhanced by simultaneous administration of cortical extracts.

Growth ceases or is greatly retarded in the absence of, or in deficiency of, insulin from the islets within the pancreas. This hormone exerts qualitatively the same type of action on blood N.P.N. as does the anterior lobe growth 
hormone. Testosterone injections are also known to be followed by some nitrogen retention and weight gain.

Some of the roles of hormones in mammals in influencing growth through protein metabolism are diagrammed in Figure 277.

It was discovered many years ago that the feeding of thyroid gland material to amphibians hastens metamorphosis ${ }^{66}$ and, conversely, that removal of the rudiment of the thyroid from larval forms prevents metamorphosis. ${ }^{7}, 80$ Metamorphosis can be obtained in thyroidectomized individuals by administration of thyroglobulin or thyroxin. Less effective is di-iodotyrosine, and still less effective are inorganic salts of iodine or elemental iodine, which, however, will induce metamorphosis if they are present in sufficient quanti-

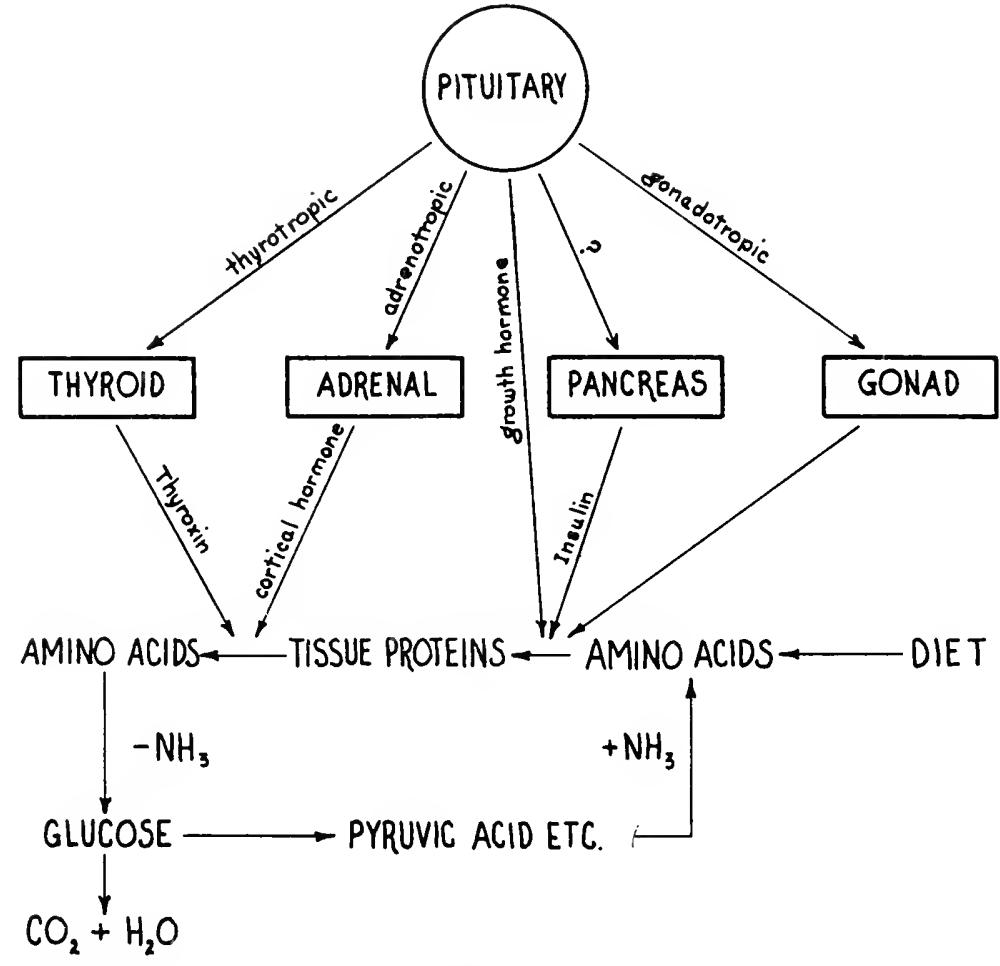

Fig. 277. Diagram summarizing the major influences of hormones on growth in manmals. Modified from Long. ${ }^{104}$

ties. ${ }^{11}$ This response to thyroxin is quite distinct from the increase in the general rate of metabolism. Dinitrophenol, a powerful stimulant of metabolism, has no influence on metamorphosis. Acetylated thyroxin, on the other hand, retains its normal capacity to induce metamorphosis although it has lost entirely its metabolism-stimulating action.

Various amphibian species differ greatly among themselves in their responsiveness to thyroxin. During their early development, none of them shows ability to respond to this hormone, but most acquire the capacity at some particular stage in their development. Some species, like the Mexican axolotl, never develop any considerable degree of reactivity to this metamorphosis- 
inducing factor, and hence normally do not metamorphose but can be made to do so by large doses of thyroxin; others, such as Necturus, appear never to develop the reactivity and never metamorphose. That the failure of metamorphosis in Necturus is the result of failure of tissue response rather than of the absence of an appropriate thyroid principle is shown by the fact that Necturus thyroid will accelerate metamorphosis in Rana clamitans. ${ }^{1.50}$

In metamorphosis there is an orderly sequence of changes. This sequence appears due, at least in part, to a graded responsiveness of the various tissues of the organism to the hormone. ${ }^{10,29.59}$

The capacity of amphibians to metamorphose is also lost after hypophysectomy. ${ }^{5}, 7,1+8$ This activity has been traced to the anterior lobe; the principle involved is ineffective in inducing metamorphosis after complete thyroidectomy. "The thyroids are also known not to accumulate any colloid in the absence of the hypophysis. This demonstrates that the production and liberation of the thyroid hormone in amphibians, and hence metamorphosis, is under the control of the hypophysis.

Among teleost fishes it is known that the thyroid gland is concerned in metamorphosis in eels and in certain flatfishes, ${ }^{69,} 117$ and that changes in the amount of thyroxin available will, in other species, result in alterations in growth rates and in body form. ${ }^{65},{ }^{72}, 103$ Furthermore, as in higher vertebrates, the pituitary yields a thyrotropic principle on whose activity the development and secretion of the thyroid depends. ${ }^{6},{ }^{63}$ It is interesting that, despite these clearly evident influences of the thyroid on teleost growth and development, within this group of animals the thyroid appears to have no influence on the general metabolic rate. ${ }^{60,113,145}$

Invertebrates: Gene Hormones. The color of wild-type eye of Drosophila is due to the presence of two types of colored granules: purplish red and brownish yellow. The chemical composition of these pigments is unknown, but they are considered to belong to a new group of substances named ommatins, of low molecular weight. ${ }^{20}$ The reddish pigment is called erythrommatin; the brownish, phaeommatin. In the eye these pigment molecules are believed to be conjugated with proteins. These retinal pigments differ greatly in composition among the insects thus far investigated. The absence of either one or both of these pigments in Drosophila is observed in certain mutant stocks, ${ }^{108}$ the vermilion group of mutants possessing only the red, the mutant brown possessing only the brownish, and the mutant white possessing neither of these two pigments.

In Drosophiia the eyes differentiate during pupal development from the optic imaginal discs. When the differentiation of the eye is nearly complete, frrst the brownish pigment makes its appearance and later the red. The developing eyes of the wild-type flies thus pass through a brownish phase in their development. The stimulus for development of the eve discs, as with all other aspects of pupal development, appears to require the presence of the general growth and differentiation hormone, ${ }^{49}$ which is treated in the section on molt, pupation, and metamorphosis in insects.

It has been clearly established that the development of brown pigment in the eye depends on the presence of a blood-born factor which has been named the $V+$ hormone. $^{1 \mathrm{~s}, 57}$ Eye discs from a vermilion larva, normally producing only red pigment, when transplanted into a wild-type larva develop 
into wild-type eyes, containing the brownish pigment in addition to the red. Furthermore, blood from a wild-type pupa transfused into a vermilion one will induce development of the wild-type pigmentation. The $\mathrm{V}^{+}$hormone is known to be released from the malpighian tubules and the fat bodies. ${ }^{16}$ That the eye-discs themselves may also produce some hormone is demonstrated by transplanting wild-type or brown eye discs into vermilion larvae, the discs in these cases developing to a greater or less extent their normal wild-type or brown coloration, respectively.

The $\mathrm{V}+$ hormone is neither species-specific nor genus-specific; ${ }^{17}$ it is present in several species of Drosophila as well as in Ephestia, Bombyx, and other insects. In Ephestia and Bombyx, the same hormone thus operates on other pigmentary types in eye-color determination. It is believed that this hormone is identical with the substance kynurenin, ${ }^{19}$ a product of general tryptophane metabolism, and considered to arise in the insects in association with the extensive protein degradation accompanying metamorphosis.

Two mutants of the vermilion group, vermilion and cinnabar, both possess eyes containing only red pigment. The eyes of the two are distinguishable only with difficulty. It can be shown, however, by reciprocal eye-disc transplantation between larvae of these two mutant types that the reason for the failure to form brown pigment differs in the two. Vermilion discs transplanted into cinnabar larvae develop wild-type pigmentation, thus indicating the presence of $\mathrm{V}+$ hormone. Cinnabar discs transplanted into vermilion larvae, on the other hand, develop only cinnabar pigmentation. Since both vermilion and cinnabar discs develop wild-type pigmentation in wild. type hosts, it is clear that cinnabar lacks a second factor normally found in wild-type blood, a factor which has been called the $\mathrm{Cn}+$ hormone. ${ }^{18,57} \mathrm{An}$ assay of the blood of various mutant types for $\mathrm{V}+$ and $\mathrm{Cn}+$ hormones by the general technique of implanting vermilion and cinnabar eyediscs shows that the $\mathrm{Cn}+$ hormone is never found in the absence of $\mathrm{V}+$ hormone and that as $\mathrm{V}+$ decreases in amount so always does $\mathrm{Cn}+$. Furthermore, mutants without $\mathrm{Cn}+$ may possess the ability to produce this latter if $\mathrm{V}+$ is supplied. It has therefore been assumed that in the normal metabolism of the wild-eye type of Drosophila $\mathrm{V}+$ and $\mathrm{Cn}+$ are formed sequentially, the former being a normal precursor of the latter, with the vermilion mutant unable to form $\mathrm{V}+$ but nevertheless able to make the conversion of $\mathrm{V}+$ to $\mathrm{Cn}+$ and with cinnabar mutants able to produce $\mathrm{V}+$ but unable to make the conversion to $\mathrm{Cn}+$. The $\mathrm{Cn}+$ hormone, like the $\mathrm{V}+$ hormone, is found in genera other than Drosophila and has been shown to be produced in the malpighian tubules and in the eye discs themselves. It is not found, however, in the fat bodies. Its chemical composition is not yet known.

Just as we are now well aware that a single gene may determine the presence or absence of such blood-borne integrating factors in development as $\mathrm{V}+$ and $\mathrm{Cn}+$ hormones, so is the evidence clear that single gene changes may determine the abilities of the target organs to respond to the hormonal substances. The mutant scarlet can be shown, by implantations of their eye discs into other test larvae, to produce both $\mathrm{V}+$ and $\mathrm{Cn}+$ hormones and yet produce no brown pigment, ${ }^{58}$ and the mutant white produces neither brown nor red. This indicates the need for the presence of specific "substrate" substances within the eye to permit the normal color responses. All 
of the relationships discussed here are brought together in Figure 278.56

There is substantial evidence that the foregoing gene hormones are used up in the reactions in which they are involved, probably entering directly

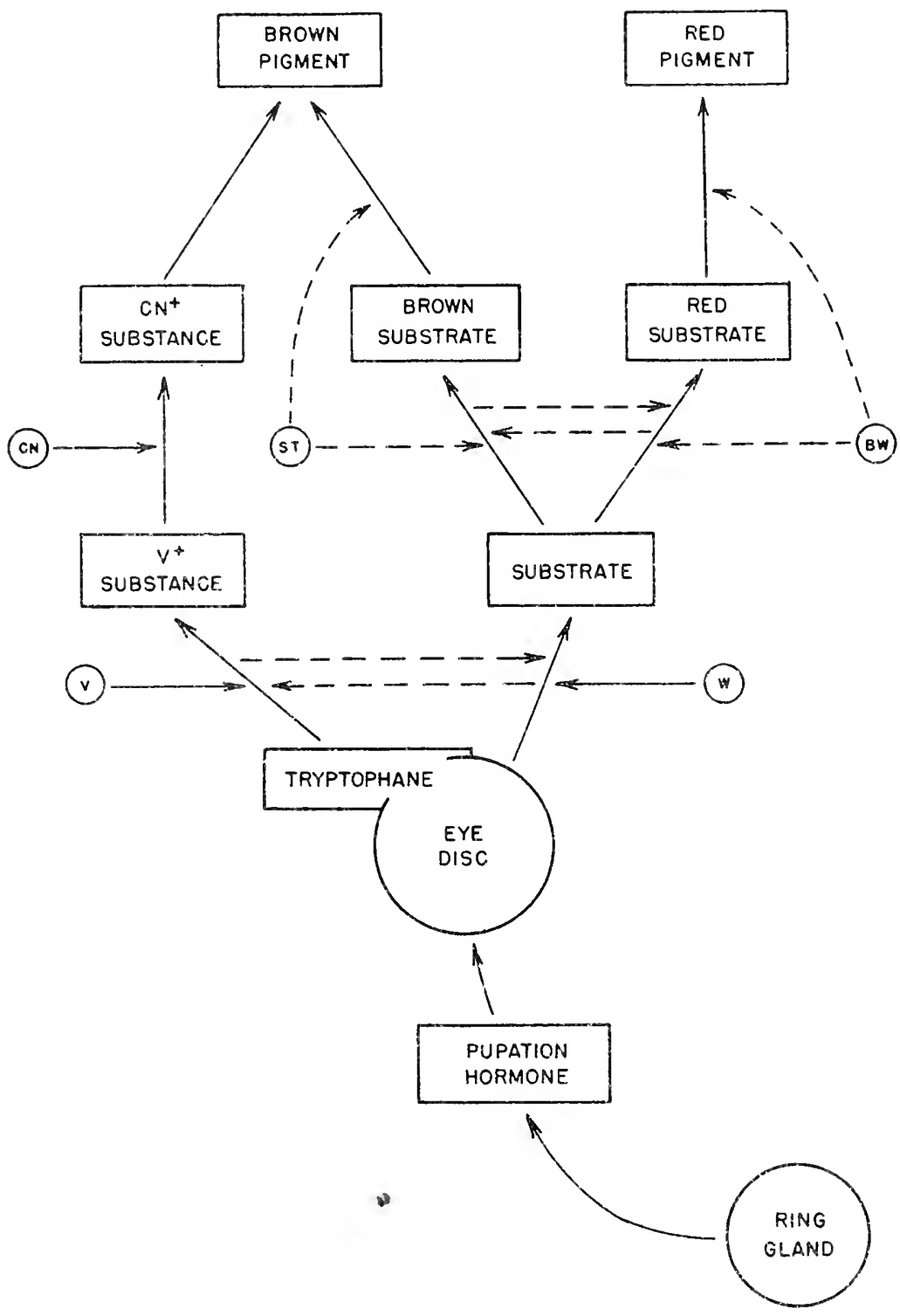

Fig. 278. Diagram summarizing the roles of gene hormones and certain other factors in the development of eye pigments in Drosophila. From Ephrussi. ${ }^{5 \theta}$

into certain of the reactions involved in pigment production. There seems to be a direct relationship between the quantity of hormone made available and the amount of eye pigment produced. However, since the same hormones operating in different insect groups seem to be responsible for the de- 
velopment of pigments of quite different chemical composition, it would appear doubtful that the substances actually become a portion of the chromophore molecule.

We see from the preceding discussion that in the production and operation of gene hormones we have a most favorable opportunity to investigate in some detail instances of the production and action of hormones in terms of the metabolic transformations proceeding within cells.

Molt, Pupation, and Metamorphosis in Insects. The post-embryonic development of insects comprises an orderly series of stages in the course of which the insect becomes transformed from a larva to an adult or imago (Fig. 279). The process involves growth by means of a series of molts, and a metamorphosis in which the larval characters are lost and imaginal ones

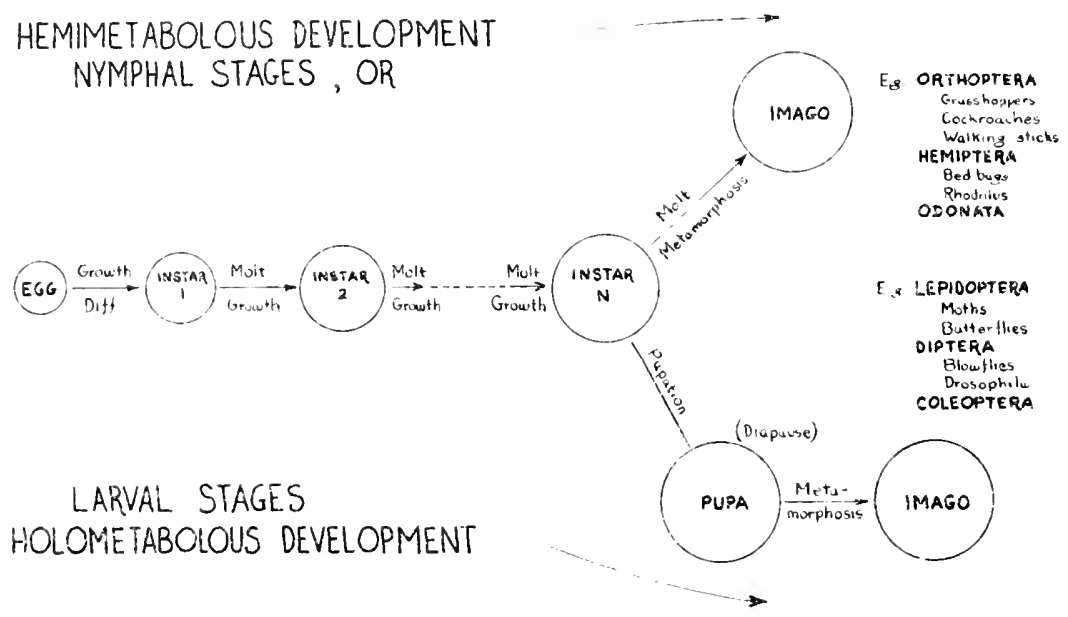

Fig. 279. Diagram representing the principal stages and processes involved in the developinent of hemimetabolous and of holometabolous insects.

differentiated. In the hemimetabolous insects, such as the orthopterans (grasshoppers, cockroaches, walking sticks, etc.), the hemipterans (bedbugs, Rhodninis, etc.), and Odonata, the transition from larval to adult condition is typically a gradual one, in which the developing young, typically known as nymphs, in succeding molts come gradually to resemble more and more nearly the adult. In such hemimetabolous insects, however, the last nymphal molt is usually assuciated with by far the most striking transformation and hence is commoniy considered the metamorphic molt. In holonetabolous insects, such as the Lepidoptera (moths, bufterflies, etc.), Diptera (Drosophila, blowflies, eic.), and Coleoptera, very little change of form in the direction of the adult occurs during the larval series of molts, these being primarily grow th changes, but the last larval molt is associated with the formation of a pupa. During the pupal stage the larval organism becones completely transformed into an adult, a spectacular metanorphosis. There is no fundamental difference between the two foregoing types of development, the observed diflerences being primarily the result of differences in the times at which the processes of imaginal differentiation oceur. These latter are extended to a considerable extent over the whole larval period in the 
hemimetabola and largely confined to the pupal stage in the holometabola.

The number of larval or nymphal stages in development differs from species to species, but is usually a constant for each species. The bug Rhodnins, for example, has five nymphal stages or instars, the orthopteran, Dixippus, has six, and the dipteran, Drosophila, has three. It is now well established that the sequence of erents in the growth and differentiation in the post-embryonic insect is in good measure under the integrating action of hormones derived from glandular sources in the anterior region of the insect. ${ }^{31}$

Experiments on the tropical bug, Rhodnins prolixus, have provided a clear demonstration of the action of two developmental hormones, ${ }^{16 \times .}{ }^{169 ?, 170}$ one inducing molt accompanied by metamorphosis, and the other inhibiting metamorphosis. The former has been called the molting hormone or the growth and differentiation (GD) hormone, and the latter the inhibitory or juvenile hormone. The roles of these hormones are readily investigated in developing Rhodnius. This insect, at each nymphal instar, molts a definite number of days after a meal of blood. The distention of the abdomen appears to serve as the adequate stimulus. Following decapitation these insects will survive for 6 to 10 months. It has been shown by decapitation at different intervals after feeding that there is a "critical period" occurring a few days after the feeding. Nymphs decapitated before this period never undergo molt; those decapitated afterward do. That the critical period constitutes a time when a blood-borne molt-inducing hormone is being liberated from some organ in the head is shown by experimental telobiosis (Fig. 280, A) of two decapitated individuals. Under such conditions, for example, molting in a fourth stage nymph decapitated before the critical period will be induced by a fourth stage nymph decapitated after the critical period. Both thereby become fifth stage nymphs.

The fifth nymphal stage, upon molt, typically metamorphoses at that time into an adult. If a fifth stage nymph is decapitated after its critical period it. can be shown by telobiotic union with a first or second stage nymph decapitated before the critical period, that the fifth stage nymph possesses within its blood factors which determine molt with metamorphosis. The attached first or second stage nymph becomes in this instance a diminutive adult. If a fourth stage nymph which would normally molt to become a fifth stage nymph is decapitated after the critical period and united with a fifth stage nymph decapitated before the critical period, the latter molts and becomes a giant, supernumerary sixth stage nymph instead of an imago. Furthermore, although the adult normally does not molt again, it can be made to do so by telobiotic and parabiotic union to two or three fifth stage nymphs decapitated after the critical period. If fourth stage instead of fifth stage nymphs are used, the adult on molt shows a partial return to the nymphal condition (Fig. 280, B).

From such experiments it is readily seen that the blood of a nymph after the critical period contains factors that determine molting either without or with an accompanying extensive metamorphosis, the blood of the molting fifth-stage nymph, alone, inducing metamorphosis. A careful study of the results of decapitation of third and fourth instars at different times during the critical period itself shows that, of those molting after decapitation, those 
decapitated early in the period show a significant tendency toward premature metamorphosis, while those decapitated later do not do so. This observation has been interpreted as indicating that during the critical period of the first four instars a molting and metamorphosis hormone is first liberated and that quickly thereafter a metamorphosis-inhibiting principle is also secreted. The fifth instar alone in Rhodnius fails to produce the latter.

The source of the metamorphosis-inhibiting or juvenile hormone is the corpus allatum, a median unpaired gland in Rhodnins located in the posterior region of the head. The source of the hormone inducing molting and differentiation is in the pars intercerebralis of the dorsal mid-region of the brain, probably in certain neurosecretory cells shown histologically to be located there. The elongated form of the head of Rhodnius renders it very

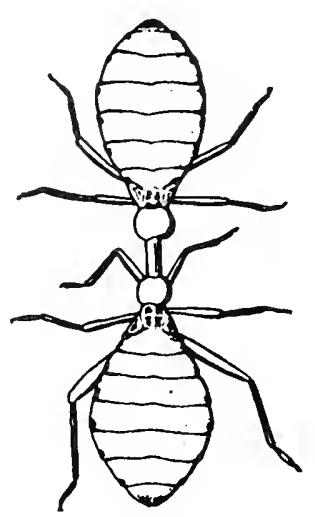

A

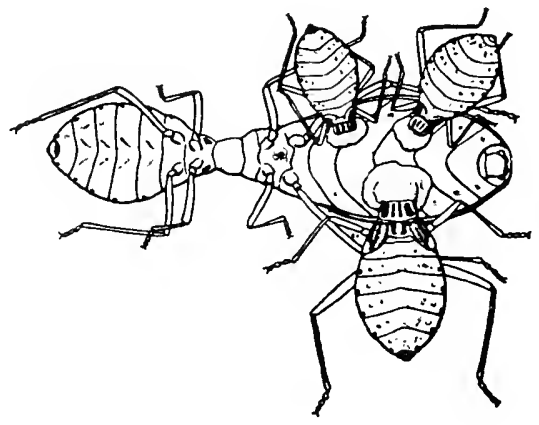

B

Fig. 280. Experimental telobiosis and parabiosis in the bug, Rhodnius. A, Telobiosis involving only nymphal instars. $B$, Fourth and fifth instar nymphs united telobiotically and parabiotically with an imago. From Wigglesworth. ${ }^{165,170}$

simple to cut the head transversely in such a manner as to retain the corpus allatum while the brain is removed. Utilizing this technique in conjunction with telobiotic experiments, as well as by implanting corpora allata and pars intercerebralis regions of brains into nymphs decapitated before the critical period, one can obtain at will either a nymphal molt or a metamorphosis in Rhodnius.

The metamorphosis and the juvenile hormones are not species-specific, and the hormones of Rhodnizis will induce similar changes in other hemipterans, suclı as Triatoma and Cimex.

Among the orthopterans, Dixippus, ${ }^{12 \pi,}, 1: 2$ Leucophaea, ${ }^{13:}$ and Melanoplus, $1: 3$ which have been investigated at some length and which also show hemimetabolous development, there is as yet no clear proof of the existence or site of origin of a molting or CD hormone, although it is generally assumed one is present. The corpora allata in all, however, produce a juvenile hormone. In Dixippus, which normally has six nymplal stages, removal of the corpora allata in the third nymphal stage results in a premature metamorphosis, although two nymphal molts usually intervene between the op- 
eration and the metamorphosis. This latter is interpreted to mean that the hormone from the gland is stored somewhere in the body over this period Transplantation of corpora allata from third or fourth instar nymphs into sixth instar nymphs of Dixiputs may produce giant imagos which have undergone as many as three or four additional nymphal molts. In Leucophaea which normally possess eight nymphal instars allatectomy of a seventh instar before the critical period results in an adult-like stage following the molt. Such an individual has been termed an "adultoid" and differs from adults in having a smaller size and shorter wings. Allatectomy in fifth or sixth instars results on molt in "pre-adultoids" which require one additional molt to produce "adultoids."

In the holometabolous dipterans the ring gland of the larva, located dorsally to the brain and between the brain lobes, is the source of a hormone inducing pupation. ${ }^{67,68}$ The role of this gland is readily demonstrated by ligating the larva to constrict it into two portions, one containing the brain, and the other without it. ${ }^{61}$ If this operation is performed before a certain "critical period," only that portion containing the ring gland pupates; if constriction is produced after the critical period, both portions pupate. Transplantation of a ring gland from a last larval instar into a first larval instar produces premature pupation in the latter. Ring gland implants will also induce pupation in a portion of a larva cut off by ligation from its own ring gland before the critical period. This hormone appears also to be the effective one operating in metamorphosis as well as in pupation itself. ${ }^{21}$ Imaginal discs implanted into the hemocoele of adult flies will not differentiate unless ring glands of late larvae are also implanted. ${ }^{68}$ Furthermore, the metamorphosis hormone of Lepidoptera will induce pupation in dipterans. ${ }^{21}$

The corpora allata of the Lepidoptera, like those of the hemipterans and orthopterans, are the source of a juvenile hormone. Allatectomy in caterpillars of younger instars is followed by a premature pupation, and, conversely, implantation of corpora allata from early instars into caterpillars ready to pupate will significantly delay the pupation. ${ }^{33}$

From the time of the original experiments of Kopec ${ }^{99}$ on lepidopteran pupation and metamorphosis it has been known that at a certain "critical period" prior to pupation a hormone is liberated from the anterior portion of the larva and that this is essential for pupation and metamorphosis. In the silkworm moth, Bombyx, this hormone is produced in the prothoracic gland located in the prothoracic segment. ${ }^{62}$ If the prothoracic segment is cut off by ligation from the more posterior regions of the body immediately after pupation, the posterior portion fails to develop further. If the constriction is made 12 to 18 hours after pupation the posterior portion metamorphoses normally. If prothoracic gland is implanted into the posterior portion of a pupa ligated before the 12 to 18 hour "critical period" metamorphosis of this part also proceeds normally; or an abdomen cut off by constriction may be induced to metamorphose by connection of its hemolymph cavity with that of a normal metamorphosing specimen, even when the connection is made by way of a glass capillary tube.

In the giant silkworm moth, Platysamia, after pupation, a dormant period of diapause exists for some 5 to 6 months in pupa maintained at $25^{\circ} \mathrm{C}$. If the brain is removed from such diapausing pupa the insect never metamorphoses 

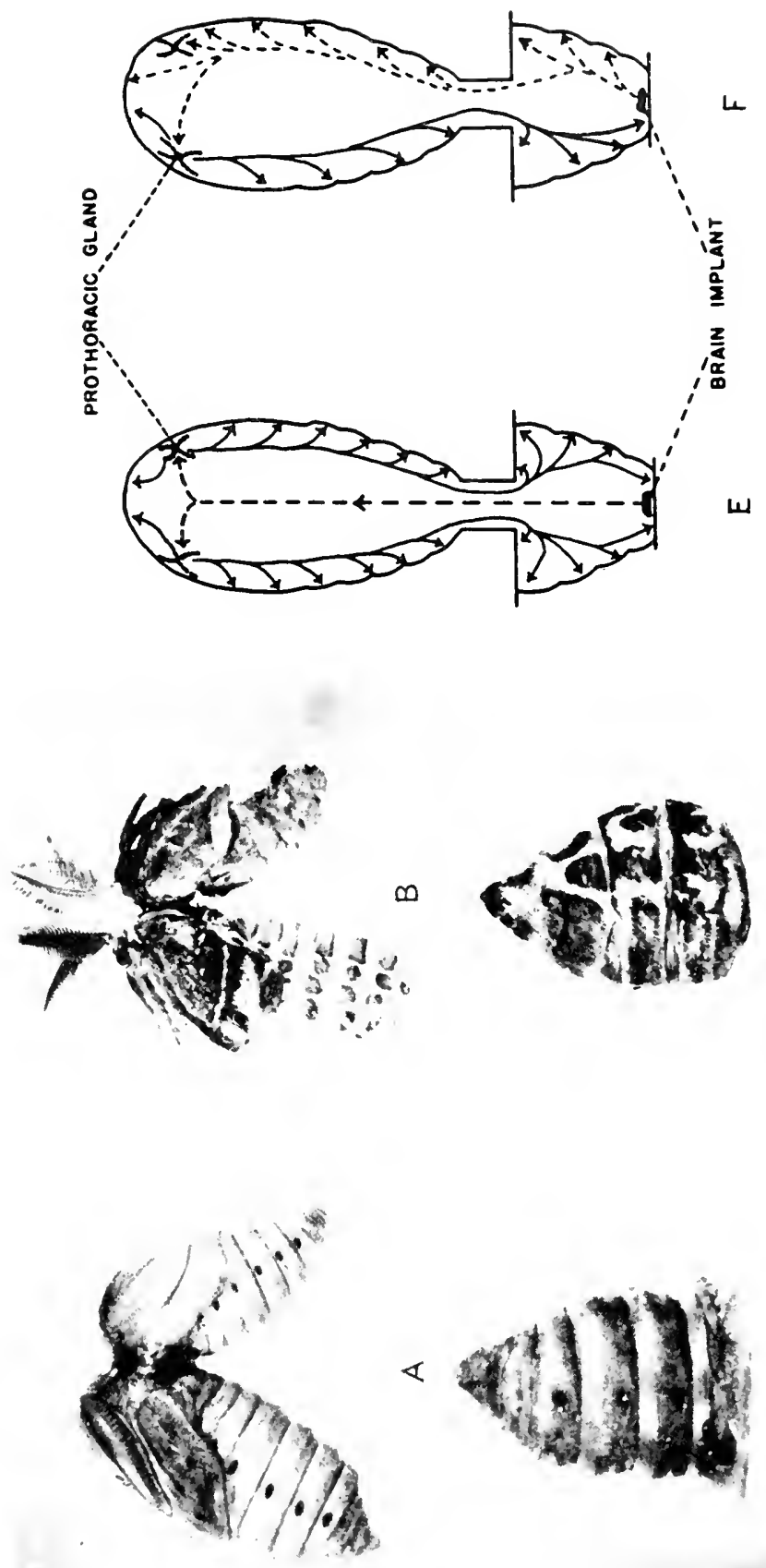

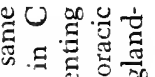

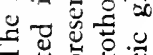

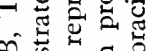
$\infty$ 政 陦灵交总

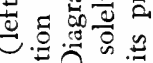
용

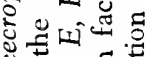
政 눙 है. 훙ㅎㅇ 녕 द 녀요 范究点 ว

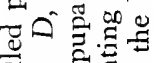

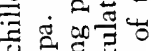

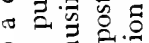
$\therefore x$ o 马.7.

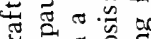

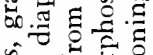
今 है山े 若 궁 उ.요 \& 马 등

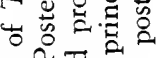
की 要证

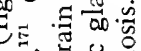

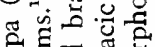

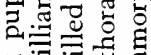
舟䆑焉 ज的

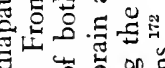

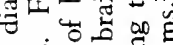

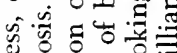
等 U

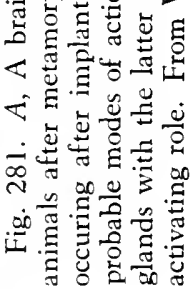


although the animal usually survives for about a year. ${ }^{171}$ If a pupa is chilled by exposure to a temperature of $3-5^{\circ} \mathrm{C}$. for $1 \frac{1 / 2}{2}$ months metamorphosis will then occur in a little over one month after the pupa is restored to the higher temperature. When a chilled pupa is united parabiotically with an unchilled one, both will metamorphose synchronously in about $1 \frac{1}{2}$ months (Fig. 281, $A$ and $B$ ). Implants of brain from a chilled pupa will induce metamorphosis in an unchilled pupa.

A reconciliation of the observed influences on metamorphosis of the prothoracic gland, on the one hand, and of the brain, on the other, has recently' been effected. ${ }^{172,} 175$ In Platysamia cecropia the active fraction from the brain arises in the inner mass of each cerebral lobe of the brain, within which are found two groups of neurosecretory cells, a median and a lateral group. The median group of cells corresponds with the pars intercerebralis cells of the hemipterans. ${ }^{174}$ To induce netamorphosis of a brainless pupa a portion of a brain containing both groups of neurosecretory cells must be implanted, suggesting that these two groups collaborate in the production of the material involved. Killed brain, or brain extract, has thus far proved ineffective as a substitute for living brain tissue in inducing metamorphosis.

If one transects pupae of Platysamia just anterior to the sixth abdominal segment and then seals a plastex cover slip over the cut end of each portion one obtains preparations which are viable for eight months or more and development can be readily observed in them. Chilled anterior portions, or brainless anterior portions which have received an implant of a chilled brain, undergo normal metamorphosis. The posterior portion will not undergo metamorphosis even when a number of chilled brains are implanted into a single abdomen. Such abdomens will metamorphose, however, when grafted to metamorphosing anterior portions. An endocrine factor in addition to that from the brain is obviously essential. That the source of the second factor is the prothoracic gland can be shown by the induction of pupation of isolated abdomens by the implantation of both chilled brains and prothoracic glands (Fig. 281, C and D). The latter may come from either chilled or unchilled pupae. Prothoracic glands by themselves are not adequate. It would therefore appear that metamorphosis normally depends on the production of at least two factors. The first, produced from the brain, is apparently required to activate the prothoracic glands (Fig. 281, $E$ and $F$ ).

Other experiments ${ }^{173}$ suggest that the prothoracic gland principle influences termination of pupal diapause and consequent metamorphosis through action on the cytochrome system of the larval tissues.

Molting in Crustaceans. The decapod crustaceans in their development typically undergo a number of molts, passing through a series of characteristic larval stages, and after having achieved the body-form of the adult they continue to grow through periodic molting of the exoskeleton. Little or nothing is known regarding the integrating factors concerned in the larval development, although it seems probable that the endocrine factors operating here differ little from those known to be operating in later molting and growth.

The molting process is a complex one. The molting cycle may be divided into four periods: (1) premolt, a period of active preparation for molt, including gradual thinning of the cuticle and storage in the gastroliths or 
hepatopancreas of inorganic constituents for a new exoskeleton; (2) molt, the splitting and shedding of the old, partially resorbed cuticle, and an abrupt size increase largely due to absorption of water; (3) postmolt, a period of rapid redeposition of chitin and inorganic salts to produce a new cuticle, and of protoplasmic growth; and (4) intermolt, a period during which physiological processes normally associated with the molting process are largely held in abeyance. In the fresh-water crayfish, Cambarus, on which most of our knowledge of hormonal integration of the molting phenomenon is based, there is no true larval stage; the individual hatches as a diminutive adult. During its first year of life it molts at intervals of about 12 to 13 days, probably without intervention of any significant intermolt period. After the first growth season there are usually two molts a year, one occurring in the spring, in late April or May, and the other in the summer, in July or August. In these older, mature crayfishes the premolt period is 3 to 5 weeks. During this time there is a gradual resorption of the exoskeleton and a deposition of calcium salts in the form of gastroliths in the antero-lateral walls of the cardiac stomach. There is also a gradual increase in the rate of oxygen consumption and of water content for a week or so prior to molt, reaching a peak at the time of molt. The period of postmolt is one in which these changes proceed in the opposite direction and require approximately the same time as the corresponding processes of premolt. Postmolt is followed by intermolt, which is of longer duration after the summer than after the spring postmolt.

Observations on changes associated with the molting cycle in other crustaceans show that the hepatopancreas is a site of storage of calcium salts in certain of the crabs. The stored salts are not sufficient to account for the normal hardening of the exoskeleton, thus making it necessary that the postmolt period is one of rapid absorption of calcium, both directly from the external medium and from ingested food. A study of apparent respiratory quotients of crayfishes shows that although animals in intermolt have a quotient of about 0.8 , freshly molted crayfishes show values as low as 0.1-0.2 during the first few postmolt hours because of $\mathrm{CO}_{2}$ fixation during carapace hardening, and that this value gradually increases to 0.7-0.8 during the first postmolt week. This obviously indicates that calcium is very avidly taken up from the surrounding medium immediately after molt, and that the rate declines rapidly during the first few days.

It is known that removal of the eyestalks from Astacus, ${ }^{114} U_{c a},{ }^{4,}{ }^{94}$ Eriocheir, ${ }^{71}$ Palaemonetes, ${ }^{36}$ or Cambarus ${ }^{39,}{ }^{149}$ results in a more rapid onset of the following and succeeding molts. In young Cambarus, in their first year of life, removal of the eyestalks results in a shortening of the period between molts at 20 to $22^{\circ} \mathrm{C}$. from about 12 days to about 8 days. ${ }^{149}$ That this influence is not the result of general operative injury is seen in that other operative injuries, such as destruction of the retinas, which are at least as severe, do not result in such acceleration; if any influence is seen there is a retardation. The remaining two possibilities, that the results are due to (1) the destruction of important nerve centers, or (2) the removal of endocrine organs important in molt regulation, have been resolved in favor of the latter. If both eyestalks are removed from mature crayfishes molt will occur a significant time in advance of that of unoperated controls. If, however, sinus 
glands from other crayfishes are implanted into the abdomen of such eyestalkless specimens, molting will be delayed beyond the time of that of the controls. ${ }^{36}, 39$ Similar implantations of eyestalk tissue from which the sinus gland has been removed are without appreciable molt-retarding influence. Removal of only one evestalk results in a slightly accelerated molt, suggesting the effect to be quantitative in character.

A molt-inhibiting role of the crustacean sinus gland has received confirmation in studies of the control of gastrolith formation in crayfishes (Fig. 282). These concretions, normally produced only during the premolt periods, may be caused to form at any other period of the year by excision of both eyestalks or by surgical extirpation of the sinus glands by themselves. ${ }^{101}$, $1: 11$ After eyestalk removal during a non-molting period, such as between September and March, the gastroliths commence to form in less than 24 hours at about $20^{\circ} \mathrm{C}$. and then increase in size slowly during eight to ten days,

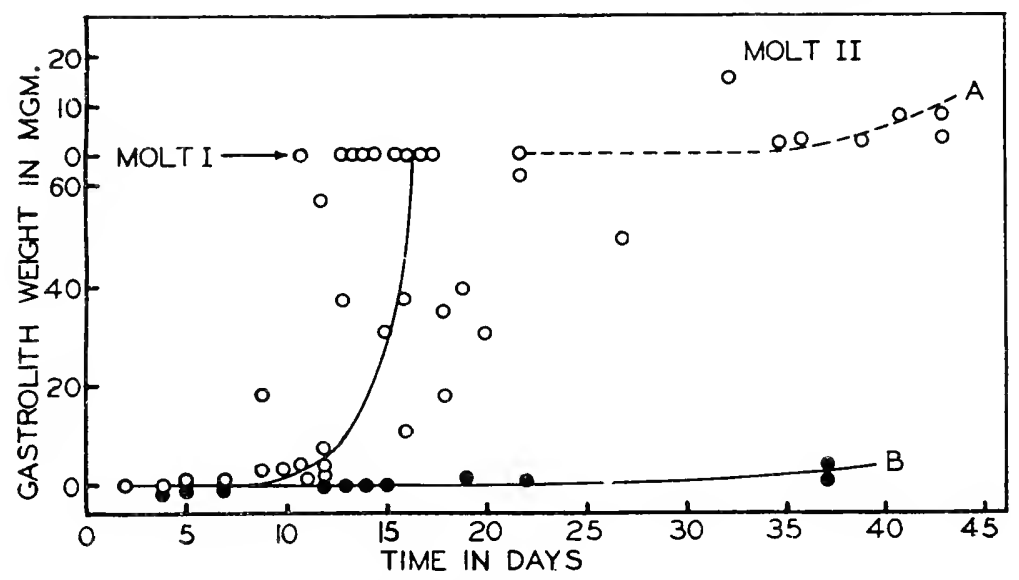

Fig. 282. A, Increase in gastrolith size and molting in crayfishes after removal of the eyestalks with their included sinus glands. Note that an animal once molted proceeds almost immediately to prepare for another molt. B, Eyestalkless crayfishes into which sinus glands are implanted at three or four-day intervals show no gastrolith production. From Scudainore. ${ }^{1 \star 1}$

thereafter accelerating rapidly up to the time of molt, which usually occurs between the fifteenth and twentieth days. Those individuals which survive the molt immediately proceed to form a new set of gastroliths. If, however, one implants a sinus gland into the abdominal region of eyestalkless animals at three- or four-day intervals gastrolith formation is suppressed. Eyestalk tissues other than the sinus gland have no such action. When sinus gland implantations are discontinued, gastroliths begin to form about a week after the last implant, indicating that the implanted glands are no longer effective.

Histological changes in the sinus glands of crustaceans have been shown to be correlated with the molt cycle. ${ }^{128}$ Just prior to molt, acidophilic secretory granules appear to be the predominant ones; after molt completion, basophilic ones are more prevalent.

The molting process which is set into operation by eyestalk removal resembles still further the changes observed in normal premolt animals. ${ }^{141}$ 
There is a greatly accelerated rate of oxvgen consumption and an increase in water uptake. There is also the typical thinning of the exoskeleton resulting from the dissolving and resorptive activity of the hypodermal cells. All of these results of eyestalk removal are prevented by implantation of sinus glands, and it therefore appears probable that all are under the influence of a single molt-inhibiting hormone from the sinus gland.

Comparing the abrupt increase in oxygen consumption after eyestalk removal with the gradual increase during normal premolt, it appears evident that the normal molt, unlike the experimentally induced one, results from a gradual and slow decrease in the blood titer of the molt-inhibiting principle from the sinus gland. This difference between normal and experimentally induced molt might account in part for the often-reported observation that eyestalkless animals show greatly reduced viability, usually succumbing about the time of the first succeeding molt, or, if surviving that, commonly dying during the second. A rare animal survives to the third. It has been shown that the average survival time of eyestalkless animals can be extended from about 17 days to more than 38 by periodic implants of sinus glands. It seems a reasonable supposition that in molting in evestalkless animals some uncurbed molting processes greatly outrun other more basic underlying ones. That some differences from normal molt do occur is seen in that the exuvia of eyestalkless crustaceans have less inorganic salt content than those from unoperated molting specimens. ${ }^{97}$ The high mortality is associated with factors other than the mere molting.

The molt-inhibiting hormone from the sinus glands appears to be responsible for the failure of egg-bearing female Crangon to molt until the young, have hatched, ${ }^{76}$ and for the fact that at the annual spring molt of Cambarus the egg-bearing females molt several weeks later than the males and only after the young have left the maternal pleopods. Sinus gland removal is just as effective in inducing molt in egg-bearing female crayfishes as is a similar operation in males. ${ }^{111}$

There is reason to suspect that at least one additional factor, arising in some region. of the body other than the eyestalks, cooperates in the control of molt. A careful study of the deposition of calcium salts in the gastroliths shows that this process is rhythmical, with rapid deposition during the night and little deposition during the day. Suggestive in this regard, injection of extract of the brain tissue or stiong electrical stimulation of the cut ends of the optic nerves of eyestalkless animals will cause a transitory acceleration in rate of oxygen consumption. ${ }^{141}$

\section{HORMONES AND SEX AND REPRODUCTION}

Vertebrates. The subject of hormones and sex and reproduction in higher vertebrate has been comprehensively reviewed. ${ }^{12.45}$

The hormones whose primary functions are concerned with sexual reproduction and care of the developing young may be divided in vertebrates into three general groups. The first of these, the gonadotropins, are hormones which principally govern the development and activities of the primary sex organs, the gonads. The second general group comprises those hormones, of gonadal or other origin, which typically regulate the male and female secondary and accessory sex characters of the body and exercise a control oves 
the sexual and reproductive impulse and behavior. These latter hormones are known as the gonadal hormones and include the androgens, estrogens, and progestins. A third group we shall call the lactogens. These hormones, of pituitary origin, are essential to the secretion of milk by the crop glands of pigeons and by the mammae of mammals and probably are influential in determining maternal behavior.

Gonadotropins. It has been known for some years that pituitary deficiency or hypophysectomy in the mammal results in an atrophy of the gonads and certain accessory genital structures, and that these changes can be reversed by pituitary implants or extract injections. The gonadotropins to which these influences of the pituitary are due always exhibit the common properties of proteins. Factors which destroy protein structure effectively inactivate these hormones. It would therefore appear that the gonadotropins are themselves proteins or consist of a prosthetic group intimately associated with a protein to which the specific activities of the hormones are due.

The anterior lobe of the pituitary is the principal source of gonadotropins. From this site arise two gonadotropins, the follicle-stimulating hormone or FSH, and the hiteinizing hormone or LH. Another gonadotropin, called chorionic gonadotropin, is known to arise not from the pituitary but from the placenta and to resemble LH rather closely in function. The bulk of the evidence at hand suggests very strongly that these three gonadotropins are three different compounds.

In the absence of gonadotropins, such as normally accompanies hypophysectomy, the ovary shows an arrest of development in young animals and a reduction in size in adults. In the latter instances existing follicles become atretic. FSH in excess, on the other hand, will stimulate the simultaneous maturation of an excessive number of follicles. In some mammals, such as the rabbit, ferret, etc., ovulation from mature follicles occurs only after mating or after some other effective stimulus. Such stimulation is known to induce the liberation of $\mathrm{LH}$, which causes the ovulation. In other mammals, including the human, no special stimulation of the accessory complex is essential to induce ovulation. In all mammals LH is normally responsible for the formation of corpora lutea in the ruptured follicles.

The gonadotropins are not sex specific. They regulate the activity of the testis as well as of the ovary. Removal of the pituitary in the male results in an arrest of testicular function and reduction in size of the testis. Activity of the testes can be maintained in hypophysectomized individuals by pituitary implants or extract injection. Many mammals which breed only at certain sharply delimited periods of the year may have their testes rendered unseasonably active by treatment with gonadotropins. This has been demonstrated for such species as the ground squirrel, Citellus, and the alpine marmot, Marmota. After the mating season in such species the testes normally recede into the abdomen and spermatogenesis ceases. Administration of pituitary gonadotropin, or even chorionic gonadotropin, found normally only in pregnant females, will reverse these processes at any season.

An enlargement of the ovaries of the horned lizard, Phrynosoma, has been obtained by FSH and LH from hog pituitary and by serum from pregnant mares. ${ }^{115}$

Female frogs and toads may be induced to ovulate at any time of the 
year, except immediately after an egg-laying period, by injection of macerated anterior lobe of the pituitary. ${ }^{133}, 134,135,136$, Preparations of pituitaries from females are about twice as effective as those from males. The ovulation, which will occur between the second and fourth days, includes rupture of the follicles and expulsion of the eggs by contraction of smooth muscle fibers in the follicle wall. Injection of pituitary extract into male frogs induces amplexus. These sex reactions in amphibians may also be induced by pituitary extracts from other amphibians, from fishes, ${ }^{176}$ mammals, ${ }^{134}$ or may even occur in response to antuitrin-S from pregnancy urine.

Premature spawning has been induced in several species of fishes after injection of fresh pituitary glands from other fishes. The ovoviviparous fish Cnasterodon was caused to spawn more than two weeks before the normal time after treatment with pituitaries from Micropagon and Luciopimelodus. ${ }^{8 \cdot 2}$ The Brazilian species, Pimelodus ${ }^{48}$ and Prochilodus, ${ }^{122}$ which normally spawn after the heavy rains that in Brazil follow a long period of drought, were induced to produce eggs and sperm during the period of drought within 1 to 3 days after an intramuscular injection of pituitaries from these same species. Rainbow and brown trout, Salmo gairdnerii and S. fario, in Wisconsin, were caused to produce mature eggs and sperm 6 to 7 weeks before the normal spawning season by intraperitoneal injections of fresh or acetone-dried pituitary glands from the carp. ${ }^{7+}$ FSH from sheep and serum from pregnant mares were without such effect. Increase in size of the ovocytes and ovaries of the lamprey, Petromyzon, and precocious sexual maturity were observed after treatment with human pregnancy urine. ${ }^{47,51}$ Hypophysectomy in the killifish, Fundulus, is followed by regressive changes in the ovaries and testes, compared with controls. ${ }^{111}$ When the gland is removed in the autumn the gonads fail to undergo their normal spring enlargement. Furthermore, implantation of adult pituitaries at 3 day intervals into immature Fundulus induces within 4 weeks a considerable degree of gonadal activation in both sexes, together with production of secondary' sexual pigmentary changes in the males characteristic of the breeding season. ${ }^{112}$

Gonadal Hormones. Hormones arising in the gonads regulate the male and female characters of the body. They stimulate the development and activity of the accessory reproductive organs, and control the sexual behavior of the animal. Although the influences of these hormones are basically similar among all vertebrates there is considerable difference among species, and even among individuals, as to their detailed actions.

There are three types of gonadal hormones, androgens, estrogens, and progestins, all three types being elaborated by both sexes. The chief sources of these hormones, as the name gonadal hormones implies, are the ovaries and testes, although other organs such as the placenta and adrenals may also contribute significant quantities. All the naturally occurring gonadal hormones possess a steroid nucleus, but recent studies of active synthetic substances appear to indicate that their activities do not depend on this structure.

The androgens mainly regulate the development and activities of those accessory reproductive structures functionally important in the male. The estrogens are mainly concerned with the development and activity of those 
structures functionally important in the female. The progestins function primarily in providing adequately for the intimate relationship between the parent and developing young. The principal naturally occurring substance in each class is: (1) androgen-testosterone; (2) estrogen-estradiol; and (3) progestin-progesterone, which have the chemical structures shown.

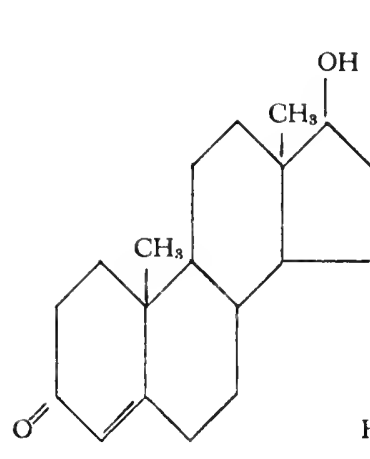

Testosterone

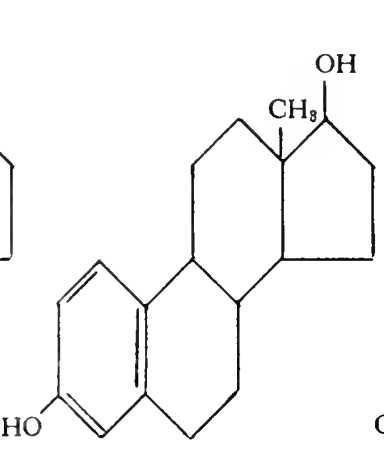

Estradiol

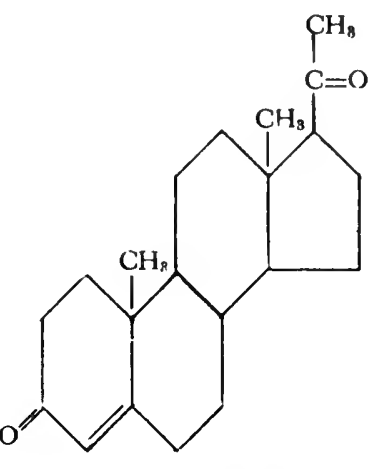

Progesterone

Just as these three classes of hormones show a basic similarity to one another in their chemical organization, so is there also considerable functional overlapping, and, in many instances, a ready conversion within the body from one type to another. These facts have made the acquisition of knowledge of their normal function somewhat difficult.

ANDRogens. Androgen is a term used to describe all those compounds whose biological actions resemble in general those of testosterone. The primary function of androgens is to stimulate the development and activity of the male accessory reproductive organs. They are also responsible in part for differences in the conformation of the male body, the lower pitch of the human male voice, the characteristic distribution of hair, male pugnacious assertiveness, and many special male secondary sex characteristics such as the swollen clasping digits of the male frog, the comb of the cock, the crest of the newt, and the nuptial coloration and gonopodal appendages of certain viviparus fishes. Many of these are characters the presence of which is largely restricted to the breeding season.

The various androgens differ from one another in their overall potency and their relative effects on the numerous individual characters within the body and these differences in turn may vary from species to species. This complicates greatly the problem of the roles of androgens within the body.

The testes comprise the principal sources of androgens; the principal one is testosterone. This organ, furthermore, shows the capacity to extract less potent androgens from the circulating blood and convert them to substances of increased potency. Numerous observations have indicated the interstitial glandular cells to be the specific source. Reduction or disappearance of the seminal epithelium following $x$-radiation, ligation of the vasa efferentia, or in cryptorchidism has been reported to cause no atrophy of the interstitial glandular tissue and no loss of androgenic potency. In the newt, Triton cristatus, there is no interstitial glandular tissue in the testes until the approach of the breeding season. At this time certain portions of the semin- 
iferous epithelial tissue become transformed to produce a glandular body, and this transformation is temporally associated with the differentiation of the characteristic seasonal secondary sexual adornments in the male. Destruction of this glandular tissue is the equivalent of total castration in preventing development of these characters. In the stickleback, Gasterosteus, there is very close correlation between the annual cycle of differentiation of interstitial glandular cells and the cycle of secondary sex characters and behavior such as skin-coloration, secretion of nest-building mucus, and mating behavior. The latter characteristics in this species, on the other hand, show no relationship to amount of spermatogenic activity. That the seminal epithelium is not a significant source of androgen has also been demonstrated by removal of the greater part of the testes of cocks and assay of these organs after regeneration. The regenerated organs aro principally seminal epithelial tissue with a great paucity of interstitial tissue, and, correspondingly, there is evidence of only little androgen production by such organs.

These and numerous other experiments indicate that the interstitial gland ular cells are the points of origin of testicular androgens.

Androgens are also produced by organs other than the testes. Male accessory reproductive structures have been maintained in full functional activity in castrated specimens by grafted ovarian tissue. Among fowl, the combs of both the cock and the hen are stimulated by androgens. The ovaries of the hen normally produce enough androgen to maintain a certain degree of development of the comb; after ovariectomy there is atrophy of the comb. The seasonal yellowing of the bills of both male and female starlings and blackening of the bills of male and female English sparrows are responses to androgens, estrogens being ineffective in this respect. Black-crowned night herons show, during the breeding season, certain changes in plumage coloration common to both sexes, an effect due to androgens. Many observations such as the foregoing establish decisively the ovaries as a site of androgen production.

Another source of androgens is the adrenal gland. Implantation of adrenal tissue in young cocks results in precocious development of male sex characters and behavior. Castration of young rats does not result in atrophy of accessory genital structures for many days unless there is simultaneously complete adrenalectomy. Androgens are still excreted in the urine of animals after castration. Extracts of adrenal tissue have been found to yield the androgens adrenosterone and desoxycorticosterone, the latter having an activity about the equivalent of that of androsterone, and the former having activity of about one fifth of this value.

There is little evidence of any significant storage of androgens in the body. They are usually rapidly destroyed and therefore must be constantly produced if their influence is to be maintained. Certain poeciliid fishes ${ }^{154}$ can be treated with androgens for only a few hours but the activity of the andrugens continues for many days. This observation appears to indicate either an actual or an effective storage within the body. The pigmented fat-body of the hibernating woodchuck, the so-called hibernating gland, has a remarkably high androgen content. The androgens are inactivated principally in the liver. The androgenic activity of blood leaving the liver is much less than that of blood entering this organ. Products of the inactivation are both in- 
active substances and androgens of somewhat lower activity, such as androsteronc. Estrogens are also commonly produced in the process. These products of metabolic conversion are excreted by the kidney.

The relation of androgens to the development of the characteristic male suspensor organs, gonopodia, and to the smaller body size in male poeciliid fishes has been carefully analyzed. ${ }^{154}$ Castration of males during the metamorphosis of the anal fin into a gonopodium results in immediate cessation of the process. Application of androgenic hormones to castrated males or to normal females can induce full development of the male characters which are typical of the species. It has been shown that progressively larger concentrations of hormones are required for consecutive steps in the development of the gonopodium, with a concentration of one part of hormone in $4.2 \times 10^{10}$ parts of water being sufficient to induce the first step. The gonopodium normally develops under the influence of a gradually increasing concentration in the blood of androgen from the fish's own testes. A certain optimum concentration is required for normal development of each step, higher concentrations inhibiting growth and inducing precocious differentiation.

ESTROGENS. Estrogen is a term applied to any substance which will produce the characteristics of normal estrus, including cornification in the vagina, in an adult mouse. The estrogens include a number of naturally occurring compounds which differ slightly from one another chemically and in effectivencss: estradiol, estrone, estriol, equilenin, and equilin. The principal functions of the estrogens are to stimulate the growth and functioning of those accessory reproductive organs which are characteristically female, to contribute to the production of the conformation of the body which renders it typically female, including the characteristically smaller size for such species as man, and to influence in a characteristic manner the psychology and behavior of the organism.

The exact source of estrogen within the ovary is still the subject of considerable controversy. Early observations that the follicular fluid was rich in estrogen led to the hypothesis that the granulosa cells were the sites of estrogen formation. This view has been supported by demonstration that these cells contain estrogen. However, destruction of the follicles by $\mathrm{x}$-ray does not deprive the ovary of its estrogen-producing capacity under the influence of gonadotropins. After intensive treatment with $x$-radiation, the ovary comes to consist of little other than interstitial tissue. Gonads of fetal horses before follicle formation have proved rich in estrogens. There appears therefore to be convincing evidence that interstitial tissue, including the thecal cells, produces the hormone, and that there is also a possibility that the granulosa and luteal cells manufacture it. It is often difficult to establish that a substance is actually formed within a tissue in which it is found, instead of arriving there secondarily.

The placenta, both the maternal and the embryonic portion, contains abundant estrogen of much the same character as ovarian estrogen. Furthermore, oophorectomy in certain pregnant mammals does not result in significant change in the term of pregnancy or in any permanent reduction in the amount of estrogens excreted in the urine. In such cases the symphyses also become separated normally, a change ordinarily conditioned by joint action of estrogen and progestin. 
Estrogens are also produced by the testes of males. In fact the tissue with the highest known estrogen yield is the stallion testis, in which estrone is the substance present. The testicular source appears to be, as for the androgens, the interstitial glandular cells. Estrogens have also been extracted from the adrenals.

Estrogens are very rapidly inactivated in the living body. Studies on the relation between site of transplantation of ovarian tissue and the physiological actions of the implants indicate the liver to be the principal site of destruction. Estrogen-containing blood passing through the liver reappears largely free of active concentrations of the hormone. Destruction of hepatic cells by carbon tetrachloride permits the hormone titer in the blood to rise with expected effects on estrus of the animal. Liver slices inactivate estradiol in vitro through the action of some $\mathrm{CN}$-sensitive mechanism. Progestin, on the other hand, diminishes the rate of estrogen inactivation and thus permits a higher titer to be maintained in the blood, from which much is then able to escape into the urine.

The kidneys and liver are the principal organs of estrogen excretion, although the relative importance of the two organs is not yet known.

The organ and tissue changes induced by estrogens are characterized by large variations in degree of responsiveness of the different cells and tissues to the hormone, thereby giving rise to gradients of response. Also characterizing the responses is a very high degree of reversibility of the changes when the concentration of the hormone declines. Such reversibility of the changes is most evident in many of the secondary and accessory sex character changes paralleling the reproductive cycles in many species.

As with the androgens, the specific threshold and character of the responses are determined by the inherent cellular characters, as can be seen in the unchanged character of the response as the individual tissues are transplanted to novel sites within the body. The relation of the response to the genetic constitution of the species is seen in reciprocal transplantations of feather-bearing skin between the sexes in fowl, resulting in the characteristic feather types for the host sex.

PROGESTINS. The progestins include all those compounds exhibiting an action on the organism like that produced by progesterone. These substances are concerned primarily with those changes in the organism associated with pregnancy and parturition. The chief sources of progestin are the corpora lutea of the ovary. The source would therefore appear to be cells derived from the granulosal layer of the follicle, although some investigators believe that thecal tissue participates in lutea formation. It is possible that other cells of the ovary also contribute progestins, with perhaps the same cells which at other times produce estrogens gradually becoming transformed in the reproductive cycle to liberate progestins.

Progestins are also produced in the adrenals. Not only has progesterone been demonstrated in this organ, but desoxycorticosterone also acts as a progestin. Extracts of adrenals can produce typical progestational alterations in the uterus in rabbits if, as a preliminary, they are treated with estrogen.

The placenta is an important natural source of progesterone. In the mammal the placenta is believed normally to take over after a time the major share of production of the normal progesterone of pregnancy. Even after the 
total removal of the ovaries of pregnant rats sufficient progestin is liberated from the placentae to carry the animals to normal terms. In man this changeover of control from corpora lutea to placenta is considered to occur between the 79th and the 90th day of pregnancy.

Progestins are not stored in the body; they disappear very rapidly from the blood. Injection of progesterone, or the normal production of this hormone, is followed by the appearance of corresponding amounts of a relatively inert alcohol, pregnanediol, in the urine. The preponderance of evidence points to the progestational endometrium of the uterus as the principal organ concerned in this conversion, which is principally in evidence in the progestational phases of the sexual cycle and usually ceases on hysterectomy. Furthermore, it is greatly reduced by injections of estradiol, which is known to suppress the progestational condition. That the progestational endometrium is not the sole site of the conversion is seen in the excretion of pregnanediol in males, and occasionally in females after hysterectomy.

The corpora lutea which are formed from the follicles, either without ovulation, as in the case of atretic follicles, or after ovulation, vary considerably in the extent of their development. Their greatest development accompanies gestation; lesser development is associated with lactation and pseudo-pregnancy and the typical corpora lutea of ovulation are still smaller. The corpora lutea are dependent for their development on a supply of LH from the pituitary. Mammals appear to vary considerably in their need for external stimuli to encourage the necessary production of LH. An adequate stimulus has been shown to be copulation in the case of the rabbit. Suckling and lactation have been shown to stimulate its production in the rat, and psychic stimuli, such as the mere presence of a second individual, have been found effective in such animals as the pigeon and the rabbit. That the last stimulus is visual, at least for the pigeon, is demonstrated by the fact that a mirror image is often sufficient to produce the effect.

While all investigators are agreed that $\mathrm{LH}$ is essential for the initial development of the corpora lutea, there is much controversy as to the endocrine factors participating in the maintenance of these bodies. There appears to be good evidence from a number of types of experiments that the absence of FSH contributes significantly to their maintenance, and that LH is nonessential. Gonadal hormones assist in their maintenance, probably through a suppression of FSH liberation by the pituitary. Comparison of experiments in which the embryos have been removed, leaving the placenta in situ, with those in which the placentae as well are excised, clearly demonstrates that the latter organs yield hormonal material which can operate to maintain the corpora lutea. These results also appear capable of interpretation in terms of suppression of FSH production by placentally-derived gonadal hormones That still other factors may contribute to the total explanation is seen in the observation that the corpora lutea may be maintained even after complete removal of the fetuses and placentae, provided the uteri are kept distended by such inert bodies as pellets of paraffin wax.

The presence of corpora lutea, at least among the higher vertebrates, appears to be associated with viviparity. They are said to be absent in birds and oviparous reptiles, but present in certain pregnant viviparous snakes, such as Crotolus and Bothrops. 
Relaxin. Relaxin, a non-steroidal substance, insoluble in fat solvents, is

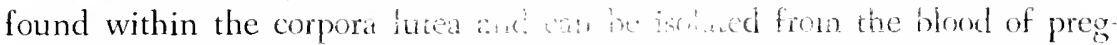

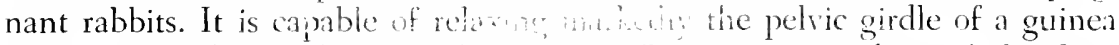
pig in 6 to 12 hours after a single wection. Bugestereme and estradiol induce the secretion of relaxin in female rabbits unless they are castrated and hysterectomized. The latter observations indicate these organs to be essential to this response to the gonadal hormones.

Lactogenic Hormones. The production of milk is an adaptation of all mammals and such birds as the pigeon for postpartum nutrition of their young. Hence it is not surprising to find that there is a considerable influence of gonadal hormones on milk-producing glands of the organism. The mammary glands of the mammalian fetus become enlarged under the influence of the hormonal complex of the maternal blood supply, but regress on parturition and typically remain so until sexual maturity, when the animal's own hormonal supply becomes adjusted for their development. The mammae of some mammals, such as the monkey, Macacus, develop fully under the administration of estrogens alone. Other species, such as the rabbit, require administration of both estrogens and progestins for similar development. Since in many species mammary growth cannot be induced by the foregoing hormones after hypophysectomy it would appear that at least one additional substance, a lactogen from the pituitary, is also essential to normal mammary development.

Whatever the nature of the hormonal complex required for the normal development of the mammary glands, it is clearly evident that lactogen from the pituitary is essential to stimulate the actual secrction of milk. This principle in the mammal has been called galactogen or prolactin, although some investigators prefer to restrict the latter term to the analogous principle in the pigeon, which siimulates the production of pigeon's-milk by the crop gland. Lactating mammary glands cease activity at once after hypophysectomy, but may be maintained by administration of lactogen and either adrenotropin or extracts of the adrenal cortex. Normal adrenal functioning is thus also essential to continued lactation.

In the normal regulation of lactation it is generally believed that the estrogens present in large amounts during gestation inhibit lactogen liberation. With the disappearance of the estrogens on parturition the lactogen appears in the blood. Strongly supporting this hypothesis is the well known observation that injection of estrogens will rapidly terminate mammary secretion. The stimulus of suckling, through some neural mechanism, seems also to stimulate the liberation of lactogen, at last in many species.

Control of Reproductive Cycles and Behavior. The pituitary gland, through the production of gonadotropins, is the principal cndocrine organ through which reproductive activities are governed. We, therefore, with reason, look to a study of factors controlling this organ to supply us with fundamental information as to the mechanism of control of reproductive rhythms and adaptive reproductive responses of the organism to its external environment.

The pituitaries of young mammals are bipotential organs which normally develop into either a male or female type, under the influence of the particular gonad present. The gland is typically a relatively larger organ in the 
adult female than in the male. Both female and male pituitaries normally produce FSII and LII in relatively large amounts. The primary difference between male and female pituitaries is one of cycle differences. Ovariar implants, but not testis implants, suppress FSH production by the adult pituitary in castrated females; in castrated males only testis implants will suppress FSH production. Thus, the adult pituitary is diflerentiated into differently responding male and female types, in contrast with its sexually indifferent condition in new-born mammals.

During the growth of a young mammal there is a gradual increase in the production of gonadotropins up to the time of sexual maturity, when the gonads are consequently brought to their completely functional state and

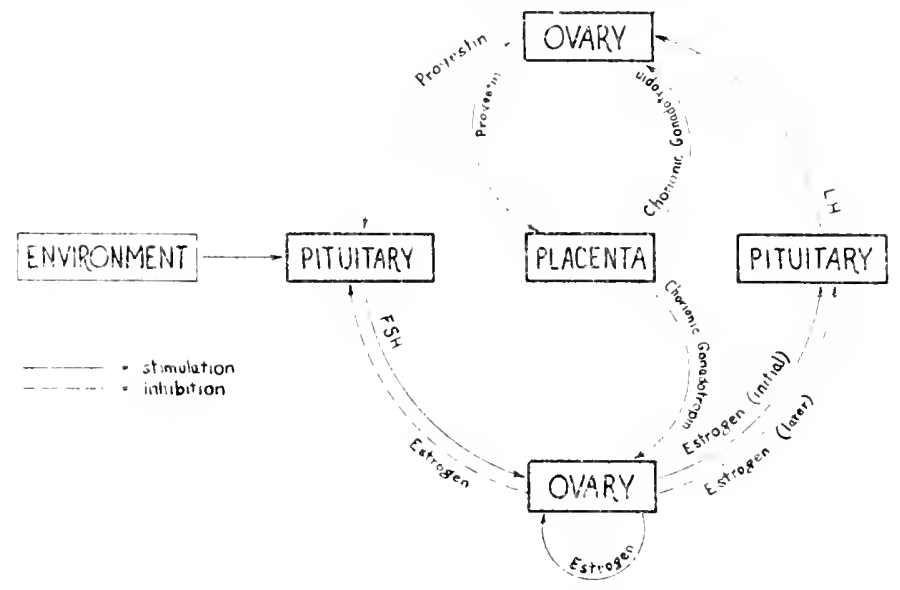

Fig. 283. Diagram representing the major endocrine infuences operating during the normal reproductive cycle of the adult female mammal.

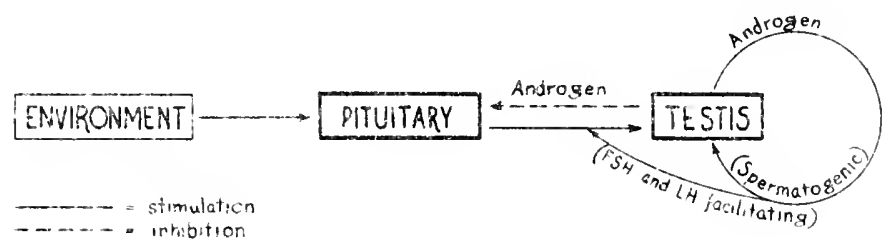

Fig. 284. Diagram representing the major endocrine influences operating during the normal reproductive cycle of the adult male mammal.

their estrogens and ardiogens contribute to the fuil differentiation of the secondary and accessory sexual characteristics. The animal is now capable of nomal reproductive activity. This is, in the vertebrates, typically a cyclical phenomenon with the periodicity often correlated with the annual solar crcle in such an adaptive fashion as to assure the young of a favorable time of year for birth and early postnatal development. In some species the reproductive periodicity bears much less or no relationship to external environmental stimuli and appears to result from a wholly inherent rhythmical mechanism, as in the case of the rat, mouse, man, etc. 
A vast amount of research has been done on the contributions of the interactions of endocrine sources to the maintenance of the normal cycles of mammals. The endocrine actions and interactions of the major organs participating in the normal reproductive cycles are indicated for the female inammal in Figure 283, and for the male in Figure 284.

In the female the pituitary, through FSH production, stimulates the development of the ovarian follicles and interstitial tissues, with a consequent increase in estrogen production. The rising concentration of estrogen in the blood mimics FSH and furthers the ovarian development. At the same time the estrogen gradually suppresses FSH production by the pituitary while at first strongly encouraging LH liberation by the pituitary. Over a longer period estrogen suppresses LH liberation. LII liberated from the pituitary stimulates ovulation in many mammals, and in all it stimulates the differentiation of corpora lutea with the subsequent production by them of progester one. Some investigators, notably Astwood, believe that LH simply induces differentiation of the corpora lutea, an additional pituitary principle, luteotropin, being needed for stimulating the actual secretion of progesterone. Luteotropin is claimed to show many properties very similar to the lactogenic principle and may, in fact, be identical with it. Progesterone cooperates with estrogen in the further suppression of the production of FSH by the pituitary. In case of pregnancy, the placentae of the developing embryos are activated in the presence of progesterone and proceed to liberate substantial amounts of chorionic gonadctropin the activity of which very closely resembles that of pituitary $\mathrm{LH}$. This gonadotropin contributes to the suppression of production of both pituitary gonadotropins through its stimulation of greater gonadal hormone production.

The corresponding endocrine interactions participating in the male reproductive eycle are relatively much simpler. Gonadotropin from the pituitary activates the interstitial and spermatogenic tissues of the testes, with the resultant increase in testosterone production in the former. This androgen simultaneously stimulates further development of the spermatogenic epithelium and facilitates the influences of both FSH and LII. As the blood titer of androgen gradually rises this hormone suppresses pituitary gonadotropin production. The decline of andiogen production after pituitary suppression eventually leaves the latter free to initiate a new cycle of reproductive activity.

In a few species there tends to be an annual reproductive period at a particular scason of the year, without any regard to any particular factor of the external environment. Such an operation of an inherent rhythm has been clearly demonstrated for certain organisms which continue to breed indefi. nitely cluring the same calendar months after transfer from the northern to the southern hemisphere, or vice versa. Other species on similar transfer more or less rapidly undergo a readjustment of their breeding period to the corresponding scason for their new locality. ${ }^{10: 9}$ The readiness with which the organism makes such an adjustment is obviously a function of the relative degree to which the breeding cycle depends on external factors.

Of all the external stimuli known to exert an influence on reproductive cycles, one of the most definitely influential so far demonstrated is light and photoperiodism. 1:32 Among certain north temperate zone birds it has been 
shown that the testis of the male normally reaches a peak of its annual cyclical activity in the late spring. Experiments on male juncos, sparrows, and starlings have clearly shown that gradual increase in length of the daily period of illumination through supplementary artificial lighting will bring the activity of the testes to a maximum at a time not typical for the species Properly controlled experiments have shown that it is the light itself rather than the resulting longer daily periods of activity or fecding which is the actual determining factor. A similar subjection to increased periods of illumination over a period of about two months has been found to bring female ferrets into estrus in winter, a time at which in their normal reproductive crcle they are in anestrus. ${ }^{-4}$ Similar observations have been made on mice (Peromyscus). ${ }^{16 i}$ Among the fishes the reproductive cycle of the trout has also been shown to be importantly influenced by photoperiodism. ${ }^{79}$

In the field mouse, Microtus, the gonads have been shown to diminish in size during treatment of the animal with gradually decreasing periods of illumination. ${ }^{15}$

Such experiments as these appear to indicate that the gradual changes in day-length in the annual solar cycle constitute a very effective stimulus in determining the normal annual reproductive cycles of many species of verte. brates.

In the immature duck whose gonads are normally stimulated by increased illumination, it has been shown that pituitaries from illuminated specimens show increased gonadotropic activity over those of untreated controls when implanted into immature mice. Red and orange lights are more effective than other colors in inducing the effect. The eyes are not essential to the reaction; direct illumination of the pituitary resulting from illumination of the orbit after enucleation effectively activates the gland. When light is conducted directly to the pituitary through a quartz rod, blue light, otherwise showing only small influence, becomes quite as effective as red and orange. Similar action of light on the reproductive cycle through other routes than the eyes has been shown for sparrows. In the ferret, on the other hand, division of the optic nerves completcly inhibits the action of light, indicating the retina of the eyes to be the effective receptor agency involved in this species.

Nervous connection of the hypothalamus with other parts of the nervous system is usually essential to normal sexual functioning. Nervous connection between the hypothalamus and the pituitary is essential for certain aspects of control of the reproductive cycle, as for example ovulation in the rabbit, but appears non-essential in numerous other instances, the conduction in these latter cases apparently being humoral.

The temperature of the external environment has been shown to play an important role in determining the time of gonadal development and appearance of secondary sex characters in the stickleback (Gasterosteus), a sufficient rise in temperature rapidly inducing these changes. These fish show no significant responses to increasing illumination. Low temperature and darkness such as normally obtain during its annual period of hibernation will activate the gonads in the ground squirrel, Citellus, at any season.

The general state of nutrition of an organism, as well as the sufficiency of specific vitamins such as $A, E$, and the $B$ complex, have an important ef- 
fect on the reproductive activities of the animal, although we know too little about their roles to attempt any generalizations.

Invertebrates: Hormones and Secondary and Accessory Sex Characters. Many species of invertebrate organisms representing many phyla possess a sexual dimorphism which has long been suspected to owe its origin, in some measure at least, to hormones comparable to the gonadal hormones of vertebrates. ${ }^{11}$. $9 \times$ Even to the present, however, no incontrovertible evidence has been advanced to prove this is so. Nevertheless, there are numerous reports in the literature suggesting hormones to be playing roles in this regard. These come from observations on (1) parallel effects of parasites on gonads and sexual differentiations; (2) parallel cyclical changes in the degree of gonadal activity and certain seconclary sexual characters; (3) results of irradiation of the gonads or of the whole organism with $x$-rays or radium rays; and (4) results of surgical extirpation of gonads.

It has been observed after operative destruction that the normal regeneration of the copulatory apparatus of planarians requires the presence of the gonads, particularly the testes. ${ }^{1.5 .}$. 1.ti $\ln$ earthworms which lack the normal secondary sex character, the clitellum, it has been observed that their testes, but not their ovaries, had been destroyed by the sporozoan, Monocystis. Surgical extirpation of the segments containing the testes, but not the ovaries, was reported by one investigator to be followed by production of a reduced clitellum or none at all, 7:2 whereas similar surgical castration was reported by another investigator to be without any influence on either develpoment of the clitellum or the mating behavior. ${ }^{1: 3}$, it Since differentiation of the clitellum normally commences simultaneously with the beginning of spermatogenesis, it has been suggested that both of these phenomena may be parallelly influenced by an extragonadal hormonal factor, although completion of development of the clitellum may be hormonally determined by a chemical factor liberated by the ripe sperm cells. This latter hypothesis finds support in the observation that renoval from mature Eisenia of the anterior twelve segments containing the seminal vesicles results in the rapid disappearance of the clitellum and its subsequent failure to redifferentiate. ${ }^{77}$

The periwinkle, Littorina, exhibits an annual cycle of development of gonads and aceessory characters. Observations on specimens whose gonads were considerably damaged by trematode larval stages revealed incomplete differentiation of their accessory characters. The copulatory arm, or hectocotylus, of cephalopods has been reported as failing to show its characteristic differentiation after surgical castration. ${ }^{116,143}$

There have been numerous observations on the influence of parasitic castration of male decapod crustaceans upon the size and forms of the pleopods, chelipeds, and abdomen which typically show distinct differences between the two sexes. The partial to complete castration which normally results from parasitization by rhizocephalans (parasitic Cirripedia) or bopyrids (parasitic isopods) is commonly accompanied by the failure of these portions of the body to assume their typical masculine form, the specimens approaching the female form in their secondary sexual differentiation.

The interpretations of these results of parasitic sastration of male crustaceans in general lall into either one of two major categories: (I) those 
which assume that the presence of the parasite has influenced the expression of the genetic mechanism of sex determination, and (2) those which assume that the testes or some other tissue produced an endocrine possessing androgenic activity. In support of the first of these two types of explanations is the observation that certain decapod crustaceans normally possess hermaphroditic gonads, or are readily induced to develop them under the influence of parasitization. Some species, such as Leander, which show no secondary sexual character changes on castration may be thought to possess a relatively stable mechanism of sex determination, not easily influenced by the parasite, ${ }^{46}$ whereas others like Upogebia possess very labile mechanisms and consequently show considerable feminization after parasitic infestation. A number of investigators have noticed that the higher fat content of the blood and liver characteristic of normal females is also often observed in parasitized males. ${ }^{84}$ It is postulated by these investigators that the parasite imposes much the same metabolic demands on the host as are normally made by the developing eggs of the ovary, and that associated with the increased fat metabolism is the production of a "sexual formative" stuff which parallelly influences the development of both the gonads and the secondary s. $x$ characters.

The second type of explanation of the observed influences of parasitic castration in males assumes the operation of a masculinizing hormone. According to this hypothesis the animal after parasitization does not become feminized but assumes a neutral form, which chances to resemble more clearly the female than the male. Considerable support is given this view in observations on the influence of three species of parasites on the crab, Munida sarsi. Two smaller parasitic crustaceans, Triangulus munidae and Lernaeodiscus ingolfi, totally or partially castrate the crab and produce strik. ing modifications in the male secondary sex characters; a much larger parasite, Triangulus boschmai, leaves the gonads functional and does not modify the sex characters. Such observations appear to exclude in these instances a direct influence of the metabolic demands of the parasite in inducing the observed changes. Other investigators, however, have failed to find a correlation between the degree of gonadal atrophy and the extent of suppression of the male characters and suggest that a tissue other than the testis produces the hormone in question.

Another kind of observation suggesting an action of a hormone in male decapod crustaceans is the seasonal cycle of changes in the copulatory appendages of the crayfish. These appendages assume a sexually functional form (form I) at the time of the late summer molt. This is a time when the testes are large and active. At the time of the spring molt, a period of low gonadal activity, they revert to a non-functional condition (form II). Experimental induction of molt during the winter months when testis activity is similarly low always produces form II. ${ }^{1+1}$ These observations may be equally well explained in terms either of the influence of a gonodal hormone on the appendages or of the parallel action of a hormone from some other source in the body on both gonad and appendages.

Parasitic castration of female crustaceans is in general accompanied by little or no change in the general form of the body and appendages. It has been observed, however, that the brood pouch of Asellus and that of Daphrita 
fail to develop after injury to the ovaries by irradiation. In the amphipod, Gammarus pulex, suppression of the ovaries by a parasitic worm, Polymorphus minutus, or by irradiation, has been observed to be associated with failure of the typical marginal bristles of the oostegites to develop. The ability to develop the marginal bristles was restored parallelly with oogenesis after cessation of the irradiation treatments. Female shrimp, Leander, castrated by bopyrids or by $x$-ray irradiation, showed absence of development of the abdominal incubatory chamber and the special guanophores associated with the corresponding abdominal segments. ${ }^{46,96}$ These observations strongly support the hypothesis that in these crustaceans the ovaries may produce a hormone normally influencing certain bodily modifications concerned with provision for the developing young.

In insects, despite extensive observations on the effects of parasitic castration, surgical castration, and gonad implantation, there is no reliable suggestion as yet that gonadal or other blood-borne hormones significantly influence the differentiation of secondary sex characters. In fact, strong evidence to the contrary is seen in the frequently observed occurrence of gynandromophism.

Gonadotropic Hormonal Activity. Many invertebrates, like the vertebrates, show annual or other reproductive rhythms, with periods of sexual activity alternating with periods of inactivity. In most instances there is as yet no knowledge of the pathways through which the gonads are activated or inhibited.

Some recent experiments have indicated that oogenesis in female shrimp of the genus, Leander, is under the control of a hormone originating in the sinus glands. ${ }^{119}, 120,121$ This shrimp reaches the end of its breeding season late in the summer, and its ovaries become tremendously reduced in size and activity and normally remain so during the fall, winter, and early spring. Removal of the eyestalks or even removal of only the sinus glands from the eyestalks in such a non-breeding season as September or October results in a very rapid increase in weight of the ovaries, these organs increasing about seventy-fold in 45 days (Fig. 285). Normal eggs may be laid at the end of this period. Unoperated controls show almost no increase during the same period. Implantation of sinus glands into the abdomens of eyestalkless animals will inhibit ovarian development, depressing it even more than is observed in unoperated controls. A similar sort of hormonal relationship between the sinus gland and the ovary, with the sinus gland acting as inhibitor for ovarian maturation, has been demonstrated for the fiddler crab, Uca. ${ }^{40 a}$ A similar situation appears to obtain in the crayfish. ${ }^{40}$

A further reproductive function of the crustacean. sinus gland is observed in the fact that female crayfishes bearing eggs on their pleopods normally postpone their spring molt beyond the time of molting of males, and until the young have become free. This adaptive response is apparently the result of activity of a sinus gland hormone, inasmuch as egg-bearing females after sinus gland extirpation molt as readily as do males.

The majority of species of insects so far investigated show a hormonal relationship between the corpora allata and the ovaries. Allatectomy in late larval stages or young adults is accompanied by a failure of the eggs in the ovary to undergo their normal growth and development. This has been dem- 
onstrated for the hemipteran, Rhodnius; ${ }^{169}$ the dipterans, Calliphora, ${ }^{152,153}$ Lucilia, ${ }^{54},{ }^{152}$ Sarcophaga, ${ }^{54}$ and Drosophila; ${ }^{157}, 158,159$ and the orthopterans, Melanoplus, ${ }^{123,} 161$ and Leucophaea. ${ }^{139}$ Implantation of corpora allata into allatectomized individuals restores the ability to produce normal eggs. On the other hand, no such relationship appcars to exist in the orthopteran, Dixippus, ${ }^{125,} 126,127$ or in Lepidoptera. ${ }^{34}$ Evidence for the humoral nature of this relationship was clearly demonstrated by telobiotic experiments in Rhodnius, in which the factor concerned was shown to be blood-borne, and by transplantation of the corpora allata into allatectomized individuals, which indicated that the corpora allata exert their typical action irrespective of their new location. ${ }^{169}$ The influence of the active allatum principle appears to operate through its influence on the deposition of yolk within the eggs rather than on the earlier development of the oocytes. The corpora allata are

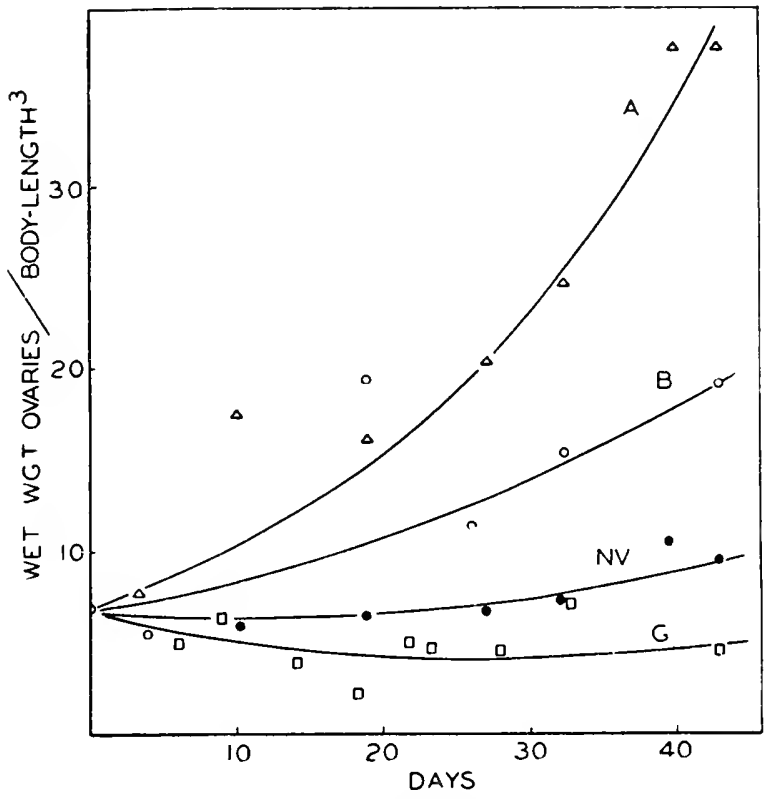

Fig. 285. Rate of increase in ovarian weight in the shrimp, Leander, after: $A$, eyestalk amputation; $B$, sinus gland extirpation; $G$, eyestalk amputation followed by sinus gland implantation. $N V$, Normal control. From Brown, ${ }^{38}$ redrawn from Panouse. ${ }^{120}$

apparently essential only throughout the period of oocyte growth and yolk deposition of each successive reproductive cycle. It has been shown that allatectomy is followed by profound alterations in the general metabolism of Melanoplus. ${ }^{124}$ This has led some investigators to support the view that the reproductive functions of the principle from the corpora allata are secondary to more basic metabolic ones.

Allatectomy also depresses the growth and activity of certain female accessory organs in Calliphora, Melanoplus, and Leucophaea, this influence being independent of the presence of the gonads and hence not operating through them.

There is considerable evidence that the ovary also exerts an action on the 
corpora allata in female insects. Ovariectomy in Melanoplus, Calliphora, and Lucilia leads to hypertrophy of the allata, and perhaps leads to a functional alteration in stiil other insects, such as Sarcophaga and Leucophaea.

Allatectomy leads to less distinct results in male insects than in females. The operation in no manner interferes with the production of sperm cells; in fact, allatectomized males of Leucophaea show ability to mate with, and effectively fertilize the eggs of, normal females. There are reports, however, that the male accessory glands of Rhodnius and of Calliphora fail to show normal development after allatectomv. Castration of male Lencophaea or Lucilia has led to no observable modifications in the corpora allata.

There is some evidence that the roaches, Blatta and Blattella, produce within their ovaries a functional counterpart of the mammalian corpus luteum, which contributes to normal egg-producing rhythms of these species. ${ }^{89}$ In this rhythm the mature ova are laid in cocoons which are carried about at the genital opening of the female. The ovaries are inhibited while these cocoons are being borne, but are released to further activity after deposition of the cocoons. Implantation of actively growing ovaries into the body of cocoon-bearing females is followed by a rapid change of certain characteristics of the implanted oocytes to resemble those seen in immature ovaries. Extracts of ovaries from animals in the inhibited stage of the normal reproductive cycle yield the same results. Histological examination of ovaries in their normally inhibited stage showed the presence of yellowish granules in the follicles formerly occupied by the developing ova.

\section{MORMONES AND GENERAL MAINTENANCE AND METABOLISM}

Intermediary Metabolism: Vertebrates. A number of hormones within the body, arising in the anterior pituitary, thyroid, adrenals, pancreas, and other organs, cooperate in their activities to help maintain the delicate balances obtaining in normal metabolism. The exact modes of action of these hormones involved in general metabolic homeostasis are still far from completely known, although the general outlines of their mechanisms of action have become clear.

Much more is known about the intermediary metabolism of carbohydrates than about that of the other two major categories of organic substances, the proteins and lipids. In the higher vertebrate, at least, the amount of blood glucose is maintained within a relatively narrow range of concentration as a result of a dynamic balance between blood glucose formation in the liver from glycogen stores, on the one hand, and utilization of glucose by the body tissues, on the other. Muscle, despite its large reservoirs of glycogen, is unable to produce glucose rapidly enough to maintain normal blood sugar levels in hepatectomized individuals. These latter become profoundly hypoglycemic. Muscle, however, does contribute some glucose to blood indirectly, through the escape of excess lactic acid by way of the blood to the liver, where it is converted into glycogen. Glycogen is also formed in the liver from the deaminized products of protein catabolism within the organism, this process thus providing indirectly an additional endogenous source of blood sugar. The foregoing relationships are depicted in Figure 286.

The steps in the process of reversible glycogen formation from glucose and the enzymes concerned have been considerably elucidated ${ }^{50}$ (see also 
Chapter 8). Glycogen is reversibly broken down to produce glucose-1-phosphate under the influence of an enzyme phosphorylase, a process which has been referred to as phosphorolysis because of its similarity to hydrolysis. In phosphorolysis $\mathrm{H}_{3} \mathrm{PO}_{4}$ participates in the reaction in a manner comparable to that of $\mathrm{H}_{2} \mathrm{O}$ in hydrolysis. Under the influence of the enzyme phosphoglucomutase the phosphate group is reversibly shifted within the molecule to the 6-position. The resulting glucose-6-phosphate is reversibly split by the action of phosphatase to yield glucose and inorganic phosphate. Glucose-6phosphate and fructose-6-phosphate, with which the former comes into equilibrium under the influence of the enzyme isomerase, comprise the primary substrates in the aerobic and anaerobic energy-yielding oxidative metabolism of carbohydrates.

A number of endocrine glands in the mammal are known to influence the intermediary metabolism of carbohydrates and consequently the carbohydrate contents of body tissues. Adrenin, from the adrenal medulla, elevates blood sugar by accelerating the formation of lactic acid from glycogen in muscle and of glucose from glycogen in the liver. In muscle, after adren-

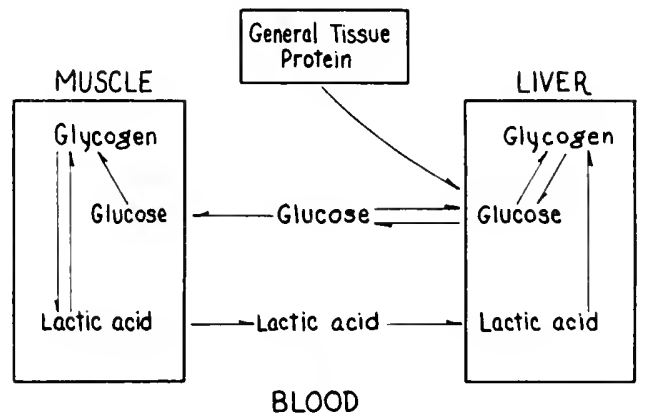

Fig. 286. Diagram representing the gross metabolic transformations of carbohydrate in muscle and liver occurring in the higher vertebrate.

alin administration, hexose monophosphate increases and inorganic phosphate decreases. It would appear that adrenalin increases phosphorylase activity, since this is the predominant reaction common to both muscle and liver in these changes. Adrenalin accelerates this reaction only in living cells, being ineffective in enzyme solutions.

Insulin from the islets of Langerhans of the pancreas appears to affect carbohydrate metabolism primarily through acceleration of the change, glucose to glycogen, in both muscle and liver. ${ }^{106}$ Insulin is not essential to this process at high blood sugar levels but is essential within the physiological range of blood sugar concentrations. In fact glycogen may be formed from glucose even in hypoglycemic animals, after insulin treatment. There is also some evidence that insulin has an inhibitory action on adrenalin-accelerated phosphorolysis in the liver.

Hormones of the adrenal cortex influence the intermediary metabolism involving carbohydrates in mammals. ${ }^{85},{ }^{105}$ After adrenalectomy rats and mice may be maintained in health by administration of sodium salts and glucose. However, such individuals, when fasted, suffer a rapid drop in 
stored glycogen in the body and in blood sugar, and exhibit greatly decreased nitrogen excretion, the latter indicating decreased protein catabolism. Conversely, after injection of cortical extracts normal specimens show an increase in both stored glycogen and blood sugar, while exhibiting increased nitrogen excretion. Since these changes occur even in fasting specimens, it is reason: able to consider that all the carbohydrate increase has occurred at the expense of body protein. In fact, the amount of nitrogen excreted under these circumstances appears to be of the proper order of magnitude to render this explanation highly probable.

In partially depancreatized rats with glycosuria maintained by appropriate feeding, adrenalectomy is followed by a marked reduction in glycosuria, which may be restored to its original high level by cortical extracts. Hypophysectomy of depancreatized or normal rats is also accompanied by carbohydrate reduction in the body, an effect which may in part be reversed by administration of cortical extracts. It would therefore appear that at least a part of the influence of the pituitary on carbohydrate metabolism is exerted through its adrenotropic factor. Of the cortical principles, corticosterone and dehydrocorticosterone influence carbohydrate metabolism much more strongly than do desoxycorticosterone and progesterone, although the latter are very influential in maintaining the electrolyte balance and life of the organism.

It would appear from the available evidence that the adrenal cortex influences carbohydrate metabolism largely through the stimulation of production of carbohydrate at the expense of protein.

The thyroid of the mammal through the action of its hormone, thyroxin, exerts a powerful influence on the basal metabolism and hence on the oxidation of such normal substrates as hexosemonophosphate. Its mode of action would therefore appear to be through the activation of those enzyme systems which limit the rate of the process-probably dehydrogenases.

A number of principles from the anterior lobe of the pituitary influence various aspects of intermediary metabolism. ${ }^{181,83}$ Through the action of a pancreatropic principle the normal amount of islet tissue and hence of normal insulin production is maintained. An adrenotropic principle is essential to continued normal production of carbohydrate-influencing cortical principles. A diabetogenic principle is believed to inhibit insulin secretion and hence give rise to the characteristic symptoms of diabetes-namely hyperglycemia, glycosuria, and ketonuria. Through a thyrotropic principle the thyroid is caused to continue its normal production and liberation of thyroxin. Other principles of anterior pituitary origin which more questionably possess separate identities are believed by many to influence metabolism more directly. A heat-stable glycotropic principle appears to act as an anti-insulin, having no influence on metabolism apart from its action relative to insulin. A glycostatic principle may retard or inhibit the oxidation of hexosemonophosphate. A ketogenic factor (possibly identical with the glycostatic, adrenotropic, or diabetogenic factor) is believed to influence fat metabolism in such a manner as to result in the production of ketone bodies, organic acids characteristically found in the blood of diabetics.

The observation of Houssay and his associates that removal of the anterior lobe of the pituitary from depancreatized mammals diminishes the intensity of the diabetic condition of these animals, thereby demonstrating 
a diabetogenic action of the pituitary, has been confirmed in lower vertebrates. Removal of the whole pituitary or of only the anterior lobe in toads ameliorates pancreatic diabetes. ${ }^{144}$ The anterior lobe of the dogfish, Mustelus, possesses a similar principle." Hypoglycemia follows hypophysectomy in this fish, an effect reported as following removal of only the anterior lobe. Furthermore, removal of the anterior lobe decreases the severity of diabetes experimentally induced in these fishes by removal of the pancreas.

Experiments involving thyroid administration to fishes appear to indicate that this hormone has no influence on $\mathrm{O}_{2}$ consumption in the guppies and goldfish. ${ }^{60},{ }^{145}$ Treatment with thiourea does not significantly alter $\mathrm{O}_{2}$ consumption in Fundulus. ${ }^{113}$ Injection of extracts of thyroid glands of Bermuda parrot fish increased $\mathrm{O}_{2}$ consumption in white grunts, but only when the latter fish weighed more than about 15 grams. ${ }^{147}$ As in most fish, administration of thyroid to Rana pipiens tadpoles prior to changes of metamorphosis resulted in no alteration in $\mathrm{O}_{2}$ consumption. ${ }^{75}$ In adult Rana pipiens, on the other hand, administration of thyroid increased $\mathrm{O}_{2}$ consumption substantially, ${ }^{160}$ and if the animals were kept at temperatures higher than about $13^{\circ} \mathrm{C}$. caused reduction in body weight. Among the reptiles and birds the effect of thyroid administration on the basal metabolic rate appears quite similar to that observed in mammals. Pigeons show a marked reduction in basal heat production after complete thyroidectomy. ${ }^{110}$

Young Python, during long-continued thyroid feeding, show greatly increased excitability and weight reduction. ${ }^{100}$

A further suggestion that endocrine control of intermediary metabolism of fishes differs markedly from that in higher vertebrates is seen in the comparative effects of adrenalin on $\mathrm{O}_{2}$ consumption. Whereas administration of adrenalin to normal mammals produces a considerable elevation, in the fish Girella there is only a reduction, and this latter occurs only in response to doses which are huge in comparison with effective doses in the mammal. ${ }^{1+6}$

Invertebrates. There is no conclusive evidence that any of the hormones of the vertebrates which have a dufinite influence on intermediary metabolism within that group have any comparable actions in any invertebrate. It seems well established, however, that among the arthropods, at least, hormones do have an influence on general metabolism. Removal of the eyestalks or of the sinus glands of these stalks of Cambarus is promptly followed by an elevation in basal metabolism, as evidenced by the observed increase in $\mathrm{O}_{2}$ consumption. ${ }^{1+1}$ This can be reduced by injection of aqueous extracts of the glands. Injection of extracts of eyestalks of $U_{c a}$, the fiddler crab, into Callinectes, the blue crab, results in a rapid rise in the blood sugar from about $20 \mathrm{mg} . / 100 \mathrm{cc}$. to more than $80 .^{3}$ The maximum is reached in one hour, and then there is a slow decline to normal. This latter action has been attributed to the presence of a diabetogenic factor in the crustacean sinus gland, and recent work has confirmed this conclusion for the spider crab Libinia. ${ }^{95 a}$

In the grasshopper, Melanoplus, the corpora allata appear to be essential for normal fat metabolism in the female. ${ }^{124}$ The female in adult development first shows a rapid rise in fatty-acid content and much fat storage in the fat bodies. This period, also one of the early growth of oocytes in the 
ovary, is followed by a period of rapid yolk deposition. During this latter period the fatty stores are rapidly reduced and stabilized at a new lower level. These changes occur in a closely similar manner in castrated animals, indicating that the control of these changes resides outside of these organs. However, when allatectomy is performed in young adult females, the operated animals continue to deposit fat for a long time, the fat bodies becoming tremendously hypertrophied. It would thus appear that during normal development of the corpora allata these organs undergo an endocrine alteration with respect to factors controlling fat metabolism, in a manner directly adapted to normal yolk deposition. Furthermore, in castrated but otherwise normal females there is observed an accumulation of non-fatty material which does not occur in allatectomized-castrated specimens. This suggests that the corpora allata are responsible for the accumulation of "precursory materials" normally used up in the production of yolk but which, in the absence of the ovaries, are forced to accumulate in other regions of the body, such as the hemolymph.

Water and Salt Regulation. The maintenance of the normal water content and the characteristic percentage compositions of various essential inorganic constituents in the blood, tissue, and intracellular fluids is dependent on a regulation of the relative rates at which these materials enter the body and leave it, and also on a regulation of the distribution of the materials among blood, tissue fluids, intracellular contents, and storage reservoirs. Important participants in such regulatory roles are a number of endocrine organs of the body of the mammal, notably the pituitary, the adrenal cortex, the parathyroids, and the kidneys.

Extirpation of the cortical tissue of the adrenals of the mammal is rapidly fatal. ${ }^{85}$ There are an increase in the rate of loss of sodium and chloride ions in the urine and a decrease in the rate of their absorption from the gut. Potassium ions accumulate in the blood. These effects of adrenalectomy may in some measure be compensated by administration of a diet low in potassium and high in sodium. Sodium salts appear to leave the cells of the tissues as well. In the absence of the cortex the water content of the blood declines, the blood becoming concentrated as water passes to the tissue fluids and also out of the body through the kidneys. Administration of extracts of the cortex, and especially of one of the numerous steroids isolated from it, namely desoxycorticosterone, prevents occurrence of these changes in completely adrenalectomized individuals. These hormones apparently diminish the absorptive capacity of the gut, kidney tubules, and tissue cells for sodium and chloride ions.

The parathyroids, through the action of their hormone, parathormone, are essential to the maintenance of the normal calcium and phosphate ion content and distribution within the body. ${ }^{151}$ In this role the hormone functions chiefly through governing the movement of these ions between bone and body fluids. The presence of the hormone favors the movement in the direction of the blood; its absence favors movement from blood to bone. The hormone also appears essential for the normal resorption of these ions by the kidney tubules. In its absence the ions are largely lost from the body through kidney filtration and a subsequent failure of resorption of these ions by the 
tubules. Parathyroidectomy is rapidly lethal; there is a rapid decline in blood calcium and finally death in tetanus.

The posterior lobe influences the rate of water loss through the kidneys. ${ }^{86}$ Destruction of this portion of the pituitary results in an increased urine flow and an increase in the consumption of water by the organism (diabetes insipidus). A hormone, the antidiuretic principle, is essential for the normal extensive water resorption by the kidney tubules.

The kidney, on reduction of its blood supply by any means, liberates a globulin protein, renin, into the blood. ${ }^{11 \times, 35}$ Renin is a proteolytic enzyme which acts on a serum globulin, hypertensinogen, converting it into a fraction, hypertensin. Hypertensin, a powerful agent in elevating blood pressure

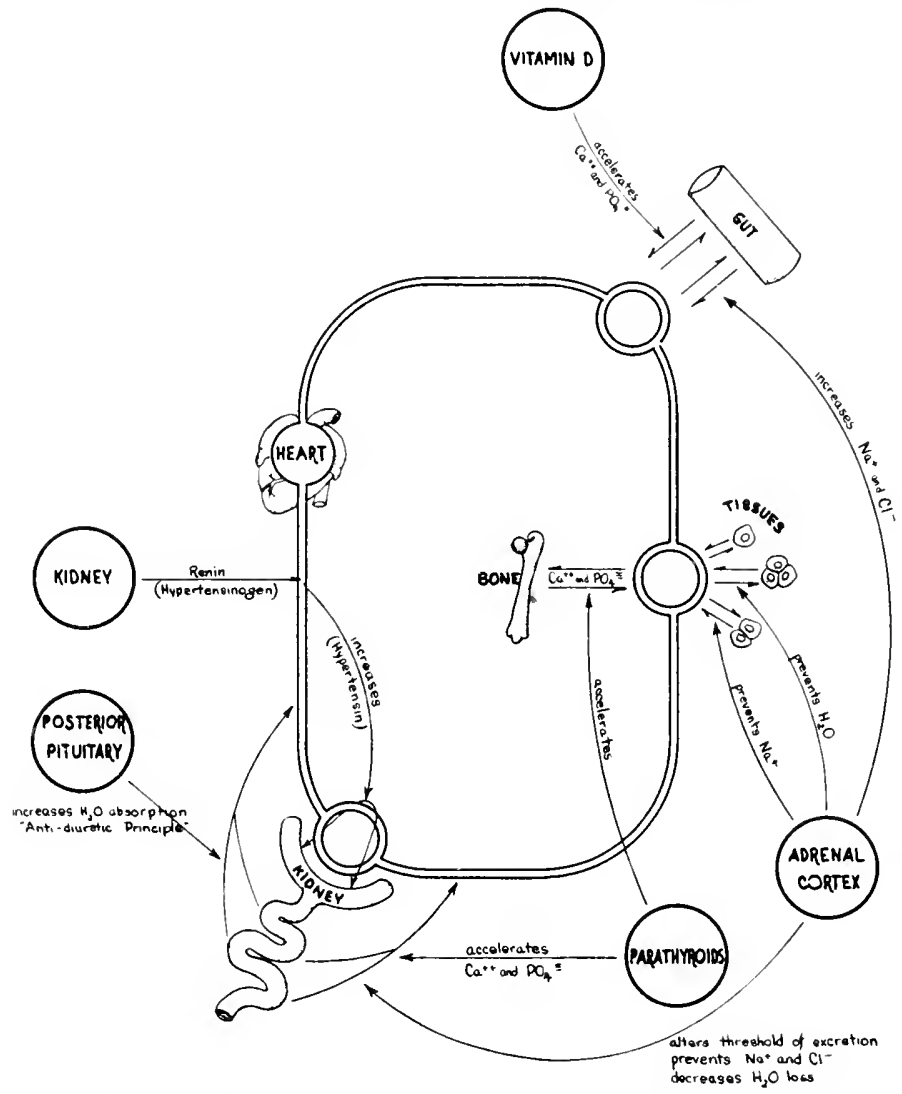

Fig. 287. Diagram summarizing influences of hormones on maintenance of the normal water and salt balance of the mammalian organism.

within the animal, appears to be a protein derivative of polypeptide character which is normally destroyed slowly in the blood stream by an enzyme, hypertensinase. The production of renin by the kidney would therefore appear to be an adaptive reaction to assure the organism of sufficiently high blood pressure to permit adequate kidney function.

Most of the more important influences of hormones on water and salt balance are diagrammatically portrayed in Figure 287. 


\section{HORMONES AND GASTROINTESTINAL COORDINATION}

In the mammal the integration of the activities of various parts of the digestive system in dealing with ingested food is in part endocrine in character $^{64,65 a, 87,88}$ The major hormones and their general sites of formation and regions of action are shown diagrammatically in Figure 288.

The pyloric mucosa on appropriate stimulation secretes into the blood a hormone, gastrin. Non-innervated, transplanted gastric pouches are caused to secrete in response to the presence of food in the stomach of the animal. For a long time gastrin was believed to be identical with histamine, but it is now known that histamine-free extracts of the pyloric mucosa will stimulate secretion of a highly acid gastric juice with very little peptic activity and simultaneously stimulate pancreatic secretion. By fractional precipitation the gastric stimulant, gastrin, has been separated from the activator of the pancreas; the latter resembles very closely the secretin obtained from the duodenal mucosa. Gastrin and gastric secretin appear to have protein-like properties.

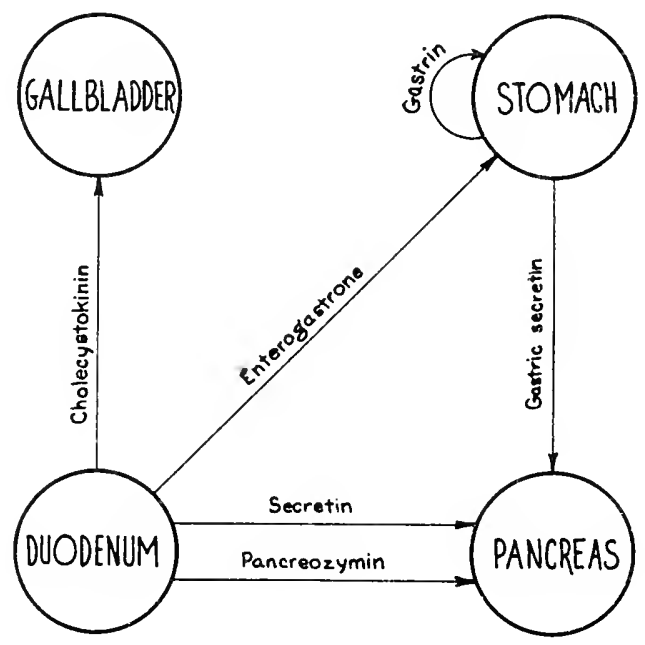

Fig. 288. Diagrammatic representation of the sources and points of action of the principal hormones operating in gastrointestinal coordination.

The duodenal mucosa quantitatively stimulates the pancreas to liberate its digestive juice through action of the hormone secretin. It was formerly thought that secretin stimulated mainly the secretion of water and bicarbonate by the pancreas and that the production of enzymes was nervously controlled, but no consistent data were obtained to support this hypothesis. The variable concentrations of enzyme in pancreatic juice that have been reported to be produced in response to secretin injections appear to find their explanation in terms of the presence of a second hormone in the crude extract. One fraction stimulates the secretion chiefly of water and inorganic salts from a dencrvated pancreas. A second fraction will stimulate pancreatic enzyme secretion. The active factor of this latter fraction has been termed pancreozymin. 
Another active principle from the duodenum, and one which has been separated from crude secretin extracts, is cholecystokinin a powerful agent in producing gallbladder contraction.

A fourth factor, enterogastrone, has been isolated from the duodenal mucosa. Crude extracts containing this factor inhibit gastric secretion and gastric motility, but, after purification, the extract largely loses the latter capacity. The activity of enterogastrone therefore appears to be principally
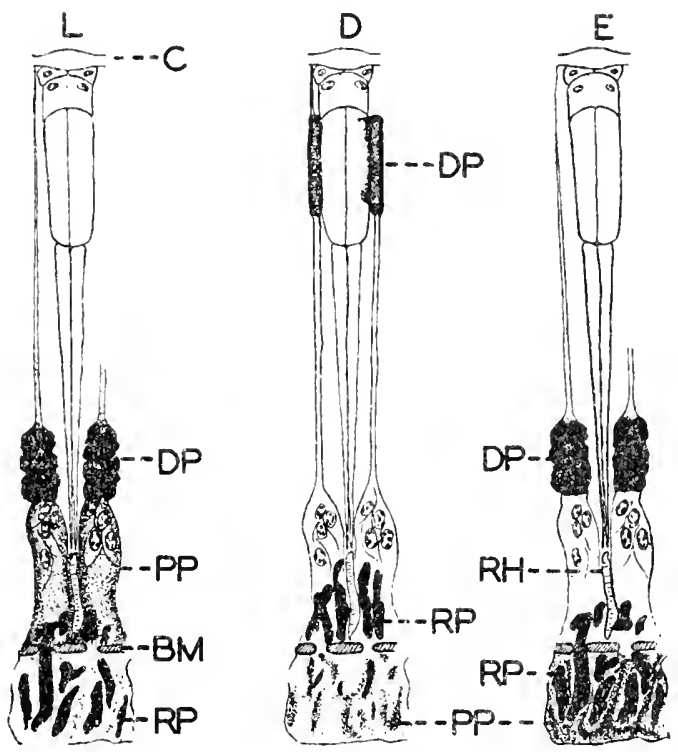

Fig. 289. Position of the retinal pigments in the ommatidium of the eye of the shrimp, Palaemonetes, $L$, in the light-adapted state, $D$, in the dark-adapted state, and $E$, after injection of eyestalk-extract into a dark-adapted specimen kept in darkness. C, Cornea; $D P$, distal retinal pigment; $P P$, proximal retinal pigment; $R P$, reflecting pigment; $R H$, rhabdome; and $B M$, basement membrane. From Kleinholz.:"

antisecretory, and since in the presence of enterogastrone the gastric juice produced under the influence of gastric stimulants such as histamine is low in acid content and rich in pepsin it would appear that enterogastrone preferentially inhibits the acid-secreting parietal cells of the stomach.

Other gastrointestinal chemical coordinators have been proposed on the basis of brief experiments but these appear to have a more questionable existence than the aforementioned ones.

\section{HORMONES AND PIGMENT-CELL ACTIVITIES}

Integumentary Chromatophores. The roles of hormones in the control of the integumentary color changes in cephalopods, crustaceans, insects, fishes, amphibians, and reptiles have been dealt with at some length in Chapter 21 and will not be reviewed here.

Retinal Pigment Migration. The movements of pigments within the eyes of many animals such as vertebrates, insects, and crustaccans contributes im- 
portantly to the mechanical adaptation of these organs to changes in light intensity. Only among the crustaceans, however, has clear evidence been presented that hormones are involved in the control of these movements (see Chapter 11, p. 388).

The compound eye of crustaceans is made up of a number of units, the ommatidia (Fig. 289). Each ommatidium possesses three functionally distinct groups of pigments. The distal retinal pigment, melanin, is located in cells which surround the distally placed dioptric apparatus of the ommatidium. In the dark-adapted eye this pigment occupies only a distal position; the pigment disperses proximally in daylight to envelope the whole of the ommatidium as far as the retinula elements. The proximal pigment, which is also melanin, is located in the retinula cells and migrates to a position proximal to the basement membrane in the dark, at night, and distally to meet the distal retinal pigment in daylight. In the light-adapted state the whole ommatidium is therefore enclosed in a light-absorbing sleeve of pigment. A third pigment, the reflecting white, appears to be particulate guanin. In darkness this pigment occupies a position surrounding the retinula elements, thus constituting a functional tapetum. It migrates to a position proximal to the basement membrane in daylight.

Investigations to determine the extent to which the pigment cells of the right and left eyes of an individual are capable of independent responses to illumination have led to various results. The more recent experiments of this type have led to the conclusion that there is at least a partial interdependence between the two eyes, a darkened eye becoming more or less light-adapted when the contralateral eye is subjected to illumination. ${ }^{22} \mathrm{Num}$ erous observations have also indicated that one or more of the retinal pigments of numerous species of crustaceans may undergo diurnally rhythmic alterations in their position in animals kept in constant conditions in respect to illumination, especially in constant darkness. ${ }^{23,}{ }^{162}$ Leander kept on an illuminated black background has been observed to show a dorso-ventral differentiation in position of retinal pigment, apparently the result of the considerably lesser illumination of the ventral than of the dorsal elements of the eyes. ${ }^{95}$ These various responses of the retinal pigments appear to suggest that the control of the retinal pigments is not a simple one, but probably involves a direct reaction of the retinal pigment cells to illumination and, in addition, endocrine and possibly also nervous activities.

There appears to be good evidence that the sinus gland of the eyestalk produces a hormone which influences the position of the retinal pigment. ${ }^{91}$ 163,164 This has been called retinal pigment hormone or RPH. ${ }^{38}$ Injection of extracts of the eyestalks of light-adapted Palaemonetes into dark-adapted specimens kept in darkness induces in the latter a movement of the distal and reflecting pigments to the position characteristic of the light-adapted state. The eyestalks of all crustaceans which have been examined show the presence of RPH in larger or smaller quantities. That this hormonal principle is normally concerned with the retinal pigment movements is indicated by the fact that extracts of eyestalks taken from light-adapted individuals show a much greater RPH content than do eyestalks taken from dark-adapted ones. ${ }^{91}$ However, it has been reported that the diurnal variation in retinal pigments of certain grapsoid crabs persists even following removal of the 
sinus glands. ${ }^{149 a}$ More recently evidence has been presented to support the view that a hormone from the sinus glands contributes at least in part to the. control of the distal retinal pigment of Leander. $5 \%$ in

The retinal pigments of Cambarus show different thresholds of response to RPH. ${ }^{163}$ In low concentrations of the hormone only the distal pigment is influenced; with higher concentration both distal and proximal retinal pigments move to the light-adapted state.

The origin of RPH is the sinus gland of the eyestalk. This organ, when extracted alone, is able to induce a strong retinal pigment response. No other tissue or organ of the evestalk approaches it in effectiveness.

The retinal pigment hormone of the sinus gland will withstand boiling, as in the case of the sinus gland chromatophorotropins, but nothing else is yet known of its properties. There is ample reason to believe that it is not identical with any of the dominant chromatophorotropins, inasmuch as the pigmentary system of the integument ordinarily undergoes its complete gamut of activities in color changes in response to illuminated backgrounds while the eve remains continuously light-adapted. ${ }^{92}$ This latter is true despite the fact that the threshold of response of the retinal pigments to eyestalk extract is substantially higher than the threshold of response to the body chromatophores. Such a situation obviously could not obtain were RPH identical with one of the dominant chromatophorotropins.

A search for a possible comparable endocrine influence on the state of the retinal pigments of the insect, Ephestia, disclosed no evidence of such an endocrine activity. ${ }^{\overline{3}}$ Injection of extracts of the heads of light-adapted moths into either dark- or light-adapted specimens produced no modification in the state of the pigments. Extracts of crustacean sinus glands also showed no activity on the retinal pigments of the moth.

The movement of pigments and the changes in lengths of the contractile fibrils (myoids) of the rods and cones of the eyes of lower vertebrates have long been known, but there was no reason to suspect that they were other than direct responses to light until it was shown that the lengths and positions of the rods and cones of the eyes of catfishes kept in constant darkness still undergo a diurnally rhythmic change. ${ }^{165}$ It is not yet known whether the control of these changes involves chemical coordinators or only nervous activity.

Coloration and Seasonal Color Changes of Birds and Mammals. Among the numerous species of birds which show differentiation of hen and cock plumages a wide variety of mechanisms are involved. ${ }^{52,}$ 55 In the English sparrow the control of plumage type is exclusively genetic. Among the pheasants, the plumage type is determined by simultaneous action of genes and hormones. In perhaps the majority of birds, however, the plumage typical of one sex is neutral; that of the other sex is determined by blood-borne hor mones. In the common domestic fowl the neutral type appears to be the cock plumage, with the hen type the result of action of estrogens. In African weaver finches, the neutral type is the hen plumage; the cock plumage is the result of action of hypophyseal hormones. Only in the herring gull, among the birds thus far studied, does the cock type of plumage depend on action of androgens. ${ }^{32}$

In the majority of common birds the adults undergo a rather complete 
post-nuptial molt immediately after the breeding period; the new regenerating plumage becomes the winter or eclipse plumage. A second, usually much less extensive molt, the pre-nuptial, occurs in the spring. The regenerating plumage constitutes the breeding or nuptial plumage. This latter molt is most extensive in those species which exhibit conspicuous breeding coloration. The times at which birds assume the nuptial and winter plumages are governed by a number of factors. In some species, such as the African weaver finch, Pyromelanox, and the starling, the changes appear due to an inherent cyclically rhythmic hypophyseal activity, ${ }^{17 i}$ although the occurrence of the rhythm may depend in part on length of daily light periods. ${ }^{41,44,102}$ Some other species, such as mallard ducks, Anas platyrhyncha, the white-throated sparrow, and the bob-white, can be induced to molt and undergo a plumagetype change at a time other than their regular season by subjection to artificially increasing or decreasing light periods. Therefore, the annual plumage changes of most birds, like migrating and breeding activity, appear to be controlled in good measure by the annual cycle of day lengths.

Certain northern birds and mammals show a seasonal color change from brown in summer to white in winter. The times of these changes in such forms as the ptarmigan, Lagopus, ${ }^{81}$ the ermine, Mustela, ${ }^{28}$ and the varying hare, Lepus, ${ }^{107}$ have been shown to be determined by the seasonal changes in day lengths; the animals could be caused to whiten out of season by appropriate experimental alteration of the daily lighting. Experiments involving masking of the varying hare ${ }^{107}$ indicate that the eyes are the normal receptors. Both in the natural cycle of color change and in color changes induced artificially by modification of illumination, the varying hare is physiologically brown when large amounts of gonadotropic hormones are present in the biood and is physiologically white when these hormones are low in concentration. Molting in a physiologically brown animal is followed by production of brown hair; in a physiologically white one, by production of white hair. Extracts of whole pituitary containing gonadotropic hormones will convert physiologically white animals into physiologically brown ones and simultaneously induce shedding of the white hair. Hypophysectomy in ferrets abolishes the cyclic molting. ${ }^{25}, 27$ No endocrine gland other than the pituitary appears to be involved in these color changes. Castrated and thyroidectomized hares undergo the normal seasonal color changes. ${ }^{107}$

\section{HORMONES AND BIRD MIGRATION}

Some years ago it was postulated that the migrational behavior of birds was under the control of hormones from the gonads. ${ }^{129,}$ 130, 131 Northward migration of birds in the northern hemisphere was considered a result of the recrudescence of the gonads in the spring; regression of the gonads after the breeding season was correlated with migration southward. In the junco, the northward migration appeared correlated with periods of greater interstitial cell activity of the gonads. In the crow, southward migration appeared independent of gonadal regression, as indicated by castration. In the European starling, northward migration appeared correlated with gonadal secretion and southward migration with absence of secretion. ${ }^{42,43}$

Other evidence seems to indicate with moderate clarity that clianges in gonadal activity are not causally related to migrational behavior. Removal of 
gonads does not appear to abolish migrational behavior. ${ }^{70}$ Furthermore many birds migrate while they are still sexually immature. ${ }^{1 \times 0}$ Migrant juncos retained as long as two months after their normal time of migration would still move northward on release, although their gonads were already in breeding condition. ${ }^{178}$ Birds retained in their wintering grounds during the summer, and thus prevented from spring migrating and breeding, nonetheless underwent the typical gonadal regression and fat deposition observed in birds preparing for the annual fall migration.

Examination of the pituitaries of juncos showing increase in testis size, and of birds later during breeding, revealed cytological evidence of much more active secretion of this gland than during the winter when testis size was minimal. Furthermore, injections of antuitrin-G containing several anterior lobe principles will induce a condition--increased gonad size and heavy fat deposition-which resembles closely those characteristics in a bird ready to migrate. These observations strongly suggest that the pituitary is an important conditioning agent for migration. ${ }^{179}$

Within many species of birds, including juncos, it is possible to differentiate two types of individuals. Some individuals are normal migrants, and others are non-migrants or residents. These two types can sometimes be clearly differentiated into morphologically different subspecies or varieties, whereas again they may comprise quite similar individuals. An individual bird may belong to both types at different times during its lifetime. There appears to be good evidence that similar treatment of "residents" and "migrants" with increased light periods will result in gonadal recrudescence in both instances, yet only the migrants will exhibit fat deposition and subsequent migration.

Such observations as these lead to the concept that an inherent potentiality to migrate exists in certain birds, but that the behavior patterns which initiate and carry out the migratory flight are activated or influenced by seasonally varying external factors, principally temperature, ${ }^{30 .}{ }^{90}$ (see p. 371) and changing day lengths. These factors appear to operate initially by way of the anterior lobe of the pituitary ${ }^{26}$ Hormones from this organ then gradually alter the physiological state of the bird, one or more of these latter alterations probably serving as the immediate stimulus to migration. The data suggest that the fundamental alteration in physiological state involved here is the deposition of fat which would provide the energy for the migrational flight.

\section{GENERAL CONSIDERATIONS}

In any comparative survey of endocrine mechanisms one is impressed by the fact that, in all those animal groups in which endocrine systems have differentiated, the same general types of functions are being subserved by them. In the two very widely separated animal groups, the Arthropoda and the Vertebrata, which are considered by most zoologists to possess no common ancestry short of relatively primitive forms of life, we see the same general distribution of coordinatory functions between nervous and endocrine systems. In both groups growth, differentiation, reproduction, metabolism, and pigment cells are influenced by hormones. In brief, the endocrine system seems to influence primarily those functions in which the time to induce a response is long (as in those activities involving growth 
and cell differentiation), those processes in which the controlling influence needs to be maintained over long periods of time (as in the control of general processes in basic maintenance and metabolism), and situations in which effector organs are maintained in one or another condition for very long periods of time (as with chromatophores).

Within even relatively large natural groups of animals there is commonly a lack of specificity of the hormonal substances. Among the vertebrates, hormones such as the gonadal hormones, insulin, thyroxin, and others seem rather widely distributed, typically producing within any species a type of response characteristic for that species. Similarly, among the insects, the juvenile hormone and the GD hormone (see p. 733) appear interchangeable among the various species, and often even among orders. The same situation appears also to obtain for the chromatophorotropins, retinal pigment hormone, and molt-inhibiting hormone among various species of the Crustacea.

Beyond these groups little is known as to chemical or physiological similarities of the active principles. The corpora cardiaca of insects yield a principle highly active on the chromatophores of crustaceans. A rather extensive literature has developed regarding the influence of invertebrate hormones on vertebrates and especially of vertebrate hormones on invertebrates. ${ }^{71}, 166$ Much of the work is confusing and contradictory and gives us little reason for believing that the results have anything other than interesting pharmacological value. The vertebrate chromatophorotropin, intermedin, and the crustacean hormone, UDH (see p. 698), appear qualitatively to resemble one another in their chromatophorotropic action and in many of their physicochemical properties. ${ }^{1}$ A critical examination, however, gives us strong reason for believing they are not identical. ${ }^{37}$

The nervous systems of a wide variety of animals show histological and, in certain cases, good physiological evidence of the differentiation of endocrine elements or neurosecretory cells. ${ }^{140}$ Such cells occur in certain portions of the nervous systems of worms, molluscs, arthropods, and vertebrates. In the last group, they are located in the nucleus preopticus of fishes and amphibians, and in the homologous nuclei supraopticus and paraventricularis of reptiles and mammals. Only among certain invertebrates is there evidence of a functional role of these neurosecretory cells within the organism. The pars intercerebralis of the insect brain contains knots of large neurosecretory cells and the endocrine activity of this part of the nervous system has ailready been described. A certain chromatophorotropically active agent for crustacean chromatophores has been found to have a quantitative distribution in the nervous system of Limulus closely paralleling the frequency of neurosecretory cells known to occur there. ${ }^{39}, 137$ Certain large ganglion cells in annelids give a chromaffin-staining reaction characteristic of adrenalinproducing tissues, and extraction and assay of such nervous tissue gives positive physiological tests for adrenalin.

It is interesting to point out that the insect corpora cardiaca and allata are so closely associated with the nervous system that they were formerly known respectively as the anterior and posterior esophageal ganglia; the corpora cardiaca contain ganglion as well as secretory cells. The sinus gland of crustaceans appears to be a derivative of the neurilemmal sheath of the anterior central nervous system, as does also the glandular source of the hormones of 
the tritocerebral commissure of Crago. Among the vertebrates the medulla of the adrenal and the posterior lobe of the pituitary are considered to be derived embryologically from nervous tissue. The latter is believed to be homologous with the neural gland of tunicates, which, however, in addition to possessing oxytocic, pressor, and melanophore activating agents, appears also to have some gonadotropic activity.

In brief, it would seem that there has been an evolution of certain endocrine tissues from tissues possessing a simple nervous or conductile function and located within the central nervous system, through a condition where the cells exhibited secretary function while still retaining the functional properties of nerve cells, to a condition in which the cells have become specialized for endocrine activity alone. In the last instances the cells have commonly become separated from the nervous system to form definite glandular tissues or organs. In such organisms as the arthropods, which possess an open circulatory system, no better access to a rich hemolymph supply for such organs is to be found in the organism than the surfaces of the central nervous organs which are bathed directly by the fluid contents of a large blood sinus.

Other endocrine organs have developed ontogenetically and phylogenetically independently of the nervous system.

It is interesting to note that, in the vertebrates, those hormones from sources of ectodermal or endodermal origin are proteins (pituitary principles, thyroglobulin, insulin) or at least contain nitrogen (adrenin); hormones arising from tissues of mesodermal origin are characteristically steroids (sex hormones, cortical principles).

There has been some speculation in the literature as to which is probably the primitive integrating mechanism within animals-nervous or endocrine. Obviously both inechanisms, when broadly interpreted, extend to all forms of living organisms, both unicellular and multicellular. The phenomena of excitation and conduction, the basic underlying activities in the physiology of the nervous system, are common to all cells, as are also the intracellular influences of numerous chemical products of activity of every cell. As was pointed out in the introduction to this chapter, some organizing and differentiating forces obviously had to precede both of these coordinating mechanisms in both phylogeny and ontogeny. In development gene-induced differentiation precedes organizer activity. There is no good reason to postulate that either the excitatory or the chemical coordinating factor is phylogenetically the more primitive. Both types of coordinatory mechanisms probably evolved simultaneously and entirely parallelly for the most part; and, in response to the functional needs of larger size, and later the multicellular character, of the organisms, highly specialized nervous and endocrine systems differentiated.

\section{REFERENCES}

1. Aвramowitz, A. A., Physiol. Zool. 11:299-311 (1938). Similarity of crustacean sinus gland hormone to intermedin.

2. Abramowitz, A. A., Hisaw, F. L., Boettiger, E., and Papandrea, D. N., Biol. Bull. 78:189-201 (1940). Hypophysis and pancreatic diabetes in the dogfish.

3. Abramowitz, A. A., Hisaw, F. L., and Papandrea, D. N., Biol. Bull. 86:1-5 (1944). Diabetogenic factor in crustacean sinus glands. 
4. Abramowitz, R. K., and Abramowitz, A. A., Biol. Bull. 78:179-188 (1940). Eyestalks and molting in crabs.

5. Adeter, L., Arch. Entw. Mech. 39:21-45 (1914). Hypophysis in amphibian netamorphosis.

6. Alzent, A., Endocrinology 37:389-406 (1945). Thyrotropic principle in fish pituitary.

7. AlleN, B. M., Science 44:755-757 (1916). 'Thyroid and amphibian metamorphosis.

8. Allen, B. M., Science 52:274-276 (1920). Pituitary and thyroid in amphibian metamorphosis.

9. Allen, B. M., Univ. Calif. Publ. Zool. 31:53-78 (1927). Anterior lobe and amphibian metamorphosis.

10. Allen, B. M., Anat. Rec. 54:45-64, 65-81 (1932). Hormones and amphibian metamorphosis.

11. Allen, B. M., Biol. Rev. 13:1-19 (1938). Hormones and amphibian metamorphosis, review.

12. Allen, E., Sex and Internal Secretions (1939). Baltimore, Williams and Wilkins $1346 \mathrm{p}$.

13. Avel, M., Bull. Soc. Zool. France 53:322-323 (1928). Testes and clitellum fonnation in earthworms.

14. Aver, M., C. R. Soc. Biol. Paris 99:616-618 (1929). Testes and clitellum formation in earthworm.

15. Baker, J. R., and Ranson, R. M., Proc. Roy. Soc. Lond., B. 110:313-322 (1932). Light anó breeding in Microtus.

16. Beadle, G. W., Genetics 22:587-611 (1937). Sources of Vt-hormone in Drosophila.

17. Beadle, G. W., Anderson, R. L., and Maxwell, J., Proc. Nat. Acad. Sci. 24: 80-85 (1938). Lack of species-specificity of insect $V+$-hormone.

18. Beadle, G. W., and Ephrussi, B., Genetics 21:225-247 (1936). Cnt- and $\mathrm{V}+$-hormone in Drosophila eye pigment development.

19. Beadle, G. W., Tatum, E. L., and Clancy, C. W., Biol. Bull. 77:407-414 (1939). Chemical nature of insect $V$ t-hormone.

20. Becker, E., Biol. Zentralbl. 59:597-627 (1939). Eye pigments of Drosophila.

21. Becker, E., and Plagge, E., Biol. Zentralbl. 59:326-341 (1939). Hormonal control of dipteran metamorphosis.

22. Bennitt, R., Physiol. Zool. 5:49-64 (1932). Interrelation of eyes of crustaceans in light adaptation.

23. Bennitt, R., Physiol. Zool. 5:65-69 (1932). Diurnal rhythm in crustacean retinal pigment movements.

24. Bissonnette, "Г. H., Proc. Roy. Soc. Lond., B. 110:322-336 (1932). Photoperiodism and sexual cycle of ferret.

25. Bissonnette, T. H., Anat. Rec. 63:159-168 (1935). Hypophysis and molting in ferrets.

26. Bissonnette; T. H., Wilson Bull. 49:241-270 (1937). Hormones in bird migration.

27. Bissonnette, T. H., Endocrinology 22:92-103 (1938). Influence of the hypophysis upon molting in the ferret.

28. Bissonnette, T. H., and Bailey, E. E., Ann. New York Acad. Sci. 45:221-260 (1944). Control of seasonal changes in coat color of the ermine, Mustela.

29. Blacher, L. J., Trav. Lab. Zool. Exp. Morph. Anim. 3:172-173 (1928). Hormones and amphibian metamorphosis.

30. Blanchard, B. D., Univ. Calif. Publ. Zool. 46:1-178 (1941). Temperature change and bird migration.

31. Bodenstein, D., Cold Spring Harbor Symp. Quant. Biol. 10:17-26 (1942). Hormones in insect development, review.

32. Boss, W. R., J. Exper. Zool. 94:181-209 (1943). Hormones and plumage type in gulls.

33. Bounhol, J. J., C. P. Soc. Biol. Paris 126:11.89-1191 (1937). Corpora allata and lepidopteran development. 
34. Bountuor, I. J., Arch. Zool. Exper. Gen. 81:54-64 (1939). Hormones in lepidopteran reproduction.

35. Braun-Menendez, E., Ann. Rev. Physiol. 6:265-294 (1944). Physiological action of renin, revicw.

36. Brown, F. A., JR., Anat. Rec. (suppl.) 75:129 (1939). Sinus gland and molting in Palacmonetes.

37. Brown, F. A., Jr., Quart. Rev. Biol. 19:32-46, 118-143 (1944). Endocrines in crustaceans, review.

38. Brown, F. A., Jr., Chemistry and Physiology of the Hormones (1948). New York, Academic Press. Vol. I, ch. 5. Crustacean hormones, review.

39. Brown, F. A., Jk., and Cunningham, O., Biol. Bull. $77: 104-114$ (1939). Sinus gland hormone and molting in Crustacea.

40. Brown, F. A., Jr., and Jones, G. M., Anat Rec. 99:657 (1947). Eyestalks and ovarian growth in crayfishes.

40a. Brown, F. A., Jr., and Jovrs, G. M., Biol. Bull. 96:228-232 (1949). Sinus gland hormone in Uca.

41. Brown, F. A., Jr., and Rollo, M., Auk 57:485-498 (1940). Influence of light periods upon plumage types in birds.

42. Bullough, W. S., Phil. Tr. Roy. Soc. Lond. B. $231: 165-246$ (19+2). Gonadal hormones in bird migration.

43. Bullouch, W. S., Biol. Rev. 20:89-99 (1945). Hormones and bird migration.

44. Bunger, J. W., Bird Banding 12:27-29 (19+1). Infuence of light periods upon plumage type in birds.

45. Burnows, H., Biological Actions of Sex Homnnes (1949). 2nd Ed. Cambridge Univ. Press. 615 p.

46. Callan, H. G, J. Exper. Biol. 17:168-179 (1940). Parasitic castration and secondary sex characters in Crustacea.

47. CaI vet, J., C. R. Soc. Biol., Paris 109:595-597 (1932). Influence of gonadotropins on cyclostomes.

48. Cardoso, D. M., Arch. Inst. Biol. São Paulo 5:133-136 (1934). Hypophyseal gonadotropins in fishes.

49. Chevais, S., C. R. Soc. Biol. Paris 127:1005-1007 (1938). GD hormone and development of eye discs in Drosophila.

50. Coni, C. F., Endocrinology 26:285-296 (1940). Carbohydrate metabolism, review.

51. Damas, H., Bull. Roy. Soc. Liege 2:94-98 (1933). Influence of gonadotropins on cyclostomes.

52. Danforth, C. H., Biol. Symp. 9:67-80 (1942). Hormones and plumage changes in birds.

53. Day, M. F., Biol. Bull. $80: 275-291$ (19+1). Control of retinal pigment migration in insects.

54. Day, M. F., Biol. Bull. 84:127-140 (1943). Corpus allatum hormone and dipteran reproduction.

55. Domm, L. V., Sex and Internal Secretions (1939). Baltimore, Williams and Wilkins. Ch. V, pp. 227-327. Hormones and plumage changes in birds.

56. Ephrussi, B., Cold. Spring Harbor Symp. Quant. Biol. 10:4048 (1942). Hormores and eye pigment development in Drosophila, review.

57. Ephrussi, B., and Beaile, G. W., Genetics 22:65-75 (1937). Cnt- and Vthormones in Drosophila eye pigment development.

58. Ephrussi, B., and Chevais, S., Biol. Bull. 72:48-78 (1938). Role of hormones and substrate in Drosophila eye pigment development.

59. Eтkin, W. N., J. Exper. Zool. 71:317-340 (1935). Hormones and amphibian metamorphosis.

60. Erkin, W., Root, R. W., and Mofskin, B. P., Plysiol. Zool. 13:415-429 (1940). Thyroid and $\mathrm{O}_{2}$ consumption in tishes.

61. Fraenkel, G., Proc. Roy. Soc. London 118:1-12 (1935). Hormonal control of dipteran development.

62. Fuxuda, S., Annot. Zool. Japan. 20:9-13 (1941). Prothoracie glands and lepidopteran metamorphosis.

63. Gorbman, A., Proc. Soc. Exper. Biol. \& Med. 45:772-773 (1940). Thyrotropic principle in fish pituitary. 
64. Greengard, H., The Chemistry and Physiology of Hormones (1944). Washington, A.A.A.S. pp. 174-178. Hormones and digestion, review.

65. Grobstein, C., and Bellamy, A. W., Proc. Soc. Exper. Biol. E Med. 41:363-365 (1939). Thyroid and growth in fishes.

65a. Grossman, M. I., Physiol. Rev. 30:33-90 (1950). Review of gastrointestinal hormones.

66. Gudernatsch, J. F., Arch. Entw. Mech. 35:457-483 (1912). Thyroid and amphibian metamorphosis.

67. Hadorn, E., Proc. Nat. Acad. Sci. 23:478-484 (1937). Larval ring-gland and dipteran development.

68. Hadonn, E., and Neel, J., Arch. Entw. Mech. 138:281-304 (1938). Larval ring gland and dipteran development.

69. von Hagen, F., Zool. Jahrb., Abt. Anat. 61:467-538 (1936). Thyroid and metamorphosis in fishes.

70. HanN, H. W., Bird Banding 10:122-124 (1939). Castration and bird migration.

71. Hanstrom, B., Hormones in Invertebrates (1939). Oxford, Clarendon. 198 p.

72. Harms, J. W., Ztschr. wiss. Zool. 146:417-462 (1935). Thyroid and growth in fishes.

73. Harms, W., Arch. Entw. Mech. 34:90-131 (1912). Testes and clitellum production in earthworms.

74. Hasler, A. D., Meyer, R. K., and Field, H. M., Endocrinology 25:978-983 (1939). Hypophyseal gonadotropins in fishes.

75. Helff, O. M., J. Exper. Zool. 45:69-93 (1926). Influence of thyroxin on $\mathrm{O}_{2}$ consumption of frog tadpoles.

76. Hess, W. N., Biol. Bull. 81:215-220 (1941). Inhibition of molting in egg-bearing female crustaceans.

77. Hess, W. N., and Bacon, R. L., Anat. Rec. (suppl.) 78:150 (1940). Relation of testes and seminal vesicles to clitellum production in earthworms.

78. Heumann, A., Ztschr. wiss. Zool. 138:515-554 (1931). Testes and clitellum production in earthworms.

79. Hoover, E. E., and Hubbard, H. E., Copeia, pp. 206-210 (1937). Photoperiodism and sexual cycle of trout.

80. Hoskins, E. R., and Monrıs, M., Anat. Rec. 11:363 (1917). Thyroid and amphibian metamorphosis.

81. Host, P., Auk. 59:388-403 1942). Light periods and seasonal plumage changes in the ptarmigan.

82. Houssax, B. A., C. R. Soc. Biol. Paris 106:1-377 (1931). Hypophyseal gonadotropins in fishes.

83. Houssay, B. A., Endocrinology 30:884-897 (1942). Hypophysis and carbohydrate metabolism, review.

84. Hughes, T. E., J. Exper. Biol. 17:331-336 (1940). Influence of parasitic castration in crustaceans.

85. INGLE, D. J., in The Chemistry and Physiology of Hormones (F. R. Moulton, ed.) (1944). Washington, A.A.A.S. pp. 83-103. Physiology of adrenal hormones, review.

86. Irving, G. W., Jr., in The Chemistry and Physiology of Hormones (F. R. Moul. ton, ed.) (1944). Washington, A.A.A.S. pp. 28-46. Functions of posterior lobe of pituitary, review.

87. Ivy, A. C., Physiol. Rev. 10:282-335 (1930). Hormones in digestion, review.

88. Ivy, A. C., in Glandular Physiology and Therapy (1942). Chicago, American Medical Association. Ch. 30. Hormones. in digestion, review.

89. Iwanoff, P. P., and Mestrscherskoja, K. A., Zool. Jahrb., Abt. allg. Zool. $\boldsymbol{u}$. Physiol. 55:281-348 (1935). Gonadal hormone in insect reproduction.

90. Kendeig11, S. C., Ecol. Monog. 4:299-417 (1934). Environmental factors and bird migration.

91. Kleiniolz, L. H., Biol. Bull. 70:159-184 (1936). Eyestalk-hormone and retinal pigment migration in crustaccans.

92. Kleinholz, L. H., Biol. Bull. 75:510-532 (1938). Hormonal control of crustacean retinal-pigment movements. 
93. Kleinholz, L. H., Biol. Rev. 17:91-119 (1942). Endocrines in crustaceans, review.

94. Kleiniolz, L. H., and Bourquin, E., Proc. Nat. Acad. Sci. 27:145-149 (1941). Eyestalks and molting in crabs.

95. Kleiniolz, L. H., and Knowles, F. G. W., Biol. Bull. 75:266-273 (1938). Movements of retinal pigments of Crustacea in response to backgrounds.

95a. Kleinilolz, L. H., and Little, B. C., Biol. Bull. 96:218-227 (1949). Diabetogenic factor in spider crab.

95b. Knowles, F. G. W., Biol. Bull. 98:66-80 (1950). Sinus gland hormone in Leander.

96. Knowles, F. G. W., and Callan, H. G., J. Exper. Biol. 17:262-266 (1940). Castration in female crustaceans and sccondary sex characters.

97. Koller, G., Ztschr. vergl. Physiol. 12:632-667 (1930). Calcium exchange in crustacean molt.

98. Koller, G., Hormone bei wirbellosen Ticren (1938). Leipzig, Akad. Verlags. $143 \mathrm{p}$.

99. Kopec, S., Biol. Bull. 42:324-342 (1922). Endocrine control of insect development.

100. Квоскевт, G., Vitamine u. Hormone 1:24-31 (1941). Thyroxin and metabolism in Python.

101. Kyer, D. L., Biol. Bull. 82:68-78 (1942). Hormonal control of gastrolith formation in crayfishes.

102. Lesher, S. W., and Kendeigh, S. C., Wilson Bull. 53:169-180. (1941). Influence of light periods on plumage types in birds.

103. Lieber, A., Ztschr. wiss. Zool. 148:364-400 (1936). Thyroid and growth in fishes.

104. Long, C. N. H., Cold Spring Harbor Symp. Quant. Biol. 10:91-103 (1942). Hypophysis and growth in mammals.

105. Long, C. N. H., Katzin, B., and Fry, E. G., Endocrinology 26:309-344 (1940). Adrenal cortex and carbohydrate metabolism, review.

106. Lukens, F. D. W., in The Chemistry and Physiology of Hormones (F. R. Moulton, ed.) (1944). Washington, A.A.A.S. pp. 74-82. Physiological action of insulin, review.

107. Lyman, C. P., Bull. Mus. Comp. Zool. Harvard 93:391-461 (1943). Control of seasonal changes in coat color of the varying hare, Lepus.

108. Mainx. F., Ztschr. induk. Abstam. Vererb. 75:256-276 (1938). Eye pigments in mutant stocks of Drosophila.

109. Marsiall, F. H. A., Biol. Rev. 17:68-90 (1942). Factors in sexual periodicity.

1 10. Marvin, H. N., and Smith, G. C., Endocrinology 32:87-91 (1943). Thyroid and heat production in pigeons.

111. Matthews, S. A., Biol. Bull. 76:241-250 (1939). Role of gonadotropins in teleosts.

112. Matthews, S. A., Biol. Bull. 79:207-214 (1940). Role of gonadotropins in teleosts.

113. Mattiews, S. A., and Smith, D. C., Physiol. Zool. 20:161-164 (1947). Thyroid and fish metabolism.

114. Megusar, F., Arch. Entw. Mech. 33:462-665 (1912). Eyestalks and molting in Crustacea.

15. Mellish, C. H., and Meyer, R. K., Anat. Rec. 69:179-189 (1937). Gonadotropic activity in reptiles.

116. Montalenti, G., and Vitagliano, G., Pub. Staz. Zool. Napoli 20:1-18 (1946). Spermatogenesis and hectocotylus development in cephalopods.

117. Murr, E., and Sklower, A., Ztschr. vergl. Physiol. 7:279-288 (1928). Thyroid and metamorphosis in fishes.

118. Page, 1. H., Bull. New York Acad. Med. 19:461-477 (1943). The physiological action of renin.

119. Panouse, J., C. R. Acad. Sci. 217:553-555 (1943). Eyestalks and ovarian growth in crustaceans.

120. Panouse, J., C. R. Acad. Sci. 218:293-294 (1944). Sinus glands and ovarian growth in crustaceans. 
121. Panouse, J., Ann. Inst. Oceanogr. 23:65-147 (1946). Sinus glands and ovarian growth in crustaceans.

122. Periera, J., Jr., and Cardoso, D. M., C. R. Soc. Biol. 116:1133-1134 (1934). Hypophyseal gonadotropins in fishes.

123. Pfeiffer, I. W., J. Exper. Zool. 82:439-461 (1939). Corpora allata and reproduction in Orthoptera. Hormonal control of development.

124. Pfeiffer, I. W., J. Exper. Zool. 99:183-233 (1945). Corpus allatum and general metabolism in insects.

125. Pflugfelder, O., Ztschr, wiss. Zool. 149:477-512 (1937). Hormonal control of orthopteran development and reproduction.

126. Pflugfelder, O., Ztschr. wiss. Zool. 152:384-408 (1939). Hormones in Dixippus reproduction.

127. Pflugfelder, O., Ztschr. wiss. Zool. 153:108-135 (1940). Hormones in Dixippus development and reproduction.

127a. Pincus, G., and Thimann, K. U., Editors, Chemistry and Physiology of the Hormones. Vol. I (1948), Vol. II (1950). New York, Academic Press.

128. Pyle, R. W., Biol. Bull. 85:87-102 (1943). Cycles in histological picture in sinus glands and molting in crustaceans.

129. Rowan, W., Proc. Boston Soc. Nat. Hist. 39:151-208 (1929). Factors controlling bird migration.

130. Rowan, W., The Riddle of Migration (1931). Baltimore, Williams and Wilkins. $151 \mathrm{p}$.

131. Rowan, W., Proc. Nat. Acad. Sci. 18:639-654 (1932). Factors determining bird migration.

132. Rowan, W., Biol. Rev. 13:374-402 (1938). Light and reproductive cycles.

133. Rugh, R., Biol. Bull. 66:22-29 (1934). Gonadotropic activity of hypophysis in amphibia.

134. Rugh, R., Biol. Bull. 68:74-81 (1935). Anterior lobe extract and ovulation in amphibians. Mammalian pituitary extract in amphibian ovulation.

135. Rugh, R., J. Exper. Zool. 71:149-162 (1935). Gonadotropic activity of hypophysis in Amphibia.

136. Rugi, R., J. Exper. Zool. 71:163-193 (1935). Gonadotropic activity of hypophysis in Amphibia.

137. Scharrer, B., Physiol. Rev. 21:383-409 (1941). Endocrines in invertebrates, review.

138. Scharrer, B., Endocrinology 38:35-45 (1946). Hormonal control of orthopteran development.

139. SCharRer, B., Endocrinology 38:46-55 (1946). Corpus allatum hormone and ovarian growth in Orthoptera.

140. Scharrer, E., and Scharrer, B., Physiol. Rev. 25:171-181 (1945). Neurosecretion, review.

141. Scudamore, H. H., Physiol. Zool. 20:187-208 (1947). Sinus gland and $O_{2}$ consumption in crayfish. Hormonal control of gastrolith formation in crayfishes.

142. Scudamore, H. H., Biol. Bull. 95:229-237 (1948). Molting and seasonal sex cycle in Cambarus.

143. Sereni, E., Am. J. Physiol. 90:512 (1929). Relation of testes to hectocotylus development in cephalopods.

144. Slome, D., J. Exper. Biol. 13:1-6 (1936). Hypophysis and pancreatic diabetes in toads.

145. Smith, D. C., and Evenetr, G. M., J. Exper. Zool. 94:229-240 (1943). Thyroxin and $\mathrm{O}_{2}$ consumption in fishes.

146. Smith, D. C., and Matthews, S. A., Am. J. Physiol. 137:533-538 (1942). Effect of adrenalin on $\mathrm{O}_{2}$ consumption in fishes.

147. Smith, D. C., and Matthews, S. A., Am. J. Physiol. 153:215-221 (1948). Thyroid and $\mathrm{O}_{2}$ consumption in fishes.

148. Smith, P. E., Anat. Rec. 11:57-64 (1916). Hypophysis in amphibian metamorphosis.

149. Smitr, R. I., Biol. Bull. 79:145-152 (1940). Eyestalks and molting in young crayfishes.

149a. Smith, R. I., Biol. Bull. 95:169-185 (1948). Retinal pigment in grapsoid crab. 
150. Swingle, W. W., J. Exper. Zool. 36:397-421 (1922). Thyroid and amphibian metamorphosis.

151. Thomsen, D. L., and Collip, J. B., Physiol. Rev. 12:309-383 (1932). Action of parathyroid lormone, review.

152. Thomsen, E., Nature 145:28-29 (1940). Corpus allatum hormones and insect reproduction.

153. Thomsen, E., Vidensk. Meddel. Dansk. Naturhist. Foren. 106:320-405 (1942). Corpus allatum hormone and dipteran reproduction.

154. Turner, C. L., Physiol. Zool. 15:263-280 (1942). Gonadal hormones and secondary sex characters of fishes.

155. Vandel, A., C. R. Acad. Sci. 170:249-251 (1920). Gonads and development of planarian copulatory apparatus.

156. Vandel, A., Bull. Biol. France e. Belg. 55:343-518 (1921). Gonads and development of planarian copulatory apparatus.

157. Vogt, M., Biol. Zentralbl. 60:479-484 (1940). Corpora allata and reproduction in Drosophila.

158. Vogt, M., Biol. Zentralbl. 61:242-252 (1941). Corpora allata and reproduction in Drosophila.

159. Vogt, M., Arch. Entw. Mech. 141:424-454 (1942). Corpora allata and reproduction in Drosophila.

160. Warren, M. R., J. Exper. Zool. 83:127-156 (1940). Thyroxin and Oz consumption in frogs.

161. Weed, I. G., Proc. Soc. Exper. Biol. E Med. 34:883-885 (1936). Corpora allata and reproduction in orthopterans.

162. Welsh, J. H., Proc. Nat. Acad. Sci. 16:386-395 (1930). Diurnal rhythm in crustacean retinal pigment inovements.

163. Welsh, J. H., Biol. Bull. 77:119-125 (1939). Eyestalk-hormone and retinal pigment migration in crayfish.

164. Welsh, J. H., J. Exper. Zool. 86:35-49 (1941). Sinus glands and retinal pigment rnigration in crayfishes.

165. Welsh, J. H., and Osborn, C. M., J. Comp. Neurol. 66:349-359 (1937). Diurnal rhythm in activity of elements in catfish retina.

166. VON DER WENSE, T. F., Wirkungen und Vorkommen von Hormonen bei wirbellosen Tieren (1938). Leipzig, J. A. Barth. 80 p.

167. Whitaker, W. L., Proc. Soc. Exper. Biol. \& Med. 34:329-330 (1936). Photoperiodism and breeding in Peromyscus.

168. Wigglesworth, V., Quart. J. Micr. Sci. 77:191-222 (1934). Hormones in insect molt and metamorphosis: Hemiptera.

169. Wigglesworth, V., Quart. J. Micr. Sci. 79:91-121 (1936). Hormones in molt and metamorphosis and reproduction: Hemiptera.

170. Wiggleswortif, V., J. Exper. Biol. 17:201-222 (1940). Hormones in insect molt and metamorphosis: Hemiptera.

171. Williams, C. M., Biol. Bull. 90:234-243 (1946). Brain-hormones in lepidopteran metamorphosis.

172. Williams, C. M., Biol. Bull. $93: 89-98$ (1947). Roles of brain and prothoracic gland principles in lepidopteran metamorphosis.

173. Williams, C. M., Anat. Rec. 99:591 (1947). Cytochrome system in diapause and development in Cecropia silkworm.

174. Williams, C. M., Anat. Rec. 99:671 (1947). Brain and termination of pupal diapause.

175. Williams, C. M., Biol. Bull. 94:60-65 (1948). Prothoracic glands and insect development.

176. Wills, I. A., Riley, G. M., and Stubbs, E. M., Proc. Soc. Exper. Biol. E Med. 30:411-412. 784-786 (1933). Hypophysis and sex behavior in Amphibia.

177. Witschr, E., Wilson Bull. 47:177-188 (1935). Hypophyseal control of plumage in weaver finches.

178. Wolfson. A., Condor 44:237-263 (1942). Factors controlling bird migration.

179. Wolfson, A., Condor 47:95-127 (1945). Controlling factors in bird migration.

180. Woodbury, A. M., Auk 58:463-505 (1941). Temperature change and bird migration.

181. Young, F. G., Endocrinology 26:345-351 (1940). Pituitary gland and carbohy- 


\section{Nervous Systems}

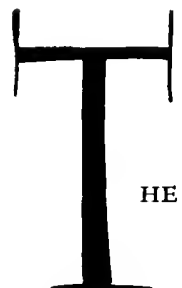

HE MIETABOLIC FUNCTIONS of an animal can proceed without specialized conducting tissue. The specialization of conducting tissue, particularly of nervous tissue, gives an animal a certain freedom from the environment which has survival value, permitting a combination of large size and high motility. Many Protozoa and free-swimming larvae are small and have little rapid conduction from one part to another, yet are motile. Many sessile animals have slightly developed conducting mechanisms and may be large, but they are not motile. All of those animals which are both large and motile have rapidly conducting nervous tissue.

In the evolution of nervous tissues four trends are apparent: (1) increasing speed of conduction; (2) integration, or making one impulse count for more or less than one; (3) cephalic dominance; and (4) modifiability of the pattern of response.

\section{NON-NERVOUS CONDUCTION}

Among the animals which do not have nervous systems some coordinating mechanisms have been described. Probably all living cells have the ability to conduct waves of excitation. Conduction in many plant cells, eggs, and body cells of higher animals has been described as a wave of depolarization passing along the plasma membrane. Conduction from cell to cell, as in ciliated epithelium, is well known. Conduction by way of many intracellular fibrils has been claimed for a few Protozoa. ${ }^{396}$

Many ciliates have a regular network of fibrils connecting the basal granules of the cilia. The form of this network varies, but in general the basal granules are connected in longitudinal rows with some cross connections. ${ }^{161}$ This peripheral network is connected to the central neuromotor system, which may have a center or motorium and has internal fibrils which extend to membranelles, cytopharynx, and cirri when these are present. The entire fibrillar system has been variously claimed to be supporting, contractile, and conducting. Experimental evidence for a conducting function came from Taylor, ${ }^{395}$ who with microneedles cut fibrils between the motorium and the cirri in Euplotes (and cut the peripheral fibrils as well). Coordination between the cirri and membranelles in the oral region disappeared. Coordination of membranelles was disrupted after destruction of the motorium in Chlamydodon, ${ }^{272}$ and ciliary movement was disorganized after fibrillar injury in Ichthyophthicus. ${ }^{275}$ However in Paramecium lesions near the peristome did not destroy ciliary coordination, ${ }^{291}$ even though a motorium is said to be located there. ${ }^{271}$ 
Conduction in higher animals is predominantly a function of cell membranes, and in neurones the fibrils per se probably have nothing to do with conduction. Taylor ${ }^{396}$ suggests that the fibrillar system in ciliates actually may have several different functions. Ciliates are highly specialized acellular organisms. Hence, the ciliate fibrillar system is not a precursor of nervous structures.

In sponges there are no nerve cells, but spindle-shaped muscle cells bring about closure of oscula. These muscle cells have been called independent effectors, ${ }^{317}$ because they combine sensory and motor functions. There is a slow conduction (about $1 \mathrm{~cm} . / \mathrm{min}$.) from cell to cell in a sponge, as shown by the response of the osculum to a prick a short distance away. ${ }^{316}$ In some coelenterate tentacles there may be a few two-celled arcs (sense cell-muscle cell), ${ }^{317}$ but beginning with the coelenterates coordination between sense organs and effectors is principally nervous.

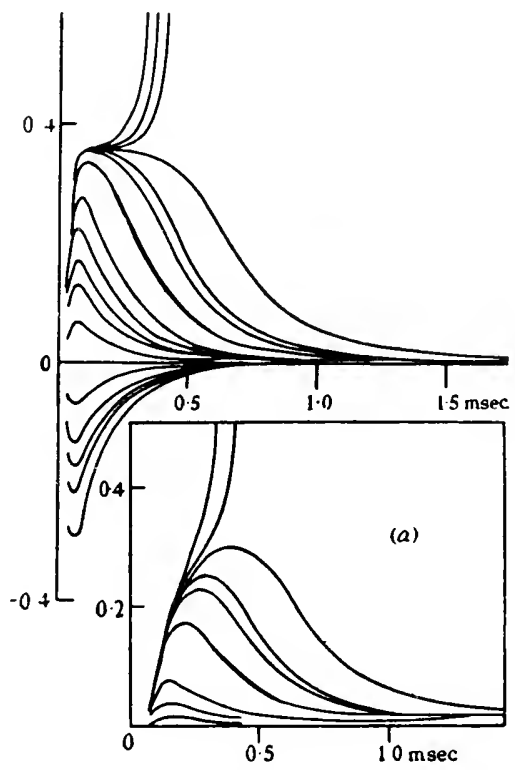

Fig. 290. Electrical changes in crab nerve at the stimulating electrodes to shocks of increasing intensity. Electrotonic potential seen at anode (below zero) and in first five records at cathode (above zero), local response only in upper records 6-9, and beginning of action potential spike in upper three responses. Upper six curves at threshold intensity. Insert $a$, another series at cathode. From Hodgkin. ${ }^{107}$

\section{NERVOUS CONDUCTION}

The Action Potential Wave. A nerve impulse is the sum total of chemical and physical events in the propagation of a wave of physiological activity along a nerve fiber. The electrical components of a nerve impulse have been measured most precisely and are of interest in comparative physiology.

A nerve trunk is composed of many nerve fibers, each fiber conducting independently of the others. Each nerve fiber is polarized when at rest by about 60 millivolts, the inside of the cell membrane being negative to the outside. Excitation consists, in part, in the breakdown of this polarized, 
relatively impermeable membrane so that it is momentarily permeable to ions; membrane resistance decreases during conduction.

In conduction the membrane shows a series of some five different electrical phases. Some types of nerve fiber show certain of these electrical waves more clearly than others; a composite picture of the electrical disturbance in a nerve impulse follows:

(1) The membrane has electrical capacitance and resistance, and when subthreshold current is passed through the nerve there is current spread according to the electrical constants of the membrane. This electrotonic wave declines exponentially along the fiber from the source of stimulation and

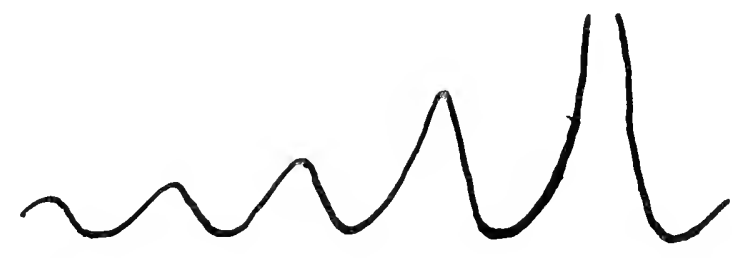

Fig. 291. Local graded oscillations with emergent spike in decalcified giant axons of squid. From Arvanitaki. ${ }^{17}$

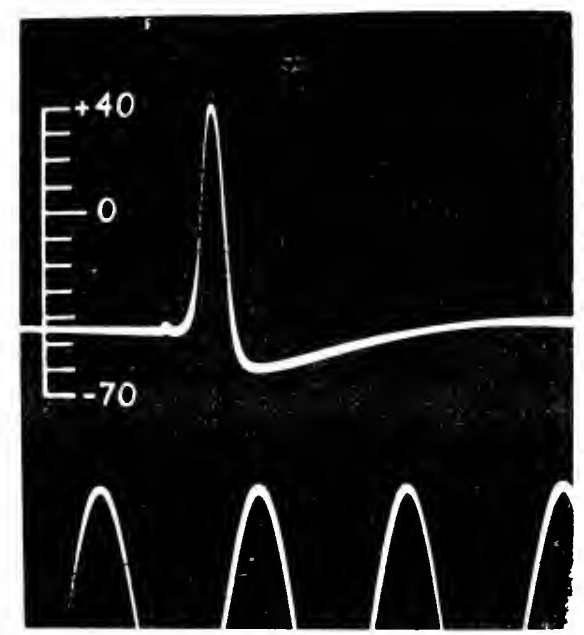

Fig. 292. Action potential recorded between the inside and outside of giant axon of squid showing initial resting potential level and overshoot of action potential. From Hodgkin and Huxley. ${ }^{198}$

can be detected $0.5-3 \mathrm{~mm}$. away from a stimulating electrode; the electrotonic (polarization) potential is best seen with stimuli of half rheobasic (threshold) strength. It varies with stimulus intensity and is normally symmetrical, but in reverse polarities at cathode and anode (Fig. 290). An electrotonic wave spreads ahead of a propagated impulse and can be detected beyond a region where conduction is blocked by cold.

(2) As the stimulus strength increases and the polarization (electrotonic) potential grows, out of it appears a local response or prepotential. This ap- 
pears only at the cathode (on the make), is reduced or absent if the nerve is refractory, and is graded in size. Its increase is non-linear, the local response increasing out of proportion to the stimulus strength. Local responses have been seen in thin-sheathed fibers of Crustacea ${ }^{197}$ and of Cephalopoda, ${ }^{17}$ and in myelinated fibers of the frog ${ }^{234}$ (Fig. 290). Lorente de $\mathrm{No}^{265}$ classes this graded response as part of the electrotonic potential.

(3) When the stimulus is of threshold magnitude the local graded potential reaches a critical height and a propagated nerve impulse (spike) arises out of it. The critical height of the local potential in crustacean fibers is about 20 per cent of the spike height. ${ }^{197}$ Under conditions of membrane instability, as in decalcified giant nerve fibers of the squid, electrical oscillations of variable size appear, and out of the largest of these conducted impulses arise ${ }^{\mathbf{i} 7,1 \mathrm{~s}}$ (Fig. 291). The spike is all-or-none, rises rapidly to its crest, and then declines at a decreasing rate. The spike duration varies with

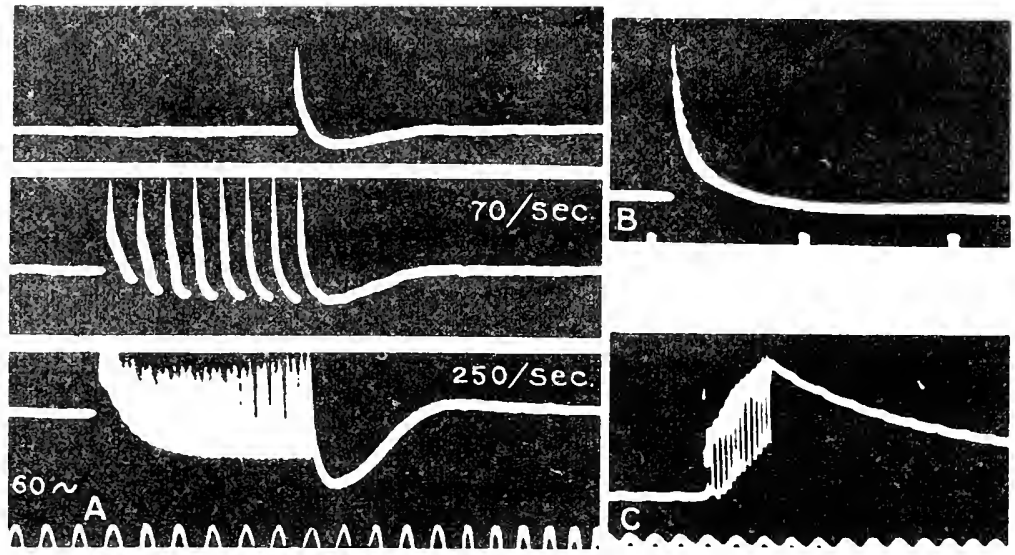

Fig. 293. A, Changes produced in the after-potentials of a phrenic nerve (A fibers) by short tetanic stimulations. The spike potentials are not visible so the records begin with the negative after-potential and continue into positive after-potential. The top record shows the after-potential response evoked by a single stimulus, for comparison with the curves below at the designated frequencies of stimulation. Time line $=60$ cycles. $B, C$, Negative after-potentials in response to single and tetanic stimulation in veratrinized nerve. Time 60 cycles. From Gasser and Grundfest. ${ }^{15}$

conduction velocity and is roughly $1.5 \mathrm{msec}$. for fast frog and crustacean fibers. It was formerly believed that the action potential spike was merely a reduction in the resting potential of the nerve fiber. However, in giant nerve fibers of the squid it has been possible to insert an electrode inside the fiber and thus record the maximum potential developed across the membrane. In such fibers with resting potentials of about $55 \mathrm{mv}$. where the inside is negative, the outside may become negative, sometimes by an additional 50 or $60 \mathrm{mv}^{109,198}$ (Fig. 292). Thus the spike is more than a mere reduction of resting potential; it is also an active reversal in potential.

(4) Following the spike and delaying its downward deflection is a negative after-potential (Fig. 293, B, C). The negative after-potential represents a process of negativity which starts during the spike and reaches its peak much later ( $4-6$ msecs. in frog A fibers, which have a spike crest time 
of $0.4 \mathrm{msec}$.). The negative after-potential is increased by veratrine, by the alkaline carths, and by tetanization of a nerve trunk. In crab nerve fibers the negative after-potential persists for 15 minutes or more. ${ }^{155}$, 254 The afternegativity in crab fibers, as in vertebrate fibers, is prolonged by veratrine ${ }^{40}$ and by repetitive stimulation. ${ }^{50}$

(5) A positive after-potential follows the negative after-potential (Fig. 293, A). It may persist for $100 \mathrm{msec}$. in small mammalian fibers and for several seconds in frog fibers. It may last for many minutes in crab fibers. ${ }^{445}$ After-positivity is enhanced by yohimbine, by low calcium, and by a tetanus.

A nerve fiber recovers its excitability rapidly so that after the spike its membrane is again ready to conduct another impulse. The after-potentials are related in some unknown way to oxidative recovery and to ionic unbalance. In addition, the cxcitability of a nerve is enhanced during the negative afterpotential (catelectrotonus) and is diminished during the positive after-potential (anelectrotonus) (Fig. 294).

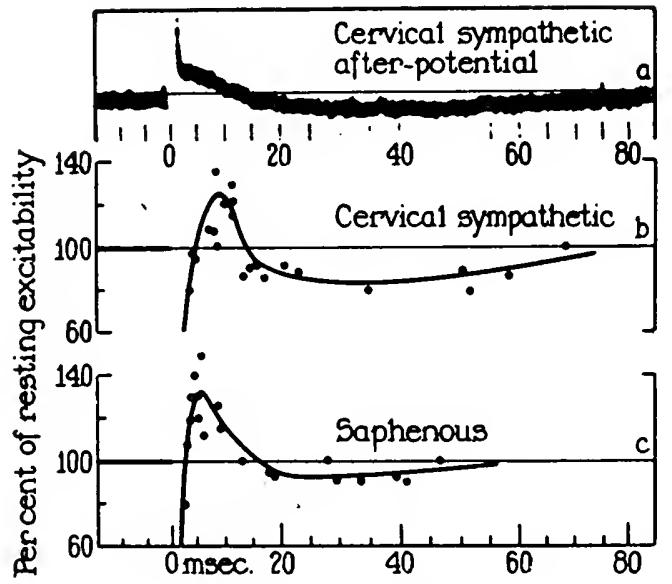

Fig. 294. After-potentials and excitability cycle following a response. $a$, after-potential, and $b$, excitability cycle in cervical sympathetic fibers (cat). $c$, excitability cycle in saphenous nerve. From Gasser. ${ }^{157}$

Ionic Basis of Nerve Action Potentials. There are few fields of cellular physiology in which it is so difficult to fit the varied chemical and physical observations into a reasonable pattern as in nervous propagation. There is little doubt that oxidations supply the recovery energy, probably by restoring and maintaining the molecular structure of the plasma membrane. Thiamine is involved in activity. ${ }^{300}$ Encrgy appears to be made available by phosphate transfer, as in muscle.

A part of the action potential spike consists of reduction in the resting potential, and this probably results from a net increased movement of potassium outward, diminishing the ratio of potassium inside to that outside. It has been demonstrated with thin-sheathed nerves (crustacean) that during propagation the rate of exchange of potassium is greatly increased, with the net effect of an outward movement of potassium. ${ }^{105}, 199.238,368$ The overshoot whereby the outside becomes negative to the inside may result from simultancous inward movement of sodium. ${ }^{368}$ 
The plasma membrane is lipo-protein, as indicated by much indirect evidence. Certain electrochemical properties of the lipo-protein membrane seem to be imparted by a substance that is a quaternary ammonium base, the synthesis of which requires sodium. ${ }^{26 i}$ Specific aromatic hydrocarbons and other non-polar molecules depolarize, apparently by adsorbing on receptor areas of less than $8 \AA \AA$; calcium stabilizes against such depolarization. ${ }^{4: 7}$ The insecticides DDT, pyrethrin, and naphthalene have non-polar groups which are very active in reducing membrane stability. ${ }^{127}$

Acetylcholine $\left(\mathrm{ACl}_{1}\right)$ is involved in neuro-transmission in one or more unknown ways. It is normally bound, presumably to protein, and may be released in excitation. However, the distribution of acetylcholine is not well correlated with nervous function (see Table 77), and its high concentration in such organs as spleen and placenta can hardly be connected

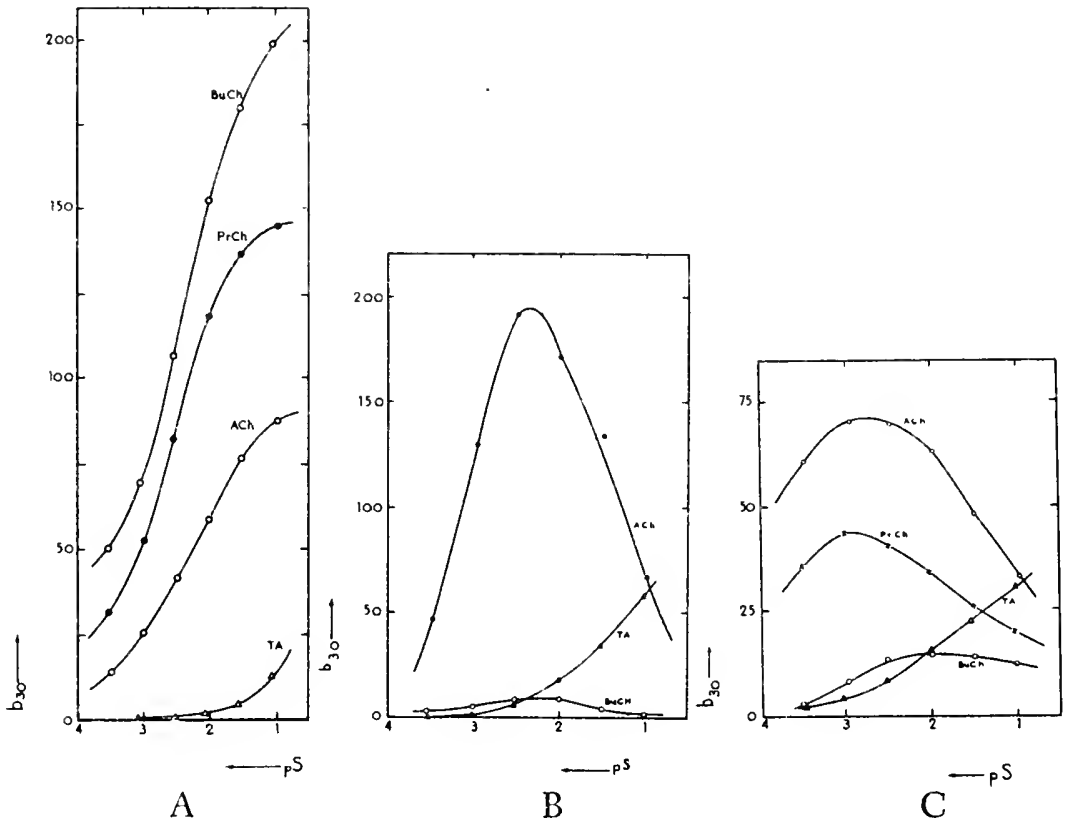

Fig. 295. Types of cholinesterase. Enzyme from, $A$, human serum, B, squid ganglion, and $C$, nucleus caudatus (beef). $b$, Activity of enzyme; $\mathrm{pS}$, negative log molar concentration of substrate. Substrates: Ach, acetylcholine; $\mathrm{PrCh}$, propionylcholine; $\mathrm{BuCh}$, butyrylcholine; TA, triacetin. Modified from Augustinson. ${ }^{24}$

with propagation. The lowest concentrations of $\mathrm{ACh}$ are in those parts of the nervous system which are most sensitive to asphyxia and several bits of evidence suggest that it may be involved in some oxidative system. ${ }^{426}$ Another hypothesis is that $\mathrm{ACh}$ is important in determining permeability and that liberated ACh makes the membrane permeable to potassium and sodium. ${ }^{305}$ This hypothesis is supported by evidence regarding permeability of nerve and red blood cells. A third hypothesis ${ }^{37}$ is based on the fact that ACh has a powerful electrochemical action at certain oil-saline interfaces and suggests that released ACh may cause the local electronegativity. 
Whatever the function of $\mathrm{ACh}$, it is certain that even the molecular lipoid layer of squid giant axons is virtually impermeable to $\mathrm{ACh}$ (a quaternary amine), whereas it is readily penetrated by trimethyl amine. In regions where lipoid membranes are absent (or altered), as at junctions, low concentrations of $\mathrm{ACh}$ are exciting and higher concentrations are depressant. Complete (more than 90 per cent) inhibition of cholinesterase abolishes nerve conduction, presumably by permitting accumulation of depressant concentrations of acetylcholine. ${ }^{84}$ Quaternary anti-esterases such as prostigmine and curare fail to penetrate nerve fibers but physostigmine and dipropylfluoro phosphate (DFP) penetrate and block conduction. The action of the toxic agent, DFP, and of the insecticides hexaethyl tetraphosphate and tetraethyl pyrophosphate appears to be due to their anti-esterase properties. $^{93}$ Cholinesterase is widely distributed, is concentrated in the axon sheath (squid giant fiber), and has very high turnover number. ${ }^{305}$

Two types of cholinesterase have been identified. One (Fig. 295, A) is found in mammalian blood serum and in the dart sac of Helix. This is not specific for acetylcholine; it splits propionylcholine and butyrylcholine at higher rates, and it is not inhibited by high concentrations of acetylcholine. The other type (Fig. 295, B, C) is characteristic of all excitable tissues, nerve, muscle, and electric organs; this splits acetylcholine at a higher rate than other choline esters and shows a sharp optimal ACh concentration. ${ }^{24}$

The chemical events underlying the electrical activity are probably similar in all nerves. However, quantitative differences among nerves and animals may be used in learning the significance of different steps in the complex chain of events.

Speed of Conduction. The speed of conduction in nerve fibers and nerve tracts differs from animal to animal and in different parts of the same animal. Five morphological adaptations of nerves correlate with increased speed of reaction among animals: length of nerve processes, fiber diameter, myelin sheath, nodes, and giant neurones. There are probably intrinsic differences among the nerve fiber nembranes as well.

Length of Nerve Processes. Animals increase their speed of reaction by the lengthening of conducting processes. A message travels more quickly by a single long fiber than when it has to pass through numerous synapses, with a delay at each. In many coelenterates the nervous system consists of a network of multipolar and bipolar neurones whose processes have been traced for several millimeters. ${ }^{55}$ Conduction in this network is slow, less than $0.5 \mathrm{M}$./sec. (Table 75). In some coelenterates through tracts of continuous fibers exist for rapid conduction. ${ }^{312}$ In general, fast-moving animals have some nerve tracts consisting of long single fibers. Giant fiber conduction in annelids and arthropods is more rapid than conduction through the neuropile or small fiber mass in their ganglion chains.

Fiber Diameter. In mixed nerves of frogs and mammals speed of conduction is proportional to some function of the fiber diameter. The fiberdiameter/velocity correspondence has not been investigated for the entire spectrum of any invertebrate nerves. However, there is no doubt that faster animals have some large motor nerve fibers, and in slower animals most nerve fibers are small. 
TABLE 75.

VELOCITY OF CONDUCTION IN NERVE NETS AND GANGLIONIC CHAINS

\begin{tabular}{|c|c|c|}
\hline Animal & Temperature $\left({ }^{\circ} \mathrm{C}.\right)$ & Velocity $\mathrm{m} . / \mathrm{sec}$. \\
\hline & \multicolumn{2}{|c|}{ Nerve nets of coelenterates (velocity of contractile waves) } \\
\hline \multirow{12}{*}{$\begin{array}{l}\text { Metridium } \\
\text { Calliactis }^{312} \\
\quad \text { (net) } \\
\quad \text { (through tracts) } \\
\text { Physalia filaments }^{319} \\
\text { Cassiopea }^{154} \\
\text { Cassiopea }^{255} \\
\text { Mastigias }^{240} \\
\text { Renilla }^{318} \\
\text { (muscular wave) } \\
\text { (luminescent wave) }\end{array}$} & 21 & $0.121-0.146$ \\
\hline & & $0.121-0.140$ \\
\hline & & $0.04-0.15$ \\
\hline & & 1.2 \\
\hline & 26 & 0.121 \\
\hline & 18 & $0.136-0.234$ \\
\hline & & $0.15-1.2$ \\
\hline & 25.5 & 0.57 \\
\hline & & \\
\hline & 21 & 0.078 \\
\hline & 21 & 0.074 \\
\hline & \multicolumn{2}{|c|}{ Ganglionic cords (neuropile conduction) } \\
\hline \multicolumn{3}{|l|}{ Annelids } \\
\hline Earthworm ${ }^{217}$ & & 0.6 \\
\hline Leech $^{247}$ & & 0.4 \\
\hline Earthworm $^{53}$ & & $0.02-0.03$ \\
\hline Cerebratulus $^{223}$ & & $0.059-0.09$ \\
\hline Aphrodite ${ }^{223}$ & & 0.545 \\
\hline \multicolumn{3}{|l|}{ Myriapods } \\
\hline Scolopendra ${ }^{223}$ & & 2.5 (giant?) \\
\hline Julus 228 & & 0.2 \\
\hline Himantarium ${ }^{223}$ & & 0.28 \\
\hline \multicolumn{3}{|l|}{ Crustacea } \\
\hline Cambarus ${ }^{331}$ & & 1.2 \\
\hline
\end{tabular}

The numerical relationship of velocity to fiber size differs according to fiber type and sheath characteristics. In mammalian A fibers the velocity in meters per second numerically equals the diameter in microns times a constant (approximately 6 or 7 ). ${ }^{158,}, 217,157$ In giant fibers of the squid velocity increases as the diameter raised to the 0.61 power ${ }^{340}$ Nerve fibers are attenuated near their terminations, and here conduction is slow.

Table 76 gives the velocities in moist air and, where possible, the total fiber diameters in a variety of nerve fibers. The general relationship between diameter and velocity holds. Velocities in situ are slightly greater. The most striking instance of large fiber diameter and rapid conduction is in the giant fibers of crustaceans, cephalopods, and annelids, which may have diameters of tens of microns or even several hundred microns, and conduct many times faster than the smaller fibers of these animals. Insects in general have very small nerve fibers but conduction distances are short, hence fast conduction is less important to the animal than a large number of fibers. Mosquito larvae have fibers less than $1-2 \mu$ in diameter, although cockroaches have some fibers as large as $10 \mu$ in diameter. ${ }^{347}$

Excitation times also vary inversely with fiber diameters, i.e., fast fibers have short chronaxies although for a given fiber type chronaxies are relatively constant over wide ranges in size. ${ }^{139,} 157$ 
TABLE 76. VELOCITY OF CONDUCTION IN SELECTED NERVE FIBERS AS RELATED TO FIBER SIZE AND SHEATH THICKNESS

(VELOCITY IN MOIST AIR)

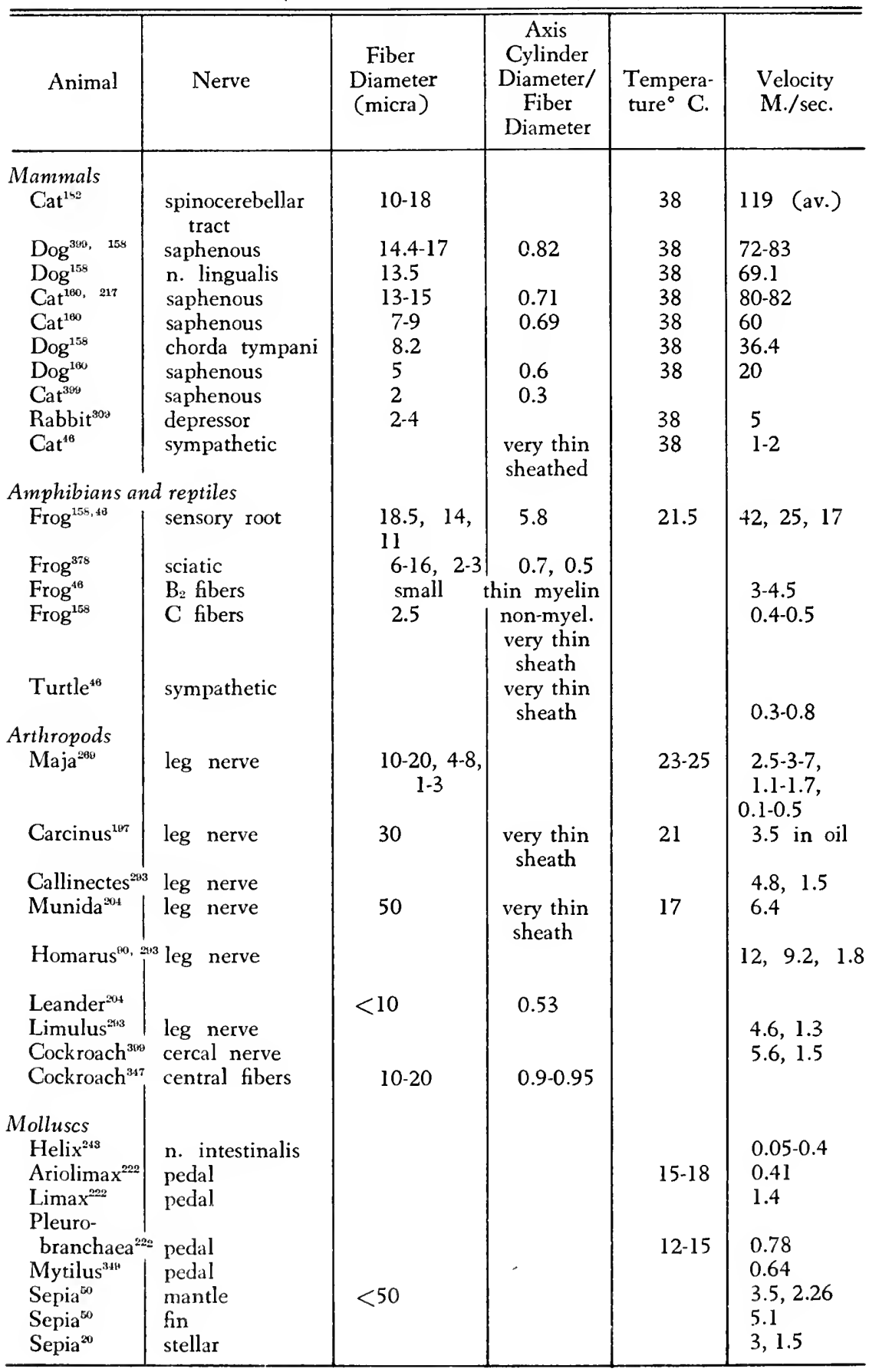


TABLE 76 (continued)

VELOCITY OF CONDUCTION IN SELECTED NERVE FIBERS AS RELATED

TO FIBER SIZE AND SHEATH THICKNESS (VELOCITY IN MOIST AIR)

\begin{tabular}{|c|c|c|c|c|c|}
\hline Animal & Nerve & $\begin{array}{c}\text { Fiber } \\
\text { Diameter } \\
\text { (micra) }\end{array}$ & $\begin{array}{c}\text { Axis } \\
\text { Cylinder } \\
\text { Diameter/ } \\
\text { Fiber } \\
\text { Diameter }\end{array}$ & $\begin{array}{l}\text { Tempera- } \\
\text { ture }^{\circ} \mathrm{C} \text {. }\end{array}$ & $\begin{array}{l}\text { Velocity } \\
\text { M./sec. }\end{array}$ \\
\hline \multirow[t]{2}{*}{$\begin{array}{l}\text { Loligo }^{340} \\
\text { Loligo }^{222} \\
\text { Loligo }^{298}\end{array}$} & \multirow[t]{2}{*}{$\begin{array}{l}\text { fin } \\
\text { fin }\end{array}$} & 42.6 (av.) & & $21-22$ & $\begin{array}{l}0.32,0.22 \\
4.6 \text { (av.) } \\
4.3 \\
16,2.5\end{array}$ \\
\hline & & \multicolumn{4}{|c|}{ Giant nerve fibers } \\
\hline $\begin{array}{l}\text { Vertebrates } \\
\text { Ameiurus }^{109}\end{array}$ & Mauthner & $22-43$ & $0.59-0.56$ & $10-15$ & $50-60$ \\
\hline $\begin{array}{l}\text { Camba- } \\
\text { rus }^{432 .} \quad 433\end{array}$ & median & $100-250$ & & 20 & $15-20$ \\
\hline Leander $^{201}$ & lateral & $\begin{array}{l}70-150 \\
35\end{array}$ & 0.77 & $\begin{array}{l}20 \\
17\end{array}$ & $\begin{array}{l}10-15 \\
18-23 \text { in } \\
\text { oil }\end{array}$ \\
\hline $\begin{array}{l}\text { Munidaa } \\
\text { Shrimp }\end{array}$ & & $\begin{array}{r}50 \\
>25 \\
<8\end{array}$ & $\begin{array}{l}\text { thin sheath } \\
0.87 \\
\text { metatropic }\end{array}$ & 17 & 6.4 \\
\hline Annelids & & & & & \\
\hline $\begin{array}{l}\text { Lumbricus } \\
347,134,391 \\
63,370\end{array}$ & median & $\begin{array}{r}>40 \\
64\end{array}$ & 0.9 & $10-12$ & $\begin{array}{l}17-25 \\
15-45 \\
20\end{array}$ \\
\hline Neanthes ${ }^{\text {sn }}$ & lateral & $\begin{array}{r}37 \\
<11\end{array}$ & metatropic & $10-12$ & $\begin{array}{l}7-12 \\
5-15 \\
10 \\
15,5.4 \\
2.5\end{array}$ \\
\hline $\begin{array}{l}\text { Molluscs } \\
\text { Sepia }^{340} \\
\text { Loligo }^{340}\end{array}$ & & $\begin{array}{l}147 \\
718,589 \\
\quad 335\end{array}$ & & & $\begin{array}{l}7.7 \\
22.3,20.6 \\
13.7\end{array}$ \\
\hline $\begin{array}{l}\text { Loligo }^{350} \\
\text { Loligo }^{41}\end{array}$ & & $\begin{array}{l}280-400 \\
400-500\end{array}$ & $\begin{array}{l}>0.99 \\
\text { metatropic }\end{array}$ & 22 & 20 \\
\hline
\end{tabular}

Nerve Fiber Sheath. In addition to fiber diameter, the existence of a lipoid sheath around the axon is directly correlated with velocity of conduction. Among vertebrates the larger fast motor and sensory fibers have myelin sheaths which blacken with osmic acid and can be readily measured, whereas the slow small sympathetic fibers are said to be non-myelinated. When examined in polarized light the larger fibers are seen to be positively birefringent with respect to their radially directed optic axis; this is due to the lipoid sheath and the fibers are called myelotropic. The birefringence in frog fibers is nearly constant among fibers $9 \mu$ or more in diameter but diminishes in smaller fibers until it is zero at $2 \mu$, and below that size it reverses sign. ${ }^{378}$ The birefringence of the "non-medullated" fibers, on the other hand, is normally negative with respect to the radial axis, is due to proteins, and can 
be counterbalanced by immersion in glycerin of the same refractive index, whereupon a slight positive birefringence appears; these fibers are said to be metatropic.

Most invertebrate nerve fibers are said to be "non-myelinated," but in many of them examination with polarized light reveals a thin outer layer which is positively birefringent with respect to the radial axis. ${ }^{41,}{ }^{379}$ Certain fibers of crabs and shrimps, and of cockroaches and other insects, Nereis, etc., are metatropic. ${ }^{347,398}$ The protein layer has a refractive index of 1.58 in fibers from lobster cord, frog motor root, and cat corpus callosum. ${ }^{96}$ The reversal in sign in glycerin reveals a thin lipoid layer which in insects may even stain with sudan dyes. ${ }^{347}$ This layer can be removed with fat solvents. As the diameter increases, the positive birefringence decreases so that earthworm giant fibers are weakly myelotropic above $11 \mu$ and shrimp giant fibers above $8 \mu,{ }^{398}$ as compared with frog fibers, above $2 \mu .{ }^{378}$ In giant fibers of the prawn, Leander, the ratio of axis cylinder to total fiber diameter is 0.53 for fibers smaller than $10 \mu, 0.69$ for $10-20 \mu$ fibers, and 0.77 for $20-50 \mu$ fibers. ${ }^{204}$ Squid giant fibers are uniformly positive with respect to length (negative to radial axis) regardless of diameter, but when immersed in glycerin a lipoid sheath is demonstrated which is less than 1 per cent of the fiber diameter. ${ }^{41}$ In summary, most invertebrate fibers, except large giant fibers of earthworm and shrimp, resemble vertebrate thinly myelinated fibers in optical propertiesa thin lipoid sheath masked by protein. Some lipoid is probably present outside all nerve fibers.

The fastest vertebrate fibers are of medium size $(4-15 \mu)$ but have thick sheaths ( $30-50$ per cent of total fiber diameter), whereas the fastest fibers of invertebrates are large ( 50 to several hundred microns) but have thin sheaths $(1-10$ per cent of the diameter-Table 76$)$. In both these types of fibers the ratio of axis cylinder to total fiber diameter usually increases with increase in size of the fibers, up to some critical size. Taylor ${ }^{399}$ summarized the relation between diameter, sheath, and velocity as follows: $4 \mu$ fiber of the cat saphenous at $38^{\circ}$ conducts at about the same rate $(25 \mathrm{M}$./sec.) as a $650 \mu$ squid giant axon at $20^{\circ}$. Assuming a $Q_{10}$ of 1.5 , an $8 \mu$ cat saphenous fiber would conduct at $20^{\circ}$ at the same rate as the $650 \mu$ squid giant fiber. The myelin sheath of the mammalian fiber constitutes some 42 per cent of its total diameter, whereas in the squid giant fiber the sheath is about 1 per cent of the fiber diameter.

Many invertebrate nerve fibers also have a connective tissue and glial sheath. In some invertebrate fibers (prawn, ${ }^{204}$ squid $^{4+3}$ ), connective tissue cells lie between the thin lipoid sheath and the axon. There is no evidence that the connective tissue sheath speeds conduction.

Nodes. Some effects of polarization can be explained by the assumption that impulses in frog medullated nerve travel in a saltatory fashion from node to node. ${ }^{138}$ There are regions of thinning of the sheath in shrimp giant fibers. ${ }^{203,}{ }^{204}$ However, dependence on sheath and nodes for speed is largely a vertebrate adaptation. Myelinated spinal tracts conduct rapidly but lack nodes. ${ }^{111}$

Giant Fibers. There are two types of giant fiber systems. Each has apparently evolved independently several times. The first type consists of a single large or giant cell which gives rise to a single large axon. The second 
type consists of a syncytium in which processes from a number of nerve cells fuse to form a single giant axon. Unicellular giant fibers are found in nemerteans and cestode worms, in numerous polychactes, in balanoglossids, and in lower vertebrates. In the polychaete Halla, ${ }^{21}$ for example, there are groups of unipolar giant cells $30-150 \mu$ in diameter in each ganglion of the first few segments; these give rise to fibers up to $40 \mu$ thick which may run the length of the worm. Similar unicellular giant fibers have been described in other worms. Typically, in balanoglossids there are about a dozen to 150 giant cells, usually in the collar nerve cord; these give rise to large (3-6 $\mu$ ) fibers which run back in the nerve cord and eventually dwindle in size to merge with the ordinary $(1 \mu)$ fibers. ${ }^{75}, 193$ Nerve cells of the unicellular type of giant fiber, Mauthner and Müller cells, occur in the base of the medulla as part of the vestibular reflex system of bony fishes, urodeles, and tadpoles of anurans. These fibers, $22-43 \mu$ in diameter, conduct at 50-60 M./sec. at $10-15^{\circ} \mathrm{C}$. (Table 76)..$^{169}$

The multicellular or syncytial type of giant fiber system has been described in annelids, crustaceans, and cephalopod molluscs.

In the ventral nerve cord of the earthworm there are in each segment several cells which send axons to the three dorsal giant fibers, which occur as one median and two smaller lateral fibers. These giant fibers run the full length of the ventral nerve cord and give off segmental branches to motor neurones in the cord. The median giant fiber is connected in each segment to approximately two pairs of giant cells and each lateral giant fiber to approximately one pair of giant cells. ${ }^{392}$ The lateral giant fibers are also connected with each other. In addition to the three dorsal giant fibers there are two smaller ventral ones. ${ }^{383}, 391$ At each segment there is in the giant fibers an oblique partition, the two sides of which stain differently. ${ }^{76,392}$

Evidence from cutting the giant fibers, from regeneration, from blocking by drugs, and from embryonic development indicates that in the earthworm the giant fibers are responsible for quick end-to-end "startle" contractions. ${ }^{53,328}$ Stough ${ }^{392}$ cut single giant fibers and concluded that the median one conducts posteriorly and the two lateral ones conduct anteriorly. Action potentials, ${ }^{134}$ however, showed that conduction is equally good in either direction in each fiber. Bullock ${ }^{76}$ settled the disagreement by showing that the median fiber is normally excited by sensory stimulation in the anterior forty segments, whereas the lateral fibers are excited by sense organs behind that level. The two lateral fibers and the median fiber form two separate conduction and excitability systems; cross connections between the lateral fibers were shown by conduction which continued when right and left fibers were alternately cut. $^{370}$ Usefulness of the giant fiber system, particularly in protective withdrawal reactions, is indicated by the great difference in speed of conduction: conduction in the smaller fibers of the cord (including synapses) is about $0.025 \mathrm{M} . / \mathrm{sec}^{.}{ }^{53}$ whereas in the lateral giant fibers it is $7-12 \mathrm{M} . / \mathrm{sec}$. and in the median fiber it is $17-45 \mathrm{M}$./sec., depending on fiber size. ${ }^{76,}{ }^{13 *}$ Some polychaete worms have no giant nerve fibers (e.g., Aphrodita, Chaetopterus), others have only one (Arenicola, Pista), and still others have several, as shown both.by the action potentials in their nerve cords and by histological examination (Neanthes, Glycera). ${ }^{80}$ The junctions in Neanthes are complex and are polarized histologically but not physiologically. 
Among Crustacea giant fiber systems have been described in the crayfish, the lobster, and the prawns Palaemonetes and Leander. In the crayfish ${ }^{225}$ there are three kinds of giant fiber: median, lateral, and motor. The paired median giant fibers start in the brain (at least in the crayfish), decussate there, and pass to the ventral nerve cord. The lateral fibers occur in thoracic and abdominal ganglia and consist of segmental units which appear to overlap in long side-by-side contacts; they presumably are made up of processes from ganglion cells in each segment. Branches are given off from the laterals and medians to motor giant fibers in each segment. The motor fibers have their cell bodies in one segment, decussate, and pass out in the posterior nerve of that segment. ${ }^{225}$ Stimulation of the giant fiber system elicits quick flipping of the abdomen ${ }^{226}$ and causes extension and drawing together of antennae. ${ }^{42,433}$ The median and lateral systems independently activate the same motor fibers. The median and lateral giant fibers of the crayfish conduct equally well in both directions, but transmission to the motor giant fibers is polarized. Crossing occurs from one lateral giant to the other in 0.5 msec., but there is no crossing between the two median fibers. There is no appreciable delay (less than $0.1 \mathrm{msec}$.) at the segmental junctions of the lateral giants. Summation of the motor response occurs if the faster median fibers are stimulated before the laterals. Probably the median giants are normally activated only in the brain, and the laterals at any ganglion behind the fourth thoracic.

In the prawn, Leander, ${ }^{203}$ two types of synapse exist: oblique regions of apposition between the lateral fibers in each segment, and typical boutor.s at the points of contact of processes from both median and lateral with the motor fibers. The median giant fibers show incomplete septa. Transection of the cord at various points failed to produce degeneration of the median or lateral giant fibers. In Leander the motor giant fibers do not show myelination from the cell body to their synapse but are myelinated from that point out to the muscles.

The cephalopod molluscs have a giant fiber system which is intermediate between the unicellular type and the segmentally fused type. The cephalopod system has been studied by Young. ${ }^{41,} 442,443$ In the decapods (squids) a pair of giant cells lie at the posterior end of the pedal ganglion portion of the brain; their processes fuse and cross, then synapse in the visceral part of the brain with cell processes which run out in mantle nerves. These synapse with a third set of giant fibers in the stellate or mantle ganglion. From the stellate ganglion some dozen nerves pass out to the muscles of the mantle; each nerve contains one giant fiber, which may be as much as $800 \mu$ in diameter, plus many $1 \mu$ fibers. Each third order giant axon arises by the fusion of processes from $300-1500$ cells in the stellate ganglia. Each giant axon serves the circular muscles of a large area of the mantle, and the largest fibers pass to the posterior part of the mantle so that impulses arrive nearly simultaneously in the entire mantle. ${ }^{42}$ Velocities of conduction in fibers $30-718 \mu$ in diameter range from 2.7 to $22.8 \mathrm{M}$./sec. at $20^{\circ}$ (Table 76). Stimulation of a giant fiber elicits a maximal contraction at threshold, ${ }^{442}$ and there is no increase in tension with increasing frequency of stimulation, hence the one syncytial fiber with a large area of circular muscle constitutes a motor unit. The contractile response forces water out 
of the mantle cavity, thus sending the animal backward or forward, according to the funnel angle; this escape or predatory reaction is, according to Young, twice as fast by virtue of the giant fiber system as it would be with. out it.

Young ${ }^{41}$ has described an interesting series among cephalopods (Fig. 296). In the decapod Sepia the cells contributing to the large giant fibers are scattered through the stellate ganglion. In Loligo these cells are confined to one posterior lobe, whereas in the octopods Eledone and Octopus the lobe is present and contains cells which may resemble neurones but which lack true processes. This lobe, the epistellar body, receives the secondary giant fibers from the visceral ganglion; its possible neurosecretory function is indicated by an atonia of the mantle after removal of the epistellar body.

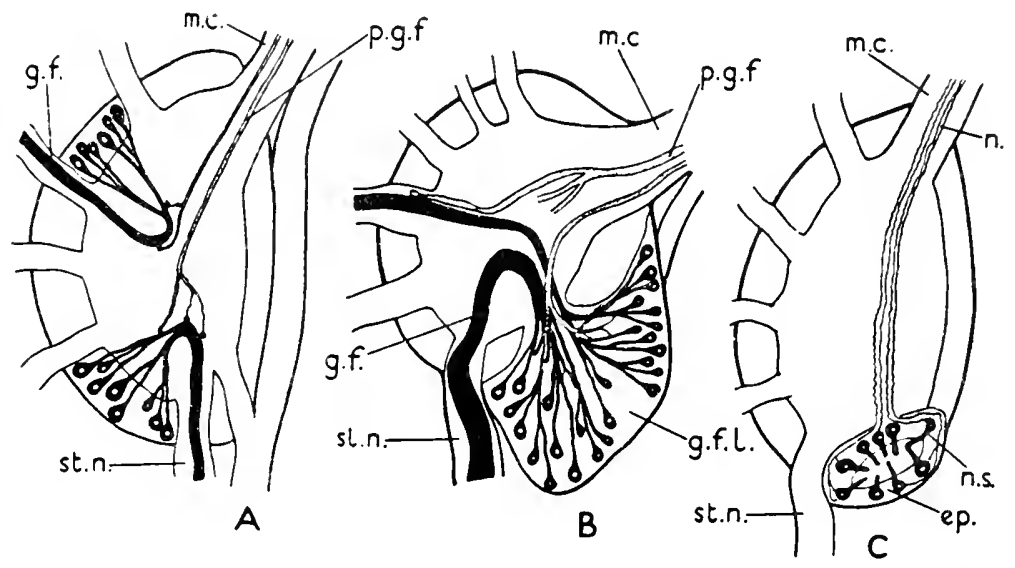

Fig. 296. Comparison of stellate ganglion of, A, Sepia, B, Loligo, and C, Eledone. m.c., Mantle connective (from brain mass); p.g.f., preganglionic fiber; ep. epistellar lobe. From Young. ${ }^{441}$

Giant nerve fibers have evolved many times. They are absent from animals closely related to animals which have them. For example, giant fibers are found in only a few polychaetes, are absent from crabs but present in shrimps and prawns, absent from octopods but present in decapods, absent from adult anurans but present in urodeles. Wherever they are present they seem to function in rapid escape reactions; they are efficient, since one nerve impulse in a giant fiber can activate muscles of a wide body area. A single nervous unit carries out a complex fast response.

Interneuronic Transmission. Continuity versus Contiguity. In all nerve centers the axons branch extensively and terminate on the dendrites and cell body of the receiving neurone-rarely also on its axon. The pattern of dendrites differs greatly according to the nerve center, and for many years histologists disagreed as to whether there is continuity or contiguity between neurones. With the introduction of cytological fixation methods and degeneration experiments the argument seems well settled in favor of contiguity. The fine axon branches often terminate in enlargements, boutons; in numerous preparations two separate membranes can be seen-Mauthner cells of urodeles ${ }^{39,47,48}$ (Fig. 297, A), oculomotor nucleus of fish (Fig. 297, 
B), anterior horn cells of spinal cord, ${ }^{200}$ and giant cells in stellate ganglion of cephalopods. ${ }^{43}$

Even in nerve nets of coelenterates, where anastomoses have been described many times in silver-stained network, vital stains show that the processes of the neurones actually intertwine or run along parallel with one another, but the fibers do not fuse. These net "synapses" are not structurally polarized, as are synaptic boutons; as much surface of one neurone as of the other is exposed at an apposition synapse. Similarly, the junctions between segments of giant fibers in earthworms and crustaceans are contiguous but the surfaces are equal; these are monosynaptic junctions. ${ }^{48}$ In bouton
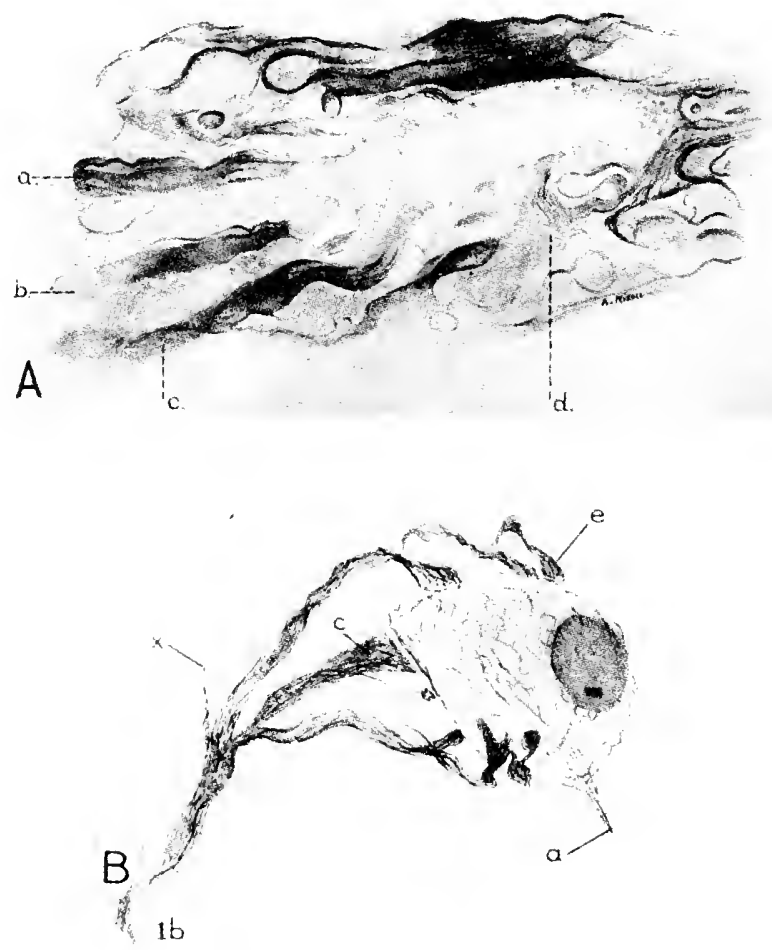

Fig. 297. Figures of polysynaptic junctions. $A$, Endings of axons on dendrite of a giant Mauthner cell of catfish, Ameiurus. From Bartelmez and Hoerr. ${ }^{\text {so }} B$, Branching axon ending in bulbs and club endings on a cell in the oculomotor nucleus of the goldfish. From Bodian. ${ }^{47}$

and basket synapses (polysynaptic junctions) the branches from several axons converge onto one receiving neurone, and the total active surface of the receiving neurone is much greater than that of any one incoming axon.

Polarity. Synaptic transmission usually occurs in only one direction, orthodromically; antidromic (backward) impulses stop at the receiving neurone soma (cell body and dendrites). One explanation of polarized transmission lies in the fact that in such centers as sympathetic ganglia acetylcholine is liberated in appreciable amounts only at preganglionic axon terminations, not by antidromic impulses. A second explanation of polarity is that a cer- 
tain area of a neurone must be depolarized for it to be excited. ${ }^{2 \times 7}$ The area of the boutons of one axon is insufficient for antidromic excitation of that axon. Hence impulses can summate from various converging axons and excite a motoneurone, but they cannot pass in the opposite dircction. ${ }^{264}$

In synaptic systems, which are not structurally polarized, impulses can be conducted in either direction, as across giant fiber septa and nerve net junctions. In coelenterate nerve nets, conduction is diffuse and essentially cqual in all directions. ${ }^{137,366}$ If interdigitating lateral or central incisions are made in a jellyfish or if the animal is cut into a zig-zag strip or into a central disc connected by a narrow neck to an outer ring, impulses pass around corners and in both directions, so long as nervous tissue is present. That this diffuse conduction is nervous is shown by conduction in areas where muscles are absent but nerve fibers present, ${ }^{285}$ in regenerating areas where conducting but not contracting tissue is present, and in preparations paralyzed with curare $^{366}$ or magnesium. ${ }^{74}, 285,386$ Similar conduction across narrow nerve bridges occurs in sea anemones ${ }^{315}$ and from zooid to zooid in colonial coelenterates such as Renilla. ${ }^{318}$ In sea anemones, however, there is some polarity. Responses of the free end and of the central cut end of tentacles differ. ${ }^{313}$, 315 Through conducting tracts occur, particularly in mesenteries, where velocities of $1.2 \mathrm{M}$./sec. exist, in contrast to local conduction at $0.04-0.15 \mathrm{M}$. $/ \mathrm{sec}$. The velocity of conduction varies in different regions of an anemone. ${ }^{312}$ It is possible that polarity is imposed by differences in facilitation for conduction in different directions.

The transverse septa of giant fibers are not polarized in Lumbricus, nor are the more complex overlapping junctions of giant fibers in Neanthes ${ }^{80}$ and Cambarus. ${ }^{433}$ It is likely that all non-polarized junctions are monosynaptic, and that polarized junctions are polysynaptic.

The Synaptic Transmitter. Synaptic transmission is a complex series of events and little is really known of the sequence and role of the different agents. Several suggestions have been made as to the mode of transmission of excitation from the axon to the receiving neurone. Several agents and mechanisms have been proposed, including: (1) acetylcholine; (2) inorganic ionic changes; and (3) electric current.

ACETYLCHOLINE. In previous chapters it was shown that many cardioregulator nerve endings and some motor endings on muscles are cholinergic (acetylcholine liberated at the junction), and some others are adrenergic (adrenin liberated at the junction). The only interneuronic junctions at which liberation of acetylcholine has been definitely demonstrated during transmission are those of sympathetic ganglia. The superior cervical ganglion of the cat can be perfused by a branch of the carotid artery and the perfusate collected from the internal jugular vein. When the preganglionic nerve trunk is stimulated and the ganglion eserinized, acetylcholine is liberated into the perfusate in amounts corresponding to the number of impulses entering the ganglion. ${ }^{142}$ Injected acetylcholine stimulates ganglion cells to discharge. Acetylcholine is not liberated in appreciable amounts by antidromic impulses, but it is still liberated on preganglionic stimulation after transmission to the postganglionic neurones is blocked by nicotine. curare, etc. Physostigmine (eserine) protects against destruction by cholinesterase of the liberated $\mathrm{ACh}$ and sensitizes the ganglion cells to ACh. ${ }^{143}$ 


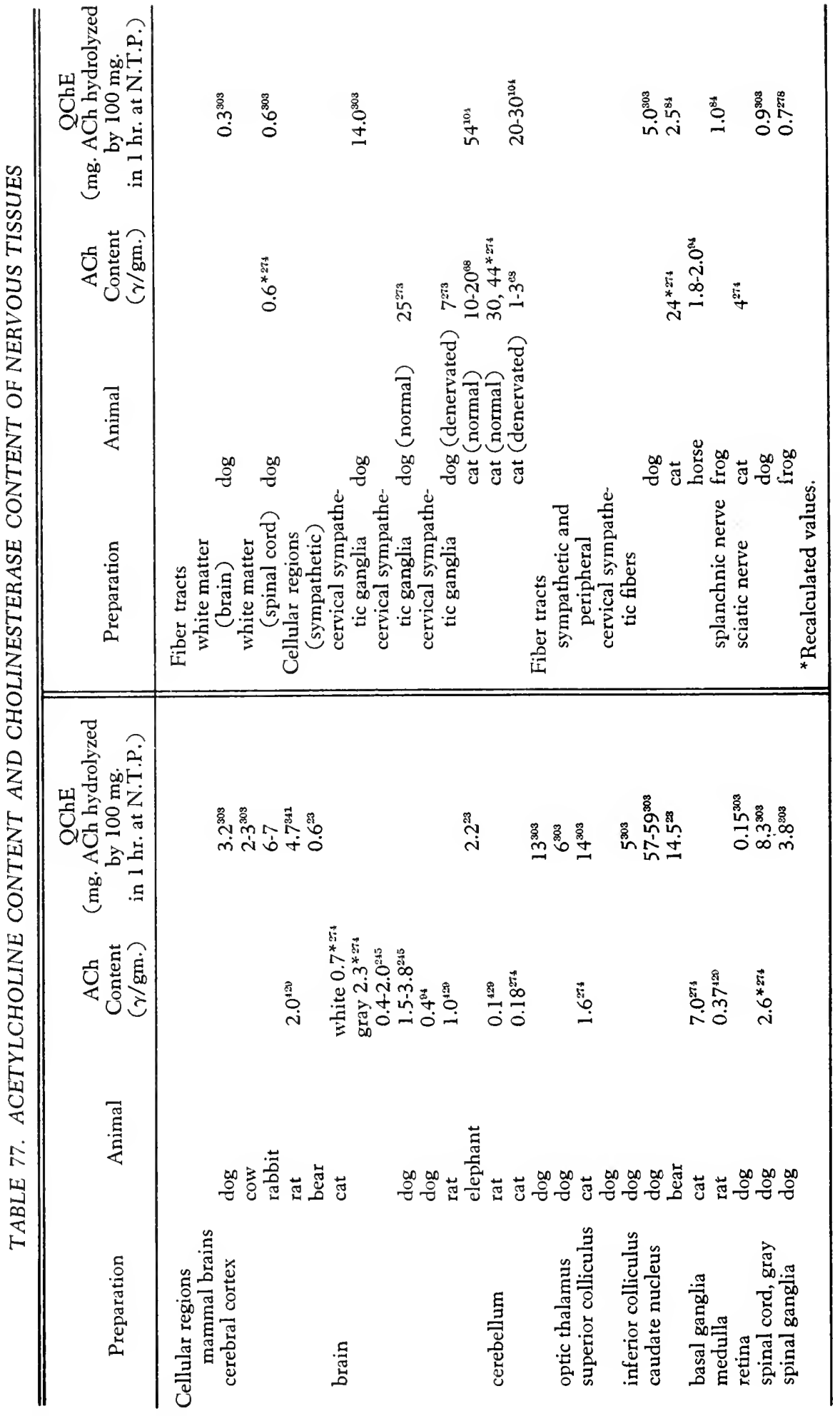




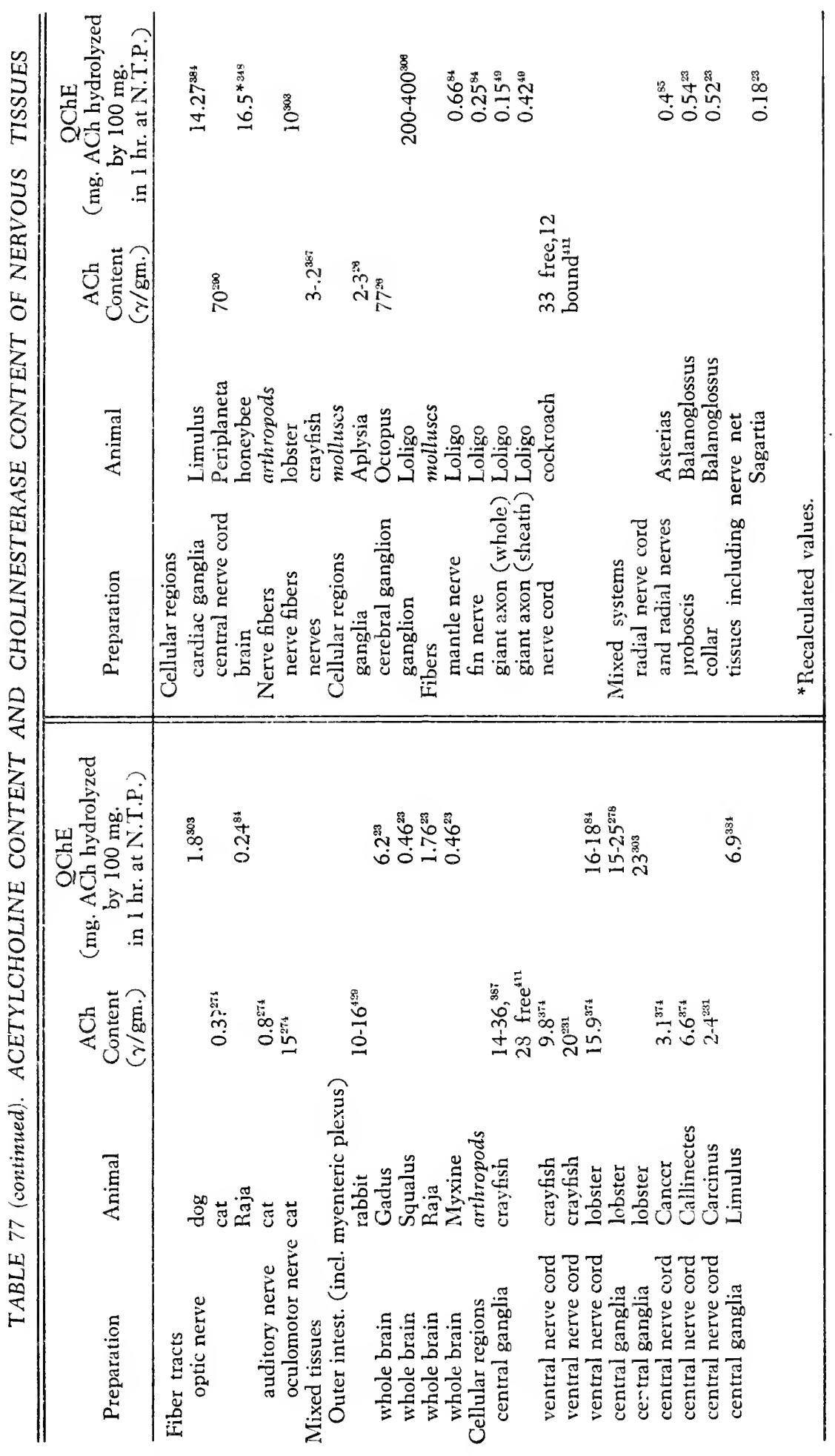


When small amounts of $\mathrm{ACh}$ are injected and at the same time a few preganglionic fibers are stimulated, the two stimuli add, producing a response in more ganglion cells." There is no doubt that acetylcholine is liberated from some structure in sympathetic ganglia and that it excites ganglionic neurones, but it does not follow that it is the transmitter.

In central nervous systems there are suggestions but no proof that acetylcholine functions in synaptic transmission. The literature is extensive on the effects of $\mathrm{ACh}$ in mammalian and amphibian nervous systems ${ }^{1+1}$ and in invertebrate nervous systems. ${ }^{337,430}$ Acetylcholine applied to the cerebral cortex of mammals is excitatory, particularly in high concentrations and after eserine. High amplitude spike-like brain waves are set up, and these may be associated with motor activity. ${ }^{292}$ The spinal cord is stimulated to motor discharge when perfused with acetylcholine, particularly after sensitization with adrenalin, ${ }^{71}$ and there are numerous instances of potentiation of central action of acetylcholine by adrenalin. Acetylcholine potentiates salivary reflexes. ${ }^{51}$ Some spinal reflexes are depressed (e.g., knee jerk), others are enhanced (e.g., flexors) by eserine. ${ }^{71,394}$ Eserine is without effect on the potentials associated with synaptic transmissions in the spinal cord, and high concentrations of $\mathrm{ACh}$ depress the synaptic potentials. ${ }^{131}$ When the lower portion of the spinal cord of a dog is perfused with eserinized Ringer solution, ACh appears on stimulation of the sciatic nerve. ${ }^{71}$ When eserine is injected into cats ACh appears in the cerebrospinal fluid, and there is evidence for liberation of $\mathrm{ACh}$ when the vagus nerve is stimulated. ${ }^{1+1}$ The variable and negative effects of eserine applied to vertebrate nervous systems probably result from the very low permeability of nerve membranes to eserine and the fact, as shown in peripheral nerve, that practically all of the cholinesterase must be inactivated before conduction is affected. 305,84

Measurements of the acetylcholine content of many nerve centers are not always comparable because of the rapid inactivation of the ester and the uncertainty regarding the manner in which acetylcholine is bound, and because of the variable amounts free or bound. In general (Table 77) there is more $\mathrm{ACh}$ in gray than white matter. Regions which are sensitive to hypoxia, such as the cerebrum, contain little ACh. ${ }^{+29}$ The concentration in sympathetic ganglia is higher than in brain, and sensory fibers contain much less than do motor fibers. ${ }^{.73,274}$ The enzyme system which synthesizes $\mathrm{ACh}$ is very active in cholinergic neurones (such as lower motor and preganglionic sympathetic neurones) and not so active in non-cholinergic nerves (such as sensory roots and the optic nerve). ${ }^{1+4}$ Among invertebrates the central nervous systems often contain more ACh than mammalian nervous systems (Table 77). Crustacean ganglia compare favorably with mammalian sympathetic ganglia in ACh content, and insect and cephalopod ganglia contain the highest concentrations yet observed. The correlation between $A C h$ content and nervous activity, however, is not good.

The ganglia of arthropods, molluses, and annelids are relatively insensitive to acetylcholine with or without eserine treatment. In Octopus and Eledone acetylcholine and eserine had no apparent eflect on the stellate ganglion. ${ }^{2 x}$ When applied to arthropod central nervous systems acetylcholine is ineffective in causing the discharge of impulses except at concentrations as 
high as 0.1 per cent in the crayfish, ${ }^{334}$ and in the cockroach. ${ }^{358}$ Injections of large amounts (up to $40 \mathrm{mg}$.) into crabs (Carcinus and Panulirus) cause no change in reflex activity, ${ }^{26}$ although eserine and acetylcholine facilitate autotomy in crabs. ${ }^{428}$ Synaptic transmission in the sixth abdominal ganglion of the cravfish is unaffected by large amounts of acetylcholine, physostigmine, and prostigmine. ${ }^{334}$ Acetylcholine in a crayfish perfusion saline does, however, maintain the ganglia in a normal state of excitability for potassium. ${ }^{355}$ Transmission across the junctions from giant fibers to motor fibers in the crayfish is not affected by a series of drugs, including $\mathrm{ACh}$, adrenalin, prostigmine, and curare, but is blocked by DFP and eserine; nicotine initially facilitates and then blocks transmission. ${ }^{375}$ Absence of effect of a drug may result from lack of penetration rather than from absence of specific action. In cockroaches the anti-esterase DFP causes alternate facilitation and blocking of synaptic transmission at concentrations which permit fiber conduction, and in the presence of DFP added ACh blocks the synapses. ${ }^{360,361}$ In the stellate ganglion of the squid, synaptic transmission is blocked by DFP at about the same concentrations at which fiber conduction is blocked. ${ }^{84}$

The cholinesterase content of central nervous tissues varies as much as the ACh content (Table 77). Ganglia of squid, honeybee, lobster, and Limulus approach mammalian sympathetic ganglia in QChE. The mammalian cerebral cortex is surprisingly low in QChE. In lower invertebrates it is difficult to separate nervous from muscular tissue as regards cholinesterase distribution (see also Chapter 16), but there is little doubt that cholinesterase is present in all nervous tissue.

In general, the distribution of $\mathrm{ACh}$ and $\mathrm{ChE}$ seems not well correlated with central nervous activity; acetylcholine is excitatory to some nerve centers and inhibitory to others; the sensitivity is high in sympathetic ganglia and in the spinal cord under special conditions, but sensitivity to $\mathrm{ACh}$ is low for cerebral cortex and practically zero for arthropod central nervous systems. Liberation of acetylcholine has been demonstrated at axon terminations in sympathetic ganglia. Evidence regarding the meaning of the high concentrations of $A C h$ and $\mathrm{ChE}$ in invertebrate central ganglia might elucidate the role of acetylcholine in synaptic transmission in general. It is becoming more likely that "the transmitter" is electrical but that acetylcholine is one substance associated with the production of the action potential. ${ }^{130}$, 30t

INORGANIC IONIC CHANGES. Synaptic transmission is profoundly affected by the potassium and calcium in the bathing fluids. In the perfused superior cervical ganglion of the cat, potassium is given off on preganglionic stimulation and the potassium content of the ganglion is greatly reduced; ${ }^{+19}$ potassium also moves outward from stimulated nerve fibers (p. 780). Injection of srnall amounts of potassium increases the ganglionic response to submaximal stimulation. ${ }^{\text {it }}$ Like acetylcholine, potassium excites in low concentrations and inhibits in larger amounts. Addition of potassium to the perfusate causes ACh liberation, and ACh injection causes K liberation. Perfusion with a saline deficient in calcium or containing five times the normal concentration of potassium increases both the number of active cells and the frequency of discharge in sympathetic ganglia. ${ }^{6: 2}$ Brain potentials are sensitive to potassium/calcium balance. Excess potassium causes fast irregular waves, and calcium in excess elicits large slow waves in the isolated frog brain. ${ }^{255}$ 
Many invertebrate ganglia are likewise initially stimulated by high potassium or low calcium (see Table 69, Ch. 15, for heart ganglia). In crayfish abdominal ganglia in situ the effect of high potassium is to depress spontaneous activity, whereas low potassium increases it. After a half hour of bathing in saline the reverse effects of potassium are observed. ${ }^{334,336,357}$ Addition of acetylcholine to the bathing solution prevents the reversal in potassium action and suggests that differences in behavior of intact and isolated ganglia may result from loss of $\mathrm{ACh}$ on isolation. ${ }^{358}$ Transmission across synapses of the cockroach is remarkably unaffected by changes in potassium and calcium. ${ }^{359}$

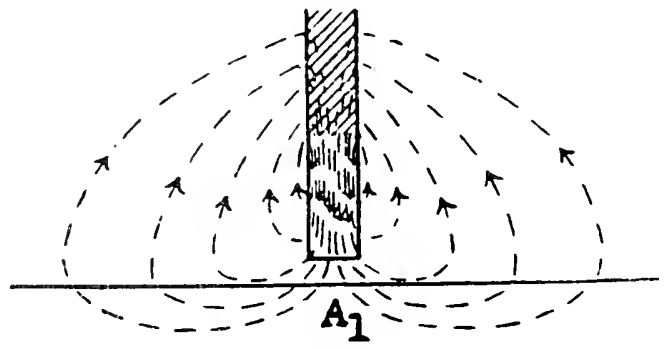

(a)

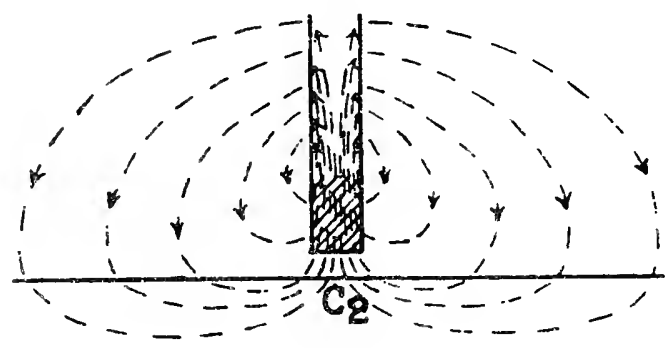

(b)

Fig. 298. Current flow at a synaptic junction; presynaptic fiber represented by vertical block and postsynaptic neurone by horizontal line. Current flow with presynaptic impulse $(a)$ approaching synapse and $(b)$ ar synapse. Focal anode $\left(A_{1}\right)$ followed by focal cathode $\left(\mathrm{C}_{2}\right)$. Cross hatching, region of the presynaptic impulse. Current flow represented as toward region of negativity. From Eccles. ${ }^{132}$

ELECTRIC CURRENT. The action potential wave of the discharging axons may itself be an adequate stimulus for the recciving neurones. An electric shock of time relations similar to the action potential is an adequate nerve stimulus. In the oculomotor nucleus of the rabbit a rolley of inspuises in ascending axons, subthreshold for the motoneurones, can sum with a subliminal electric shock applied directly to the motor cells during the rising phase of the action potential. ${ }^{262}$ When two nerves are placed in proper contact side by side, an impulse can be transmitted from fibers of one nerve to the other. Transmission in such an artificial synapse, or ephapse, occurs if the impulse in the pre-cphaptic fiber stops at the junction so that the final phase of its potential change is negative or catelectrotonic. ${ }^{18}$ Delays vary 
with conditions, but may be as brief as 0.1 to $0.3 \mathrm{msec}$. for certain mammalian preparations, ${ }^{172}$ or 2 to $5 \mathrm{msec}$. between squid giant axons. ${ }^{18}$ The excitability of the receiving nerve fiber can be altered by subthreshold impulses, and transmission is facilitated when the afferent impulse coincides with oscillations in local graded potentials in the efferent fiber. ${ }^{18}$ Similar electrical transmission across points of contact occurs between two separate lobes of the brain of frogs. ${ }^{163}$ The current fields set up in nerve centers are such that electrical stimulation could occur at synaptic junctions. ${ }^{266}$

Eccles $^{127}$ has analyzed the electrical pattern at a nerve terminal and demonstrated its adequacy for stimulation. As an impulse approaches a nerve terminal it sets up an anodal (positive) focus, which, when the peak of the impulse reaches the terminal, reverses (downsweep of potential), causing a cathodal focus with anodal surround on the receiving neurone. Current flow at the cathodal focus causes a local response which depolarizes an area depending in size on the current magnitude, and if it is large enough sets off a propagated impulse.

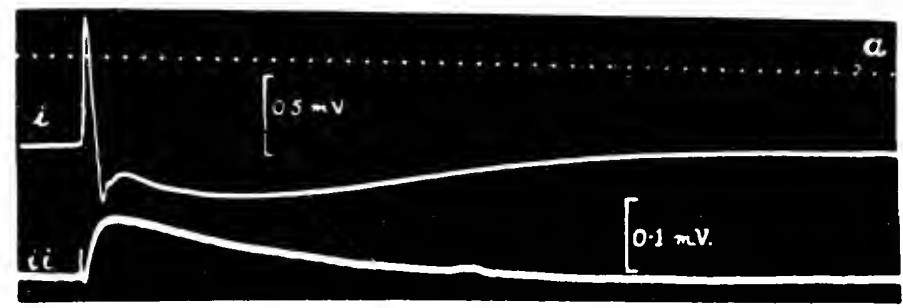

Fig. 299. Action potential from the stellate sympathetic ganglion of a cat (i) before and (ii) after curarization. Synaptic potential only remains after curarization. From Eccles. ${ }^{128}$

We are left with numerous agents-acetylcholine, potassium, electric current-each present and capable of stimulating nerve cells. Whether or not a nerve cell responds is determined by the environment of the cell, and one or several components of this environment may constitute adequate exciting agents. ${ }^{62}$ The old distinction between electrical and chemical transmission is superseded by two other hypotheses. One is that axon propagation is electrical, involving potassium and other ions. and that at synaptic transmission either chemical agents (ACh or others) are liberated (Dale-Loewi), or that synaptic transmission is also electrical (Eccles). The other theory (Nachmansohn) states that axon and synaptic transmission are not different, that both are electrical, and that ACh is an essential link in the electrical picture.

Synaptic and Ganglionic Potentials. The interpretation of potentials recorded trom nerve centers is difficult because of the small dimensions of the separate parts of neurones. Evidence is accumulating that conduction in the different parts of a neurone occurs at different rates.

SYNAPTIC POTENTIALS. At interneuronic junctions, and presumably associated with a transmission process, is a synaptic potential analogous to the end-plate potential of muscle (Ch. 16). In sympathetic ganglia, stimulation of pre-ganglionic axons sets up a complex propagated electrical response in postganglionic neurones; after curarization this propagated response is replaced by a graded potential which is conducted decrementally away from 
the ganglion. This graded synaptic potential is normally masked by the propagated response. In the superior cervical ganglion of the cat this synaptic potential begins after a latency of $5 \mathrm{msec}$., reaches its peak at $10-20$ msec. and falls to about $1 / 3$ in $60-90$ msec., many units rsponding out of phase (Fig. 299).129 The synaptic potential is absent when antidromic impulses enter the ganglion and is graded in size with the number of pre-ganglionic impulses; it can summate on repeated subthreshold stimulation. When transmission to interneurones and motoneurones in the spinal cord is blocked by nembutal a sensory volley sets up a synaptic potential which can be picked up either by focal microelectrodes in the cord or conducted electrotonically over ventral roots. In monosynaptic reflexes of cats the synaptic potential recorded focally in the spinal cord shows a delay of 0.3 to $0.45 \mathrm{msec}$. and peak time of 0.8 to $1.5 \mathrm{msec}$. Similar graded potentials have been recorded from the stellate ganglion of Loligo (Fig. 300). ${ }^{7 \times}, 81$ The

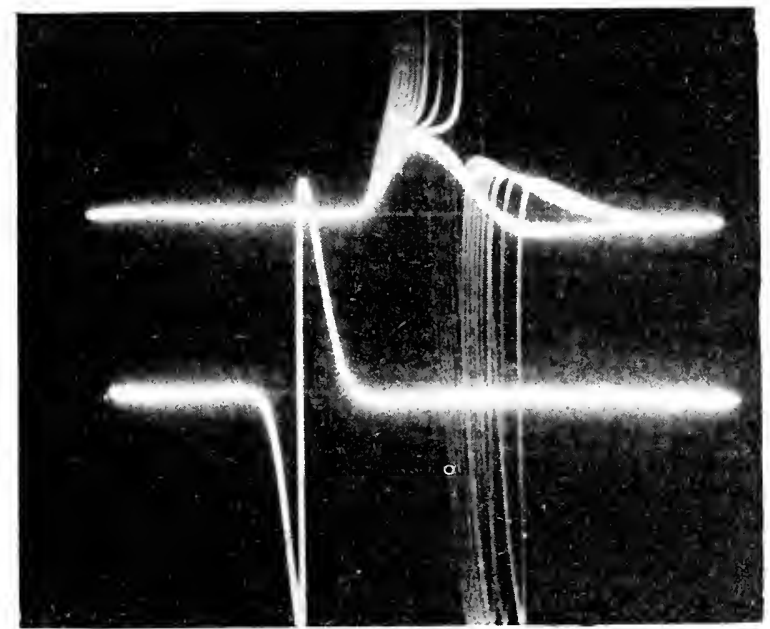

Fig. 300. Synaptic potentials and emergent action potential spikes from stellate ganglion of the squid. Lower record-spike in preganglionic fiber. Upper record-postganglionic spike developing later and later out of synaptic potential as fatigue occurs finally leaving only synaptic potential. From Bullock. ${ }^{75, ~ s t}$

propagated postganglionic spike arises out of the synaptic potential, and when motoneurones are fatigued only the synaptic potential remains. Direct stimulation of the ganglion can elicit a synaptic potential, but antidromic impulses cannot do so. It is postulated ${ }^{63}$ that the transmitter passes its peak before the beginning of the synaptic potential, and that the synaptic potential must be of a critical size before the post-synaptic spike is evoked.

SOMA RESPONSES. The soma of a central neurone refers to the non-axon portions, i.e., the dendrites and cell body. The potentials which arise in the soma appear to be slow compared with those in axons. Sympathetic ganglion potentials show several spikes corresponding to activity in different cell groups, prolonged as compared with axon spikes, and they also show slow negative and positive after-potentials ${ }^{126.257}$ (Fig. 299). Records from the mammalian oculomotor nucleus show fast axon spikes followed by slowed motoneurone potentials; the latter only are found in response to antidromic 
volleys ${ }^{262}$ (Fig. 300). Similarly in the hypoglossal nucleus deep electrodes detect slow waves which can be separated experimentally from the axon impulse, conduction in the soma being not more than one-tenth as fast as in the axons. ${ }^{266}$ These slow soma potentials can be observed when the cells are excited antidromically.

The ganglia of invertebrate animals are complex in organization. Soma potentials lasting $30 \mathrm{msec}$. have been recorded from isolated cell bodies of the visceral ganglion of the snail Aplysia. Their origin is not clear. ${ }^{19,}$, 89 ,A closer correlation between the histology and physiology of nerve centers is needed.

Graded localized synaptic potentials precede the discharge of motoneurones, and soma potentials are slower than axon spikes; the soma potentials must have significant influence on the excitability of surrounding neurones.

Symaptic Delay. At all interneuronic junctions an appreciable time is taken for setting up an efferent impulse. Synaptic delays are longer in centers receiving impulses over slow afferent fibers than over fast ones. The older measurcments of "central time" in spinal reflexes give little information regarding synaptic delays because the number of interneurones interposed between afferent and motor neurones is not known. In two-neurone reflexes of the cat Lloyd finds central delays of 0.7 msec.; these can be reduced by facilitation to a minimum of 0.3 msec. ${ }^{63}, 258$ And in the oculomotor nucleus of the rabbit, delays at synaptic junctions averaged $0.7 \mathrm{msec}^{262}$ Delays in the slower sympathetic ganglia are much longer. The superior cervical ganglion of the cat shows delays of 3 and 6 msec., respectively, for two groups of units, ${ }^{125}$ and interneurones probably do not occur. In the stellate ganglion of the squid a single synaptic junction of the giant fiber system has a delay of 0.5 msec. at $24^{\circ} .81$ The sixth abdominal ganglion of the crayfish gives a ganglionic delay of $2-5 \mathrm{msec}$; this may include interneurones. ${ }^{333}$ In the last abdominal ganglion of the cockroach some sensory fibers from the cerci synapse with ascending giant neurones; the synaptic delay of this system is 0.6-1.5 msec., but there may be one interneurone. ${ }^{361}$

Several explanations of synaptic delay have been suggested:

1. Axon branches are usually greatly attenuated; conduction is slow in small fibers. This must be particularly important in sympathetic ganglia where there is a basket-like arrangement of axon and dendrite branches. When allowance is made for terminal slow conduction, the synaptic delay is somewhat reduced.

2. There may be asynchrony in arrival of impulses over the different branches of an axon, hence time is consumed in their spatial summation.

3. Motoneurones have a true excitation time, like the "utilization time" of axons.

4. Time is required for liberation and diffusion of acetylcholine or other chemical transmitter from one membrane to another. Shortening of the delay by facilitation may be explained by assuming that a subliminal quantity of transmitter persists for a time so that a threshold concentration is at tained sooner with a second volley. Diffusion is not a factor if transmission is electrical.

5. The synaptic potential rises at a certain rate and the time when an impulse is started depends on when the synaptic potential reaches a critical size, just as with end-plate potentials. In the squid giant-fiber synapse, for 
example, the synaptic potential reaches its peak in $0.6 \mathrm{msec}$; in fatigue this local response does not change in latency but falls in height, the propagated spike arising later on the synaptic potential. ${ }^{81}$ In the cat spinal cord the synaptic potential begins about $0.5 \mathrm{msec}$. after arrival of afferent impulses; 0.05-0.1 msec. is needed for conduction in the small afferent fibers, and the delay can be shortened by facilitation to $0.2-0.3 \mathrm{msec}$. Motor impulses can be started only during part of the rising phase of the synaptic potential, $1 \mathrm{msec}$. or less according to facilitation. ${ }^{132}$ A synaptic delay is required to remove the intial anodal focus and permit catelectrotonic spread of the local synaptic potential. ${ }^{127}$

Refractory Period. In general the absolute refractory period at synapses is similar to the refractory period of the efferent nerve fibers. Measurements of motoneurone refractory periods for several mammalian centers have been made by preceding an afferent volley at varying time intervals by an antidromic volley (impulses entering the center through the efferent neurones). By this method the refractory period of the oculomotor nucleus is 0.5-0.6 msec.; ${ }^{263}$ that of the cervical sympathetic ganglion is about $2 \mathrm{msec}$. for the fastest units. ${ }^{66}$

Some centers fail to follow repetitive stimuli at lower frequencies than do isolated axons, but this failure is not a direct measure of refractoriness. In the crayfish, for example, transmission across the sixth abdominal ganglion stops at $10 / \mathrm{sec}$. while sensory impulses enter up to $100 / \mathrm{sec}^{333}$ In fresh preparations the junctions from giant fibers to motoneurones of the crayfish show no longer refractoriness than the giant fibers, but with slight fatigue they are blocked at 30-50/sec. ${ }^{433}$ In the cockroach sixth ganglion the synapse between cercal sensory fibers and ascending giant fibers follows up to $400 /$ sec. $^{361}$

The refractory period of the giant fiber synapse in the stellate ganglion of the squid is less than that of preganglionic fibers. Impulses can follow across the junction at rates as high as $475 / \mathrm{sec}$., and a local response, too small for spike initiation, may be elicited with no absolute refractoriness. By analogy with muscle, where the graded response shows no refractory period, the synaptic potential also probably has no true refractory period.

Facilitation. By synaptic facilitation is meant the requirement that more than one incoming impulse is needed to excite a post-synaptic neurone. One incoming impulse may be ineffective alone, but when accompanied by others it is effective. Most synaptic transmission requires facilitation; there are, however, some interneuronic junctions as in invertebrate giant fiber systems in which conduction is one-to-one, and no facilitation is required for transmission. ${ }^{82}$

Some of these junctions are non-polarized, as in the longitudinal conduction in giant fibers of annelids and crustaceans. Others are polarized, as in the squid stellate ganglion and the connection between lateral giants and motor fibers in the crayfish. Facilitation does appear, however, when these systems are fatigued.

In the coelenterates, facilitation in the nerve net is marked and often necessary for the elicitation of a mechanical response. The excitatory process persists for several seconds (see p. 595). It appears likely that primitive interneuronic junctions required facilitation for transmission and that the 
one-to-one relay conduction of giant ncurone systems is a relatively evolutionary development.

When an interneurone or motoneurone is stimulated by two incoming volleys -separated by different times, the second volley is usually found to be most effective at two periods after the first; these are periods of early and of delayed facilitation.

1. Early facilitation (spatially determined). Two afferent volleys converging over different axons on one motoneurone are most effective when synchronous. One afferent volley alone may not be able to elicit a motor response, but together they summate. If one volley is delayed after the first, it becomes less effective as the excitatory process from the first decays. Varying the interval, then, between spatially converging subliminal impulses is useful in showing the time course of the excitatory process. Temporal summation at one synapse (bouton) is impossible because of refractoriness. Spatial summation is generally required for synaptic excitation. This is well shown in the tail of the crayfish, where flexion of one sensory hair on a uropod scnds one impulse into the sixth abdominal ganglion; stimulation of several, usually four, adjacent hairs is necessary to elicit one efferent (post synaptic) impulse. ${ }^{33+}$ In vertebrates it is not probable that a single impulse in one afferent axon ever elicits a reflex response.

In the superior cervical sympathetic ganglia of the cat two submaximal volleys in different preganglionic branches sum maximally at synchrony, the summation declines to zero at $4.5 \mathrm{msec}^{125,367}$ In the oculomotor nucleus of the rabbit summation between two converging volleys declines to a minimum

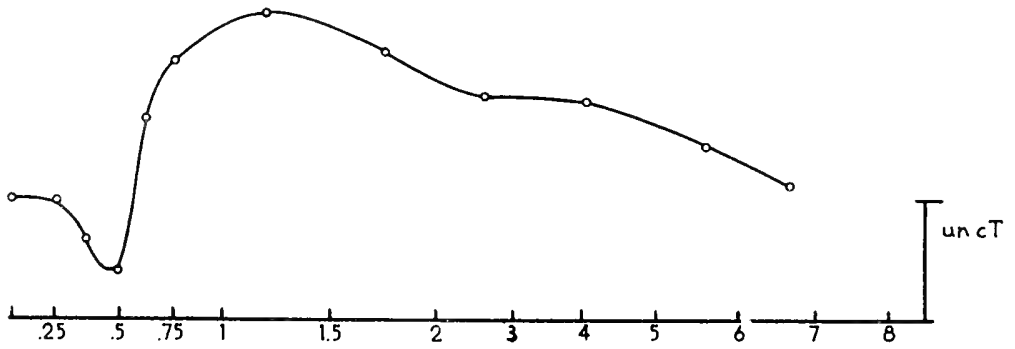

Fig. 301. Facilitation curve of motoneurones of oculomotor nucleus of rabbit. Motor response in mv. to a test shock to presynaptic (floor) neurones at intervals in msec. (abscissa) after a conditioning shock. uncT, Height of response of unconditioned test shock. From Lorente de Nó.:02

at 0.5 msec. separation ${ }^{262}$ (Fig. 301). In monosynaptic spinal reflexes of the cat the facilitation falls to half the maximum of simultaneity at about 2.8 msec. $^{6+}$ The early facilitation represents the time course of decay of the synaptic transmitter.

2. Delayed facilitation. The second type of facilitation represents enhanced excitability of receiving neurones after the initial excitatory process has passed; this facilitation can be detected by test volleys in either the same or different incoming pathways. In flexor reflexes of the cat, for example, Eccles and Sherrington ${ }^{135}$ observed a maximum response to a second afferent volley 12-20 msec. after an initial volley. The enhanced excitability after re- 
covery resembles the supernormal period in cardiac muscle (Ch. 15). Explanations have been suggested as follows:

(a) It is difficult to account for this delayed facilitation on the basis of persistence of any chemical transmitter unless it is assumed that a second excitatory substance is liberated.

(b) In nerve fibers the excitability returns during the refractory period and is supernormal during the negative after-potential. Similar enhanced excitability can be produced by cathodal polarization, catelectrotonus. In some nerve centers the late facilitation is at a time of delayed negativity. A relation between reflex facilitation and slow negative potentials in the spinal

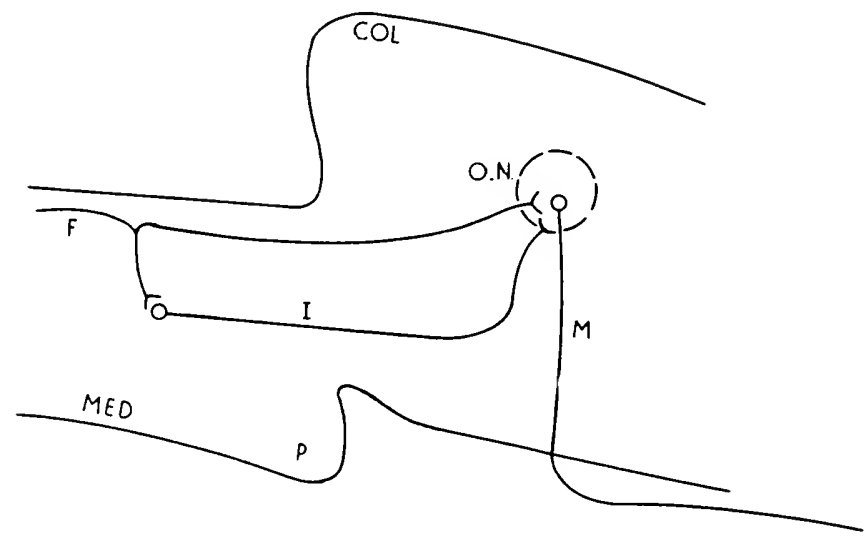

(A)

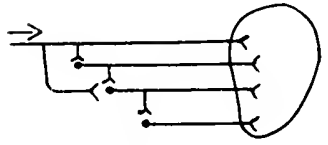

(B)

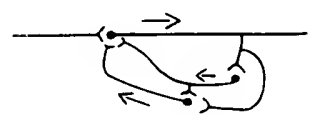

(C)

Fig. 302. (A) Diagram of delay path circuit in oculomotor nucleus. F, Floor neurone; I, interneurone; $M$, motoneurone; O.N., oculomotor nucleus; COL, colliculus; MED., medulla. Modified from Lorente de Nó. ${ }^{263}$ (B) Schema of a delay path (multiple chain) circuit with three successive interneurones. (C) Schema of a closed reverberating chain circuit of internuncial neurones. From Lorente de Nó. ${ }^{204}$

cord is indicated. ${ }^{215}$ In the superior cervical sympathetic ganglion the ganglionic response consists of one or more spikes, lasting 6-8 msec., followed by a slow negative wave persisting 50-100 msec. ${ }^{12 \pi}$, 2.77 When two preganglionic volleys enter the ganglion at such an interval that the second enters during the slow negative wave due to the first, a spike results which is facilitated and has shorter latency than when the second stimulus is applied alone. The time course of this facilitation follows the slow negative wave according to Eccles, ${ }^{125}$ but not according to Rosenblueth and Simeonc.: ${ }^{367}$

(c) A third explanation for delayed facilitation is by-pass conduction in interneurones. In the oculomotor nucleus $26: 2,264$ e.g., neurones are ar- 
ranged with direct connections of ascending "floor" axons to the motoneurones and indirect connections via many parallel interneurones (Fig. 302, A). The late facilitation is maximal at about 1 msec. (Fig. 301). The time course of this late facilitation is such that internuncial impulses are arrising at the motoneurone at the peak of the facilitation, and the internuncial impulse from volley one arrives at the same time as the direct impulse from volley two. Prolonged facilitation can occur if there is a chain of successive inter. neurones (Fig. 302, B). Facilitation can be altered by lesions to side pathways, and when motor responses are asynchronous the latencies of the late waves can be altered by facilitation. In the oculomotor nucleus, then, submaximal internuncial bombardment seems to account for late facilitation, impulses from one indirect path adding to later ones from another and direct path. Temporal summation is in this sense spatial summation. Such an explanation of delayed facilitation is not applicable to centers like sympathetic ganglia which lack interneurones.

Curves of delayed facilitation have not been obtained for any non-mammalian nerve center.

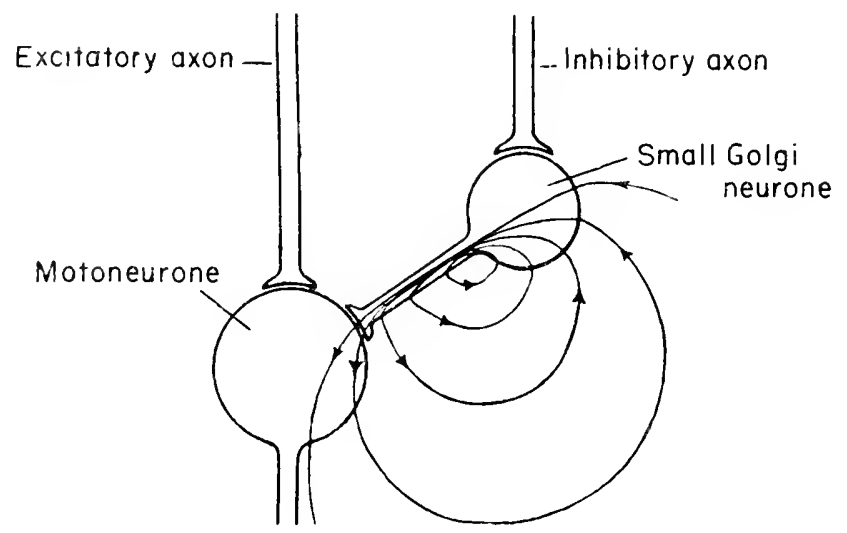

Fig. 303. Schema illustrating electrical theory of direct inhibition. It is assumed that the small golgi neurone is excited subliminally and that its synaptic potential creates a negative sink, causing outflowing current in the motoneurone and there producing a region of anelectrotonus. From Eccles. ${ }^{133}$

Inhibition. In a series of classical experiments Sherrington demonstrated that under certain circumstances reflex responses can be diminished by sensory impulses. He proposed that a central inhibitory state (CIS) counteracts a central excitatory state (CES). Some afferent nerve fibers are inhibitory, others excitatory. One afferent neurone can probably be excitatory and inhibitory to different neurones, although this is not proved. Single inhibitory fibers have been isolated in arthropod motor nerves (Ch. 16) and in frog sensory nerves. $: 233$ Just as with facilitation, there are two times of synaptic inhibition, early and delayed. Inhibitory impulses may prevent motoneurone stimulation if they arrive simultaneously with, or immediately before, the excitatory impulses. The two-neurone reflexes are thus inhibited by afferents in specific ipsilateral sensory fibers. 258

A specific inhibitory substance might be liberated at synapses; a given 
agent may be excitatory for some, and inhibitory for other receptor cells. Inhibitory mediators occur in the heart and in smooth muscle, but there is no evidence for inhibitory agents in nerve centers.

An electrical hypothesis of direct inhibition makes use of the short (2-3 $\mathrm{mm}$.) "Golgi" interneurones which are found in many parts of the central nervous system, particularly in the spinal cord (Fig. 303). ${ }^{133}$ Such a neurone might be excited by an inhibitory axon to produce a graded synaptic potential which would be conducted electronically over the short neurone and on entering the motoneurone produce there regions of decreased excitability or anelectrotonus. These would compete with excitatory synaptic potentials set up on the motoneurone and prevent the initiation of a propagated impulse.

Another postulate is that inhibitory endings are so distributed that excitatory fibers are unable to depolarize a sufficient area for excitation.

The second type of inhibition has a much longer latency. Sherrington obtained maximum inhibition of flexor reflexes in cats if the inhibitory volley preceded the excitatory volley by $30-80 \mathrm{msec}$. This inhibitory process builds up and declines more slowly than the excitatory process.

The delayed inhibition may result from slow positive potentials in nerve centers. It was stated above (p. 780) that, during the positive after-potential, nerve fibers are depressed in excitability. This is similar to lowered excitability in anodal polarization, anelectrotonus. Slow positive waves have been recorded from numerous centers, but their interpretation is difficult because they vary with electrode placement and the origin of slow potentials in nerve centers is not well known. Correspondence between diminished excitability and positivity in the anterior horn of the spinal cord has been indicated. ${ }^{215}$ In sympathetic ganglia a long-lasting positive wave is maximal at 100-200 msec. ${ }^{125}, 257$ The excitability of the ganglion for preganglionic volleys is diminished in a time course corresponding well with the slow positive potential. ${ }^{125}$ Such correspondence did not appear when the ganglion was conditioned by tetanic preganglionic stimulation. ${ }^{367}$ In the vertebrate retina a correlation has been suggested between positivity of the retinal potential (negativity of the ganglion cells of the inverted retina) and the setting up of optic nerve impulses, also between the reverse state (positivity of the ganglion cells) and cessation of optic nerve discharge (inhibition). ${ }^{170}$ Whatever the temporal relation between inhibition and positive after-potentials in nerve centers, any prolonged positivity will certainly lower the excitability of synapses within its electric field.

The neurone circuits of parts of central nervous systems are very complex. It is possible to construct circuits which are reasonable anatomically and which are properly timed to account for inhibition. ${ }^{139}$ Impulses entering a center over one path and acting through interneurones may be cancelled by impulses from another pathway which arrive at such a time earlier as to leave the interneurones inexcitable.

The time course of inhibition has not been examined in any non-mammalian center. There are many examples, however, of the inhibitory action of one center on another. Integrative centers maintain a balance between inhibition and excitation of motor centers. Examples of these will be considered below. 
Inhibition is essential in the antagonistic action of muscles. An afferent rolley which stimulates flexor motoneurones normally inhibits the motoneurones of antagonistic extensor muscles, and vice versa. Such reciprocal ex citation probably occurs in many metazoans. The longitudinal and circular muscles of an earthworm are reciprocally innervated. Even in medusac, where the nervous system is a diffuse nerve net, there are reciprocal contractions of circular and radial muscles. ${ }^{54}$ Whether reciprocal inhibition accompanies these alternate contractions is not known, but it is likely.

The term inhibition has also been applied to the cancellation of one wave of activity by another. For example a doughnut-shaped ring can be cut out of a jellyfish and a wave of contraction started in both directions from a stimulus at a point; these waves will cancel when they meet. ${ }^{2 \times 5}$ Two opposing waves of luminescence in sea fans cancel when they meet. ${ }^{294}$ No evidence for periods of depressed excitability has come from the use of paired or repetitive stimulation of coelenterates. The cancellation of colliding waves is probably due to refractoriness of conducting pathways.

After-discharge. Frequently a single afferent volley sets off a repetitive discharge which long outlasts the stimulus. Some reflexes are brief responses, as the knee jerk, others persistent, as the scratch reflex. Three explanations of after-discharge may be considered:

1. After-discharge may result from the persistence or continued liberation of liminal amounts of a chemical mediator, such as acetylcholine. An eserinized sympathetic ganglion, for example, may give repetitive response to a single preganglionic volley. ${ }^{62,367}$

2. After-discharge may result from delay paths and circus conduction. A message may pass around a ring of neurones, returning periodically to excite the same motoneurone over a side branch. Lorente de No has described such pathways in several centers and has demonstrated their action in the oculomotor nucleus preparation ${ }^{262,264}$ (Fig. 302, C). A gross example of circus conduction is afforded by cutting a doughnut-shaped ring out of a jellyfish. ${ }^{184,285}$ If a wave is started in both directions, then blocked on one side by pressure and allowed to continue on the other side, a circusing self-perpetuating wave can be kept going for many hours; the same units are reexcited repeatedly.

3. A third explanation of after-discharge is based on the property of many centers of showing spontaneous rhythmicity. Often neurones are capable of rhythmic activity and need only a slight stimulus to trip them off. Sympathetic ganglion cells can be made to fire repetitively by slight environmental changes, as with acetylcholine, low calcium, etc. ${ }^{62}$ There is a fine balance between rest and activity.

"Spontaneous" Activity of Nerve Centers. Many nerve centers isolated from incoming sensory messages exhibit activity. In general, integrative centers such as the cerebrum, midbrain nuclei, and the central ganglia of annelids and arthropods show spontaneity more than do distributing centers such as sympathetic ganglia and sensory nuclei. There is no final clear-cut proof that any mammalian nerve center is spontaneously active, independent of sensory stimulation or reverberating chains, although synchronized rhythms have been recorded from the spinal cord of curarized cats, ${ }^{57}$ and activity is seen in isolated bits of cerebellum (Snider, personal communication). Probably 
most nerve cells have the ability to discharge without stimulation by other neurones if the chemical environment is suitable. The "spontaneously" active cells can be considered as relaxation oscillators where a continuous source of energy, e.g., oxidative system, stores up potential to a critical value and then the cell membrane breaks down. In this sense such activity is not spontaneous but rather a response to the chemical environment. Nerrous pacemakers in hearts have been discussed above (Ch. 15). A classification of nervous rhythms is given by Bullock. ii. i?

FROG

a

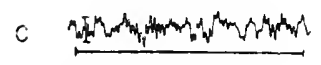

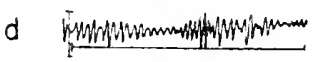

e

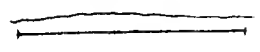

A

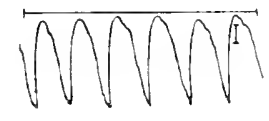

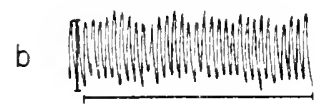

MAN

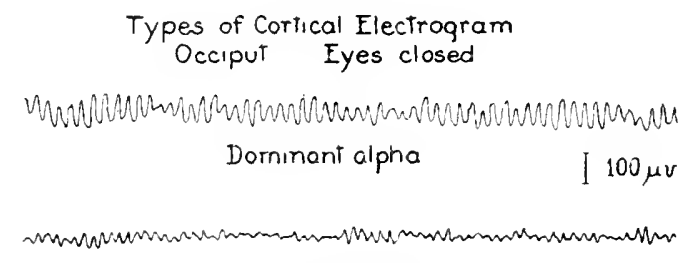

Subdominant alpha
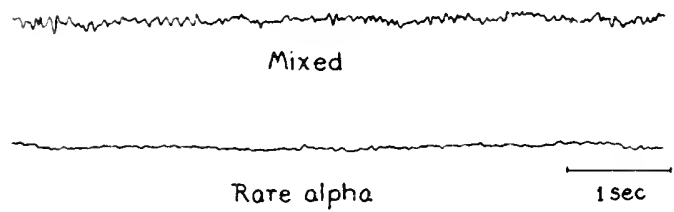

$B$

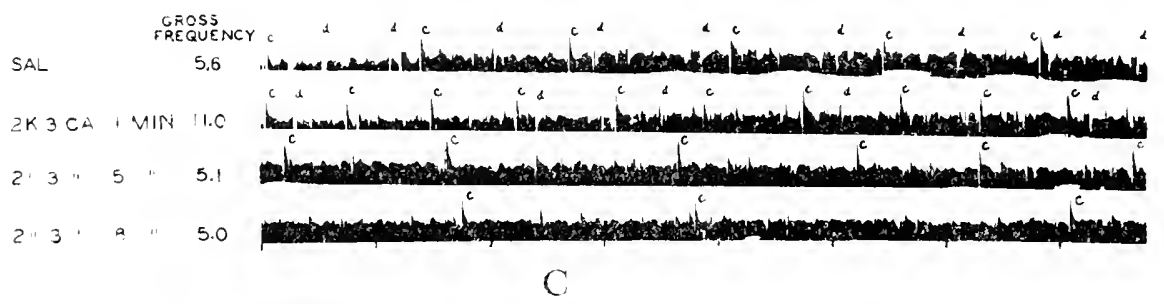

Fig. 30+. Types of rhytlmic activity in central nervous systems: A, Synchronized spontaneous rhythm from isolated olfactory bulbs of irog: $a$, freshly isolated bulb; $b$, small isolated bit of bulb; $c$, after $30 \mathrm{~min}$. in Ringer; $d$, single separated bulb; $e$, control on optic lube some time after isolation. Time, horizontal line-1 sec. From Libet and Gerard. ${ }^{25} B$, Electroencephalograms of different inen recorded from skin orer occipital lobes with eyes closed. From Davis and Davis."1" C, Spontaneuus activity in isolated abdoninal ganglion of crayfish, showing asynchronous rhythmis in several designated neurones. Increase in potassium content of saline by two times and in calcium content by three times causes initially an increase and later decrease in frequency. Letters identify imnulses in individual neurnnes. From Prosser.

Asynchronous Spontaneons Actuit). In some nerve centers each neurone is independent of other ncurones, although a given cell may discharge more or less rhythmically, difterent neurones having different frequencies. Such asynchronous activity is found in ganglionic nervous systems of anne 
lids and arthropods ${ }^{329},{ }^{332}$ (Fig. 304, C). The spontancous impulses find their way out to body muscles and are tonic in character; incoming messages are superimposed on the spontaneous background (Fig. 304). The spontaneous activity in the different units originates in the non-axon portion of the neurone, as is shown by the cutting of interganglionic commissures.

Synchronous Spontaneous Rhythms. In most nerve centers, however, there is some synchrony among the different neurones. A synchronized rhythm may be a coordinated periodic discharge of many units, each unit being active once for each wave, as may hold for "brain waves." Or the rhythm may be slower, each unit discharging several times for each wave, as in the ganglionic pacemakers of arthropod hearts or in respiratory centers.

The forebrain of fish, ${ }^{10}$ of frogs (Fig. 304, A), ${ }^{255}$ and of mammals, ${ }^{11}$ all show rhythmic waves of much longer duration than axon spikes. Just what is the "unit discharge" in a wave is not clear. Probably each cell depolarization lasts much longer than an axon spike but not so long as a full brain wave. Gerard ${ }^{16: 3}$ has suggested that the brain wave represents electrical oscillations of soma (non-axon) potentials rather than propagated discharges. The cortical rhythm in sereral mammals is predominantly $10 / \mathrm{sec}$, with faster and slower components superposed. However, this rhythm can be broken up into short-duration components, can be replaced by irregular asynchronous activity, and can change in frequency and pattern as a result of mental activity, sensory stimulation, or the influence of various salts and drugs.

Electrical activity, as shown by the electroencephalogram (EEG), remains in monkeys after removal of the cortex, but is profoundly altered by lesions to basal ganglia and is abolished if both thalamus and hypothalamus are removed. ${ }^{236}$

Synchronized rhythms resembling vertebrate brain waves have been seen occasionally in invertebrates, as in ganglia of the slug, Ariolimax. ${ }^{77}$ The optic lobes of the brain of Dytiscus, when the eyes are illuminated, show a rhythm of 20-40 waves per second, whereas in darkness a synchronized rhythm of 7-10 per sec. appears. ${ }^{7}$

The slow rhythms in which each neurone clearly discharges several times for each wave are best illustrated in respiratory and cardiac pacemakers. Isolated nerve cords of some insects show rhythmic discharges corresponding to breathing rate; each axon discharges several times during each respiratory burst. Similar repetitive activity is found in single units of the mammalian respiratory center, as indicated in phrenic nerve axons or muscle units. ${ }^{165}, 324$ In the Limulus heart ganglion each unit usually discharges several times per heart beat (Fig. 206, Ch. 15). It is possible to convert the synchronous rhythm into continuous asynchronous activity by alterations in salt balance. ${ }^{335}$

Synchronizution. The ease with which synchronous waves can be converted to asynchronous activity suggests that synchrony is a pattern imposed on a more or less continuous background. Two suggested mechanisms of synchronization may be mentioned:

1. Neurones may be caused to fire together by slow electrical waves, catelectrotonically. In the Limulus heart ganglion each large pacemaker cell appears to give rise to a slow wave of negativity which tends to coordinate 
the discharge of the smaller motor cells. ${ }^{188,335}$ When certain nerves are cut (crustacean nerves), or when one cut end from each of many types of nerve is dipped into a citrate solution, the injury sets up activity which becomes synchronized, possibly by electrical means. ${ }^{6}$ Electrical polarization of pacemaker neurones in the Limulus cardiac ganglion increases the synchronization and frequency of discharge. ${ }^{83}$

2. Circus conduction in a closed ring with side-branching neurones can maintain a synchronized rhythm (Fig. 302, C). Interruption of subcortical tracts in mammals alters the brain-wave synchrony. Similarly the respiratory center is composed of several centers, and interconnections among these are essential for synchronized respiratory discharge. ${ }^{324}$

The significance of "spontaneous" activity in central nervous systems is not clear. It may have a motor effect, although muscle tone is largely reflex. More probably spontaneity represents a state of "tone" or high excitability in the central neurones.

\section{NERVE NETS}

In the preceding discussion of the properties of synaptic conduction many examples were chosen from coelenterate nerve nets. It is thus evident that coordinative nervous functions evolved very early. Nerve nets, or diffuse nervous systems, have been best studied functionally in coelenterates, both polyps and medusae, and in ctenophores. Early histologists ${ }^{43}, 182$ pictured the nerve net as anastomosing fibers of multipolar and bipolar neurones. Evidence ${ }^{55,435}$ from vital staining shows that the fibers do not fuse; the junctions are not structurally polarized but the nerve net is probably not a syncytium.

Conduction in a coelenterate nerve net proceeds freely in all directions; impulses pass around corners and across narrow bridges. Further, a contractile wave dies out with distance of conduction in some cases, and this has been cited as evidence that nerve net conduction is decremental. Actually, the picture of a non-polarized diffuse conducting system is too simple for all the facts. There is condensation of fibers into nerve trunks in sea anemones and in medusae. Conduction in some trunks is fast, also speed of conduction in the subepidermal net in anemones varies greatly in different regions. ${ }^{312}$ Thus definite conduction pathways serve specific functions, even in coelenterate nervous systems.

Nerve nets also show facilitation. Repeated impulses are needed to produce threshold excitation (Fig. 227, Ch. 16) in anemones, ${ }^{311}$ and responsiveness is enhanced after a volley in medusae. ${ }^{74}$ The strength and extent of a contractile response depends on the number of impulses delivered to a given region of the nerve net. The decremental appearance of conduction in a nerve net can be explained in terms of decreasing facilitation at junctions, as a wave spreads out from its point of origin. Facilitation may sometimes lead to supernumerary discharges, a sort of after-discharge.

Two waves of action in a nerve net cancel when they meet, whether indicated by blocking of contraction ${ }^{285}$ or of luminescence ${ }^{294}$ (see p. 805), probably because of refractoriness. In a jellyfish ring, ${ }^{240}$ if the absolute refractory period of the nerve elements $\left(0.25 \mathrm{sec}\right.$. at $24.5^{\circ}, 0.8-0.62 \mathrm{sec}$. at $15^{\circ}$ ) exceeded the circulation time, the wave stopped. Cancellation of a wave by mechanical pressure raises the possibility that some of the net con- 
duction is reflex in nature; i.e., that contracting muscles re-excite conducting fibers. Reciprocal contraction of circular and radial muscles does occur. ${ }^{54}$ The possibility of such reflex arcs in nerve net systems seems not to have been explored.

\section{PERIPHERAL VERSUS CENTRAL CONTROL OF LOCOMOTION}

A subepidermal nerve network exists in many animal groups-echinoderms, balanoglossids, annelids, and molluscs. Jordan ${ }^{230}$ has called those animals in which behavior is under peripheral control the "hohlorganartige Tiere." Jordan and his students think of the peripheral network in invertebrates as analogous to autonomic ganglia, centers of distribution of certain centrally originating messages. To what extent can the peripheral system act independently? It is appropriate to compare locomotion in many invertebrates with movement of the mammalian intestine, in which the nerve network is essential for coordinated movements, even though the muscle may be capable of spontaneous movement. The subepidermal nervous system and the central nervous system may be in competition with respect to locomotor control. The competition between peripheral and central nervous components is of phylogenetic interest. In general, in more sluggish animals, the peripheral system is more important, and in more active forms central reflexes take over locomotor reactions. Even in animals where locomotion is entirely centrally controlled, visceral plexi remain autonomous, e.g., the myenteric plexus of the vertebrate intestine and the stomatogastric system of annelids and arthropods.

The peripheral portion of the nervous system is more important for locomotion in echinoderms and in balanoglossids than in any of the worms, molluscs, or arthropods. The nervous system of echinoderms consists of a central oral ring from which the radiating ambulacral nerves originate. These radial nerve trunks contain cell bodies and connect by branches to the peripheral network which innervates tube feet, spines, etc. ${ }^{385}$ The peripheral network contains ganglion cells, in Echini in rings at the base of large spines. The peripheral nerve network can conduct impulses independently of the ring and radial nerves. Jennings ${ }^{224}$ cut through a radial nerve of a starfish and found that a prick at the tip of the injured ray caused pedicellariae of other rays to rise. In a piece of the dorsal body wall of a starfish, stimuli in any part excite all of the pedicellariae on the piece. If one arm of an ophiuroid is placed under tension, excitation of the ring goes to the stretched arm rather than to other arms. ${ }^{416}$ Similar observations were made with fragments of sea urchin shells, in which spine movement in response to $\mathrm{NaCl}$ stimulation was studied. ${ }^{241}$ A piece of paper soaked in $\mathrm{NaCl}$ caused a nearby spine to bend toward the stimulus; if a cut was made between the paper and the spine, there was no response. If one spine was pulled, a nearby one was stimulated to bend; if a cut was then made between the two, the excitation still spread from one to the other, provided a third spine was at the end of the cut. If a spine was held so that it could not move, waves were not conducted past it. These results indicate that the ganglion cells at the base of each spine constitute local reflex centers, and that the peripheral system is not a continuous network.

The starfish central ring has a directive function. A starfish with radial 
nerres cut eventually rights itself but shows poor coordination. ${ }^{99}$ If all elements of the ring are removed, an isolated arm travels toward its base; if a bit of the ring remains, the arm moves toward its distal end; if the ring is cut in two places, the starfish pulls itself in two. Ophiuroids show no fixed central control but a "plastic reciprocal relation between peripheral conditions and central functioning". ${ }^{246}$ In two monographs Smith ${ }^{385,} 386$ has described the interrelations between peripheral plexi, and radial and ring nerves as a series of reflex arcs. In general, the central ring of echinoderms is directive, the radial nerves are necessary for true locomotion and righting, but alone the peripheral system can permit a certain amount of coordination between pedicellariae, spines, etc.

The nervous system of balanoglossids (Enteropneusta) consists of a subepidermal plexus, together with dorsal and ventral cords and collar connectives. ${ }^{73}$ Experiments in which the cords were cut showed that the subepidermal network can conduct both circularly and longitudinally but conducts more readily circularly. Conduction in the proboscis is entirely by way of the subepidermal network. The dorsal, ventral, and collar trunks seem to be essentially conducting cords and show little integrative activity.

In tunicates a single ganglion lies between the two siphons. Experimental work has been done largely on Ciona and Ascidia, in which a peripheral nerve net has not been adequately demonstrated. When the ganglion is removed or cut transversely, intersiphonal reflexes are abolished.111,186, 239 Strong protective reflexes are also abolished. Local responses of a single siphon and of the body to mechanical stimuli remain, but reaction time is increased and excitability diminished. Opinions are equally divided as to whether tonus is increased or decreased by extirpation of the ganglion. ${ }^{337} \mathrm{~A}$ contraction wave was conducted around a cut which slit a siphon. ${ }^{11}$ It seems certain that a direct connection exists from epidermal receptors to underlying muscles in ascidians, but conduction and tonus properties of the muscle need elucidation.

Among higher chordates peripheral nerve networks persist only for coordination of smooth muscle movement, particularly in the gut. On the other side of the phylogenetic tree (worms, molluscs, arthropods), peripheral nets are less important in controlling locomotion than on the echinoderm-chordate side.

Among flatworms dependence on central neurones is striking, in view of the primitiveness of these worms in other respects. In free-living flatworms the nerve trunks ( 2 to 8 in number) run posteriorly from the brain and contain nerve cells which are not grouped into ganglia. As long as a portion of one of these nerve cords is left in a piece of a planarian, spontaneous movement and some coordinated responses persist. ${ }^{36} \mathrm{~A}$ bit of remaining nerve cord is necessary also in nemerteans. ${ }^{136}$ The only example of independent function of the peripheral system is in the planarian proboscis where, if the two connections to the central nerve trunks are severed, the proboscis autoamputates and shows food-seeking reactions. ${ }^{237}$

Annelids, being tubular and containing a movable coelomic fluid, might well be expected to show peripheral coordination. In the earthworm a subepidermal plexus of branching fibers and scattered nerve cells lies outside the circular muscles; each central ganglion gives rise to three pairs of seg- 
mental nerves, containing both sensory and motor fibers, which connect with the subepidermal plexus. Can this peripheral network conduct impulses along the body? Can a strip of earthworm body wall move spontaneously? Are epidermal sense cells connected through the peripheral network directly to muscles?

It has long been known that if the ventral nerve eord is cut or is removed from the earthworm for several segments, peristaltic waves still pass from one end of the worm to the other. Peristaltic transmission occurs if the two pieces of a transected worm are connected by a thread or when a few seg. ments are anesthetized. ${ }^{14:}$ if part of the nerve cord is removed, a wave of mucus secretion fails to pass from normal to denervated segments. ${ }^{101}$ If the nerve cord is removed from more than three segments and those segments are firmly pinned down so that no pull can be exerted bevond them, no peristaltic wave passes, but if fewer than three segments are denervated and these segments pinned down, a wave is conducted. ${ }^{3: 3}$ Action potentials in the segmental nerves show that each nerve serves three segments, the different nerves serving overlapping fields which are fairly discrete. When one nerve is stimulated there is contraction in its own segment and, to a less extent, in adjacent segments. ${ }^{330}$ When a T-shaped cut is made in the lateral body wall of an earthworm, transmission of a contraction wave occurs "around the corner" if the nerve cord is intact, but not when the nerve cord is lacking. 219

Spontaneous contractions have been seen in strips of earthworm body wall lacking the ventral nerve cord and have been denied."33 Janzen ${ }^{21: 9}$ obtained spontaneous periodic contractions in strips of earthworm body wall which had been narcotized during dissection, but not in non-narcotized strips; the contractions were usually local but could be propagated; the two muscle layers could contract separately. Hatai ${ }^{1 \mathrm{n}}$ reported spontaneous activity in dorsal strips from the posterior end of Allolobophora, but not from the anterior portion, and not at all from Perichaeta. When dorsal pieces are isolated in Lumbricus the muscles of segments anterior to and including the clitellum often contract at the edges for hours after excision, this probably being a response of the muscle to injury; these excised segments fail to show spontaneous movements. Postclitellar strips relax but are not spontaneously active. Dorsal strips consisting of about twenty extreme posterior segments, however, sometimes show spontaneous contractions. ${ }^{337}$ It appears then that posterior regions of the dorsal body wall of earthworms are capable of spontaneous movement and that contractions can occur in conducted waves, but there is no reason to implicate the subepidermal plexus. Conduction may be from muscle cell to cell as in vertebrate smooth muscle. The bodr wall muscle may, like the smooth muscle of the mammalian intestine, be capable of some intrinsic movement.

Janzen ${ }^{219}$ found that tactile stimulation of dorsal body strips elicited contractions, usually local but sometimes propagated; spontaneously active strips showed the best responses. The body wall muscle may be responding to direct stimulation. However, in a few of the preparations from the posterior end of Lumbricus a clear-cut response to bright illumination was obtained after dark-adaptation. ${ }^{337}$ It is probable that in the response to illumination and to very light touch there is some direct connection between sense end- 
ings and muscle. However, the weakness and the local nature of the response and its virtual absence in anterior segments show that it can hardly be of importance in behavior.

The preceding evidence indicates that the subepidermal plexus of the earthworm is not a true nerve net for general conduction, that although there may be connections through it from sense cells to muscle these cannot be of importance in behavior, and that there is no indication that the network plays any part in initiating spontaneous movement of the body wall. The peripheral plexus is probably a sensory relay area since there are many more epidermal and muscle sense cells than there are segmental nerve fibers. ${ }^{330}$ It is possible that among more primitive annelids the connection through the peripheral plexus from sense organs to muscle was important but that in earthworms the central nerve cord has now taken over complete control. Certainly amung polychaetes (e.g., Arenicola ${ }^{232}$ ) there is no evidence of any conduction of peristaltic waves except by way of the nerve cord.

In gastropod molluscs a subepidermal plexus has been described, particularly in the foot. ${ }^{43}, 45$ Small pieces of the foot of a slug show peristaltic movement. ${ }^{45}$ Jordan ${ }^{229}$ removed the paired pedal ganglion from Aplysia and noted a marked increase in tonus of the "wings"; this was arbitrarily attributed to a release of the peripheral net from inhibition by the pedal ganglion. When ganglionic connections to the "wings" of Aplysia were cut ${ }^{201}$ and one of the miotor nerves was stimulated, there was an immediate contraction of the area served by that nerve, followed by a peristaltic wave which spread out from this area. This wave was said to be reflex in nature, with the peripheral network conducting the impulses. Excised labial palps of Anodonta show autonomous responses to stimulation by light, touch, and chemicals. ${ }^{97}$ These palps must co tain direct connection through the peripheral nervous network from sense organs to muscle. In general, molluscan subepidermal networks relay messages from central ganglia but are incapable of any independent locomotor control. There is little doubt that normal coordinated locomotion of the foot requires the presence of the pedal ganglia. More knowledge of the conduction properties of the muscle is needed before functional importance can be assigned to the peripheral network.

The use of a peripheral nerve network in locomotion appears with slight success in the echinoderm-chordate line, and with still less success in the annelid-arthropod-mollusc line. In the higher groups-vertebrates, polychaetes, cephalopod molluscs, and arthropods-there is no evidence of a peripheral network for locomotor control. Yet in most of these a nerve network is retained for regulation of movement in the digestive system. Central nervous reflexes have become dominant patterns in animal behavior.

\section{GANGLIONIC FUNCTION IN INVERTEBRATES}

Locomotion is under reflex control in annelids, arthropods, molluscs, and chordates, but ganglionic reflexes in invertebrates have not been subjected to such complete analysis as spinal reflexes in mammals.

Annelids. A number of segments of the ventral nerve cord of an annelid, isolated from the body musculature, can conduct a peristaltic wave to the region beyond. Normally, however, the wave is reinforced by the segmental reflexes. 
Collier ${ }^{100}$ suspended 20-40 intact segments of earthworms in a saline bath and found no peristalsis if the worms were carefully balanced. If, however, a slight stretch (0.1-1.0 gm. on the recording lever) or a tactile stimulus was applied, peristalsis resulted (Fig. 305). Tension and touch elicited peristalsis only if the preparation was normally innervated. When the tactile receptors are anesthetized, peristalsis can be intiated by tension.

A tension-induced peristaltic reflex can be inhibited by strong tactile stimulation, e.g., by touching the anterior end during a tension wave ${ }^{100}$ (Fig. 305). Whether the blocking effect of an antiperistaltic wave is due to central inhibition or to refractoriness somewhere in the reflex chain is not clear. Maintained tension sets up rhythmic contractions at a maximum frequency of one every two seconds; the frequency increases to a maximum as the applied weight is increased. If the weight is applied for only 0.5 second, a rhythmic response occurs, a sort of after-discharge which may last as long as 3 minutes. In the leech not only tension and contact but also exteroceptive stimuli from the sucker are capable of eliciting peristaltic reflexes. ${ }^{178}$ The leech nerve cord shows asynchronous spontaneous electrical activity when isolated, and a

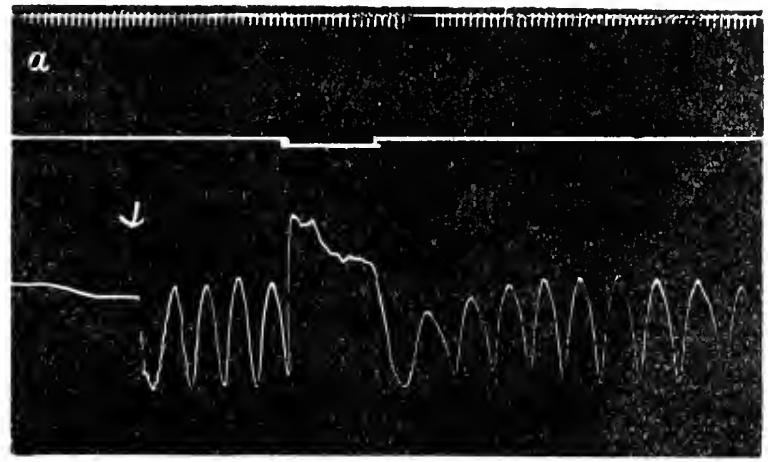

Fig. 305. Reflex rhythmic contractions in an earthworm in response to tension applied at arrow and inhibited by tactile stimulation of dorsal surface at time indicated by signal magnet record above response. From Collier. ${ }^{300}$

rhythm corresponding to the contractile rhythm when connected to an active piece of the body wall. Peristalsis can be started in an earthworm ${ }^{175}$ by electrical polarization with the anterior end positive and peristalsis can be inhibited by reverse polarization.

Peristaltic locomotion in the polychaete Arenicola is likewise controlled by segmental reflexes, and stimuli at the anterior and posterior ends cause opposite parapodial movements. ${ }^{232}$ Motion pictures of Nereis in locomotion ${ }^{17:}$ show patterns of movement successively involving clumps of 4 to 8 parapodia. Accompanying the wave of parapodial beat the longitudinal muscles on the same side contract while those on the opposite side relax. Thus the Nereis crawls in the direction in which the wave travels over the body and not, as does the earthworm, in the opposite direction.

The earthworm peristaltic reflex is segmental, bi-segmental or tri-segmental in character, with reciprocal innervation of longitudinal and circular muscles. Circular muscles contract while longitudinal muscles relax, and the diameter of the animal is reduced. Then longitudinal muscles contract, 
shortening the worm. Strychnine stimulates the motor system for both sorts of muscle, so that under strychnine the worm shortens and also constricts; ${ }^{242}$ normally one group of motoneurones is inhibited while the antagonists are excited. Janzen ${ }^{219}$ described correlative reflexes controlling the setae. The locomotor reflexes have both homolateral and crossed nervous components.

The rhythmic contraction waves of peristaltic locomotion in annelids require chain reflexes involving tactile or muscle or sucker receptors and antagonistic central arcs. What recovery mechanisms are so slow as to determine the frequency of contraction under continuous stimulation is not clear, but they may well be in the muscle rather than in the central nervous system.

Arthropods. Arthropod locomotion depends on central conduction and on segmental reflexes which show more complex connections than in annelids. In insect larvae such as Lucanus, peristalsis depends on the integrity of the nerve cord. ${ }^{37.3}$ When a segmental nerve is cut, its segment is paralyzed but conducts a wave to succeeding segments if the cord remains intact. Tonus is lost in skeletal muscles of a silkworm if ganglia are removed. ${ }^{299}$ In some lepidopteran larvae (noctuids), each segmental ganglion serves its own segment and the one ahead. ${ }^{207}$

The reflex control of insect walking and flight and of breathing is very precise. In 1899 Pompilian noted some automatism of thoracic ganglia for leg movements in Dytiscus. Flight can be initiated reflexly by removing the legs from support; i.e., tarsal contact inhibits flight. ${ }^{1+6}$ Leg muscles of scorpions, crayfish, and some beetles show tonic low potential discharges at $30-40 / \mathrm{sec}$. during rest, and muscle potentials of higher amplitude and frequency during activity. 351 When one or two segments-ganglion and limbs-are isolated, reciprocal alternate contractions of flexors and extensors in successive seginents occur. Pringle ${ }^{325}$ made similar observations on the cockroach and noted crossed inhibition. A variety of operations on the nervous system of the walking stick Dixippus show that cach leg by its motion has a reflex stimulating effect on neighboring legs. ${ }^{69}$

When the normal sequence of leg movement is upset by removing legs from various spiders, crabs, beetles, etc., changes in coordination occur at once. ${ }^{4,}, 207$ Legs which previously alternated now work together, pedipalps may function in locomotion, swimming is carried out by other legs than it is normally. Ilolding a leg is not equivalent to removing it. The changes occur too fast for learning and must represent pre-existing pathways and an adaptive property of the ganglia. Ten Cate ${ }^{405}$ obtained alterations in coordination in a cricket if he removed a foot only. He concluded that the normal sequence and rhythm is set up reflexly. However, the new response does occur over central connections already established. Not only can changes in leg rhythm be brought about by amputation, but they also occur with normal changes in gait. The mantis leg order ${ }^{355}$ for example, when the insect is quictly walking, is $\mathrm{L}$ (left) $3, \mathrm{~L} 2, \mathrm{R}$ (right) $3, \mathrm{R} 2$; when it is excited and walling last, the rhythm is $\mathrm{L} 2+\mathrm{R} 2, \mathrm{~L} 3+\mathrm{R} 3$; when climbing, $\mathrm{L} 3+\mathrm{R} 1, \mathrm{~L} 2+\mathrm{R} \mathrm{3,L} \mathrm{l}+\mathrm{R} 2$. In some insects the order of leg movement depends on the kind of surface; i.e., the order is determined by proprioceptive reflexes. $=07$ 
Prosser ${ }^{331}$ recorded action potentials from various segmental nerves and from the central nerve cord of the crayfish. By stimulating different appendages and making appropriate cuts, he obtained a functional map of the nerve cord. The available connections are multiple; which ones are used in a given behavior pattern must depend on the strength of stimulus and on the case of passage through a particular reflex arc.

Locomotor rhythms in insects and crustacea, as in annclids, are reflex in nature, and a variety of patterns of locomotion may be used, depending on external requirements.

Molluscs. In gastropod and pelecypod molluscs, locomotor reflexes are mediated by three pairs of ganglia-cerebral, pedal, and visceral.

Pavlov in 1885 experimented with the mussel Anodonta. Spontaneous activity of the font stopped when the visceral ganglion was removed, but tonus could still be maintained in the adductor muscles after they were denervated. The posterior adductor muscle received motor (exciting) impulses from the visceral ganglion, the anterior adductor from the cerebral ganglion; both adductors received inhibitory fibers from the cerebral ganglion. In the razorshell clam Ensis ${ }^{122}$ local stimulation of the foot failed to elicit general responses if the sensory connections to the cerebral ganglia were eliminated. Crossed responses go from one cerebral via the visceral back to the other cerebral ganglion. ${ }^{155}$ In Mytilus ${ }^{438}$ all of the ganglia act somewhat independently; e.g., if the cerebral ganglion is removed the foot still spins and creeps, by pedal control. If the visceral ganglion is removed, opening and closing reactions to changes in freshness of water are lost. All of these observations argue for fairly restricted control of local areas by specific ganglia in pelecypods.

In gastropods the pedal ganglion gives off a series of nerves which appear to discharge successively to elicit peristalsis along the foot. ${ }^{45}$ When the pedal ganglion of Aplysia was removed ${ }^{2: 29}$ the foot showed more resistance to stretch (tonus) and contractions occurred, particularly at the foot margins. When the cerebral ganglion was removed there was increased locomotor activity and increased excitability of the "wings" (foot). It has been suggested therefore, that the cerebral ganglion inhibits locomotor functions of the pedal ganglion and that the pedal ganglion inhibits tonic mechanisms in the foot. Each nerve from the "wings" contains sensory fibers which reflexly through the pedal ganglion elicit contractions in certain areas of the opposite side of the foot. The cerebral ganglion is not necessary for this response. If the interpedal commissure is cut, only the homolateral responses persist. In Helix, foot potentials indicate that the cerebral ganglion inhibits motor cells of the pedal ganglion. ${ }^{190}$

Ganglionic reflexes control the muscles and chromatophores of the mantle and fin of cephalopods. The mantle nerve goes from the brain mass to the mantle or stellate ganglion and from this the stellar nerves pass to the mantle. The mantle ganglia maintain tonus of the muscles and chromatophores of the mantle. ${ }^{152}$ Stimulation of the mantlc nerve at low intensity inhibits the stellate ganglion, at higher intensities excites it. Ganglionic delay of 10 msec. has been reported and summation indicated. Fatigue of a stellar nerve did not affect the mantle nerve. When the mantle nerve was cut, a weak reflex response could be elicited through the stellate ganglion alone. Reflex 
contraction can be elicited over either of two pathways from the mantle, via the brain mass (traversing the stellate ganglion for both afferent and efferent fibers), or via the stellate ganglion alone. ${ }^{152,400}$

Molluscs, in general, have well developed local reflex centers which may show some interaction but which have relatively restricted control of particular sensory and motor areas. In this respect molluscs are even more high. ly specialized than the segmental arthropods.

\section{CEPHALIC DOMINANCE IN INVERTEBRATES}

A step in complexity of nervous function beyond local or segmental reflexes is the increasing importance of centers near the anterior end of the animal. This importance depends first on the concentration of sense organs at the front end of the animal and second on coordinative functions of the "brain" ganglia.

Flatworms. In the free-living flatworms the brain is associated with eyes and other sense organs. In the polyclad Thysanozoon the brain is needed for spontaneous locomotion, ${ }^{260}$ and after removal of the brain or head the polyclad Yungia is inactive and less sensitive than it is normally but it can be stimulated mechanically or chemically to perform swimming movements. ${ }^{295}$ In Yungia, some hours after the operation, spontaneous and coordinated but undirected movement occurs. In some marine polyclads swimming stops but ciliary action persists if the brain is removed or split; ${ }^{310}$ the rippling movement (ataxic locomotion) continues, but extension, placing, and release (ditaxic locomotion) and peristalsis stop since these require conduction from the brain back over lateral cords. Righting appears to require the brain in the polyclad Planocera but not in the triclad Planaria. ${ }^{200}$ In Planaria, locomotion and reflex extension of the pharynx and swallowing continue after removal of the head, but normal behavior to food reappears only after the brain regenerates. ${ }^{36}$

In cestodes, a wave of contraction progresses backward across a cut through lateral cords, being conducted reflexly by tension applied behind the cut. . $^{3+6}$ Cutting one lateral cord results in circus movements in those species of nemerteans in which transverse connections between lateral cords are incomplete, but not where the cords are connected. ${ }^{150}$ In summary, the flatworm brain relays sensory messages and coordinates motor responses, there being no other ganglia; its directive function is, therefore, very important.

Annelids. An earthworm has sensory cells of various types-tactile, chemical, and light receptors, scattered throughout the epidermis, but most concentrated in the prostomium and anterior segments. Only the sense cells which are sensitive to dryness seem restricted to the prostomium. 320 Nereis (Neanthes), on the other hand, has fewer epidermal receptors; its effective chemoreceptors are on palps and tentacles, and instead of scattered photosensitive cells it has several pairs of eyes on its head. After the brain is removed from an earthworm the anterior segments are lifted upward, it crawls normally, appears restless and active, it can right itself, can copulate, eat, and burrows in half an hour as compared with a normal time of one to two minutes. $2.3,3.4 \times$ After the brain is removed from a nereid worm, it no longer feeds, does not burrow, is over-active and has lost its light sensitivity and most of its chemical sensitivity. ${ }^{260}, 2 \times 3$ When the subesophageal ganglion is 
removed from either type of worm there is no burrowing and the worms are very quiet. The brain, then, is a sensory center, and it normally has an inhibitory or restraining control over the motor centers in the subesophageal ganglion.

An example of integrative cephalic dominance which has a simple morphological basis is found in the responses of an earthworm to light. An earth-

A
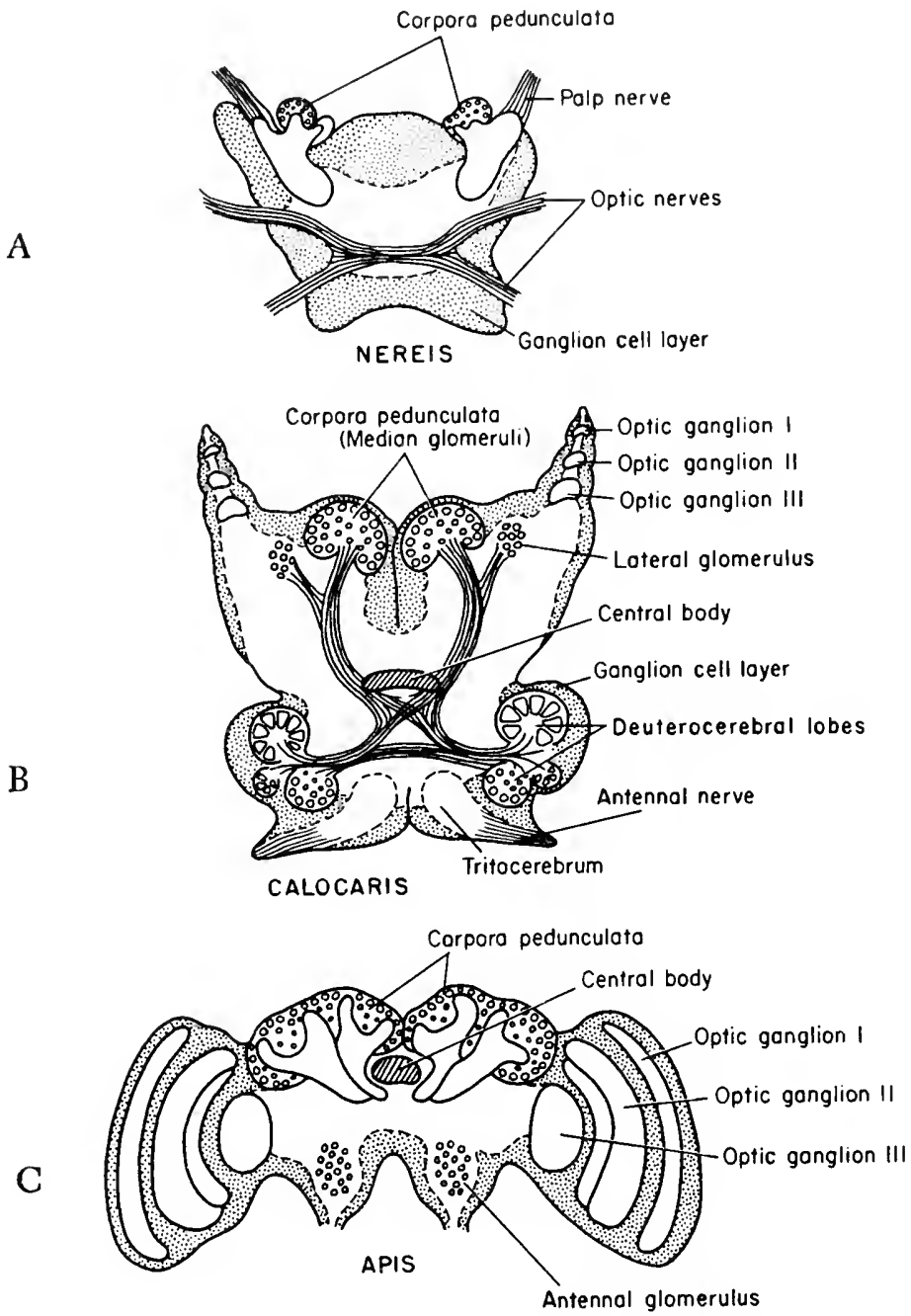

Fig. 306. Sections of brains (supraesophageal ganglia) of several invertebrate animals. $A$, Nereis; $B$, the crustacean Calocaris; $C$, a worker honeybee. From Hanström. ${ }^{183}$

worm normally responds negatively to lateral illumination. When the brain is removed the direction of its response is reversed. ${ }^{192}$ If the ventral cord is transected a few segments behind the subesophageal ganglion, the anterior tip turns away from the light, whereas the region behind the cut tends to bend toward the light. If one esophageal connective is cut, the worm tends to circus toward the normal side and when illuminated from the intact side 
turns toward the light, but when illuminated from the operated side it turns away from the light. ${ }^{327,213}$ Apparently the scattered photoreceptors set up a homolateral reflex in the ventral ganglia, a positive response to light; the tracts from the prostomium and first two segments. however, cross in the brain and cause a negative response. The brain normally dominates, but at very low light intensities a positive response may occur.

The brains of some polychaete worms are complex and have lobes corresponding to the specialized forebrain centers of arthropods. ${ }^{183}$ These lobes (corpora pedunculata) are best developed in free-swimming polychaetes which have cuticular eyes (Fig. 306, A); they do not occur among oligochaetes. An investigation of integrative functions of the polychaete brain should be very interesting.

Arthropods. There is much variation in complexity of the brain among different groups of arthropods. In general there are three regions: the protocerebrum, the deutocerebrum, and the tritocerebrum. The bulk of the lateral portions of the protocerebrum is devoted to vision centers which are directly connected to the eyes; the middle and anterior portions contain the association areas, the protocerebral bridge, the central body, and the large cellular corpora pedunculata (Fig. 306, B, C). There are no corpora pedunculata among some lower Crustacea. The deutocerebrum usually lies ventro-anteriorly and may contain large antennal centers as well as "olfactory" lobes. The tritocerebrum lies behind and customarily gives rise to certain nerves going to mouthparts as well as to the stomatogastric nerves; the tritocerebrum is continuous with the circumesophageal connectives.

In view of the complex instinctive behavior and limited but real modifiability of behavior in arthropods, especially among insects, an understanding of the function of different parts of the brain should be useful. Hanström ${ }^{183}$ has compared the relative portions of the brain mass occupied by different regions in various groups as in Table 78.

Since the days of Aristotle, the observation of behavior of arthropods from which parts of the body have been removed has been a favorite pastime of naturalists. Reviewers ${ }^{337}, 402$ have listed fifteen papers dealing with brain operations in crustaceans, sixteen on insects, and five on myriapods. The findings are roughly similar in all of these studies.

Removal of any part of the brain which receives tracts from any sense organ is equivalent to removing that sense organ. Immediately after removal of a sense organ or injury to the brain, there may be a shock reaction of gen. eral incoordination. Thereafter, specific behavioral deficiencies appear. Most arthropods can eventually compensate for the removal of one eye or of an antenna, whereas injury to the brain leaves a more permanent behavior de. ficiency. If both circumpharyngeal connectives are transected, reflexes of the head, such as antennary movements, remain; otherwise the effect is like total brain removal. Spontaneous directed locomotion ceases, although locomotion is possible under stimulation. Locomotion is less impaired by brain removal in some species (Carcinus and Eupagurus) than in others (Astacus). Mouthparts may move, but usually there is no coordinated feeding. Some animals can chew but cannot swallow after brain removal. Righting and leg reflexes are unimpaired. Usually there is extensive random activity of legs.

When one circumesophageal connective is cut, or one lateral half of the 
TABLE 78. RELATIVE PORTIONS OF BRAIN MASS OCCUPIED

BY DIFFERENT REGIONS (FROM HANSTRÖMI'ts.s)

\begin{tabular}{|c|c|c|c|}
\hline Region of Brain & Animal Group & Type of Eye & $\begin{array}{l}\text { Percent of Brain } \\
\text { Occupied by Region }\end{array}$ \\
\hline Vision centers & $\begin{array}{l}\text { Arachnoidea and } \\
\text { Myriapoda }\end{array}$ & $\begin{array}{l}\text { epithelial photorecep } \\
\text { tors and ocelli }\end{array}$ & $0.3-2.8$ \\
\hline Vision centers & Crustacea and Insecta & $\begin{array}{l}\text { rudimentary com- } \\
\text { pound eycs }\end{array}$ & 2.9-9.9 \\
\hline Vision centers & Crustacea and Insecta & $\begin{array}{l}\text { higher compound } \\
\text { eyes }\end{array}$ & $33.0-80.0$ \\
\hline Olfactory centers & Crustacea & & $10.0-30.0$ \\
\hline Olfactory centers & day-flying insects & & $1.3-4.4$ \\
\hline Olfactory centers & one night-flying insect & & 13.4 \\
\hline Olfactory centers & worker ant (Formica) & & 18.1 \\
\hline Central body & Arachnoidea & & $1.6-15$ \\
\hline Central body & Crustacea & & $0.2-0.5$ \\
\hline Central body & insect & & $0.2-0.6$ \\
\hline Corpora pedunculata & Limulus & & 78.7 \\
\hline Corpora pedunculata & Arachnoidea & & $2.0-49.0$ \\
\hline Corpora pedunculata & decapod Crustacea & & $10.0-30.0$ \\
\hline Corpora pedunculata & isopod Crustacea & & 0.0 \\
\hline Corpora pedunculata & $\begin{array}{l}\text { Odonata, Lepidoptera, } \\
\text { Diptera }\end{array}$ & & $2.0-9.6$ \\
\hline Corpora nedunculata & worker ant (Formica) & & 40.4 \\
\hline
\end{tabular}

brain destroyed, the arthropod circuses toward the intact side. Legs on the operated side tend to be flexed and low in tonus, so that the animal leans toward that side.

There are many reports that removal of the subesophageal ganglion or cutting behind this ganglion stops spontaneous activity. Local segmental reflexes, however, persist. The subesophageal ganglion is apparently the principal motor center, but the other thoracic and abdominal ganglia are capable of carrying out reflexes of locomotion, autotomy, and the like; these ganglia are normally regulated by the subesophageal ganglion.

The increased leg activity after brain removal suggests the release of the ventral motor centers from inhibition by the brain. When the brain is removed from dragon-fly larvae there is an increase in breathing frequency, $2 \times 2$. 119 whereas when the subesophageal ganglion is removed the breathing frequency (determined by posterior ganglia) is decreased. Death-feigning is a function of the nervous system as a whole rather than of any particular ganglion; in some insects and in spiders it is relatively unaffected by brain removal; in others, as in the spider Celaenia, ${ }^{353}$ there is no death-feigning after brain removal. In Ranatra, the death-feigning reaction is shortened after brain removal; the posterior half of the body with nerve-cord cut comes out of a feint sooner than the part of the body containing the brain. ${ }^{202}$ The most convincing evidence for inhibitory action of the brain on the ventral ganglia comes from electrical stimulation. Jordan 228 stopped circus movement in a crab by a weak electrical stimulation of a transected circumesophageal connective. Flexion of the abdomen of Palinurus and Homarus was elicited by tetanic stimulation of the abdominal nerve-cord; if during this stimulation the brain or circumesophageal connectives were also stimulated, the abdominal response was diminished. ${ }^{\mathbf{4 0 2}}$ 
The interaction between the brain and subesophageal ganglia is clearly shown by the work of Roeder on the praying mantis. ${ }^{355}$ When one side of the protocerebrum is removed the legs lose tone on the opposite side; the legs on the operated side are more active, and the leg sequence changes. Circusing is to the normal side. When both protocerebral lobes are destroyed, leg tonus is lost on both sides, and there is locomotor restlessness. The mantis walks straight ahead, fails to avoid objects, and shows no head motility or backward walking. When the protocerebrum is split there is decreased leg movement, high neck and prothoracic tonus, and active visual responses (following of moving objects). When the subesophageal ganglion is removed there is no locomotion except in response to strong stimulation. Roeder concludes that the subesophageal ganglion excites locomotor activity in thoracic ganglia, that the protocerebral ganglion has inhibitory centers controlling the irritability of the subesophageal locomotor center and excitatory centers maintaining tonus and activity in neck and prothoracic muscles; these protocerebral centers are homolateral, are inhibited contralaterally by each other, and are strengthened by homolateral sensory impulses. Sometimes in mating, after clasping, the female eats the male, head first, and copulation continues actively. If only the brain is removed there is no sexual activity, but if the subesophageal ganglion also is removed the male makes copulatory movements and the female will receive the male. ${ }^{35}+$ The copulatory center is in the last abdominal ganglion and this is normally inhibited by the subesophageal ganglion. Thus the eating of the head, including the subesophageal ganglion, by the female actually releases copulatory activity!

Some arthropods exhibit very complex behavior, some of which may require the presence of the brain. A brainless hermit crab will not reenter its shell unless it is helped by having the telson pushed in; ${ }^{401}$ the hermit crab without brain will grasp a new shell but fails to investigate it as a possible dwelling-place. In some arthropods (female Bombyx and Carcinus), mating occurs in brainless individuals, whereas in others (various butterflies) it does not.

There is ample evidence that some insects have capacity for learning. Experiments concerning the possible relation of parts of the brain to learning should be very profitable. The functions of the specific lobes of the brain can be partly inferred from their connections as shown in Table 78. Removal of the globuli (the cellular portion of the corpora pedunculata) in Carcinus causes the loss of certain responses to light, and of sidewise locomotion; the globuli are inhibitory centers of the brain. ${ }^{42}$ In the myriapod Lithobius $^{221}$ the protocerebrum inhibits the ventral motor centers and controls the sense of direction; the protocerebrum is an inhibitory center in the mantis. ${ }^{356}$

In summary, then, the brains of arthropods not only are connected with the most important sense organs of the body, but they also exert important integrative, particularly inhibitory, control of the ventral motor centers. It should be profitable to investigate localization of function within the arthropod brain and the mechanisms of interaction between the brain and ventral ganglia, and between the subesophageal and other ventral ganglia.

Cephalopods. Cephalic dominance has developed in a somewhat different manner in cephalopod molluscs. The brain and some thoracic ganglia of 
arthropods represent fusion of several ganglia. The cephalopod brain-mass represents fusion of paired cerebral, pedal, and visceral ganglia, and completely surrounds the esophagus. ${ }^{372 .}{ }^{+15}$ Paul Bert in 1867 was the first to show that much of the supraesophageal portion of the brain-mass was, like the association areas in the mammalian cerebral cortex, electrically incxcitable with respect to behavior. He also showed that a respiratory center exists in the subesophageal portion of the brain-mass. This respiratory center has regions for inspiration and expiration. ${ }^{415} \mathrm{~A}$ center in the subesophageal portion regulates chromatophores. The subesophageal portion of the brain-mass ${ }^{372}$ contains other local centers; the brachial and pedal ganglia controlling arms and tentacles, the pedal ganglion controlling funnel and eye-muscles, and the palliovisceral ganglion controlling mantle, fins, and viscera. In Octopus a center for pupillary closure is found in the subesophageal portion. . $^{2: 2}$

The supraesophageal portion ${ }^{372}$ contains: (1) Higher motor centers in the circumesophageal region-lobus basalis anterior, posterior, and lateralis, lobus pedunculi; stimulation of these lobes elicits movements of large groups of muscles, and unilateral extirpation results in circus movements which may be almost continuous. (2) Primary sensory centers such as the olfactory lobes and the optic lobes in the optic stalk; stimulation of the latter may result in chromatophore expansion and some mantle and fin movement. (3) The dorsally located verticalis complex of three lobes; electrical stimulation of this results in no motor responses. After removal of the entire verticalis complex Sepia can see, swim, steer properly, capture and eat prawns, etc., but fails to follow prawns as they disappear out of sight. Removal of part of the verticalis complex from Octopus hampered its ability to escape from a cage and from a pan of shallow water. ${ }^{\times 7}$ The verticalis complex, then, is an integrative portion of the cephalopod brain-mass.

\section{SPINAL REFLEX CONTROL OF VERTEBRATE LOCOMOTION}

In the more efficient, better coordinated invertebrates the peripheral portions of the nervous system have lost autonomy, and behavior depends on central nervous reflexes. In all classes of vertebrates locomotion is mediated by spinal reflexes. In embryonic development, mass resnonses of large muscle groups become differentiated into more precisely localized responses. In phylogenetic development the spinal cord is less autonomous as the control of locomotion is pushed cephalad. Concurrent with cephalad development of reflex centers there appears more antagonism between regions of the central nervous system, one region inhibiting another, usually cephalad regions inhibiting "lower" or caudal centers.

The basic organization of the spinal cord changes with the functions it must perform. ${ }^{15}$ In general, each segment of the cord receives two pairs of roots; the dorsal roots contain afferent (sensory) fibers, and a few efferent (motor) fibers, while the ventral roots contain efferent (motor) fibers, both somatic and visceral (autonomic), whose cell bodies are in the ventral horn of the cord. In Amphioxus there are very many uniform segments, dorsal and ventral roots emerging alternately. In cyclostomes also the dorsal and ventral roots alternate; they unite, forming mixed nerves in myxinoids but not in petromyzonts. From lower to higher classes of vertebrates there is a reduction in number of spinal segments and in the size of the caudal portion of 
the cord, and an increase in the number of spinal tracts to and from the brain.

Many afferent fibers cross or connect with arcuate interneurones. In cyclostomes there are ascending bundles of short fibers, and most spinal correlation can be carried out in a few segments; the principal descending fibers are of the reticular system. In elasmobranchs and teleosts ascending spinobulbar (medullary) and spino-mesencephalic tracts are present; in teleosts spino-cerebellar tracts and the sympathetic ganglion chains appear, and the

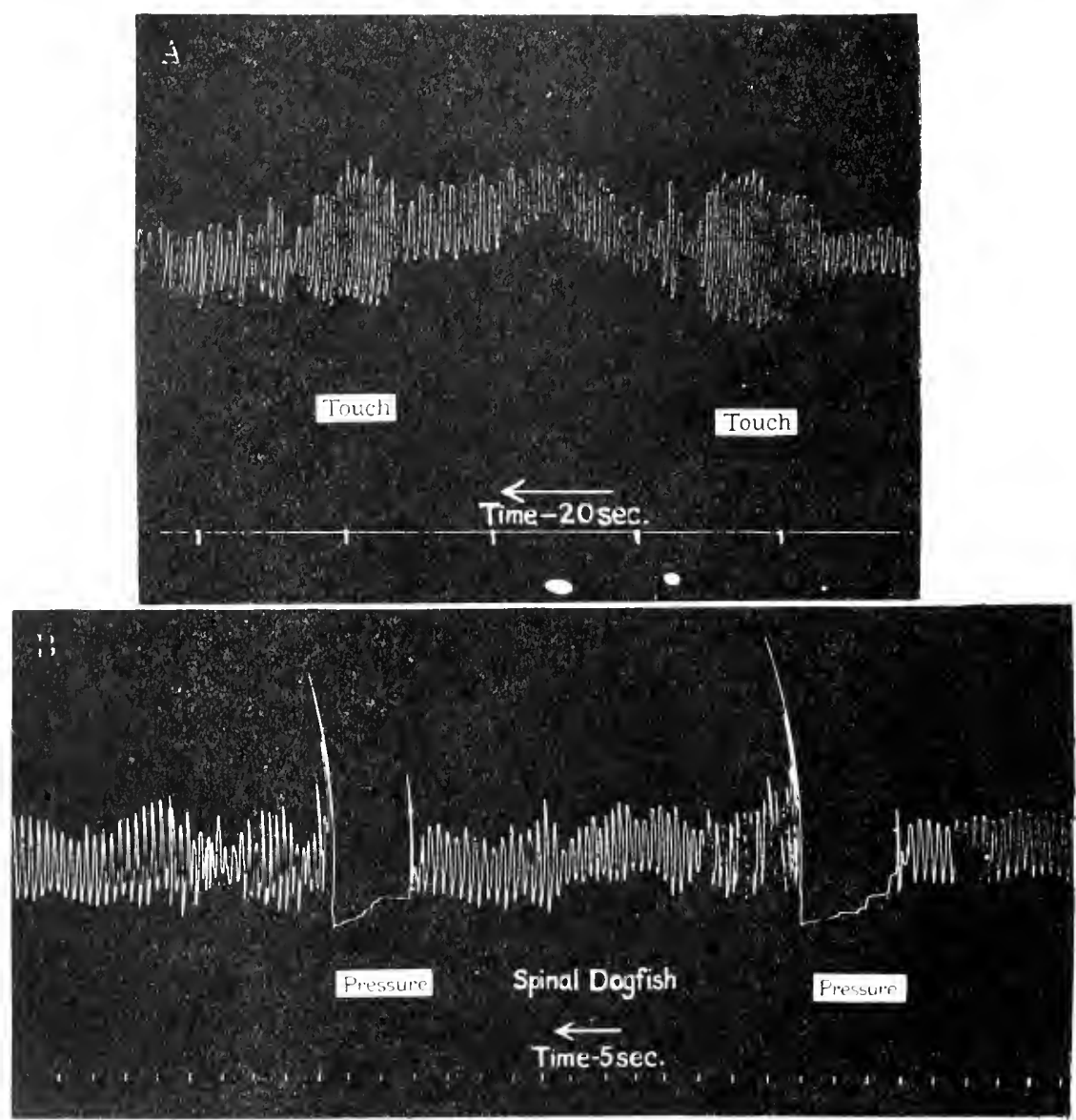

Fig. 307. A, Rhythmic undulations in dorsal fin of a spinal dogfish; rhythm increased when body teuched lightly behind point of transection. B. Rhythmic movement of dorsal fin in a spinal dogfish; brief struggle followed by inhibition when strong pressure was applied loy a clams to the body. From Gray and Sand. ${ }^{170}$

descending vestibulo-spinal tract is added. As limbs appear, the cord enlarges in cervical and lumbar regions. Amphibans show a well defined central grey II and great reduction in the number of spinal segments. In birds a specific tecto-spinal tract appears, whercas in lower vertebrates the tectum (part of midbrain) connects with the cord via the reticulospinal system. In mammals the corticompinals, particularly the pyramidals, appear as dominant 
descending tracts; also the spinothalamic tracts are differentiated and the long ascending tracts of the posterior columns appear. Associated with these morphological changes there is a striking change of function of the spinal cord. In the transition from water to land, locomotion changed from swimming, a mass movement, to the much more complicated quadripedal locomotion. Lower vertebrates are characterized by behavior involving mass movements of a total-pattern type as distinguished from localized reflexes of higher vertebrates. ${ }^{189}$

There has been much disagreement over the autonomy of the spinal cord and the extent to which locomotor behavior is fixed by sensory stimulation rather than following a set pattern of neurones in the cord. What the cord can do seems to vary from animal class to class and from one related genus to another. In a general way the cord carries out more independent action in the lower vertebrates than in higher forms. Also the shock resulting from spinal transection is less in lower vertebrates. In higher groups (mammals particularly), the more primitive part of the central nervous system, the spinal cord, is less sensitive to asphyxiation but more sensitive to strychnine than is the cerebrum..$^{57}$ Evidence regarding the potentialities of the cord is obtained by isolating all or part of the spinal cord from the rest of the central nervous system and removing sensory influence by cutting dorsal roots.

A swimming or crawling animal with many segments often locomotes by undulatory waves which show remarkably precise timing. In an eel, for example, the phase of movement of each segment is just behind the phase of the segment ahead, and the muscles on the two sides differ in phase by onehalf cycle. ${ }^{173}$ Is the nervous basis for such a coordinated locomotor wave inherent in the spinal cord or is it dependent on chain reflexes? It has been suggested that the central pattern for walking evolved from this basic locomotor plan.

In Amphioxus any local stimulus elicits a general avoiding response. Lesions to the cord show that propagation of strong undulations over the whole body and end-to-end startle responses require the median giant neurones but that the rest of the isolated cord can reflexly evoke secondary superficial waves. ${ }^{406}$

Spinal hagfish (Polystotrema) are inactive for long periods but when stimulated they swim normally. Normal undulating waves start at the anterior end and / the point / of 'transection iof the cord becomes a pacemaker for initiating swimming waves. When the stimulation is vigorous, a wave may pass a cut by reflexly exciting the region beyond. ${ }^{88}$ Similarly an elasmobranch made spinal by transection behind the head swims much like a normal fish. Ten Cate ${ }^{403}$ maintained that a wave of contraction could pass a cut in the cord by virtue of overlap of proprioceptive fields in several segments and stretch of one segment by the preceding one. Thus the propagation would be a chain reflex. Gray and his associates, ${ }^{179}, 256$ on the other hand, found that transmission could proceed in an intact cord even through 12 denervated segments, and that transmission failed when the cord was cut. So long as the spinal dogfish is free from contact, a locomotor rhythm at about 40 waves per minute occurs, but a gentle tactile stimulation, particularly of the ventral surface, increases or inhibits this rhythm (Fig. 307).

The rhythmic movement of body and fins of a dogfish is abolished by 
complete deafferentation but the rhythm can be transmitted across a deafferented region of the cord. About 25 continuous intact segments in any part of the cord are needed to maintain the rhythm. Swimming in elasmobranchs apparently depends on reflex excitation, and peripheral connections are essential, but the cord is capable of transmitting the rhythm through a region where the nerve roots have been cut. Stimuli which accelerate the swimming rhythm in normal fish inhibit it in spinal or fatigued fish. Spinal dogfish show a variety of reflexes, particularly of fins; a single segment isolated from the rest of the cord can mediate homolateral and crossed responses. ${ }^{256,} 403$

Spinal teleosts also show fin reflexes elicited from localized sensory areas, ${ }^{\mathbf{1 1 4}}$ some of them enhanced, that is, released from cephalic inhibition by the spinal section. ${ }^{210}$ There appears to be much difference in autonomy of the cord from genus to genus. In fish which swim by undulatory waves, as the

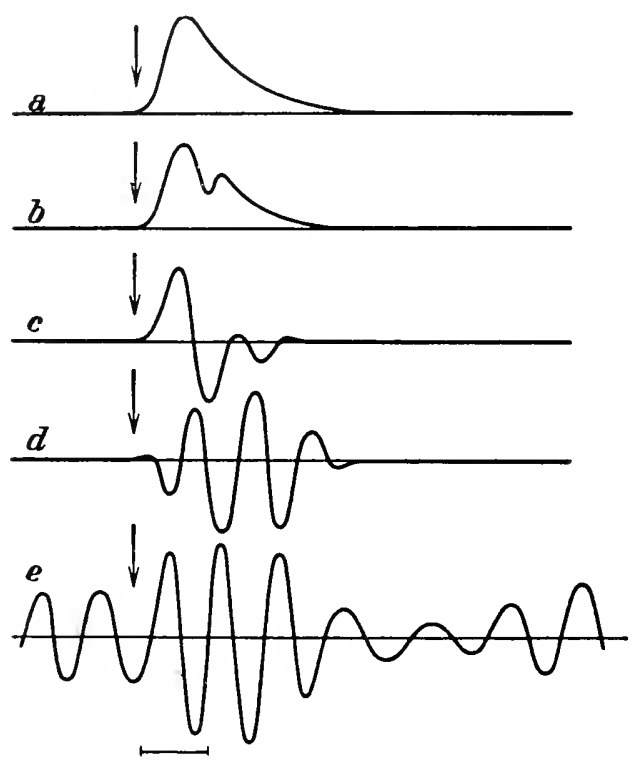

Fig. 308. Rhythmic responses of posterior abdomen of goldfish (Carassius) to stream of water applied at side of body, after transection of cord, $a$, at about vertebra $16 ; b$, at vertebra 7; $c$, between vertebra 7 and medulla; $d$ and $e$, at anterior medulla, ahead of vagus root. From Holst. ${ }^{205}$

eel, section of the cord interferes very little with swimming. An eel still swims when the skin is removed and when the muscles of a quarter of the body are removed. It is as if the undulatory rhythm were inherent in the cord and normally released by the medulla or by peripheral stimulation. ${ }^{173}$ Fish which swim with fins show little or no swimming when the cord is transected anteriorly.209, 210 Simple reflex responses of the fins and tail region are easily elicited and the sensitivity of the skin to a stream of water may actually be enhanced after spinal section, as demonstrated in studies on Carassius. ${ }^{205}, 414$ When spinal section is made close to the medulla the reflex response becomes prolonged, and when the cut is at the level of the vagal 
roots in the anterior medulla fish often show a continuous rapid rhythmic movement of fins (Fig. 308). ${ }^{205}$ The pectoral fin rhythm is normally slower than the dorsal; there is interaction between the two rhythms, but the rhythm of one fin can be altered reflexly without affecting the other. A series of rhythmic centers in the medulla is postulated but the part played by reflex re-excitation is not well known.

In amphibians the spinal cord alone permits considerable locomotion. A spinal frog jumps in coordinated fashion when stimulated but not spontaneously; reflex responses, particularly of protractor muscles, remain coordinated. $^{176}$ If one or two legs are deafferented ( $B u f o$ ) there is little interference with coordinated ambulation; when three are deafferented coordination is strikingly reduced. After deafferentation of all four limbs no locomotor movements occur, although the toad can swim, provided the labyrinths are intact, If only one leg retains its sensory and motor nerve supply and all other sensory nerves are cut, there are normal diagonal limb movements; if the motor root of the fourth leg is then cut, all ambulatory movements cease, and stimulation of this fourth leg elicits only simple monophasic responses in the other three limbs. In the toad, therefore, sensory and motor supply of one segment is necessary for the diagonal pattern of ambulation. ${ }^{176,177}$

In contrast to this strictly reflex picture of ambulation in Amphibia is the concept of a fixed pattern in the central nervous system. The basic patterns of coordination arise by self-differentiation within nerve centers prior to experience. ${ }^{424}$ Embryos kept narcotized for several days emerge from their narcosis at the behavior stage for their age without ever having experienced the motions of the developmental stages corresponding to the period of narcosis. In an extended research on salamanders Weiss ${ }^{423,424}$ has examined the movement of limbs transplanted to abnormal body regions. When a supernumerary fore-leg is transplanted to a position near a normal one and innervated by one or a few nerves of the brachial plexus, the corresponding muscles of the two legs always contract simultaneously. If a limb is rotated by $180^{\circ}$ when transplanted to replace a normal limb, the grafted limb moves in perfect temporal coordination but in reverse direction to normal, with the result that the transplanted forelimbs move the salamander backward. The effects are similar when the limbs are deafferented. Each re-innervated muscle of the extra limb contracts synchronously with the corresponding muscle of the normal limb. The spinal cord signals a particular muscle irrespective of its innervation, as if there were for a particular muscle a sort of "resonance" pattern in the cord as a whole. Basic motor patterns owe their organization to the intrinsic properties of the central nervous system rather than to peripheral influences; furthermore, the patterns are permanent, stable, and nonmodifiable in their essential feaures. This contrasts with the mammals, in which corrective influences (probably of cortical origin) can supersede the original patterns of coordination if the latter have become inadequate.

The two viewpoints, reflex control and intrinsic patterns, are not mutually exclusive. The intrinsic pattern limits the range within which reflex control can operate. The physiological basis for such limitation is unknown, and there is a wide gap between our knowledge of simple spinal reflexes and integrated locomotion. It is certain, however, that the spinal cord is much more than a complicated switchboard and terms such as homologous func- 
tion, resonance, and plasticity merely cover our ignorance of the nature of nervous integration.

In mammals the spinal cord shows less autonomy, and shock following cord section lasts much longer in higher than in lower orders. ${ }^{153}$ When the cord of a cat or dog is transected in the upper thoracic region so that respiration continues, all motor reflexes are at first depressed. Flexor reflexes return in a cat in a few hours, extensor reflexes after several days. In man spinal shock, when even flexor responses are unobtainable, lasts 1 to 6 weeks after spinal transection. It was formerly noted that good stretch reflexes did not return, and that for many weeks a light tactile stimulus might elicit flexor spasms of the legs along with defecation, urination, and sweating, the socalled "mass reflex." Observations on a large number of war casualties who received superior care showed that flexor spasms predominate from six weeks to one year, and that extensor spasms predominate thereafter, with much individual variability. Many reflexes reappear, and the spasms can be stopped by cutting dorsal roots. ${ }^{24+}$ Spinal shock results from interruption of the corticospinal system, specifically the pyramid tract, and is greater in chimpanzees than in baboons and macaques, and less in monkeys. ${ }^{153}$

Reflex potentialities of the cord are well seen in animals such as cats and dogs, in which recovery from shock occurs after a few days or weeks. A spinal cat or dog cannot stand, yet when suspended it may make rhythmic treading motions. A variety of intersegmental movements, both contralateral and ipsilateral, can be elicited, many of obviously primitive protective value to the organism. Single limb reflexes involving many muscles, such as the rhythmic scratch response to a stimulus to the skin of the back, show much central coordination. Visceral functions such as urination and defecation are elicited reflexly; when one leg is warmed vasodilatation may occur in the opposite one. Sexual functions such as erection of the penis and ejaculation, and assumption of copulatory posture occur, and spinal bitches have been impregnated and delivered of puppies after a normal gestation period. ${ }^{153}$

Short pieces of the cord, ${ }^{+12}$ isolated by two cuts and appropriate root section, are capable of some reflexes. The sensory areas of single dorsal roots have been mapped ${ }^{352}$ in dogs. An isolated lumbo-sacral region of a puppy showed no autonomous movements, although rhythmic flexions might be elicited by pressure stimulation of the cord. ${ }^{+12}$ However, deafferented sections of the cord and sections in curarized and narcotized cats showed much "spontaneous" discharge, particularly in the ventral columns. ${ }^{57}$ Some stimulus seems to be necessary to integrate this "spontaneous" activity into coordinate movements. A single sensory volley may elicit rhythmic responses in the legs, even in completely deafferented hind limbs. Isolated segments of the frog spinal cord show less electrical activity than when they are connected to the brain, but show much more activity than when the spinal nerves of the isolated segments are cut. ${ }^{409}$

The literature dealing with interaction between regions of the cord and between cord and brain is very extensive. In general, this interaction increases with increase of ascending and descending columns in higher vertebrates. Lower cord centers may even inhibit higher ones, as in hyperreflexia of forelimbs after lumbar spinal section, the Schiff-Sherrington phenome- 
non. ${ }^{369}$ As the brain gains importance the spinal cord is less capable of independent control of locomotion.

\section{BEHAVIOR OF EMBRYOS}

The sequence of development of behavior, both "spontaneous" movements and responses to stimuli, can be correlated with the development of sensory, central nervous, and effector structures. Usually the necessary structures are present well before a given behavior pattern is manifest. In those animals in which behavior development has been studied, several gradients exist, the primary gradient being backward from the cephalad end, and a secondary gradient being laterally from proximal to distal effectors.

The extensive literature on embryonic behavior emphasizes a controversy whether specific reflexes appear first and are later integrated, or whether an integrated pattern precedes the reflexes which individuate out from it. Diverging opinions result from striking differences in the speed of emergence of adult behavior patterns in different species and in the rather futile attempt to compare those embryos which move first without appendages with those whose first movement is of appendages. The important generalization emerges, however, that reflex behavior is integrated from the beginning.

The only invertebrate in which the pattern of behavior has been correlated with histological development is the earthworm, Eisenia foetida. ${ }^{326}$ The first motile stage is a ciliated gastrula, the second is a stage of contractions localized around the stomodaeum while the embryo is swallowing quantities of albumin. Next the local contractions lead to peristaltic waves which, when unequal, result in rolling of the body; at this time mechanical stimuli elicit localized contractions, at first antero-ventrally, later laterally and posteriorly; all of these are myogenic responses. Then, when nervous mechanisms are developed, regular flexion of the head begins and the anterior end turns away from a point of stimulation. Head extension and flexion initiate the peristalsis of crawling, and withdrawal from stimuli develops backward until the whole animal is sensitive. The worm is capable of burrowing before it hatches. In Limulus embryos ${ }^{322}$ kicking of the legs begins soon after the appearance of abdominal respiratory movements, and the trilobite larvae are able to swim well before they hatch.

In extensive studies on behavior in embryos of Amblystoma, Coghill98 identified the following stages: a non-motile stage when the muscles of the somites contract in response to direct stimulation, a stage of simple flexure of the body in response to tactile stimulation, spontaneous bending into a coil and uncoiling, an S-stage, and finally a stage when the S-contractions are executed to effect locomotion. Motor and sensory neurones are present in the non-motile stage, but no bipolar commissural neurones are present until the coil stage. All limb and gill movements appear first in conjunction with trunk movements. Parts of limbs move first with the entire limb and later independently. In general, the first movements are of gross regions, the total behavior complex appears early, and simple reflexes are individuated from it. The general pattern in anuran embryos is similar to that in Amblysto$m a,{ }^{444}$ but there are species differences in rate of development. Bufo is premotile at hatching; Acris and Pseudacris, like Amblystoma, are free-swim- 
ming and sensitive over the whole body. Young ( $2 \mathrm{~mm}$.) tadpoles of Rana show longer lasting responses to tactile stimulation than do $4 \mathrm{~mm}$. tadpoles; lesion to the midbrain, however, removes an inhibiting influence in the older tadpoles and leads to more persistent responses. ${ }^{20}$

The development of behavior in birds and mammals shows great species variability and differs from behavior in Amblystoma in that there is no movements of the trunk before limbs appear. The literature on various animals has been summarized: rats, ${ }^{13}$ sheep, ${ }^{31}{ }^{32}$ guinea pigs and rats, ${ }^{92}$ cats, ${ }^{\mathbf{4 3}}$ and general summary. ${ }^{38}$

In general, the latent period and contraction time of embryonic muscle are long. There is an initial pre-motile stage when local contractions are elicited by direct stimulation of some muscles. The first spontaneous movements, usually in the neck, are of uncertain origin but appear to be reflexes. ${ }^{38}$ The earliest definite responses to mechanical stimulation are gross reflexes of the neck (head movement), and then of the forelimbs. In the rat, limb movements occur first in conjunction with movements of the trunk; in the cat, sheep, and guinea pig they appear to be independent. However, these limb movements are gross but integrated, and separate segmental movements develop later. Movement appears relatively earlier in the rat, while the sheep starts movement at a later stage. ${ }^{43 *}$ The segregation of reflexes depends on interneurone development and the appearance of inhibitory mechanisms.

Development of cephalad inhibition of lower motor centers is well shown in the sheep fetus. ${ }^{31,32}$ Movements are at first (at 40 days gestation) jerky, later smooth and sustained, indicating repetitive nerve cell discharge; still later (at 60 days gestation), the fetus is inert to stimulation on the snout. Anoxia inhibits the movements acquired last; for example, it reverses the response when an animal is in the inert stage, resulting first in sustained and then in jerky movements. Also brain transections show that the jerky movements are mediated by bulbospinal systems; these are converted to sustained movement by the midbrain, and inhibited by cephalad portions of the brain. In newborn monkeys tonic innervation of muscles and grasp reflexes are dominant, then in a spastic stage limbs resist passive movement; finally discrete use of muscles appears. If in the adult a premotor area of the cerebral cortex (area 6 ) is removed the grasp reflexes seen in the newborn return. ${ }^{196}$

In some species of animals, then, the first reflex responses are generalized, in others they are somewhat localized, but in both types the movements are integrated from the start, and restricted movements become individuated later. Behavior development follows morphological gradients and cephalic dominance is important in the sequence of responses.

\section{GROSS FUNCTIONAL EVOLUTION OF THE BRAIN}

Neuroanatomists, particularly under the influence of Ariens-Kappers, ${ }^{15}$ picture the anterior enlargement of the neural tube phylogenetically and the expansion of the medulla as a response to the increase in sense organs in the head. The medulla shows greater development of sensory roots than the spinal cord. Thure is a progressive phylogenetic reduction in the number of branchial arches; there are 36 in Amphioxus, 8 in Petromyzon, and 5 in the sharks. Many changes in the sensory components occur from group to group. For example, in fish the numbers of visceral sensory and gustatory 
fibers in the facial nerve are large; in higher vertebrates the visceral sensory centers are reduced, and in birds taste sense is atrophicd. The lateral line is an important component of the tenth nerve from cyclostomes through aquatic amphibia, the cochlea from land amphibians through mammals,

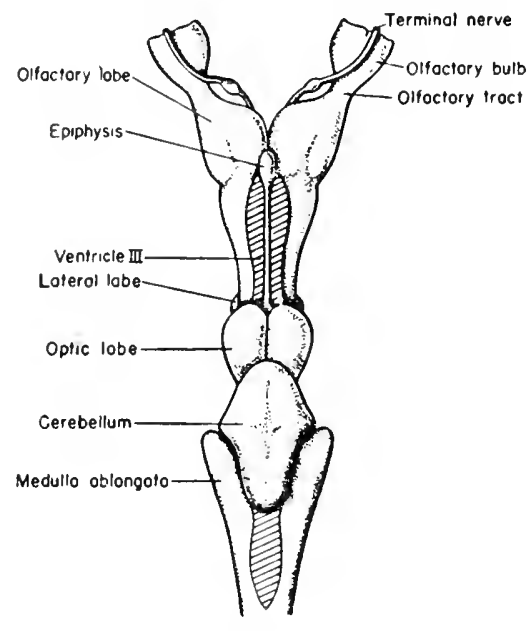

A, Scymnus

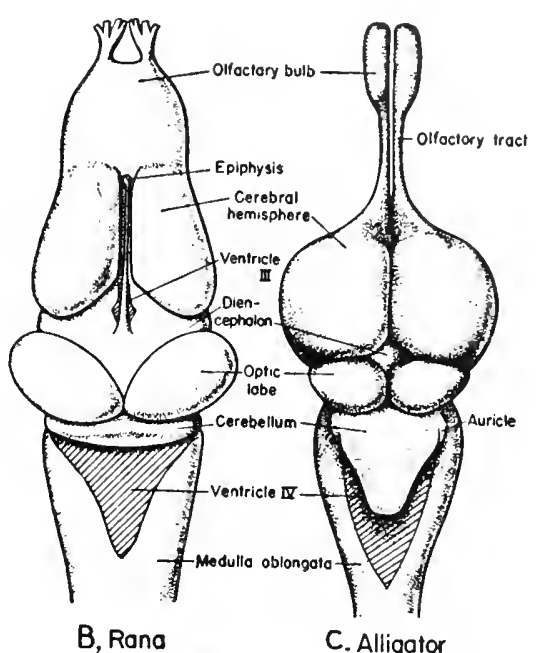

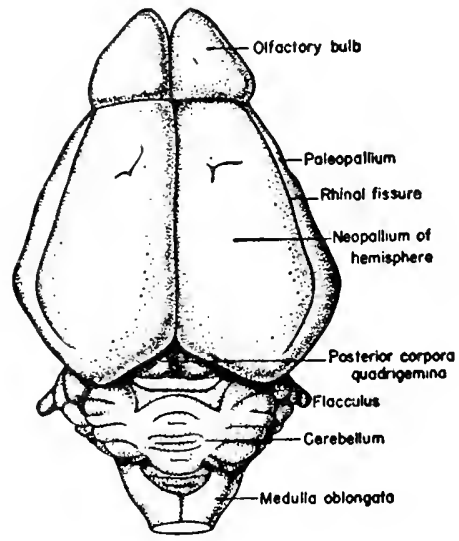

D, Gymnura

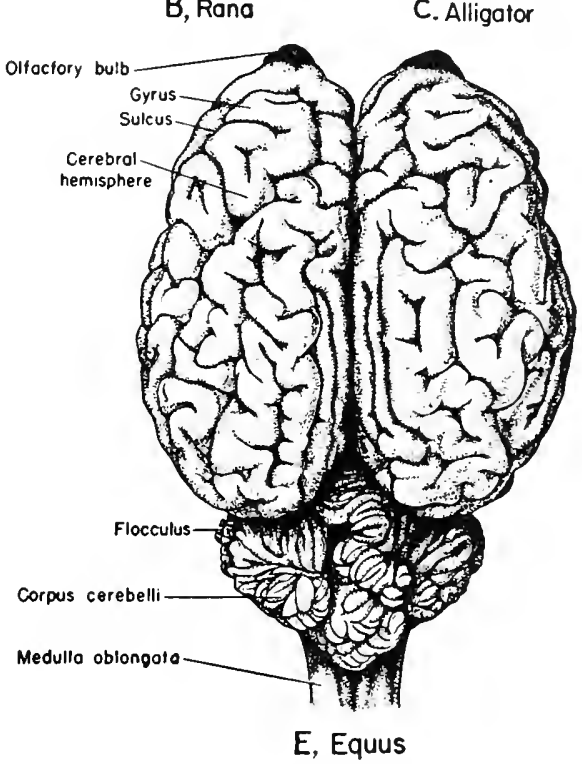

Fig. 309. Dorsal views of brains of a number of vertebrates: $A$, Shark (Scymnus); B, frog (Rana); C, alligator; $D$, tree shrew (Gymnura); E, horse (Equus). From Romer, The Vertebrate Body (1949). Philadelphia, W. B. Saunders.

whereas the vestibular nerve is present in all classes. Each of these sensory differences is reflected in medullary and midbrain centers.

Figure 309 shows diagramatically the brains of representatives of various classes of vertebrates. The cephalad shift in dominance parallels the anatomical development, and as the cerebellum and cerebrum become more im- 
portant the opportunity for lateral connections is increased by surface folding.

Medulla Oblongata. In addition to serving as the point of entrance and exit for most of the cranial nerves, and providing way-stations for their fibers in numerous sensory nuclei, the medulla has some integrative functions. It contains the respiratory center in all vertebrates, the motor outflow differing from group to group. Respiratory outflow in elasmobranchs is largely in the tenth cranial nerve, partly in the seventh; ${ }^{390}$ in mammals it is in the phrenic arising from the third to fifth cervical roots and in nerves to the intercostal muscles (thoracic roots). In the skate the right and left halves control respiration homolaterally. ${ }^{218}$ That the respiratory center is spontaneously rhythmic was shown by Adrian and Buytendijk, who recorded rhythmic electrical waves corresponding to breathing frequency from the medulla of an isolated goldfish brain; this observation has not been confirmed.

The respiratory center in mammals lies in the mid-dorsal part of the medulla at about the level of entrance of the eighth cranial nerve, and consists (in the cat, monkey, and dog) of a lower inspiratory portion and an upper expiratory center. ${ }^{165}, 323$ Anterior to the respiratory center, at the level of the pons, is a pneumotaxic or tonic center. The respiratory center receives sensory impulses over several routes, primarily via the vagus from the lung. The breathing cycle $e^{324}$ is as follows: The inspiratory center is spontaneously active continuously, it discharges impulses to the spinal motor centers, which cause the inspiratory movements of diaphragm, and of intercostal and abdominal muscles; it also sends impulses to the pneumotaxic center. As the lungs are distended afferent impulses via the vagus, together with impulses from the now excited pneumotaxic center, inhibit the inspiratory center, stopping its discharge. The expiratory center acts principally by inhibiting the inspiratory center but partly by inducing active exhalation.

The organization of the respiratory centers in non-mammalian groups is not so well known. In turtles, breathing is apneustic (prolonged inspiration); there is evidence for centers of inspiration and expiration but not for a pneumotaxic center. ${ }^{270}$ In amphibians and reptiles, lungs have an outer layer of smooth muscle, and this shows spontaneous tonic activity. In frogs and salamanders efferent impulses of the vagi inhibit the spontaneous lung contraction during breathing, ${ }^{91}$ and breathing is a swallowing act. In the reptiles (turtle and snake), on the other hand, the vagi are excitatory to the lung musculature, causing lung contraction between respirations, and the center for this contraction is inhibited during the respiratory act. ${ }^{91}$ The respiratory center is a swallowing center in adiaphragmatic animals, and in its evolution it is associated with movements of esophagus and glottis. The chemical and reflex control of the respiratory center is considered in Chapter 8.

The medulla also contains other regions which regulate autonomic functions. A vasomotor center is found in mammals in the anterior mid-medulla, just caudal to the pons; this center contains neurones which have a vasoconstrictor action, also probably some vasodilator cells. Stimulation of the vasomotor center is by specific sensory paths, descending tracts, and by direct chemical excitation, there being also some interaction with the respiratory center. The comparative physiology of vasomotor reflexes has been discussed previously (Ch. 15), also the temperature sensitivity of the vasomotor center in the turtle (Ch. 10). 
The reticular formation also contains centers for salivation and vomiting ${ }^{52}$

Of the various intermediary nuclei associated with postural control we have previously noted the center for rhythmic fin movements in teleosts, 209 which is released from inhibition by transection in the anterior part of the medulla. The most important integrative function of the medulla through all classes of vertebrates is associated with the control of equilibrium and the function of vestibular centers. A comparative study of the functioning of vestibular centers would be useful. Vestibular nuclei are extensive and are often associated with proprioceptors and with midbrain centers. If the eighth nerve of a dogfish is cut or the medulla injured on one side the fish swims in a spiral, rolling toward the operated side; the eyes deviate toward the opcrated side, the pectoral and pelvic fins are elevated on the operated and lowered on the normal side, and the dorsal fin is bent toward the intact side. ${ }^{284}$ Electrical stimulation of the medulla dorsally behind the vagal lobes elicits downward movement of homolateral pectoral fins and movement of dorsal fins toward the side stimulated. Similar movements are obtained with toadfish. Associated with vestibular, lateral line, and other afferent pathways in teleosts and urodeles are two large Mauthner cells. When these cells are destroyed in larval salamanders, equilibrium and swimming are normal but there is a tendency for early exhaustion during maintained activity. ${ }^{112}$

Vestibular nuclei take on a special function with respect to posture control in birds and mammals. Varying degrees of extensor rigidity appear when the brainstem is sectioned anterior to the vestibular nuclei but posterior to the upper midbrain (superior colliculus). A dog or cat made decerebrate by transection in the lower mesencephalon shows enhancement of all extensor reflexes, diminution of flexor responses, and increased tone in all the antigravity systems of musculature. The decerebrate animal may stand but falls over if it is pushed slightly off balance. The animal carries out many sympathetic reflexes, breathes, and shows reflexes of neck, trunk, and limbs. The extensor rigidity results from interruption of extrapyramidal tracts from high. er centers which normally inhibit the vestibular nuclei. The red nucleus, in the floor of the midbrain, is the source of one important inhibitory tract. The exaggerated extension is reflex in nature, mostly from stretch receptors, and is diminished by cutting of dorsal roots, particularly of the neck. Decerebrate mammals have been particularly useful for the study of postural reflexes. ${ }^{154}$ Animals like the three-toed sloth and bats, which normally have a flexor rather than extensor anti-gravity response, often show flexor rigidity when decerebrated;60, 277, 350 decerebrated humans show extensor rigidity of the legs and flexor rigidity of arms. ${ }^{153}$

In general, fish and amphibians transected just ahead of the medulla show nearly normal locomotion, whereas for birds and mammals no locomotion is possible with the medulla only. In all of these animals the basic respiratory and cardiovascular reflexes remain.

Cerebellum. The cerebellum is an outgrowth of the dorsal lip of the fourth ventricle. Phylogenetically, it is a derivative of the vestibular system and is a region of interaction among afferents from vestibular and lateral line organs, descending fibers from the midbrain and ascending fibers from the spinal cord. The two lateral lobes receive fibers mainly from vestibule and lateral line, whereas the central part (corpus) receives spino- and olivo- 
tract connections from the medulla. The cerebellum is lacking in myxinoids; it shows much variation among both fishes and reptiles, and has a very extensive surface in birds and mammals. The functions of the various nuclei in the mammalian cerebellum have been extensively discussed ${ }^{121}$ but are yet poorly known, in that the cerebellum is a true integrating organ and is not a distributing or reflex structure. Since it has evolved in close association with the vestibular system it is not surprising that the cerebellum has to do with the finer shades of posture regulation.

Removal of the cerebellum from dogfish fails to interfere with swimming, although when one half is removed the dogfish tend to circus because of less movement of the fins on the operated side. In certain teleosts (Lophius, flounder), there is a very poorly developed cerebellum, but in others (goldfish) removal of the cerebellum leads to disordered movements, rolling from side to side, and low tonus, whereas after unilateral decerebellation the goldfish rolls toward the operated side and may lie with the operated side down. ${ }^{413}$ In the frog, also, the cerebellum aids in tonus regulation and seems necessary for normal spontaneous jumping. Removal of one lateral half of the cerebellum from a frog results in tilting of the head and flexion of the limbs on one side of the lesion, with recovery in a few weeks. Similar postural deficiency results in the frog when one side of the medulla or one vestibular nerve is injured, and no recovery occurs. ${ }^{286}$ Removal of the entire cerebellum causes no such deficiency. One side of the cerebellum can compensate, then, for a defect in the other so long as the vestibular (and to less extent the visual) sense is unimpaired. When the entire cerebellum is removed from frogs there is some muscular weakness and the frogs are no longer able to catch files. ${ }^{261}$ Stimulation of the frog cerebellum is excitatory to muscles contralaterally and may be inhibitory homolaterally. ${ }^{164}$ In reptiles the cerebellum is important not only in equilibrium, but in giving smoothness to movements. A decerebellate lizard shows less spontaneity than normal, and walks "like a drunken man."70

The cerebellum is highly developed in birds and is important in their sense of balance. For some days after removal of the cerebellum a bird can hardly fly, walk, or stand; it falls readily to one side, but can eat and drink. ${ }^{70}$ The vestibular center in the medulla is normally inhibited by the cerebellum. removal of which gives effects similar to stimulation of the vestibular nucleus. Stimulation of the cerebellum in birds inhibits postural tonus homolaterally, and destruction of the anterior lobe produces extensor spasticity. ${ }^{56}$ When the cerebellum is removed from a mammal, no single reaction is lost, but ability to balance precisely is impaired. Freshly-operated cats and dogs may show some opisthotonus (strong extension of neck and head), ${ }^{1.53}$ Bats are said to lose their ability to fly after losing the cerebellum, and the effect of removal is greater in cats and dogs than in apes. One of the most conspicuous deficiencies in monkeys after ablation of the posterior (flocculonodular) lobes is a disturbance of equilibrium, accompanied by tremor in volitional movement. ${ }^{153}$

Stimulation of the anterior lobe of the cerebellum diminishes decerebrate rigidity (birds and mammals); removal augments stretch reflexes. The cerebellum shows an electrical rhythm of higher frequency than that of the 
cerebrum. ${ }^{121}$ Some localization of electrical responses to sensory stimulation of the feet and vibrissae has been described in the cat. ${ }^{3 n \times}$

In general, the cerebellum in all classes is intimately concerned with vestibular and other postural reflexes. Through the vertebrate series the cere bellum increases in inhibitory control. Localization of function is of a grosser sort in the cerebellum than in the cerebrum.

Midbrain. The midbrain is an important integrating center in lower verte brates; it retains nuclei of the optic system and adds auditory centers in high. er animals (Fig. 309). In cyclostomes, elasmobranchs, bony fishes, and amphibians the dorsal portion of the midbrain is known as the tectum and receives terminal fibers from the optic tracts. There is sensory localization on the surface of the tectum, also a pattern of lamination, at least in the salamander. ${ }^{1 \times 9}$ The tectum also integrates other vital systems, as in the proprioceptive and exteroceptive systems of urodeles. Efferent tracts go from tectum to cerebellum and to motor nuclei in the brainstem. The ventral portion of the midbrain is the tegmentum, and in addition to ascending and descending tracts this region contains the nuclei of the nerves to the eve muscles. The nucleus of the trochlear nerve, for example, receives afferent impulses from optic, vestibular, lateral line, and various trigeminal tracts. In reptiles, birds, and mammals the optic tecta become the superior colliculi and behind these lobes appear cochlear centers, the inferior colliculi. The tectum has many cell layers and is important for correlating several sensory modalities. The tegmentum receives fibers from thalamus and forebrain, and beginning with reptiles the reticular portion of it becomes differentiated as the red nucleus.

When the midbrain is removed from a dogfish, swimming is good except for poor sensory coordination, particularly with respect to equilibrium and vision. Removal of both tecta hampers righting and vision. If one tectum, e.g., the left, is removed, there is increased extensor tonus on the intact side, with flexion on the operated side so that the fish is curved to the left (operated side). The fish circuses toward the operated side. Stimulation of the lateral midbrain results in homolateral eye movements. Electrical stimulation of the midbrain of fish and amphibians elicits good responses of fins, opercula, and legs, much as does stimulation of the motor cortex of mammals. ${ }^{95} .302$ There is evidence that the tectum can function in learning in goldfish. Removal of optic lobes, leaving the floor of the midbrain intact, leaves normal vision. ${ }^{261}$ The midbrain originates impulses which inhibit the lower motor centers, as shown by the increased activity, such as rhythmic fin movements in fish and decerebrate rigidity in mammals, after transection behind the midbrain, but not after transection ahead of it. An Amblystoma larva reaches a stage (Harrison's stage 40) when the bulbo-spinal apparatus loses its autonomy in controlling locomotion and the midbrain is essential: then tecto-bulbar and tecto-spinal connections are present. ${ }^{11+, 1: 9} \mathrm{~A}$ center for the warning croak of a mating male frog is located in the base of the tectum; ${ }^{16}$ also, a portion of the tegmentum is needed for normal spawning movements of male frogs.

In mammals one can destroy the superior colliculus and still get pupillary light reactions. When the brain is transected anterior to the midbrain, dogs and cats do not show decerebrate rigidity, and by a series of maneuvers the animals may be able to right and to stand. A midbrain primate, however, 
is unable to stand. Preparations of this kind have been used extensively in the study of righting reflexes.

The inferior colliculus can mediate some reflex shocklike responses to sound, and action potentials from the midbrain of the cat in response to sound show that the inferior colliculus is an important auditory reflex center." Similar electrical recording shows that the superior colliculus is a center for mediating responses to light, ${ }^{14}$ with a considerable degree of point-topoint representation for the retina. Local stimulation of the superior colliculus by strychnine elicits discrete eve movements. Mammals lacking the visual (striate) cortex show limited visual responses mediated by the midbrain; cats and dogs still show some intensity discrimination, monkeys show less discrimination, and in man vision for light and dark seems to be lost. A variety of evidence indicates that in most mammals rod vision depends less than cone vision on the cortex, but that in man neither can be subserved by subcortical levels.

The midbrain of fishes and amphibians is the region of highest integration and controls the most complex behavior which these animals show. In higher animals, particularly mammals, the midbrain retains the regulation of restricted visual and auditory reflexes, and complex integration has moved forward to areas of greater surface.

The Forebrain. Diencephalon. The various portions of the thalamus and associated structures show great variations in structure from one vertebrate class to another. The function of this part of the brain is little known in nonmammalian groups. The diencephalon gives rise to the posterior pituitary gland, also to the pineal and contains (in fish) many neurosecretory cells. ${ }^{376}$ The thalamus consists of dorsal and ventral thalamus and the hypothalamus, which is increased greatly in birds and mammals.

The thalamus is not known functionally in fish. Frogs from which the entire thalamus has been removed (also the forebrain connections) are inactive and do not jump voluntarily. ${ }^{70}$ They appear to be blind. ${ }^{261}$ Release of the female by a male frog after oviposition requires a region in the dorsal hypothalamus and preoptic forebrain. ${ }^{16}$ There is evidence that water transfer through the skin may also be regulated by the thalamus. In birds and mammals the thalamus becomes important as an autonomic center. In birds temperature regulation is localized in this region, ${ }^{365}$ and stimulation causes a rise in blood pressure and slowing of respiration. ${ }^{116}$

In mammals the dorsal thalamus is important in correlating some sensory functions, particularly those of taste and temperature. The hypothalamus is essential to life and is the seat of many vegetative functions, such as water regulation by eliciting liberation of a pituitary hormone which decreases reabsorption of water by kidncy tubules, and regulation of temperature, sleep, and blood sugar level, and other functions. There is some cortical regulation of the autonomic centers of the thalamus, and removal of parts of the cortex from cats releases a so-called center of sham rage. ${ }^{33}$ The anterior part of the hypothalamus prevents abnormally high body temperatures; the posterior part prevents chilling. Damage to the hypothalamus removes emotional drive and induces somnolence: ${ }^{: 3+4}$

Telencephalon. The telencephalon is absent from Amphioxus but is present in all classes of vertebrates, where it has evolved in association with the 
paired olfactory nerves. In cyclostomes and elasmobranchs the primitive pallium (cortex or cover) known as the paleocortex contains secondary olfactory fibers, and this paleocortex is represented in higher vertebrates by the piriform lobes, the primary olfactory region of the brain in higher vertebrates. The archipallium of fishes contains tertiary olfactory neurones and becomes the hippocampus of higher classes: Both the paleocortex and archicortex receive some non-olfactory fibers and have integrative functions to varying degrees in different animal groups. ${ }^{15}$ A neurological summary of the origin of the cerebral cortex is given by Herrick. ${ }^{189}$ In the sluggish dipnoan fish, relatives of the likely ancestors of amphibians, enlarged vascular choroid plexuses insure a supply of oxygen to the brain and the ventricles are dilated. The expanded hemispheric vesicles persist in amphibians, and there is addition of some non-olfactory fibers entering the forebrain, but there is no superficial gray matter. In reptiles a cortex appears, with penetration into the pallium of localized thalamic projection fibers. In birds the olfactory system is greatly enlarged; there is a massive corpus striatum and a limited primitive neocortex.

The neocortex is already well developed in the most primitive mammals; it is non-olfactory in its connections and lies between the paleocortex and archicortex. Higher mammals are characterized by an increase in complexity of this cortex, six cellular layers normally being recognized in the mammalian neocortex. These six layers are about 100 cells (or 100 synapses) thick in higher primates but are much thinner in lower mammals. ${ }^{2 \$ 8}$

As one goes from the primitive orders (monotremes and marsupials) to the higher (primates), there is a gradual increase in total surface by folding and formation of sulci between gyri. The total number of neurones in the human cortex has been estimated at $10^{10}$ and that of extrinsic ascending or descending neurones at $10^{8} .{ }^{288}$ Most of the cortical connections, are therefore, within the cortex itself, horizontal, or vertical from one cellular level to another. The system of relay paths must be exceedingly complex. If we assume all of the properties of synaptic transmission to apply at each cortical junction it is little wonder that the total configuration shows unique "psychic" properties.

Inside the hemispheres is the massive corpus striatum, present first as a solid structure in fishes. Beside the primitive striatum develops in higher vertebrates a neostriatum which contains the various basal nuclei. These are intimately connected with the thalamus; for many functions the thalamus is best considered a part of the forebrain.

The function of regions of the cerebral cortex has been studied by various means: by ascertaining anatomical connections; by electrical and chemical (strychnine) stimulation, with observation of motor and autonomic responses; by extirpation, with observation of deficiencies in behavior; sensory and strychnine stimulation. None of these methods is adequate to of locating and seizing food is unaffected; the variability in frequency of measure the functioning of the system as a whole.

The forebrain of fishes is essentially an olfactory brain, although some olfactory discrimination is said to remain when the forebrain is removed. The forebrain shows some spontaneous electrical rhythms ${ }^{10}$ and responds well to chemical stimulation of the olfactory organs. Removal of the fore- 
brain from elasmobranchs and teleosts causes no disturbance of posture and locomotion. Also the fish forebrain has integrative functions, as indicated by operative studies on goldfish (Carassius ${ }^{220}$ ) and on Phoxinus and Gobio. 211 Fish without forebrains show nearly normal "spontaneous" activity and change in position without specific environmental stimulation, but lose "in. itiative"-the ability to react to stimuli in a specific, non-reflex manner. Speed of locating and seizing food is unaffected; the variability in frequency of spontaneous movements of opercula and eves is diminished, and the operated fish after being on a given background tend to select a background of the same brightness rather than a dark background, as preferred by normal fish. Fleeing is elicited by general exciting stimulation in both normal and operated fish; but the fish without forebrains fail to aggregate as much as normal fish, particularly when individuals are separated by wide-mesh screening. Holocentrus lacking all of the forebrain can still learn to feed from the cxperimenter's hand. ${ }^{2} \$ 9$

The forebrain of a frog shows much spontaneous electrical activity even when it is isolated. ${ }^{162}$ Electrical stimulation of the forebrain, as in fishes, elicits no motor response. ${ }^{95}$ Lessening of both spontaneity and initiative as a result of removal of the forebrain from frogs and salamanders is both affirmed ${ }^{115}$ and denied; ${ }^{261}$ active feeding diminishes, although chewing and swallowing are normal. Comparable reduction in foraging does not result when eyes and nasal organs are removed from amphibian embryos without brain interference. ${ }^{113}$ Some conditioned responses are lost after forebrain removal. ${ }^{115}$ The swimming of a male frog toward a female at the time of spawning is a midbrain response which is facilitated by the forebrain. ${ }^{16}$ Thus the amphibian forebrain appears to be more important in integration than is the fish forebrain.

Little is known of forebrain function in reptiles, although an electrically excitable area has been described for turtles and lizards ${ }^{227}$ and for alligators, ${ }^{29}$ but its presence was not confirmed in alligators and Iguana (Straus, personal communication), or in the lizard Lacerta. ${ }^{16 i}$ However, in Lacerta the motor responses elicited by stimulation of the tectum were modified by simultaneous stimulation of the cortex. Straus reports no visible response in four alligators on stimulation of the cerebral cortex (with a unipolar electrode). All reactions secured could be interpreted as the result of spread of current or as "pain" reactions. In three iguanas similar stimulation produced inconstant and variable contractions of muscles of the head, neck, and trunk, but none at all of the muscles of the extremities or tail. It seems doubtful whether the results secured in Iguana can be regarded as constituting proof of the existence of a true "motor" cortex.

There appear to be no recent studies on electrical stimulation of the nonlaminated cortex in birds, and older experimenters disagree regarding the existence of excitable areas. It appears certain, however, that removal of the hemispheres from pigeons makes the birds listless and uninterested in their environment; the operation interferes somewhat with reproductive behavior, although the birds can maintain their equilibrium and can erect their feathers and maintain body temperature. ${ }^{365}$ With cortex gone but the entire striatum intact, some birds mated and reared young. ${ }^{363}$ The operated birds learned to eat after some weeks. Forebrainless pigeons were able to avoid 
obstacles, selected small bits of food visually, but paid little attention to a threatening approach. ${ }^{418}$ Stimulation of the striatum elicits movements of the beak and other structures, and removal of large parts of the striatum causes severe behavior deficiencies. ${ }^{363}$

The non-laminated neocortex of submammalian classes deserves more study, particularly observations of electrical responses; at present it is not possible to assign to it any specific function.

The mammals present a wide range of cortical function which is well il lustrated by the increasing complexity of responses to electrical stimulation

A
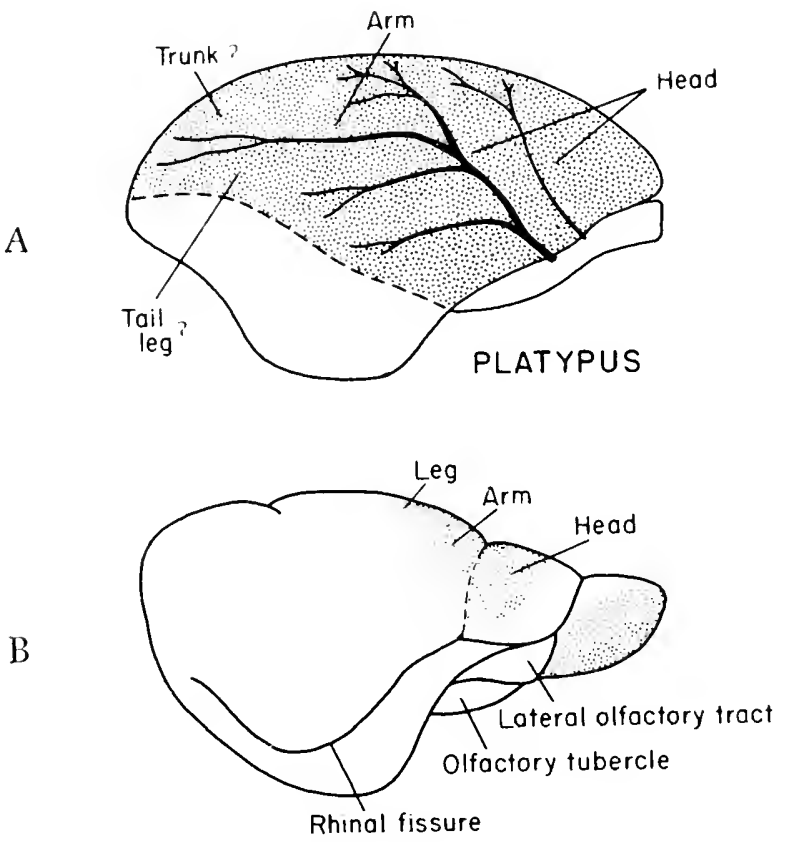

MARSUPIAL

(Trichosurus)

Fig. 310. Lateral views of distribution of total excitable (motor) cortex. $A$, In the monotreme Ornithorhynchus. From Abbie. ${ }^{1}$ B, In the marsupial Trichosurus. From Abbie. ${ }^{2}$

in the series from monotremes to primates. The number of discrete movements that can be elicited on electrical stimulation is quoted as less than 10 in monotremes and marsupials, ${ }^{70} 28$ in an ant-eater, ${ }^{11 .}$ and several hundred in higher primates. The monotremes Echidnatis and Tachyglossus ${ }^{1}$ have a band of motor cortex with gross representation from above downward of tail, hind legs, trunk, fore-legs, and head: the motor cortex activates a long cortico-spinal tract, there being no pyramidal tract. In Ornithorhynchus (Fig. $310, A$ ), on the other hand, a large portion of the cortex is excitable with overlapping representation of fore limbs, head, and eyelids, and with no good representation of tail and hind limbs. In marsupials also the excitable areas are poorly defined and there is much overlap (Fig. 310, B); the fore limbs and head are represented and in only one of the three marsupials 
which have been studied is there certain representation of the hind limbs and tail. ${ }^{1 .}=$ Remoral of the excitable area from the opossum causes temporary weakness of the fore feet, and stimulation of the striatum then elicits movement of contralateral legs. ${ }^{36+1}$ Removal of cortex plus striatum causes partial contralateral paralysis, and failure to climb or maintain balance. However, the animals can eat if food touches the mouth. The face is well repre-
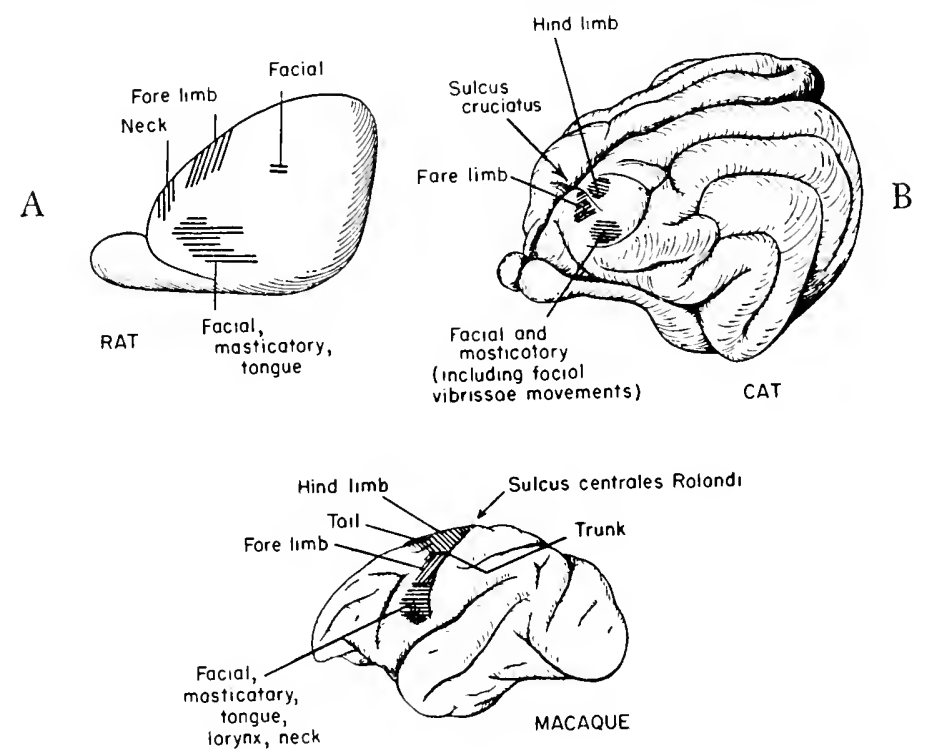

C

Fig. 311. Diagrams showing distribution of excitable cortex. $A$, in the laboratory rat. $B$, In the cat. $C$, In the macaque monkey (Pitnecus). From Huber. ${ }^{21}$

sented in the motor cortex. In the hedgehog Erinaceus the neocortex is relatively small, and electrical responses to chemical stimulation of the olfactory organ $^{9}$ are observed in both the pyriform area and the olfactory bulb. The motor cortex is less active and less defined than in more specialized mammals; ${ }^{15}$ the cortex of the sheep is not much better.

In rodents there are widely dispersed excitable areas, electrical stimulation of which elicits discrete movements of groups of muscles (Fig. 311, A). Decorticate rabbits survive well; they appear blind at first, but later can distinguish light and darkness. ${ }^{404}$ In dogs, when all of the cortex is removed. the deficiencies are more severe. The animals are blind, although the pupils constrict to bright light, and they are nearly deaf; they lose all those responses which depend on past training, but are able to stand and to make many postural responses.

Localization in the cortex of primates, which is distinctly superior to that in any other mammal, has been extensively studied, ${ }^{15+4}$ only a few aspects of it can be mentioned here. There is a striking series from lemurs through the anthropoids to man. One viewpoint is that specific functions are precisely localized; another opinion is that the cortex functions as a whole, or at least by areas in which finer localization is not rigidly fixed. In a general way the 
lobes of the forebrain have specific functions: occipital lobe-vision; temporal lobe-hearing; parietal lobe (post-central gyrus)-proprinceptive and exteroceptive sense; frontal lobe (precentral gyrus)-motor control. The functions named are not restricted to their lobes and in each part of the cortex there are also areas of integration. The sensory areas have been mapped by
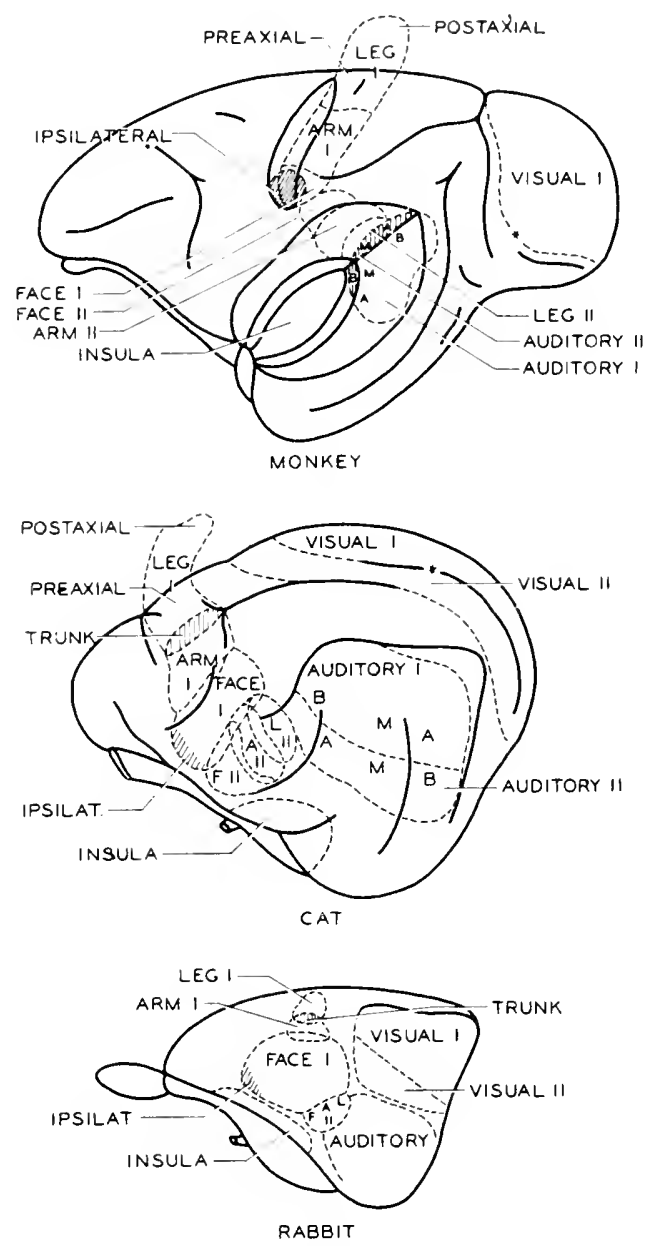

Fig. 312. Maps of the somatic sensory, auditory, and visual areas of the cerebral cortex of monkey, cat, and rabbit as determined by action potentials. I primary, II secondary areas for each sensory modality. Hatched lines, ipsilateral face areas. $A M B$ in auditory areas indicate projection of apical, middle, and basal turns of cochlea. In diagram of monkey brain the sylvian fissure is represented as spread apart to reveal the insula. Redrawn from Woolsey. ${ }^{438}$

local application of strychnine in animals, by electrical stimulation in conscious humans, by extirpation and observation of sensory deficiencies, and by recording electrical responses to sensory stimulation. To a localized sensory stimulus there are usually two or more cortical regions of response, primary and secondary (Fig. 312). ${ }^{+36}$ The retina is represented point for point on the 
contralateral occipital cortex (area striata in rats). ${ }^{50}$ Areas responding to tactile stimulation are not confined to the postcentral gyrus but also extend somewhat anteriorly. The sensory areas correspond in size to the more important parts of the sensory pattern, as the mouth parts in rabbits, claws and dorsal and lateral forelimb in cats, face in dog, hands and face in monkey. ${ }^{8}$ Auditory areas show sharp projection from regions of the cochlea, hence of sound frequency, in monkeys, ${ }^{30}$ less of point-to-point projection in cats, and probably still less in rats. $\overline{1}$

The motor area is best known from direct stimulation. Cortical stimulation usually elicits responses not of single muscles but rather of groups of muscles, exciting some and inhibiting their antagonists, the delineation of cortical areas corresponding to discrete movements rather than to individual muscles. The motor points which on stimulation yield discrete movements in higher primates lie in the precentral gyrus (area 4), with areas for feet and legs dorsally, then, progressing downward, for body, arms, neck, face, tongue. Corresponding sensory points lie opposite on the postcentral gyrus (Fig. 312).

Ahead of the motor cortex lies the premotor area (area 6), which effects movement by way of the motor area. Also anterior to the motor area are regions with specific regulatory functions, such as pupillary constriction, eye movements, and respiration. Overlapping these and the motor and premotor areas are regions concerned with autonomic regulation, cardiovascular and gastrointestinal control, sweating, salivation, etc. The extreme rostral portion of the cortex, the frontal areas (prefrontal of many authors), are "association" regions, and stimulation here elicits no motor response. Removal of these regions, of frontal lobotomy, as practiced in certain neuropsychiatric patients, has little postural effect but results in a distinct "intellectual deficit" in respect to insight and foresight, and reduces the responses to emotional tension and the "worry tendency." $1+$ s

Areas which are "silent" with respect to electrical stimulation also occur in other lobes, parietal and temporal. Aphasia of various types results from ablations in several regions. Hence, although intelligence in primates has been interfered with most by prefrontal lesions, strict association function is widely distributed in the cortex with recent evidence emphasizing the importance of the temporal lobes.

Quadrupedal vertebrates (also to some extent arthropods) display placing reactions of their legs which are initiated by tactile, visual, or proprioceptive stimuli. Also when their feet are dragged laterally against a plane surface they show a hopping reaction. In cats, dogs, and monkeys hopping reactions are lost when the contralateral sensori-motor cortex is removed and are retained when all but these gyri are ablated. In the rat, placing reactions are controlled by a larger area, approximately one third of the cortex, and hopping may be subcortical. In the opossum, placing reactions are controlled by the contralateral excitable cortex, as in higher mammals. ${ }^{61}$ In the alligator and lizard, proprioceptive placing remains when all of the brain ahead of the mesencephalon is removed, but placing fails contralaterally after removal of a lateral half of the midbrain (not tectum alone). ${ }^{34,35}$ These reactions illustrate different degrees of dependence on the forebrain, and degrees of localization within the cerebral cortex. In man, the only subcortical 
visual response is pupillary, whereas in fish and amphibia all vision may be mediated by the tectum. Dogs and monkeys have subcortical perception of light and dark, but not of pattern. ${ }^{2 \times 0}$

The cortex is also distinguished by a certain equipotentiality of function. Although there is point-for-point projection on the visual cortex (Fig. 140), deficiencies in learned visual reactions occur in rats in proportion to the amount of cortex removed, largely irrespective of location. ${ }^{249,}, 250$ Removal of a visual arca from monkevs causes a loss of a learned visual habit but the habit is readily relearned, presumably by a new area. ${ }^{4}$ Auditory learning in dogs can occur in substitute areas, any of three regions on both sides being required in varying amounts. $: 2$ When high intensities of electrical stimulation and facilitation are used instead of threshold stimuli for mapping motor areas, there appears to be more overlap of the areas eliciting movements of different limbs.:31 Histological identification of different areas of cortex in monkeys shows much variation among the individuals of a species, differences which may even be the basis for individual variation in behavior." 5 : The histological localization of specific cortical areas is by no means as precise as was formerly believed::30

Mammals, as compared with birds and reptiles, show striking increase in specificity of function of the neocortex, with lamination and the development of cortico-spinal, particularly pyramidal, tracts. Motor areas are present even in monotremes and marsupials, but localization is less precise, fewer kinds of movement are elicited, and there is more overlap than in higher mammals. The basic response to electrical stimulation of a motor area is a gross movement; increase in the number of neurone layers and increase in cortical folding permit more discrete localization and more modifiability of response. The evolution of the ncocortex in mammals has involved an increase in number of "computer circuits," a greater possible variety of combinations, and a greater memory factor.

Behavior mediated by the cortex differs from responses of lower centers in consisting of delayed reactions and in showing considerable lability of response. The interconnections of cerebral neurones are multiple. The true picture of cortical function combines gross localization and lability of finer units; it is doubtful that the mechanisms of cortical lability can be understood by any of the experimental techniques now available.

\section{PERSISTENT PHENOMENA IN CENTRAL NERVOUS SYSTEMS}

Most of the behavior described in the preceding sections can be accounted for by messages acting for brief periods over fixed anatomical pathways. Nerve physiologists have concentrated on mechanisms of transmission, facilitation, and inhibition, which are transient, lasting at most a few hundred milliseconds. Less understond is the capacity of the nervous system to retain patterns of response for long times-seconds, days, and a lifetime. We know virtually nothing of the physiological basis for complex "instinctive" behavior, conditioning, learning, memory, and abstract reasoning. Tropistic behavior, direct reaction, rarely occurs in nature, where animals are subjected to multiple stimuli and where reactions depend on past experience.

Changes in excitability persisting for seconds in mammalian nerve cen ters have been described. The summation in the lumbar spinal cord associ- 
ated with copulation is an example. Another example is facilitation and extinction in the cerebral cortex. ${ }^{1: 3,1: 24}$

Brief repetitive stimulation of a local area of the cortex may reduce the electrical response to another brief stimulus applied seconds later at the same point. In a monkey the extinction is maximal in the unanesthetized state after 4 seconds, under moderate anesthesia after 13-15 seconds, and under deep anesthesia after 1-3 minutes. The first stimuli may even be subliminal for a cortical response, and the extinction effect depends on deep layers of the cortex. A faster effect, facilitation, is an increase in cortical response if preceded a few seconds earlier by cutaneous or adjacent cortical stimulation. In sympathetic ganglia a single preganglionic volley may cause facilitation for a few seconds, but after a series of volleys facilitation lasts longer than five minutes. ${ }^{24 *}$ The mechanism of these persistent effects must be very different from synaptic excitability changes described above.

Classification of Conditioned Responses. The literature of comparative psychology is extensive and comprises observations of social behavior of animals and of habits of hunting, feeding, reproduction, and care of young, and some experimental studies. An extensive bibliography of conditioning (1111 references) is given by Razran, ${ }^{34.5}$ and summaries of the literature may be found in books by numerous authors, Maier and Schneirla, ${ }^{276}$ and Warner, ${ }^{+21}$ among others. Modern theory of conditioning is well presented by Hilgard and Marquis. ${ }^{19+}$ We are here concerned with adaptive behavior only when it gives evidence concerning neural mechanisms.

In classical conditioning one stimulus, the conditioned stimulus (CS), is usually followed by another stimulus, the unconditioned stimulus (UnCS), which alone elicits a given response (RUnCS); after sufficient presentations of both stimuli in proper sequence, the first (CS) alone elicits the response (RCS). If CS is then applied repeatedly, without reinforcement by $\mathrm{UnCS}_{n}$, the response to CS may become extinguished; recovery may, however, occur spontaneously or as the result of some strong generalized stimulation, disinhibition. Many levels of conditioning exist, and methods for distinguishing among different kinds of neural modification are needed before any functional classification of types of conditioning can be made. $A$ comparison of the modifiability of different kinds of nerve centers is needed. The following "levels of conditioning" may be recognized.

1. Neural modification may result from repeated stimulation over one sensory pathway, such that there occur changes in the quantitative relationship between the intensity of a stimulus $(S)$ and the size of response ( $R$ ). The effectiveness of the stimulus may be either increased or decreased so that, for example, $1 / 2 S \rightarrow R$, or $2 S \rightarrow R$. Central modilication must be distinguished from sensory adaptation by making certain that the repeated stimulation does not alter the sense organ response. An example of clecreased central excitability is extinction or "fatigue" of a behavior response as the sense organ is repeatedly stimulated. Also, animals may respond differently to different intensities of stimulus, as of light, and the neural modification on repetition may consist of raising the range in which the response to the low intensity is elicited. Possibly such modification occurs in sensory centers.

2. The effect of one sensory pathway may be altered by messages over a second sensory pathway, even though the two are not presented in a spe- 
cific sequence. After repetition of $S_{1} \rightarrow R_{1}$ a second stimulus, $S_{2}$, may now elicit its own response, $R_{2.2}$, although before the repetition of $\mathrm{S}_{1}$, stimulus $\mathrm{S}_{2}$ failed to evoke any overt response. A weak tactile stimulation to a rat, for cxample, mav elicit no motor response unless it follows strong visual stimulation. The stimulation by light lowers the threshold for tactile stimulation, cren though the responses are different. A response which has been extinguished by repetition can be "disinhibited" or brought back by generatized sensory stimulation. One sense modality directly modilies the response to another.

3. In pseudoconditioning the stimulus $\mathrm{S}_{2.2}$ comes to elicit the same response, $R_{1}$, as does $S_{1}$. In some instances the stimuli are not presented together. In other instances they are presented in combination. The conditioned response (RC) is very similar in latency and form to the unconditioned response ( $\mathrm{RUnC}$ ); persistence is limited. Examples are conditioning of the knee jerk or of the pupillary reflex. Trial or error learning by lower animals and much "instinctive" behavior appear to depend on pseudoconditioning mechanisms; this type of modification of behavior is definitely limited by existing neural connections.

4. True conditioning depends on temporal contiguity of the unconditioned stimulus UnCS, and the conditioned stimulus CS. The RCS differs somewhat from $R \operatorname{In}_{n} C S$; it has a longer latency, and the new response may persist for days unless it is extinguished. The RCS may be anticipatory in character. Conditioned salivary reflexes of the classic Pavloy type and the neural modifiability for maze learning and problem-box solving (instrument. al conditioned reflexes) represent true conditioning

5. The most complex behavior changes require motivation separated in time and space from the CS. Such behavior is "insightful," and the animal responds to a new situation, such as a multiple choice problem, in the light of memory of complex past experience. Complex learning may involve symbolic processes, as in reasoning.

The preceding classification is arbitrary and has no experimental physiological basis, but it may provide a background for considering different kinds of animal "learning." Three theories of conditioning have been proposed: (1) New svnaptic connections may be established during conditioning; the time required for conditioning is so brief that morphological change seems unlikely. (2) Persistent synaptic facilitation may occur; electrical studies have not given evidence for long-lasting changes in neurone excitability. (3) Conditioning may start conduction in reverberating chains and thereafter impulses pass continually around the chain, giving off motor discharges as called for; opposed to this is the fact that memory continues after anesthesia. None of these hypotheses is satisfactory for long-term learning, and a new approach is nceded if the physiology of conditioning is to be un derstood. Examination of the kinds of conditioning which can occur in different animals and in various nerve centers may aid in understanding the mechanisms of persistent responses.

Neural Basis for Conditioned Responses in Animals. In Protozoa altcration in sign and rate of response to repeated stimulation has been reported; an amoeba avoids a bright light by decreasing pseudopodial contacts, $\stackrel{-\infty 1}{ }$ and a paramecium turns around in a capillary tube with fewer partial turns after 
several tries. A conditioning experiment which should be repeated was performed by Plavilstchikov (reported by Razran ${ }^{345}$ ) on the colonial peritrich Carchesium with colored light as CS and a mechanical prod as UnCS. All of 82 colonies were "conditioned" to respond to the light after an average of 138 trials at 15 to 20 per day, and the habit was reported to be retained and carried along when part of the colony was transplanted to another colony!

There is no evidence of conditioning among coelenterates, although the tentacles of fed actinians fail to accept paper after several repetitions; this may be sensory adaptation. Echinoderms, particularly starfish, show complex behavior in feeding, righting, and escaping from narrow confines, but there is no evidence for lasting modifiability except perhaps in changing dominant arms after restraint. ${ }^{2.4}$ No conditioning experiments have been reported on coelenterates or echinoderms in which different sense modalities have been used as CS and UnCS.

In the flatworms and annelids there is clear evidence for dominance of cephalic sense organs and nerve centers. There are numerous examples of reversal of a response to one sensory stimulus by combining such a stimulus with another which is normally more potent. A marine flatworm, for example, which was quiet in darkness and active in light, was "trained" by touching each time it moved in the light to remain quiet on illumination. ${ }^{212}$ Specimens of Neanthes (Nereis) which normally responded negatively to illumination or to touch "learned" to come out of their tubes in the light by virtue of an UnCS of mussel juice presented at the time of the light or touch. ${ }^{102.103}$ Reversals of normal sign of response to light were elicited by tactile stimulation in Hydroids ${ }^{439}$ and in Lumbriculus. ${ }^{342}$ The earthworm is normally positive to low light intensities and negative to high; if this is also true for other species the reversal in response (as in Neanthes) may be a rise in threshold for negative response. This hypothesis could be tested by measuring conditioned responses at several light intensities. Quite a different order of conditioning was achieved on one individual earthworm by Yerkes ${ }^{440}$ and on several by Heck $^{1 \times 7}$ and by Swartz, ${ }^{393}$ using a $T$-shaped maze with electric shock on one arm. The worms formed an association with the "correct" (shockless) direction of turning, the habit was not lost after removal of the supraesophageal ganglion, and worms from which several anterior segments had been removed learned the habit. Apparently the ventral ganglia can be conditioned.

Sensory adaptation is familiar in molluscs, and in one conditioning experiment on a snail, Physa, tactile stimulation of the foot, which normally inhibited chewing movements, presented in combination with UnCS of a lettuce leaf, elicited chewing movements. ${ }^{410}$ Experiments with snails in mazes have not been very successful. Among the cephalopod molluscs, individuals of Sepia and Octopus learned not to eject their tentacles at a prawn behind a glass plate on which a white circle was painted, while they continued to shoot at prawns not accompanied by glass and circle. ${ }^{372,}{ }^{407} \mathrm{~A}$ normal sepia goes around a corner after a prawn which has disappeared behind a barrier; when the dorsal integrative portion of the brain mass, the verticalis complex, is removed (p. 821), the sepia no longer hunts around a corner for a prawn, although it can still learn not to eject its tentacles at a prawn be- 
hind a glass.:" Eledone has been conditioned to give a chromatophore response with colored light as the CS.

In no other group of invertebrates is instinctive behavior so highly developed as in the arthropods, particularly in hymenopteran insects. The literature on social behavior of bees, wasps, and ants is more extensive than for any other group exeept primates. Experimental studies are fewer. Light has been used successfully as a CS, associated with prod or shocking as UnCS, in arthropods such as the water mite Limnesia,:31 Cyclops, hermit crabs (Eupagumus), :": Pagurus and Leander (Mickhailoff in Razran'34.;), and cockroaches by several authors (e.g., Munter ${ }^{216}$ ). Larvae and adults of Tenebrio were trained to avoid rough or smooth surfaces and to make a correct turn in a $\mathrm{T}$-maze. ${ }^{417}$ A crude correlation has been suggested between the relative size of the mushroom bodies (corpora pedunculata) and intelligence of insects (Table 78), but no one seems to have tested the effect on learning and memory of the removal of these bodies. Limulus, an animal with very large corpora pedunculata, has not been studied for "intelligence." Arthropod behavior is modifiable within limits.

Fish have been conditioned in many experiments, particularly to color, form, and sound as the conditioned stimuli, and the habit has been retained for several weeks. ${ }^{22}$ Training in mazes has also been successful. Some fish show social behavior, nest building, and care of the young, and some migrate great distances. Removal of the forebrain from sticklebacks resulted in no loss in behavior or in ability to learn color or form discrimination; also after removal of the cerebellum these fish learned and retained their habits well. ${ }^{30 s}$ Goldfish have been trained to second-order responses, i.e., first to visual stimulus with food as the UnCS and then to the olfactory stimulus of amyl acetate with the visual stimulus as reward. 371 When parts of the tectum were removed, particularly its anterior border, the second-order learning was upset; hence the site of conditioning was the tectum. There appears to be much variation in the function of the forebrain (principally striatum) among fish. Cichlid fish, for example, after loss of the entire forebrain showed sex recognition, learned new territories, but failed to deposit or fertilize eggs or to go in schools. In Lebistes, however, mating persisted; Hemichromis, 19 months after a lesion to the striatum, failed to guard its eggs. ${ }^{307}$ Both the tectum and striatum, then, function in certain species for learned and complex instinctive behavior.

Amphibians refuse distasteful food after a few trials. Both urodeles ${ }^{297}$ and anurans $^{1+7}$ have been trained successfully in simple mazes. Conditioning to visual and auditory stimuli with food as $\mathrm{UnCS}_{n}$ occurs if the stimuli are no more than 2 seconds apart. ${ }^{115}$ Associations are still formed with one side of the forebrain removed but not with both halves lacking.

Turtles have been trained in mazes nearly as readily as rats. Birds perform complex feeding, mating, nest-building, and rearing of young. Migration and homing are poorly understood, but certainly experience and good memory of places are contributory. Many studies by conditioning and mazelearning techniques have demonstrated the high order of learning in birds, ${ }^{+21}$ but few correlations with neural structures have been made. Discrimination between a circle and triangle continues in the fowl after removal of the cortical surface of the forebrain. ${ }^{253}$ 
In contrast to reptiles and birds, mammals have been conditioned in ways which indicate the general site of persistent neural modification, if not its nature. Reflexes which can occur without the cortex are susceptible of conditioning, for example, eyelid reactions, pupillary response, psycho-galvanic reflex and knee jerk. However it is not proved that the conditioning per se is not in higher centers. A word association may be formed in man on a single trial, but spinal reflexes (in intact animals, including man) are very difficult to condition. ${ }^{377,431}$ Ability to learn appears to increase in the fo! lowing order: rats (and some other rodents), cats, dogs, raccoons. Primates excel in problem-solving and use of tools; cortical conditioning in them is rapid and varied, and usually the RC differs in detectable manner from the RUnCS.

There is no evidence for conditioning of sympathetic ganglia or various sensory nuclei. It has been claimed that in acutely spinal dogs certain spinal reflexes can be conditioned. $3 \times 2,107$ However, in chronic preparations, alterations in leg response by conditioning methods do not occur according to a typical learning curve; the response is variable in form and may be either extension or flexion. ${ }^{235}$ In chronically spinal rats crossed extension, responses of the rump to tail stimulation, and some other reflexes are gradually "extinguished" after many stimulations at the rate of one every fifteen seconds; these responses return (disinhibition) after general vigorous excitation. 338 Truc conditioning of the spinal cord has not been demonstrated, but persistent alterations in spinal excitability certainly do occur.

Subcortical centers of the brain can be conditioned in certain mammals. Decorticate $\operatorname{dogs}^{166}$ and rabbits, ${ }^{404}$ as well as animals lacking the striate or visual cortex (rabbits, ${ }^{40 s}$ rats $^{249,}, 2.11$ ), have been conditioned to light and to sound. Even some responses acquired prior to decortication can be retained after the operation. The kinds of response that can be conditioned in decorticate dogs are limited, and are of widespread activity rather than restricted response. ${ }^{108}$ Numerous experiments after removal of the visual cortex indicate conditioning of generalized responses to light but not detailed visual discrimination. In cats auditory conditioning normally involves the auditory cortex; if this is lacking some "learning" occurs at the level of the inferior colliculus, and lacking this a modified, low sensitivity response occurs at even lower levels. ${ }^{342}$ The dependence on specific cortex decreases in the order: man, monkey, dog, rabbit. Startle responses to sound are made by cats after transection at the level of the superior colliculus. In non-operated rats startle responses to sound and light can be extinguished by repeating stimuli at intervals of many seconds; the extinguished response can be disinhibited, and the threshold for startle responses can be raised by simultaneous shocks to the feet.

What are the probable loci of persistent modifications in response? Intervals between stimuli are so great that sense organ responses cannot have been altered. It is possible to substitute stimulation anywhere on the sensory pathway (spinal cord, sensory cortex) for the CS. However, no conditioning occurs if the motor ncrve or motor cortex is stimulated as the UnCS. ${ }^{268}$ The motor (mechanical) response need not be made during conditioning; the ventral roots to the leg of a dog were cut and the dog conditioned by a sound plus shock to the leg, then after nerve regeneration the muscular 
response occurred to the CS (sound). Much evidence indicates that conditioning and extinction occur not in sensory or motoneurones but rather in interneurones.

\section{CONCLUSIONS}

Nervous conduction is a cellular process which varies little among different groups of animals. High speed of reaction is associated with long fiber tracts, large fiber diameter, and nerve sheaths. Thick myelin sheaths and nodes are primarily vertebrate developments. Syncytial giant fibers evolved at least twice-with cell bodies scattered along the nervous system in annelids and crustaceans, and with cell bodies grouped in single ganglia in cephalopod molluses. Unicellular giant fibers are large neurones, and they grade in a series into small fibers; enlargement of neurones has occurred many times.

Synapses may be of two types, relay and integrative. Relay synapses are regions of redistribution, are usually polarized, but do not require facilitation. Polarization appears to depend on convergence of axons; in non-polarized junctions equal surfaces are presented. Integrative synapses make incoming impulses count for more or less than one in eliciting outgoing messages; reinforcement occurs by facilitation and diminution by inhibition.

The correlation between chemical and electrical events of synaptic transmission is not clear, but limited sampling shows synaptic potentials followed by soma potentials of slower time course than axon potentials. Most of the distributional properties of synapses evolved very early. In the coelenterate nerve net, for example, there is already facilitation in such high degree that it determines a sort of polarity at junctions. Central antagonism with reciprocal innervation of muscles is well developed in annelids and may occur in lower phyla. Facilitation may be early or delayed; early facilitation represents addition of converging subliminal impulses and gives the time course of decay of the excitatory process, whereas late facilitation may be associated with convergence via delay-paths or with after-negativity of motoneurones. Inhibition also may be direct or delayed: direct inhibition may result from anelectrotonic conduction in short interneurones: delayed inhibition may result from blocking by impulses in suitably timed delay-paths, or from after-positivity.

Peripheral conducting systems or subepidermal networks have been largely replaced by local reflex centers for activating fast muscles. It is quite possible that the subepidermal plexus in the earthworm and in molluscan feet (but not in echinoderms) is even now vestigial. In most phyla, diffuse networks for the control of visceral musculature persist.

Among invertebrates central nervous and neuromuscular mechanisms are closely interconnected in evolution. In the coelenterate nerve net system there is little distinction as to whether facilitation is neuroneural or neuromuscular. Some of the so-called peripheral conduction in worms and molluscs may be muscular. In arthropods the neuromuscular junctions with multiple innervation, facilitation, and inhibition are as important as the central nervous system in gradation of movement. (Ch. 16)

As one passes toward the most specialized groups-cephalopod molluscs, insects and higher crustaceans, birds, and mammals-one sees increasing cephalization in the nervous system. The sensory basis for cephalization is clear, 
the intergrative basis is less so. In each of the most specialized animal groups are regions of the "brain" which are not sensory centers and which may mediate no direct motor response when stimulated-the verticalis complex of cephalopods, "association areas" of the cerebral cortex of mammals, and probably the corpora pedunculata of arthropods. As cephalization proceeds there is increasing control of one level of the nervous system by another level, the cephalic levels usually inhibiting and regulating the lower motor levels.

All integrative nerve centers which have been examined show "spontaneous" electrical activity, which is not shown in distributing or sensory centers. The integrative centers - earthworm ventral ganglia, goldfish tectum, and mammalian cortex - show true conditioning.

Both phylogenetically and ontogenetically two trends in development of nervous systems are evident: (1) from mass reactions to restricted complicated behavior patterns, and (2) from fixed rigidity of response to lability of response. The movements of most worms, of lower fish, and of early embryos of all animals are gross total patterns, whereas restricted movements appear as locomotor appendages develop. The nervous system constantly integrates the behavior of each animal as a whole. Neural rigidity implies a simple stimulus-response system, lability requires integrative mechanisms. In the vertebrate series the primitive neuropile may show some lability, but as the series progresses the associative regions are pushed cephalad and increase greatly in amount. New regions of the brain take over functions served by lower regions in less specialized and less versatile vertebrates.

In a survey of comparative neurophysiology one is impressed by how much is known about transmission in axons and across synapses and how little is known about central nervous phenomena that persist longer than a few milliseconds. There are many examples of behavior for which no physiological mechanism is known. Animals such as arthropods immediately alter leg coordination when one appendage is interfered with; this is plasticity of the nervous system. In other animals, particularly in amphibians, the whole spinal cord contains some means of "tuning" a specific muscle-resonance of the nervous system. Locomotion is more than a sequence of chain reflexes. The specific behavior associated with feeding, mating, nest-building, care of young, etc., in such animals as insects, fishes, birds, and even polychaete worms, is unlearned and very complex. Modifiability of response by repeated or by associated sensory stimulation is varied, and no adequate classification of types of "conditioning" exists. Finally learning, abstract reasoning, and in man the evolution of speech are neural phenomena whose nature remains for the future to explain.

\section{REFERENCES}

1. Abrie, A. A., Austral. J. Exper. Biol. E Med. Sci. 16:143-152 (1938). Excitable cortex of monotremes.

2. Aвнie, A. A., J. Comp. Neurol. $72: 469-485$ (1940). Excitable cortex of malsupials.

3. AdEs, H. WV., J. Neurophysiol. 2:415-424 (19+4). Midbrain auditory mechanisms, cats.

4. Ades, H. W., J. Neuropath. E Exper. Neurol. 5:60-65 (1946). Cortical function in visual learning, monkey.

5. Ades, H. W., and Felder, R. E., J. Neurol. 8:464-470 (1945). Acoustic projection system. 
6. Adrian, E. D., Proc. Roy. Soc. Lond., B. 106:596-618 (1930). Injury discharges from mammalian axons.

7. Adrian, E. D., J. Physiol. 91:66-89 (1937). Synchronized responses of optic ganglion of Dytiscus.

8. Adprian, E. D., J. Physiol. 100:159-191 (1941). Sensory areas in mammalian cortex.

9. Adrian, E. D., J. Physiol. 100:459-473 (1942). Olfactory reactions in brain of hedgehog.

10. Adrian, E. D., and Ludwig, C., J. Physiol. 94:441-460 (1938). Electrical activity, olfactory brain of fish.

11. Adrian, E. D., and Matrnews, B. H. C., J. Physiol. 81:440-47I (1934). Rhythmic activity from the cerebral cortex.

12. Allen, W. F., Am. J. Physiol. 144:415-428 (1945). Cortical localization of auditory conditioning, dog.

13. Angulo, A. W., J. Comp. Neurol. 55:395-472 (1932). Development of behavior in rat embryos.

14. Apter, J. T., J. Neurophysiol. 8:123-134 (1945). Retinal projection on cat superior colliculus; ibid. 9:73-86 (1946). Eye movements elicited from superior colliculus.

15. Ariens-Kappers, C. U., Huber, G. C., and Crosby, E. C., Comparative Anatomy of the Nervous System of Vertebrates, Including Man (1936). New York, Macmillan. 2 vols., 1845 p.

16. Aronson, L. R., and Noble, G. K., Bull. Am. Mus. Nat. Hist. 86:83-140 (1945). Neural basis of sex behavior, male frog.

17. Anvanitaki, A., Arch. Internat. Physiol. 49:209-256 (1939). Local responses in decalcified giant axons.

18. Arvanitak1, A., J. Neurophysiol. 5:89-108 (1942). Ephapse transmission; local potentials, Sepia giant axons.

19. Arvanitaki, A., and Cardot, H., Bull. Histol. 18:133-144 (1941). Potentials on soma of single unipolar ganglion cells of Aplysia.

20. Anvanitak1, A., Fessard, A., and Kruta, V., C. R. Soc. Biol. Paris 122:1204. 1206 (1936). Conduction in visceral nerves, Sepia.

21. Asuworth, J. H., Phil. Trans. Roy. Soc. Lond., B. 200:427-521 (1909). Giant neurones in polychaete, Halla.

22. Augustinsson, K., Acta Physiol. Skand. 11:146-150 (1945). Cholinesterase activity and distribution.

23. Aucustinsson, K., Acta Physiol. Skand. Vol. 15, Suppl. 52, 1-182 (1948). Cholinesterase activity.

24. Augustinsson, K. B., Arch. Biochem. 23:111-126 (1949). Substrate specificity for choline ester-splitting enzymes.

25. Augustinsson, K. and Nachmansohn, D., Science 110:98-99 (1949). Acetylcholine-esterase and choline-esterase.

26. Bace, Z. M., Arch. Internat. Physiol. 42:24-46 (1935). Acetylcholine in tissues of invertebrate animals.

27. Bace, Z. M., Arch. Internat. Physiol. 42:47-60 (1935). Insensitivity of crustaceans to Ach.

28. Bace, Z. M., Arch. Internat. Physiol. 44:174-181 (1937). Action of Ach and eserine on various invertebrates.

29. Bagley, C., and Langworthy, O. R., Arch. Neurol. Psychol. 16:154-166 (1926). Forebrain and midbrain, alligator.

30. Bailey, P., Bonin, G. v., Garol, H. W., and McCulloch, W. S., J. Neurophysiol. 6:121-128 (1943). Topography of auditory cortex, monkey.

31. Barcroft, J., and Barron, D. H., J. Physiol. 91:329-351 (1937). Movements of sheep embryos.

32. Barcroft, J., and Barron, D. H., J. Comp. Neurol. 70:477-502 (1939). Behavior development in fetal sheep.

33. Bard, P., Am. J. Physiol. 84:490-515 (1928). Sham rage in cats.

34. Bard, P., Harvey Soc. Lect. 33:143-169 (1937). Cortical sensory areas.

35. Bard, P., Brooks, C. M., and Lowry, T., Am. J. Physiol. 101:3-4 (1932). Localization of "placing" reactions. 
36. Bardeen, C. R., Am. J. Physiol. 5:175-179 (1901). Function of brain in Planaria.

37. Barnes, T. C., and Beutner, R., Anat. Rec. 94:369, 430, also Am. J. Med. Sci. 212:125 (1946). Electrogenic action of acetylcholine in an oil cell.

38. Barron, D. H., Biol. Rev. 16:1-33 (1941). Review, behavior development.

39. Bartelmez, G. W., and Hoerr, N. L., J. Comp. Neurol. 57:401-428 (1933). Mauthner cell synapses.

40. Bayliss, L. E., ET al., J. Physiol. 83:439-454 (1935). Veratrine and yohimbine on action potentials of Maja nerve.

41. Bear, R. S., Schmitt, F. O., and Young, J. Z., Proc. Roy. Soc. Lond., B. 123: 496-504 (1937). Sheath components of giant nerve fibers.

42. Bethe, A., Pflïg. Arch. ges. Physiol. 68:449-545 (1897). Function of central nervous system of arthropods (Translated in J. Comp. Neurol. 8:232-238). (1898).

43. Betrie, A., Allgemeine Anatomie und Physiologie des Nervensystems (1903). Leipzig, Thième. $448 \mathrm{p}$.

44. Betre, A., Pflïg. Arch. ges. Pliysiol. 224:793-820, $821-835$ (1930). Plasticity of nervous function in arthropods.

45. Biedermann, W., Pflïg. Arch. ges. Physiol. 111:251-297 (1906). Innervation and peristalsis of mollusc foot.

46. Bishop, G. H., and Heinbecker, P., Am. J. Physiol. 94:170-200 (1930). Fiber types, frog and turtle nerves.

47. Bodian, D., J. Comp. Neurol. 68:117-159 (1937), ibid. 73:323-343 (1940). Synaptic structure, brain of goldfish.

48. Bodian, D., Physiol. Rev. 22:146-169 (1942). Review, structure of synapses.

49. Boell, E. J., and Nachmansohn, D., Science 92:513-514 (1940). Cholinesterase in squid nerve fibers.

50. Bogue, J. Y., and Rosenberg, H., J. Physiol. 87:158-178 (1936). Conduction rates, after-potentials in Maja nerve.

51. Bonvallet, M., and Mınz, B., C. R. Soc. Biol. Paris 128:158-162 (1938). Stimulation of salivary reflexes by acetylcholine.

52. Borison, H. L., and WANG, S. C., Fed. Proc. 8:13 (1949). Emesis center in medulla.

53. Bovand, J. F., Univ. Calif. Publ. Zool. 18:103-134 (1918). Nervous conduction in earthworm.

54. Bozlen, E., Ztschr. vergl. Physiol. 4:37-80 (1926). Motor coordination in medusae.

55. Bozler, E., Ztschr. Zellforsch. mikr. Anat. 5:244-262 (1927). Contact between cells in nerve net.

56. Bremer, F., C. R. Soc. Biol. Paris 90:381-384 (1924). Cerebellar function in pigeon.

57. Bremer, F., Arch. Internat. Physiol. 51:51-84 (1941). Synchronization; drug effects in spinal cord of curarized cat.

58. Bremer, F., Bull. Acad. Roy. de Med. de Belg. 9:148-173 (1944). "Spontaneous" activity of mammalian brain centers.

59. Bremer, F., J. Belge Neurol. E Psychiat. 47:542-561 (1947). Activity in deafferented brains.

60. Britton, S. W., and Kline, R. F., J. Nearophysiol. 6:65-69 (1943). Decerebrate rigidity in sloth.

61. Bromiley, R. B., and Вrooks, C. M., J. Neurophysiol. 3:339-346 (1940). Cortex and placing reactions, opossum.

62. Bronk, D. W., J. Neuroplyysiol. 2:380-401 (1939). Review of theories of synaptic transmission.

63. Brooks, C. M., and Eccles, J. C., J. Neurophysiol. 10:251-273 (1947). Electrical activity in monosynaptic path in spinal cord.

64. Brooks, C. M., and Eccles, J. C., J. Neurophysiol. 11:365-376 (1948). Synaptic excitation in the spinal cord.

65. Brown, G. L., Proc. Roy. Soc. Lond., B. 85:278-289 (1912). Spinal rhythms.

66. Brown, G. L., J. Physiol. 81:228-242 (1934). Refractory period of sympathetic ganglia. 
67. Brown, G. L., and Feldrerg, W., J. Physiol. 86:290-305 (1936). Action of potassium on sympathetic ganglion.

68. Brown, G. L., and Feldberg, WV., J. Physiol. 88:265-283 (1936). Acetylcholine content and output, sympathetic ganglia.

69. Buddenbrock, W. v., Biol. Zentralbl. 41:41-48 (1921). Locomotor rhythm in Dixippus.

70. Budpenbrock IV., v., Grundriss der vergleichenden Physiologie (1937). Berlin, Borntraeger. Bd. 2. pp. 301-546. Nervous system physiology.

71. Bulbring, E., and Burn, J. H., J. Physiol. 100:337-368 (1941). Effects of acetylcholine on spinal reflexes.

72. Bull, H., J. Mar. Biol. Assn. 15:485-533 (1928). Conclitioned responses, fish.

73. Bulzock, T. H., Biol. Bull. 79:91-113 (1940). Organization of nervous system, Enteropneusta.

74. Bullock, T. H., J. Cell. \& Comp. Physiol. 22:251-272 (1943). Facilitation in medusae.

75. Bullock, T H., J. Comp. Neurol. 80:355-367 (1944). Giant fibers in balanoglossids.

76. Bullock, T. H., J. Neurophysiol. 8:55-72 (1945). Function of giant fibers of Lumbricus.

77. Bullock, T. H., Yale J. Biol. \& Med. 17:657-679 (1945). Comparative study of brain waves.

78. Bullock, T. H., Nature 158:555-556 (1946). Synaptic potential in squid stellate ganglion.

79. Bullock, T. H., Physiol. Rev. 27:6+3-66+ (1947). Electrophysiology of invertebrates.

80. Bullock, T. H., Physiol. Comp. e. Oecol. 1:1-14 (1948). Giant fiber systems of polychaetes.

81. Bullock, T. H., I. Ncurophysiol. 11:343-364 (1948). Synaptic transmission, squid mantle ganglion.

82. Bullock, T. H., Biol. Bull. 95:249 (1948). Facilitatory and non-facilitatory synapses.

83. Bullock, T., Burr, H. S., and Nims, L. F., J. Neurophysiol. 6:85-97 (1943). Synchronization of ganglion cells by polarization.

84. Bullock, T. H., Grundfest, H., et. Al., J. Neurophysiol. 10:11-21, 63-78 (1947). DFP on action potential and cholinesterase of various nerves.

85. Bullock, T. H., and Nachmansohn, D., I. Cell. E Comp. Plysiol. 20:239-242 (1942). Cholinesterase in tissues of invertebrates.

86. Burn, J. H., Physiol. Rev. 25:377-394 (1945). Relation of adrenalin to ACh in nervous system.

87. Buytendijk, F. J. J., Arch. Néerl. Physiol. 18:24-70 (1933). Effects of brain lesions on behavior of Octopus.

88. Campbell, B., J. Neurophysiol. 3:323-328 (1940). Locomotor integration in hagfish, Polistotrema.

89. Cardot, H., Arvanitaki, A., and Ho, T. S., C. R. Soc. Biol. Paris 136:367-369. (1942). Soma potentials in single giant ganglion cells.

90. Carlson, A. J., Am. J. Physiol. 15:136-143 (1906). Conduction velocity, invertebrate nerves.

91. Carlson, A. J., and Luckhardt, A. B., Am. J. Physiol. 54:55-95, 261-306 (1920). Lung reflexes in amphibians and reptiles.

92. Carmichael, L., Psych. Bull. 38:1-28 (1941). Behavior development in guinea pigs.

93. Chadwick, L. E., and Hill, D. L., J. Neurophysiol. 10:235-246 (1947). Antiesterase action of pyrophosphates.

94. Chang, H. C., and Gaddum, J. H., J. Physiol. 79:255-285 (1933). Acetylcholine in dog brain and mammalian tissues.

95. Chauchard, A., and Chauchard, B., C. R. Acad. Sci. Paris 184:696-698, 185 : 667-669 (1927). Local stimulation of brain in fish and amphibians.

96. Chinn, P., and Schmitr, F. O., J. Cell. \& Comp. Physiol. 9:289-296 (1937). Birefringence of nerves in cross sections.

97. Coвb, P., Proc. Nat. Acad. Sci. 4:234-235 (1918). Autonony of labial palps of Anodonta. 
98. Cognul, G. E., Anatomy and the Problem of Behavior (1929). New York, Macmillan. 113 p. Neurological basis of behavior of Amblystoma embryos.

99. Cole, L. J., Biol. Bull. 24:362-369 (1913). Coordination in starfish.

100. Collier, H. O., J. Exper. Biol. 16:286-299, 300-312 (1939). Central reflexes of earthworms.

101. Coonfield, B. R., J. Comp. Neurol. 55:7-17 (1932). Peripheral nervous conduction in earthworms.

102. Copeland, M., J. Comp. Psych. 10:339-354 (1930). Conditioned response, Nereis.

103. Copeland, M., and Brown, F. A., Biol. Bull. 67:356-364 (1934). Conditioned response, Nereis.

I04. Couteaux, R., Bull. Biol. France e. Belg. 76:14-57 (1942). Cholinesterase distribution in muscle and in sympathetic ganglia.

105. Cowan, S. L., Proc. Roy. Soc. Lond., B. 115:216-260 (1934). Loss of K from stimulated nerves.

106. Creed, R. S., Eccles, J. C., Denny-Brown, D., et. Al., Reflex Activity of the Spinal Cord (1938). London, Oxford Univ. Press. 183 p.

107. Culler, E., Psych. Bull. 34:742 (1937). Conditioning, spinal dog.

108. Culler, E., and Metrler, F. A., J. Comp. Psych. 18:291-303 (1934). Conditioned behavior, decorticate dog.

109. Cuntis, H. J., and Cole, K. S., J. Cell. E Comp. Physiol. 19:135-144 (1942). Resting and action potentials of giant axons.

110. Davis, H., and Davis, P., Arch. Neurol. E Psychiat. 36:1214-1224 (1936). Electroencephalograms, man.

111. DAY, E. C., J. Exper. Zool. 28:307-335 (1919). Physiology of nervous system of tunicates.

112. Detwiler, S. R., Neuroembryology (1936). New York, MacMillan. 218 p. Ch. 13, pp. 167-181. Function of Mauthner cells.

113. Detwiler, S. R., J. Exper. Zool. 100:103-117 (1945). Forebrain lesions in Amblystoma.

114. Detwiler, S. R., Ann. New York Acad. Sci. 49:834-855 (1948). Nervous control, locomotion Amblystoma larvae.

115. Diebschlag, E., Ztschr. vergl. Physiol. 21:343-394 (1934). Forebrain lesions;

116. Dirning; frogs.
Di k, J. A. v., Arch. Néerl Physiol. 17:495-503 (1932), ibid. 16:567-573 (1931). Stimulation of diencephalon, birds.

117. Dikshit, B. B., J. Physiol. 80:409-421 (1934). Acetylcholine in cat brain.

118. Dios, M. V., Rev. Brasil. Biol. 5:377-382 (1945). Motor cortex, ant-eaters.

II9. Dotterweich, H., Zool. Jahrb., Abt. allg. Zool. u. Physiol. 44:426-450 (1928). Effects of nervous system lesions on dragonfly larvae.

120. Dow, R. S., J. Physiol. 94:67-86 (1938). Electrical activity of cerebellum.

121. Dow, R. S., Biol. Rev. 17:179-220 (1942). Evolution and anatomy of cerebellum.

122. Drew, G., J. Exper. Zool. 5:311-326 (1907). Motor functions of ganglia in clam, Ensis.

123. Dusser de Barenne, J. G., and McCulloch, W. S., Am. J. Physiol. 118:5I0524 (1937). Extinction in cerebral cortex.

124. Dusser de Barenne, J. G., and McCulloch, W. S., J. Neurophysiol. 2:319-355 (1939). Facilitation and extinction in cortex.

125. Eccles, J. C., J. Physiol. 85: 179-206, 207-238, 464-501 (1935). Action potentials, facilitation and inhibition in sympathetic ganglion, cat.

126. Eccles, J. C., J. Physiol. 88:1-38 (1936). Action potentials of sympathetic ganglia.

127. Eccles, J. C., Nature 156:680-683 (1943). Electrical hypothesis of synaptic transmission.

128. Eccles, J. C., J. Physiol. 101:465-483 (1943). Synaptic potentials, sympathetic ganglia.

129. Eccles, J. C., J. Physiol. 103:27-54 (1944). Synaptic transmission in sympathetic ganglia.

130. Eccles, J. C., Nature 156:680-683 (1945). Electrical theory of synaptic and neuromuscular transmission. 
131. Eccles, J. C., J. Neurophysiol. 9:87-120 (1946). Synaptic potentials of spinal cord.

132. Eccles, J. C., Ann. New York Acad. Sci. 47:429-455 (1946). Electrical hypothesis of synaptic transmission.

133. Eccles, J. C., Nature 159:760-771 (1947). Electrical theory of inhibition.

134. Eccles, J. C., Granit, R., and Young, J. Z., J. Physiol. 77:23P-25P (1933). Two-way conduction in earthworm giant fibers.

135. Eccles, J. C., and Sherrington, C. S., J. Physiol. 69:1-28 (1930). Summation in Hexor reflex.

136. Eggers, F., Ztschr. vergl. Physiol. 1:579-589 (1924). Coordination in nemerteans.

137. Ermer, T., Verl. Physiol. Med. Ges. 6:45-69 (1874). Conduction in medusae.

138. Erlanger, J., and Bla1r, E. A., Am. J. Physiol. 110:287-311 (1934). Segmentation in myelinated axons.

139. Erlanger, J., and Gasser, H., Electrical Signs of Nervous Activity (1937). Philadelphia, Univ. Pennsylvania Press. 221 p.

140. Feld, E. A., Grundfest, H., ET. Al., J. Neuroplysiol. $11: 123-132$ (1948). DFP on action potential and cholinesterase of squid giant axons:

141. Feldberg, W., Physiol. Rev. 25:596-642 (1945). Role of acetylcholine synthesis and function in vertebrate nervous systems.

142. Feldberg, W., and Gaddum, J. H., J. Physiol. $81: 305-319$ (1934). Acetylcholine liberation at sympathetic ganglia.

143. Feldberc, W., and Vartialnen, A., J. Physiol. 83:103-128 (1935). Physiology and pharmacology of sympathetic ganglia.

144. Feldberg, W., and Vogt, M., J. Physiol. 107:372-381 (1948). Acetylcholine syntheses by regions of central nervous system, dog.

145. Fraenkel, G., Ztschr. vergl. Physiol. 6:167-220 (1927). Burrowing reflexes, razor clam.

146. Fraenkel, G., Ztschr. vergl. Physiol. 16:371-393, 394-417, 418-443 (1932). Flight reflexes of insects.

147. Franz, V., Biol. Zentralbl. 47:1-12 (1927). Conditioning of anurans.

148. Freeman, W., and Watts, J. W., Arch. Neurol. E Psych. 58:417-425 (1947). Psychosurgery.

149. Friediänder, B., Pflüg. Arch. ges.,Physiol. 58:168-207 (1894). Central nervous coordination of earthworm movement.

150. Friedrich, H., Zool. Jahrb., Abt. allg. Zool. u. Physiol. 52:537-560 (1932): Locomotion and coordination in nemerteans.

151. Friedrich, H., Ztschr. vergl. Physiol. 18:536-561 (1933). Neuromuscular inhibition in Dixippus.

152. Fröнlıch, F. W.,Ztschr. allg. Physiol. 10:384-390, 391-395 (1910). Function of mantle ganglia in cephalopod molluscs.

153. Fulton, J. F., The Physiology of the Nervous System (1943). London, Oxford Univ. Press. 614 p.

154. Fulton, J. F., in Howell's Textbook of Physiology (1946). Phila., Saunders. Ch. 8. Spinal and decerebrate mammals.

155. Furusawa, K., J. Physiol. 67:325-342 (1929). After-potential in crustacean nerve; effect of anoxia.

156. Gasser, H. S., Am. J. Physiol. 121:193-202 (1938). Negative after potentials, recruitment.

157. Gasser, H. S., Ohio J. Sci. 41:145-159 (1941). Velocity and fiber diameter.

158. Gasser, H. S., and Erlanger, J., Am. J. Physiol. 80:522-547 (1927). Relation between fiber size and velocity of nerve propagation.

159. Gasser, H. S., and Grundfest, H., Am. J. Physiol. 117:113-133 (1936). Action potential and excitability, A-fibers.

160. Gasser, H. S., and Grundfest, H., Am. J. Physiol. 127:393-414 (1939). Conduction and fiber diameter, cat saphenous.

161. Gelei, J. v., Ztschr. Zellforsch. mikr. Anat. 22:244-254 (1935). Interciliary fibers of ciliates.

162. Gerard, R. W., Cold Spring Harbor Symp. 4:292-304 (1936). Control of brain potentials. 
163. Gerard, R. W., Ohio J. Sci. 41:160-172 (1941). Rhythmicity and interaction of neurones.

164. Gerebtzoff, M. A., Arch. Internat. Physiol. 52:249-260 (1942). Stimulation of frog cerebellum.

165. Gesell, R., Am. J. Physiol. 116:228-238 (1936). Single unit pattern of respiratory discharge.

166. Ginden, E., ET. Al., J. Comp. Psych. $21: 367-385$ (1936). Conditioning of decorticate dogs.

167. Goldby, F., J. Anat. 71:332-355 (1937). Experiments on brain of lizard.

168. Goldey, F., J. Anat. 73:509-524 (1939). Motor cortex, Echidna.

169. Graham, H. T., and O'Leary, J. L., J. Neurophysiol. 4:224-252 (1941). Conduction in Mautliner-Müller fibers of catfish.

170. Granit, R., Rubenstein, B., and Therman, P. O., J. Physiol. 85:34P-36P (1935). Retinal potentials and optic nerve stimulation.

171. Granit, R., and'Skoglund, C. R., J. Physiol. 103:435-448 (1945). Conduction at an artificial synapse.

172. Granit, R., and Skoglund, C. R., J. Neurophysiol. 8:211-217 (1945). Artificial synapse, cut mammalian nerve.

173. Gray, J., J. Exper. Biol. 13:181-191 (1936). Locomotor reflexes in eel.

174. Gray, J., J. Exper. Biol. 16:9-17 (1939). Locomotion of Nereis.

175. Gray, ]., and Lissmann, H. W., Exper. Biol. 15:506-517 (1938). Locomotor retlexes in earthworm.

176. Gray, J., and Lissmann, H. W., J. Exper. Biol. 17:227-236, 237-251 (1940). Deafferentation and locomotor reflexes of amphibia.

177. Gray, J., and Lissmann, H. W., J. Exper. Biol. 23:121-132, 133-142 (1946). Afferent control of locomotor reflexes of toad.

178. Gray, J., .Lissmann, H. W., and Pumphrey, R. J., J. Exper. Biol. 15:408-430 (1938). Mechanism of locomotion in leech.

179. Gray, J., and Sand, A., J. Exper. Biol. 13:200-209, 210-218 (1936). Locomotor reflexes in dogfish.

180. Grundfest, H., Ann. Rev. Physiol. 9:477-506 (1947). Review, bioelectric potentials in nerve, role of acetylcholine.

181. Grundfest, H., and Campbell, B., J. Neurophysiol. 5:275-294 (1942). Speed of conduction in spinal tracts.

182. Hadz1, J., Arb. Zool. Inst. Wien 17:225-268 (1909). Nervous system of Hydra.

183. Hanström, B., Vergleichende Anatomie des Nervensystems der wirbellcsen Tiere (1928). Berlin, Springer. 628 p.

184. Harvey, E. N., Carnegie Inst., Wash., Tortugas Papers 132:29-39 (1911). Conduction in nerve net, Cassiopea.

185. Hatal, S., Jap. J. Zool. 1:1-21 (1922). Rhythmic activity in oligochaetes.

186. Hecht, S., J. Exper. Biol. 25:229-260, 261-299 (1918). Coordination of responses in Ascidia.

187. HECK, L., Lotos 67/68:168-189 (1920). Associative behavior in earthworm.

188. Heinbeckek, P., Am. J. Physiol. 117:686-700 (1936). Potentials of Limulus. heart ganglion.

189. Henruck, C. J., The Brain of the Tiger Salamander (1947). Univ. Chicago Preșs. $409 \mathrm{p}$.

190. Henter, K.,Ztschr. vergl. Physiol. 14:609-628 (1931). Ganglionic function in Helix.

191. Hertwig, O., and Hertwig, R., Das Nervensystem und die Sinnesorgane der Medusen (1878). Leipzig, Vögel. 186 p.

192. Hess, W. N., J. Morph. 39:515-542 (1924). Reactions to light in Lumbricus.

193. Hess, W. N., J. Comp. Neurol. 68:161-171 (1937). Nervous system of Dolichoglossus.

194. Hilgard, E. R., and Marquis, D. C., Conditioning and Learning (1940). New York, Appleton-Century. $429 \mathrm{p}$.

195. Hines, M., J. Neurophysiol. 3:442-466 (1940). Excitation of precentral gyrus of chimpanzee.

196. Hines, M., Carnegie Inst. Contr. to Embryol. 196:153-209 (1942). Neuromotor development in macaque monkeys. 
197. Hodgkin, A. L., Proc. Roy. Soc. Lond., B. 126:87-121 (1938). Subthreshold potentials in crustacean nerves.

198. Hodgkin, A. L., and Huxley, A. F., I. Physiol. 104:176-195 (1945). Resting and action potentials in giant axons.

199. Hodgkin, A. L., and Rushton, W. A. H., Proc. Roy. Soc. Lond., B. 133:444-479 (1946). K leakage and membrane conductance.

200. Hof F, E. C., Proc. Roy. Soc. Lond., B. $111: 175-188,226-237$ (1932). Synaptic boutons, spinal cord.

201. Hofmann, F. B., Pfliig. Arch. ges. Physiol. 132:43-81 (1910). Tonic functions of ganglia in molluscs.

202. Holmes, S. J., J. Comp. Neurol. 16:200-216 (1906). Death feigning in Ranatra.

203. Holmes, W., Phil. Tr. Roy. Soc. Lond., B. $231: 293-311$ (1942). Structure of giant fibers of prawn, Leander.

204. Holmes, W., Pumphrey, R. J., and Young, J. Z., J. Exper. Biol. 18:50-54 (1941). Velocity of medullated nerve of prawns.

205. Holst, E. v., Ztschr. vergl. Physiol. 20:582-599 (1934). Reflexes of goldfish.

206. Holst, E. v., Ztschr. vergl. Physiol. 21:395-414 (1934). Central nervous function in lepidopteran larvae.

207. Holst, E. v., Biol. Rev. 10:234-261 (1935). Review, ganglionic control of locomotion, arthropods.

208. Hoist, E. v., Pflïg. Arch. ges. Physiol. 236:149-158 (1935). Central rhythms in bony fish.

209. Holst, E. v., Ztschr. vergl. Physiol. 26:481-528 (1939). Locomotor rhythms in fish.

210. Ноoker, D., Proc. Soc. Exper. Biol. E Med. 28:89-90 (1930). Spinal goldfish.

211. Hosch, L., Zool. Jahrb., Abt. allg. Zool. u. Physiol. 57:57-98 (1936). Forebrain function, Phoxinus, Gobio.

212. Hovey, H. B., Physiol. Zool. 2:322-333 (1929). Association reactions in flatworms.

213. Howell, C. D., J. Exper. Zool. 81:231-259 (1939). Nervous mechanisms in responses of Pheretima to light.

214. Huber, E., Quart. Rev. Biol. 9:55-91 (1934). Phylogeny of motor cortex, mammals.

215. Hughes, J., and Gasser, H. S., Am. J. Physiol. 108:295-306, 307-321 (1934). Spinal cord potentials.

216. Hunter, W. S., J. Genet. Psychol. 41:253-266 (1932). Conditioning of cockroach.

217. Hursh, J. B., Am. J. Physiol. 127:131-139 (1939). Velocity and fiber diameter in cat nerves.

218. Hyde, I., Am. J. Physiol. 10:236-258 (1903). Respiratory center of skate.

219. Janzen, R., Zool. Jahrb., Abt. allg. Zool. u. Physiol. 50:51-149 (1931). Conduction in peripheral nervous system of earthworm.

220. Janzen, W., Zool. Jahrb., Abt. allg. Zool. u. Physiol. 52:591-628 (1933). Forebrain function in goldfish.

221. JAwlowski, H., Acta Biol. Exper. 3:289-316 (1929). Central nervous function in myriapod, Lithobius.

222. Jenkins, O. P., and Carlson, A. J., Am. J. Physiol. 8:251-268 (1903). Conduction rate, molluscan nerves.

223. Jenkins, O. P., and Carlson, A. J., Comp. Neurol. 13:259-289 (1903). Velocity of conduction, nerve cords of annelids.

224. Jennings, H. S., Univ. Calif. Publ. Zool. 4:53-185 (1907). Behavior of starfish.

225. Johnson, G. E., J. Comp. Nentrol. 36:323-374 (1924). Giant nerve fibers of crustaceans.

226. Jornson, G. E., J. Comp. Neurol. 42:19-35 (1927). Function of giant fibers in Crustacea.

227. Johnston, J. B., J. Comp. Neurol. 26:475-479 (1916). Motor pallium in reptiles.

228. Jordan, H. J., Pflïg. Arch. ges. Physiol. 131:317-386 (1910). Effects of lesions to nervous system on behavior of crabs.

229. Jordan, H. J., Ztschr. allg. Physiol: 17:146-163 (1916). Tonus in Aplysia.

230. Jondan, H. J., Allgemeine vergleichende Physiologie der Tiere (1929). Berlin, Gruyter. $761 \mathrm{p}$. 
231. Jullien, A., and Vincent, D., C. R. Soc. Biol. Paris 129:845-848 (1938). Acetylcholine and cholinesterase in crustacean tissues.

232. Just, B., Ztschr. vergl. Physiol. 2:155-183 (1925). Coordination in Arenicola.

233. Kato, G., Ho, W. Y., ET. Al., Keijo Med. Sci. 11:1137-1147 (1931). Specific inhibitory afferent fibers, frog.

234. Katz, B., J. Physiol. 106:66-79 (1947). Subthreshold responses in medullated nerve.

235. Kellogg, W. N., Pronko, N. H., et. Al., Science 103:49-50 (1946). Spinal "conditioning," dogs.

236. KenNARD, M. A., J. Neurophysiol. 6:233-242 (1943). Electroencephalogram, decorticate monkey.

237. Kepner, W. A., and Rich, A., J. Exper. Zool. 26:83-100 (1918). Reactions of proboscis of Planaria.

238. Keynes, R. D., J. Physiol. 107:35P-36P (1948). Leakage of K from stimulated nerve.

239. Kinoshita, T., Pflïg. Arch. ges. Physiol. 134:501-530 (1910). Motor responses of ascidian, Ciona.

240. Kinosita, H. J., Jap. J. Zool. 9:209-220 (1941). Refractory period of jellyfish nerve net.

241. Kinosita, H. J., Jap. J. Zool. 9:221-232 (1941). Conduction in sea urchin nerves.

242. Knowlton, F. P., and Moone, A. R., Am. J. Physiol. 44:490-491 (1917). Strychnine on locomotor reflexes in Lumbricus.

243. Krijgsman, B. J., Ztschr. vergl. Physiol. 28:286-324 (1941). Conduction velocity, Helix nerves.

244. Kuns, R. A., and Mаснт, M. B., Bull. Johns Hopkins Hosp. 84:43-75 (1949). Reflexes of spinal man.

245. Kwiatowski, H., Arch. Exper. Path.-Pharm. 177:154-158 (1935). Acetylcholine in brain, mammals.

246. Langeloh, H. P., Zool. Jahrb., Abt. allg. Zool. u. Physiol. 57:235-279 (1937). Coordination in ophiuroids.

247. Lapicque, L., and Veil, C., C. R. Soc. Biol. Paris 93:1590-1591 (1925). Conduction rate, leech, earthworm.

248. Larrabee, M. G., and Bronk, D. W., J. Neurophysiol. 10:139-154 (1947). Prolonged facilitation in sympathetic ganglia.

249. Lashley, K. S., Am. J. Physiol. 59:44-71 (1922). Vicarious cortical function in vision.

250. Lashley, K. S., J. Comp. Neurol. 60:57-59 (1934). Retinal projection on cortex of rat.

251. Lashley, K. S., Comp. Psych. Monog. 11:3-42, 43-79 (1935). Neural basis of learning, vision in rat.

252. Lashley, K. S., and Clark, L., J. Comp. Neurol. 85:223-305 (1946). Cytoarchitecture of spider monkey cortex.

253. Layman, J. D., Comp. Psych. Monog. 12:1-36 (1936). Subcortical pattern discrimination, fowl.

254. Levin, A., J. Physiol. 63:113-129 (1927). After-potentials of crustacean nerve.

255. Libet, B., and Gerard, R. W., J. Neurophysiol. 2:153-169 (1939). Effects of ions on brain activity.

256. Lissman, H. W., J. Exper. Biol. 23:143-161, 162-176 (1946). Locomotor activity of spinal dogfish.

257. LloYd, D. P. C., J. Physiol. 96:118-129 (1939). After-potentials in sympathetic ganglia.

258. Lloyd, D. P. C., J. Neurophysiol. 6:111-120 (1943). Two-neurone spinal reflexes.

259. Lloyd, D. P. C., in Howell's Textbook of Physiology (John R. Fulton, ed.) (1946). Philadelphia, Saunders. Ch. 6, pp. 146-177. Spinal retlexes.

260. Loeb, J., Pflïg. Arch. ges. Physiol. 56:247-269 (1894). Function of brain in flatworms and polychaetes.

261. Loesser, W., J. Comp. Neurol. Psych. 15:355-374 (1905). Functional localization in frog brain.

262. Lonente de No, R., Am. J. Phy'siol. 112:595-609, 113:505-523, 524-528 (1935). Responses of oculomotor nucleus, antidromic stimulation, facilitation, delay paths. 
263. Lorente de No, R., J. Cell. E Comp. Physiol. 7:47-71 (1935). Electrical excitability of motoneurones.

264. Lonento de No, R., J. Neurophysiol. 1:187-244 (1938). Polarization and facilitation of synapses.

265. Lonente de No, R., Studies from Rockefeller Inst. Med. Res. 131:496 p., 132: 537 p. (1947). Relation betwcen electrotonic and resting potentials.

266. . Lorente de No, R., J. Cell. E Comp. Physiol. 29:207-299 (1947). Electrical fields in hypoglossal nucleus.

267. Lonente DE No, R., Bull. Johns Hopkins Hosp. 83:497-529 (1948). Quaternary ammonium ions and sodium ions in nerve physiology.

268. Loucks, R. B., J. Psych. 1:5-44 (1935). Neural delimitation of cortical learning.

269. Lullies, H., Pflïg. Arch. ges. Physiol. 233:584-606 (1934). Conduction rates, crustacean nerves.

270. Lumsden, T., J. Physiol. 58:259-266 (1924). Respiratory center, turtle.

271. Lund, E. E., Univ. Calif. Publ. Zool. 39:35-76 (1933). Neuromotor system of Paramecium.

272. MacDougall, M. S., Biol. Bull. 54:471-484 (1928). Neuromotor apparatus of Chlamydodon.

273. MacIntosil, F. C., Arch. Internat. Physiol. 47:321-324 (1938). Acetylcholine content, sympathetic ganglia.

274. Maclntosil, F. C., J. Physiol. 99:436-442 (1941). Distribution of ACh.

275. MacLennan, R. R., Arch. Protist. 86:191-210 (1935). Neuromotor apparatus of Ichthyophthicus.

276. Maier, N. R., and Schneinla, T. C., Principles of Animal Psychology (1935). New York, McGraw Hill. 529 p.

277. Maluf, N. S. R., J. Cell. E Comp. Physiol. 20:243-245 (1942). Decerebrate flexor rigidity in bat.

278. Marnay, A., and Nachmansohn, D., C. R. Soc. Biol. Paris 125:1005-1007 (1937). Cholinesterase in lobster.

279. Marnay, A., and Nachmansohn, D., J. Physiol. 92:37-47 (1938). Cholinesterase in muscle and nerve, frog.

280. Marquis, D. G., Arch. Neurol. E Psych. 33:807-815 (1935). Phylogeny of visual cortex.

281. Mast, S. O., and Pusch, F., Biol. Bull. 46:55-60 (1924). Modification of response to light, Amoeba proteus.

282. Matula, J., Pflïg. Arch. ges. Physiol. 138:388-456 (1911). Ganglionic function in dragonfly nymphs.

283. Maxwell, S. S., Pflüg. Arch. ges. Physiol. 67:263-297 (1897). Brain function in annelids.

284. Maxwell, S. S., Labyrinth and Equilibrium (1923). Philadelphia, Lippincott. $163 \mathrm{p}$. Vestibular and brain lesions, fish.

285. Mayer, A. G., Carnegie Inst., Wash., Publ. 102, pp. 115-131 (1908). Rhythmic contraction and conduction in medusae.

286. Mayer, S., and Heldfond, A., J. Comp. Neurol. 64:523-527 (1936). Cercbellar lesions in frogs.

287. McCulloch, W. S., Science 87:65-66 (1938). Theory of polarization of synapses.

288. McCulloch, W. S., Fed. Proc. 6:448-452 (1947). Summary of cortical organization.

289. Meader, R. G., Zoologica 24:11-14 (1939). Forebrain lesions, teleosts.

290. Mikalonis, S. J., and Brown, R. H., J. Cell. E Comp. Physiol. 18:401-403 (1941). Acetylcholine in insect nervous system.

291. Milucen, W., Acta Biol. Exper. 9:174-194 (1935). Experiments on neuromotor apparatus in Paramecium.

292. Miller, F. R., J. Physiol. $91: 212-221$ (1937). Action of eserine and acetylcholine on mammalian cortex.

293. Monnier, A. M., and Dubuisson, M., Arch. Internat. Physiol. 34:25 (1931). Conduction rate, crustacean nerve.

294. Moore, A. R., Am. J. Physiol. 76:112-115 (1922). Cancellation of luminescent waves in Pennatula. 
295. Mooke, A. R., J. Gen. Physiol. 6:73-76 (1923). Function of brain in polyclad worm, Yungia.

296. Moore, A. R., Sci. Rep. Tohoku Imp. Univ. IV, 6:193 (1933). Brain function, polyclad Planocera.

297. Moore, A. R., J. Comp. Psych. 29:283-292 (1940). Conditioning in Amblystoma larvae.

298. Moore, A. R., and Turner, R. S., Physiol. Zool. $21: 224-231$ (1948). Conduction, narcosis, fin nerve, Loligo.

299. Mori, S., Dobuts. Zasshi. Tok. 47:553-557 (1913). Function of nervous system in silkworm.

300. Muralt, A. v., Die Signalübermittlung in Nerven (1945). Basel, Birkhauser. pp. 293-301. Thiamin liberation in conduction.

301. Murphy, J. P., and Gellhorn, E., Arch. Neurol. \& Psych. 54:256-273 (1945). Multiple motor representation in cortex.

302. Musken, L. J. J., J. Comp. Neurol. 50:289-331 (1930). Centers of eye movement in birds.

303. Nachmansohn, D., Bull. Soc. Chim. Biol. $21: 761-796$ (1939). Cholinesterase in central nervous system, dog.

304. Nachmansonn, D., Ann. New York Acad. Sci. 47:375-602 (1946). Theory of acetylcholine in nerve transmission.

305. Nachmansohn, D., Bull. lohns Hopkins Hosp. 83:463-493 (1948). Acetylcholine in nerve conduction.

306. Nachnansohn, D., and Meyerhof, B., J. Neurophysiol. 4:348-361 (1941) Cholinesterase in squid ganglia.

307. Noble, G. K., Anat. Rec. (suppl.) 64:34 (1936), ibid. (suppl.) 70:58 (1937). Brain lesions, reproductive behavior, fish.

308. Nolte, W., Ztschr. vergl. Physiol. 18:255-278 (1932). Neural basis of learning in fish.

309. O'Leary, J. L., Heinbecker, P., et. al., Am. J. Physiol. 109:274-285 (1934). Fiber constitution, depressor nerve of rabbit.

310. Olmsted, J. M. D., J. Exper. Zool. 36:57-66 (1922). Nervous system in locomotion in polyclad worms.

311. Pantin, C. F. A., J. Exper. Biol. 12:119-138 (1935). Facilitation in nerve nets.

312. Pantin, C. F. A., J. Exper. Biol. 12:139-155 (1935). Plan of nerve net of actinians.

313. Pantin, C. F. A., J. Exper. Biol. 12:156-164 (1935). Polarity and after-discharge in nerve nets.

314. Papez, J. W., Comparative Neurology (1929). New York, T. Crowell Co. 518 p.

315. Parker, G. H., J. Exper. Zool. 22:87-94 (1917). Nervous transmission in actinians.

316. Parker, G. H., J. Gen. Physiol. 1:231-236 (1918). Nerve net transmission, Metridium.

317. Parker, G. H., The Elementary Nervous System (1919). Philadelphia, Lippincott. 229 p.

318. Parker, G. H., J. Exper. Zool. $31: 475-515$ (1920). Nervous conduction in Renilla.

319. Parker, G. H., J. Cell. E Comp. Physiol. 1:53-63 (1932). Nerve net conduction, Physalia.

320. Parker, G. H., and Parshley, H. M., J. Exper. Zool. 11:361-364 (1911). Reactions of earthworms to moisture.

321. Pavlov, J., Pfliig. Arch. ges. Physiol. 37:6-31 (1885). Control of movement in Anodonta.

322. Pearl, R., J. Comp. Neurol. 14:138-165 (1904). Behavior development in Limulus embryos.

323. Pitts, R. W., J. Neurophysiol. 6:439-454 (1943). Repetitive activity in neurones of respiratory center.

324. Pitss, R. W., in Howell's Textbook of Physiology (John R. Fulton, ed.) (1946). Philadelphia, Saunders. Ch. 41, 42; pp. 896-930. Analysis of respiratory center.

325. Pringle, J. W. S., J. Exper. Biol. 16:220-231 (1939), ibid. 17:8-17 (1940). Reflex mechanisms of cockroach leg. 
326. Prosser, C. L., J. Comp. Neurol. 58:603-630 (1934). Behavior development, earthworm embryos.

327. Prosser, C. L., J. Comp. Neurol. 59:61-91 (1934). Nervous mechanisms in reactions of earthworms to light.

328. Prosser, C. L., Quart. Rev. Biol. 9:181-200 (1934). Physiology of earthworm nervous system.

329. Prosser, C. L., J. Cell. E Comp. Physiol. 4:185-209 (1934). Spontaneous impulses in crayfish ganglia.

330. Prosser, C. L., J. Exper. Biol. 12:95-104 (1935). Impulses in segmental nerve of earthworm.

331. Prosser, C. L., J. Comp. Neurol. 62:495-505 (1935). Functional tracts in nervous system of crayfish.

332. Prosser, C. L., Cold Spring Harbor Symp. Quant. Biol. 4:339-346 (1936). Rhythmicity in nerve centers.

333. Prosser, C. L., J. Cell. E Comp. Physiol. 7:95-111 (1939). Synaptic delay and facilitation in crayfish ganglia.

334. Prosser, C. L., J. Cell. E Comp. Physiol. 16:25-38 (1940). Drugs and salts on synaptic transmission in crayfish.

335. Prosser, C. L., J. Cell. E Comp. Physiol. 21:295-305 (1943). Unit discharge of Limulus heart ganglion.

336. Prosser, C. L., J. Cell. \& Comp. Physiol. 22:131-145 (1943). Ions on activity of crayfish abdominal ganglia.

337. Prosser, C. L., Physiol. Rev. 26:337-382 (1946). Physiology of nervous systems of invertebrate animals.

338. Prosser, C. L., and Hunter, W., Am. J. Physiol. 117:609-618 (1936). Extinction of startle and spinal reactions, rats.

339. Pumphrey, R. J., and Rawdon-Smith, A. F., Proc. Roy. Soc. Lond., B. 122:106I 18 (1937). Synaptic transmission, conduction in cercal nerve, cockroach.

340. Pumphrey, R. J., and Younc, J. Z., J. Exper. Biol. 15:453-466 (1938). Velocity of fibers of various diameters.

341. Quastel, J. H., ET. Al., Biochem. J. 30:1668-1681 (1936). Choline esterase in rat tissues.

342. RaAb, D. H., Am. J. Psych. 59:59-83 (1946). Auditory conditioning, decorticate cats.

343. RaAbe, S., Ztschr. vergl. Physiol. 26:611-643 (1939). Associations in annelid Lumbriculus.

344. Ranson, S., Harvey Soc. Lectures 33:92-121 (1937). Review, functions of hypothalamus.

345. Razran, G. H. S., Psych. Bull. 34:191-256 (1937). Bibliography of conditioned responses.

346. Reitschel, P. E., Zool. Anz. 111:109-111 (1935). Locomotion mechanisms in cestodes.

347. Richards, A. G., J. New York Ent. Soc. 52:286-310 (1944), ibid. 51:55-69 (1943). Fiber diameter and sheath thickness, insect nerve fibers.

348. Richards, A. G., and Cutкomp, L. N., J. Cell. E Comp. Physiol. 26:57-61 (1945). Cholinesterase in insect nervous tissues.

349. Richards, O. W., Biol. Bull. 56:32-40 (1929). Nerve conduction, Mytilus.

350. Richter, C., and Bartemeier, L. H., Brain 49:207-225 (1926). Flexor rigidity, sloth.

351. Rijlant, P., C. R. Soc. Biol. Paris 111:631-635, 636-639 (1932). Electrical signs of locomotor reflexes in arthropods.

352. Rijnberk, G. v., and Ten Cate, J., Arch. Néerl. Physiol. 18:291-336 (1933). Reflexes of isolated segment of dog spinal cord.

353. Robertson, T. B., J. Physiol. 31:410-417 (1904). Sham-death reflex in spiders.

354. Roeder, K. D., Biol. Bull. 69:203-220 (1935). Nervous basis of sexual behavior in Mantis.

355. Roeder, K. D., J. Exper. Zool. 76:353-374 (1937). Control of tonus and locomotion in Mantis.

356. Roeder, K. D., J. Cell. E Comp. Physiol. 14:1-12 (1939). Drugs on electrical activity of roach nervous system. 
357. Roeder, K. D., J. Cell. E Comp. Physiol. 18:1-9 (1941): Effects of salts on cray. fish ganglia.

358. Roeder, K. D., Fed. Proc. 3:40 (1944). Effect of Ach on responses to potassium in crayfish nerve cord.

359. Roeder, K. D., J. Cell. \& Comp. Physiol. $31: 327-338$ (1948). Ions on activity of roach nervous system.

360. Roeder, K. D., Bull. Johns Hopkins Hosp. 83:587-600 (1948). Antiesterases on nervous system, cockroach.

361. Roeder, K. D., Kennedy, N. K., and Samson, E. A., J. Neurophysiol. 10:1-10 (1947). Anticholinesterases and synaptic transmissión, cockroach.

362. Rogers, F. T., Am. J. Physiol. 55:310 (1920). Lesions in forebrain of birds.

363. Rogers, F. T., J. Comp. Neurol. 35:61-65 (1922). Stimulation of forebrain of pigeon.

364. Rogers, F. T., J. Comp. Neurol. 37:265-315 (1924). Cortical function in opossum.

365. Rogers, F. T., Am. J. Physiol. 86:639-650 (1928). Temperature regulation and other functions in bird thalamus.

366. Romanes, G. J., Phil. Tr. Roy. Soc. Lond. 166:269-313 (1876), ibid. 167:659752 (1877). Mechanisms of locomotion in medusae.

367. Rosenblueth, A., and Simeone, F. A., Am. J. Physiol. 122:688-707 (1938). Potentials in sympathetic ganglia.

368. Rothenberg, M., Biochim. et Biophys. Acta 4:96-114 (1950). Ionic movements across axonal membranes.

369. Ruch, T. C., Am. J. Physiol. 114:457-467 (1936). Non-segmental factors, spinal reflexes.

370. Rushton, W. A. H., Proc. Roy. Soc. Lond., B. 132:423-437 (1945). Action potentials in earthworm giant fibers.

371. Sanders, F. K., J. Exper. Biol. 17:416-434 (1940). Second order learning in tectum of goldfish.

372. Sanders, F. K., and Young, J. Z., J. Neurophysiol. 3:501-525 (1940). Functions of higher nerve centers in Sepia.

373. SASSE, E., Ztschr. allg. Physiol. 13:69-104 (1912). Function of insect nervous system.

374. Schallek, W., J. Cell. E Comp. Physiol. 26:15-24 (1945). Acetylcholine in lobster nerve cord.

375. Schallek, W., and Wiersma, C. A. G., J. Cell. E Comp. Physiol. 31:35-47 (1948). Drugs on crustacean synapse.

376. Scharrer, E., and Scharrer, B., Physiol. Rev. $25: 171-181$ (1945). Neurosecretion.

377. Schlossberg, H., J. Gen. Psychol. 7:328-342 (1932). Conditioned reflexes, man.

378. Schmitt, F. O., and Bear, R. S., J. Cell. E Comp. Physiol. 9:261-273 (1937). Birefringence of frog nerve fibers.

379. Schmitr, F. O., and Bear, R. S., Biol. Rev. 14:27-80 (1939). Ultrastructure of nerve axon sheath.

380. Sсhмitт, F. O., and Schmitt, O., J. Physiol. 98:26-46 (1940). Conduction in Loligo nerves.

381. Scunabel, W., Ztschr. vergl. Physiol. 30:194-226 (1943). Conditioning in water mite.

382. Shurrager, P. S.; and Culler, W., Am. J. Physiol. 123:186-187 (1938), also Psych. Bull. 34:742-743 (1937). Spinal conditioning, dog.

383. Smallwood, W. M., and Holmes, M. T., J. Comp. Neurol. 43:327-345 (1927). Cytology of giant fibers in earthworm.

384. Smith, C., and Glick, D., Biol. Bull. 77:321-322 (1939). Cholinesterase in nerve, muscle and cardiac tissues of invertebrate animals.

385. Smiti, J. E., Phil. Tr. Roy. Soc. Lond., B. 227:111-173 (1937). Nervous system of starfish.

386. Smitir, J. E., Biol. Rev. 20:29-43 (1945). Function of nervous system of starfish.

387. Smitif, R. I., J. Cell. E Comp. Physiol. 13:335-344 (1939). Acetylcholine in nervous tissue, crayfish. 
388. Snider, R. S., and Stowell, A., J. Neurophysiol. 7:331-357 (1947). Sensory localization in cerebellum, cat.

389. Spaulding, E. G., J. Comp. Neurol. 14:49-61 (1904). Learning by hermit crabs.

390. Springer, M. G., Arch. Neurol. \& Psychiat. 19:834-864 (1928). Respiratory center, elasmobranchs.

391. Stough, H. B., J. Comp. Neurol. 40:409-463 (1926). Giant fiber system, earthworm.

392. Stough, H. B., J. Comp. Neurol. 50:217-230 (1930). Polarization of giant fibers in earthworm.

393. Swartz, R. D., J. Comp. Psych. 9:17-33 (1929). Modification of behavior, earthworm.

394. Sweitzer, A., and Wright, S., J. Physiol. 89:165-197 (1937). Effects of eserine, acetylcholine on knee jerk.

395. Taylor, C. V., Univ. Calif. Publ. Zool. 19:403-470 (1920). Microdissection of neuromotor system in Euplotes.

396. Taylor, C. V., in Protozoa in Biological Research (G. Calkins and F. M. Summers, eds.) (1941). Columbia Univ. Press. pp. 191-270. Fibrillar systems in ciliates.

397. Taylor, G. W., J. Cell. E Comp. Physiol. 15:363-372 (1940). Birefringence of earthworm giant fibers.

398. Taylon, G. W., J. Cell \& Comp. Physiol. 18:233-242 (1941). Optical properties of shrimp nerve fibers.

399. Taylon, G. W., J. Cell. \& Comp. Physiol. 20:359-372 (1942). Birefringence and conduction particularly in cat fibers.

400. 'Ten Cate, J., Arch. Néerl Physiọl. 14:1-6 (1929). Reflexes of stellate ganglia of Sepia.

401. Ten Cate, J., Arch. Néerl. Physiol. 15:242-253 (1930). Function of nervous system in hermit crabs.

402. Ten Cate, J., Ergebn. Physiol. 33:137-336 (1931). Review of ganglion function in invertebrate animals.

403. Ten Cate, J., Arch. Néerl. Physiol. 18:497-502 (1933). Wave transmission in elasmobranch, Scylium.

404. Ten Cate, J., Arch. Néerl Physiol. 20:467-476 (1935). Visual association after removal of striate area, rabbits.

405. Ten Cate, J., Arch. Néerl. Physiol. 21:562-566 (1936). Coordination of leg movements in cricket.

406. Ten Cate, J., Arch. Néerl Physiol. 23:409-415 (1938). Central reflexes in Amphioxus.

407. Ten Cate, J., and Ten Cate, K. B., Arch. Néerl. Physiol. 23:541-552 (1938). Pattern learning by Octopus.

408. Ten Cate, J., and Van Herk, A. W. H., Arch. Nóerl. Physiol. 18:337-386 (1933). Vision in rabbits without striate area.

409. Ten Cate, J., Walter, W. S., et. Al., J. Neurophysiol. 10:223-233 (1947). Spontaneous activity, frog spinal cord.

410. Thompson, E. L., Behav. Monog. 3, no. 3, 97 p. (1917). Conditioning of Physa.

411. Tobras, S. M., Kollross, J. I., and Savit, I., J. Cell. E Comp. Physiol. 28:159. 186 (1946). Acetylcholine in nervous system of cockroach and crayfish.

412. Tower, S. S., J. Comp. Neurol. 67:107-131 (1937). Activity of isolated portions of spinal cord of dog.

413. Tuge, H., J. Comp. Neurol. 60:201-224 (1934). Cerebellar ablation in goldfish.

414. Tuge, H., and Hanzawa, S., Sci. Rep. Tohoku Univ., Ser. IV, 10:589-606 (1935). Movements of spinal fish.

415. Uexkull, J. v., Ztschr. Biol. 31:584-609 (1895). Anatomy and function of brain mass in cephalopods.

416. Uexkull, J. v., Ergebn. Physiol. 3:1-11 (1904). Control of behavior in echinoderms.

417. Vernay, W. B. du, Ztschr. vergl. Physiol. 30:84-116 (1942). Conditioning in Tenebrio.

418. Visser, J. A., and Rademaker, G. G., Arch. Néerl Physiol. 20:284-295 (1935). Visual reactions after forebrain removal, birds. 
419. Vogr, M., J. Physiol. 86:258-263 (1936). Potassium in synaptic transmission in sympathetic ganglia.

420. WANG, G., and Lu, T., J. Neurophysiol. 7:147-162 (1943). Behavior development, frog.

421. Warden, C. J., Jenkins, T. N., and Warner, L. H., Comparative Psychology (1940). New York, Ronald. 1070 p. Vol. I Vertebrates, Vol. II Plants and Invertebrates, Vol. III Theory.

422. Weel, P. B., and Thore, S., Ztschr. vergl. Physiol 23:26-33 (1936). Mechanism of pupillary reaction in Octopus.

423. WeIss, P., J. Comp. Neurol. 67:269-315 (1937). Responses of transplanted amphibian limbs.

424. Weiss, P., Comp. Psych. Monog. 17:1-96 (1941). Review of experimental evidence for fixed patterns of coordination.

425. Welsh, J. H., J. Exper. Biol. 16:198-219 (1939). ACh in tissues of Carcinus.

426. Welsh, J. H., Bull. Johns Hopkins Hosp. 83:568-579 (1948). Theories of action of acetylcholine.

427. Welsh, J. H., and Gondon, H. T., J. Cell. E Comp. Physiol. 30:147-171 (1947). Mode of action of insecticides on crustacean axons.

428. Welsh, J. H., and Haskin, H. H., Biol. Bull. 76:405-415 (1939). ACh and autotomy in Crustacea.

429. Welsh, J. H., and Hyde, J. E., J. Neurophysiol. 7:41-49 (1944). Acetylcholine in rat brain; also Proc. Soc. Exper. Biol. \& Med. 55:256-257 (1944). ACh in myenteric plexus.

430. Welsh, J. H., and Schallek, W., Physiol. Rev. 26:447-478 (1946). Review of physiology of arthropod nervous systems.

431. Wendt, G. W., Arch. Psychol. 19:1-97 (1930). Conditioning of knee-jerk, man.

432. Wiersma, C. A. G., Proc. Soc. Exper. Biol. \& Med. 38:661-662 (1938). Function of giant fibers in crayfish.

433. Wiersma, C. A. G., J. Neurophysiol. 10:23-38 (1947). Giant fiber function in crayfish.

434. Windle, W. F., Physiology of the Fetus (1940). Philadelphia, Saunders. 249 p.

435. Woolard, H. H., and Harfman, J. A., J. Anat. 73:559-562 (1939). Discontinuity of coelenterate nerve net.

436. Woolsey, C. N., Fed. Proc. 6:437-441 (1947). Sensory cortex.

437. Woolsey, C. N., Marshall, W. H., and Bard, P., Bull. Johns Hopkins Hosp. 70:399-44l (1942). Sensory responses of monkey cortex.

438. Woortmann, K., Ztschr. vergl. Physiol. 4:488-527 (1926). Function of ganglia of Mytilus.

439. Yerkes, A. W., J. Comp. Neurol. Psych. 16:441-449 (1906). Conditioning in Hydroides.

440. Yerkes, R. M., J. Anim. Behav. 2:332-352 (1912). Conditioned response in earthworm.

441. Younc, J. Z., Quart. J. Micr. Sci. 78:367-386 (1936). Giant fiber system of cephalopods:

442. Young, J. Z., J. Exper. Biol. 15:170-185 (1938). Giant nerve fiber activation of squid mantle.

443. Young, J. Z., Phil. Tr. Roy. Soc. Lond., B. 229:465-501 (1939). Gross and histological anatomy of giant fiber system, squid.

444. Youngstrom, K. A., J. Comp. Neurol. 68:351-379 (1938). Behavior development in Anura.

445. Zhuкov, E. K., Bull. Biol. Med. Exper. Moscow 3:297-299 (1937). After-positivity in crab nerve. 


\section{Index}

Absorption, ionic, 91 of food, body region for, 149

Àcidity, gastric. See Hydrogen ion concentration.

Acclimatization, temperature, 346-349 metabolic changes in, 349

Accommodation, 399 range of vertebrate eye, 395

Acetylcholine, 540 action of, 546, 548, 557, 558, 559, 560 content of nervous tissue, 792 effect on inelanophores in fish, 708 effect on visceral muscles, 606 in electric organs, 620 in neuromuscular transmission, 608 nerve conduction and, 781

Acetylcholinesterase, 608

Actin, 578

Actiniohematin, 294

Action potential, muscular, 587 nerve, ionic basis of, 780 wave, 777

retinal, 421

effect of temperature on, 423 optic nerve activity and, 434

Actomyosin, 578

Adaptation, dark, electrical measurement of, 426

in Mya, 406

kinetics of, 415

nerve impulse in, 432

genetic, 3

metabolic, 268-277

of mechanoreceptors, 510

physiological, 2

to temperature, 374

visual, in lower vertebrates, $397-405$

Adenine, excretion of, 189

Adenosine triphosphate, breakdown of, 615

Adjustment, osmotic, 10-14

Adrenal gland, androgen production by, 744

cortex of, effect on water and salt balance, 760

growth and, 727

hormones, effect on carbohydrate metabolism, 757

progestin production by, 746
Adrenalin, 537

effects of, 561

on heart, 557

on spinal cord, 794

on visceral muscles, 606

in neuromuscular transmission, 612

regulation of heart and, 548

Adrenergic regulation of hearts, 557

systems in muscle, 612

Adrenin, 537

effect on carbohydrate metabolism, 757

See also Adrenalin.

Aedes, larvae of, osmoregulation in, 33

aegypti, essential amino acids for, 117, 120

After-discharge in nerve centers, 805

Age, critical oxygen tension and, 250 oxygen consumption and, 228

Agglutinins, 106

Air, dry, poikilotherms inhabiting, 353 moist, poikilotherms inhabiting, 351 temperature of, as related to body temperature in mammals, 363

body temperature and, 351 vision in, 403

Alanine, structural formula, 119

Albumin, serum, molecular size, 105

Alimentary tract, diagrammatic representation of, 148

functional regions of, 147

mucosa of, ds respiratory mechanism, 218

Allantoic acid, 189

Allantoicase, distribution of, 203-205

Allantoin, 189

Allantoinase, distribution of, 203-205

Allatectomy, effect on reproductive cycles of insects, 754

Altitude, effect on oxygen capacity of blood, 312

oxygen capacity and saturation and, 316

Amblystoma, embryo of, respiration in, 229, 232

embryonic behavior in, 827

phonoreception in, 487

Ameiurus, color changes in, 710

Amino acids, essential, 117

excretion of, 187 
Amino acids, in various animals, 192-196 growth of Euglena in, 116

Aminopeptidases, 159

Ammonia, excretion of, 187, 189, 191 in fish, 197

in various animals, 192-196

Ammonotelic animals, 19l

Amoeba, cell forms of, 631 efficiency of contractile vacuole in, 46 fluid excretion in, 41 mechanism of filling contractile vacuole in, 45

light sensitivity in, 382

osmotic adaptation in, $38,39,40$

permeability in, 39

Amoeboid movement, 630-639

theories of, 634

types of cells in, 630

Amphibians, chemoreception in, 456

chromatophores in, 703

conditioned responses in, 845

digestive secretion in, 157

erythrocytes in, 295

forebrain in, 836

gills in, 216

heart in, 544, 551

hemoglobin content in, 295

hormones and growth in, 728

kidney in, 48

nerve conduction in, 784

nitiogen excretion in, 195, 198

osmoregulation in, 52-55

oxygen dissociation curves for, 314,321

phonoreception in, 487

respiratory characteristics of blood of, 307

respiratory responses in, 266

serologic relationships in, 107, 108

spinal reflexes in, 825

temperature relations of, 351

uricolytic enzymes in, 204, 205

water loss in, 54

Amphioxus, gills in, 215

Amylase, distribution of, 170

Anaerobic metabolism in diving, 259

Anaerobiosis, 268, 271-274

degrees of, 272

oxygen utilization in, 272

Anchistioides, eye pigment migration in, 388

Androgens, 743

Anelectrotonus, 780

Anemia, macrocytic, 129

Anemone, muscle responses in, 594

Anemonia, nematocysts in, 657

Anemotaxis, 509

Angle, visual, 399

Anguilla vulgaris, osmoregulation in, 50

Animal kingdom, phylogenetic tree, 4

Annelids, blood pressure in, 539
Annelids, brain in, 816

chemoreception in, 450

cholinergic systems in muscles in, 610 , 612

chromatophores in, 688

conditioned responses in, 844

ganglionic reflexes in, 812

heart in, 545, 551

ions in body fluids in, 82

light sensitivity in, 383

luminescence in, 663

nerve conduction in, 785

nervous regulation of heart in, 559

nervous system in, 810

nitrogen excretion in, 192

ocelli in, 384

oxygen supply of, 211

oxygen withdrawal by, 251

$\mathrm{pH}$ of digestive organs, 152

protein in coelomic fluids in, 104

proteinases of, 160

respiratory responses in, 265

Anobiidae, cellulose digestion in, 172

Anodonta, cilia in, 648 osmoregulation in, 35,36

Anolis, chromatophores in, 681 color changes in, 712

Ant, light orientation in, 393

Antigens, 106

Aphrophora spumaria, ocellus of, 392

Aplysia, ionic regulation in, 89 osmotic adjustment in, 12, 13

Arachnoids, oxygen transport in, 326

Arbacia, eggs of, volume changes in relation to tonicity of sea water, 10,11 pustulosa, chromatophores in, 689

Archicortex, 835

Area centralis, 396

Arenicola, effect of acetylcholine on heart of, 560

heart in, 545

hemoglobin function in, 324

osmotic adjustment in, 12

stomatogastric nerve plexus in, 607

Arginase, distribution of, 201

Arginine, structural formula, 118

Arginine phosphate. See Phosphoarginine.

Arrhenius $\mu, 343$

Artemia, osmoregulation in, 32

Arthropods, blood picssure in, 539

brain in, 818

portions occupied by different regions, 819

chemoreception in, 450

compound eye in, 387

conditioned responses in, 845

electrocardiograms in, 555

exopeptidases in, 166

fat digestion in, 177

ganglionic reflexes in, 814 
Arthropods, hormones and metabolism in, 759

ionic regulation in, 84

ions in body fluids in, 82,83

luminescence in, 663

motor innervation in, 596

nerve conduction in, 784,785

nervous regulation of heart in, 559

ocelli in, 391

oxygen transport in, 326

$\mathrm{pH}$ of digestive organs, 152

phonoreception in, 492

photic behavior of, 392

proteinases of, 161

terrestrial, osmoregulation in, 55-58

tracheae in, 222

Ascaris, osmotic regulation in, 17

Ascidia mentula, osmotic adjustment in, 14

Ascidians, heart in, 551

ions in body fluids in, 83

nervous regulation of heart in, 559

oxygen withdrawal by, 252

vanadium chromogens in, 305

Ascorbic acid, 130

Aspartic acid, structural formula, 119

Astacus, blood chloride in, 86, 88

breathing movements in, 263

changes in body fluids in, 85

electrocardiogram from, 555

Asterias, osmotic adjustment in, 14

Astigmatism, 395

Atropine, 540

action of, 561

effect on visceral muscles, 606 regulation of heart and, 548

Attagenas, essential amino acids for, 117

Autotrophic organisms, 113

Avena, carotenoids in, 409

BABAK's law, 683

Bacteria, thiamine requirements in, 123

Balance, ionic, 94 water. See Water balance.

Balanoglossids, peripheral nervous system in, 810

Baldes-Hill method of measurement of osmotic pressure, 8

Barger's method of measurement of osmotic pressure, 8

Basal metabolic rate in homoiotherms, 370 . See also Metabolism, basal.

Bats, hearing in, 491

Becs, body temperature in, 356 brain in, 817 oxygen consumption in, 233,234

sensillum placodeum in, 457

Beetles, dietary factors of, 136

Behavior, development of, 827

Betaine, chromatophores in cephalopods and, 690
Bioluminescence, 660-676

control of, 671

extracellular, 660

functional significance of, 673

intracellular, 660, 671

mechanism of, 667

occurrence of, 661

physical characteristics of, 666

Biotin, 128

Birds, androgens in, 744

body temperatures in, 362

cerebellum in, 832

chemoreception in, 456

conditioned responses in, 845

digestive secretion in, 156

erythrocytes in, 295

forebrain in, 836

fovea in, 401

hemoglobin content in, 295

kidney in, 48, 58

lungs in, 221

migration in, 371

hormones and, 766

nitrogen excretion in, 195, 200

osmoregulation in, 58

phonoreception in, 490

respiratory characteristics of blood, 307

respiratory control in, 258

respiratory responses in, 267

seasonal color changes in, 765

temperature regulation in, chemical mechanisms, 369

uricolytic enzymes in, 204

visual field in, 400

Black tongue, 127

Bladder, gas, 218

Blind spot, 396

Blindness, color, 437

Blood, ammonia in, 187

carbon dioxide content, 329, 330

cells, red. See Erythrocytes.

circulation of, $531-575$

concentration of, in fresh-water vertebrates, 52

ions in. See Ions, inorganic.

mammalian, reactions to carbon dioxide transport in, 331

oxygen capacity of, 306

pigments, oxygen tension and, 247. See also Pigments.

pressure, in closed systems, 534

in open systems, 540

measurement and regulation, 534

values in various aninials, 538

proteins in, concentration in, 103, 104

pigments as, 334

size of, 105

respiratory characteristics of in various animals, 307-309

salinity of. See Osmoregulation. 
Blood, transport by, 533

types, 106

vessels, pulsating, 545

volume, 533

values in various animals, 535

Body fluids. See Fluids, body.

Body temperatures in hibernation, 372

in homoiotherms, 362

in poikilotherms, 349, 351, 353, 358

Bombyx, developmental hormone in, 735

Boring mechanisms for feeding, 146

Bostrychidae, wood digestion in, 172

Bradycardia during diving, 260

Brain, functional evolution of, 828-841

in invertebrates, 816

in vertebrates, 829

Branchial tufts, 213, 214

Breathing. See Respiration.

Brightness discrimination, 415

explanation of, 418

Brine shrimps, osmoregulation in, 32

B-substance in amphibian color changes, 704

in color changes in fish, 709

Buffering in invertebrates, 333

in lower vertebrates, 332

Bunsen-Roscoe reciprocity law, 406

Busycon, oxygen transport in, 328

\section{Calciferol, 132}

Calcium, absorption of, in postmolt in crustaceans, 738

functions of, 97

in nerve transmission, 795

ions, effects on hearts, 564, 565

in sea water and body lluids, 78

in tissues and body fluids, 76

ratios of concentration, 82

Calocaris, brain in, 817

Calories produced by various foods, 237

Cambarus, molting in, 738

sinus gland in, 696

Canals, semicircular, 475, 517

Cancer pagurus, body fluids in, 91 osmoregulation in, 24

Carassius, oxygen consumption in, 241 spinal reflexes in, 824

Carbohydrases, specificity of, 170

Carbohydrates, conversion to fat, 269

digestion of, 168-176 age and, 176

metabolism of, hormonal influences in, 757

nature of, 168

oxygen consumption in utilization of 237

spced of utilization of, 237

utilization in Protozoa, 114, 115

Carbon requirements, $114-115$

in Protozoa, 113, 114
Carbon dioxide, combining power of, 329, 333

dissociation curve of, 329

effect on oxygen dissociation, 311 , $313,314,315$

effect on respiratory center, 255

elimination of in anaerobiosis, 273

exchange in vertebrates, 212 . See also Respiration.

increase in, respiratory responses to, 265

tensions, 330

tolerance in diving mammals, 260

transport of, 329-334

in lower vertebrates, 332

reactions in mammalian blood, 331

Carbonic anhydrase, 331

distribution, 333

Carbonyl-proteinases, 158

Carboxyhemoglobin, absorption spectra of, 298, 299

Carboxypeptidases, 159, 167

Carcinus, blood ions in, 87,89

muscle responses in, 594

osmoregulation in, 26

respiratory quotient in, 277

Cardiac cycle, 543. See also Heart.

Carnivores, digestive enzymes in, 178

Carotene, 131, 132

Carotenoids in vision, 408

Cat, muscle in, 589

nictitating membrane in, 591 pacinian corpuscle in, 504

Catelectrotonus, 780

Caterpillars, phonoreception in, 498

Catfish, color changes in, 710

Cathepsin, distribution of, 163

Cathepsin I, 158

Cathepsin II, 159

Catocala, tympanal organs of, 494

Caudina, ionic regulation in, 89 osmotic adjustment in, 13 salts in body fluids in, 85

CBLH, 700

$\mathrm{CDH}, 700$

Cell(s), giant nerve, 786 membrane, electrical phases of, 778

Cellular physiology, 1

Cellulase, distribution of, 171

Cellulose, digestion of, 169, 171

Central excitatory state, 803 inhibitory state, 803

Centrostephanus longispinus, chromatophores in, 689

Cephalic dominance in invertebrates, 816

Cephalopods, blood and oxygen capacity in, 304

brain in, 820

chemoreception in, 450

chromatophores in, 678,690 
Cephalopods, ganglionic reflexes in, 815 glant tibers in, 788

luminescence in, 662, 664

nervous regulation of heart in, 558

neuromuscular transmission in, 613

oxygen transport in, 326

oxygen withdrawal by, 252

respiratory control in, 263

Cerambycidae, cellulose digestion in, 172

Cerebellum, functions of, 831

Cerebral cortex, areas of, 839

equipotentiality of function in, 841

in mammals, 837

motor area, 840

premotor area, 840

CES, 803

Cestodes, locomotion in, 816

Chaetopterus, luminescence in, 661, 662, 663

Chameleons, color changes in, 714

Chelonia, nitrogen excretion in, 198

Chemical sense, common, sensitivity of, 459. See also Chemoreception.

Chemoreception, 447-470

behavior and, $448-457$

in arthropods, 450

in invertebrates, 448

in vertebrates, 453

intensity discrimination in, 465

physiology of, $457-468$

Chemoreceptors, 459

activation of, 465

classification of, 447

contact, 448

control of respiration by, 255

in insects, 451

in vertebrates, 453

sensitivity of, 457

Chemotrophic organisms, 112

Chiasma, optic, 435

Chick embryo, nitrogen excretion in, 200

Chicken, essential amino acids for, 117

metabolism in, 232

Chilomonas paramecium, carbon requirements of, 114

oxygen consumption in, 229

thiamine and, 123

Chironomus, hemoglobin function in, 321, 322,323

Chitin, 170

digestion of, 173

Chloride ions in blood in relation to chloride in medium, 86

in sea water and body fluids, 78

in tissues and body fluids, 76

ratios of concentration, 82

Chlorocruorin, 291, 293, 302, 303

absorption spectra, 300

oxygen transport by, 326

Cholecystokinin, 156, 763
Cholesterol, dietary requirements, 134

Choline, 129

Cholinergic regulation of heart, 546, 548, 557

systems in muscles, 608

Cholinesterase content of nervous tissue, 792

in electric organs, 620

types of, 781, 782

Chordates, blood pressure in, 538

exopeptidases in, 167

proteinases of, 162

Chordotonal organs, 493, 494, 495

Chromatophores and color change, 677-724

aneuronic, 707

dineuronic, 707

factors affecting, 684

functional organization of, 688

functional significance of, 715

in amplibians, 703

in annelids, 688

in cephalopods, 690

in crustaceans, 693

in echinoderms, 689

in fish, 706

in insects, 691

in reptiles, 712

in vertebrates, 703

index of, 682

light and, 684

mononeuronic, 707

structure and methods of action, 677 684

Chromatophorotropins in central nervous system of crustaceans, 700

in eyestalks of crustaceans, 694-699

Chromatosomes, 711

Chymotrypsin, 159

Cilia, 640-653

activity of, characteristics of, 643

environmental factors and, 649

reversal of, 645

theories of, 650

as feeding mechanism, 145

coordination and control of, 646

functions of, 640

metachronism in, 646

propulsion of food by, 149

responses to chemicals by, 448

structure of, 642

Ciliates, conduction in, 776

Circulation, 531-575. See also Bluod and Heart.

summary, 567

CIS, 803

Clam, motor innervation in, 601 photoreception in, 406

Clarias lazera, branchial diverticula in, 217

Cnidoblast, 656

Cnidocil, 656 
Cochlea, 474

structure of, 475

Coelenterates, chemoreception in, 449

exopeptidases in, 166

fresh-water, osmoregulation in, 35

ionic regulation in, 84

ions in body fluids in, 82

luminescence in, 661

nematocysts of, 655

nerve nets in, 808

nitrogen excretion in, 192

ocelli in, 384

oxygen supply of, 211

$\mathrm{pH}$ of digestive organs, 152

proteinases of, 160

site of digestion in, 150

speed of nerve conduction in, 782, 783

uricolytic enzymes in, 203

Coelom, 533

Coelomic fluid, protein concentration in, 104

Cold, adaptation to, 241

metabolic response to, 369

narcosis, 371

receptors for, 364

Cold-blooded animals. See Poikilotherms.

Coleoptera, gills in, 215

luminescence in, 664

Colliculus, inferior, 834

superior, 833

Color blindness, 437

Color change and chromatophores, 677-724 diurnal rhythms and, 687

factors affecting, 684

functional significance of, 715

light and, 684

morphological, 683

physiological, 678

measurement of, 682

primary, 687

seasonal, hormonal control, 765

secondary, 685

Color vision, $436 \cdot 440$

abnormal, 436

"dominator-modulator" theory of, 439

normal, 436

peripheral mechanism of, 437

phylogenetic distribution of, 404

Coloration, hormonal control, 765

Comparative physiology, defined, 1

ecological aspects, 3

phylogenetic aspects, 3

Compound eye in arthropods, image

formation in, 389

Conditioned responses, classification of, 842

neural basis for, 843

Conditioning, levels of, 842

theories of, 843

Conduction in nerve nets, 808
Conduction, non-nervous, 776

nervous, $777-808$

speed of, 782

of food, 147

body region for, 149

Contact stimuli, orientation to, 507

Contractile vacuole. See Vacuole.

Cooling, lethal effects, 343

Coordination, non-nervous, 776

Copepods, proteinases in, 161

Copper, blood, in various animals, 304

Corpuscles, pacinian, adaptation in, 511

Corpus allatum, 734

in grasshopper, fat metabolism and, 759

in insects, gonadotropic activity of, 754

Corpus luteum, development of, 747

progestin production by, 746

Corpus striatum, 835

Cortex, visual, 435. See also Cerebral cortex.

Corti, organ of, 475

Cothurnia, contrastile vacuole in, 44 osmoregulation in, 42

Crabs, blood concentrations of, $22,23,24$, 25

gills in, 214

kelp, oxygen consumption and body weight of, 228

motor innervation in, 597

osmoregulation in both hypertonic and

hypotonic media, 27

in either fresh or sea water, 28

respiratory quotient in, 277

rock, osmoregulation in, 24

shore, osmoregulation in, 26

volume changes in sea water, 21

Crago, color changes in, 695, 700

Crayfish, breathing movements in, 263

giant fibers in, 788

kidney of, 30

molting in, 738

osmoregulation in, 29

oxygen consumption in, 239

Creatine as muscle phosphagen, 615

excretion of, 189

Creatinine, excretion of, 189

Crista, 517

Cromofora, 677

Crop, funciions of, 147

Crustaceans, anaerobiosis in, 273

blood and oxygen capacity in, 304

blood pressure in, 541

blood protein in, 104

breathing movements in, 263

cardiac pacemaker in, 547

chemoreception in, 453

chromatophores in, 680, 693

digestive tract muscles in, 607 
Crustaceans, eye in, 388

fresh-water, 29

giant fibers in, 788

gills in, 214

ionic concentration in, 86

male hormone in, 752

marine, 29

molting in, 737

motor innervation in, 596

nervous regulation of hearts in, 558

nitrogen excretion in, 191, 192

osmoregulation in, 20-33

oxygen consumption in, 242

oxygen withdrawal by, 251

respiratory mechanisms in, 214

respiratory response in, 265

retinal pigment control in, 764

uricolytic enzymes in, 203

Crystal structure of hemoglobins, 105

Ctenophores, cholinergic systems in muscles in, 611

luminescence in, 662

statocyst of, 514

Cupula, 517

Curare, effect on muscle, 588

neuromuscular transmission and, 612

Cutaneous sensation, 509. See also Skin.

Cyanea, osmotic adjustment in, 14

Cyanide sensitivity in relation to oxygen consumption, 233

Cyclostomes, ions in body fluids in, 83

kidney in, 48

osmoregulation in, 51

photosensitive pigments in, 412

Cypridina, luciferin in, 668

Cy'sticercus tenuicollis, osmoregulation in, 18

Cystine, structural formula, 119

Cystophora, blood changes in during diving, 260

Cytochrome, 294

absorption spectrum of, 300

Daphnia, eggs of, osmoregulation in, 20

hemoglobin function in, 323

Dark adaptation, electrical measurement of, 426

in $M y a, 406$

kinetics of, 415

nerve impulse in, 432

Deaminases, distribution of, 201

Debt, oxygen, 274

Decibel, 472

7-Dehydrocholesterol, 132

Delay, synaptic, 799

Dendrocoelum, osmoregulation in, 35 photoreception in, 387

Depth perception, 401

Desert, water balance of mammals in, 60

Desiccation, prevention of in insects, 56
Desoxycorticosterone, as progestin, 746 effect on water and salt balance, 760

Deuteranopia, 437

Dcutoccrebrum, 818

Diabetes, effect of pituitary on, 758

Diencephalon, functions of, 834

Diet, restricted, 136. See also Nutrition.

Differentiation, growth and, hormones and, $726-740$

Diffusion constants of oxygen, 210

Diffusion lungs, 219

Digestion, body region for, 147 correlation with feeding habits, 178 extracellular, 150

fluids of, hydrogen ion concentration of, 15 I-155

stimulation of secretion, 155

hormones and, 762

intracellular, 150

mechanical factors in, 147

movement of food mass in, 149

of carbohydrates, 168-176

age and, 176

of fats, 176-178

of proteins, 157-168

site of, 150

Digestive tract. See Alimentary tract.

Dinitrophenol, stimulation of oxygen consumption by, 238

Diopter, definition, 395

Dipeptidases, 159

Dissociation curve, carbon dioxide, 329

Dissociation, oxygen, 306

Diurnal rhythms, color change and, 687

Diving, respiratory mechanisms in, 258

Dixippus, chromatophores in, 680 color changes in, 692 metamorphosis in, 734

Dogfish, nitrogen excretion in, 197 spinal, behavior in, 822,823

Dogs, olfactory sense in, 457 spinal reflexes in, 826 uric acid in, 205

Donnan equilibrium in ionic regulation, 90

Doris, osmotic adjustment in, 12, 13

Drosophila, gene hormones in, 729 variations in oxygen consumption in species of, 235

Drugs, effects on hearts, 559 effects on visceral muscles, 606

Duodenum, hormones of, 762

Dytiscus marginatus, retinal action potentials of, 427

EAR, external, 473

function of, 477

in fish, 484

inner, 474

middle, 473

morphology of, 473 
Earthworm, brain in, 817 calciferous glands in, 97 chemoreception in, 450 conditioned responses in, 844 embryonic behavior in, 827 epithelial sense organ of, 504 giant fibers in, 787

heart in, 545 hemoglobin function in, 321 nervous system in, 810 nitrogen excretion in, 197 osmoregulation in, 36

Echinochrome, 292, 293

Echinoderms, blood pressure in, 539, 542 cholinergic systems in muscles in, 611, 613

chromatophores in, 689 exopeptidases in, 167 ions in body fluids in, 82 light sensitivity in, 382 nitrogen excretion in, 193 osmotic adjustment in, 14 oxygen withdrawal by, 252 peripheral nervous system in, 809 $\mathrm{pH}$ of digestive organs, 152 protein in blood in, 104 proteinases of, 162 respiratory papulae in, 213 site of digestion in, 151 uricolytic enzymes in, 203

Echinus esculentus, body fluids in, 91

Echiurids, respiratory pigments in, 294

Ecology, comparative physiology and, 3

Eel, electric, 617, 619

electrocardiogram in, 553, 554

melanophores in, 710

osmoregulation in, 50

Eisenia, chemoreception in, 450 embryonic behavior in, 827

Elasmobranchs, body fluids in, 51 digestive secretion in, 157 ions in body fluids in, 83 kidney in, 48, 51 luminescence in, 665 nitrogen excretion in, 197 osmoregulation in, 50 spinal reflexes in, 823 temperature receptors in, 350

Electric organs, 617-621

Electrical activity of optic nerve, 428-435 of photoreceptors, 420 diurnal rhythms in, 427

Electrical potential. See Potential.

Electrical stimulation of heart muscle, 565

Electrocardiogram, 553

Electroencephalogram, 806, 807

Electrophoresis, protein separation by, 103 Electrophorus, electric organs of, 617, 619 Electroplaxes, 617

Electroretinogram, 422
Eledone, giant fibers in, 789

Elements essential for growth, 94 trace, 94

Embryos, behavior of, 827 hemoglobin in, 313, 320

oxygen consumption in, 230

Emplectonema kandai, luminescence in, 663

End-plate potential, 588

Endocrine glands, temperature regulation and, 369

Endocrine mechanisms, 725-775 general considerations, 767

Endocrine tissues, evolution of, 769

Endolymph, 475

Endoparasites, anaerobiosis in, 273 dietary factors of, 135

Endopeptidases, 158

Energy conversion, oxygen requirements and, 268

sources of, 112

Enterocrinin, 156

Enterogastrone, 763

Environment, organism and, 2

Enzymes, 150, 151 digestive, correlation with food habits, 178

in carbohydrate digestion, 168-176

lipolytic, 176

of protein degradation products, 201

proteolytic. See Proteases.

uricolytic, distribution of, 203

Ephemeridae, gills in, 215 oxygen consumption in, 239, 245

Ephestia, linoleic acid deficiency in, 133, 134

Epinephrine. See Adrenalin.

Equilenin, 745

Equilibrium, nervous control of, 831

Equilibrium orientation in vertebrates, 520

Equilibrium reception, 514-527

Equilibrium receptors, types of, 514

Equilin, 745

Eriocheir, blood chloride in, 88 osmoregulation in, 28 salts in body fluids in, 85

Erytlirinus, respiration in, 256, 257

Erythrocruorin. See Hemoglobin.

Erythrocytes, composition of, 294 numbers of, 293, 295 permeability of, 109 size of, 293, 295

Erythrommatin, 729

Erythrophores, 681 control of in fish, 711

Eserine, 558, 559 effect on nervous systems, 794 neuromuscular transnission and, 613

Esophagus, 147

Estivation, 371 
Estradiol, 745

structure of, 743

Estriol, 745

Estrogens, 745

Estrone, 745

Euglena, flagclla in, 642 growth in amino acids, 116 photoreceptor in, 383, 384, 385

Euryhaline, definition, 20

Excretion, chloride, in crayfish, 31 Huid, in Amoeba, 41 in Paramecium, 43 ionic, 92

nitrogen, 187-208

distribution of products, $190-200$

protein, conclusions on, 201

salts, in marine fish, 48

soxlium chloride, in Carcinus, 26

Exbalation, 254

Exopepticlases, 159

characteristics of, 166

distribution of, 165

Fxpiration, 254

Eyc, adaptation in, 397-405

to arrhythmic activity, 397

to day-time activity, 397

to media and substrate, 402

to night-time activity, 398

to photic quality, 404

to space and motion, 398

appositional, 388, 390

color in Drosopinila, 729

color changes and, 685

compound, in arthropods, 387

structure of, 387

in molluscs, 384

in vertebrates, 393-405

morphology of, 393

retina in, 395

movements, 401

pigment of, hormonal control of, 763

in compound eye, diurnal movements of, 388

superpositional, 388, 390. See also Photoreceptors.

Eyeshine, 398

Eyestalk, color changes and, 694

effect on molting in crustaceans, 738

retinal pigment hormone in, 764

Facilitation, delayed, 801

early, 801

neuromuscular, 594

synaptic, 800

Fasciola hepatica, osmoregulation in, 18

Fat, as dietary essential, 133

digestion of, 176-178

metabolism in grasshopper, hormones and, 759
Fat, oxygen consumption in utilization of, 237

synthesis from carbohydrate, 269

Fatty acids in diet, 133

Feathers in temperature regulation, 367

Feces, region of formation, 149

Feeding and digestion, 144-186

cilia in, 641

habits, correlation with digestive enzymes, 178

mechanisms, 144-147

Female hormones. See Estrogens.

Female reproductive cycle, endocrine inHuences on, 749

Fibers, nerve. See Nerve fibers.

Fibrimogen, scrum, molecular size, 105

Field, visual, 399

Firetly, 664

control of Hashing in, 672

Fish, acclimatization in, 348

ammonotelic, 197

anadromous, 49

blood pressure in, 537

carbon dioxide content of blood in, 330

catadromous, 49

chemoreception in, 456

chromatophores in, 685, 686, 706

color changes in, 715

conditioned responses in, 845

distribution of in relation to respiratory pigments, 315

eften. we mysuid hormone on, 759

electric, 617

erythrocytes in, 296

forebrain in, 835

fresh-water, osmoregulation in, 47

gas bladder in, 218

gills in, 216

heart in, 544

hemoglobin content in, 296, 315

hormones and growth in, 729

kidney in, 48

luminescence in, 665

marine, osmoregulation in, 48

nitrogen excretion in, 194, 197

osmoregulation in, $46-52$

in eggs of, 19

oxygen consumption of, 239

oxygen dissociation curves of, 315

oxygen tension in relation to $\mathrm{pH}$ in, 249

oxygen withdrawal by, 252

phonoreception in, 484-488

photosensitive pigments in, 412

respiratory characteristics of blood, 308

respiratory control in, 257

responses to hydrostatic pressure, 509

spinal reflexes in, 824

temperature receptors in, 350

ureotelic, 197 
Fish, uricolytic enzymes in, 204, 205 vibration sense in, 507 visual field in, 402

Flagella, 640. See also Cilia.

Flagellates, carbon requirements in, 114 cellulose digestion in, 172 photoreceptors in, 383 thiamine requirements in, 123

Flatworms, brain in, 816 cholinergic neuromuscular systems in, 614

conditioned responses in, 844

locomotion in, 816

nervous system in, 810

ocelli in, 384

osmoregulation in, 35

Flavone glycosides, 130

Flicker fusion frequency, 415 explanation of, 418,419

Flight, in insects, orientation during, 523 oxygen consumption during, 238

respiration during, 261 respiratory quotsent and, 276

Fluid(s), body, before and after dialysis against sea water, 90, 91

circulation of, 531-575 summary, 567

distribution of pigments in, 290-293 hydrogen ion concentration of, 80,81 in elasmobranchs, 51

in Pheretima posthuma, 37

ions in, 75

in relation to external media, 77

responses to alterations in medium, 84-90

respiratory functions of, 290-340

coelomic, protein concentration in, 104 digestive, hydrogen ion concentration of, 151-155

stimulation of secretion, 155 mechanisms for ingesting, 146

Fluid balance, maintenance of, endocrine influences, 760

Fly, olfactory pit in, 451

Folic acid, 128

Follicle-stimulating hormone, 741, 747, 750

Food, digestion of. Sce Digestion. inactive, swallowing mechanisms for, 146

movement in alimentary tract, 149

orientation to, 449

particulate, mechanisms for dealing with, 145

retention in alimentary tract, 149

soluble, absorption of, 144

vacuoles in protozoa, 150,155

Forebrain, functions of, 834

Fovea centralis, 396

Freezing, 343
Freezing point, measurement of, 7 sodium chloride and, 7

Frequency discrimination in man, 478

Frequency, flicker fusion, 415

Frogs, cerebellum in, 832

cutaneous vs. pulmonary respiration in, 212

digestive secretion in, 156

forebrain in, 836

heart in, electrical stimulation of, 566

kidney in, 54, 55

mechanoreception in, 510

muscle in, 578, 588

muscle contractions in, 593

muscle responses in, 596

optic nerve fibers in, 430

osmoregulation in, 52

phonoreception in, 488

respiratory control in, 257

temperature relations of, 351

FSH, 741, 747, 750

Fructosidase, $\beta$-, distribution of, 175

Fructosides, 169

Fundulus, color changes in, 707, 710 eggs of, osmoregulation in, 19

lateral line nerve responses in, 507 osmoregulation in, 49

xanthophores in, 711

Fur in temperature regulation, 367, 368

Galactogen, 748

Galactosidases, distribution of, 175

Galactosides, 169

Gammarus, blood chloride in, 87, 88 oxygen consumption in, 32

Ganglia, potentials in, 797

Gas bladder, 218

Gases, partial pressures in dry air, 210

Gastrin, 156, 762

Gastrointestinal hormones, 762

Gastrointestinal tract, muscles of, 605

Gastropods, blood and oxygen capacity in, 304

ganglionic reflexes in, 815

nitrogen excretion in, 198

oxygen transport in, 326

oxygen withdrawal by, 251

Geese, respiratory quotients of, 276

Gelation in amoebae, 636

Gel-sol ratio, amoeboid movement and, 636

Gene hormones, 729

Geotaxis, 515

negative, 516

positive, 515

Giant nerve fiber systems, 786

Gills, in crustaceans, 214

in marine fish, 49

respiration by, 213

Girella, temperature reactions in, 350

Globulin, serum, molecular size, 105 
Glomerulus in amphibia, 54 in marine fish, 48

Glucose, $\alpha$ and $\beta$, structure of, 168

Glucosidase, $\beta$, 173

Glucosidases, distribution of, 175

Glucosides, 169

Glutamic acid, structural formula, 119

Glycine, structural formula, 119

Glycogen as energy source, 269 digestion of, 169, 173

Glycolysis, 268 pathway of, 270

Glycosidases, distribution of, 174-176

Glycosides, classification of, 169

Goat, oxygen dissociation curves in, 320

Goldfish, acclimatization of, 347,348

oxygen consumption in, 241

spinal reflexes in, 824

Gonadotropin, chorionic, 741

Gonadotropins, 741

Gonads, hormones of, 742

Grasshopper, electrocardiogram of, 556

fat metabolism in, hormones and, 759

respiration in, 264

Gravity, orientation to, 515 receptors, 514

Gregarines, movement in, 638

Growth and differentiation, hormones and, 726-740

essential salts for, 94

hormones and, in vertebrates, 726

Gryllus, phonoreception in, 498

Guanine, excretion of, 189

Guanophores, control of, 711

Gunda ulvae, osmoregulation in, 18

Gustation. See Taste.

Habitat, oxygen consumption and, 239

Hair sensilla as phonoreceptors, 497

Halteres, 523

Hamsters, hibernation in, 372, 373

Hare, varying, color changes in, 766

Harmonics, 472

Hearing, place theory of, 479

range of, 478

See also Phonoreception.

Heart(s), ampullar, 545

chambered, 542

cholinergic mechanisms in regulation of, 546,548

cycle in, 543

direct responses of, $559-567$

effects of drugs on, 559

effects of salts on, 562

lymph, 551

muscle, effects of electrical stimulation on, 565

myogenic, 546

nervous regulation of, 557

neurogenic, 547
Heart, physiology of, 542-559

pulsating vessel type, 545

rates, 552

slowing of, in diving, 260

tubular, 544

types of, 542

Heat, adaptation to, 241

conductivity of water, 342

lethal effects of, 346

production of, 241

in homoiotherms, 370

receptors for, 364

Helicorubin, 294

absorption spectrum of, 300

Helix, electrocardiogram from, 555

muscle action potentials from, 590

osmoregulation in, 36

Helminths, parasitic, osmoregulation in, 17

Hemagglutinogens, 106

Hematin as dietary essential, 131

Heme, structural formula of, 297

Hemerythrin, 291, 293, 302, 303

absorption spectrum of, 300

oxygen transport by, 325

Hemicellulose, digestion of, 173

Hemochromogens, 294

Hemocoel, 531

Hemocyanin, 292, 293, 303, 304

absorption spectra of, 300

buffering capacity of, 333

dissociation rates of, 298

oxygen dissociation curves in Limulus, 328

oxygen transport by, 326

Hemoglobin, absorption spectra of, 297, 299

chemical composition, 294

content of blood, 295

crystal structure of, 105

dissociation rates of, 298

distribution of, 290,291

in diving mammals, 259

in embryos, 313,320

in invertebrates, 320

molecular size of, 298

oxygen dissociation curves of, 306

oxygen tension and, 248

oxygen transport by, 306-325

sedimentation rates of, 301

tissue, 292

Hemolysis, time required for, 109

Herbivores, digestive enzymes in, 179

Hering-Breuer reflex, 254, 255

Heterotherms, temperature relations in, 361

Heterotrophic organisms, 113

Hibernation, 371

Hippuric acid, excretion of, 189

Hirudinea, osmoregulation in, 36

Histidine, structural formula, 119 
Holocentrus, erythrophores in, 711

Holometabolous insects, 732

Holothuria, light sensitivity in, 382

Homarus vulgaris, body fluids in, 91

Homoiosmotic animals, 14-20 definition, 8

Homoiothermism, 240-244

Homoiotherms, acclimatization in, 348

body temperatures in, 362

development of, 363

lethal temperatures in, 362

temperature relations in, $361-370$

variations in, 361

Homostrophic reflex, 508

Honeybee, brain in, 817

Hormone(s), bird migration and, 766

carbohydrate metabolism and, 757

$\mathrm{Cn}+, 730$

color changes in crustaceans and, 698

coloration and seasonal color changes and, 765

developmental, in insects, 733

digestive, 156

effects on water and salt balance, 760

follicle-stimulating, 741

gastrointestinal coordination and, 762

GD, in insects, 733

gene, 729

general considerations, 767

general maintenance and metabolism and, $756-761$

gonadal, 742

growth and differentiation and, 726-740

in color changes in fish, 709

inhibitory, 733

lactogenic, 748

luteinizing, 741

molt-inhibiting, in crustaceans, 739

molting, 733

Palaemonetes lightening, 698

pigment-cell activities and, 763-766

pituitary, affecting color changes in amphibians, 704

pupation, 735

retinal pigment, 764

sex and reproduction and, 740-756

specificity in, 768

$\mathrm{V}+, .729$

Horseshoe crab. Sce Limulus.

Humidity, effect on color change, 684

effect on water balance in insects, 56, 57

receptors in insects, 57

sensitivity to in insects, 453

Hydra, osmoregulation in, 35

Hydrogen ion concentration, amoeboid movement and, 636

effect on ciliary activity, 649

effect on oxygen tension, 315

minimun oxygen tension and, 249

of body fluids, 80,81
Hydrogen ion concentration of digestive Aluids, 151-155

Hydrostatic pressure, responses to, 509

Hydroxyproline, structural formula, 119

Hymenoptera, body temperature in, 355

Hypermetropia, 395

Hypertensin, 761

Hypertensinogen, 761

Hypertonicity, definition, 6

Hypophysis. See Pituitary.

Hypothalamus, effect on reproductive cycle, 751

functions of, 834

Hypotonicity, definition, 6

Illumination, intensity of, and frequency of nerve impulse discharge, 432. See also Light.

Image, apposition, 390, 392

formation in compound eye, 389

mosaic, 391

superposition, 390, 392

Imido-proteinase, 159

Immunological specificity of proteins, 105

Incus, 473

Inhibition in nerve transmission, 803

Inorganic ions, 75-102

Inositol, 130

Insects, aquatic, osmoregulation in, 33

blood pressure in, 541

blood proteins in, 104

body temperature of, 353

chemoreception in, 450

behavior and, 452

chemoreceptors in, 451

cholesterol requirements in, 134

chromatophores in, 680, 691

digestive tract muscles in, 607

eye in, 387, 388, 390, 391

fat digestion in, 177

fying, orientation in, 523

ganglionic reflexes in, 814

gills in, 215

gonadotropic hormones in, 754

heart in, 551

heat absorption in, 356

hemimetabolous, 732

holometabolous, 732

humidity sensitivity in, 453

ionic concentration in, 77

leg movements in, 814

luminescence in, 663

metabolic heat in, 355

molt, pupation and metamorphosis in, 732

motor innervation in, 601

nitrogen excretion in, 194, 199

nitrogen requirements, 120

olfaction in, 461

osmoregulation in, 55 
Insects, oxygen consumption in, 233 during flight, 238

phonoreception in, 492-499

respiration in, 264

respiratory responses in, 266

restricted diets in, 136

temperature "sense" in, 357

thermoreception in, 358

tracheae in, 223

tymuancic ungans in, function, 495 morphology, 493

uricolytic enzymes in, 204, 205

vitamin requirements of, 125

wood, nutrition of, 172

Inspiration, 254

Insulin, effect on carbohydrate metabolism, 757

growth and, 727

Integument, as respiratory mechanism, 211. See also Skin.

Intensity of sound, discrimination of, 482

Intestines, respiration through, 218

lnvertase, 174

Invertebrates, anaerobiosis in, 273

blood pressure in, 540

buffering in, 33.3

carbon dioxide content of blood in, 330

cephalic dominance in, 816

chemoreception in, 448

ganglionic function in, 812

gonadotropic hormonal activity in, 754

hemoglobin function in, 320

hormones in, growth and 729

metabolism and, 759

secondary and accessory sex characters and, 752

ions in body fluids in, 78

marine, nitrogen excretion in, 191, 192

muscles in, 583

optic nerve discharge patterns in, 430

$\mathrm{pH}$ of digestive fluids in, 154

respiratory characteristics of blood of, 308

secretion of digestive fluids in, 157

serological relations among, 107

site of digestion in, 150

See also Annelids, Arthropods, Coelenterates, etc.

Iodine, permeability to, in crabs, 24,25

Iodopsin, 395, 397, 411

Ionic regulation, $80-84$

active absorption and, 91

excretion and, 92

mechanisms of, 90-94

physical factors in, 90

responses to alterations in medium, $84-90$

summary of, 97

Ions, inorganic, 75-102

balance of, 94 lons, inorganic, concentration in urine and plasma, 93

in sea water and body fluids, 78

in tissues and body fluids, 75

ratios of concentration, 82

See also specific substances.

Iridocytes, 681

lridosomes, 711

Iris cells, primary and secondary, 388

Iron content of hemoglobin, 296

Isoleucine, structural formula, 118

Isopods, oxygen consumption in, 239

respiratory mechanisms in, 215

water loss in, 57

Isotonicity, definition, 6

JeLLYFISH, muscle responses in, 595 osmotic adjustment in, 14

Johnston's organ, 509

KidNEX, amphibian, 54

effect on blood pressure, 761

in birds, 58

in crayfish, 30

in elasmobranchs, 51

in fresh-water fish, 47

in marine fish, 48

in molluscs, 36

in vertebrates, 48

Killifish, 49

Kinesis, 381

Klinokinesis, 387, 449

Klinotaxis, 383, 385, 449

Krause, bulbs of, 364

Krebs cycle, 188

Krogh's method of measurement of osmotic pressure, 8

Kynurenin, 730

LABYRINTH in vertebrates, 483

functions of, 516

osseous, 474

Labyrinthectomy, effects of, 520

Lactation, 748

Lactic acid tolerance in diving mammals, 260

Lactogens, 748

Lagena, 483

Lakes, salt, osmoregulation to life in, 32

Lateral line organs, 486, 507

Leander, giant fibers in, 788 gonadotropic hormone in, 754

Learning, 843. See also Conditioned responses.

Leech, chromatophores in, 688 osmoregulation in, 36

Leg movements in insects, 814

Lemaecocera branchialis, blood in, 22

Lepidoptera, developmental hormones in, 735 
Lepus, color changes in, 766

Leucine, structural tormula, 118

Leucophores, control of, 711

Leucylpeptidase, 167

LH, 741, 747, 750

Lichenin, digestion of, 173

Light, animal, intensity of, 666

physical characteristics of, 666

wave lengths of, 666

cells, 383

compass reaction, 393

effect on color change, 684

effect on reproductive cycles, 750

intensity, magnitude of retinal action potential and, 425

orientation to, 381

production of. See Bioluminescence.

protection from, color change and, 716

reaction, dorsal, 393

sensitivity to, 381-446

diffuse, 381

localized, 383

See also Photoreception.

Ligia, melanophore clanges in, 702, 703

Limnaea, osmoregulation in, 20, 36

Limnophilus, larvae of, 35

Limulus, dark adaptation in, 432

electrocardiogram of, 556

electroretinogram in, 422

heart in, 544, 547

electrical stimulation of, 566

optic nerve fibers in, $429,430,431$

optic nerve impulses in, 428

osmoregulation in, 26

oxygen transport in, 326, 327, 328

Linoleic acid deficiency in Ephestia, 133

Lipases, 176

Lipophores, 681

Littorina, reproductive cycle in, 752

Lizards, nitrogen excretion in, 200

Lobster, effect of acetylcholine on heart of, 560

Locomotion, amoeboid, 630, 632

ciliary, 640

in invertebrates, reflex control of, 812

in vertebrates, spinal reflex control of, 821-827

peripheral vs. central control of, 809

Locusta migratoria, color change in, 691

Loligo, giant fibers in, 789

oxygen consumption in, 233

Luciferase, 667

Luciferin, 667 absorption spectrum of, 669, 670

Lucilia sericata, haltere of, $\mathbf{5 2 4}$

Lumbricus, hemoglobin function in, 321, 322

light sensitivity in, 383

nervous system in, 811

osmoregulation in, 36,37
Lumbricus. See also Earthworm.

Luminescence, 660-676. See also Bioluminescence.

extracellular, 671

Lungfish, nitrogen excretion in, 197

Lungs, 217-222

diffusion, 219

in divıng mammals, 259

respiration by, 212

ventilation, 220

water-, 217

Luteinizing hormone, 741, 747, 750

Luteotropin, 750

Lyctidae, wood digestion in, 172

Lymph hearts, 551

Lysine, structural formula, 118

Magnesium ions, effects on hearts, 564, 565

in sea water and body fluids, 78

in tissues and body fluids, 76

ratios of concentration, 82

Maja, volume changes in sea water in, 21

Male hormones. See Androgens.

Male reproductive cycle, endocrine influences on, 749

Malleus, 473

Maltase, distribution of, 174

Mammals, acclimatization in, 348

blood of, reactions to carbon dioxide in, 331

body temperatures in, 362

cerebellum in, 832

chemoreception in, 457

conditioned responses in, 846

cortical function in, 837

digestive secretion in, 156

diving, duration of dives in, 258

embryonic, oxygen consumption in, 230

erythrocytes in, 295

fat digestion in, 178

hemoglobin content in, 295

hibernation in, 371

hormones and growth in, 726

kidney in, 48

lactation in, 748

lungs in, 220

marine, blood and urine concentrations in, 62

osmoregulation in, 60

neocortex in, 841

nerve conduction in, 784

nitrogen excretion in, 196

nitrogen requirements of, 121

osmoregulation in, 58-62

$\mathrm{pH}$ of digestive fluids in, 154

phonoreception in, 491

respiratory characteristics of blood of, 307 
Mammals, respiratory control in, 257 seasonal color change in, 766 skeletal muscle of, nerve endings in, 505 small, oxygen consumption of, 226, 227 spinal reflexes in, 826

temperature regulation in, physical mechanisms in, 366 urea excretion in, 198 uricolytic enzymes in, 204, 205

Man, amino acid requirements for, 121 carbon dioxide combining power in, 329,330

electrocardiograms in, 553

essential amino acids for, 117

eye in, 393

olfaction in, 454, 459

phonoreception in, $473-483$

range, 478

requirement of linoleic acid in, 134

spinal reflexes in, 826

taste sense in, 455

thermoreception in, 364

See also Mammals.

Mantis, praying, brain in, 820

Mating, color change and, 716

luminescence and, 673

Mechanoreception, 502-514

Mechanoreceptors, adaptation of, 510 histological types, 503

Medulla oblongata, functions of, 830

Melanin as retinal pigment, 764

Melanophores, 681. See also Chromatophores.

in fish, 706

in reptiles, 712

Melanotus, oxygen consumption in, 243

Membrane, basilar, 475

cell, electrical phases of, 778

permeability in Protozoa, 39. See also Permeability.

Reissner's, 475

tympanic, 473

Mesidotea, blood chloride in, 86, 87

osmoregulation in, 26

Mesotrophic organisms, 113

Metabolism, adaptations in, 268-277

and respiration, 209-289

basal, in arthropods, hormones and, 759

in temperature regulation, 369

intermediary, hormones and, 756

See also Oxygen consumption.

Metachronism of cilia, 646

Metamorphosis in amphibians, hormones and, 728

in insects, 732

Metatrophic organisms, 113

Methemoglobin, absorption spectra of, 298, 299

Methionine, structural formula, 119

Microphonic effect of cochlea, 477
Midbrain, functions of, 833

Migration, 371

hormones and, 766

Modiolus, 475

Molds, thiamine requirements in, 123

Molgula, osmotic adjustment in, 14

Molluscs, anaerobiusis in, 273

blood pressure in, 539

blood protein in, 104

chemorcception in, +49

cholinergic systems in muscles in, 610

conditioned responses in, 844

exopeptidases in, 166

fat digestion in, 177

fresh-water osmoregulation in, 35

function of shell in, 97

ganglionic reflexes in, 815

giant fibers in, 788

heart in, 544, 546

ions in body fluids in, 82

light sensitivity in, 383

luminescence in, 664

nerve conduction in, 784, 785

nervous regulation of heart in, 558

nervous system in, 812

neuromuscular transmission in, 613

nitrogen excretion in, 191, 193

osmotic adjustment in, 12

oxygen transport in, 326

$\mathrm{pH}$ of digestive organs, 152

photoreception in, 383, 384

proteinases of, 160

respiratory control in, 263

respiratory mechanisms in, 214

respiratory responses in, 265

site of digestion in, 151

uricolytic enzymes in, 203, 204, 205

Molpadin, 293

absorption spectrum of, 300

Molt in crustaceans, 737

in insects, 732

seasonal, hormonal control, 765

Monas, flagellum in, 644, 645

Mosaic image, 391

Mosquito, larvae of, ionic concentration in, 88

osmoregulation in, 33

Mouse, oxygen consumption of, 227, 248

Mouth, 147

Movement, amoeboid, 630-639. See also Amoeboid movement.

ciliary, 640-653

characteristics of, 643

theories of, 650

in gregarines, 638

perception of, 402

Mucus, as feeding mechanism, 145

Muscarine, 561

Muscle(s), 576-629

cholinergic systems in, 608 
Muscle(s), classification of, 576 conduction in, 584, 587 contraction time, 584, 586 electron microscopy of, 577 fast and slow responses in, 597 fibers, types of, 581, 582

frequency of stimulation of, 592 gross functions of, 576

heart, effects of electrical stimulation on, 565

hemoglobin in, 292

histological types, 580

inhibition in action of, 805

innervation, multiple, 595 inhibition by, 597

iterative, 592

microscopic histology of, 578

non-iterative, 592

origin of cardiac beat in, 546

phosphagens of, distribution of, 615

propulsion of food by, 149

red, 582

relaxation rates of, $58+, 586,601$

repetitive excitation in, 592

rhythmic activity in, 605

sense receptors, adaptation in, 512

smooth, conduction in, 590

striations in, 578

summary, 621

time constants of, 584, 586

time relations of, 577

histological correlations, 577

tonus of, 601

transmission of impulse to, chemical agents in, 608-614.

use of in feeding, 145

visceral, effect of drugs on, 606

viscosity of, 602

white, 582

Mussel, electrocardiogiam from, 555. See also Mytilus.

$M y a$, motor innervation in, 601 photoreception in, 406

Myelination of nerve fibers, speed of conduction and, 785

Myoglobin, 292 absorption spectra of, 298

Myopia, 395

Myosin, 578

Myoxocephalus, chloride excretion in, 94

Mytilus, blood chloride in, 88

cilium in, 644

muscle relaxation in, 603

osmotic adjustment in, 12, 13

Naphthoguinone, methyl phytyl, 132

Neanthes virens, osmotic regulation in, 17

Necturus, kidney in, 54

Nematocyte, 656

Nematocysts, 655-658
Nemertea, oxygen supply of, 211

Neocortex, 835

Neoechinorhy'nchus inydis, osmoregulation in, 18

Nephridia in earthworm, 37

Nephron, vertebrate, schematic representation, 48

Nereis, brain in, 817 osmoregulation in, $14,15,16,17,18$ peristaltic locomotion in, 813

Nereis cultrifera, osmotic adjustment in, 12

Nereis diversicolor, hemoglobin function in, 322

Nereis pelagica, osmotic adjustment in, 12

Nerophis, osmoregulation in embryo of, 19

Nerve(s), control of respiration by, 254 control of thermostimulation by, 365 motor, muscular conduction and, 587 optic. See Optic nerve.

regulation of hearts by, 557

stimulation of secretion of fluids by, 156

transmission of hearing impulse by, 480

transmission to muscle, chemical agents in, 608,614

Nerve action potentials, ionic basis of, 780

Nerve centers, "spontaneous" activity of, 805

asynchronous, 806

synchronous, 807

Nerve fibers, diameter of, speed and, 782 electrical phases in, 778

giant, 786

length of, speed and, 782

myelotropic, 785

nodes in, 786

sheath of, speed and, 784, 785

speed of conduction in, 782

Nerve impulse, auditory, 477

nature of, $777-781$

in photoreception, 428

Nerve nets, 808

Nervous conduction, interneuronic transmission in, 789

Nervous systems, 776-862

conduction in, 777-808

speed of, 782

development of, 848

endocrine activity' of, 768

peripheral vs. central, 809

persistent phenomena in, 841-847

rhythmic activity in, 806, 807

summary. of, 847

Nervous rissue, acetylcholine and cholinesterase content, 792

Nets, nerve, 808

Neuromuscular facilitation, 594

Neuromuscular junctions, 587

Neuromuscular transmission, chemical agents in, 608-614

Neurones, contiguity between, 789 
Neurones, sensory, 454, 455

soma of, responses in, 798

transmission between, 789

polarity in, 790

Neurosecretory cells, 768

Niacin, requirements of, 126

Nicotine, action of on heart, 561

Nicotinic acid. See Niacin.

Nitrogen, content of, in relation to metabolic rate, 229

excretion of, 187-208

distribution of products, $190-200$

protein, conclusions on, 201

requirements of, $115-122$

sources of, 113

Nitrogenous products, chemical nature of, 187-190

Noctiluca, 10

bioluminescence in, 661

Nocturnal activity, eye adaptation to, 398

Nucleic acid metabolism, products of, 189, 203

Nutrition, 112-143

oxygen consumption and, 236

specific factors in, 122-136

summary of, 136

unknown factors in, 135

Nymphs, metamorphosis of, 732

Nystagmus, 522

Ocean, salinity of, 9. See also Sea water.

Ocelli in arthropods, 391

in flagellates, 383

Octopine, 617

Octopus, electrocardiogram in, 554

Oculomotor nucleus, facilitation in, 801

Odonata, gills in, 215

Odor, chemical structure and, 466

classification of, 460

receptors for. See Olfaction and Chemoreceptors.

Olfaction, receptors for, 451, 453, 459

activation of, 465

in insects, 450

sensitivity of, 457

thresholds of, 458

See also Chemoreception.

Oligochaeta, osmoregulation in, 36

Onmatidia, 763, 764

in compound eye, 387, 389, 390

Onchidium, osmotic adjustment in, 12, 13

Opalinidae, water entrance in, 43

Ophiuroidea, luminescence in, 664

Ophryoscolecidae, water intake in, 44

Opossum, body temperature in, 363, 364

Optic nerve activity, retinal activity and, 433

discharge, measurement of visual functions by, 431
Optic nerve, electrical activity of, 428-435

Orange, transient, 411

Organ of Corti, 475

Orientation, equilibrium, in vertebrates, 520 in Aying insects, 523

photorcceptors and, 381-405

in arthropods, 393

to gravity, 515

Ornithine cycle, 188 phylogeny of, 201

Ornithorhynchus, motor cortex in, 837

Ornithuric acid, excrction of, 189

Orthokinesis, $4+49$

Orthoptera, phonoreception in, 498, 499

Osmoregulation, 8, 14-20

by exclusion of water, 19

by storage of water, 18

in Crustacea, 20-33

in fish, 46-52

in land arthropods, 55

in reptiles, birds, and mammals, 58-62

simple types, 14

summary of conclusions, $62-66$

Osmotic behavior, nitrogen excretion in relation to, 201

Osmotic characteristics of various animals, 63

Osmotic concentration, adjustment to mcdium, 10-14

with volume regulation, 13

without volume regulation, 10

of cytoplasm of Protoza, 39

regulation to medium, 14-20

water content of tissues and, 8

Osmotic pressure, definition, 6 methods of measuring, 8

Osmotic regulation. See Osmoregulation.

Ossicles, Weberian, 486

Ostariophysina, phonoreception in, 486

Osteichthyes, respiratory responses in, 266

Ovary, androgen production by, 744

effect of gonadotropins on, 741

estrogen production by, 745

Ovulation, gonadotropins and, 741

Oxidation, sources of, 268

Oxygen, availability of, effects of, 209

capacity, 306

altitude and, 316

consumption, activity and, 237

age and, 228

ciliary activity and, 651

dependence on oxygen tension, 244

determination of, 224

habitat and, 239

in Gammarus, 32

in hibernation, 372

levels of, 223-244

nutrition and, 236

relation to oxygen tension, $244-254$ 
Oxygen consumption, season and, 240

sex and, 235

size and, 225

species and, 235

temperature and, 240

debt, 274

deficiency. See Anaerobiosis.

diffusion as means of supply of, 211

diffusion constants of, 210

dissociation curve, 306

altitude and, 313

effect of carbon dioxide on, 311, $313,314,315$

effect of temperature changes on, 312

in embryos, 313,320

in relation to distribution of amphibians and fishes, 314

rates, 297, 298

exchange in vertebrates, 212 . See also

Respiration.

lack, adaptation to, 252

requirements of, energy conversion and, 268

saturation, critical oxygen tension and, 250

solubility in biological fluids, 210

storage in diving mammals, 259

supply, low, adaptation to, 252

tension, blood pigments and, 247

critical, 246

size and age and, 250

tissue oxygen saturation and, 250

for half saturation, effect of $\mathrm{CO}_{2}$ on, 315

minimum, $\mathrm{pH}$ and, 249

relation to oxygen consumption, 244254

respiratory quotient and, 275

temperature and, 248, 317, 318, 319

transport by blood pigments, 306-329

by hemoglobin, 306

hemerythrins in, 325

in invertebrates, 320

utilization in anaerobiosis, 272

want; respiratory responses to, 265

withdrawal, 250

Oxyhemoglobin, absorption spectra of, 298, 299

Oyster, respiratory control in, 263

Pacemakers, cardiac, 545

Pacinian corpuscles, adaptation in, 511

Pain impulses, 513

Palaemonetes, chromatophores in, 679 color changes in, 697, 698

Paleocortex, 835

Pancreozymin, 762

Panting, 367

Pantothenic acid, 127
Papillae, anal, in mosquito, 33, 34 tongue, in man, 455

Para-aminobenzoic acid, 130

Parallax, 401

Paramecium, chemoreception in, 448 cilium in, 642, 643

contractile vacuole in, 44

oxygen deficiency and, 253

trichocysts in, 655

vacuolar excretion of fluid in, 43

Paramyosin, 578

Parasites, intestinal, osmoregulation in, 17

Parathyroid glands, effect on calcium and phosphate balance, 760

Particles, food, large, mechanisms for dealing with, 146

small, mechanisms for dealing with, 145

Pattern vision, localized photoreceptors and, 387

Pecten, adductor muscle in, 604 optic nerve fibers in, 431

Pectin, digestion of, 173

Pelecypoda, oxygen withdrawal by, 251

Pellagra, 127

Pepsin, 158 distribution of, 165

Peptidase. See Exopeptidases.

Perception, depth and solidity, 401 movement, 402

Perch, swim bladder in, 218, 220

Perilymph, 475

Peripheral control of locomotion, 809

Peristalsis in annelids, 813

Periwinkle, reproductive cycle in, 752

Permeability, in Protozoa, 39 of erythrocytes, 109

to water and salts, in rock crabs, 24

Peritricha, fresh-water, water uptake in, 44

marine, osmoregulation in, 42

$\mathrm{pH}$. See Hydrogen ion concentration.

Phaeommatin, 729

Phascolosoma, osmotic adjustment in, 11 salt regulation in, 89

Phengodes, luminescence in, 664, 665

Phenylalanine, structural formula, 118

Pheretima posthuma, composition of body fluids, 37

Phonoreception, 471-501

in amphibia, 487

in arthropods, 492-499

in birds, 490

in fish, 484

in insects, 492

in mammals, 491

in man, 473-483

functional aspects, 477

in reptiles, 489

in vertebrates, 483 
Phonoreception, problems of, 473

transmission in nervous system, 480

Phosphagens, muscle, distribution of, 614

Phosphoarginine, distribution of, 615 structural formula, 616

Phosphocreatine, distribution of, 615 structural formula, 616

Phosphorolysis, 757

Photochemical effect, illuminating intensity and, 407

Photochemical nature of photoreception, 405

Photochemical reaction, reactions follow. ing, 408

of photoreception, 440

temperature coefficient, 406

Photoreception, 381-446. See also Vision. carotenoids in, 408

measurement of functions of, 414

nonphotochemical theories of, 418

photochemical nature of, 405

physiology of, 405-440

summary of, 439

Photoreceptors, electrical activity of, 420 localized, 383

function in orientation, 385

morphology, 383

pattern vision and, 387

See also Eye.

Photosensitivity. See Light sensitivity.

Phototrophic organisms, 113

Phototropism, 381

Phrixothrix, luminescence in, 664

Phrynosoma, color changes in, 713

Phylogeny, comparative physiology and, 3

Physiology, fields of, 1

Physostigmine. See Eserine.

Phytomonadida, photoreceptors in, 383

Piercing and sucking mechanisms, 147

Pieris brassicae, chromatophores in, 691

Pigeon, lungs and air sacs of, 221

Pigments, absorption spectra of, 299

as blood proteins, 334

blood, oxygen tension and, 247

carotenoid, in vision, 408

chemistry of, 294-306

dissociation rates of, 297, 298

distribution of, 290, 293

in worms, 294

eye, in compound eye, diurnal movements of, 388

in Drosophila, 730

in chromatophores, 678, 680

in annelids, 688

in Dixippus, 692

light sensitivity and, 382

molecular weights of, 301

nitrogenous, excretion of, 190

oxygen transport by, 306-329

photosensitive, chemical evolution of, 412
Pigments, retinal, hormonal control of, 763 sedimentation rates of, 301

Pilocarpine, action of, 561

Pinna, 473

Pinnaglobin, 292, 293

Pituitary gland, active principle of, effect on amphibia, 53

anterior, adrenotropic principle, 758

bird migration and, 767

diabetogenic principle, 758

effect on carbohydrate metabolism, 758

gonadotropins in, 741

growth and, 726

thyrotropic principle, 758

control of color changes in reptiles, 712

control of reproductive cycles by, 748

effect on color changes in amphibians, 704

in fish, 709

posterior, effect on water balance, 761

Placenta, estrogen in, 745

progestin production by, 746

Placobdella parasitica, chromatophores in, 688

Planaria, dietary factors of, 135

osmoregulation in, 35

photoreception in, 386, 387

Planorbis, hemoglobin function in, 324

Plasma, concentration of ions in, 93

Plasmagel in amoeba, 632

Plasmalemma in anoeba, 632

Plasmalemma in amoeba, 632

Plastron, 223

Platyhelminths, oxygen supply of, 211

Platysamia, metamorphosis in, 735

PLH, 698

Plumage, hormonal control of, 765

Poikilosmotic animals, 10-14 definition, 8

Poikilothermism, 240-244

Poikilotherms, acclimatization in, 348

aquatic, body temperature of, 349 thermal reception and orientation in, 350

inhabiting dry air, 353

inhabiting moist air, body temperature of, 351

lethal temperatures of, 344

temperature relations in, 349-361

Polarity in synaptic transmission, 790

Polychaetes, gills in, 213

respiratory pigments in, 294

ventilation in, 262

Polypus, digestion of gelatin by, 164

Polysaccharides, 169

digestion of, 173

distribution of, 170 
Porifera, cholinergic systems in muscles in, 611

oxygen supply of, 211

oxygen withdrawal by, 251

See also Sponges.

Porphyropsin, phylogenetic origin of, $4 \mathrm{I} 2$

Porpoise, respiration in, 260

Posture, nervous control of, 831, 832

Potassium, effect on visceral muscles, 606

in synaptic transmission, 795

ions, effects on hearts, 562, 563

in sea water and body fluids, 78

in tissues and body fluids, 76

ratios of concentration, 82

nerve conduction and, 780

Potential, action, nerve, 777

end-plate, 588

ganglionic, 797

synaptic, 797

Praying mantis, brain in, 820

Precipitins, immune, 107

Pregnanediol, 747

Presbyopia, 395

Pressure, blood, measurement and regulation of, 534. See also Blood pressure. hydrostatic, effects on various gel systems, 635

responses to, 509

osmotic. See Osmotic pressure.

oxygen. See Oxygen tension.

partial, of gases in dry air, 210

Prey, mechanisms for seizing, 146

Primates, cortical lecalization in, 838

Prochordates, proteinases in, 162

Progesterone, structure of, 743

Progestins, 746

Prolactin, 748

Proline, structural formula, 119

Proprioceptors, 503

Protanopia, 437

Proteases, classification of, 157

Protein(s), blood, pigments as, 334 degradation of, compared with purine breakdown, 202

products, enzymes of, 201

types of, 187

digestion of, 157-168

electrophoretic separation of, 103

immunological specificity of, 105

in blood, 103, 104

size of, 105

in hemoglobin, 297

metabolism of, distribution of excretory products, $190-200$

oxygen consumption in utilization of, 237

sedimentation of, 105

specificity, 103-111

Proteinases, characteristics of, 160-163

distribution of, 163
Proteinases. See also Protease.

Prothrombin, vitamin $\mathrm{K}$ and, 133

Protocerebrum, 818

Protopterus annectens, lungs of, 219

Protozoa, adaptation to marine, fresh-water and parasitic life, 38-46

anaerobiosis in, 273

avenues of water entrance in, 43

bioluminescence in, 661

carbon and nitrogen requirements in, 113

carbon requirements in, 114

chemoreception in, 448

conditional responses in, 843

contractile vacuoles of, 38

efficiency of, 46

function of, 40

mechanism of filling, 44

essential elements for, 94

exopeptidases in, 166

fat digestion in, 177

membrane permeability in, 39

nitrogen excretion in, 191, 192

osmotic adjustment in, 10

osmotic concentration of cytoplasm of, 39

oxygen supply of, 211

$\mathrm{pH}$ of digestive organs, 152

$\mathrm{pH}$ of food vacuoles in, 155

photoreceptors in, 383

proteinases of, 160

site of digestion in, 150

thiamine requirements, 123

trichocysts in, 654

Pseudocoelom, 531

Pseudopodia, as feeding mechanism, 145 types of, 630, 631

Psychic stimuli, effect on color change, 684

Pterines, excretion of, 190

Pteroylglutamic acid, 128

Puffer fish, oxygen withdrawal by, 252, 253

respiration in, 261

Pugettia, oxygen consumption and body weight of, 228

Pupation in insects, 735

Purines, breakdown of, 190

excretion of in various animals, 192-196 in diet, 130

inetabolism of, compared with protein breakdown, 202

distribution of enzymes and products of, 203

evolutionary trends in, 205

products, excretion of, 189

Purkinje tissue, 546

Pyridoxal, 127

Pyridoxamine, 127

Pyridoxine, 127 
Pyrimidines, excretion of, 190 in diet, 130

requirements for, 123

Pyrophorus, luminescence in, 664

Pyrosoma, luminescence in, 665

Pyruvic acid, oxidation of, 269, 271

$Q_{10}, 3+2$

Quotient, respiratory, 274

Radiation, absorption of heat from, 356

Raja clavata, equilibrium reception in, 517

Rana. See also Frogs.

Rana fusca, cutaneous vs. pulmonary respiration in, 212

Rana esculenta, cutaneous vs. pulmonary respiration in, 212

Rana temporaria, respiration in, 213

Rat, essential amıno acids for, 117 muscular action potentials from, 591 oxygen consumption of, 227

phonoreception in, 491

Ratio, U/P, 697

Reception of food, 147

Reciprocity law, 406

determined from electrical measure ments, 425

in measurement of visual functions, 431

Red blood cells. See Erythrocytes.

Reflexes, conditioning of, 846

ganglionic, in invertebrates, 812

Refractory period in synaptic transmission, 800

Regulation, ionic, 80-84

mechanisms of, 90-94

responses to alterations in medium, 84-90

See also Ionic regulation.

osmotic, 8, 14-20. See also Osmoregula. tion.

Reissner's membrane, 475

Relaxation of muscle, 601

Relaxin, 748

Renin, 761

Rennin, 163

Reproduction, hormones and, 740-756

Reproductive cycles, control of, 748

factors influencing, 750

Reptiles, body temperature of, 358

erythrocytes in, 295

forebrain in, 836

hemoglobin content in, 295

kidney in, 48

melanophores in, 712

nitrogen excretion in, 195

osmoregulation in, 58

phonoreception in, 489
Reptiles, respiratory characteristics of blood of, 307

respiratory control in, 257

respiratory responses in, 266

uricolytic enzymes in, 204, 205

Respiration and metabolism, 209-289

sumnary of, 277

control of, in diving birds and manmals, 258

in molluscs, 263

cutaneous, 211

flight and, 261, 262

gills in, 213

in insects, 264

in polychaetes, 262

in Tubifex, 262

lungs in, 212, 217

nervous control of, 830

pulmonary, 212

regulation of, $254-268$

skin in, 211

temperature and, 261

tracheae in, 222

types of mechanisms for, 211-223

See also Oxygen consumption.

Respiratory center, organization of, 830

Respiratory quotient, 274

factors affecting, 275

Respirometers, 224

Response, conditioned, $8+2$

neural basis for, $8+3$

Retina, action potential of, 421 effect of temperature on, 423

activity of, optic nerve activity and, 423

electrical activity in, +20

in color vision, 438

in compound eyc, 387

pigment of, hormonal control, 763

vertebrate, structure of, 395

Retinene, 410

Rhabdom in compound eye, 387

Rheotaxis, 508

Rhodnins, developmental hormones in, 733 experimental parabiosis and telobiosis in, 734

Rhodopsin, 395, 397, 410, 411 phylogenetic origin of, 412

Rhythm, cardiac, 545 diurnal, color change and, 687 in nervous systems, 806,807 muscular, 605

Ribollavin, requirements of, 124

Rodents, water balance in, 59

Rondeletia, luminescence in, 662

Rotatoria, oxygen supply of, 211

RPH, 764

R.Q. See Respiratory quotient.

Ruffini organs, 367

Ruminants, cellulose digestion in, 172

Rutin, 130 
Sabella pavonia, oxygen consumption by, 322,326

Saccharase, 174

Sacculus, 475

Salamanders, gills in, 216

Salmon, blood concentration in, 49

Salt balance, maintenance of, endocrine influences, 760

Salt lakes, osmoregulation to life in, 32

Salt water. See Sea water.

Salts, effects on hearts, 562. See also Ions and Sodium.

Saturation, oxygen, 310 critical oxygen tension and, 250 effect of $\mathrm{CO}_{2}$ on, 315

Schiff-Sherrington phenomenon, 826

Schistocephalus, osmoregulation in, 18

Schistocerca gregaria, respiration during flight, 262

Scolopidium, 493, 494, 495

Scolytidae, wood digestion in, 172

Scraping mechanisms for feeding, 146

Sea water, carbon dioxide content, 330 concentration of ions in, 78

copper content of, 304

crabs in, 21

hydrogen ion concentration of, 80

fish in, osmoregulation of, 48

mammals in, osmoregulation in, 60

osmotic adjustment to, 10

salinity of, 9

Seal, osmoregulation in, 61, 62

respiratory mechanisms in, 259

Seasons, oxygen consumption and, 240

Secretin, 156, 762

Secretion of digestive fluids, stimulation of, 155

oxidative, in filling of contractile vacuole, 45

Sedimentation of proteins, 105

Seizure of prey, mechanisms for, 146

Semicircular canals, 517

Sensilla basiconica, 451

campaniform, 506

adaptation in, 512

coeloconica, 451

hair, as phonoreceptors, 497

placodea, 451

Sensitivity, spectral, electrical measurement of, 422

measurement of, 431

to light, 381-446. See also Photoreception.

Sepia, eye in, 384

giant fibers in, 789

Sergestes, luminescencc in, 662

Serine, structural formula, 119

Serological relationships of various animals, 105

Serum protein. Sce Blood protein.
Setae, as feeding mechanism, 146

Sex, hormones and, 740-756

oxygen consumption and, 235

Sheep, embryonic behavior in, 828

Shivering, 368

Shrew, oxygen consumption of, 226, 227

Shrimps, brine, osmoregulation in, 32 gonadotropic hormone in, 754

luminescence in, 662, 663

Sigura lugubris, osmoregulation in, 33

Silkworm, developmental hormone in, 735

Simocephalus, oxygen consumption in, 233

Sinus gland in crustaceans, as source of chromatophorotropins, 696

effect on molting, 739

gonadotropic hormone in, 754

retinal pigment hormone in, 764

Sipunculids, blood pressure in, 540, 542

cholinergic systems in muscles in, 612

ions in body fluids in, 82

nitrogen excretion in, 192

protein in body fluids in, 104

respiratory pigments in, 294

uricolytic enzymes in, 203

Sipunculus, osmotic adjustment in, 12 oxygen transport in, 325

Size, critical oxygen tension and, 250 oxygen consumption and, 225

Skate, equilibrium reception in, 517

Skin, as respiratory mechanism, 211 thermal receptors in, 364

Smell. See Odor and Olfaction.

Snail, digestive secretion in, 157 hemoglobin function in, 324 muscle tension in, 602 osmoregulation in, 36 uric acid in nephridium of, 198, 199

Snakes, nitrogen excretion in, 200 phonoreception in, 489

Sodium ions, effects on hearts, 562, 563 in sea water and body fluids, 78 in tissues and body fluids, 76 ratios of concentration, 82

Solation in amoebae, 636

Solidity perception, 401

Solubility of oxygen in biological fluids, 210

Solutions, physiological, for different species, 95

properties of, 7

Soma, neuronic, responses of, 798

Sound discrimination in insects, 496

intensity of, discrimination of, 482

localization of, 482

in insects, 497

waves, 771

See also Phonoreception.

Spawning, gonadotropins and, $7+2$

Species, oxygen consumption and, 235 
Specificity, enzyme, 170 protein, 103-111

immunological, 105

Spectacles, 404

Spectrum sensitivity, electrical measurement of, 422

measurement of visual function and, 431

Spiders, ocelli in, 391

phonoreception in, 499

Spinal cord, control of locomotion by, 823 organization of, 821

reflexes, conditioning of, 846

Spirocysts, 656

Spirostomum, 44

Sponges, chemoreception in, 449' conduction in, 777

fresh-water, osmoregulation in, 35

See also Porifera.

Spongilla, osmoregulation in, 35

Squid, muscle contraction in, 593

oxygen consumption in, 233

photosensitive pigment in, 410

Stapes, 473

Starch, digestion of, 169, 170, 173

Starfish, digestion of gelatin by, 164 peripheral nervous system in, 809

Statocyst, 512

Statolith, 514

Stemmata, 391

Stenohaline, definition, 20

Stereotaxis, 507

Sterols, in various animals, 134

Stigma in flagellates, 383

Stimulation, tactile, effect on color change, 684

therma!, 364

Stimulus, conditioned, 842

unconditioned, 842

Stomach, contents of, $\mathrm{pH}$ of, 154 hormones of, 762

Storage of food, 147

Streaming, protoplasmic, 630. See also Amoeboid movement.

Style, amylase in, 171

Submergence. See Diving.

Sucking mechanisms, 147

Sucrose, structure of, 168

Sugar utilization in flagellates, 114

Sugars. See Carbohydrates and under specific compounds.

Sulfate ions in sea water and body fluids, 78

in tissues and body fluids, 76

ratios of concentration, 82

Summation, muscular, 592

Surface tension, amoeboid movement and, 634

Sweating, 367

Swim bladder, 218
Sympathin, 537, 557

Synapses, 789

delay at, 799

facilitation in, 800

integrative, 847

mode of transmission in, 791

potential in, 797

refractory period at, 800

relay, 847

Synchronization of nerve activity, 807

Syncytium, giant fibers in, 787

TACTILE receptors, adaptation in, 510 types of, 503

sense, 509

stimulation, effect on color change, 684

Talorchestia, oxygen consumption in, 243

Tapeworms, dietary factors of, 135

Taste, classification of, 462

receptors for, 461,462

activation of, 467

sensitivity of, 458

See also Chemoreception thresholds of, 459

Taxis, 381

Taxonomy, stereochemical basis for, 109

Tectum, 833

Tegmentum, 833

Telencephalon, functions of, 834

Telotaxis, 393

Telphusa fluviatilis, osmoregulation in, 29

Temperature, adaptation to, 346

changes in, adaptation to, 240-244

effect on oxygen dissociation curve, 312

characteristics, 342

coefficient of photochemical reactions, 406

effect on ciliary activity, 649

effect on color thange, 684

effect on reproductive cycles, 751

effect on retinal action potential, 423

high, lethal effects of, 346

low, 343

metabolic response to, 369

metabolic aspects and perception, 341380

summary of, 373

oxygen consumption and, 240

oxygen tension and, $317,318,319$

oxygen tension and consumption and,

248

reception. See Thermoreception.

regulation of, chemical mechanisms of, 369

color change and, 716

development of in homoiotherms, 363

physical mechanisms of, 366

relations in poikilotherms, 349-361

respiration and, 261 
Temperature, respiratory quotient and, 276 "sense" in insects, 357

Tenebrio, linoleic acid synthesis in, 134 stereotropic orientation in, 508

Tension of saturation, 310 oxygen, 244-254. See also Oxygen tension.

receptors, adaptation in, 512 surface, amoeboid movement and, 634

Tentacles as feeding mechanism, 145

Termites, digestion in, 172

Testis, effect of gonadotropins on, 741 estrogen production by, 746 hormones of, 743

Testosterone, structure of, 743

Tetrahymena, dietary factors of, 135 essential amino acids for, 117 substances needed for growth, 120

Thalamus, functions of, 834

Thermoreception in aquatic poikilotherms, 350

in insects, 358

in $\operatorname{man}, 364$

in poikilotherms inhabiting moist air, 353

in reptiles, 360

Thermoregulation. See Temperature regulation.

Thiamine, requirements for, 122

Thiazole, requirements for, 123

Thigmotaxis, 507

Threonine, structural formula, 118

Thresholds, sensory, 458

Thyroid gland, effect on metabolism, 238, 758

growth and, 727

in amphibians, 728

Thyroxin, effect on amphibians, 728

Tissues, body, ions in, 75 soft, mechanisms for ingesting, 146

Tocopherol, 132

Tonus, muscle, 601

Torpedo, electric organs of, 617,619

Touch, receptors of, 503

adaptation in, 510

sense of, 509

Trace elements, 94

Tracheae, 222 respiratory control and, 267

Tracheole, 222

Transmission, interneuronic, 789 after-discharge in, 805 inhibition in, 803

synaptic, 791 acetylcholine and, 791 delay in, 799 electric current in, 796 ionic changes in, 795 refractory period in, 800

Transport in fluid-filled body spaces, 531
Transport, intracellular, 531

mechanisms, extra-organismic, 531 morphological types of, 531

Tricarboxylic acid cycle, 269, 271

Trichocysts, 654

Trichoptera, gills in, 215

Trichosurus, motor cortex in, 837

Tritocerebrum, 818

Trimethylamine oxide, excretion of, 189 in fish, 197

Tritanopia, 437

Trituration, 147

Tropism, 381

Tropotaxis, 386, 449

Trout, dietary factors of, 135 phonoreception in, 485

Trypsin, 158 distribution of, 164

Tryptophane, structural formula, 118

Tubifex, hemoglobin function in, 321, 322,323 respiration in, 262

Tufts, branchial, 213, 214

Tunicates, peripheral nervous system in, 810. See also Ascidians.

Tunicin, 305

Turtles, blood and urine concentrations in, 58

effect of acetylcholine on heart of, 560 nitrogen excretion in, 198

Tympanic membrane, 473

Tympanic organs in insects, function, 495 morphology of, 493

Tyramine, chromatophores in cephalopods and, 690

neuromuscular transmission and, 613

Tyrosine, structural formula, 119

UcA, color changes in, 695, 701

UDH, 698

$\mathrm{U} / \mathrm{P}$ ratio, 697

Urea, excretion of, 188, 189, 198

in fish, 197

in various animals, 192-196

production of, 201

Urease, distribution of, 203-205

Urechis, hemoglobin function in, 324, 325

Ureotelic animals, 198

Uric acid, excretion of, 188, 189, 199 in aquatic vs. land animals, 198 in various animals, 192-196

formation of, 189

in body fluids, 200

Uricase, distribution of, 203-205

Uricotelic animals, 199

Urine, concentration of ions in, 93 nitrogen in. See Nitrogen excretion and Excretion.

Urodeles, phonoreception in, 487 
Urodeles, serologic relationships in, 107, 108 Utriculus, 475,517

Vacuoles, contractile, in Protozoa, 38 efficiency of, 46

function of, 40 mechanism of filling, 44

food, in Protozoa, 150, 155

Vagus nerve, effect on gastric section, 156, 157

effect on heart, 557

Vahlkampfia calkensi, contractile vacuole in, 42

Valine, structural formula, 118

Vanadium chromogen, 292, 293

as blood pigment, 305

Vanadocytes, 305

Ventilation in polychaete worms, 262

Ventilation lungs, 220

Venus, calcium in, 97

effect of acetylcholine on heart of, 560 muscle in, 579

osmotic adjustment in, 13

Vertebrates, blood protein concentration in, 104

brain in, various types, 829

cardiac rhythm in, 546

chemoreception in, 453

behavior and, 456

cholinergic systems in muscles in, 609

chromatophores in, 681, 703

control of secretion in, 156

cutaneous respiration of, 212

equilibrium orientation in, 520

eye in, 393-405

fat digestion in, 177

gills in, 215

growth in, hormones and, 726

hormones and sex and reproduction and, 740

intermediary metabolism and hormones in, 756

ions in body fluids in, 80

kidney in, 48

labyrinth in, 516

locomoxion in, spinal reflex control of, 821-827

lower, carbon dioxide transport in, 332 marıne, ions in body fluids in, 83

$\mathrm{pH}$ of digestive organs, 153

phonoreception by, 483

respiratory movements in, 254

site of digestion in, 151

See also Amphibians, Mammals, etc.

Vestibular nuclei, 831

Vestibule, 475

Vibration sense, 506

Viscosity of muscle, 602, 604

Vision, acuity of, 417

explanation of, 418
Vision, aerial, 403

air- and water-, 403

acpuatic, 402

central mechanism of, 435

color, 436-440. See also Color vision.

dichromatic, 437

functions, electrical measurement of, 422

measurement of functions of, 414

by optic nerve discharge, 431

nonphotochemical theories of, 418

pattern, localized photoreceptors and, 387

peripheral mechanism of, 405

See also Eye and Photoreception.

Visual angle, 399

Visual field, 399

Visual purple. See Phodopsin.

Vitamin(s), defined, 122

fat-soluble, 131-135

requirements of insects, 125

water-soluble, 122

Vitamin A, 131

in vision, 410

Vitamin $B_{1}$. See Thiamine.

Vitamin $B_{2}$. See Riboflavin.

Vitamin $\mathrm{B}_{\mathrm{i}}, 127$

Vitamin $B_{12}, 129$

Vitamin C. See Ascorbic acid.

Vitamin D, 132

Vitamin E, 132

Vitamin K, 132

Vitamin $\mathrm{P}, 130$

Vitrification at low temperatures, 345

Volume, regulation of, in osmotic adjustment, 13

in Protozoa, 38

Whrm-BloOded animals. See Homoiotherms.

Wasp, respiration during flight, 262

Water, 6-74

avenues of entrance in Protozoa, 43

balance, maintenance of, 6 endocrine influences, 760

relation to nitrogen excretion, 201

brackish, Crustacea in, 24

content of tissucs, osmotic concentration and, 8

fresh, adaptation to, $33-38$

crustaceans in, 29

osmoregulation of fish in, 47

loss in insects, 56

in mammals, 59

in temperature regulation, 366

oxygen withdrawal from, 251

poikilotherms inhabiting, 349

salt. See Sea water.

thermal properties of, 341

vision in, 402 


\section{Water-lungs, 217}

Waves, sound, 471

Weberian ossicles, 486

Weight, oxygen consumption and, 226

Whale, blood and urine concentrations in, 62

Wood, digestion of by insects, 172

Woodchuck, hibernation in, 371, 372

Worms, parasitic, osmoregulation in, 17 polychaete, ventilation in, 262 respiratory pigments in, 294 uricolytic enzymes in, 203
Worms. See also Annelids.

Wren, temperature regulation in, 364, 365

$\mathrm{W}$-substance in amphibian color changes, 705

Xanthine oxidase, distribution of, 203

Xanthophores, 681 control of in fish, 711

Xenopus, color changes in, 704

Xylan, digestion of, 173

YARROw, respiration in, 256, 257 





13,

If

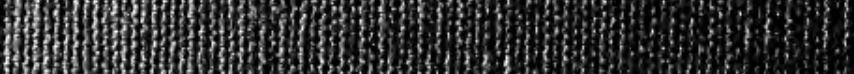

alo

S3

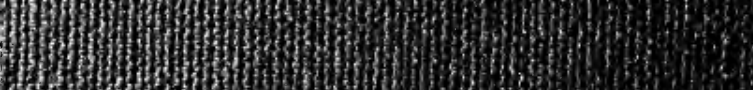

s.

13 13)

(6)

3.

If

3is

Hats

Hon

Ifos

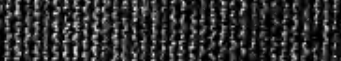

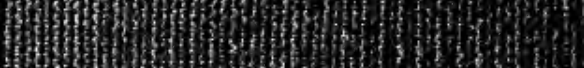

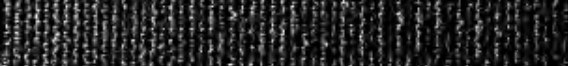

ats

In

4 (19)

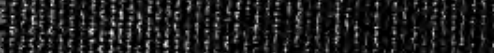

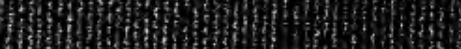

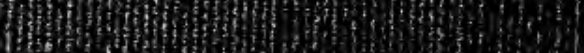

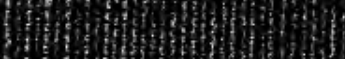

Hing

ation

Ely

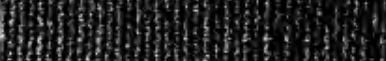

if

fof

1960)

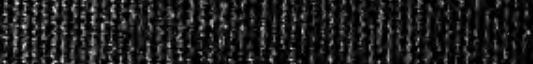

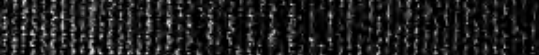

3 3)

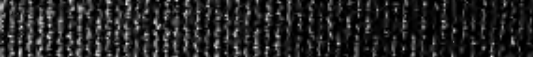

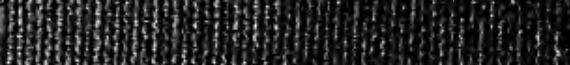

Hiflos

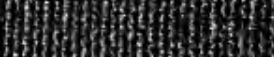

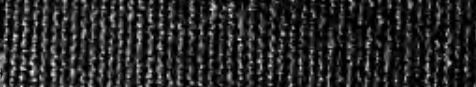

15)

Hols

Hens

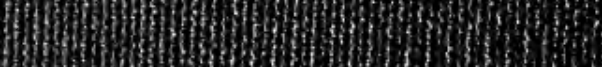

130 (3)

istos

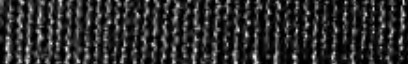

